\title{
STEREOCHEMICAL EFFECTS ON \\ INTERVALENCE CHARGE TRANSFER
}

\author{
Thesis submitted by \\ Deanna Michelle D’Alessandro B.Sc. (Hons) \\ September 2005
}

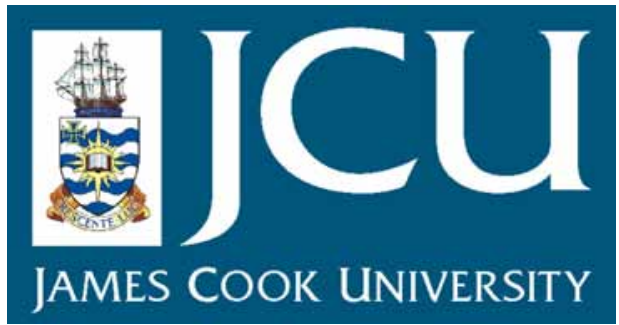

For the degree of Doctor of Philosophy

School of Pharmacy and Molecular Sciences

James Cook University, Townsville, Queensland, Australia 


\section{DECLARATION}

I declare that this thesis is my own work and has not been submitted in any form for another degree or diploma at any university or other institution of tertiary education. Information derived from the published or unpublished work of others has been acknowledged in the text and a list of references is provided.

Deanna M. D’Alessandro

September 2005 


\section{STATEMENT OF ACCESS}

I, the undersigned, author of this work, understand that James Cook University will make this thesis available for use within the University Library and via the Australian Digital Theses network, for use elsewhere.

I understand that, as an unpublished work, a thesis has significant protection under the Copyright Act and all users consulting this thesis will be required to sign the following statement:

"In consulting this thesis I agree not to copy or closely paraphrase it in whole or in part without the written consent of the author, and to make proper public written acknowledgment for any assistance which I have obtained from it."

Beyond this, I do not wish to place any restriction on access to this thesis.

Deanna M. D’Alessandro

September 2005 


\section{STATEMENT OF CONTRIBUTION OF OTHERS}

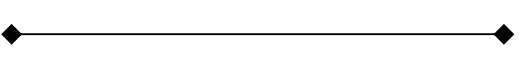

The work reported in this thesis was conducted under the supervision of Prof. Richard Keene, using the facilities in the Department of Chemistry at James Cook University.

Collaborations with Prof. Joe Hupp (Centre for Nanofabrication and Materials Science, Northwestern University, U.S.A.), Prof. Peter Steel (University of Canterbury, N.Z.), and Dr Jeff Reimers (University of Sydney) have been instrumental in providing intellectual and experimental support for this work. Electroabsorption (Stark effect) measurements were conducted by the candidate under the skilful guidance of Dr Peter Dinolfo and Dr Keith Walters during a three-month research visit to Prof. Hupp’s laboratory. Prof. Steel supplied a number of novel bridging ligands which have been employed in this work. The preliminary Density Functional Theory calculations reported in Chapter 2 were conducted by the candidate under Dr Reimers' expert guidance.

The collection and analysis of X-ray crystal structure data were performed by Dr Murray Davies (JCU) and A/Prof. Peter Junk (JCU and Monash University) in the Advanced Analytical Centre at JCU. Preliminary infrared spectroelectrochemical measurements were performed by the candidate in the laboratory of Dr Stephen Best (University of Melbourne). Dr Graham Heath (Research School of Chemistry, The Australian National University) provided custom-made spectroelectrochemical equipment. A number of complexes and ligands employed in the work were kindly supplied by other collaborators, and their contributions have been fully acknowledged where appropriate in the text.

This work was funded by an Australian Research Council (ARC-DP) grant to Prof. Keene. Travel to the laboratories of Dr Reimers and Dr Best was undertaken with the support of the ARC, and travel to Prof. Hupp's laboratory was jointly funded by a JCU Doctoral Research Scheme grant (to the candidate) and grant No. DE-FG02-8713808 from the Basic Energy Sciences Program, Office of Science, U.S. Department of Energy to Prof. Hupp. Financial support for the candidate was obtained from an Australian Postgraduate Award, and funding during the compilation and writing of this thesis was obtained from a JCU Ph.D. Completion Scholarship.

Deanna M. D’Alessandro

September 2005 


\section{ACKNOWLEDGMENTS}

"While the difficult takes time, the impossible just takes a little longer."

Art E. Berg

The finalisation of this thesis would not have been possible without contributions from a large number of people who have provided both scientific and personal support during my Ph.D.

I am sincerely grateful to my supervisor Prof. Richard Keene for his patience, understanding and for the generosity of his time and knowledge. I am indebted to Richard for instilling in me his passion for chemistry, and for allowing me the freedom to pursue a field of research that has challenged me both intellectually and experimentally. The scientific, professional and personal experiences which I have gained will remain with me far beyond my time at JCU. I am also grateful to Richard for facilitating interactions with our talented collaborators which has broadened the dimensions of this work.

To Richard's family - Cheryl, Nicola, Martin and Andrew - thank you for your kindness and hospitality, and for the many memorable evenings of Keene cuisine that I have been fortunate to have enjoyed over the years. Special thanks must also go to Nicola for dealing with my bottomless pile of references!

To all the members of the Keene group both past and present - Dr Brad Patterson for passing on his experimental expertise and Dr Joy Morgan, Jayden Smith, Dr Laurie Kelso, Dr Rob Gauci, Fiona Foley, Amy Topley, Caitriona Spillane and Dean Richards - thank you for providing a pleasant lab environment.

My sincere thanks also go to the academic, technical and administrative staff in the JCU Chemistry Department for their advice and expertise. I am particularly grateful to Dr Murray Davies and A/Prof. Peter Junk for their patience with the collection of crystallographic data, Dr Ken Adam for his advice on computational aspects of the work, and Dr Bruce Bowden for his assistance with NMR spectroscopy. In addition, thank you to A/Prof. Michael Ridd, A/Prof. George Meehan, Dr Brian McCool and Prof. David Yellowlees - the encouragement, support and understanding you have all provided throughout my time at JCU is very much appreciated. I am also grateful for the interactions with many fellow students within the department (especially Ronelle, Dave, Ces, Dianne and Madhavi), which have contributed to a warm and constructive working environment. My appreciation also goes to the technical and secretarial staff for their assistance and kindness over the years - thank you Sonia Dalla Pozza, Dr Sherryl Robertson, Dr Steve Gheller, Maree Hines, Sharryn Gleeson, Max Shaw, Dave Soper, John Sweet, Dr Moira McCann and Debbie Saunders.

I am grateful to our collaborators both within Australia and abroad who have provided intellectual insights and experimental skills which have enabled the depth of this project to evolve. I thank Prof. Peter Steel (University of Canterbury, N.Z.) and all the members of the Steel group (Dr Chris Sumby, Dr Jon Slater and Dr Chris Richardson) for designing and synthesising some of the novel 
bridging ligands used in this work. My sincere thanks to Prof. Joe Hupp (Northwestern University, USA) for inspiring some of the preliminary experiments and for many helpful discussions on theoretical aspects during the early stages of the project. My appreciation also to the members of the Hupp group for their hospitality during my visit to Northwestern, and for introducing me to the essentials of American lifestyle (especially the Chicago-style pizza!). I am especially grateful to Dr Peter Dinolfo and Dr Keith Walters for their time and assistance with the collection and analysis of the electroabsorption data, and to Dr Jerry Sando for his advice on spectral simulation techniques.

My appreciation goes to Dr Jeff Reimers and Prof. Noel Hush (University of Sydney) for many helpful and insightful discussions, and for their continued interest in this research. In particular, I thank Dr Reimers for his expert guidance on computational aspects, and for challenging me to think more deeply about theoretical issues which have been paramount to the methods employed for the spectral analyses. I am very grateful to both Dr Reimers and Prof. Hush for giving me the opportunity to present some aspects of this work at a recent symposium to honour the $80^{\text {th }}$ Birthday of Prof. Hush and his seminal contributions to the field of electron transfer research. My thanks to the members of the Reimers group, and to Jeff's family for their hospitality during my visits.

I am thankful to Dr Stephen Best (University of Melbourne) for providing access to his FT-IR instrument for some last-minute experiments, and to Dr Graham Heath (Resarch School of Chemistry, Australian National University) for his assistance with the UV/Vis/NIR instrumentation. I also acknowledge and thank Prof. Tom Meyer for a helpful discussion on the interpretation of some aspects of this work during the final stages of the writing of this thesis.

On a personal note, I am indebted to all my family and friends who have shared (and at times, endured!) this Ph.D. with me. I am eternally grateful to my parents for their never-ending love, motivation and understanding - thank you for always encouraging me to "have a go", and for instilling in me persistence and perseverance, without which this work would not have come to fruition. Thanks also for helping me to see the lighter side of "dielectric salvation effects in pilyporidyl complexes” whilst I was emersed in Chapter 2! A special thank you also to Craig, who has helped me to put things into perspective on numerous occasions - thank you for your friendship, kindness and frequent computerrelated advice. I am deeply grateful to you all for your faith and conviction in my abilities, and your resolution in seeing the finalisation of this thesis. 


\section{ABStract}

This thesis reports the first observation of stereochemical effects on intervalence charge transfer (IVCT) in di- and trinuclear mixed-valence complexes. The differential IVCT characteristics of the diastereoisomers of polypyridyl complexes of ruthenium and osmium offer a new and intimate probe of the fundamental factors that govern the extent of electronic delocalisation and the barrier to electron transfer. These findings challenge prior assertions that the inherent stereochemical identity of such complexes would have no influence on the intramolecular electron transfer properties of polymetallic assemblies. Chapter 1 addresses these issues within the context of the existing theoretical and experimental framework for IVCT.

Solvatochromism studies on the meso and rac diastereoisomers of $\left[\left\{\mathrm{Ru}(\mathrm{bpy})_{2}\right\}_{2}(\mu \text {-bpm })\right]^{5+}$ \{bpy = 2,2'-bipyridine; bpm = 2,2'-bipyrimidine $\}$ reported in Chapter 2, reveal striking differences between their IVCT characteristics due to stereochemically-directed specific solvent interactions. Such effects are inconsistent with dielectric continuum theories of solvation which are typically used to assess the contribution of the Franck-Condon outer-sphere reorganisational energy to the electron transfer barrier. Solvent proportion experiments demonstrate that the magnitude of the specific interaction is enhanced for the rac relative to the meso form, as the dimensionality of the "clefts" between the planes of the terminal polypyridyl ligands are ideally disposed to accommodate discrete solvent molecules. Subtle and systematic variations in the size and shape of the clefts through bridging ligand modification and the judicious positioning of alkyl substituents on the terminal ligands reveal that the magnitudes of the effects are dependent on the different cavity dimensions, and the number, size, orientation and location of solvent dipoles within the clefts.

Chapter 3 discusses stereochemically-directed solvent and anion interactions in systems of the type $\left[\left\{\mathrm{M}(\mathrm{bpy})_{2}\right\}_{2}(\mu-\mathrm{BL})\right]^{5+}\{\mathrm{M}=\mathrm{Ru}$, Os $\}$ where BL denotes an extensive series of $\mathrm{N}$-heterocyclic di- and tri-bidentate polypyridyl bridging ligands. NIR region electroabsorption (Stark effect) measurements of the mixed-valence complexes reveal small dipole moment changes for the IVCT transitions. In all cases, the effective charge transfer distances are negligible compared with the geometrical metal-metal separations, in support of a moderately- to strongly-delocalised assignment for the systems. This contrasts previous assertions in the literature which favoured a localised (“Class II”) classification for complexes of the genre.

IVCT solvatochromism and thermochromism studies on the mixed-valence species reveal that a subtle increase in the extent of inter-metal coupling with bridging ligand modification reduces the reorganisational barrier to electron transfer and leads to a transition between the localised ("Class II") and localised-to-delocalised (“Class II-III”) regimes. The importance of the bridging ligand in mediating the IVCT process necessitates a three-state theoretical analysis for the IVCT line-shape which explicitly includes the symmetric vibration mode. 
The comproportionation constants $\left(\mathrm{K}_{\mathrm{c}}\right)$ which are typically used to assess the degree of metalmetal coupling are markedly dependent on the electrolyte anion and the stereochemical identity of the complex. This emphasises the need for standard conditions for data from which analyses based on the magnitude of $\mathrm{K}_{\mathrm{c}}$ are made, and the danger of over-interpretation of the values. The differential anion interactions between the diastereoisomers are also manifested in their IVCT characteristics and represent a redox asymmetry contribution to the electron transfer barrier.

The magnitude of the differences between the IVCT characteristics of the diastereoisomeric forms of the same complex are more pronounced in the presence of inherent structural distortions in the bridging ligands, which are evident in their solid-state X-ray crystal structures. Such distortions decrease the extent of delocalisation through their redox asymmetry contribution to the electron transfer barrier. The interconfigurational (IC) transitions in the fully-oxidised forms of the dinuclear osmium complexes indicate that stereochemical effects modulate the energy levels of the metal-based $\mathrm{d} \pi$ orbitals themselves, which are split by spin-orbit coupling and ligand field asymmetry.

Chapter 4 extends the IVCT probe to stereochemically-pure trinuclear assemblies through a systematic investigation of the influence of the oxidation state, nuclearity and overall geometry of the systems on their intramolecular electron transfer processes. The IVCT properties of the dinuclear complexes $\left[\left\{\mathrm{Ru}(\mathrm{bpy})_{2}\right\}(\mu-\mathrm{HAT})\left\{\mathrm{M}(\mathrm{bpy})_{2}\right\}\right]^{5+}\{\mathrm{M}=\mathrm{Ru}, \mathrm{Os}\}$ and $\left[\left\{\mathrm{Ru}(\mathrm{bpy})_{2}\right\}_{2}(\mu \text {-ppz) }]^{5+}\right.$ are contrasted with their trinuclear analogues, which are "cluster-type" $\left[\left\{\mathrm{Ru}(\mathrm{bpy})_{2}\right\}_{2}\left\{\mathrm{M}\left(\mathrm{bpy} \mathrm{b}_{2}\right)\right\}(\mu-\mathrm{HAT})\right]^{n+}$ or "chainlike” $\left[\left\{\operatorname{Ru}(\mathrm{bpy})_{2}\right\}_{2}\left\{\mathrm{Ru}(\mathrm{bpy})(\mu-\mathrm{ppz})_{2}\right\}\right]^{n+}\{n=7,8 ; \mathrm{M}=\mathrm{Ru}, \mathrm{Os}$; BL = HAT $(1,4,5,8,9,12-$ hexaazatriphenylene) and ppz (4,7-phenanthrolino-5,6:5',6'-pyrazine) $\}$. While the diastereoisomers of the dinuclear complexes possess similar electrochemical and IVCT characteristics, the trinuclear "clustertype” system bridged by HAT exhibits significantly greater electronic coupling than the "chain-like" assembly based on ppz. The IVCT transitions in the singly-oxidised (+7) and doubly-oxidised (+8) trinuclear mixed-valence species are markedly different to those in their dinuclear analogues due to appreciable second-order interactions which depend on the overall geometry and oxidation state of the assemblies.

The observation of stereochemical effects on IVCT illustrates the subtle interplay of factors that govern the localised-to-delocalised transition, and addresses the limited experimental data which exist to probe the microscopic factors that facilitate this transition. The recognition of such effects on intramolecular electron transfer processes has significant implications for the elucidation of spatial influences on electron migration in biological systems such as metalloenzymes in nature. Ultimately, stereochemical modifications may be exploited in materials science applications to "fine-tune" the physical properties of novel molecular devices such as artificial photosynthetic systems for solar energy harvesting. 


\section{TABLE OF CONTENTS}

Declaration ..ii

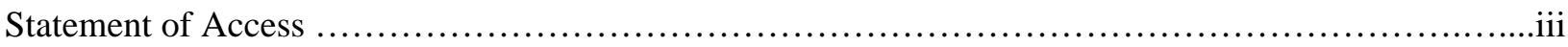

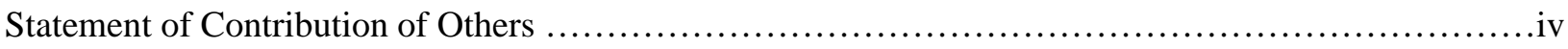

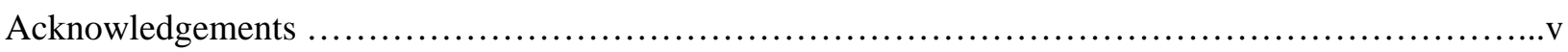

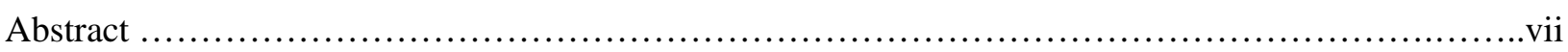

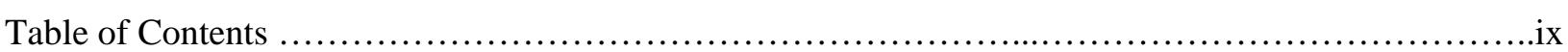

CHAPTER 1 INTRODUCTION

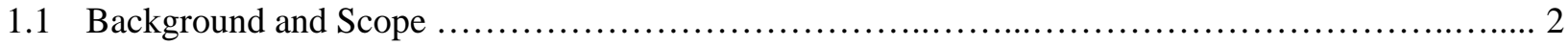

1.2 IVCT in Dinuclear Mixed-Valence Complexes ............................................. 4

1.2.1 Two-State Classical and Semi-Classical Theories of IVCT .............................. 4

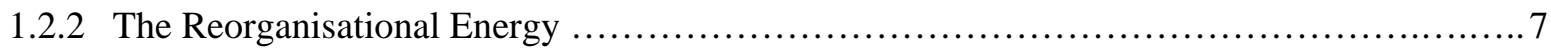

1.3 The Analysis of IVCT Transitions According to Two-State Classical and Semi-Classical Models... 8

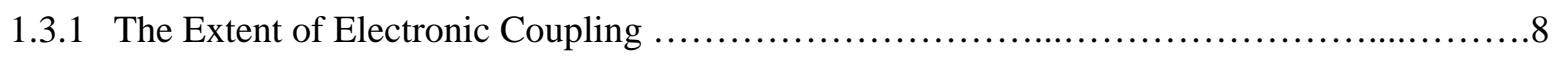

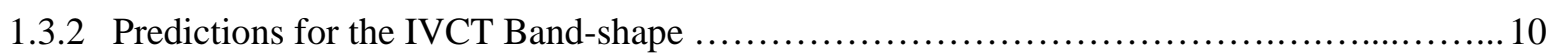

1.3.3 Classifying Mixed-Valence Systems between the Class II and Class III Regimes ............ 12

1.4 Theoretical Models for the Localised-to-Delocalised Transition in IVCT ........................13

1.4.1 Three- and Four-State Classical Models ..................................................... 13

1.4.2 Vibronic Coupling Models: the "PKS Model" .............................................. 15

1.4.3 Molecular Orbital Models: the "Ondrechen Model" ...................................... 16

1.4.4 Computational Approaches and Quantum Mechanical Methods ............................. 18

1.4.5 Towards a "Unifying Theory” for IVCT ............................................. 18

1.5 The Fundamental Factors Governing the Localised-to-Delocalised Transition ....................... 19

1.5.1 Dynamic Considerations and the "Class II-III” Classification .................................. 19

1.5.2 Experimental Studies of Mixed-Valence Systems in the Class II-III Regime ...............20

1.5.3 Probing the Factors which Govern the Localised-to-Delocalised Transition ................. 23

1.6 IVCT in Stereoisomeric Mixed-Valence Complexes ........................................ 31

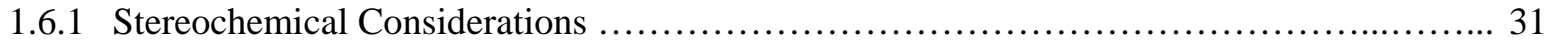

1.6.2 Solutions to the "Stereochemical Problem" ........................................ 33

1.6.3 Stereochemical Effects on Intramolecular Electron Transfer ................................ 34

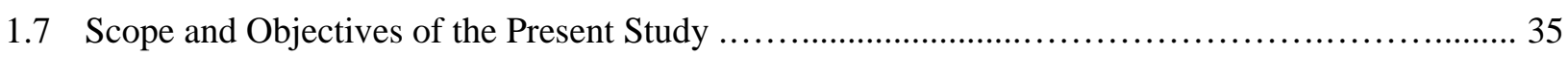

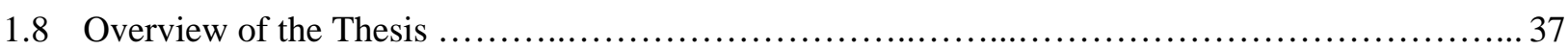

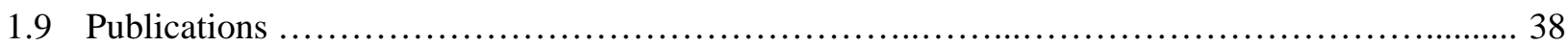

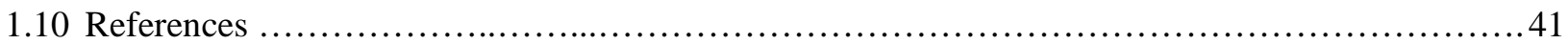




\section{Chapter 2 Diastereoisomers as Probes For Solvent} REORGANISATIONAL EFFECTS IN IVCT $\quad 46$

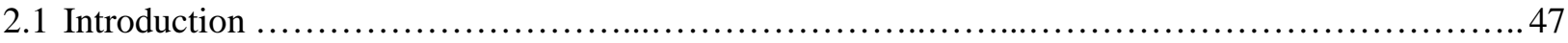

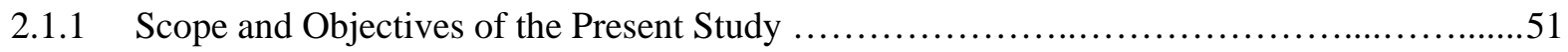

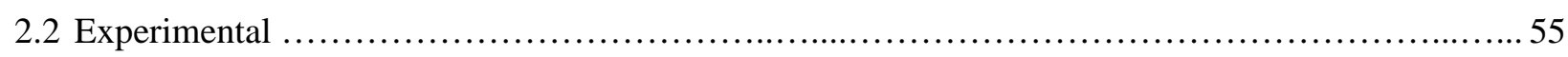

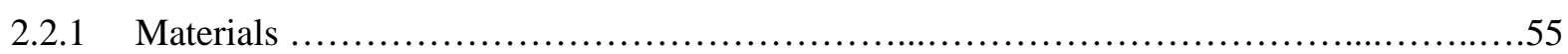

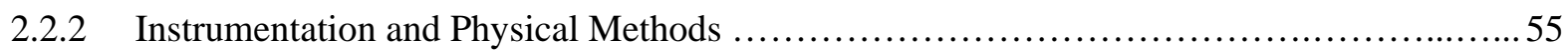

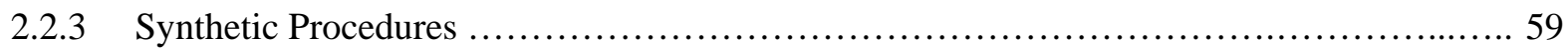

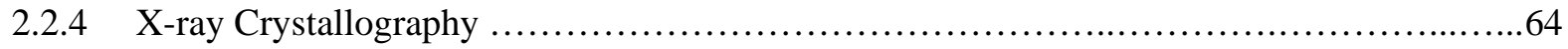

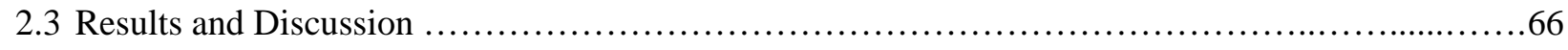

2.3.1 Diastereoisomer Synthesis, Separation and Structural Characterisation ..................... 66

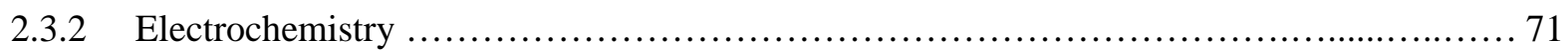

2.3.3 Electronic Spectroscopy and Spectroelectrochemistry ….............................. 73

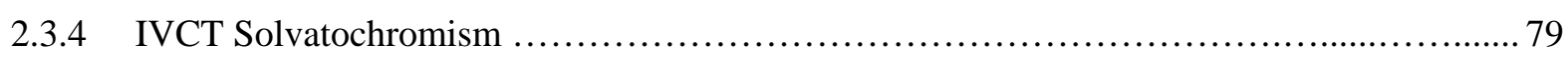

2.4 Conclusions and Future Prospects ........................................................ 96

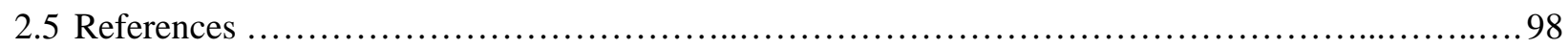

\section{ChAPTER 3 DiAstereoisomers as Probes For THE} LOCALISED-TO-DELOCALISED TRANSITION IN IVCT 101

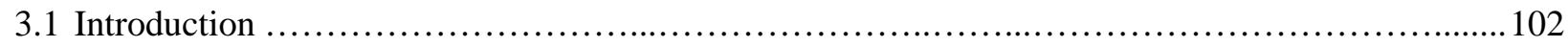

3.1.1 The Localised-to-Delocalised Transition in IVCT .................................. 103

3.1.2 Scope and Objectives of the Present Study .............................................105

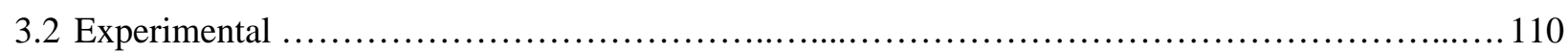

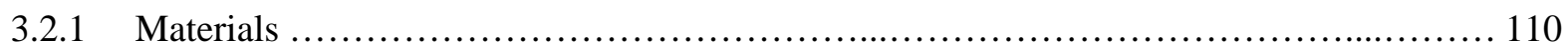

3.2.2 Instrumentation and Physical Methods ................................................ 111

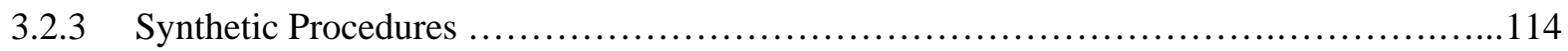

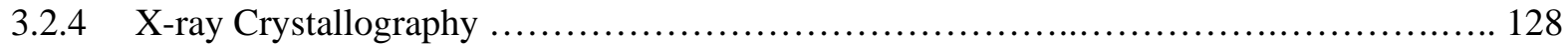

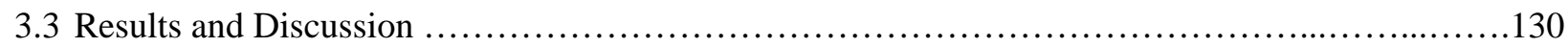

3.3.1 Diastereoisomer Synthesis, Separation and Structural Characterisation .................... 130

3.3.2 Diastereoisomers of Ruthenium Complexes Incorporating a Series of Angular

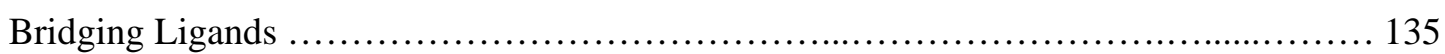

3.3.3 Two Examples of Complete Delocalisation ........................................ 150

3.3.4 Differential Redox Asymmetry Contributions in the Diastereoisomers of Unsymmetrical Mixed-Valence Complexes ............................................ 152

3.3.5 Dinuclear Osmium Complexes as Probes for Spin-Orbit Coupling Contributions ............ 158

3.3.6 The Effective Electron Transfer Distance: Stark Absorption Spectroscopy ...................... 169 
3.3.7 Diastereoisomers as Probes for Redox Asymmetry Contributions:

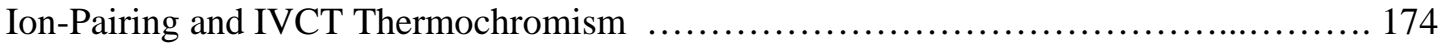

3.3.8 Diastereoisomers as Probes for Solvent Reorganisational Contributions ..................180

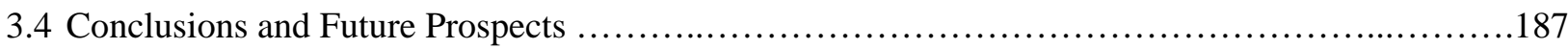

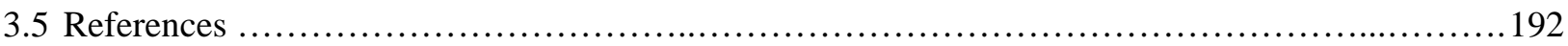

CHAPTER 4 EXTENDING THE IVCT PROBE TO TRINUCLEAR Polymetallic Assemblies 196

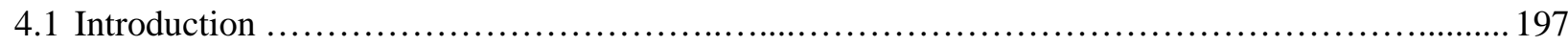

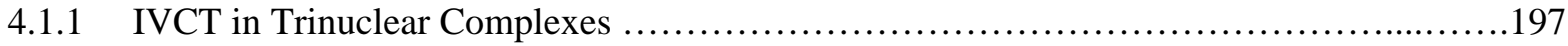

4.1.2 Trinuclear Polymetallic Assemblies: Molecular Architectures and Applications .............199

4.1.3 Stereochemical Ambiguities in Higher Nuclearity Polymetallic Assemblies .................200

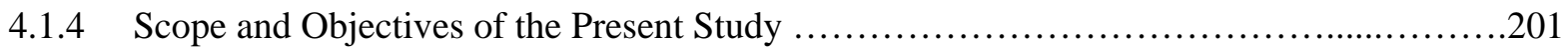

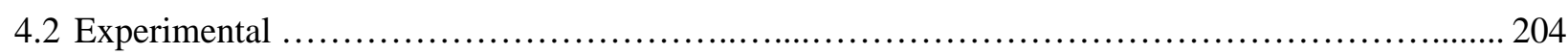

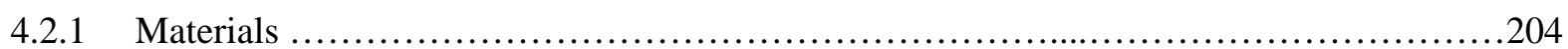

4.2.2 Instrumentation and Physical Methods ................................................204

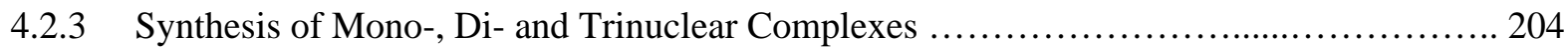

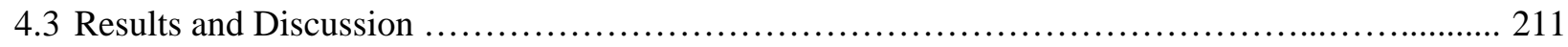

4.3.1 Homo-nuclear Ruthenium Complexes based on the Bridging Ligand HAT ................211

4.3.2 Homo- and Hetero-nuclear Osmium Complexes based on HAT …........................222

4.3.3 Mono-, Di- and Trinuclear Assemblies based on the Bridging Ligand ppz.................. 236

4.4 Conclusions and Future Prospects ....................................................... 250

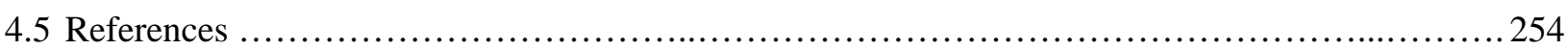

$\begin{array}{lr}\text { EPILOGUE } & 258\end{array}$

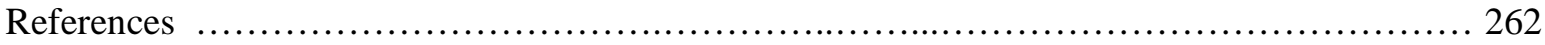

$\begin{array}{lr}\text { APPENDICES } & 263\end{array}$

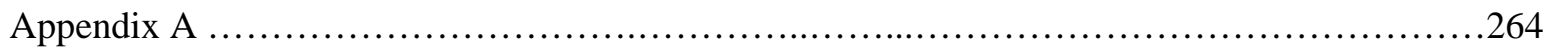

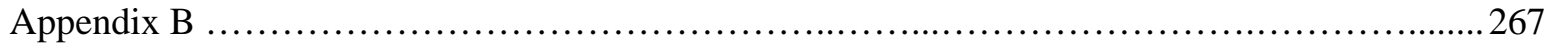

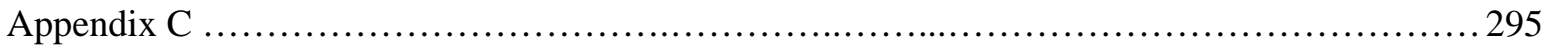

\section{ACCOMPANIMENT}

X-ray Crystallography Data and Publications 
ChAPTER 1 


\section{Chapter 1}

\section{INTRODUCTION}

\subsection{Background and Scope}

Electron transfer is ubiquitous in chemical, physical and biological systems. The phenomenon is fundamental in natural processes such as photosynthesis and in materials science applications such as light-activated devices and non-linear optical materials. The importance of electron transfer underlies the extensive multidisciplinary research efforts which have led to the discovery of mixed-valence complexes, a class of transition metal-based compounds which contain ions of the same element in different formal oxidation states. The significance of the mixed-valence phenomenon was elucidated in the early twentieth century with the realisation that the valences are in rapid oscillation between the metal centres (rather than being uniquely fixed, one to each ion), ${ }^{1,2}$ giving rise to the absorption of light in the visible region of the electromagnetic spectrum.

The first synthetic mixed-valence compound, "Prussian Blue", $\mathrm{Fe}^{\mathrm{II}} \mathrm{Fe}^{\mathrm{III}}(\mathrm{CN})_{6} \cdot x \mathrm{H}_{2} \mathrm{O}^{3,4}$ has been prized industrially for centuries as an ink and dye-stuff. This infinite three-dimensional cubic array of alternate $\mathrm{Fe}^{\mathrm{II}}$ and $\mathrm{Fe}^{\mathrm{III}}$ centres linked by bridging cyanide $\left(\mathrm{CN}^{-}\right)$groups ${ }^{*}$ exhibits an intense blue colouration which cannot be attributed to a combination of the individual absorption spectra of the constituent ions. $^{2}$ The mixed-valence nature of the species gives rise to an intervalence charge transfer ${ }^{\dagger}$ (IVCT) transition between the metal centres which corresponds to the absorption of light in the visible region of the spectrum.

The mixed-valence phenomenon has also been invoked to explain the extraordinary colours displayed by many transition-metal containing gemstones including sapphire and aquamarine, which incorporate $\mathrm{M}^{n}$ and $\mathrm{M}^{n+1}$ centres (e.g. $\mathrm{M}=\mathrm{Fe}^{\mathrm{II}}$, $\mathrm{Ti}^{\mathrm{III}}$ ). ${ }^{1,2,6,7}$ The spectral, electrical and magnetic properties of naturally-occurring and synthetic mixed-valence compounds are related to the additive properties of their constituent metal ions, and to cooperative effects which originate from electronic coupling between the metal centres across a bridging ligand. This interplay of effects endows the systems with profoundly new properties which are not indicative of their constituent metal ions.

The reaction centres in bacterial, algal and plant systems constitute elaborate examples of biological mixed-valence systems. In the natural photosynthetic process, the primary electron donor is the "special-pair”, $\mathrm{P}$ - a membrane-bound protein comprising two bacteriochlorophyll molecules. Photoexcitation of the reaction centre gives rise to a long-lived charge-separated state via a series of rapid electron transfer relay processes between the excited dimer $\mathrm{P}^{*}$ and neighbouring pigments. The resultant special-pair radical cation $\mathrm{P}^{+}$is a mixed-valence species. ${ }^{8}$ While several fundamental features have been elucidated regarding the kinetics, energetics and structural aspects of the electron transfer reactions in

\footnotetext{
${ }^{*}$ The three-dimensional X-ray crystal structure contains cyanide C-bonded to $\mathrm{Fe}^{\mathrm{II}}$ and $\mathrm{N}$-bonded to $\mathrm{Fe}^{\mathrm{III}}$.

${ }^{\dagger}$ The alternate terminology metal-to-metal charge transfer (MMCT) is also commonly employed.
} 
photosynthetic reaction centres, several questions remain unclear. ${ }^{9}$ Of pivotal importance is the elucidation of the factors that govern the efficiency of the primary charge separation process. These include the relative orientations and distances between the chromophores, protein residues and solvent molecules, and the degree of charge delocalisation. In addition, the understanding of the inherent stereochemical complexities has, to date, been limited. ${ }^{10,11}$ In photobiological systems, there is no ambiguity in the geometry of the natural assembly, as the building blocks are chiral amino acids or phosphate sugars which are organised in definitive orientations by an enzymic matrix.

The elegance and efficiency of the charge separation function in photosynthetic organisms has provided the inspiration for the design and synthesis of many ingenious model systems. ${ }^{12,13}$ These include covalently-linked porphyrin arrays, ${ }^{14-19}$ chromophore-quencher assemblies utilising transition metal complexes ${ }^{13,20,21}$ and polymetallic ligand-bridged assemblies. ${ }^{22-24}$ Polypyridyl complexes of the $d^{6}$ metals ruthenium(II) and osmium(II) have been extensively employed as the basis for such assemblies due to their unique combination of photochemical, photophysical and redox properties, including the stability of their ground and excited states and their chemical inertness in a variety of oxidation states. ${ }^{25}$

Dinuclear mixed-valence complexes of ruthenium and osmium provide ideal model systems for elucidating the fundamental factors that govern inter-molecular electron transfer reactions in natural and artificial systems. In the late 1960's, Allen and Hush ${ }^{1}$ and Robin and Day ${ }^{2}$ published seminal reviews of mixed-valence materials. Hush ${ }^{26}$ subsequently provided a theoretical model linking the physical properties of dinuclear mixed-valence complexes (i.e. the parameters of their IVCT absorption bands) to the activation barriers for electron transfer from Marcus theory. ${ }^{26,27}$ Of particular significance was the prediction of a relationship between the energy of the intervalence transition $\left(v_{\max }\right)$ and several factors which govern the activation barrier:

$$
v_{\max }=h v=\lambda_{i}+\lambda_{0}+\Delta \mathrm{E}_{0}+\Delta \mathrm{E}^{\prime}
$$

The Franck-Condon factors, $\lambda_{i}$ and $\lambda_{0}$, correspond to the reorganisational energies within the inner- and outer-sphere (respectively), the redox asymmetry, $\Delta \mathrm{E}_{0}$, is the thermodynamic energy difference between the two metal-based chromophores, and $\Delta \mathrm{E}^{\prime}$ reflects any additional energy contributions due to spin-orbit coupling and ligand field asymmetry. To date, these factors have been probed by varying the "global" features of dinuclear complexes such as the identity and coordination environments of their constituent metal centres, or through the introduction of redox asymmetry. The reorganisational energy contributions are typically assessed by varying the macroscopic features of the external environment such as the solvent, anions and temperature.

The present study represents the first investigation of IVCT in stereochemically-pure di- and trinuclear polypyridyl complexes of ruthenium and osmium, and addresses the limited experimental data available to probe the influence of the inherent stereochemical complexities in polymetallic assemblies on their physical properties. ${ }^{10,11}$ The subtle and systematic variations in the geometries of the stereoisomers provide a new experimental probe for the factors which govern the barriers to electron transfer at the 
molecular level. The extension of the IVCT probe to trinuclear systems provides the link between the understanding of dinuclear mixed-valence complexes, and higher nuclearity metallosupramolecular systems. This has important implications for the elucidation of spatial influences on electron migration in natural processes such as photosynthesis, and for exploiting stereochemical effects in artificial materials capable of performing useful light- and redox-induced functions.

\subsection{IVCT in Dinuclear Mixed-Valence Complexes}

\subsubsection{Two-State Classical and Semi-classical Theories of IVCT}

The IVCT transition in dinuclear mixed-valence species may be examined by considering a complex of the type $\left[\left\{M_{1}(L)_{n}\right\}(\mu-B L)\left\{M_{2}(L)_{n}\right\}\right]^{4+}$ \{where $M_{1}$ and $M_{2}$ represent the metal centres; $L$ and BL represent the terminal and bridging ligands respectively\}, hereafter abbreviated $\left[\mathrm{M}_{1}{ }^{\mathrm{II}} \mathrm{M}_{2}{ }^{\mathrm{II}}\right]$, where both metal centres are in the +2 oxidation state. The oxidation of one metal centre gives rise to the mixedvalence species $\left[\mathrm{M}_{1}{ }^{\mathrm{II}} \mathrm{M}_{2}{ }^{\mathrm{III}}\right]$, where the overall +5 charge corresponds to $\left[\mathrm{M}_{1}{ }^{\mathrm{II}} \mathrm{M}_{2}{ }^{\mathrm{III}}\right]$ in a fully localised description and $\left[\mathrm{M}_{1}{ }^{\mathrm{II} / 2} \mathrm{M}_{2}{ }^{\mathrm{II} 1 / 2}\right]$ in a fully delocalised description. ${ }^{26,27}$ A symmetrical dinuclear complex is typically described by the parabolic potential energy surfaces depicted in Figure 1.1 where the dimensionless reaction coordinate $(X)$ represents an anti-symmetric combination of the metal-ligand and solvent stretching vibrations. ${ }^{28}$ The dotted curves correspond to the wavefunctions $\Psi_{\mathrm{a}}$ and $\Psi_{\mathrm{b}}$ for the valence-localised "electronic isomers" $\left[\mathrm{M}_{1}{ }^{\mathrm{II}} \mathrm{M}_{2}{ }^{\mathrm{III}}\right]_{0}$ and $\left[\mathrm{M}_{1}{ }^{\mathrm{II}} \mathrm{M}_{2}{ }^{\mathrm{III}}\right]_{0}$, respectively, where the odd electron is completely localised on either metal centre. These "diabatic" states are centred at $X_{\min }=0$ and 1 on the reaction coordinate and are assumed to be harmonic with identical force constants. Their energies are given by $\mathrm{H}_{\mathrm{a}}$ and $\mathrm{H}_{\mathrm{b}}$ in equations 1.2(a) and (b), where $\hat{\mathrm{H}}$ is the effective two-state Hamiltonian operator and $\lambda$ is the reorganisational energy.

$$
\begin{aligned}
& \mathrm{H}_{\mathrm{a}}=\left\langle\Psi_{\mathrm{a}}|\hat{\mathrm{H}}| \Psi_{\mathrm{a}}\right\rangle=\lambda X^{2} \\
& \mathrm{H}_{\mathrm{b}}=\left\langle\Psi_{\mathrm{b}}|\hat{\mathrm{H}}| \Psi_{\mathrm{b}}\right\rangle=\lambda(X-1)^{2}
\end{aligned}
$$

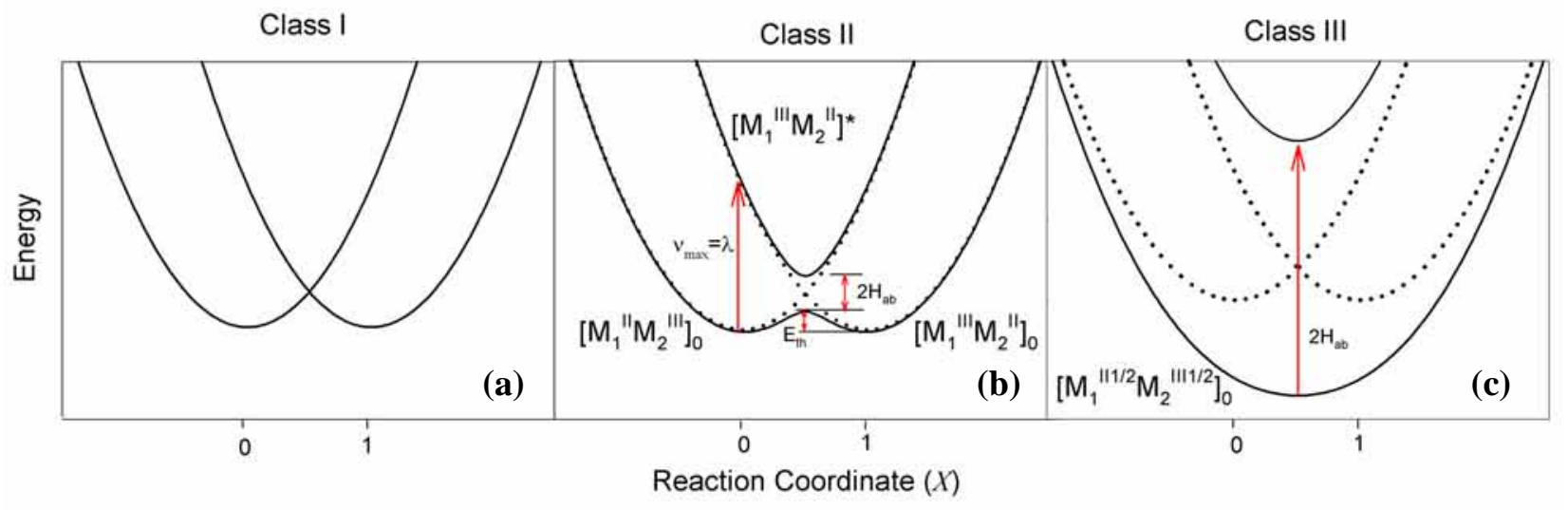

Figure 1.1 Potential energy curves for electron transfer in ligand-bridged dinuclear complexes with (a) negligible, (b) weak $\left(\mathrm{H}_{\mathrm{ab}}=\lambda / 4\right)$ and (c) strong $\left(\mathrm{H}_{\mathrm{ab}}=3 \lambda / 4\right)$ electronic coupling. The dotted and solid curves represent the diabatic and adiabatic states, respectively. 
At the intersection of the diabatic surfaces $(X=0.5)$, the mixing between the wavefunctions removes the degeneracy and gives rise to two new "adiabatic" surfaces, depicted by the solid curves in Figure 1.1. The energies of the lower and upper adiabatic states, $\mathrm{E}_{-}$and $\mathrm{E}_{+}$(respectively), are given by equation $1.3,{ }^{28,29}$ and the splitting between the surfaces at $X=0.5$ defines the electronic coupling parameter, $\mathrm{H}_{\mathrm{ab}}=\left\langle\Psi_{\mathrm{a}}|\hat{\mathrm{H}}| \Psi_{\mathrm{b}}\right\rangle$.

$$
\mathrm{E}_{ \pm}=\frac{\left[\lambda\left(2 X^{2}-2 X+1\right)\right]}{2}-\frac{\left\{[\lambda(2 X-1)]^{2}+4 \mathrm{H}_{\mathrm{ab}}{ }^{2}\right\}^{1 / 2}}{2}
$$

The optically-induced vertical transition between the adiabatic states is the IVCT transition (equation 1.4), which corresponds to the formation of the vibrationally excited state of the ion, $\left[\mathrm{M}_{1}{ }^{\mathrm{III}} \mathrm{M}_{2}{ }^{\mathrm{II}}\right]^{*}$. Since this process proceeds faster than nuclear motion (in accordance with the FranckCondon Principle), $\left[\mathrm{M}_{1}{ }^{\mathrm{III}} \mathrm{M}_{2}{ }^{\mathrm{II}}\right] *$ contains $\left[\mathrm{M}_{1}{ }^{\mathrm{III}}\right] *$ in a coordination environment appropriate for $\left[\mathrm{M}_{1}{ }^{\mathrm{II}}\right]_{0}$, and $\left[\mathrm{M}_{2}{ }^{\mathrm{II}}\right] *$ in a coordination environment appropriate for $\left[\mathrm{M}_{2}{ }^{\mathrm{III}}\right]_{0}$.

$$
\left[\mathrm{M}_{1}^{\mathrm{II}} \mathrm{M}_{2}{ }^{\mathrm{III}}\right]_{0} \stackrel{h v}{\longrightarrow}\left[\mathrm{M}_{1}{ }^{\mathrm{III}} \mathrm{M}_{2}{ }^{\mathrm{II}}\right]^{*}
$$

The degree of electronic coupling between $\mathrm{M}_{1}$ and $\mathrm{M}_{2}$, and the resultant splitting between the adiabatic surfaces dictates the "class" of a mixed-valence system. When $\mathrm{M}_{1}$ and $\mathrm{M}_{2}$ are far apart or when their interaction is symmetry or spin forbidden, the electronic coupling is negligible $\left(\mathrm{H}_{\mathrm{ab}}=0\right)$ and IVCT transitions are not possible. This situation is illustrated in Figure 1.1(a) and corresponds to Class I in the Robin and Day classification scheme. ${ }^{2}$ Class II systems are characterised by weak to moderate electronic coupling between $\mathrm{M}_{1}$ and $\mathrm{M}_{2}$, and exhibit IVCT bands due to the vertical transition between the adiabatic states at $X_{\min }$ \{Figure 1.1(b)\}. As $\mathrm{H}_{\mathrm{ab}}$ increases, the greater vertical splitting between the adiabatic surfaces is compensated for by the reactant and product minima moving closer together such that the energy of the transition ( $\left.v_{\max }\right)$ is equal to $\lambda$ within the Class II regime (equation 1.5). ${ }^{28,30,31}$ While $\lambda$ is a property of the diabatic states and corresponds to the transfer of unit charge, the adiabatic reorganisational energy, $\lambda^{\prime}$ ', corresponds to the "actual" charge transferred, as expressed in equation $1.6{ }^{28,30,31} \lambda^{\prime}$ is reduced relative to $\lambda$ by delocalisation, and vanishes when $\lambda=2 \mathrm{H}_{\mathrm{ab}}$.

$$
\begin{aligned}
v_{\max } & =\lambda=\lambda^{\prime}+4 \mathrm{H}_{\mathrm{ab}}{ }^{2} / \lambda \\
\lambda^{\prime} & =\lambda\left(1-4 \mathrm{H}_{\mathrm{ab}}{ }^{2} / \lambda^{2}\right)
\end{aligned}
$$

In addition to the optically-activated IVCT process, electron transfer can occur by thermal activation and surface crossing on the ground state adiabatic surface in Figure 1.1(b). The energies of the thermal and optical processes are directly related, ${ }^{26,27}$ and the energetic barrier for thermal electron transfer, ${ }^{28,30,31} \mathrm{E}_{\mathrm{th}}$, is given by equation 1.7.

$$
\mathrm{E}_{\mathrm{th}}=\lambda / 4-\mathrm{H}_{\mathrm{ab}}+\mathrm{H}_{\mathrm{ab}}{ }^{2} / \lambda
$$


IVCT measurements on dinuclear complexes provide a sensitive and powerful probe to elucidate aspects of intramolecular electron transfer processes. The parameters of the absorption band specifically, their energy $\left(v_{\max }\right)$, intensity $\left(\varepsilon_{\max }\right)$ and bandwidth at half-height $\left(\Delta v_{1 / 2}\right)$ - may be quantitatively related to the factors which influence the barrier to intramolecular electron transfer according to the classical theory developed by Hush. ${ }^{26,27}$ Within the Class II regime, the IVCT bands are typically weak ( $\varepsilon_{\max } \leq 5000 \mathrm{M}^{-1} \mathrm{~cm}^{-1}$ ), solvent dependent, and exhibit large bandwidths $\left(\Delta v_{1 / 2} \geq 2000 \mathrm{~cm}^{-1}\right)$. In the two-state limit, the predicted bandwidth, ${ }^{26,27} \Delta v_{1 / 2}{ }^{\circ}$, is given by equation 1.8 , where $\mathrm{R}$ is the gas constant, $\mathrm{T}$ is the temperature (in $\mathrm{K}$ ), and the term $16 \mathrm{RT} \ln 2$ takes a value of $2310 \mathrm{~cm}^{-1}$ at $298 \mathrm{~K}$.

$$
\begin{aligned}
\Delta v_{1 / 2}{ }^{0} & =[16 \mathrm{RT} \ln 2(\lambda)]^{1 / 2} \\
& =\left[16 \mathrm{RT} \ln 2\left(v_{\max }-\Delta \mathrm{E}_{0}-\Delta \mathrm{E}^{\prime}\right)\right]^{1 / 2}
\end{aligned}
$$

For Gaussian-shaped IVCT bands, $\mathrm{H}_{\mathrm{ab}}$ is given by equation $1.9,{ }^{26,27}$ where $r_{\mathrm{ab}}$ is the distance between the two diabatic states. A more rigorous quantum mechanical formulation is given by equation $1.10,{ }^{32,33}$ where $\left|\mu_{12}\right|$ is the adiabatic transition dipole moment and $e$ is the unit electronic charge. This form of the equation for $\mathrm{H}_{\mathrm{ab}}$ has the advantage that no implicit assumption is made regarding the shape of the IVCT band, as $\left|\mu_{12}\right|$ may be calculated from the integrated intensity of the absorption band. ${ }^{33}$

$$
\begin{aligned}
& \mathrm{H}_{\mathrm{ab}}=\frac{2.06 \times 10^{-2}\left(v_{\max } \varepsilon_{\max } \Delta v_{1 / 2}\right)^{1 / 2}}{r_{a b}} \\
& \mathrm{H}_{\mathrm{ab}}=\frac{\left|\mu_{12}\right|}{e r_{a b}} \nu_{\text {max }}
\end{aligned}
$$

According to the Robin and Day classification scheme, ${ }^{2}$ the abovementioned classes of mixedvalence systems are distinguished by the relative magnitudes of $\lambda$ and $2 \mathrm{H}_{\mathrm{ab}}$. For weakly-coupled Class II systems, $2 \mathrm{H}_{\mathrm{ab}}$ « $\lambda$. When $\mathrm{M}_{1}$ and $\mathrm{M}_{2}$ are strongly electronically coupled ( $2 \mathrm{H}_{\mathrm{ab}}$ » $\left.\lambda\right)$, the thermal barrier to intramolecular electron transfer vanishes and the ground state adiabatic surface exhibits a single minimum at $X_{\min }=0.5$ \{Figure 1.1(c)\}. In these delocalised Class III systems, both metal centres posses a partial oxidation state of +2.5 and the IVCT transitions* occur within the molecular orbital manifolds of the systems. The transitions are typically intense $\left(\varepsilon_{\max } \geq 5000 \mathrm{M}^{-1} \mathrm{~cm}^{-1}\right)$, solvent independent and exhibit narrow bandwidths $\left(\Delta v_{1 / 2} \leq 2000 \mathrm{~cm}^{-1}\right)$. The energies of the IVCT bands provide a direct measure of $\mathrm{H}_{\mathrm{ab}}$ according to equation 1.11 . $^{26,27}$

$$
v_{\max }=2 \mathrm{H}_{\mathrm{ab}}
$$

\footnotetext{
* The terms "IVCT transition" and "mixed-valence" are retained although the transitions do not involve net charge transfer and the systems are more accurately defined as "average valence". ${ }^{34}$
} 


\subsubsection{The Reorganisational Energy}

The reorganisational energy, $\lambda$, is partitioned into two independent contributions corresponding to the inner- $\left(\lambda_{i}\right)$ and outer-sphere $\left(\lambda_{o}\right)$ reorganisational energies (equation 1.12). ${ }^{26,27} \lambda_{i}$ represents the energy required for reorganisation of the metal-ligand and intra-ligand bond lengths and angles, and $\lambda_{0}$ is the energy required for reorganisation of the surrounding solvent medium. Classical expressions for $\lambda_{i}$ are formulated in terms of the equilibrium bond length changes and force constants of the reactants and products according to equation $1.13,{ }^{26,27}$ where $d_{n}$ and $f_{n}$ denote the metal-ligand bond lengths and the force constants, respectively, for the metal centres in oxidation states $(+n)$ of +2 or +3 .

$$
\begin{aligned}
& \lambda=\lambda_{i}+\lambda_{0} \\
& \lambda_{i}=\frac{n\left(2 f_{2} f_{3}\right)\left(d_{2}-d_{3}\right)}{f_{2}+f_{3}}
\end{aligned}
$$

The classical model is appropriate for low frequency modes which are coupled to the electron transfer, such as vibrations in polar solvents. However, the model is inadequate for systems which exhibit nuclear tunnelling through the activation barrier due to high frequency internal modes ${ }^{* 35-37}$ which require explicit treatment through a quantum mechanical approach. $\lambda_{i}$ may be determined from equation 1.14, where $v_{i}$ and $\Delta_{i}$ are the vibrational frequency and the dimensionless normal coordinate displacement, respectively, for each vibrational mode coupled to the electronic transition. These mode-specific contributions may be evaluated experimentally by resonance Raman spectroscopy. ${ }^{38,39}$

$$
\lambda_{i}=\frac{1}{2} \sum_{i} \Delta_{i}^{2} v_{i}
$$

The $\lambda_{0}$ contribution is generally treated as a one-dimensional classical mode due to the low frequencies of coupled solvent vibrations. ${ }^{26,27,40,41}$ According to the spherical cavity dielectric continuum model given in equation 1.15, the solvent is modelled as a structureless dielectric continuum and has no specific interactions with itself or with the redox sites. ${ }^{42}$ The two metal centres of a symmetrical dinuclear complex are assumed to be centrally located in two non-interpenetrating spheres $(d » 2 a)$ embedded in the dielectric. The parameters $a$ and $d$ define the molecular radii and distance between the donor and acceptor, and $\mathrm{D}_{\mathrm{s}}$ and $\mathrm{D}_{\mathrm{op}}$ are the static and optical dielectric constants of the solvent, respectively.

$$
\lambda_{\mathrm{o}}=e^{2}\left(\frac{1}{a}-\frac{1}{d}\right)\left(\frac{1}{\mathrm{D}_{\mathrm{op}}}-\frac{1}{\mathrm{D}_{\mathrm{s}}}\right)
$$

In accordance with equations $1.1,1.5$ and $1.15, v_{\max }$ should vary linearly with the solvent dielectric function $\left(1 / \mathrm{D}_{\mathrm{op}}-1 / \mathrm{D}_{\mathrm{s}}\right)$, with slope $e^{2}(1 / a-1 / d)$ and intercept $\lambda_{i}+\Delta \mathrm{E}$ '. The validity of the

\footnotetext{
${ }^{*}$ For low frequency modes, $\hbar \omega$ « $k_{B} T$, where $k_{B} \mathrm{~T} \sim 200 \mathrm{~cm}^{-1}$ at room temperature and $\hbar \omega \sim 1-10 \mathrm{~cm}^{-1}$ for polar solvents. For highfrequency modes, $\hbar \omega » k_{B} T$. $\hbar, \omega$ and $k_{B}$ denote Planck’s constant, the vibrational frequency and Boltzmann's constant, respectively.
} 
dielectric continuum model is typically assessed experimentally through IVCT solvatochromism measurements. 2, 26, 27, 34, 37, 43-45 The sensitivity of IVCT bands to solvent variation is often employed as a criterion for the class of a mixed-valence species: a solvent dependence signals valence localisation (Class II), while a solvent independence signals delocalisation (Class III). ${ }^{34,43,46}$

\subsection{The Analysis of IVCT Transitions According to Two-State Classical and Semi-classical Models}

\subsubsection{The Extent of Electronic Coupling}

\subsubsection{Spectral Methods}

The classification of mixed-valence complexes as localised (Class II) or delocalised (Class III) is dependent on the extent of delocalisation which is governed by the competition between $\lambda$ and $\mathrm{H}_{\mathrm{ab}}: \lambda$ exceeds $2 \mathrm{H}_{\mathrm{ab}}$ in valence localised systems, whereas $\lambda$ is less than $2 \mathrm{H}_{\mathrm{ab}}$ in the delocalised case. The determination of the class of a mixed-valence system based on this criterion is often complicated by the inability to accurately determine $\mathrm{H}_{\mathrm{ab}}$ from equations 1.9 and 1.10. While $r_{\mathrm{ab}}$ is typically equated with the through-space geometrical distance between the metal centres, the effective charge transfer distance is decreased relative to the geometric distance as electronic coupling across the bridge increases and these equations provide a lower limit only for $\mathrm{H}_{\mathrm{ab}}{ }^{37}$ Electroabsorption (Stark effect) measurements on the IVCT bands of dinuclear mixed-valence complexes have revealed that the actual (adiabatic) chargetransfer distances are often significantly less than the geometric metal-metal distances. ${ }^{47-51}$ This issue is addressed further in §1.5.3.2.

In addition to electronic delocalisation effects, band-shape analyses are frequently complicated by the presence of multiple overlapping IVCT and interconfigurational (IC) transitions. These arise from the effects of low symmetry and spin-orbit coupling in transition metal complexes of Ru and Os. ${ }^{37,52-54}$ Importantly, only the lowest energy IVCT transition corresponds to the thermal electron transfer pathway and can be used to assess the extent of delocalisation in the ground state. ${ }^{37}$ Since the multiple IVCT transitions are generally unresolved, the $\mathrm{H}_{\mathrm{ab}}$ values obtained by assuming a single IVCT band provides an upper limit only to the ground state $\mathrm{H}_{\mathrm{ab}}$ value. This issue is the subject of §1.5.3.5.

While the Hush model ${ }^{26,27}$ is the preferred method of analysis for IVCT bands, alternate spectral methods for extracting the electronic coupling parameter have been sought which do not rely primarily on the characteristics of the bands. The "CNS" approach ${ }^{28,32}$ introduced by Creutz, Newton and Sutin is based upon a superexchange formalism (§1.5.3.1), in which the metal-metal coupling is facilitated by mixing with a metal-to-bridging ligand charge transfer (MLCT) and/or bridging ligand-to-metal charge transfer (LMCT) state. For a dinuclear system $\mathrm{M}_{1}-\mathrm{BL}-\mathrm{M}_{2}$, the electronic coupling parameter (denoted by $\mathrm{H}_{M_{1} M_{2}}$ instead of $\mathrm{H}_{\mathrm{ab}}$ ) may be calculated from equation 1.16(a). 


$$
\begin{aligned}
\mathrm{H}_{M_{1} M_{2}} & =\frac{\mathrm{H}_{M_{1} L} \mathrm{H}_{M_{2} L}}{2 \Delta E_{M L}}+\frac{\mathrm{H}_{L M_{1}} \mathrm{H}_{L M_{2}}}{\Delta E_{L M}} \\
\frac{1}{\Delta E_{M L}} & =\frac{1}{2}\left(\frac{1}{\Delta E_{M L C T}}-\frac{1}{\Delta E_{M L C T}-\Delta E_{I V C T}}\right)
\end{aligned}
$$

Here, $\mathrm{H}_{M L L}$ is the $\mathrm{M}_{1}$-BL coupling for the $\mathrm{M}_{1}{ }^{\mathrm{II}}$ site (determined from the characteristics of the MLCT band by an expression analogous to equation 1.9), $\mathrm{H}_{M_{2} L}$ is the corresponding quantity for an $\mathrm{M}_{2}{ }^{\mathrm{II}}$ site at the $\mathrm{M}_{2}{ }^{\mathrm{III}}$ geometry, and $\Delta E_{M, L}$ is the effective $\mathrm{M}_{1}-\mathrm{BL}$ energy gap. The latter is determined from equation 1.16(b), where $\Delta E_{\text {MLCт }}$ and $\Delta E_{\text {IVCT }}$ are the energies of the MLCT and IVCT transitions, respectively. The quantities denoted by the subscripts $L M_{1}$ and $L M_{2}$ are the corresponding values for the LMCT transition. Typically, the superexchange coupling of the metal centres is dominated by either an electron or hole transfer superexchange mechanism, and equation 1.16(a) is reduced to the first or second term only. The equations are further simplified by assuming that the quantities denoted by the subscripts $M_{1} L$ and $L M_{1}$ are equivalent to those denoted by $M_{2} L$ and $L M_{2}$, respectively, which is reasonable given the small differences in geometry between the two redox sites. ${ }^{55}$ Indeed, for weakly-coupled systems such as $\left[\left\{\mathrm{Ru}^{\mathrm{II}}\left(\mathrm{NH}_{3}\right)_{5}\right\}\left(\mu-4,4^{\prime}-\text { bpy }\right)\left\{\mathrm{Ru}^{\mathrm{III}}\left(\mathrm{NH}_{3}\right)_{5}\right\}\right]^{5+}$, the value of the electronic coupling parameter obtained from equation $1.9\left(\mathrm{H}_{\mathrm{ab}}=900 \mathrm{~cm}^{-1}\right)$ is in satisfactory agreement with the value calculated from equations 1.16(a) and (b) \{i.e. $\mathrm{H}_{M_{1} M_{2}}=800 \mathrm{~cm}^{-1}$, where $\mathrm{H}_{M_{1} L}=\mathrm{H}_{M_{2} L}=4.4 \times 10^{3} \mathrm{~cm}^{-1}$ and $\Delta E_{M_{1} L}=12.7 \times 10^{3}$ $\left.\mathrm{cm}^{-1}\right\}{ }^{28,32}$ For a number of Class II systems, the magnitudes of the electronic coupling parameters calculated from the Hush and CNS methods, were in close agreement. ${ }^{56-58}$ The validity of the CNS method has been questioned due to the need for empirical parameterisation and assumptions regarding the charge transfer distance, a single orbital interaction directed along the $\mathrm{M}_{1}-\mathrm{BL}-\mathrm{M}_{2}$ axis, and the treatment of a single MLCT or LMCT excited state only. ${ }^{32,56,57}$

\subsubsection{Electrochemical Methods and the Comproportionation Equilibrium}

The comproportionation constant, $\mathrm{K}_{\mathrm{c}}$, and the free energy of comproportionation, $\Delta \mathrm{G}_{\mathrm{c}}{ }^{\circ}$, can be deduced from electrochemical measurements, and provide alternate measures for determining the degree of electronic delocalisation. ${ }^{43,59-61}$ In the absence of electronic coupling, the two metal centres in a symmetrical dinuclear complex undergo oxidation at approximately the same potential; however, any electronic interaction between the centres leads to the existence of discrete oxidation waves. ${ }^{43-45}$ In the potential domain between these two processes the complex is in the mixed-valence state, $\left[\mathrm{M}_{1}{ }^{\mathrm{II}} \mathrm{M}_{2}{ }^{\mathrm{III}}\right]$ (designated [2,3] to indicate the respective formal oxidation states of the two metal centres). The $\mathrm{K}_{\mathrm{c}}$ values for the comproportionation equilibrium express the stability of the $[2,3]$ species relative to the fully-reduced, [2,2], and fully-oxidised, [3,3], forms:

$$
[2,2]+[3,3] \stackrel{K_{c}}{=} 2[2,3] \quad \text { where, } \quad K_{c}=\frac{[2,3]^{2}}{[2,2][3,3]}
$$


$\mathrm{K}_{\mathrm{c}}$ can be measured spectrally ${ }^{59,60}$ or electrochemically from the separation between the two redox potentials for the successive oxidation processes $\left(\Delta \mathrm{E}_{\mathrm{ox}}\right)$ according to equation 1.18(a), where F/RT takes the value $38.92 \mathrm{~V}^{-1}$ at $298 \mathrm{~K}$ ( $\mathrm{F}$ is the Faraday constant). ${ }^{* 43,61}$ Large values of $\mathrm{K}_{\mathrm{c}}$ and $\Delta \mathrm{E}_{\mathrm{ox}}$ are essential requirements for the isolation of a complex in its mixed-valence state.

$$
\begin{aligned}
\mathrm{K}_{\mathrm{c}} & =\exp \left(\Delta \mathrm{E}_{\mathrm{ox}} \mathrm{F} / \mathrm{RT}\right) \\
\Delta \mathrm{G}_{\mathrm{c}}{ }^{\circ} & =-\mathrm{RT} \ln \mathrm{K}_{\mathrm{c}}=-\Delta \mathrm{E}_{\mathrm{ox}} \mathrm{F}
\end{aligned}
$$

Four factors contribute to the magnitude of $\Delta \mathrm{G}_{\mathrm{c}}{ }^{\circ}$ \{equation 1.18(b)\}: a statistical contribution $(1 / 2 \mathrm{RT} \ln 1 / 4)$; an electrostatic factor $\left(\Delta \mathrm{G}_{\mathrm{e}}{ }^{\circ}\right)$ arising from the repulsion of the two similarly charged metal centres linked by the bridging ligand; a synergistic factor $\left(\Delta \mathrm{G}_{\mathrm{s}}{ }^{\circ}\right)$ due to metal-ligand backbonding interactions; and a resonance stabilisation factor $\left(\Delta \mathrm{G}_{\mathrm{r}}{ }^{\circ}\right)$ due to electronic delocalisation. ${ }^{43,59-62}$ For localised mixed-valence systems, the first three contributions can be readily estimated. ${ }^{43-45,61} \Delta \mathrm{G}_{\mathrm{r}}{ }^{\circ}$ is the only component that represents the “actual” metal-metal coupling, and is related to $H_{a b}$ by equation $1.19^{\dagger}$ :

$$
-\Delta \mathrm{G}_{\mathrm{r}}^{\circ}=2 \mathrm{H}_{\mathrm{ab}}{ }^{2} / \lambda=2 \mathrm{H}_{\mathrm{ab}}{ }^{2} / v_{\max }
$$

The comparison of $\Delta \mathrm{E}_{\mathrm{ox}}$ and $\mathrm{K}_{\mathrm{c}}$ values determined from electrochemical data have been widely used for the assessment of the extent of coupling in mixed-valence complexes. ${ }^{43-45,63}$ However, the comparison between the electronic coupling parameters derived from the semi-classical Hush model, ${ }^{26,27}$ the CNS model, ${ }^{28,32}$ and the electrochemical treatment, are frequently in poor agreement. ${ }^{57,58}$ Previous reports have emphasised the need for caution in the interpretation of electrochemical data, due to the significant dependence of redox potentials on the identity of the solvent and anions in the electrolyte medium. ${ }^{56}$ Spectral measurements of the IVCT transitions provide a more accurate and systematic probe of the degree of electronic coupling. ${ }^{26,27}$

\subsubsection{Predictions for the IVCT Band-shape}

The transition between the localised and delocalised regimes is driven by an increase in $\mathrm{H}_{\mathrm{ab}}$. The predicted effect of increasing delocalisation on the IVCT band-shape for a symmetrical dinuclear mixedvalence complex is illustrated in Figure 1.2. ${ }^{31}$ The band is calculated by assuming a Boltzmann distribution over the energies on the ground state adiabatic surface. ${ }^{26,27}$ For $2 \mathrm{H}_{\mathrm{ab}}$ « $\lambda$, this produces a Gaussian-shaped contour that is symmetrical about $v_{\max }$, with a bandwidth of $\Delta v_{1 / 2}{ }^{\circ}$ \{Figures 1.2(a) and (b)\}. As $\mathrm{H}_{\mathrm{ab}}$ increases, the Gaussian shape is retained, but is truncated on the low energy side below $2 \mathrm{H}_{\mathrm{ab}}$, i.e. the minimum difference between the ground and excited states at $X=0.5$, as shown in Figure $1.2(\mathrm{c}) .^{57,64}$

\footnotetext{
* Due to comproportionation of the mixed-valence species, the proportion of the complex in the mixed-valence form (at equilibrium) is $\mathrm{K}_{\mathrm{c}}{ }^{1 / 2} /\left(2+\mathrm{K}_{\mathrm{c}}{ }^{1 / 2}\right)$. This must be accounted for in measurements of the intensities of the IVCT bands. ${ }^{56}$

${ }^{\dagger}$ Equation 1.19 relates to localised systems. In the delocalised case, $-\Delta \mathrm{G}_{\mathrm{r}}{ }^{\circ}=2\left(\mathrm{H}_{\mathrm{ab}}-\lambda / 4\right)=v_{\max }-\lambda / 2 .^{28}$
} 
(a)
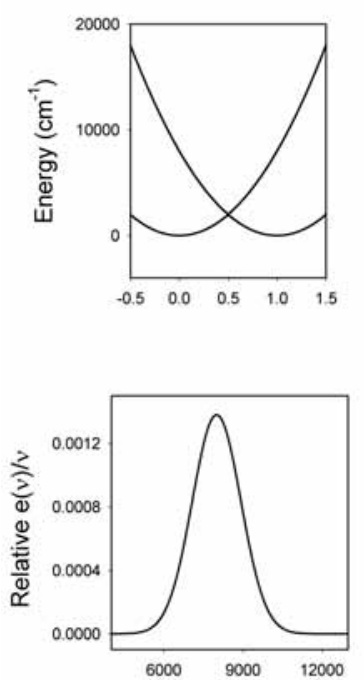

(b)

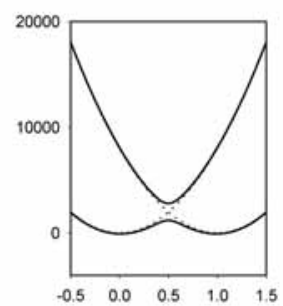

(c)
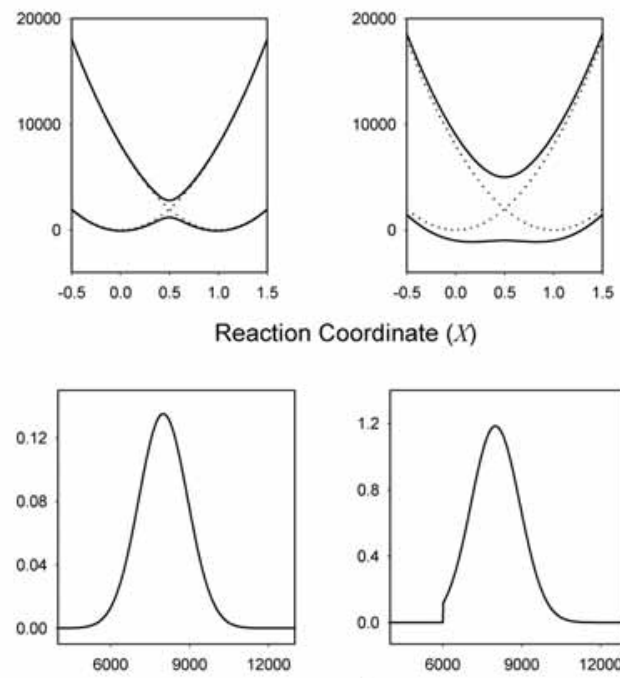

Reaction Coordinate $(X)$ (d)
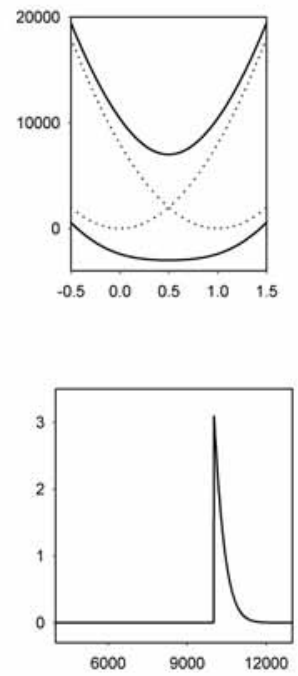

Figure 1.2 Potential energy surfaces and band-shape predictions from the two-state classical model. ${ }^{31}$ Plots of the energy vs. reaction coordinate (top panel) and the relative reduced absorption spectra $\varepsilon / v$ vs. $v^{*}$ (lower panel) for a symmetrical mixed-valence system with increasing $2 \mathrm{H}_{\mathrm{ab}} / \lambda\left(\lambda=8000 \mathrm{~cm}^{-1}\right)$. The values of $2 \mathrm{H}_{\mathrm{ab}} / \lambda\left(\mathrm{H}_{\mathrm{ab}}\right.$, in $\left.\mathrm{cm}^{-1}\right) \mathrm{are}$ (a) 0.02 (80), (b) 0.2 (800), (c) 0.75 (3000), and (d) 1.25 (5000).

The "band cut-off effect" is manifested by IVCT bands which are Gaussian on the high energy side and skewed on the low energy side. The physically-unrealistic nature of the sharp spectral cut-off is attributed to the neglect of quantum effects and solvent broadening of the individual vibronic components which "round-off" the abrupt cut-off. As indicated in Figure 1.2, the effect does not become significant until $\mathrm{H}_{\mathrm{ab}}$ exceeds $\lambda / 4$, and $\Delta \mathrm{v}_{1 / 2}$ is equivalent to $\Delta \mathrm{v}_{1 / 2}{ }^{\circ}$ for $2 \mathrm{H}_{\mathrm{ab}}<\lambda-[4 \mathrm{RT} \ln 2]^{1 / 2}$. $^{31}$ Beyond this regime, $\Delta v_{1 / 2}$ decreases relative to $\Delta v_{1 / 2}{ }^{o}$ with increasing $\mathrm{H}_{\mathrm{ab}}$ and the IVCT bands increase in intensity until $2 \mathrm{H}_{\mathrm{ab}}=$ $\lambda$ at the transition between Classes II and III. At this point, the band retains intensity in the higher energy half only. The $H_{a b}$ values determined from equation 1.9 are increasingly underestimated as the system approaches the Class II-III borderline, and this underestimation reaches $50 \%$ when $2 \mathrm{H}_{\mathrm{ab}}=\lambda .^{57}$ For $2 \mathrm{H}_{\mathrm{ab}}>\mathrm{v}_{\max }$, only the high energy side remains and the asymmetrically-shaped band shifts to higher energy, increases in intensity, and narrows due to the greater curvature at the $\mathrm{E}_{+}$minimum \{Figure 1.2(d) $\}^{31,57}$ An IVCT band which exhibits a cut-off effect should become increasingly symmetrical as the temperature is decreased. ${ }^{37}$

The explicit consideration of this effect is required for the accurate analysis of the IVCT bands of moderately- to strongly-coupled systems. Brunschwig, Creutz and Sutin have proposed relationships between the IVCT energies and bandwidths which enable the calculation of "corrected" values for $\mathrm{H}_{\mathrm{ab}}$ through the introduction of an "error function". ${ }^{31}$ The treatment of symmetrical mixed-valence systems ${ }^{31}$ has subsequently been extended to unsymmetrical systems ${ }^{66}$ in which the Gaussian-shaped band is centred at $\lambda+\Delta \mathrm{E}_{0}$ and exhibits a cut-off at $2 \mathrm{H}_{\mathrm{ab}}$.

\footnotetext{
* Wavelength-dependent charge transfer intensities scale with the inverse of the absolute absorption energy $\left(v^{-1}\right),{ }^{26,65}$ and the energy maximum of the "reduced" absorption spectrum (i.e. $\varepsilon / v v s v$ ) is identified with the vertical separation between the lower and upper surfaces and is the quantity most appropriately employed in the analysis of IVCT bands.
} 


\subsubsection{Classifying Mixed-Valence Systems between the Class II and Class III Regimes}

The cut-off effect has been observed in the spectra of both organic and transition metal-centred mixed-valence species, and has been utilised as a diagnostic marker for systems which lie close to the transition between the localised (Class II) and delocalised (Class III) regimes. ${ }^{31,34,37,43,57,64}$ While the majority of reports have considered the relative magnitudes of $2 \mathrm{H}_{\mathrm{ab}}$ and $\lambda$ as the criterion for defining the position of a mixed-valence system between the two regimes, Nelsen ${ }^{57}$ and Brunschwig, Creutz and Sutin ${ }^{31}$ have recently proposed alternate criteria.

Nelsen ${ }^{57}$ considered the disappearance of the electron transfer barrier as an indication of the transition from Class II to the Class II-III borderline. By factoring the semi-classical expression for $\mathrm{E}_{\mathrm{th}}$ in equation 1.7, the parameter $F$ was defined as the fraction of the electron transfer barrier remaining after the inclusion of electronic coupling (equation 1.20). The magnitude of $F$ varies between 1 (in the limit of complete localisation) and 0 (at the Class II-III borderline).

$$
\mathrm{E}_{\mathrm{th}}=F\left(v_{\max } / 4\right) \quad \text { where, } \quad F=\left(1-2 \mathrm{H} / v_{\max }\right)^{2}
$$

The magnitudes of $F$ determined on the basis of electronic coupling values from the Hush model $^{26,27}$ (with $\mathrm{H}=\mathrm{H}_{\mathrm{ab}}$ ) and CNS model ${ }^{28,32}$ (with $\mathrm{H}=\mathrm{H}_{M_{1} M_{2}}$ ) were compared ${ }^{57}$ for the series of 1,4dicyanamidobenzene complexes ${ }^{58,67-71}$ including $\left[\left\{\mathrm{Ru}\left(\mathrm{NH}_{3}\right)_{5}\right\}_{2}(\mu-\mathrm{BL})\right]^{3+}$, trans,trans$\left[\left\{\mathrm{Ru}\left(\mathrm{NH}_{3}\right)_{4}(\mathrm{py})\right\}_{2}(\mu-\mathrm{BL})\right]^{3+}$ and mer,mer- $\left.\left[\mathrm{Ru}\left(\mathrm{NH}_{3}\right)_{3}(\mathrm{bpy})\right\}_{2}(\mu-\mathrm{BL})\right]^{3+}$ \{where BL represents the dianions $\operatorname{dicyd}^{2-}$ (1,4-dicyanamidobenzene), $\mathrm{Me}_{2}$ dicyd $^{2-}$ (1,4-dicyanamido-2,5-dimethylbenzene) and $\mathrm{Cl}_{4} \mathrm{dicyd}^{2-}$ (2,3,5,6-tetrachloro-1,4-dicyanamidobenzene) $\}$. F decreased with progressively stronger electronic coupling as BL was varied through the series $\mathrm{Cl}_{4} \mathrm{dicyd}^{2-}$, dicyd ${ }^{2-}$ and $\mathrm{Me}_{2} \mathrm{dicyd}^{2-}$. While the values of $F_{\text {Hush }}$ and $F_{\text {CNS }}$ were comparable in the absence of significant IVCT band cut-off effects, the CNS method provided more reasonable estimates of the degree of coupling near the Class II to III borderline. ${ }^{57}$ The $\mathrm{H}_{\mathrm{ab}}$ values determined from the Hush model (equation 1.9) were increasingly underestimated as the borderline was approached, due to cut-off effects and discrepancies between the effective charge transfer distance and the geometrical metal-metal distance. While the magnitude of $F$ provides a suitable means to assess the relative degree of electronic coupling within a series of closely-related complexes in the localised regime,${ }^{57}$ the criterion is inappropriate for strongly-coupled systems which exhibit highly asymmetric IVCT bands. The latter typically require at least a three-state analysis.

As an alternative classification scheme, Brunschwig, Creutz and Sutin ${ }^{31}$ introduced a criterion based upon the experimental and predicted IVCT bandwidths, ${ }^{26,27}$ according to equation 1.21. The magnitude of the $\Gamma$ parameter distinguishes the class of a mixed-valence system: $0<\Gamma<0.1$ for weaklycoupled Class II systems, $0.1<\Gamma<0.5$ for moderately-coupled Class II systems, $\Gamma \approx 0.5$ at the transition between Classes II and III, and $\Gamma>0.5$ for Class III systems.

$$
\Gamma=1-\left(\Delta v_{1 / 2}\right) /\left(\Delta v_{1 / 2}{ }^{\circ}\right)
$$


The classification of a number of mixed-valence systems based on the magnitudes of $\Gamma$ were shown to be in favourable agreement with the results from prior experimental studies. ${ }^{31}$ For $\left[\left\{\mathrm{Ru}\left(\mathrm{NH}_{3}\right)_{5}\right\}_{2}\left(\mu-4,4^{\prime} \text {-bpy }\right)\right]^{5+}\left\{4,4^{\prime}\right.$-bpy $=4,4^{\prime}$-bipyridine $\}$, for example, the $\Gamma$ value of -0.1 was consistent with a Class II assignment, as established previously from the broad, Gaussian-shaped and solvent dependent IVCT band. ${ }^{60}$ A delocalised assignment was favoured for the Creutz-Taube ion, $\left[\left\{\mathrm{Ru}\left(\mathrm{NH}_{3}\right)_{5}\right\}_{2}(\mu \text {-pyz })\right]^{5+}\{$ pyz $=$ pyrazine $\}$, on the basis of the $\Gamma$ value of 0.63 for the narrow $\left(\Delta v_{1 / 2}=1480\right.$ $\mathrm{cm}^{-1}$ ) asymmetrically-shaped IVCT band at $6320 \mathrm{~cm}^{-1}$ (in DCl/D $\mathrm{D}_{2} \mathrm{O}$ ). ${ }^{72,73}$ This classification was consistent with the findings from an extensive series of physical techniques of varying timescales $^{34,37,43,74}$ including IR, ${ }^{75,76}$ single crystal EPR, ${ }^{77}$ Stark effect $^{78,79}$ and Raman spectroscopy. ${ }^{80}$

While the $\Gamma$ bandwidth criterion provides a reasonable qualitative measure for the classification of mixed-valence systems between the localised and delocalised limits, band-shape analyses are frequently complicated by a number of factors. These include multiple overlapping IVCT and IC transitions, ${ }^{37,52-54}$ differences in the extent of electronic delocalisation between the ground and mixedvalence excited states, contributions from vibronic progressions, and multiple nuclear and solvent vibrations which are coupled to the electron transfer. ${ }^{37}$

\subsection{Theoretical Models for the Localised-to-Delocalised Transition in IVCT}

\subsubsection{Three- and Four-State Classical Models}

Classical models ${ }^{26,27}$ are the preferred method of analysis for IVCT transitions due to the facility of their application and their overlap with the Marcus theory of electron transfer. ${ }^{40,41}$ However, the classical two-state approximation is based upon the Born-Oppenheimer approximation and is strictly valid in the strongly localised and delocalised limits. ${ }^{26,27}$ In real systems, the inclusion of additional electronic states is often necessary to account for the role of the bridging ligand in mediating the superexchange coupling between the metal-based sites.

The three-state model proposed by Brunschwig, Creutz and Sutin ${ }^{31}$ provides an extension to the two-state classical model by explicitly including a third electronic state formed by charge transfer to or from the bridging ligand. The influence of this additional state depends upon its energy $\left(\mathrm{H}_{\mathrm{c}}\right.$ in equation 1.22) relative to the other two diabatic states $\left\{\mathrm{H}_{\mathrm{a}}\right.$ and $\mathrm{H}_{\mathrm{b}}$ in equations 1.2(a) and (b) $\} . \Delta \mathrm{E}_{\mathrm{ac}}$ represents the energy difference between the minima of the potential energy surfaces for states ' $a$ ' or ' $b$ ' and the bridge state 'c'.

$$
\mathrm{H}_{\mathrm{c}}=\lambda(X-0.5)^{2}+\Delta \mathrm{E}_{\mathrm{ac}}
$$

The salient features of the three-state model are considered here for a symmetrical mixed-valence system, where the mediating state lies above the intersection of the reactant and product diabatic states at $X=0.5 .^{31}$ The adiabatic surfaces are obtained solving a $3 \times 3$ Hamiltonian analytically or numerically. The electron transfer process involves superexchange coupling of the three states, where states ' $a$ ' and ' $b$ ' couple to state ' $c$ ' through the electronic coupling parameter $\mathrm{H}_{\mathrm{ac}}\left(=\mathrm{H}_{\mathrm{bc}}\right)$, and the direct $\mathrm{H}_{\mathrm{ab}}$ coupling is 
zero. The diabatic and adiabatic potential energy surfaces, and the corresponding absorption spectra for a series of $\mathrm{H}_{\mathrm{ac}}$ coupling strengths are illustrated in Figure $1.3,{ }^{*}$ where $\lambda$ and $\Delta \mathrm{E}_{\mathrm{ac}}$ are 8000 and $16000 \mathrm{~cm}^{-1}$, respectively.

(a)
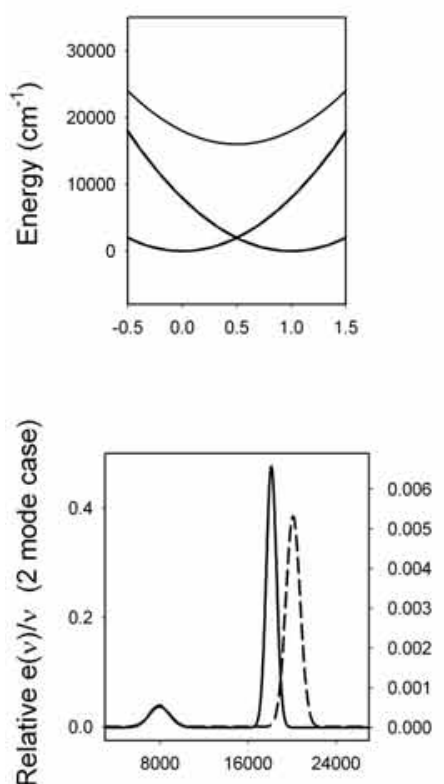

(b)

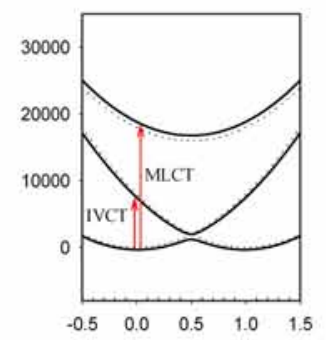

(c)
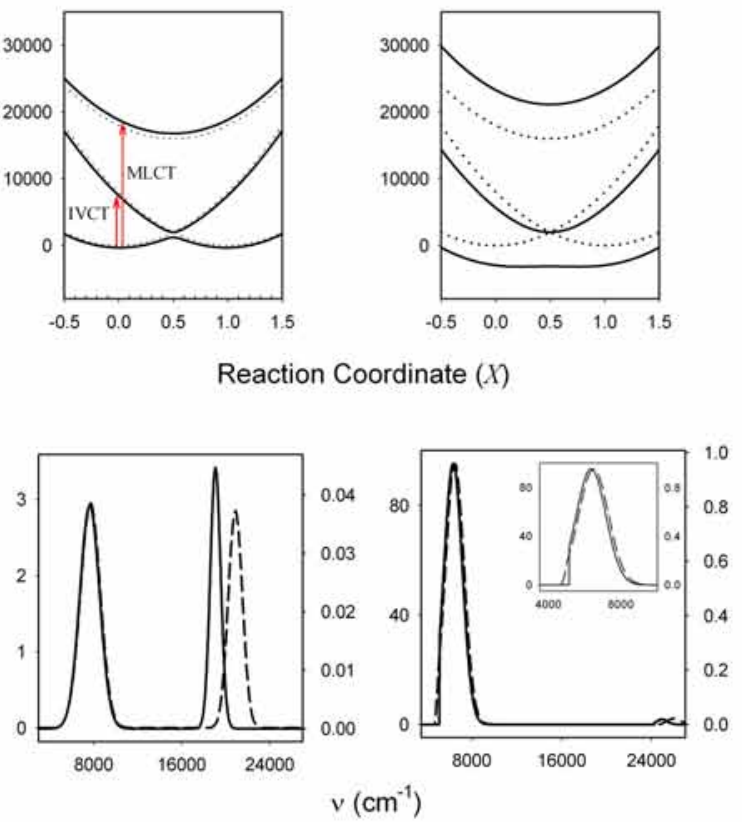

(d)
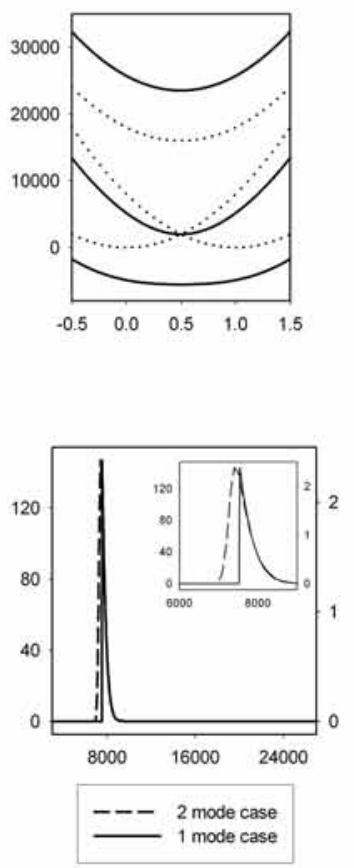

Figure 1.3 Potential energy surfaces and band-shape predictions from the three-state classical model. ${ }^{31,66}$ Plots of the energy $v s$. reaction coordinate for the three-state one-mode model (top panel) and overlay of the reduced absorption spectra $\varepsilon / v$ vs. $v^{\dagger}$ (lower panel) for a symmetric mixed-valence system with increasing values of $\mathrm{H}_{\mathrm{ac}}$ $\left(\lambda=8000 \mathrm{~cm}^{-1}, \Delta \mathrm{E}_{\mathrm{ac}}=16000 \mathrm{~cm}^{-1}\right)$ for the one- (---) and two-mode (-) cases. The values of $\mathrm{H}_{\mathrm{ac}}$ in $\mathrm{cm}^{-1}$ are (a) 800, (b) 2400, (c) 7000, and (d) 9000. The class II-III transition occurs at $\mathrm{H}_{\mathrm{ac}}=8000 \mathrm{~cm}^{-1}$.

The major difference between the two- and three-state models is the allowance in the latter for a second absorption (generally MLCT) at higher energy than the IVCT. As $\mathrm{H}_{\mathrm{ac}}\left(=\mathrm{H}_{\mathrm{bc}}\right)$ increases in the Class II regime \{Figures 1.3(a) and (b)\}, the intensity of the IVCT transition increases. The MLCT intensity initially increases before reaching a maximum and decreasing to the Class II-III borderline, at which point the band vanishes \{Figure 1.3(c)\}. $v_{\max }$ is initially equal to $\lambda$ in the Class II limit, but decreases as the Class II-III borderline is approached. This contrasts the two-state model where $v_{\max }=\lambda$ throughout the Class II regime until the Class II-III borderline is reached. For the three-state case, $v_{\max }$ increases as does $\mathrm{H}_{\mathrm{ac}}$ in the Class III regime \{Figure 1.3(d)\}. The inclusion of a fourth electronic state is required to qualitatively reproduce the experimental observation of MLCT transitions for Class III complexes. $^{31}$

\footnotetext{
* The numerical codes used for the construction of the potential energy surfaces and resulting spectra based on the three- and four-state models of Brunschwig, Creutz and Sutin ${ }^{31}$ were kindly provided by Dr Gerald Sando. ${ }^{66}$ The routines were implemented within Mathcad 2001 Professional using internal algorithms for matrix diagonalisation.

${ }^{\dagger}$ Wavelength-dependent charge transfer intensities scale with the inverse of the absolute absorption energy $\left(v^{-1}\right),{ }^{26,65}$ and the energy maximum of the "reduced" absorption spectrum (i.e. $\varepsilon / v v s v$ ) is identified with the vertical upper-/lower-surface energy separation and is the quantity most appropriately employed in the analysis of IVCT bands.
} 
Within a three-state two-mode approach, ${ }^{31}$ the addition of a second vibrational mode representing a symmetrical combination of stretches results in a smooth spectral cut-off. Figure 1.3 depicts the potential energy surfaces and corresponding absorption spectra. The energies of the diabatic states are expressed in equation 1.23 where $X_{1}$ and $X_{2}$ represent the anti-symmetric and symmetric reaction coordinates, respectively. In the Class II regime, the addition of the symmetric vibration mode has a modest effect only on $v_{\max }$ and $\Delta v_{1 / 2}$ \{Figures 1.3(a) and (b) \}. In Class III, the IVCT band is broadened and the spectral cut-off is smoothed out relative to the one-mode two- and three-state models \{Figure $1.3(\mathrm{~d})\}$.

$$
\begin{aligned}
& \mathrm{H}_{\mathrm{a}}=\lambda X_{1}^{2}+\lambda X_{2}^{2} \\
& \mathrm{H}_{\mathrm{b}}=\lambda\left(X_{1}-1\right)^{2}+\lambda X_{2}^{2} \\
& \mathrm{H}_{\mathrm{c}}=\lambda\left(X_{1}-0.5\right)^{2}+\lambda\left(X_{2}-0.5\right)^{2}+\mathrm{E}_{\mathrm{ac}}
\end{aligned}
$$

The central role of the symmetric vibration mode for moderately- to strongly-coupled mixedvalence systems was first discussed by Hush, ${ }^{74}$ and arises from the symmetrical nature of the electronic redistribution that occurs on charge excitation. The IVCT transition originates from a state in which the electron is predominantly localised on a metal site, to one on the bridge. The latter is displaced from the metal-localised state along the totally symmetric vibration coordinate, and the vertical IVCT excitation occurs from the ground state surface to a region on the upper surface that is higher up, and steeper. The addition of the symmetric mode results in a broadening in the IVCT and MLCT bands with increasing delocalisation.

\subsubsection{Vibronic Coupling Models: the "PKS Model”}

Quantum mechanical treatments account for the integral role of vibronic coupling which becomes increasingly important as the degree of electronic coupling increases due to the dynamic interaction between the vibrational and electronic motions (i.e. the breakdown in the Born-Oppenheimer approximation). The "PKS model” proposed by Piepho, Krausz and Schatz. ${ }^{81}$ was originally formulated as a two-state, one-dimensional model along an anti-symmetric vibrational coordinate, $q{ }^{82,83}$ The vibronic energy levels are determined using wavefunctions which allow for the dependence on both the nuclear and electronic coordinates, and the IVCT manifold is calculated as a superposition of the transitions between these levels. The model was the first to investigate the full IVCT absorption profile by iteratively fitting the band to three parameters: the electronic and vibronic coupling parameters ( $\varepsilon$ and $\lambda$, respectively) and the wavenumber of the totally symmetric metal-ligand stretching vibration $(\bar{v})$. The energies of the surfaces, $E_{1,2}(\mathrm{PKS})$, and the expressions for the PKS parameters are given by equations 1.24(a) and (b), where $c$ is the speed of light, $f$ is the metal-ligand force constant and $\mu$ is the reduced metal-ligand mass. ${ }^{43}$ The localised limit occurs when $\varepsilon \approx 0$ and $\mathrm{E}_{2}-\mathrm{E}_{1} \approx 2 \lambda q$, and the delocalised limit when $\lambda \approx 0$ and $\mathrm{E}_{2}-\mathrm{E}_{1} \approx 2 \varepsilon$. 


$$
\begin{gathered}
\mathrm{E}_{1,2}(\mathrm{PKS})=\frac{q^{2}}{2} \pm\left(\varepsilon^{2}+\lambda^{2} q^{2}\right)^{1 / 2} \\
v_{\max }=2 \lambda^{2} \bar{v}, \quad \mathrm{H}_{\mathrm{ab}}=\varepsilon \bar{v} \quad \text { and } \quad \bar{v}=1 / c\left(f / 4 \pi^{2} \mu\right)
\end{gathered}
$$

The PKS treatment has been successfully applied to simulate the IVCT bands of a range of systems which lie between the fully localised and delocalised regimes. ${ }^{81,82,84-86}$ While the method reproduces the salient features of the localised-to-delocalised transition - including the increasing intensity and narrowness of the IVCT bands - Reimers and Hush have argued that the treatment is flawed in several fundamental aspects of its design and application. ${ }^{74,87}$ Most importantly, the model neglects the central role of the bridging ligand in mediating the charge transfer transition between the metal centres. In response to these criticisms, the PKS model has been extended to include coupling to multicentre vibrations, solvent ${ }^{82}$ and symmetric vibration modes. ${ }^{88,89}$

\subsubsection{Molecular Orbital Models: the “Ondrechen Model”}

The three-state molecular orbital model proposed by Ondrechen and coworkers ${ }^{90-102}$ for delocalised mixed-valence systems was the first to accommodate the effects of excitations in the totally symmetric vibration modes. The two metal-based states of the dinuclear complex are each coupled to one local vibrational degree of freedom, and interact indirectly via a third electronic state localised on the bridging ligand. Each terminal state is separated from the bridging ligand state by an energy gap, $\alpha{ }^{*}$ and the coupling between the states is represented by the electron-exchange coupling constant, $J$. The latter quantifies the donor-bridge and acceptor-bridge interactions, rather than the direct donor-acceptor interaction. The three-state delocalised system is constructed from symmetric and anti-symmetric combinations of the initial unperturbed electronic states, as depicted schematically in Figure 1.4. The IVCT transition arises from electron transfer between the bonding (B) and non-bonding (N) orbitals within the molecular orbital manifold of the mixed-valence system. Like the PKS model, the Ondrechen model involves parameter fitting through the simulation of the absorption spectrum for the mixed-valence species.

The energy of the IVCT transition $\left(\mathrm{E}_{\mathrm{B} \rightarrow \mathrm{N}}\right)$ is given by equation 1.25. In the limit of large exchange coupling, the magnitude of $\mathrm{E}_{\mathrm{B} \rightarrow \mathrm{N}}$ is dominated by the value of $J$ and modified only slightly by $\alpha$. This represents a key difference between the Ondrechen model and the two-state semi-classical model in which the energy of the IVCT band is $\lambda$ (equation 1.5). In the former, equation 1.25 accounts for the appearance of high energy IVCT bands for delocalised complexes with minimal reorganisational energies.

\footnotetext{
* $\alpha$ is typically estimated from the difference in potentials between the metal-based oxidation and ligand-based reduction potentials.
} 


$$
E_{B \rightarrow N}=-\frac{1}{2}\left(\alpha-\sqrt{\alpha^{2} 8 J^{2}}\right)
$$

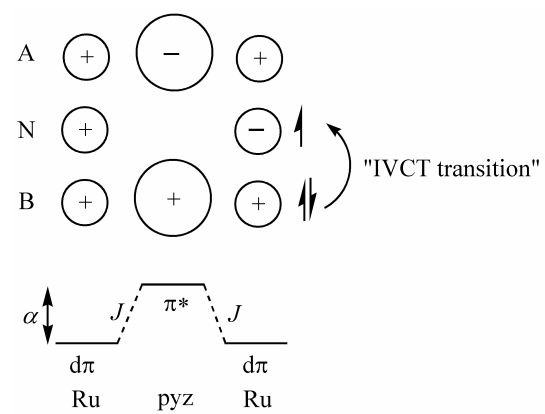

Figure 1.4 Qualitative molecular orbital diagram for a mixed-valence complex such as $\left[\left\{\operatorname{Ru}(\mathrm{bpy})_{2}\right\}_{2}(\mu-\mathrm{pyz})\right]^{5+}$ according to the three-state model. The bonding, B (symmetric), non-bonding, $\mathrm{N}$ (node on the bridging ligand) and antibonding, A (anti-symmetric) molecular orbitals are shown. The "IVCT transition" corresponds to a bonding $\rightarrow$ non-bonding transition with energy $\mathrm{E}_{\mathrm{B} \rightarrow \mathrm{N}}$.

The Ondrechen model has been applied to simulate the IVCT line-shape for the Creutz-Taube ion, ${ }^{92-95}$ in which the two $\mathrm{Ru}(\mathrm{d} \pi)$ and vacant pyz $\left(\pi^{*}\right)$ orbitals form bonding (fully-occupied), non-bonding (half-occupied) and antibonding (unoccupied) combinations of molecular orbitals (Figure 1.4). The "IVCT band" at $6320 \mathrm{~cm}^{-1}$ was assigned as a B $\rightarrow \mathrm{N}$ transition. Importantly, this transition exhibits a dual nature: it contains IVCT character due to the transition from a sum to a difference combination of $\operatorname{Ru}(\mathrm{d} \pi)$ orbitals, in addition to LMCT character due to the redistribution of charge from the node on pyz in the non-bonding state to the two metal centres. ${ }^{94}$ The latter is accompanied by a change in the metalligand bond lengths on both sides of the bridge. The symmetric vibrations coupled to the two sites and the symmetric modes of the bridging ligand itself are vibrationally-coupled to the electronic transition. ${ }^{74}$ Qualitatively, this coupling is manifested by a small blue-shift and increased line-width in the absorption band. ${ }^{92}$ Resonance Raman experiments on delocalised mixed-valence systems with excitation in the B $\rightarrow$ $\mathrm{N}$ transition reveal significant resonant enhancement of symmetric bridging ligand modes. Such enhancements have been observed for both the Creutz-Taube ion ${ }^{103,104}$ and for the pyz-bridged "dimersof-trimers”, $\left[\left\{\mathrm{Ru}_{3}\left(\mu_{3}-\mathrm{O}\right)\left(\mu-\mathrm{O}_{2} \mathrm{CCH}_{3}\right)_{6}(\mathrm{CO})(\mathrm{L})\right\}_{2}(\mu-\mathrm{pyz})\right]^{-}\{\mathrm{L}=4$-(dimethylamino)pyridine, pyridine, 3- or 4-cyanopyridine.$^{105-112}$ The results indicated that a three-site, rather than two-site, mixing mechanism was operative for ground state delocalisation in these systems. The Ondrechen model has been extended to include vibronic coupling, and to a five-state treatment with two coupled vibrations. ${ }^{94,105}$ However, the model has been criticised due to complications with the treatment of near-degenerate orbitals and spin-orbit coupling effects. ${ }^{99,105}$ 


\subsubsection{Computational Approaches and Quantum Mechanical Methods}

Semiempirical computational methods such as INDO (intermediate neglect of differential overlap), ${ }^{113,}{ }^{114}$ AM1 and PM3, and $a b$ initio methods such as Density Functional Theory (DFT) ${ }^{115-118}$ have been extensively employed to provide rapid calculations of the structural and electronic properties of mixed-valence systems. ${ }^{119-124}$ The electronic structures of the systems are generally treated by a two-state model in which the donor-acceptor interaction is quantified by an electronic coupling parameter, and the environment surrounding the system is treated as a dielectric continuum. ${ }^{56,125}$ However, such approaches obscure the physical insight into the factors that govern electronic delocalisation and the barriers to intramolecular electron transfer ${ }^{36,53,126,127}$ as they often involve empirical parameterisation, and neglect the central role of the bridging ligand as well as non-continuum solvation effects in real systems. ${ }^{126-128}$

Reimers, Hush and coworkers have recently proposed an extensive theoretical and computational framework for calculating solvent shifts on electronic absorption bands, ${ }^{126,127,129-132}$ and for quantitatively modelling the electronic characteristics of mixed-valence systems in the localised-to-delocalised transition. ${ }^{74}$ The formalism accounts for the key features of the transition, including the central role of both anti-symmetric and symmetric modes within the full treatment of the vibronic coupling problem. The application of these formalisms has been facilitated by the development of fast numerical methods using high level a priori computational calculations such as DFT and $a b$ initio techniques. ${ }^{65,74,128,133-135}$

Such quantum mechanical models are intuitively appealing in that they provide a systematic theoretical basis for understanding the transition between the localised and delocalised regimes, and can be extended to include multiple coupled electronic states and spin-orbit coupling. The success of the methodology has recently been demonstrated by providing a "unified description" to correlate the molecular structure and photosynthetic function of the "special pair" in bacterial photosynthesis using a four-state seventy-mode model. ${ }^{8,9,87,136-138}$ The model has also been applied to mixed-valence systems such as the Creutz-Taube ion, ${ }^{74}$ and has enabled the elucidation of several key features regarding the interplay of symmetric and anti-symmetric modes. Importantly, in the weak-coupling limit, displacements in the anti-symmetric modes control the energy levels and the presence of vibronic coupling through the symmetric modes is of minor importance. By comparison, in the strong coupling limit, displacements in the symmetric modes dictate the energy levels, and vibronic coupling through the anti-symmetric modes is relatively less important. ${ }^{74}$

\subsubsection{Towards a "Unifying Theory" for IVCT}

The development of a conceptual framework for the treatment of systems between the fully localised and delocalised limits is at the forefront of current experimental, theoretical and computational research in electron transfer. The abovementioned models (\$1.4.1-1.4.4) capture the essence of the localised-to-delocalised transition, and demonstrate that a "unifying theory" must embody all of the features; i.e. the explicit inclusion of the bridging ligand and anti-symmetric and symmetric modes within the full treatment of a vibronic coupling problem. ${ }^{37,74}$ Importantly, such a theory must be parameterised 
in terms of physically transparent quantities which are amenable to experimental analysis. ${ }^{36,37,53}$ A key realisation is that nuclear dynamics are important even for formally "delocalised" complexes which can display a large range of behaviours depending on their "degree" of delocalisation. ${ }^{105}$ In reality, the transition between localisation and delocalisation is not abrupt, and the explicit distinction between the two regimes is a semantic rather than physical one.

\subsection{The Fundamental Factors Governing the Localised-to-Delocalised Transition}

\subsubsection{Dynamic Considerations and the “Class II-III" Classification}

The elucidation of the factors which govern the localised-to-delocalised transition in mixedvalence systems is complicated by the interplay between the time-scales for intramolecular electron transfer and the coupled nuclear and solvent vibrations. A recent seminal review by Meyer and coworkers $^{37}$ addressed these issues from a semi-classical perspective, by defining mixed-valence systems in the localised-to-delocalised transition as “Class II-III”. This classification poses a further complexity as two transition regions - between Classes II and II-III, and between Classes II-III and III - are additionally required.

The distinction between the various classes is based on the dynamic characteristics of the systems and the relative time-scales of the solvent, vibrational and electronic motions. ${ }^{31,37}$ Within the semiclassical formalism, the lower frequency solvent modes are treated classically, while the collective higher frequency inner-shell vibrations are treated quantum mechanically (i.e. $\lambda=\lambda_{0}+\Sigma \lambda_{i}$ ). As $H_{a b}$ increases in a closely-related series of complexes, the barrier to electron transfer decreases, as does the time-scale for this process. If the solvent barrier is initially eliminated $\left(\lambda_{0}<\Sigma \lambda_{i}\right)$, then the solvent modes for the donor and acceptor sites are averaged in the region where $\lambda_{0}<2 \mathrm{H}_{\mathrm{ab}}<\lambda_{\mathrm{o}}+\Sigma \lambda_{i}$. However, the two sites remain structurally distinct and will exhibit localised oxidation states. This scenario occurs if the rate of electron transfer, $k_{\mathrm{et}}$, is intermediate between the frequencies for solvent reorientations $\left(10^{11}-10^{12} \mathrm{~s}^{-1}\right)$ and bond vibrations $\left(10^{13}-10^{14} \mathrm{~s}^{-1}\right)$, such that $k_{\mathrm{et}} \sim 10^{12}-10^{13} \mathrm{~s}^{-1}$ and $0.7<2 \mathrm{H}_{\mathrm{ab}} / \lambda<1$. On this basis, operational definitions ${ }^{37}$ for the various classes have been proposed: in Class II the solvent and exchanging electron are localised; in Class II-III the solvent modes are averaged but the exchanging electron is localised; and in Class III, the exchanging electron is delocalised and the solvent and vibrational modes are averaged. Although a system is considered to be fully delocalised when $2 \mathrm{H}_{\mathrm{ab}}>\lambda$, the effects of transient charge localisation may still be observed on the IR time-scale for a borderline Class III system. ${ }^{31,37}$

The classification of mixed-valence systems is not straightforward and relies on observations from several experimental techniques with widely different time-scales. ${ }^{37}$ The appearance of IVCT bands and their solvent dependence provides the most useful experimental criterion for distinguishing between Classes II (broad, solvent dependent, localised oxidation states) and II-III (narrow, solvent independent, localised oxidation states). The appearance of symmetrical bridging ligand stretch vibrations s such as $v\left(\right.$ pyz), $v\left(\mathrm{~N}_{2}\right)$ or $\left.v(\mathrm{CO})\right\}$ on the $\leq 10^{-11}$ s IR time-scale provides one of the most reliable markers for electronic localisation. 


\subsubsection{Experimental Studies of Mixed-Valence Systems in the Class II-III Regime}

Clear examples of localised and delocalised dinuclear systems have been reported in several comprehensive surveys of mixed-valence complexes. ${ }^{34,36,43-45,53,56,139,140}$ Class II behaviour has been observed in species of the type $\left[\mathrm{Ru}\left(\mathrm{NH}_{3}\right)_{5}\right\}_{2}(\mu \text {-BL) }]^{5+}$ containing the series of 4,4'-bipyridyl-type bridging ligands shown in Figure 1.5(a), ${ }^{59,60}$ and in $\left[\left\{\mathrm{Ru}(\mathrm{bpy})_{2}(\mathrm{Cl})\right\}_{2}(\mu-\mathrm{pyz})\right]^{5+} \cdot 34,37,43-45$ These systems are characterised by broad Gaussian-shaped and solvent dependent IVCT bands in the near-infrared (NIR) region, which are indicative of weakly-coupled $\mathrm{Ru}^{\mathrm{II}}$ and $\mathrm{Ru}^{\mathrm{III}}$ centres. For $\left[\left\{\mathrm{Ru}\left(\mathrm{NH}_{3}\right)_{5}\right\}_{2}\left(\mu-4,44^{\prime}-b p y\right)\right]^{5+}$, the experimental bandwidth $\left(5200 \mathrm{~cm}^{-1}\right.$ in $\left.0.1 \mathrm{M} \mathrm{DCl} / \mathrm{D}_{2} \mathrm{O}\right)$ is slightly broader than that predicted from equation $1.8\left(4900 \mathrm{~cm}^{-1}\right) .^{60,141}$

Dinolfo and coworkers ${ }^{142-144}$ have recently reported rare examples of ligand-based mixed-valency in a series of singly-reduced molecular rectangles, $\left[\left\{\operatorname{Re}(\mathrm{CO})_{3}\right\}_{2} \mathrm{X}\right]_{2}-\mu, \mu^{\prime}-(\mathrm{LL})_{2}$ shown in Figure 1.5(b), where $\mathrm{X}$ is one of two bridging units (alkoxides, thiazolates or 2,2'-bisbenzimidazolates) and LL is one of a pair of cofacially aligned redox active ligands. The IVCT transitions in the singly-reduced forms originate from direct donor-orbital overlap between the LL moieties, rather than superexchange coupling. The electronic interaction is facilitated by a decrease in the LL/LL approach distance, and an increase in the extent of van der Waals contact and the degree of lateral alignment of LL/LL. ${ }^{142-144}$ These two-state mixed-valence systems lack the complexities in metal-based complexes which occur due to spin-orbit coupling and overlapping IVCT components.

(a)
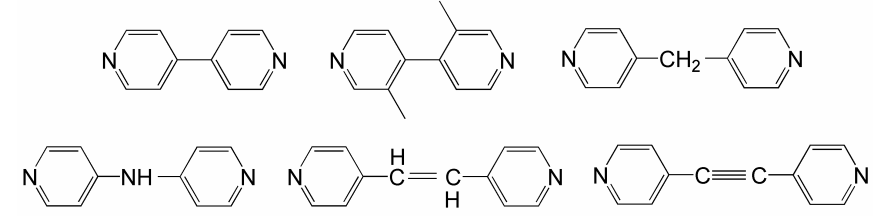

LL

(b)
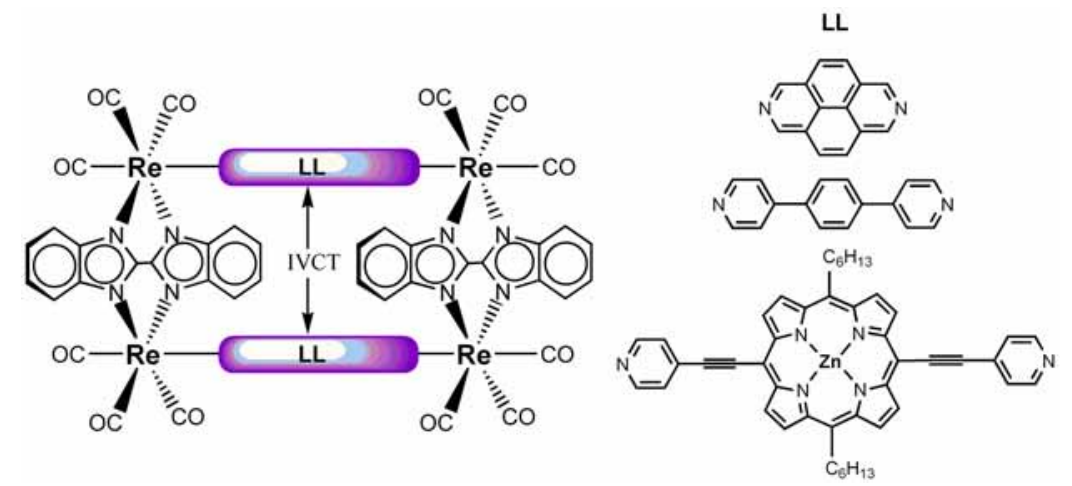

Figure 1.5 (a) Examples of 4,4'-bipyridyl-type bridging ligands used in the construction of Class II mixed-valence systems. $^{59,60}$ (b) Molecular rectangles of the form $\left[\left\{\operatorname{Re}(\mathrm{CO})_{3}\right\}_{2} \mathrm{BiBzIm}\right]_{2}-\mu, \mu$ '- $(\mathrm{LL})_{2}$, where BiBzIm is 2,2'-bisbenzimidazolate and LL are the cofacially aligned ligands. ${ }^{142-144}$

In the Class III systems $\left[\left\{\mathrm{Os}\left(\mathrm{NH}_{3}\right)_{5}\right\}_{2}(\mu-\mathrm{BL})\right]^{5+}\left\{\mathrm{BL}=\mathrm{N}_{2},{ }^{145} \mathrm{pyz}^{146}\right\}$, the enhanced back-bonding ability of Os relative to Ru leads to electronic delocalisation and averaged valences of +2.5 . Symmetrical bridging ligand vibrations $\left\{v\left(\mathrm{~N}_{2}\right)\right.$ or $\left.v(\mathrm{pyz})\right\}$ are of low intensity or absent in the IR region, and the spectra do not display the expected IC marker bands for Os ${ }^{\mathrm{III}}$ which would signal localised oxidation states. $^{37}$ 
By comparison with these well-defined examples of Class II and Class III behaviour, the classification of the Creutz-Taube ion ${ }^{72,73}$ has been the subject of much controversy. ${ }^{147}$ The complex exhibits a narrow $\left(\Delta v_{1 / 2}=1480 \mathrm{~cm}^{-1}\right)$, asymmetrically-shaped IVCT band at $6320 \mathrm{~cm}^{-1}$ which is composed of two underlying components centred at 6320 and $7360 \mathrm{~cm}^{-1}$. Relatively weaker narrow bands also appear at 2000, 3200 and $4700 \mathrm{~cm}^{-1} .{ }^{72,73,75-77}$ The results from an extensive series of experiments including IR, ${ }^{75,76}$ single crystal $\mathrm{EPR}^{77}$ and Stark spectroscopy, ${ }^{78,79}$ led to a consensus in favour of a delocalised ground state for the complex. Resonance Raman measurements ${ }^{39,80}$ provided definitive evidence for a three-state delocalisation mechanism ${ }^{89}, 94$ on the basis of the strong coupling of the IVCT transition to a symmetric vibration mode of pyz. A three-state vibronic coupling model, ${ }^{89,94}$ which explicitly includes an electronic state localised on the bridging ligand, is required to fully explain the spectral properties of the ion. ${ }^{147}$

Meyer and coworkers ${ }^{37}$ have suggested that the spectroscopic properties of the Creutz-Taube ion may also be consistent with a Class II-III classification. On the basis of a valence-localised ground-state and an electronically delocalised excited state, spin-orbit coupling and ligand-field asymmetry could give rise to three IVCT bands. The two lowest energy transitions were assigned as IC transitions, while the bands at 4700, 6320 and $7360 \mathrm{~cm}^{-1}$ were identified as three IVCT transitions \{IVCT(1), (2) and (3), in order of increasing energy $\}$. The $\mathrm{H}_{\mathrm{ab}}$ value of $300 \mathrm{~cm}^{-1}$ for IVCT(1) was indicative of a small degree of residual localisation in the ground state, while the mixed-valence excited states $\{\operatorname{IVCT}(2)$ and (3) $\}$ were relatively more delocalised.

Several recent reviews ${ }^{31,37,57}$ have highlighted a number of systems which exhibit Class II-III behaviour. In the mixed-valence complex $\left[\left\{\mathrm{Os}(\text { bpy })_{2}(\mathrm{Cl})\right\}(\mu-\mathrm{pyz})\left\{\mathrm{Ru}\left(\mathrm{NH}_{3}\right)_{5}\right\}\right]^{4+}$, there is significant electronic coupling, but also evidence of localised oxidation states in the $\mathrm{Os}^{\mathrm{III}}-\mathrm{Ru}^{\mathrm{II}}$ isomer from the appearance of NIR and IR spectral markers for Os ${ }^{\text {III }}{ }^{148,149}$ Despite the strong electronic coupling between the $\mathrm{d} \pi$ obitals along the pyrazine axis, a residual barrier to electron transfer exists because the transferring electron at $\mathrm{Ru}^{\mathrm{II}}$ occupies an orbital which is orthogonal, and only weakly-coupled, to Os ${ }^{\mathrm{III}}$.

Meyer and coworkers have reported an extensive series of Class II-III dinuclear osmium complexes. The symmetrical $\mathrm{N}_{2}$-bridged ${ }^{150,151}$ systems incorporating cis,cis-Os(bpy) $2(\mathrm{Cl})$, trans,trans$\mathrm{Os}(\mathrm{bpy})_{2}(\mathrm{Cl}), \mathrm{Os}(\mathrm{tpm})(\mathrm{Cl})_{2}$ and $\mathrm{Os}(\mathrm{Tp})(\mathrm{Cl})_{2}$ terminal units $\{\mathrm{tpm}=\operatorname{tris}(1$-pyrazoyl $)$ methane, $\mathrm{Tp}=\operatorname{tris}(1-$ pyrazoyl)borate exhibit intense $v\left(\mathrm{~N}_{2}\right)$ stretching vibrations in the IR region which are consistent with electronic localisation on the IR time-scale. Each complex displayed five absorption bands in the region $2500-8500 \mathrm{~cm}^{-1}$. The two lowest energy bands were assigned as $\mathrm{d} \pi \rightarrow \mathrm{d} \pi$ transitions at Os ${ }^{\mathrm{III}}$ and provided additional evidence for localised oxidation states. The three IVCT transitions at higher energy were narrow and solvent independent. The related pyz-bridged mixed-valence system, cis,cis$\left[\left\{\mathrm{Os}(\mathrm{bpy})_{2}(\mathrm{Cl})\right\}_{2}(\mu-\mathrm{pyz})\right]^{3+}$, was also assigned to Class II-III on the basis of the characteristic five-band pattern, and the appearance of an intense $v(\mathrm{pyz})$ vibration. ${ }^{152,153}$ Non-averaged $v(\mathrm{bpy})$ ring-stretching vibrations were observed in the region $1400-1500 \mathrm{~cm}^{-1}$, and were indicative of the localised oxidationstate distribution. This contrasts the situation in the structurally-related Class II system bridged by 
4,4'-bpy in which the $v$ (bpy) vibrations are an average of those in the $\mathrm{Os}^{\mathrm{II}} \mathrm{Os}^{\mathrm{II}}$ and $\mathrm{Os}^{\mathrm{III}} \mathrm{Os}^{\mathrm{III}}$ spectra. $^{37}$ While the $v$ (bpy) and $v$ (pyz) "spectator vibrations" provide useful oxidation state markers, they are only weakly-coupled to the electron transfer and provide a minimal contribution to the barrier. ${ }^{37}$

One of the most comprehensive sets of experimental data for the transition between the localised and delocalised regimes has been observed by variation of the peripheral ligands in the series of the Creutz-Taube ion derivatives, trans- $\left[\left\{\mathrm{Ru}\left(\mathrm{NH}_{3}\right)_{5}\right\}\left(\mu \text {-pyz) }\left\{\mathrm{Ru}\left(\mathrm{NH}_{3}\right)_{4}(\mathrm{~L})\right\}\right]^{5+}\left\{\mathrm{L}=\mathrm{NH}_{3}\right.\right.$, py (pyridine), 3,5-Me 2 py (3,5-dimethylpyridine), 3-Cl-py (3-chloropyridine), 2,6-Me $2 \mathrm{pyz}$ (2,6-dimethylpyrazine) ${ }^{154}$ illustrated in Figure 1.6(a). As shown by Curtis and coworkers, ${ }^{154}$ the introduction of redox asymmetry $\left(\Delta \mathrm{E}_{0}\right)$ by the incorporation of increasingly stronger back-bonding ligands results in a broadening and blue-shifting of the IVCT band.

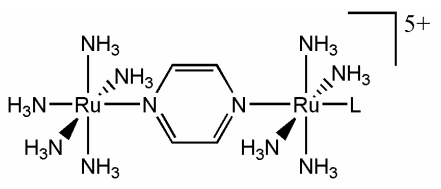

(a)

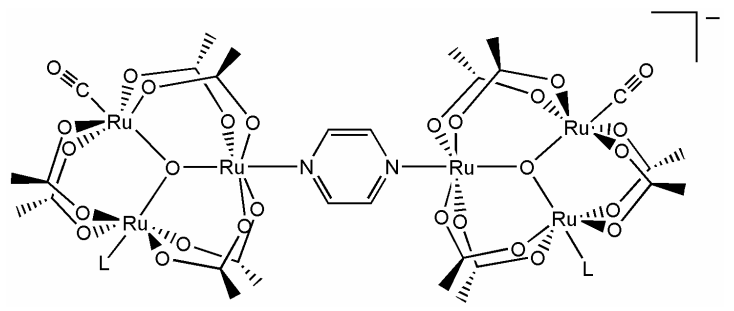

(b)

Figure 1.6 (a) Trans- $\left[\left\{\mathrm{Ru}\left(\mathrm{NH}_{3}\right)_{5}\right\}\left(\mu \text {-pyz) }\left\{\mathrm{Ru}\left(\mathrm{NH}_{3}\right)_{4}(\mathrm{~L})\right\}\right]^{5+}\left\{\mathrm{L}=\mathrm{py}, 3,5-\mathrm{Me} \mathrm{e}_{2} \mathrm{py}, 3-\mathrm{Cl}-\mathrm{py}, 2,6-\mathrm{Me}_{2} \mathrm{pyz}\right\} .{ }^{154}\right.$ (b) Pyrazine-bridged clusters $\left[\left\{\mathrm{Ru}_{3}\left(\mu_{3}-\mathrm{O}\right)\left(\mu-\mathrm{O}_{2} \mathrm{CCH}_{3}\right)_{6}(\mathrm{CO})(\mathrm{L})\right\}_{2}(\mu \text {-pyz) }]^{-}\{\mathrm{L}=4\right.$-(dimethylamino)pyridine, pyridine, 4-cyanopyridine $\}^{106,107}$

Kubiak and coworkers have investigated the IR line broadening and coalescence behaviour due to the dynamic coupling of the $v(\mathrm{CO})$ "spectator vibrations" to electron transfer in the pyrazine-bridged mixed-valence clusters $\left[\left\{\mathrm{Ru}_{3}\left(\mu_{3}-\mathrm{O}\right)\left(\mu-\mathrm{O}_{2} \mathrm{CCH}_{3}\right)_{6}(\mathrm{CO})(\mathrm{L})\right\}_{2}(\mu-\mathrm{pyz})\right]^{-}\{\mathrm{L}=4$-(dimethylamino)pyridine, pyridine, 4-cyanopyridine shown in Figure 1.6(b). ${ }^{106,107}$ Varying $L$ through the series 4(dimethylamino)pyridine, pyridine and 4-cyanopyridine results in an increase in $\mathrm{H}_{\mathrm{ab}}$ and a change in the pattern of CO stretching vibrations from two discrete bands to a single averaged band. This is suggestive of increasing solvent averaging and demonstrates that the rate constant for electron transfer is determined by the solvent reorganisation frequency within the Class II-III region. ${ }^{106-112}$

The localised-to-delocalised transition has also been investigated in the series of 1,4dicyanamidobenzene complexes ${ }^{58,67-71}\left[\left\{\mathrm{Ru}\left(\mathrm{NH}_{3}\right)_{5}\right\}_{2}(\mu-\mathrm{BL})\right]^{3+}$, trans,trans- $\left[\left\{\mathrm{Ru}\left(\mathrm{NH}_{3}\right)_{4}(\mathrm{py})\right\}_{2}(\mu-\mathrm{BL})\right]^{3+}$ and mer,mer- $\left.\left[\mathrm{Ru}\left(\mathrm{NH}_{3}\right)_{3}(\mathrm{bpy})\right\}_{2}(\mu-\mathrm{BL})\right]^{3+}$ \{where BL represents the dianions dicyd ${ }^{2-}(1,4-$ dicyanamidobenzene), $\mathrm{Me}_{2} \mathrm{dicyd}^{2-}$ (1,4-dicyanamido-2,5-dimethylbenzene) and $\mathrm{Cl}_{4}$ dicyd $^{2-}$ (2,3,5,6tetrachloro-1,4-dicyanamidobenzene)\}, and in purely organic mixed-valence systems such as the bistriaryalamine derivatives. ${ }^{64,155,156}$

The complexity of factors which influence intramolecular electron transfer in the localised-todelocalised transition underlie the importance of systematic experimental studies of mixed-valence complexes in this region. However, a limited number of studies exist in which the subtleties in behaviour 
in this transition have been explored. ${ }^{31,36,37,57,74}$ Consequently, there is extensive interest in the preparation of systems to guide and test the development of a "unifying theory" of electron transfer. ${ }^{37,53}$

\subsubsection{Probing the Factors which Govern the Localised-to-Delocalised Transition}

Experimentally, the factors which govern the transition have been probed by the dependence of the IVCT characteristics on structural and substitutional changes in mixed-valence complexes, such as the distance between the metal centres, ${ }^{139}$ the ability of the bridging ligand to delocalise the electronic charge, ${ }^{57}$ as well as the identity and coordination environments of the metal centres. ${ }^{34,37,43-45,56,140}$ Variations in the external environment such as the identity and composition of the solvent, ${ }^{42,53,141,157-167}$ the temperature, ${ }^{168-172}$ and anions in the surrounding medium ${ }^{173-178}$ also provide critical contributions to the electron transfer barrier.

\subsubsection{The Role of the Bridging Ligand: Structural and Electronic Considerations}

Bridging ligands provide the linkage of the metal centres in polynuclear complexes and control the distance and relative orientation of the components, as well as the inter-component electron and energy transfer processes. The role of the bridging ligand has been extensively documented and reviewed $^{24,44,45,61,63,125,179}$ and the pertinent theoretical issues only are mentioned here. Assemblies comprising flexible bridging ligands which contain saturated alkyl or ether linkages $\left\{\right.$ e.g. - $\left.\left(\mathrm{CH}_{2}\right)_{n^{-}},-\mathrm{O}-\right\}$ permit significant rotational and translational movement between components. Since the treatment of electron transfer processes is dependent on well-defined geometries, the theoretical analysis of these systems is complicated by their structural ambiguity. Extended polypyridyl-based bridging ligands which link the component metal centres via a conjugated $\pi$-system are desirable in this regard, as they permit the assembly of rigid systems.

For a dinuclear ligand-bridged complex, the coupling between the donor and acceptor can occur directly via a "through-bond" mechanism, or indirectly via a "through-space" superexchange process where the bridging ligand mediates the donor-acceptor coupling. In reality, the operative mechanism of delocalisation can involve both contributions. Superexchange theory ${ }^{180}$ provides a conceptual framework for bridging ligand effects in intramolecular electron transfer. ${ }^{61,63}$ Electronic coupling between the two metal-based units may comprise bridge-mediated electron transfer (which utilises the lowest unoccupied molecular orbitals, LUMOs, of the bridge) or hole transfer (which utilises the highest occupied molecular orbitals, HOMOs, of the bridge) as shown in Figure 1.7. The parameters which are effective to describe the through-bond interaction are the overlap between the frontier orbitals of the metals $\left\{d \pi\left(M_{1}\right)\right.$ and $\left.\mathrm{d} \pi\left(\mathrm{M}_{2}\right)\right\}$ and the bridging ligand ( $\pi_{\mathrm{BL}}$ and $\pi_{\mathrm{BL}}{ }^{*}$ ), and the electronic interactions between the atoms of the bridge connecting the frontier units to one another.

Class I or weakly-interacting Class II polymetallic complexes comprising two or more noninteracting sites will exhibit a superposition of the individual properties of the constituent sub-units and 
are known as "supramolecular assemblies". ${ }^{25}$ Interacting sites permit the possibility of long-range and vectorial energy or electron transfer processes and are generally Class II or III systems.

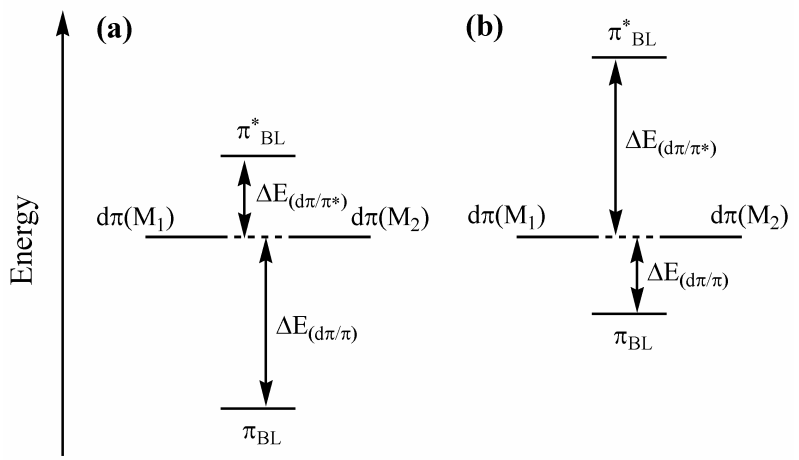

Figure 1.7 Schematic energy level diagrams for superexchange-assisted electronic coupling via (a) an electron transfer mechanism or (b) a hole transfer mechanism. ${ }^{63} \mathrm{M}_{1}$ and $\mathrm{M}_{2}$ denote the metal centres and BL denotes the bridging ligand.

\subsubsection{The Effective Electron Transfer Distance: Stark Absorption Spectroscopy}

As mentioned in §1.3.1.1, the effective (adiabatic) charge transfer distance $\left(r_{12}\right)$ is decreased relative to the geometric distance as electronic coupling across the bridge increases. Equations 1.9 and 1.10 provide a lower limit only for $\mathrm{H}_{\mathrm{ab}}$ when $r_{\mathrm{ab}}$ is identified as the through-space geometrical distance between the metal centres. ${ }^{37}$ Stark effect measurements on the IVCT bands of dinuclear mixed-valence complexes provide a powerful method to determine the effective electron transfer distances by yielding the change in dipole moment for the IVCT transition, $\left|\Delta \mu_{12}\right|$, according to equation 1.26.

$$
r_{12}=\left|\Delta \mu_{12}\right| / e
$$

Stark absorption spectroscopy is the measurement of the change in the absorption spectrum, $\Delta \mathrm{A}(\mathrm{v})$, for an isotropic and immobilised sample in response to an applied electric field, $\mathbf{F}_{\text {ext }}$ (typically 1-4 $\left.\mathrm{MVcm}^{-1}\right){ }^{34,47-51}$ The internal electric field, $\mathbf{F}_{\text {int }}$ experienced by the chromophore is given by equation 1.27 , where $f$ is the local-field correction (typically assumed to be 1.3 for organic solvents ${ }^{47}$ ).

$$
\mathbf{F}_{\text {int }}=f \times \mathbf{F}_{\text {ext }}
$$

The origins of the electric field-induced perturbations on a Gaussian-shaped band are shown schematically in Figure 1.8. The Stark absorption analysis is typically performed according to the classical treatment of Liptay, ${ }^{181}$ in which the $\Delta \mathrm{A}(v)$ vs. $v$ spectrum is a linear combination of the zeroth-, first- and second-order derivatives of the unperturbed absorption spectrum, according to equation 1.28. 

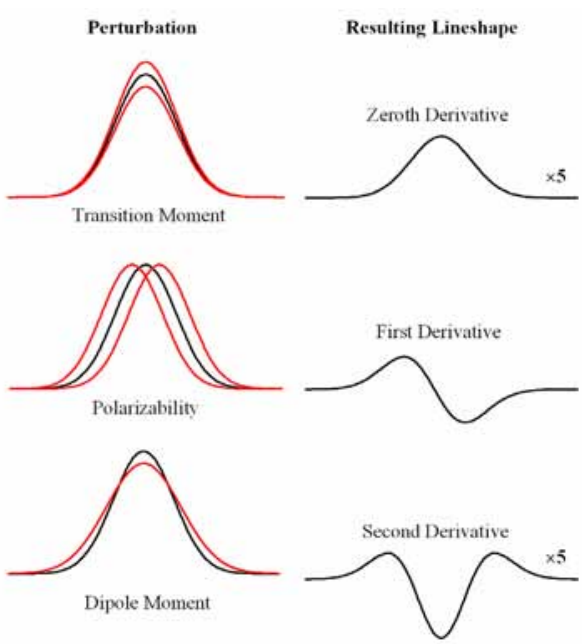

Figure 1.8 Origins of the electric field-induced perturbations on a Gaussian-shaped absorption band in Stark spectroscopy. ${ }^{49}$ Left: Unperturbed absorption band, $\mathrm{A}(v) v s . v(-)$, and the change in this band in response to the applied field (一). Right: The resulting line-shape in the $\Delta \mathrm{A}(v) v$ s. $v$ spectrum.

$$
\Delta \mathrm{A}(v)=\left\{A_{\chi} \mathrm{A}(v)+\frac{B_{\chi} v}{15 h c} \frac{d[\mathrm{~A}(v) / v]}{d v}+\frac{C_{\chi} v}{30 h^{2} c^{2}} \frac{d^{2}[\mathrm{~A}(v) / v]}{d v^{2}}\right\} \mathbf{F}_{\mathrm{int}}{ }^{2}
$$

Here, $\chi$ is the experimental angle between $\mathbf{F}_{\text {ext }}$ and the polarisation of the incident light, $h$ is Planck's constant, and $c$ is the speed of light in a vacuum. The coefficients $A_{\chi}, B_{\chi}$, and $C_{\chi}$ provide information on changes in the transition dipole moment, and the excited state/ground state polarisability and dipole moment differences, respectively, according to:

$$
\begin{aligned}
& A_{\chi}=\frac{\left\langle\alpha_{m}\right\rangle}{3}+\frac{1}{30}\left(3 \cos ^{2} \chi-1\right)\left[3\left\langle\beta_{m}\right\rangle-2\left\langle\alpha_{m}\right\rangle\right] \\
& B_{\chi}=\frac{5}{2} \operatorname{Tr}(\Delta \alpha)+\left(3 \cos ^{2} \chi-1\right)\left[\frac{3}{2} \hat{\mathbf{g}} \cdot \Delta \alpha \cdot \hat{\mathbf{g}}-\frac{1}{2} \operatorname{Tr}(\Delta \alpha)\right] \\
& C_{\chi}=\left|\mu_{v}\right|^{2}\left[5+\left(3 \cos ^{2} \xi-1\right)\left(3 \cos ^{2} \chi-1\right)\right]
\end{aligned}
$$

In these equations, $\left\langle\alpha_{\mathrm{m}}\right\rangle$ and $\left\langle\beta_{\mathrm{m}}\right\rangle$ are the scalar functions of the transition moment polarisability and hyperpolarisability tensors, $\operatorname{Tr}(\Delta \alpha)$ is the trace of the polarisability change between the ground and excited electronic states, $\hat{\mathbf{g}} \cdot \Delta \alpha \cdot \hat{\mathbf{g}}$ is the polarisability change along the transition moment $(\hat{\mathbf{g}}$ is the unit vector), $\left|\mu_{v}\right|$ is the vector change in dipole moment (the sign is not determined), and $\xi$ is the angle between the transition dipole moment and change in dipole moment vectors. ${ }^{49}$ Stark spectra are measured at two or more values of $\chi$ (the incident angle of polarised light in relation to the electric field) and are fitted to a sum of the zeroth-, first- and second-order derivatives of the absorption spectrum to obtain values for the molecular parameters.

For IVCT transitions, $\left|\Delta \mu_{12}\right|$ is derived from the second derivative component and is related to $r_{12}$ via equation 1.26. Since $\left|\mu_{12}\right|$ is known from the unperturbed absorption spectrum, the diabatic dipole 
moment change, $\left|\Delta \mu_{\mathrm{ab}}\right|$, (or equivalently, $r_{\mathrm{ab}}$ since $r_{\mathrm{ab}}=\left|\Delta \mu_{\mathrm{ab}}\right| / e$ ) is determined from equation 1.30. Stark effect spectroscopy thus permits an independent determination of $H_{a b}$ via equation $1.10 .^{32,33,182,183}$

$$
\left|\Delta \mu_{\mathrm{ab}}\right|=e r_{\mathrm{ab}}=\left[\left(\Delta \mu_{12}\right)^{2}+4\left(\mu_{12}\right)^{2}\right]^{1 / 2}
$$

The extent of delocalisation may be assessed by the comparison of $r_{\mathrm{ab}}$ and $r_{12}$ according to equation 1.31. $\Delta q$ is the "actual" amount of charge transferred in the IVCT process and $b^{2}$ is the amount of charge delocalisation in the ground state. $b^{2}$ values of 0 and 0.5 correspond to the limits of complete localisation and delocalisation, respectively. ${ }^{31,48,49}$ As the degree of delocalisation increases, the dipole moment change decreases, and the effective charge transfer distance is shortened relative to the geometrical metal-metal distance. Qualitatively, this scenario will be manifested by a small second derivative contribution to the Stark absorption line-shape.

$$
e r_{12}=(\Delta q) r_{\mathrm{ab}} \quad \text { where, } r_{12}=r_{\mathrm{ab}}\left(1-2 b^{2}\right)
$$

Stark spectroscopic measurements for dinuclear mixed-valence ruthenium and osmium complexes frequently yield effective charge transfer distances which are a fraction only of the geometrical inter-metal distances. ${ }^{47-51}$ For example, the IVCT band at $6320 \mathrm{~cm}^{-1}$ in the Creutz-Taube ion yielded a dipole moment change of $0.7 \pm 0.1 \mathrm{D}$, compared with the value of $32.7 \mathrm{D}$ expected for unit charge transfer across the full geometrical distance. ${ }^{47,78,79}$ The negligible second derivative behaviour of the Stark absorption line-shape and the resulting $b^{2}$ value of $0.46^{48}$ were consistent with the delocalised classification of the system. Stark effect measurements on analogues of the Creutz-Taube ion such as trans- $\left[\left\{\mathrm{Ru}\left(\mathrm{NH}_{3}\right)_{4}(\mathrm{py})\right\}_{2}(\mu-\mathrm{pyz})\right]^{5+},{ }^{184}$ in addition to cyanide-bridged systems such as $\left[\left\{\mathrm{M}(\mathrm{CN})_{5}\right\}_{2}(\mu-\mathrm{CN})\right]^{-}$ $\{\mathrm{M}=\mathrm{Fe}, \mathrm{Ru}, \mathrm{Os}\}^{185-187}$ revealed $r_{12}$ values that corresponded to less than half the inter-metal distances. The consequence of this smaller effective distance is a severe underestimation of the $\mathrm{H}_{\mathrm{ab}}$ values derived from equations 1.9 and 1.10. For example, the $\mathrm{H}_{\mathrm{ab}}$ value of $460 \mathrm{~cm}^{-1}$ for $\left[\left\{\mathrm{Ru}\left(\mathrm{NH}_{3}\right)_{5}\right\}_{2}\left(\mu-4,4^{\prime}-\mathrm{bpy}\right)\right]^{5+}$ which was based on the geometrical distance of $11.3 \AA{ }^{60},{ }^{60}$ was revised upwards to $1020 \pm 130 \mathrm{~cm}^{-1}$ when the effective distance of $5.1 \pm 0.7 \AA$ from Stark effect measurements was employed. ${ }^{188}$ However, the introduction of an effective distance in two-state classical expressions may not provide an accurate measure of the actual degree of electronic coupling if the explicit inclusion of additional electronic states is required. ${ }^{74}$

While the classical Liptay analysis ${ }^{181}$ is applicable to complexes in the weak-coupling Class II and fully-delocalised Class III regimes, the approach is inappropriate for systems in the intermediate localised-to-delocalised regime due to violation of the Born-Oppenheimer approximation. Several studies have attempted to model the "non-Classical" Stark effects for systems in the transition region using threeand four-state semi-classical models ${ }^{66}$ and quantum mechanical vibronic coupling treatments. ${ }^{74,189}$ Stark spectroscopy has also been extended to the IR region, ${ }^{47,189,190}$ and higher order Stark spectroscopy has been introduced ${ }^{47,191}$ to provide more accurate measurements of overlapping absorption bands. 


\subsubsection{Solvent Reorganisational Contributions}

The sensitivity of the IVCT transition to solvent variation is generally accepted as a criterion for distinguishing between localisation or delocalisation. ${ }^{34,36,37,43-45,53}$ The latter is exemplified by the negligible solvent dependence of the IVCT band for $\left[\left\{\mathrm{Ru}(\mathrm{bpy})_{2}\right\}_{2}(\mu-\mathrm{pyz})\right]^{5+}, 72,73$ in contrast to the solvent dependent band for the localised system $\left[\left\{\operatorname{Ru}(\mathrm{bpy})_{2}\right\}_{2}\left(\mu-4,4^{\prime}-\mathrm{bpy}\right)\right]^{5+} \cdot{ }^{60,141}$

While the predictions of the dielectric continuum model (equation 1.15) have been verified in a number of IVCT solvatochromism studies of mixed-valence ruthenium complexes, ${ }^{42,192-198}$ the model breaks down when the underlying assumptions of the classical treatment are invalidated, or in the presence of dielectric saturation effects or specific solvent-solute interactions. The failure of dielectric continuum models has motivated extensive experimental, theoretical and computational studies on the microscopic basis of solvent reorganisation. ${ }^{36,53}$ IVCT solvatochromism studies on complexes of the type $\left[\left\{\mathrm{Ru}(\mathrm{bpy})_{2} \mathrm{Cl}\right\}\left(\mu \text {-pyz) }\left\{\mathrm{Ru}\left(\mathrm{NH}_{3}\right)_{4} \mathrm{~L}\right\}\right]^{4+}\left\{\mathrm{L}=\mathrm{NH}_{3}\right.\right.$, py (pyridine), 3,5-Me 2 py (3,5-dimethylpyridine), 3-Clpy (3-chloropyridine), 2,6-Me ${ }_{2}$ pyz (2,6-dimethylpyrazine) $\}^{199}$ have revealed correlations between $v_{\max }$ and the Gutmann donor number. ${ }^{200}$ Similar effects have been observed in symmetrical mixed-valence species such as $\left[\left\{\mathrm{Ru}\left(\mathrm{NH}_{3}\right)_{4}(\mathrm{~L})\right\}_{2}(\mu-\mathrm{BL})\right]^{5+141,201,202}$ and were ascribed to H-bonding interactions between the ammine ligands of the chromophore and the electron-rich moieties on discrete solvent molecules. ${ }^{203}$ The specific solvation effects occur preferentially at the $\mathrm{Ru}^{\mathrm{III}}$ coordination sphere, and introduce an additional "solvent trapping barrier" which represents a redox asymmetry contribution $\left(\Delta \mathrm{E}_{0}\right)$.

Continuum theories are successful for the treatment of weakly-coupled systems such as $\left[\left\{\mathrm{Ru}\left(\mathrm{NH}_{3}\right)_{5}\right\}_{2}\left(\mu-4,4^{\prime}-\text {-bpy }\right)\right]^{5+}$ when solvents of comparable molecular volumes are used. However, the treatments systematically fail for water and hexamethylphosphoramide (HMPA), which possess significantly different molecular volumes compared with most common solvents. Matyushov and coworkers ${ }^{162,204,205}$ have proposed a molecular solvation theory in which $\lambda_{0}$ is parameterised in terms of the dipole moments $(\mu)$ and core diameters $(\sigma)$ of discrete solvent molecules. This theory accounts for the results of IVCT solvatochromism measurements over a broad range of solvents with significantly different molecular volumes, including $\mathrm{H}_{2} \mathrm{O}$ and HMPA (where $\sigma=2.84$ and $6.87 \AA$, compared with $\sigma=$ 4-5 $\AA$ for most common solvents). ${ }^{162}$

\subsubsection{Structural and Environmental Contributions to the Redox Asymmetry}

Redox asymmetry $\left(\Delta \mathrm{E}_{0}\right)$ contributions are typically assessed experimentally within the classical framework, and provide a means of inducing localisation in a valence-delocalised complex. ${ }^{43}$ Central to the two-state classical analysis is the assumption that a $\Delta \mathrm{E}_{0}$ contribution is manifested spectroscopically as an equivalent variation in $v_{\max }$ (according to equation 1.1) at constant $\mathrm{H}_{\mathrm{ab}}$ and $\lambda .^{31}$ Redox asymmetry effects have been probed through direct perturbation of the inner coordination sphere by terminal ligand substitution, ${ }^{154}$ conformational variations in the bridging ligand structures, ${ }^{45}$ the incorporation of unsymmetrical bridging ligands, or the use of hetero-dinuclear complexes. Environmental $\Delta \mathrm{E}_{0}$ 
contributions include ion-pairing, encapsulation, $\mathrm{pH}$ and temperature effects, in addition to specific solvation effects involving $\mathrm{H}$-bonding interactions with the bridging or terminal ligands. ${ }^{43}, 45,53$

Curtis and coworkers ${ }^{206-209}$ have proposed a semi-quantitative interpretation for $\Delta \mathrm{E}_{0}$ effects, based on a classical two-state perturbation treatment and electrochemical potential measurements. According to equation 1.32, a quantity $m^{\prime}$ is defined as the ratio of the shift in the electrochemical potential at the indirectly perturbed redox site ' $\mathrm{a}$ ' $\left\{\delta \mathrm{E}_{\mathrm{ox}}\left(\mathrm{Ru}_{\mathrm{b}}\right)\right\}$ to the shift at the directly perturbed redox site 'b' $\left\{\delta \mathrm{E}_{\mathrm{ox}}\left(\mathrm{Ru}_{\mathrm{a}}\right)\right\}$, and a quantity $\rho$ is defined as the ratio of the coefficients for the diabatic wavefunctions $\left(\Psi_{\mathrm{a}}\right.$ and $\left.\Psi_{\mathrm{b}}\right)$. The electrochemical shifts are assessed relative to the redox potentials in the unperturbed complex.

$$
\rho=\left(m^{\prime}\right)^{1 / 2}=\frac{b}{a}=\left(\frac{\delta \mathrm{E}_{\mathrm{ox}}\left(\mathrm{Ru}_{\mathrm{b}}\right)}{\delta \mathrm{E}_{\mathrm{Ox}}\left(\mathrm{Ru}_{\mathrm{a}}\right)}\right)^{1 / 2}
$$

The parameters $a^{2}$ and $b^{2}$ represent the fractional valences on the directly and indirectly perturbed metal centres, respectively. Assuming that the overlap between the diabatic wavefunctions is negligible, $a^{2}$ and $b^{2}$ are obtained from the normalisation condition $a^{2}+b^{2}=1$. $\rho$ is determined from the slope $(m)$ of a plot of the change in the formal potentials of the acceptor versus the donor, which are given by $E_{o x}\left(R_{b}\right)$ and $\mathrm{E}_{\mathrm{ox}}\left(\mathrm{Ru}_{\mathrm{a}}\right)$, respectively. From the slope, $b^{2}=$ slope/(1+slope), where $b^{2}$ has been discussed previously in relation to equation $1.31^{*} .31,48$ The parameter $\rho$ provides an alternate measure of the extent of electronic coupling between the metal centres, where $0 \leq \rho \leq 1$, and 0 and 1 are the limits of complete localisation and delocalisation, respectively. The validity of equation 1.32 is expected to become increasingly invalid as the degree of electronic coupling increases. ${ }^{206-209}$ Application of the electrochemical analysis to the series of mixed-valence complexes trans-[\{Ru(NH$\left.)_{5}\right\}\left(\mu \text {-pyz) }\left\{\mathrm{Ru}\left(\mathrm{NH}_{3}\right)_{4}(\mathrm{~L})\right\}\right]^{5+}\left\{\mathrm{L}=\mathrm{NH}_{3}\right.$, py, 3,5$\mathrm{Me}_{2}$ py, 3-Cl-py, 2,6-Me $\mathrm{p}_{2} \mathrm{pyz},{ }^{154}$ yielded a value of $\rho=0.74$ for the Creutz-Taube ion $\left\{\mathrm{L}=\mathrm{NH}_{3}\right\}$, consistent with a delocalised classification.

Similar yet more subtle $\Delta \mathrm{E}_{0}$ contributions may arise from environmental perturbations such as encapsulation effects. ${ }^{104,163,210}$ The addition of the crown ether dibenzo-36-crown-12 to $\left[\left\{\mathrm{Ru}\left(\mathrm{NH}_{3}\right)_{5}\right\}_{2}(\mu-\right.$ pyz) $]^{5+}$ leads to dramatic changes in the IVCT band due to H-bonding interactions between the ammine ligands and the crown ether oxygen atoms. The introduction of the $\Delta \mathrm{E}_{0}$ component on formation of the unsymmetrical 1:1 adduct results in a broader and blue-shifted IVCT band as the degree of localisation increases in the nominally valence-delocalised complex. Further addition of the crown ether forms the symmetrical 2:1 adduct, and the original line-shape which is indicative of delocalisation is largely restored.

While the majority of IVCT measurements have been performed at high electrolyte concentrations of the tetra-n-butylammonium salts of $\mathrm{PF}_{6}{ }_{6}^{-}, \mathrm{ClO}_{4}{ }^{-}$and halide anions $\left(\mathrm{Cl}^{-}, \mathrm{Br}^{-}, \mathrm{I}^{-}\right),{ }^{43,45,53}$ the analyses have often been conducted without regard for potentially significant ion-pairing and ionic strength effects. ${ }^{173-178,202,211,212}$ The IVCT bands for the $\mathrm{I}_{3}{ }^{-}$salt of the biferrocenium cation, ${ }^{213,214}$ and the

\footnotetext{
${ }^{*} b^{2}$ is equivalent to the delocalisation parameter, $\alpha^{2}$, defined by Hush. ${ }^{139}$
} 
$\mathrm{PF}_{6}{ }^{-}$salt of the biferrocenylacetylene cation ${ }^{201}$ were blue-shifted with an increase in the ionic strength of the medium, due to ion-pairing between the complex cations and the counter and electrolyte anions. Similar effects have been observed for dinuclear ruthenium ${ }^{168,177,178,214}$ and iron ${ }^{175,212,214}$ systems. In all cases, the effects were rationalised on the basis of preferential ion-pairing interactions at the smaller more highly charged $\mathrm{M}^{\mathrm{III}}$ terminus of $\left[\mathrm{M}^{\mathrm{II}} \mathrm{M}^{\mathrm{III}}\right]$, resulting in electrostatic stabilisation of the ground state and the introduction of a $\Delta \mathrm{E}_{0}$ component. The ion-pairing contribution has also been assigned as a reorganisational energy due to translation of the counter-ion between the donor and acceptor. ${ }^{53}$

The temperature dependence of the IVCT characteristics for unsymmetrical mixed-valence complexes has been used to assess the fundamental form of the redox asymmetry term; viz. whether this should be viewed as the energy difference between the minima of free energy surfaces, ${ }^{215}$ or as the difference between potential energy surfaces. ${ }^{26,27}$ The temperature dependencies of the IVCT energies for $\left[\left\{\mathrm{Ru}(\mathrm{bpy})_{2} \mathrm{Cl}\right\}\left(\mu \text {-pyz) }\left\{\mathrm{Ru}\left(\mathrm{NH}_{3}\right)_{5}\right\}\right]^{4+}\right.$ and $\left[\left\{\mathrm{Ru}\left(\mathrm{NH}_{3}\right)_{5}\right\}(\mu-\mathrm{NC})\left\{\mathrm{Fe}(\mathrm{CN})_{5}\right\}\right]^{-}$were consistent with an entropic contribution to $v_{\max }$, and the identification of the redox asymmetry contribution as a free energy change. ${ }^{168,170}$ The difference between $\mathrm{d} v_{\max } / \mathrm{dT}$ for $\left[\left\{\mathrm{Ru}(\mathrm{bpy})_{2} \mathrm{Cl}\right\}\left(\mu \text {-pyz) }\left\{\mathrm{Ru}\left(\mathrm{NH}_{3}\right)_{5}\right\}\right]^{4+}\left(-18 \mathrm{~cm}^{-1} \mathrm{~K}^{-1}\right)\right.$ and $\left[\left\{\mathrm{Ru}\left(\mathrm{Me}_{2} \mathrm{bpy}\right)_{2} \mathrm{Cl}\right\}(\mu \text {-pyz })\left\{\mathrm{Ru}\left(\mathrm{NH}_{3}\right)_{4}(4-\mathrm{Mepy})\right\}\right]^{4+}\left(+2 \mathrm{~cm}^{-1} \mathrm{~K}^{-1}\right)$ were also used as arguments for localisation and delocalisation, respectively. ${ }^{53}$ The IVCT bands of symmetrical Class II, and both symmetrical and unsymmetrical Class III complexes, were shown to exhibit relatively weak temperature dependencies. This was speculatively attributed to a weak temperature dependence of $\mathrm{H}_{\mathrm{ab}}$ in the latter case. $^{34}$

There is currently significant interest in elucidating the microscopic origins of redox asymmetry effects for the extension of vibronic coupling models, ${ }^{53,74}$ and the potential application of such effects in molecular switching devices. ${ }^{45}$ Recently, the elucidation of $\Delta \mathrm{E}_{0}$ contributions to the IVCT properties of mutants of the photosynthetic reaction centre in purple bacteria has allowed insights into the relationship between structurally-induced perturbations and the mechanism of the photosynthetic function. ${ }^{8,74}$

\subsubsection{Spin-Orbit Coupling Contributions}

A complication that has not always been acknowledged in mixed-valence analyses is the existence of multiple IVCT transitions. ${ }^{37}$ Due to the effects of ligand field asymmetry and spin-orbit coupling at low-spin $\mathrm{M}^{\mathrm{III}}\left(\mathrm{d} \pi^{5}\right)$ centres $\{\mathrm{M}=\mathrm{Fe}, \mathrm{Ru}, \mathrm{Os}\}$, the $\mathrm{t}_{2 \mathrm{~g}}$ orbitals are split into three $\mathrm{d} \pi$ levels (Kramer's doublets) which are linear combinations of the $\mathrm{d}_{x y}, \mathrm{~d}_{x z}$ and $\mathrm{d}_{y z}$ atomic orbitals. ${ }^{37,52,216-218}$ As shown in Figure 1.9(a), the configurations of the three orbitally non-degenerate states are $\mathrm{d} \pi_{1}{ }^{2} \mathrm{~d} \pi_{2}{ }^{2} \mathrm{~d} \pi_{3}{ }^{1}$, $\mathrm{d} \pi_{1}{ }^{2} \mathrm{~d} \pi_{2}{ }^{1} \mathrm{~d} \pi_{3}{ }^{2}$ and $\mathrm{d} \pi_{1}{ }^{1} \mathrm{~d} \pi_{2}{ }^{2} \mathrm{~d} \pi_{3}{ }^{2}$, in order of increasing energy. The compositions and energy splittings between the levels are governed by the magnitude of the spin-orbit coupling constant of the metal and the electronic character of the surrounding ligands. Two $\mathrm{d} \pi \rightarrow \mathrm{d} \pi$ IC transitions between the Kramer's doublets arise due to the formation of the excited states $\mathrm{d} \pi_{1}{ }^{2} \mathrm{~d} \pi_{2}{ }^{1} \mathrm{~d} \pi_{3}{ }^{2}$ or $\mathrm{d} \pi_{1}{ }^{1} \mathrm{~d} \pi_{2}{ }^{2} \mathrm{~d} \pi_{3}{ }^{2}$. These transitions are nominally parity or LaPorte forbidden and are typically observed in the NIR region as narrow $\left(\Delta v_{1 / 2}<1000 \mathrm{~cm}^{-1}\right)$ solvent independent bands, e.g. for [Os $\left.{ }^{\mathrm{III}}(\mathrm{bpy})_{3}\right]^{3+}$ the IC bands occur at $4580 \mathrm{~cm}^{-1}$ 
$\left(\varepsilon=450 \mathrm{M}^{-1} \mathrm{~cm}^{-1}\right)$ and $5090 \mathrm{~cm}^{-1}\left(\varepsilon=360 \mathrm{M}^{-1} \mathrm{~cm}^{-1}\right)$ in $\mathrm{CD}_{3} \mathrm{CN}^{217}$ In dinuclear mixed-valence systems such as cis,cis- $\left[\left\{\mathrm{Os}(\mathrm{bpy})_{2}(\mathrm{Cl})\right\}_{2}(\mu \text {-pyz })\right]^{3+152,153}$ electronic delocalisation across the bridging ligand mixes charge-transfer character into the $\mathrm{d} \pi \rightarrow \mathrm{d} \pi$ transitions. The IC bands are typically red-shifted and exhibit significantly enhanced intensities compared with their mononuclear analogues.

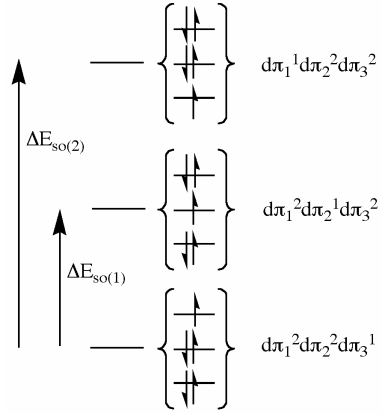

(a)

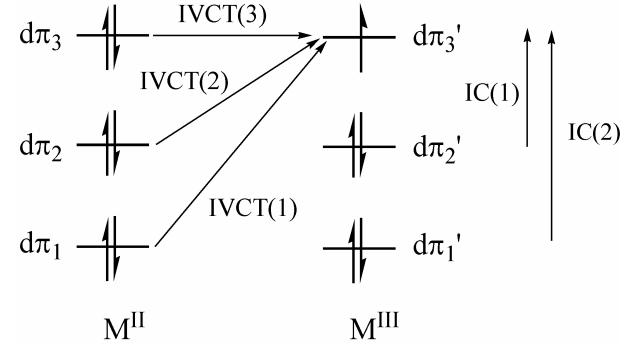

(b)

Figure 1.9 Schematic illustrations of (a) the IC transitions between the energy levels at an $\mathrm{M}^{\mathrm{III}}$ centre; and (b) the IVCT and IC transitions in a mixed-valence dinuclear complex, $\left[\mathrm{M}^{\mathrm{II}} \mathrm{M}^{\mathrm{III}}\right]$.

In addition to two IC transitions, three IVCT transitions arise due to excitation from each of the $\mathrm{M}^{\mathrm{II}}(\mathrm{d} \pi)$ orbitals to the hole at $\mathrm{M}^{\mathrm{III}}$, as shown in Figure 1.9(b). If the spacing between the spin-orbit states is sufficiently large, three distinguishable IVCT transitions will be observed in the spectra of dinuclear mixed-valence complexes. The larger magnitude of the spin-orbit coupling for $\mathrm{Os}^{\mathrm{III}}$ relative to $\mathrm{Ru}^{\mathrm{III}}$ and $\mathrm{Fe}^{\mathrm{III}}\left\{\xi \sim 3000\left(\mathrm{Os}^{\mathrm{III}}\right), 1000\left(\mathrm{Ru}^{\mathrm{III}}\right), 800 \mathrm{~cm}^{-1}\left(\mathrm{Fe}^{\mathrm{III}}\right)\right\}^{37}$ typically leads to the observation of a five-band pattern in the IR and NIR regions, as described in §1.5.2. The appearance of two discernable IC transitions provides a marker for localisation and a Class II or Class II-III classification. ${ }^{37}$ Discrete IVCT components have been observed in a limited number of cases for dinuclear mixed-valence complexes of $\mathrm{Ru}$ and $\mathrm{Fe},{ }^{219}$ as the lesser $\mathrm{d} \pi$ - $\mathrm{d} \pi$ energy gaps typically result in a broad manifold of three closely-spaced IVCT transitions. The IC transitions are weak, and occur at significantly lower energies compared with their Os analogues. Meyer and coworkers ${ }^{37,52}$ have proposed semi-quantitative relationships between the energies of the IC and IVCT bands in weakly-coupled Class II systems:

$$
\begin{aligned}
& \Delta \mathrm{E}_{\mathrm{so}(1)} \approx v_{\max }\{\operatorname{IVCT}(2)\}-v_{\max }\{\operatorname{IVCT}(1)\} \\
& \Delta \mathrm{E}_{\mathrm{so}(2)} \approx v_{\max }\{\operatorname{IVCT}(3)\}-v_{\max }\{\operatorname{IVCT}(1)\}
\end{aligned}
$$

The energy splittings $\Delta \mathrm{E}_{\mathrm{so}(1)}$ and $\Delta \mathrm{E}_{\mathrm{so}(2)}$ represent the $\Delta \mathrm{E}^{\prime}$ contribution to the intramolecular electron transfer barrier in equation 1.1. The separation between the IVCT components in mixed-valence Ru systems may be estimated from the splitting between the IC bands in their dinuclear Os analogues. ${ }^{37}$

The recognition of the existence of IVCT spectral components and knowledge of their relative intensities is crucial for the accurate analysis of IVCT bands. ${ }^{37} \lambda_{i}, \lambda_{0}$ and $H_{a b}$ may differ for the three transitions, and only the lowest energy transition, IVCT(1), corresponds to the thermal electron transfer pathway and can be used to assess the extent of delocalisation in the ground state. 


\subsection{IVCT in Stereoisomeric Mixed-Valence Complexes}

\subsubsection{Stereochemical Considerations}

Polymetallic assemblies which comprise octahedral coordination centres with bidentate ligands exhibit stereoisomerism due to the inherent chirality of the component metal centres. An octahedral metal centre with at least two symmetrical bidentate ligands, such as the archetypal tris(bidentate) species $\left[\mathrm{Ru}(\mathrm{bpy})_{3}\right]^{2+}$, possesses two chiral forms (known as enantiomers), designated $\Lambda$ (left-handed) and $\Delta$ (right-handed), as shown in Figure 1.10. The UV/Vis/NIR spectral, electrochemical and NMR characteristics of the enantiomeric forms are identical in an achiral environment, and differ only when the technique used to probe them is itself chiral, e.g. circular dichroism (CD) spectroscopy. ${ }^{220}$

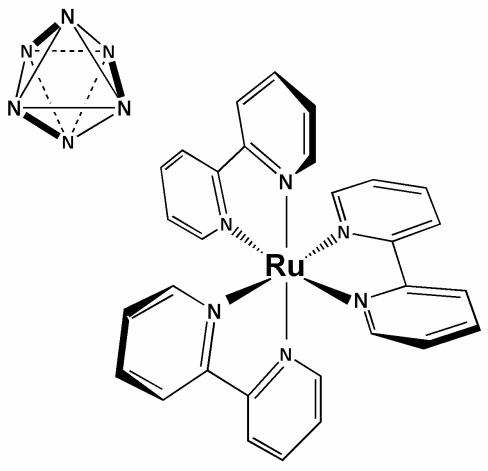

$\Lambda$

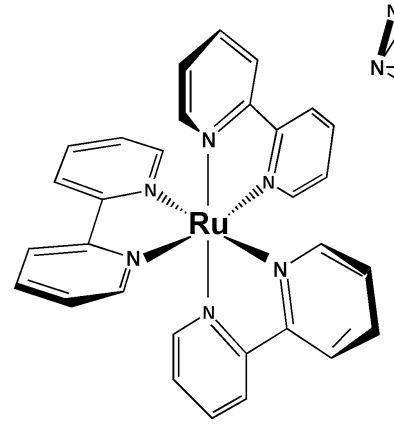

$\Delta$

Figure 1.10 Schematic representations of the enantiomeric forms of $\left[\mathrm{Ru}(\mathrm{bpy})_{3}\right]^{2+}$.

A homo-dinuclear complex of the form $\left[\left\{\mathrm{M}(\mathrm{pp})_{2}\right\}_{2}(\mu-\mathrm{BL})\right]^{4+}$ constitutes the simplest example of a polymetallic assembly, where pp is a symmetrical bidentate ligand such as bpy, and BL is a symmetrical di-bidentate bridging ligand such as bpm (2,2'-bipyrimidine), as shown in Figure 1.11. ${ }^{10,11}$

(a)

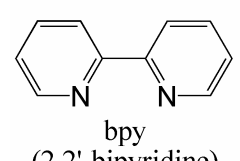

(2,2'-bipyridine)

(b)

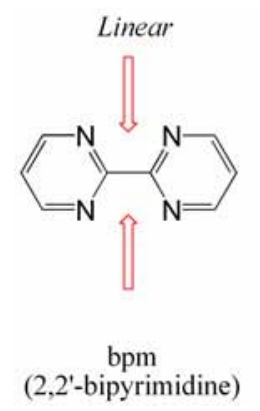

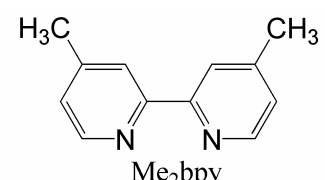

(4,4'-dimethyl-2,2'-bipyridine)

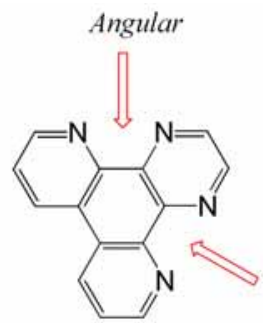

$\mathrm{ppz}$

(4,7-phenanthrolino-5,6:5'6'-pyrazine)

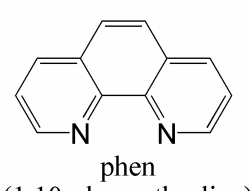

(1,10-phenanthroline)

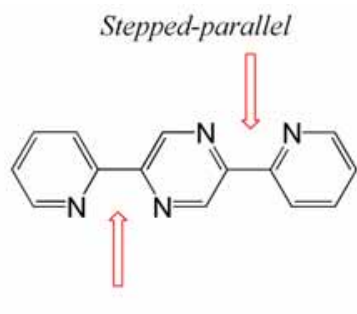

2,5-dpp

(2,5-bis(2-pyridyl)pyrazine)

Figure 1.11 Examples of (a) terminal bidentate and (b) bridging di-bidentate ligands commonly used in the construction of polymetallic assemblies. The bridging ligands may be categorised as linear, angular or steppedparallel based on the relative disposition of the bidentate coordination sites. 
The dinuclear complex $\left[\left\{\mathrm{Ru}(\mathrm{bpy})_{2}\right\}_{2}(\mu-\mathrm{bpm})\right]^{4+}$ may exist in three possible stereoisomeric forms - a meso form $\left(\Delta \Lambda \equiv \Lambda \Delta\right.$; point group symmetry $\mathbf{C}_{2 \mathrm{~h}}$ ), and a racemic (rac) form (point group symmetry $\mathbf{D}_{2}$ ), which comprises the enantiomeric pair $\Delta \Delta$ and $\Lambda \Lambda$ \{Figure 1.12(a)\}. ${ }^{10,11}$ As shown in Figure 1.12(b), the relative positions and coordination environments of the metal centres are identical for each diastereoisomer, however a significant difference may be discerned in the relative orientations of the terminal bpy ligands. ${ }^{10,11}$ When the relationship between the axes of the "bites" of the two coordination sites of the bridging ligand are linear (e.g. bpm) or stepped-parallel (e.g. 2,5-dpp), the terminal ligands "above" and "below" the plane of the bridge are approximately parallel in the rac $(\Delta \Delta / \Lambda \Lambda)$ form and orthogonal in the meso $(\Lambda \Delta)$ diastereoisomer. However, when the "bites" are angular (e.g. ppz), this description is reversed.

(a)
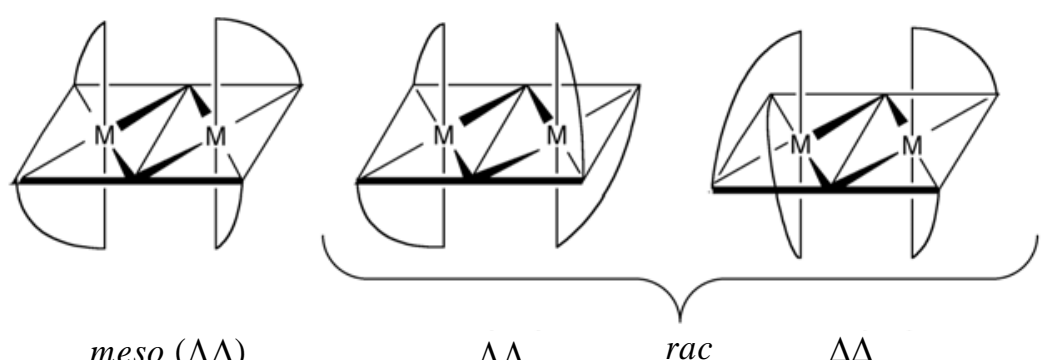

(b)

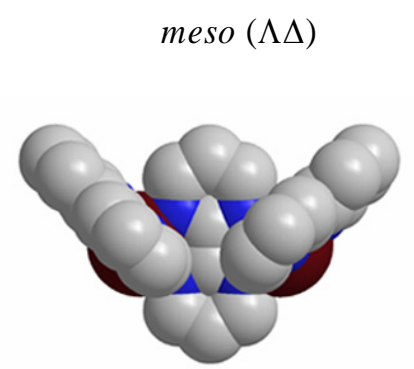

meso $(\Lambda \Delta)$
$\Lambda \Lambda$

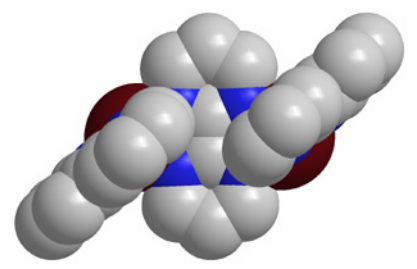

$\operatorname{rac}(\Delta \Delta)$

Figure 1.12 (a) Schematic representation of the two diastereoisomeric forms of $\left[\left\{\mathrm{M}(\mathrm{pp})_{2}\right\}_{2}(\mu-\mathrm{BL})\right]^{4+}\{\mathrm{M}=\mathrm{Ru}$ or Os; $\mathrm{pp}=$ a symmetrical bidentate ligand; $\mathrm{BL}=$ a linear bridging ligand $\}$. (b) Chem3D representations of the meso $(\Lambda \Delta)$ and $\operatorname{rac}(\Delta \Delta)$ diastereoisomers of $\left[\left\{\operatorname{Ru}(\mathrm{bpy})_{2}\right\}_{2}(\mu \text {-bpm) }]^{4+}\right.$. Views from "above" the plane of the bridging ligand. The terminal bpy ligands "below" the plane omitted for clarity.

The stereoisomeric complexity of polymetallic assemblies increases with the nuclearity of the system, and in the presence of unsymmetrical bridging or peripheral ligands. ${ }^{10,11,221}$ In addition to the diastereoisomers induced by the chirality of the tris(bidentate) centres, the presence of any unsymmetrical component introduces geometric isomerism (meridional/facial and/or cis/trans). For homo-dinuclear complexes of the form $\left[\left\{\mathrm{M}(\mathrm{pp})_{2}\right\}(\mu-\mathrm{BL})\left\{\mathrm{M}\left(\mathrm{pp}^{\prime}\right)_{2}\right\}\right]^{4+}\left(\mathrm{pp} \neq \mathrm{pp}^{\prime}\right)$, and hetero-dinuclear complexes of the form $\left[\left\{\mathrm{M}(\mathrm{pp})_{2}\right\}(\mu-\mathrm{BL})\left\{\mathrm{M}^{\prime}(\mathrm{pp})_{2}\right\}\right]^{4+}$ and $\left[\left\{\mathrm{M}(\mathrm{pp})_{2}\right\}(\mu-\mathrm{BL})\left\{\mathrm{M}^{\prime}\left(\mathrm{pp}^{\prime}\right)_{2}\right\}\right]^{4+}$, the $\Delta \Lambda$ and $\Lambda \Delta$ forms are no longer equivalent. These complexities are exemplified for higher nuclearity assemblies such as the dendritic decanuclear complex $\left[\mathrm{Ru}_{10}(2,3-\mathrm{dpp})_{9}(\mathrm{bpy})_{12}\right]^{20+}\{2,3$-dpp $=2$,3-bis(2-pyridyl)pyrazine $\}{ }^{222}$ The ten stereocentres each possess $\Delta$ or $\Lambda$ chirality, and the unsymmetrical bridging ligand 2,3-dpp introduces the possibility of mer/fac isomerism. While the resultant 6144 possible stereoisomers may not all be represented in a synthesised mixture, the presence of as few as two diastereoisomers introduces considerable complexity into the structural characterisation process, as the forms exhibit different NMR spectra. $^{10}$ 


\subsubsection{Solutions to the "Stereochemical Problem"}

The development of general synthetic methodologies using enantiometrically pure "building blocks”, 10, 11, 223, 224 and chromatographic techniques, ${ }^{10,11}$ pioneered by Keene and coworkers have permitted the isolation of the individual stereoisomers (geometric isomers, diastereoisomers and enantiomers) of mono-, di- and trinuclear polypyridyl complexes, as well as helicate species. ${ }^{225}$

(1) Stereoselective Synthetic Strategies. The principle of stereoselective synthesis is based on the construction of stereochemically-pure polymetallic complexes in which the chirality of each metal centre is predetermined by the use of the chiral forms of the precursor building blocks. For example, the complexes $\Delta$ - or $\Lambda$ - $\left[\mathrm{Ru}(\mathrm{pp})_{2}(\mathrm{py})_{2}\right]^{2+}\{\mathrm{pp}=$ bpy, phen $\}$ - which are resolved by diastereoselective crystallisation with $O, O^{\prime}$-dibenzoyltartrate and arsenyl-(+)-tartrate, respectively - retain their chiral integrity upon replacement of the two pyridyl groups. ${ }^{223,224,226,227}$ The development of a general synthetic methodology for tris(heteroleptic) complexes of ruthenium(II), [Ru(pp)(pp')(pp") $]^{2+}$ \{where pp, pp', pp" are bidentate polypyridyl ligands\} is based on the sequential addition of the ligands to the oligomeric precursor $\left[\mathrm{Ru}(\mathrm{CO})_{2} \mathrm{Cl}_{2}\right]_{\mathrm{n}}{ }^{228,229}$ This method provides an additional strategy for the stereoselective synthesis of polynuclear complexes via the $\Delta / \Lambda$-[Ru(pp)(pp')(CO) $\left.)_{2}\right]^{2+}$ intermediate, since the decarbonylation reactions proceed with retention of the stereochemistry. ${ }^{230-235}$ A number of other approaches have been demonstrated for the predetermination of the stereochemistry in polymetallic assemblies, including (i) condensation reactions of chiral monomers of the type $\Delta / \Lambda$ - $\left[\operatorname{Ru}(\mathrm{phen})_{2}(1,10\right.$ phenanthroline-5,6-dione) $]^{2+}$, 236-240 (ii) the use of “chiragens” based on [4,5]-pineno-2,2'-bipyridine which impose a chirality on the attached metal centres ("stereospecificity"), ${ }^{241-243}$ and (iii) the chiral resolution of mononuclear polypyridylruthenium(II) complexes using TRISPHAT anions as resolving agents. ${ }^{24-246}$ The stereochemical complexities have been avoided in a number of cases by the synthesis of achiral mononuclear species such as $\left[\mathrm{Ru}(\text { tpy })_{2}\right]^{n+}\left\{\right.$ tpy $=2,2^{\prime}: 6,2$ "-terpyridine $\}$, in which the tridentate tpy ligands coordinate in a meridional fashion about the metal centre. ${ }^{16,24,247}$

(2) Chromatographic Methodologies. A general cation-exchange chromatographic method for the separation of stereoisomeric mixtures has been developed using SP Sephadex C-25 as the support. ${ }^{10,11}$ The mechanism for the separation involves the differential association between the diastereoisomers of the same complex and eluent anions such as toluene-4-sulfonate $\left\{\mathrm{CH}_{3}\left(\mathrm{C}_{6} \mathrm{H}_{4}\right) \mathrm{SO}_{3}{ }^{-}\right\}$. The nature of the interaction has been ascribed to specific $\pi$-stacking and hydrophobic effects. ${ }^{248}$ Enantiomeric resolutions are enabled by the use of chiral eluent anions such as (-)-O,O'-dibenzoyltartrate, or by virtue of the inherent chirality of the Sephadex support itself.

The combination of the abovementioned stereoselective synthetic and chromatographic techniques permits the subtle and designed control of the coordination identities, geometries and electronic characteristics of the resulting polymetallic assemblies. This provides a unique opportunity to synthesise target assemblies in which the spatial consequences of intramolecular electron transfer can be intimately probed. 


\subsubsection{Stereochemical Effects on Intramolecular Electron Transfer}

Over the past two decades polymetallic assemblies incorporating ligands such as those in Figure 1.11 have been the subject of extensive research efforts due to their potential as the basis of novel molecular materials. ${ }^{24,179,249-251}$ However, there has been little recognition of the stereochemical complexities which are inherent in such assemblies, and measurements on their physical properties have often been conducted on stereoisomeric mixtures. ${ }^{10,11}$

Keene and coworkers reported the first four examples of differences in the spectral, electrochemical and photophysical properties of the stereoisomers in mono-, di- and trinuclear systems. ${ }^{252-255}$ Recent studies have also demonstrated the differential association between the enantiomeric and diastereoisomeric forms of such systems with DNA. ${ }^{256-260}$

(1) A mononuclear chromophore-quencher system. A comparative study was undertaken on the photophysical properties of the four separate geometric isomers (one trans and three cis) of the chromophore-quencher system $\left[\mathrm{Ru}\left(\mathrm{Me}_{2} \mathrm{bpy}\right)\left(\mathrm{bpy}-\mathrm{MQ}^{2+}\right)(\mathrm{bpy}-\mathrm{PTZ})\right]^{4+}\left\{\right.$ bpy-MQ $\mathrm{MQ}^{2+}=1-\left[\left(4^{\prime}-\right.\right.$ methyl-2,2'bipyridin-4-yl)methyl]-1'-methyl-4,4'-bipyridinediium cation; bpy-PTZ = 10-[(4'-methyl-2,2'-bipyridin-4yl)methyl]phenothiazine; Figure 1.13(a)\}. The study revealed differences in the lifetimes of the chargeseparated states $\left[\mathrm{Ru}^{\mathrm{II}}\left(\mathrm{Me}_{2} \mathrm{bpy}\right)\left(\mathrm{bpy}-\mathrm{MQ}^{\cdot+}\right)\left(\mathrm{bpy}-\mathrm{PTZ}^{\circ+}\right)\right]^{4+}$ for all the isomers, with the rate for backelectron transfer varying between 4.5 and $8.7 \times 10^{6} \mathrm{~s}^{-1} \cdot 253,255$

(2) Diastereoisomers incorporating the $\alpha$-azodiimine bridges. Differences in the separation of the two metal-based oxidation processes and the energies of the MLCT bands were observed between the diastereoisomeric forms of $\left[\left\{\mathrm{Ru}(\mathrm{pp})_{2}\right\}(\mu-\mathrm{BL})\left\{\mathrm{Ru}\left(\mathrm{pp}^{\prime}\right)\right]^{4+}\right.$ \{where pp, pp' = bpy and/or $\mathrm{Me}_{2} \mathrm{bpy}$; BL = apy (azobis(2-pyridine)) or mapy (azobis(4-methyl-2-pyridine)); Figure 1.13(b)\}. ${ }^{252}$ However, the origins of these differences were not elucidated.

(3) Di- and trinuclear complexes of HAT. Emission studies on $\left[\left\{\mathrm{Ru}(\mathrm{bpy})_{2}\right\}_{2}(\mu-\mathrm{HAT})\right]^{4+}$ and $\left[\left\{\mathrm{Ru}(\mathrm{bpy})_{2}\right\}_{3}(\mu \text {-HAT })\right]^{6+}\{\mathrm{HAT}=1,4,5,8,9,12$-hexaazatriphenylene; Figure 1.13(c) $\}$ revealed decreased luminescence quantum yields and emission lifetimes for the meso versus the rac forms in the dinuclear case, and between the heterochiral $\left(\Delta_{2} \Lambda / \Lambda_{2} \Delta\right)$ and homochiral $\left(\Delta_{3} / \Lambda_{3}\right)$ forms in the trinuclear case. ${ }^{254}$

(4) Diastereoisomers incorporating the $d p b$ bridge. Electrochemical studies on the diastereoisomers of $\left[\left\{\mathrm{Ru}(\mathrm{bpy})_{2}\right\}_{2}(\mu \text {-dpb) }]^{4+}\{\mathrm{dpb}=\right.$ 2,3-bis(2-pyridyl)-1,4-benzoquinoxaline; Figure 1.13(d) $\}$ in the presence of different electrolyte anions revealed cathodic shifts in the redox potentials for the metal-based oxidation processes in the sequence $\mathrm{PF}_{6}{ }^{-}<\mathrm{BF}_{4}{ }^{-} \sim \mathrm{ClO}_{4}{ }^{-}<$toluene-4-sulfonate ${ }^{-261}$ The meso diastereoisomer consistently exhibited a greater $\Delta \mathrm{E}_{\mathrm{ox}}$ compared with the rac form, which was attributed to preferential ion-pairing. 


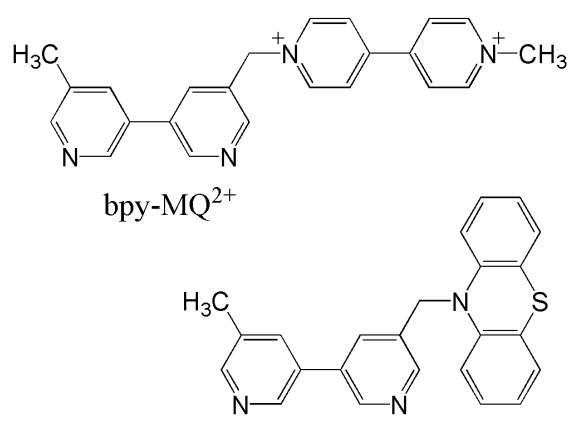

(a)

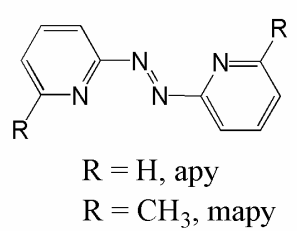

(b)

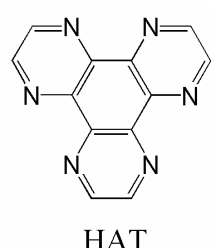

(c)

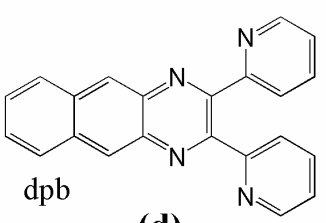

(d)

Figure 1.13 Examples of ligands used in the construction of mono-, di- and trinuclear complexes exhibiting stereochemically-dependent intramolecular electron transfer characteristics: (a) bpy-MQ ${ }^{2+}$ and bpy-PTZ, and the bridging ligands (b) apy and mapy, (c) HAT and (d) dpb.

From these initial studies, it is evident that the inherent stereochemical identity of mono-, di- and trinuclear complexes influences the fundamental electron transfer processes that occur in these systems. While the origins of such stereochemical dependencies have not been elucidated, the differential association of the meso and rac diastereoisomers in (2) and (4) with electrolyte anions may account for the variation in the electrochemical and spectral responses in each case. Indeed, these differential interactions permit the separation of the stereoisomers via cation-exchange chromatography, ${ }^{10,11}$ and it is plausible that such outer-sphere interactions at the molecular level also have significant influences on the electron transfer properties.

\subsection{Scope and Objectives of the Present Study: Stereochemical Effects on IVCT}

The elucidation of stereochemical effects on IVCT in the diastereoisomeric forms of dinuclear polypyridyl complexes of ruthenium and osmium provide a means to elucidate the microscopic origins of the factors which influence the electron transfer barrier. The two major thrusts of the present study are outlined below.

\section{(1) Stereochemical Effects on the Fundamental Factors that Govern the Electron Transfer Barrier}

As discussed in §1.5.2 and §1.5.3, experimental studies of IVCT in dinuclear mixed-valence complexes have provided crucial insights into the contributions of Franck-Condon reorganisational factors and redox asymmetry, and have enabled verification of the predictions of classical, semi-classical and quantum mechanical theoretical models of electron transfer. To date, the majority of experimental IVCT studies have been conducted by varying global features of the complexes, such as the identity and coordination environment of the metal centres, or through variations in the macroscopic features of the environment such as the solvent, anions and temperature. In many cases, the theoretical implications of the results have been complicated by ion-pairing, and ambiguities in the geometries of the complexes due to a lack of structural rigidity and/or stereoisomeric purity. ${ }^{10,11}$ 
The present study represents the first systematic investigation of the IVCT properties in the mixed-valence forms of the diastereoisomers of $\left[\left\{\mathrm{M}(\mathrm{pp})_{2}\right\}_{2}(\mu-\mathrm{BL})\right]^{5+}$ \{where $\mathrm{M}=\mathrm{Ru}$ or Os; pp denotes bidentate ligands such as those shown in Figure 1.11(a), and BL represents linear, angular and steppedparallel bridging ligands such as those shown in Figure 1.11(b)\}. These systems exhibit several attractive features over dinuclear complexes which have been employed to date for IVCT studies: (i) the complexes are structurally rigid; and (ii) the dimensions of the clefts may be varied in a subtle and systematic manner through stereochemical variation, bridging ligand modification, or the judicious positioning of substituents on the terminal polypyridyl ligands - while maintaining the identity and coordination environments of the component metal centres. A further important feature is the possibility of extensive tuning of the ground state electronic properties by the incorporation of bridging and terminal ligands possessing different $\pi$-accepting and $\sigma$-donating properties. ${ }^{24,25,228,251,262}$

(i) Solvent Reorganisational Energy Contributions. The subtle variations in the dimensions of the clefts between the diastereoisomers of symmetrical dinuclear complexes offer a new and intimate probe of reorganisational contributions to intramolecular electron transfer due to solvent association at the molecular level. $\lambda_{i}$ and $\Delta \mathrm{E}^{\prime}$ are expected to be identical for the diastereoisomers of the same complex, so that IVCT solvatochromism studies of the forms permit a direct probe of spatially-directed solvent effects on $\lambda_{0}$.

(ii) Mapping the Localised-to-Delocalised Transition in IVCT. The variation in the electronic properties of the mixed-valence complexes $\left[\left\{\mathrm{M}(\mathrm{pp})_{2}\right\}_{2}(\mu-\mathrm{BL})\right]^{5+}$ provides a means for examining changes in the IVCT characteristics whilst traversing the localised-to-delocalised transition. The validity of the two-state classical model for determining the extent of delocalisation between the different complexes is assessed by application of the electrochemical and spectral analysis proposed by Curtis and coworkers. ${ }^{207}$, 263 As discussed in \$1.5.3.4, this method is based upon the study of a closely-related series of complexes in which the redox asymmetry is systematically varied through synthetic modifications or environmental influences. An examination of anion and temperature effects on the IVCT parameters as a function of their stereochemical identity, offers further detailed insights into redox asymmetry contributions to the electron transfer barrier.

The consideration of the multiple IVCT transitions in the spectra of the mixed-valence species, and the effective distance transferred by the electron, are essential for the accurate analysis of IVCT transitions and the degree of ground state delocalisation. Stark effect measurements on $\left[\left\{\mathrm{M}(\mathrm{bpy})_{2}\right\}_{2}(\mu-\right.$ $\mathrm{BL})]^{5+}\{\mathrm{M}=\mathrm{Ru}, \mathrm{Os}\}$ provide unambiguous determinations of the effective electron transfer distances, and subsequently, independent measures of $\mathrm{H}_{\mathrm{ab}}$. The stereochemical dependence of $\Delta \mathrm{E}^{\prime}$ is probed through the analysis of the IC transitions in the series of dinuclear osmium complexes.

\section{(2) Extending the IVCT Probe to Trinuclear Polymetallic Assemblies}

To date, IVCT studies have focused predominantly on dinuclear mixed-valence complexes, while experimental and theoretical studies on higher nuclearity polymetallic assemblies are relatively scarce. 
The elucidation of the origins of stereochemical effects on the factors that influence the electron transfer barrier in dinuclear complexes has important implications for controlling inter-metal interactions in higher nuclearity systems. Through a systematic investigation of IVCT in di- and trinuclear polypyridyl complexes of ruthenium and osmium, the present study addresses the following influences on IVCT: (i) the increasing nuclearity and oxidation state of the assembly; (ii) the nature of the bridging ligand; (iii) the relative distance, orientation and stereochemical relationship of the component metal centres; and (iv) the overall geometry of the assembly.

\subsection{Overview of the Thesis}

The work reported herein addresses these thrusts by systematically probing stereochemical effects on the fundamental factors that govern electronic delocalisation and the barrier to electron transfer.

Chapter 2 examines the IVCT solvatochromism properties of meso- and rac-[ $\left\{\mathrm{Ru}(\mathrm{pp})_{2}\right\}_{2}(\mu-$ $\mathrm{BL})]^{5+}$ incorporating linear bridging ligands. The dependence of the stereochemically-directed solvent effects on the dimensionality of the clefts, and the number, size, orientation and location of the solvent dipoles within the clefts is examined using a homologous series of nitrile solvents.

Chapter 3 extends the investigation of solvent reorganisational effects to complexes of the form $\left[\left\{\mathrm{M}(\mathrm{bpy})_{2}\right\}_{2}(\mu-\mathrm{BL})\right]^{5+}(\mathrm{M}=\mathrm{Ru}, \mathrm{Os})$, where BL represents a series of angular and stepped-parallel bridging ligands. Variations in the electronic properties of the bridging ligands provide insights into the subtle influence of stereochemical and structural factors on the electron transfer barrier. The IVCT and IC characteristics in the series of osmium complexes are contrasted with their dinuclear ruthenium analogues. Stark effect spectroscopy provides measures of the effective versus the geometrical metalmetal distances.

Chapter 4 examines IVCT in stereochemically-unambiguous di- and trinuclear assemblies based on the HAT and ppz bridging ligands. The degree to which the IVCT process is influenced by the nuclearity, oxidation state and overall geometry of the systems is elucidated through the comparative study of the mixed-valence diastereoisomeric forms of $\left[\left\{\mathrm{M}_{1}(\mathrm{bpy})_{2}\right\}(\mu-\mathrm{BL})\left\{\mathrm{M}_{2}(\mathrm{bpy})_{2}\right\}\right]^{5+}\left(\mathrm{M}_{1} \mathrm{M}_{2}=\mathrm{RuRu}\right.$, RuOs, OsOs), and the mixed-valence forms of the trinuclear complexes, which are "cluster-type" $\left[\left\{\mathrm{Ru}(\mathrm{bpy})_{2}\right\}_{3}(\mu-\mathrm{HAT})\right]^{n+}$ and $\left[\left\{\mathrm{Ru}(\mathrm{bpy})_{2}\right\}_{2}\left\{\mathrm{Os}(\mathrm{bpy})_{2}\right\}(\mu-\mathrm{HAT})\right]^{n+}$ or "chain-like” $\left[\left\{\operatorname{Ru}(\mathrm{bpy})_{2}\right\}_{2}\left\{\mathrm{Ru}(\mathrm{bpy})(\mu-\mathrm{ppz})_{2}\right\}\right]^{n+}(n=7,8)$. The study provides the link between the IVCT studies on dinuclear systems in Chapters 2 and 3, and the understanding of inter-component electron transfer in extended arrays and supramolecular systems.

The Epilogue consolidates the major findings of the study, highlights the shortcomings of the experimental and theoretical analysis, and emphasises the implications of the research within the context of the existing framework of IVCT. 


\subsection{Publications}

Some aspects of the work described in this thesis have been published, the references of which are cited below, and are included on the accompanying CD.

- "Stereochemical Influences on Intervalence Charge Transfer in Homo-dinuclear Complexes of Ruthenium”, D’Alessandro, D. M.; Kelso, L. S.; Keene, F. R. Inorganic Chemistry, 2001, 40, 6841-6844.

- "Mono- and Dinuclear Complexes of the Ligands 3,4-di(2-pyridyl)-1,2,5-oxadiazole and 3,4di(2-pyridyl)-1,2,5-thiadiazole: New Bridges Allowing Unusually Strong Metal-Metal Interactions”, Richardson, C.; Steel, P. J.; D’Alessandro, D. M.; Junk, P. C.; Keene, F. R. Journal of the Chemical Society, Dalton Transactions, 2002, 2775-2785.

- "Ruthenium(II) Complexes of Multidentate Ligands Derived from Di(2-pyridyl)methane”, D’Alessandro, D.M.; Keene, F. R., Steel, P. J.; Sumby, C. J. Australian Journal of Chemistry, 2003, 56(7), 657-664.

- "A Cautionary Warning on the Use of Electrochemical Measurements to Calculate Comproportionation Constants for Mixed-Valence Compounds”, D’Alessandro, D. M.; Keene, F. R. Dalton Transactions, 2004, 23, 3950-3954.

- "Intervalence Charge Transfer in the Stereoisomers of a Dinuclear Ruthenium Complex Containing the Bridging Ligand Dibenzoeilatin”, D’Alessandro, D. M.; Keene, F. R.; Bergman, S. D.; Kol, M. Dalton Transactions, 2005, 2, 332-337.

- "Intervalence Charge Transfer (IVCT) in Ruthenium Dinuclear and Trinuclear Assemblies Containing the Bridging Ligand HAT (1,4,5,8,9,12-hexaazatriphenylene)”, D’Alessandro, D. M.; Keene, F. R. Chemistry, A European Journal, 2005, 11(12), 3679-3688.

- "Ion-Pairing and Thermochromism in a Series of Dinuclear Ruthenium Complexes", D’Alessandro, D. M.; Junk, P. C.; Keene, F. R. Supramolecular Chemistry, 2005, in press. \{Invited contribution in special Australasian issue.\}

- “Diastereoisomers as Probes for Solvent Reorganisational Effects in IVCT”, D’Alessandro, D. M.; Keene, F. R. Chemical Physics, 2005, in press. \{Invited contribution for inclusion in a special issue in honour of Professor Noel Hush.\}

- "Driving the Localised-to-Delocalised Transition in the Diastereoisomers of Unsymmetrical Dinuclear Ruthenium Mixed-Valence Complexes”, D’Alessandro, D. M.; Keene, F. R., Australian Journal of Chemistry, 2005, in press.

- "Intervalence Charge Transfer in a "Chain-like” Ruthenium Trinuclear Assembly Based on the Bridging Ligand 4,7-phenanthrolino-5,6:5',6'-pyrazine (ppz)”, D’Alessandro, D. M.; Keene, F. R., Dalton Transactions, 2005, in press. 
- "The Effective Electron Transfer Distance in Dinuclear Ruthenium Complexes Containing the Unsymmetrical Bridging Ligand 3,5-bis(2-pyridyl)-1,2,4-triazolate”, D’Alessandro, D. M.; Dinolfo, P. H.; Hupp, J. T.; Junk, P.C.; Keene, F. R. European Journal of Inorganic Chemistry, accepted for publication.

- "Metal-Metal Interactions in Dinuclear Ruthenium Complexes incorporating "Stepped-Parallel” Bridging Ligands: Synthesis, Stereochemistry and Intervalence Charge Transfer”, D’Alessandro, D. M.; Keene, F. R. New Journal of Chemistry, accepted for publication.

- "Intervalence Charge Transfer (IVCT) in Trinuclear and Tetranuclear Complexes of Iron, Ruthenium and Osmium”, D’Alessandro, D. M.; Keene, F. R., proposal accepted for submission to Chemical Reviews, submitted for publication.

- "Current Trends and Future Challenges in the Experimental, Theoretical and Computational Analysis of Intervalence Charge Transfer (IVCT) Transitions”, D’Alessandro, D. M.; Keene, F. R., Chemical Society Reviews, invited review, submitted for publication.

- “The Underlying Spin-Orbit Coupling Structure of the Intervalence Charge Transfer Bands in Dinuclear Polypyridyl Complexes of Ruthenium and Osmium”, D’Alessandro, D. M.; Dinolfo, P. H.; Hupp, J. T.; Davies, M.S.; Keene, F. R., submitted for publication to Inorganic Chemistry.

- "Solid-State Anion Interactions in the Diastereoisomers of Dinuclear Ruthenium Complexes Based on 2,2'-bipyrimidine”, D’Alessandro, D. M.; Foley, F. M.; Davies, M.S.; Junk, P. C.; Keene, F. R., submitted for publication to Polyhedron.

- "Metal-Metal Interactions in Dinuclear Ruthenium Complexes Containing Bridging 4,5-di(2pyridyl)imidazolates”, Slater, J. W.; D’Alessandro, D. M.; Steel, P. J.; Keene, F. R., submitted for publication to Dalton Transactions.

- "Multi-site Effects on Intervalence Charge Transfer in a "Cluster-like” Trinuclear Assembly Containing Ruthenium and Osmium”, D’Alessandro, D. M.; Davies, M. S.; Keene, F. R., submitted for publication to Inorganic Chemistry.

- "Probing the Transition between the Localised ("Class II”) and Localised-to Delocalised ("Class II-III”) Regimes using Intervalence Charge Transfer Solvatochromism in a Series of MixedValence Dinuclear Ruthenium Complexes”, D’Alessandro, D. M.; Topley, A.C.; Davies, M.S.; Keene, F. R., to be submitted for publication.

This work has also been the subject of a number of oral and poster presentations by the candidate:

- Poster and Invited Oral Presentation: "Stereochemical Effects on Intervalence Charge Transfer in Polymetallic Supramolecular Assemblies”, D’Alessandro, D. M.; Keene, F. R., National Conference of the Division of Inorganic Chemistry, Royal Australian Chemical Institute, 
Melbourne, Australia, February 2003. \{This presentation received one of two Don Stranks Awards for the best student presentation at the conference.\}

- Poster Presentation: “Stereochemical Effects on Intervalence Charge Transfer: New Insights into the Factors which Affect Intramolecular Electron Transfer in Metallosupramolecular Assemblies”, D’Alessandro, D. M.; Keene, F. R., International Conference of Macrocyclic Chemistry, Cairns, Australia, July 2004.

- Poster Presentation: "New Insights into the Factors which Affect Intramolecular Electron Transfer in Metallosupramolecular Assemblies”, D’Alessandro, D. M.; Keene, F. R., Townsville Festival of Life Sciences, Townsville, Australia, November 2004. \{This presentation received two poster prizes sponsored by Progen Biosciences and Livingstone International.\}

- Invited Oral Presentation: "Fundamental Stereochemical Effects on Intervalence Charge Transfer in Polymetallic Assemblies”, D’Alessandro, D. M.; Keene, F. R., Symposium in Honour of the 80 ${ }^{\text {th }}$ Birthday of Professor Noel Hush, Sydney, Australia, July 2005.

- Oral Presentation: "Stereochemical Effects on Intervalence Charge Transfer in Polymetallic Assemblies”, D’Alessandro, D. M.; Keene, F. R.; Dinolfo, P. H.; Hupp, J. T., National Conference of the Royal Australian Chemical Institute, Sydney, Australia, July 2005.

- Poster Presentation: "Extending the Intervalence Charge Transfer Probe to Trinuclear Polymetallic Assemblies”, D’Alessandro, D. M.; Keene, F. R, National Conference of the Royal Australian Chemical Institute, Sydney, Australia, July 2005. \{This presentation received an Inorganic Chemistry Division poster prize.\} 


\subsection{References}

1. $\quad$ Allen, G. C.; Hush, N. S. Prog. Inorg. Chem. 1967, 8, 357-389.

2. $\quad$ Robin, M. B.; Day, P. Adv. Inorg. Chem. Radiochem. 1967, 10, 247-403.

3. $\quad$ Glauser, R.; Hauser, U.; Herren, F.; Ludi, A.; Roder, P.; Schmidt, E.; Siegenthaler, H.; Wenk, F. J. Am. Chem. Soc. 1973, 95, 8457-8458.

4. $\quad$ Haim, A.; Wilmarth, W. K. J. Am. Chem. Soc. 1961, 83, 509-516.

5. Vahrenkamp, H.; Gieß, A.; Richardson, G. N. J. Chem. Soc., Dalton Trans. 1997, 3643-3651.

6. $\quad$ Day, P. In Mixed-Valence Compounds, Brown, D. B. Ed.; D. Reidel Publishing Company: Oxford, 1979; pp 3-24.

7. $\quad$ Ludi, A. In Mixed-Valence Compounds, Brown, D. B. Ed.; D. Reidel Publishing Company: Oxford, 1979; pp 25-71.

8. $\quad$ Reimers, J. R.; Hush, N. S. J. Am. Chem. Soc. 2004, 126, 4123-4144.

9. $\quad$ Hughes, J. M.; Hutter, M. C.; Reimers, J. R.; Hush, N. S. J. Am. Chem. Soc. 2001, 123, 8550-8563.

10. Keene, F. R. Coord. Chem. Rev. 1997, 166, 122-159.

11. Keene, F. R. Chem. Soc. Rev. 1998, 27, 185-193.

12. Juris, A.; Campagna, S.; Bidd, I.; Lehn, J.-M.; Ziessel, R. Inorg. Chem. 1988, 27, 4007-4011.

13. Meyer, T. J. Acc. Chem. Res. 1989, 22, 163-170.

14. Gust, D.; Moore, T. A.; Moore, A. L.; Kang, H. K.; Degraziano, J. M.; Liddell, P. A.; Seely, G. R. J. Phys. Chem. 1993, 97, 13637-13642.

15. Wasielewski, M. R. Chem. Rev. 1992, 92, 435-461.

16. Sauvage, J.-P.; Collin, J.-P.; Chambron, J. C.; Guillerez, S.; Coudret, C.; Balzani, V.; Barigelletti, F.; De Cola, L.; Flamigni, L. Chem. Rev. 1994, 94, 993-1019.

17. Wasielewski, M. R.; Gaines, G. L.; Wiederrecht, G. P.; Svec, W. A.; Niemczyk, M. P. J. Am. Chem. Soc. 1993, 115, 10442-10443.

18. $\quad$ Kurreck, H.; Huber, M. Angew. Chem., Int. Ed. Engl. 1995, 34, 849-866.

19. Crossley, M. J.; Sintic, P. J.; Walton, R.; Reimers, J. R. Org. Biomol. Chem. 2003, 1.

20. $\quad$ Fox, M. A.; Jones, W. E.; Watkins, D. M. Chem. Eng. News 1993, 38-48.

21. $\quad$ Larson, S. L.; Elliott, C. M.; Kelley, D. F. J. Phys. Chem. 1995, 99, 6530-6539.

22. Bignozzi, C. A.; Argazzi, R.; Scandola, F. Gazz. Chim. Ital. 1996, 126, 199-210.

23. Scandola, F.; Argazzi, R.; Bignozzi, C. A.; Chiorboli, C.; Indelli, M. T.; Rampi, M. A. In Supramolecular Chemistry; Balzani, V., De Cola, L., Eds.; Kluwer Academic Publishers: Dordrecht; 1992, pp 235-248.

Sutin, N. Acc. Chem. Res. 1982, 15, 275-282.

Brunschwig, B. S.; Creutz, C.; Sutin, N. Chem. Soc. Rev. 2002, 31, 168-184.

Creutz, C.; Newton, M. D.; Sutin, N. J. Photochem. 1994, 82, 47-59.

Cave, R. J.; Newton, M. D. Chem. Phys. Lett. 1996, 249, 15-19.

Crutchley, R. J. Adv. Inorg. Chem. 1994, 41, 273-325.

Meyer, T. J. In Mixed-Valence Compounds, Brown, D. B. Ed.; D. Reidel Publishing Company: 1980; pp 75-113.

Barbara, P. F.; Meyer, T. J.; Ratner, M. A. J. Phys. Chem. 1996, 100, 13148-13168.

Demadis, K. D.; Hartshorn, C. M.; Meyer, T. J. Chem. Rev. 2001, 101, 2655-2685.

Myers, A. B. Chem. Rev. 1996, 96, 911-926.

Hupp, J. T.; Williams, R. D. Acc. Chem. Res. 2001, 34, 808-817.

Marcus, R. A. J. Chem. Phys. 1957, 26, 867-871.

Marcus, R. A. J. Chem. Phys. 1956, 24, 966-978.

Sullivan, B. P.; Curtis, J. C.; Kober, E. M.; Meyer, T. J. Nouv. J. Chim. 1980, 4, 643-650.

Creutz, C. Prog. Inorg. Chem. 1983, 30, 1-73.

Kalyanasundaram, K.; Nazeeruddin, M. K. Inorg. Chim. Acta 1994, 226, 213-230.

Ward, M. D. Chem. Soc. Rev. 1995, 24, 121-134.

Creutz, C.; Chou, M. H. Inorg. Chem. 1987, 26, 2995-3000.

Bublitz, G. U.; Boxer, S. G. Annu. Rev. Phys. Chem. 1997, 48, 213-242.

Brunschwig, B. S.; Creutz, C.; Sutin, N. Coord. Chem. Rev. 1998, 177, 61-79.

Walters, K. A. In Comprehensive Coordination Chemistry II; Meyer, T. J., McCleverty, J. A. Eds.;

Elsevier: Oxford, 2004; Vol.2, pp 303-313.

50. Ferretti, A. Coord. Chem. Rev. 2003, 238-239, 127-141.

51. Vance, F. W.; Williams, R. D.; Hupp, J. T. Int. Rev. Phys. Chem. 1998, 17, 307-329. 
52. $\quad$ Kober, E. M.; Goldsby, K. A.; Narayana, D. N. S.; Meyer, T. J. J. Am. Chem. Soc. 1983, 105, $4303-4309$.

53. $\quad$ Chen, P.; Meyer, T. J. Chem. Rev. 1998, 98, 1439-1477.

54. Powers, M. J. J. Am. Chem. Soc. 1980, 102, 1289-1297.

55. Shin, Y. G. K.; Brunschwig, B. S.; Creutz, C.; Newton, M. D.; Sutin, N. J. Phys. Chem. 1996, 100, 1104-1110.

56. Launay, J.-P. Chem. Soc. Rev. 2001, 30, 386-397.

57. Nelsen, S. F. Chem. Eur. J. 2000, 6, 581-588.

58. $\quad$ Evans, C. E. B.; Naklicki, M. L.; Rezvani, A. R.; White, C. A.; Kondratiev, V. V.; Crutchley, R. J. J. Am. Chem. Soc. 1998, 120, 13096-13103.

59. $\quad$ Sutton, J. E.; Sutton, P. M.; Taube, H. Inorg. Chem. 1979, 18, 1017-1021.

60. Sutton, J. E.; Taube, H. Inorg. Chem. 1981, 20, 3125-3134.

61. Richardson, D. E.; Taube, H. Coord. Chem. Rev. 1984, 60, 107-129.

62. Goldsby, K. A.; Meyer, T. J. Inorg. Chem. 1984, 23, 3002-3010.

63. Giuffrida, G.; Campagna, S. Coord. Chem. Rev. 1994, 135, 517-531.

64. Lambert, C.; Nöll, G. J. Am. Chem. Soc. 1999, 121, 8434-8442.

65. Reimers, J. R.; Hush, N. S. Inorg. Chem. 1990, 29, 3686-3697.

66. Sando, G. M. Ph.D. Thesis, Northwestern University (Evanston, I.L., USA), 2003.

67. Aquino, M. A. S.; Lee, F. L.; Gabe, E. J.; Bensimon, C.; Greedan, J. E.; Crutchley, R. J. J. Am. Chem. Soc. 1992, 114, 5130-5140.

68. Naklicki, M. L.; Crutchley, R. J. J. Am. Chem. Soc. 1994, 116, 6045-6046.

69. Naklicki, M. L.; Crutchley, R. J. Inorg. Chim. Acta 1994, 225, 123-127.

70. Mosher, P. J.; Yap, G. P. A.; Crutchley, R. J. Inorg. Chem. 2001, 40, 1189-1195.

71. Al-Noaimi, M.; Yap, G. P. A.; Crutchley, R. J. Inorg. Chem. 2004, 43, 1773-1778.

72. Creutz, C.; Taube, H. J. Am. Chem. Soc. 1969, 91, 3988-3989.

73. Creutz, C.; Taube, H. J. Am. Chem. Soc. 1973, 95, 1086-1094.

74. Reimers, J. R.; Hush, N. S. Chem. Phys. 1996, 208, 177-193.

75. Best, S. P.; Clark, R. J. H.; McQueen, R. C. S.; Joss, S. J. Am. Chem. Soc. 1989, 111, 548-550.

76. Fürholz, U.; Bürgi, H.-B.; Wagner, F. E.; Stebler, A.; Ammeter, J. H.; Krausz, E.; Clark, R. J. H.; Stead, M. J.; Ludi, A. J. Am. Chem. Soc. 1984, 106, 121-123.

77. Beattie, J. K.; Hush, N. S.; Taylor, P. R. Inorg. Chem. 1976, 15, 992-993.

78. Oh, D. H.; Boxer, S. G. J. Am. Chem. Soc. 1990, 112, 8161-8162.

79. Oh, D. H.; Sano, M.; Boxer, S. G. J. Am. Chem. Soc. 1991, 113, 6880-6890.

80. Lu, H.; Petrov, V.; Hupp, J. T. Chem. Phys. Lett. 1995, 235, 521-527.

81. Piepho, S. B.; Krausz, E. R.; Schatz, P. N. J. Am. Chem. Soc. 1978, 100, 2996-3005.

82. Wong, K. Y.; Schatz, P. N. Prog. Inorg. Chem. 1981, 28, 369-449.

83. $\quad$ Prassides, K.; Schatz, P. N. J. Phys. Chem. 1989, 93, 8387-8387.

84. Tanner, M.; Ludi, A. Inorg. Chem. 1981, 20, 2350-2352.

85. Wong, K. Y.; Schatz, P. N. Chem. Phys. Lett. 1984, 108, 484-489.

86. Wong, K. Y. Inorg. Chem. 1984, 23, 1285-1290.

87. Reimers, J. R.; Shapley, W. A. J. Chem. Phys. 2003, 119, 3249-3261.

88. Piepho, S. B. J. Am. Chem. Soc. 1988, 110, 6319-6326.

89. Piepho, S. B. J. Am. Chem. Soc. 1990, 112, 4197-4206.

90. Root, L. J.; Ondrechen, M. J. Chem. Phys. Lett. 1982, 93, 421-424.

91. Ko, J.; Ondrechen, M. J. Chem. Phys. Lett. 1984, 112, 507-512.

92. Ondrechen, M. J.; Ko, J.; Root, L. J. J. Phys. Chem. 1984, 88, 5919-5923.

93. Ko, J.; Ondrechen, M. J. J. Am. Chem. Soc. 1985, 107, 6161-6167.

94. Ondrechen, M. J.; Ko, J.; Zhang, L.-T. J. Am. Chem. Soc. 1987, 109, 1672-1676.

95. Zhang, L.-T.; Ko, J.; Ondrechen, M. J. J. Am. Chem. Soc. 1987, 109, 1666-1671.

96. Zhang, L.-T.; Ko, J.; Ondrechen, M. J. J. Phys. Chem. 1989, 93, 3030-3034.

97. Zhang, L.-T.; Ondrechen, M. J. Inorg. Chim. Acta 1994, 226, 43-51.

98. $\quad$ Ondrechen, M. J.; Ferretti, A.; Lami, A.; Villani, G. J. Phys. Chem. 1994, 98, 11230-11232.

99. $\quad$ Ferretti, A.; Lami, A.; Ondrechen, M. J.; Villani, G. J. Phys. Chem. 1995, 99, 10484-10491.

100. Murga, L. F.; Ondrechen, M. J. Theor. Chim. Acta. 1995, 90, 331-339.

101. Murga, L. F.; Ferretti, A.; Lami, A.; Ondrechen, M. J.; Villani, G. Inorg. Chem. Commun. 1998, 1, 137-140.

102. Ferretti, A.; Lami, A.; Murga, L. F.; Shehadi, I. A.; Ondrechen, M. J.; Villani, G. J. Am. Chem. Soc. 1999, 121, 2594-2596.

103. Petrov, V.; Hupp, J. T.; Mottley, C.; Mann, L. C. J. Am. Chem. Soc. 1994, 116, 2171-2172.

104. Hupp, J. T.; Dong, Y. Inorg. Chem. 1994, 33, 4421-4424.

105. Londergan, C. H.; Kubiak, C. P. J. Phys. Chem. A 2003, 107, 9301-9311.

106. Ito, T.; Hamaguchi, T.; Nagino, H.; Yamaguchi, T.; Kido, H.; Zavarine, I. S.; Richmond, T.; Washington, J.; Kubiak, C. P. J. Am. Chem. Soc. 1999, 121, 4625-4632. 
107. Ito, T.; Hamaguchi, T.; Nagino, H.; Yamaguchi, T.; Washington, J.; Kubiak, C. P. Science 1997, 277, 660-663.

108. Londergan, C. H.; Kubiak, C. P. Chem. Eur. J. 2003, 9, 5962-5969.

109. Ito, T.; Imai, N.; Yamaguchi, T.; Hamaguchi, T.; Londergan, C. H.; Kubiak, C. P. Angew. Chem., Int. Ed. 2004, 43, 1375-1381.

110. Londergan, C. H.; Salsman, C.; Ronco, S.; Kubiak, C. P. Inorg. Chem. 2003, 42, 926-928.

111. Londergan, C. H.; Salsman, J. C.; Ronco, S.; Dolkas, L. M.; Kubiak, C. P. J. Am. Chem. Soc. 2002, 124, $6236-6237$.

112. Yamaguchi, T.; Imai, N.; Ito, T.; Kubiak, C. P. Bull. Chem. Soc. Jpn. 2000, 73, 1205-1212.

113. Pople, J. A.; Beveridge, D. L.; Dobosh, P. A. J. Chem. Phys. 1967, 47, 2026-2033.

114. Pople, J. A.; Berendge, D. L., Aproximate Molecular Orbital Theory; McGraw Hill: New York, 1970.

115. Bencini, A.; Ciofini, I.; Daul, C. A.; Ferretti, A. J. Am. Chem. Soc. 1999, 121, 11418-11424.

116. Braun-Sand, S. B.; Wiest, O. J. Phys. Chem. A 2003, 107, 285-291.

117. Estiú, G.; Cukiernik, F. D.; Maldivi, P.; Poizat, O. Inorg. Chem. 1999, 38, 3030-3039.

118. Patra, S.; Sarkar, B.; Ghumaan, S.; Fiedler, J.; Zális, S.; Kaim, W.; Lahiri, G. K. Dalton Trans. 2004, 750-753.

119. Constantino, V. R. L.; Toma, H. E.; de Oliveira, L. F. C.; Rein, F. N.; Rocha, R. C.; de Oliveira Silva, D. J. Chem. Soc., Dalton Trans. 1999, 1735-1740.

120. Harden, N. C.; Humphery, E. R.; Jeffery, J. C.; Lee, S.-M.; Marcaccio, M.; McCleverty, J. A.; Rees, L. H.; Ward, M. D. J. Chem. Soc., Dalton Trans. 1999, 2417-2426.

121. Behrendt, A.; Couchman, S. M.; Jeffery, J. C.; McCleverty, J. A.; Ward, M. D. J. Chem. Soc., Dalton Trans. 1999, 4349-4355.

122. Metcalfe, R. A.; Vasconcellos, L. C. G.; Mirza, H.; Franco, D. W.; Lever, A. B. P. J. Chem. Soc., Dalton. Trans. 1999, 2653-2667.

123. Masui, H.; Freda, A. L.; Zerner, M. C.; Lever, A. B. P. Inorg. Chem. 2000, 39, 141-152.

124. da Cunha, C. J.; Dodsworth, e. D.; Monteiro, M. A.; Lever, A. B. P. Inorg. Chem. 1999, 38, 5399-5409.

125. Newton, M. D. Chem. Rev. 1991, 91, 767-792.

126. Hush, N. S.; Reimers, J. R. Coord. Chem. Rev. 1998, 177, 37-60.

127. Hush, N. S.; Reimers, J. R. Chem. Rev. 2000, 100, 775-786.

128. Reimers, J. R.; Hush, N. S. J. Phys. Chem. A 1999, 103, 3066-3072.

129. Zeng, J.; Hush, N. S.; Reimers, J. R. J. Am. Chem. Soc. 1996, 118, 2059-2068.

130. Zeng, J.; Craw, J. S.; Hush, N. S.; Reimers, J. R. J. Phys. Chem. 1994, 98, 11075-11088.

131. Zeng, J.; Hush, N. S.; Reimers, J. R. J. Phys. Chem. 1996, 100, 9561-9567.

132. Zeng, J.; Hush, N. S.; Reimers, J. R. J. Phys. Chem. 1995, 99, 10459-10470.

133. Reimers, J. R.; Hush, N. S. Chem. Phys. 1989, 134, 323-354.

134. Reimers, J. R.; Hush, N. S. J. Photochem. 1994, 82, 31-46.

135. Reimers, J. R.; Hush, N. S. Inorg. Chem. 1990, 29, 4510-4513.

136. Reimers, J. R.; Hughes, J. M.; Hush, N. S. Biochemistry 2000, 39, 16185-16189.

137. Reimers, J. R.; Shapley, W. A. J. Chem. Phys. 2003, 119, 3240-3248.

138. Reimers, J. R. J. Chem. Phys. 2003, 119, 3262-3277.

139. Hush, N. S. Coord. Chem. Rev. 1985, 64, 135-157.

140. Kaim, W.; Klein, A.; Glöckle, M. Acc. Chem. Res. 2000, 33, 755-763.

141. Hupp, J. T.; Weydert, J. Inorg. Chem. 1987, 26, 2657-2660.

142. Dinolfo, P. H.; Williams, M. E.; Stern, C. L.; Hupp, J. T. J. Am. Chem. Soc. 2004, 126, 12989-13001.

143. Dinolfo, P. H.; Hupp, J. T. J. Am. Chem. Soc. 2004, 126, 16814-16819.

144. Dinolfo, P. H.; Lee, S. J.; Coropceanu, V.; Brédas, J.-L.; Hupp, J. T. Inorg. Chem. 2005, 44, 5789-5797.

145. Dubicki, L.; Ferguson, J.; Krausz, E. R.; Lay, P. A.; Maeder, M.; Magnuson, R. H.; Taube, H. J. Am.

Chem. Soc. 1985, 107, 2167-2171.

146. Dubicki, L.; Ferguson, J.; Krausz, E. R. J. Am. Chem. Soc. 1985, 107, 179-182.

147. Hupp, J. T. In Comprehensive Coordination Chemistry II, Meyer, T. J. McCleverty, J. A. Eds.; Elsevier Ltd.: Oxford, 2004; Vol. 2, pp 709-716.

148. Neyhart, G. A.; Hupp, J. T.; Curtis, J. C.; Timpson, C. J.; Meyer, T. J. J. Am. Chem. Soc. 1996, 118, 3724-3729.

149. Neyhart, G. A.; Timpson, C. J.; Bates, W. D.; Meyer, T. J. J. Am. Chem. Soc. 1996, 118, 3730-3737.

150. Demadis, K. D.; El-Samanody, E.-S.; Coia, G. M.; Meyer, T. J. J. Am. Chem. Soc. 1999, 121, 535-544.

151. Demadis, K. D.; Meyer, T. J.; White, P. S. Inorg. Chem. 1997, 36, 5678-5679.

152. Demadis, K. D.; Neyhart, G. A.; Kober, E. M.; Meyer, T. J. J. Am. Chem. Soc. 1998, 120, 7121-7122.

153. Demadis, K. D.; Neyhart, G. A.; Kober, E. M.; White, P. S.; Meyer, T. J. Inorg. Chem. 1999, 38, 5948-5959.

154. de la Rossa, R.; Chang, P. J.; Salaymeh, F.; Curtis, J. C. Inorg. Chem. 1985, 24, 4229-4231.

155. Lambert, C.; Nöll, G. Angew. Chem., Int. Ed. Engl. 1998, 37, 2107-2110.

156. Lambert, C.; Nöll, G.; Schelter, J. Nature Mater. 2002, 1, 69-76.

157. Nelsen, S. F.; Trieber, D. A., II; Ismagilov, R. F.; Teki, Y. J. Am. Chem. Soc. 2001, 123, 5684-5694. 
158. Hupp, J. T.; Weaver, M. J. Inorg. Chem. 1984, 23, 3639-3644.

159. Katriel, J.; Ratner, M. A. J. Phys. Chem. 1989, 93, 5065-5070.

160. Drago, R. S.; Richardson, D. E.; George, J. E. Inorg. Chem. 1997, 36, 25-32.

161. Barthel, E. R.; Martini, I. B.; Schwartz, B. J. J. Phys. Chem. B 2001, 2001, 12230-12241.

162. Matyushov, D. V.; Schmid, R. J. Phys. Chem. 1994, 98, 5152-5159.

163. Roberts, J. A.; Hupp, J. T. Inorg. Chem. 1992, 31, 157-160.

164. Blackbourn, R. L.; Hupp, J. T. J. Phys. Chem. 1988, 92, 2817-2820.

165. Blackbourn, R. L.; Hupp, J. T. Inorg. Chem. 1989, 28, 3786-3790.

166. Ennix, K. S.; McMahon, P. T.; de la Rosa, R.; Curtis, J. C. Inorg. Chem. 1987, 26, 2660-2666.

167. Brunschwig, B. S.; Ehrenson, S.; Sutin, N. J. Phys. Chem. 1986, 90, 3657-3668.

168. Hupp, J. T.; Neyhart, G. A.; Meyer, T. J.; Kober, E. M. J. Phys. Chem. 1992, 96, 10820-10830.

169. Hupp, J. T.; Dong, Y. H. J. Am. Chem. Soc. 1993, 115, 6428-6429.

170. Dong, Y.; Hupp, J. T. Inorg. Chem. 1992, 31, 3322-3324.

171. Catterjee, D.; Bajaj, H. C.; Das, A. Inorg. Chim. Acta 1994, 224, 189-192.

172. D'Alessandro, D. M.; Kelso, L. S.; Keene, F. R. Inorg. Chem. 2001, 40, 6841-6844.

173. Nelsen, S. F.; Ismagilov, R. F. J. Phys. Chem. A 1999, 103, 5373-5378.

174. Marcus, R. A. J. Phys. Chem. B 1998, 102, 10071-10077.

175. Pereztejeda, P.; Neto-Ponce, P.; Sánchez, F. J. Chem. Soc., Dalton Trans. 2001, 1686-1691.

176. Lau, K. W.; Hu, A. M. H.; Yen, M. H. J.; Fung, E. Y.; Grzybicki, S.; Matamoros, R.; Curtis, J. C. Inorg. Chim. Acta 1994, 226, 137-143.

177. Lewis, N. A.; Obeng, Y. S.; Purcell, W. L. Inorg. Chem. 1989, 28, 3796-3799.

178. Lewis, N. A.; Obeng, Y. S. J. Am. Chem. Soc. 1988, 110, 2306-2307.

179. Garcia, C. G.; de Lima, J. F.; Iha, N. Y. M. Coord. Chem. Rev. 2000, 196, 219-247.

180. McConnell, H. M. J. Chem. Phys. 1961, 35, 508-515.

181. Liptay, W. Angew. Chem., Int. Ed. Engl. 1969, 8, 177-188.

182. Shin, Y.-g. K.; Brunschwig, B. S.; Creutz, C.; Sutin, N. J. Phys. Chem. 1996, 100, 8157-8169.

183. Reimers, J. R.; Hush, N. S. J. Phys. Chem. 1991, 95, 9773-9781.

184. Williams, R. D.; Hupp, J. T. In Book of Abstracts, Proceedings of the $215^{\text {th }}$ ACS National Meeting, Dalas, Texas, USA, 1998; American Chemical Society: Washington, D. C., 1998.

185. Karki, L.; Lu, H. P.; Hupp, J. T. J. Phys. Chem. 1996, 100, 15637-15639.

186. Karki, L.; Hupp, J. T. J. Am. Chem. Soc. 1997, 119, 4070-4073.

187. Vance, F. W.; Karki, L.; Reigle, J. K.; Hupp, J. T.; Ratner, M. A. J. Phys. Chem. A 1998, 102, 8320-8324.

188. Hupp, J. T.; Dong, Y. H.; Blackbourn, R. L.; Lu, H. J. Phys. Chem. 1993, 97, 3278-3282.

189. Treynor, T. P.; Boxer, S. G. J. Phys. Chem. B 2004, 108, 13513-13522.

190. Chattopadhyay, A.; Boxer, S. G. J. Am. Chem. Soc. 1995, 117, 1449-1450.

191. Bublitz, G. U.; Laidlaw, W. M.; Denning, R. G.; Boxer, S. G. J. Am. Chem. Soc. 1998, 120, 6068-6075.

192. Powers, M. J.; Salmon, D. J.; Callahan, R. W.; Meyer, T. J. J. Am. Chem. Soc. 1976, 98, 6731-6733.

193. Callahan, R. W.; Brown, G. M.; Meyer, T. J. Inorg. Chem. 1975, 14, 1443-1453.

194. Callahan, R. W.; Meyer, T. J. Chem. Phys. Lett. 1976, 39, 82-84.

195. Powers, M. J.; Callahan, R. W.; Salmon, D. J.; Meyer, T. J. Inorg. Chem. 1976, 15, 1457-1459.

196. Curtis, J. C.; Meyer, T. J. J. Am. Chem. Soc. 1978, 100, 6284-6286.

197. Callahan, R. W.; Keene, F. R.; Meyer, T. J.; Salmon, D. J. J. Am. Chem. Soc. 1977, 99, 1064-1073.

198. Powers, M. J.; Meyer, T. J. Inorg. Chem. 1978, 17, 1785-1790.

199. Chang, J. P.; Fung, E. Y.; Curtis, J. C. Inorg. Chem. 1986, 25, 4233-4241.

200. Gutmann, V. Electrochim. Acta 1976, 21, 661-670.

201. Blackbourn, R. L.; Hupp, J. T. Chem. Phys. Lett. 1988, 150, 399-405.

202. Blackbourn, R. L.; Hupp, J. T. J. Phys. Chem. 1990, 94, 1788-1793.

203. Curtis, J. C.; Sullivan, B. P.; Meyer, T. J. Inorg. Chem. 1983, 22, 224-250.

204. Elliott, c. M.; Derr, D. L.; Matyushov, D. V.; Newton, M. D. J. Am. Chem. Soc. 1998, 120, 11714-11726.

205. Matyushov, D. V. Chem. Phys. 1993, 174, 199-218.

206. Curtis, J. C.; Bernstein, J. S.; Schmehl, R. H.; Meyer, T. J. Chem. Phys. Lett. 1981, 81, 48-52.

207. Salaymeh, F.; Berhane, S.; Yusof, R.; Delarosa, R.; Fung, E. Y.; Matamoros, R.; Lau, K. W.; Zheng, Q.; Kober, E. M.; Curtis, J. C. Inorg. Chem. 1993, 32, 3895-3908.

208. Dong, Y.; Hupp, J. T. Inorg. Chem. 1992, 31, 3170-3172.

209. Mines, G. A.; Roberts, J. A.; Hupp, J. T. Inorg. Chem. 1992, 31, 125-128.

210. Zhang, X. L.; Yoon, D. I.; Hupp, J. T. Inorg. Chim. Acta 1995, 240, 285-289.

211. Khoshtariya, D. E.; Bajaj, H. C.; Tregloan, P. A.; van Eldik, R. J. Phys. Chem. A 2000, 104, 5535-5544.

212. Blackbourn, R. L.; Dong, Y.; Lyon, L. A.; Hupp, J. T. Inorg. Chem. 1994, 33, 4446-4452.

213. Hammack, W. S.; Drickamer, H. G.; Lowery, M. D.; Hendrickson, D. N. Chem. Phys. Lett. 1986, 132 , 231-235.

214. Lowery, M. D.; Hammack, W. S.; Drickamer, H. G.; Hendrickson, D. N. J. Am. Chem. Soc. 1987, 109, 8019-8024.

215. Marcus, R. A.; Sutin, N. Comments Inorg. Chem. 1986, 5, 119-133. 
216. Kober, E. M.; Meyer, T. J. Inorg. Chem. 1982, 21, 3967-3977.

217. Kober, E. M.; Meyer, T. J. Inorg. Chem. 1983, 22, 1614-1616.

218. Neyhart, G. Ph. D. Thesis, University of North Carolina (Chapel Hill, USA), 1988.

219. Joss, S.; Reust, H.; Ludi, A. J. Am. Chem. Soc. 1981, 103, 981-982.

220. Moffitt, W. J. Chem. Phys. 1956, 25, 467.

221. von Zelewsky, A. Stereochemistry of Coordination Compounds; Wiley: Chichester, 1995.

222. Denti, G.; Campagna, S.; Serroni, S.; Ciano, M.; Balzani, V. J. Am. Chem. Soc. 1992, 114, $2944-2950$.

223. Hua, X.; von Zelewsky, A. Inorg. Chem. 1995, 34, 5791-5797.

224. von Zelewsky, A.; Belser, P.; Hayoz, P.; Dux, R.; Hua, X.; Suckling, A.; Stoecklievans, H. Coord. Chem. Rev. 1994, 132, 75-85.

225. Rapenne, G.; Patterson, B. T.; Sauvage, J.-P.; Keene, F. R. Chem. Commun. 1999, 1853-1854.

226. Hua, X.; von Zelewsky, A. Inorg. Chem. 1991, 30, 3796-3798.

227. Hua, X. Ph.D. Thesis, University of Fribourg (Fribourg, Switzerland), 1993.

228. Anderson, P. A.; Deacon, G. B.; Haarmann, K. H.; Keene, F. R.; Meyer, T. J.; Reitsma, D. A.; Skelton, B. W.; Strouse, G. F.; Thomas, N. C.; Treadway, J. A.; White, A. H. Inorg. Chem. 1995, 34, 6145-6157.

229. Strouse, G. F.; Anderson, P. A.; Schoonover, J. R.; Meyer, T. J.; Keene, F. R. Inorg. Chem. 1992, 31, 3004-3006.

230. Reitsma, D. A.; Keene, F. R. J. Chem. Soc., Dalton Trans. 1993, 2859-2860.

231. Rutherford, T. J.; Reitsma, D. A.; Keene, F. R. J. Chem. Soc., Dalton Trans. 1994, 3659-3666.

232. Rutherford, T. J.; Quagliotto, M. G.; Keene, F. R. Inorg. Chem. 1995, 34, 3857-3858.

233. Patterson, B. T.; Keene, F. R. Inorg. Chem. 1998, 37, 645-650.

234. Fletcher, N. C.; Keene, F. R. J. Chem. Soc., Dalton Trans. 1998, 2293-2301.

235. Rutherford, T. J.; Pellegrini, P. A.; Aldrich-Wright, J.; Junk, P. C.; Keene, F. R. Eur. J. Inorg. Chem. 1998, 1677-1688.

236. Hiort, C.; Nordén, B.; Rodger, A. J. Am. Chem. Soc. 1990, 112, 1971-1982.

237. Lincoln, P.; Nordén, B. J. Chem. Soc., Chem. Commun. 1996, 2145-2146.

238. Warnmark, K.; Thomas, J. A.; Heyke, O.; Lehn, J.-M. Chem. Commun. 1996, 701-702.

239. MacDonnell, F. M.; Bodige, S. Inorg. Chem. 1996, 35, 5758-5759.

240. Bodige, S.; Torres, A. S.; Maloney, D. J.; Tate, D.; Kinsel, G. R.; Walker, A. K.; MacDonnell, F. M. J. Am. Chem. Soc. 1997, 119, 10364-10369.

241. Fletcher, N. C.; Keene, F. R.; Ziegler, M.; Stoecklievans, H.; Viebrock, H.; Vonzelewsky, A. Helv. Chim. Acta 1996, 79, 1192-1202.

242. Fletcher, N. C.; Keene, F. R.; Viebrock, H.; von Zelewsky, A. Inorg. Chem. 1997, 36, 1113-1121.

243. Murner, H.-R.; Belser, P.; von Zelewsky, A. J. Am. Chem. Soc. 1996, 118, 7989-7994.

244. Lacour, J.; Torche-Haldimann, S.; Jodry, J. J.; Ginglinger, C.; Favarger, F. Chem. Commun. 1998, 1733-1734.

245. Lacour, J.; Jodry, J. J.; Ginglinger, C.; TorcheHaldimann, S. Angew. Chem., Int. Ed. Engl. 1998, 37, 2379-2380.

246. Maury, O.; Lacour, J.; Le Bozec, H. Eur. J. Inorg. Chem. 2001, 201-204.

247. Constable, E. C.; Thompson, A. M. W. C. J. Chem. Soc., Dalton Trans. 1992, 3467-3475.

248. Fletcher, N. C.; Keene, F. R. J. Chem. Soc., Dalton Trans. 1999, 683-689.

249. Belser, P.; Bernhard, S.; Blum, C.; Beyeler, A.; De Cola, L.; Balzani, V. Coord. Chem. Rev 1999, 190-192, 155-169.

250. Belser, P.; Dux, R.; Baak, M.; De Cola, L.; Balzani, V. Angew. Chem., Int. Ed. Engl. 1995, 34, 595-598.

251. Balzani, V.; Scandola, F., Supramolecular Photochemistry; Ellis Horwood: Chichester, 1991.

252. Kelso, L. S.; Reitsma, D. A.; Keene, F. R. Inorg. Chem. 1996, 35, 5144-5153.

253. Rutherford, T. J.; Keene, F. R. Inorg. Chem. 1997, 36, 2872-2878.

254. Rutherford, T. J.; Van Gijte, O.; Kirsch-De Mesmaeker, A.; Keene, F. R. Inorg. Chem. 1997, 36, 4465-4474.

255. Treadway, J. A.; Chen, P.; Rutherford, T. J.; Keene, F. R.; Meyer, T. J. J. Phys. Chem. A 1997, 101, 6824-6826.

256. $\quad$ Patterson, B. T.; Foley, F. M.; Richards, D.; Keene, F. R. Dalton Trans. 2003, 709-714.

257. Brodkorb, A.; Kirsch-De Mesmaeker, A.; Rutherford, T. J.; Keene, F. R. Eur. J. Inorg. Chem. 2001, 2151-2160.

258. Foley, F. M.; Keene, F. R.; Collins, J. G. J. Chem. Soc., Dalton Trans. 2001, 2968-2974.

259. Patterson, B. P.; Collins, J. G.; Foley, F. M.; Keene, F. R. J. Chem. Soc., Dalton Trans. 2002, 4343-4350.

260. Smith, J. A.; Collins, J. G.; Patterson, B. T.; Keene, F. R. Dalton Trans. 2004, 1277-1283.

261. Yeomans, B. D.; Kelso, L. S.; Tregloan, P. A.; Keene, F. R. Eur. J. Inorg. Chem. 2001, 239-246.

262. Anderson, P. A.; Keene, F. R.; Meyer, T. J.; Moss, J. A.; Strouse, G. F.; Treadway, J. A. J. Chem. Soc., Dalton Trans. 2002, 3820-3831.

263. Curtis, J. C.; Blackbourn, R. L.; Ennix, K. S.; Hu, S.; Roberts, J. A.; Hupp, J. T. Inorg. Chem. 1989, 28, 3791-3795. 
ChAPTER 2 


\section{Chapter 2}

\section{DiAstereoisomers as Probes For SOLVENT REORGANISATIONAL EFFECTS IN IVCT}

\subsection{Introduction}

Dinuclear ligand-bridged mixed-valence complexes have played a pivotal role in the assessment of activation barriers to intramolecular electron transfer since the disclosure of the Creutz-Taube ion, $\left[\left\{\mathrm{Ru}\left(\mathrm{NH}_{3}\right)_{5}\right\}(\mu \text {-pyz })\left\{\mathrm{Ru}\left(\mathrm{NH}_{3}\right)_{5}\right\}\right]^{5+}\{$ pyz $=$ pyrazine $\}$, in 1973. ${ }^{1}$ Systems of this genre have provided important experimental insights into the roles of solvent dynamics, ${ }^{2-15}$ ion-pairing, ${ }^{16-21}$ encapsulation, ${ }^{22,23}$ temperature, ${ }^{24-28}$ and redox asymmetry, ${ }^{29,} 30$ and they have been used as model systems to verify the salient predictions of several important theoretical models that describe the activation barrier to electron transfer. $^{31-35}$

The particular appeal of mixed-valence complexes of the form $\left[\left\{L_{n} M^{\mathrm{II}}\right\}(\mu-B L)\left\{M^{\mathrm{III}} L_{n}\right\}\right]^{5+}\{M=$ metal centres, $\mathrm{L}=$ terminal ligands and $\mathrm{BL}=$ bridging ligand $\}$ is the observation of an absorption band in the near-infrared (NIR) region of the electronic spectrum which is identified as the optically-induced IVCT transition. IVCT measurements provide a sensitive and powerful probe to elucidate aspects of intramolecular electron transfer processes as the energy $\left(v_{\max }\right)$, intensity $(\varepsilon)$ and bandwidth $\left(\Delta v_{1 / 2}\right)$ of these transitions can be quantitatively related to the factors which influence the activation barrier to electron transfer. $^{31,32}$

For symmetrical, valence-localised mixed-valence systems, Hush ${ }^{31,32}$ proposed the relationship

$$
v_{\max }=\lambda_{i}+\lambda_{0}+\Delta \mathrm{E}^{\prime}
$$

where $\lambda_{i}$ and $\lambda_{o}$ are the inner- and outer-sphere reorganisational (Franck-Condon) parameters, respectively, and $\Delta \mathrm{E}^{\prime}$ represents any additional energy contributions due to spin-orbit coupling and/or ligand field asymmetry. $\lambda_{i}$ corresponds to the energy required for reorganisation of the metal-ligand and intra-ligand bond lengths and angles, and $\lambda_{0}$ is the energy required for reorganisation of the surrounding solvent medium. The solvent contribution is generally modelled as a one-dimensional classical mode due to the low frequencies of the coupled vibrations. ${ }^{31-34}$ The spherical cavity dielectric continuum model given by equation 2.2 provides a framework for the calculation of the this contribution in which the electron donor and acceptor are modelled as two non-interpenetrating spheres, embedded in the dielectric continuum. $^{31,32}$

$$
\lambda_{\mathrm{o}}=e^{2}\left(\frac{1}{a}-\frac{1}{d}\right)\left(\frac{1}{\mathrm{D}_{\mathrm{op}}}-\frac{1}{\mathrm{D}_{\mathrm{s}}}\right)
$$


The parameters $a$ and $d$ define the molecular radii and distance between the donor and acceptor, $e$ is the unit electronic charge, and $D_{s}$ and $D_{o p}$ are the macroscopic static and optical dielectric constants of the solvent respectively. In accordance with equations 2.1 and 2.2, (i) $v_{\max }$ should vary linearly with the solvent dielectric function $\left(1 / \mathrm{D}_{\mathrm{op}}-1 / \mathrm{D}_{\mathrm{s}}\right)$, with slope $e^{2}(1 / a-1 / d)$ and intercept $\lambda_{i}+\Delta \mathrm{E}^{\prime}$ at $\left(1 / \mathrm{D}_{\mathrm{op}}-1 / \mathrm{D}_{\mathrm{s}}\right)=0$, and (ii) $v_{\max }$ should vary linearly with $1 / d$, with slope $e^{2}\left(1 / \mathrm{D}_{\mathrm{op}}-1 / \mathrm{D}_{\mathrm{s}}\right)$ in a given solvent in which the length of the bridging ligand is varied (at fixed $a$ ).

In the elegant pioneering work of Meyer and coworkers, ${ }^{9,36-42}$ the inverse dependence of $\lambda_{0}$ on the inter-metal distance was verified for the polypyridyl complexes $\left[\left\{\mathrm{Ru}(\mathrm{bpy})_{2} \mathrm{~L}\right\}_{2}(\mu-\mathrm{BL})\right]^{n+}\{\mathrm{L}=\mathrm{Cl}$ or py (pyridine) $\}$. Plots of $v_{\max }$ as a function of $1 / \mathrm{D}_{\mathrm{op}}-1 / \mathrm{D}_{\mathrm{s}}$ were linear for the series of complexes in which the length of the bridging ligand was systematically increased through the series pym, pyz, 4,4'-bpy, BPE, BPA, shown in Figure 2.1. The predicted linear trend was also verified from the plot of $v_{\max }$ as a function of 1/d for the complexes incorporating the pyz, 4,4'-bpy, and BPE bridging ligands, in which the bridge length increases in the order 6.9, 11.1 and 13.2 A, respectively. ${ }^{36,43}$

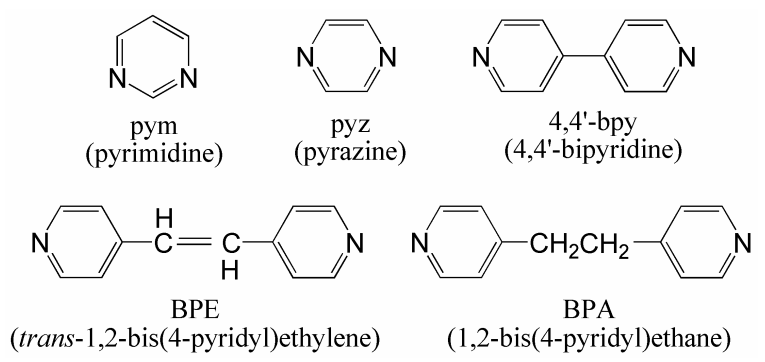

Figure 2.1 Bridging ligands employed to verify the inverse dependence of $\lambda_{0}$ on $d$ in complexes of the type $\left[\left\{\mathrm{Ru}(\mathrm{bpy})_{2} \mathrm{~L}\right\}_{2}(\mu-\mathrm{BL})\right]^{n+}\{\mathrm{L}=\mathrm{Cl} \text { or py (pyridine) }\}^{9,36-42}$

While the predictions of the dielectric continuum model have been verified in a number of IVCT solvatochromism studies of mixed-valence dinuclear ruthenium complexes, ${ }^{2,44,45}$ the treatment breaks down when the underlying assumptions of the classical model are invalidated, or in the presence of specific solvent-solute interactions or dielectric saturation effects which often confound experimental solvatochromism studies. ${ }^{2}$ Theoretically, the dielectric continuum model is formulated in terms of a onedimensional classical mode for the solvent, and is inadequate for systems that exhibit coupled highfrequency quantum modes which must be explicitly treated through a quantum mechanical approach. ${ }^{15}$ Equation 2.2 also neglects the volume occupied by the donor and acceptor (the "excluded volume”) and is valid only when the distance between the redox centres exceeds the sum of their radii $(d » 2 a)$. The corrections due to cut-out effects and non-spherical fields around the metal centres become increasingly important as the distance between the metal centres is decreased.

As an alternative to the spherical cavity approximation, ellipsoidal cavity models have been developed to provide a more physically realistic description for the non-spherical structures of dinuclear complexes. Such models account for solvent effects in terms of the change in electronic distribution within a regular ellipse enclosing the complex..$^{13,15,46,47}$ However, as for the spherical cavity dielectric continuum model, ellipsoidal cavity models are derived within the classical formalism and assume 
idealised shapes for the molecules which are approximations only of the actual geometries involved. The solvent reorganisational contribution is thus modelled in terms of the macroscopic dielectric properties of the solvent, and the $1 / \mathrm{D}_{\mathrm{op}}-1 / \mathrm{D}_{\mathrm{s}}$ dependence of $\lambda_{\mathrm{o}}$ dominates the mathematical expression of the model. ${ }^{13,15,46,47}$

Experimentally, the analysis of IVCT solvatochromism data according to the spherical and ellipsoidal dielectric continuum models has often been severely complicated by non-continuum effects. These issues have been addressed in an extensive review of medium effects on the IVCT properties of mixed-valence complexes by Chen and Meyer, ${ }^{2}$ and include specific solvent-solute interactions and dielectric saturation effects, in addition to ion-pairing contributions from the counter and electrolyte ions, the concentration of the chromophore and the chemical oxidant used for the generation of the mixedvalence complex.

\section{Towards a Molecular Theory of Solvation}

The elucidation of the relative contributions of continuum and non-continuum effects is the subject of considerable experimental interest in the attempt to develop more sophisticated theoretical models for solvent reorganisational contributions to the electron transfer barrier. ${ }^{2,48}$ Dielectric continuum theory obscures the "molecularity" of the solvent by neglecting the properties of individual solvent molecules, and this underpins the recent theoretical interest towards understanding the molecular basis of reorganisational effects. ${ }^{2,48}$

The experimental strategy for extracting information at the molecular level using IVCT solvatochromism studies involves probing the first solvation shell separately from the bulk solution. Dinuclear ruthenium complexes incorporating ammine and cyano ligands have been extensively investigated in this regard, due to the existence of strong directional H-bonding and donor-acceptor interactions between the chromophore ligands and individual solvent molecules. ${ }^{6,11-15,49}$ These specific solvent interactions coexist with, and often dominate dielectric continuum effects. ${ }^{2}$ Correlations have been found between the IVCT solvent shifts and empirical solvent parameters such as the Gutmann donor and acceptor numbers... ${ }^{50}$ In studies of dinuclear ruthenium mixed-valence complexes based on $\mathrm{Ru}\left(\mathrm{NH}_{3}\right)_{5}$, trans-Ru( $\left.\mathrm{NH}_{3}\right)_{4}$ (py) and $\mathrm{Ru}(\mathrm{bpy})\left(\mathrm{NH}_{3}\right)_{3}$ with pyz, 4-cyanopyridine and 4,4'-bpy bridging ligands, ${ }^{19,51,52}$ the IVCT energies correlated linearly with the Gutmann solvent donor number (DN) due to specific H-bonding interactions between the ammine ligands and the solvent molecules. In each case, the magnitude of the specific interaction increased with the donor number of the solvent, and the number of $\mathrm{NH}_{3}$ ligands in the chromophore.

IVCT solvatochromism studies in solvent mixtures have demonstrated that the solvent reorganisational process occurs predominantly within the first solvation layer, and may be profoundly influenced by the systematic replacement of individual solvent molecules in the immediate vicinity of the

\footnotetext{
* The Gutmann donor number (DN) provides a measure of the ability of individual solvent molecules to donate an electron pair to $\mathrm{SbCl}_{5}$, and to engage in $\mathrm{H}$-bonding to $\mathrm{NH}_{3}$. The acceptor number provides a measure of the ability of individual solvent molecules to act as electron pair acceptors. ${ }^{50}$
} 
mixed-valence chromophore. 2, 5, 7, 13, 14, 19, 53 In mixed solvents containing varying proportions of acetonitrile and dimethylsulfoxide (DMSO), selective solvation by DMSO occurs preferentially at the more highly-charged $\mathrm{Ru}^{\mathrm{III}}\left(\mathrm{NH}_{3}\right)_{5}$ fragment in $\left[\left\{\mathrm{Ru}^{\mathrm{II}}\left(\mathrm{NH}_{3}\right)_{5}\right\}\left(\mu-4,4^{\prime}-\text {-bpy }\right)\left\{\mathrm{Ru}^{\mathrm{III}}\left(\mathrm{NH}_{3}\right)_{5}\right\}\right]^{5+}$. 11-14 This gives rise to an additional energy contribution, and hence a blue-shift in the IVCT energy for dilute solutions of DMSO in acetonitrile. This strategy allowed the reorganisational energies for the first molecular layer of solvent to be examined separately from the bulk solvent composition. Despite the correlations with empirical solvent scales, no general theory exists to explain these effects. ${ }^{2,54}$

In parallel with experimental investigations, considerable attention has been directed to the development of semi-classical ${ }^{55}$ and quantum mechanical ${ }^{56-61}$ computational methods to achieve a higher level of quantification in the theoretical treatment of solvent molecularity effects on the electron transfer barrier. To date, semiempirical computational methods such as INDO (intermediate neglect of differential overlap), ${ }^{62,63}$ AM1 and PM3 have been extensively employed due to their ability to provide rapid calculations of the structural and electronic properties of molecular systems. ${ }^{64-69}$ However, these methods are dependent on empirical parameterisation and often fail to provide adequate quantitative descriptions of the electronic structures of open-shell systems such as dinuclear mixed-valence complexes. In addition, they also fail to provide an adequate representation of intermolecular interactions due to the treatment of the solvent as a dielectric continuum. ${ }^{56,60}$ With the advent of high-performance computing, ab initio methods such as Density Functional Theory (DFT) have permitted a priori calculations of the electronic properties of transition metal chromophores, and have been applied to a number of dinuclear mixed-valence complexes of ruthenium. ${ }^{70-73}$ The development of reliable $a b$ initio methods require parameterisation to include continuum and non-continuum solvation effects in order to provide a physically realistic treatment of solvation under experimental conditions. ${ }^{74}$

A method for calculating solvent shifts on metal-to-ligand charge transfer (MLCT) and other electronic absorption bands involving charge transfer has been developed by Zeng, Reimers and Hush. ${ }^{56,60}$ The "ZHR-SS" method is parameterised in terms of explicit solvent dipoles for the first solvation shell, while the bulk solvent is treated as a dielectric continuum. ${ }^{56-61}$ The equilibrium groundstate structure of the solvent around the chromophore is determined by standard Monte Carlo or molecular dynamics simulations. Sampled configurations are subsequently taken from which $a b$ initio methods are employed to calculate the energy, charge distribution and polarisability of the solute. The strength of the ZHR-SS method lies in the explicit solvent structure approach, albeit at the cost of a full liquid structure simulation. A detailed discussion of the ZHR-SS method, in addition to the relative merits of various computational approaches for the treatment of solvation effects on the electronic spectra of dinuclear transition metal complexes, is outside the scope of this thesis, but has been provided by Reimers and Hush. ${ }^{56,60}$

Unfortunately, the development of practical calculation procedures for the quantitative modelling and prediction of intramolecular electron transfer processes, using input from electronic structure calculation methods for the complexes and molecular theories of solvation, have been impeded by limited 
experimental data. ${ }^{2,48,56,60}$ There is clearly a need for experimental studies of IVCT solvatochromism which provide insights into microscopic solvent reorganisational contributions.

\subsubsection{Scope and Objectives of the Present Study}

Historically, the Franck-Condon reorganisational contributions to the electron transfer barrier have been probed by IVCT solvatochromism studies of symmetrical dinuclear mixed-valence complexes, through the treatment of the solvent as a structureless dielectric continuum (equation 2.2). The impetus towards the development of a molecular theory of solvation has motivated experimental studies to probe the microscopic basis of solvent reorganisation by the investigation of specific solvation effects. ${ }^{2}$ In the present study, the investigation of the IVCT solvatochromism in the diastereoisomers of the mixedvalence complex $\left[\left\{\mathrm{Ru}(\mathrm{pp})_{2}\right\}_{2}(\mu \text {-bpm) }]^{5+}\left\{\mathrm{pp}=2,2^{\prime}\right.\right.$-bipyridine and its derivatives; bpm = 2,2'bipyrimidine\} provides a new experimental approach to probe such effects.

As discussed in §1.6, the dinuclear species $\left[\left\{\mathrm{Ru}(\mathrm{bpy})_{2}\right\}_{2}(\mu \text {-bpm })\right]^{4+}$ exists in two diastereoisomeric forms - meso, $\Lambda \Delta(\equiv \Delta \Lambda)$ and racemic (rac) \{illustrated in Figure 2.2\}, the latter comprising the enantiomeric pair $\Delta \Delta$ and $\Lambda \Lambda$. While the identity and coordination environments of the metal centres are identical in each diastereoisomeric form, a significant difference may be discerned in the nature of the "clefts" formed between the planes of the terminal bpy ligands. ${ }^{75}$ "Interior clefts" are formed between the bpy ligands immediately "above" and "below" the plane of the bridging ligand, and are approximately orthogonal in the meso form and parallel in the rac form. In addition, "exterior clefts" are evident between the planes of the terminal bpy ligands at either end of the complex, and are identical for two diastereoisomeric forms. $\lambda_{i}$ and $\Delta \mathrm{E}^{\prime}$ are expected to be identical for the diasterereoisomers of the same complex, so that IVCT solvatochromism studies of the two forms permit a direct probe of stereochemically-induced $\lambda_{0}$ variations on the intramolecular electron transfer barrier.

(a)
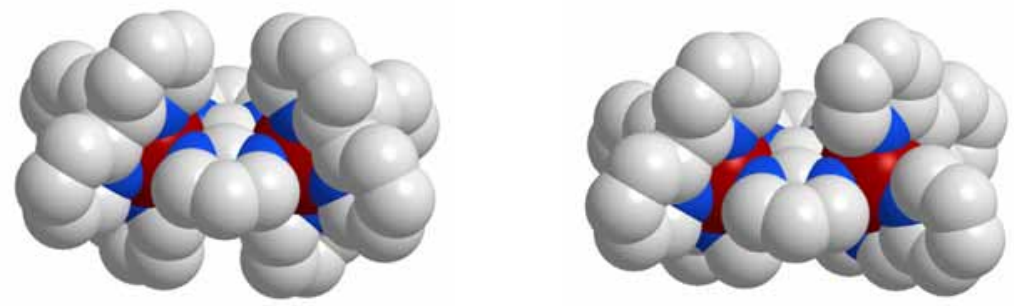

(b)

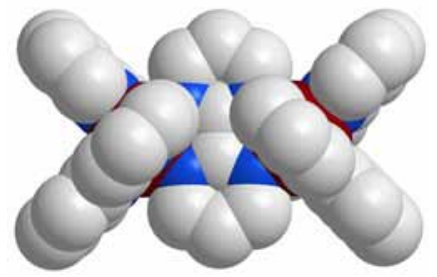

meso $(\Delta \Lambda)$

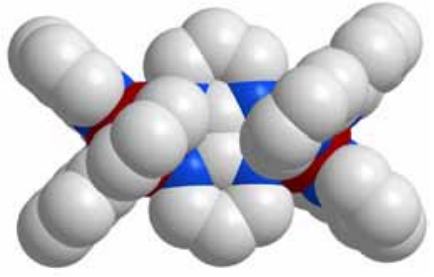

$\operatorname{rac}(\Delta \Delta)$

Figure 2.2 (a) Front view and (b) top view of the meso $(\Lambda \Delta)$ and $\operatorname{rac}(\Delta \Delta)$ diastereoisomers of $\left[\left\{\mathrm{Ru}(\mathrm{bpy})_{2}\right\}_{2}(\mu-\mathrm{bpm})\right]^{4+}$ illustrating the subtle variation in the dimensions of the clefts above and below the plane of the bridging ligand. Hydrogen atoms are omitted for clarity. 
The variation in the dimensions of the clefts between the diastereoisomeric forms of the same complex may have significant consequences for differential solvent and anion association at the molecular level. Indeed, the differential association of eluent anions such as toluene-4-sulfonate $\left\{\mathrm{CH}_{3}\left(\mathrm{C}_{6} \mathrm{H}_{4}\right) \mathrm{SO}_{3}{ }^{-}\right.$; tosylate $\}$gives rise to the separation of the diastereoisomeric forms in the chromatographic cation-exchange separation process. ${ }^{75-78}$

\section{IVCT Solvatochromism Studies}

The present study involves the investigation of the IVCT solvatochromism for the diastereoisomeric forms of $\left[\left\{\mathrm{Ru}(\mathrm{bpy})_{2}\right\}_{2}(\mu \text {-bpm })\right]^{5+}$ in the homologous series of the nitrile solvents acetonitrile $\left\{\mathrm{CH}_{3} \mathrm{CN}\right.$; $\left.\mathrm{AN}\right\}$, propionitrile $\left\{\mathrm{CH}_{3} \mathrm{CH}_{2} \mathrm{CN} ; \mathrm{PN}\right\}, n$-butyronitrile $\left\{\mathrm{CH}_{3}\left(\mathrm{CH}_{2}\right)_{2} \mathrm{CN} ; \mathrm{BN}\right\}$, iso-butyronitrile $\left\{\left(\mathrm{CH}_{3}\right)_{2} \mathrm{HCCN}\right.$; $\left.{ }^{\mathrm{B} N}\right\}$ and benzonitrile $\left\{\mathrm{C}_{6} \mathrm{H}_{5} \mathrm{CN}\right.$; $\left.\mathrm{BzN}\right\}$, shown in Figure 2.3. The macroscopic properties of the solvents (as defined by the solvent parameter $1 / \mathrm{D}_{\mathrm{op}}-1 / \mathrm{D}_{\mathrm{s}}$ ) vary over the range 0.5127 (AN) to $0.3897(\mathrm{BzN})$, while the subtle variation in the molecular shape, size and symmetry through the series of aliphatic and aromatic nitriles allows for a detailed analysis of the microscopic origins of the solvent reorganisational energy due to stereochemically-directed solvent effects.

To eliminate ion-pairing artefacts - which are dependent on the concentrations of the mixedvalence chromophore and the supporting electrolyte $20,21,79,80-$ the IVCT solvatochromism measurements were performed in $\left[\left(n-\mathrm{C}_{4} \mathrm{H}_{9}\right)_{4} \mathrm{~N}\right]\left\{\mathrm{B}\left(\mathrm{C}_{6} \mathrm{~F}_{5}\right)_{4}\right\}$ electrolyte containing a uniform, low concentration of the given diastereoisomer. The $\left\{\mathrm{B}\left(\mathrm{C}_{6} \mathrm{~F}_{5}\right)_{4}\right\}^{-}$anion is known to associate weakly, in comparison with $\mathrm{PF}_{6}^{-}$and $\mathrm{ClO}_{4}{ }^{-}$counter-ions which have been traditionally used for electrochemical and spectroelectrochemical measurements. ${ }^{81}$

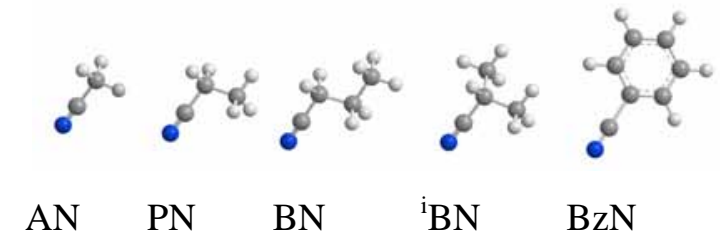

Figure 2.3 Homologous series of nitrile solvents used in the IVCT solvatochromism studies.

To gain further insights into the modification of solvent reorganisational effects induced by subtle and systematic chemical variations in the bridging or terminal ligands, and stereochemical variations, two additional strategies are pursued in the present study. The first strategy involves the examination of the IVCT solvatochromism properties in the diastereoisomeric forms of $\left[\left\{\mathrm{Ru}(\mathrm{bpy})_{2}\right\}_{2}(\mu \text {-dbneil })\right]^{5+}\{\mathrm{dbneil}=$ dibenzoeilatin, Figures 2.4 and 2.5\} to assess the effect of increasing the dimensions of the interior clefts by comparison with the diastereoisomers of $\left[\left\{\mathrm{Ru}(\mathrm{bpy})_{2}\right\}_{2}(\mu \text {-bpm })\right]^{5+}$. In a recent publication, Kol et al. ${ }^{82}$ described the properties of the dbneil bridging ligand in which a number of issues were addressed, including diastereoisomeric preference in dinuclear complex formation, the effect of coordination of the second metal centre on the low-lying metal-to-ligand charge transfer (MLCT) band, the nature of the excited state, and the degree of inter-metal communication in the dinuclear species. 

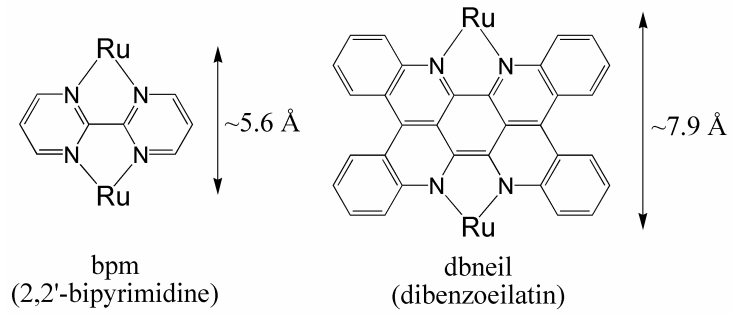

Figure 2.4 The bpm and dbneil bridging ligands with the crystallographically-determined Ru-Ru distances in their dinuclear complexes meso- $\left[\left\{\mathrm{Ru}\left(\mathrm{Me}_{2} \mathrm{bpy}\right)_{2}\right\}_{2}(\mu \text {-bpm })\right]^{4+76}$ and meso- $\left[\left\{\mathrm{Ru}(\mathrm{bpy})_{2}\right\}_{2}(\mu \text {-dbneil })\right]^{4+82}\left\{\mathrm{Me}_{2} \mathrm{bpy}=4,44^{\prime}-\right.$ dimethyl-2,2'-bipyridine\}.

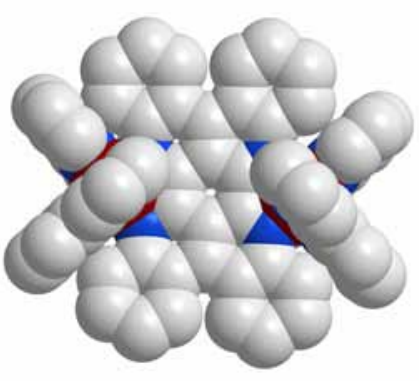

$\operatorname{meso}(\Delta \Lambda)$

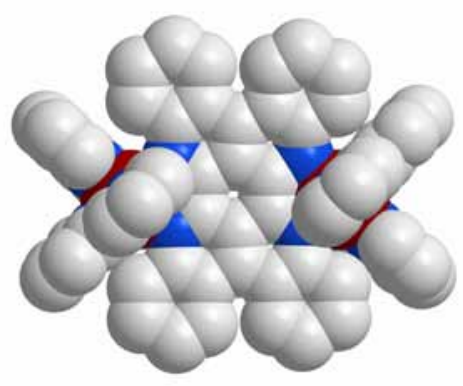

$\operatorname{rac}(\Delta \Delta)$

Figure 2.5 Chem 3D representations of the diastereoisomeric forms of $\left[\left\{\mathrm{Ru}(\mathrm{bpy})_{2}\right\}_{2}(\mu \text {-dbneil })\right]^{n+}$ illustrating the subtle variations in the dimensions of the interior clefts above and below the plane of the bridging ligand. Hydrogen atoms are omitted for clarity.

The second strategy involves the systematic modification of the dimensions of the interior and exterior clefts by the judicious positioning of substituents on the terminal ligands in the series $\left[\left\{\mathrm{Ru}(\mathrm{pp})_{2}\right\}_{2}(\mu \text {-bpm) }]^{5+}\right.$, where pp = 5,5'-dimethyl-2,2-bipyridine (5,5'-Me $\left.\mathrm{bpy}\right), 4,4$ ',5,5'-tetramethyl-2,2'bipyridine ( $\mathrm{Me}_{4} \mathrm{bpy}$ ), 2,9-dimethyl-1,10-phenanthroline ( $\mathrm{Me}_{2} \mathrm{phen}$ ) and 4,4'-di-tert-butyl-2,2'-bipyridine ( ${ }^{\mathrm{B}} \mathrm{Bu}_{2} \mathrm{bpy}$ ), shown in Figures 2.6 and 2.7. In each case, the IVCT characteristics of the separated diastereoisomers are investigated in the homologous series of nitrile solvents.

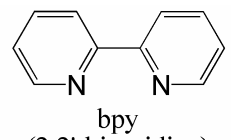

(2,2'-bipyridine)

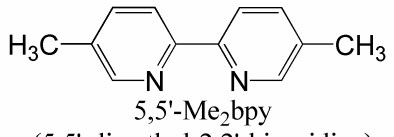

(5,5'-dimethyl-2,2'-bipyridine)<smiles>Cc1cnc(-c2cc(C)c(C)cn2)nc1</smiles>

(4,4',5,5'-tetramethyl-2,2'-bipyridine)

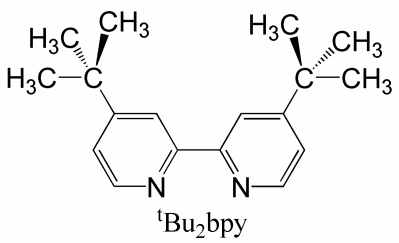

(4,4'-di-tert-butyl-2,2'-bipyridine)

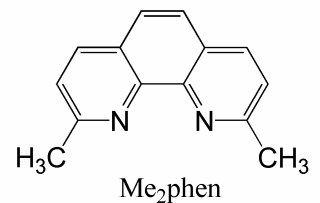

(2,9-dimethyl-1,10-phenanthroline)

Figure 2.6 Terminal polypyridyl ligands used in the present study. 


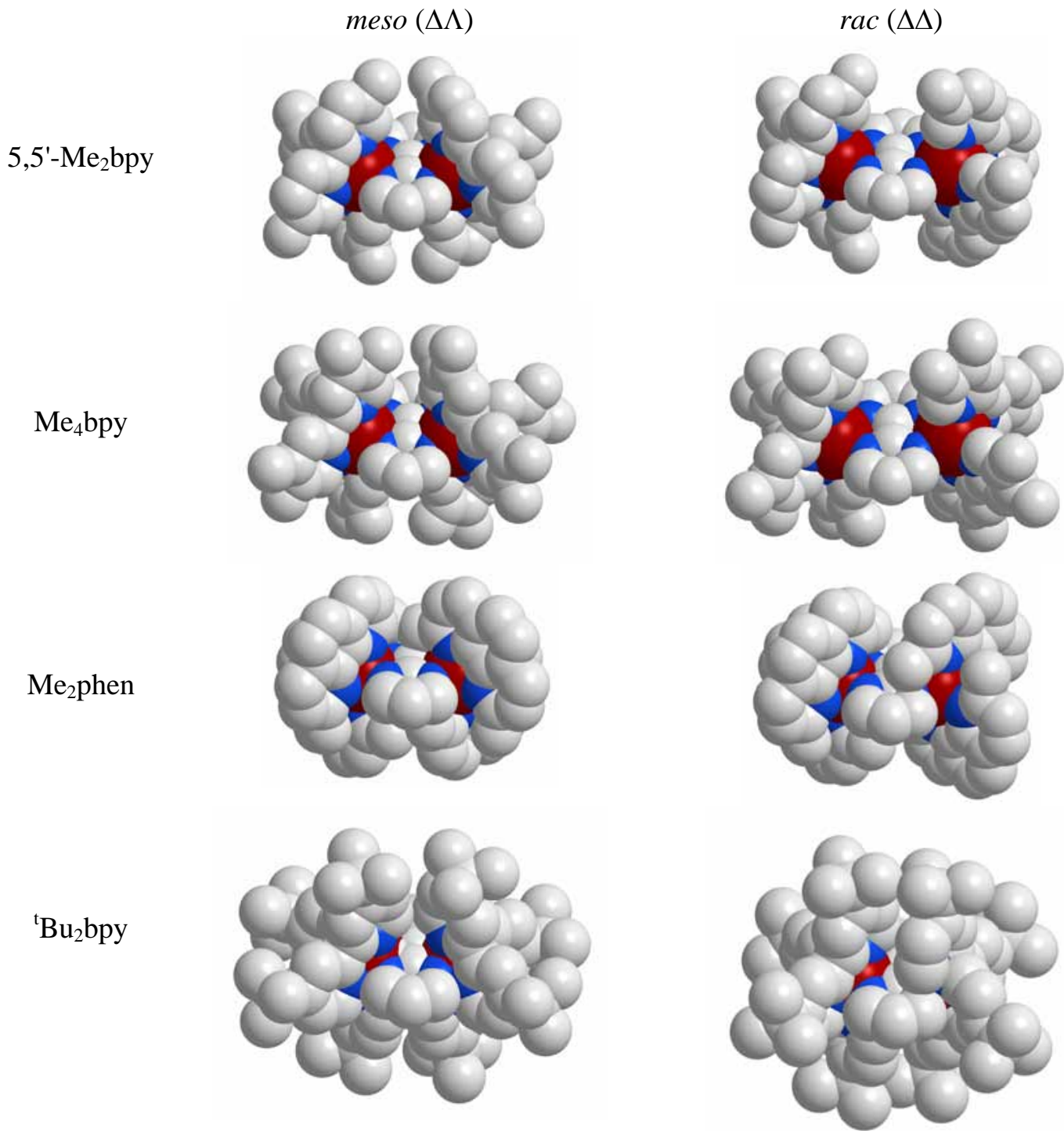

Figure 2.7 Chem 3D representations of the diastereoisomers of $\left[\left\{\mathrm{Ru}(\mathrm{pp})_{2}\right\}_{2}(\mu \text {-bpm) }]^{5+}\left\{\mathrm{pp}=5,5^{\prime}-\mathrm{Me}_{2} \mathrm{bpy}, \mathrm{Me}_{4} \mathrm{bpy}\right.\right.$, $\left.\mathrm{Me}_{2} \mathrm{phen},{ }^{\mathrm{t}} \mathrm{Bu}_{2} \mathrm{bpy}\right\}$ illustrating the variation in the nature of the clefts above and below the plane of the bridging ligand. Hydrogen atoms are omitted for clarity.

Preliminary ab initio computational calculations on selected systems have been performed to gain a physical insight into the nature of stereochemically-directed solvent interactions and provide a basis for the quantitative modelling of solvent-solute interactions. 


\subsection{Experimental}

\subsubsection{Materials}

Hydrated ruthenium trichloride $\left(\mathrm{RuCl}_{3} .3 \mathrm{H}_{2} \mathrm{O}\right.$; Strem, 99\%), 2,2'-bipyrimidine (bpm; Lancaster), 2,2'-bipyridine (bpy; Aldrich, 99+\%), 4,4'-dimethyl-2,2'-bipyridine ( $\mathrm{Me}_{2} \mathrm{bpy}$; Aldrich), 5,5'-dimethyl-2,2'bipyridine (5,5'-Me 2 bpy; Aldrich), 2,9-dimethyl-1,10-phenanthroline ( $\mathrm{Me}_{2}$ phen; Monsanto), 4,4'-di-tertbutyl-2,2'-bipyridine ( ${ }^{\mathrm{B}} \mathrm{Bu}_{2}$ bpy; Aldrich, 98\%), stannous chloride $\left(\mathrm{SnCl}_{2} \cdot 2 \mathrm{H}_{2} \mathrm{O}\right.$; Ajax), lithium chloride ( $\mathrm{LiCl}$; Aldrich, 99+\%), ammonium hexafluorophosphate $\left(\mathrm{NH}_{4} \mathrm{PF}_{6}\right.$; Aldrich, 99.99\%), potassium hexafluorophosphate ( $\mathrm{KPF}_{6}$; Aldrich, 98\%), lithium tetrakis(pentafluorophenyl)borate diethyletherate ( $\mathrm{Li}\left\{\mathrm{B}\left(\mathrm{C}_{6} \mathrm{~F}_{5}\right)_{4}\right\}$.Et $\mathrm{Et}_{2} \mathrm{O}$; Boulder Scientific), ethylene glycol (Ajax, 95\%), sodium octanoate (Aldrich), sodium benzoate (Aldrich, 98\%), sodium toluene-4-sulfonate (Natos, sodium tosylate; Aldrich, 98\%), DOWEX $^{\circledR} 1 \times 8,50-100$ mesh (Aldrich) and Amberlite ${ }^{\circledR}$ IRA-400 (Aldrich) $\mathrm{Cl}^{-}$anion exchange resins and laboratory reagent solvents were used as received. Tetra- $n$-butylammonium hexafluorophosphate $\left(\left[\left(n-\mathrm{C}_{4} \mathrm{H}_{9}\right)_{4} \mathrm{~N}\right] \mathrm{PF}_{6}\right.$; Fluka, 99.9+\%) was dried in vacuo at $60^{\circ} \mathrm{C}$ prior to use and ferrocene (Fc; BDH) was purified by sublimation prior to use. SP Sephadex C-25, QAE Sephadex A-25, Sephadex LH-20 (Amersham Pharmacia Biotech) and silica gel (200-400 mesh , $60 \AA$, Aldrich) were employed for the chromatographic separation and purification of the complexes. ${ }^{75}$ Aqueous solutions of (-)-O,O-di-4toluoyl-L-tartrate were prepared by neutralisation of the corresponding acid (Fluka) using $\mathrm{NaOH}$.

The following solvents were distilled prior to use: acetonitrile (Aldrich, 99.9+\%) and propionitrile (PN; Aldrich) were distilled over $\mathrm{CaH}_{2}$; acetone (BDH, HPLC grade) was distilled over $\mathrm{K}_{2} \mathrm{CO}_{3}$; dichloromethane was distilled over $\mathrm{CaCl}_{2}$; and $N$, $N$-Dimethylformamide (DMF; Ajax) was distilled under reduced pressure ${ }^{83}\left(76^{\circ} \mathrm{C}\right.$ at $\left.39 \mathrm{mmHg}\right)$. $n$-Butyronitrile (BN; Aldrich, 99+\%), iso-butyronitrile ( ${ }^{\mathrm{i}} \mathrm{BN}$; Aldrich), benzonitrile (BzN; Aldrich), and 2,2,2-trifluoroethanol (Aldrich) were used as received.

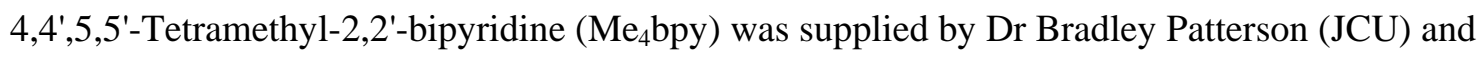
was prepared according to the literature method. ${ }^{84}$

\subsubsection{Instrumentation and Physical Methods}

\section{NMR Spectroscopy}

1D and 2D ${ }^{1} \mathrm{H}$ NMR spectra were performed on a Varian Mercury $300 \mathrm{MHz}$ spectrometer. Chemical shifts for all complexes are reported relative to $99.9 \% \mathrm{~d}_{3}$-acetonitrile $\left\{\mathrm{CD}_{3} \mathrm{CN}\right.$; Cambridge Isotope Laboratories (CIL) $\}$ at $\delta=1.93 \mathrm{ppm} .{ }^{1} \mathrm{H}$ NMR assignments were performed with the assistance of COSY experiments to identify each pyridine ring system. 


\section{Elemental Microanalyses}

Elemental microanalyses were performed at the Microanalytical Unit in the Research School of Chemistry, Australian National University. For some complexes, an allowance for hydration was necessary to account for analysis figures within the acceptable limits $( \pm 0.4 \%)$.

\section{Circular Dichroism}

Circular dichroism (CD) spectra were recorded in acetonitrile solution at concentrations of ca. 2-3 $\times 10^{-5} \mathrm{M}$ in a $1.0 \mathrm{~cm}$ pathlength cell, using a JASCO J-715 spectropolarimeter. CD spectra are presented as $\Delta \varepsilon\left(\mathrm{M}^{-1} \mathrm{~cm}^{-1}\right) v s$. wavelength, $\lambda(\mathrm{nm})$.

\section{Electrochemical Measurements}

Electrochemical measurements were performed under argon using a Bioanalytical Systems BAS 100A Electrochemical Analyser. Cyclic and differential pulse voltammograms were recorded under $\mathrm{Ar}$ in $0.02 \mathrm{M}\left[\left(n-\mathrm{C}_{4} \mathrm{H}_{9}\right)_{4} \mathrm{~N}\right]\left\{\mathrm{B}\left(\mathrm{C}_{6} \mathrm{~F}_{5}\right)_{4}\right\} / \mathrm{CH}_{3} \mathrm{CN}$ at $+25^{\circ} \mathrm{C}$ using a glassy carbon working electrode, a platinum wire auxiliary electrode and an $\mathrm{Ag} / \mathrm{AgCl}(0.02 \mathrm{M}$ $\left.\left[\left(n-\mathrm{C}_{4} \mathrm{H}_{9}\right)_{4} \mathrm{~N}\right]\left\{\mathrm{B}\left(\mathrm{C}_{6} \mathrm{~F}_{5}\right)_{4}\right\} / \mathrm{CH}_{3} \mathrm{CN}\right)$ reference electrode. Ferrocene was added as an internal standard on completion of each experiment the ferrocene/ferrocenium couple $\left(\mathrm{Fc}^{+} / \mathrm{Fc}^{0}\right)$ occurred at +550 $\mathrm{mV}$ vs. $\mathrm{Ag} / \mathrm{AgCl}\}$ and all potentials are quoted in $\mathrm{mV}$ versus $\mathrm{Fc}^{+} / \mathrm{Fc}^{0}{ }^{85}$ Cyclic voltammetry was performed with a sweep rate of $100 \mathrm{mV} \mathrm{s}^{-1}$; differential pulse voltammetry was conducted with a sweep rate of $4 \mathrm{mV} \mathrm{s}^{-1}$ and a pulse amplitude, width and period of $50 \mathrm{mV}, 60 \mathrm{~ms}$ and $1 \mathrm{~s}$, respectively. Potentials from DPV experiments are reported $\pm 3 \mathrm{mV}$.

In order to obtain reasonable electrochemical responses, measurements in the $0.02 \mathrm{M}$ $\left[\left(n-\mathrm{C}_{4} \mathrm{H}_{9}\right)_{4} \mathrm{~N}\right]\left\{\mathrm{B}\left(\mathrm{C}_{6} \mathrm{~F}_{5}\right)_{4}\right\} / \mathrm{CH}_{3} \mathrm{CN}$ electrolyte required a concentration of complex which was approximately double that in $0.1 \mathrm{M}\left[\left(n-\mathrm{C}_{4} \mathrm{H}_{9}\right)_{4} \mathrm{~N}\right] \mathrm{PF}_{6} / \mathrm{CH}_{3} \mathrm{CN}$. $i R$ compensation was not employed for the electrochemical measurements.

\section{UV/Vis/NIR Spectroelectrochemisty}

Electronic spectra were recorded using a CARY 5E UV/Vis/NIR spectrophotometer interfaced to Varian WinUV software. The absorption spectra of the electrogenerated mixed-valence species were obtained in situ, by the use of a cryostatted Optically Semi-Transparent Thin-Layer Electrosynthetic (OSTLE) cell (path length $0.685 \mathrm{~mm}$ ) mounted in the light-path of the spectrophotometer. ${ }^{86}$

The OSTLE cell, which was mounted in a hollow teflon block secured in the beam of the spectrophotometer, was of fused-silica construction and embodied a conventional-geometry optical cuvette (Suprasil W) of sub-millimetre path-length, expanding into a tubular upper section to accommodate the counter and reference electrodes (Figure 2.8). A platinum gauze working electrode (70\% transmittance) was located centrally in the optical beam in the lower section of the cell. To ensure electrolysis occurred only at the platinum gauze, the section of wire passing to the top of the cell was 
sheathed by poly(tetrafluoroethylene) $\{\mathrm{PTFE}\}$ tubing. A platinum wire auxiliary electrode and $\mathrm{Ag} / \mathrm{AgCl}$ reference electrode were positioned in the upper section of the cell and were separated from the solution by salt bridges containing electrolyte solution. A matching section of platinum gauze was placed in the reference beam.

The PTFE cell block comprised gas-tight double-glazed (Suprasil W) windows enabling the cell (and its contents) to be cryostatted by the passage of helium or hydrogen gas, pre-cooled by passing it through a copper coil immersed in liquid $\mathrm{N}_{2}$. Room temperature $\mathrm{N}_{2}$ gas was passed between the inner and outer cell-block windows to prevent fogging.

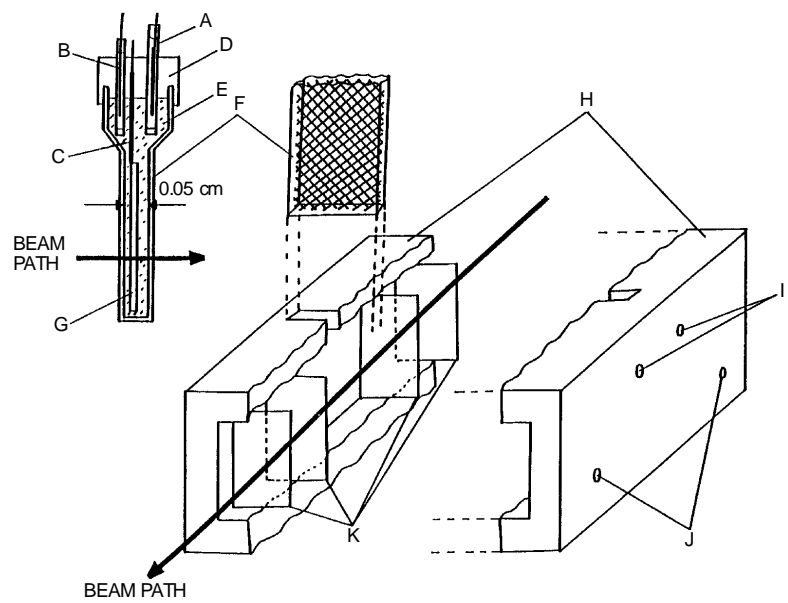

$\begin{aligned} \text { KEY } & \text { Counter electrode } \\ \text { A } & \text { Reference electrode } \\ \text { B } & \text { Working electrode connection protected from bulk solution by PTFE sleeve } \\ \text { C } & \text { Cell cap } \\ \text { E } & \text { Sample solution, degassed with } \mathrm{N}_{2} \\ \text { F } & \text { 0.05 cm Suprasil W quartz cell containing Pt gauze working electrode } \\ \text { G } & \text { Pt gauze working electrode } \\ \text { H } & \text { PTFE cell block } \\ \text { I } & \text { Cold He or } \mathrm{H}_{2} \text { inlet port } \\ \text { J } & \text { Dry (298 K) } \mathrm{N}_{2} \text { inlet ports (to prevent fogging of inner quartz windows) } \\ \text { K } & \text { Supasil W quartz cell block windows }\end{aligned}$

Figure 2.8 Schematic representation of the Optically Semi-Transparent Thin-Layer Electrosynthetic (OSTLE) cell employed in the spectroelectrochemical experiments. ${ }^{86}$

All solutions were purged with $\mathrm{N}_{2}$ prior to transferral into the OSTLE cell (via syringe). Appropriate potentials were applied using a BAS CV27 Voltammograph coupled to a digital LCD multimeter to permit control of both the current and potential during the electrolysis. By this method, the electrogenerated species (which are otherwise unstable) were obtained in situ, and their absorption spectra recorded at regular intervals throughout the electrolysis. The attainment of a steady-state spectrum and the decay of the current to a constant minimum at a potential appropriately beyond $\mathrm{E}_{1 / 2}$ (for the redox process in question) are indicative of the complete conversion of the starting material. For the mixedvalence species, a potential intermediate between the two metal-centred redox processes was employed.

The reversibility of the spectral data was confirmed by the observation of stable isosbestic points, and the regeneration of the starting spectrum following the attainment of the steady-state spectrum for the partially-oxidised mixed-valence $(+5)$ or fully-oxidised $(+6)$ species. For the systems under investigation 
in the present study which possess multiple redox steps, this procedure was repeated for each step before continuing to subsequent processes.

Solutions for the solvent proportion experiments were prepared by serial dilution of solvent mixtures containing acetonitrile and propionitrile. To minimise artefacts in the NIR spectral data due to ion-pairing and concentration effects which are known to influence the IVCT transitions of dinuclear complexes, ${ }^{20,21,28,79,87}$ the spectra were measured using a constant concentration of complex $\left(0.40 \times 10^{-3} \mathrm{M}\right)$ in $0.02 \mathrm{M}\left[\left(n-\mathrm{C}_{4} \mathrm{H}_{9}\right)_{4} \mathrm{~N}\right]\left\{\mathrm{B}\left(\mathrm{C}_{6} \mathrm{~F}_{5}\right\}_{4}\right\}$ at $+25^{\circ} \mathrm{C}$. Spectroelectrochemical experiments were repeated three times at each concentration, and the results reported as an average of the triplicate experiments.

\section{Analysis of Spectroelectrochemical Data}

Since wavelength-dependent charge transfer intensities scale with the inverse of the absolute absorption energy $\left(v^{-1}\right),{ }^{88}$ plots of $\varepsilon / v v s v$ are expected to exhibit a Gaussian shape rather than plots of $\varepsilon$ vs v (i.e. the "raw" experimental data). The energy maximum of the "reduced" absorption spectrum (i.e. $\varepsilon / v v s v$ ) is identified with the vertical upper-/lower-surface energy separation and is the quantity most appropriately employed analysis of IVCT bands. The "raw" absorption spectra ( $\varepsilon v s v)$ were thus scaled as $\int \varepsilon(v) / v d v^{31,88}$ and all data reported in both tabulated and graphical format are presented as $\varepsilon / v v s v$.

Due to comproportionation of the mixed-valence species ${ }^{89}$ the proportion (P) of the complex in the mixed-valence form is given by equation 2.3 , where $\mathrm{K}_{\mathrm{c}}$ is the comproportionation constant derived from electrochemical measurements (§1.3.1.2).

$$
\mathrm{P}=\frac{\mathrm{K}_{\mathrm{c}}^{1 / 2}}{2+\mathrm{K}_{\mathrm{c}}^{1 / 2}}
$$

Spectral deconvolution of the NIR transitions was performed using the curve-fitting subroutine implemented within the GRAMS32 commercial software package. For the dinuclear complexes, convergence of the iteration procedure was generally achieved for three Gaussian-shaped bands under the IVCT manifold. The following procedure was employed for the analysis of all spectroelectrochemical data, and incorporates similar features to previous literature reports. ${ }^{90}$ The positions of the underlying IVCT components were estimated by examining the first- and second-order derivatives of the bands as a guide to the energies of the major components. The energies of the major components were subsequently fixed, and the spectral deconvolution procedure performed to obtain the parameters for the band intensities and bandwidths. The fixed parameters were successively removed as the fit was iteratively refined. In each case, deconvolution was performed with the minimum number of components required to obtain convergence of the fitting procedure.

The band manifolds were approximated by a Gaussian component which closely matched the maximum energy and band-shape on the low-energy side for asymmetrically-shaped IVCT bands. Additional Gaussian-shaped components were added to reproduce the band-shape of the broad, high- 
energy tail. While the band parameters for these components varied slightly over repeat iterations, their relative energies, intensities and bandwidths remained constant. Based on the reproducibility of the parameters obtained from the deconvolutions, the uncertainties in the energies $\left(v_{\max }\right)$, intensities $\left\{(\varepsilon / v)_{\max }\right\}$ and bandwidths $\left(\Delta v_{1 / 2}\right)$ were estimated as $\pm 10 \mathrm{~cm}^{-1}, \pm 0.0001 \mathrm{M}^{-1}$ and $\pm 10 \mathrm{~cm}^{-1}$, respectively. In all cases, the correlation coefficient $\left(R^{2}\right)$ for the fits reported was $>0.995$.

\section{Computational Details}

Computational studies were performed in collaboration with Dr Jeff Reimers and Dr Zhengli Cai in the School of Chemistry at the University of Sydney.

Gas phase geometry optimisations on the $n=4$ and 6 forms of $r a c-\left[\left\{\operatorname{Ru}(\mathrm{bpy})_{2}\right\}_{2}(\mu \text {-bpm) }]^{n+}\right.$ were performed using VASP5 (Vienna Ab initio Simulation Package). ${ }^{91,92}$ Nuclear coordinates were obtained from the Chem3D representation of the structure. The structure of the +5 mixed-valence species was taken as the composition of half of the optimised structures of the +4 and +6 forms. Gas phase geometry optimisations of the solvent molecules were performed at the same level using nuclear coordinates derived from the Chem3D programme.

Geometry optimisations of the structures involving associated solvent molecules were performed by positioning the molecules within the clefts between the planes of the terminal polypyridyl ligands as described in $\S 2.4$.

\subsubsection{Synthetic Procedures}

Metathesis of $\left[\left(\boldsymbol{n}-\mathbf{C}_{4} \mathbf{H}_{\mathbf{9}}\right)_{\mathbf{4}} \mathbf{N}\right]\left\{\mathbf{B}\left(\mathbf{C}_{6} \mathbf{F}_{5}\right)_{4}\right\} .{ }^{81} \mathrm{Li}\left\{\mathrm{B}\left(\mathrm{C}_{6} \mathrm{~F}_{5}\right)_{4}\right\}$. Et ${ }_{2} \mathrm{O}(2.00 \mathrm{~g}, 2.4 \mathrm{mmol})$ was dissolved in $50: 20 \mathrm{H}_{2} \mathrm{O} /$ methanol $\left(14 \mathrm{~cm}^{3}\right)$ and the solution was added dropwise with stirring to [ $\left.\left(n-\mathrm{C}_{4} \mathrm{H}_{9}\right)_{4} \mathrm{~N}\right] \mathrm{Br}$ ( $0.773 \mathrm{~g}, 2.4 \mathrm{mmol})$ in methanol $\left(3 \mathrm{~cm}^{3}\right)$. The white precipitate was stirred for one hour, filtered through a coarse frit, washed with water and dried in vacuo. Recrystallisation of $\left[\left(n-\mathrm{C}_{4} \mathrm{H}_{9}\right)_{4} \mathrm{~N}\right]\left\{\mathrm{B}\left(\mathrm{C}_{6} \mathrm{~F}_{5}\right)_{4}\right\}$ was achieved by dissolving the solid in a minimum volume $\left(\mathrm{ca} .1 \mathrm{~cm}^{3}\right)$ of dichloromethane, followed by the addition of $\mathrm{Et}_{2} \mathrm{O}\left(\mathrm{ca} .2 \mathrm{~cm}^{3}\right)$. The solution was warmed and hexane $\left(3 \mathrm{~cm}^{3}\right)$ added dropwise to induce precipitation. After cooling in the freezer overnight, the white crystalline solid was isolated by filtration, washed with chilled hexane and dried in vacuo at $30^{\circ} \mathrm{C}$ prior to use. Yield: $1.56 \mathrm{~g}$ (75\%).

\section{Microwave techniques}

Microwave-assisted syntheses were conducted in a round bottom flask fitted with condenser, mounted within a modified microwave oven (Sharp, Model R-2V55; 600W, $2450 \mathrm{MHz}$ ) on medium-high power. ${ }^{93-95}$ 


\section{Column Chromatography}

Routine purifications of the crude mixtures of the complexes obtained following syntheses was performed via a gradient elution procedure using SP Sephadex C-25 cation-exchange support (column dimensions were typically 20-30 $\mathrm{cm}$ in length $\times 2-4 \mathrm{~cm}$ in diameter). Complexes were loaded onto columns in aqueous solutions $\left(\mathrm{Cl}^{-}\right.$form, obtained directly from the reaction mixture or by stirring an aqueous suspension with DOWEX ${ }^{\circledR}$ or Amberlite ${ }^{\circledR}$ anion-exchange resins) and eluted with aqueous $\mathrm{NaCl}$ (0.1-0.5 M). Complexes were separated on the basis of their nuclearity, with mononuclear complexes typically eluting with 0.1-0.3 M NaCl, followed by dinuclear complexes with 0.4-0.5 M NaCl.

The separation and resolution of the diastereoisomeric forms was performed using SP Sephadex C-25 cation-exchange support (column dimensions were $c a$. $96 \mathrm{~cm}$ in length $\times 1.6 \mathrm{~cm}$ in diameter) with aqueous sodium tosylate, sodium octanoate, sodium benzoate or (-)-O, $\mathrm{O}^{\prime}$-di-4-toluoyl-L-tartrate as eluents. When the separation of the stereoisomers was not achieved in a single passage down the column, the column was sealed, and the substrate recycled several times down its length with the aid of a peristaltic pump. The "effective column length" (ECL) for the separation represents the length of support travelled by the sample for visual band separation. A detailed account of the cation-exchange chromatographic procedures has been reported previously. ${ }^{76,77}$

\section{Purification of complexes}

Rigorous purification methods were employed prior to structural elucidation (via elemental and/or NMR analyses) and physical characterisation due to the potentially strong associations between the complex cations and the anions present in the eluents used for the chromatographic separations. ${ }^{76,77}$ The impure complex was dissolved in a minimum volume of acetone and loaded onto a short column of silica gel (200-400 mesh; equilibrated with AR acetone, $c a .3 \mathrm{~cm}$ in length $\times 1.5 \mathrm{~cm}$ in diameter), washed alternately with copious amounts of water and acetone, and then eluted with AR acetone containing $5 \%$ $\mathrm{NH}_{4} \mathrm{PF}_{6}$. Following the addition of an equal volume of water, and removal of the acetone under reduced pressure, the precipitate was isolated by vacuum filtration, washed with chilled water and diethyl ether and dried in vacuo.

\subsubsection{Synthesis of Mononuclear Precursors}

The mononuclear ruthenium complexes $\left[\mathrm{Ru}(\mathrm{DMSO})_{4} \mathrm{Cl}_{2}\right],{ }^{96}$ cis-[Ru(pp $\left.)_{2} \mathrm{Cl}_{2}\right] \cdot 2 \mathrm{H}_{2} \mathrm{O}\{\mathrm{pp}=$ bpy, $\left.\mathrm{Me}_{2} \mathrm{bpy}\right\}^{97}$ and cis-[Ru($\left.\left(\mathrm{Me}_{4} \mathrm{bpy}\right)_{2} \mathrm{Cl}_{2}\right]^{98}$ were synthesised according to the literature procedures.

[Ru(5,5'- $\left.\mathbf{M e}_{2} \mathbf{b p y}\right)_{2} \mathbf{C l}_{2}$ ] was prepared according to an adaptation of the "ruthenium blue" method reported by Togano et al. ${ }^{97}$ Following the dissolution of the $\left[\mathrm{Ru}^{\mathrm{III}}\left(5,5^{\prime}-\mathrm{Me}_{2} \mathrm{bpy}\right)_{2} \mathrm{Cl}_{2}\right] \mathrm{Cl}$ intermediate $(1.0 \mathrm{~g}, 1.74 \mathrm{mmol})$ in a boiling solution of dilute $\mathrm{HCl}\left(3 \mathrm{M}, 250 \mathrm{~cm}^{3}\right), \mathrm{SnCl}_{2} .2 \mathrm{H}_{2} \mathrm{O}(20 \mathrm{mg}, 0.0886 \mathrm{mmol})$ was added and the mixture boiled for a further $2.5 \mathrm{~h}$ during which time the solution attained a black colouration. The volume of the solution was reduced by rotary evaporation to precipitate a black 
microcrystalline solid which was isolated by filtration and washed with chilled water $\left(5 \mathrm{~cm}^{3}\right)$.

Yield: 0.935 g (100 \%). Anal. Calcd for $\mathrm{C}_{24} \mathrm{H}_{48} \mathrm{~N}_{4} \mathrm{Cl}_{2} \mathrm{Ru}_{2}$ : C, 51.1; H, 8.51; N, 9.93\%; Found: C, 51.1; $\mathrm{H}$, 8.50; N, 9.94\%.

$\left[\mathbf{R u}\left(\mathbf{M e}_{2} \mathbf{p h e n}\right)_{2} \mathbf{C l}_{2}\right.$ ] was synthesised via a modification of a procedure reported by Sullivan et al. ${ }^{99}$ for the synthesis of [Ru(bpy) ${ }_{2} \mathrm{Cl}_{2}$ ]. $\mathrm{RuCl}_{3} \cdot 3 \mathrm{H}_{2} \mathrm{O}$ (1.386 g, $5.3 \mathrm{mmol}$ ), $\mathrm{Me}_{2}$ phen (2.51 g, $\left.12.1 \mathrm{mmol}\right)$ and $\mathrm{LiCl}$ $(0.274 \mathrm{~g}, 6.47 \mathrm{mmol})$ were heated at reflux in DMF $\left(27.5 \mathrm{~cm}^{3}\right)$ for $10 \mathrm{~h}$. The dark purple solution was cooled to room temperature, poured into a solution of acetone $\left(50 \mathrm{~cm}^{3}\right)$ and chilled by refrigeration overnight. A black solid was isolated by vacuum filtration, washed with diethyl ether $\left(3 \times 5 \mathrm{~cm}^{3}\right)$ and dried in vacuo at $50^{\circ} \mathrm{C}$ for three days. Yield: 0.700 g (22\%). Anal. Calcd for $\mathrm{C}_{28} \mathrm{H}_{24} \mathrm{~N}_{4} \mathrm{Cl}_{2} \mathrm{Ru}$ : C, 57.1; $\mathrm{H}$, 4.08; N, 9.52\%; Found: C, 57.0; H, 4.03; N, 9.50\%.

[Ru( $\left.{ }^{\mathrm{t}} \mathbf{B u}_{2} \mathbf{b p y}\right)_{2} \mathbf{C l}_{2}$ ] was synthesised according to the method for $\left[\mathrm{Ru}\left(\mathrm{Me}_{2} \mathrm{phen}\right)_{2} \mathrm{Cl}_{2}\right]$, using ${ }^{\mathrm{t}} \mathrm{Bu}_{2} \mathrm{bpy}$ (7.76 g, 28.9 mmol) instead of $\mathrm{Me}_{4}$ phen, with $\mathrm{RuCl}_{3} .3 \mathrm{H}_{2} \mathrm{O}$ (3.0 g, $0.0145 \mathrm{~mol}$ ), $\mathrm{LiCl}(0.748 \mathrm{~g}, 17.6 \mathrm{mmol}$ ) and DMF $\left(25 \mathrm{~cm}^{3}\right.$ ). Yield: 7.86 g (77\%). Anal. Calcd for $\mathrm{C}_{36} \mathrm{H}_{48} \mathrm{~N}_{4} \mathrm{Cl}_{2} \mathrm{Ru}$ : C, 61.0; H, 6.78; N, 7.91\%; Found: C, 61.0; H, 6.80; N, 7.92\%.

[Ru(Me $\left.\left.\mathbf{M e n}_{2} \mathbf{p h e n}\right)_{2}(\mathbf{b p m})\right]\left(\mathbf{P F}_{\mathbf{6}}\right)_{2}$. [Ru( $\left.\left.\mathrm{Me}_{2} \mathrm{phen}\right)_{2} \mathrm{Cl}_{2}\right]$ (500 mg, $\left.0.850 \mathrm{mmol}\right)$ and bpm (123 mg, 0.776 mmol) were heated at reflux in 3:1 EtOH/water $\left(200 \mathrm{~cm}^{3}\right)$ for $5.5 \mathrm{~h}$ during which time the solution attained an orange coloration. Upon cooling, the mixture was diluted with distilled water $\left(50 \mathrm{~cm}^{3}\right)$ and loaded onto a column $(15 \mathrm{~cm} \times 3.5 \mathrm{~cm})$ containing SP Sephadex C-25 support. Separation of the mononuclear product from the crude mixture was achieved via a gradient elution procedure using aqueous $0.1-0.4 \mathrm{M} \mathrm{NaCl}$ as the eluent. The orange band of mononuclear material was precipitated as the $\mathrm{PF}_{6}{ }^{-}$salt from the eluate by addition of a saturated solution of aqueous $\mathrm{KPF}_{6}$. The solid was isolated by vacuum filtration and washed with diethyl ether. Yield $433 \mathrm{mg}$ (53\%). Anal. Calcd for $\mathrm{C}_{36} \mathrm{H}_{30} \mathrm{~F}_{12} \mathrm{~N}_{8} \mathrm{P}_{2} \mathrm{Ru}$ : C, 44.8; H, 3.11; N, 11.6\%; Found: C, 44.8; H, 3.05; N, 11.5\%.

[Ru( $\left.\left.\left.{ }^{\mathrm{t}} \mathbf{B u}_{2} \mathbf{b p y}\right)_{2} \mathbf{( b p m}\right)\right]\left(\mathbf{P F}_{6}\right)_{2}$. A suspension of bpm (120 mg, $\left.0.760 \mathrm{mmol}\right)$ in ethylene glycol $\left(3 \mathrm{~cm}^{3}\right)$ was heated in a modified microwave oven on medium-high power for $20 \mathrm{~s}$ to complete dissolution. $\left[\mathrm{Ru}\left({ }^{\mathrm{t}} \mathrm{Bu}_{2} \mathrm{bpy}\right)_{2} \mathrm{Cl}_{2}\right]$ (700 mg, $\left.0.988 \mathrm{mmol}\right)$ was added and the mixture heated at reflux for a further $5 \mathrm{~min}$ during which time the solution attained an orange coloration. Upon cooling, the mixture was diluted with distilled water $\left(50 \mathrm{~cm}^{3}\right)$ and loaded onto a column $(15 \mathrm{~cm} \times 3.5 \mathrm{~cm})$ containing SP Sephadex C-25 support. Separation of the mononuclear product from the crude mixture was achieved via a gradient elution procedure using aqueous $0.1-0.4 \mathrm{M} \mathrm{NaCl}$ as the eluent. The orange band of mononuclear material was precipitated as the $\mathrm{PF}_{6}{ }^{-}$salt by addition to the eluate of a saturated solution of aqueous $\mathrm{KPF}_{6}$. The solid was isolated by vacuum filtration and washed with diethyl ether. Yield $609 \mathrm{mg}$ (74\%). Anal. Calcd for $\mathrm{C}_{44} \mathrm{H}_{54} \mathrm{~F}_{12} \mathrm{~N}_{8} \mathrm{P}_{2} \mathrm{Ru}$ : C, 48.7; H, 5.01; N, 10.3\%; Found: C, 48.6; H, 4.99; N, 10.3\%. 
[Ru(bpy $\left.\left.)_{3}\right]_{(\mathbf{P F}}\right)_{2}$ was synthesised in a similar manner to the literature method, ${ }^{97}$ and isolated as the $\mathrm{PF}_{6}{ }^{-}$ salt by addition of $\mathrm{KPF}_{6}$. The ${ }^{1} \mathrm{H}$ NMR and electronic spectrum were in agreement with those reported previously. ${ }^{100-102}$

\subsubsection{Synthesis of Dinuclear Complexes, and Separation of Diastereoisomers}

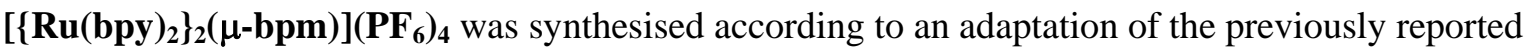
procedures. ${ }^{30,103}\left[\mathrm{Ru}(\mathrm{bpy})_{2} \mathrm{Cl}_{2}\right] .2 \mathrm{H}_{2} \mathrm{O}$ (300 mg, $0.5765 \mathrm{mmol}$ ) and bpm (41.4 mg, $0.262 \mathrm{mmol}$ ) were heated at reflux $\left(\sim 120^{\circ} \mathrm{C}\right)$ in $10 \%$ water/ethylene glycol $\left(20 \mathrm{~cm}^{3}\right)$ for $5 \mathrm{~h}$. Upon cooling, the dark green solution was diluted with distilled water $\left(50 \mathrm{~cm}^{3}\right)$ and loaded onto a column $(15 \mathrm{~cm} \times 3.5 \mathrm{~cm})$ containing SP Sephadex C-25 support. Separation of the mono- and dinuclear products from the crude mixture was achieved via a gradient elution procedure using aqueous $0.1-0.5 \mathrm{M} \mathrm{NaCl}$ as the eluent. An orange band of mononuclear material eluted first (0.2-0.3 M NaCl), followed by a dark green band of the dinuclear material $(0.5 \mathrm{M} \mathrm{NaCl})$. The complexes were precipitated as their $\mathrm{PF}_{6}{ }^{-}$salts by addition of a saturated aqueous solution of $\mathrm{KPF}_{6}$. The orange mononuclear, and dark green dinuclear solids were isolated by vacuum filtration, washed with chilled water and diethyl ether, and dried in vacuo at $40^{\circ} \mathrm{C}$ for $4 \mathrm{~h}$. Yields: $\left[\mathrm{Ru}(\mathrm{bpy})_{2}(\mathrm{bpm})\right]\left(\mathrm{PF}_{6}\right)_{2}, 15 \mathrm{mg} ;\left[\left\{\mathrm{Ru}(\mathrm{bpy})_{2}\right\}_{2}(\mu-\mathrm{bpm})\right]\left(\mathrm{PF}_{6}\right)_{4}, 385 \mathrm{mg}(94 \%)$. The NMR spectra of the dinuclear products were identical to those reported previously by Hua. ${ }^{103}$

The diastereoisomeric mixture of the dinuclear complex was converted to the chloride salt by stirring an aqueous suspension with DOWEX ${ }^{\circledR}$ anion exchange resin (50-100 mesh; $\mathrm{Cl}^{-}$form). The complex was sorbed onto the column containing SP Sephadex C-25 as the support, and separation of the diastereoisomers was achieved by cation exchange chromatography using aqueous $0.25 \mathrm{M}$ sodium tosylate solution as the eluent. ${ }^{76}$ The diastereoisomers separated after passing through an ECL ${ }^{77}$ of approximately $180 \mathrm{~cm}$. The two dark green bands were collected and precipitated as the $\mathrm{PF}_{6}{ }^{-}$salts by addition of a saturated solution of $\mathrm{KPF}_{6}$. The solid products from each band were purified on silica gel as described above. Bands 1 and 2 exhibited different NMR spectra, and were assigned as the meso and rac diastereoisomers, respectively (Table 2.1, §2.3.1.1).

$\left[\left\{\mathbf{R u}\left(\mathbf{M e}_{2} \mathbf{b p y}\right)_{2}\right\}_{2}(\mu-\mathbf{b p m})\right]\left(\mathbf{P F}_{6}\right)_{4}$ was prepared via an analogous procedure to that reported above for $\left[\left\{\mathrm{Ru}(\mathrm{bpy})_{2}\right\}_{2}(\mu-\mathrm{bpm})\right]\left(\mathrm{PF}_{6}\right)_{4}$. Diastereoisomeric separation was achieved within one pass down the column (ECL $\sim 50 \mathrm{~cm}$ ) using $0.25 \mathrm{M}$ sodium tosylate solution as eluent. The ${ }^{1} \mathrm{H}$ NMR spectra of the diastereoisomeric forms were in agreement with those reported previously. ${ }^{76,77}$

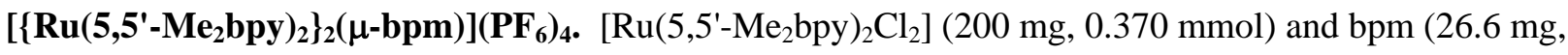
$0.168 \mathrm{mmol})$ were heated at reflux in ethylene glycol $\left(2 \mathrm{~cm}^{3}\right)$ in a modified microwave oven on mediumhigh power for $10 \mathrm{~min}$. The separation of the orange mononuclear material from the desired dark green product was achieved via a gradient elution procedure as described above for 
$\left[\left\{\mathrm{Ru}(\mathrm{bpy})_{2}\right\}_{2}(\mu-\mathrm{bpm})\right]\left(\mathrm{PF}_{6}\right)_{4}$. Yield $226 \mathrm{mg}$ (85\%). Anal. Calcd for $\mathrm{C}_{56} \mathrm{H}_{56} \mathrm{~F}_{24} \mathrm{~N}_{12} \mathrm{P}_{4} \mathrm{Ru}_{2}$ : C, 40.1; H, 3.37; N, 10.0\%; Found: C, 40.0; H, 3.34; N, 10.0\%.

The separation and purification of the diastereoisomeric forms was achieved as described above, however $0.25 \mathrm{M}$ sodium benzoate solution was used as the eluent instead of sodium tosylate solution. ${ }^{1} \mathrm{H}$ NMR ( $\left.\delta \mathrm{ppm} ; \mathrm{CD}_{3} \mathrm{CN}\right)$ : (Band 1; meso) $2.20\left(\mathrm{CH}_{3}{ }_{3}\right.$ at $\left.\mathrm{H}^{\prime}, 12 \mathrm{H}, \mathrm{s}\right), 2.35\left(\mathrm{CH}_{3}\right.$ at $\left.\mathrm{H} 5,12 \mathrm{H}, \mathrm{s}\right), 7.32$ (H6', 4H, s), 7.45 (H5 bpm, 2H, $J=5.7 \mathrm{~Hz}, \mathrm{t}), 7.72$ (H6, 4H, s), 7.88 (H4', 4H, $J=5.0 \mathrm{~Hz}, \mathrm{~d}), 7.90$ (H4, 4H, $J=5.0 \mathrm{~Hz}, \mathrm{~d}), 7.96$ (H4, H6 bpm, 4H, $J=5.7 \mathrm{~Hz}, \mathrm{~d}), 8.29$ (H3, 4H, $J=3 \mathrm{~Hz}, \mathrm{~d}), 8.32$ (H3', 4H, $J=3$ Hz, d); (Band 2; rac) 2.15 ( $\mathrm{CH}_{3}{ }_{3}$ at H5', 12H, s), 2.18 ( $\mathrm{CH}_{3}$ at H5, 12H, s), 7.27 (H6', 4H, s), 7.46 (H5 bpm, 2H, $J=5.7 \mathrm{~Hz}, \mathrm{t}), 7.46(\mathrm{H} 6,4 \mathrm{H}, \mathrm{s}), 7.89$ (H4', 4H, $J=5.0,1.0 \mathrm{~Hz}, \mathrm{dd}), 7.96$ (H4, 4H, $J=5.0,1.0$ Hz, dd), 8.07 (H4, H6 bpm, 4H, $J=5.7$ Hz, d), 8.35 (H3', 4H, $J=3$ Hz, d), 8.37 (H3', 4H, $J=3$ Hz, d).

$\left[\left\{\mathbf{R u}\left(\mathbf{M e}_{4} \mathbf{b p y}\right)_{2}\right\}_{2}(\mu-\mathbf{b p m})\right]\left(\mathbf{P F}_{6}\right)_{4}$ was synthesised according to the microwave methodology reported above for $\left[\left\{\mathrm{Ru}\left(5,5^{\prime}-\mathrm{Me}_{2} \mathrm{bpy}\right)_{2}\right\}_{2}(\mu-\mathrm{bpm})\right]\left(\mathrm{PF}_{6}\right)_{4}$ using $\left[\mathrm{Ru}\left(\mathrm{Me}_{4} \mathrm{bpy}\right)_{2} \mathrm{Cl}_{2}(188 \mathrm{mg}, 0.315 \mathrm{mmol})\right.$ and bpm (19.4 mg, $0.122 \mathrm{mmol}$ ) in ethylene glycol $\left(3 \mathrm{~cm}^{3}\right)$. Yield: $1.286 \mathrm{~g}(59 \%)$. Anal. Calcd for $\mathrm{C}_{64} \mathrm{H}_{70} \mathrm{~F}_{24} \mathrm{~N}_{12} \mathrm{P}_{4} \mathrm{Ru}_{2}$ : C, 43.0; H, 3.91; N, 9.40\%; Found: C, 42.9; H, 3.87; N, 9.37\%.

The separation and purification of the diastereoisomeric forms was achieved as described above, using 0.25 M sodium benzoate solution as the eluent. ${ }^{1} \mathrm{H}$ NMR ( $\delta \mathrm{ppm} ; \mathrm{CD}_{3} \mathrm{CN}$ ): (Band 1; meso) 2.12 $\left(\mathrm{CH}_{3}{ }_{3}\right.$ at H5', 12H, s), $2.24\left(\mathrm{CH}_{3}\right.$ at $\left.\mathrm{H} 5,12 \mathrm{H}, \mathrm{s}\right), 2.49\left(\mathrm{CH}_{3}^{\prime}\right.$ at $\mathrm{H} 4$ ', $\left.12 \mathrm{H}, \mathrm{s}\right), 2.67\left(\mathrm{CH}_{3}\right.$ at $\left.\mathrm{H} 4,12 \mathrm{H}, \mathrm{s}\right), 7.17$ (H6', 4H, s), 7.51 (H6, 4H, s), 7.42 (H5 bpm, 2H, J = 5.7 Hz, t), 8.02 (H4, H6 bpm, 4H, J = 5.7 Hz, d), 8.20 (H3', 4H, s), 8.25 (H3, 4H, s); (Band 2; rac) $2.03\left(\mathrm{CH}_{3}^{\prime}\right.$ at H5', 12H, s), $2.07\left(\mathrm{CH}_{3}\right.$ at $\left.\mathrm{H} 5,12 \mathrm{H}, \mathrm{s}\right)$, $2.44\left(\mathrm{CH}_{3}{ }_{3}\right.$ at $\mathrm{H} 4$ ', 12H, s), $2.50\left(\mathrm{CH}_{3}\right.$ at H4, 12H, s), $7.12(\mathrm{H6}$ ', 4H, s), 7.25 (H6, 4H, s), 7.42 ( $\mathrm{H} 5 \mathrm{bpm}$, 2H, $J=5.7 \mathrm{~Hz}, \mathrm{t}$ ), 8.02 (H4, H6 bpm, 4H, $J=5.7 \mathrm{~Hz}, \mathrm{~d}), 8.25$ (H3', 4H, s), 8.27 (H3, 4H, s).

[ $\left\{\mathbf{R u}\left(\mathbf{M e}_{2} \mathbf{p h e n}\right)_{2}\right\}_{2}(\mu$-bpm $\left.)\right]\left(\mathbf{P F}_{6}\right)_{4}$ was synthesised according to the microwave methodology reported above for $\left[\left\{\mathrm{Ru}\left(5,5^{\prime}-\mathrm{Me}_{2} \mathrm{bpy}\right)_{2}\right\}_{2}(\mu\right.$-bpm) $]\left(\mathrm{PF}_{6}\right)_{4}$ using $\left[\mathrm{Ru}\left(\mathrm{Me}_{2} \mathrm{phen}\right)_{2} \mathrm{bpm}\right]\left(\mathrm{PF}_{6}\right)_{2}(150 \mathrm{mg}, 0.155 \mathrm{mmol})$ and $\left[\mathrm{Ru}\left(\mathrm{Me}_{2} \mathrm{phen}\right)_{2} \mathrm{Cl}_{2}\right]$ (160 mg, $\left.0.250 \mathrm{mmol}\right)$ in ethylene glycol $\left(5 \mathrm{~cm}^{3}\right)$. Yield $208 \mathrm{mg}$ (76\%). Anal. Calcd for $\mathrm{C}_{64} \mathrm{H}_{54} \mathrm{~F}_{24} \mathrm{~N}_{12} \mathrm{P}_{4} \mathrm{Ru}_{2}$ : C, 43.4; H, 3.05; N, 9.48\%; Found: C, 43.3; H, 3.01; N, 9.40\%.

The separation and purification of the diastereoisomeric forms was achieved as described above using $0.20 \mathrm{M}$ sodium benzoate solution as the eluent. Bands 1 and 2 were identified as the meso and rac diastereoisomers, respectively, as established by ${ }^{1} \mathrm{H}$ NMR and COSY experiments. ${ }^{1} \mathrm{H}$ NMR $(\delta \mathrm{ppm}$; $\mathrm{CD}_{3} \mathrm{CN}$ ): (Band 1; meso) $1.40\left(\mathrm{CH}_{3}\right.$ at $\left.\mathrm{H} 2,12 \mathrm{H}, \mathrm{s}\right), 1.42\left(\mathrm{CH}_{3}\right.$ at $\left.\mathrm{H} 9,12 \mathrm{H}, \mathrm{s}\right), 7.43(\mathrm{H} 3,4 \mathrm{H}, J=6.2,1.5$, dd), 7.49 (H5 bpm, 2H, $J=5.7 \mathrm{~Hz}, \mathrm{t}), 7.51(\mathrm{H} 4,4 \mathrm{H}, J=6.5 \mathrm{~Hz}, \mathrm{~d}), 7.55$ (H8, 4H, $J=6.2,1.5$, dd), 7.84 (H7, 4H, $J=6.5 \mathrm{~Hz}, \mathrm{~d}), 8.00$ (H4, H6 bpm, 4H, $J=5.7 \mathrm{~Hz}, \mathrm{~d}), 8.42$ (H5, 4H, $J=9 \mathrm{~Hz}, \mathrm{~d}), 8.44$ (H6, 4H, $J=9 \mathrm{~Hz}$, d); (Band 2; rac) $1.40\left(\mathrm{CH}_{3}\right.$ at $\left.\mathrm{H} 2,12 \mathrm{H}, \mathrm{s}\right), 1.48\left(\mathrm{CH}_{3}\right.$ at $\left.\mathrm{H} 9,12 \mathrm{H}, \mathrm{s}\right), 7.27(\mathrm{H} 3,4 \mathrm{H}, J=6.2,1.5$, dd), 7.42 (H8, 4H, $J=6.2,1.5$, dd), 7.45 (H4, 4H, $J=6.5 \mathrm{~Hz}, \mathrm{~d}), 7.49$ (H5 bpm, 2H, $J=5.7 \mathrm{~Hz}, \mathrm{t}), 7.57$ (H7, 4H, $J=6.5 \mathrm{~Hz}, \mathrm{~d}), 8.04$ (H4, H6 bpm, 4H, $J=5.7 \mathrm{~Hz}, \mathrm{~d}), 8.49$ (H5, 4H, $J=9 \mathrm{~Hz}, \mathrm{~d}), 8.49$ (H6, 4H, $J=9 \mathrm{~Hz}, \mathrm{~d}$ ). 
$\left[\left\{\mathbf{R u}\left({ }^{t} \mathbf{B u}_{2} \mathbf{b p y}\right)_{2}\right\}_{2}(\mu-\mathbf{b p m})\right]\left(\mathbf{P F}_{6}\right)_{4}$. An analogous procedure was employed to that described above for the synthesis of $\left[\left\{\mathrm{Ru}\left(\mathrm{Me}_{2} \mathrm{phen}\right)_{2}\right\}_{2}(\mu\right.$-bpm $\left.)\right]\left(\mathrm{PF}_{6}\right)_{4}$ using the precursors $\left[\mathrm{Ru}\left({ }^{\mathrm{t}} \mathrm{Bu}_{2} \mathrm{bpy}\right)_{2}(\mathrm{bpm})\right]\left(\mathrm{PF}_{6}\right)_{2}(200 \mathrm{mg}$, $0.184 \mathrm{mmol})$ and $\left.\left[\mathrm{Ru}^{(}{ }^{\mathrm{B}} \mathrm{Bu}_{2} \mathrm{bpy}\right)_{2} \mathrm{Cl}_{2}\right](212 \mathrm{mg}, 0.313 \mathrm{mmol})$. Yield $251 \mathrm{mg}(68 \%)$. Anal. Calcd for $\mathrm{C}_{80} \mathrm{H}_{102} \mathrm{~F}_{24} \mathrm{~N}_{12} \mathrm{P}_{4} \mathrm{Ru}_{2}$ : C, 47.7; H, 5.11; N, 8.35\%; Found: C, 47.5; H, 5.25; N, 7.99\%. ${ }^{1} \mathrm{H}$ NMR ( $\delta$ ppm; $\left.\mathrm{CD}_{3} \mathrm{CN}\right)$ : (Band 1; meso) $1.40\left(\left(\mathrm{CH}_{3}^{\prime}\right)_{3}\right.$ at $\mathrm{H} 4$ ', 36H, s), $1.42\left(\left(\mathrm{CH}_{3}\right)_{3}\right.$ at $\left.\mathrm{H} 4,36 \mathrm{H}, \mathrm{s}\right), 7.45(\mathrm{H} 5$ ', $4 \mathrm{H}, J=8$, $1.5 \mathrm{~Hz}, \mathrm{dd}), 7.49$ (H5 bpm, 2H, $J=5.7 \mathrm{~Hz}, \mathrm{t}), 7.54$ (H6', 4H, $J=5 \mathrm{~Hz}, \mathrm{~d}), 7.57$ (H5, 4H, $J=8,1.5 \mathrm{~Hz}$, dd), 7.84 (H6, 4H, $J=5$ Hz, d), 8.01 (H4, H6 bpm, 4H, $J=5.7 \mathrm{~Hz}, \mathrm{~d}), 8.42$ (H3, 4H, $J=3 \mathrm{~Hz}, \mathrm{~d}$ ), 8.44 (H3', 4H, $J=3 \mathrm{~Hz}, \mathrm{~d})$; (Band 2; rac) $1.40\left(\left(\mathrm{CH}_{3}^{\prime}\right)_{3}\right.$ at $\mathrm{H} 4$ ', 36H, s), $1.48\left(\left(\mathrm{CH}_{3}\right)_{3}\right.$ at $\left.\mathrm{H} 4,36 \mathrm{H}, \mathrm{s}\right), 7.27(\mathrm{H} 5$ ', 4H, $J=5,1.5 \mathrm{~Hz}$, dd), 7.43 (H5, 4H, $J=5,1.5 \mathrm{~Hz}$, dd), 7.43 (H6, 4H, $J=5 \mathrm{~Hz}, \mathrm{~d}$ ), 7.48 (H5 bpm, 2H, $J$ = $5.7 \mathrm{~Hz}, \mathrm{t}), 7.57$ (H6', 4H, $J=5 \mathrm{~Hz}, \mathrm{~d}), 8.03(\mathrm{H} 4, \mathrm{H} 6 \mathrm{bpm}, 4 \mathrm{H}, J=5.7 \mathrm{~Hz}, \mathrm{~d}), 8.49(\mathrm{H} 3,4 \mathrm{H}, J=3 \mathrm{~Hz}$, d), $8.50(\mathrm{H3}$ ', 4H, $J=3 \mathrm{~Hz}, \mathrm{~d})$.

[\{Ru(bpy $\left.)_{2}\right\}_{2}(\mu$-dbneil) $]\left(\mathbf{P F}_{6}\right)_{4}$ and $\left[\mathrm{Ru}(\mathrm{bpy})_{2}(\mathrm{dbneil})\right]\left(\mathrm{PF}_{6}\right)_{2}$ were supplied by Prof. Moshe Kol (The School of Chemistry, Tel Aviv University, Israel). ${ }^{82}$ The separation and purification of the diastereoisomeric forms of the dinuclear complex were achieved using the chromatographic techniques described above, with $0.25 \mathrm{M}$ sodium octanoate solution as the eluent. The separated bands were collected following two passages through the column (ECL $=2 \mathrm{~m}$ ). Bands 1 and 2 were identified as the meso and rac diastereoisomers, respectively, as established by ${ }^{1} \mathrm{H}$ NMR and COSY experiments. The ${ }^{1} \mathrm{H}$ NMR spectra are reported in Table 2.1, §2.3.1.1.

The material from the second band was re-applied to an identical column, and following elution with an aqueous solution of $0.15 \mathrm{M}$ sodium (-)-O,O'-di-4-toluoyl-L-tartrate, two bands were eluted (ECL $=1 \mathrm{~m}$ ), from which the enantiomers of the rac form were isolated and purified. The CD spectra in $\mathrm{CH}_{3} \mathrm{CN}$ are provided in Figure A2.1 (Appendix A); $\lambda / \mathrm{nm}\left\{\Delta \varepsilon / \mathrm{M}^{-1} \mathrm{~cm}^{-1}\right.$ (Band 1, Band 2) $\}: 219(+25,-23$ ), $237(-4.0,+4.4), 250(+8.8,-8.0), 283(+66,-67), 301(-76,+75), 318(-59,+59), 380(+2.8,-2.1), 420(-$ $10,+11), 450(-4.3,+5.4), 684(-10,+11) .^{104}$

\subsubsection{X-ray Crystallography}

The collection and structural refinement of the X-ray crystal data was performed by Dr Murray Davies (Department of Chemistry, James Cook University).

Crystals of meso-[\{Ru(bpy $\left.\left.)_{2}\right\}_{2}(\mu-b p m)\right]\left(\mathrm{PF}_{6}\right)_{4}$ were grown by slow evaporation of a 70:30 2,2,2-trifluoroethanol $/ \mathrm{H}_{2} \mathrm{O}$ solution of the complex under ambient conditions in the absence of light. The rod-shaped dichroic cyrystals exhibited a deep green/red colouration. A single crystal of dimensions $0.2 \times 0.2 \times 0.15 \mathrm{~mm}$ was coated with paratone- $\mathrm{N}$ and mounted onto a glass fibre.

Single crystals of the related complex meso- $\left[\left\{\mathrm{Ru}\left(\mathrm{Me}_{2} \mathrm{bpy}\right)_{2}\right\}_{2}(\mu\right.$-bpm $\left.)\right](\text { tos })_{4}$ were obtained by preliminary anion exchange of the $\mathrm{PF}_{6}{ }^{-}$to the tosylate salt using QAE Sephadex A-25 anion-exchange support. The support was initially equilibrated with $1 \mathrm{M}$ sodium tosylate by the passage of $c a .100 \mathrm{~cm}^{3}$ of the aqueous eluent through the short column (dimensions $10 \mathrm{~cm}$ length $\times 2 \mathrm{~cm}$ diameter) containing the 
support (as the $\mathrm{Cl}^{-}$form), then washed with copious amounts of water to remove excess sodium tosylate. An aqueous solution of the $\mathrm{Cl}^{-}$form of meso-[\{Ru(Me $\left.\left.2 \mathrm{bpy}\right)_{2}\right\}_{2}(\mu \text {-bpm) }]^{4+}\left(c a .10 \mathrm{mg}\right.$ in $\left.2 \mathrm{~cm}^{3}\right)-$ obtained by stirring an aqueous suspension with $\mathrm{DOWEX}^{\circledR}$ anion-exchange resin - was subsequently loaded onto the column. The tight green band of the dinuclear complex was eluted with water and the solution permitted to evaporate under ambient conditions the absence of light for several weeks to yield dichroic dark green/red plate-like crystals. A single crystal of dimensions $0.2 \times 0.2 \times 0.02 \mathrm{~mm}$ was quickly coated with polyacrylamide (to avoid rapid solvent loss) and mounted onto a glass fibre for X-ray structural determination.

X-ray data ( $\mathrm{T}=293 \mathrm{~K}$, Mo K $\alpha$ radiation $\lambda=0.71073 \AA$ ) was collected on a Bruker AXS CCD diffractometer using the SMART software package. The data sets were corrected for absorption using SADABS. ${ }^{105}$ The structures were solved by direct methods and refined on $F^{2}$ using SHELXL-97 ${ }^{106}$ within the WING-X programme. All non-hydrogen atoms were refined aniostropically and hydrogen atoms were located but were refined with isotropic thermal parameters using SHELXS-97 ${ }^{106}$ also within the WING-X software package. In both cases, the unambiguous determination of the crystal structures confirmed the identity of the first band eluted in the diastereoisomer separation as the meso forms (consistent with a previous literature report). ${ }^{76}$ The structures were plotted using ORTEP-3 and atoms are shown using $50 \%$ anisotropic thermal ellipsoids. A summary of the data collection parameters and structural refinement details is provided in Table A2.1 (Appendix A) and the full details are provided on the CD accompaniment to this thesis. 


\subsection{Results and Discussion}

\subsubsection{Diastereoisomer Synthesis, Separation and Structural Characterisation}

The complexes $\left[\left\{\mathrm{Ru}(\mathrm{bpy})_{2}\right\}_{2}(\mu \text {-bpm })\right]^{4+}$ and $\left[\left\{\mathrm{Ru}\left(\mathrm{Me}_{2} \mathrm{bpy}\right)_{2}\right\}_{2}(\mu \text {-bpm })\right]^{4+}$ have been synthesised previously ${ }^{30,76,77,103,107-113}$ utilising a thermal method involving the reaction of cis$\left[\mathrm{Ru}(\mathrm{bpy})_{2} \mathrm{Cl}_{2}\right] .2 \mathrm{H}_{2} \mathrm{O}$ precursor with bpm in aqueous methanol or ethanol/water (1:1) under reflux, and a microwave-assisted synthetic procedure. In the present study, the latter method was used for the complexes $\left[\left\{\mathrm{Ru}(\mathrm{pp})_{2}\right\}_{2}(\mu \text {-bpm })\right]^{4+}\left\{\mathrm{pp}=\right.$ bpy, Me $\mathrm{Mepy}$, 5,5'-Me ${ }_{2}$ bpy, $\mathrm{Me}_{4}$ bpy $\}$, which resulted in comparable yields to those obtained from the thermal methods but with a significant reduction in the reaction times (typically $\sim 10 \mathrm{~min}$ rather than $2 \mathrm{~h}$ for the thermal procedure).

For the complexes $\left[\left\{\mathrm{Ru}(\mathrm{pp})_{2}\right\}_{2}(\mu \text {-bpm })\right]^{4+}\left\{\mathrm{pp}={ }^{\mathrm{t}} \mathrm{Bu}_{2}\right.$ bpy and $\mathrm{Me}_{2}$ phen $\}$, the one-step microwaveassisted method produced lower yields than a two-step procedure involving the initial synthesis of $\left[\mathrm{Ru}(\mathrm{pp})_{2}(\mathrm{bpm})\right]^{2+}$, followed by its reaction with an excess of $\left[\mathrm{Ru}(\mathrm{pp})_{2} \mathrm{Cl}_{2}\right]$. This probably reflects the unfavourable steric interactions between the methyl substituents in the ${ }^{t} \mathrm{Bu}_{2} \mathrm{bpy}$ and $\mathrm{Me}_{2}$ phen terminal ligands in the formation of the dinuclear complex.

Separation of the diastereoisomeric forms of $\left[\left\{\mathrm{Ru}(\mathrm{pp})_{2}\right\}_{2}(\mu-\mathrm{bpm})\right]^{4+}$ and $\left[\left\{\mathrm{Ru}(\mathrm{bpy})_{2}\right\}_{2}(\mu-\right.$ dbneil) $]^{4+}$ was achieved by cation-exchange chromatography using SP Sephadex C-25 support with aqueous sodium tosylate, sodium benzoate or sodium octanoate solutions as the eluents. ${ }^{75-78,104}$ The latter was found to provide a more efficient separation (i.e. shorter ECL) for the complexes incorporating alkylated terminal ligands ( $\mathrm{Me}_{2}$ bpy, 5,5'- $\mathrm{Me}_{2}$ bpy, ${ }^{\mathrm{t}} \mathrm{Bu}_{2} \mathrm{bpy}$ and $\mathrm{Me}_{2}$ phen). In all cases, the diastereoisomers of $\left[\left\{\mathrm{Ru}(\mathrm{pp})_{2}\right\}_{2}(\mu \text {-bpm) }]^{4+}\right.$ were obtained in a ratio of approximately 1:1 from the chromatographic procedure.

\subsubsection{1 ${ }^{1}$ H NMR Studies}

Structural characterisation of the meso and rac forms of $\left[\left\{\mathrm{Ru}(\mathrm{pp})_{2}\right\}_{2}(\mu-\mathrm{bpm})\right]^{4+}\{\mathrm{pp}=\mathrm{bpy}$, 5,5'-Me ${ }_{2}$ bpy, $\mathrm{Me}_{4}$ bpy, $\mathrm{Me}_{2}$ phen, $\left.{ }^{\mathrm{t}} \mathrm{Bu} \mathrm{u}_{2} \mathrm{bpy}\right\}$ and $\left[\left\{\mathrm{Ru}(\mathrm{bpy})_{2}\right\}_{2}(\mu \text {-dbneil })\right]^{4+}$ was performed using one- and two-dimensional ( ${ }^{1} \mathrm{H}$ COSY) NMR techniques. The ${ }^{1} \mathrm{H}$ NMR chemical shift assignments for meso- and rac- $\left[\left\{\mathrm{Ru}(\mathrm{bpy})_{2}\right\}_{2}(\mu-\mathrm{BL})\right]^{4+}\{\mathrm{BL}=\mathrm{bpm}$ and dbneil $\}$ are provided in Table 2.1. The coordinated bpy ligands exhibit the expected coupling constant values ${ }^{101,114}\left\{J_{3,4}=8 \mathrm{~Hz}, J_{3,5}=1.5 \mathrm{~Hz}, J_{4,5}=8 \mathrm{~Hz}, J_{4,6}=\right.$ $1.5 \mathrm{~Hz}$ and $J_{5,6}=5 \mathrm{~Hz}$ \} and coupling patterns based on the symmetry requirements of the complexes. The conventional numbering schemes are employed in the discussion of ${ }^{1} \mathrm{H}$ NMR spectra, as shown in Figure 2.9.
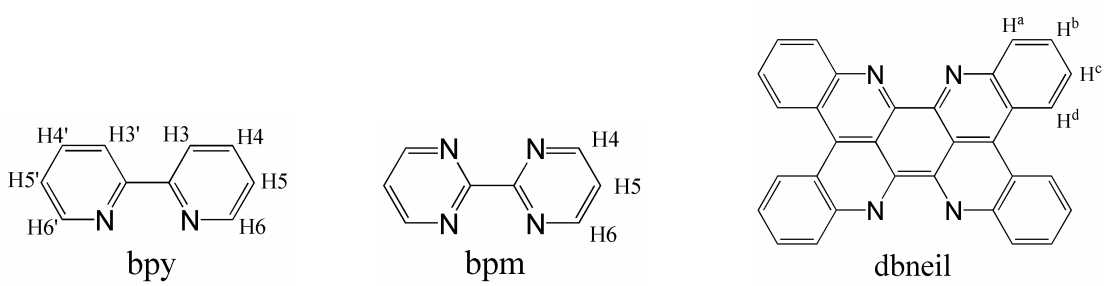

Figure 2.9 Proton numbering scheme for the bpy, bpm and dbneil ligands. 
The assignment of the ${ }^{1} \mathrm{H}$ resonances associated with individual terminal ligands was based on the relative degree of diamagnetic anisotropic interactions between the terminal and bridging ligands. Methods for the assignment of the ${ }^{1} \mathrm{H}$ NMR spectra of dinuclear complexes incorporating the bridging ligand bpm are well established, ${ }^{76,103,111-113}$ and the present discussion emphasises the distinctive chemical shifts which differ between the two diastereoisomers.

Meso- and rac- $\left[\left\{\mathrm{Ru}(\mathrm{bpy})_{2}\right\}_{2}(\mu \text {-bpm) }]^{4+}\right.$ possess $\mathbf{C}_{2 \mathbf{h}}$ and $\mathbf{D}_{2}$ point group symmetries, respectively, and exhibit different ${ }^{1} \mathrm{H}$ NMR spectra, as shown in Figure 2.10. As a consequence of the symmetry, the bridging bpm ligand exhibits three inequivalent proton resonances, and the four peripheral bpy ligands are equivalent although the two halves of each remain magnetically non-equivalent. The aromatic region of the ${ }^{1} \mathrm{H}$ NMR spectrum thus consists of 3 non-equivalent bpm resonances and 8 non-equivalent bpy resonances. The notation for the terminal bpy ligands in $\left[\left\{\mathrm{Ru}(\mathrm{bpy})_{2}\right\}_{2}(\mu-\mathrm{bpm})\right]^{4+}$ is shown in Figure 2.11 where ring $a$ is oriented away from the bridging ligand, over the plane of the bpy coordinated to the same ruthenium centre on the opposite side of the bpm-plane, and ring $b$ is oriented over the plane of the bridging ligand.

$\left[\left\{\operatorname{Ru}(\mathrm{bpy})_{2}\right\}_{2}(\mu-\mathrm{bpm})\right]^{4+}$

(a)

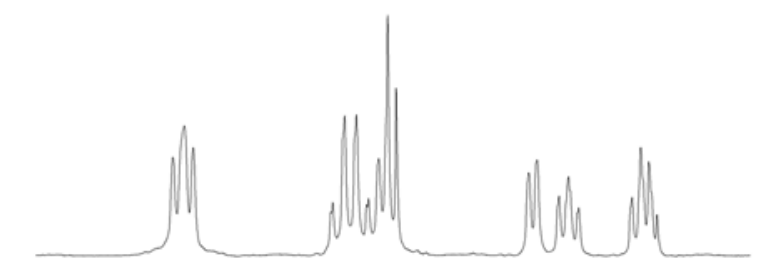

(b)

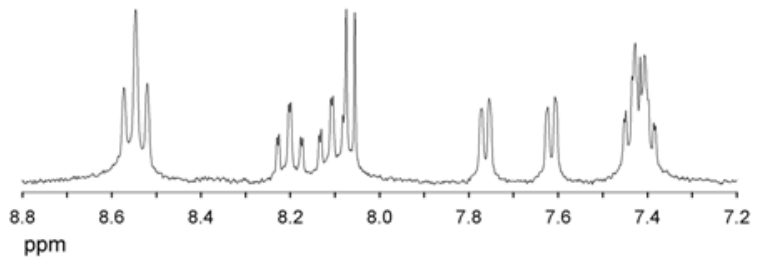

$\left[\left\{\operatorname{Ru}(\text { bpy })_{2}\right\}_{2}(\mu-d b n e i l)\right]^{4+}$

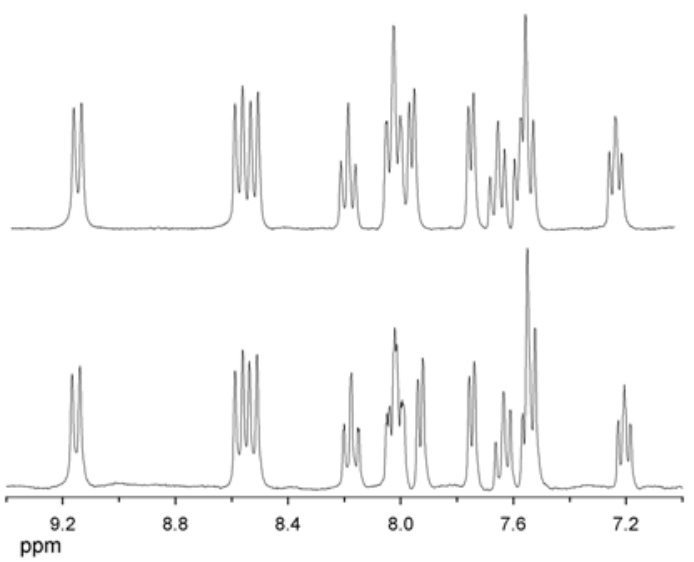

Figure 2.10 ${ }^{1} \mathrm{H}$ NMR spectra (aromatic region only) of the (a) meso and (b) rac diastereoisomers of $\left[\left\{\mathrm{Ru}(\mathrm{bpy})_{2}\right\}_{2}(\mu-\mathrm{bpm})\right]^{4+}$ and $\left[\left\{\mathrm{Ru}(\mathrm{bpy})_{2}\right\}_{2}(\mu \text {-dbneil })\right]^{4+}\left(\mathrm{PF}_{6}{ }^{-}\right.$salts; $\mathrm{CD}_{3} \mathrm{CN}$ solution $)$.

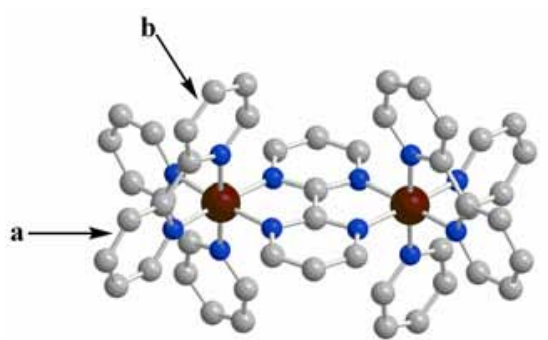

(a)

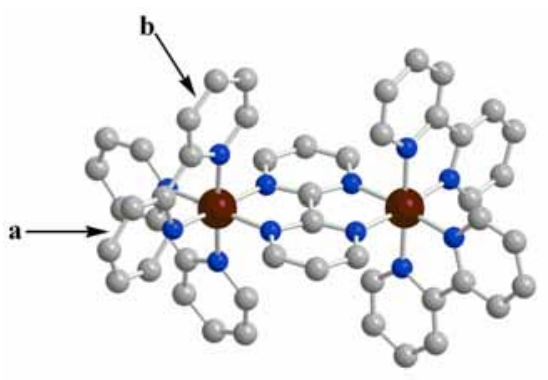

(b)

Figure 2.11 Chem 3D representations of (a) meso-( $\Lambda \Delta)$ - and (b) rac-( $\Delta \Delta)-\left[\left\{\operatorname{Ru}(\mathrm{pp})_{2}\right\}_{2}(\mu-\mathrm{bpm})\right]^{4+}$ and $\left[\left\{\mathrm{Ru}(\mathrm{bpy})_{2}\right\}_{2}(\mu \text {-bpm })\right]^{4+}$. Hydrogen atoms are omitted for clarity. 
The H5' and H6' protons of ring a experience slightly increased diamagnetic anisotropic effects (and hence more upfield chemical shifts) relative to the corresponding $\mathrm{H} 5$ and $\mathrm{H} 6$ protons of ring $b$, due to the increased shielding influence of bpy relative to bpm. The chemical shift values for the H5'/H5 and H6 $/ \mathrm{H} 6$ protons also exhibit the most pronounced difference between the two diastereoisomeric forms. In the rac diastereoisomer, ring $b$ is oriented over the plane of the bpm ligand, parallel to the bpy ligand across the bridge, while ring $b$ is oriented over the plane of the bpm ligand, towards the deshielding cone of the bpy ligand across the bridge in the meso form. As a result, the $\mathrm{H} 5$ (ring $b$ ) proton in the meso form is assigned to the resonance at $7.61 \mathrm{ppm}(J=8.0,1.2 \mathrm{~Hz}, \mathrm{dd})$, and occurs relatively downfield of the H5 (ring $b$ ) resonance in the rac form which occurs at $7.43 \mathrm{ppm}(J=8.0,1.2 \mathrm{~Hz}$, dd). The H5' protons of ring a occupy similar chemical environments in both diastereoisomeric forms, and exhibit similar chemical shift values of 7.44 and $7.41 \mathrm{ppm}(J=8.0,1.2 \mathrm{~Hz}$, dd) in the meso and rac forms, respectively.

The ${ }^{1} \mathrm{H}$ NMR spectra for the diastereoisomeric forms of $\left[\left\{\mathrm{Ru}(\mathrm{pp})_{2}\right\}_{2}(\mu \text {-bpm })\right]^{4+}\left\{\mathrm{pp}=5,5^{\prime}-\right.$ $\mathrm{Me}_{2}$ bpy, $\mathrm{Me}_{2} \mathrm{bpy}, \mathrm{Me}_{2}$ phen and ${ }^{\mathrm{t}} \mathrm{Bu}_{2} \mathrm{bpy}$ \} are reported in §2.2.3.2 and were assigned on the basis of the spectra of meso- and rac-[\{Ru(bpy $\left.)_{2}\right\}_{2}(\mu \text {-bpm) }]^{4+}$, the relative degree of diamagnetic anisotropic interactions between the stereochemically-related ligands, and ${ }^{1} \mathrm{H}$ COSY spectra. For the methylsubstituted pyridyl rings the methyl chemical shift is indicated in place of the corresponding proton resonance. Over the series of complexes, the ${ }^{1} \mathrm{H}$ NMR spectrum for a given diastereoisomer exhibited similar features, allowing for the 0.2-0.3 ppm upfield shift of protons adjacent to a methyl group and the different coupling pattern of the methyl-substituted pyridyl rings.

Table 2.1 ${ }^{1} \mathrm{H}$ Chemical shifts (ppm) for the diastereoisomeric forms of $\left[\left\{\mathrm{Ru}(\mathrm{bpy})_{4}\right\}_{2}(\mu \text {-bpm) }]^{4+}\right.$ and $\left[\left\{\mathrm{Ru}(\mathrm{bpy})_{2}\right\}_{2}(\mu-\mathrm{dbneil})\right]^{4+}\left(\mathrm{PF}_{6}{ }^{-}\right.$salts; $\mathrm{CD}_{3} \mathrm{CN}$ solution $)$.

\begin{tabular}{|c|c|c|c|c|c|}
\hline & \multicolumn{2}{|c|}{$\left[\left\{\mathrm{Ru}(\mathrm{bpy})_{2}\right\}_{2}(\mu-\mathrm{bpm})\right]^{4+\mathrm{a}}$} & \multicolumn{2}{|c|}{$\left[\left\{\mathrm{Ru}(\mathrm{bpy})_{2}\right\}_{2}(\mu-\mathrm{dbneil})\right]^{4+\mathrm{b}}$} \\
\hline & & meso & rac & meso & rac \\
\hline \multirow{4}{*}{$\begin{array}{l}\text { bpy ring a } \\
\text { (over bpy) }\end{array}$} & H3' & 8.45 & 8.54 & 8.49 & 8.49 \\
\hline & H4' & 8.08 & 8.11 & 7.99 & 7.99 \\
\hline & H5' & 7.44 & 7.41 & 7.19 & 7.18 \\
\hline & H6' & 7.68 & 7.61 & 7.71 & 7.72 \\
\hline \multirow{4}{*}{$\begin{array}{l}\text { bpy ring } b \\
\text { (over bpm } \\
\text { or dbneil) }\end{array}$} & H3 & 8.48 & 8.56 & 8.54 & 8.55 \\
\hline & $\mathrm{H} 4$ & 8.11 & 8.20 & 8.15 & 8.15 \\
\hline & H5 & 7.61 & 7.43 & 7.53 & 7.52 \\
\hline & H6 & 8.00 & 7.76 & 7.92 & 7.90 \\
\hline \multirow[t]{3}{*}{ bpm } & H4 & 8.03 & 8.08 & & \\
\hline & H5 & 7.43 & 7.42 & & \\
\hline & H6 & 8.00 & 8.07 & & \\
\hline \multirow[t]{4}{*}{ dbeil } & $\mathrm{H}^{\mathrm{a}}$ & & & 7.49 & 7.50 \\
\hline & $\mathrm{H}^{\mathrm{b}}$ & & & 7.61 & 7.61 \\
\hline & $\mathrm{H}^{\mathrm{c}}$ & & & 7.99 & 7.99 \\
\hline & $\mathrm{H}^{\mathrm{d}}$ & & & 9.13 & 9.12 \\
\hline \multicolumn{6}{|c|}{$\begin{array}{l}\text { For the bpy ligands, H3,3' (d, } J=8.0 \mathrm{~Hz}, 4 \mathrm{H}), \mathrm{H4}, 4^{\prime}(\mathrm{dd}, J=8.0,1.5 \mathrm{~Hz}, 4 \mathrm{H}), \mathrm{H} 5,5^{\prime}(\mathrm{dd}, J=8.0,1.2 \mathrm{~Hz} \text {, } \\
4 \mathrm{H}), \mathrm{H6}, 6^{\prime}(\mathrm{dd}, J=6.3,1.5,0.6 \mathrm{~Hz}, 4 \mathrm{H}) \text {. For bpm, } \mathrm{H} 4(\mathrm{~d}, J=5.7 \mathrm{~Hz}, 2 \mathrm{H}), \mathrm{H} 5(\mathrm{t}, J=5.7 \mathrm{~Hz}, 2 \mathrm{H}), \mathrm{H} 6 \text { (d, } \\
J=5.7 \mathrm{~Hz}, 2 \mathrm{H}) .\end{array}$} \\
\hline \multicolumn{6}{|c|}{$\begin{array}{l}\text { b For the bpy ligands, H3,3' (d, } J=8.1 \mathrm{~Hz}, 4 \mathrm{H}), \mathrm{H} 4,4^{\prime}(\mathrm{dd}, J=7.8,1.2 \mathrm{~Hz}, 4 \mathrm{H}), \mathrm{H} 5,5^{\prime}(\mathrm{dd}, J=6.9,1.5 \mathrm{~Hz} \text {, } \\
4 \mathrm{H}), \mathrm{H6}, \mathrm{G}^{\prime}(\mathrm{dd}, J=5.0,1.5 \mathrm{~Hz}, 4 \mathrm{H}) \text {. For dbneil, protons at the same position in symmetrically-related } \\
\text { spin systems experience slightly different anisotropic interactions: }(\text { meso }) \mathrm{H}^{\mathrm{a}}(\mathrm{d}, J=8.1 \mathrm{~Hz}, 4 \mathrm{H}), \mathrm{H}^{\mathrm{b}}(\mathrm{dd} \text {, } \\
J=7.5,1.0 \mathrm{~Hz}, 4 \mathrm{H}), \mathrm{H}^{\mathrm{c}}(\mathrm{dd}, J=8.0,1.5 \mathrm{~Hz}, 4 \mathrm{H}), \mathrm{H}^{\mathrm{d}}(\mathrm{d}, J=8.1 \mathrm{~Hz}, 4 \mathrm{H}) \text { and }(\mathrm{rac}) \mathrm{H}^{\mathrm{a}}(\mathrm{dd}, J=7.8,1.0 \mathrm{~Hz} \text {, } \\
4 \mathrm{H}), \mathrm{H}^{\mathrm{b}}(\mathrm{dd}, J=7.2,1.5 \mathrm{~Hz}, 4 \mathrm{H}), \mathrm{H}^{\mathrm{c}}(\mathrm{dd}, J=7.5,1.2 \mathrm{~Hz}, 4 \mathrm{H}), \mathrm{H}^{\mathrm{d}}(\mathrm{d}, J=8.4 \mathrm{~Hz}, 4 \mathrm{H}) .\end{array}$} \\
\hline
\end{tabular}


As shown in Figure 2.10, the ${ }^{1} \mathrm{H}$ NMR spectra of the diastereoisomers of $\left[\left\{\mathrm{Ru}(\mathrm{bpy})_{2}\right\}_{2}(\mu\right.$ dbneil) $]^{4+}$ are almost identical and only slight differences in the chemical shifts are evident between them (Table 2.1). However, the peaks designated as multiplets (m) for the isomeric mixture in an earlier report ${ }^{82}$ were resolved on diastereoisomer separation. The anisotropic interactions between the terminal bpy rings located on different ruthenium centres, which give rise to the observed differences between the ${ }^{1} \mathrm{H}$ NMR spectra of the diastereoisomers of $\left[\left\{\mathrm{Ru}(\mathrm{bpy})_{2}\right\}_{2}(\mu \text {-bpm })\right]^{4+}$, are reduced for $\left[\left\{\mathrm{Ru}(\mathrm{bpy})_{2}\right\}_{2}(\mu-\right.$ dbneil) $]^{4+}$ due to the relatively large distance (ca. $7.9 \AA^{82}$ ) between the metal centres. The dbneil ligand is not planar due to steric interactions between the $\mathrm{H}^{\mathrm{d}}$ protons in the "bay regions", and instead exhibits a curved surface. ${ }^{82}$ Only four signals appear for the dbneil ligand for both diastereoisomeric forms: the fact that a single set of peaks is observed for this ligand on the NMR time-scale suggests that dbneil undergoes a fast "wagging" motion. As discussed previously, ${ }^{82}$ the dbneil flips and the two metal fragments move up and down - a motion which maintains time-averaged symmetries of $\mathbf{C}_{\mathbf{2 h}}$ and $\mathbf{D}_{\mathbf{2}}$ for the meso and rac diastereoisomers, respectively. The spectra were found not to be concentration or temperature dependent (as was the case for mononuclear complexes of dbneil), ${ }^{115}$ as $\pi$-stacking interactions are not possible in the dinuclear complexes for steric reasons. ${ }^{82}$ Since a distinction between the diastereoisomers on the basis of the NMR studies was precluded due to the similarity of their spectra, the distinction of the two forms was achieved by the resolution of the rac form and determination of the corresponding CD spectra of the enantiomeric $\Delta \Delta$ and $\Lambda \Lambda$ forms, as shown in Figure A2.1 (Appendix A). ${ }^{104}$

\subsubsection{X-ray Crystallography}

Meso- $(\Lambda \Delta)-\left[\left\{\mathrm{Ru}(\mathrm{bpy})_{2}\right\}_{2}(\mu\right.$-bpm $\left.)\right]\left(\mathrm{PF}_{6}\right)_{4}$ crystallised in the monoclinic space group $\mathrm{C}_{2} / c$ and meso- $(\Lambda \Delta)-\left[\left\{\mathrm{Ru}\left(\mathrm{Me}_{2} \mathrm{bpy}\right)_{2}\right\}_{2}(\mu\right.$-bpm) $](\text { tos })_{4}$ crystallised in the orthorhombic space group Pbca, with four dinuclear cations in the unit cell in both cases. The asymmetric unit of meso- $\left[\left\{\mathrm{Ru}(\mathrm{bpy})_{2}\right\}_{2}(\mu-\mathrm{bpm})\right]\left(\mathrm{PF}_{6}\right)_{4}$ contains one dinuclear cation and four $\mathrm{PF}_{6}{ }^{-}$counter-ions. The asymmetric unit of meso$\left[\left\{\mathrm{Ru}\left(\mathrm{Me}_{2} \mathrm{bpy}\right)_{2}\right\}_{2}(\mu \text {-bpm })\right]^{4+}$ contains one-half of the dinuclear cation which is located on a crystallographic axis. The data collection parameters and structural refinement details are provided in Table A2.1 (Appendix A).

The perspective views (ORTEP) of the dinuclear cations meso- $(\Lambda \Delta)-\left[\left\{\operatorname{Ru}(\mathrm{bpy})_{2}\right\}_{2}(\mu-\mathrm{bpm})\right]^{4+}$ and meso- $(\Lambda \Delta)-\left[\left\{\mathrm{Ru}\left(\mathrm{Me}_{2} \mathrm{bpy}\right)_{2}\right\}_{2}(\mu \text {-bpm })\right]^{4+}$ with partial atom labelling are shown in Figures 2.12(a) and 2.13(a), respectively. Both complexes exhibit comparable Ru-N distances and N-Ru-N angles which are consistent with those reported previously for meso- $(\Lambda \Delta)-\left[\left\{\mathrm{Ru}\left(\mathrm{Me}_{2} \mathrm{bpy}\right)_{4}\right\}_{2}(\mu-\mathrm{bpm})\right](\mathrm{Cl})_{4}{ }^{76}$ and related polypyridyl complexes of ruthenium. ${ }^{68,116-120}$ In both structures, the Ru centres reside in slightly distorted octahedral environments. The "bite angles" of the terminal chelating ligands vary between $78.1(3)^{\circ}$ and $79.4(3)^{\circ}$, which is typical for the polypyridyl ligands bpy and $\mathrm{Me}_{2} \mathrm{bpy},{ }^{118,121-123}$ and the metal centres are coordinated at each bidentate coordination site of bpm with average N-Ru-N angles of 
$79.9(3)^{\circ}$ and $80.2(3)^{\circ}$, for the complexes incorporating the bpy and $\mathrm{Me}_{2}$ bpy terminal ligands, respectively. The bridging bpm ligand maintains planarity in both structures, with $\mathrm{Ru} \cdots \mathrm{Ru}$ separations of 5.565(5) and 5.530(5) $\AA$, for the bpy and $\mathrm{Me}_{2}$ bpy complexes.

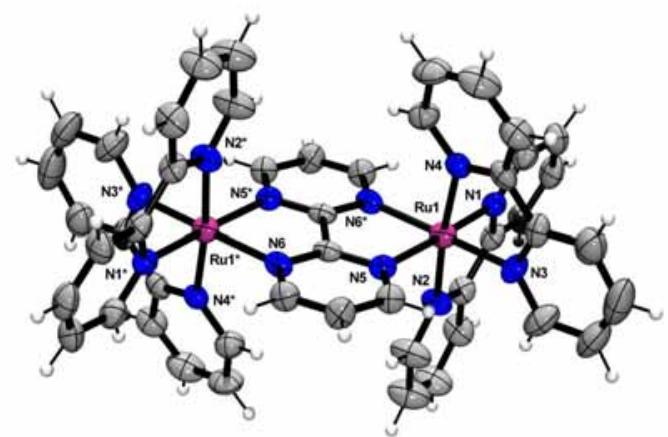

(a)

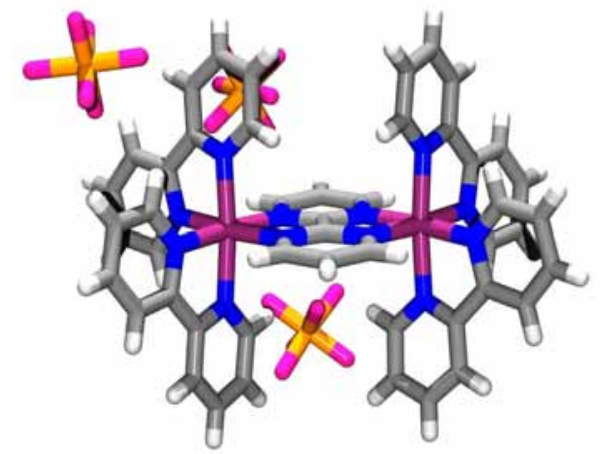

(b)

Figure 2.12 (a) ORTEP view (50\% probability) of the cation in meso- $(\Lambda \Delta)-\left[\left\{\mathrm{Ru}(\mathrm{bpy})_{2}\right\}_{2}(\mu\right.$-bpm) $]\left(\mathrm{PF}_{6}\right)_{4}$ with atom labelling. (b) The meso- $(\Lambda \Delta)-\left[\left\{\mathrm{Ru}(\mathrm{bpy})_{2}\right\}_{2}(\mu \text {-bpm) }]^{4+}\right.$ cation showing the proximity of the $\mathrm{PF}_{6}{ }^{-}$anions to the clefts between the bpy ligands.

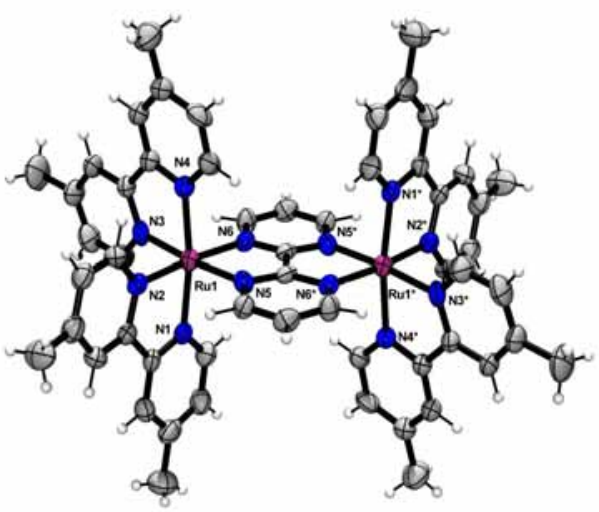

(a)

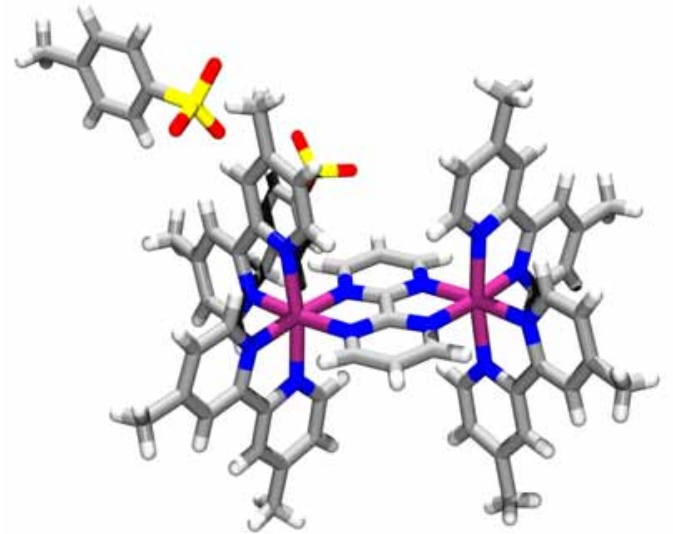

(b)

Figure 2.13 (a) ORTEP view (50\% probability) of the cation in meso- $(\Lambda \Delta)-\left[\left\{\operatorname{Ru}\left(\mathrm{Me}_{2} \mathrm{bpy}\right)_{2}\right\}_{2}(\mu\right.$-bpm) $](\text { tos })_{4}$ with atom labelling. (b) The meso- $(\Lambda \Delta)-\left[\left\{\mathrm{Ru}\left(\mathrm{Me}_{2} \mathrm{bpy}\right)_{2}\right\}_{2}(\mu-\mathrm{bpm})\right]^{4+}$ cation showing the proximity of the tos ${ }^{-}$anions to the clefts between the $\mathrm{Me}_{2}$ bpy ligands.

Figure 2.12(b) shows the close proximity of three $\mathrm{PF}_{6}{ }^{-}$counter-ions to the clefts between the bpy ligands in meso- $(\Lambda \Delta)-\left[\left\{\mathrm{Ru}(\mathrm{bpy})_{2}\right\}_{2}(\mu\right.$-bpm $\left.)\right]\left(\mathrm{PF}_{6}\right)_{4}$. The crystal packing diagram reveals that the additional $\mathrm{PF}_{6}{ }^{-}$counter-ions reside in layers between the predominantly organic layers of the complex. The presence of these distinct layers may account for the rapid solvent loss and brittle nature of the crystals. Similar observations were noted previously in the crystal structure determination of meso- $(\Lambda \Delta)-\left[\left\{\mathrm{Ru}\left(\mathrm{Me}_{2} \mathrm{bpy}\right)_{2}\right\}_{2}(\mu\right.$-bpm $\left.)\right](\mathrm{Cl})_{4},{ }^{76}$ and may account for the limited number of crystal structures for complexes of this genre in the literature.

The proximities of two tosylate counter-ions (tos') to the cation in the meso-( $\Lambda \Delta$ )$\left[\left\{\mathrm{Ru}\left(\mathrm{Me}_{2} \mathrm{bpy}\right)_{2}\right\}_{2}(\mu\right.$-bpm) $](\text { tos })_{4}$ are featured in Figure 2.13(b). Both counter-ions reside close to the cleft between the $\mathrm{Me}_{2}$ bpy ligands "above" the plane of the bridging ligand, with the hydrophilic $\mathrm{SO}_{3}{ }^{-}$terminus of the anion oriented towards the relatively open orthogonal shaped cavity of the cation. The additional 
counter-ions do not engage in any intermolecular interactions with the complex cation. The orientation adopted by the tosylate counter-ions in the solid-state is in contrast to that in solution where the counterion is positioned with the hydrophobic aryl ring in the cavity and the $\mathrm{SO}_{3}{ }^{-}$terminus orientated away from the bridge, as determined by NMR titration studies. ${ }^{76}$

A limited number of crystal structures of dinuclear polypyridyl ruthenium complexes have been reported to date. Three examples of dinuclear species containing symmetrical bridging ligands have been described $^{68,82,120}$ where crystallisation afforded the meso forms preferentially, and Ward ${ }^{116,117}$ described the isolation of the rac form of an alkoxide-bridged species. Keene et al. ${ }^{76}$ reported the first example of a crystal structure in which the meso diastereoisomer of $\left[\left\{\mathrm{Ru}\left(\mathrm{Me}_{2} \mathrm{bpy}\right)_{2}\right\}_{2}(\mu \text {-bpm })\right]^{4+}$ was isolated via chromatography prior to crystal growth. Three examples of dinuclear species incorporating unsymmetrical bridging ligands ${ }^{118,119,124}$ have been reported, including two containing the 3,5-bis(2pyridyl)-1,2,4-triazolate (bpt') bridging ligand. In all cases, the crystals were obtained as the meso diastereoisomeric forms during attempts to separate the diastereoisomers of the complexes via fractional crystallisation. The existence of a mixture of stereoisomeric forms may contribute towards the difficulties encountered in the growth of crystals of such species. The present examples therefore represent one of the few reports ${ }^{76,125,126}$ of selective diastereoisomer isolation prior to crystal growth.

\subsubsection{Electrochemistry}

The electrochemical properties of the diastereoisomeric forms of $\left[\left\{\mathrm{Ru}(\mathrm{pp})_{2}\right\}_{2}(\mu-\mathrm{bpm})\right]^{4+}\{\mathrm{pp}=$ bpy, 5,5'-Me $\mathrm{Me}_{2}$ bpy, $\mathrm{Me}_{4}$ bpy, $\mathrm{Me}_{2}$ phen and $\left.{ }^{\mathrm{t}} \mathrm{Bu}_{2} \mathrm{bpy}\right\}$ and $\left[\left\{\mathrm{Ru}(\mathrm{bpy})_{2}\right\}_{2}(\mu \text {-dbneil) }]^{4+}\right.$ were investigated by cyclic and differential pulse voltammetry in acetonitrile containing $0.1 \mathrm{M}\left[\left(n-\mathrm{C}_{4} \mathrm{H}_{9}\right)_{4} \mathrm{~N}\right] \mathrm{PF}_{6}$, and are reported in Table 2.2. The electrochemical and spectroelectrochemical characteristics of $\left[\left\{\mathrm{Ru}(\mathrm{bpy})_{2}\right\}_{2}(\mu-\right.$ $\mathrm{bpm})]^{4+}$ (as a diastereoisomeric mixture) have been detailed in a number of previous studies. ${ }^{30,107-110,127-132}$

Table 2.2 Electrochemical data (in $\mathrm{mV}$ relative to the $\mathrm{Fc}^{+} / \mathrm{Fc}^{0}$ couple) for $\left[\left\{\mathrm{Ru}(\mathrm{pp})_{2}\right\}_{2}(\mu-\mathrm{BL})\right]^{4+}\{\mathrm{pp}=\mathrm{bpy}$, 5,5'-Me 2 bpy, $\mathrm{Me}_{4}$ bpy, ${ }^{\mathrm{t}} \mathrm{Bu}_{2}$ bpy, $\mathrm{Me}_{2}$ phen; BL = bpm, dbneil\} in $0.1 \mathrm{M}\left[\left(n-\mathrm{C}_{4} \mathrm{H}_{9}\right)_{4} \mathrm{~N}\right] \mathrm{PF}_{6} / \mathrm{CH}_{3} \mathrm{CN}$. ${ }^{\mathrm{a}, \mathrm{b}}$

\begin{tabular}{|c|c|c|c|c|c|c|c|}
\hline Complex & $\Delta \mathrm{E}_{\mathrm{ox}}$ & $E_{0 \times 2}$ & $E_{0 \times 1}$ & $\mathrm{E}_{\text {red1 }}$ & $\mathrm{E}_{\text {red2 }}$ & $\mathrm{E}_{\text {red3 }}$ & $\mathrm{E}_{\text {red4 }}$ \\
\hline $\begin{array}{l}\text { meso- }\left[\left\{\mathrm{Ru}(\mathrm{bpy})_{2}\right\}_{2}(\mu-\mathrm{bpm})\right]^{4+} \\
\text { rac- }\left[\left\{\mathrm{Ru}(\mathrm{bpy})_{2}\right\}_{2}(\mu-\mathrm{bpm})\right]^{4+}\end{array}$ & $\begin{array}{l}192 \\
188\end{array}$ & $\begin{array}{l}1384 \\
1380\end{array}$ & $\begin{array}{l}1192 \\
1192\end{array}$ & $\begin{array}{l}-794 \\
-792\end{array}$ & $\begin{array}{l}-1466 \\
-1480\end{array}$ & $\begin{array}{l}-1948^{\mathrm{C}} \\
-1928^{\mathrm{c}}\end{array}$ & $\begin{array}{l}-2368^{\mathrm{c}, \mathrm{e}} \\
-2360^{\mathrm{c}, \mathrm{e}}\end{array}$ \\
\hline $\begin{array}{l}\text { meso- }\left[\left\{\mathrm{Ru}\left(5,5^{\prime}-\mathrm{Me}_{2} \mathrm{bpy}\right)_{2}\right\}_{2}(\mu-\mathrm{bpm})\right]^{4+} \\
\text { rac- }\left[\left\{\mathrm{Ru}\left(5,5^{\prime}-\mathrm{Me}_{2} \mathrm{bpy}\right)_{2}\right\}_{2}(\mu-\mathrm{bpm})\right]^{4+}\end{array}$ & $\begin{array}{l}188 \\
176\end{array}$ & $\begin{array}{l}1260 \\
1248\end{array}$ & $\begin{array}{l}1072 \\
1072\end{array}$ & $\begin{array}{l}-824 \\
-824\end{array}$ & $\begin{array}{l}-1508 \\
-1532\end{array}$ & $\begin{array}{l}-2032^{\mathrm{c}} \\
-2032^{\mathrm{c}}\end{array}$ & $\begin{array}{l}-2272 \\
-2272\end{array}$ \\
\hline $\begin{array}{l}\text { meso- }\left[\left\{\mathrm{Ru}\left(\mathrm{Me}_{4} \mathrm{bpy}\right)_{2}\right\}_{2}(\mu-\mathrm{bpm})\right]^{4+} \\
\text { rac- }\left[\left\{\mathrm{Ru}\left(\mathrm{Me}_{4} \mathrm{bpy}\right)_{2}\right\}_{2}(\mu-\mathrm{bpm})\right]^{4+}\end{array}$ & $\begin{array}{l}200 \\
192 \\
\end{array}$ & $\begin{array}{l}1208 \\
1200 \\
\end{array}$ & $\begin{array}{l}1008 \\
1008 \\
\end{array}$ & $\begin{array}{l}-872 \\
-880 \\
\end{array}$ & $\begin{array}{ll}-1460 \\
-1464 \\
\end{array}$ & $\begin{array}{l}-2055^{\mathrm{C}} \\
-2056^{\mathrm{c}} \\
\end{array}$ & $\begin{array}{l}-2315 \\
-2320 \\
\end{array}$ \\
\hline $\begin{array}{l}\text { meso- }\left[\left\{\mathrm{Ru}\left({ }^{\mathrm{t}} \mathrm{Bu}{ }_{2} \mathrm{bpy}\right)_{2}\right\}_{2}(\mu-\mathrm{bpm})\right]^{4+} \\
\text { rac- }\left[\left\{\mathrm{Ru}\left({ }^{\mathrm{B}} \mathrm{Bu}_{2} \mathrm{bpy}\right)_{2}\right\}_{2}(\mu-\mathrm{bpm})\right]^{4+}\end{array}$ & $\begin{array}{l}192 \\
184\end{array}$ & $\begin{array}{l}1280 \\
1280\end{array}$ & $\begin{array}{l}1088 \\
1088\end{array}$ & $\begin{array}{l}-816 \\
-816\end{array}$ & $\begin{array}{l}-1488 \\
-1490\end{array}$ & $\begin{array}{l}-2008^{\mathrm{C}} \\
-2015^{\mathrm{c}}\end{array}$ & $\begin{array}{l}-2281 \\
-2292\end{array}$ \\
\hline $\begin{array}{l}\text { meso- }\left[\left\{\mathrm{Ru}\left(\mathrm{Me}_{2} \mathrm{phen}\right)_{2}\right\}_{2}(\mu-\mathrm{bpm})\right]^{4+} \\
\text { rac- }\left[\left\{\mathrm{Ru}\left(\mathrm{Me}_{2} \mathrm{phen}\right)_{2}\right\}_{2}(\mu-\mathrm{bpm})\right]^{4+}\end{array}$ & $\begin{array}{l}192 \\
184\end{array}$ & $\begin{array}{l}1270 \\
1266\end{array}$ & $\begin{array}{l}1078 \\
1082\end{array}$ & $\begin{array}{l}-816 \\
-816\end{array}$ & $\begin{array}{l}-1488 \\
-1512\end{array}$ & $\begin{array}{l}-2008^{\mathrm{d}} \\
-2016^{\mathrm{d}}\end{array}$ & $\begin{array}{l}-2272 \\
-2264^{d}\end{array}$ \\
\hline $\begin{array}{l}\text { meso- }\left[\left\{\mathrm{Ru}(\mathrm{bpy})_{2}\right\}_{2}(\mu \text {-dbneil })\right]^{4+} \\
\text { rac- }\left[\left\{\mathrm{Ru}(\mathrm{bpy})_{2}\right\}_{2}(\mu-\mathrm{dbneil})\right]^{4+}\end{array}$ & $\begin{array}{l}180 \\
182\end{array}$ & $\begin{array}{l}1268 \\
1270\end{array}$ & $\begin{array}{l}1088 \\
1088\end{array}$ & $\begin{array}{l}-544 \\
-540\end{array}$ & $\begin{array}{l}-945 \\
-946\end{array}$ & $\begin{array}{l}-1676 \\
-1668\end{array}$ & $\begin{array}{l}-1972^{\mathrm{e}} \\
-1965\end{array}$ \\
\hline
\end{tabular}

${ }^{a}$ All potentials are reported $\pm 3 \mathrm{mV}$.

b $\Delta \mathrm{E}_{\mathrm{ox}}=\mathrm{E}_{\mathrm{ox} 2}-\mathrm{E}_{\mathrm{ox} 1}$

c Two-electron reduction process.

d Process complicated by adsorption/desorption peaks.

e Irreversible reduction process. 
The $\left[\left\{\mathrm{Ru}(\mathrm{pp})_{2}\right\}_{2}(\mu-\mathrm{bpm})\right]^{4+}\left\{\mathrm{pp}=\right.$ bpy, 5,5'-Me $\mathrm{Me}_{2}$ bpy, $\mathrm{Me}_{4} \mathrm{bpy}, \mathrm{Me}_{2}$ phen and ${ }^{\mathrm{t}} \mathrm{Bu}_{2}$ bpy $\}$ complexes are characterised by two reversible one-electron redox processes corresponding to successive oxidation of the metal centres, in addition to multiple reversible ligand-based reductions. In the cathodic region, the first two reduction processes are assigned as successive one-electron reduction processes localised on the bridging ligand (i.e. $\mathrm{bpm}^{\mathrm{o}-}$ and $\mathrm{bpm}^{-/ 2-}$ ), due to the stronger $\pi$-acceptor nature of bpm relative to the terminal pp ligands. ${ }^{30,107-110,127-132}$ The subsequent reduction processes are localised on the terminal pp ligands. For $\left[\left\{\mathrm{Ru}(\mathrm{bpy})_{2}\right\}_{2}(\mu \text {-bpm) }]^{4+}\right.$, the two two-electron redox processes correspond to the simultaneous reduction of the bpy ligands coordinated to different ruthenium centres according to $\left[\left\{\operatorname{Ru}\left(\mathrm{bpy}^{0}\right)_{2}\right\}_{2}\left(\mu-\mathrm{bpm}^{2-}\right)\right]^{2+} \rightarrow\left[\left\{\operatorname{Ru}\left(\mathrm{bpy}^{0}\right)\left(\mathrm{bpy}^{\bullet-}\right)\right\}_{2}\left(\mu-\mathrm{bpm}^{2-}\right)\right]^{0} \rightarrow\left[\left\{\operatorname{Ru}\left(\mathrm{bpy}^{\bullet-}\right)\right\}_{2}\left(\mu-\mathrm{bpm}^{2-}\right)\right]^{2-} \cdot{ }^{110}$ By analogy, $\mathrm{E}_{\mathrm{red} 3}$ corresponds to a simultaneous two-electron reduction process for the pp ligands bound to different ruthenium centres in $\left[\left\{\mathrm{Ru}(\mathrm{pp})_{2}\right\}_{2}(\mu-\mathrm{bpm})\right]^{n+}\left\{\mathrm{pp}=5,5^{\prime}-\mathrm{Me}_{2} \mathrm{bpy}, \mathrm{Me}_{4} \mathrm{bpy}, \mathrm{Me}_{2}\right.$ phen and ${ }^{t} \mathrm{Bu}_{2}$ bpy $\}$. The simultaneous two-electron redox process $\mathrm{E}_{\text {red } 4}$ in $\left[\left\{\mathrm{Ru}(\mathrm{bpy})_{2}\right\}_{2}(\mu-\mathrm{bpm})\right]^{4+}$ is split into two closely-spaced one-electron steps in the substituted complexes. Such splittings have been noted previously ${ }^{133}$ for related dinuclear polypyridyl complexes of ruthenium such as [ $\left\{\mathrm{Ru}(\mathrm{bpy})_{2}\right\}_{2}(\mu-2,3-$ $\mathrm{dpp})]^{4+}\{2,3-\mathrm{dpp}=2,3-(2-p y r i d y l) p y r a z i n e\}$, and have been attributed to electronic delocalisation between the peripheral ligands bound to different metal centres. ${ }^{110}$ In the present case, a combination of steric effects between the alkyl substituents of the peripheral ligands and differing solvation and ionpairing interactions may also contribute to the observed splitting of the bpy-based reduction potentials.

The influence of the peripheral ligands on the electrochemical characteristics of this series of substituted complexes $\left[\left\{\mathrm{Ru}(\mathrm{pp})_{2}\right\}_{2}(\mu \text {-bpm })\right]^{4+}$ are consistent with the variation in the relative $\pi$-accepting abilities of the terminal ligands. ${ }^{134}$ For the diastereoisomers of a given complex, $\mathrm{E}_{\mathrm{ox} 1}$ and $\mathrm{E}_{\mathrm{ox} 2}$ shift cathodically as the peripheral ligands are varied through the series bpy $>{ }^{t} \mathrm{Bu}_{2} \mathrm{bpy} \approx \mathrm{Me}_{2}$ phen $>$ $5,5^{\prime}-\mathrm{Me}_{2}$ bpy $>\mathrm{Me}_{4}$ bpy. Minor variations in $\Delta \mathrm{E}_{\mathrm{ox}}$ (defined as $\mathrm{E}_{\mathrm{ox} 2}-\mathrm{E}_{\mathrm{ox} 1}$ ) are also evident across the series of complexes, and between the diastereoisomeric forms of the same complex. $\Delta \mathrm{E}_{\mathrm{ox}}$ is greatest for the diastereoisomers of $\left[\left\{\mathrm{Ru}\left(\mathrm{Me}_{4} \mathrm{bpy}\right)_{2}\right\}_{2}(\mu \text {-bpm) }]^{4+}\right.$ and least for the diastereoisomers of $\left[\left\{\operatorname{Ru}\left(5,5^{\prime}-\mathrm{Me}_{2} \mathrm{bpy}\right)_{2}\right\}_{2}(\mu \text {-bpm) }]^{4+}\right.$. The potentials of the ligand-based reduction processes shift cathodically through the series bpy $>\mathrm{Me}_{2}$ phen $={ }^{\mathrm{t}} \mathrm{Bu}_{2}$ bpy $>$ 5,5'-Me $-\mathrm{Me}_{2} \mathrm{by}>\mathrm{Me}_{4} \mathrm{bpy}$. The results are consistent with the increase in the $\pi$-acceptor ability of the pp ligands in the order $\mathrm{Me}_{4} \mathrm{bpy}>5,5^{\prime}-\mathrm{Me}_{2} \mathrm{bpy}$ $>\mathrm{Me}_{2}$ phen $\approx{ }^{\mathrm{t}} \mathrm{Bu}_{2} \mathrm{bpy}>$ bpy. For a given complex, $\Delta \mathrm{E}_{\mathrm{ox}}$ is slightly greater for the meso compared with the rac diastereoisomeric form, however this difference is significant for the $\mathrm{Me}_{2}$ phen and ${ }^{\mathrm{t}} \mathrm{Bu}_{2} \mathrm{bpy}$ species only.

In an earlier study of the electrochemical behaviour of $\left[\left\{\mathrm{Ru}(\mathrm{bpy})_{2}\right\}_{2}(\mu \text {-dbneil) }]^{4+}\right.$ (as a diastereoisomeric mixture), ${ }^{82}$ it was noted that the difference in the redox potentials of the $4+\rightarrow 5+$ and $5^{+} \rightarrow 6^{+}$oxidations for the isomeric mixture was $160 \mathrm{mV}$ \{measured in $\left[\left(n-\mathrm{C}_{4} \mathrm{H}_{9}\right)_{4} \mathrm{~N}\right] \mathrm{PF}_{6} / \mathrm{CH}_{3} \mathrm{CN}$ \} which was indicative of substantial electronic communication between the metal centres. The first two reduction processes are associated with the dbneil ligand, while subsequent processes correspond to 
reduction of the terminal bpy ligands. ${ }^{82,124}$ The potentials of the metal-based oxidation processes are shifted cathodically (by ca. $100 \mathrm{mV}$ ) relative to the corresponding processes in $\left[\left\{\mathrm{Ru}(\mathrm{bpy})_{2}\right\}_{2}(\mu \text {-bpm) }]^{4+}\right.$, and $\Delta \mathrm{E}_{\text {ox }}$ is reduced from $192 \mathrm{mV}$ in the meso-diastereoisomer to $180 \mathrm{mV}$ in meso- $\left[\left\{\mathrm{Ru}(\mathrm{bpy})_{2}\right\}_{2}(\mu-\right.$ dbneil) $]^{4+}$. The first reduction potential centred on the dbneil ligand occurs $250 \mathrm{mV}$ anodic of the corresponding bpm-based process in $\left[\left\{\mathrm{Ru}(\mathrm{bpy})_{2}\right\}_{2}(\mu \text {-bpm })\right]^{4+}$, which is indicative of the enhanced stabilisation of the $\pi^{*}(\mathrm{dbneil})$ orbitals relative to $\pi^{*}(\mathrm{bpm})$.

The separation in the potentials between the metal-based oxidation processes permitted the generation of the mixed-valence forms of the complexes. The relative magnitudes of $\Delta \mathrm{E}_{\mathrm{ox}}$ suggest that the stabilities of the mixed-valence species are comparable for the series of complexes. ${ }^{30,135}$ Some caution must be exercised in the interpretation of the electrochemical data as the $\Delta \mathrm{E}_{\mathrm{ox}}$ values conceal a number of factors, ${ }^{30}$ such as ion-pairing and solvation energies in addition to electronic delocalisation which may differ appreciably for the diastereoisomeric forms of the same complex. The issue of differential ion-pairing effects on the electrochemical potentials of a series of dinuclear complxes including $\left[\left\{\mathrm{Ru}(\mathrm{bpy})_{2}\right\}_{2}(\mu \text {-bpm) }]^{4+}\right.$ and $\left[\left\{\mathrm{Ru}(\mathrm{bpy})_{2}\right\}_{2}(\mu \text {-dbneil })\right]^{4+}$ is the subject of $\S 3.3 .2 .2 .^{136}$

\subsubsection{Electronic Spectroscopy and Spectroelectrochemistry}

The UV/Vis/NIR spectral data for the un-oxidised (+4) and mixed-valence $(+5)$ forms of the meso and rac diastereoisomers of $\left[\left\{\mathrm{Ru}(\mathrm{bpy})_{2}\right\}_{2}(\mu \text {-bpm) }]^{n+}\right.$ and $\left[\left\{\mathrm{Ru}(\mathrm{bpy})_{2}\right\}_{2}(\mu \text {-dbneil })\right]^{n+}$ over the range $3050-30000 \mathrm{~cm}^{-1}$ are reported in Table 2.3, and the spectral progressions accompanying the formation of the mixed-valence forms of the rac diastereoisomers are shown in Figures 2.14 and 2.15, respectively. The spectral features for the diastereoisomeric forms of the complexes are consistent with previous literature reports for $\left[\left\{\mathrm{Ru}(\mathrm{bpy})_{2}\right\}_{2}(\mu \text {-bpm) }]^{4+30,107-110,137}\right.$ and $\left[\left\{\mathrm{Ru}(\mathrm{bpy})_{2}\right\}_{2}(\mu \text {-dbneil) }]^{4+82}\right.$ (as diastereoisomeric mixtures).

The UV/Vis spectra over the region $10000-30000 \mathrm{~cm}^{-1}$ for $\left[\left\{\mathrm{Ru}(\mathrm{bpy})_{2}\right\}_{2}(\mu-\mathrm{BL})\right]^{4+}\{\mathrm{BL}=\mathrm{bpm}$, dbneil $\}$ are characterised by a combination of overlapping $\mathrm{d} \pi\left(\mathrm{Ru}^{\mathrm{II}}\right) \rightarrow \pi^{*}(\mathrm{BL})$ and $\mathrm{d} \pi\left(\mathrm{Ru}^{\mathrm{II}}\right) \rightarrow \pi^{*}(\mathrm{bpy})$ MLCT transitions. The lowest energy transitions at 16775 and $16730 \mathrm{~cm}^{-1}$ in the meso and rac diastereoisomers of $\left[\left\{\mathrm{Ru}(\mathrm{bpy})_{2}\right\}_{2}(\mu-\mathrm{bpm})\right]^{4+}$, respectively, and at 14300 and $14290 \mathrm{~cm}^{-1}$ in the meso and rac diastereoisomers of $\left[\left\{\mathrm{Ru}(\mathrm{bpy})_{2}\right\}_{2}(\mu \text {-dbneil })\right]^{4+}$, respectively, are assigned as $\mathrm{d} \pi\left(\mathrm{Ru}^{\mathrm{II}}\right) \rightarrow \pi^{*}(\mathrm{BL})$ transitions. These assignments are supported by comparisons with the mononuclear complexes $\left[\mathrm{Ru}(\mathrm{bpy})_{2}(\mathrm{bpm})\right]^{2+108,109,129}$ and $\left[\mathrm{Ru}(\mathrm{bpy})_{2}(\mathrm{dbneil})\right]^{2+82}$ (Table 2.3 and Figures A2.1-3, Appendix A), and the well-documented transitions in $\left[\mathrm{Ru}(\mathrm{bpy})_{3}\right]^{2+} .102,138$

In the spectra of $\left[\mathrm{Ru}(\mathrm{bpy})_{2}(\mathrm{bpm})\right]^{2+}$ and $\left[\mathrm{Ru}(\mathrm{bpy})_{2}(\mathrm{dbneil})\right]^{2+}$, the lowest energy absorption bands at 20900 and $16595 \mathrm{~cm}^{-1}$ respectively, are assigned as $\mathrm{d} \pi\left(\mathrm{Ru}^{\mathrm{II}}\right) \rightarrow \pi^{*}(\mathrm{BL})$ singlet MLCT transitions. ${ }^{82,108-}$ 110, 129, 138 The energy of this transition in the latter is consistent with the greater stabilisation of the $\pi^{*}$ (dbneil) orbitals relative to the $\pi^{*}(\mathrm{bpm})$ orbitals. The band at $23105 \mathrm{~cm}^{-1}$ is assigned as an $\mathrm{d} \pi\left(\mathrm{Ru}^{\mathrm{II}}\right) \rightarrow \pi^{*}(\mathrm{bpm}) \mathrm{MLCT}$ transition in $\left[\mathrm{Ru}(\mathrm{bpy})_{2}(\mathrm{bpm})\right]^{2+}$, and the bands in the region 25000-30000 
$\mathrm{cm}^{-1}$ are assigned as overlapping $\mathrm{d} \pi\left(\mathrm{Ru}^{\mathrm{II}}\right) \rightarrow \pi^{*}$ (bpm,bpy) MLCT transitions. [Ru(bpy $\left.)_{2}(\mathrm{dbneil})\right]^{2+}$ is characterised by overlapping $\mathrm{d} \pi\left(\mathrm{Ru}^{\mathrm{II}}\right) \rightarrow \pi^{*}$ (dbneil,bpy) MLCT and $\pi \rightarrow \pi^{*}$ (dbneil) transitions in the region $22000-26000 \mathrm{~cm}^{-1}$. The transitions in the region $20000-24000 \mathrm{~cm}^{-1}$ for both complexes shift to longer wavelengths upon oxidation, consistent with their $\mathrm{d} \pi\left(\mathrm{Ru}^{\mathrm{III}}\right) \rightarrow \pi^{*}(\mathrm{BL}, \mathrm{bpy})$ assignment. The weak $\left\{(\varepsilon / v)_{\max } \approx 0.05 \mathrm{M}^{-1}\right\}$ bands which appear at the tail of the lowest energy MLCT transition in the oxidised complexes $\left[\mathrm{Ru}(\mathrm{bpy})_{2}(\mathrm{BL})\right]^{3+}$ are tentatively assigned as ligand-to-metal charge transfer (LMCT) transitions involving the $\mathrm{Ru}^{\mathrm{III}}$ centres. The energies and relatively weak intensities of these bands are consistent with the LMCT transitions at 14815 and $17160 \mathrm{~cm}^{-1}$ in $\left[\mathrm{Ru}(\mathrm{bpy})_{3}\right]^{3+}{ }^{338,139}$

Table 2.3 UV/Vis/NIR spectral data of the reduced absorption spectra $(\varepsilon / v v s . v)$ for the $\left[\mathrm{Ru}(\mathrm{bpy})_{2}(\mathrm{BL})\right]^{n+}$ and $\left[\left\{\mathrm{Ru}(\mathrm{bpy})_{2}\right\}_{2}(\mu-\mathrm{BL})\right]^{n+}\{\mathrm{BL}=$ bpm, dbneil $\}$ in $0.02 \mathrm{M}\left[\left(n-\mathrm{C}_{4} \mathrm{H}_{9}\right)_{4} \mathrm{~N}\right]\left\{\mathrm{B}\left(\mathrm{C}_{6} \mathrm{~F}_{5}\right)_{4}\right\} / \mathrm{CH}_{3} \mathrm{CN}$ at $+25^{\circ} \mathrm{C}$. ${ }^{\mathrm{a}}$ The NIR spectral data are indicated in bold type. Spectral data for the mononuclear complex $\left[\mathrm{Ru}(\mathrm{bpy})_{3}\right]^{n+}$ in $0.02 \mathrm{M}$ $\left[\left(n-\mathrm{C}_{4} \mathrm{H}_{9}\right)_{4} \mathrm{~N}\right]\left\{\mathrm{B}\left(\mathrm{C}_{6} \mathrm{~F}_{5}\right)_{4}\right\} / \mathrm{CH}_{3} \mathrm{CN}$ is included for comparison.

\begin{tabular}{|c|c|c|c|}
\hline \multirow[t]{2}{*}{ Complex } & \multirow[t]{2}{*}{$n$} & \multicolumn{2}{|c|}{$\begin{aligned} v_{\max } & \pm 10 / \mathrm{cm}^{-1} \\
\left\{(\varepsilon / v)_{\max }\right. & \left. \pm 0.0001 / \mathrm{M}^{-1}\right\}\end{aligned}$} \\
\hline & & bpm & dbeil \\
\hline \multirow[t]{2}{*}{ meso- $\left[\left\{\mathrm{Ru}(\mathrm{bpy})_{2}\right\}_{2}(\mu-\mathrm{BL})\right]^{n+}$} & 4 & $\begin{array}{l}16775(0.6142) \\
\text { sh } 18200(0.4173) \\
24270(1.550)\end{array}$ & $\begin{array}{l}14290(1.975) \\
\text { sh } 22270(1.131) \\
23700(1.591)\end{array}$ \\
\hline & 5 & $\begin{array}{l}\mathbf{5 0 5 5} \mathbf{( \mathbf { 0 . 1 7 8 1 } )} \\
13540(0.2463) \\
16440(0.2880) \\
24340(0.6772)\end{array}$ & $\begin{array}{l}\mathbf{4 6 5 0}(\mathbf{0 . 2 1 1}) \\
14045(1.007) \\
23660(1.005)\end{array}$ \\
\hline \multirow[t]{2}{*}{ rac- $\left[\left\{\mathrm{Ru}(\mathrm{bpy})_{2}\right\}_{2}(\mu-\mathrm{BL})\right]^{n+}$} & 4 & $\begin{array}{l}16730(0.6143) \\
\text { sh } 18200(0.3895) \\
24230(1.551)\end{array}$ & $\begin{array}{l}14290(3.219) \\
\text { sh } 22195(1.813) \\
23780(2.590) \\
\end{array}$ \\
\hline & 5 & $\begin{array}{l}\mathbf{5 0 8 0}(\mathbf{0 . 1 7 8 1}) \\
13540(0.2504) \\
16395(0.2952) \\
24300(0.6801) \\
\end{array}$ & $\begin{array}{l}\mathbf{4 5 6 0}(\mathbf{0 . 2 0 6 4 )} \\
14070(1.660) \\
23070(1.660)\end{array}$ \\
\hline \multirow[t]{2}{*}[\mathrm{Ru}(\mathrm{bpy})_{2}(\mathrm{BL})]{$^{n+}$} & 2 & $\begin{array}{l}\text { sh } 20900(0.2001) \\
23105(0.4120) \\
27780(0.2502)\end{array}$ & $\begin{array}{l}16595(1.122) \\
22835(0.9872) \\
23715(0.9283) \\
\operatorname{sh} 26570(0.8335) \\
\end{array}$ \\
\hline & 3 & $\begin{array}{l}13970(0.01705) \\
23915(0.08067)\end{array}$ & $\begin{array}{l}\text { sh } 11450(0.0926) \\
15260(0.6029) \\
20870(0.8218) \\
22100(0.7928) \\
25355(0.8626)\end{array}$ \\
\hline \multirow[t]{2}{*}[\mathrm{Ru}(\mathrm{bpy})_{3}]{$^{n+}$} & 2 & \multicolumn{2}{|c|}{$\begin{array}{c}22145(0.6281) \\
\text { sh } 23360(0.5000) \\
28480(0.1912)\end{array}$} \\
\hline & 3 & \multicolumn{2}{|c|}{$\begin{array}{l}14810(0.0268) \\
23960(0.0818)\end{array}$} \\
\hline
\end{tabular}

Spectroelectrochemical generation of the mixed-valence forms of meso- and rac- $\left[\left\{\operatorname{Ru}(\mathrm{bpy})_{2}\right\}_{2}(\mu-\right.$ $\mathrm{BL})]^{5+}\{\mathrm{BL}=\mathrm{bpm}$ and dbneil $\}$ revealed stable isosbestic points in the spectral progressions, as shown in Figures 2.14 and 2.15, respectively. The MLCT absorption bands decreased in intensity, and experienced 
a slight red-shift following one-electron oxidation to the mixed-valence species, with the appearance of a new band in the NIR region 3000-9000 $\mathrm{cm}^{-1}$. The NIR bands are absent in the spectrum of the +4 species, and decrease in intensity as the potential is held at a value beyond that required for generation of the +6 species. On this basis the bands at 4900 and $4890 \mathrm{~cm}^{-1}$ in meso- and $\mathrm{rac}-\left[\left\{\mathrm{Ru}(\mathrm{bpy})_{2}\right\}_{2}(\mu \text {-bpm })\right]^{5+}$ and at 4650 and $4560 \mathrm{~cm}^{-1}$ in meso- and rac-[\{Ru(bpy $\left.)_{2}\right\}_{2}(\mu$-dbneil $\left.)\right]^{5+}$ are assigned as IVCT transitions.

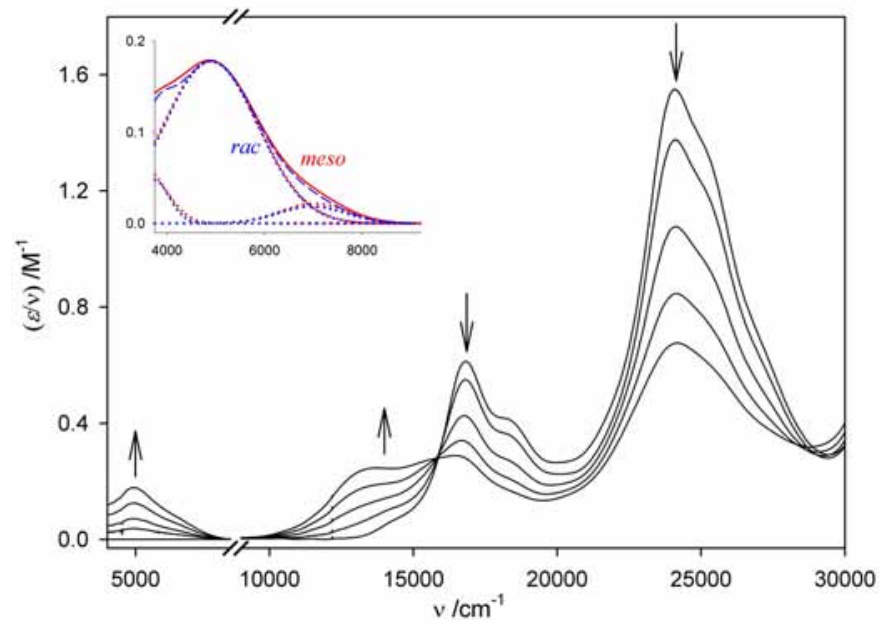

Figure 2.14 UV/Vis/NIR spectroelectrochemical progression for the oxidation reaction rac- $\left[\left\{\mathrm{Ru}(\mathrm{bpy})_{2}\right\}_{2}(\mu-\mathrm{bpm})\right]^{4+} \rightarrow$ rac- $\left[\left\{\mathrm{Ru}(\mathrm{bpy})_{2}\right\}_{2}(\mu-\mathrm{bpm})\right]^{5+}$ in $0.02 \mathrm{M}\left[\left(n-\mathrm{C}_{4} \mathrm{H}_{9}\right)_{4} \mathrm{~N}\right]\left\{\mathrm{B}\left(\mathrm{C}_{6} \mathrm{~F}_{5}\right)_{4}\right\} / \mathrm{CH}_{3} \mathrm{CN}$ at $+25^{\circ} \mathrm{C}$. The break in the axis signifies that the spectra were obtained at different scan rates. Inset: overlay of IVCT bands for meso ( - ) and rac ( ---- ) diastereoisomers and the components obtained by Gaussian deconvolution.

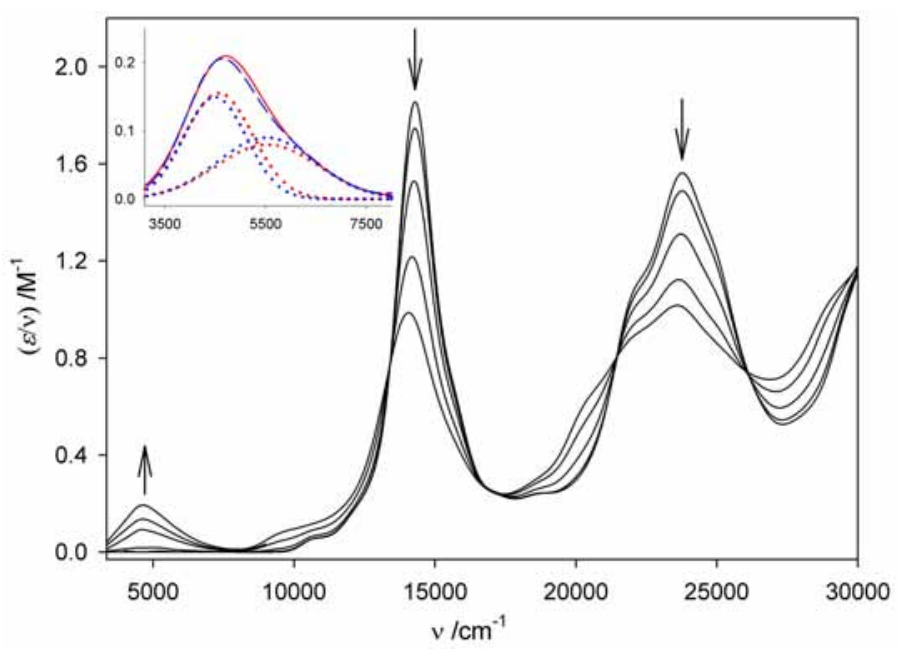

Figure 2.15 UV/Vis/NIR spectroelectrochemical progression for the oxidation reaction rac- $\left[\left\{\mathrm{Ru}(\text { bpy })_{2}\right\}_{2}(\mu \text {-dbneil })\right]^{4+} \rightarrow$ rac- $\left[\left\{\mathrm{Ru}(\text { bpy })_{2}\right\}_{2}(\mu \text {-dbneil })\right]^{5+}$ in $0.02 \mathrm{M}\left[\left(n-\mathrm{C}_{4} \mathrm{H}_{9}\right)_{4} \mathrm{~N}\right]\left\{\mathrm{B}\left(\mathrm{C}_{6} \mathrm{~F}_{5}\right)_{4}\right\} / \mathrm{CH}_{3} \mathrm{CN}$ at $+25^{\circ} \mathrm{C}$. Inset: overlay of IVCT bands for meso ( - ) and rac (---- ) diastereoisomers and the components obtained by Gaussian deconvolution.

The characterisation of the +6 states of the complexes were not possible as the complete generation of the fully-oxidised forms could not be achieved reversibly. The energies of the $\mathrm{Ru}^{\mathrm{III}}$-based LMCT transitions in the dinuclear species could not be established, however a comparison with the mononuclear species $\left[\mathrm{Ru}(\mathrm{bpy})_{2}(\mathrm{bpm})\right]^{3+}$ and $\left[\mathrm{Ru}(\mathrm{bpy})_{2}(\mathrm{dbneil})\right]^{3+}$ (Table 2.3 and Figures A2.2-2.4, 
Appendix A) suggests that such transitions, if present, should occur in the visible region, between 10000 and $15000 \mathrm{~cm}^{-1}$. This provides further support for the assignment of the NIR bands as IVCT, rather than LMCT transitions.

In previous work on $\left[\left\{\mathrm{Ru}(\mathrm{bpy})_{2}\right\}_{2}(\mu \text {-bpm) }]^{5+}\right.$ (as a diastereoisomeric mixture), ${ }^{30,127,140}$ an analysis of the IVCT characteristics was precluded as the mixed-valence form was not sufficiently stable for a meaningful bandwidth to be obtained. In the present case, a fast scanning technique was employed over the wavelength range $3200-9200 \mathrm{~cm}^{-1}$ (at a rate of $8000 \mathrm{~cm}^{-1} / \mathrm{min}$ ), which permitted the generation of the mixed-valence species for a sufficient time to provide characterisation of the IVCT bands before appreciable net reduction of the $5+$ to the $4+$ ion occurred. Chemical decomposition was eliminated as a source of the instability of the mixed-valence species as the regeneration of the un-oxidised +4 species was achieved with $>98 \%$ reversibility, and the decrease in the IVCT intensity (following the attainment of the maximum intensity) was consistent with the disproportionation of the mixed-valence species according to expected second-order kinetics.

\subsubsection{Intervalence Charge Transfer}

The NIR spectra of the dinuclear systems were scaled as $\int \varepsilon(v) / v d v^{31,88}$ and deconvoluted by use of the software package GRAMS32. The results of the band maxima, $v_{\max }$, intensities, $(\varepsilon / v)_{\max }$, and bandwidths, $\Delta v_{1 / 2}$, are summarised in Table 2.4.

\section{A Classical Analysis}

The NIR band manifolds appear asymmetrical and slightly narrower on the lower energy side with bandwidths at half-height $\left(\Delta v_{1 / 2}\right)$ of 2800 and $2620 \mathrm{~cm}^{-1}$ for meso- and rac-[\{Ru(bpy $\left.)_{2}\right\}_{2}(\mu \text {-bpm) }]^{5+}$, respectively, and 1970 and $2050 \mathrm{~cm}^{-1}$ for meso- and rac-[\{Ru(bpy) $\}_{2}(\mu \text {-dbneil) }]^{5+}$, respectively. On the basis of a classical two-state model, the theoretical bandwidth at half-height $\left(\Delta v_{1 / 2}{ }^{\circ}\right)$ is given by equation 1.8 for a weakly-coupled system, where $16 \mathrm{RT} \ln 2=2310 \mathrm{~cm}^{-1}$ at $298 \mathrm{~K}^{.143}$ The relatively narrow observed bandwidths suggest that the localised Class II description may be inappropriate for these systems. $^{144}$

As described in $\S 1.3 .3$, the parameter $\Gamma$ provides a criterion for the degree of electronic coupling in the system. ${ }^{35}$ According to equation $1.21, \Gamma=0.175$ and 0.229 for meso- and rac- $\left[\left\{\operatorname{Ru}(\mathrm{bpy})_{2}\right\}_{2}(\mu\right.$ bpm) $]^{5+}$ respectively, and $\Gamma=0.40$ and 0.37 for meso- and $r a c-\left[\left\{\mathrm{Ru}(\mathrm{bpy})_{2}\right\}_{2}(\mu \text {-dbneil })\right]^{5+}$ respectively. Accordingly, all the systems appear to lie between the localised (Class II) and delocalised (Class III) regimes, in the Class II-III transition region. It must be noted that the assignment of the mixed-valence systems as Class II-III is strictly valid only when there is clear evidence of (i) localisation on the relatively infrared time-scale $\left(\leq 10^{-11} \mathrm{~s}\right)$, and (ii) delocalisation on relatively longer time-scale of the

\footnotetext{
* The mixed-valence species disproportionates according to the "disproportionation" $2[2,3]=[2,2]+[3,3]$ where the rate (proportional to the change in absorbance with time $(t)$ is given by $\mathrm{d}[2,3] / \mathrm{d} t=k[2,2][3,3]$ ( $k$ is a constant). ${ }^{44,141,142}$
} 
UV/Vis experiments, giving rise to IVCT bands which are narrow and asymmetrically-shaped compared with the predicted Gaussian-shaped contour for a weakly-coupled system. ${ }^{145}$

Table 2.4 Characteristics of the IVCT bands (for the absorption spectra scaled as ( $\varepsilon / v v s . v$ ) for the dinuclear mixed-valence complexes in $0.02 \mathrm{M}\left[\left(n-\mathrm{C}_{4} \mathrm{H}_{9}\right)_{4} \mathrm{~N}\right]\left\{\mathrm{B}\left(\mathrm{C}_{6} \mathrm{~F}_{5}\right)_{4}\right\} / \mathrm{CH}_{3} \mathrm{CN}$ at $+25^{\circ} \mathrm{C}$. Parameters for overall envelope are shown in bold type and details of the deconvoluted bands are in normal type. ${ }^{\mathrm{a}, \mathrm{b}}$

\begin{tabular}{|c|c|c|c|c|c|c|}
\hline Complex & $\begin{array}{l}v_{\max } \\
\pm 10 \\
/ \mathrm{cm}^{-1}\end{array}$ & $\begin{array}{c}(\varepsilon / \nu)_{\max } \\
\pm 0.0001 \\
/ \mathrm{M}^{-1}\end{array}$ & $\begin{array}{l}\Delta v_{1 / 2} \\
\pm 10 \\
/ \mathrm{cm}^{-1}\end{array}$ & $\begin{array}{l}\Delta v_{1 / 2}{ }^{0} \\
/ \mathrm{cm}^{-1}\end{array}$ & $\Gamma$ & $\begin{array}{c}\mathrm{H}_{\mathrm{ab}} \\
/ \mathrm{cm}^{-1}\end{array}$ \\
\hline \multirow{4}{*}{ meso- $\left[\left\{\mathrm{Ru}(\mathrm{bpy})_{2}\right\}_{2}(\mu-\mathrm{bpm})\right]^{5+}$} & 5055 & 0.1781 & 2800 & 3395 & 0.175 & 420 \\
\hline & 3615 & 0.0664 & 995 & 2870 & 0.347 & 110 \\
\hline & 5055 & 0.1779 & 2145 & 3395 & 0.368 & 365 \\
\hline & 7185 & 0.0193 & 1505 & 4050 & 0.372 & 145 \\
\hline \multirow[t]{4}{*}{ rac- $\left[\left\{\mathrm{Ru}(\mathrm{bpy})_{2}\right\}_{2}(\mu-\mathrm{bpm})\right]^{5+}$} & 5080 & 0.1781 & 2620 & 3400 & 0.229 & 405 \\
\hline & 3920 & 0.0479 & 710 & 2990 & 0.237 & 280 \\
\hline & 5090 & 0.1775 & 2145 & 3405 & 0.370 & 370 \\
\hline & 7160 & 0.0187 & 1520 & 4040 & 0.376 & 140 \\
\hline \multirow[t]{3}{*}{ meso-[\{Ru(bpy $\left.)_{2}\right\}_{2}(\mu$-dbneil $\left.)\right]^{5+}$} & 4650 & 0.2112 & 1970 & 3270 & 0.398 & 250 \\
\hline & 4570 & 0.1549 & 1595 & 3240 & 0.508 & 190 \\
\hline & 5530 & 0.0795 & 2390 & 3265 & 0.268 & 200 \\
\hline \multirow[t]{3}{*}{ rac-[\{Ru(bpy $\left.)_{2}\right\}_{2}(\mu$-dbneil $\left.)\right]^{5+}$} & 4560 & 0.2064 & 2050 & 3232 & 0.366 & 220 \\
\hline & 4495 & 0.1493 & 1465 & 3215 & 0.544 & 155 \\
\hline & 5515 & 0.0898 & 2290 & 3560 & 0.357 & 185 \\
\hline
\end{tabular}

$\Delta v_{1 / 2}{ }^{o}=\left[2310\left(v_{\max }\right)\right]^{1 / 2}$ at $298 \mathrm{~K} \cdot{ }^{31,32}$

${ }^{\mathrm{b}}$ Lower limits for $\mathrm{H}_{\mathrm{ab}}$ using $r_{\mathrm{ab}}=7.9 \AA$ for $\mathrm{dbneil}^{82}$ and 5.565(5) $\AA$ (the $\mathrm{Ru} \cdots \mathrm{Ru}$ distance obtained from the crystal structure of $\left[\left\{\mathrm{Ru}(\mathrm{bpy})_{2}\right\}_{2}(\mu \text {-bpm) }]^{4+}\right.$ complex $)$ for bpm. These may differ for the two diastereoisomers.

Within the framework of the classical model, the asymmetric appearance of the bands is ascribed to the "band cut-off effect" $35,144,146$ which occurs at $h v=2 \mathrm{H}_{\mathrm{ab}}$ (§1.3.2), where the electronic coupling parameter, $\mathrm{H}_{\mathrm{ab}}$, is given by equation 1.9, §1.2.1. As detailed in $\S 1.5 .3 .2, r_{\mathrm{ab}}$ is often equated with the through-space geometrical distance between the metal centres. ${ }^{145}$ However, the effective charge transfer distance is decreased relative to the geometric distance as electronic coupling across the bridge increases, and equation 1.9 provides a lower limit only for $\mathrm{H}_{\mathrm{ab}}{ }^{* 145}$ With this caveat noted, $\mathrm{H}_{\mathrm{ab}}$ values for the meso and rac diastereoisomers are shown in Table 2.4, with $r_{\mathrm{ab}}$ equated with the approximate geometric metalmetal separation of $7.9 \AA^{82}$

The relatively larger $\Gamma$ values obtained for the dbneil-bridged diastereoiosmers suggest that the diastereoisomers of $\left[\left\{\mathrm{Ru}(\mathrm{bpy})_{2}\right\}_{2}(\mu \text {-dbneil })\right]^{5+}$ exhibit a greater degree of delocalisation relative to their bpm-bridged analogues. Since the IVCT characteristics of the diastereoisomers display evidence of both localised and delocalised behaviour, a localised model and orbital geometry considerations may be invoked to rationalise the origins of the IVCT components. Such localised models have been proposed by Meyer and coworkers ${ }^{53,145,147-149}$ to rationalise the origins of the IVCT bands in a series of dinuclear ruthenium and osmium $\mathrm{N}_{2}$ - and pyrazine-bridged mixed-valence systems which display evidence for both

\footnotetext{
* The notion of the "effective charge transfer distance" as opposed to the geometric distance between the metal centres is speculative, however this argument is invoked in a number of literature reports of IVCT with the qualification that equation 1.9 provides a lower limit only for $\mathrm{H}_{\mathrm{ab}}$ when the geometric metal-metal distance is used in its determination.
} 
localised and delocalised behaviour. ${ }^{145}$ For the present systems, three IVCT transitions are anticipated due to separate electronic excitations from the $\mathrm{d} \pi_{1}, \mathrm{~d} \pi_{2}$ and $\mathrm{d} \pi_{3}$ orbitals at $\mathrm{Ru}^{\mathrm{II}}$ to the hole in the corresponding $\mathrm{d} \pi_{\mathrm{n}}$ orbital which is present in any of the three spin-orbit states at the $\mathrm{Ru}^{\mathrm{III}}$ centre, as discussed in §1.5.3.5. ${ }^{145}$ The non-degeneracy of the $\mathrm{d} \pi$ levels at $\mathrm{Ru}^{\mathrm{II} / \mathrm{III}}$, and the appearance of multiple IVCT components, arises due to the combined effects of low symmetry and spin-orbit coupling. The skewed nature of the IVCT bands may also arise from (i) underlying transitions that constitute a vibronic progression, ${ }^{150}$ however, further computational efforts are required to address this possibility; or (ii) a "band cut-off effect" due to the population of molecules at or near the top of the electron transfer barrier. $^{145}$

The IVCT components may be assigned by considering the dominant Cartesian character of the $\mathrm{d} \pi(\mathrm{Ru})$ orbitals. If the $x$ axis defines the direct metal-metal axis, $y$ is the long axis of the bpm or dbneil bridging ligand, and $z$ is perpendicular to the plane of the bridging ligand, then the $x y$ plane defines the mean plane of the bridging ligand, as shown for $r a c-(\Delta \Delta)-\left[\left\{\operatorname{Ru}(\mathrm{bpy})_{2}\right\}_{2}(\mu-\mathrm{bpm})\right]^{4+}$ in Figure 2.16. The $\mathrm{d}_{x z}$ and $\mathrm{d}_{y z}$ orbitals are overlapped with the bridging $\pi^{*}$ system to a greater extent than the $\mathrm{d}_{x y}$ orbitals which lie in the plane of the bridging ligand. On this basis, the $d_{x z}$ and $d_{y z}$ orbitals will be stabilised by backbonding to a greater extent than the $\mathrm{d}_{x y}$ orbitals which are less strongly coupled across the bridge. In order of increasing energy, the three $d \pi$ orbitals may be more closely represented by $\mathrm{d}_{x z}, \mathrm{~d}_{y z}\left(\mathrm{~d} \pi_{1}, \mathrm{~d} \pi_{2}\right)$ and $d_{x y}\left(d \pi_{3}\right)$. The intensities of the three IVCT components should be proportional to the degree of orbital overlap between the three $\mathrm{d} \pi$ orbitals at the formally $\mathrm{Ru}^{\mathrm{II}}$ centre and the $\mathrm{d} \pi$ orbital containing the hole at the formally $\mathrm{Ru}^{\mathrm{III}}$ centre. Transitions between the $\mathrm{d}_{x z}, \mathrm{~d}_{y z}\left(\mathrm{~d} \pi_{1}, \mathrm{~d} \pi_{2}\right)$ orbitals on the two metal centres should provide the majority of the intensity to the IVCT manifold, and the transitions $\pi_{1}\left(\mathrm{Ru}^{\mathrm{II}}\right) \rightarrow \mathrm{d} \pi_{3}\left(\mathrm{Ru}^{\mathrm{III}}\right)$ and $\mathrm{d} \pi_{2}\left(\mathrm{Ru}^{\mathrm{II}}\right) \rightarrow \mathrm{d} \pi_{3}\left(\mathrm{Ru}^{\mathrm{III}}\right)$ should occur on the high-energy side of the IVCT manifold. Based on these ligand field considerations, the two components obtained by decovolution of the IVCT manifolds for the $\left[\left\{\mathrm{Ru}(\mathrm{bpy})_{2}\right\}_{2}(\mu \text {-dbneil })\right]^{5+}$ diastereoisomers are assigned as $\pi_{1}\left(\mathrm{Ru}^{\mathrm{II}}\right) \rightarrow \mathrm{d} \pi_{3}\left(\mathrm{Ru}^{\mathrm{III}}\right)$ and $\mathrm{d} \pi_{2}\left(\mathrm{Ru}^{\mathrm{II}}\right) \rightarrow \mathrm{d} \pi_{3}\left(\mathrm{Ru}^{\mathrm{III}}\right)$ transitions. The $\pi_{3}\left(\mathrm{Ru}^{\mathrm{II}}\right) \rightarrow \mathrm{d} \pi_{3}\left(\mathrm{Ru}^{\mathrm{III}}\right)$ transition is of low intensity as the large inter-metal separation of $7.9 \AA^{82}$ gives rise to the weak coupling of the $d_{x y}$ orbitals.
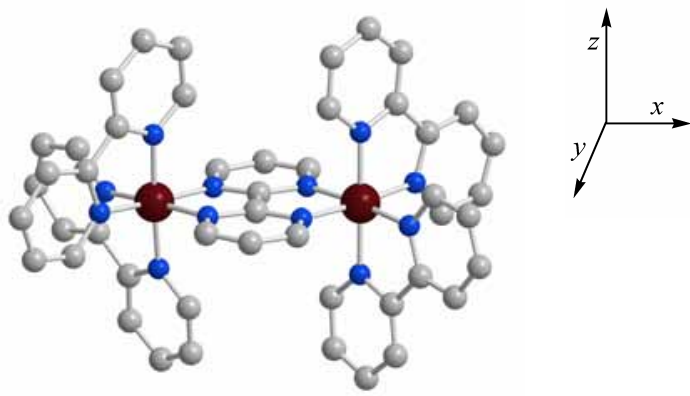

Figure 2.16 Coordinate axes for the $\operatorname{rac}(\Delta \Delta)$ diastereoisomer of $\left[\left\{\mathrm{Ru}(\mathrm{bpy})_{2}\right\}_{2}(\mu \text {-bpm })\right]^{4+}$. 
For the diastereoisomeric forms of $\left[\left\{\mathrm{Ru}(\mathrm{bpy})_{2}\right\}_{2}(\mu \text {-bpm })\right]^{5+}$, the IVCT bands are dominated by a single Gaussian-shaped component, with evidence for a weak transition on the high energy side. The dominant component of the IVCT manifold observed in Figure 2.13 is thus assigned to a convolution of $\pi_{1}\left(\mathrm{Ru}^{\mathrm{II}}\right) \rightarrow \mathrm{d} \pi_{3}\left(\mathrm{Ru}^{\mathrm{III}}\right)$ and $\mathrm{d} \pi_{2}\left(\mathrm{Ru}^{\mathrm{II}}\right) \rightarrow \mathrm{d} \pi_{3}\left(\mathrm{Ru}^{\mathrm{III}}\right)$ interactions, which are unresolvable by Gaussian deconvolution. The origin of the weak component is ambiguous, since the $\pi_{3}\left(\mathrm{Ru}^{\mathrm{II}}\right) \rightarrow \mathrm{d} \pi_{3}\left(\mathrm{Ru}^{\mathrm{III}}\right)$ interation should lie on the lower energy side of the IVCT manifold which is obscured in the present case by the detector limit. The possibility of effects arising from direct (in addition to superexchange-assisted) coupling for the bpm-bridged diastereoisomers due to the close proximity of the metal centres may also be possible. ${ }^{130-132}$

\subsubsection{IVCT Solvatochromism}

\subsubsection{The Diastereoisomers of $\left[\left\{\mathrm{Ru}(\mathrm{bpy})_{2}\right\}_{2}(\mu-\mathrm{bpm})\right]^{5+}$ as Probes for Solvent Reorganisational Effects in IVCT}

The NIR spectra for the diastereoisomers of $\left[\left\{\mathrm{Ru}(\mathrm{bpy})_{2}\right\}_{2}(\mu \text {-bpm })\right]^{5+}$ were measured in the range of solvents AN, PN, BN, ${ }^{\mathrm{B}} \mathrm{BN}$ and BzN. The energies of the IVCT band maxima $\left(v_{\max }\right)$ as a function of $1 / \mathrm{D}_{\mathrm{op}}-1 / \mathrm{D}_{\mathrm{s}}$ are presented in Table 2.5 and Figure 2.17.

Table 2.5 IVCT solvatochromism data of the reduced absorption spectra $(\varepsilon / v v s$. $v)$ for the diastereoisomers of $\left[\left\{\mathrm{Ru}(\mathrm{bpy})_{2}\right\}_{2}(\mu-\mathrm{bpm})\right]^{5+}$ in $0.02 \mathrm{M}\left[\left(n-\mathrm{C}_{4} \mathrm{H}_{9}\right)_{4} \mathrm{~N}\right]\left\{\mathrm{B}\left(\mathrm{C}_{6} \mathrm{~F}_{5}\right)_{4}\right\} /$ solvent at $+25^{\circ} \mathrm{C}$. MLCT energies for the +4 states are also tabulated. ${ }^{\mathrm{a}, \mathrm{b}}$

\begin{tabular}{|c|c|c|c|c|c|c|c|c|}
\hline \multirow[b]{2}{*}{ Solvent } & \multirow[b]{2}{*}{$1 / \mathrm{D}_{\mathrm{op}}-1 / \mathrm{D}_{\mathrm{s}}$} & \multicolumn{3}{|c|}{ meso } & \multicolumn{3}{|c|}{ rac } & \multirow{2}{*}{$\begin{array}{c}\Delta v_{\max } \\
(m e s o-r a c) \\
\pm 10 \\
/ \mathrm{cm}^{-1}\end{array}$} \\
\hline & & $\begin{array}{l}v_{\max } \\
\pm 10 \\
/ \mathrm{cm}^{-1}\end{array}$ & $\begin{array}{l}\Delta v_{1 / 2} \\
\pm 10 \\
/ \mathrm{cm}^{-1}\end{array}$ & $\begin{array}{c}v_{\mathrm{MLCT}(1)} \\
\pm 10 \\
/ \mathrm{cm}^{-1}\end{array}$ & $\begin{array}{l}v_{\max } \\
\pm 10 \\
/ \mathrm{cm}^{-1}\end{array}$ & $\begin{array}{l}\Delta v_{1 / 2} \\
\pm 10 \\
/ \mathrm{cm}^{-1}\end{array}$ & $\begin{array}{c}v_{\text {MLCT }(1)} \\
\pm 10 \\
/ \mathrm{cm}^{-1}\end{array}$ & \\
\hline AN & 0.5127 & 5055 & 2800 & 16775 & 5080 & 2620 & 16775 & -25 \\
\hline PN & 0.5011 & 5435 & 2710 & 16900 & 5470 & 2770 & 16900 & -35 \\
\hline${ }^{\mathrm{i}} \mathrm{BN}$ & 0.4795 & 5420 & 3200 & 16850 & 5425 & 2952 & 16780 & -5 \\
\hline $\mathrm{BN}$ & 0.4762 & 5395 & 2686 & 16800 & 5375 & 2860 & 16750 & 20 \\
\hline BzN & 0.3897 & 5065 & 2570 & 16695 & 5095 & 2674 & 16660 & -30 \\
\hline
\end{tabular}

${ }^{\mathrm{a}}$ IVCT characteristics are reported as an average of three repeat experiments.

${ }^{\mathrm{b}}$ The absolute intensities of the IVCT bands, $(\varepsilon / v)_{\max }$, are not tabulated as all spectra were normalised at the maximum intensity of the IVCT manifold.

The plot in Figure 2.17 reveals the predicted linear trend for both diastereoiosmers in all solvents except AN. From the data (excluding AN), the following values were obtained for the slope and intercept: (meso) slope $=3545 \pm 390 \mathrm{~cm}^{-1} \AA^{-1}$ and intercept $=3390 \pm 245 \mathrm{~cm}^{-1}\left(R^{2}=0.98\right)$; $(\mathrm{rac})$ slope $=$ $3690 \pm 180 \mathrm{~cm}^{-1} \AA^{-1}$ and intercept $=3780 \pm 115 \mathrm{~cm}^{-1}\left(R^{2}=0.99\right)$. The slopes of the plots are identical (within experimental error) for both diastereoisomers, while the intercept for the rac form is marginally greater than the meso form. The similar results for the two forms reflect the comparable electronic and structural characteristics (in terms of bond lengths and angles). Minor differences in the energies of the 
IVCT bands are apparent between the diastereoisomers of a given complex $\left\{v_{\max }(\right.$ meso-rac $)$ in Table 2.5$\}$ across the series of solvents.

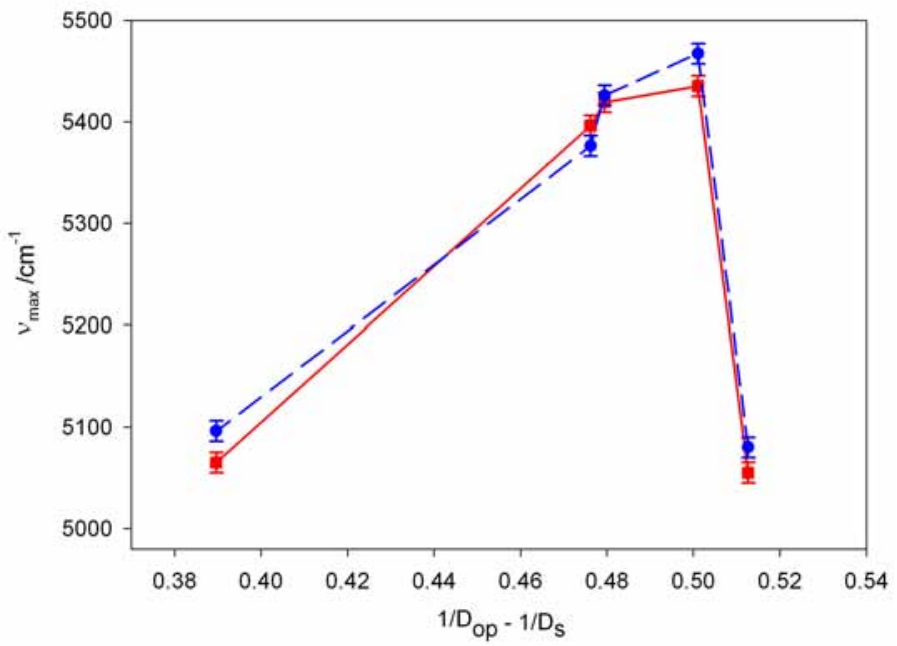

Figure 2.17 $v_{\max }$ as a function of the solvent parameter $1 / \mathrm{D}_{\mathrm{op}}-1 / \mathrm{D}_{\mathrm{s}}$ for the meso $(-)$ and $\mathrm{rac}(----)$ diastereoisomeric forms of $\left[\left\{\mathrm{Ru}(\mathrm{bpy})_{2}\right\}_{2}(\mu-\mathrm{bpm})\right]^{5+}$ in $0.02 \mathrm{M}\left[\left(n-\mathrm{C}_{4} \mathrm{H}_{9}\right)_{4} \mathrm{~N}\right]\left\{\mathrm{B}\left(\mathrm{C}_{6} \mathrm{~F}_{5}\right)_{4}\right\} /$ solvent at $+25^{\circ} \mathrm{C}$.

The origin of the disparity in AN may lie in the invalidity of the assumptions underlying equation 2.2. In particular, the assumption $d » 2 a$ is likely to be invalid for the bpm-bridged dimers due to interpenetration of the coordination spheres. The definition of an idealised radius, $a$, for the redox sites is somewhat ambiguous due to the non-spherical nature of the sites. The average radii for dinuclear complexes incorporating $\mathrm{Ru}(\mathrm{bpy})_{2}$ units may be estimated ${ }^{151}$ by considering the coordinate system described for $\left[\left\{\mathrm{Ru}(\mathrm{bpy})_{2}\right\}_{2}(\mu \text {-bpm })\right]^{4+}$ in Figure 2.16, and the bond distances from the X-ray crystal structure of the meso- $(\Lambda \Delta)-\left[\left\{\operatorname{Ru}(\mathrm{bpy})_{2}\right\}_{2}(\mu-\mathrm{bpm})\right]^{4+}$ cation (§2.3.1.2). From the average of the four distances along the Ru-N(bpy) axes from the Ru ion to the end of the van der Waals radii of the remote hydrogen atoms ${ }^{152}$ (ca. $6.75 \AA$ ) and the two distances along the Ru-N(bpm) axes, where each is taken as half the Ru-Ru distance ( $2.78 \AA$ ), an estimate of $5.43 \AA$ is obtained for $a$. By comparison, equation $2.2^{*}$ yields a value of $4.76 \pm 0.52 \AA$ for $a$ based on the slope of $3545 \mathrm{~cm}^{-1}\left\{=e^{2}(1 / a-1 / d)\right\}$ for the meso diastereoisomer with the crystallographically-determined inter-metal distance $(d)$ of 5.565(5) $\AA$. Using the latter estimate for $a$ based on the dielectric continuum analysis of the solvent dependence of the IVCT band yields a value for the outer-sphere contribution to the reorganisational energy $\left(\lambda_{0}\right)$ of $1820 \pm 200 \mathrm{~cm}^{-1}$ in AN and $1775 \pm 195 \mathrm{~cm}^{-1}$ in PN for the meso diastereoisomer.

The intercept of the IVCT solvatochromism plot in Figure 2.17 provides the sum of the contributions from the solvent independent parameters (assumed to be $\lambda_{i}$ and $\Delta \mathrm{E}^{\prime}$ ) to $v_{\max }$. The $\Delta \mathrm{E}^{\prime}$ contribution arises from the non-degeneracy of the $\mathrm{Ru}(\mathrm{d} \pi)$ levels due to the combined effects of spin-orbit coupling and lower symmetry perturbations. ${ }^{153}$ The non-degeneracy of the three $\mathrm{d} \pi$ levels results in the possibility of three separate IVCT transitions, which if unresolved also contribute to the observed IVCT

\footnotetext{
${ }^{*}$ From equation $2.2, \lambda_{\mathrm{o}}=116000 \mathrm{~cm}^{-1}(1 / a-1 / d)\left(1 / \mathrm{D}_{\mathrm{op}}-1 / \mathrm{D}_{\mathrm{s}}\right)$ with $a$ and $d$ in $\AA$.
} 
bandwidth. In the present case, the IVCT band is reasonably approximated by a dominant Gaussian component corresponding closely to the maximum energy, intensity and bandwidth of the observed IVCT transition. Evidence for a relatively less intense component at higher energy, and the presence of band(s) at lower energy (which are obscured by the detector limit) cannot be discounted. The lack of discernable structure in the NIR band indicates that the IVCT transition is dominated by a single donor-acceptor orbital pair, or if multiple orbital contributions exist, that the energy separations between the levels are small compared with the overall bandwidth of the NIR manifold. Figure 2.17 depicts the solvent dependence of the component or convolution of components that contribute to the IVCT energy. A classical analysis based on geometry considerations (§2.3.3) argues that the dominant Gaussian component of the IVCT manifold represents a contribution from two overlapping transitions assigned as $\operatorname{IVCT}(1)$ and IVCT(2).

In the absence of resolved transitions, the energy separations between the IVCT components are approximated by the energies of the $\mathrm{d} \pi\left(\mathrm{Ru}^{\mathrm{III}}\right)$ spin orbit states, $\mathrm{E}_{\mathrm{so}(1)}$ and $\mathrm{E}_{\mathrm{so}(2)}$, given by equation 2.4(a)-(c). ${ }^{15,46,135}$

$$
\begin{aligned}
& v_{\max }\{\operatorname{IVCT}(1)\}=\lambda_{i}\{\operatorname{IVCT}(1)\}+\lambda_{o} \\
& v_{\max }\{\operatorname{IVCT}(2)\}=\lambda_{i}\{\operatorname{IVCT}(2)\}+\lambda_{\mathrm{o}}+\mathrm{E}_{\mathrm{so}(1)} \\
& v_{\max }\{\operatorname{IVCT}(2)\}=\lambda_{i}\{\operatorname{IVCT}(3)\}+\lambda_{\mathrm{o}}+\mathrm{E}_{\mathrm{so}(2)}
\end{aligned}
$$

Assuming that $\lambda_{\mathrm{o}}$ is independent of the spin-orbit state (which is reasonable in view of the $\mathrm{d} \pi \rightarrow \mathrm{d} \pi$ nature of the transitions ${ }^{135}$ ) $\lambda_{i}$ for each component may be extracted from the intercept of the solvent dependence plot.

Invoking a classical localised argument, geometrical considerations proposed in §2.3.3 suggested that three IVCT transitions for the bpm-bridged dimer $\{\operatorname{IVCT}(n), n=1-3\}$ may be ascribed in order of increasing energy to $\mathrm{d} \pi_{3}\left(\mathrm{Ru}^{\mathrm{II}}\right) \rightarrow \mathrm{d} \pi_{3}\left(\mathrm{Ru}^{\mathrm{III}}\right), \mathrm{d} \pi_{2}\left(\mathrm{Ru}^{\mathrm{II}}\right) \rightarrow \mathrm{d} \pi_{3}\left(\mathrm{Ru}^{\mathrm{III}}\right)$ and $\mathrm{d} \pi_{1}\left(\mathrm{Ru}^{\mathrm{II}}\right) \rightarrow \mathrm{d} \pi_{3}\left(\mathrm{Ru}^{\mathrm{III}}\right)$ transitions. Considering the deviation of the sites from octahedral symmetry and the proposed coordinate system, the dominant Cartesian components of the $\mathrm{d} \pi$ orbitals are $\mathrm{d}_{x z} \mathrm{~d}_{y z}\left(\mathrm{~d} \pi_{1} \mathrm{~d} \pi_{2}\right)$ and $\mathrm{d}_{x y}\left(\mathrm{~d} \pi_{3}\right)$. If the IVCT band is skewed towards IVCT(2) and IVCT(3) with energies given by equations 2.4(b) and (c), respectively, then the energy of the IVCT band is given by IVCT(1) $+\Delta$, where $\Delta$ is the energy difference between the absorption band maximum arising from the higher energy components and IVCT(1), ${ }^{46}$ as expressed in equation 2.5 .

$$
v_{\max }=\lambda_{i}+\lambda_{0}+\Delta
$$

Assuming that $\mathrm{E}_{\mathrm{so}(1)}$ and $\mathrm{E}_{\mathrm{so}(2)}$ are the energies of the interconfigurational (IC) transitions at 1560 and $1910 \mathrm{~cm}^{-1}$ in $\left[\mathrm{Ru}(\mathrm{bpy})_{3}\right]^{3+154}$, respectively, and that the intensity of the IVCT manifold is dominated by the $\mathrm{d} \pi_{1}$ and $\mathrm{d} \pi_{2}$ transitions, $\Delta$ is approximated as $1735 \mathrm{~cm}^{-1}$. From the intercept $\left(\lambda_{i}\right.$ and $\left.\Delta\right)$ in Figure 2.17, $\lambda_{i}$ is determined as $1655 \pm 245$ and $2045 \pm 115 \mathrm{~cm}^{-1}$ for the meso and rac disatereoisomers, respectively. The large magnitude of $\lambda_{i}$ is surprising in view of the small bond distance changes that 
occur with oxidation and which give rise to the inner-sphere barrier. ${ }^{44,153}$ In the absence of detailed vibrational information and structural data for the mixed-valence complex, quantification of $\lambda_{i}$ is not possible. However, estimates based on Ru-N bond distance changes between $\left[\mathrm{Ru}(\mathrm{bpy})_{3}\right]^{2+}$ and $\left[\mathrm{Ru}(\mathrm{bpy})_{3}\right]^{3+}$ lead to a prediction for the inner-sphere barrier of less than $400 \mathrm{~cm}^{-1}$, due to the small changes in bond lengths on oxidation. ${ }^{155}$ In the present case, this estimate is not compatible with the predicted values for $\lambda_{i}$. Such anomalies have been noted previously for related complexes such as $\left[\left\{\operatorname{Ru}(\text { bpy })_{2}(\text { py })\right\}_{2}\left(\mu-4,4^{\prime}-\text { bpy }\right)\right]^{5+}{ }^{42}$ The slope of $3.1 \times 10^{3} \mathrm{~cm}^{-1} \AA^{-1}$ was significantly smaller than the value calculated on the basis of the spherical and ellipsoidal dielectric continuum models ${ }^{15}$ and the relatively large intercept of $6.0 \times 10^{3} \mathrm{~cm}^{-1}$ was also difficult to rationalise from the small bond-length variations with oxidation. ${ }^{44,153}$ The origin of the anomalous behaviour was attributed to delocalisation of the electron between the two sites which invalidates the weak-coupling approximation which is implicit in equation 2.2. Electronic coupling effects have also been advanced to rationalise the lower than expected solvent dependence for $\left[\left\{\mathrm{Ru}\left(\mathrm{NH}_{3}\right)_{5}\right\}_{2}\left(\mu-4,4^{\prime} \text {-bpy }\right)\right]^{5+}{ }^{156}$ A revision in the charge transfer distance $(d$ in equation 2.1) from $5.1 \AA$ from Stark effect measurements ${ }^{157,158}$ compared with the geometrical intrametal distance of $11.3 \AA$, led to agreement with both spherical cavity and ellipsoidal cavity models. In the present case, the non-negligible electronic delocalisation (and the invalidity of the localised assumption of equation 2.2) may also provide an explanation for the smaller than expected slope.

Clearly, the intercepts conceal additional energy contributions that are not accounted for by the dielectric continuum model. In a proper treatment, all relevant modes must be considered to account for the role of inner-sphere vibrations, and quantum mechanical treatments are necessary when vibrational spacings are appreciable compared with $k_{B} T$. In the absence of vibrational information from resonance Raman studies which would permit an accurate calculation of $\lambda_{i}$, the estimates of $\lambda_{i}$ from the intercepts of the solvatochromism plots are identical for both diastereoisomers of $\left[\left\{\operatorname{Ru}(\mathrm{bpy})_{2}\right\}_{2}(\mu \text {-bpm) }]^{5+}\right.$. Since the inner-sphere vibrational energies are identical for both diastereoisomeric forms, the differences in the energies of the IVCT bands arise primarily from different outer-sphere solvent interactions with the two forms.

While an ellipsoidal cavity mode ${ }^{15}$ may provide a more physically realistic description for the dinuclear complexes, the treatment is based on the macroscopic dielectric properties of the solvent (as for the spherical cavity model), and would not account for the anomalous results in AN.

\section{Continuum versus Non-continuum Solvent Reorganisational Effects}

The origin of the energy disparity in AN is attributed to a specific solvent effect which overwhelms the dielectric continuum description. The clefts between the terminal bpy rings in the diastereoisomeric forms of $\left[\left\{\mathrm{Ru}(\mathrm{bpy})_{2}\right\}_{2}(\mu \text {-bpm) }]^{5+}\right.$ permit the AN molecules to approach the metal centres more closely than is permitted by the theoretical model: the clefts between the ligands at the two $\mathrm{Ru}(\mathrm{bpy})_{2}$ termini (the "exterior clefts”) are identical in both diastereoisomeric forms while the dimensions of the clefts above and below the plane of the bridging ligand (the "interior clefts") are distinguishable in 
the two forms, as shown in Figure 2.2. The minor differences between the energies of the IVCT bands for the diastereoisomers suggest that specific "stereochemically-directed" interactions may be present in all solvents due to solvent penetration within the interior clefts. The red-shifts in $v_{\max }$ of $380 \pm 10$ and $390 \pm 10 \mathrm{~cm}^{-1}$ between PN and AN for the meso and rac diastereoisomers, respectively, are surprising in view of the similar bulk dielectric properties of AN and PN, and the similarity of their structures which differ only in the presence of an additional methyl group for PN. Despite the subtle structural difference between the two solvents, the results suggest that the relatively small AN molecules may approach the metal centres more closely than is permitted for PN due to steric hindrance of that additional methyl group.

Two issues arise in the attempt to rationalise these observations. Firstly, the red-shift of the IVCT energy in AN relative to $\mathrm{PN}$ is unexpected since the general expectation is that increased specific solvation induces an additional contribution to the reorganisational energy, and hence a blue-shift of the IVCT energy. Previous solvatochromism experiments for complexes based on $\mathrm{Ru}\left(\mathrm{NH}_{3}\right)_{5}$ have revealed correlations between the energies of the IVCT bands and empirical solvent basicity parameters such as DN due to specific solvent-ammine H-bonding. ${ }^{2}$ In the present case, the solvent-solute interactions are likely to involve electrostatic interactions between the solvent molecules and pockets of electron density on the bpy and bpm ligands. Such interactions have been observed previously for tris( $\alpha, \alpha^{\prime}$-diimine) chelates such as $\left[\mathrm{Ru}(\mathrm{bpy})_{3}\right]^{2+}{ }^{159}$ Secondly, specific solvation effects will be present in addition to dielectric continuum solvation. In previous studies of complexes based on $\mathrm{Ru}\left(\mathrm{NH}_{3}\right)_{5}$, trans- $\mathrm{Ru}\left(\mathrm{NH}_{3}\right)_{4}(\mathrm{py})$ and $\mathrm{Ru}$ (bpy)( $\left(\mathrm{NH}_{3}\right)_{3}$ with pyz, 4-cyanopyridine and 4,4'-bpy bridging ligands, ${ }^{19} v_{\max }$ was fitted to a dual parameter equation including both $1 / \mathrm{D}_{\mathrm{op}}-1 / \mathrm{D}_{\mathrm{s}}$ and $\mathrm{DN}$. In the present case, the apparent success of dielectric continuum theory in explaining the linear solvent dependence of $v_{\max }$ on $1 / \mathrm{D}_{\mathrm{op}}-1 / \mathrm{D}_{\mathrm{s}}$ in $\mathrm{PN}, \mathrm{BN}$, ${ }^{\mathrm{i}} \mathrm{BN}$ and $\mathrm{BzN}$ is surprising if all the solvents engage in some form of specific solvation. In polar solvents, $1 / D_{\text {op }}$ » $1 / D_{s}$, and the value of $D_{o p}$ in the first solvation shell may be similar to that in bulk solution. This would explain the apparent success of the continuum theory, even in the presence of specific solvation effects for these solvents. The anomalous result in AN suggests that these molecules engage in a different form of specific interaction with the diastereoisomers, compared with PN, BN, ${ }^{\mathrm{i}} \mathrm{BN}$ and BzN.

To address the issue of continuum versus specific solvation, the IVCT characteristics of the diastereoisomeric forms of $\left[\left\{\mathrm{Ru}(\mathrm{bpy})_{2}\right\}_{2}(\mu \text {-bpm) }]^{5+}\right.$ were investigated as a function of solvent composition in a series of solvent mixtures containing varying mole fractions of $\mathrm{AN}\left(n_{\mathrm{AN}}\right)$ and $\mathrm{PN}\left(n_{\mathrm{PN}}=\right.$ 1- $n_{\mathrm{AN}}$ ) \{Figure 2.18 and Table 2.6\}. 


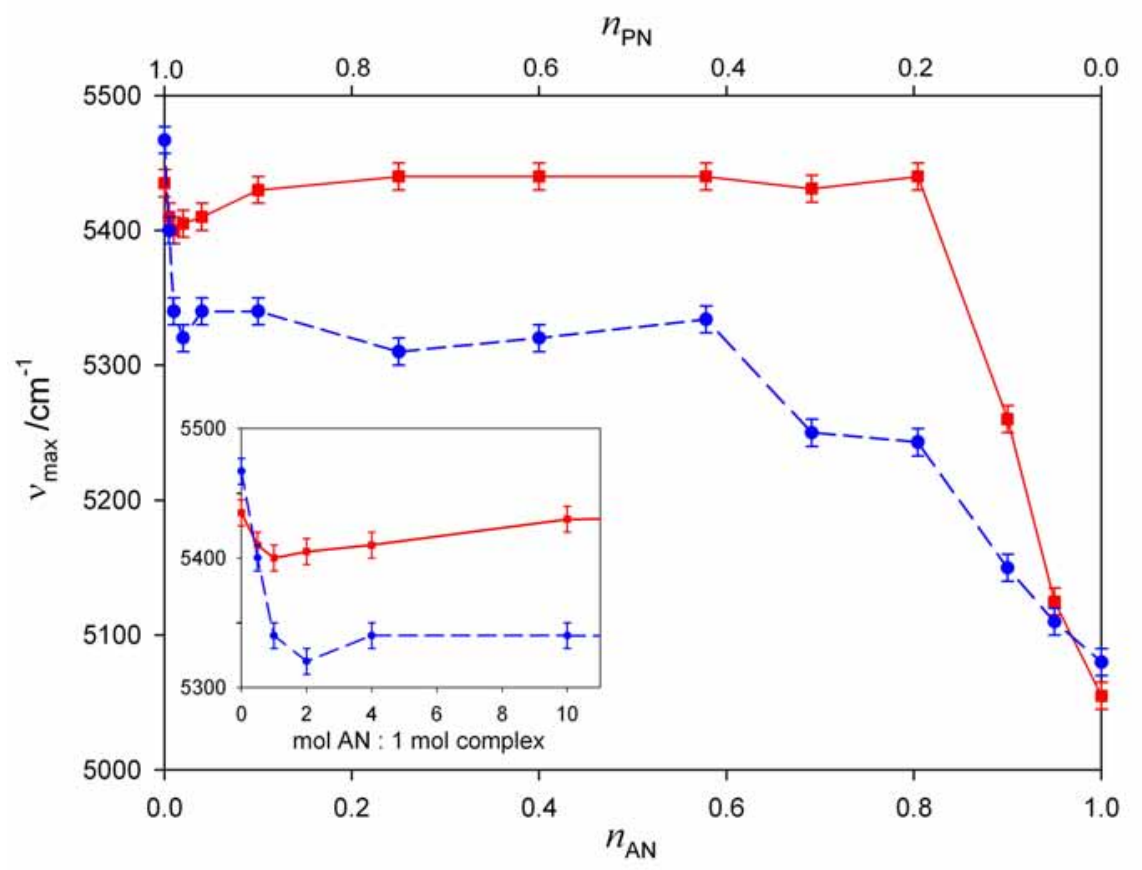

Figure 2.18 $v_{\max }$ as a function of solvent composition for the meso ( - ) and rac (---- ) diastereoisomers of $\left[\left\{\mathrm{Ru}(\mathrm{bpy})_{2}\right\}_{2}(\mu-\mathrm{bpm})\right]^{5+}$ in AN and PN mixtures containing $0.02 \mathrm{M}\left[\left(n-\mathrm{C}_{4} \mathrm{H}_{9}\right)_{4} \mathrm{~N}\right]\left\{\mathrm{B}\left(\mathrm{C}_{6} \mathrm{~F}_{5}\right)_{4}\right\} /$ solvent at $+25^{\circ} \mathrm{C}$.

Table 2.6 IVCT solvatochromism data of the reduced absorption spectra $(\varepsilon / v v s$. $v)$ for the diastereoisomers of $\left[\left\{\mathrm{Ru}(\mathrm{bpy})_{2}\right\}_{2}(\mu \text {-bpm })\right]^{5+}$ in $0.02 \mathrm{M}\left[\left(n-\mathrm{C}_{4} \mathrm{H}_{9}\right)_{4} \mathrm{~N}\right]\left\{\mathrm{B}\left(\mathrm{C}_{6} \mathrm{~F}_{5}\right)_{4}\right\} /$ solvent at $+25^{\circ} \mathrm{C}$. The lowest energy MLCT transition for the +4 state is also tabulated. ${ }^{\text {a }}$

\begin{tabular}{|c|c|c|c|c|c|c|c|}
\hline \multirow[b]{2}{*}{$n_{\mathrm{AN}}$} & \multicolumn{3}{|c|}{ meso } & \multicolumn{3}{|c|}{ rac } & \multirow{2}{*}{$\begin{array}{c}\Delta v_{\max } \\
(\text { meso-rac) } \\
\pm 10 \\
/ \mathrm{cm}^{-1}\end{array}$} \\
\hline & $\begin{array}{l}v_{\max } \\
\pm 10 \\
/ \mathrm{cm}^{-1}\end{array}$ & $\begin{array}{l}\Delta v_{1 / 2} \\
\pm 10 \\
/ \mathrm{cm}^{-1}\end{array}$ & $\begin{array}{c}v_{\mathrm{MLCT}(1)} \\
\pm 10 \\
/ \mathrm{cm}^{-1}\end{array}$ & $\begin{array}{l}v_{\max } \\
\pm 10 \\
/ \mathrm{cm}^{-1}\end{array}$ & $\begin{array}{l}\Delta v_{1 / 2} \\
\pm 10 \\
/ \mathrm{cm}^{-1}\end{array}$ & $\begin{array}{c}v_{\text {MLCT(1) }} \\
\pm 10 \\
/ \mathrm{cm}^{-1}\end{array}$ & \\
\hline 0 & 5435 & 2710 & 16900 & 5470 & 2770 & 16900 & -32 \\
\hline 0.005 & 5410 & 2500 & 16890 & 5400 & 2130 & 16880 & 60 \\
\hline 0.010 & 5400 & 2370 & 16885 & 5340 & 2605 & 16850 & 85 \\
\hline 0.020 & 5405 & 2450 & 16860 & 5320 & 2635 & 16875 & 70 \\
\hline 0.040 & 5410 & 2590 & 16860 & 5340 & 2490 & 17000 & 90 \\
\hline 0.10 & 5430 & 2630 & 16852 & 5340 & 2820 & 17475 & 130 \\
\hline 0.25 & 5440 & 2650 & 16870 & 5310 & 2750 & 17200 & 120 \\
\hline 0.40 & 5440 & 2635 & 16886 & 5320 & 2780 & 17111 & 106 \\
\hline 0.60 & 5440 & 2640 & 16904 & 5334 & 2700 & 17180 & 197 \\
\hline 0.80 & 5431 & 2600 & 16860 & 5250 & 2720 & 16817 & 110 \\
\hline 0.90 & 5440 & 2610 & 16815 & 5243 & 2710 & 16765 & 15 \\
\hline 0.95 & 5260 & 2650 & 16754 & 5150 & 2670 & 16782 & -25 \\
\hline 1.0 & 5055 & 2798 & 16775 & 5080 & 2622 & 16775 & 197 \\
\hline
\end{tabular}

${ }^{\mathrm{a}}$ The absolute intensities of the IVCT bands, $(\varepsilon / v)_{\max }$, are not tabulated as all spectra were normalised at the maximum intensity of the IVCT manifold. 
Figure 2.18 reveals striking differences in the dependence of $v_{\max }$ on solvent composition for the two diastereoisomeric forms. Three regions are discernable:

(i) For $0 \leq n_{\mathrm{AN}} \leq 0.02$, the rac diastereoisomer exhibited a $150 \pm 10 \mathrm{~cm}^{-1}$ red-shift with the addition of two mole equivalents of AN while the meso form exhibited a $30 \pm 10 \mathrm{~cm}^{-1}$ red-shift over the same range. The ratio of the number of moles of $\mathrm{AN}$ to $\left[\left\{\mathrm{Ru}(\mathrm{bpy})_{2}\right\}_{2}(\mu-\mathrm{bpm})\right]^{5+}$ is $2: 1$ at $n_{\mathrm{AN}}=0.02$. The results suggest that the specific solvent effect in dilute AN occurs within the interior clefts, since the exterior clefts are identical in both diastereoisomeric forms. The specific effect for the rac diastereoisomer may well correspond to the association of two AN molecules, one within each identical cleft either side of the bpm plane. The closer distance of approach of the AN molecules in the rac form gives rise to the larger magnitude of the specific effect.

(ii) For $0.02 \leq n_{\mathrm{AN}} \leq 0.8$ (meso) and $0.2 \leq n_{\mathrm{AN}} \leq 0.6$ ( $\mathrm{rac}$ ), $v_{\max }$ is relatively invariant to solvent composition. In the rac form, the AN molecules located in the interior clefts block access to association by additional solvent molecules, while the composition of molecules within the exterior clefts remains essentially constant. The specific association of solvent molecules within the first solvation shell increases the effective radius and decreases the sensitivity of $v_{\max }$ to variation in the solvent composition. The composition in the exterior clefts remains constant over the same range for the meso form, and the composition at the interior clefts also remains relatively constant due to the larger orthogonal-shaped cleft compared with the rac form. In the latter the AN molecules associated within the relatively smaller interior clefts are not accessible to interaction with molecules in the bulk solvent. Given the more open nature of the interior clefts in the meso diastereoisomer, the solvent molecules associated within these clefts are more accessible to solvent molecules in the outer solvation shells and the bulk solution. The increased solvent-solvent interactions may give rise to the consistently higher $v_{\max }$ for the meso compared with the rac form.

(iii) For $0.8 \leq n_{\mathrm{AN}} \leq 1.0$ (meso) and $0.6 \leq n_{\mathrm{AN}} \leq 1.0$ ( $\mathrm{rac}$ ), $v_{\max }$ decreases for both diastereoisomers. The dependence of $v_{\max }$ on solvent composition is greater for the meso form, as quantified by the slope of $-2020 \pm 130 \mathrm{~cm}^{-1}$ per unit $n_{\mathrm{AN}}$ (meso) versus $-843 \pm 60 \mathrm{~cm}^{-1}$ per unit $n_{\mathrm{AN}}$ (rac) over the range $0.8 \leq n_{\mathrm{AN}} \leq 1.0$. At $n_{\mathrm{AN}}=0.2$ (and $n_{\mathrm{PN}}=0.8$ ), the ratios of AN and PN molecules to the mixedvalence complex are 36:1 and 8.8:1, respectively. The sharp red-shift in $v_{\max }$ with increasing concentration of AN occurs at higher $n_{\mathrm{AN}}$, and more rapidly for the meso diastereoisomer. This form exhibits a greater sensitivity to solvent structure effects compared with the rac diastereoisomer, and these effects are more pronounced for solvent mixtures containing high concentrations of AN. ${ }^{160}$ The parameter $v_{\max }$ decreases more gradually for the rac diastereoisomer as the solvent molecules associated within the interior clefts are relatively more restricted towards interactions with the bulk solvent, and hence to solvent structure effects.

The results indicate that the specific solvent effect of discrete solvent molecules in the immediate vicinity of the complex dominate the solvent reorganisational energy. The diastereoisomers offer a 
detailed insight into the solvent reorganisational contribution from specific solvent molecules on $\lambda_{0}$, and the results support the general hypothesis that the distance of approach and orientation of the discrete solvent molecules dictate the solvent shifts. However, the clefts between the bpy rings are "open" structures and the interaction of solvent molecules situated in these clefts with the first and second solvation shells are as important as their interaction with the complex itself.

\section{Theoretical Considerations and Implications}

Due to the specific nature of the solvent interactions, a quantitative model for such effects must treat the solvent on the molecular level as discrete entities. The specific solvent effect $\left(\Delta \mathrm{E}_{\mathrm{s}}\right)$, due to the presence of an oriented solvent molecule on a charge transfer transition, may subsequently be inferred from London dipole solvation theory ${ }^{56,60}$ (equation 2.6), where $\mu_{\mathrm{s}}$ is the ground state dipole moment of the solvent molecule, $\Delta \mu$ is the dipole change due to charge transfer, $r$ is the cavity radius and $f(\in)$ is a function of the solvent dielectric properties.

$$
\Delta \mathrm{E}_{\mathrm{s}}=-\frac{\mu_{\mathrm{s}} \cdot \Delta \mu}{r^{3} f(\in)}
$$

Equation 2.6 provides a more general formulation of the quantitative analytical theory that is generally employed ${ }^{56,60}$ to treat the gas-phase to solution-phase frequency change, $\Delta v$, for a non-ionic chromophore which resides in a spherical cavity of radius $a$, given by equation 2.7 . The solvent is treated as a dielectric continuum with dielectric constant, $\in$, and refractive index, $n$.

$$
\Delta v=-\frac{2 \in-2}{2 \in+2} \frac{1}{a^{3}} \mu \cdot \Delta \mu-\frac{n^{2}-1}{2 n^{2}+1} \frac{1}{a^{3}}|\Delta \mu|^{2}
$$

The first term generally dominates the observed shift: in polar solvents the initial state dipole moment $|\mu|$, which arises from the equilibrium polarisation induced in the solvent by the solute, dominates the dipole change accompanying the transition, $|\Delta \mu|$.

The $1 / r^{3}$ dependence of $\Delta \mathrm{E}_{\mathrm{s}}$ in equation 2.6 is such that solvent molecules located in closer proximity to the metal centres will dominate the specific solvent effect. The distance dependence may account (in part) for the larger energy shifts in AN. Due to their relatively small size, the discrete AN molecules can approach the metal centres more closely than the other solvents of the series. The greater magnitude of the specific effect for the rac versus the meso diastereoisomer at low AN concentration may reflect the ability of the AN molecules to associate more strongly within the clefts between the metal centres.

The dependence of $\Delta \mathrm{E}_{\mathrm{s}}$ on the vector dot product of $\mu_{\mathrm{s}}$ and $\Delta \mu$ gives rise to the orientation dependence of the solvent molecules located within the clefts of the chromophore. All else being constant, $\left|\Delta \mathrm{E}_{\mathrm{s}}\right|$ is smallest when the dipole moment of the solvent molecule is oriented perpendicularly to the plane of the bridging ligand and greatest when oriented parallel to this plane. Assuming that the red- 
shift of $150 \pm 10 \mathrm{~cm}^{-1}$ in $v_{\max }$ corresponds to the formation of a 2:1 complex between AN and the dinuclear cation for the rac diastereoisomer, the results suggest that each AN dipole is oriented with the nitrogen of $-\mathrm{C} \equiv \mathrm{N}$ directed inwards towards the clefts at an angle $0 \leq \theta \leq 90^{\circ}$ to the Ru-Ru charge transfer axis. The small sizes of the AN molecules are such that they may associate sufficiently close to solvate both metal centres simultaneously. By comparison, the additional methyl group in PN may restrict the close approach to the metal centres. The dipole is thus oriented towards the metal centre with the higher partial positive charge $\left(\delta^{+}\right)$, giving rise to a greater positive reorganisational contribution to $v_{\max }$. For PN and the other members of the nitrile series, the magnitudes of the specific effect are relatively smaller compared with AN, such that when they are superimposed on their continuum contributions, apparent conformity with the theoretical prediction is obtained.

Clearly, a quantitative test of the applicability of equation 2.6 depends on a realistic estimation of the dimensions of the clefts (which is ambiguous due to their non-spherical nature). However, the qualitative predictions for the orientation and distance dependence are compatible with the experimental results. $\Delta \mathrm{E}_{\mathrm{s}}$ is thus superimposed on the $1 / \mathrm{D}_{\mathrm{op}}-1 / \mathrm{D}_{\mathrm{s}}$ continuum theory prediction and can be regarded as an additional energy contribution to $\lambda_{0}$.

\section{Assumptions and Shortcomings of the Present Study}

The theoretical assumptions which are implicit in the application of the dielectric continuum model have been discussed previously. For the $\left[\left\{\mathrm{Ru}(\mathrm{bpy})_{2}\right\}_{2}(\mu \text {-bpm) }]^{5+}\right.$ diastereoisomers, two major assumptions may not be valid. Firstly, estimates of the radii of the redox sites suggest that $2 a$ » is not valid, and the clefts between the terminal bpy rings permit specific solvent interactions which are not accounted for by the dielectric continuum model. Secondly, it is not clear that the valence-localised assumption is valid as the bandwidths are narrower than those predicted by the classical two-state theory, ${ }^{31,32}$ and electronic delocalisation effects may account for the larger than anticipated intercept (and smaller than expected slope) obtained in Figure 2.17. Dielectric saturation effects may also account for the discrepancies, ${ }^{43}$ although such effects should be minimised under the conditions of low chromophore concentration and employment of the weakly-associating $\left\{\mathrm{B}\left(\mathrm{C}_{6} \mathrm{~F}_{5}\right)_{4}\right\}^{-}$electrolyte.

Despite these shortcomings, the predicted linear trend is evident for four of the nitrile solvents, and the deviations in AN may be adequately described by a specific solvation effect which dominates the continuum contribution. Equation 2.6 may provide a mathematical description of this specific effect, but the quantitative validity of the equation is speculative at present. Application of the equation requires the definition of the dimensions of the interior and exterior clefts in the diastereoisomeric forms, and knowledge of the number, orientation and distance of approach of the solvent molecules. The relative contributions of solvent-solvent and solvent-solute interactions to the reorganisational energy are also required. The insights provided by the solvent proportion experiments provide compelling evidence for stereochemically-directed specific effects between discrete solvent molecules and the diastereoisomers of $\left[\left\{\operatorname{Ru}(\mathrm{bpy})_{2}\right\}_{2}(\mu-\mathrm{bpm})\right]^{5+}$. Chemical modification of the systems through bridging and terminal ligand 
variations afford additional opportunities for probing the detailed nature of specific solvation effects between the diastereoisomeric forms of symmetrical dinuclear mixed-valence complexes.

\subsubsection{Varying the Selectivity of Solvent Association: Influence of the Bridging Ligand}

The IVCT solvatochromism properties of the diastereoisomeric forms of $\left[\left\{\mathrm{Ru}(\mathrm{bpy})_{2}\right\}_{2}(\mu-\right.$ dbneil) $]^{5+}$ were examined to assess the effect of increasing the dimensions of the interior clefts by comparison with the diastereoisomers of $\left[\left\{\mathrm{Ru}(\mathrm{bpy})_{2}\right\}_{2}(\mu-\mathrm{bpm})\right]^{5+}$. The variation in $v_{\max }$ as a function of $1 / \mathrm{D}_{\mathrm{op}}-1 / \mathrm{D}_{\mathrm{s}}$ is presented in Table 2.7 and Figure 2.19. The complete UV/Vis/NIR spectral data for the unoxidised (+4) and mixed-valence $(+5)$ forms of $\left[\left\{\mathrm{Ru}(\mathrm{bpy})_{2}\right\}_{2}(\mu \text {-dbneil })\right]^{n+}$ are provided in Table A2.2 (Appendix A).

Table 2.7 IVCT solvatochromism data of the reduced absorption spectra ( $\varepsilon / v$ vs. $v)$ for the diastereoisomers of $\left[\left\{\mathrm{Ru}(\text { bpy })_{2}\right\}_{2}(\mu \text {-dbneil })\right]^{5+}$ in $0.02 \mathrm{M}\left[\left(n-\mathrm{C}_{4} \mathrm{H}_{9}\right)_{4} \mathrm{~N}\right]\left\{\mathrm{B}\left(\mathrm{C}_{6} \mathrm{~F}_{5}\right)_{4}\right\} /$ solvent at $+25^{\circ} \mathrm{C}$. MLCT energies for the +4 states are also tabulated. Parameters for overall envelope are shown in bold type: details of deconvoluted bands (in AN only) are in normal type.

\begin{tabular}{|c|c|c|c|c|c|c|c|c|c|}
\hline \multirow[b]{2}{*}{ Solvent } & \multicolumn{4}{|c|}{ meso } & \multicolumn{4}{|c|}{ rac } & \multirow{2}{*}{$\begin{array}{c}\Delta v_{\max } \\
(\text { meso-rac) } \\
\pm 10 \\
/ \mathrm{cm}^{-1}\end{array}$} \\
\hline & $\begin{array}{l}v_{\max } \\
\pm 10 \\
/ \mathrm{cm}^{-1}\end{array}$ & $\begin{array}{c}(\varepsilon / v)_{\max } \\
\pm 0.0001 \\
/ \mathrm{M}^{-1}\end{array}$ & $\begin{array}{l}\Delta v_{1 / 2} \\
\pm 10 \\
/ \mathrm{cm}^{-1}\end{array}$ & $\begin{array}{l}v_{\mathrm{MLCT}(1)} \\
\pm 10 \\
/ \mathrm{cm}^{-1}\end{array}$ & $\begin{array}{l}v_{\max } \\
\pm 10 \\
/ \mathrm{cm}^{-1}\end{array}$ & $\begin{array}{c}(\varepsilon / v)_{\max } \\
\pm 0.0001 \\
/ \mathrm{M}^{-1}\end{array}$ & $\begin{array}{l}\Delta v_{1 / 2} \\
\pm 10 \\
/ \mathrm{cm}^{-1}\end{array}$ & $\begin{array}{l}v_{\mathrm{MLCT}(1)} \\
\pm 10 \\
/ \mathrm{cm}^{-1}\end{array}$ & \\
\hline \multirow[t]{3}{*}{$\mathrm{AN}$} & 4650 & 0.2112 & 1970 & \multirow[t]{3}{*}{14290} & 4560 & 0.2064 & 2050 & \multirow[t]{3}{*}{14290} & \multirow[t]{3}{*}{90} \\
\hline & 4570 & 0.1549 & 1595 & & 4495 & 0.1493 & 1465 & & \\
\hline & 5530 & 0.07953 & 2390 & & 5515 & 0.08983 & 2290 & & \\
\hline PN & 4610 & 0.2081 & 1929 & 14335 & 4670 & 0.1515 & 1764 & 14335 & -60 \\
\hline BN & 4590 & 0.1890 & 1884 & 14340 & 4585 & 0.0855 & 1948 & 14340 & 5 \\
\hline${ }^{\mathrm{i}} \mathrm{BN}$ & 4610 & 0.1953 & 1871 & 14350 & 4600 & 0.1929 & 1917 & 14350 & 10 \\
\hline BzN & 4600 & 0.1800 & 1880 & 14170 & 4590 & 0.1500 & 1880 & 14170 & 10 \\
\hline
\end{tabular}

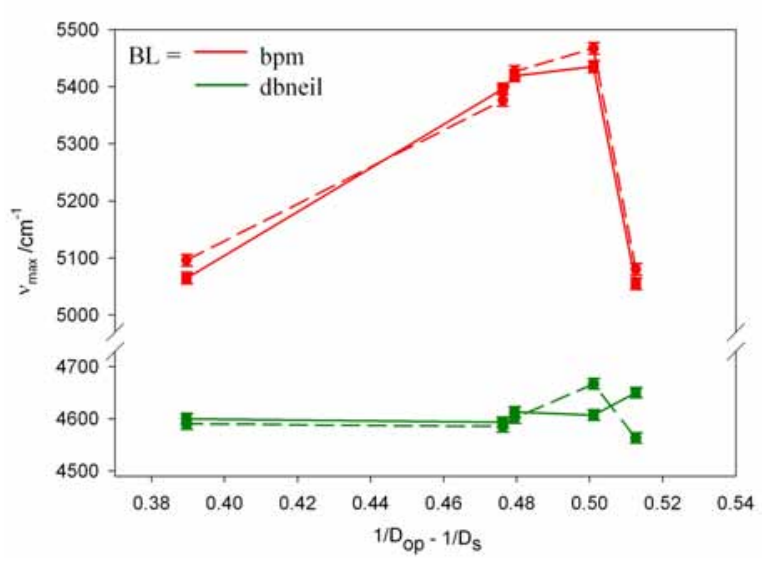

(a)

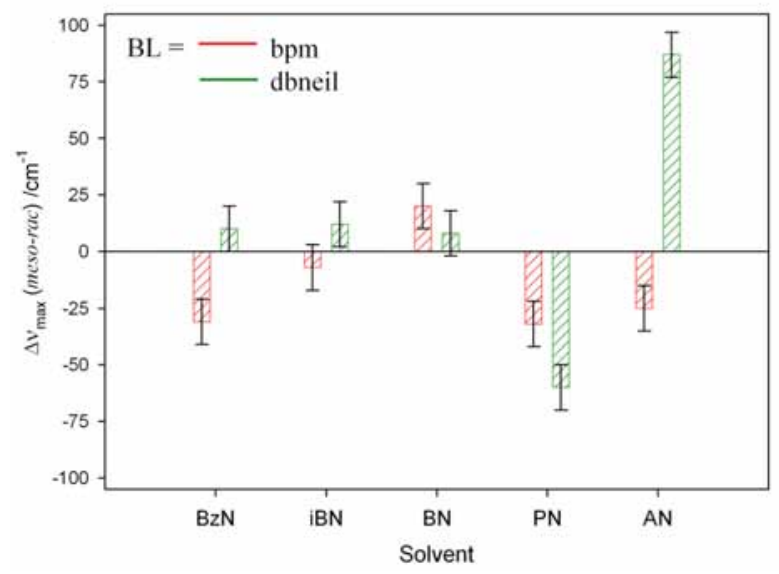

(b)

Figure 2.19 (a) $v_{\max }$ as a function of the solvent parameter $1 / \mathrm{D}_{\mathrm{op}}-1 / \mathrm{D}_{\mathrm{s}}$ for the meso and rac diastereoisomers of $\left[\left\{\mathrm{Ru}(\mathrm{bpy})_{2}\right\}_{2}(\mu \text {-bpm) }]^{5+}\right.$ and $\left[\left\{\mathrm{Ru}(\mathrm{bpy})_{2}\right\}_{2}(\mu \text {-dbneil })\right]^{5+}$ in $0.02 \mathrm{M}\left[\left(n-\mathrm{C}_{4} \mathrm{H}_{9}\right)_{4} \mathrm{~N}\right]\left\{\mathrm{B}\left(\mathrm{C}_{6} \mathrm{~F}_{5}\right)_{4}\right\} /$ solvent at $+25^{\circ} \mathrm{C}$. (b) Differential energies of the IVCT bands $\left\{\Delta v_{\max }\right.$ (meso-rac) $\}$ in a given solvent. 
The energies of the IVCT bands of $\left[\left\{\mathrm{Ru}(\mathrm{bpy})_{2}\right\}_{2}(\mu \text {-dbneil })\right]^{5+}$ are essentially solvent independent for both diastereoisomers, as shown in Figure 2.19(a). The analysis reveals the following values for the slope and intercept: for the meso diastereoisomer; slope $=250 \pm 220 \mathrm{~cm}^{-1} \AA^{-1}$ and intercept $=4500 \pm 105 \mathrm{~cm}^{-1}\left(R^{2}=0.29\right)$; for the rac diastereoisomer, slope $=130 \pm 460 \mathrm{~cm}^{-1} \AA^{-1}$ and intercept $=$ $4540 \pm 220 \mathrm{~cm}^{-1}\left(R^{2}=0.03\right)$. The energies of the IVCT bands for the two diastereoisomers in AN and PN $\left\{\Delta v_{\max }\right.$ (meso-rac) in Table 2.7 and Figure 2.19(b) $\}$ differ by $90 \pm 10$ and $60 \pm 10 \mathrm{~cm}^{-1}$, respectively.

The decreased slope of the solvatochromism plots compared with bpm-bridged analogues contradicts the prediction from equation 2.2 that $\lambda_{0}$ increases as the intra-metal distance is increased from 5.565(5) $\AA$ for the bpm-bridged systems to $7.9 \AA^{82}$ for the dbneil-bridged systems (at fixed $a$ ). The interior clefts are of similar dimension for the dbneil-bridged diastereoisomers (Figure 2.5), but larger for a given diastereoisomer compared with their bpm-bridged analogues. Since the exterior clefts are identical for the diastereoisomers of both complexes, the results suggest that solvation in the interior clefts is more important than solvation about the entire dimer. In particular, the solvent reorganisational contribution to the IVCT energy is greater for the bpm-bridged diastereoisomers which contain the relatively smaller interior clefts.

Alternatively, the dbneil-bridged diastereoisomers may exhibit relatively greater delocalisation than their bpm-bridged analogues, due to the extensive aromatic framework of the bridging ligand in the former case. The slope of the sovlatochromism plot is given by $e^{2}(1 / a-1 / d)$ according to equation 2.2, however the equation is frequently expressed in terms of the effective amount of charge transferred, and the slope is given instead as $(\Delta e)^{2}(1 / a-1 / d)$. Since the effective amount of charge transferred is reduced from unit charge transfer by delocalisation, this explanation may provide a qualitative rationale for the lesser slope for the dbneil-bridged systems. The data for the IVCT parameters in Table 2.7 do indeed suggest that the latter are more delocalised as the bands are narrower and more intense.

The solvent dependence of $v_{\max }$ for the dbneil-bridged diastereoisomers arises from a superposition of the solvent reorganisational contributions due to continuum and specific solvation effects. The difference in $v_{\max }$ between the diastereoisomers in a given solvent $\left\{\Delta v_{\max }\right.$ (meso-rac) $\}$ provides a measure of the specific solvation effect due to interactions at the interior clefts. A comparison of $\Delta v_{\max }$ (meso-rac) for the dbneil- and bpm-bridged complexes reveals that differences in the specific solvent interactions between the diastereoisomeric forms are present in each solvent \{Figure 2.19(b)\}. In particular, the difference in the specific solvent interaction with the diastereoisomers of the same complex in AN and PN is greater for the dbneil-bridged complex. This reflects the larger cleft available for solvent penetration relative to that in the bpm-bridged species.

As noted previously for the bpm-bridged diastereoisomers, the magnitudes of the intercepts are larger than expected given the minor bond length changes on oxidation from $\mathrm{Ru}^{\mathrm{II}}$ to $\mathrm{Ru}^{\mathrm{III}} .{ }^{153,155}$ Deconvolution of the IVCT manifolds revealed the presence of two underlying components due to the combined effects of spin-orbit coupling and ligand-field asymmetry. Assuming that the two components correspond to IVCT(2) and IVCT(3) in a localised model (§2.3.3.1), and taking $\Delta$ as $1735 \mathrm{~cm}^{-1}$ in 
equation 2.5 (as discussed previously for the bpm-bridged case), yields $\lambda_{i}$ estimates of $2765 \pm 105 \mathrm{~cm}^{-1}$ and $2805 \pm 220 \mathrm{~cm}^{-1}$ for the meso and rac diastereoisomers, respectively. The larger estimate of $\lambda_{i}$ for the dbneil- versus bpm-bridged complexes may reflect the higher degree of structural distortion in the former: the crystal structure of meso- $\left[\left\{\mathrm{Ru}(\mathrm{bpy})_{2}\right\}_{2}(\mu \text {-dbneil) }]^{4+82}\right.$ revealed a slight structural distortion in the bridging ligand from planarity due to steric interactions between the $\mathrm{H}^{\mathrm{d}}$ protons in the "bay regions" (Figure 2.9) and the NMR spectra were consistent with a fast "wagging” motion of the ligand on the NMR time-scale (\$2.3.1.1).

\subsubsection{Varying the Selectivity of Solvent Association: Influence of the Terminal Ligands}

The final investigation involved the systematic modification of the interior and exterior clefts by the incorporation of substituents on the terminal ligands in the series $\left[\left\{\operatorname{Ru}(p p)_{2}\right\}_{2}(\mu-b p m)\right]^{5+}\left\{p p=5,5^{\prime}-\right.$ $\mathrm{Me}_{2}$ bpy, $\mathrm{Me}_{4}$ bpy, $\mathrm{Me}_{2}$ phen and ${ }^{\mathrm{t}} \mathrm{Bu}_{2}$ bpy $\}$. The results for $v_{\max }$ as a function of $1 / \mathrm{D}_{\mathrm{op}}-1 / \mathrm{D}_{\mathrm{s}}$ for the diastereoisomeric forms are reported in Table 2.8 and shown in Figure 2.20.

Table 2.8 IVCT solvatochromism data of the reduced absorption spectra ( $\varepsilon / v v s . v$ ) for the diastereoisomers of $\left[\left\{\mathrm{Ru}(\mathrm{pp})_{2}\right\}_{2}(\mu-\mathrm{bpm})\right]^{5+}$ in $0.02 \mathrm{M}\left[\left(n-\mathrm{C}_{4} \mathrm{H}_{9}\right)_{4} \mathrm{~N}\right]\left\{\mathrm{B}\left(\mathrm{C}_{6} \mathrm{~F}_{5}\right)_{4}\right\} /$ solvent at $+25^{\circ} \mathrm{C}$. MLCT energies for the +4 states are also tabulated. ${ }^{\text {a }}$

\begin{tabular}{|c|c|c|c|c|c|c|c|c|}
\hline \multirow[b]{2}{*}{ pp } & \multirow[b]{2}{*}{ Solvent } & \multicolumn{3}{|c|}{ meso } & \multicolumn{3}{|c|}{ rac } & \multirow{2}{*}{$\begin{array}{c}\Delta v_{\max } \\
(m e s o-r a c) \\
\pm 10 \\
/ \mathrm{cm}^{-1}\end{array}$} \\
\hline & & $\begin{array}{l}v_{\max } \\
\pm 10 \\
/ \mathrm{cm}^{-1}\end{array}$ & $\begin{array}{l}\Delta v_{1 / 2} \\
\pm 10 \\
/ \mathrm{cm}^{-1}\end{array}$ & $\begin{array}{c}v_{\text {MLCT }(1)} \\
\pm 10 \\
/ \mathrm{cm}^{-1}\end{array}$ & $\begin{array}{l}v_{\max } \\
\pm 10 \\
/ \mathrm{cm}^{-1}\end{array}$ & $\begin{array}{l}\Delta v_{1 / 2} \\
\pm 10 \\
/ \mathrm{cm}^{-1}\end{array}$ & $\begin{array}{c}v_{\text {MLCT(1) }} \\
\pm 10 \\
/ \mathrm{cm}^{-1}\end{array}$ & \\
\hline \multirow[t]{5}{*}{ 5,5'-Me $\mathrm{Me}_{2}$ bpy } & AN & 4960 & 2520 & 16680 & 4990 & 2195 & 16560 & -30 \\
\hline & PN & 5188 & 1855 & 16650 & 5208 & 1910 & 16610 & -20 \\
\hline & ${ }^{\mathrm{i}} \mathrm{BN}$ & 5280 & 2450 & 16610 & 5265 & 1970 & 16505 & 15 \\
\hline & BN & 5200 & 2070 & 16660 & 5110 & 1850 & 16505 & 90 \\
\hline & $\mathrm{BzN}$ & 5060 & 1604 & 16580 & 5110 & 1620 & 16470 & -50 \\
\hline \multirow[t]{5}{*}{$\mathrm{Me}_{4} \mathrm{bpy}$} & AN & 4823 & 2620 & 16176 & 4900 & 2965 & 16072 & -77 \\
\hline & PN & 5130 & 2235 & 16315 & 5097 & 2400 & 16320 & 33 \\
\hline & ${ }^{\mathrm{i}} \mathrm{BN}$ & 5083 & 2850 & 16160 & 5027 & 1930 & 16165 & 56 \\
\hline & $\mathrm{BN}$ & 5060 & 2270 & 16090 & 5095 & 1885 & 16090 & -35 \\
\hline & $\mathrm{BzN}$ & 5038 & 2120 & 16176 & 5013 & 1710 & 16040 & 25 \\
\hline \multirow[t]{5}{*}{$\mathrm{Me}_{2}$ phen } & $\mathrm{AN}$ & 4790 & 2780 & 16160 & 4820 & 2380 & 16190 & -30 \\
\hline & PN & 5200 & 2660 & 16200 & 5137 & 2580 & 16290 & 63 \\
\hline & ${ }^{\mathrm{i}} \mathrm{BN}$ & 5240 & 2630 & 16230 & 5060 & 2330 & 16190 & 180 \\
\hline & $\mathrm{BN}$ & 5027 & 2370 & 16260 & 5065 & 2285 & 16140 & -38 \\
\hline & $\mathrm{BzN}$ & 5000 & 2330 & 16230 & 5050 & 2200 & 16175 & -50 \\
\hline \multirow[t]{5}{*}{${ }^{t} \mathrm{Bu}_{2} \mathrm{bpy}$} & $\mathrm{AN}$ & 5090 & 2524 & 16315 & 5145 & 3518 & 16210 & -55 \\
\hline & PN & 5180 & 3294 & 16260 & 5132 & 2676 & 16265 & 48 \\
\hline & ${ }^{\mathrm{i}} \mathrm{BN}$ & 5106 & 2464 & 16124 & 5140 & 2518 & 16367 & -34 \\
\hline & $\mathrm{BN}$ & 5085 & 2580 & 16263 & 5150 & 2470 & 16332 & -65 \\
\hline & BzN & 5065 & 2414 & 16315 & 5065 & 2284 & 16193 & 0 \\
\hline
\end{tabular}

${ }^{a}$ The intensities of the IVCT bands, $(\varepsilon / v)_{\max }$, are not tabulated as all spectra were normalised at the maximum intensity of the IVCT manifold. 


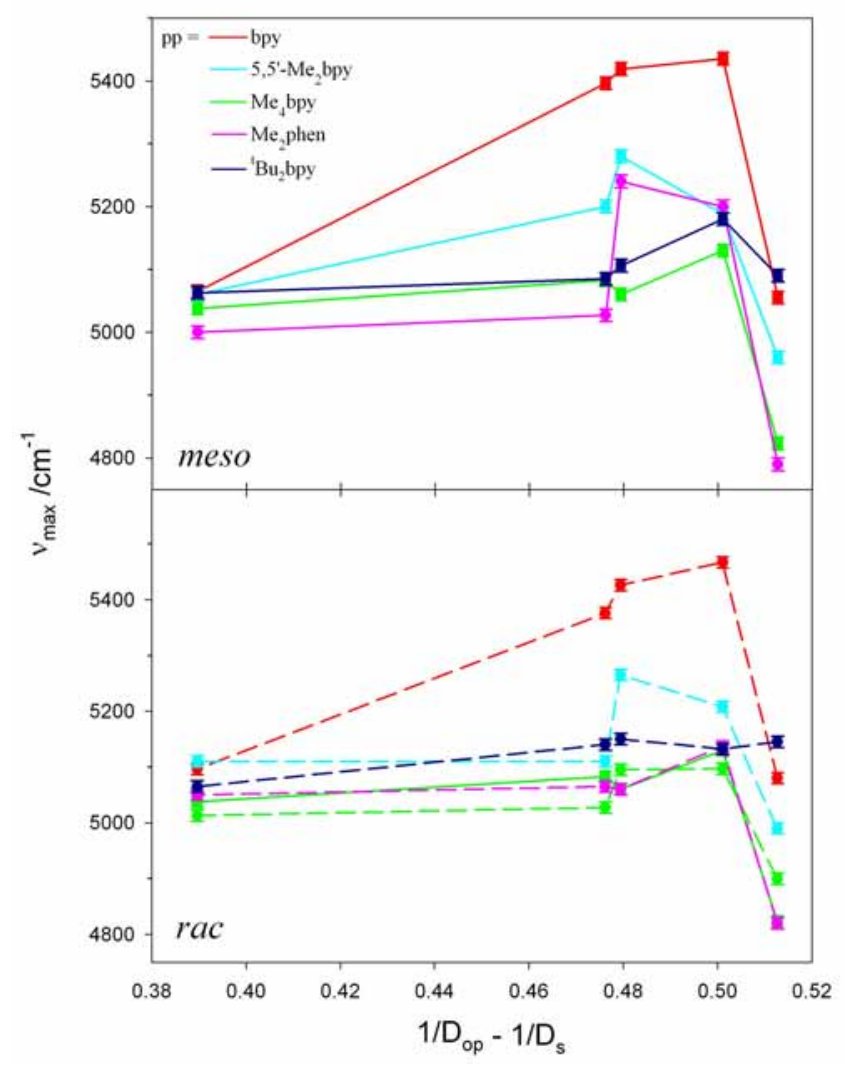

Figure 2.20 $v_{\max }$ as a function of the solvent parameter $1 / \mathrm{D}_{\mathrm{op}}-1 / \mathrm{D}_{\mathrm{s}}$ for the meso and rac diastereoisomers of $\left[\left\{\mathrm{Ru}(\mathrm{pp})_{2}\right\}_{2}(\mu-\mathrm{bpm})\right]^{5+}\left\{\mathrm{pp}=5,5^{\prime}-\mathrm{Me}_{2}\right.$ bpy, $\mathrm{Me}_{4}$ bpy, $\mathrm{Me}_{2}$ phen and $\left.{ }^{\mathrm{t}} \mathrm{Bu}_{2} \mathrm{bpy}\right\}$ in $0.02 \mathrm{M}[(n-$ $\left.\left.\mathrm{C}_{4} \mathrm{H}_{9}\right)_{4} \mathrm{~N}\right]\left\{\mathrm{B}\left(\mathrm{C}_{6} \mathrm{~F}_{5}\right)_{4}\right\} /$ solvent at $+25^{\circ} \mathrm{C}$.

In accordance with equation 2.2, an increase in the effective radii $(a)$ of the redox sites at constant $d$ should be manifested by a decrease in the $\lambda_{0}$ contribution to $v_{\max }$, and a decrease in the slope of the solvatochromism plot. While the definition of an "effective" radius is somewhat ambiguous in view of the non-spherical nature of the sites, the radii increase approximately in the order bpy $<5,5^{\prime}-\mathrm{Me}_{2} \mathrm{bpy}<$ $\mathrm{Me}_{4}$ bpy $<\mathrm{Me}_{2}$ phen $<{ }^{\mathrm{t}} \mathrm{Bu}_{2}$ bpy. The most severe assumption for the series of substituted derivatives would appear to be the $2 a » d$ approximation, which suggests that consideration of an ellipsoidal cavity mode ${ }^{15}$ may provide a more realistic physical description of the dinuclear complexes. Like the spherical cavity model, the ellipsoidal model is formulated in terms of bulk dielectric properties which are inappropriate to account for the $\lambda_{0}$ variation for the series of complexes. Specific solvation effects clearly dominate the magnitude of $\lambda_{0}$, and the consideration of the subtle and systematic structural variation between the different complexes and between the diastereoisomeric forms of the same complex provide insights into the reorganisational contributions of specific solvent molecules to $v_{\max }$.

The energies of the IVCT bands for the substituted derivatives exhibit a lesser but scattered dependence on $1 / \mathrm{D}_{\mathrm{op}}-1 / \mathrm{D}_{\mathrm{s}}$ relative to the linear dependence of $v_{\max }$ on $1 / \mathrm{D}_{\mathrm{op}}-1 / \mathrm{D}_{\mathrm{s}}$ for the diastereoisomers of $\left[\left\{\mathrm{Ru}(\mathrm{bpy})_{2}\right\}_{2}(\mu-\mathrm{bpm})\right]^{5+}$ in PN, BN, ${ }^{\mathrm{i}} \mathrm{BN}$ and BzN. Negligible slopes are obtained for all complexes (neglecting the results in AN). The striking discontinuity between PN and AN in the plot of $v_{\max }$ versus $1 / \mathrm{D}_{\mathrm{op}}-1 / \mathrm{D}_{\mathrm{s}}$ for the $\left[\left\{\mathrm{Ru}(\mathrm{bpy})_{2}\right\}_{2}(\mu \text {-bpm) }]^{5+}\right.$ diastereoisomers is also evident for the substituted analogues, with the notable exceptions being the diastereoisomers of $\left[\left\{\mathrm{Ru}\left({ }^{t} \mathrm{Bu} \mathrm{u}_{2} \mathrm{bpy}\right)_{2}\right\}_{2}(\mu-\mathrm{bpm})\right]^{5+}$. The observation 
may be rationalised by the steric restriction of the bulky tert-butyl substituents to the access of solvent molecules to the interior and exterior clefts. However, minor differences in the IVCT energies are evident between the two diastereoisomeric forms in all solvents, as quantified by $\Delta v_{\max }$ (meso-rac) \{Table 2.8 and Figure 2.21\}. The rac diastereoisomer exhibits a negligible solvent shift $\left(13 \pm 10 \mathrm{~cm}^{-1}\right)$ between $\mathrm{AN}$ and PN due to the smaller, less solvent-accessible interior cleft compared with the meso form where the shift is $90 \pm 10 \mathrm{~cm}^{-1}$.

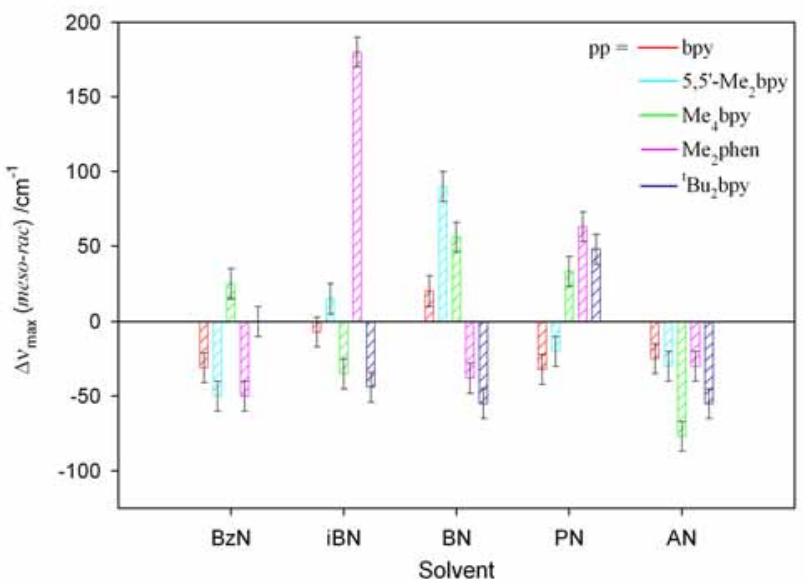

Figure 2.21 Differential energies of the IVCT bands, $\Delta v_{\max }($ meso-rac) in a given solvent.

Despite the significant scatter in the solvent dependence plots, the intercept of the plots are comparable across the full series of complexes, as shown in Table 2.9 (excluding the data for AN). Assuming that $\Delta \mathrm{E}^{\prime}$ is identical to the value of $\Delta=1735 \mathrm{~cm}^{-1}$ determined for $\left[\left\{\mathrm{Ru}(\mathrm{bpy})_{2}\right\}_{2}(\mu-\mathrm{bpm})\right]^{5+}$, the $\lambda_{i}$ estimates are significantly greater than the values of $1655 \pm 245$ and $2045 \pm 115 \mathrm{~cm}^{-1}$ for the meso and rac diastereoisomers of $\left[\left\{\mathrm{Ru}(\mathrm{bpy})_{2}\right\}_{2}(\mu-\mathrm{bpm})\right]^{5+}$, respectively. This may reflect the additional energy required for bond length and angle rearrangement due to steric interactions between the methyl substituents of the terminal polypyridyl ligands. The presence of moderate-electronic coupling that invalidates the two-state weak-coupling approximation may also account for the larger than expected intercepts.

Table 2.9 Intercepts $\left(=\lambda_{i}+\Delta \mathrm{E}^{\prime}\right)$ in $\mathrm{cm}^{-1}$ of the solvatochromism plots for the series $\left[\left\{\mathrm{Ru}(\mathrm{pp})_{2}\right\}_{2}(\mu \text {-bpm })\right]^{5+}$.

\begin{tabular}{ccc}
\hline pp & meso & rac \\
\hline $5,5 '-\mathrm{Me}_{2}$ bpy & $4480 \pm 345$ & $4750 \pm 410$ \\
$\mathrm{Me}_{4}$ bpy & $4780 \pm 155$ & $4740 \pm 190$ \\
$\mathrm{Me}_{2}$ phen & $4320 \pm 570$ & $4830 \pm 200$ \\
${ }^{\mathrm{t}} \mathrm{Bu}_{2}$ bpy & $4855 \pm 210$ & $4810 \pm 80$ \\
\hline
\end{tabular}

The subtle structural differences between the various $\left[\left\{\mathrm{Ru}(\mathrm{pp})_{2}\right\}_{2}(\mu-\mathrm{bpm})\right]^{5+}$ complexes provide further opportunities to quantitatively assess of stereochemically-directed specific solvent effects. The diastereoisomers of the respective complexes are shown in Figure 2.7. 


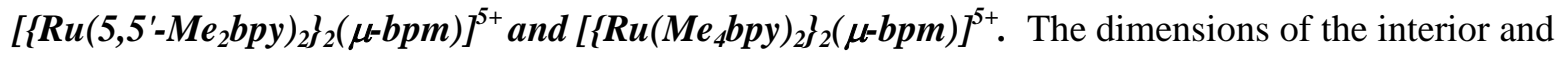
exterior clefts between the same diastereoisomeric form of the two complexes differ only with respect to the additional methyl substituents at the 4,4 ' positions in $\left[\left\{\mathrm{Ru}\left(\mathrm{Me}_{4} \mathrm{bpy}\right)_{2}\right\}_{2}(\mu \text {-bpm })\right]^{5+}$, which may hinder the access of solvent molecules from directly above the interior clefts. For both complexes, the methyl substituents at the 5,5' positions restrict solvent access at the convergence of the terminal ligands in the meso form, while the dimensions of the clefts in the rac form are comparable to those of the unsubstituted bpy complex. Despite the presence of the methyl substituents, the magnitude of the specific solvent effect due to AN is maintained for both diastereoisomers of both complexes. The difference between the diastereoisomers $\left\{\Delta v_{\max }\right.$ (meso-rac) in Figure 2.21\} is more pronounced in $\mathrm{AN}$ for the $\mathrm{Me}_{4}$ bpy derivatives compared with all the substituted complexes. This suggests that substitution at the 4,4' positions of the terminal bpy-type ligands results in the most pronounced difference in the specific interaction of the AN molecules at the interior clefts. For a given diastereoisomer, the energies of the IVCT transitions in PN, $\mathrm{BN},{ }^{\mathrm{i}} \mathrm{BN}$ and $\mathrm{BzN}$ are greater for the 5,5'-Me 2 bpy derivative, which may reflect the smaller $a$ (and hence larger continuum contribution, according to equation 2.2) for the diastereoisomers compared with the corresponding forms of $\left[\left\{\mathrm{Ru}\left(\mathrm{Me}_{4} \mathrm{bpy}\right)_{2}\right\}_{2}(\mu \text {-bpm })\right]^{5+}$. For both diastereoisomers of the latter, $v_{\max }$ remains essentially constant over the series $\mathrm{PN}, \mathrm{BN},{ }^{\mathrm{i}} \mathrm{BN}$ and $\mathrm{BzN}$ compared with the relatively greater solvent effects for the 5,5'-Me 2 bpy derivative. The results indicate that the specific solvent interactions may occur via penetration from directly above or below the plane of the bridging ligand, since the only difference between the same diastereoisomer for the two complexes is substitution at the 4,4' positions of the $\mathrm{Me}_{2}$ bpy rings. When tert-butyl substituents occupy these positions, solvent access is severely restricted, even for the relatively small AN molecules.

$\left[\left\{\mathbf{R u}\left(\mathbf{M e} \boldsymbol{e}_{2} \text { phen }\right)_{2}\right\}_{2}(\boldsymbol{\mu} \text {-bpm })\right]^{5+}$. The methyl substituents at the 2,9 positions of 1,10-phenanthroline (phen) induce a steric crowding close to the metal centres which should restrict the solvent access in the immediate vicinity of the metal centres to a greater extent than substitution at the 4,4' and/or 5,5' positions of the bpy-based ligands. In the meso diastereoisomer, the methyl substituents induce a crowding at the convergence of the terminal rings on either side of the bridging ligand plane, and the size of the orthogonal shaped clefts are comparable to those in meso- $\left[\left\{\mathrm{Ru}(\mathrm{pp})_{2}\right\}_{2}(\mu \text {-bpm })\right]^{5+}\left\{\mathrm{pp}=\mathrm{bpy}, 5,5^{\prime}-\mathrm{Me}_{2} \mathrm{bpy}\right.$ and $\left.\mathrm{Me}_{4} \mathrm{bpy}\right\}$. The close resemblance of the interior clefts for the complexes would account for the similar nature of the specific solvent effect observed in AN. The magnitude of the specific effect between $\mathrm{AN}$ and $\mathrm{PN}$ for rac- $\left[\left\{\mathrm{Ru}\left(\mathrm{Me}_{2} \mathrm{phen}\right)_{2}\right\}_{2}(\mu \text {-bpm })\right]^{5+}$ is comparable to that for rac- $\left[\left\{\mathrm{Ru}(\mathrm{bpy})_{2}\right\}_{2}(\mu \text {-bpm })\right]^{5+}$, which suggests that the AN and PN dipoles may assume similar orientations and distances of approach in both complexes.

Substitution at the 2 and 9 positions gives rise to a dramatic difference in $v_{\max }$ between the meso and rac diastereoisomers in ${ }^{\mathrm{i}} \mathrm{BN}$, where $\Delta v_{\max }$ (meso-rac) is $180 \pm 10 \mathrm{~cm}^{-1}$. The difference is striking in view of the subtle structural variation between ${ }^{\mathrm{i}} \mathrm{BN}, \mathrm{PN}$ and $\mathrm{BN}$. The additional steric bulk provided by the branched position of the methyl group in ${ }^{\mathrm{i}} \mathrm{BN}$ compared with PN and BN may be sufficient to inhibit 
penetration of these solvent molecules within the interior clefts of the rac form compared with the relatively more open clefts in the meso diastereoisomer. The magnitude of the difference between the two forms is significantly greater than that observed for the 5,5'-Me $\mathrm{Me}_{2} \mathrm{bpy}$ and $\mathrm{Me}_{4} \mathrm{bpy}$ derivatives. This suggests that the presence of methyl substituents at the 2,9 positions in $\mathrm{Me}_{2}$ phen gives rise to the greatest stereochemically-induced differentiation between the diastereoisomeric forms.

$\left[\left\{\boldsymbol{R u}\left({ }^{t} \mathbf{B} \mathbf{u}_{2} \mathbf{b p y}\right)_{2}\right\}_{2}(\boldsymbol{\mu} \text {-bpm })\right]^{5+}$. The tert-butyl substituents at the $4,4{ }^{\prime}$ positions of the terminal ${ }^{\mathrm{t}} \mathrm{Bu} \mathrm{z}_{2} \mathrm{bpy}$ ligands in $\left[\left\{\mathrm{Ru}\left({ }^{\mathrm{t}} \mathrm{Bu}_{2} \mathrm{bpy}\right)_{2}\right\}_{2}(\mu \text {-bpm })\right]^{5+}$ provide significantly greater steric hindrance to solvent penetration into the internal (and to a lesser extent, external) clefts relative to the diastereoisomers of $\left[\left\{\mathrm{Ru}(\mathrm{pp})_{2}\right\}_{2}(\mu-\mathrm{bpm})\right]^{5+}\left\{\mathrm{pp}=\right.$ bpy, 5,5'-Me $\mathrm{Me}_{2}$ by, $\mathrm{Me}_{4}$ bpy, $\left.\mathrm{Me}_{2} \mathrm{phen}\right\}$. Most notably, the specific solvent effect described previously for the latter group of complexes in AN is absent as the tert-butyl substituents block access to the interior clefts for the smallest solvent of the nitrile series. Small differences in $v_{\max }$ are evident between the diastereoisomeric forms of $\left[\left\{\mathrm{Ru}\left({ }^{\mathrm{t}} \mathrm{Bu}_{2} \mathrm{bpy}\right)_{2}\right\}_{2}(\mu-\mathrm{bpm})\right]^{5+}$, as the solvent molecules may approach the interior clefts more readily in the meso diastereoisomer compared with the rac form.

\section{Theoretical Implications and Considerations}

The solvent dependence of the IVCT energies for the diastereoisomers of the series $\left[\left\{\mathrm{Ru}(\mathrm{pp})_{2}\right\}_{2}(\mu \text {-bpm) }]^{5+}\left\{\mathrm{pp}=\right.\right.$ bpy, 5,5'-Me $\mathrm{bpy}, \mathrm{Me}_{4}$ bpy, $\left.\mathrm{Me}_{2} \mathrm{phen},{ }^{\mathrm{t}} \mathrm{Bu} \mathrm{u}_{2} \mathrm{bpy}\right\}$ are consistent with the superposition of continuum and specific solvation effects, in which the magnitude of the latter dominates the $\lambda_{0}$ contribution to $v_{\max }$. The subtle variations in the dimensions of the clefts between the diastereoisomeric forms of the same complex, and between both the interior and exterior clefts between the same diastereoisomer for the series of complexes, give rise to a marked distance and orientation dependence of the specific effects on $\lambda_{0}$. The solvent dependence of the IVCT energies for the diastereoisomers of $\left[\left\{\mathrm{Ru}(\mathrm{bpy})_{2}\right\}_{2}(\mu \text {-bpm) }]^{5+}\right.$ exhibits the theoretically-predicted linear dependence on $1 / D_{o p}-1 / D_{s}$ for all solvents except AN. The striking discontinuity in the latter arises from penetration of the small AN molecules within the clefts between the terminal bpy rings. This specific interaction is also evident for the substituted variants $\left[\left\{\mathrm{Ru}(\mathrm{pp})_{2}\right\}_{2}(\mu \text {-bpm })\right]^{5+}\left\{\mathrm{pp}=5,5^{\prime}-\mathrm{Me}_{2} \mathrm{bpy}, \mathrm{Me}_{4} \mathrm{bpy}, \mathrm{Me}_{2} \mathrm{phen}\right\}$, however the effect is absent when solvent penetration to the clefts is restricted by bulky tert-butyl substituents. The comparable magnitudes of the specific effects for both the unsubstituted bpy, and methyl-substituted $\left\{\mathrm{pp}=5,5^{\prime}-\mathrm{Me}_{2} \mathrm{bpy}, \mathrm{Me}_{4} \mathrm{bpy}, \mathrm{Me}_{2} \mathrm{phen}\right\}$ complexes, suggest that the AN dipoles must assume a similar orientation to the bpm plane in each case. The red-shift of $v_{\max }$ between PN and AN indicates that the AN molecules are associated within the interior clefts so as to solvate both metal centres simultaneously, as discussed previously for the $\left[\left\{\mathrm{Ru}(\mathrm{bpy})_{2}\right\}_{2}(\mu-\mathrm{bpm})\right]^{5+}$ diastereoisomers. By comparison, the slightly larger PN molecules are oriented towards the metal centre with the higher partial 
positive charge $\left(\delta^{+}\right)^{*}$, giving rise to a greater positive reorganisational contribution to $v_{\max }$. The nature of the specific solvent interactions is markedly dependent on the position of the methyl substituents on the terminal ligands, and the size of the solvent molecules. In general, the relatively larger orthogonal-shaped interior clefts in the meso diastereoisomers permit greater access of solvent molecules to the metal centres compared with the parallel-shaped interior clefts in the rac forms. The difference in the IVCT energies between the meso and rac forms of $\left[\left\{\mathrm{Ru}\left(\mathrm{Me}_{2} \mathrm{phen}\right)_{2}\right\}_{2}(\mu \text {-bpm })\right]^{5+}$ in ${ }^{\mathrm{i}} \mathrm{BN}$ provides the most pronounced example of differential stereochemically-directed solvent effect on $\lambda_{0}$.

Quantitatively, the relative contributions of specific solvent effects on $\lambda_{0}$ due to solvent penetration at the exterior versus the interior clefts is difficult to discern given that the dimensions of both clefts vary between the same diastereoisomer across the series of complexes. The solvent shifts between the diastereoisomeric forms of the same complex provide a direct measure of the contribution of specifically-oriented solvent molecules within the interior clefts on $\lambda_{0}$. While equation 2.6 provides a quantitative measure for this specific effect, its verification is dependent on an accurate knowledge of the number, orientation and distance of solvent molecules within the interior clefts, in addition to the contribution of solvent-solvent interactions between molecules in the first solvation layer with those in the bulk solution. Nevertheless, the solvent proportion experiment for the diastereoisomers of $\left[\left\{\mathrm{Ru}(\mathrm{bpy})_{2}\right\}_{2}(\mu-\mathrm{bpm})\right]^{5+}$ suggests that the penetration of two AN molecules between the interior clefts induces a $150 \pm 10 \mathrm{~cm}^{-1}$ red-shift in $\lambda_{0}$. The additional $240 \pm 10 \mathrm{~cm}^{-1}$ red-shift in $\lambda_{\mathrm{o}}$ (which gives rise to a total $390 \pm 10 \mathrm{~cm}^{-1}$ shift between PN and AN) arises from the combined effects of specific solvent-solute interactions within the exterior clefts, and solvent-solvent interactions. Clearly, the majority of the specific reorganisational contribution to $\lambda_{0}$ arises in the first solvation layer, and predominantly from discrete solvent molecules located within the interior clefts.

\section{Correlations with Existing Molecular Solvation Theories and Empirical Solvent Scales}

Matyushov and coworkers ${ }^{8,161,162}$ have proposed a molecular treatment of solvation in which $\lambda_{0}$ is separated into components from the orientational fluctuations of solvent dipoles, $\mathrm{E}_{\mathrm{p}}$, and density fluctuations, $E_{d}$, according to $\lambda_{\mathrm{o}}=\mathrm{E}_{\mathrm{p}}+\mathrm{E}_{\mathrm{d}}$. The molecular size of the discrete solvent molecules is accounted for by the treatment of individual molecules as spheres and defined by their dipole moments $(\mu)$ and core diameters $(\sigma)$. In the present case, major variations in $\lambda_{0}$ occur with subtle variations in the molecular size, shape and volume of solvent molecules for the homologous series of nitrile solvents, and it would appear that a treatment based on the assumption of spherical-shaped molecules would not be adequate to explain the observed solvent effects.

Specific solvent effects on the IVCT properties arising from electron pair donor-acceptor interactions and H-bonding in ammine or cyano complexes have been shown to correlate with empirical

\footnotetext{
${ }^{*}$ The formal oxidation state distribution for the mixed-valence complex is $\left[\left\{\mathrm{Ru}^{\mathrm{II}}(\mathrm{pp})_{2}\right\}(\mu-\mathrm{bpm})\left\{\mathrm{Ru} \mathrm{u}^{\mathrm{III}}(\mathrm{pp})_{2}\right]^{5+}\right.$. Due to electronic delocalisation, the actual oxidation states of the formally $\mathrm{Ru}^{\mathrm{II}}$ and $\mathrm{Ru}^{\mathrm{III}}$ centres are II $+\Delta q$ and III- $\Delta q$, respectively, where $\Delta q$ is the fraction of a unit electronic charge transferred between the metal centres in the ground state. Providing that $0 \leq \Delta q \leq 0.5$, the $\mathrm{Ru}^{(\mathrm{III}-\Delta q)}$ centre possess the higher partial positive charge $\left(\delta^{+}\right)$.
} 
solvent scales such as the Gutmann donor or acceptor numbers. ${ }^{50}$ Such effects are additive in the number of $\mathrm{NH}_{3}$ or $\mathrm{CN}^{-}$ligands present in the dinuclear complex, and influence the redox asymmetry $(\Delta \mathrm{E})$ and $\lambda_{0}$ contributions to the electron transfer barrier differently. ${ }^{2}$ The absence of any discernable correlations between the magnitudes of the specific solvent effects observed in the present study, and the empirical solvent parameters is not surprising since the polypyridyl ligands do not engage in H-bonding interactions of the type described for complexes containing $\mathrm{NH}_{3}$ and $\mathrm{CN}^{-}$ligands.

\subsection{Conclusions and Future Prospects}

IVCT solvatochromism studies on the meso and rac diastereoisomers of $\left[\left\{\mathrm{Ru}(\mathrm{bpy})_{2}\right\}_{2}(\mu \text {-bpm })\right]^{5+}$ in a homologous series of nitrile solvents reveal that stereochemically-directed specific solvent effects in the first solvation shell dominate the outer-sphere contribution to the reorganisational energy for intramolecular electron transfer. Solvent proportion experiments in AN/PN solvent mixtures demonstrate that the magnitude and direction of the specific effect is dependent on the relative abilities of discrete solvent molecules to penetrate the clefts between the planes of the terminal polypyridyl ligands. In particular, the specific effects are dependent on the dimensionality of the clefts, and the number, size, orientation and location of the solvent dipoles within the interior and exterior clefts.

IVCT solvatochromism studies on the diastereoisomeric forms of $\left[\left\{\mathrm{Ru}(\mathrm{bpy})_{2}\right\}_{2}(\mu \text {-dbneil })\right]^{5+}$ and $\left[\left\{\mathrm{Ru}(\mathrm{pp})_{2}\right\}_{2}(\mu \text {-bpm) }]^{5+}\left\{\mathrm{pp}=5,5^{\prime}-\mathrm{Me}_{2} \mathrm{bpy}, \mathrm{Me}_{4} \mathrm{bpy}, \mathrm{Me}_{2} \mathrm{phen},{ }^{\mathrm{t}} \mathrm{Bu}_{2} \mathrm{bpy}\right\}\right.$ reveal that the subtle and systematic changes in the nature of the clefts by the variation of the bridging ligand, and the judicious positioning of substituents on the terminal ligands profoundly influence the magnitude of the reorganisational energy contribution to the electron transfer barrier.

Quantitatively, the effects cannot be rationalised in terms of existing theoretical solvation models or empirical solvent scales. A more sophisticated molecular solvation model is required, which is parameterised in terms of microscopic solvent properties, including the relative orientation and distance of discrete solvent molecules from the charge transfer axis. The present study provides an experimental platform which addresses the paucity of experimental data available to guide and test developing solvation models which link the dynamics of individual solvent molecules and the Marcus-Hush theory of electron transfer. ${ }^{2,8,48,60,163}$

\section{Future Prospects}

$A b$ initio computational methods provide a powerful platform to quantify the predictions of the present experimental study, and test mathematical formulations to model the individual and collective contributions of solvent molecules to $\lambda_{0}$. The "ZHR-SS" method proposed by Zeng, Reimers and Hush. ${ }^{56 \text {, }}$

${ }^{60}$ for calculating solvent shifts on electronic absorption bands is parameterised in terms of explicit solvent dipoles, and provides an opportunity to quantify the specific solvent effects observed in the present study. Preliminary ab initio VASP computational studies were performed in collaboration with Dr Jeffrey 
Reimers in the School of Chemistry at the University of Sydney, and represent an important basis for future work towards the development of a molecular solvation theory.

Geometry optimisations on the $n=4$ and 6 forms of $\operatorname{rac}-(\Delta \Delta)-\left[\left\{\operatorname{Ru}(\text { bpy })_{2}\right\}_{2}(\mu-\mathrm{bpm})\right]^{n+}$ in $\mathbf{D}_{2}$ symmetry were performed using VASP. The structure of the +5 mixed-valence species was subsequently constructed from half of the optimised structures of the +4 and +6 forms. On the basis of the experimental evidence for a red-shift in the IVCT energy on formation of a 2:1 complex between AN and rac- $\left[\left\{\mathrm{Ru}(\mathrm{bpy})_{2}\right\}_{2}(\mu-\mathrm{bpm})\right]^{5+}(\S 2.3 .4 .1)$, a geometry optimisation was performed on the +5 species including two AN molecules in the interior clefts. The solvent dipoles were positioned symmetrically on both sides, and perpendicular to the bridging ligand plane, at the mid-point of the $\mathrm{C}$-C bond linking the two pyrimidine moieties of bpm. As shown in Figure 2.22, the positions of the AN molecules within the clefts were maintained in the VASP-optimised structure, in which the terminal bpy rings were distorted outwards to accommodate the molecules. Clearly, the specific effects may have important fundamental consequences in that the solvent is coupled to the internal electronic structure of complex which mixes the solvent and electronic coordinates and invalidates the Franck-Condon approximation. ${ }^{2}$

From the experimental studies, the striking difference in the energies of the IVCT bands for the meso and rac diastereoisomers on addition of two equivalents of AN to PN was rationalised by the differential solvent association within the interior clefts of the diastereoisomers. While solvent molecules located within the first solvation shell dominate the outer-sphere reorganisational contribution, both solvent-solute and solvent-solvent interactions are of importance. This provides a motivation for computational simulations of the full liquid structure.

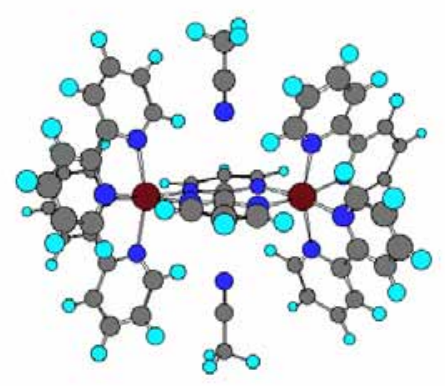

Figure 2.22 VASP-optimised structure of the $\operatorname{rac}-(\Delta \Delta)-\left[\left\{\mathrm{Ru}(\mathrm{bpy})_{2}\right\}_{2}(\mu-\mathrm{bpm})\right]^{4+}$ cation showing the two AN molecules associated within the interior clefts.

Future calculations at the DFT level are required to investigate and quantify the effects of the orientations and distances of the solvent dipoles on the single point energies. Of particular interest is the comparison of the energies of the optimised structures in AN and PN by the application of molecular dynamics approaches. The extension of the calculations to quantify the dependence of the energies on the nature of the discrete solvent interactions in the methyl-substituted complexes $\left[\left\{\mathrm{Ru}(\mathrm{pp})_{2}\right\}_{2}(\mu \text {-bpm })\right]^{4+}$ $\left\{\mathrm{pp}=5,5^{\prime}-\mathrm{Me}_{2} \mathrm{bpy}, \mathrm{Me}_{4} \mathrm{bpy}, \mathrm{Me}_{4}\right.$ phen, $\left.{ }^{\mathrm{t}} \mathrm{Bu}_{2} \mathrm{bpy}\right\}$ represents an important thrust of future computational studies. While the contributions from solvent molecules located in the first and second solvation shells must be explicitly included, the remainder of the solvent reorganisational energy arising from diffusive motions in the bulk liquid may be adequately modelled as a bulk dielectric continuum. 


\subsection{References}

1. $\quad$ Creutz, C.; Taube, H. J. Am. Chem. Soc. 1973, 95, 1086-1094.

2. $\quad$ Chen, P.; Meyer, T. J. Chem. Rev. 1998, 98, 1439-1477.

3. Nelsen, S. F.; Trieber, D. A., II; Ismagilov, R. F.; Teki, Y. J. Am. Chem. Soc. 2001, 123, 5684-5694.

4. $\quad$ Hupp, J. T.; Weaver, M. J. Inorg. Chem. 1984, 23, 3639-3644.

5. $\quad$ Katriel, J.; Ratner, M. A. J. Phys. Chem. 1989, 93, 5065-5070.

6. $\quad$ Drago, R. S.; Richardson, D. E.; George, J. E. Inorg. Chem. 1997, 36, 25-32.

7. $\quad$ Barthel, E. R.; Martini, I. B.; Schwartz, B. J. J. Phys. Chem. B 2001, 105, 12230-12241.

8. $\quad$ Matyushov, D. V.; Schmid, R. J. Phys. Chem. 1994, 98, 5152-5159.

9. $\quad$ Sullivan, B. P.; Curtis, J. C.; Kober, E. M.; Meyer, T. J. Nouv. J. Chim. 1980, 4, 643-650.

10. Roberts, J. A.; Hupp, J. T. Inorg. Chem. 1992, 31, 157-160.

11. $\quad$ Blackbourn, R. L.; Hupp, J. T. J. Phys. Chem. 1988, 92, 2817-2820.

12. Blackbourn, R. L.; Hupp, J. T. Inorg. Chem. 1989, 28, 3786-3790.

13. Hupp, J. T.; Weydert, J. Inorg. Chem. 1987, 26, 2657-2660.

14. Ennix, K. S.; McMahon, P. T.; de la Rosa, R.; Curtis, J. C. Inorg. Chem. 1987, 26, 2660-2666.

15. $\quad$ Brunschwig, B. S.; Ehrenson, S.; Sutin, N. J. Phys. Chem. 1986, 90, 3657-3668.

16. $\quad$ Nelsen, S. F.; Ismagilov, R. F. J. Phys. Chem. A 1999, 103, 5373-5378.

17. Marcus, R. A. J. Phys. Chem. B 1998, 102, 10071-10077.

18. Pereztejeda, P.; Neto-Ponce, P.; Sánchez, F. J. Chem. Soc., Dalton Trans. 2001, 1686-1691.

19. Lau, K. W.; Hu, A. M. H.; Yen, M. H. J.; Fung, E. Y.; Grzybicki, S.; Matamoros, R.; Curtis, J. C. Inorg. Chim. Acta 1994, 226, 137-143.

20. Lewis, N. A.; Obeng, Y. S.; Purcell, W. L. Inorg. Chem. 1989, 28, 3796-3799.

21. Lewis, N. A.; Obeng, Y. S. J. Am. Chem. Soc. 1988, 110, 2306-2307.

22. Hupp, J. t.; Dong, Y. Inorg. Chem. 1994, 33, 4421-4424.

23. Ferretti, A.; Lami, A.; Villani, G. Inorg. Chem. 1998, 37, 4460-4465.

24. Hupp, J. T.; Neyhart, G. A.; Meyer, T. J.; Kober, E. M. J. Phys. Chem. 1992, 96, 10820-10830.

25. Hupp, J. T.; Dong, Y. H. J. Am. Chem. Soc. 1993, 115, 6428-6429.

26. $\quad$ Dong, Y.; Hupp, J. T. Inorg. Chem. 1992, 31, 3322-3324.

27. $\quad$ Catterjee, D.; Bajaj, H. C.; Das, A. Inorg. Chim. Acta 1994, 224, 189-192.

28. D'Alessandro, D. M.; Kelso, L. S.; Keene, F. R. Inorg. Chem. 2001, 40, 6841-6844.

29. de la Rossa, R.; Chang, P. J.; Salaymeh, F.; Curtis, J. C. Inorg. Chem. 1985, 24, 4229-4231.

30. Goldsby, K. A.; Meyer, T. J. Inorg. Chem. 1984, 23, 3002-3010.

31. Hush, N. S. Prog. Inorg. Chem. 1967, 8, 391-444.

32. Hush, N. S. Electrochim. Acta 1968, 13, 1005-1023.

33. Marcus, R. A. J. Chem. Phys. 1957, 26, 867-871.

34. Marcus, R. A. J. Chem. Phys. 1956, 24, 966-978.

35. Brunschwig, B. S.; Creutz, C.; Sutin, N. Chem. Soc. Rev. 2002, 31, 168-184.

36. $\quad$ Powers, M. J.; Salmon, D. J.; Callahan, R. W.; Meyer, T. J. J. Am. Chem. Soc. 1976, 98, 6731-6733.

37. Callahan, R. W.; Brown, G. M.; Meyer, T. J. Inorg. Chem. 1975, 14, 1443-1453.

38. Callahan, R. W.; Meyer, T. J. Chem. Phys. Lett. 1976, 39, 82-84.

39. Powers, M. J.; Callahan, R. W.; Salmon, D. J.; Meyer, T. J. Inorg. Chem. 1976, 15, 1457-1459.

40. $\quad$ Curtis, J. C.; Meyer, T. J. J. Am. Chem. Soc. 1978, 100, 6284-6286.

41. Callahan, R. W.; Keene, F. R.; Meyer, T. J.; Salmon, D. J. J. Am. Chem. Soc. 1977, 99, 1064-1073.

42. $\quad$ Powers, M. J.; Meyer, T. J. Inorg. Chem. 1978, 17, 1785-1790.

43. Powers, M. J. J. Am. Chem. Soc. 1980, 102, 1289-1297.

44. Creutz, C. Prog. Inorg. Chem. 1983, 30, 1-73.

45. Crutchley, R. J. Adv. Inorg. Chem. 1994, 41, 273-325.

46. Hupp, J. T.; Meyer, T. J. Inorg. Chem. 1987, 26, 2332-2334.

47. Hupp, J. T.; Meyer, T. J. J. Phys. Chem. 1987, 91, 1001-1003.

48. Barbara, P. F.; Meyer, T. J.; Ratner, M. A. J. Phys. Chem. 1996, 100, 13148-13168.

49. Curtis, J. C.; Sullivan, B. P.; Meyer, T. J. Inorg. Chem. 1983, 22, 224-250.

50. Gutmann, V. Electrochim. Acta 1976, 21, 661-670.

51. Chou, M. H.; Creutz, C.; Sutin, N. Inorg. Chem. 1992, 31, 2318-2327.

52. Creutz, C.; Chou, M. H. Inorg. Chem. 1987, 26, 2995-3000.

53. Neyhart, G. A.; Timpson, C. J.; Bates, W. D.; Meyer, T. J. J. Am. Chem. Soc. 1996, 118, 3730-3737.

54. Lay, P. A. J. Phys. Chem. 1986, 90, 878-885.

55. Brunschwig, B. S.; Logan, J.; Newton, M. D.; Sutin, N. J. Am. Chem. Soc. 1980, 102, 5798-5809.

56. Hush, N. S.; Reimers, J. R. Coord. Chem. Rev. 1998, 177, 37-60.

57. Zeng, J.; Hush, N. S.; Reimers, J. R. J. Am. Chem. Soc. 1996, 118, 2059-2068.

58. Zeng, J.; Craw, J. S.; Hush, N. S.; Reimers, J. R. J. Phys. Chem. 1994, 98, 11075-11088.

59. Z Zeng, J.; Hush, N. S.; Reimers, J. R. J. Phys. Chem. 1996, 100, 9561-9567.

60. Hush, N. S.; Reimers, J. R. Chem. Rev. 2000, 100, 775-786. 
61. Zeng, J.; Hush, N. S.; Reimers, J. R. J. Phys. Chem. 1995, 99, 10459-10470.

62. $\quad$ Pople, J. A.; Beveridge, D. L.; Dobosh, P. A. J. Chem. Phys. 1967, 47, 2026-2033.

63. Pople, J. A.; Berendge, D. L., Aproximate Molecular Orbital Theory; McGraw Hill: New York, 1970.

64. Constantino, V. R. L.; Toma, H. E.; de Oliveira, L. F. C.; Rein, F. N.; Rocha, R. C.; de Oliveira Silva, D. J. Chem. Soc., Dalton Trans. 1999, 1735-1740.

65. Harden, N. C.; Humphery, E. R.; Jeffery, J. C.; Lee, S.-M.; Marcaccio, M.; McCleverty, J. A.; Rees, L. H.; Ward, M. D. J. Chem. Soc., Dalton Trans. 1999, 2417-2426.

66. Behrendt, A.; Couchman, S. M.; Jeffery, J. C.; McCleverty, J. A.; Ward, M. D. J. Chem. Soc., Dalton Trans. 1999, 4349-4355.

67. Metcalfe, R. A.; Vasconcellos, L. C. G.; Mirza, H.; Franco, D. W.; Lever, A. B. P. J. Chem. Soc., Dalton. Trans. 1999, 2653-2667.

68. Masui, H.; Freda, A. L.; Zerner, M. C.; Lever, A. B. P. Inorg. Chem. 2000, 39, 141-152.

69. da Cunha, C. J.; Dodsworth, e. D.; Monteiro, M. A.; Lever, A. B. P. Inorg. Chem. 1999, 38, 5399-5409.

70. Bencini, A.; Ciofini, I.; Daul, C. A.; Ferretti, A. J. Am. Chem. Soc. 1999, 121, 11418-11424.

71. $\quad$ Braun-Sand, S. B.; Wiest, O. J. Phys. Chem. A 2003, 107, 285-291.

72. $\quad$ Estiú, G.; Cukiernik, F. D.; Maldivi, P.; Poizat, O. Inorg. Chem. 1999, 38, 3030-3039.

73. $\quad$ Patra, S.; Sarkar, B.; Ghumaan, S.; Fiedler, J.; Zális, S.; Kaim, W.; Lahiri, G. K. Dalton Trans. 2004, 750-753.

74. $\quad$ Reimers, J. R.; Hush, N. S. J. Phys. Chem. A 1999, 103, 3066-3072.

75. Keene, F. R. Chem. Soc. Rev. 1998, 27, 185-193.

76. $\quad$ Fletcher, N. C.; Junk, P. C.; Reitsma, D. A.; Keene, F. R. J. Chem. Soc., Dalton Trans. 1998, 133-138.

77. $\quad$ Fletcher, N. C.; Keene, F. R. J. Chem. Soc., Dalton Trans. 1999, 683-689.

78. Keene, F. R. Coord. Chem. Rev. 1997, 166, 122-159.

79. $\quad$ Blackbourn, R. L.; Hupp, J. T. J. Phys. Chem. 1990, 94, 1788-1793.

80. Blackbourn, R. L.; Hupp, J. T. Chem. Phys. Lett. 1988, 150, 399-405.

81. LeSuer, R.; Geiger, W. E. Angew. Chem., Int. Ed. Engl. 2000, 39, 248-250.

82. Bergman, S. D.; Goldberg, I.; Barbieri, A.; Barigelletti, F.; Kol, M. Inorg. Chem. 2004, 43, $2355-2367$.

83. Armarego, W. L.; Perrin, D. D. Purification of Laboratory Chemicals. 4th ed.; Butterworth-Heinemann: Oxford, 1996.

84. Patterson, B. T.; Keene, F. R. Inorg. Chem. 1998, 37, 645-650.

85. Connelly, N. G.; Geiger, W. E. Chem. Rev. 1996, 96, 877-910.

86. Duff, C. M.; Heath, G. A. Inorg. Chem. 1991, 30, 2528-2535.

87. Lowery, M. D.; Hammack, W. S.; Drickamer, H. G.; Hendrickson, D. N. J. Am. Chem. Soc. 1987, 109, 8019-8024.

88. Reimers, J. R.; Hush, N. S. Inorg. Chem. 1990, 29, 3686-3697.

89. Launay, J.-P. Chem. Soc. Rev. 2001, 30, 386-397.

90. Seneviratne, D. S.; Uddin, M. J.; Swayambunathan, V.; Schlegel, H. B.; Endicott, J. F. Inorg. Chem. 2002, 41, 1502-1517.

91. $\quad$ Kresse, G.; Hafner, J. J. Phys. Rev. B 1993, 48, 13115.

92. $\quad$ Kresse, G.; Hafner, J. J. Phys.: Condens. Matter 1994, 6, 8245.

93. Mingos, D. M. P.; Baghurst, D. R. D. Chem. Soc. Rev. 1991, 20, 1-47.

94. Jandrasics, E. Z. Ph.D. Thesis, University of Fribourg (Fribourg, Switzerland), 1995.

95. Jandrasics, E. Z.; Keene, F. R. J. Chem. Soc., Dalton Trans. 1997, 153-159.

96. $\quad$ Evans, I. P.; Spencer, A.; Wilkinson, G. J. Chem. Soc., Dalton Trans. 1973, 204-209.

97. Togano, T.; Nagao, N.; Tsuchida, M.; Kumakura, H.; Hisamatsu, K.; Howell, F. S.; Mukaida, M. Inorg. Chim. Acta 1992, 195, 221-225.

98. Anderson, P. A.; Anderson, R. F.; Furue, M.; Junk, P. C.; Keene, F. R.; Patterson, B. T.; Yeomans, B. D. Inorg. Chem. 2000, 39, 2721-2728.

99. Sullivan, B. P.; Salmon, D. J.; Meyer, T. J. Inorg. Chem. 1978, 17, 3334-3341.

100. McClanahan, S. F.; Dallinger, R. F.; Holler, F. J.; Kincaid, J. R. J. Am. Chem. Soc. 1985, 107, 4853-4860.

101. Constable, E. C.; Lewis, J. Inorg. Chim. Acta 1983, 70, 251-253.

102. Juris, A.; Barigelletti, S.; Campagna, S.; Balzani, V.; Belser, P.; von Zelewsky, A. Coord. Chem. Rev. 1988, 84, 85-277.

103. Hua, X. Ph.D. Thesis, University of Fribourg (Fribourg, Switzerland), 1993.

104. D’Alessandro, D. M.; Keene, F. R.; Bergman, S. D.; Kol, M. Dalton Trans. 2005, 332-337.

105. Blessing, R. H. Acta. Cryst. 1995, A51, 33-38.

106. Sheldrick, G. M. SHELXL-97, Program for Crystal Structure Solution; University of Göttingen: Germany, 1997; Sheldrick, G. M. SHELXS-97, Program for Crystal Structure Refinement; University of Göttingen: Germany, 1997.

108. Dose, E. V.; Wilson, L. J. Inorg. Chem. 1978, 17, 2660-2666.

109. Rillema, P.; Mack, K. B. Inorg. Chem. 1982, 21, 3849-3854.

110. Krejcik, M.; Vlcek, A. A. Inorg. Chem. 1992, 31, 2390-2395.

111. Hua, X.; von Zelewsky, A. Inorg. Chem. 1991, 30, 3796-3798. 
112. Hua, X.; von Zelewsky, A. Inorg. Chem. 1995, 34, 5791-5797.

113. Reitsma, D. A.; Keene, F. R. J. Chem. Soc., Dalton Trans. 1993, 2859-2860.

114. Hua, X.; Lappin, A. G. Inorg. Chem. 1995, 34, 992-994.

115. Bergman, S. D.; Reshef, D.; Groysman, S.; Goldberg, I.; Kol, M. Chem. Commun. 2002, 2374-2375.

116. Bardwell, D.; Jeffery, J. C.; Joulie, L.; Ward, M. D. J. Chem. Soc., Dalton Trans. 1993, 2255-2256.

117. Bardwell, D. A.; Horsburgh, L.; Jeffery, J. C.; Joulié, L. F.; Ward, M. D.; Webster, I.; Yellowlees, L. J. J Chem Soc Dalton Trans 1996, 2527-2531.

118. Hage, R.; Haasnoot, J. G.; Nieuwenhuis, H. A.; Reedijk, J.; De Ridder, D. J. A.; Vos, J. G. J. Am. Chem. Soc. 1990, 112, 9245-9251.

119. Balzani, V.; Bardwell, D. A.; Barigelletti, F.; Cleary, F. L.; Guardigli, M.; Jeffery, J. C.; Sovrani, T.; Ward, M. D. J. Chem. Soc., Dalton Trans 1995, 3601-3608.

120. Baitalik, S.; Flörke, U.; Nag, K. J. Chem. Soc, Dalton Trans. 1999, 719-727.

121. Hage, R.; Turkenburg, J. P.; de Graaff, R. A. G.; Haasnoot, J. G.; Reedijk, J. Acta Cryst. 1989, 45, 381-383.

122. Rillema, D. P.; Taghdiri, D. G.; Jones, D. S.; Keller, C. D.; Worl, L. A.; Meyer, T. J.; Levy, H. A. Inorg. Chem. 1987, 26, 578-585.

123. Rillema, D. P.; Jones, D. S.; Levy, H. A. J. Chem. Soc., Chem. Commun. 1979, 849-851, 849-851.

124. Gut, D.; Goldberg, I.; Kol, M. Inorg. Chem. 2003, 42, 3483-3491.

125. Richardson, C.; Steel, P. J.; D'Alessandro, D. M.; Junk, P. C.; Keene, F. R. J. Chem. Soc., Dalton Trans. 2002, 2775-2785.

126. Zampese, J. A.; Keene, F. R.; Steel, P. J. Dalton Trans. 2004, 4124-4129.

127. Pavinato, R. A.; Walk, J. A.; McGuire, M. E. Inorg. Chem. 1993, 32, 4982-4984.

128. Ruminski, R. R.; Petersen, J. D. Inorg. Chem. 1982, 21, 3706-3708.

129. Rillema, D. P.; Allen, G.; Meyer, T. J.; Conrad, D. Inorg. Chem. 1983, 22, 1617-1622.

130. Ernst, S.; Kasack, V.; Kaim, W. Inorg. Chem. 1988, 27, 1146-1148.

131. Ernst, S. D.; Kaim, W. Inorg. Chem. 1989, 28, 1520-1528.

132. Kaim, W.; Kohlmann, S. Inorg. Chem. 1987, 26, 68-77.

133. Marcaccio, M.; Paolucci, F.; Paradisi, C.; Roffia, S.; Fontanesi, C.; Yellowlees, L. J.; Serroni, S.;

Campagna, S.; Denti, G.; Balzani, V. J. Am. Chem. Soc 1999, 121, 10081-10091.

134. Giuffrida, G.; Campagna, S. Coord. Chem. Rev. 1994, 135, 517-531.

135. Kober, E. M.; Goldsby, K. A.; Narayana, D. N. S.; Meyer, T. J. J. Am. Chem. Soc. 1983, 105, $4303-4309$.

136. D'Alessandro, D. M.; Keene, F. R. Dalton Trans. 2004, 3950-3954.

137. Nallas, G. N. A.; Jones, S. W.; Brewer, K. J. Inorg. Chem. 1996, 35, 6974-6980.

138. Lytle, F. E.; Hercules, D. M. J. Am. Chem. Soc. 1969, 91, 253-257.

139. Kalyanasundaram, K.; Zakeeruddin, S. M.; Nazeeruddin, M. K. Coord. Chem. Rev. 1994, 132, 259-264.

140. Baumann, F.; Kaim, W.; Posse, M. C.; Katz, N. E. Inorg. Chem. 1998, 37, 658-660.

141. Ward, M. D. Chem. Soc. Rev. 1995, 24, 121-134.

142. Kalyanasundaram, K.; Nazeeruddin, M. K. Inorg. Chim. Acta 1994, 226, 213-230.

143. Hush, N. S. Coord. Chem. Rev. 1985, 64, 135-157.

144. Nelsen, S. F. Chem. Eur. J. 2000, 6, 581-588.

145. Demadis, K. D.; Hartshorn, C. M.; Meyer, T. J. Chem. Rev. 2001, 101, 2655-2685.

146. Robin, M. B.; Day, P. Adv. Inorg. Chem. Radiochem. 1967, 10, 247-403.

147. Lambert, C.; Nöll, G. J. Am. Chem. Soc. 1999, 121, 8434-8442.

148. Demadis, K. D.; El-Samanody, E.-S.; Coia, G. M.; Meyer, T. J. J. Am. Chem. Soc. 1999, 121, $535-544$.

149. Demadis, K. D.; Neyhart, G. A.; Kober, E. M.; White, P. S.; Meyer, T. J. Inorg. Chem. 1999, 38, 59485959.

150. Piepho, S. B. J. Am. Chem. Soc. 1990, 112, 4197-4206.

151. Hupp, J. T.; Zhang, X. L. J. Phys. Chem. 1995, 99, 853-855.

152. Pauling, L., The Nature of the Chemical Bond. 3rd ed.; Cornell University Press: Ithaca, New York, 1960. 153. Creutz, C. Inorg. Chem. 1978, 17, 3723-3725.

154. Kober, E. M.; Meyer, T. J. Inorg. Chem. 1983, 22, 1614-1616.

155. Brunschwig, B. S.; Creutz, C.; Macartney, D. H.; Sham, T.-K.; Sutin, N. Faraday Discuss. Chem. Soc. 1982, 74, 113-127.

156. Hupp, J. T.; Dong, Y. H.; Blackbourn, R. L.; Lu, H. J. Phys. Chem. 1993, 97, 3278-3282.

157. Oh, D. H.; Sano, M.; Boxer, S. G. J. Am. Chem. Soc. 1991, 113, 6880-6890.

158. Oh, D. H.; Boxer, S. G. J. Am. Chem. Soc. 1990, 112, 8161-8162.

159. La Mar, G. N.; Van Hecke, G. R. Inorg. Chem. 1973, 12, 1767-1773.

160. Reimers, J. R.; Hall, L. E. J. Am. Chem. Soc. 1999, 121, 3730-3744.

161. Elliott, c. M.; Derr, D. L.; Matyushov, D. V.; Newton, M. D. J. Am. Chem. Soc. 1998, 120, 11714-11726.

162. Matyushov, D. V. Chem. Phys. 1993, 174, 199-218.

163. Small, D. W.; Matyushov, D. V.; Voth, G. A. J. Am. Chem. Soc. 2003, 125, 7470-7478. 
ChApter 3 
Chapter 3

\section{DiASTEREOISOMERS AS PROBES FOR THE FACTORS GOVERNING THE LOCALISED-TO-DELOCALISED TRANSITION IN IVCT}

\subsection{Introduction}

A pivotal problem in the analysis of mixed-valence complexes is the extent of electronic delocalisation between the metal centres - which is governed by competition between the electronic coupling $\left(\mathrm{H}_{\mathrm{ab}}\right)$ and the sum of the Franck-Condon reorganisational energy, the redox-asymmetry $\left(\Delta \mathrm{E}_{0}\right)$ and additional contributions due to spin-orbit coupling and ligand field asymmetry $\left(\Delta \mathrm{E}^{\prime}\right){ }^{1,2}$ Classically, the reorganisational energy is composed of an inner-sphere vibrational component, $\lambda_{i}$, corresponding to the energy required for reorganisation of the metal-ligand and intra-ligand bond lengths and angles, and an outer-sphere component, $\lambda_{\mathrm{o}}$, corresponding to the energy required for reorganisation of solvent and anion molecules in the surrounding medium. According to Marcus-Hush theory, ${ }^{1-4}$ these fundamental parameters govern the activation barrier to intramolecular electron transfer, and may be assessed by probing the characteristics of the IVCT transitions observed in dinuclear mixed-valence complexes. In weakly-coupled $\left(\right.$ Class II $\left.{ }^{5}\right)$ systems, the energy of the IVCT band $\left(v_{\max }\right)$ is given by equation 3.1: $:^{1,2}$

$$
v_{\max }=\lambda_{i}+\lambda_{0}+\Delta \mathrm{E}_{0}+\Delta \mathrm{E}^{\prime}
$$

$\mathrm{H}_{\mathrm{ab}}$ is given by equation 3.2, ${ }^{6,7}$ where $r_{\mathrm{ab}}$ is the distance between the two diabatic states, $e$ is the unit electronic charge, and $\left|\mu_{12}\right|$ is the adiabatic transition moment which may be calculated from the integrated intensity of the absorption band.

$$
\mathrm{H}_{\mathrm{ab}}=\frac{\left|\mu_{12}\right|}{e r_{a b}} v_{\max }
$$

For a generalised mixed-valence species of the form $\left[\left\{L_{n} M^{I I}\right\}(\mu-B L)\left\{M^{I I I} L_{n}\right\}\right]^{5+}\{M=$ metal centres, $\mathrm{L}=$ terminal ligands and $\mathrm{BL}=$ bridging ligand $\}$ electronic delocalisation is promoted by mixing between the donor $\left(\mathrm{M}^{\mathrm{II}}\right)$ and acceptor $\left(\mathrm{M}^{\mathrm{III}}\right)$ wavefunctions. In the limit of strong overlap, delocalisation is complete and both metal centres possess valences of +2.5 . In this case, there is no longer a thermal barrier to electron transfer, and the process involves a transition within the molecular orbital manifold of the species. For symmetrical delocalised (Class $\mathrm{III}^{5}$ ) systems, the energy of the transition provides a direct measure of $\mathrm{H}_{\mathrm{ab}}$ according to equation 1.11. ${ }^{1,2}$ The degree of delocalisation is dependent on the relative magnitudes of $\mathrm{H}_{\mathrm{ab}}$ and the sum of the factors which govern the activation barrier to electron 
transfer in equation 3.1: valence localisation occurs when this sum exceeds $2 \mathrm{H}_{\mathrm{ab}}$, whereas delocalisation occurs when this sum is less than $2 \mathrm{H}_{\mathrm{ab}}$.

As described in $\$ 1.2$ and $\S 1.3$, the two-state Hush model ${ }^{1,2}$ has provided the preferred method of analysis for mixed-valence complexes and the validity of the model for the determination of the relative contributions of the fundamental parameters expressed in equations 3.1 and 3.2 has been extensively tested and reviewed. ${ }^{8-17}$ Experimentally, these contributions have been probed by the dependence of the IVCT characteristics on structural and substitutional changes in the mixed-valence systems. These include the distance between the metal centres, ${ }^{8}$ the ability of the bridging ligand to delocalise the electronic charge,$^{18}$ and the coordination environment of the metal centres ${ }^{9-12,14-16}$ which can be controlled through variations in the metal centers and the bridging or terminal ligands. The characteristics of the external medium such as the identity of the solvent ${ }^{17,19-31}$ and anions ${ }^{32-37}$ also constitute critical contributions to the electron transfer barrier. While the classical two-state model ${ }^{1,2}$ has been successfully applied to the analysis of complexes in the strongly localised and delocalised limits, apparent breakdowns occur between these two extremes ${ }^{17}$ in the presence of significant electronic delocalisation, or specific solvent-solute interactions - such as those observed for $\left[\left\{\mathrm{Ru}(\mathrm{bpy})_{2}\right\}_{2}(\mu \text {-bpm })\right]^{5+}\left\{\mathrm{bpm}=2,2^{\prime}-\right.$ bipyrimidine\} in Chapter 2.

\subsubsection{The Localised-to-Delocalised Transition in IVCT}

\subsubsection{Theoretical Models}

The development of theoretical models to describe the transition between the localised and delocalised regimes represents a long-standing issue in mixed-valence chemistry. ${ }^{8-17}$ Three- and fourstate classical models have been proposed ${ }^{38}$ in which the bridging ligand is explicitly included as an additional electronic state. These models provide a more physically-realistic basis for bridging-ligand mediated superexchange effects in systems with appreciable electronic coupling. While the classical model is appropriate for low frequency modes such as solvent vibrations, the model is inadequate for systems in which high frequency internal modes are coupled to the electron transfer. The latter require explicit treatment through a quantum mechanical approach. Semi-classical models ${ }^{6,38-42}$ combine a classical treatment of the solvent reorganisational contributions with a quantum mechanical description of inner-sphere modes. Such formalisms are remarkably successful in reproducing the results of a full quantum mechanical treatment by permitting nuclear tunnelling transitions below the classical barrier. ${ }^{43}$ The localised-to-delocalised transition has also been treated through quantum mechanical formalisms such as the "PKS model". ${ }^{4-47}$ This model explicitly includes vibronic coupling, and accounts for the inter-dependence of the electronic and nuclear motions that occur with increasing electronic delocalisation. A more detailed discussion of these theoretical methods was presented in §1.4. 


\section{The Symmetric Vibration Mode}

The central role of symmetric vibration modes in the theoretical treatment of the localised-todelocalised transition was first discussed by Hush, ${ }^{48}$ and was a key conceptual development in the threestate molecular orbital model proposed by Ondrechen and coworkers for delocalised mixed-valence systems (§1.4.3). ${ }^{49-53}$ In the delocalised limit, transitions occur between delocalised electronic levels ${ }^{9,14}$ which correspond to symmetric and anti-symmetric combinations of the initial unperturbed electronic wave functions. The redistribution of electron density from the bridging ligand to the terminal metal centres is accompanied by a change in the metal-ligand bond distances on both sides of the bridge which corresponds to vibrational excitation in the symmetric mode. ${ }^{48}$ The significance of both symmetric and anti-symmetric modes has led to the extension of the two-site classical model ${ }^{1,2}$ to the two-mode threestate classical model (§1.4.1). ${ }^{38}$ Reimers and Hush have provided a conceptual framework for the inclusion of both anti-symmetric and symmetric modes within the full treatment of the vibronic coupling problem (§1.4.4). ${ }^{48}$

These theoretical formalisms describe the key features of the localised-to-delocalised transition, including the contributions from vibronic coupling as well as symmetric and anti-symmetric modes. ${ }^{14,48}$ However, the experimental elucidation of the factors which govern the localised-to-delocalised transition in real mixed-valence systems has proven somewhat elusive due to the complexity of contributing factors. These include (i) multiple through-bridge interactions (due to the effects of low symmetry and spin-orbit coupling, which lead to multiple overlapping IVCT and interconfigurational (IC) bands in the mixedvalence spectra ${ }^{14,17,54,55}$ which, if not separately resolved, also contribute to the bandwidth); (ii) multiple nuclear and solvent vibrations coupled to the electron transfer which may have different time-scales; ${ }^{14}$ and (iii) contributions from specific solvation, ion-pairing and additional medium effects. ${ }^{17}$

The complexity of factors which influence intramolecular electron transfer, and their sensitivity to the properties of the environment, underlie the need for systematic experimental studies to probe the factors which govern the localised-to-delocalised transition. These studies are crucial to address the deficiency of experimental data for the transition region, and to guide and test the development of a "unifying theory” which is parameterised in terms of physically transparent quantities (§1.4.5). ${ }^{13,14,17}$

\subsubsection{Experimental Considerations}

Mixed-valence systems are characterised by transitions which typically appear at low energy in the near-infrared (NIR) or mid-infrared (mid-IR) regions of their spectra. ${ }^{1,2,8-13,56}$ In localised systems, the IVCT transitions are of low intensity and broad $\left(\varepsilon_{\max } \leq 5000 \mathrm{M}^{-1} \mathrm{~cm}^{-1}, \Delta v_{1 / 2} \geq 2000 \mathrm{~cm}^{-1}\right)$ due to coupling with solvent. In delocalised species, the bands arise from transitions within the molecular orbital manifold of the systems and the bands are intense, narrow ( $\left.\varepsilon_{\max } \geq 5000 \mathrm{M}^{-1} \mathrm{~cm}^{-1}, \Delta v_{1 / 2} \leq 2000 \mathrm{~cm}^{-1}\right)$ and solvent independent. For systems near the localised-to-delocalised transition, the IVCT bands are frequently asymmetrically-shaped and intense. ${ }^{14,18,38}$ 
The various theoretical treatments attribute asymmetry to different effects, and previous modeling studies have attempted to reproduce the line-width by several methods. ${ }^{14}$ Within the two-state classical model, the asymmetry is a natural consequence of the "band cut-off effect" in which the IVCT band is Gaussian-shaped on the high-energy side, skewed on the low energy side, and truncated at $v \leq 2 \mathrm{H}_{\mathrm{ab}}$ (§1.3.2). ${ }^{14,18,38,57}$ Alternatively, there may be contributions from overlapping mixed-valence transitions, differences in the extent of electronic delocalisation between the ground and mixed-valence excited states, or contributions from a vibronic progression or progressions. ${ }^{14}$ While the application of vibronic coupling theories with the inclusion of symmetric and anti-symmetric mode contributions is necessary for the band-shape analysis of mixed-valence systems in the localised-to-delocalised transition, the classical analysis may be adequate if the widths of the separate vibronic components exceed their vibrational separation.

Experimentally, the classification of mixed-valence complexes as localised (Class II), delocalised (Class III), or intermediate localised-to-delocalised systems is generally based upon the observed bandwidth at half-height $\left(\Delta v_{1 / 2}\right)$ compared with that calculated from equation $1.8^{1,2}$ in the limit of a localised two-site transition $\left(\Delta v_{1 / 2}{ }^{\circ}\right)$. A system is localised if $\Delta v_{1 / 2} \geq \Delta v_{1 / 2}{ }^{\circ}$ and delocalised if $\Delta v_{1 / 2}<\Delta v_{1 / 2}{ }^{\circ}$. However, the discrimination between the two regimes is not straightforward as band-shape analyses are frequently complicated by multiple overlapping transitions, and a significant number of mixed-valence systems exhibit the characteristics of both localised and delocalised systems. Meyer and coworkers ${ }^{14}$ addressed this conundrum by defining systems in the localised-to-delocalised transition as "Class II-III”. In reality, the transitions between the classes are not abrupt, and their distinction is based on the relative time-scales of the solvent, vibrational and electronic motions, as detailed in $§ 1.5 .1{ }^{14}$ The challenging nature of classification is reminiscent of the arguments involved in the study of the CreutzTaube ion, $\left[\left\{\mathrm{Ru}\left(\mathrm{NH}_{3}\right)_{5}\right\}_{2}(\mu-\mathrm{pyz})\right]^{5+}\{$ pyz $=$ pyrazine $\},{ }^{58,59}$ and its derivatives (§1.5.2). Due to the limited number of experimental studies in which the subtleties in behaviour in the localised-to-delocalised transition have been systematically explored, there is currently extensive interest in the preparation of systems which exhibit Class II-III behaviour. ${ }^{13,14,18,38,48}$

\subsubsection{Scope and Objectives of the Present Study}

The present study represents the first investigation of IVCT in the diastereoisomers of $\left[\left\{\mathrm{M}(\mathrm{bpy})_{2}\right\}_{2}(\mu-\mathrm{BL})\right]^{5+}\{\mathrm{M}=\mathrm{Ru}$, Os; and BL represents the angular and stepped-parallel polypyridyl bridging ligands shown in Figure 3.1\}. While the electrochemical, photochemical and photophysical properties of many of these complexes have been the subject of extensive research efforts over the past two decades, ${ }^{60-64}$ the measurements have often been conducted without regard for the inherent stereoisomeric complexities $(\S 1.6 .3){ }^{65,66}$ 
Linear
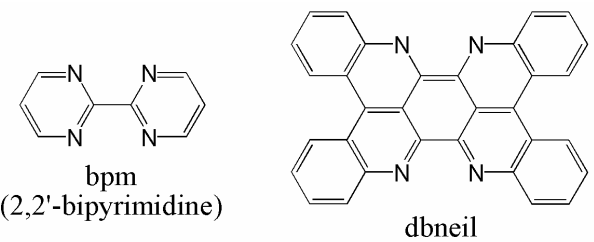

(dibenzoeilatin)

SteppedParallel

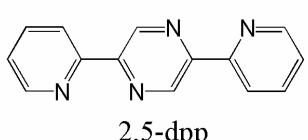

(2,5-bis(2-pyridyl)pyrazine)

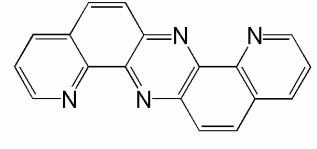

dpop

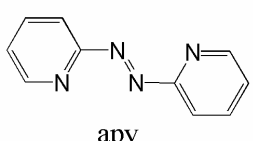

apy

(azobis(2-pyridine))

(dipyrido(2,3-a;2',3'-h)phenazine)

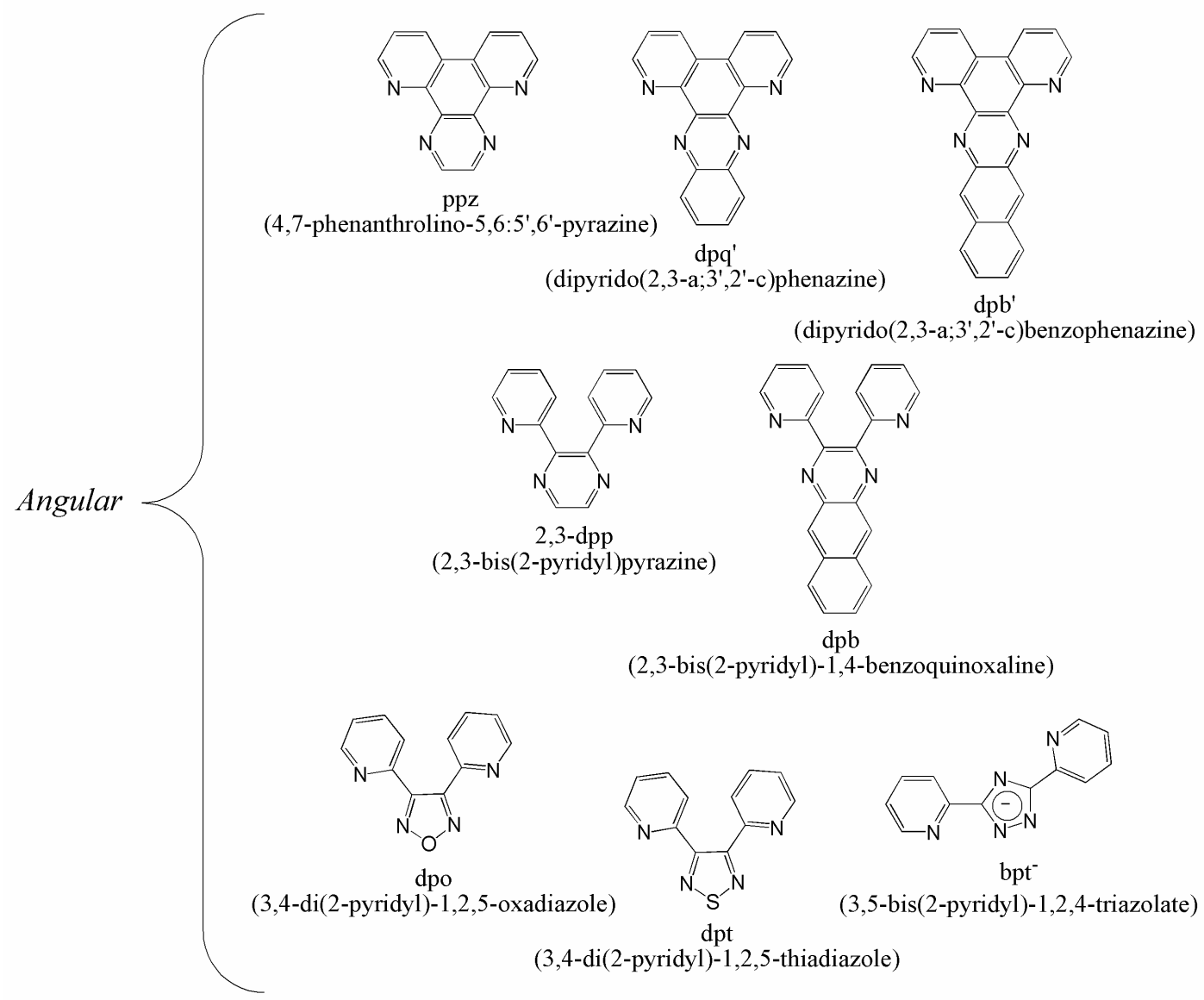

Figure 3.1 Angular and stepped-parallel polypyridyl bridging ligands employed in the present study. The linear bridging ligands employed in Chapter 2 are shown for comparison.

In addition to systematic electronic variations in the series of complexes, the angular or steppedparallel geometries of the bridging ligands permit subtle changes in the dimensions of the clefts. For the complexes based on the stepped-parallel ligands, the terminal polypyridyl ligands "above" and "below" the plane of the bridging ligand are approximately orthogonal in the meso $(\Lambda \Delta \equiv \Delta \Lambda)$ diastereoisomer while they are approximately parallel in the $\operatorname{rac}(\Delta \Delta / \Lambda \Lambda)$ form, as shown in Figure 3.2(a). ${ }^{65,66}$ When the relationship of the axes of the two "bites" is angular this description is reversed \{Figure 3.2(b)\}. 
(a)

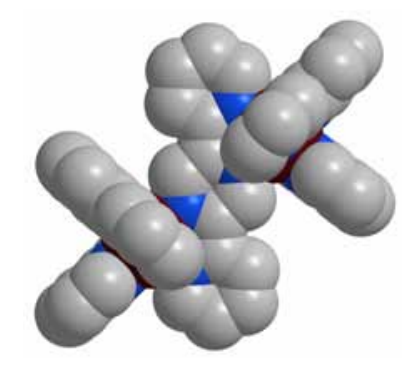

meso $(\Lambda \Delta)$

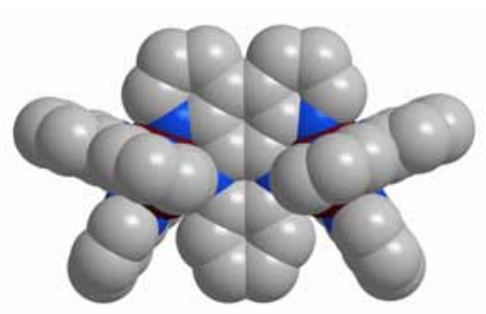

$\operatorname{meso}(\Lambda \Delta)$

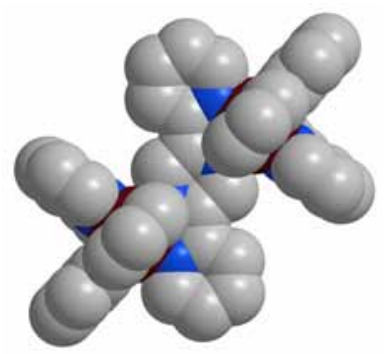

$\operatorname{rac}(\Delta \Delta)$

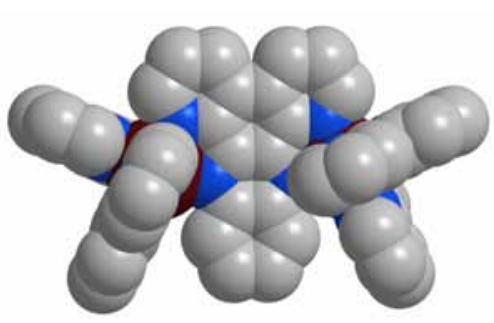

$\operatorname{rac}(\Delta \Delta)$

Figure 3.2 Chem3D representations (view from above the plane of the bridging ligand) of the meso $(\Lambda \Delta)$ and rac $(\Delta \Delta)$ diastereoisomers of (a) $\left[\left\{\mathrm{Ru}(\mathrm{bpy})_{2}\right\}_{2}(\mu-2,5-\mathrm{dpp})\right]^{4+}$ and (b) $\left.\left[\left\{\mathrm{Ru}(\mathrm{bpy})_{2}\right\}_{2}(\mu-\mathrm{dpq})^{\prime}\right)\right]^{4+}$ showing the different dimensions of the clefts "above" and "below" the plane of the bridging ligand.

\subsubsection{Approach}

\section{(1) Electronic and Structural Consequences of Bridging Ligand Modification}

(i) Electronic Aspects. The redox and spectral properties for the diastereoisomeric forms of $\left[\left\{\mathrm{Ru}(\mathrm{bpy})_{2}\right\}_{2}(\mu-\mathrm{BL})\right]^{4+}\left\{\mathrm{BL}=\mathrm{dpb}, \mathrm{dpb}\right.$ and apy, $\left.{ }^{67-70} \mathrm{BL}=\mathrm{dpo}, \mathrm{dpt}^{71}\right\}$ and for diastereoisomeric mixtures of $\left[\left\{\mathrm{Ru}(\mathrm{bpy})_{2}\right\}_{2}(\mu-\mathrm{BL})\right]^{4+}\left\{\mathrm{BL}=\mathrm{dpb}\right.$ and $2,3-\mathrm{dpp}^{72-82}$ and $\mathrm{BL}=2,5-\mathrm{dpp}$ and $\left.\mathrm{dpop}^{72,77,81,83-86}\right\}$ have been reported previously. However, their IVCT properties have received limited attention. ${ }^{67-69}$ The bridging ligands possess unoccupied low-lying $\pi^{*}$ orbitals and mediate electron transfer between the metal centres via a superexchange-assisted electron transfer mechanism (§1.5.3.1). ${ }^{* 81}$ By the systematic variation in the energy of the lowest unoccupied molecular orbital (LUMO) of the bridging ligands, the change in the degree of electronic delocalisation $\left(\mathrm{H}_{\mathrm{ab}}\right)$ on the IVCT properties may be assessed. The variations in the bridging ligands include the addition of electron-withdrawing phenyl groups fused to the side of the pyrazine ring through the series ppz, dpq' and dpb' or the modification of the heteroatom in dpo and dpt.

(ii) Structural Aspects. Since the planarity of the bridging ligand influences its degree of electronic delocalisation, a comparison of the IVCT properties of the diastereoisomers of $\left[\left\{\mathrm{Ru}(\mathrm{bpy})_{2}\right\}_{2}(\mu-\right.$ $\left.\left.\mathrm{dpb}^{\prime}\right)\right]^{5+}$ and $\left[\left\{\mathrm{Ru}(\mathrm{bpy})_{2}\right\}_{2}(\mu-\mathrm{dpb})\right]^{5+}$ incorporating the "fused" and "unfused" bridging ligands dpb' and dpb, respectively, provides insights into the influence of stereochemically-induced structural distortions on the degree of delocalisation. X-ray crystallographic studies provide information on the structural characteristics of the systems.

\footnotetext{
* By comparison, the electron-rich high-lying $\pi *$ orbitals in bpt ${ }^{-}$mediate the metal-metal interaction via a hole transfer mechanism. ${ }^{81}$
} 


\section{(2) Redox Asymmetry ( $\left.\Delta E_{0}\right)$ Contributions.}

The introduction of redox asymmetry into the dinuclear complexes $\left[\left\{\mathrm{Ru}(\mathrm{bpy})_{2}\right\}_{2}(\mu-\mathrm{dpb})\right]^{5+}$ and $\left[\left\{\mathrm{Ru}(\mathrm{bpy})_{2}\right\}_{2}(\mu-\mathrm{dpb})\right]^{5+}$ by the substitution of the terminal bpy ligands at one end by $\mathrm{Me}_{2}$ bpy $\left(4,4^{\prime}-\right.$ dimethyl-2,2'-bipyridine) and $\mathrm{Me}_{4}$ bpy (4,4',5,5'-tetramethyl-2,2'-bipyridine) diminishes the degree of delocalisation, and provides a strategy for examining the transition between the delocalised and localised regimes within a closely-related series of complexes. In the present study, the electrochemical and spectral analysis by Curtis and coworkers $(\S 1.5 .3 .4)^{87-90}$ is applied to investigate the onset of localisation in the series of unsymmetrical complexes $\left[\left\{\mathrm{Ru}(\mathrm{bpy})_{2}\right\}(\mu-\mathrm{BL})\left\{\mathrm{Ru}(\mathrm{pp})_{2}\right\}\right]^{5+}\left\{\mathrm{BL}=\mathrm{dpb}\right.$ ', dpb; pp $=\mathrm{Me}_{2} \mathrm{bpy}$, $\mathrm{Me}_{4}$ bpy . The method is formulated within the classical framework, ${ }^{1,2}$ and provides a means to assess the validity of the two-state analysis to determine the relative degree of ground state delocalisation between the different complexes, and between the diastereoisomeric forms of the same complex.

\section{(3) IVCT Solvatochromism, Thermochromism and Ion-Pairing Contributions.}

Environmental effects such as solvent and anion rearrangement, and the temperature of the medium, constitute critical contributions to the activation barrier to electron transfer through their influence on $\lambda_{0}$ and $\Delta \mathrm{E}_{0}{ }^{9}, 10,12,17$ In the present study, an examination of the solvent, temperature and anion dependencies of the IVCT process in the diastereoisomers of a series of structurally-related complexes provides a means to evaluate the modification of reorganisational effects and redox asymmetries induced by stereochemical and substitutional changes in the complexes.

(i) IVCT solvatochromism studies on the diastereoisomeric forms of $\left[\left\{\mathrm{Ru}(\mathrm{bpy})_{2}\right\}_{2}(\mu-\mathrm{BL})\right]^{5+}$ (where BL represents the angular bridging ligands dpb, dpb' and dpq', and the stepped-parallel bridging ligands 2,5-dpp, dpop and apy) provide insights into the modification of solvent reorganisational effects $\left(\lambda_{0}\right)$. In addition to the systematic electronic variations in the bridging ligands, the subtle structural and stereochemical variations within the series of complexes permit insights into solvent and anion interactions at the molecular level.

(ii) IVCT thermochromism studies. The differences in the degree of electronic communication between the metal centres are related to the rate of electron transfer, and the temperature dependence of the IVCT parameters. Comparisons between the IVCT thermochromism properties of the diastereoisomers of $\left[\left\{\mathrm{Ru}(\mathrm{bpy})_{2}\right\}_{2}(\mu-\mathrm{BL})\right]^{5+}$ based on the "fused” bridging ligands dpb' and dpq' with those incorporating the "unfused" ligands dpb and 2,3-dpp will provide more detailed insights into the microscopic origins of the reorganisational and redox asymmetry contributions., 17, 91-99

(iii) Ion-pairing studies. Differential ion-pairing interactions with the diastereoisomers represent a crucial factor in the chromatographic separation of the forms. ${ }^{65,66}$ An investigation of the IVCT properties of the diastereoisomeric forms of $\left[\left\{\mathrm{Ru}(\mathrm{bpy})_{2}\right\}_{2}(\mu-\mathrm{BL})\right]^{5+}\{\mathrm{BL}=\mathrm{dpb}$ ', dpq' and dpb $\}$ in the presence of relatively strongly, $\mathrm{PF}_{6}^{-}$, and weakly, $\left\{\mathrm{B}\left(\mathrm{C}_{6} \mathrm{~F}_{5}\right)_{4}\right\}^{-}$, ion-pairing anions ${ }^{100}$ afford an analysis of 
ion-pairing effects at the molecular level. ${ }^{32-37,101-103}$ The examination of ion-pairing influences on the electrochemical properties of the diastereoisomers in the presence of $\mathrm{PF}_{6}{ }^{-}$and $\left\{\mathrm{B}\left(\mathrm{C}_{6} \mathrm{~F}_{5}\right)_{4}\right\}^{-}$provides insights into the sensitivity of the comproportionation constants $\left(\mathrm{K}_{\mathrm{c}}\right)$ to ion-pairing effects. The $\mathrm{K}_{\mathrm{c}}$ values are typically used to assess the degree of inter-metal electronic coupling, as discussed in §1.3.1.2.

\section{(4) Spin-Orbit Coupling ( $\left.\Delta E^{\prime}\right)$ Contributions.}

Gaussian deconvolution of the IVCT bands in the dinuclear ruthenium complexes provides information on the individual characteristics of the three underlying transitions (§1.5.3.5). The larger magnitude of the spin-orbit coupling for osmium results in three distinguishable IVCT components in $\left[\left\{\mathrm{Os}(\mathrm{bpy})_{2}\right\}_{2}(\mu-\mathrm{BL})\right]^{5+}\{\mathrm{BL}=\mathrm{dpb}$ ', dpq', ppz, dpb, 2,3-dpp $\}$, and the separation of the IC bands in the fully-oxidised species enables quantification of the $\Delta \mathrm{E}^{\prime}$ contribution to the electron transfer barrier.

\section{(5) The Effective Electron Transfer Distance $\left(r_{a b}\right)$.}

Stark effect spectroscopy on the meso diastereoisomers of $\left[\left\{\mathrm{Ru}(\mathrm{bpy})_{2}\right\}_{2}(\mu-\mathrm{BL})\right]^{5+}\{\mathrm{BL}=\mathrm{dpq}$, $\mathrm{dpb}\}$ and $\left[\left\{\mathrm{Os}(\mathrm{bpy})_{2}\right\}_{2}(\mu-\mathrm{BL})\right]^{5+}\{\mathrm{BL}=\mathrm{dpb}$ ', dpq', ppz, dpb $\}$ provides an independent determination of $\mathrm{H}_{\mathrm{ab}}$ via the measurement of the effective electron transfer distances (§1.5.3.2).

The present study addresses the limited experimental data which exist to probe the physical characteristics of polymetallic assemblies as a function of their stereochemical identity. This broadens the investigation of solvent reorganisational effects presented in Chapter 2, and provides a basis for extending the IVCT probe to trinuclear systems in Chapter 4. 


\subsection{Experimental}

\subsubsection{Materials}

Hydrated ruthenium trichloride $\left(\mathrm{RuCl}_{3} .3 \mathrm{H}_{2} \mathrm{O}\right.$; Strem, 99\%), 2,2'-bipyrimidine (bpm; Lancaster), 2,2'-bipyridine (bpy; Aldrich, 99+\%), 4,4'-dimethyl-2,2-bipyridine ( $\mathrm{Me}_{2} \mathrm{bpy}$; Aldrich), 2,3-bis(2pyridyl)pyrazine (2,3-dpp; Aldrich, 98\%), 2,3-diaminonaphthalene (Fluka), 4,7-phenanthroline (Aldrich), 3-nitrobenzenesulfonic acid ( $\mathrm{Na}^{+}$salt, Aldrich, 98\%), 2-methoxy-1,4-phenylenediamine hydrate (Aldrich, 95\%), glycerol (APS Finechem), sulfuric acid ( $\mathrm{H}_{2} \mathrm{SO}_{4}$; Ajax, 98\%), ammonium hydrogen carbonate $\left(\mathrm{NH}_{4} \mathrm{HCO}_{3}\right.$; May and Baker), stannous chloride $\left(\mathrm{SnCl}_{2} \cdot 2 \mathrm{H}_{2} \mathrm{O}\right.$, Ajax), lithium chloride (LiCl; Aldrich, 99+\%), ammonium chloride $\left(\mathrm{NH}_{4} \mathrm{Cl}\right.$; $\left.\mathrm{BDH}, 98 \%\right)$, ammonium hexafluorophosphate $\left(\mathrm{NH}_{4} \mathrm{PF}_{6}\right.$; Aldrich, 99.99\%), potassium hexafluorophosphate ( $\mathrm{KPF}_{6}$; Aldrich, 98\%), zinc chloride $\left(\mathrm{ZnCl}_{2} \cdot \mathrm{xH}_{2} \mathrm{O}\right.$; Fluka, 98\%), lithium tetrakis(pentafluorophenyl)borate $\left(\mathrm{Li}\left\{\mathrm{B}\left(\mathrm{C}_{6} \mathrm{~F}_{5}\right)_{4}\right\}\right.$. Et $\mathrm{t}_{2} \mathrm{O}$; Boulder Scientific), nitrosonium hexafluorophosphate ( $\mathrm{NOPF}_{6}$ stored under Ar; Alfa Aesar, 96\%), hydrazine hydrate (Aldrich), ethylene glycol (Ajax 95\%), sodium octanoate (Aldrich, 98\%), sodium benzoate (Aldrich, 98\%), sodium toluene4-sulfonate (sodium tosylate; Aldrich, 98\%), DOWEX ${ }^{\circledR} 1 \times 8,50-100$ mesh (Aldrich) and Amberlite ${ }^{\circledR}$ IRA-400 (Aldrich) $\mathrm{Cl}^{-}$anion exchange resins, Celite (Aldrich) and laboratory reagent solvents were used as received. Tetra-n-butylammonium hexafluorophosphate $\left(\left[\left(n-\mathrm{C}_{4} \mathrm{H}_{9}\right)_{4} \mathrm{~N}\right] \mathrm{PF}_{6}\right.$; Fluka, 99+\%) was dried in vacuo at $60^{\circ} \mathrm{C}$ prior to use and ferrocene (Fc; BDH) was purified by sublimation prior to use. SP Sephadex C-25, Sephadex LH-20 (Amersham Pharmacia Biotech), and silica gel (200-400 mesh , $60 \AA$, Aldrich) were employed for the chromatographic separation and purification of ruthenium complexes. ${ }^{65}$ Silica gel (Kieselgel 60H; 5-40 $\mu \mathrm{m}$ ) was used for vacuum liquid chromatography. ${ }^{104}$

Prior to use, acetonitrile $\left(\mathrm{CH}_{3} \mathrm{CN}\right.$; Aldrich, 99.9+\%) and propionitrile (PN; Aldrich) were distilled over $\mathrm{CaH}_{2}$, while acetone (BDH, HPLC grade) was distilled over $\mathrm{K}_{2} \mathrm{CO}_{3}$ and dichloromethane over $\mathrm{CaCl}_{2}$. n-Butyronitrile (BN; Aldrich, 99+\%), iso-butyronitrile ('BN; Aldrich), benzonitrile (BzN; Aldrich), 2,2,2-trifluoroethanol (Aldrich, 98\%) and 1-methyl-2-pyrrolidinone (Aldrich, 99.8\%) were used as received. $N, N$-Dimethylformamide (DMF; AR, Ajax) was stirred with 4A molecular sieves and distilled under reduced pressure $\left(76^{\circ} \mathrm{C}\right.$ at $\left.39 \mathrm{mmHg}\right)$, and ethylenediamine (Aldrich, 99\%) was freshly distilled from KOH immediately prior to use. ${ }^{105}$ Fuming nitric acid (>90\%) was prepared in an all-glass apparatus by the distillation of a mixture of $70 \% \mathrm{HNO}_{3}\left(100 \mathrm{~cm}^{3}\right)$ and $98 \% \mathrm{H}_{2} \mathrm{SO}_{4}\left(100 \mathrm{~cm}^{3}\right)$ at $125^{\circ} \mathrm{C}$. ${ }^{106}$

4,4',5,5'-Tetramethyl-2,2'-bipyridine ( $\mathrm{Me}_{4} \mathrm{bpy}$ ) and 2,5-bis(2-pyridyl)pyrazine (2,5-dpp) were supplied by Dr Bradley Patterson, ${ }^{107}$ and $\left(\mathrm{NH}_{4}\right)_{2}\left[\mathrm{Os}^{\mathrm{IV}} \mathrm{Cl}_{6}\right]$ was supplied by Dr Eric Jandrasics. [( $n$ $\left.\left.\mathrm{C}_{4} \mathrm{H}_{9}\right)_{4} \mathrm{~N}\right]\left\{\mathrm{B}\left(\mathrm{C}_{6} \mathrm{~F}_{5}\right)_{4}\right\},{ }^{100}$ was prepared by metathesis from $\mathrm{Li}\left\{\mathrm{B}\left(\mathrm{C}_{6} \mathrm{~F}_{5}\right)_{4}\right\} . \mathrm{Et}_{2} \mathrm{O}$ as described previously (§2.2.3). 


\subsubsection{Instrumentation and Physical Methods}

Details of the structural characterisation methods $\left({ }^{1} \mathrm{H}\right.$ NMR spectroscopy, elemental microanalyses) and instrumentation and physical measurements (electronic spectroscopy, electrochemistry, UV/Vis/NIR spectroelectrochemistry) are provided in §2.2.2.

\section{FT-IR Spectroelectrochemistry}

Infrared spectroelectrochemistry was performed with the assistance of Dr Stephen Best and Dr Tom Behrsing in the School of Chemistry at the University of Melbourne using the reflection/absorptionspectroelectrochemical cell described previously, ${ }^{108,109}$ which incorporates a multi-electrode assembly comprising 1 and $3 \mathrm{~mm}$ diameter Pt working electrodes, a Pt ring counter electrode and a $2 \mathrm{~mm}$ diameter Ag wire pseudo-reference electrode. Samples (ca. $1.5 \times 10^{-3} \mathrm{M}$ complex and $0.1 \mathrm{M}\left[\left(n-\mathrm{C}_{4} \mathrm{H}_{9}\right)_{4} \mathrm{~N}\right] \mathrm{PF}_{6}$ as the supporting electrolyte) were prepared using anhydrous $\mathrm{CH}_{3} \mathrm{CN}$ and thoroughly deoxygenated with an $\mathrm{N}_{2}$ stream prior to each measurement.

Cyclic voltammetry measurements were initially performed at the $1 \mathrm{~mm}$ working electrode to examine the electrochemical properties of the solution independently from the $3 \mathrm{~mm}$ electrode used for the spectroelectrochemical measurements. The cell potential was controlled using a Princeton Applied Research model 362 scanning potentiostat which was interfaced with EChem software (Version 1.5.2, AD Instruments).

The spectroelectrochemical measurements were conducted using a BioRad FTS 175C FT-IR spectrometer using a $3 \mathrm{~mm}$ working electrode, and the potentiostat was coupled to a digital multimeter to permit monitoring of the current throughout the electrolysis. Near-infrared (NIR) spectra (2000$8000 \mathrm{~cm}^{-1}$ ) were collected with a W-lamp source, $\mathrm{Si} / \mathrm{CaF}_{2}$ beamsplitter, and a high-sensitivity narrowband MCT detector cooled with liquid nitrogen, while mid-IR spectra $\left(1000-4000 \mathrm{~cm}^{-1}\right)$ were obtained with a Glowbar source and $\mathrm{Ge} / \mathrm{KBr}$ beamsplitter using the MCT detector.

The spectral characteristics of the electrogenerated mixed-valence $(+5)$ species were followed by applying appropriate potentials determined from the preliminary electrochemical measurements, intermediate between the two metal-based redox processes. Following the generation of the fullyoxidised (+6) species, the cell potential was reversed to regenerate the +4 species. Identical time-frames were used for the collection of the NIR and mid-IR spectra to permit seamless visualisation of the spectral progressions over the region from 1000 to $8000 \mathrm{~cm}^{-1}$.

For selected systems, FT-IR spectra were also recorded on a Perkin Elmer FT-IR spectrometer over the range $1000-7000 \mathrm{~cm}^{-1}$ using a liquid FT-IR cell which incorporated $\mathrm{CaF}_{2}$ windows. The mixedvalence species were generated by chemical oxidation with $\mathrm{NOPF}_{6}$ in $\mathrm{CD}_{3} \mathrm{CN}$, and their FT-IR spectra were recorded following the sequential addition of small aliquots of the oxidant to a solution of the +4 species (ca. $3 \mathrm{mg}$ in $0.5 \mathrm{~cm}^{3} \mathrm{CD}_{3} \mathrm{CN}$ ). 


\section{UV/Vis/NIR Spectroelectrochemisty}

The instrumentation and details for the spectroelectrochemical generation of the mixed-valence species are detailed in §2.2.2. Solutions for the spectroelectrochemical experiments typically contained $\left[\left(n-\mathrm{C}_{4} \mathrm{H}_{9}\right)_{4} \mathrm{~N}\right] \mathrm{PF}_{6}(0.1 \mathrm{M})$ supporting electrolyte and the complex $\left(\mathrm{ca} .10^{-3} \mathrm{M}\right)$ under investigation in the given solvent, while solutions for the solvatochromism and thermochromism measurements were performed using a constant, low concentration of both the complex $\left(0.4 \times 10^{-3} \mathrm{M}\right)$ and supporting electrolyte (0.02 M $\left.\left[\left(n-\mathrm{C}_{4} \mathrm{H}_{9}\right)_{4} \mathrm{~N}\right]\left\{\mathrm{B}\left(\mathrm{C}_{6} \mathrm{~F}_{5}\right)_{4}\right\}\right)$ in order to minimise inhomogeneous broadening effects due to ion-pairing. ${ }^{100,110,111}$ All solutions were purged with $\mathrm{N}_{2}$ prior to transference (via syringe) into the OSTLE cell. The low temperature facility enabled in situ spectroelectrochemical characterisation of the electrogenerated species which were otherwise unstable at room temperature. The temperature was stabilised to $\pm 0.3^{\circ} \mathrm{C}$ prior to commencing electrolysis. The dinuclear systems required approximately $6 \mathrm{~h}$ for data collection at $-35^{\circ} \mathrm{C}$.

To minimise artefacts in the NIR spectral data due to ion-pairing and concentration which are known to influence the IVCT transitions of dinuclear complexes ${ }^{36,37,69,103,112}$ the spectra were measured using a constant concentration of complex under identical conditions of electrolyte and temperature.

\section{Analysis of Spectroelectrochemical Data}

The analysis and spectral deconvolution of the data were performed as described previously (§2.2.2). For asymmetrically-shaped IVCT bands, the application of the equations derived from the classical analysis of $\mathrm{Hush}^{2}$ are not valid as they assume a Gaussian band-shape. Accordingly, an examination of the first-order moments $\left(M_{1}\right)$ for the absorption bands are required, rather than the experimentally-observed quantities $\left\{v_{\max }, \Delta v_{1 / 2}\right.$ and $\left.(\varepsilon / v)_{\max }\right\} . M_{1}$ defines the average energy of the absorption manifold. While an examination of the moments to first-order (i.e. $M_{1}$ ) only was undertaken in the present study, the consideration of the higher-order moments would be necessary in an exact treatment. $f(v)$ is the line-shape function of the reduced absorption spectrum $\{(\varepsilon / v) v s . v\}$ and the limits of the region are $v_{1}$ and $v_{2}$. The denominator in equation 3.3 is the area under the band of the reduced absorption spectrum, i.e. the zeroth-moment, $M_{0}$. For a Gaussian-shaped band, $M_{0}$ is approximated as $\left\{(\varepsilon / v)_{\max } \Delta v_{1 / 2} / v_{\max }\right\}$.

$$
M_{1}=\frac{\int_{v_{1}}^{v_{2}} f(v) v d v}{\int_{v_{1}}^{v_{2}} f(v) d v}
$$

The transition moment $\left(\left|\mu_{12}\right|\right.$ in $\left.\mathrm{e} \AA\right)$ is subsequently defined as

$$
\left|\mu_{12}\right|=0.0206 \AA \times M_{0}^{1 / 2}
$$




\section{Stark Absorption Spectroscopy of Mixed-Valence Compounds}

Electroabsorption (electronic Stark effect) measurements were performed by the author with the assistance of Dr Peter Dinolfo and Dr Keith Walters under the supervision of Prof. Joseph Hupp in the Centre for Nanofabrication and Materials Science, Northwestern University, Evanston, IL, USA.

(i) Sample Preparation and Absorption Measurements. In a typical experiment, the un-oxidised complex (ca. $5 \mathrm{mg}$ ) was dissolved in $n$-butyronitrile $\left(1 \mathrm{~cm}^{3}\right)$ and the mixed-valence species was generated via chemical oxidation with $\mathrm{NOPF}_{6}$ \{ saturated solution in $n$-butyronitrile $\left(1 \mathrm{~cm}^{3}\right)$ \}.

The spectrum of the sample solution was recorded \{with background subtraction of the glassing solvent ( $n$-butyronitrile) $\}$ in a $1 \mathrm{~mm}$ quartz cuvette mounted in the path of the CARY 5E UV/Vis/NIR spectrophotometer interfaced to Varian WinUV software. The oxidising agent was added to the sample solution via syringe in $0.01 \mathrm{~cm}^{3}$ aliquots, and the absorption spectrum monitored between each addition until the absorbance of the NIR band was maximised (to $c a$. 1 absorbance unit). The solutions were filtered through a $0.22 \mu \mathrm{m}$ teflon filter and loaded rapidly into the electroabsorption cell by capillary action. The film was glassed immediately by immersion in liquid nitrogen within the Stark dewar. Due to rapid disproportionation of the mixed-valence species in solution (which was significantly greater for the ruthenium systems relative to their osmium analogues), this procedure was performed within 1-2 min at ambient temperature. Exposure to air was minimised during the manipulations.

Stark absorption measurements were performed in a dual liquid nitrogen immersion cryostat (Janis Research Corporation) at $77 \mathrm{~K} .{ }^{113}$ The electroabsorption cell consisted of two indium-tin oxide (ITO) coated quartz slides (Delta Technologies) separated by $100 \mu \mathrm{m}$ Kapton spacers (DuPont). The preparation of the electrodes has been detailed by Dinolfo et al. ${ }^{114}$

The absorption spectrum of the solvent matrix was initially measured for use as a background subtraction and for conformation of the cell pathlength. The latter was measured interferometrically via equation 3.5, where $\lambda_{1}$ and $\lambda_{2}$ are two adjacent fringe peaks, $\eta$ is the refractive index of $n$-butyronitrile (1.38) and the thickness of the cell is in $\mathrm{nm} .{ }^{115}$

$$
\text { Thickness }=\frac{\lambda_{1} \lambda_{2}}{2 \eta\left(\lambda_{2}-\lambda_{1}\right)}
$$

(ii) Stark Absorption Measurements. Electroabsorption measurements were performed at $77 \mathrm{~K}$ and a field strength of $4.0 \mathrm{MVcm}^{-1}$ using a CARY-14 spectrophotometer featuring OLIS control software. Dry nitrogen gas was blown over the dewar windows to prevent fogging. A detailed account of the instrumentation is provided elsewhere. ${ }^{114,116}$

Electroabsorption measurements over the range $3850-12500 \mathrm{~cm}^{-1}$ were recorded with a photovoltaic HgCdTe detector that was thermoelectrically cooled to $-40^{\circ} \mathrm{C}$ (Judson Technologies), and a DE110 Silicon photodiode for measurements over the range $9090-20000 \mathrm{~cm}^{-1}$. Stark absorption spectra 
for the IVCT bands of meso- $\left[\left\{\mathrm{Ru}(\mathrm{bpy})_{2}\right\}_{2}(\mu-\mathrm{BL})\right]^{5+}\{\mathrm{BL}=\mathrm{dpb}, \mathrm{dpq}\}^{*}$ were obtained over the range $3850-12500 \mathrm{~cm}^{-1}$, while the spectra of meso- $\left[\left\{\mathrm{Os}(\mathrm{bpy})_{2}\right\}_{2}(\mu-\mathrm{BL})\right]^{5+}\{\mathrm{BL}=\mathrm{dpb}, \mathrm{dpb}$ ', dpq', ppz $\}$ were obtained over the range $3850-20000 \mathrm{~cm}^{-1}$.

In a typical experiment, the sinusoidal oscillating electric field was applied across the sample, and the field dependent and independent transmittance signals were detected over the given spectral range. The field dependent signal was demodulated using a lock-in amplifier (Stanford Research Systems SRS 850) locked at twice the field modulation frequency (typically $200 \mathrm{~Hz}$ ). The $\Delta \mathrm{A}(v)$ spectrum was derived from equation 3.6, where $V_{\mathrm{AC}}$ and $V_{\mathrm{DC}}$ are the AC (change in absorption) and DC (unperturbed absorption) signals, respectively. ${ }^{117}$

$$
\Delta \mathrm{A}=-\log \left(\frac{2 \sqrt{2} V_{\mathrm{AC}}}{V_{\mathrm{DC}}}+1\right)
$$

Measurements were collected at 90 and $45^{\circ}$ with the orientation of the sample set by rotation of the mounting rod. Measurements were duplicated at each angle.

(iii) Analysis of Stark Absorption Data. Analysis of the data was performed according to the method of Liptay ${ }^{118}$ as described in detail elsewhere ${ }^{114,116,119}$ and outlined in §1.5.3.2. Briefly, each Stark spectrum \{obtained from duplicate experiments at two angles $\left(90\right.$ and $\left.\left.45^{\circ}\right)\right\}$ was fitted to a linear combination of the zeroth-, first- and second-order derivatives of the absorption spectrum (at $77 \mathrm{~K}$ ) to yield values for the coefficients, $\mathrm{A}_{\chi}, \mathrm{B}_{\chi}$ and $\mathrm{C}_{\chi}$ in equation 1.28. The molecular parameters were subsequently obtained from equation 1.29. A value of 1.3 was assumed for the local-field correction factor $(f)$ in equation $1.27 .^{120}$

\subsubsection{Synthetic Procedures}

\subsubsection{Ligand Synthesis}

2,3-bis(2-pyridyl)-1,4-benzoquinoxaline (dpb), ${ }^{67}$ dipyrido(2,3-a;3',2'-c)benzophenazine $(\mathrm{dpb}),{ }^{68,69}$ and dipyrido(2,3-a;3',2'-c)phenazine $\left(\mathrm{dpq}^{\prime}\right)^{68}$ were prepared according to the literature methods.

4,7-Phenanthrolino-5,6:5',6'-pyrazine (ppz). The synthesis involves a three-stage procedure:

(a) 5-Methoxy-4,7-phenanthroline hydrogen sulfate was prepared via a modification of the literature procedure. ${ }^{121}$ A two-necked $3 \mathrm{dm}^{3}$ round bottom flask was fitted with a mechanical stirrer and condenser, and 3-nitrobenzenesulfonic acid ( $\mathrm{Na}^{+}$salt; $\left.100 \mathrm{~g}, 0.444 \mathrm{~mol}\right)$, 2-methoxy-1,4pheneylenediaminesulfate ( $50 \mathrm{~g}, 0.212 \mathrm{~mol}$ ) and glycerol (934.7 g, $9.53 \mathrm{~mol}$ ) were added to the flask. A solution of $98 \% \mathrm{H}_{2} \mathrm{SO}_{4}\left(510 \mathrm{~cm}^{3}\right)$ in $\mathrm{H}_{2} \mathrm{O}\left(270 \mathrm{~cm}^{3}\right)$ was prepared, allowed to cool to room temperature, and added in a single portion. The mixture was heated at reflux $\left(\sim 140^{\circ} \mathrm{C}\right)$ for $6 \mathrm{~h}$, during which time the

\footnotetext{
${ }^{*}$ Attempts to apply the technique to meso-[\{Ru(bpy $\left.\left.)_{2}\right\}_{2}(\mu-\mathrm{BL})\right]^{5+}\{\mathrm{BL}=\mathrm{dpb}$ ', ppz $\}$ were unsuccessful due to rapid disproportionation of the mixed-valence species.
} 
mixture attained a dark red colouration. The solution was cooled to room temperature, poured over ice (540 g) and basified to $\mathrm{pH} 10$ with 50\% $\mathrm{NaOH}\left(c a .1200 \mathrm{~cm}^{3}\right)$. The aqueous solution was extracted with chloroform until the extracts were pale yellow. Since a large amount of solid $\mathrm{Na}_{2} \mathrm{SO}_{4}$ precipitated during the extraction, the solids were filtered off and washed with chloroform until the organic extracts were pale yellow. The extracts were combined, dried over anhydrous $\mathrm{Na}_{2} \mathrm{SO}_{4}$ and the solvent removed in vacuo. The resulting dark red oil was evaporated to a paste in the fumehood.

The isolation of the solid as the hydrogen sulfate salt was conducted according to a modification of the method of Bonhôte. ${ }^{122}$ The residue was dissolved in a minimum volume of methanol $\left(120 \mathrm{~cm}^{3}\right)$ and $98 \% \mathrm{H}_{2} \mathrm{SO}_{4}$ added to precipitate a brown solid. The precipitate was isolated by vacuum filtration, washed with ethanol $\left(50 \mathrm{~cm}^{3}\right)$ followed by diethyl ether $\left(20 \mathrm{~cm}^{3}\right)$ and dried in vacuo. Yield: $40.6 \mathrm{~g}$ (60\%). The ${ }^{1} \mathrm{H}$ NMR spectrum and melting point were identical to those reported previously. ${ }^{122}$

(b) 4,7-phenanthroline-5,6-dione was prepared according to a modification of the literature method. ${ }^{122}$ 5-Methoxy-4,7-phenanthroline hydrogen sulfate (40.63 g; $0.132 \mathrm{~mol}$ ) was dissolved in 98\% $\mathrm{H}_{2} \mathrm{SO}_{4}\left(150 \mathrm{~cm}^{3}\right)$. With ice bath cooling, fuming $\mathrm{HNO}_{3}\left(60 \mathrm{~cm}^{3}\right)$ was added dropwise with stirring. The mixture was refluxed at $96^{\circ} \mathrm{C}$ for $10 \mathrm{~h}$, then cooled to room temperature, poured onto ice (ca. $900 \mathrm{~g}$ ), and neutralised to $\mathrm{pH}$ 6.8-7.0 ( $\mathrm{pH}$ meter) by the stepwise addition of ammonium bicarbonate, maintaining the temperature below $10^{\circ} \mathrm{C}$ throughout the procedure. After $2 \mathrm{~h}$, the pale yellow precipitate was isolated by vacuum filtration, washed with chilled $\mathrm{H}_{2} \mathrm{O}\left(1500 \mathrm{~cm}^{3}\right)$, acetone $\left(250 \mathrm{~cm}^{3}\right)$ and diethyl ether $\left(250 \mathrm{~cm}^{3}\right)$, and dried in vacuo overnight. Yield: $24.0 \mathrm{~g}$ (86\%). The ${ }^{1} \mathrm{H}$ NMR and melting point were identical to those reported previously. ${ }^{122}$

(c) 4,7-phenanthrolino-5,6:5',6'-pyrazine (ppz) was prepared according to a modification of the literature procedures. ${ }^{121,122}$ 4,7-phenanthroline-5,6-dione (21.95 g, $0.104 \mathrm{~mol}$ ) was dissolved in methanol $\left(380 \mathrm{~cm}^{3}\right)$ at $30^{\circ} \mathrm{C}$. Freshly distilled ethylenediamine $\left(7.3 \mathrm{~cm}^{3}, 0.110 \mathrm{~mol}\right)$ was added in a single portion, at which point the mixture attained a red colouration. The reaction was stirred at $30^{\circ} \mathrm{C}$ for $2 \mathrm{~h}$, and the solvent removed by rotary evaporation to yield an orange solid. The residue was dissolved in $\mathrm{H}_{2} \mathrm{O}$ (500 $\left.\mathrm{cm}^{3}\right)$ and extracted with chloroform $\left(3 \times 100 \mathrm{~cm}^{3}\right)$. The solution volume was reduced in vacuo and chromatographed on silica gel via gradient elution using $10 \%$ methanol $/ 1 \% \mathrm{NH}_{4} \mathrm{OH}$ in dichloromethane. ppz was isolated as the light yellow band which eluted prior to a deep purple band (which presumably contained the non-aromatic tetrahydroquinoxaline analogue). ${ }^{68}$ The solvent was removed in vacuo and the beige solid washed with acetone. Yield: $15.7 \mathrm{~g}$ (65\%). The ${ }^{1} \mathrm{H}$ NMR and melting point were identical to those reported previously. ${ }^{121,122}$

\subsubsection{Synthesis of Mononuclear Precursors}

The mononuclear ruthenium complexes $\left[\mathrm{Ru}(\mathrm{DMSO})_{4} \mathrm{Cl}_{2}\right],{ }^{123}$ cis- $\left[\mathrm{Ru}(\mathrm{pp})_{2} \mathrm{Cl}_{2}\right] \cdot 2 \mathrm{H}_{2} \mathrm{O}\{\mathrm{pp}=\mathrm{bpy}$, $\left.\mathrm{Me}_{2} \mathrm{bpy}\right\},{ }^{124}$ and cis-[Ru(Me$\left.\left(\mathrm{Me}_{4} \mathrm{bpy}\right)_{2} \mathrm{Cl}_{2}\right]^{125}$ were synthesised according to the literature methods. 


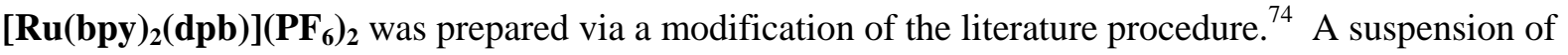
dpb (64.2 mg, $0.192 \mathrm{mmol})$ in ethylene glycol $\left(4 \mathrm{~cm}^{3}\right)$ was heated in a modified microwave oven on medium high power for $20 \mathrm{~s}$ to complete dissolution. cis-[Ru(bpy $\left.)_{2} \mathrm{Cl}_{2}\right] \cdot 2 \mathrm{H}_{2} \mathrm{O}(100 \mathrm{mg}, 0.192 \mathrm{mmol})$ was added and the mixture heated at reflux for a further 2 min on medium-high power during which time the solution attained a deep purple colouration. The mixture was diluted with water $\left(c a .50 \mathrm{~cm}^{3}\right)$ and separation of the desired mononuclear product from the crude mixture was achieved via a gradient elution procedure on SP Sephadex C-25 support using aqueous 0.1-0.3 $\mathrm{M} \mathrm{NaCl}$ solution as the eluent. The major claret-red band eluted with $0.2 \mathrm{M} \mathrm{NaCl}$, and the complex was precipitated by the addition of saturated aqueous $\mathrm{KPF}_{6}$. The purple solid was isolated by vacuum filtration, washed with chilled water $\left(3 \mathrm{~cm}^{3}\right)$ followed by copious diethyl ether, and dried in vacuo. Yield: $178 \mathrm{mg}$ (89\%). The ${ }^{1} \mathrm{H}$ NMR and UV/Vis spectral characteristics were identical to those reported previously. ${ }^{74}$ Anal. Calcd for $\mathrm{C}_{42} \mathrm{H}_{30} \mathrm{~F}_{12} \mathrm{~N}_{8} \mathrm{P}_{2} \mathrm{Ru}$ : C, 48.6; H, 2.91; N, 10.8\%. Found: C, 48.4; H, 2.90; N, 10.8\%.

$\left[\mathbf{R u}(\mathbf{b p y})_{\mathbf{2}}\left(\mathbf{d p b}^{\prime}\right)\right]\left(\mathbf{P F}_{\mathbf{6}}\right)_{2}$ was prepared according to the method described above for $\left[\mathrm{Ru}(\mathrm{bpy})_{2}(\mathrm{dpb})\right]\left(\mathrm{PF}_{6}\right)_{2}$ using dpb' (128 mg, $0.384 \mathrm{mmol})$ and cis-[Ru(bpy $\left.)_{2} \mathrm{Cl}_{2}\right] .2 \mathrm{H}_{2} \mathrm{O}(200 \mathrm{mg}, 0.384 \mathrm{mmol})$. The mononuclear complex was precipitated as green solid. Yield: $358 \mathrm{mg}$ (90\%). Anal. Calcd for $\mathrm{C}_{42} \mathrm{H}_{28} \mathrm{~F}_{12} \mathrm{~N}_{8} \mathrm{P}_{2} \mathrm{Ru}$ : C, 48.7; H, 2.72; N, 10.8\%. Found: C, 48.5; H, 2.71; N, 10.8\%.

cis-[Os(bpy $\left.)_{2} \mathbf{C l}_{2}\right] .2 \mathbf{H}_{2} \mathbf{O}$ was prepared via a modification of the literature procedure. ${ }^{126,127}$ $\left(\mathrm{NH}_{4}\right)_{2}\left[\mathrm{Os}^{\mathrm{IV}} \mathrm{Cl}_{6}\right](1.10 \mathrm{~g}, 2.51 \mathrm{mmol})$ was suspended in anhydrous DMF $\left(20 \mathrm{~cm}^{3}\right)$ and refluxed at $160^{\circ} \mathrm{C}$ with bpy (0.822 g, $5.26 \mathrm{mmol}$ ) for $1 \mathrm{~h}$. During this time, crystals of $\mathrm{NH}_{4} \mathrm{Cl}$ began to separate (after ca. 15 min) and the initially dark red solution attained a brown colouration. After $1 \mathrm{~h}$, the solution was filtered to remove $\mathrm{NH}_{4} \mathrm{Cl}$ and anhydrous methanol $\left(15 \mathrm{~cm}^{3}\right)$ added. cis-[Os $\left.{ }^{\mathrm{III}}(\mathrm{bpy})_{2} \mathrm{Cl}_{2}\right] \mathrm{Cl}$ was precipitated from the filtrate as a light brown solid by addition of diethyl ether $\left(10 \mathrm{~cm}^{3}\right)$. The solid was isolated by vacuum filtration and washed with diethyl ether $\left(3 \times 10 \mathrm{~cm}^{3}\right)$. Yield: $1.50 \mathrm{~g}(98 \%)$. The ${ }^{1} \mathrm{H}$ NMR, UV/Vis spectral characteristics were identical to those reported previously. ${ }^{126,127}$

The cis-[Os ${ }^{\text {III }}$ (bpy) $\left.{ }_{2} \mathrm{Cl}_{2}\right] \mathrm{Cl}$ product (1.00 g, $1.64 \mathrm{mmol}$ ) was dissolved in a minimum volume of warm methanol (ca. $100 \mathrm{~cm}^{3}$ ) and hydrazine monohydrate (5 drops) added dropwise with stirring to yield a dark purple/black solution. Addition of diethyl ether $\left(100 \mathrm{~cm}^{3}\right)$ and ice-bath cooling afforded a deep red/purple microcrystalline product of cis-[Os $\left.{ }^{\mathrm{II}}(\mathrm{bpy})_{2} \mathrm{Cl}_{2}\right]$ which was isolated by vacuum filtration and washed with diethyl ether $\left(3 \times 10 \mathrm{~cm}^{3}\right)$. Yield: $0.661 \mathrm{~g}(75 \%)$. The ${ }^{1} \mathrm{H}$ NMR, UV/Vis spectral characteristics were identical to those reported previously. ${ }^{126,127}$

\subsubsection{Synthesis of Dinuclear Complexes, and Separation of Diastereoisomers}

A detailed account of the microwave techniques and column chromatographic procedures employed for the separation and purification of the diastereoisomers is provided in §2.2.3. 
$\left[\left\{\mathrm{Ru}(\mathrm{bpy})_{2}\right\}_{2}(\mu\right.$-dpop) $]\left(\mathrm{PF}_{6}\right)_{4}$ was supplied by Dr Dana Dattelbaum and Prof. Tom Meyer (Los Alamos National Laboratory, U.S.A.). $\left[\left\{\mathrm{Ru}(\mathrm{bpy})_{2}\right\}_{2}(\mu-\mathrm{BL})\right]\left(\mathrm{PF}_{6}\right)_{4}\{\mathrm{BL}=\mathrm{dpo}$, dpt $\}$ were supplied by Prof. Peter Steel ${ }^{71}$ (University of Canterbury, New Zealand). [\{Ru(bpy $\left.)_{2}\right\}_{2}(\mu$-dpo $\left.)\right]\left(\mathrm{PF}_{6}\right)_{4}$ was also synthesised via the alternate method reported below.

\section{(i) Dinuclear Ruthenium Complexes Incorporating Angular Bridging Ligands}

[\{Ru(bpy $\left.\left.)_{2}\right\}_{2}(\mu-\mathbf{d p b})\right]\left(\mathbf{P F}_{6}\right)_{4}$. A suspension of dpb (44.1 $\left.\mathrm{mg}, 0.132 \mathrm{mmol}\right)$ in ethylene glycol $\left(3 \mathrm{~cm}^{3}\right)$ was heated in a modified microwave oven on medium high power for $20 \mathrm{~s}$ to complete dissolution. cis[Ru(bpy) $\left.)_{2} \mathrm{Cl}_{2}\right] \cdot 2 \mathrm{H}_{2} \mathrm{O}(150 \mathrm{mg}, 0.288 \mathrm{mmol}$ ) was added and the mixture heated at reflux for a further $8 \mathrm{~min}$ on high power during which time the solution attained a dark green colouration. The mixture was diluted in water $\left(c a .50 \mathrm{~cm}^{3}\right)$ and the dinuclear product was separated from the crude mixture via a gradient elution procedure using aqueous $0.1-0.5 \mathrm{M} \mathrm{NaCl}$ as the eluent. A claret-red band of mononuclear material eluted first $(0.2 \mathrm{M} \mathrm{NaCl})$ followed by the desired dark green product $(0.4 \mathrm{M} \mathrm{NaCl})$ which was precipitated as the $\mathrm{PF}_{6}^{-}$salt by addition of a saturated solution of aqueous $\mathrm{KPF}_{6}$. The solid was isolated by vacuum filtration and washed with diethyl ether $\left(3 \times 10 \mathrm{~cm}^{3}\right)$. Yield: $207 \mathrm{mg}(90 \%)$. A sample for microanalysis was purified by passage through a short column (dimensions $20 \mathrm{~cm}$ length $\times 2 \mathrm{~cm}$ diameter) of Sephadex LH-20 (eluent 50\% methanol-acetone). Anal. Calcd for $\mathrm{C}_{62} \mathrm{H}_{46} \mathrm{~F}_{24} \mathrm{~N}_{12} \mathrm{P}_{4} \mathrm{Ru}_{2}$ : C, 42.8; H, 2.66; N, 9.65\%. Found: C, 42.7; H, 2.60; N, 9.42\%.

Separation of the diastereoisomers was achieved by cation exchange chromatography on SP Sephadex C-25 support (dimensions $96 \mathrm{~cm}$ length $\times 1.6 \mathrm{~cm}$ diameter). The complex (ca. $100 \mathrm{mg}$ ) was loaded onto the column in aqueous solution (as the $\mathrm{Cl}^{-}$form, obtained by stirring an aqueous suspension of the complex with DOWEX ${ }^{\circledR}$ anion exchange resin) and eluted with $0.25 \mathrm{M}$ sodium tosylate solution. ${ }^{128}$ The diastereoisomers separated after passing through an effective column length of $2 \mathrm{~m}$. The two bands were collected, saturated aqueous $\mathrm{KPF}_{6}$ solution added and the products extracted with dichloromethane. The organic extracts were dried with anhydrous $\mathrm{Na}_{2} \mathrm{SO}_{4}$ and the solvent removed by rotary evaporation.

Rigorous purification methods were employed prior to characterisation and physical measurements due to the potentially strong associations between the complex cations and the anions present in the eluents employed in the chromatographic separations. ${ }^{128,129}$ Each product was dissolved in a minimum volume of acetone and loaded onto a short column of silica gel (dimensions $3 \times 2.5 \mathrm{~cm}$ ), washed with acetone, water and acetone and eluted with acetone containing $5 \% \mathrm{NH}_{4} \mathrm{PF}_{6}$. Addition of water and removal of the acetone under reduced pressure afforded dark green solids which were collected by filtration through Celite and washed with diethyl ether $\left(3 \times 5 \mathrm{~cm}^{3}\right)$. In each case the precipitate was washed off the Celite into a test tube with acetone $\left(c a .2 \mathrm{~cm}^{3}\right)$, evaporated under a stream of dry $\mathrm{N}_{2}$ and dried in vacuo for $3 \mathrm{~h}$ at $50^{\circ} \mathrm{C}$. Bands 1 and 2 were determined to be the meso and rac diastereoisomers, respectively, as established by X-ray crystallography and NMR characterisation. ${ }^{1} \mathrm{H}$ NMR $(\delta$ ppm; 
$\mathrm{CD}_{3} \mathrm{CN}$ ): (Band 1; meso $\left.{ }^{*}\right) 6.58$ (H6d, 1H, $J=5,1.5 \mathrm{~Hz}$, dd), 6.97 (H6d', 1H, $J=5,1.5 \mathrm{~Hz}$, dd), 7.18 (H5d, 1H, $J=8,5$ Hz, dd), 7.26 (H5d', 1H, $J=8,5$ Hz, dd), 7.38-7.68 (10H, m), 7.72-8.40 (12H, m), 8.49 (H3d, 1H, $J=8,1.5 \mathrm{~Hz}$, dd), 8.58 (H3d', 1H, $J=8,1.5 \mathrm{~Hz}, \mathrm{dd}$ ), 8.60 (H3a, 1H, $J=8,1.5 \mathrm{~Hz}, \mathrm{dd}$ ), 8.75 (H3c, H3a', 2H, $J=8,1.5$ Hz, dd), 8.80 (H3c', 1H, $J$ = 8, 1.5 Hz, dd); (Band 2; rac) 7.09 (H5b, 2H, $J=8$, 5 Hz, dd), 7.34 (H3/6 dpb, 2H, $J=10,8$ Hz, dd), 7.45 (H5c, H5d, 4H, $J=8,5$ Hz, dd), 7.53 (H4/5 dpb, 2H, $J=10,8 \mathrm{~Hz}, \mathrm{dd}), 7.62$ (H5a, 2H, $J=8,5 \mathrm{~Hz}, \mathrm{dd}), 7.64$ (H6d, 2H, $J=5,1.5 \mathrm{~Hz}, \mathrm{dd}$ ), 7.70 (H6b, 2H, $J$ = 5, $1.5 \mathrm{~Hz}, \mathrm{dd}$ ), 7.79 (H6c, 2H, $J=5,1.5 \mathrm{~Hz}, \mathrm{dd}), 8.00$ (H4a, 2H, $J=8,8 \mathrm{~Hz}, \mathrm{dd}$ ), 8.06 (H4d, 2H, $J=8$, $8 \mathrm{~Hz}, \mathrm{dd}), 8.06$ (H2/7 dpb, 2H, s), 8.07-8.10 (6H, m), 8.11 (H4b, 2H, $J=8,8 \mathrm{~Hz}, \mathrm{dd}), 8.20$ (H4c, 2H, $J=$ 8, $8 \mathrm{~Hz}, \mathrm{dd}$ ), 8.23 (H6a, 2H, $J=5,1.5 \mathrm{~Hz}, \mathrm{dd}), 8.31$ (H3a, 2H, $J=8,1.5 \mathrm{~Hz}, \mathrm{dd}), 8.33$ (H3b, 2H, $J=8$, $1.5 \mathrm{~Hz}, \mathrm{dd}), 8.67$ (H13/14 dpb, 2H, dd), 8.68 (H3d, 2H, $J=8,1.5 \mathrm{~Hz}, \mathrm{dd}), 8.71$ (H3c, 2H, $J=8,1.5 \mathrm{~Hz}$, dd).

$\left[\left\{\mathbf{R u}(\mathbf{b p y})_{2}\right\}(\mu-\mathbf{d p b})\left\{\mathbf{R u}\left(\mathbf{M e}_{2} \mathbf{b p y}\right)_{2}\right\}\right]\left(\mathbf{P F}_{6}\right)_{4}$ was synthesised via an adaptation of the literature procedure for $\left[\left\{\mathrm{Ru}(\mathrm{bpy})_{2}\right\}(\mu\right.$-HAT $\left.)\left\{\mathrm{Ru}(\mathrm{phen})_{2}\right\}\right]\left(\mathrm{PF}_{6}\right)_{4}{ }^{130}\left[\mathrm{Ru}(\mathrm{bpy})_{2}(\mathrm{dpb})\right]\left(\mathrm{PF}_{6}\right)_{2}(60 \mathrm{mg}, 0.0578 \mathrm{mmol})$ was added to a $\mathrm{N}_{2}$-purged solution of aqueous methanol $\left(1: 1,40 \mathrm{~cm}^{3}\right)$ and dissolved by heating the mixture at reflux for $5 \mathrm{~min}$. [Ru( $\left.\mathrm{Me}_{2} \mathrm{bpy}\right)_{2} \mathrm{Cl}_{2}$ ] (33.4 mg, $0.0578 \mathrm{mmol}$ ) was added and the solution heated at reflux for $16 \mathrm{~h}$. During this time the initially purple solution attained a green colouration, suggesting the formation of a dinuclear species. The progress of the reaction was monitored by the TLC analysis of small aliquots of the reaction mixture during the synthesis using thin-layer silica gel plates with $0.1 \mathrm{M} \mathrm{NH}_{4} \mathrm{Cl}$ in 4:1 $\mathrm{H}_{2} \mathrm{O} / \mathrm{DMF}$. The mixture was cooled to room temperature and the methanol removed by rotary evaporation. A green solid was precipitated on addition of a saturated solution of aqueous $\mathrm{KPF}_{6}$ and the mixture chilled by refrigeration overnight. The solid was isolated by filtration and washed with diethyl ether.

Isolation of the desired dinuclear product from the crude mixture (converted to the $\mathrm{Cl}^{-}$form by stirring an aqueous suspension of the complex with DOWEX ${ }^{\circledR}$ anion exchange resin) was achieved by cation-exchange chromatography on SP Sephadex C-25 using a gradient elution procedure with 0.1-0.5 M $\mathrm{NaCl}$ solution. A claret-red band of unreacted mononuclear material eluted first (0.1-0.2 M NaCl) followed by the desired dark green product $(0.4 \mathrm{M} \mathrm{NaCl})$ which was precipitated as the $\mathrm{PF}_{6}{ }^{-}$salt by addition of a saturated solution of aqueous $\mathrm{KPF}_{6}$. The dinuclear complex was isolated by vacuum filtration and washed with diethyl ether $\left(3 \times 10 \mathrm{~cm}^{3}\right)$. Yield: $80.1 \mathrm{mg}(77 \%)$. Anal. Calcd for $\mathrm{C}_{66} \mathrm{H}_{54} \mathrm{~F}_{24} \mathrm{~N}_{12} \mathrm{P}_{4} \mathrm{Ru}_{2}$ : C, 44.1; H, 3.03; N, 9.35\%. Found: C, 44.0; H, 3.05; N, 9.42\%.

Separation of the diastereoisomers was achieved by cation exchange chromatography on SP Sephadex C-25 support using $0.25 \mathrm{M}$ sodium octanoate solution as eluent. The isolation and purification of the diastereoisomers from the eluted bands was achieved as described above for [ $\left\{\operatorname{Ru}(\mathrm{bpy})_{2}\right\}_{2}(\mu-$ $\mathrm{dpb})]^{4+}$. Bands 1 and 2 were determined to be the $\Delta \Delta / \Lambda \Lambda$ and $\Delta \Lambda / \Lambda \Delta$ diastereoisomers, respectively. ${ }^{1} \mathrm{H}$

\footnotetext{
${ }^{*}$ Complete spectral assignments were not possible due to the convoluted nature of the $1 \mathrm{D}$ and 2D spectra. However, X-ray crystallography provided unambiguous structural identification of the meso diastereoisomer.
} 
NMR ( $\delta$ ppm; $\mathrm{CD}_{3} \mathrm{CN}$ ): (Band 1; $\left.\Delta \Lambda / \Lambda \Delta\right) 2.42$ (3H, s), 2.45 (3H, s), 2.58 (3H, s), $2.60(3 \mathrm{H}, \mathrm{s}), 6.42$ (H6d $\mathrm{Me}_{2}$ bpy, 1H, $J=5 \mathrm{~Hz}, \mathrm{~d}$ ), 6.69 (H6d, 1H, $J=5,1.5 \mathrm{~Hz}, \mathrm{dd}$ ), 6.95 (H5d, 1H, $J=8,5 \mathrm{~Hz}, \mathrm{dd}$ ), 6.96 (H5d $\mathrm{Me}_{2}$ bpy, 1H, $J=5 \mathrm{~Hz}, \mathrm{~d}$ ), 6.96 (H6b Me 2 bpy, 1H, s), 7.05 (H6b, 1H, $J=5,1.5 \mathrm{~Hz}$, dd), 7.12 (H6d, 1H, $J$ = 8, $5 \mathrm{~Hz}, \mathrm{dd}$ ), 7.25 (H5b, H5a, 2H, $J=8,5 \mathrm{~Hz}, \mathrm{dd}$ ), 7.31 (H6c Me $\mathrm{bppy}_{1}$ H, $J=5 \mathrm{~Hz}, \mathrm{~d}$ ), 7.40-7.70 (8H, m), 7.71 (H4b, 1H, $J=8,8 \mathrm{~Hz}, \mathrm{dd}), 7.87$ (H4b, 1H, $J=8,8 \mathrm{~Hz}, \mathrm{dd}), 7.94$ (H4a, 1H, $J=8,8 \mathrm{~Hz}, \mathrm{dd})$, 7.95-8.01 (8H, m), 8.03 (H2 dpb, 1H, s), 8.05 (H7 dpb, 1H, s), 8.10 (H4d, 1H, J = 8, 8 Hz, dd), 8.15 (H3a Me 4 bpy, 1H, s), 8.26 (H3a, 1H, $J=8,1.5$ Hz, dd), 8.35 (H3d Me 4 bpy, 1H, s), 8.41 (H13/H14 dpb, 2H, d), 8.55 (H3d, 1H, $J=8,1.5$ Hz, dd), 8.58 (H3b, H3c Me 2 bpy, 2H, s), 8.73 (H3b, H3c, 2H, $J=8,1.5$ Hz, dd); (Band 2; $\Delta \Delta / \Lambda \Lambda$ ) 2.40 (3H, s), 2.50 (3H, s), 2.52 (3H, s), 2.61 (3H, s), 6.87 (H6b Me $\mathrm{Me}_{2}$ bpy, 1H, $J=5$ Hz, d), 7.08 (H5b, 1H, $J=8,5$ Hz, dd), 7.28 (H3 dpb, 1H, $J=5$ Hz, d), 7.32 (H5b Me $b p y, 1 \mathrm{H}, J=5 \mathrm{~Hz}$, d), 7.39 (H6 dpb, 1H, $J=5 \mathrm{~Hz}, \mathrm{~d}$ ), 7.46 (H6c Me $\mathrm{bpy}, 1 \mathrm{H}, J=5 \mathrm{~Hz}, \mathrm{~d}$ ), 7.52 (H5c, 1H, $J=8,5 \mathrm{~Hz}$, dd), 7.54 (H4/5 dpb, 2H, $J=8,8$ Hz, dd), 7.61 (H5a, 1H, $J=8,5$ Hz, dd), 7.68 (H5d, 1H, $J=8,5$ Hz, dd), 7.70-7.81 (7H, m), 7.83 (H6b, 1H, J = 5, 1.5 Hz, dd), 7.96-8.07 (10H, m), 8.08 (H2/H7 dpb, 2H, s), 8.14 (H4b, 1H, $J=8,8 \mathrm{~Hz}$, dd), 8.17 (H4a, 1H, $J=8,8 \mathrm{~Hz}$, dd), 8.22 (H4d, 1H, $J=8,8 \mathrm{~Hz}, \mathrm{dd}$ ), 8.28 (H3a, 1H, $J=8,1.5 \mathrm{~Hz}$, dd), 8.31 (H3b, 1H, $J=8,1.5 \mathrm{~Hz}, \mathrm{dd}$ ), 8.33 (H3c Me ${ }_{4} \mathrm{bpy}, 1 \mathrm{H}, \mathrm{s}$ ), 8.52 (H3d Me $\mathrm{Me}_{4} \mathrm{bpy}$, 1H, s), 8.63 (H13 dpb, 1H, d), 8.66 (H14 dpb, 1H, d), 8.66 (H3d, 1H, $J=8,1.5$ Hz, dd), 8.69 (H3c, 1H, $J$ $=8,1.5 \mathrm{~Hz}, \mathrm{dd})$.

$\left[\left\{\mathbf{R u}(\mathbf{b p y})_{2}\right\}(\mu-\mathbf{d p b})\left\{\mathbf{R u}\left(\mathbf{M e}_{4} \mathbf{b p y}\right)_{2}\right\}\right]\left(\mathbf{P F}_{6}\right)_{4}$ was synthesised according to the procedure reported above for $\left[\left\{\mathrm{Ru}(\mathrm{bpy})_{2}\right\}(\mu-\mathrm{dpb})\left\{\mathrm{Ru}\left(\mathrm{Me}_{2} \mathrm{bpy}\right)_{2}\right\}\right]\left(\mathrm{PF}_{6}\right)_{4}$ using $\left[\mathrm{Ru}(\mathrm{bpy})_{2}(\mathrm{dpb})\right]\left(\mathrm{PF}_{6}\right)_{2}(70 \mathrm{mg}, 0.0674 \mathrm{mmol})$ and [Ru(Me ${ }_{4}$ bpy $\left.)_{2} \mathrm{Cl}_{2}\right]$ (40.3 mg, $0.0674 \mathrm{mmol}$ ) Yield: $110 \mathrm{mg}$ (88\%). Anal. Calcd for $\mathrm{C}_{70} \mathrm{H}_{62} \mathrm{~F}_{24} \mathrm{~N}_{12} \mathrm{P}_{4} \mathrm{Ru}_{2}: \mathrm{C}$, 45.4; H, 3.37; N, 9.07\%. Found: C, 45.0; H, 3.25; N, 8.99\%. ${ }^{1} \mathrm{H}$ NMR ( $\delta$ ppm; $\left.\mathrm{CD}_{3} \mathrm{CN}\right)$ : (Band 1; $\Delta \Lambda / \Lambda \Delta) 1.73$ (3H, s), 1.89 (3H, s), 2.14 (3H, s), 2.23 (3H, s), 2.37 (3H, s), 2.41 (3H, s), 2.48 (3H, s),

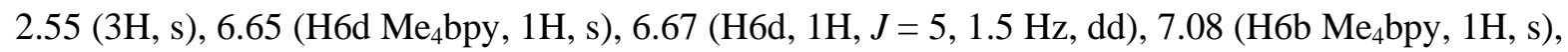
7.13 (H5d, 1H, $J=8,5$ Hz, dd), 7.25 (H5b, 1H, $J=8,5$ Hz, dd), 7.39 (H6b, 1H, $J=5,1.5$ Hz, dd), 7.40 (H6c Me $\mathrm{Me}_{4}$ ppy, 1H, s), 7.45 (H5a, 1H, $J=8,5$ Hz, dd), 7.47 (H6a Me 4 bpy, 1H, s), 7.51 (H4/5 dpb, 2H, $J=$ 8, 8 Hz, dd), 7.54 (H5c, H5a, 1H, $J=8,5$ Hz, dd), 7.55-7.80 (9H, m), 7.82 (H2 dpb, 1H, s), 7.98 (H7 dpb,

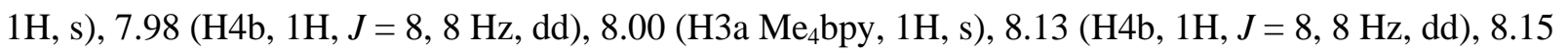
(H4a, 1H, $J=8,8 \mathrm{~Hz}, \mathrm{dd}$ ), 8.23 (H4d, 1H, $J=8,8$ Hz, dd), 8.31 (H3d Me $\mathrm{H}_{4}$ bpy, 1H, s), 8.46 (H3c Me $\mathrm{M}_{4}$ bpy, 1H, s), 8.38 (H3a, 1H, $J=8,1.5$ Hz, dd), 8.49 (H3d, 1H, $J=8,1.5$ Hz, dd), 8.59 (H13/H14 dpb, 2H, d), 8.71 (H3b, H3c, 2H, $J$ = 8, 1.5 Hz, dd); (Band 2; $\Delta \Delta / \Lambda \Lambda$ ) 1.74 (3H, s), 2.09 (3H, s), 2.11 (3H, s), 2.24 (3H, s), 2.30 (3H, s), 2.40 (3H, s), 2.44 (3H, s), 2.53 (3H, s), 7.08 (H5b, 1H, J = 8, 5 Hz, dd), 7.18

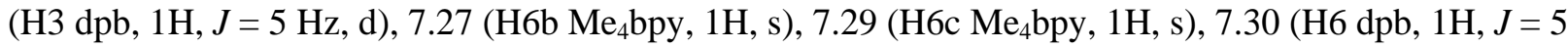
Hz, d), 7.42 (H5c, 1H, $J=8,5$ Hz, dd), 7.44 (H5a, 1H, $J=8,5$ Hz, dd), 7.51 (H4/5 dpb, 2H, $J=8,8 \mathrm{~Hz}$, dd), 7.53 (H5d, 1H, $J=8,5$ Hz, dd), 7.61 (H6b, 1H, $J=5,1.5$ Hz, dd), 7.62 (H6a Mesbpy, 1H, s) 7.74 (H6d, 1H, $J$ = 5, 1.5 Hz, dd), 7.76 (H6c, 1H, $J=5,1.5$ Hz, dd), 7.78 (H6d Me 4 bpy, 1H, s), 7.96 (H2 dpb, 1H, s), 7.98 (H7 dpb, 1H, s), 8.00-8.10 (10H, m), 8.11 (H4b, 1H, $J=8,8$ Hz, dd), 8.12 (H4a, 1H, $J=8,8$ 
Hz, dd), 8.18 (H4d, 1H, $J=8,8$ Hz, dd), 8.22 (H3a, 1H, $J=8,1.5 \mathrm{~Hz}, \mathrm{dd}$ ), 8.31 (H3b, 1H, $J=8,1.5 \mathrm{~Hz}$, dd), 8.43 (H3c Me 4 bpy, 1H, s), 8.44 (H3d Me 4 bpy, 1H, s), 8.59 (H13 dpb, 1H, d), 8.64 (H14 dpb, 1H, d), 8.66 (H3d, 1H, $J=8,1.5 \mathrm{~Hz}$, dd), 8.69 (H3c, 1H, $J=8,1.5 \mathrm{~Hz}$, dd).

$\left[\left\{\mathbf{R u}(\mathbf{b p y})_{2}\right\}_{2}(\mu-\mathbf{d p b})\right]\left(\mathbf{P F}_{6}\right)_{4}$ was synthesised from dpb' (25 mg; $\left.0.075 \mathrm{mmol}\right)$ and $\left[\mathrm{Ru}(\mathrm{bpy})_{2} \mathrm{Cl}_{2}\right] \cdot 2 \mathrm{H}_{2} \mathrm{O}$ (85 mg, $0.165 \mathrm{mmol})$ in an analogous manner to that for $\left[\left\{\mathrm{Ru}(\mathrm{bpy})_{2}\right\}_{2}(\mu-\mathrm{dpb})\right]\left(\mathrm{PF}_{6}\right)_{4} \cdot{ }^{67,69}$ The dinuclear species was isolated as an olive green solid. Yield: $93 \mathrm{mg}$ (71\%). Anal. Calcd for $\mathrm{C}_{62} \mathrm{H}_{44} \mathrm{~F}_{24} \mathrm{~N}_{12} \mathrm{P}_{4} \mathrm{Ru}_{2}$ : $\mathrm{C}$, 42.8; H, 2.55; N, 9.70\%. Found: C, 42.6; H, 2.56; N, 9.65\%. Separation and purification of the diastereoisomeric forms were achieved as described above. Bands 1 and 2 were determined to be the meso and rac diastereoisomers, respectively, as established by X-ray crystallography and NMR characterisation (Table 3.1, §3.3.1.1).

$\left[\left\{\mathbf{R u}(\mathbf{b p y})_{2}\right\}\left(\mu-\mathbf{d p b} \mathbf{b}^{\prime}\right)\left\{\mathbf{R u}\left(\mathbf{M e}_{2} \mathbf{b p y}\right)_{2}\right\}\right]\left(\mathbf{P F}_{6}\right)_{4}$ was synthesised according to the procedure described previously for the analogous dpb-bridged complex using $\left[\mathrm{Ru}(\mathrm{bpy})_{2}\left(\mathrm{dpb}^{\prime}\right)\right]\left(\mathrm{PF}_{6}\right)_{2}(90 \mathrm{mg}, 0.0868 \mathrm{mmol})$ and $\left[\mathrm{Ru}\left(\mathrm{Me}_{2} \mathrm{bpy}\right)_{2} \mathrm{Cl}_{2}\right]$ (50 mg, $\left.0.0868 \mathrm{mmol}\right)$. Given the identical colours of the $\left[\mathrm{Ru}(\mathrm{bpy})_{2}\left(\mathrm{dpb}^{\prime}\right)\right]\left(\mathrm{PF}_{6}\right)_{2}$ precursor and the desired dinuclear product, the progress of the reaction was more effectively established from the TLC analysis. Yield: $125 \mathrm{mg}(80 \%)$. Anal. Calcd for $\mathrm{C}_{66} \mathrm{H}_{52} \mathrm{~F}_{24} \mathrm{~N}_{12} \mathrm{P}_{4} \mathrm{Ru}_{2}$ : C, 44.2; H, 2.92; N, 9.36\%. Found: C, 44.0; H, 2.89; N, 9.34\%.

Separation of the diastereoisomeric forms was performed as described above using aqueous $0.25 \mathrm{M}$ sodium benzoate solution as the eluent. ${ }^{1} \mathrm{H}$ NMR ( $\left.\delta \mathrm{ppm} ; \mathrm{CD}_{3} \mathrm{CN}\right)$ : (Band 1; $\left.\Delta \Lambda / \Lambda \Delta\right) 2.34$ (3H, s), 2.58 (3H, s), 2.59 (3H, s), 2.67 (3H, s), 6.77 (H6d Me 2 bpy, 1H, $J=5$ Hz, d), 6.95 (H5d Me 2 bpy, 1H, $J=5$ Hz, d), 7.02 (H6d, 1H, $J=5 \mathrm{~Hz}, \mathrm{~d}$ ), 7.12 (H6b Me 2 bpy, 1H, $J=5 \mathrm{~Hz}, \mathrm{~d}$ ), 7.19 (H5d, 1H, $J=8,5 \mathrm{~Hz}, \mathrm{dd}$ ), 7.31 (H5b, 1H, $J=8,5$ Hz, dd), 7.34 (H6c, H6a Me $b p y, 2 H, J=5$ Hz, d), 7.56 (H3, H6 dpb', 2H), 7.59 (H5c, 1H, $J=8,5$ Hz, dd), 7.65 (H5a, 1H, $J=8,5$ Hz, dd), 7.65 (H4/5 dpb', 2H, s), 7.74 (H6b, H6c, 2H, $J=5 \mathrm{~Hz}, \mathrm{~d}), 7.83$ (H4d Me $\mathrm{H}_{2}$ py, 1H, $\left.J=8,8 \mathrm{~Hz}, \mathrm{dd}\right), 7.95-8.05$ (8H, m), 8.10-8.40 (12H, m), 8.59 (H3b, H3c $\mathrm{Me}_{4}$ bpy, 2H, $J=8 \mathrm{~Hz}, \mathrm{~d}$ ), 8.73 (H3c, 1H, $\left.J=8 \mathrm{~Hz}, \mathrm{~d}\right), 8.76$ (H3b, 1H, $J=8 \mathrm{~Hz}, \mathrm{~d}$ ), 9.31 (H12 dpb', 1H, $J=8$ Hz, d), 9.34 (H13 dpb', 1H, $J=8$ Hz, d); (Band 2; $\Delta \Delta / \Lambda \Lambda$ ) 2.49 (3H, s), 2.51 (3H, s), 2.65 (3H, s), 2.69 (3H, s), 6.84 (H6b Me 2 bpy, 1H, $J=5$ Hz, d), 7.06 (H5b, 1H, $J=8,5$ Hz, dd), 7.13 (H6d Me bpy, $1 \mathrm{H}, J=5 \mathrm{~Hz}, \mathrm{~d}), 7.19$ (H3/6 dpb', 2H), 7.19 (H6c Me dd), 7.21 (H5d, H5b Me 2 bpy, 2H, $J=5,1.5$ Hz, dd), 7.30 (H6a Me bpy, $_{1 H} J=5$ Hz, d), 7.36 (H5a $\mathrm{Me}_{2}$ bpy, $\left.1 \mathrm{H}, J=5 \mathrm{~Hz}, \mathrm{~d}\right), 7.41$ (H6b, 1H, $J=5 \mathrm{~Hz}, \mathrm{~d}$ ), 7.48 (H6c, 1H, $\left.J=5 \mathrm{~Hz}, \mathrm{~d}\right), 7.52$ (H5d, 1H, $J=8$, $5 \mathrm{~Hz}, \mathrm{dd}), 7.54$ (H5c, 1H, $J=8,5 \mathrm{~Hz}$, dd), 7.56 (H4/5 dpb', 2H), 7.63 (H5a, 1H, $J=8,5 \mathrm{~Hz}, \mathrm{dd}), 7.66$ (H6a, $1 \mathrm{H}, J=5 \mathrm{~Hz}, \mathrm{~d}), 7.72-7.78$ (4H, m), 8.01 (H4d, 1H, $J=8,8 \mathrm{~Hz}, \mathrm{dd}), 8.04$ (H4b, 1H, $J=8,8 \mathrm{~Hz}$, dd), 8.08 (H11/14 dpb', 2H, $J=10,9$ Hz, dd), 8.12 (H4c, 1H, $J=8$, 8 Hz, dd), 8.21 (H2/7 dpb', $2 \mathrm{H}), 8.25$ (H4a, 1H, $J=8,8$ Hz, dd), 8.29 (H3b Me 2 bpy, 1H, s), 8.35 (H3a Me $\mathrm{M}_{2} \mathrm{bpy}, 1 \mathrm{H}, \mathrm{s}$ ), 8.44 (H3c Me $\mathrm{bpy}, 1 \mathrm{H}$, s), 8.46 (H3d, 1H, $J=8$ H, d), 8.59 (H3a, 1H, $J=8$ Hz, d), 8.62 (H3b, 1H, $J=8$ Hz, d), 8.67 (H3c, 1H, $J$ $=8 \mathrm{~Hz}, \mathrm{~d}), 9.27$ (H12 dpb', 1H, $J=8 \mathrm{~Hz}, \mathrm{~d}), 9.30(\mathrm{H} 13 \mathrm{dpb}, 1 \mathrm{H}, J=8 \mathrm{~Hz}, \mathrm{~d})$. 
$\left[\left\{\mathbf{R u}(\mathbf{b p y})_{2}\right\}\left(\mu-\mathbf{d p b} \mathbf{b}^{\prime}\right)\left\{\mathbf{R u}\left(\mathbf{M e}_{4} \mathbf{b p y}\right)_{2}\right\}\right]\left(\mathbf{P F}_{6}\right)_{4}$ was synthesised according to the procedure described previously for $\left[\left\{\mathrm{Ru}(\mathrm{bpy})_{2}\right\}\left(\mu-\mathrm{dpb}^{\prime}\right)\left\{\mathrm{Ru}\left(\mathrm{Me}_{2} \mathrm{bpy}\right)_{2}\right\}\right]\left(\mathrm{PF}_{6}\right)_{4}$ using $\left[\mathrm{Ru}(\mathrm{bpy})_{2}\left(\mathrm{dpb}^{\prime}\right)\right]\left(\mathrm{PF}_{6}\right)_{2}$ (70 mg, $0.0675 \mathrm{mmol})$ and [Ru( $\left.\left.\mathrm{Me}_{4} \mathrm{bpy}\right)_{2} \mathrm{Cl}_{2}\right]$ (40.3 mg, $\left.0.0674 \mathrm{mmol}\right)$. Yield: $91 \mathrm{mg}$ (76\%). Anal. Calcd for $\mathrm{C}_{70} \mathrm{H}_{60} \mathrm{~F}_{24} \mathrm{~N}_{12} \mathrm{P}_{4} \mathrm{Ru}_{2}$ : C, 45.4; H, 3.27; N, 9.08\%. Found: C, 45.5; H, 3.25; N, 8.98\%. ${ }^{1} \mathrm{H}$ NMR ( $\delta$ ppm; $\mathrm{CD}_{3} \mathrm{CN}$ ): (Band 1; $\left.\Delta \Lambda / \Lambda \Delta\right) 1.85$ (3H, s), 1.88 (3H, s), 2.08(3H, s), 2.21 (3H, s), 2.30 (3H, s), 2.43 (3H, s), 2.53 (3H, s), 2.55 (3H, s), 6.61 (H6d Me 4 bpy, 1H, s), 7.08 (H6b Me 4 bpy, 1H, s), 7.10 (H6c $\mathrm{Me}_{4}$ bpy, 1H, s), 7.10 (H6d, 1H, $J=5$ Hz, d), 7.23 (H6a Me 4 bpy, 1H, s), 7.25 (H5d, 1H, $J=8,5$ Hz, dd), 7.49 (H5b, 1H, $J=8,5$ Hz, dd), 7.54 (H6 dpb', 1H), 7.60 (H5c, 1H, $J=8,5$ Hz, dd), 7.68 (H3 dpb', 1H), 7.81 (H5a, 1H, $J=8,5$ Hz, dd), 7.89 (H4/5 dpb', 2H, s), 7.95 (H6b, 1H, $J=5$ Hz, d), 7.97 (H6c, 1H, $J=5$ Hz, d), 8.05-8.30 (12H, m), 8.15 (H10/15 dpb', 2H, $J=8$ Hz, d), 8.36 (H3a, 1H, $J=8$ Hz, d), 8.38 (H3c $\mathrm{Me}_{4}$ bpy, 1H, s), 8.42 (H3b Me 4 bpy, 1H, s), 8.66 (H3c, 1H, $J=8$ Hz, d), 8.68 (H3b, 1H, $J=8$ Hz, d), 9.24 (H12 dpb', 1H, $J=8$ Hz, d), 9.27 (H13 dpb', 1H, $J=8$ Hz, d); (Band 2; $\Delta \Delta / \Lambda \Lambda$ ) 1.63 (3H, s), 1.88 (3H, s), 2.11 (3H, s), 2.15 (3H, s), 2.35 (3H, s), 2.52 (3H, s), 2.57 (3H, s), 2.59 (3H, s), 6.92 (H6b Me 4 bpy, 1H, s), 6.99 (H6d Me $\mathrm{M}_{4} \mathrm{bpy}, 1 \mathrm{H}, \mathrm{s}$ ), 7.03 (H5b, 1H, J = 8, 5 Hz, dd), 7.18 (H6c Me 4 bpy, 1H, s), 7.22 (H3/6 dpb', 2H), 7.28 (H6d, 1H, $J=5,1.5$ Hz, dd), 7.49 (H6a Me 4 bpy, 1H, s), 7.53 (H5d, 1H, $J=8,5$ Hz, dd), 7.57 (H5c, 1H, $J=8,5 \mathrm{~Hz}$, dd), 7.65 (H6b, 1H, $J=5,1.5 \mathrm{~Hz}, \mathrm{dd}$ ), 7.67 (H4/5 dpb', 2H), 7.77 (H6c, 1H, $J=5$, $1.5 \mathrm{~Hz}$, dd), 7.94 (H5a, 1H, $J=8,5$ Hz, dd), 7.96 (H11/14 dpb', 2H, $J=10,9 \mathrm{~Hz}$, dd), 8.06 (H4d, 1H, $J=$ 8, $8 \mathrm{~Hz}$, dd), 8.07 (H6a, 1H, J = 5, 1.5 Hz, dd), 8.15 (H4b, 1H, J = 8, 8 Hz, dd), 8.20 (H2/7 dpb', 2H),

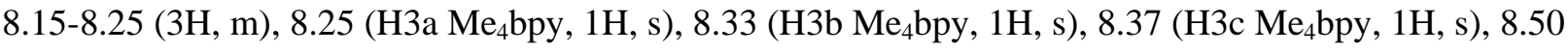
(H3d, 1H, $J=8 \mathrm{H}, \mathrm{d}$ ), 8.59 (H3a, 1H, $J=8 \mathrm{~Hz}, \mathrm{~d}$ ), 8.59 (H3b, 1H, $J=8 \mathrm{~Hz}, \mathrm{~d}$ ), 8.63 (H3c, 1H, $J=8 \mathrm{~Hz}$, d), 9.22 (H12 dpb', 1H, $J=8$ Hz, d), 9.24 (H13 dpb', 1H, $J=8$ Hz, d).

$\left[\left\{\mathbf{R u}(\mathbf{b p y})_{2}\right\}_{2}(\mu-\mathbf{d p q})\right]\left(\mathbf{P F}_{6}\right)_{4}$. The synthesis, diastereoisomeric separation and purification were performed under similar conditions to those described above for $\left[\left\{\mathrm{Ru}(\mathrm{bpy})_{2}\right\}_{2}(\mu-\mathrm{dpb})\right]\left(\mathrm{PF}_{6}\right)_{4}$ involving the reaction of $c i s-\left[\mathrm{Ru}(\mathrm{bpy}){ }_{2} \mathrm{Cl}_{2}\right] .2 \mathrm{H}_{2} \mathrm{O}(132.7 \mathrm{mg}, 0.255 \mathrm{mmol})$ with dpq' (32.8 $\left.\mathrm{mg}, 0.116 \mathrm{mmol}\right)$. The dinuclear species was isolated as an olive green solid. Yield: $170 \mathrm{mg}$ (87\%). Anal. Calcd for $\mathrm{C}_{58} \mathrm{H}_{42} \mathrm{~F}_{24} \mathrm{~N}_{12} \mathrm{P}_{4} \mathrm{Ru}_{2}$ : C, 41.2; H, 2.51; N, 10.0\%. Found: C, 41.0; H, 2.48; N, 9.88\%. ${ }^{1} \mathrm{H}$ NMR ( $\delta$ ppm; $\mathrm{CD}_{3} \mathrm{CN}$ ): (Band 1; meso) 6.91 (H6d, 2H, $J=5,1.5 \mathrm{~Hz}$, dd), 7.15 (H5d, 2H, $J=8,5 \mathrm{~Hz}$, dd), 7.30 (H5b, $2 \mathrm{H}, J=8,5 \mathrm{~Hz}, \mathrm{dd}), 7.44$ (H5c, 2H, $J=8,5 \mathrm{~Hz}, \mathrm{dd}), 7.53$ (H5a, 2H, $J=8,5 \mathrm{~Hz}, \mathrm{dd}), 7.56$ (H2/5 dpq', 2H, $J=3 \mathrm{~Hz}, \mathrm{~d}$ ), 7.66 (H6c, 2H, $J=5,1.5 \mathrm{~Hz}, \mathrm{dd}), 7.70$ (H3/4 dpq', 2H, $J=10,8 \mathrm{~Hz}, \mathrm{dd}), 7.78$ (H4d, 2H, $J=8,8 \mathrm{~Hz}, \mathrm{dd}), 7.99$ (H6a/H6b, 4H, $J=5,1.5 \mathrm{~Hz}, \mathrm{dd}),(\mathrm{H9} / 12 \mathrm{dpq}, 2 \mathrm{H}), 8.05$ (H4a, 2H, $J=8,8 \mathrm{~Hz}$, dd), 8.16 (H4b/H4c, 4H, $J=8$, 8 Hz, dd), 8.16 (H8/13 dpq', 2H), 8.19 (H4c, 2H, $J=8,8$ Hz, dd), 8.28 (H3a, 2H, $J=8,1.5 \mathrm{~Hz}$, dd), 8.68 (H3c/H3b, 4H, $J=8,1.5 \mathrm{~Hz}$, dd), 9.35 (H10/11 dpq', $J=8,1.5 \mathrm{~Hz}, \mathrm{dd}$ ); (Band 2; rac) 7.03 (H5b, 2H, $J=8,5 \mathrm{~Hz}, \mathrm{dd}$ ), 7.25 (H5d, 2H, $J=8,5 \mathrm{~Hz}, \mathrm{dd}$ ), 7.33 (H6d, 2H, $J=5,1.5$ Hz, dd), 7.45-7.52 (H6b/H5c/H5a, H2/5 dpq', H3/4 dpq', 5H, m), 7.63 (H6a, 2H, J = 5, 1.5 Hz, dd), 7.85 (H6c, 2H, $J=5,1.5$ Hz, dd), 7.98 (H9/12 dpq', 2H, $J=10,8$ Hz, dd), 8.03 (H4b, 2H, $J=8,8$ Hz, dd), 8.07 (H4d, 2H, $J=8,8 \mathrm{~Hz}$, dd), 8.09 (H4c, 2H, $J=8,8 \mathrm{~Hz}, \mathrm{dd}$ ), 8.13 (H4a, 2H, $J=8,8 \mathrm{~Hz}, \mathrm{dd}$ ), 8.17 
(H8/13 dpq', 2H), 8.49 (H3d, 2H, $J=8,1.5$ Hz, dd), 8.54 (H3a/H3b, 4H, $J=$ 8, 1.5 Hz, dd), 8.58 (H3c, 2H, $J=8,1.5 \mathrm{~Hz}, \mathrm{dd}), 9.31$ (H10/11 dpq', 2H, $J=8,1.5 \mathrm{~Hz}, \mathrm{~d})$.

$\left[\left\{\mathbf{R u}(\mathbf{b p y})_{2}\right\}_{2}(\mu-\mathbf{p p z})\right]\left(\mathbf{P F}_{6}\right)_{4}$. The synthesis and purification were performed under similar conditions to those described above for $\left[\left\{\mathrm{Ru}(\mathrm{bpy})_{2}\right\}_{2}(\mu-\mathrm{dpb})\right]\left(\mathrm{PF}_{6}\right)_{4}$ involving the reaction of cis-[Ru(bpy) $\left.{ }_{2} \mathrm{Cl}_{2}\right] \cdot 2 \mathrm{H}_{2} \mathrm{O}$ (111 mg, $0.213 \mathrm{mmol}$ ) with ppz (24 mg, $0.103 \mathrm{mmol}$ ). The dinuclear species was isolated as a purple solid. Yield: 140 mg (83\%). Anal. Calcd for $\mathrm{C}_{54} \mathrm{H}_{40} \mathrm{~F}_{24} \mathrm{~N}_{12} \mathrm{P}_{4} \mathrm{Ru}_{2}$ : C, 39.6; H, 2.46; N, 10.3\%. Found: C, 39.4; H, 2.44; N, 10.1\%. ${ }^{1} \mathrm{H}$ NMR ( $\delta$ ppm; $\mathrm{CD}_{3} \mathrm{CN}$ ): (Band 1; meso) 7.25 (H5d, 2H, $J=8,5 \mathrm{~Hz}, \mathrm{dd}$ ), 7.39 (H5b, 2H, $J=8,5 \mathrm{~Hz}$, dd), 7.42 (H5c, 2H, $J=8,5 \mathrm{~Hz}$, dd), 7.50 (H5a, 2H, $J=8,5 \mathrm{~Hz}$, dd), 7.54 (H6d, 2H, $J=5,1.5 \mathrm{~Hz}$, dd), 7.73 (H6a, 2H, $J=5,1.5 \mathrm{~Hz}$, dd), 7.75 (H6c, 2H, $J=5,1.5 \mathrm{~Hz}$, dd), 7.85 (H6b, 2H, $J=5,1.5 \mathrm{~Hz}, \mathrm{dd}$ ), 7.93 (H2/3 ppz, 2H, s), 7.99 (H4d, 2H, $J=8,8 \mathrm{~Hz}, \mathrm{dd}), 8.01$ (H7/10 ppz, $J$ = 10, $8 \mathrm{~Hz}, \mathrm{dd}$ ), 8.06 (H4c, 2H, $J=8,8 \mathrm{~Hz}, \mathrm{dd}), 8.09$ (H4b, 2H, $J=8,8 \mathrm{~Hz}, \mathrm{dd}), 8.15$ (H4a, 2H, $J=8,8$ Hz, dd), 8.23 (H6/11 ppz, $J$ = 5, 1.5 Hz, dd), 8.39 (H3d, 2H, $J=8,1.5$ Hz, dd), 8.43 (H3c, 2H, $J=8,1.5$ Hz, dd), 8.52 (H3b, 2H, $J=8,1.5$ Hz, dd), 8.58 (H3a, 2H, $J=8,1.5$ Hz, dd), 9.32 (H8/9 ppz, $J=8,1.5$ Hz, dd); (Band 2; rac) 7.02 (H5b, 2H, $J=8,5 \mathrm{~Hz}$, dd), 7.36 (H5d, 2H, $J=8,5 \mathrm{~Hz}$, dd), 7.38 (H5c, 2H, $J$ = 8, $5 \mathrm{~Hz}, \mathrm{dd}$ ), 7.42 (H6d, 2H, $J=5,1.5 \mathrm{~Hz}, \mathrm{dd}$ ), 7.51 (H5a, 2H, $J=8,5 \mathrm{~Hz}, \mathrm{dd}$ ), 7.61 (H6b, 2H, $J=5$, $1.5 \mathrm{~Hz}, \mathrm{dd}), 7.66$ (H6c, 2H, $J$ = 5, $1.5 \mathrm{~Hz}, \mathrm{dd}), 7.76$ (H6a, 2H, $J$ = 5, $1.5 \mathrm{~Hz}, \mathrm{dd}), 7.96$ (H2/3 ppz, 2H, s), 8.00 (H4d, 2H, $J=8,8$ Hz, dd), 8.01 (H7/10 ppz, $J=10,8$ Hz, dd), 8.07 (H4b, 2H, $J=8,8$ Hz, dd), 8.13 (H4a, 2H, $J=8,8 \mathrm{~Hz}, \mathrm{dd}$ ), 8.15 (H4c, 2H, $J=8,8 \mathrm{~Hz}, \mathrm{dd}$ ), 8.23 (H6/11 ppz, $J=5,1.5 \mathrm{~Hz}, \mathrm{dd}$ ), 8.48 (H3d, 2H, $J=8,1.5 \mathrm{~Hz}, \mathrm{dd}$ ), 8.51 (H3b, 2H, $J=8,1.5 \mathrm{~Hz}$, dd), 8.53 (H3a, 2H, $J=8,1.5 \mathrm{~Hz}$, dd), 8.55 (H3c, 2H, $J=8,1.5 \mathrm{~Hz}, \mathrm{dd}), 9.31$ (H8/9 ppz, $J=8,1.5 \mathrm{~Hz}, \mathrm{dd})$.

[\{Ru(bpy $\left.\left.)_{2}\right\}_{2}(\mu-2,3-d p p)\right]\left(\mathbf{P F}_{6}\right)_{4}$. The synthesis and purification were performed under similar conditions to those described above for $\left[\left\{\mathrm{Ru}(\mathrm{bpy})_{2}\right\}_{2}(\mu\right.$-dpb) $]\left(\mathrm{PF}_{6}\right)_{4}$ involving the reaction of $\operatorname{cis}$ - $\left[\mathrm{Ru}(\mathrm{bpy})_{2} \mathrm{Cl}_{2}\right] \cdot 2 \mathrm{H}_{2} \mathrm{O}$ (100 mg, $0.192 \mathrm{mmol}$ ) with 2,3-dpp (18 mg, $0.0769 \mathrm{mmol})$. The dinuclear species was isolated as a purple solid. Yield: $122 \mathrm{mg}$ (97\%). Anal. Calcd for $\mathrm{C}_{54} \mathrm{H}_{42} \mathrm{~F}_{24} \mathrm{~N}_{12} \mathrm{P}_{4} \mathrm{Ru}_{2}$ : C, 39.5; H, 2.58; N, 10.2\%. Found: C, 39.3; H, 2.22; N, 9.98\%. ${ }^{1} \mathrm{H}$ NMR ( $\delta \mathrm{ppm}$; $\left.\mathrm{CD}_{3} \mathrm{CN}\right)$ : (Band 1; meso) $7.41(2 \mathrm{H}, J=8 \mathrm{~Hz}, \mathrm{~d})$, 7.48 (2H, $J=8,5 \mathrm{~Hz}, \mathrm{dd}), 7.50-7.60(6 \mathrm{H}, \mathrm{m}), 7.85-7.95$ (8H, m), 8.07 (2H, $J=8,8 \mathrm{~Hz}, \mathrm{dd}), 8.12(2 \mathrm{H}, J=$ 8, 8 Hz, dd), 8.15 (2H, $J=8$, 8 Hz, dd), 8.20 (2H, $J=8$, 8 Hz, dd), 8.07 (H7/12 2,3-dpp, 2H, m), 8.388.46 (8H, m), 8.59 (4H, $J=8,1.5 \mathrm{~Hz}$, dd), 8.50 (H9/10 2,3-dpp, 2H, $J=8,1.5 \mathrm{~Hz}$, dd); (Band 2; rac) 7.26 (2H, $J=8 \mathrm{~Hz}, \mathrm{~d}), 7.38-7.42(6 \mathrm{H}, \mathrm{m}), 7.48(4 \mathrm{H}, J=5 \mathrm{~Hz}, \mathrm{~d}), 7.55-7.65(6 \mathrm{H}, \mathrm{m}), 7.67(2 \mathrm{H}, J=8 \mathrm{~Hz}$, dd), $7.78(2 \mathrm{H}, \mathrm{m}), 7.92(2 \mathrm{H}, J=8,5 \mathrm{~Hz}, \mathrm{dd}), 7.91(2 \mathrm{H}, J=8,5 \mathrm{~Hz}, \mathrm{dd}), 8.07$ (2H, $J=8,8 \mathrm{~Hz}, \mathrm{dd}), 8.15$ (2H, $J=8,8 \mathrm{~Hz}, \mathrm{dd}), 8.22$ (2H, $J=8,8 \mathrm{~Hz}, \mathrm{dd}), 8.43(2 \mathrm{H}, J=8 \mathrm{~Hz}, \mathrm{~d}), 8.50(2 \mathrm{H}, J=8 \mathrm{~Hz}, \mathrm{~d}), 8.54(2 \mathrm{H}$, $J=8 \mathrm{~Hz}, \mathrm{~d}), 8.59(2 \mathrm{H}, J=8 \mathrm{~Hz}, \mathrm{~d}), 8.76(2 \mathrm{H}, J=8 \mathrm{~Hz}, \mathrm{~d})$.

[\{Ru(bpy $\left.)_{2}\right\}_{2}(\boldsymbol{\mu}$-dpo $\left.) \mathbf{C P F}_{6}\right)_{4}$. The bridging ligand dpo (36.6 mg, $\left.0.163 \mathrm{mmol}\right)$ was refluxed with $\left[\mathrm{Ru}(\mathrm{bpy})_{2} \mathrm{Cl}_{2}\right] \cdot 2 \mathrm{H}_{2} \mathrm{O}(187 \mathrm{mg}, 0.359 \mathrm{mmol})$ in 3:1 ethanol/water $\left(20 \mathrm{~cm}^{3}\right)$ for $24 \mathrm{~h}$ under $\mathrm{N}_{2}$. Ethanol was 
removed via rotary evaporation and the crude product precipitated from the aqueous solution by addition of a saturated solution of $\mathrm{KPF}_{6}$. A dark red solid was isolated by vacuum filtration and washed with diethyl ether. Purification was achieved by cation exchange chromatography (SP Sephadex C-25; eluent $0.5 \mathrm{M} \mathrm{NaCl}$ ). An orange band of mononuclear material eluted first, followed by the desired dark red product, which was isolated as the $\mathrm{PF}_{6}{ }^{-}$salt. Yield: $130 \mathrm{mg}$ (49\%). The ${ }^{1} \mathrm{H}$ NMR spectrum was identical to that reported previously. ${ }^{71}$

Separation of the diastereoisomers was achieved using $0.20 \mathrm{M}$ sodium tosylate solution as the eluent. Bands 1 (red) and 2 (purple) were determined to be the rac and meso diastereoisomers, respectively, as established by X-ray crystallography and NMR characterisation. ${ }^{1} \mathrm{H}$ NMR $(\delta \mathrm{ppm}$; $\mathrm{CD}_{3} \mathrm{CN}$ ): (Band 1; rac) 7.09 (H6b, 2H, $J=8,5 \mathrm{~Hz}$, dd), 7.11 (H5b, 2H, $J=8,5 \mathrm{~Hz}$, dd), 7.23 (H5d, 2H, $J=8,5 \mathrm{~Hz}, \mathrm{dd}), 7.37$ (H6d, 2H, $J=5,1.5 \mathrm{~Hz}, \mathrm{dd}), 7.51$ (H5c, 2H, $J=8,5 \mathrm{~Hz}, \mathrm{dd}), 7.61$ (H5a, 2H, $J=5$, $1.5 \mathrm{~Hz}, \mathrm{dd}$ ), 7.62 (H5 dpo, 2H, $J$ = 5, $1.5 \mathrm{~Hz}$, dd), 7.71 (H6c, 2H, $J=5,1.5 \mathrm{~Hz}, \mathrm{dd}$ ), 7.88 (H6 dpo, 2H, $J$ = 5, $1.5 \mathrm{~Hz}, \mathrm{dd}), 7.91$ (H6a, 2H, $J=8,5 \mathrm{~Hz}, \mathrm{dd}), 7.95$ (H4d, 2H, $J=8,8 \mathrm{~Hz}, \mathrm{dd}), 8.05$ (H4b, 2H, $J=8,8$ Hz, dd), 8.16 (H4c, 2H, $J=8,8 \mathrm{~Hz}, \mathrm{dd}$ ), 8.25 (H4a, 2H, $J=8,8 \mathrm{~Hz}, \mathrm{dd}$ ), 8.26 (H3d, 2H, $J=8 \mathrm{~Hz}, \mathrm{~d}$ ), 8.27 (H4 dpo, 2H, $J=8,8$ Hz, dd), 8.44 (H3a, 2H, $J=8 \mathrm{~Hz}, \mathrm{~d}$ ), 8.46 (H3b, 2H, $J=8 \mathrm{~Hz}, \mathrm{~d}$ ), 8.53 (H3c, 2H, $J=8 \mathrm{~Hz}, \mathrm{~d}$ ), 8.86 (H3 dpo, 2H, $J=8 \mathrm{~Hz}$, d); (Band 1; meso) 7.19 (H5d, 2H, $J=8,5 \mathrm{~Hz}$, dd), 7.23 (H5b, 2H, $J=8,5 \mathrm{~Hz}, \mathrm{dd}$ ), 7.42 (H6b, 2H, $J=5,1.5 \mathrm{~Hz}$, dd), 7.45 (H5c, 2H, $J=8,5 \mathrm{~Hz}$, dd), 7.52 (H6d, 2H, $J=5,1.5 \mathrm{~Hz}, \mathrm{dd}), 7.53$ (H5a, 2H, $J=8,5 \mathrm{~Hz}, \mathrm{dd}$ ), 7.59 (H5 dpo, 2H, $J=8,5 \mathrm{~Hz}, \mathrm{dd}$ ), 7.62 (H6c, 2H, $J=5,1.5 \mathrm{~Hz}, \mathrm{dd}$ ), 7.83 (H6 dpo, 2H, $J=5,1.5 \mathrm{~Hz}, \mathrm{dd}$ ), 7.98 (H4d, 2H, $J=8,8 \mathrm{~Hz}$, dd), 8.02 (H4b, 2H, $J$ = 8, $8 \mathrm{~Hz}, \mathrm{dd}$ ), 8.16 (H4a, 2H, $J=8,8 \mathrm{~Hz}, \mathrm{dd}), 8.18$ (H4c, 2H, $J=5,1.5 \mathrm{~Hz}, \mathrm{dd}), 8.19$ (H6a, 2H, $J=8,8$ Hz, dd), 8.27 (H4 dpo, 2H, $J=8,8$ Hz, dd), 8.31 (H3d, 2H, $J=8$ Hz, d), 8.34 (H3b, 2H, $J=8$ Hz, d), 8.52 (H3c, 2H, $J=8 \mathrm{~Hz}, \mathrm{~d}$ ), 8.56 (H3a, 2H, $J=8 \mathrm{~Hz}, \mathrm{~d}$ ), 8.87 (H3 dpo, 2H, $J=8 \mathrm{~Hz}, \mathrm{~d}$ ).

$\left[\left\{\mathbf{R u}(\mathbf{b p y})_{2}\right\}_{2}(\mu-\mathbf{d p t}) \mathbf{P F}_{6}\right)_{4}$. The separation and purification of the diastereoisomeric forms was achieved using 0.20 M sodium benzoate solution as the eluent. Bands 1 (purple) and 2 (brown) were identified as the rac and meso diastereoisomers, respectively, as established by ${ }^{1} \mathrm{H}$ NMR and COSY experiments. ${ }^{1} \mathrm{H}$ NMR ( $\delta$ ppm; $\mathrm{CD}_{3} \mathrm{CN}$ ): (Band 1; rac) 7.19 (H5b, 2H, $\left.J=8,5 \mathrm{~Hz}, \mathrm{dd}\right), 7.27$ (H5d, 2H, $J=8,5 \mathrm{~Hz}$, dd), 7.27 (H6b, 2H, $J=5,1.5 \mathrm{~Hz}$, dd), 7.30 (H5c, 2H, $J=8,5 \mathrm{~Hz}, \mathrm{dd}$ ), 7.45 (H6d, 2H, $J=5,1.5 \mathrm{~Hz}, \mathrm{dd}$ ), 7.49 (H5 dpt, 2H, $J=8,5$ Hz, dd), 7.58 (H5a, 2H, $J=8,5$ Hz, dd), 7.63 (H6c, 2H, $J=5,1.5$ Hz, dd), 7.87 (H6 dpt, 2H, $J=5,1.5 \mathrm{~Hz}, \mathrm{dd}$ ), 7.97 (H6a, 2H, $J=5,1.5 \mathrm{~Hz}, \mathrm{dd}$ ), 8.00 (H4d, 2H, $J=8,8 \mathrm{~Hz}$, dd), 8.08 (H4c, 2H, $J=8,8 \mathrm{~Hz}, \mathrm{dd}$ ), 8.14 (H4b, 2H, $J=8,8 \mathrm{~Hz}, \mathrm{dd}$ ), 8.21 (H4 dpt, 2H, $J=8,8 \mathrm{~Hz}, \mathrm{dd}$ ), 8.26 (H4a, 2H, $J$ = 8, $8 \mathrm{~Hz}, \mathrm{dd}$ ), 8.44 (H3d, 2H, $J=8 \mathrm{~Hz}, \mathrm{~d}$ ), 8.52 (H3b, 2H, $J=8 \mathrm{~Hz}, \mathrm{~d}), 8.55$ (H3a, H3c, 4H, $J=8 \mathrm{~Hz}$, d), 8.92 (H3 dpt, 2H, $J=8 \mathrm{~Hz}$, d); (Band 2; meso) 7.32 (H5d, 2H, $J=8,5 \mathrm{~Hz}, \mathrm{dd}$ ), 7.36 (H5b, 2H, $J=8$, $5 \mathrm{~Hz}$, dd), 7.45 (H5c, 2H, $J=8,5 \mathrm{~Hz}$, dd), 8.24 (H6b, 2H, $J=5,1.5 \mathrm{~Hz}, \mathrm{dd}$ ), 7.55 (H6c, 2H, $J=5,1.5$ Hz, dd), 7.61 (H5a, H5 dpt, 4H, $J$ = 8, 5 Hz, dd), 7.65 (H6d, 2H, $J=5,1.5$ Hz, dd), 7.90 (H6 dpt, 2H, $J=$ 5, $1.5 \mathrm{~Hz}, \mathrm{dd}$ ), 8.01 (H4d, 2H, $J=8,8 \mathrm{~Hz}, \mathrm{dd}), 8.02$ (H6a, 2H, $J=8,8 \mathrm{~Hz}, \mathrm{dd}$ ), 8.06 (H4b, 2H, $J=8,8$ 
Hz, dd), 8.14 (H4c, 2H, $J=8,8$ Hz, dd), 8.19 (H4a, 2H, $J=8,8$ Hz, dd), 8.24 (H4 dpt, 2H, $J=8,8$ Hz, dd), 8.37 (H3b, H3d, 4H, $J=8$ Hz, d), 8.57 (H3a, H3c, 4H, $J=8$ Hz, d), 8.94 (H3 dpt, 2H, $J=8$ Hz, d).

$\left[\left\{\mathbf{R u}(\mathbf{b p y})_{2} \boldsymbol{\}}_{2}(\mu\right.\right.$-bpt $\left.)\right]\left(\mathbf{P F}_{6}\right)_{4}$. The synthesis and diastereoisomer separation was performed according to the literature procedure. ${ }^{131}$

\section{(ii) Dinuclear Osmium Complexes Incorporating Angular Bridging Ligands}

[\{Os(bpy $\left.\left.)_{2}\right\}_{2}(\mu-\mathbf{d p b})\right]\left(\mathbf{P F}_{6}\right)_{4}$. A suspension of dpb (25 mg, $\left.0.0747 \mathrm{mmol}\right)$ in ethylene glycol $\left(1.5 \mathrm{~cm}^{3}\right)$ was heated in a modified microwave oven on medium high power for $20 \mathrm{~s}$ to complete dissolution. cis[Os(bpy) $\left.)_{2} \mathrm{Cl}_{2}\right] .2 \mathrm{H}_{2} \mathrm{O}(94.3 \mathrm{mg}, 0.155 \mathrm{mmol})$ was added and the mixture heated at reflux for a further 5 min on high power during which time the solution attained a dark green colouration. The crude mixture was diluted with distilled water $\left(50 \mathrm{~cm}^{3}\right)$ and loaded onto a column of SP Sephadex C-25 (dimensions 25 $\times 3 \mathrm{~cm}$ ). Separation of the desired dinuclear product from the mixture was achieved via gradient elution procedure with aqueous $\mathrm{NaCl}$ solution as the eluent. A purple band of mononuclear material eluted first $(0.2 \mathrm{M} \mathrm{NaCl})$ followed by the desired dark green product $(0.4 \mathrm{M} \mathrm{NaCl})$ which was precipitated as the $\mathrm{PF}_{6}{ }^{-}$ salt by addition of a saturated solution of aqueous $\mathrm{KPF}_{6}$. The solid was isolated by vacuum filtration and washed with diethyl ether $\left(3 \times 10 \mathrm{~cm}^{3}\right)$. Yield: $131 \mathrm{mg}(92 \%)$. Anal. Calcd for $\mathrm{C}_{62} \mathrm{H}_{46} \mathrm{~F}_{24} \mathrm{~N}_{12} \mathrm{P}_{4} \mathrm{Os}_{2}$ : C, 38.8; H, 2.42; N, 8.75\%. Found: C, 38.6; H, 2.41; N, 8.67\%. Further characterisation was performed following diastereoisomer separation.

Separation of the diastereoisomers was achieved as described above. Bands 1 and 2 were determined to be the meso and rac diastereoisomers, respectively, as established by X-ray crystallography and NMR characterisation. ${ }^{1} \mathrm{H}$ NMR ( $\delta \mathrm{ppm}$; $\mathrm{CD}_{3} \mathrm{CN}$ ): (Band 1; meso) 6.41 (H6d, $\left.1 \mathrm{H}, J=5 \mathrm{~Hz}, \mathrm{~d}\right), 6.94$ (H6b, 1H, $J=5,1.5$ Hz, dd), 7.09 (H5d, 1H, $J=8,5$ Hz, dd), 7.14 (H5d', 1H, $J=8,5$ Hz, dd), 7.23 (H5c, $1 \mathrm{H}, J=8,5 \mathrm{~Hz}, \mathrm{dd}), 7.33$ (H5b, 2H, $J=8,5 \mathrm{~Hz}, \mathrm{dd}), 7.43-7.61$ (13H, m), 7.69-8.07 (14H, m), 8.13 (2H, $J$ = 8, $8 \mathrm{~Hz}, \mathrm{dd}), 8.28$ (1H, $J=8 \mathrm{~Hz}, \mathrm{~d}), 8.30(1 \mathrm{H}, J=8 \mathrm{~Hz}, \mathrm{~d}), 8.33(1 \mathrm{H}, J=8 \mathrm{~Hz}, \mathrm{~d}), 8.36(1 \mathrm{H}, J=8 \mathrm{~Hz}$, d), $8.49(1 \mathrm{H}, J=8 \mathrm{~Hz}, \mathrm{~d}), 8.58(1 \mathrm{H}, J=8 \mathrm{~Hz}, \mathrm{~d}), 8.60(1 \mathrm{H}, J=8 \mathrm{~Hz}, \mathrm{~d}), 8.75(1 \mathrm{H}, J=8 \mathrm{~Hz}, \mathrm{~d}), 8.83(1 \mathrm{H}$, $J=8 \mathrm{~Hz}, \mathrm{~d}$ ), 8.90 (1H, $J=8 \mathrm{~Hz}, \mathrm{~d}$ ); (Band 2; rac) 6.98 (H5b, 2H, $J=8,5 \mathrm{~Hz}, \mathrm{dd}$ ), 7.21 (H5d, 2H, $J=8$, $5 \mathrm{~Hz}, \mathrm{dd}$ ), 7.32 (H3/6 dpb, 2H, $J=10,8 \mathrm{~Hz}, \mathrm{dd}$ ), 7.47 (H5c, 2H, $J=8,5 \mathrm{~Hz}, \mathrm{dd}$ ), 7.49 (H6b, 2H, $J=5$, $1.5 \mathrm{~Hz}, \mathrm{dd}), 7.55$ (H6d, 2H, $J=$ 5, $1.5 \mathrm{~Hz}, \mathrm{dd}), 7.56$ (H4/5 dpb, 2H, $J=10,8 \mathrm{~Hz}, \mathrm{dd}), 7.58$ (H6c, 2H, $J=$ 5, $1.5 \mathrm{~Hz}, \mathrm{dd}), 7.64$ (H5a, 2H, $J=8,5 \mathrm{~Hz}, \mathrm{dd}), 7.76$ (H2/7 dpb, 2H, s), 7.87 (H4d, 2H, $J=8,8 \mathrm{~Hz}, \mathrm{dd}$ ), 7.84-7.91 (10H, m), 7.96 (H4b, 2H, $J=8,8$ Hz, dd), 8.08 (H4c, 2H, $J=8,8$ Hz, dd), 8.29 (H10/17 dpb, 2H, $J=8 \mathrm{~Hz}, \mathrm{~d}), 8.33$ (H13/14 dpb, 2H, $J=8 \mathrm{~Hz}, \mathrm{~d}), 8.68$ (H3b, 2H, $J=8 \mathrm{~Hz}, \mathrm{~d}), 7.77$ (H3c, H3d, 4H, $J$ $=8 \mathrm{~Hz}, \mathrm{~d})$.

$\left[\left\{\mathbf{O s}(\mathbf{b p y})_{2}\right\}_{2}(\mu-\mathbf{d p b})\right]\left(\mathbf{P F}_{6}\right)_{4}$. The synthesis, diastereoisomeric separation and purification were performed via a similar procedure to that described above for $\left[\left\{\mathrm{Os}(\mathrm{bpy})_{2}\right\}_{2}(\mu\right.$-dpb) $]\left(\mathrm{PF}_{6}\right)_{4}$ involving the reaction of cis-[Os(bpy) $\left.{ }_{2} \mathrm{Cl}_{2}\right] .2 \mathrm{H}_{2} \mathrm{O}$ (114 mg, $0.187 \mathrm{mmol}$ ) with dpb' (30 mg, $\left.0.0903 \mathrm{mmol}\right)$. The 
dinuclear species was isolated as an olive green solid. Yield: $147 \mathrm{mg}$ (85\%). Anal. Calcd for $\mathrm{C}_{62} \mathrm{H}_{44} \mathrm{~F}_{24} \mathrm{~N}_{12} \mathrm{P}_{4} \mathrm{Os}_{2}$ : C, 38.8; H, 2.31; N, 8.77\%. Found: C, 38.5; H, 2.46; N, 8.53\%. ${ }^{1} \mathrm{H}$ NMR ( $\delta \mathrm{ppm}$; $\mathrm{CD}_{3} \mathrm{CN}$ ): (Band 1; meso) 6.71 (H6d, 2H, $J=5 \mathrm{~Hz}, \mathrm{~d}$ ), 7.12 (H5d, 2H, $J=8,5 \mathrm{~Hz}$, dd), 7.17 (H5b, 2H, $J$ = 8, 5 Hz, dd), 7.29 (H3/6 dpb', 2H), 7.49 (H5c, 2H, J = 8, 5 Hz, dd), 7.55 (H2/7 dpb', 2H), 7.57 (H4/5 dpb', 2H, s), 7.58 (H6c, 2H, $J=5$ Hz, d), 7.63 (H4d, 2H, $J=8,8$ Hz, dd), 7.66 (H5a, 2H, $J=8,5$ Hz, dd), 7.76 (H11/14 dpb', 2H, $J=10,9 \mathrm{~Hz}, \mathrm{dd}), 7.91$ (H4c, 2H, $J=8$, 8 Hz, dd), 7.94 (H6a, 2H, $J=5 \mathrm{~Hz}, \mathrm{~d}$ ), 7.98 (H6b, 2H, $J=5$ Hz, d), 8.03 (H4b, 2H, $J=8,8$ Hz, dd), 8.05 (H10/15 dpb', 2H), 8.06 (H3d, H10/15 dpb', 4H), 8.08 (H4a, 2H, $J=8,8 \mathrm{~Hz}, \mathrm{dd}$ ), 8.26 (H3c, 2H, $J=8 \mathrm{~Hz}, \mathrm{~d}), 8.69$ (H3b, 2H, $J=8 \mathrm{~Hz}, \mathrm{~d}$ ), 8.78 (H3a, 2H, $J=8 \mathrm{~Hz}, \mathrm{~d}), 9.06$ (H12/13 dpb', 2H, $J=8,1.5 \mathrm{~Hz}$, d); (Band 2; rac) 6.93 (H5b, 2H, $J=8,5$ Hz, dd), 7.12 (H3/6 dpb', 2H), 7.09 (H6d, 2H, $J=5,1.5$ Hz, dd), 7.20 (H5d, 2H, $J=8,5$ Hz, dd), 7.36 (H6b, 2H, $J=5,1.5 \mathrm{~Hz}, \mathrm{dd}), 7.51$ (H2/7 dpb', 2H), 7.54 (H6a, 2H, $J=5,1.5 \mathrm{~Hz}, \mathrm{dd}), 7.55$ (H5a, 2H, $J=$ 8, 5 Hz, dd), 7.56 (H5c, 2H, J = 8, 5 Hz, dd), 7.70 (H4/5 dpb', 2H, s), 7.76 (H6c, 2H, $J=$ 5, 1.5 Hz, dd), 7.79 (H11/14 dpb', 2H, $J=10,9$ Hz, dd), 7.89 (H4b, 2H, $J=8,8$ Hz, dd), 7.93 (H4d, 2H, $J=8,8 \mathrm{~Hz}$, dd), 8.04 (H4a, 2H, $J=8,8$ Hz, dd), 8.06 (H4c, 2H, $J=8,8$ Hz, dd), 8.05 (H10/15 dpb', 2H, $J=8,1.5$ Hz, dd), 8.45 (H3b, 2H, $J=8$ Hz, d), 8.54 (H3d, 2H, $J=8$ H, d), 8.59 (H3a, 2H, $J=8$ Hz, d), 8.67 (H3c, 2H, $J=8 \mathrm{~Hz}, \mathrm{~d}), 9.02(\mathrm{H} 12 / 13 \mathrm{dpb}$ ', 2H, $J=8,1.5 \mathrm{~Hz}, \mathrm{~d})$.

$\left[\left\{\mathbf{O s}(\mathbf{b p y})_{2}\right\}_{2}(\mu-\mathbf{d p q})\right]\left(\mathbf{P F}_{6}\right)_{4}$. The synthesis and purification were performed under similar conditions to those described above for $\left[\left\{\mathrm{Os}(\mathrm{bpy})_{2}\right\}_{2}(\mu-\mathrm{dpb})\right]\left(\mathrm{PF}_{6}\right)_{4}$ involving the reaction of $c i s$-[Os(bpy $\left.)_{2} \mathrm{Cl}_{2}\right] \cdot 2 \mathrm{H}_{2} \mathrm{O}$ (100 mg, $0.164 \mathrm{mmol}$ ) with dpq' (22.3 mg, $0.0791 \mathrm{mmol})$. The dinuclear species was isolated as a dark green solid. Yield: $145 \mathrm{mg}$ (98\%). Anal. Calcd for $\mathrm{C}_{58} \mathrm{H}_{42} \mathrm{~F}_{24} \mathrm{~N}_{12} \mathrm{P}_{4} \mathrm{Os}_{2}$ : C, 37.3; H, 2.27; N, 9.00\%.

Found: C, 37.0; H, 2.15; N, 8.87\%. ${ }^{1} \mathrm{H}$ NMR ( $\left.\delta \mathrm{ppm} ; \mathrm{CD}_{3} \mathrm{CN}\right)$ : (Band 1; meso) 6.68 (H6d, $2 \mathrm{H}, J=5 \mathrm{~Hz}$, d), 7.10 (H5d, 2H, $J=8,5 \mathrm{~Hz}$, dd), 7.21 (H5b, 2H, $J=8,5 \mathrm{~Hz}$, dd), 7.45 (H5c, 2H, $J=8,5 \mathrm{~Hz}, \mathrm{dd}$ ), 7.47 (H5a, 2H, $J=8,5$ Hz, dd), 7.46 (H2/5 dpq', 2H), 7.54 (H6c, 2H, $J=5$ Hz, d), 7.50 (H6a, 2H, $J=5$ Hz, d), 7.50 (H3/4 dpq', 2H), 7.65 (H4d, 2H, $J=8,8$ Hz, dd), 7.77 (H6b, 2H, $J=5$ Hz, d), 7.81 (H9/12 dpq', 2H), 7.85 (H4a, 2H, $J=8,8$ Hz, dd), 7.99-8.05 (H4b, H8/13 dpq', 4H, m), 8.05 (H4c, 2H, $J=8,8$ Hz, dd), 8.09 (H3d, 2H, $J=8 \mathrm{~Hz}, \mathrm{~d}), 8.27$ (H3a, 2H, $J=8 \mathrm{~Hz}, \mathrm{~d}$ ), 8.66 (H3b, 2H, $J=8 \mathrm{~Hz}, \mathrm{~d}), 8.73$ (H3c, 2H, $J=$ 8 Hz, d), 9.12 (H10/11 dpq', 2H, $J=8 \mathrm{~Hz}, \mathrm{~d}$ ); (Band 2; rac) 6.95 (H5b, 2H, $J=8,5 \mathrm{~Hz}, \mathrm{dd}$ ), 7.15 (H5d, 2H, $J=8,5 \mathrm{~Hz}, \mathrm{dd}), 7.20$ (H6d, 2H, $J=5,1.5 \mathrm{~Hz}, \mathrm{dd}), 7.22$ (H3/4 dpq', 2H), 7.28 (H6b, 2H, $J=5,1.5$ Hz, dd), 7.35 (H2/5 dpq', 2H), 7.43 (H5a, 2H, $J=8,5$ Hz, dd), 7.47 (H6c, 2H, $J=5,1.5$ Hz, dd), 7.51 (H5c, 2H, $J=8,5 \mathrm{~Hz}, \mathrm{dd}), 7.63$ (H6a, 2H, $J=5,1.5 \mathrm{~Hz}, \mathrm{dd}), 7.83$ (H9/12 dpq', 2H, $J=10,8 \mathrm{~Hz}, \mathrm{dd}$ ), 7.88 (H4b, 2H, $J=8,8 \mathrm{~Hz}, \mathrm{dd}$ ), 7.93 (H4a, 2H, $J=8,8 \mathrm{~Hz}, \mathrm{dd}$ ), 7.96 (H4d, 2H, $J=8,8 \mathrm{~Hz}, \mathrm{dd}$ ), 8.03 (H4c, 2H, $J=8,8$ Hz, dd), 8.09 (H8/13 dpq', 2H, $J=6$ Hz, dd), 8.48 (H3d, 2H, $J=8$ H, d), 8.51 (H3b, 2H, $J=8 \mathrm{~Hz}, \mathrm{~d}$ ), 8.54 (H3a, 2H, $J=8 \mathrm{~Hz}, \mathrm{~d}), 8.60$ (H3c, 2H, $J=8 \mathrm{~Hz}, \mathrm{~d}), 9.06$ (H10/11 dpq', 2H, $J=8$ $\mathrm{Hz}, \mathrm{d})$. 
[\{Os(bpy $\left.\left.)_{2}\right\}_{2}(\mu-\mathbf{p p z})\right]\left(\mathbf{P F}_{6}\right)_{4}$. The synthesis and purification were performed under similar conditions to those described above for $\left[\left\{\mathrm{Os}(\mathrm{bpy})_{2}\right\}_{2}(\mu-\mathrm{dpb})\right]\left(\mathrm{PF}_{6}\right)_{4}$ involving the reaction of cis-[Os $\left.(\mathrm{bpy})_{2} \mathrm{Cl}_{2}\right] \cdot 2 \mathrm{H}_{2} \mathrm{O}$ (54.3 mg, $0.0947 \mathrm{mmol}$ ) with ppz (10 mg, $0.0431 \mathrm{mmol}$ ). The dinuclear species was isolated as a turquoise solid. Yield: $60 \mathrm{mg}$ (77\%). Anal. Calcd for $\mathrm{C}_{54} \mathrm{H}_{40} \mathrm{~F}_{24} \mathrm{~N}_{16} \mathrm{P}_{4} \mathrm{Os}_{2}$ : C, 34.6; H, 2.15; N, 12.0\%. Found: C, 34.4; H, 2.28; N, 11.8\%. ${ }^{1} \mathrm{H}$ NMR ( $\delta$ ppm; $\mathrm{CD}_{3} \mathrm{CN}$ ): (Band 1; meso) 7.18 (H5d, 2H, J = 8, 5 Hz, dd), 7.32 (H5b, 2H, $J=8,5$ Hz, dd), 7.37 (H5c, 2H, $J=8,5 \mathrm{~Hz}, \mathrm{dd}), 7.44$ (H6d, 2H, $J=5,1.5 \mathrm{~Hz}$, dd), 7.46 (H5a, 2H, $J=8,5$ Hz, dd), 7.60 (H6a, 2H, $J=5,1.5$ Hz, dd), 7.65 (H6c, 2H, $J=5,1.5$ Hz, dd), 7.71 (H2/3 ppz, 2H, s), 7.75 (H6b, 2H, $J=5,1.5 \mathrm{~Hz}, \mathrm{dd}$ ), 7.85 (H4d, 2H, $J=8,8 \mathrm{~Hz}, \mathrm{dd}$ ), 7.88 (H4c, 2H, $J=8,8 \mathrm{~Hz}, \mathrm{dd}), 7.90$ (H7/10 ppz, 2H, $J=10,8 \mathrm{~Hz}, \mathrm{dd}), 7.90$ (H4b, 2H, $J=8,8 \mathrm{~Hz}, \mathrm{dd}), 7.97$ (H4a , 2H, $J=8,8 \mathrm{~Hz}, \mathrm{dd}), 8.16$ (H6/11 ppz, 2H, $J=5,1.5 \mathrm{~Hz}, \mathrm{dd}$ ), 8.38 (H3d, 2H, $J=8,1.5 \mathrm{~Hz}, \mathrm{dd}$ ), 8.42 (H3c, 2H, $J=8,1.5 \mathrm{~Hz}, \mathrm{dd}$ ), 8.49 (H3b, 2H, $J=8,1.5 \mathrm{~Hz}, \mathrm{dd}$ ), 8.57 (H3a, 2H, $J=8,1.5 \mathrm{~Hz}, \mathrm{dd}$ ), 9.09 (H8/9 ppz, 2H, $J=8,1.5 \mathrm{~Hz}$, dd); (Band 2; rac) 7.00 (H5b, 2H, $J=8,5 \mathrm{~Hz}, \mathrm{dd}$ ), 7.29 (H5d, 2H, $J=8,5 \mathrm{~Hz}$, dd), 7.30 (H6b, 2H, $J=5,1.5 \mathrm{~Hz}, \mathrm{dd}$ ), 7.31 (H5c, 2H, $J=8,5 \mathrm{~Hz}, \mathrm{dd}$ ), 7.47 (H5a, 2H, $J=8,5 \mathrm{~Hz}, \mathrm{dd}$ ), 7.51 (H6d, 2H, $J=5,1.5 \mathrm{~Hz}$, dd), 7.53 (H6c, 2H, $J=5,1.5 \mathrm{~Hz}$, dd), 7.64 (H6a, 2H, $J=5,1.5 \mathrm{~Hz}$, dd), 7.74 (H2/3 ppz, 2H, s), 7.83 (H4b, 2H, $J=8,8$ Hz, dd), 7.89 (H7/10 ppz, 2H, $J=10,8$ Hz, dd), 7.89 (H4c, 2H, $J=8,8 \mathrm{~Hz}, \mathrm{dd}), 7.97$ (H4a, 2H, $J=8,8 \mathrm{~Hz}, \mathrm{dd}$ ), 8.01 (H4d, 2H, $J=8,8 \mathrm{~Hz}$, dd), 8.17 (H6/11 ppz, 2H, $J=5,1.5 \mathrm{~Hz}$, dd), 8.45 (H3b, 2H, $J=8,1.5 \mathrm{~Hz}, \mathrm{dd}$ ), 8.52 (H3c, 2H, $J=8,1.5 \mathrm{~Hz}$, dd), 8.52 (H3a, 2H, $J=8,1.5 \mathrm{~Hz}, \mathrm{dd}$ ), 8.56 (H3d, 2H, $J=8,1.5 \mathrm{~Hz}, \mathrm{dd}$ ), 9.09 (H8/9 ppz, 2H, $J=8,1.5 \mathrm{~Hz}, \mathrm{dd}$ ).

[\{Os(bpy $\left.\left.)_{2}\right\}_{2}(\mu-2,3-\mathbf{d p p})\right]\left(\mathbf{P F}_{6}\right)_{4}$. The synthesis and purification were performed under similar conditions to those described above for $\left[\left\{\mathrm{Os}(\mathrm{bpy})_{2}\right\}_{2}(\mu-\mathrm{dpb})\right]\left(\mathrm{PF}_{6}\right)_{4}$ involving the reaction of cis-[Os(bpy $\left.)_{2} \mathrm{Cl}_{2}\right] \cdot 2 \mathrm{H}_{2} \mathrm{O}$ (94.3 mg, $0.164 \mathrm{mmol})$ with 2,3-dpp (17.5 $\mathrm{mg}, 0.0747 \mathrm{mmol})$. The dinuclear species was isolated as a dark purple solid. Yield: $130 \mathrm{mg}$ ( 96\%). Anal. Calcd for $\mathrm{C}_{54} \mathrm{H}_{42} \mathrm{~F}_{24} \mathrm{~N}_{12} \mathrm{P}_{4} \mathrm{Os}_{2}$ : C, 35.7; H, 2.33; N, 9.24\%. Found: C, 35.5; H, 2.14; N, 9.20\%. ${ }^{1} \mathrm{H}$ NMR ( $\delta \mathrm{ppm} ; \mathrm{CD}_{3} \mathrm{CN}$ ): (Band 1; meso) 7.16 (H5d, 2H, $J=8 \mathrm{~Hz}$, d), 7.33 (H5b, 2H, $J$ = 8, 5 Hz, dd), 7.40 (H8/11 2,3-dpp, 2H, m), 7.42 (H5c, 2H, $J=8,5$ Hz, dd), 7.49 (H6d, 2H, $J=5,1.5 \mathrm{~Hz}$, dd), 7.51 (H5a, 2H, $J=8,5 \mathrm{~Hz}, \mathrm{dd}$ ), 7.69 (H6a, 2H, $J=5,1.5 \mathrm{~Hz}, \mathrm{dd}), 7.72$ (H6b, H6c, 4H, $J$ = 5, 1.5 Hz, dd), 7.78 (H2/3 2,3-dpp, 2H, s), 7.85 (H4d, 2H, $J=8,8$ Hz, dd), 7.95 (H4b, H4c, 4H, $J=8,8$ Hz, dd), 7.98 (H7/12 2,3-dpp, 2H, $J=10,8$ Hz, dd), 8.00 (H4a , 2H, $J=8,8$ Hz, dd), 8.39 (H6/13 2,3-dpp, 2H, m), 8.39 (H3c, H3d, 4H, $J=8,1.5$ Hz, dd), 8.56 (H3b, 2H, $J=8,1.5$ Hz, dd), 8.58 (H3a, 2H, $J=8,1.5$ Hz, dd), 8.74 (H9/10 2,3-dpp, 2H, $J=8,1.5$ Hz, dd); (Band 2; rac) 6.98 (H5b, 2H, $J=8,5 \mathrm{~Hz}, \mathrm{dd}$ ), 7.30 (H5d, 2H, $J=8,5 \mathrm{~Hz}, \mathrm{dd}$ ), 7.33 (H6b, 2H, $J=5,1.5 \mathrm{~Hz}, \mathrm{dd}), 7.29$ (H5c, 2H, $J$ = 8, 5 Hz, dd), 7.50 (H8/11 2,3-dpp, 2H, m), 7.48 (H5a, 2H, $J=8,5$ Hz, dd), 7.51 (H6d, 2H, $J=5,1.5$ Hz, dd), 7.53 (H6c, 2H, J = 5, 1.5 Hz, dd), 7.64 (H6a, 2H, $J=5,1.5$ Hz, dd), 7.77 (H2/3 2,3-dpp, 2H, s), 7.90 (H4b, 2H, J = 8, 8 Hz, dd), 8.40 (H6/13 2,3-dpp, 2H, m), 7.89-7.99 (6H, m), 8.00 (H7/12 2,3-dpp, 2H, $J=10,8 \mathrm{~Hz}, \mathrm{dd}$ ), 8.30 (H3b, 2H, $J=8,1.5 \mathrm{~Hz}, \mathrm{dd}$ ), 8.35 (H3c, 2H, $J=8,1.5 \mathrm{~Hz}, \mathrm{dd}$ ), 8.53 (H3a, 2H, $J=8,1.5 \mathrm{~Hz}, \mathrm{dd}$ ), 8.56 (H3d, 2H, $J=8,1.5 \mathrm{~Hz}, \mathrm{dd}), 8.70$ (H9/10 2,3-dpp, 2H, $J=8,1.5 \mathrm{~Hz}, \mathrm{dd}$ ). 


\section{(iii) Dinuclear Ruthenium Complexes Incorporating Stepped-Parallel Bridging Ligands}

$\left[\left\{\mathbf{R u}(\mathbf{b p y})_{2}\right\}_{2}(\mu-\mathbf{2 , 5}-\mathbf{d p p})\right]\left(\mathbf{P F}_{6}\right)_{4}$ was synthesised by an adaptation of the literature procedure. ${ }^{132}$ The synthesis and purification were performed according to the microwave methodology described above for $\left[\left\{\mathrm{Ru}(\mathrm{bpy})_{2}\right\}_{2}(\mu-\mathrm{dpb})\right]\left(\mathrm{PF}_{6}\right)_{4}$ involving the reaction of cis-[Ru(bpy) $\left.)_{2} \mathrm{Cl}_{2}\right] \cdot 2 \mathrm{H}_{2} \mathrm{O}(100 \mathrm{mg}, 0.192 \mathrm{mmol})$ with 2,5-dpp (18.3 mg, $0.0873 \mathrm{mmol}$ ). The dinuclear species was isolated as a purple solid. Yield: $130 \mathrm{mg}$ (92\%). Anal. Calcd for $\mathrm{C}_{52} \mathrm{H}_{42} \mathrm{~F}_{24} \mathrm{~N}_{12} \mathrm{P}_{4} \mathrm{Ru}_{2}$ : C, 38.6; H, 2.62; N, 10.4\%. Found: C, 38.4; H, 2.45; N, $10.3 \%$.

Separation of the diastereoisomers was achieved as described above using $0.25 \mathrm{M}$ sodium tosylate. Bands 1 and 2 were determined to be the rac and meso diastereoisomers, respectively, as established by NMR characterisation and comparison with the ${ }^{1} \mathrm{H}$ NMR spectra for $\left[\left\{\mathrm{Ru}(\mathrm{phen})_{2}\right\}_{2}(\mu-2,5-\mathrm{dpp})\right]\left(\mathrm{PF}_{6}\right)_{4}$ reported by Hua. ${ }^{132}{ }^{1} \mathrm{H}$ NMR ( $\delta$ ppm; $\mathrm{CD}_{3} \mathrm{CN}$ ): (Band 1; rac) 7.31 (H5b, 2H, $J=8,5 \mathrm{~Hz}$, dd), 7.46 (H5b, H5c, 4H, $J$ = 8, 5 Hz, dd), 7.51 (H6d, H6b, 4H, $J$ = 5, 1.5 Hz, dd), 7.59 (H5a, H4 2,5-dpp, 4H, $J=$ 8, $5 \mathrm{~Hz}$, dd), 7.77 (H6c, 2H, $J$ = 5, $1.5 \mathrm{~Hz}$, dd), 7.83 (H6a, 2H, $J$ = 5, 1.5 Hz, dd), 7.90 (H6 2,5-dpp, 2H, $J$ $=8 \mathrm{~Hz}, \mathrm{~d}), 7.98$ (H4d, 2H, $J=8,8 \mathrm{~Hz}, \mathrm{dd}), 8.03$ (H6b, 2H, $J=5,1.5 \mathrm{~Hz}, \mathrm{dd}), 8.10$ (H4c, 2H, $J=8,8 \mathrm{~Hz}$, dd), 8.13 (H4a, 2H, $J=8,8 \mathrm{~Hz}$, dd), 8.17 (H4, 2H, $J=8,8 \mathrm{~Hz}, \mathrm{dd}$ ), 8.20 (H4, 2H, $J=8,8 \mathrm{~Hz}, \mathrm{dd}$ ), 8.38 (H7/H14 2,5-dpp, 2H, s), 8.54 (H3d, 2H, $J=8,1.5$ Hz, dd), 8.56 (H3b, H3c, H3a, 6H, $J=8$ Hz, d); (Band 2; meso) 7.19 (H4 2,5-dpp, 2H, $J=8,5 \mathrm{~Hz}$, dd), 7.48 (H5d, H5b, 4H, J = 8, 5 Hz, dd), 7.50 (H5c, 2H, $J=8,5$ Hz, dd), 7.56 (H3 2,5-dpp, H6d, 4H, $J=5,1.5$ Hz, dd), 7.65 (H6c, H6a, 4H, $J=5,1.5$ Hz, dd), 7.76 (H6d, H6b, 4H, $J=5,1.5$ Hz, dd), 7.90 (H4 2,5-dpp, 2H), 7.96 (H4d, 2H, J = 8, 8 Hz, dd), 8.06 (H4b, 2H, $J=8,8 \mathrm{~Hz}, \mathrm{dd}$ ), 8.11 (H4c, 2H, $J=8,8 \mathrm{~Hz}$, dd), 8.17 (H5 2,5-dpp, 2H, $J=8,8 \mathrm{~Hz}, \mathrm{dd}$ ), 8.21 (H4a, 2H, $J=8,8$ Hz, dd), 8.42 (H7/H14 2,5-dpp, 2H, s), 8.50 (H6/13 2,5-dpp, 2H, $J=8,1.5$ Hz, dd), 8.54 (H3d, 2H, $J=8,1.5 \mathrm{~Hz}$, dd), 8.56 (H3b, 2H, $J$ = 8, $1.5 \mathrm{~Hz}, \mathrm{dd}$ ), 8.59 (H3c, 2H, $J$ = 8, 1.5 Hz, dd).

$\left[\left\{\mathbf{R u}(\mathbf{b p y})_{2}\right\}_{2}(\mu\right.$-dpop) $]\left(\mathbf{P F}_{6}\right)_{4}$. The separation and purification of the diastereoisomeric forms were achieved as described previously, using $0.25 \mathrm{M}$ sodium tosylate solution as the eluent. ${ }^{1} \mathrm{H}$ NMR ( $\delta$ ppm; $\mathrm{CD}_{3} \mathrm{CN}$ ): (Band 1; rac) 6.97 (H5d, 2H, $J=8,5 \mathrm{~Hz}$, dd), 7.21 (H6d, 2H, $J=5,1.5 \mathrm{~Hz}$, dd), 7.28 (H5b, 2H, $J$ = 5, $1.5 \mathrm{~Hz}$, dd), 7.29 (H6b, 2H, $J=5,1.5 \mathrm{~Hz}, \mathrm{dd}$ ), 7.45 (H6/13 dpop, 2H, $J=8 \mathrm{~Hz}, \mathrm{~d}$ ), 7.51 (H5a, 2H, $J=5,1.5 \mathrm{~Hz}, \mathrm{dd}$ ), 7.57 (H5c, 2H, $J=5,1.5 \mathrm{~Hz}, \mathrm{dd}$ ), 7.66 (H6a, 2H, $J=5,1.5 \mathrm{~Hz}, \mathrm{dd}$ ), 7.86 (H7/14 dpop, 2H, $J=10,8$ Hz, dd), 7.93 (H6c, 2H, $J=5,1.5$ Hz, dd), 8.05 (H4d, 2H, $J=8,8$ Hz, dd), 8.08c (H4X, 2H, $J=8,8 \mathrm{~Hz}, \mathrm{dd}), 8.14-8.20$ (2H, m), 8.23 (H4a, 2H, $J=8,8 \mathrm{~Hz}, \mathrm{dd}), 8.49$ (H3a, 2H, $J=$ 8, $1.5 \mathrm{~Hz}, \mathrm{dd}$ ), 8.51 (H3c, 2H, $J=8,1.5 \mathrm{~Hz}, \mathrm{dd}), 8.57$ (H3d, 2H, $J=8,1.5 \mathrm{~Hz}, \mathrm{dd}$ ), 8.61 (H3a, 2H, $J=8$, $1.5 \mathrm{~Hz}, \mathrm{dd}$ ); (Band 2; meso) 6.51 (H6b, 2H, $J=5,1.5 \mathrm{~Hz}, \mathrm{dd}$ ), 6.77 (H5b, 2H, $J=8,5 \mathrm{~Hz}, \mathrm{dd}$ ), 7.31 (H5d, 2H, $J=8,5 \mathrm{~Hz}$, dd), 7.43 (H5c, 2H, $J=8,5 \mathrm{~Hz}$, dd), 7.59 (H6/13 dpop, 2H, $J=8$ Hz, d), 7.62 (H6c, 2H, $J=5,1.5 \mathrm{~Hz}, \mathrm{dd}$ ), 7.65 (H6a, 2H, $J=5,1.5 \mathrm{~Hz}, \mathrm{dd}$ ), 7.82 (H7/14 dpop, 2H, $J=10,8 \mathrm{~Hz}, \mathrm{dd}$ ), 7.82 (H5a, 2H, $J=8,5 \mathrm{~Hz}$, dd), 8.04 (H6d, 2H, $J=5,1.5 \mathrm{~Hz}, \mathrm{dd}$ ), 8.10 (H4b, 2H, $J=8,8 \mathrm{~Hz}, \mathrm{dd}$ ), 8.11 (H4a, 2H, $J=8,8 \mathrm{~Hz}, \mathrm{dd}$ ), 8.14-8.22 (10H, m), 8.40 (H3a, 2H, $J=8,1.5 \mathrm{~Hz}, \mathrm{dd}$ ), 8.62 (H3b, 2H, $J=8$, $1.5 \mathrm{~Hz}, \mathrm{dd}$ ), 8.68 (H3b, H3c, 4H, $J=8,1.5 \mathrm{~Hz}, \mathrm{dd}$ ). 
$\left[\left\{\mathbf{R u}(\mathbf{b p y})_{2}\right\}_{2}(\mu\right.$-apy $\left.)\right]\left(\mathbf{P F}_{6}\right)_{4}$. The synthesis and diastereoisomer separation was performed according to the literature procedure. ${ }^{70}$

\subsubsection{X-ray Crystallography}

\section{(i) Ruthenium and Osmium Complexes Incorporating the Bridging Ligands dpb, dpb' and dpq'}

Single crystals of meso-[\{Ru(bpy $\left.\left.)_{2}\right\}_{2}(\mu-\mathrm{dpb})\right]\left(\mathrm{PF}_{6}\right)_{4} \cdot 5 \mathrm{H}_{2} \mathrm{O}$ (dark red rod-shaped crystals) and meso-[\{Ru(bpy $\left.)_{2}\right\}_{2}\left(\mu\right.$-dpb')] $\left(\mathrm{PF}_{6}\right)_{4} \cdot 2 \mathrm{H}_{2} \mathrm{O} .2\left\{\left(\mathrm{CH}_{3}\right)_{2} \mathrm{CO}\right\}$ (dichroic red/green crystals) were grown by slow evaporation of a solution of $c a .1 \mathrm{mmol}$ of the complex in acetone/water $\left(1: 1,2 \mathrm{~cm}^{3}\right)$ under ambient conditions in the absence of light. Single crystals of meso- $\left[\left\{\mathrm{Ru}(\mathrm{bpy})_{2}\right\}_{2}(\mu\right.$-dpb) $]\left(\mathrm{ZnCl}_{4}\right)_{2} \cdot 5 \mathrm{H}_{2} \mathrm{O}$, meso$\left[\left\{\mathrm{Ru}(\mathrm{bpy})_{2}\right\}_{2}(\mu-\mathrm{dpq})\right]\left(\mathrm{ZnCl}_{4}\right)_{2} \cdot 3 \mathrm{H}_{2} \mathrm{O}$ and meso-[\{Os(bpy $\left.\left.)_{2}\right\}_{2}(\mu-\mathrm{dpb})\right]\left(\mathrm{ZnCl}_{4}\right)_{2} \cdot 6 \mathrm{H}_{2} \mathrm{O}$ were obtained by initially stirring a suspension of the hexafluorophosphate salt $(10 \mathrm{mg})$ in distilled water $\left(1 \mathrm{~cm}^{3}\right)$ with DOWEX ${ }^{\circledR}$ anion exchange resin $\left(\mathrm{Cl}^{-}\right.$form), to afford the corresponding chloride salt. Following the addition of two molar equivalents of $\mathrm{ZnCl}_{2}$ and aqueous $\mathrm{HCl}$ (3 drops, $2 \mathrm{M}$ ), the solutions were allowed to evaporate slowly at room temperature to yield dichroic red/green rod-shaped crystals of meso$\left[\left\{\mathrm{Ru}(\mathrm{bpy})_{2}\right\}_{2}(\mu-\mathrm{dpb})\right]\left(\mathrm{ZnCl}_{4}\right)_{2} \cdot 5 \mathrm{H}_{2} \mathrm{O}$ and meso-[ $\left\{\mathrm{Ru}(\mathrm{bpy})_{2}\right\}_{2}\left(\mu \text {-dpq')]( } \mathrm{ZnCl}_{4}\right)_{2} \cdot 3 \mathrm{H}_{2} \mathrm{O}$, and deep green rodshaped crystals of meso-[\{Os(bpy $\left.)_{2}\right\}_{2}(\mu$-dpb) $]\left(\mathrm{ZnCl}_{4}\right)_{2} \cdot 6 \mathrm{H}_{2} \mathrm{O}$ suitable for $\mathrm{X}$-ray determination. Deep green, octahedral-shaped crystals of meso-[\{Os(bpy $\left.\left.)_{2}\right\}_{2}\left(\mu-\mathrm{dpq}^{\prime}\right)\right]\left(\mathrm{PF}_{6}\right)_{4}$ were grown by slow evaporation of a $70: 30$ 2,2,2-trifluoroethanol/ $\mathrm{H}_{2} \mathrm{O}$ solution $\left(1.5 \mathrm{~cm}^{3}\right)$ of the complex under ambient conditions in the absence of light.

The collection and refinement of X-ray data were performed by Dr Murray Davies and Dr Peter Junk in the Advanced Analytical Centre at James Cook University. Hemispheres of data were collected (capillary sealed specimens) at room temperature on a Bruker SMART CCD diffractometer using the omega scan mode. Summaries of the data collection and refinement details are provided in Tables B3.1 and B3.15 (Appendix B) and the full details are included on the CD accompaniment to this thesis. Data sets were corrected for absorption using the program SADABS. ${ }^{133}$ The solution and refinement for all structures was carried out using SHELXL-97 ${ }^{134}$ utilising the graphical interface X-Seed. ${ }^{135}$

For meso- $\left[\left\{\mathrm{Ru}(\mathrm{bpy})_{2}\right\}_{2}(\mu-\mathrm{dpb})\right]\left(\mathrm{PF}_{6}\right)_{4} \cdot 5 \mathrm{H}_{2} \mathrm{O}$, all four $\mathrm{PF}_{6}{ }^{-}$anions were disordered and these were successfully modelled with partial occupancies. The hydrogen atoms on the lattice water molecules were not located. For meso- $\left[\left\{\mathrm{Ru}(\mathrm{bpy})_{2}\right\}_{2}(\mu\right.$-dpb) $]\left(\mathrm{ZnCl}_{4}\right)_{2} \cdot 5 \mathrm{H}_{2} \mathrm{O}$, the water molecules exhibited high thermal motion and their hydrogen atoms were not located. All non-hydrogen atoms were refined anisotropically. For meso-[ $\left.\left\{\mathrm{Ru}(\mathrm{bpy})_{2}\right\}_{2}(\mu-\mathrm{dpb})\right]\left(\mathrm{PF}_{6}\right)_{4} \cdot 2 \mathrm{H}_{2} \mathrm{O} .2\left\{\left(\mathrm{CH}_{3}\right)_{2} \mathrm{CO}\right\}$, the oxygen atoms of the water molecules and all atoms of the acetone molecules were isotropically refined. The hydrogen atoms of the lattice water molecules were not located. For meso-[ $\left\{\mathrm{Ru}(\mathrm{bpy})_{2}\right\}_{2}(\mu$-dpq') $)\left(\mathrm{ZnCl}_{4}\right)_{2} \cdot 3 \mathrm{H}_{2} \mathrm{O}$, the oxygen atoms of the water molecules were isotropically refined and their hydrogen atoms were not located. 


\section{(ii) Ruthenium Complexes Incorporating the Bridging Ligand dpo}

Single crystals of meso-[\{Ru(bpy $\left.)_{2}\right\}_{2}(\mu$-dpo $\left.)\right]\left(\mathrm{ZnCl}_{4}\right)_{2} \cdot 6 \mathrm{H}_{2} \mathrm{O}$ and $\mathrm{rac}-\left[\left\{\mathrm{Ru}(\mathrm{bpy})_{2}\right\}_{2}(\mu-\right.$ dpo) $]\left(\mathrm{ZnCl}_{4}\right)_{2}\left(\mathrm{ZnCl}_{2}\right) \cdot 2 \mathrm{H}_{2} \mathrm{O}$ were obtained by initially stirring a suspension of $c a .10 \mathrm{mg}$ of the $\mathrm{PF}_{6}^{-}$salt in distilled $\mathrm{H}_{2} \mathrm{O}\left(1 \mathrm{~cm}^{3}\right)$ with DOWEX ${ }^{\circledR} \mathrm{Cl}^{-}$anion exchange resin to afford the corresponding chloride salt. Following the addition of two molar equivalents of $\mathrm{ZnCl}_{2}$ and aqueous $\mathrm{HCl}$ (2 $\mathrm{M}, 3$ drops), the solution was allowed to evaporate slowly at room temperature to yield deep red rod-shaped crystals suitable for Xray determination.

The collection and refinement of X-ray data were performed as described above. For the rac diastereoisomer, the $\left[\mathrm{ZnCl}_{2}\left(\mathrm{H}_{2} \mathrm{O}\right)_{2}\right]$ entity was disordered over two sites, but this was successfully refined. Summaries of the data collection and refinement details and the selected bond lengths and angles are provided in Tables B3.2(a) and B3.2(b) \{Appendix B\}. A complete listing of the latter is included on the CD accompaniment to this thesis. 


\subsection{Results and Discussion}

\subsubsection{Diastereoisomer Synthesis, Separation and Structural Characterisation} (i) Symmetrical Dinuclear Ruthenium and Osmium Complexes Incorporating Angular and

\section{Stepped-Parallel Bridging Ligands}

The complexes $\left[\left\{\mathrm{Ru}(\mathrm{bpy})_{2}\right\}_{2}(\mu-\mathrm{BL})\right]^{4+}\{\mathrm{BL}=\mathrm{dpb}, \mathrm{dpb}$ ', dpq', ppz, 2,3-dpp, 2,5-dpp $\}$ and $\left[\left\{\mathrm{Os}(\mathrm{bpy})_{2}\right\}_{2}(\mu-\mathrm{BL})\right]^{4+}\{\mathrm{BL}=\mathrm{dpb}, \mathrm{dpb}$ ', dpq', ppz, 2,3-dpp $\}$ were synthesised by the reaction of 2.2 equivalents of cis-[M(bpy $\left.)_{2} \mathrm{Cl}_{2}\right]\{\mathrm{M}=\mathrm{Ru}$ or Os $\}$ with the bridging ligand in ethylene glycol, using the microwave-assisted methodology which is well established for the synthesis of a range of mono-, ${ }^{125,127,130}$ di-, ${ }^{67-69,128,130}$ and trinuclear ${ }^{130,136}$ polypyridyl complexes of ruthenium and osmium. This technique produced equivalent or increased reaction yields of the dinuclear species compared with previously reported thermal methods for the syntheses of $\left[\left\{\mathrm{Ru}(\mathrm{bpy})_{2}\right\}_{2}(\mu-\mathrm{BL})\right]^{4+}\left\{\mathrm{BL}=\mathrm{dpb},{ }^{74} \mathrm{ppz},{ }^{137,138}\right.$ 2,3-dpp, $\left.{ }^{72,75-79}\right\}$ and $\left[\left\{\mathrm{Os}(\mathrm{bpy})_{2}\right\}_{2}(\mu-\mathrm{BL})\right]^{4+}\{\mathrm{BL}=\mathrm{dpb}, 2,3-\mathrm{dpp}\}{ }^{72,139-141}$ However, there was a significant reduction in the reaction times (typically from $45 \mathrm{~min}$ for the osmium complexes and 2-24 $\mathrm{h}$ for the ruthenium complexes under the thermal refluxing procedure) to $c a .10$ min under microwaveassisted conditions.

The separation of the diastereoisomeric forms of the dinuclear complexes was achieved by cation-exchange chromatography using SP Sephadex C-25 as the support with aqueous solutions of sodium tosylate or sodium benzoate as the eluents. In all cases, the Band 1 and 2 eluates were determined to be the meso and rac diastereoisomers, respectively, for the dinuclear ruthenium and osmium complexes incorporating the angular bridging ligands. For the complexes incorporating the stepped-parallel bridging ligands 2,5-dpp and dpop, the opposite order of elution was observed.

In the past, the structural determinations of the dinuclear complexes investigated herein were achieved predominantly using mass spectroscopy and elemental microanalyses, ${ }^{67,68}$ as unambiguous NMR analyses were complicated by the stereoisomeric complexity of the systems. ${ }^{72,74-79,137,139-141}$ In the present study, X-ray crystallography and ${ }^{1} \mathrm{H}$ NMR analyses permitted unambiguous characterisation of the separated diastereoisomers.

\section{(ii) Unsymmetrical Dinuclear Ruthenium Complexes Incorporating Angular Bridging Ligands}

The unsymmetrical complexes $\left[\left\{\mathrm{Ru}(\mathrm{bpy})_{2}\right\}(\mu-\mathrm{BL})\left\{\mathrm{Ru}(\mathrm{pp})_{2}\right\}\right]^{4+} \quad\left\{\mathrm{BL}=\mathrm{dpb}, \mathrm{dpb} ; \mathrm{pp}=\mathrm{Me}_{2} \mathrm{bpy}\right.$, $\mathrm{Me}_{4}$ bpy $\}$ were synthesised by the reaction of equimolar amounts of the mononuclear precursors $\left[\mathrm{Ru}(\mathrm{bpy})_{2}(\mathrm{BL})\right]^{2+}$ and $\left[\mathrm{Ru}(\mathrm{pp})_{2} \mathrm{Cl}_{2}\right]$ in ethylene glycol$/ 10 \%$ water for 11 hours at $120^{\circ} \mathrm{C}$. The reactions were also attempted under microwave conditions but with limited success as the preliminary column chromatographic purification of the complexes following synthesis revealed a number of bands which were indicative of ligand scrambling.

The separation of the unsymmetrical complexes from their reaction mixtures was achieved using cation-exchange chromatography with a gradient elution of aqueous sodium chloride solution 
(0.1-0.5 M). The major green band eluted from the column was the desired unsymmetrical complex. The diastereoisomers $\Delta \Lambda / \Lambda \Delta$ - and $\Delta \Delta / \Lambda \Lambda$ - $\left[\left\{\mathrm{Ru}(\mathrm{bpy})_{2}\right\}(\mu-\mathrm{BL})\left\{\mathrm{Ru}(\mathrm{pp})_{2}\right\}\right]^{4+}$ were separated by cation exchange chromatography using SP Sephadex C-25 as the support with aqueous $0.25 \mathrm{M}$ sodium octanoate ffor BL $=\mathrm{dpb}, \mathrm{pp}=\mathrm{Me}_{2} \mathrm{bpy}$ and $\left.\mathrm{Me}_{4} \mathrm{bpy}\right\}$ and aqueous $0.25 \mathrm{M}$ sodium benzoate $\left\{\right.$ for $\mathrm{BL}=\mathrm{dpb}$ ', $\mathrm{pp}=\mathrm{Me}_{2} \mathrm{bpy}$ and $\mathrm{Me}_{4}$ bpy solutions as eluents. An additional level of stereoisomeric complexity is present in the $\left[\left\{\operatorname{Ru}(\mathrm{bpy})_{2}\right\}(\mu-\mathrm{BL})\left\{\mathrm{Ru}(\mathrm{pp})_{2}\right\}\right]^{4+}$ complexes because the $\Delta \Lambda$ and $\Lambda \Delta$ forms are no longer equivalent as they are when pp = bpy (i.e. a meso form). While differences may exist between the physical properties of the stereoisomeric forms, the present study involves the investigation of the mixtures of the enantiomers $\Delta \Lambda / \Lambda \Delta$ (“pseudo-meso"), and $\Delta \Delta / \Lambda \Lambda$ (“pseudo-rac").

\subsubsection{1 ${ }^{1} \mathrm{H}$ NMR Studies}

\section{(i) Symmetrical Dinuclear Complexes of Ruthenium and Osmium Incorporating Angular Bridging}

\section{Ligands}

The ${ }^{1} \mathrm{H}$ NMR data for the meso and rac diastereoisomers of $\left[\left\{\mathrm{Ru}(\mathrm{bpy})_{2}\right\}_{2}(\mu-\mathrm{dpb})\right]^{4+}$ are presented in Table 3.1, and the data for the complexes $\left[\left\{\mathrm{Ru}(\mathrm{bpy})_{2}\right\}_{2}(\mu-\mathrm{BL})\right]^{4+}\{\mathrm{BL}=\mathrm{dpb}, \mathrm{dpq}$ ', ppz, 2,3-dpp $\}$ and $\left[\left\{\mathrm{Os}(\mathrm{bpy})_{2}\right\}_{2}(\mu-\mathrm{BL})\right]^{4+}\{\mathrm{BL}=\mathrm{dpb}, \mathrm{dpb}$ ', dpq', ppz, 2,3-dpp $\}$ were reported in §3.2.3.3. The ${ }^{1} \mathrm{H}$ numbering schemes for the diastereoisomers of $\left[\left\{\mathrm{Ru}(\mathrm{bpy})_{2}\right\}_{2}\left(\mu-\mathrm{dpb}^{\prime}\right)\right]^{4+}$ are shown in Figure 3.3, and the designations for the full series of terminal and bridging ligands are shown in Figure B3.1 (Appendix B).

(a)

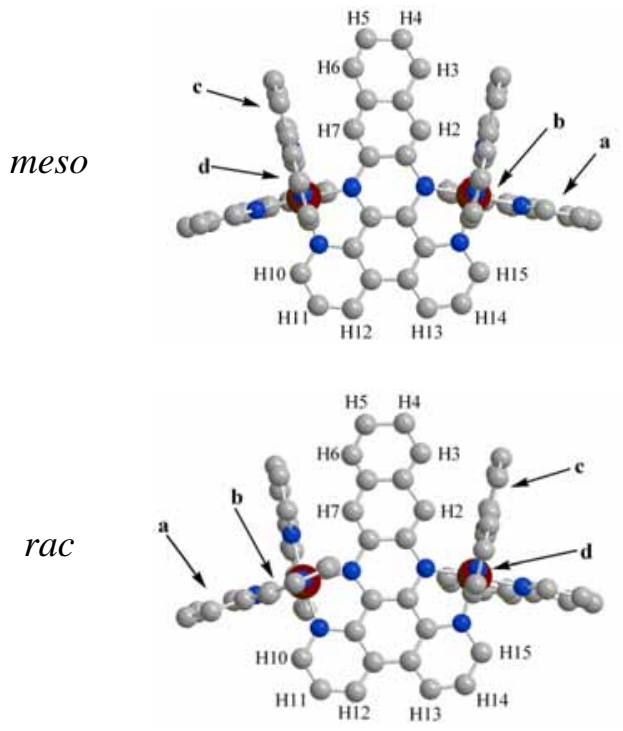

(b)

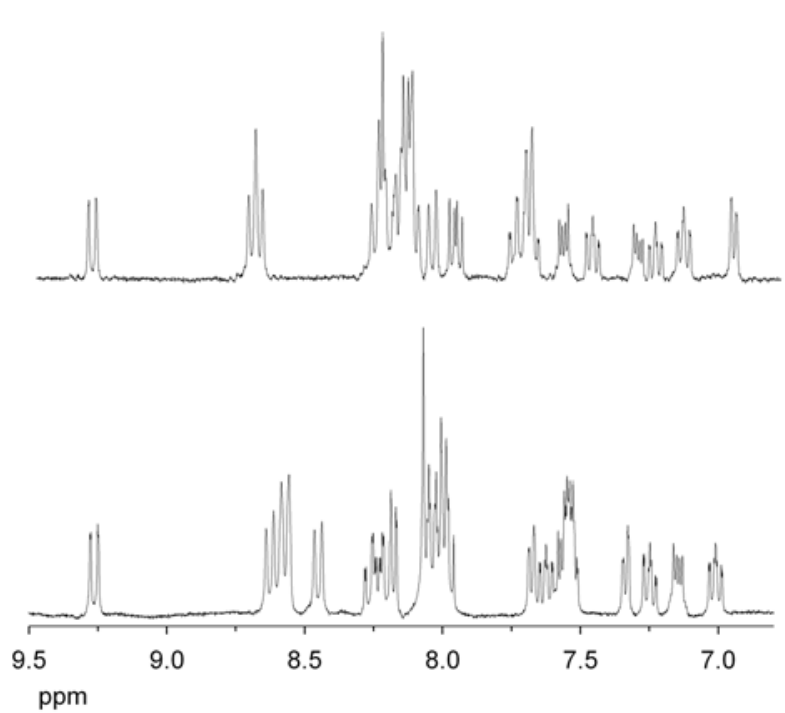

Figure 3.3 (a) Chem 3D representations of meso- $(\Lambda \Delta)$ - and rac- $(\Delta \Delta)-\left[\left\{\operatorname{Ru}(\mathrm{bpy})_{2}\right\}_{2}(\mu \text {-dpb') }]^{4+}\right.$. Hydrogen atoms are omitted for clarity. (b) ${ }^{1} \mathrm{H}$ NMR spectra (aromatic region only) for the diastereoisomers $\left(\mathrm{PF}_{6}{ }^{-}\right.$salts; $\mathrm{CD}_{3} \mathrm{CN}$ solution). 
Table 3.1 ${ }^{1} \mathrm{H}$ Chemical shifts (ppm) for the diastereoisomeric forms of $\left.\left[\left\{\mathrm{Ru}(\mathrm{bpy})_{2}\right\}_{2}(\mu-\mathrm{dpb})^{\prime}\right)\right]^{4+}\left(\mathrm{CD}_{3} \mathrm{CN}\right)$.

\begin{tabular}{|c|c|c|c|}
\hline & & $\begin{array}{c}\text { meso } \\
(\Delta \Lambda / \Lambda \Delta)\end{array}$ & $\begin{array}{c}r a c \\
(\Delta \Delta / \Lambda \Lambda)\end{array}$ \\
\hline \multirow{4}{*}{$\begin{array}{l}\text { bpy ring a a } \\
\text { (over bpy) }\end{array}$} & H3' & 8.27 & 8.57 \\
\hline & H4' & 8.13 & 8.25 \\
\hline & H5' & 7.70 & 7.62 \\
\hline & H6' & 7.97 & 8.00 \\
\hline \multirow{4}{*}{$\begin{array}{l}\text { bpy ring } b^{\text {a }} \\
\text { (over ppz) }\end{array}$} & H3 & 8.72 & 8.57 \\
\hline & H4 & 8.19 & 8.05 \\
\hline & H5 & 7.25 & 7.01 \\
\hline & H6 & 7.72 & 7.54 \\
\hline \multirow{4}{*}{$\begin{array}{l}\text { bpy ring c }{ }^{\text {a }} \\
\text { (over bpy) }\end{array}$} & H3' & 8.69 & 8.63 \\
\hline & H4' & 8.24 & 8.21 \\
\hline & H5' & 7.48 & 7.54 \\
\hline & H6' & 7.75 & 7.68 \\
\hline \multirow{4}{*}{$\begin{array}{l}\text { bpy ring } d^{\text {a }} \\
\text { (over } \mathrm{BL} \text { ) }\end{array}$} & H3 & 8.06 & 8.45 \\
\hline & $\mathrm{H} 4$ & 7.76 & 8.02 \\
\hline & H5 & 7.15 & 7.25 \\
\hline & H6 & 6.97 & 7.34 \\
\hline \multirow[t]{6}{*}{$\mathrm{dpb}^{\prime}{ }^{\mathrm{b}}$} & $\mathrm{H} 2 / 7$ & 8.24 & 8.07 \\
\hline & Н3/6 & 7.33 & 7.15 \\
\hline & $\mathrm{H} 4 / 5$ & 7.58 & 7.57 \\
\hline & H10/15 & 8.16 & 8.18 \\
\hline & H11/14 & 7.98 & 7.99 \\
\hline & H12/13 & 9.33 & 9.26 \\
\hline
\end{tabular}

${ }^{\mathrm{a}} \mathrm{H} 6$ (dd; $J$ = 5, $1.5 \mathrm{~Hz}$ ); H5 (dd; $J=8,5 \mathrm{~Hz}$ ); H4 (dd; $J$ = 8, $8 \mathrm{~Hz}$ ); H3 (dd; $J=8,1.5 \mathrm{~Hz}$ ).

${ }^{\text {b }} \mathrm{H} 2 / 7$ (s); H3/6 (dd, $J=5,1.5 \mathrm{~Hz}$ ); H4/5 (dd, $J=10$, 8 Hz); H10/15 (d, $J=8 \mathrm{~Hz}$ ); H11/14 (dd, $J=8,1.5 \mathrm{~Hz}$ ); H12/13 (d, $J=8 \mathrm{~Hz}$ ).

The assignment of the ${ }^{1} \mathrm{H}$ NMR spectra was performed with the assistance of 2D COSY spectra, and by comparison with the diastereoisomers of the structurally-related complex $\left[\left\{\mathrm{Ru}(\mathrm{bpy})_{2}\right\}_{2}(\mu-\mathrm{HAT})\right]^{4+}$ (HAT = 1,4,5,8,9,12-hexaazatriphenylene). ${ }^{136,142}$ Since these methods are well established, the present discussion emphasises the distinctive chemical shifts which differ between the diastereoisomers. In all cases, the coordinated bpy ligands exhibited the expected coupling constant values ${ }^{143}\left\{J_{3,4}=8 \mathrm{~Hz}\right.$, $J_{3,5}=1.5 \mathrm{~Hz}, J_{4,5}=8 \mathrm{~Hz}, J_{4,6}=1.5 \mathrm{~Hz}$ and $\left.J_{5,6}=5 \mathrm{~Hz}\right\}$ and coupling patterns based on the symmetry requirements of the complexes.

\section{$\left[\left\{R u(b p y)_{2}\right\}_{2}\left(\mu-d p b^{\prime}\right)\right]^{4+}$}

The meso and rac diastereoisomers possess $\mathbf{C}_{\mathrm{s}}$ and $\mathbf{C}_{2}$ point group symmetries, respectively, and may be distinguished on the basis of differential anisotropic interactions experienced by the bpy protons depending upon the stereochemical relationship of the two metal centres. The point group symmetries of both diastereoisomers require two non-equivalent bpy ligands, with the two halves of each being magnetically non-equivalent. This gives rise to four different environments for the pyridyl rings (denoted ring $a-d$ in Figure 3.3): two over the bridge (rings $b$ and $d$ ) and two directed away from the bridge (rings $a$ and $c$ ). In addition, the dpb' ligand exhibits seven magnetically non-equivalent proton resonances. 
The $\mathrm{H} 5$ and $\mathrm{H} 6$ protons (rings $b$ and $d$ oriented over the bridge) experience the most pronounced shifts between the two diastereoisomeric forms. In the rac diastereoisomer, bpy ring $b$ is oriented over the plane of the dpb ligand and the bpy ligand across the bridge such that the $\mathrm{H} 5$ and $\mathrm{H} 6$ protons of this ring experience increased diamagnetic anisotropic effects. The H5 proton of ring $b$ was assigned as the most upfield resonance at $7.01 \mathrm{ppm}(J=8,5 \mathrm{~Hz}$, dd) while $\mathrm{H} 6$ (ring $b)$ was assigned as the $7.54 \mathrm{ppm}(J=$ 5, $1.5 \mathrm{~Hz}, \mathrm{dd}$ ) resonance. By comparison, in the meso diastereoisomer, the $\mathrm{H} 5$ and $\mathrm{H} 6$ protons of bpy ring $b$ are oriented approximately in the plane and thus in the deshielding cone of the equivalent bpy across the bridge. As a result, the H5' proton of ring d, which is situated over the plane of the dpb' ligand and approximately parallel to the magnetically equivalent bpy across the bridge, experiences the greatest anisotropic effect and is assigned as the most upfield resonance at $7.25 \mathrm{ppm}(J=8,5 \mathrm{~Hz}$, dd).

\section{$\left[\left\{R u(b p y)_{2}\right\}_{2}(\mu-B L)\right]^{4+}\{B L=d p b, d p q ', p p z, 2,3-d p p\}$}

The ${ }^{1} \mathrm{H}$ NMR spectra for a given diastereoisomer exhibit comparable features over the series of complexes, which are consistent with the symmetry requirements of the complexes $\left\{\mathbf{C}_{\mathbf{s}}\right.$ (meso) and $\mathbf{C}_{2}$ $(\mathrm{rac})\}$ and the variation in the number of resonances due to the bridging ligands. The chemical shift assignments were performed with the assistance of ${ }^{1} \mathrm{H}$ COSY spectra and by comparison with the spectral assignments for $\left[\left\{\mathrm{Ru}(\mathrm{bpy})_{2}\right\}_{2}(\mu-\mathrm{dpb})\right]^{4+}$.

The diastereoisomers of $\left[\left\{\mathrm{Ru}(\mathrm{bpy})_{2}\right\}_{2}(\mu \text {-dpb) }]^{4+}\right.$ exhibit broader ${ }^{1} \mathrm{H}$ NMR spectra than their dpb'bridged analogues. Steric interactions between the $\mathrm{H} 13$ and $\mathrm{H} 14$ protons of the two pyridyl rings of dpb (Figure B3.1, Appendix B) induce a structural distortion in the bridging ligand by forcing the rings to move out of the plane of the bridge. This distortion gives rise to conformational isomers that interchange slowly on the NMR time-scale at room temperature. The resultant spectral broadening is greater for the meso diastereoisomer compared with the rac form. A similar observation has been reported for the related system $\left[\left\{\mathrm{Ru}(\mathrm{bpy})_{2}\right\}_{2}(\mu-2,3-\mathrm{dpp})\right]^{4+}$. $^{*}$ For the diastereoisomers incorporating the "fused" bridging ligand dpb', the rigidity precludes such conformational lability. Despite the broadening of the resonances in the complexes incorporating the "unfused" dpb bridging ligand, the diastereoisomeric forms of the complex exhibit distinctive resonances which permit their characterisation.

\section{$\left[\left\{R u(b p y)_{2}\right\}_{2}(\mu-B L)\right]^{4+}\{B L=d p o, d p t\}$}

The ${ }^{1} \mathrm{H}$ NMR spectra of the separated diastereoisomers have been reported previously, ${ }^{71}$ and were assigned using a combination of one- and two-dimensional NMR techniques. Noticeable differences exist in the chemical shifts (§3.2.3.3) of some proton signals in the separate diastereoisomers which are consistent with the symmetry requirements of the complexes $\left\{\mathbf{C}_{\mathbf{s}}\right.$ (meso) and $\left.\mathbf{C}_{2}(\mathrm{rac})\right\}$.

\footnotetext{
* Steric interactions between the H9 and H10 protons of the 2,3-dpp bridging ligand give rise to non-planarity of the ligand and conformational isomerism in the bridging ligand. ${ }^{65}$
} 
$\left[\left\{O s(b p y)_{2}\right\}_{2}(\mu-B L)\right]^{4+}\{B L=d p b, d p b ', d p q ', p p z, 2,3-d p p\}$

The assignment of the ${ }^{1} \mathrm{H}$ NMR spectra were performed with the assistance of ${ }^{1} \mathrm{H}$ COSY spectra and were based on the dinuclear ruthenium analogues, allowing for the slight upfield shift of the proton resonances due to the increased shielding influence of the Os centre compared with $\mathrm{Ru} .{ }^{144}$

\section{(ii) Unsymmetrical Dinuclear Complexes of Ruthenium Incorporating Angular Bridging Ligands}

The $\Lambda \Delta / \Delta \Lambda$ and $\Delta \Delta / \Lambda \Lambda$ diastereoisomers both possess $\mathbf{C}_{\mathbf{1}}$ point group symmetry and exhibit ${ }^{1} \mathrm{H}$ NMR spectra comprising two non-equivalent bpy ligands (i.e. 16 magnetically non-equivalent proton resonances) and two non-equivalent pp ligands. In addition, 14 non-equivalent dpb proton resonances and 12 non-equivalent dpb' proton resonances are observed for the respective complexes. The four different "pyridyl" environments for each pp ligand give rise to 12 resonances in the aromatic region, and 4 singlet resonances in the aliphatic region for the unsymmetrical complexes incorporating the $\mathrm{Me}_{2} \mathrm{bpy}$ ligands. The $\mathrm{Me}_{4}$ bpy derivatives exhibit 8 resonances in both the aromatic and aliphatic regions.

The chemical shifts of the bpy protons in the diastereoisomers of the unsymmetrical complexes are approximately identical to the values in their symmetrical analogues. Minor differences were observed in the chemical shift resonances for the $\mathrm{H} 5$ and $\mathrm{H} 6$ protons of bpy rings $b$ and $d$ oriented over the bridging ligand, as they experience slightly different anisotropic interactions with the adjacent pp ligands. The chemical shifts of the protons in the methyl-substituted ligands experience a 0.2-0.3 ppm upfield shift relative to the corresponding proton resonance of the stereochemically-related bpy ligands.

\section{(iii) Dinuclear Ruthenium Complexes Incorporating Stepped-Parallel Bridging Ligands:}

\section{$\left[\{R u(b p y)\}_{2}(\mu-2,5-d p p)\right]^{4+}$ and $\left[\left\{R u(b p y)_{2}\right\}_{2}(\mu-d p o p)\right]^{4+}$}

The meso and rac diastereoisomers of $\left[\left\{\mathrm{Ru}(\mathrm{bpy})_{2}\right\}_{2}(\mu-\mathrm{BL})\right]^{4+}\{\mathrm{BL}=2$,5-dpp, dpop $\}$ possess $\mathbf{C}_{\boldsymbol{i}}$ and $\mathbf{C}_{2}$ point group symmetries, respectively. Four different "pyridyl" environments are distinguished for the two non-equivalent bpy ligands (giving rise to 16 non-equivalent bpy resonances). In addition, one set of four coupled protons and one singlet resonance arise from the bridging ligand 2,5-dpp, while five non-equivalent proton resonances arise from the dpop ligand. The notation employed for the ${ }^{1} \mathrm{H}$ NMR assignments for the diastereoisomers of $\left[\left\{\mathrm{Ru}(\mathrm{bpy})_{2}\right\}_{2}(\mu \text {-dpop) }]^{4+}\right.$ is provided in Figure 3.4.
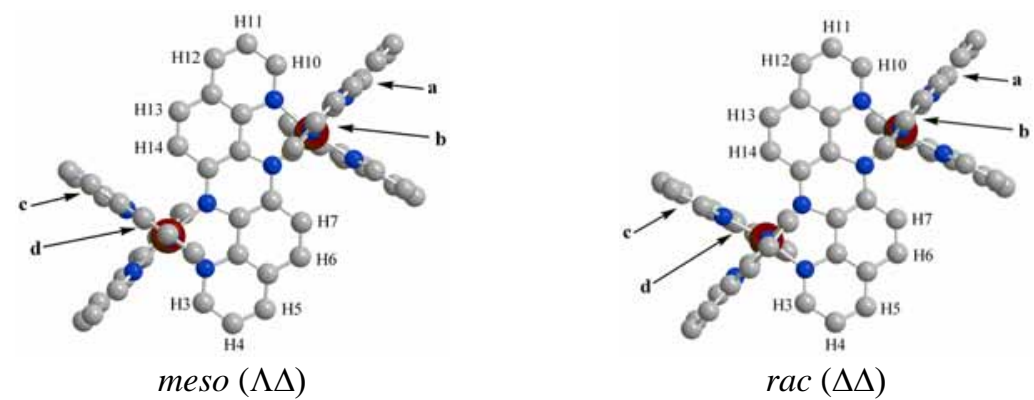

Figure 3.4 Chem 3D representations of meso- $(\Lambda \Delta)$ - and rac- $(\Delta \Delta)-\left[\left\{\operatorname{Ru}(\text { bpy })_{2}\right\}_{2}(\mu-d p o p)\right]^{4+}$. Hydrogen atoms are omitted for clarity. 
The H5 and H6 protons of the pyridyl rings situated over the plane of the bridging ligand experience the most pronounced chemical shift differences between the diastereoisomeric forms of a given complex. In the meso diastereoisomer, ring $b$ is oriented over the plane of BL and the bpy ligand across the bridge such that the $\mathrm{H} 5 / \mathrm{H} 6$ protons of this ring experience increased diamagnetic anisotropic effects (and hence occur slightly upfield) relative to the H5/H6 protons of ring $d$. In the rac diastereoisomer, the $\mathrm{H} 6$ proton of ring $b$ is situated over the plane of BL and in the deshielding cone of bpy ring $b$ across the bridge. This proton experiences a slightly decreased shielding influence relative to the $\mathrm{H} 6$ proton of ring $b$ in the meso diastereoisomer. The H6 proton of ring $d$ in the rac form is oriented over the plane of BL, parallel to the corresponding ring $d$ across the bridge, and lies in a deshielding environment compared to the $\mathrm{H} 6$ proton of ring $d$ in the meso diastereoisomer. The remaining spectral assignments were performed with the assistance of ${ }^{1} \mathrm{H}$ COSY spectra.

\subsubsection{Diastereoisomers of Ruthenium Complexes Incorporating a Series of Angular Bridging Ligands}

\subsubsection{Structural Considerations: X-ray Crystallography}

The X-ray crystal structures of the meso- $(\Lambda \Delta)-\left[\left\{\operatorname{Ru}(\mathrm{bpy})_{2}\right\}_{2}(\mu-\mathrm{dpb})\right]^{4+}$ cation were obtained as both the $\mathrm{PF}_{6}{ }^{-}$and $\left[\mathrm{ZnCl}_{4}\right]^{2-}$ salts. Meso- $(\Lambda \Delta)-\left[\left\{\mathrm{Ru}(\mathrm{bpy})_{2}\right\}_{2}(\mu-\mathrm{dpb})\right]\left(\mathrm{PF}_{6}\right)_{4} \cdot 5 \mathrm{H}_{2} \mathrm{O}$ crystallised in the orthorhombic space group Fdd2 with sixteen dinuclear cations in the unit cell and meso-( $\Lambda \Delta)$ $\left[\left\{\mathrm{Ru}(\mathrm{bpy})_{2}\right)_{2}(\mu\right.$-dpb) $]\left(\mathrm{ZnCl}_{4}\right)_{2} \cdot 5 \mathrm{H}_{2} \mathrm{O}$ crystallised in the monoclinic space group $P 2_{1} / c$ with four dinuclear cations in the unit cell. The perspective views of the dinuclear cations are shown in Figures 3.5(a) and (b), and the details of the crystal structure and refinement are provided in Table B3.1 (Appendix B). In both cases, the dpb bridging ligand exhibits a large dihedral skew of the two pyridyl ligands, as well as considerable distortion in the central pyrazine ring and a twist in the benzoquinoxaline "tail" of the bridge. The dihedral skew arises from steric hindrance between the H13 and H14 protons (denoted by asterisks in Figure 3.5) which encourages the two substituent pyridyl rings to twist out of the central plane. $\mathrm{C}(1)$ deviates from the least-squares plane defined by the benzoquinoxaline tail of the bridging ligand by $0.503(3)$ and $0.730(3) \AA$ for the structures involving $\mathrm{PF}_{6}{ }^{-}$and $\left[\mathrm{ZnCl}_{4}\right]^{2-}$ counter-ions, respectively, indicating that ion-pairing interactions between the counter-ions and cationic complex influence the degree of structural distortion in the solid-state. The $\mathrm{Ru}(1) \cdots \operatorname{Ru}(2)$ separations are $6.883(1)$ and 6.856(2) $\AA$ for the structures involving the $\mathrm{PF}_{6}{ }^{-}$and $\left[\mathrm{ZnCl}_{4}\right]^{2-}$ counter-ions, respectively (Table 3.2). In meso- $(\Lambda \Delta)-\left[\left\{\mathrm{Ru}(\mathrm{bpy})_{2}\right\}_{2}(\mu\right.$-dpb) $]\left(\mathrm{PF}_{6}\right)_{4} \cdot 5 \mathrm{H}_{2} \mathrm{O}$, a single $\mathrm{PF}_{6}{ }^{-}$counter-ion resides in the cleft between the terminal bpy ligands "below" the plane of the bridging ligand, while the additional three counter-ions and five water molecules reside within the exterior clefts. By contrast, in meso- $(\Lambda \Delta)-\left[\left\{\operatorname{Ru}(\mathrm{bpy})_{2}\right)_{2}(\mu-\right.$ $\mathrm{dpb})]\left(\mathrm{ZnCl}_{4}\right)_{2} .5 \mathrm{H}_{2} \mathrm{O}$, two $\left[\mathrm{ZnCl}_{4}\right]^{2-}$ counter-ions are located within the exterior clefts \{Figure 3.5(b)\} and engage in H-bonding interactions with the lattice water molecules. In both structures, the metal centres reside in distorted octahedral environments in which the "bite-angles" subtended by the Ru centres and nitrogen atoms of the bridging ligand are $c a .78^{\circ}$. The Ru-N(pyrazine) bond lengths of 2.080(10) and 
2.099(10) $\AA$ for $\mathrm{Ru}(1)$ and $\mathrm{Ru}(2)$ in the $\left[\mathrm{ZnCl}_{4}\right]^{2-}$ structure are slightly longer than the $\mathrm{Ru}-\mathrm{N}$ (pyridine) lengths of 2.028(10) and 2.037(11) $\AA$. A similar trend is observed for the $\mathrm{PF}_{6}{ }^{-}$structure, in which the Ru$\mathrm{N}$ (pyrazine) bond lengths of 2.074(10) and 2.101(10) $\AA$ for $\mathrm{Ru}(1)$ and $\mathrm{Ru}(2)$ are slightly longer than the Ru-N(pyridine) lengths of 2.021(10) and 2.031(11) Å.

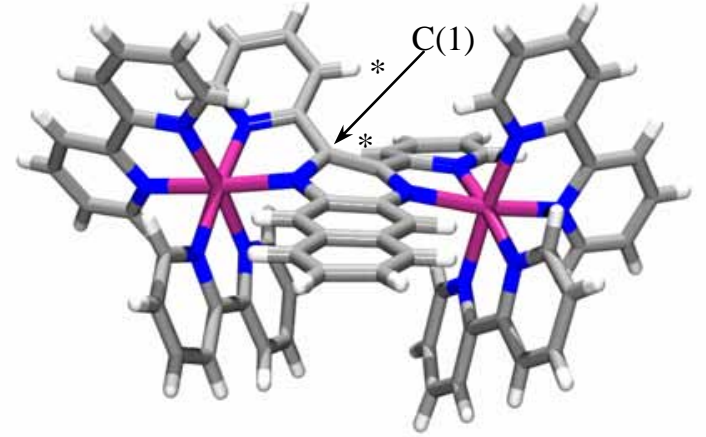

(a)

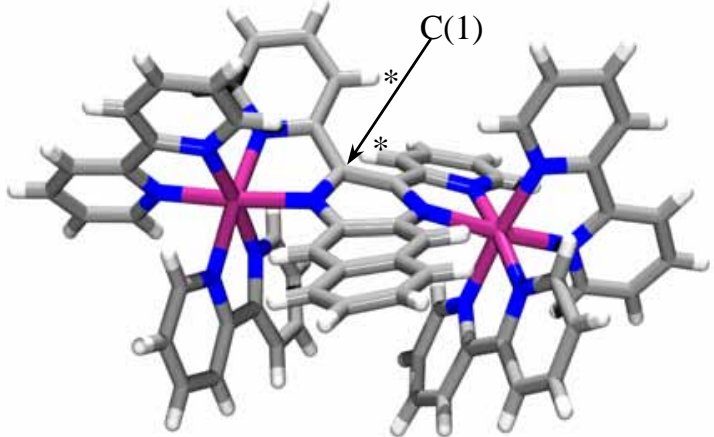

(b)

Figure 3.5 X-ray crystal structures of the cations in (a) meso- $(\Lambda \Delta)-\left[\left\{\mathrm{Ru}(\mathrm{bpy})_{2}\right\}_{2}(\mu-\mathrm{dpb})\right]\left(\mathrm{PF}_{6}\right)_{4} \cdot 5 \mathrm{H}_{2} \mathrm{O}$ and (b) meso$(\Lambda \Delta)-\left[\left\{\mathrm{Ru}(\mathrm{bpy})_{2}\right\}_{2}(\mu-\mathrm{dpb})\right]\left(\mathrm{ZnCl}_{4}\right)_{2} \cdot 5 \mathrm{H}_{2} \mathrm{O}$. The asterisks denote the $\mathrm{H}(13) / \mathrm{H}(14)$ protons that experience steric interactions.

Figures 3.6(a) and (b) present the perspective views of the dinuclear cations in meso-( $\Lambda \Delta$ )$\left[\left\{\mathrm{Ru}(\mathrm{bpy})_{2}\right\}_{2}\left(\mu-\mathrm{dpb}^{\prime}\right)\right]\left(\mathrm{PF}_{6}\right)_{4} \cdot 2 \mathrm{H}_{2} \mathrm{O} \cdot 2\left\{\left(\mathrm{CH}_{3}\right)_{2} \mathrm{CO}\right\}$ and meso- $(\Lambda \Delta)-\left[\left\{\mathrm{Ru}(\mathrm{bpy})_{2}\right\}_{2}(\mu-\mathrm{dpq})\right]\left(\mathrm{PF}_{6}\right)_{4} \cdot 3 \mathrm{H}_{2} \mathrm{O}$. The former crystallises in the orthorhombic space group Pbca with eight dinuclear cations in the unit cell, while the latter crystallises in the triclinic space group $P \overline{1}$ with two dinuclear cations in the unit cell. By comparison with the dpb ligand, the C-C bond linking the two pyridyl rings in the dpq' and dpb' bridging ligands prevents their lateral movement. In both complexes, the dpb' and dpq' bridging ligands assume a slightly arched structure arising from unfavourable steric interactions between the equatorial hydrogen atoms on the benzophenazine and phenazine "tails" of the bridging ligands, respectively, and the terminal bpy rings oriented parallel to the long axis of the bridging ligand. The N-Ru-N "bite angles" of $c a .78^{\circ}$ between the bridging ligands are comparable to those in the dpb-bridged structures. For meso-( $\Lambda \Delta)$ $\left[\left\{\mathrm{Ru}(\mathrm{bpy})_{2}\right\}_{2}\left(\mu-\mathrm{dpb}^{\prime}\right)\right]^{4+}$, the average Ru-N(pyrazine) and Ru-N(pyridine) bond lengths are 2.080(2) and 2.060(2) $\AA$, while the average lengths are 2.080(2) and 2.070(2) $\AA$, respectively, for the dpq'-bridged system. The $\mathrm{Ru}(1) \cdots \mathrm{Ru}(2)$ distances are 6.818(1) and 6.887(1) $\AA$ for the dpq'- and dpb'-bridged structures (Table 3.2). In all cases, the Ru-N distances and the N-Ru-N angles correlate with those published previously for polypyridyl complexes of ruthenium. ${ }^{128,145-150} \operatorname{In}$ meso- $(\Lambda \Delta)-\left[\left\{\operatorname{Ru}(\mathrm{bpy})_{2}\right\}_{2}(\mu-\right.$ $\left.\left.\mathrm{dpq}^{\prime}\right)\right]\left(\mathrm{ZnCl}_{4}\right)_{2} .3 \mathrm{H}_{2} \mathrm{O}$, one $\left[\mathrm{ZnCl}_{4}\right]^{2-}$ counter-ion resides in the cleft "above” the plane of the bridging ligand, while the second counter-ion resides outside the clefts and does not engage in any intramolecular interactions with the cation. In meso- $(\Lambda \Delta)-\left[\left\{\mathrm{Ru}(\mathrm{bpy})_{2}\right\}_{2}\left(\mu-\mathrm{dpb}^{\prime}\right)\right]\left(\mathrm{PF}_{6}\right)_{4} \cdot 2 \mathrm{H}_{2} \mathrm{O} \cdot 2\left\{\left(\mathrm{CH}_{3}\right)_{2} \mathrm{CO}\right\}$, one $\mathrm{PF}_{6}^{-}$ counter-ion resides in the cleft "above" the bridging ligand plane, while an acetone molecule resides in the cleft "below" the plane. An additional $\mathrm{PF}_{6}{ }^{-}$ion and acetone molecule occupy one of the exterior clefts. 


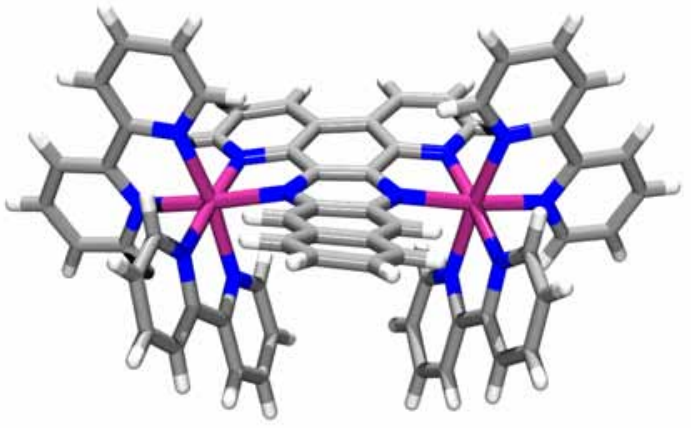

(a)

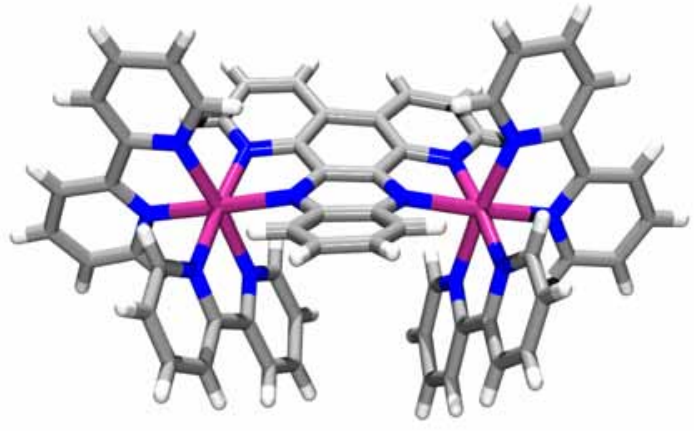

(b)

Figure 3.6 X-ray crystal structures of the cations in (a) meso- $(\Lambda \Delta)-\left[\left\{\mathrm{Ru}(\mathrm{bpy})_{2}\right\}_{2}(\mu-\mathrm{dpb})\right]\left(\mathrm{PF}_{6}\right)_{4} \cdot 2 \mathrm{H}_{2} \mathrm{O} .2\left\{\left(\mathrm{CH}_{3}\right)_{2} \mathrm{CO}\right\}$ and (b) meso-( $\Lambda \Delta)-\left[\left\{\mathrm{Ru}(\mathrm{bpy})_{2}\right\}_{2}\left(\mu-\mathrm{dpq}^{\prime}\right)\right]\left(\mathrm{ZnCl}_{4}\right)_{2} \cdot 3 \mathrm{H}_{2} \mathrm{O}$.

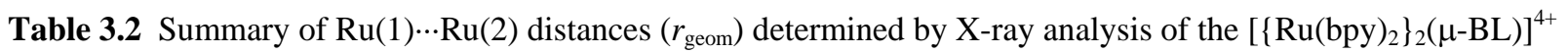
cations.

\begin{tabular}{|c|c|}
\hline Complex & $r_{\text {geom }} / \AA$ \\
\hline meso- $\left[\left\{\mathrm{Ru}(\mathrm{bpy})_{2}\right\}_{2}(\mu\right.$-dpb) $]\left(\mathrm{PF}_{6}\right)_{4}$ & $6.883(1)$ \\
\hline meso- $\left[\left\{\mathrm{Ru}(\mathrm{bpy})_{2}\right\}_{2}(\mu-\mathrm{dpb})\right]\left(\mathrm{ZnCl}_{4}\right)_{2}$ & $6.856(2)$ \\
\hline meso- $\left[\left\{\mathrm{Ru}(\mathrm{bpy})_{2}\right\}_{2}(\mu-\mathrm{dpq})\right]\left(\mathrm{ZnCl}_{4}\right)_{2}$ & $6.818(1)$ \\
\hline meso- $\left[\left\{\mathrm{Ru}(\mathrm{bpy})_{2}\right\}_{2}(\mu-\mathrm{dpb})\right]\left(\mathrm{PF}_{6}\right)_{4}$ & $6.887(1)$ \\
\hline meso- $\left[\left\{\mathrm{Ru}(\mathrm{bpy})_{2}\right\}_{2}(\mu-\mathrm{dpo})\right]\left(\mathrm{ZnCl}_{4}\right)_{2}$ & $6.014(6)$ \\
\hline rac- $\left[\left\{\mathrm{Ru}(\mathrm{bpy})_{2}\right\}_{2}(\mu\right.$-dpo $\left.)\right]\left(\mathrm{ZnCl}_{4}\right)_{2}$ & $6.016(5)$ \\
\hline
\end{tabular}

The nature of the structural distortions observed in the solid-state are qualitatively in accord with the predictions of gas-phase semiempirical PM3(tm) calculations. ${ }^{68,69}$ The dpb-bridged structures exhibited a significantly greater degree of structural distortion in both the solid-state and gas-phase compared with the dpb'- and dpq'-bridged complexes. Interestingly, the semi-empirical calculations ${ }^{68,69}$ also predicted different degrees of structural distortions in the diastereoisomers of the same complex, and these were manifested by differences in the distribution of the LUMO-based frontier orbitals that mediate the superexchange interaction between the metal centres. The presence of differential distortions in the bridging ligand between the complexes incorporating the "unfused" and "fused" ligands suggest that there may be differences in the ground-state redox asymmetry contributions across the series complexes which may be manifested in their electrochemical and IVCT properties.

X-ray crystal structures were obtained for the two separated diastereoisomers of $\left[\left\{\mathrm{Ru}(\mathrm{bpy})_{2}\right\}_{2}(\mu-\right.$ dpo) $]^{4+}$, in each case as the $\left[\mathrm{ZnCl}_{4}\right]^{2-}$ salt. ${ }^{71}$ Details regarding the crystal data and refinement and selected bond lengths and angles are presented in Tables B3.2(a) and B3.2(b) \{Appendix B\}, respectively. The meso- $(\Lambda \Delta)$ form crystallises in the monoclinic space group $\mathrm{P} 2{ }_{1} / n$ with four dinuclear cations in the unit cell while the $\operatorname{rac}-(\Delta \Delta)$ diastereoisomer crystallises in the triclinic space group $P \overline{1}$ with two molecules in the unit cell (Figure 3.7). No significant interactions are present between the dinuclear cations and the $\left[\mathrm{ZnCl}_{4}\right]^{2-}$ anions. The meso form crystallises with six molecules of water, while the rac form cocrystallises with a $\left[\mathrm{ZnCl}_{2}\left(\mathrm{H}_{2} \mathrm{O}\right)_{2}\right]$ entity in the lattice. Each Ru centre resides in a slightly distorted 
octahedral environment, with $\mathrm{Ru}(1) \cdots \cdot \mathrm{Ru}(2)$ separations of 6.016(5) (meso) and 6.014(6) $\AA$ (rac). The dpo ligand is slightly twisted in both complexes with pyridyl-pyridyl interplanar angles of 14.3(3) (meso) and $17.1(5)^{\circ}(\mathrm{rac})$. The inter-planar angles between the pyridyl rings and the oxadiazole rings are not as pronounced. For the meso and rac diastereoisomers, the deviations are 5.2(3) and 6.3(5) ${ }^{\circ}$ for the $\mathrm{N}(1)$ atom of the pyridyl ring and the five-membered ring and $10.2(3)$ and $13.0(4)^{\circ}$ for the $\mathrm{N}(4)$ atom of the pyridyl ring and the five-membered ring, respectively. In the structure of the meso form, all six lattice water molecules are involved in $\mathrm{H}$-bonding with two $\left[\mathrm{ZnCl}_{4}\right]^{2-}$ anions both involving $\left.\mathrm{Zn}(2)\right\}$. This $\mathrm{H}-$ bonding links the two $\left[\mathrm{ZnCl}_{4}\right]^{2-}$ anions via a cluster of twelve $\mathrm{H}$-bonded water molecules. The other anion \{involving $\mathrm{Zn}(1)\}$ does not engage in intermolecular interactions and resides in pockets between adjacent $\left[\left(\mathrm{ZnCl}_{4}\right)_{2}\left(\mathrm{H}_{2} \mathrm{O}\right)_{12}\right]$ clusters and $\left[\left\{\mathrm{Ru}(\mathrm{bpy})_{2}\right\}_{2}(\mu-\mathrm{dpo})\right]^{4+}$ cations. In the structure of the rac form no such H-bonding interactions are present. There is however, an unusual tetrahedral $\left[\mathrm{ZnCl}_{2}\left(\mathrm{H}_{2} \mathrm{O}\right)_{2}\right]$ species residing in the lattice. A Cambridge crystallographic database search revealed that this molecule has been observed on one occasion previously in $\left[\mathrm{ZnCl}_{2}\left(\mathrm{H}_{2} \mathrm{O}\right)_{2}\left(15\right.\right.$-crown-5)]. ${ }^{151}$ Typically, hydrated zinc salts form $\left[\mathrm{Zn}\left(\mathrm{H}_{2} \mathrm{O}\right)_{6}\right]^{2+}$ ions, but presumably in the present case, only fortuitous water exists in the organic solvent.

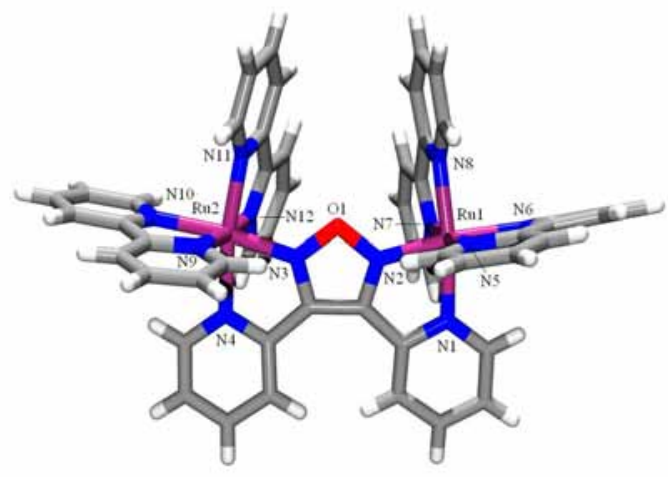

(a)

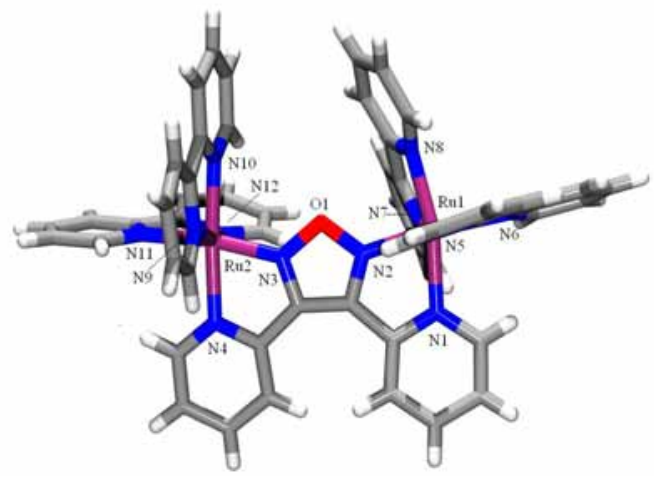

(b)

Figure 3.7 X-ray crystal structure of the cation in (a) meso- $(\Lambda \Delta)-\left[\left\{\mathrm{Ru}(\mathrm{bpy})_{2}\right\}_{2}(\mu\right.$-dpo) $)\left(\mathrm{ZnCl}_{4}\right)_{2} \cdot 6 \mathrm{H}_{2} \mathrm{O}$ and (b) rac$(\Delta \Delta)-\left[\left\{\mathrm{Ru}(\mathrm{bpy})_{2}\right\}_{2}(\mu-\mathrm{dpo})\right]\left(\mathrm{ZnCl}_{4}\right)_{2} \cdot\left[\mathrm{ZnCl}_{2}\left(\mathrm{H}_{2} \mathrm{O}\right)_{2}\right]$.

\subsubsection{Electrochemistry}

The redox potentials for the $\mathrm{E}_{\mathrm{ox1}}\left(\left[5^{+/ 4+}\right]\right.$; i.e. $\left.\mathrm{Ru}^{\mathrm{III}}-\mathrm{Ru}^{\mathrm{II}} / \mathrm{Ru}^{\mathrm{II}}-\mathrm{Ru}^{\mathrm{II}}\right)$ and $\mathrm{E}_{\mathrm{ox} 2}\left([6+/ 5+]\right.$; i.e. $\mathrm{Ru}^{\mathrm{III}}-$ $\left.\mathrm{Ru}^{\mathrm{III}} / \mathrm{Ru}^{\mathrm{III}}-\mathrm{Ru}^{\mathrm{II}}\right)$ couples for the series of complexes meso- and rac-[\{Ru(bpy $\left.\left.)_{2}\right\}_{2}(\mu-\mathrm{BL})\right]^{4+}\{\mathrm{BL}=\mathrm{dpb}$, dpb', dpq', ppz, 2,3-dpp, dpo and dpt $\}$ were investigated by cyclic and differential pulse voltammetry in acetonitrile solution containing $0.1 \mathrm{M}\left[\left(n-\mathrm{C}_{4} \mathrm{H}_{9}\right)_{4} \mathrm{~N}\right] \mathrm{PF}_{6}$, and are summarised in Tables 3.3 and B3.3 (Appendix B). Some details of the redox and spectral properties for the diastereoisomeric forms of $\left[\left\{\mathrm{Ru}(\mathrm{bpy})_{2}\right\}_{2}(\mu-\mathrm{BL})\right]^{4+}\{\mathrm{BL}=\mathrm{dpb}, \mathrm{dpb}\},{ }^{67-69}\left[\left\{\mathrm{Ru}(\mathrm{bpy})_{2}\right\}_{2}(\mu-\mathrm{BL})\right]^{4+}\{\mathrm{BL}=\mathrm{dpo}, \mathrm{dpt}\},{ }^{71}$ and for diastereoisomeric mixtures of $\left[\left\{\mathrm{Ru}(\mathrm{bpy})_{2}\right\}_{2}(\mu-\mathrm{BL})\right]^{4+}\{\mathrm{BL}=\mathrm{dpb}, 2,3-\mathrm{dpp}\}$ have been reported previously. ${ }^{72-82} \Delta \mathrm{E}_{\mathrm{ox}}$ defines the potential difference between the $\left[6+/ 5^{+}\right]$and $\left[5^{+/ 4+}\right]$ couples. 
Table 3.3 Electrochemical data (in $\mathrm{mV}$ relative to the $\mathrm{Fc}^{+} / \mathrm{Fc}^{0}$ couple) and comproportionation constants ${ }^{\mathrm{a}}\left(\mathrm{K}_{\mathrm{c}}\right)$ for $\left[\left\{\mathrm{Ru}(\mathrm{bpy})_{2}\right\}_{2}(\mu-\mathrm{BL})\right]^{4+}$ in $0.1 \mathrm{M}\left[\left(n-\mathrm{C}_{4} \mathrm{H}_{9}\right)_{4} \mathrm{~N}\right] \mathrm{PF}_{6} / \mathrm{CH}_{3} \mathrm{CN} .^{\mathrm{b}}$

\begin{tabular}{|c|c|c|c|c|c|c|c|}
\hline BL & Diastereoisomer & $\begin{array}{c}\mathrm{K}_{\mathrm{c}} \\
\left(\times 10^{-3}\right)\end{array}$ & $\Delta \mathrm{E}_{\text {ox }}$ & $\mathrm{E}_{\text {ox2 }}$ & $\mathrm{E}_{\text {ox1 }}$ & $\mathrm{E}_{\text {red1 }}$ & $\mathrm{E}_{\text {red2 }}$ \\
\hline $\mathrm{dpb}$ & meso & 2.06 & 196 & 1280 & 1084 & -624 & -1268 \\
& rac & 0.944 & 176 & 1280 & 1104 & -624 & -1260 \\
\hline dpb' $^{\prime}$ & meso & 8.35 & 232 & 1300 & 1068 & -496 & -1128 \\
& rac & 6.11 & 224 & 1304 & 1080 & -464 & -1116 \\
\hline dpq' & meso & 2.40 & 200 & 1285 & 1085 & -596 & -1332 \\
& rac & 2.40 & 200 & 1297 & 1097 & -592 & -1268 \\
\hline ppz & meso & 6.11 & 224 & 1280 & 1056 & -956 & -1704 \\
& rac & 4.48 & 216 & 1278 & 1062 & -944 & -1696 \\
\hline $2,3-d p p$ & meso & 3.83 & 212 & 1256 & 1044 & -1044 & -1540 \\
& rac & 3.83 & 212 & 1244 & 1032 & -1048 & -1560 \\
\hline dpo & meso & 1220 & 360 & 1536 & 1176 & $-820^{c}$ & -1821 \\
& rac & 478 & 336 & 1536 & 1200 & $-872^{\mathrm{c}}$ & $-1892^{\mathrm{d}}$ \\
\hline $\mathrm{dpt}$ & meso & 29.0 & 264 & 1392 & 1128 & -1032 & \\
& rac & 23.0 & 258 & 1536 & 1111 & -968 & \\
\hline
\end{tabular}

$\mathrm{K}_{\mathrm{c}}$ values determined from equation 1.18(a). ${ }^{9}$

${ }^{\mathrm{b}} \Delta \mathrm{E}_{\mathrm{ox}}=\mathrm{E}_{\mathrm{ox} 2}-\mathrm{E}_{\mathrm{ox} 1}$. Potentials are quoted $\pm 3 \mathrm{mV}$.

${ }^{\mathrm{C}}$ Irreversible reduction process.

${ }^{\mathrm{d}}$ Two-electron reduction process.

All complexes are characterised by two reversible one-electron redox processes corresponding to successive oxidation of the metal centres, in addition to multiple reversible ligand-based reductions. In the cathodic region, the first two processes are assigned to the successive one-electron reductions of the bridging ligands ( $\mathrm{BL}^{0 /-}$ and $\mathrm{BL}^{-/ 2-}$ ), consistent with the stronger $\pi$-acceptor nature of the bridging relative to the terminal bpy ligands. ${ }^{72-82}$ The subsequent four one-electron processes correspond to successive reduction of the terminal bpy ligands. The cathodic shift of $\mathrm{E}_{\text {red1 }}$ and $\mathrm{E}_{\text {red2 }}$ as the bridging ligand is varied through the series ppz, dpb, dpq', dpb' and 2,3-dpp, is consistent with the stabilisation of the $\pi^{*}(\mathrm{BL})$ LUMOs. By comparison, the potentials for the bpy-based reductions remain relatively constant with bridging ligand variation.

Measurable differences in the $\Delta \mathrm{E}_{\mathrm{ox}}$ values are evident between the different complexes and between the diastereoisomeric forms of the same complex. A summary of $\Delta \mathrm{E}_{\mathrm{ox}}$ and the resultant comproportionation constants ${ }^{9}\left(\mathrm{~K}_{\mathrm{c}}\right)$ is provided in Table 3.3. The magnitude of $\Delta \mathrm{E}_{\mathrm{ox}}$ increases as $\mathrm{BL}$ is varied through the series dpb, ppz, dpq', 2,3-dpp and dpb' and the largest difference between the values for the diastereoisomers of the same complex is observed for $\left[\left\{\mathrm{Ru}(\mathrm{bpy})_{2}\right\}_{2}(\mu-\mathrm{dpb})\right]^{4+}(14 \pm 6 \mathrm{mV}){ }^{67-69}$ The decrease in the $\Delta \mathrm{E}_{\mathrm{ox}}$ values for the dpb-bridged diastereoisomers relative to the other complexes of the series, and the significant difference in the values between the diastereoisomeric forms may reflect the presence of structural distortions. However, these differences cannot be solely ascribed to variations in the extent of electronic delocalisation, as the magnitude of $\Delta \mathrm{E}_{\mathrm{ox}}$ also reflects contributions from ionpairing interactions, ${ }^{131}$ solvation energies and statistical factors. ${ }^{152,153}$

The diastereoisomers of $\left[\left\{\mathrm{Ru}(\mathrm{bpy})_{2}\right\}_{2}(\mu-\mathrm{BL})\right]^{4+}\{\mathrm{BL}=\mathrm{dpo}, \mathrm{dpt}\}^{71}$ are characterised by two reversible one-electron redox processes corresponding to successive oxidations of the metal centres. Both 
forms of $\left[\left\{\mathrm{Ru}(\mathrm{bpy})_{2}\right\}_{2}(\mu-\mathrm{dpt})\right]^{4+}$ exhibit a single irreversible reduction process in the cathodic potential region which is assigned to the bridging ligand, while the diastereoisomers of $\left[\left\{\operatorname{Ru}(\mathrm{bpy})_{2}\right\}_{2}(\mu \text {-dpo })\right]^{4+}$ exhibit a fully reversible bridging ligand-based reduction process in this region. The electrochemistry of $\left[\left\{\mathrm{Ru}(\mathrm{bpy})_{2}\right\}_{2}(\mu-\mathrm{dpt})\right]^{4+}$ became somewhat complicated by adsorption processes at the electrode surface. This has been encountered previously in work with sulfur-containing ligands. ${ }^{154}$ However, further reduction processes were found in the region between -1800 and $-2200 \mathrm{mV}$, which were assigned to the terminal bpy ligands.

The metal-metal interactions in the dinuclear complexes are strong, with $\Delta \mathrm{E}_{\mathrm{ox}}=348 \mathrm{mV}$ (average of meso and rac diastereoisomers) for $\left[\left\{\mathrm{Ru}(\mathrm{bpy})_{2}\right\}_{2}(\mu-\mathrm{dpo})\right]^{4+}$ and $\Delta \mathrm{E}_{\mathrm{ox}}=260 \mathrm{mV}$ (average of meso and rac diastereoisomers) for $\left[\left\{\mathrm{Ru}(\mathrm{bpy})_{2}\right\}_{2}(\mu-\mathrm{dpt})\right]^{4+}$. The large separation between the metal-based redox processes indicates stronger electronic communication between the metal centres compared with the closely-related complex $\left[\left\{\mathrm{Ru}(\mathrm{bpy})_{2}\right\}_{2}(\mu-2,3-\mathrm{dpp})\right]^{4+}$. The ligands dpo and dpt have a structural similarity to 2,3-dpp, however, the replacement of the central diazine rings with a heterodiazole alters the distance between the metals, and the electronic nature of the bridging ligand. The degree of aromaticity of the bridge has been postulated to facilitate interaction between the metals. The 1,2,5-oxadiazoles are only "aromatic" in that they contain six $\pi$-electrons, with the oxygen atom contributing little of its electron density into the ring, ${ }^{155}$ so that the heterocyclic 1,2,5-oxadiazole system is perhaps better described as being "diene-like". Due to the greater polarisability of the sulfur atom, the dpt thiadiazole system may be more delocalised; however, this being the case, the low aromatic character of the dpo bridge has not restricted the inter-metal communication. On the basis of the greater $\Delta \mathrm{E}_{\mathrm{ox}}$ values for the dpo- relative to the dpt-bridged complexes, the low aromatic character of the former has served to enhance this interaction. Measurable differences were also observed in the electrochemical properties of the diastereoisomers of the same complex, with this difference being greater for the dpo-bridged forms. Indeed, the $\mathrm{K}_{\mathrm{c}}$ values suggest a significant enhancement in the stability of the meso diastereoisomer compared with the rac form.

\section{A Cautionary Warning on the use of Electrochemical Measurements to Calculate}

\section{Comproportionation Constants}

As described in §1.3.1.2, the comparison of $\mathrm{K}_{\mathrm{c}}$ values - determined from electrochemical data in accordance with equation 1.18(a) - has been widely used in assessment of the extent of inter-metal communication in dinuclear complexes., 11, 12, 81 However, a number of studies have observed a substantial dependence of redox potentials on the identity of the anion used as the electrolyte in electrochemical measurements and the implications of these results in terms of the comparison of $\mathrm{K}_{\mathrm{c}}$ values seem not to be generally appreciated

In a recent investigation of the spectral and electrochemical characteristics of the stereoisomers of a range of mono- and dinuclear ruthenium complexes by Keene et al., ${ }^{67}$ the variation of the electrolyte anion gave rise to cathodic shifts in the redox potentials for the oxidations in the sequence $\mathrm{PF}_{6}{ }^{-}<\mathrm{BF}_{4}{ }^{-} \sim$ 
$\mathrm{ClO}_{4}{ }^{-}<$tosylate. This observation was consistent with known specific interactions of anions with metal complexes of this type. ${ }^{65,129}$ For the ligand-bridged dinuclear species, the effects were manifested by a difference in the $\Delta \mathrm{E}_{\mathrm{ox}}$ values. Variation of the electrolyte cation had no effect on the redox potentials.

Geiger and coworkers have promoted the use of weakly-coordinating anions such as tetrakis(pentafluorophenyl)borate, $\left\{\mathrm{B}\left(\mathrm{C}_{6} \mathrm{~F}_{5}\right)_{4}\right\}^{-100}$, and the use of solvents with low dielectric constants such as trifluoromethylbenzene (BTF), ${ }^{110}$ as a means of probing the influence of possible ion-pairing interactions on the potentials of successive redox couples. They noted that the separation of successive oxidation waves for nickelocene, $\left[\mathrm{Cp}_{2} \mathrm{Ni}\right]$, was greater in $\mathrm{BTF}$ than in other media $-\mathrm{a}$ feature attributed to an increase in ion-pairing energies of the nickelocene dication with various electrolyte anions $\left(\mathrm{ClO}_{4}{ }^{-}\right.$, $\mathrm{PF}_{6}^{-}, \mathrm{BF}_{4}^{-}$) due to a weak association of BTF with the dication. In a subsequent paper, Geiger et al. ${ }^{156}$ also noted significant medium effects on the separation of sequential redox potentials, and commented on the need for a judicious approach to the use of $\Delta \mathrm{E}_{\mathrm{ox}}$ values for the assessment of the degree of electronic interaction between linked metal centres.

Table 3.4 presents the redox potentials for the series of complexes $\left[\left\{\mathrm{Ru}(\mathrm{bpy})_{2}\right\}_{2}(\mu-\mathrm{BL})\right]^{n+}$ $\left\{\mathrm{BL}=\mathrm{dpb} \text {, dpo, bpt }{ }^{-} \text {, bpm, dbneil }\right\}^{*}$ in acetonitrile solution containing $\left[\left(n-\mathrm{C}_{4} \mathrm{H}_{9}\right)_{4} \mathrm{~N}\right]\left\{\mathrm{B}\left(\mathrm{C}_{6} \mathrm{~F}_{5}\right)_{4}\right\}$ and $\left[\left(n-\mathrm{C}_{4} \mathrm{H}_{9}\right)_{4} \mathrm{~N}\right] \mathrm{PF}_{6}$ as the supporting electrolytes, in addition to the respective $\Delta \mathrm{E}_{\mathrm{ox}}$ and $\mathrm{K}_{\mathrm{c}}$ values in the two media. $^{\dagger}$ A full listing of the potentials for metal-based oxidation and ligand-based reduction processes for the complexes in the two media are provided in Tables B3.4 and B3.5 (Appendix B).

Table 3.4 Electrochemical data (in $\mathrm{mV}$ relative to the $\mathrm{Fc}^{+} / \mathrm{Fc}^{0}$ couple) and $\mathrm{K}_{\mathrm{c}}$ values ${ }^{\mathrm{a}}$ for $\left[\left\{\mathrm{Ru}(\mathrm{bpy})_{2}\right\}_{2}(\mu-\mathrm{BL})\right]^{4+}$ in $0.02 \mathrm{M}\left[\left(n-\mathrm{C}_{4} \mathrm{H}_{9}\right)_{4} \mathrm{~N}\right]\left\{\mathrm{B}\left(\mathrm{C}_{6} \mathrm{~F}_{5}\right)_{4}\right\} / \mathrm{CH}_{3} \mathrm{CN}$ and $0.1 \mathrm{M}\left[\left(n-\mathrm{C}_{4} \mathrm{H}_{9}\right)_{4} \mathrm{~N}\right] \mathrm{PF}_{6} / \mathrm{CH}_{3} \mathrm{CN}$. ${ }^{\mathrm{b}}$

\begin{tabular}{|c|c|c|c|c|c|c|c|c|c|}
\hline \multirow{2}{*}{ BL } & Diastereoisomer & \multicolumn{6}{|c|}{$\mathrm{B}_{\left.\left(\mathrm{C}_{6} \mathrm{~F}_{5}\right)_{4}\right\}^{-}}$} & \multicolumn{4}{|c|}{$\mathrm{PF}_{6}^{-}$} \\
\cline { 3 - 10 } & & $\mathrm{K}_{\mathrm{c}}$ & $\Delta \mathrm{E}_{\mathrm{ox}}$ & $\mathrm{E}_{\mathrm{ox} 2}$ & $\mathrm{E}_{\text {ox1 }}$ & $\mathrm{K}_{\mathrm{c}}$ & $\Delta \mathrm{E}_{\mathrm{ox}}$ & $\mathrm{E}_{\mathrm{ox} 2}$ & $\mathrm{E}_{\mathrm{ox} 1}$ \\
\hline $\mathrm{dpb}$ & meso & 5230 & 220 & 1376 & 1156 & 2060 & 196 & 1280 & 1084 \\
& rac & 2810 & 204 & 1372 & 1168 & 944 & 176 & 1280 & 1104 \\
\hline $\mathrm{dpo}$ & meso & & & $\mathrm{c}$ & 1240 & 1220000 & 360 & 1536 & 1176 \\
& rac & & & $\mathrm{c}$ & 1288 & 478000 & 336 & 1536 & 1200 \\
\hline $\mathrm{bpt}^{-}$ & meso & 409000 & 332 & 1068 & 736 & 188000 & 312 & 992 & 680 \\
& rac & 857000 & 351 & 1064 & 713 & 161000 & 308 & 992 & 684 \\
\hline bpm & meso & 5230 & 220 & 1500 & 1280 & 1760 & 192 & 1384 & 1192 \\
& rac & 5230 & 220 & 1520 & 1300 & 1510 & 188 & 1380 & 1192 \\
\hline dbneil & meso & 2500 & 201 & 1409 & 1208 & 1100 & 180 & 1268 & 1088 \\
& rac & 2920 & 205 & 1403 & 1198 & 1190 & 182 & 1270 & 1088 \\
\hline
\end{tabular}

${ }^{\mathrm{a}} \mathrm{K}_{\mathrm{c}}$ values are given by equation 1.18(a).

${ }^{\mathrm{b}} \Delta \mathrm{E}_{\mathrm{ox}}=\mathrm{E}_{\mathrm{ox} 2}-\mathrm{E}_{\mathrm{ox} 1}$. Potentials are quoted $\pm 3 \mathrm{mV}$.

${ }^{c}$ Potential more anodic than the positive potential limit of the electrolyte.

\footnotetext{
${ }^{*}$ The electrochemical properties for $\left[\left\{\mathrm{Ru}(\mathrm{bpy})_{2}\right\}_{2}(\mu-\mathrm{BL})\right]^{n+}\{\mathrm{BL}=\mathrm{bpm}$, dbneil $\}$ in $\mathrm{PF}_{6}{ }^{-}$media were reported in $\S 2.3 .2$.

${ }^{\dagger}$ The potentials are reported relative to the $\mathrm{Fc}^{+} / \mathrm{FC}^{0}$ to be consistent with the reports of Geiger and coworkers. ${ }^{100,110}$ An aspect that has not been addressed is whether the ferrocene itself is sensitive to ion-pairing in $\mathrm{PF}_{6}{ }^{-}$electrolyte. The decamethylferrocenium/decamethylferrocene redox couple has been suggested as a superior redox standard to $\mathrm{Fc}^{+} / \mathrm{Fc}^{0}:{ }^{0.15}$ In the present case, the difference of potentials between $\mathrm{Me}_{10} \mathrm{Fc}^{+} / \mathrm{Me}_{10} \mathrm{Fc}^{0}$ and $\mathrm{Fc}^{+} / \mathrm{Fc}^{0}$ couples was $510 \mathrm{mV}$ in $\mathrm{PF}_{6}^{-}$media and $528 \mathrm{mV}$ $\left\{\mathrm{B}\left(\mathrm{C}_{6} \mathrm{~F}_{5}\right)_{4}\right\}^{-}$media so that care needs to be exerted in comparison of actual potentials between the two media. Nevertheless, the comparison of the differences between the potentials in each medium is valid.
} 
While the reversibilities of the oxidation and reduction processes were comparable in both electrolytes, the relative potentials and separations of the redox processes differed markedly. Slight differences were observed in the potentials for the reduction processes in the various complexes in the two media, however the effect on the potentials for the oxidation processes was more pronounced. In all instances, the redox potentials for a particular oxidation in a specified complex occur at more positive potentials in $\left\{\mathrm{B}\left(\mathrm{C}_{6} \mathrm{~F}_{5}\right)_{4}\right\}^{-}$than in $\mathrm{PF}_{6}{ }^{-}$media. This arises from the decrease in ion-pairing between the electrolyte anions and the complex cations in the presence of the weakly-associating $\left\{B\left(\mathrm{C}_{6} \mathrm{~F}_{5}\right)_{4}\right\}^{-}$ electrolyte relative to $\mathrm{PF}_{6}{ }^{-}$. In all cases for a particular complex, the decrease is greater for $\mathrm{E}_{\mathrm{ox} 2}$ than $\mathrm{E}_{\mathrm{ox} 1}$ : consequently, the value of $\Delta \mathrm{E}_{\mathrm{ox}}$ (and therefore $\mathrm{K}_{\mathrm{c}}$ ) is invariably greater in $\left\{\mathrm{B}\left(\mathrm{C}_{6} \mathrm{~F}_{5}\right)_{4}\right\}^{-}$than in $\mathrm{PF}_{6}{ }^{-}$media. For the individual species, the following specific observations can be made:

$B L=2,3$-bis(2-pyridyl)benzoquinoxaline $(d p b) .{ }^{67}$ In this case, a difference in the value of $\mathrm{K}_{\mathrm{c}}$ between the two diastereoisomers is reflected in both media.

$B L=3,4$-bis(2-pyridyl)-1,2,5-oxadiazole (dpo). For this complex, a difference is observed between the two diastereoisomers in terms of $\Delta \mathrm{E}_{\text {ox }}$ and $\mathrm{K}_{\mathrm{c}}$ in $\mathrm{PF}_{6}{ }^{-}$media. In $\left\{\mathrm{B}\left(\mathrm{C}_{6} \mathrm{~F}_{5}\right)_{4}\right\}^{-}$media, the anodic shift in the potential of $\mathrm{E}_{\mathrm{oxz}}$ is sufficiently large that the potential is beyond the anodic limit of the $\left[\left(n-\mathrm{C}_{4} \mathrm{H}_{9}\right)_{4} \mathrm{~N}\right]\left\{\mathrm{B}\left(\mathrm{C}_{6} \mathrm{~F}_{5}\right)_{4}\right\} / \mathrm{CH}_{3} \mathrm{CN}$ solvent-electrolyte system. While the values of $\Delta \mathrm{E}_{\text {ox }}$ and $\mathrm{K}_{\mathrm{c}}$ cannot be assessed, the anodic limit of the medium is $\sim 1900 \mathrm{mV}\left(v s . \mathrm{Fc}^{+} / \mathrm{Fc}^{0}\right.$ ) and the values of $\mathrm{K}_{\mathrm{c}}$ in this medium will be considerably higher than those reported for $\mathrm{PF}_{6}^{-}$.

$B L=3,5$-bis(2-pyridyl)-1,2,4-triazolato (bpt ${ }^{-}$. The electrochemical properties of the diastereoisomeric mixture of $\left[\left\{\mathrm{Ru}(\mathrm{bpy})_{2}\right\}_{2}\left(\mu-\mathrm{bpt}^{-}\right)\right]^{4+}$ have been discussed previously. ${ }^{158,159}$ For this complex, the $\mathrm{K}_{\mathrm{c}}$ values for the two separated diastereoisomers are indistinguishable in the presence of

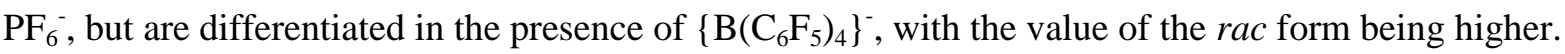

$B L=b p m$. The characteristics of the two diastereoisomers in $\left\{\mathrm{B}\left(\mathrm{C}_{6} \mathrm{~F}_{5}\right)_{4}\right\}^{-}$media are identical within the experimental uncertainty of the potential measurements $( \pm 3 \mathrm{mV})$. However, the values determined for $\mathrm{K}_{\mathrm{c}}$ in the presence of the two anions vary significantly.

$B L=$ dbneil. There are anodic shifts $(c a .160 \mathrm{mV})$ in the redox potentials associated with the respective oxidations measured in $\left\{\mathrm{B}\left(\mathrm{C}_{6} \mathrm{~F}_{5}\right)_{4}\right\}^{-}$compared with $\mathrm{PF}_{6}{ }^{-}$media. This is consistent with the stronger association of the latter with the cationic complex which lowers the effective charge of the complex and facilitates oxidation. While the $\mathrm{K}_{\mathrm{c}}$ values are indistinguishable in $\left\{\mathrm{B}\left(\mathrm{C}_{6} \mathrm{~F}_{5}\right)_{4}\right\}^{-}$media, the values are higher than in $\mathrm{PF}_{6}^{-}$media.

The results demonstrate the significant and sometimes unpredictable dependence of the redox potentials for these oxidation processes on the identity of electrolyte anions used in such electrochemical experiments. This emphasises the need for standard conditions for data from which such comparisons are made, and the danger of over-interpretation of $\Delta \mathrm{E}_{\mathrm{ox}}$ data and $\mathrm{K}_{\mathrm{c}}$ values derived from them. ${ }^{131}$ 


\subsubsection{Electronic Spectroscopy}

The UV/Vis/NIR spectral data for the un-oxidised (+4), mixed-valence $(+5)$ and fully-oxidised (+6) forms of the dinuclear systems (for the range 3050-30000 $\mathrm{cm}^{-1}$ ) are reported in Table B3.6 (Appendix B) and the spectra of meso- $\left[\left\{\mathrm{Ru}(\mathrm{bpy})_{2}\right\}_{2}(\mu-\mathrm{BL})\right]^{n+}\{\mathrm{BL}=\mathrm{dpb}, \mathrm{dpb}$ ' and dpq' $\}$ are presented in Figure 3.8.

The spectra of the +4 states of the dinuclear complexes incorporating the dpb, dpq', ppz and 2,3-dpp bridging ligands have been completely assigned previously on the basis of electrochemical, spectral, ${ }^{68,69,72-82}$ resonance Raman ${ }^{76,78,137,160-162}$ and computational calculations. ${ }^{73,162,163}$ In the present study, the spectra for the full series of complexes are characterised by a combination of overlapping $\mathrm{d} \pi\left(\mathrm{Ru}^{\mathrm{II}}\right) \rightarrow \pi^{*}(\mathrm{BL})$ and $\mathrm{d} \pi\left(\mathrm{Ru}^{\mathrm{II}}\right) \rightarrow \pi^{*}$ (bpy) singlet metal-to-ligand ( $\left.{ }^{1} \mathrm{MLCT}\right)$ transitions. The lowest energy absorption band shifts to the red as BL is varied through the series 2,3-dpp, ppz, dpb, dpq' and $\mathrm{dpb}$, consistent with the increasing stabilisation of the $\pi^{*}(\mathrm{BL})$ orbitals.

(a)

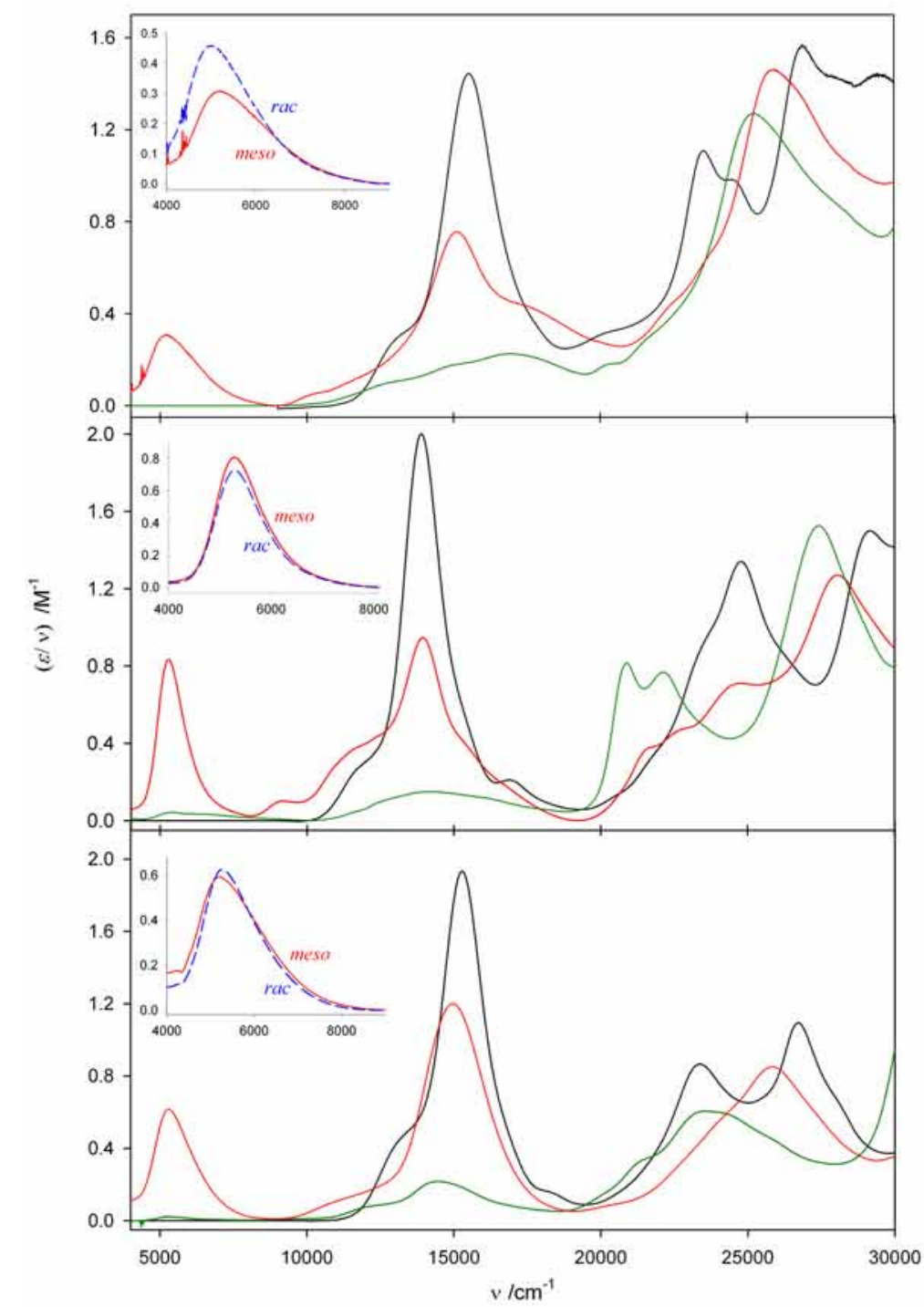

Figure 3.8 UV/Vis/NIR spectra of the reduced absorption spectra $\{(\varepsilon / v) v s . v\}$ of (a) meso-[\{Ru(bpy $\left.\left.)_{2}\right\}_{2}(\mu-d p b)\right]^{n+}$, (b) meso-[\{Ru(bpy $\left.\left.)_{2}\right\}_{2}\left(\mu-\mathrm{dpb}^{\prime}\right)\right]^{n+}$ and (c) meso-[\{Ru(bpy $\left.\left.)_{2}\right\}_{2}\left(\mu-\mathrm{dpq}^{\prime}\right)\right]^{n+}\{n=4(-), 5(-), 6(-)\}$ in $0.1 \mathrm{M}$ $\left[\left(n-\mathrm{C}_{4} \mathrm{H}_{9}\right)_{4} \mathrm{~N}\right] \mathrm{PF}_{6} / \mathrm{CH}_{3} \mathrm{CN}$ at $-35^{\circ} \mathrm{C}$. Insets: overlays of the IVCT bands for the meso and rac diastereoisomers. 
Spectroelectrochemical generation of the +5 and +6 forms of the diastereoisomers revealed stable isosbestic points in the spectral progressions accompanying both oxidation processes. The ${ }^{1}$ MLCT absorption bands decreased in intensity following one-electron oxidation and collapsed completely following oxidation to the +6 species. ${ }^{*}$ The first oxidation process was characterised by the appearance of an intense new band in the region 3500-9000 $\mathrm{cm}^{-1}$ which collapsed on removal of the second electron: on this basis the band was identified as an IVCT transition. The assignment of this band as a ligand-to-metal charge transfer transition (LMCT) was excluded on the basis of the absence of absorption bands in the NIR region for the +6 species, and the corresponding mononuclear complexes $\left[\mathrm{Ru}(\mathrm{bpy})_{2}(\mathrm{BL})\right]^{3+}\{\mathrm{BL}=$ dpb and dpb'; Table B3.7 (Appendix B)\}.

\subsubsection{Intervalence Charge Transfer}

An overlay of the IVCT bands for the full series of complexes $\{B L=d p b, d p b ', d p q$ and ppz $\}$ is shown in Figure 3.9, and the results of the band maxima $\left(v_{\max }\right)$, molar absorption coefficients $\left\{(\varepsilon / v)_{\max }\right\}$, bandwidths $\left(\Delta v_{1 / 2}\right)$ are summarised in Table 3.5. For all complexes, the bands are asymmetrically-shaped and narrower on the lower energy side. A moment analysis of the IVCT bands was pursued, ${ }^{1,164}$ and the results for the first-order moment analysis are also presented in Table 3.5, where the zeroth- $\left(M_{0}\right)$ and first-order $\left(M_{1}\right)$ moments represent the band area and average band energy, respectively, and $\left|\mu_{12}\right|$ is the transition moment (determined from equation 3.4). The electronic coupling parameters, $\mathrm{H}_{\mathrm{ab}}$, were determined from equation 3.2, where $r_{\mathrm{ab}}$ was equated with the through-space geometrical distance between the metal centres ${ }^{14}$ determined from the X-ray crystal structures of the complexes incorporating the dpb \{average of the distances for the structures involving $\mathrm{PF}_{6}{ }^{-}$and $\left[\mathrm{ZnCl}_{4}\right]^{2-}$ counter-anions of 6.870(3) $\AA$ \}, dpb' and dpq' bridging ligands (Table 3.2). An $r_{\mathrm{ab}}$ value of $6.65 \AA$ was assumed for the ppzbridged complex.

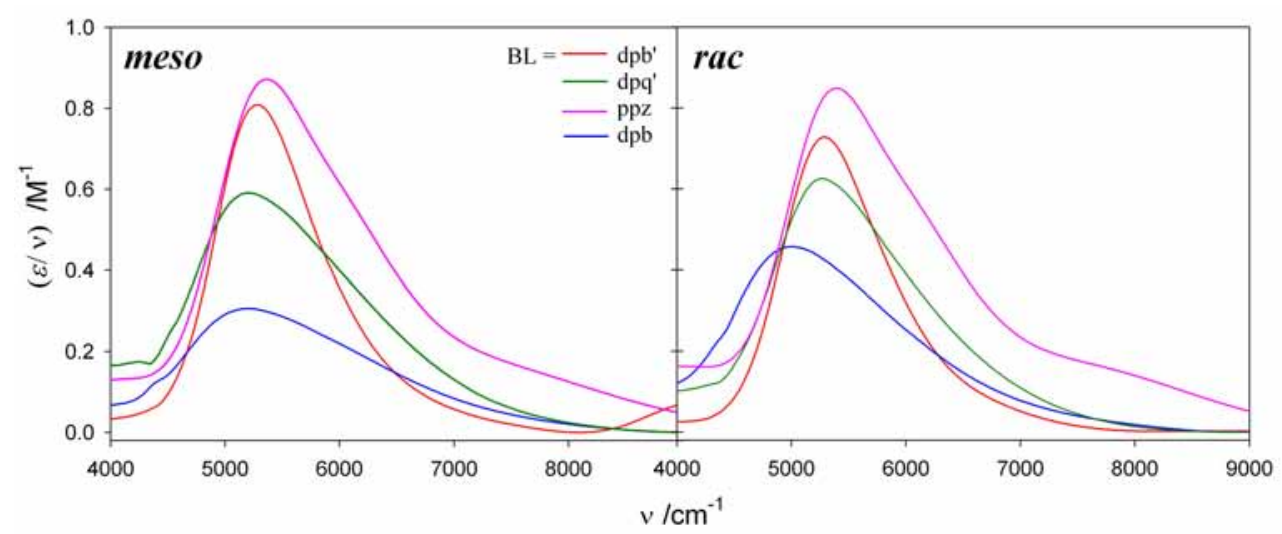

Figure 3.9 Overlay of the IVCT bands in $\mathrm{CH}_{3} \mathrm{CN}$ for the angular bridging ligands for meso- and rac$\left[\left\{\mathrm{Ru}(\mathrm{bpy})_{2}\right\}_{2}(\mu-\mathrm{BL})\right]^{5+}\{\mathrm{BL}=\mathrm{dpb}, \mathrm{dpb}$ and dpq' $\}$ in $0.1 \mathrm{M}\left[\left(n-\mathrm{C}_{4} \mathrm{H}_{9}\right)_{4} \mathrm{~N}\right] \mathrm{PF}_{6} / \mathrm{CH}_{3} \mathrm{CN}$ at $-35^{\circ} \mathrm{C}$.

\footnotetext{
${ }^{*}$ The UV/Vis/NIR spectra for the diastereoisomers of $\left[\left\{\mathrm{Ru}(\mathrm{bpy})_{2}\right\}_{2}(\mu-\mathrm{ppz})\right]^{n+}(n=4-6)$ are described further in §4.3.3.2.
} 
Table 3.5 IVCT spectral data of the reduced absorption spectra ( $\varepsilon / v v s . v)$ for the diastereoisomeric forms of $\left[\left\{\mathrm{Ru}(\mathrm{bpy})_{2}\right\}_{2}(\mu-\mathrm{BL})\right]^{5+}\{\mathrm{BL}=\mathrm{dpb}, \mathrm{dpb}$ ', dpq', ppz $\}$ in $0.1 \mathrm{M}\left[\left(n-\mathrm{C}_{4} \mathrm{H}_{9}\right)_{4} \mathrm{~N}\right] \mathrm{PF}_{6} / \mathrm{CH}_{3} \mathrm{CN}$ at $-35^{\circ} \mathrm{C}$ and parameters derived from the moment analysis of the transitions.

\begin{tabular}{|c|c|c|c|c|c|c|c|c|c|c|}
\hline BL & Diastereoisomer & $\begin{array}{c}v_{\max } \\
/ \mathrm{cm}^{-1}\end{array}$ & $\begin{array}{c}(\varepsilon / v)_{\max } \\
/ \mathrm{M}^{-1}\end{array}$ & $\begin{array}{c}\Delta v_{1 / 2} \\
/ \mathrm{cm}^{-1}\end{array}$ & $\begin{array}{c}M_{0} \\
/ \mathrm{M}^{-1}\end{array}$ & $\begin{array}{c}M_{1} \\
/ \mathrm{cm}^{-1}\end{array}$ & $\begin{array}{c}\left|\mu_{12}\right| \\
/ \mathrm{e} \AA\end{array}$ & $\begin{array}{c}\Delta v_{1 / 2}{ }^{0} \\
/ \mathrm{cm}^{-1}\end{array}$ & $\begin{array}{c}\mathrm{H}_{\mathrm{ab}} \\
/ \mathrm{cm}^{-1}\end{array}$ & $\Gamma$ \\
\hline $\mathrm{dpb}$ & meso & 5205 & 0.3062 & 1910 & 639.8 & 5655 & 0.5211 & 3090 & 395 & 0.382 \\
& rac & 5000 & 0.4572 & 1740 & 870.5 & 5450 & 0.6078 & 3030 & 440 & 0.426 \\
\hline $\mathrm{dpb}$ & meso & 5285 & 0.8084 & 1070 & 1010 & 5510 & 0.6546 & 3115 & 500 & 0.657 \\
& rac & 5290 & 0.7222 & 1080 & 903.2 & 5510 & 0.6190 & 3115 & 475 & 0.653 \\
\hline $\mathrm{dpq}$ & meso & 5195 & 0.5934 & 1710 & 1140 & 5600 & 0.6955 & 3090 & 530 & 0.447 \\
& rac & 5220 & 0.6329 & 1450 & 1050 & 5600 & 0.6675 & 3100 & 510 & 0.532 \\
\hline $\mathrm{ppz}$ & meso & 5370 & 0.8721 & 1560 & 1680 & 5930 & 0.8440 & 3140 & 680 & 0.503 \\
& rac & 5390 & 0.8494 & 1560 & 1680 & 5960 & 0.8440 & 3150 & 680 & 0.504 \\
\hline
\end{tabular}

${ }^{\mathrm{a}}$ The errors in the observed parameters are $\pm 10 \mathrm{~cm}^{-1}$ for $v_{\max }, M_{1}$ and $\Delta v_{1 / 2}, \pm 0.0001 \mathrm{M}^{-1}$ for $(\varepsilon / v)_{\max }, \pm 5 \mathrm{M}^{-1}$ for $M_{0}$ and \pm 0.0001 eÅ for $\left|\mu_{12}\right|$.

\section{(i) A Classical Two-State Analysis}

By comparison with the theoretical bandwidths $\left(\Delta v_{1 / 2}{ }^{\circ}\right)$ estimated on the basis of the classical two-state theory ${ }^{8}$ (equation 1.8, where $16 \mathrm{RT} \ln 2=1836 \mathrm{~cm}^{-1}$ at $238 \mathrm{~K}$ ), the relatively narrow bandwidths observed are indicative of significant electronic communication between the metal centres. As described in §1.3.3, the $\Gamma$ parameter (equation 1.21) provides a criterion for describing the relative degree of electronic coupling in the systems. ${ }^{38}$ For the meso diastereoisomers, $\Gamma$ increases as BL is varied through the series dpb, dpq', ppz to dpb', while for both diastereoisomers of a given complex $(\varepsilon / v)_{\max }, \Delta v_{1 / 2},\left|\mu_{12}\right|$, $M_{0}$ and $\mathrm{H}_{\mathrm{ab}}$ increase as BL is varied through the series dpb, dpb', dpq' and ppz (Table 3.5). The results suggest that all the systems lie close to the localised-to-delocalised transition.

From the moment analysis of the bands, the general trend is an increase in the extent of delocalisation for the fused bridging ligands as BL is varied from dpb' to dpq' to ppz. This observed increase in coupling as the bridging ligand LUMO is destabilised \{i.e. as the energy separation between the $\mathrm{Ru}(\mathrm{d} \pi)$ orbital and $\pi^{*}(\mathrm{BL})$ is increased \} is somewhat surprising. The LUMO on ppz is based on both the pyrazine and pyridine moieties of the ligand, ${ }^{68,69,162}$ while the LUMO localises primarily on the phenazine and benzophenazine "tails” of dpq'68, 162, 163 and dpb', ${ }^{68,69,162}$ respectively. The localisation of these orbitals away from the nitrogens coordinated to the metal centres through the series ppz to dpq' to $\mathrm{dpb}^{\prime}$ appears to counteract the destabilisation of the $\pi^{*}(\mathrm{BL})$ orbital and gives rise to the decreased delocalisation through the series. Similar trends were noted previously for dinuclear osmium complexes incorporating the series of unfused bridging ligands 2,3-dpp, dpq \{2,3-bis(2-pyridyl)quinoxaline $\}$ and dpb. ${ }^{139}$

In the present study, the IVCT parameters suggest a marginally greater degree of delocalisation for the meso diastereoisomers relative to the corresponding rac forms. While the IVCT energies ( $v_{\max }$ or $M_{1}$ ) are identical within experimental error for the diastereoisomers incorporating the series of fused bridges, the dpb-bridged forms exhibit a $205 \mathrm{~cm}^{-1}$ difference in energies. The latter also possess the most pronounced difference in $\Delta \mathrm{E}_{\mathrm{ox}}$ values for the series of complexes (Table 3.3). The results indicate that 
delocalisation is enhanced for complexes incorporating smaller, less sterically-hindered bridging ligands, and the extent of delocalisation differs for the diastereoisomeric forms of the same complex in the presence of stereochemically-induced structural distortions. The latter may be regarded as redox asymmetry contributions to the electron transfer barrier, denoted by $\Delta \mathrm{E}_{\text {struct }}$.

Since electronic coupling decreases the effective electron transfer distance relative to the geometrical metal-metal separation (which is equated with $r_{\mathrm{ab}}$ in equation 3.2), the $\mathrm{H}_{\mathrm{ab}}$ values presented in Table 3.5 represent lower limits for the electronic coupling parameter. A re-evaluation of the effective electron transfer distance from Stark effect spectroscopy ${ }^{120}$ is the subject of a subsequent study presented in §3.3.6. The effective $\mathrm{H}_{\mathrm{ab}}$ values are likely to lie closer to the values calculated in the fully-delocalised limit (i.e. $M_{1}=2 \mathrm{H}_{\mathrm{ab}}{ }^{8}$ ); e.g. for the dpb' diastereoisomers, $\mathrm{H}_{\mathrm{ab}}$ is closer to $2755 \mathrm{~cm}^{-1}$.

The theoretical analysis of the IVCT data for the borderline localised-to-delocalised systems is challenging since the application of classical models ${ }^{1,2,38,40}$ is strictly invalid due to the failure of the Born-Oppenheimer approximation upon which they are based. ${ }^{14,38}$ While the treatment of the full vibronic coupling problem ${ }^{44-46,48}$ is central to the quantitative analysis of these systems, the application of such methods is beyond the scope of the present work. Instead, the investigation considers alternate localised and delocalised models for the description of the IVCT properties of the dinuclear complexes.

\section{(ii) A Localised Model for IVCT}

From a localised viewpoint, the asymmetric appearance of the IVCT bands is attributed to the "band cut-off" effect which occurs at $h v=2 \mathrm{H}_{\mathrm{ab}}$, and is more pronounced for systems in the localised-todelocalised transition region. ${ }^{18,38,57}$ Alternatively, the asymmetry of the IVCT bands may be attributed to a manifold of discrete, underlying transitions which are identified as spin-orbit components. ${ }^{14,165-167}$

Spectral deconvolutions of the IVCT bands for each diastereoisomer of the series of complexes require a minimum of three underlying Gaussian-shaped components. However, unique fits to the IVCT manifolds could not be obtained, and in general, three different fits (differing in the relative intensities of the underlying transitions) were obtained, as illustrated for the diastereoisomers of $\left[\left\{\mathrm{Ru}(\mathrm{bpy})_{2}\right\}_{2}(\mu-\mathrm{BL})\right]^{5+}$ $\left\{\mathrm{BL}=\mathrm{dpb}, \mathrm{dpb}^{\prime}\right\}$ in Figure 3.10. The results are reported in Table B3.8 (Appendix B).

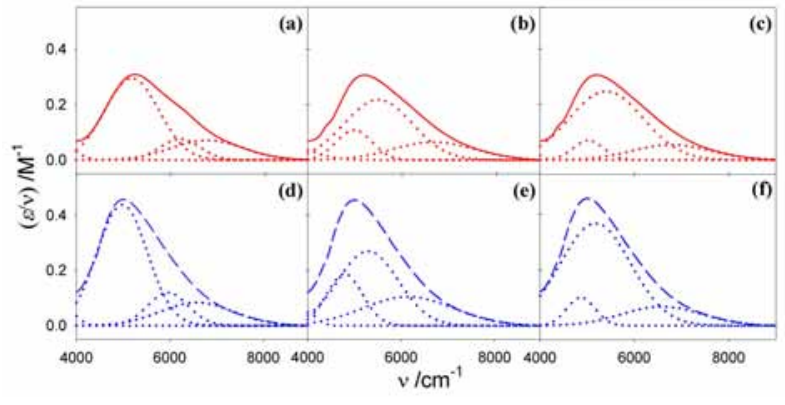

(i)

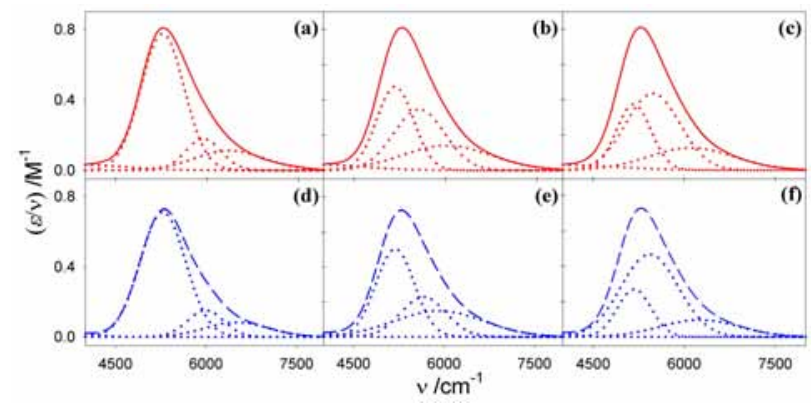

(ii)

Figure 3.10 Three fits to the IVCT bands for (i) meso- $\{(\mathrm{a})-(\mathrm{c})\}$ and $r a c-\{(\mathrm{d})-(\mathrm{f})\}\left[\left\{\mathrm{Ru}(\mathrm{bpy})_{2}\right\}_{2}(\mu-\mathrm{dpb})\right]^{5+}$ and (ii) meso- $\{(\mathrm{a})-(\mathrm{c})\}$ and rac- $\{(\mathrm{d})-(\mathrm{f})\}\left[\left\{\mathrm{Ru}(\mathrm{bpy})_{2}\right\}_{2}(\mu-\mathrm{dpb})\right]^{5+}$. 
The non-degeneracy of the $\mathrm{d} \pi$ levels at $\mathrm{Ru}^{\mathrm{II} / \mathrm{III}}$, and the appearance of multiple IVCT components, arise due to the combined effects of low symmetry and spin-orbit coupling. ${ }^{14}$ Three IVCT transitions are anticipated due to separate electronic excitations from one of the three $\mathrm{d} \pi_{\mathrm{n}}(\mathrm{n}=1-3)$ orbitals at $\mathrm{Ru}^{\mathrm{II}}$ to the hole in the corresponding $\mathrm{d} \pi_{\mathrm{n}}$ orbital which is present in any of the three spin-orbit states at $\mathrm{Ru}^{\mathrm{III}}$. These transitions are identified with the three underlying components in the IVCT band, separated by the energy differences between the ground and two excited spin-orbit states, $\Delta \mathrm{E}_{\mathrm{so}(1)}$ and $\Delta \mathrm{E}_{\mathrm{so}(2)}$, as expressed in equation 1.33, and depicted schematically in Figure 1.9. Based on the coordinate designation in Figure 3.11, qualitative considerations indicate that the metal-based orbitals with $z$ character are appropriate for overlap with the $\pi, \pi^{*}(\mathrm{BL})$ system and will be stabilised to a relatively greater extent (and will thus lie lower in energy) than those that lie orthogonal to the $\pi, \pi^{*}(\mathrm{BL})$ system; i.e. the $\mathrm{d}_{x y}$ orbitals that lie in the plane of the bridging ligand. In order of increasing energy, the three $\mathrm{d} \pi$ orbitals may be more closely represented by $\mathrm{d}_{x z}, \mathrm{~d}_{y z}\left(\mathrm{~d} \pi_{1}, \mathrm{~d} \pi_{2}\right)$ and $\mathrm{d}_{x y}\left(\mathrm{~d} \pi_{3}\right)$.
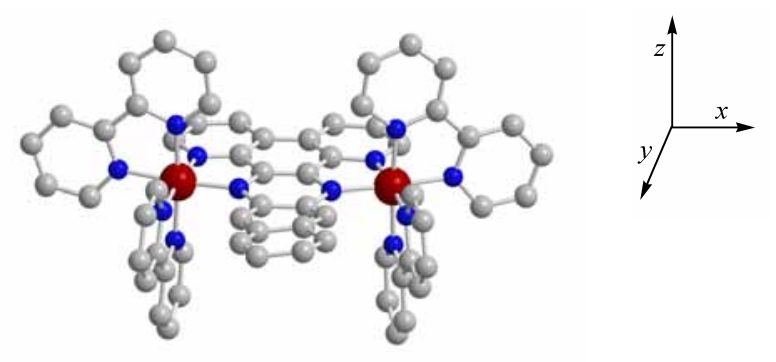

Figure 3.11 Coordinate axes for meso- $(\Lambda \Delta)-\left[\left\{\mathrm{Ru}(\mathrm{bpy})_{2}\right\}_{2}\left(\mu-\mathrm{dpb}^{\prime}\right)\right]^{4+}$.

As shown in Table B3.8 (Appendix B), the energy separations between the components $\left\{\Delta \mathrm{E}_{\mathrm{so}(1)}\right.$ and $\left.\Delta \mathrm{E}_{\mathrm{so}(2)}\right\}$ are within the range expected for spin-orbit coupling at $\mathrm{Ru}{ }^{\mathrm{III}}$ centres $\left(\xi_{\mathrm{Ru}} \sim 1000 \mathrm{~cm}^{-1}\right)$. As discussed in §2.3.3.1, the intensities of the three components should be proportional to the degree of orbital overlap between the $\mathrm{d} \pi$ orbitals at the formally $\mathrm{Ru}^{\mathrm{II}}$ centre and the $\mathrm{d} \pi$ orbital containing the hole at the formally $\mathrm{Ru}^{\mathrm{III}}$ centre. In the present case, the relative intensities of the two major components of the IVCT manifolds $\{$ IVCT(1) and (2)\} were variable for the three deconvolutions of each diastereoisomeric form, however the parameters for the highest energy component remained relatively constant. The latter can be assigned as a $\left(\mathrm{Ru}^{\mathrm{II}}\right) \mathrm{d} \pi_{3}-\pi^{*}(\mathrm{BL})-\left(\mathrm{Ru}{ }^{\mathrm{III}}\right) \mathrm{d} \pi_{3}$ transition $\left\{\right.$ abbreviated $\left.\mathrm{d} \pi_{3}-\pi^{*}(\mathrm{BL})-\mathrm{d} \pi_{3}\right\}$; this component is broad and of low absorptivity as the electronic coupling occurs between donor and acceptor orbitals which are predominantly $\mathrm{d}_{x y}$ in character, and are oriented orthogonal to the $\pi, \pi^{*}(\mathrm{BL})$ system. Based on ligand field considerations, the IVCT bands should be dominated by IVCT(1) and (2) which are associated with $d \pi_{1}-\pi^{*}(\mathrm{BL})-\mathrm{d} \pi_{1}$ and $\mathrm{d} \pi_{2}-\pi^{*}(\mathrm{BL})-\mathrm{d} \pi_{2}$ interactions across the bridge, where $\mathrm{d} \pi_{1}$ and $\mathrm{d} \pi_{2}$ are predominantly $d_{x z}$ and $d_{y z}$ in character. On this basis, deconvolutions (b) and (e) in Figure 3.10(i) represent the most reasonable solution for the three underlying components for the dpb-bridged forms; deconvolutions (b) and (e), or (c) and (d) in Figure 3.10(ii), represent the most reasonable solutions for the dpb'-bridged forms. 
The separation of the IC states in the +6 forms of the analogous dinuclear osmium systems provide a more definitive measure of the relative splittings between the underlying components for the series of complexes. This work is the focus of §3.3.5.3.

\section{(iii) Mid-IR Spectroelectrochemistry}

While the characteristics of the NIR mixed-valence bands support a localised-to-delocalised or delocalised classification for the mixed-valence species, the assignment to the former category is strictly valid only when there is clear evidence of localisation on the IR time-scale $\left(<10^{-11} \mathrm{~s}\right) .{ }^{14}$ Figure B3.2 (Appendix B) shows the differential mid-IR spectra for meso- $\left[\left\{\mathrm{Ru}(\mathrm{bpy})_{2}\right\}_{2}(\mu-\mathrm{BL})\right]^{n+}(n=5,6$; BL $=\mathrm{dpb}$, $\mathrm{dpb}$ ). On the basis of comparisons with mid-IR studies on the mixed-valence and fully-oxidised forms of related pyz- and $\mathrm{N}_{2}$-bridged polypyridyl complexes of ruthenium ${ }^{168}$ and osmium, ${ }^{165,166,169-171}$ the new vibration at $1600 \mathrm{~cm}^{-1}$ in meso-[ $\left.\left\{\mathrm{Ru}(\mathrm{bpy})_{2}\right\}_{2}(\mu-\mathrm{BL})\right]^{5+}$ coincides with the expected position for the $v_{8 a}$ (pyz) stretch. ${ }^{14}$ The appearance of this band has been employed as a diagnostic marker for localisation in related systems such as cis,cis-[\{Os(bpy $\left.)_{2}(\mathrm{Cl})\right\}_{2}(\mu \text {-pyz) }]^{3+}$ which lie in Class II-III. ${ }^{165,166}$ In the present case, the appearance of the band at $1600 \mathrm{~cm}^{-1}$ provides evidence for electronic localisation, which is consistent with a small residual barrier to intramolecular electron transfer and a localised-to-delocalised classification for the mixed-valence species.

The mid-IR difference spectra for meso- $\left[\left\{\mathrm{Ru}(\mathrm{bpy})_{2}\right\}_{2}(\mu-\mathrm{BL})\right]^{5+}\{\mathrm{BL}=\mathrm{dpb}, \mathrm{dpb}\}$ shown in Figure B3.2 (Appendix B) provide evidence for increased absorptivity at energies below $2000 \mathrm{~cm}^{-1}$ relative to the +4 and +6 states. This may reflect the presence of underlying IVCT and/or IC transitions. The possibility that the band in this region is the lowest energy IVCT transition \{i.e. IVCT(1)\} implies that the NIR bands in the region 4000-9000 $\mathrm{cm}^{-1}$ represent IVCT(2) and/or IVCT(3). Indeed, the first IVCT band in $\left[\left\{\mathrm{Ru}\left(\mathrm{NH}_{3}\right)_{5}\right\}_{2}(\mu-\mathrm{pyz})\right]^{5+}$ was observed as a broad and weak band in the IR region at $2000 \mathrm{~cm}^{-1}\left(\varepsilon_{\max } \sim 300\right.$ $\left.\mathrm{M}^{-1} \mathrm{~cm}^{-1}, \Delta \mathrm{v}_{1 / 2}=1400 \mathrm{~cm}^{-1}\right),{ }^{168}$ while the asymmetric bands in the NIR region at $4500-5000 \mathrm{~cm}^{-1}$ and 6320 $\mathrm{cm}^{-1}$ were assigned to overlapping IVCT(2) and IVCT(3) transitions. In the present case, the absorption in the mid-IR region below $2000 \mathrm{~cm}^{-1}$ cannot be definitively assigned as IVCT transition because IC transitions between the $\mathrm{d} \pi\left(\mathrm{Ru}^{\mathrm{III}}\right)$ orbitals are also expected to occur in this region at $\sim 3 / 2 \xi_{\mathrm{Ru}} \cdot{ }^{14,144}$ On this basis, the designation of the NIR bands in the region $4000-9000 \mathrm{~cm}^{-1}$ as a manifold of the three closelyspaced transitions IVCT(1), (2) and (3) is retained. Clearly, the extension of the IR measurements to the wavelength range below $1000 \mathrm{~cm}^{-1}$, in addition to IR measurements for the full series of complexes $\left[\left\{\mathrm{Ru}(\mathrm{bpy})_{2}\right\}_{2}(\mu-\mathrm{BL})\right]^{5+}\{\mathrm{BL}=\mathrm{dpq}$ ', ppz and 2,3-dpp $\}$, represents an important thrust of future work. A further discussion of the vibrational bands in the IR region is not warranted at the present time.

\section{(iv) A Qualitative Three-State Analysis}

The significant variation in the IVCT characteristics with the identity of the bridging ligand is indicative of its integral role in mediating the IVCT process, and the importance of the explicit inclusion 
of a third electronic state associated with the bridge. The trends in the IVCT parameters for the series also signal the importance of the contribution of excitation in totally symmetric vibration modes. ${ }^{38,48,172}$

The three-state model proposed by Ondrechen and coworkers for delocalised mixed-valence complexes, ${ }^{49-53,173,174}$ and the classical three-state two-mode model postulated by Brunschwig, Creutz and Sutin $^{38}$ provide quantitative treatments for the contribution of the symmetric modes within three-state formalisms. For delocalised systems, the IVCT transitions are considered as arising from electron transfer between the bonding and non-bonding molecular orbitals within the molecular orbital manifolds of the mixed-valence systems, as shown schematically in Figure 1.4. ${ }^{49-53,173,174}$

As discussed in $§ 1.4 .1$ and $§ 1.4 .3$, the inclusion of the symmetric mode is manifested by a broadening and blue-shifting in the IVCT band with increasing delocalisation. For the series of complexes investigated in the present study, the general trend is an increase in the extent of delocalisation, and $M_{1}$ as BL is varied from dpb' to dpq' to ppz (Table 3.5). This blue-shift in the IVCT energy with increasing delocalisation supports the integral role of the symmetric vibration mode and a three-state treatment for the systems. Resonance Raman studies ${ }^{175,176}$ would provide a direct indication of the symmetric mode vibrations coupled to the electronic transition, and represent an important thrust of future work.

\section{(v) Another Perspective}

Qualitatively, a localised bonding description based on the geometrical properties of the $\mathrm{d} \pi\left(\mathrm{Ru}^{\mathrm{II} / I I I}\right)$ orbitals provides a reasonable rationale for the IVCT transitions in the dinuclear systems. The asymmetry of the NIR bands may be reasonably ascribed to the overlap of three underlying IVCT bands which arise from spin-orbit coupling and lower-symmetry perturbations in a localised description. However, the results of the NIR and mid-IR spectroelectrochemical experiments are insufficient to distinguish between two- and three-centre coupling models for the analysis of the mixed-valence species. An alternate view is that the mixed-valence ground state is electronically localised, while the excited states are electronically delocalised. This possibility has been discussed by Meyer and coworkers ${ }^{14}$ in relation to the mixed-valence characteristics of $\left[\left\{\operatorname{Ru}(\mathrm{bpy})_{2}\right\}_{2}(\mu-\mathrm{pyz})\right]^{5+}$.

The following sections detail the experimental strategies which were adopted to further elucidate the origins of stereochemical influences on the IVCT properties in the series of complexes. 


\subsubsection{Two Examples of Complete Delocalisation}

The UV/Vis spectral properties of the diastereoisomeric forms of $\left[\left\{\mathrm{Ru}(\mathrm{bpy})_{2}\right\}_{2}(\mu-\mathrm{BL})\right]^{4+}\{\mathrm{BL}=$ dpo, dpt $\}$ have been discussed previously. ${ }^{71}$ In the present study, UV/Vis/NIR spectra were obtained for the diastereoisomers of $\left[\left\{\mathrm{Ru}(\mathrm{bpy})_{2}\right\}_{2}(\mu-\mathrm{BL})\right]^{n+}(n=4,5,6)$ forms over the range $3050-30000 \mathrm{~cm}^{-1}$ (Table B3.9, Appendix B). The spectral progression accompanying the one-electron oxidation of meso$\left[\left\{\mathrm{Ru}(\text { bpy })_{2}\right\}_{2}(\mu-d p o)\right]^{4+}$ in $0.1 \mathrm{M}\left[\left(n-\mathrm{C}_{4} \mathrm{H}_{9}\right)_{4} \mathrm{~N}\right] \mathrm{PF}_{6} / \mathrm{CH}_{3} \mathrm{CN}$ at $-35^{\circ} \mathrm{C}$ is shown in Figure 3.12(a).

The lowest energy absorption bands in the spectra of the +4 species are assigned as $\mathrm{d} \pi(\mathrm{Ru}) \rightarrow$ $\pi^{*}(\mathrm{BL})$ MLCT transitions. These bands decreased in energy and intensity following one-electron oxidation to the +5 species, and collapsed completely on further oxidation to the +6 state. The new bands in the regions $3050-9000 \mathrm{~cm}^{-1}$ and $14000-16000 \mathrm{~cm}^{-1}$ in the mixed-valence species are assigned as IVCT and LMCT transitions, respectively. The former are absent in the spectra of the +6 species, while the latter increase in intensity, and occur in the same region as the $\pi(\mathrm{bpy}) \rightarrow \mathrm{d} \pi\left(\mathrm{Ru}^{\mathrm{III}}\right)$ LMCT transitions at 14815 and $17160 \mathrm{~cm}^{-1}$ in $\left[\mathrm{Ru}^{\mathrm{III}}(\mathrm{bpy})_{3}\right]^{3+} .{ }^{177}$ Comparable behaviour was observed for rac-[ $\left[\mathrm{Ru}(\mathrm{bpy})_{2}\right\}_{2}(\mu-$ dpo $)]^{4+}$ and for the diastereoisomers of $\left[\left\{\operatorname{Ru}(\mathrm{bpy})_{2}\right\}_{2}(\mu-\mathrm{dpt})\right]^{4+}$.

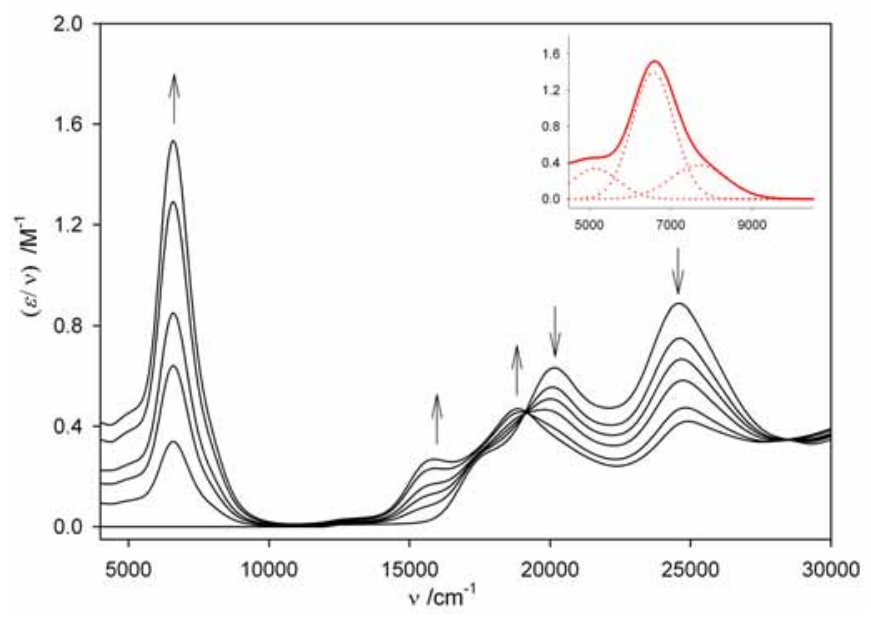

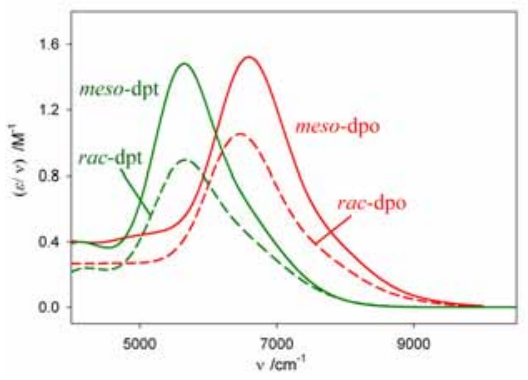

(b)

(a)

Figure 3.12 (a) Spectral progression for the oxidation reaction meso- $\left[\left\{\mathrm{Ru}(\mathrm{bpy})_{2}\right\}_{2}(\mu \text {-dpo })\right]^{4+} \rightarrow$ meso$\left[\left\{\mathrm{Ru}(\mathrm{bpy})_{2}\right\}_{2}(\mu \text {-dpo })\right]^{5+}$ in $0.1 \mathrm{M}\left[\left(n-\mathrm{C}_{4} \mathrm{H}_{9}\right)_{4} \mathrm{~N}\right] \mathrm{PF}_{6} / \mathrm{CH}_{3} \mathrm{CN}$ at $-35^{\circ} \mathrm{C}$. The inset shows the best fit Gaussian deconvolution of the IVCT band. (b) NIR spectra for meso- and rac- $\left[\left\{\mathrm{Ru}(\mathrm{bpy})_{2}\right\}_{2}(\mu-\mathrm{dpt})\right]^{5+}(\mathrm{BL}=\mathrm{dpo}$, dpt) in $0.1 \mathrm{M}$ $\left[\left(n-\mathrm{C}_{4} \mathrm{H}_{9}\right)_{4} \mathrm{~N}\right] \mathrm{PF}_{6} / \mathrm{CH}_{3} \mathrm{CN}$ at $-35^{\circ} \mathrm{C}$.

Figure 3.12(b) shows an overlay of the NIR bands for the diastereoisomeric forms of $\left[\left\{\mathrm{Ru}(\mathrm{bpy})_{2}\right\}_{2}(\mu-\mathrm{BL})\right]^{5+}\{\mathrm{BL}=\mathrm{dpo}, \mathrm{dpt}\}$, and the IVCT spectral data for the overall band manifolds are provided in Table 3.6. The parameters for the bands obtained from Gaussian deconvolution are reported in Table B3.10 (Appendix B). In each case, the IVCT bands were fitted by three Gaussian-shaped components, denoted IVCT(1), (2) and (3) in order of increasing energy. IVCT(2) exhibits the highest intensity and dominates the IVCT manifold. 
Table 3.6 NIR spectral data of the reduced absorption spectra $(\varepsilon / v v s$. $v)$ for the dinuclear complexes $\left[\left\{\mathrm{Ru}(\mathrm{bpy})_{2}\right\}_{2}(\mu-\mathrm{BL})\right]^{5+}\{\mathrm{BL}=\mathrm{dpo}$, dpt $\}$ in $0.1 \mathrm{M}\left[\left(n-\mathrm{C}_{4} \mathrm{H}_{9}\right)_{4} \mathrm{~N}\right] \mathrm{PF}_{6} / \mathrm{CH}_{3} \mathrm{CN}$ at $-35^{\circ} \mathrm{C}{ }^{\text {a }}$

\begin{tabular}{|l|c|c|c|c|c|c|}
\hline \multicolumn{1}{|c|}{ Complex } & $\begin{array}{c}v_{\max } \\
/ \mathrm{cm}^{-1}\end{array}$ & $\begin{array}{c}(\varepsilon / v)_{\max } \\
/ \mathrm{M}^{-1}\end{array}$ & $\begin{array}{c}\Delta v_{1 / 2} \\
/ \mathrm{cm}^{-1}\end{array}$ & $\begin{array}{c}\Delta v_{1 / 2}{ }^{\circ} \\
/ \mathrm{cm}^{-1}\end{array}$ & $\begin{array}{c}M_{0} \\
/ \mathrm{M}^{-1}\end{array}$ & $\begin{array}{c}\mathrm{H}_{\mathrm{ab}} \\
/ \mathrm{cm}^{-1}\end{array}$ \\
\hline meso- $\left[\left\{\mathrm{Ru}(\mathrm{bpy})_{2}\right\}_{2}(\mu-\mathrm{dpo})\right]^{5+}$ & 6590 & 1.5218 & 1470 & 3480 & 3211 & 3295 \\
rac- $\left[\left\{\mathrm{Ru}(\mathrm{bpy})_{2}\right\}_{2}(\mu-\mathrm{dpo})\right]^{5+}$ & 6460 & 1.2737 & 1492 & 3440 & 2650 & 3230 \\
\hline meso- $\left[\left\{\mathrm{Ru}(\mathrm{bpy})_{2}\right\}_{2}(\mu-\mathrm{dpt})\right]^{5+}$ & 5625 & 1.4908 & 1346 & 3210 & 2538 & 2813 \\
rac- $\left[\left\{\mathrm{Ru}(\mathrm{bpy})_{2}\right\}_{2}(\mu-\mathrm{dpt})\right]^{5+}$ & 5628 & 0.8995 & 1516 & 3215 & 1656 & 2814 \\
\hline
\end{tabular}

${ }^{\mathrm{a}}$ The errors in the observed parameters are $\pm 10 \mathrm{~cm}^{-1}$ for $v_{\max }$ and $\Delta v_{1 / 2}, \pm 0.0001 \mathrm{M}^{-1}$ for $(\varepsilon / v)_{\max }$ and $\pm 5 \mathrm{M}^{-1}$ for $M_{0}$.

The magnitude of the separation between the components $\left(\mathrm{ca} .1000 \mathrm{~cm}^{-1}\right)$ is consistent with the energy splittings expected between the three $\mathrm{d} \pi_{\mathrm{n}}(\mathrm{Ru})$ orbitals due to spin-orbit coupling ${ }^{144}\left(\xi \sim 1000 \mathrm{~cm}^{-1}\right.$ for $\left.\mathrm{Ru}^{\mathrm{III}}\right)$ and ligand-field asymmetry. These orbitals may be more closely represented by $\mathrm{d}_{x z}, \mathrm{~d}_{y z}\left(\mathrm{~d} \pi_{1}, \mathrm{~d} \pi_{2}\right)$ and $d_{x y}\left(d \pi_{3}\right)$ by analogy with the coordinate assignments in Figure 3.11 for the series of complexes incorporating angular bridging ligands. The greater stabilisation of the $\mathrm{d}_{x z}, \mathrm{~d}_{y z}\left(\mathrm{~d} \pi_{1}, \mathrm{~d} \pi_{2}\right)$ orbitals due to extensive overlap with the $\pi, \pi^{*}$ orbitals of dpo and dpt may account for the higher intensity of IVCT(2) relative to IVCT(3).

For all complexes, the bandwidths are narrow compared with the theoretical predictions for $\Delta v_{1 / 2}{ }^{\circ}$, which together with the significant intensity of the NIR manifolds suggest that the systems are delocalised (Class III). The IVCT components are more accurately assigned as transitions between bonding and non-bonding orbitals within the molecular orbital manifolds of the dinuclear complexes, as described within the three-state model of Ondrechen (\$1.4.3). ${ }^{49-53,173,174}$ As illustrated schematically in Figure 1.4, the NIR transition corresponds to promotion of an electron from the three-centre (metalligand-metal) bonding orbital to the two-centre (metal-metal) non-bonding orbital which corresponds to symmetrical charge distribution and excitation in one or more totally symmetric vibration modes. ${ }^{48}$ In the present case, the $\mathrm{d} \pi \rightarrow \pi^{*}(\mathrm{BL})$ energy gap is greater for the systems incorporating dpo due to the enhanced $\pi$-acceptor nature of this ligand compared with ppt. $^{71}$ The relatively larger separation between the bonding and non-bonding orbitals for the diastereoisomers of $\left[\left\{\mathrm{Ru}(\mathrm{bpy})_{2}\right)_{2}(\mu-\mathrm{dpo})\right]^{5+}$ is manifested as a blue-shift in the NIR band compared with their dpt-bridged analogues.

For delocalised mixed-valence complexes, the energy of the NIR transition provides a direct measure of the electronic coupling parameter, $\mathrm{H}_{\mathrm{ab}}$ according to equation 1.11. ${ }^{1,2}$ As shown in Table 3.6, the parameters of the IVCT bands differ between the same diastereoisomer of the two complexes, and the integrated intensity of the manifold is greater for the meso relative to the rac form in both cases. The differences between the diastereoisomers of the same complex are ascribed to differential ion-pairing interactions and specific solvation effects. While the dpt-bridged systems may be expected to exhibit greater delocalisation than their dpo-bridged analogues due to the larger polarisability of the sulfur atom, the relative $H_{a b}$ values suggest that the latter exhibits higher electronic coupling for a given diastereoisomer. This is consistent with the results of the electrochemical studies in §3.3.2.2. 


\subsubsection{Differential Redox Asymmetry Contributions in the Diastereoisomers of Unsymmetrical Mixed-Valence Complexes}

\subsubsection{General Electrochemical and UV/Vis/NIR Characterisation}

The electrochemical characteristics of the diastereoisomers of the unsymmetrical complexes $\left[\left\{\mathrm{Ru}(\mathrm{bpy})_{2}\right\}(\mu-\mathrm{BL})\{\mathrm{Ru}(\mathrm{pp})\}_{2}\right]^{4+}\left\{\mathrm{BL}=\mathrm{dpb}, \mathrm{dpb}\right.$ '; pp = $\mathrm{Me}_{2}$ bpy, $\mathrm{Me}_{4}$ bpy $\}$ were investigated by cyclic and differential pulse voltammetry in acetonitrile solution containing $0.1 \mathrm{M}\left[\left(n-\mathrm{C}_{4} \mathrm{H}_{9}\right)_{4} \mathrm{~N}\right] \mathrm{PF}_{6}$ as the electrolyte. The redox potentials for the metal-based oxidation processes are reported in Table 3.7, in addition to the lowest energy MLCT transitions in the +4 states. The data for the symmetrical $\left[\left\{\mathrm{Ru}(\mathrm{bpy})_{2}\right\}_{2}(\mu-\mathrm{BL})\right]^{4+}$ analogues are reported for comparison. The ligand-based reduction potentials and UV/Vis/NIR spectral data for the series of unsymmetrical complexes are provided in Tables B3.11-12 and B3.13-14 (Appendix B), respectively.

Table 3.7 Electrochemical data (in $\mathrm{mV}$ relative to the $\mathrm{Fc}^{+} / \mathrm{Fc}^{0}$ couple) and energies of the first MLCT transition in the +4 state $\left(\mathrm{E}_{\mathrm{MLCT}(1)}\right)$ for $\left[\left\{\mathrm{Ru}(\mathrm{bpy})_{2}\right\}(\mu-\mathrm{dpb})\left\{\mathrm{Ru}(\mathrm{pp})_{2}\right\}\right]^{4+}$ and $\left[\left\{\mathrm{Ru}(\mathrm{bpy})_{2}\right\}(\mu-\mathrm{dpb})\left\{\mathrm{Ru}(\mathrm{pp})_{2}\right\}\right]^{4+}$ in $0.1 \mathrm{M}[(n-$ $\left.\mathrm{C}_{4} \mathrm{H}_{9}\right)_{4} \mathrm{~N} \mathrm{PF}_{6} / \mathrm{CH}_{3} \mathrm{CN}$. ${ }^{\mathrm{a}, \mathrm{b}}$

\begin{tabular}{|c|c|c|c|c|c|c|c|c|c|}
\hline \multirow{3}{*}{ pp } & \multirow{2}{*}{ Diastereoisomer } & \multicolumn{4}{|c|}{$\mathrm{BL}=\mathrm{dpb}$} & \multicolumn{4}{c|}{$\mathrm{BL}=\mathrm{dpb}^{\prime}$} \\
\cline { 3 - 10 } & $\Delta \mathrm{E}_{\mathrm{ox}}$ & $\mathrm{E}_{\mathrm{ox} 2}$ & $\mathrm{E}_{\mathrm{ox} 1}$ & $\mathrm{E}_{\mathrm{MLCT}(1)}$ & $\Delta \mathrm{E}_{\mathrm{ox}}$ & $\mathrm{E}_{\mathrm{ox} 2}$ & $\mathrm{E}_{\mathrm{ox} 1}$ & $\begin{array}{c}\mathrm{E}_{\mathrm{MLCT}(1)} \\
/ \mathrm{cm}^{-1}\end{array}$ \\
\hline $\mathrm{Me}_{4}$ bpy & meso & 292 & 1260 & 968 & 14960 & 296 & 1252 & 956 & 13700 \\
& rac & 284 & 1248 & 964 & 14970 & 304 & 1268 & 964 & 13690 \\
\hline Me $_{2}$ bpy & meso & 244 & 1260 & 1016 & 15310 & 268 & 1268 & 1000 & 13810 \\
& rac & 224 & 1256 & 1032 & 15230 & 260 & 1268 & 1008 & 13720 \\
\hline bpy $^{\mathrm{a}}$ & meso & 196 & 1280 & 1084 & 15530 & 232 & 1300 & 1068 & 13880 \\
& rac & 176 & 1280 & 1104 & 15420 & 224 & 1304 & 1080 & 13870 \\
\hline
\end{tabular}

${ }^{\mathrm{a}}$ Data are reported in Table 3.3 and are presented here for comparison.

${ }^{\mathrm{b}} \Delta \mathrm{E}_{\mathrm{ox}}=\mathrm{E}_{\mathrm{ox} 2}-\mathrm{E}_{\mathrm{ox} 1}$. Potentials are quoted $\pm 3 \mathrm{mV}$.

The unsymmetrical complexes are characterised by two reversible one-electron processes corresponding to successive oxidation of the metal centres, in addition to multiple reversible ligand-based reductions. $\mathrm{E}_{\mathrm{ox} 1}$ corresponds to oxidation of the $\mathrm{Ru}(\mathrm{pp})_{2}$ terminus and shifts cathodically as pp is varied through the series bpy, $\mathrm{Me}_{2}$ bpy and $\mathrm{Me}_{4} \mathrm{bpy}$. Small cathodic shifts are also evident in the redox potential of the indirectly perturbed $\mathrm{Ru}(\mathrm{bpy})_{2}$ site (i.e. $\left.\mathrm{E}_{\mathrm{ox} 2}\right)$. The increasing stabilisation of the $\pi^{*}(\mathrm{BL})$ orbitals through the series is also reflected by the cathodic shifts in the first two one-electron reduction processes localised on BL, and the red-shift in $\mathrm{E}_{\mathrm{MLCT}(1)}$. By comparison with the potentials of the bpy-based reductions for $\left[\left\{\mathrm{Ru}(\mathrm{bpy})_{2}\right\}_{2}(\mu-\mathrm{BL})\right]^{n+}\left\{\mathrm{BL}=\mathrm{dpb}, \mathrm{dpb}^{\prime}\right\}$, in the unsymmetrical complexes the subsequent four one-electron redox processes are assigned to the consecutive reductions of bpy and pp ligands attached to different metal centres.

Spectroelectrochemical studies on the unsymmetrical complexes at $-35^{\circ} \mathrm{C}$ revealed stable isosbestic points for the first oxidation process, producing the mixed-valence species. The $\mathrm{d} \pi\left(\mathrm{Ru}^{\mathrm{II}}\right) \rightarrow \pi^{*}(\mathrm{BL})$ MLCT absorptions in the range 13500-15500 $\mathrm{cm}^{-1}$ decreased on one-electron oxidation and collapsed completely on removal of the second electron. The new band in the region 4000-9000 $\mathrm{cm}^{-1}$ was assigned 
as an IVCT transition. The mixed-valence complexes are formally $\left[\left\{\mathrm{Ru}^{\mathrm{II}}(\mathrm{bpy})_{2}\right\}(\mu-\mathrm{BL})\left\{\mathrm{Ru}^{\mathrm{III}}(\mathrm{pp})_{2}\right\}\right]^{5+}$ species $\left\{\mathrm{pp}=\mathrm{Me}_{2}\right.$ bpy and $\mathrm{Me}_{4}$ bpy $\}$, and the IVCT process corresponds to an $\mathrm{Ru}^{\mathrm{II}} \rightarrow \mathrm{Ru}^{\mathrm{III}}$ transition, originating from the un-oxidised $\mathrm{Ru}^{\mathrm{II}}(\mathrm{bpy})_{2}$ terminus.

\subsubsection{Comparisons with the Predictions of the Two-State Classical Model: the "Curtis Analysis"}

The electrochemical and NIR spectroscopic data for the series of unsymmetrical complexes are summarised in Tables 3.8-3.11, in addition to the data for the symmetrical complexes which are provided for comparison. The NIR spectra for the series of complexes are shown in Figure 3.13.

(a)
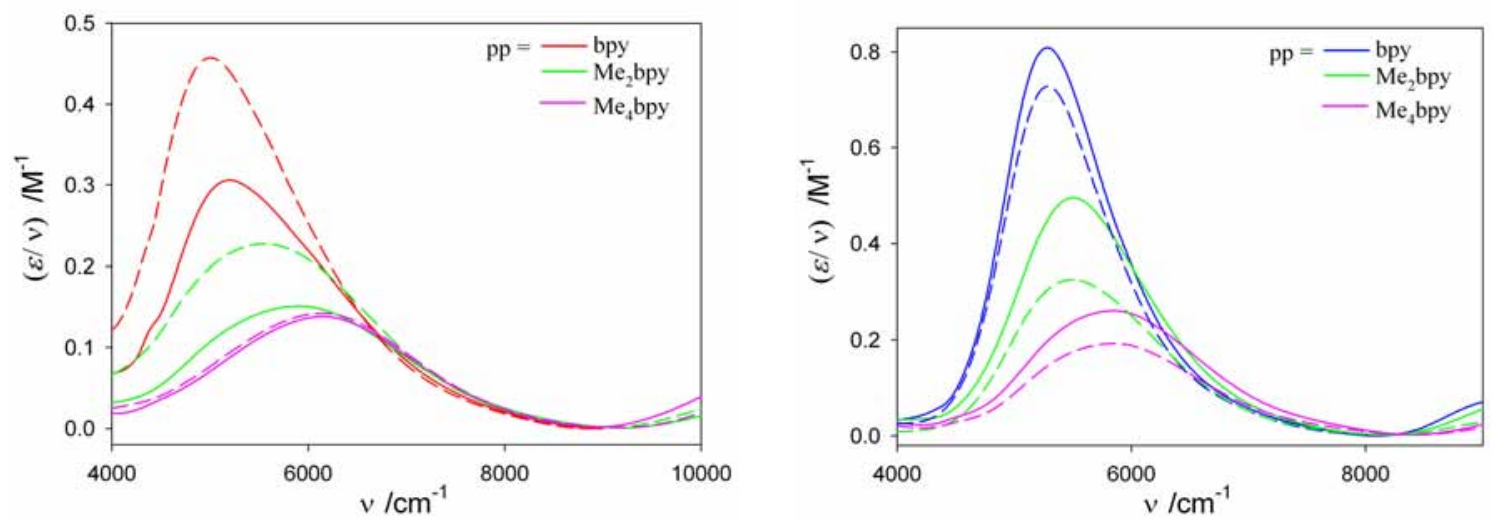

(b)

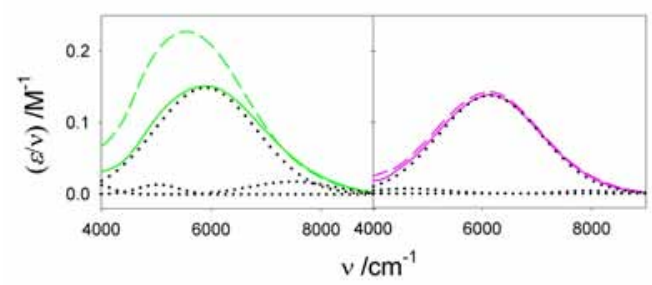

$B L=\mathbf{d p b}$

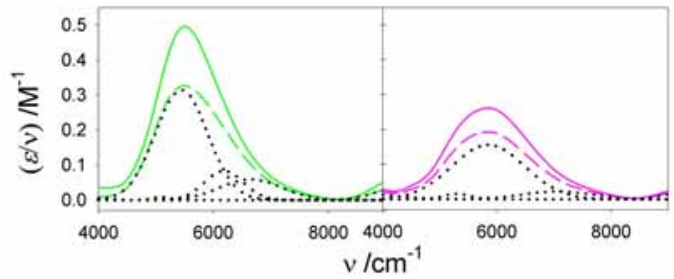

$B L=\mathbf{d p b}^{\prime}$

Figure 3.13 (a) Overlay of the IVCT bands for meso- (solid lines) and $r a c-\left[\left\{\mathrm{Ru}(\mathrm{bpy})_{2}\right\}(\mu-\mathrm{BL})\left\{\mathrm{Ru}(\mathrm{pp})_{2}\right\}\right]^{5+}$ (dashed lines) $\{\mathrm{BL}=\mathrm{dpb}$, dpb' $\}$ in $0.1 \mathrm{M}\left[\left(n-\mathrm{C}_{4} \mathrm{H}_{9}\right)_{4} \mathrm{~N}\right] \mathrm{PF}_{6} / \mathrm{CH}_{3} \mathrm{CN}$ at $-35^{\circ} \mathrm{C}$. (b) Overlays of the IVCT bands for meso- and rac- $\left[\left\{\mathrm{Ru}(\mathrm{bpy})_{2}\right\}(\mu-\mathrm{BL})\left\{\mathrm{Ru}(\mathrm{pp})_{2}\right\}\right]^{5+}$ in addition to the bands obtained from Gaussian deconvolution for the meso diastereoisomers.

On the basis of the electrochemical method reported by Curtis and coworkers, ${ }^{88-90,178}$ the extent of coupling between the metal centres is related to the ratio of the redox perturbation directly induced by ligand substitution at one end of the dimer to that indirectly induced at the other end. For both series of complexes, $\left[\left\{\mathrm{Ru}(\mathrm{bpy})_{2}\right\}(\mu-\mathrm{BL})\left\{\mathrm{Ru}(\mathrm{pp})_{2}\right\}\right]^{4+}\{\mathrm{BL}=\mathrm{dpb}, \mathrm{dpb}\}$, linearity was observed in the plots of $\mathrm{E}_{\mathrm{ox} 1}$ versus $\mathrm{E}_{\mathrm{ox} 2}\left\{\mathrm{pp}=\mathrm{bpy}, \mathrm{Me}_{2} \mathrm{bpy}, \mathrm{Me}_{4} \mathrm{bpy}\right\}$ and the slopes were identical (within experimental error) for the meso and rac diastereoisomers of each series. The slope enables an assignment of $b^{2}$ according to $b^{2}=$ slope/(1+slope), as described in §1.5.3.4. From the slopes, the $\rho^{2}$ and resulting $b^{2}$ were obtained, and are reported in Table 3.12. 
Table 3.8 Summary of the experimental electrochemical and spectral parameters for the diastereoisomers of $\left[\left\{\mathrm{Ru}(\mathrm{bpy})_{2}\right\}(\mu-\mathrm{dpb})\left\{\mathrm{Ru}(\mathrm{pp})_{2}\right\}\right]^{5+}\left\{\mathrm{pp}=\right.$ bpy, $\mathrm{Me}_{2}$ bpy, $\left.\mathrm{Me}_{4} \mathrm{bpy}\right\}$ in $0.1 \mathrm{M}\left[\left(n-\mathrm{C}_{4} \mathrm{H}_{9}\right)_{4} \mathrm{~N}\right] \mathrm{PF}_{6}$ at $-35^{\circ} \mathrm{C}^{\mathrm{a}}$

\begin{tabular}{|c|c|c|c|c|c|c|c|c|c|}
\hline \multirow[b]{2}{*}{$\mathrm{pp}$} & \multirow[b]{2}{*}{ Diastereoisomer } & \multicolumn{5}{|c|}{ Electrochemical Parameters } & \multicolumn{3}{|c|}{ Spectral Parameters } \\
\hline & & $\begin{array}{c}\delta \mathrm{E}_{\mathrm{ox} 1} \\
/ \mathrm{mV}\end{array}$ & $\begin{array}{l}\delta \mathrm{E}_{\mathrm{ox2}} \\
/ \mathrm{mV}\end{array}$ & $m^{\prime} \mathrm{b}$ & $b^{2}$ & $\begin{array}{l}\Delta \mathrm{E}_{0} \\
/ \mathrm{cm}^{-1}\end{array}$ & $\begin{array}{l}v_{\max } \\
/ \mathrm{cm}^{-1}\end{array}$ & $\begin{array}{l}\Delta v_{1 / 2} \\
/ \mathrm{cm}^{-1}\end{array}$ & $\begin{array}{c}v_{\max }-\Delta \mathrm{E}_{0} \\
/ \mathrm{cm}^{-1}\end{array}$ \\
\hline bpy & meso & - & - & - & - & 0 & 5205 & 1910 & 5205 \\
\hline \multirow{2}{*}{$\mathrm{Me}_{2} \mathrm{bpy}$} & meso & $\overline{-68.0}$ & $-\overline{-20.0}$ & $\overline{0.294}$ & $\overline{0.773}$ & 550 & $\frac{5000}{5905}$ & $\frac{1 / 40}{2415}$ & $\frac{5000}{5355}$ \\
\hline & rac & -72.0 & -24.0 & 0.333 & 0.750 & 387 & 5540 & 2345 & 5518 \\
\hline \multirow[t]{2}{*}{$\mathrm{Me}_{4} \mathrm{bpy}$} & meso & -116 & -20.0 & 0.172 & 0.853 & 935 & 6125 & 2330 & 5190 \\
\hline & rac & -140 & -32.0 & 0.229 & 0.814 & 870 & 6130 & 2270 & 5260 \\
\hline
\end{tabular}

${ }^{\mathrm{a}}$ The errors in the observed parameters are $\pm 10 \mathrm{~cm}^{-1}$ for $v_{\max }, \Delta \mathrm{E}_{0}$ and $\Delta v_{1 / 2},{ }^{b} \mathrm{~m}^{\prime}$ is determined as the potential shift ratio $\mathrm{m}^{\prime}=$ $\delta \mathrm{E}_{\mathrm{ox} 2} / \delta \mathrm{E}_{\mathrm{ox} 1}$.

Table 3.9 Comparison of the calculated parameters from the electrochemical and spectral analyses for the diastereoisomers of $\left[\left\{\mathrm{Ru}(\mathrm{bpy})_{2}\right\}(\mu-\mathrm{dpb})\left\{\mathrm{Ru}(\mathrm{pp})_{2}\right\}\right]^{5+}\left\{\mathrm{pp}=\mathrm{bpy}, \mathrm{Me}_{2} \mathrm{bpy}, \mathrm{Me}_{4} \mathrm{bpy}\right\}$. . $^{\mathrm{a}}$

\begin{tabular}{|c|c|c|c|c|c|c|c|c|}
\hline pp & Diastereoisomer & $\begin{array}{c}M_{0} \\
/ \mathrm{M}^{-1}\end{array}$ & $\begin{array}{c}M_{1} \\
/ \mathrm{cm}^{-1}\end{array}$ & $\begin{array}{c}M_{1}-\Delta \mathrm{E}_{0} \\
/ \mathrm{cm}^{-1}\end{array}$ & $\begin{array}{c}\left|\mu_{12}\right| \\
/ \mathrm{e} \AA\end{array}$ & $\Gamma$ & $\begin{array}{c}\Delta v_{1 / 2}{ }^{\circ} \\
/ \mathrm{cm}^{-1}\end{array}$ & $\begin{array}{c}\mathrm{H}_{\mathrm{ab}}{ }^{\mathrm{a}} \\
/ \mathrm{cm}^{-1}\end{array}$ \\
\hline bpy & meso & 639.8 & 5655 & 5655 & 0.5211 & 0.382 & 3090 & 395 \\
& rac & 870.5 & 5450 & 5450 & 0.6078 & 0.426 & 3030 & 440 \\
\hline $\mathrm{Me}_{2}$ bpy & meso & 386.2 & 6020 & 5470 & 0.4048 & 0.230 & 3135 & 315 \\
& rac & 559.6 & 5780 & 5392 & 0.4873 & 0.263 & 3180 & 390 \\
\hline $\mathrm{Me}_{4}$ bpy & meso & 331.7 & 6150 & 5210 & 0.3752 & 0.245 & 3085 & 280 \\
& rac & 350.0 & 6125 & 5250 & 0.3854 & 0.270 & 3110 & 295 \\
\hline
\end{tabular}

${ }^{\mathrm{a}}$ The errors in the observed parameters are $\pm 10 \mathrm{~cm}^{-1}$ for $M_{1}, \pm 5 \mathrm{M}^{-1}$ for $M_{0}$ and $\pm 0.0001 \mathrm{e} \AA$ for $\left|\mu_{12}\right| ;{ }^{\mathrm{b}} \mathrm{H}_{\mathrm{ab}}$ estimated from equation 3.2 assuming $r_{\mathrm{ab}}$ is 6.870(3) $\AA$ for both diastereoisomers of $\left[\left\{\mathrm{Ru}(\mathrm{bpy})_{2}\right\}_{2}(\mu-\mathrm{dpb})\right]^{5+}$ \{the average of the $\mathrm{Ru} \cdots \mathrm{Ru}$ distances from the crystal structure determinations of the meso diastereoisomer with the $\mathrm{PF}_{6}^{-}$and $\mathrm{ZnCl}_{4}{ }^{2-}$ counter-ions .

Table 3.10 Summary of the experimental electrochemical and spectral parameters for the diastereoisomers of $\left[\left\{\mathrm{Ru}(\mathrm{bpy})_{2}\right\}\left(\mu-\mathrm{dpb}^{\prime}\right)\left\{\mathrm{Ru}(\mathrm{pp})_{2}\right\}\right]^{5+}\left\{\mathrm{pp}=\right.$ bpy, $\mathrm{Me}_{2}$ bpy, $\mathrm{Me}_{4}$ bpy $\}$ in $0.1 \mathrm{M}\left[\left(n-\mathrm{C}_{4} \mathrm{H}_{9}\right)_{4} \mathrm{~N}\right] \mathrm{PF}_{6}$ at $-35^{\circ} \mathrm{C}^{\text {a }}$

\begin{tabular}{|c|c|c|c|c|c|c|c|c|c|}
\hline \multirow{2}{*}{ pp } & \multirow{2}{*}{ Diastereoisomer } & \multicolumn{4}{|c|}{ Electrochemical Parameters } & \multicolumn{3}{c|}{ Spectral Parameters } \\
\cline { 3 - 10 } & $\begin{array}{c}\delta \mathrm{E}_{\mathrm{ox} 1} \\
/ \mathrm{mV}\end{array}$ & $\begin{array}{c}\delta \mathrm{E}_{\mathrm{ox2}} \\
/ \mathrm{mV}\end{array}$ & $m^{\prime \mathrm{b}}$ & $b^{2}$ & $\begin{array}{c}\Delta \mathrm{E}_{0} \\
/ \mathrm{cm}^{-1}\end{array}$ & $\begin{array}{c}v_{\max } \\
/ \mathrm{cm}^{-1}\end{array}$ & $\begin{array}{c}\Delta v_{1 / 2} \\
/ \mathrm{cm}^{-1}\end{array}$ & $\begin{array}{c}v_{\max }-\Delta \mathrm{E}_{0} \\
/ \mathrm{cm}^{-1}\end{array}$ \\
\hline bpy & meso & - & - & - & - & 0 & 5285 & 1070 & 5285 \\
& rac & - & - & - & - & 0 & 5290 & 1080 & 5290 \\
\hline $\mathrm{Me}_{2} \mathrm{bpy}$ & meso & -68.0 & -32.0 & 0.471 & 0.680 & 290 & 5500 & 1415 & 5210 \\
& rac & -72.0 & -36.0 & 0.500 & 0.667 & 290 & 5420 & 1265 & 5130 \\
\hline $\mathrm{Me}_{4} \mathrm{bpy}$ & meso & -112 & -48.0 & 0.429 & 0.700 & 516 & 5810 & 1675 & 5290 \\
& rac & -116 & -116 & 0.310 & 0.763 & 645 & 5800 & 1870 & 5155 \\
\hline
\end{tabular}

${ }^{\mathrm{a}}$ The errors in the observed parameters are $\pm 10 \mathrm{~cm}^{-1}$ for $v_{\max }, \Delta \mathrm{E}_{0}$ and $\Delta v_{1 / 2} ;{ }^{b} \mathrm{~m}^{\prime}$ is determined as the potential shift ratio $\mathrm{m}^{\prime}=$ $\delta \mathrm{E}_{\mathrm{ox} 2} / \delta \mathrm{E}_{\mathrm{ox} 1}$.

Table 3.11 Comparison of the calculated parameters from the electrochemical and spectral analyses on the diastereoisomers of $\left[\left\{\mathrm{Ru}(\mathrm{bpy})_{2}\right\}\left(\mu-\mathrm{dpb}^{\prime}\right)\left\{\mathrm{Ru}(\mathrm{pp})_{2}\right\}\right]^{5+}\left\{\mathrm{pp}=\text { bpy, } \mathrm{Me}_{2} \text { bpy, } \mathrm{Me}_{4} \mathrm{bpy}\right\}^{\mathrm{a}}$

\begin{tabular}{|c|c|c|c|c|c|c|c|c|}
\hline pp & Diastereoisomer & $\begin{array}{c}M_{0} \\
/ \mathrm{M}^{-1}\end{array}$ & $\begin{array}{c}M_{1} \\
/ \mathrm{cm}^{-1}\end{array}$ & $\begin{array}{c}M_{1}-\Delta \mathrm{E}_{0} \\
/ \mathrm{cm}^{-1}\end{array}$ & $\begin{array}{c}\left|\mu_{12}\right| \\
/ \mathrm{e} \AA\end{array}$ & $\Gamma$ & $\begin{array}{c}\Delta \mathrm{v}_{1 / 2}{ }^{\circ} \\
/ \mathrm{cm}^{-1}\end{array}$ & $\begin{array}{c}\mathrm{H}_{\mathrm{ab}}{ }^{\mathrm{a}} \\
/ \mathrm{cm}^{-1}\end{array}$ \\
\hline bpy & meso & 1010 & 5510 & 5510 & 0.6546 & 0.657 & 3115 & 500 \\
& rac & 903.2 & 5510 & 5510 & 0.6190 & 0.653 & 3115 & 475 \\
\hline $\mathrm{Me}_{2}$ bpy & meso & 512.1 & 5725 & 5435 & 0.4662 & 0.542 & 3090 & 350 \\
& rac & 735.8 & 7570 & 7275 & 0.5588 & 0.588 & 3070 & 415 \\
\hline $\mathrm{Me}_{4}$ bpy & meso & 484.2 & 8640 & 8125 & 0.4524 & 0.462 & 3115 & 350 \\
& rac & 357.0 & 5930 & 5282 & 0.3892 & 0.392 & 3075 & 290 \\
\hline
\end{tabular}

${ }^{\mathrm{a}}$ The errors in the observed parameters are $\pm 10 \mathrm{~cm}^{-1}$ for $M_{1}, \pm 5 \mathrm{M}^{-1}$ for $M_{0}$ and $\pm 0.0001 \mathrm{e} \AA$ for $\left|\mu_{12}\right| ;{ }^{\mathrm{b}} \mathrm{H}_{\mathrm{ab}}$ estimated from equation 3.2 assuming $r_{\mathrm{ab}}$ is 6.887(1) $\AA$ for both diastereoisomers of $\left[\left\{\mathrm{Ru}(\mathrm{bpy})_{2}\right\}_{2}\left(\mu-\mathrm{dpb}^{\prime}\right)\right]^{5+}$ the $\mathrm{Ru} \cdots \mathrm{Ru}$ distance from the crystal structure determination of meso- $\left.\left[\left\{\mathrm{Ru}(\mathrm{bpy})_{2}\right\}_{2}\left(\mu-\mathrm{dpb}^{\prime}\right)\right]\left(\mathrm{PF}_{6}\right)_{4}\right\}$. 
Table 3.12 Comparison of the calculated parameters from the electrochemical and spectral analyses on the diastereoisomeric forms of $\left[\left\{\mathrm{Ru}(\mathrm{bpy})_{2}\right\}_{2}(\mu-\mathrm{BL})\right]^{5+}\{\mathrm{BL}=\mathrm{dpb}, \mathrm{dpb}\}$.

\begin{tabular}{|l|c|c|c|c|}
\hline \multicolumn{1}{|c|}{ Complex } & $\rho^{2}$ & $\rho$ & $b^{2}$ & $\Delta q$ \\
\hline meso- $\left[\left\{\mathrm{Ru}(\mathrm{bpy})_{2}\right\}_{2}(\mu-\mathrm{dpb})\right]^{5+}$ & $0.181 \pm 0.081$ & $0.425 \pm 0.190$ & $0.153 \pm 0.097$ & 0.659 \\
rac- $\left[\left\{\mathrm{Ru}(\mathrm{bpy})_{2}\right\}_{2}(\mu \text {-dpb) }]^{5+}\right.$ & $0.230 \pm 0.062$ & $0.479 \pm 0.129$ & $0.187 \pm 0.071$ & 0.626 \\
\hline meso- $\left[\left\{\mathrm{Ru}(\mathrm{bpy})_{2}\right\}_{2}\left(\mu-\mathrm{dpb}^{\prime}\right)\right]^{5+}$ & $0.432 \pm 0.029$ & $0.657 \pm 0.044$ & $0.302 \pm 0.029$ & 0.396 \\
rac- $\left[\left\{\mathrm{Ru}(\mathrm{bpy})_{2}\right\}_{2}(\mu-\mathrm{dpb})\right]^{5+}$ & $0.329 \pm 0.013$ & $0.574 \pm 0.023$ & $0.248 \pm 0.014$ & 0.504 \\
\hline
\end{tabular}

Physically, the $b^{2}$ parameter quantifies the fraction of a valence electronic charge that has been transferred from the donor to the acceptor metal centre in the electronic ground state, and represents the difference between the minima of the diabatic and adiabatic surfaces in the two-state model depicted in Figure 3.14. The results of the electrochemical analysis suggest that the degree of valence delocalisation in the ground state is greater for the dpb'-bridged diastereoisomers relative to their dpb-bridged analogues. In addition, the degree of delocalisation is greater for meso- $\left[\left\{\mathrm{Ru}(\mathrm{bpy})_{2}\right\}_{2}\left(\mu-\mathrm{dpb}^{\prime}\right)\right]^{5+}$ and $\mathrm{rac}-$ $\left[\left\{\mathrm{Ru}(\mathrm{bpy})_{2}\right\}_{2}(\mu-\mathrm{dpb})\right]^{5+}$ relative to their corresponding diastereoisomeric forms. $\rho$ represents the ratio of the coefficients for the diabatic wavefunctions which is unity when mixing is complete, 0.5 at the transition between the localised and delocalised regimes and zero in the absence of mixing. The magnitudes of $\rho$ in Table 3.12 support the classification of the diastereoisomers as borderline localised/delocalised systems.

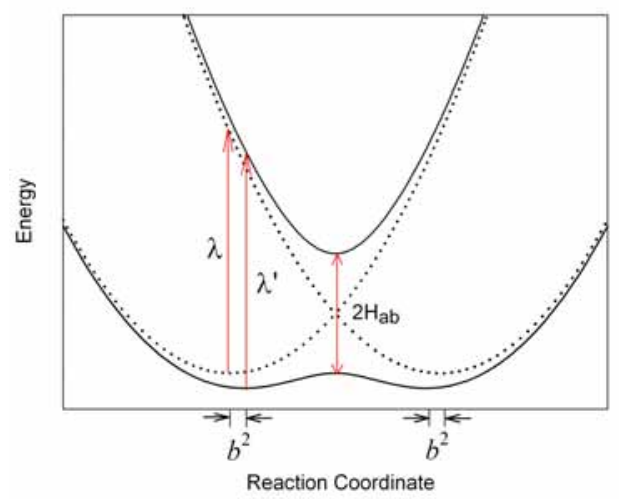

Figure 3.14 Potential energy surfaces for a symmetrical dinuclear complex. $\lambda$ and $\lambda^{\prime}$ denote the diabatic and adiabatic reorganisational energies, respectively.

According to the classical two-site model, $\mathrm{H}_{\mathrm{ab}}$ should remain constant for each series of complexes involving the dpb and dpb' bridging ligands. If this holds, the actual amount of charge transferred in the IVCT process, $\Delta q$ is given by $1-2 b^{2}$ (Table 3.12), and the adiabatic reorganisational energy $\left(\lambda^{\prime}\right)$ is a fraction only of the diabatic reorganisational energy $(\lambda)$ \{equation 3.7$\} .^{40}$

$$
\lambda^{\prime}=\lambda\left(1-2 b^{2}\right)^{2}=\lambda(\Delta q)^{2}
$$

From Table 3.12, it is evident that the actual amount of charge transferred is significantly less than a unit electronic charge $(e)$. The adiabatic $\left(r_{12}\right)$ and diabatic $\left(r_{\mathrm{ab}}\right)$ charge transfer distances are related by equation 1.31 . The difference between these distances illustrates that partial charge delocalisation decreases the effective charge transfer distance relative to the geometrical through-space distance between 
the metal centres. As a consequence, the value of $\mathrm{H}_{\mathrm{ab}}$ determined from equation 3.2 represents a lower limit only. ${ }^{14}$ A more accurate estimate of $\mathrm{H}_{\mathrm{ab}}$ may be obtained by using the effective charge transfer distance in the calculation, which results in an upwards revision of the values reported in Tables 3.9 and 3.11 to 820 and $1000 \mathrm{~cm}^{-1}$ for meso- and rac-[\{Ru(bpy)$\left.\left.\}_{2}\right\}_{2}(\mu-\mathrm{dpb})\right]^{5+}$ and 3180 and $1870 \mathrm{~cm}^{-1}$ for mesoand rac- $\left[\left\{\mathrm{Ru}(\mathrm{bpy})_{2}\right\}_{2}(\mu-\mathrm{dpb})\right]^{5+}$, respectively. These values are closer to those expected in the delocalised limit, where $\mathrm{H}_{\mathrm{ab}}$ is estimated as 2600 and $2500 \mathrm{~cm}^{-1}$ for meso- and rac- $\left[\left\{\mathrm{Ru}(\mathrm{bpy})_{2}\right\}_{2}(\mu-\right.$ $\left.\left.\mathrm{dpb}^{\prime}\right)\right]^{5+}$, and 2640 and $2645 \mathrm{~cm}^{-1}$ for meso- and rac-[\{Ru(bpy $\left.\left.)_{2}\right\}_{2}(\mu-\mathrm{dpb})\right]^{5+}$, respectively, from equation 1.11 .

The results from the electrochemical analysis indicate that the dpb'-bridged diastereoisomers exhibit a greater degree of delocalisation relative to their dpb-bridged analogues, and the degree of delocalisation differs between the diastereoisomeric forms of the same species. While the electrochemical method has been shown to provide reasonable measures of the trends in the degree of delocalisation (on the basis of the parameter $b^{2}$ ), the method is based upon the two-site model and is valid only in the limit of small overlap. As a result, the method may not yield reliable quantitative measures of the degree of delocalisation for the present systems. In addition, differential ion-pairing and solvent interactions between the diastereoisomeric forms of the same complex may confound the results.

\subsubsection{Comparisons with IVCT Spectroscopic Parameters}

A linear correlation is observed between $v_{\max }$ and $\Delta \mathrm{E}_{\mathrm{ox}}$ for the diastereoisomers of $\left[\left\{\mathrm{Ru}(\mathrm{bpy})_{2}\right\}(\mu-\mathrm{BL})\left\{\mathrm{Ru}(\mathrm{pp})_{2}\right\}\right]^{5+}\left\{\mathrm{BL}=\mathrm{dpb}, \mathrm{dpb} ;\right.$; pp = bpy, $\mathrm{Me}_{2}$ bpy, $\left.\mathrm{Me}_{4} \mathrm{bpy}\right\}$, which indicates that the peripheral ligand variation serves predominantly to change the redox asymmetry contribution $\left(\Delta \mathrm{E}_{0}\right)$ to the IVCT process. ${ }^{88-90,178}$ As shown in Figure 3.13, the introduction of $\mathrm{Me}_{2}$ bpy and $\mathrm{Me}_{4}$ bpy terminal ligands leads to increasingly localised IVCT behaviour, and the appearance of more Gaussian-shaped IVCT bands. The transition is accompanied by an increase in $v_{\max }$ and $\Delta v_{1 / 2}$, and a decrease in $(\varepsilon / v)_{\max }$ and $M_{0}$ for the IVCT band as pp is varied through the series bpy, $\mathrm{Me}_{2} \mathrm{bpy}$ and $\mathrm{Me}_{4} \mathrm{bpy}$. In addition, the observed bandwidth $\left(\Delta v_{1 / 2}\right)$ approaches the value expected in the localised limit $\left(\Delta v_{1 / 2}{ }^{\circ}\right.$, equation 1.8).

For the diastereoisomers incorporating the dpb' bridging ligand, the $v_{\max }$ and $\Delta v_{1 / 2}$ values are comparable for the two diastereoisomeric forms over the series, and $(\varepsilon / v)_{\max }$ and $M_{0}$ are consistently greater for the meso versus the rac diastereoisomer (Table 3.11). The variation in the qualitative appearance of the IVCT bands also signals the transition from the delocalised to localised regimes. The asymmetric appearance of the IVCT bands for the symmetrical complex was previously rationalised by the contributions from multiple underlying Gaussian-shaped components in the deconvolution procedure. For the unsymmetrical complex $\left[\left\{\mathrm{Ru}(\mathrm{bpy})_{2}\right\}\left(\mu-\mathrm{dpb}^{\prime}\right)\left\{\mathrm{Ru}\left(\mathrm{Me}_{2} \mathrm{bpy}\right)_{2}\right\}\right]^{5+}$, the IVCT band is also skewed towards higher energies. By comparison, the IVCT line-shape for $\left[\left\{\mathrm{Ru}(\mathrm{bpy})_{2}\right\}(\mu-\mathrm{dpb})\left\{\mathrm{Ru}\left(\mathrm{Me}_{4} \mathrm{bpy}\right)_{2}\right\}\right]^{5+}$ is approximated by a single Gaussian band. The additional minor components are required to reproduce 
the line-shape at the "tails" of the IVCT manifolds". The results for the band parameters obtained from the Gaussian deconvolution procedure are reported in Tables B3.13-14 (Appendix B).

The IVCT properties for the series of complexes bridged by dpb follow similar trends to their dpb'-bridged analogues. Qualitatively, the increase in the degree of localisation as pp is varied through the series bpy, $\mathrm{Me}_{2}$ bpy and $\mathrm{Me}_{4}$ bpy is reflected by the increasingly Gaussian-shaped appearance of the IVCT bands. While the $v_{\max }$ and $M_{0}$ values for the diastereoisomers of meso- $\left[\left\{\mathrm{Ru}(\mathrm{bpy})_{2}\right\}(\mu-\right.$ $\left.\mathrm{dpb})\left\{\mathrm{Ru}(\mathrm{pp})_{2}\right\}\right]^{5+}\left\{\mathrm{pp}=\right.$ bpy, $\left.\mathrm{Me}_{2} \mathrm{bpy}\right\}$ are greater than those of the corresponding rac forms, the IVCT band parameters for both diastereoisomers of $\left[\left\{\mathrm{Ru}(\mathrm{bpy})_{2}\right\}\left(\mu \text {-dpb) }\left\{\mathrm{Ru}\left(\mathrm{Me}_{4} \mathrm{bpy}\right)_{2}\right\}\right]^{5+}\right.$ are almost identical. The results suggest that differential stereochemically-induced structural distortions $\left(\Delta \mathrm{E}_{\text {struct }}\right)$ exist between the diastereoisomeric forms, in addition to the induced redox asymmetry contribution $\left(\Delta \mathrm{E}_{0}\right) . \Delta \mathrm{E}_{\text {struct }}$ varies over the series complexes and between the diastereoisomers of the same complex, due to differential bridging ligand distortion.

The trends in the IVCT band parameters ( $v_{\max }, \Delta v_{1 / 2}$ and $\left.M_{1}\right)$ as a function of $\Delta \mathrm{E}_{0}$ for the series of complexes are shown in Figure B3.3 (Appendix B). Estimates for the redox asymmetry contribution (reported in Tables 3.8 and 3.10) were obtained from the electrochemical measurements, as the difference between the potentials of the first metal-based oxidation process in a given diastereoisomer of the unsymmetrical complexes compared with the symmetrical analogue. These $\Delta \mathrm{E}_{0}$ estimates were determined according to the method of Curtis and coworkers ${ }^{179}$ and represent the combination of the induced redox asymmetry due to peripheral ligand variation, the structurally-induced redox asymmetry, and additional sources of splitting between the redox processes due to resonance stabilisation and differential solvation energies. ${ }^{83,88,153}$

The trends in the $\Gamma$ parameters ${ }^{38}$ (equation 1.21) reported in Tables 3.9 and 3.11 provide a further measure of redox asymmetry contributions to the line-width. The decrease in $\Gamma$ as $p p$ is varied through the series bpy, $\mathrm{Me}_{2}$ bpy and $\mathrm{Me}_{4}$ bpy demonstrates that the introduction of a relatively small redox perturbation results in a dramatic increase in the bandwidth. However, the $\Gamma$ values remain appreciable for the complexes incorporating $\mathrm{Me}_{4} \mathrm{bpy}$ ligands, which indicate that the corresponding $\Delta \mathrm{E}_{0}$ contributions are not sufficient to induce complete localisation.

\subsubsection{Theoretical Implications and Considerations}

The trends in the spectral parameters obtained from the classical analysis reveal that the introduction of redox asymmetry has profound effects: the IVCT bands shift to higher energies, broaden and decrease in intensity. The onset of localisation is accompanied by a significant decrease in $\mathrm{H}_{\mathrm{ab}}$, and an increase in $\lambda .^{38}$ Based on classical theory, both quantities should remain constant for a given series of complexes, since the expectation is that a variation in the redox asymmetry should be manifested spectroscopically as an equivalent variation in the energy of the IVCT band (equation 3.1) at constant $\mathrm{H}_{\mathrm{ab}}$

\footnotetext{
* While the IVCT manifolds were adequately fitted using a single Lorentzian band, the results of the deconvolution procedure are reported for fits using purely Gaussian-shaped bands.
} 
and $\lambda .^{38}$ In the present case, the variations in these quantities across the series suggest that different diabatic descriptions exist for each of the complexes. In addition, the results indicate that a classical twostate description is inappropriate, and electronic delocalisation is governed by superexchange effects rather than direct donor-acceptor orbital overlap. For the dpb-bridged diastereoisomers, the increase in $\Delta \mathrm{E}_{0}$ is also accompanied by a fundamental change in electronic structure, due to the contribution of $\Delta \mathrm{E}_{\text {struct. }}$ Clearly, the development of an adequate theory to describe the transition between the localised and delocalised regimes for the full series of complexes requires a model in which $\mathrm{H}_{\mathrm{ab}}$ and $\lambda$ are allowed to vary with $\Delta \mathrm{E}_{0}$.

There is significant current interest in the development of theoretical models for the localised-todelocalised transition which accurately model redox asymmetry effects on the IVCT line-shape. Reimers and $\mathrm{Hush}^{48}$ have postulated a theoretical model incorporating both symmetric and anti-symmetric modes, to model to the series of unsymmetrical analogues of the Creutz-Taube ion, trans- $\left[\left\{\mathrm{Ru}\left(\mathrm{NH}_{3}\right)_{5}\right\}(\mu-\right.$ pyz) $\left.\left\{\mathrm{Ru}\left(\mathrm{NH}_{3}\right)_{4}(\mathrm{~L})\right\}\right]^{5+}\left\{\mathrm{L}=\mathrm{NH}_{3}\right.$ (the Creutz-Taube ion itself), py, 3,5-Me $2 \mathrm{py}, 3-\mathrm{Cl}-\mathrm{py}, 2,6-\mathrm{Me}_{2} \mathrm{pyz}$; shown in Figure 1.6(a)\}. While the trends in the calculated parameters and the experimental data were in reasonable qualitative agreement, it was evident that a more sophisticated model was required in which $\mathrm{H}_{\mathrm{ab}}$ and $\lambda$ were permitted to vary with $\Delta \mathrm{E}_{0}$. The results for the present series of complexes support this contention, and provide an experimental basis for testing the validity of future theoretical models which incorporate redox asymmetry effects.

\subsubsection{Dinuclear Osmium Complexes as Probes for Spin-Orbit Coupling Contributions}

\subsubsection{Structural Considerations: X-ray Crystallography}

Meso- $(\Lambda \Delta)-\left[\left\{\mathrm{Os}(\text { bpy })_{2}\right\}_{2}(\mu-\mathrm{dpb})\right]\left(\mathrm{ZnCl}_{4}\right)_{2} \cdot 6 \mathrm{H}_{2} \mathrm{O}$ crystallised in the monoclinic space group $P 2_{1} / c$ with four dinuclear cations in the unit cell and meso- $(\Lambda \Delta)-\left[\left\{\mathrm{Os}(\mathrm{bpy})_{2}\right\}_{2}\left(\mu-\mathrm{dpq}^{\prime}\right)\right]\left(\mathrm{PF}_{6}\right)_{4}$ crystallised in the orthorhombic space group Pbca with eight dinuclear cations in the unit cell. The perspective views of the dinuclear cations are shown in Figures 3.15(a) and (b), and the crystal structure and refinement details are provided in Table B3.15 (Appendix B).

The nature of the distortions in the crystal structures of meso- $(\Lambda \Delta)-\left[\left\{\mathrm{Os}(\mathrm{bpy})_{2}\right\}_{2}(\mu-\mathrm{dpb})\right]^{4+}$ and meso- $(\Lambda \Delta)-\left[\left\{\mathrm{Os}(\mathrm{bpy})_{2}\right\}_{2}(\mu-\mathrm{dpq})\right]^{4+}$ are comparable with those observed in their ruthenium analogues (§3.3.2.1). For meso- $(\Lambda \Delta)-\left[\left\{\mathrm{Os}(\mathrm{bpy})_{2}\right\}_{2}(\mu-\mathrm{dpb})\right]^{4+}$ shown in Figure 3.15(a), steric hindrance between the H13 and H14 protons (Figure B3.1, Appendix B) encourages the two pyridyl rings to twist out of the central plane. The dpb bridging ligand exhibits considerable distortion in the central pyrazine ring, in addition to a large dihedral skew of the two pyridyl ligands and a twist in the benzoquinoxaline "tail" of the bridge. In meso- $(\Lambda \Delta)-\left[\left\{\mathrm{Os}(\mathrm{bpy})_{2}\right\}_{2}(\mu-\mathrm{dpq})\right]^{4+}$, the C-C bond linking the two pyridyl rings of the bridging ligand in dpq' prevents such lateral movements. The slight curvature of the bridging ligand shown in Figure 3.15(b) arises from unfavourable steric interactions between the equatorial hydrogen 
atoms on the phenazine "tail" of the bridging ligand and the terminal bpy rings oriented parallel to the long axis of the bridge.

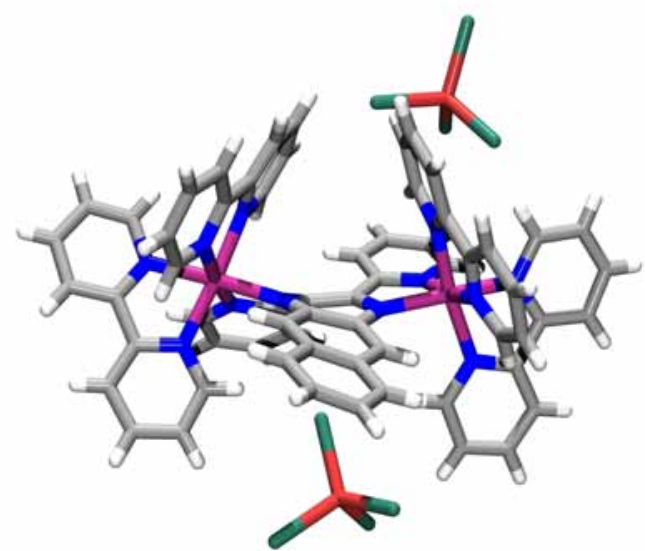

(a)

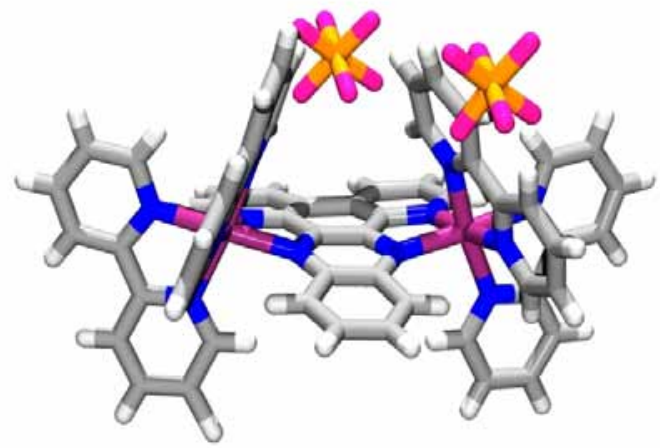

(b)

Figure 3.15 X-ray crystal structures of the cations in (a) meso- $(\Lambda \Delta)-\left[\left\{\mathrm{Os}(\mathrm{bpy})_{2}\right\}_{2}(\mu\right.$-dpb) $]\left(\mathrm{ZnCl}_{4}\right)_{2} \cdot 6 \mathrm{H}_{2} \mathrm{O}$ showing the relative positions of the $\left[\mathrm{ZnCl}_{4}\right]^{2-}$ anions, and (b) meso- $(\Lambda \Delta)-\left[\left\{\mathrm{Os}(\mathrm{bpy})_{2}\right\}_{2}(\mu-\mathrm{dpq})\right]\left(\mathrm{PF}_{6}\right)_{4}$ showing the relative positions of two $\mathrm{PF}_{6}^{-}$anions.

In both structures, the metal centres reside in distorted octahedral environments and the degree of structural distortion is more pronounced for the dpb-bridged complex. The average "bite-angles" subtended by the Os centres and nitrogen atoms of the bridging ligand are 77.3(3) and 79.2(3) ${ }^{\circ}$ for the dpb- and dpq'-bridged structures, respectively, consistent with the results for their ruthenium analogues and with those published previously for polypyridyl complexes of osmium. ${ }^{128,145-150}$ For the dpb-bridged structure, the average Os-N(pyrazine) and Os-N(pyridine) bond-lengths are 2.052(5) and 2.020(5) $\AA$, while the average bond-lengths are 2.072(5) and 2.063(5) $\AA$, respectively, for the dpq'-bridged structure. The slight shortening of the Os- $\mathrm{N}$ distances compared with the $\mathrm{Ru}-\mathrm{N}$ bond-lengths in their ruthenium analogues reflects the enhanced $\pi$-backbonding interactions for Os(II) compared with Ru(II). Such observations have been noted previously in the comparison of the isostructural ruthenium and osmium derivatives of the fully-oxidised forms of the Creutz-Taube ion, $\left[\left\{\mathrm{Os}\left(\mathrm{NH}_{3}\right)_{5}\right\}_{2}(\mu\right.$-pyz) $] \mathrm{Cl}_{6} \cdot 2 \mathrm{H}_{2} \mathrm{O}{ }^{180}$ In the

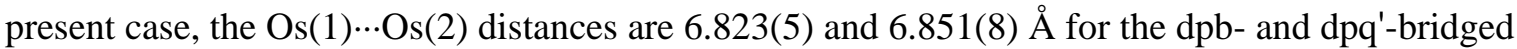
structures, respectively, which are comparable to the distances 6.856(2) and 6.818(1) $\AA$ in the crystal structures of meso- $\left[\left\{\mathrm{Ru}(\mathrm{bpy})_{2}\right\}_{2}(\mu-\mathrm{dpb})\right]\left(\mathrm{ZnCl}_{4}\right)_{2} \cdot 5 \mathrm{H}_{2} \mathrm{O}$ and meso-[ $\left.\left\{\mathrm{Ru}(\mathrm{bpy})_{2}\right\}_{2}\left(\mu-\mathrm{dpq} \mathrm{q}^{\prime}\right)\right]\left(\mathrm{ZnCl}_{4}\right)_{2} \cdot 3 \mathrm{H}_{2} \mathrm{O}$, respectively (§3.3.2.1).

The proximities of the counter-ions to the dinuclear cations are also depicted in Figures 3.15(a) and (b). In meso- $(\Lambda \Delta)-\left[\left\{\mathrm{Os}(\mathrm{bpy})_{2}\right\}_{2}(\mu-\mathrm{dpb})\right]\left(\mathrm{ZnCl}_{4}\right)_{2} \cdot 6 \mathrm{H}_{2} \mathrm{O}$, one $\left[\mathrm{ZnCl}_{4}\right]^{2-}$ counter-anion resides in the cleft between the terminal bpy ligands "below" the plane of the bridging ligand, while the additional $\left[\mathrm{ZnCl}_{4}\right]^{2-}$ ion and six lattice water molecules are located in the exterior clefts. Two $\mathrm{PF}_{6}{ }^{-}$counter-ions in meso- $(\Lambda \Delta)$-[ $\left.\left.[\text { Os(bpy })_{2}\right\}_{2}\left(\mu-\mathrm{dpq}^{\prime}\right)\right]\left(\mathrm{PF}_{6}\right)_{4}$ are in close proximity to the interior cleft "above" the plane of the bridging ligand, while the additional two $\mathrm{PF}_{6}{ }^{-}$counter-ions reside in layers between the cations. 


\subsubsection{Electrochemical and UV/Vis/NIR Spectral Characterisation}

The electrochemical and spectroelectrochemical properties of the diastereoisomeric forms of $\left[\left\{\mathrm{Os}(\mathrm{bpy})_{2}\right\}_{2}(\mu-\mathrm{BL})\right]^{4+}\{\mathrm{BL}=\mathrm{dpb}, \mathrm{dpb}$ ', dpq', ppz and 2,3-dpp $\}$ were investigated by cyclic and differential pulse voltammetry in acetonitrile containing $0.1 \mathrm{M}\left[\left(n-\mathrm{C}_{4} \mathrm{H}_{9}\right)_{4} \mathrm{~N}\right] \mathrm{PF}_{6}$, and are reported in Table 3.13. The full details of the electrochemical properties and the complete UV/Vis/NIR spectral data for the diastereoisomeric forms of $\left[\left\{\mathrm{OS}(\mathrm{bpy})_{2}\right\}_{2}(\mu-\mathrm{BL})\right]^{n+}(n=4,5,6)$ are reported in Tables B3.16 and B3.17 (Appendix B), respectively. The redox and spectral properties for the complexes $\left[\left\{\mathrm{Os}(\mathrm{bpy})_{2}\right\}_{2}(\mu-\right.$ $\mathrm{BL})]^{4+}\{\mathrm{BL}=\mathrm{dpb}$ and 2,3-dpp $\}$ as diastereoisomeric mixtures have been described previously. ${ }^{72,139,140,152}$

Table 3.13 Electrochemical data (in $\mathrm{mV}$ relative to the $\mathrm{Fc}^{+} / \mathrm{Fc}^{0}$ couple) and $\mathrm{K}_{\mathrm{c}}$ values ${ }^{\mathrm{a}}$ for the diastereoisomers of $\left[\left\{\mathrm{Os}(\mathrm{bpy})_{2}\right\}_{2}(\mu-\mathrm{BL})\right]^{4+}$ in $0.1 \mathrm{M}\left[\left(n-\mathrm{C}_{4} \mathrm{H}_{9}\right)_{4} \mathrm{~N}\right] \mathrm{PF}_{6} / \mathrm{CH}_{3} \mathrm{CN}$. ${ }^{\mathrm{b}}$ The energies $(v)$ and intensities $\{(\varepsilon / v)\}$ for the ${ }^{3} \mathrm{MLCT}$ and ${ }^{1}$ MLCT transitions in the +4 states $\left(\right.$ at $\left.-35^{\circ} \mathrm{C}\right)$ are also provided. ${ }^{\mathrm{C}}$

\begin{tabular}{|c|c|c|c|c|c|c|c|c|}
\hline BL & Diastereoisomer & $\begin{array}{c}\mathrm{K}_{\mathrm{c}} \\
\left(\times 10^{-3}\right)\end{array}$ & $\Delta \mathrm{E}_{\mathrm{ox}}$ & $\mathrm{E}_{\mathrm{ox} 2}$ & $\mathrm{E}_{\mathrm{ox} 1}$ & $\mathrm{E}_{\mathrm{red} 1}$ & $\begin{array}{c}v / \mathrm{cm}^{-1} \\
\left\{(\varepsilon / \mathrm{v}) / \mathrm{M}^{-1}\right\} \\
{ }^{\mathrm{MLCT}}\end{array}$ & $\begin{array}{c}v / \mathrm{cm}^{-1} \\
\left\{(\varepsilon / \mathrm{v}) / \mathrm{M}^{-1}\right\} \\
{ }^{1} \mathrm{MLCT}\end{array}$ \\
\hline $\mathrm{dpb}$ & meso & 256 & 320 & 956 & 636 & -664 & $9820(0.9398)$ & $14880(1.578)$ \\
& rac & 86.2 & 292 & 944 & 652 & -668 & $9780(0.8655)$ & $14840(1.592)$ \\
\hline $\mathrm{dpb}^{\prime}$ & meso & 3616 & 388 & 988 & 600 & -528 & $8700(1.141)$ & $13340(2.324)$ \\
& rac & 4225 & 392 & 992 & 604 & -528 & $8860(1.537)$ & $13440(2.256)$ \\
\hline $\mathrm{dpq}^{\prime}$ & meso & 1040 & 356 & 960 & 608 & -632 & $9730(1.454)$ & $14420(2.479)$ \\
& rac & 1660 & 368 & 976 & 616 & -640 & $9860(1.884)$ & $14540(2.816)$ \\
\hline $\mathrm{ppz}$ & meso & 890 & 352 & 916 & 568 & -968 & $12190(1.527)$ & $16690(1.906)$ \\
& rac & 1040 & 356 & 924 & 568 & -960 & $12180(1.506)$ & $16740(1.880)$ \\
\hline $2,3-\mathrm{dpp}$ & meso & 378 & 330 & 924 & 594 & -1094 & $12810(0.7952)$ & $13540(0.8155)$ \\
& rac & 237 & 318 & 912 & 594 & -1097 & $12760(0.6986)$ & $13520(0.7022)$ \\
\hline
\end{tabular}

${ }^{\mathrm{a}} \mathrm{K}_{\mathrm{c}}$ values are given by equation 1.18(a). ${ }^{9}$

${ }^{\mathrm{b}} \Delta \mathrm{E}_{\mathrm{ox}}=\mathrm{E}_{\mathrm{ox} 2}-\mathrm{E}_{\mathrm{ox} 1}$. Potentials are quoted $\pm 3 \mathrm{mV}$.

${ }^{c}$ The errors in the observed parameters are $\pm 10 \mathrm{~cm}^{-1}$ for $v$ and $\pm 0.0001 \mathrm{M}^{-1}$ for $(\varepsilon / v)$.

The dinuclear systems are each characterised by two reversible one-electron redox processes corresponding to successive oxidation of the metal centres, in addition to multiple reversible ligand-based reductions in the cathodic region. $\mathrm{E}_{\mathrm{ox} 1}$ and $\mathrm{E}_{\mathrm{ox} 2}$ are shifted cathodically by approximately $500 \mathrm{mV}$ relative to the corresponding metal-based processes in their dinuclear ruthenium analogues (Table 3.3), due to the raised $\mathrm{d} \pi$ level of Os compared with $\mathrm{Ru}^{72,139,140,144,152}$ In addition, the $\Delta \mathrm{E}_{\mathrm{ox}}$ values are increased by $100-150 \mathrm{mV}$ which results in a two-order of magnitude increase in $\mathrm{K}_{\mathrm{c}}$, for the dinuclear osmium complexes. The enhanced stability of the mixed-valence osmium species arises from the relatively greater degree of metal-ligand back-bonding. ${ }^{152,181}$ The diastereoisomers of $\left[\left\{\mathrm{Os}(\mathrm{bpy})_{2}\right\}_{2}(\mu\right.$ $\mathrm{dpb})]^{4+}$ exhibit the most pronounced difference in $\Delta \mathrm{E}_{\mathrm{ox}}$ values $\{28 \pm 6 \mathrm{mV}\}$ due to the differential structural distortions in the bridging ligand. The assignments of the BL- and bpy-based reduction processes in the cathodic region for the osmium systems are identical to those detailed for their ruthenium analogues in §3.3.2.2.

The spectra of $\left[\left\{\mathrm{Os}(\mathrm{bpy})_{2}\right\}_{2}(\mu-\mathrm{BL})\right]^{4+}$ exhibit similar features over the region $15000-30000 \mathrm{~cm}^{-1}$ which are assigned as overlapping $\mathrm{d} \pi\left(\mathrm{Os}^{\mathrm{II}}\right) \rightarrow \pi^{*}(\mathrm{BL})$ and $\mathrm{d} \pi\left(\mathrm{Os}^{\mathrm{II}}\right) \rightarrow \pi^{*}$ (bpy) singlet metal-to-ligand 
$\left({ }^{1} \mathrm{MLCT}\right)$ transitions, consistent with previous literature reports for the complexes $\left[\left\{\mathrm{Os}(\mathrm{bpy})_{2}\right\}_{2}(\mu-\mathrm{BL})\right]^{4+}$ $\{\mathrm{BL}=\mathrm{dpb}$ and 2,3-dpp $\}$ as diastereoisomeric mixtures. ${ }^{72,139,140,152}$ The lowest energy absorption band is assigned as a $\mathrm{d} \pi\left(\mathrm{Os}^{\mathrm{II}}\right) \rightarrow \pi^{*}(\mathrm{BL})$ transition to the lowest, triplet excited state $\left({ }^{3} \mathrm{MLCT}\right)$. This band undergoes a red-shift as BL is varied through the series 2,3-dpp, ppz, dpb, dpq' and dpb', consistent with the increasing stabilisation of the $\pi^{*}(\mathrm{BL})$ orbitals. ${ }^{* 2,139-141,152}$

The UV/Vis/NIR spectra for the diastereoisomers at $-35^{\circ} \mathrm{C}$ are provided in Figure 3.16. Spectroelectrochemical generation of the +5 and +6 forms of the diastereoisomers revealed stable isosbestic points in the spectral progressions accompanying both oxidation processes. The ${ }^{1}$ MLCT absorption bands decreased in intensity on one-electron oxidation and collapsed on further oxidation to the +6 species. While the energies of these transitions remain approximately constant upon oxidation for the dpb and dpb'-bridged complexes, the bands experience a blue-shift upon oxidation in the dpq'-, ppzand 2,3-dpp-bridged complexes. The behaviour of the latter is indicative of valence delocalisation. ${ }^{186}$ In contrast, the ${ }^{1}$ MLCT bands experience red-shifts upon oxidation from the +4 to the +5 species in the relatively less delocalised ruthenium complexes discussed in §3.3.2.3.

The ${ }^{3}$ MLCT absorption bands decreased in intensity upon oxidation, and small differences were observed in the energies and intensities of these transitions between the diastereoisomeric forms of the same complex. A notable feature of the spectra is the narrower ${ }^{3}$ MLCT and ${ }^{1}$ MLCT bands for the complexes incorporating the fused bridging ligands dpb' and ppz, relative to their unfused dpb and 2,3dpp analogues, respectively. For example, the ${ }^{3}$ MLCT and ${ }^{1}$ MLCT bands for meso- $\left[\{\text { Os(bpy })_{2}\right\}_{2}(\mu-$ $\mathrm{dpb})]^{4+}$ exhibit a more pronounced shoulder on their higher energy side, compared with the narrower and more intense bands for meso-[\{Os(bpy $\left.\left.)_{2}\right\}_{2}(\mu-\mathrm{dpb})\right]^{4+}$.

\subsubsection{NIR and Mid-IR Spectroelectrochemistry}

The NIR spectra for the mixed-valence complexes meso- and $r a c-\left[\left\{\mathrm{Os}(\mathrm{bpy})_{2}\right\}_{2}(\mu-\mathrm{BL})\right]^{5+}\{\mathrm{BL}=$ dpb', dpq', ppz, dpb, 2,3-dpp $\}$ are shown in Figure 3.16, and the results for the band parameters $\left\{v_{\max }\right.$ and $\left.(\varepsilon / v)_{\max }\right\}$ are summarised in Table 3.14. Due to the convoluted nature of the spectra, the $\Delta v_{1 / 2}$ values for the absorption bands were obtained by Gaussian deconvolution, and are reported in Tables B3.18-19 (Appendix B). Figure B3.4 (Appendix B), shows the overlays of the NIR spectra for meso- and rac$\left[\left\{\mathrm{Os}(\mathrm{bpy})_{2}\right\}_{2}(\mu-\mathrm{BL})\right]^{n+}(n=5,6)$.

\footnotetext{
${ }^{*}$ For the series of dinuclear osmium complexes, the expected 1:1 energy correlation between $\mathrm{E}_{\text {ox1-red } 1}$ and the energies of ${ }^{1} \mathrm{MLCT}$ and ${ }^{3}$ MLCT transitions was confirmed (meso: $R^{2}=0.998$ for ${ }^{1}$ MLCT and 0.983 for ${ }^{3}$ MLCT; rac: $R^{2}=0.997$ for ${ }^{1}$ MLCT and 0.951 for ${ }^{3}$ MLCT). ${ }^{182-185}$
} 
BL

(a)

dpb

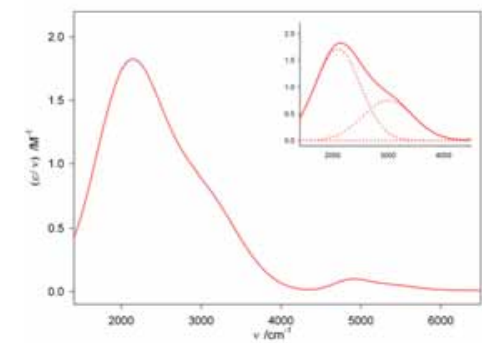

dpb'

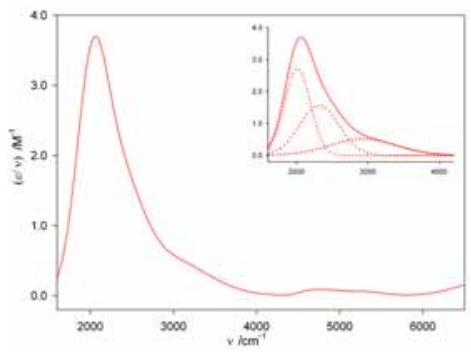

dpq'

ppz

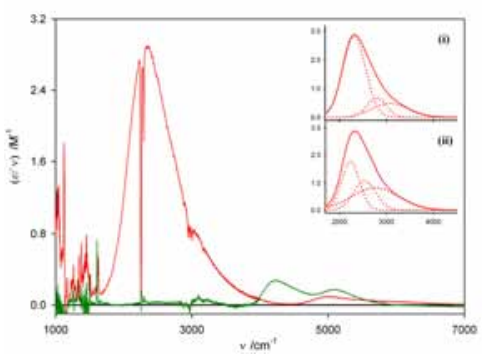

2,3-dpp (b)
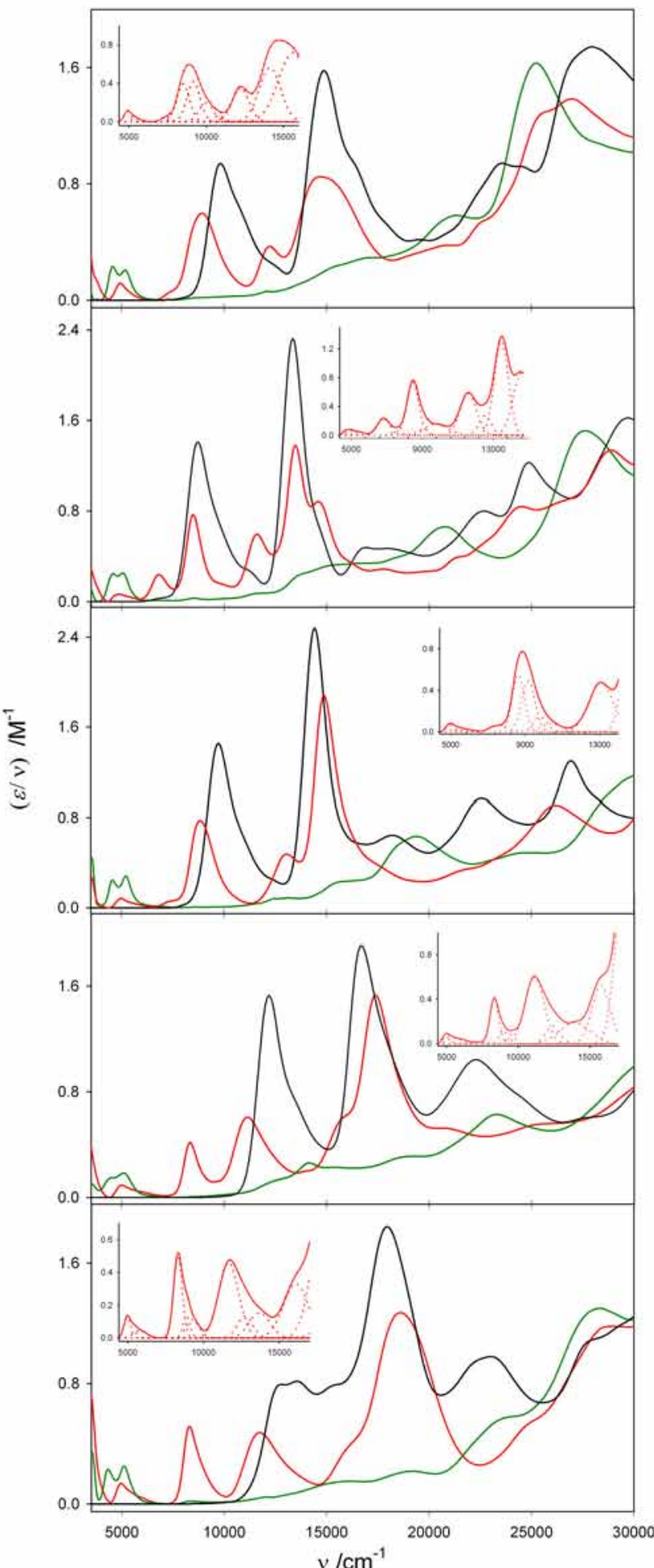

Figure 3.16 (a) Differential mid-IR spectra $\left(\mathrm{CH}_{3} \mathrm{CN}\right)$ for meso-[ $\left.\left\{\mathrm{Os}(\mathrm{bpy})_{2}\right\}_{2}(\mu-\mathrm{BL})\right]^{5+}\{\mathrm{BL}=\mathrm{dpb}$, dpb' $\}$ obtained by chemical oxidation with $\mathrm{NOPF}_{6}$, and meso- $\left[\left\{\mathrm{Os}(\mathrm{bpy})_{2}\right\}_{2}(\mu-\mathrm{ppz})\right]^{n+}\{n=5(-), 6(-)$; reference species is the +4 state $(-)\}$ obtained by spectroelectrochemical oxidation. (b) UV/Vis/NIR spectra of meso-[\{Os $\left.\left.(\mathrm{bpy})_{2}\right\}_{2}(\mu-\mathrm{BL})\right]^{n+}$ $\{\mathrm{BL}=\mathrm{dpb}, \mathrm{dpb}$ ', dpq', ppz, 2,3-dpp; $n=4(-), 5(-), 6(-)\}$ in $\left[\left(n-\mathrm{C}_{4} \mathrm{H}_{9}\right)_{4} \mathrm{~N}\right] \mathrm{PF}_{6} / \mathrm{CH}_{3} \mathrm{CN}$ at $-35^{\circ} \mathrm{C}$. The insets show the best fits of the bands obtained by Gaussian deconvolution of the spectra. ${ }^{*}$

${ }^{*}$ The deconvolutions of the mid-IR band for meso- $\left[\left\{\mathrm{OS}(\mathrm{bpy})_{2}\right\}_{2}(\mu-\mathrm{ppz})\right]^{5+}$ were not unique and the two best-fit solutions $\{(\mathrm{i})$ and (ii)\} are shown. 
Table 3.14 Summary of the mid-IR and NIR spectral data for meso- and $r a c$ - $\left[\left\{\mathrm{Os}(\mathrm{bpy})_{2}\right\}_{2}(\mu-\mathrm{BL})\right]^{5+}$ in $\left[\left(n-\mathrm{C}_{4} \mathrm{H}_{9}\right)_{4} \mathrm{~N}\right] \mathrm{PF}_{6} / \mathrm{CH}_{3} \mathrm{CN}$ at $-35^{\circ} \mathrm{C}$. ${ }^{\mathrm{a} b}$ The mid-IR spectral data is indicated in bold type.

\begin{tabular}{|c|c|c|c|c|}
\hline \multirow[b]{2}{*}{ BL } & \multicolumn{2}{|c|}{ meso } & \multicolumn{2}{|c|}{ rac } \\
\hline & $\begin{array}{l}v_{\max } \\
\pm 10 \\
/ \mathrm{cm}^{-1}\end{array}$ & $\begin{array}{c}(\varepsilon / v)_{\max } \\
\pm 0.0001 \\
/ \mathrm{M}^{-1}\end{array}$ & $\begin{array}{l}v_{\max } \\
\pm 10 \\
/ \mathrm{cm}^{-1}\end{array}$ & $\begin{array}{c}(\varepsilon / v)_{\max } \\
\pm 0.0001 \\
/ \mathrm{M}^{-1}\end{array}$ \\
\hline \multirow[t]{4}{*}{ dpb } & 2150 & 1.8262 & 4820 & 0.0888 \\
\hline & 4920 & 0.1176 & 8730 & 0.5978 \\
\hline & 8930 & 0.5979 & 12050 & 0.3346 \\
\hline & 12220 & 0.3700 & & \\
\hline \multirow[t]{5}{*}{ dpb' } & 2060 & 3.699 & 4870 & 0.0630 \\
\hline & 4870 & 0.0848 & 7045 & 0.1739 \\
\hline & 6810 & 0.2382 & 8700 & 0.6651 \\
\hline & 8480 & 0.7663 & 11860 & 0.6337 \\
\hline & 11600 & 0.5959 & & \\
\hline \multirow[t]{3}{*}{ dpq' } & 4980 & 0.1399 & 4980 & 0.1079 \\
\hline & 8840 & 0.7750 & 9190 & 0.8203 \\
\hline & 13050 & 0.4783 & 13540 & 0.6290 \\
\hline \multirow[t]{5}{*}{$\mathrm{ppz}$} & 2275 & 2.8906 & 5070 & 0.1126 \\
\hline & 5000 & 0.0930 & 8400 & 0.3014 \\
\hline & 8340 & 0.4142 & 11180 & 0.5374 \\
\hline & 11140 & 0.6074 & sh 15800 & 0.5109 \\
\hline & sh 15800 & 0.6082 & & \\
\hline \multirow[t]{4}{*}{ 2,3-dpp } & 4950 & 0.1357 & 5030 & 0.1150 \\
\hline & 8300 & 0.5164 & 8330 & 0.4519 \\
\hline & 11720 & 0.4740 & 11700 & 0.4347 \\
\hline & sh 15970 & 0.3644 & sh 16070 & 0.3640 \\
\hline
\end{tabular}

${ }^{\mathrm{a}}$ The errors in the observed parameters are $\pm 10 \mathrm{~cm}^{-1}$ for $v_{\max }$ and $\pm 0.0001 \mathrm{M}^{-1}$ for $(\varepsilon / v)_{\max }$.

${ }^{\mathrm{b}}$ Full details of the parameters of the deconvoluted bands (including $M_{0}$ and $M_{1}$ ) are reported in Tables B3.18 and B3.19 (Appendix B).

The NIR spectra of the dinuclear osmium systems exhibit an added complexity in behaviour compared to their ruthenium analogues reported in §3.3.2.4, due to the larger spin-orbit coupling constant for Os ( $\left.\xi_{\mathrm{Os}} \sim 3000 \mathrm{~cm}^{-1} c f . \xi_{\mathrm{Ru}} \sim 1000 \mathrm{~cm}^{-1}\right)$. While the maxima of the IVCT bands in the ruthenium complexes occur in the same region (5000-5400 $\mathrm{cm}^{-1}$, Table 3.5), the patterns of the energies, intensities and bandwidths of the multiple IVCT and IC transitions differ markedly within the series of osmium complexes. However, the NIR spectra of the complexes bridged by ppz and 2,3-dpp, and the complexes bridged by dpq' and dpb display a close resemblance.

The first oxidation process for the diastereoisomers of $\left[\left\{\mathrm{O}(\mathrm{bpy})_{2}\right\}_{2}(\mu-\mathrm{BL})\right]^{4+}\{\mathrm{BL}=\mathrm{ppz}$, 2,3-dpp $\}$ was characterised by the appearance of two new bands in the region $3500-10000 \mathrm{~cm}^{-1}\left\{v_{\max }\right.$ $\sim 5000$ and $\left.\sim 8300 \mathrm{~cm}^{-1}\right\}$, shown in Figure 3.16. The more intense higher energy band collapsed on removal of the second electron and was assigned as an IVCT transition. This band is asymmetrical and narrower on the lower energy side, with Gaussian deconvolution revealing the presence of three underlying transitions, as shown in the insets in Figure 3.16. The first component closely matches the energy, intensity and band-shape of the lower energy side of the IVCT manifold, while the less intense higher energy components give rise to the band asymmetry. The transitions are significantly narrower 
than those predicted on the basis of the classical two-state theory ${ }^{8}$ (equation 1.8), which indicates significant electronic communication between the metal centres. According to equation 1.11 for delocalised mixed-valence systems, ${ }^{8} \mathrm{H}_{\mathrm{ab}}$ estimates in the range $4150-4200 \mathrm{~cm}^{-1}$ are obtained for the IVCT transitions at $\sim 8300 \mathrm{~cm}^{-1}$. These values compare with the estimates of $430 \mathrm{~cm}^{-1}$ (for both diastereoisomers of ppz) and 473 and $455 \mathrm{~cm}^{-1}$ (for the meso and rac forms of the 2,3-dpp-bridged complex, respectively) as lower limits for $\mathrm{H}_{\mathrm{ab}}$ from equation 3.2 (assuming $r_{\mathrm{ab}}$ is $6.85 \AA$ ).

The mixed-valence spectra for the diastereoisomers of $\left[\left\{\mathrm{Os}(\mathrm{bpy})_{2}\right\}_{2}(\mu-\mathrm{dpb})\right]^{5+}$ are characterised by two new bands at 4870 and $\sim 7000 \mathrm{~cm}^{-1}$, as shown in Figure 3.16. The more intense, higher energy band collapsed on removal of the second electron and was assigned as an IVCT transition. Compared with the asymmetrically-shaped IVCT bands at $\sim 8300 \mathrm{~cm}^{-1}$ in the diastereoisomers of $\left[\left\{\mathrm{Os}(\mathrm{bpy})_{2}\right\}_{2}(\mu-\right.$ $\mathrm{BL})]^{5+}\{\mathrm{BL}=\mathrm{ppz}, 2,3-\mathrm{dpp}\}$, the bands at 6810 and $7045 \mathrm{~cm}^{-1}$ in meso- and rac- $\left[\left\{\mathrm{OS}(\mathrm{bpy})_{2}\right\}_{2}(\mu-\mathrm{dpb})\right]^{5+}$, respectively, were fitted by a single Gaussian-shaped component. The lower limits of the $\mathrm{H}_{\mathrm{ab}}$ values for these bands were determined as 298 and $287 \mathrm{~cm}^{-1}$ for the meso and rac diastereoisomers, respectively (from equation 3.2, assuming $r_{\mathrm{ab}}$ is $6.85 \AA$ ).

Meso- and rac-[\{Os(bpy $\left.\left.)_{2}\right\}_{2}(\mu-\mathrm{BL})\right]^{5+}\{\mathrm{BL}=\mathrm{dpb}$, dpq'\} exhibit two new bands at $\sim 5000$ and $\sim 7500 \mathrm{~cm}^{-1}$. The latter was observed as a weak shoulder on the low energy side of the band in the region $7000-9200 \mathrm{~cm}^{-1}$ and was assigned as an IVCT transition. The band in the region $8700-9200 \mathrm{~cm}^{-1}$ is composed of two underlying transitions of approximately equal intensity, one of which may also represent an IVCT transition. On the basis of the band parameters obtained from Gaussian deconvolution, the lower limits for $\mathrm{H}_{\mathrm{ab}}$ were determined as follows (equation 3.2): for meso$\left[\left\{\mathrm{Os}(\mathrm{bpy})_{2}\right\}_{2}(\mu-\mathrm{dpb})\right]^{5+}, \mathrm{H}_{\mathrm{ab}}=593$ and $800 \mathrm{~cm}^{-1}$ for the transitions at 7385 and $8500 \mathrm{~cm}^{-1}$, respectively; for meso- $\left[\left\{\mathrm{Os}(\mathrm{bpy})_{2}\right\}_{2}(\mu-\mathrm{dpq})\right]^{5+}, \mathrm{H}_{\mathrm{ab}}=197$ and $550 \mathrm{~cm}^{-1}$ for the transitions at 7570 and $8620 \mathrm{~cm}^{-1}$, respectively. In each case, $r_{\mathrm{ab}}$ was equated with the crystallographic Os $\cdots$ Os distances (§3.3.5.1).

The extension of the spectroelectrochemical measurements to the mid-IR region revealed an intense $\left\{(\varepsilon / v)_{\max } \sim 1.8-2.9 \mathrm{M}^{-1}\right\}$, asymmetrically-shaped band in the region 2000-4000 $\mathrm{cm}^{-1}$ for meso$\left[\left\{\mathrm{Os}(\mathrm{bpy})_{2}\right\}_{2}(\mu-\mathrm{BL})\right]^{5+}\{\mathrm{BL}=\mathrm{dpb}, \mathrm{dpb} ', \mathrm{ppz}\}$, as shown in Figure 3.16(a) $\{$ Tables 3.14\}. The band was composed of two-underlying Gaussian-shaped components for the dpb-bridged complex and three underlying components for the dpb'- and ppz-bridged complexes (Table B3.19, Appendix B). In the latter case, the deconvolution was not unique, and two different solutions for the three underlying components were obtained. The overall bandwidth of the manifold and the separation of the underlying components are largest for the dpb-bridged complex due to the additional redox asymmetry contribution arising from the stereochemically-induced structural distortion. The separation of the components of the mid-IR band in the Os systems ( $c a .1000 \mathrm{~cm}^{-1}$ ) are comparable to the splittings between the underlying IVCT components for their Ru analogues, whereas they might be expected to be greater for the latter due to the larger spin-orbit coupling constant. This argues against the assignment of the mid-IR band as IVCT(1) in the Os systems. 


\section{The Mechanism of Delocalisation: Two Alternate Views}

\section{(i) A Delocalised Model}

The Os complexes exhibit extensive delocalisation and the "IVCT" bands in the mixed-valence species are likely to arise from transitions between bonding and antibonding orbitals within the molecular manifolds of the systems. The intense bands in the mid-IR region are due to IC transitions between spinorbit states that are split by the coupling of the $\mathrm{Os}^{\mathrm{II.5}}$ centres. For all complexes the relatively low intensity $\left\{(\varepsilon / v)_{\max } \sim 0.1 \mathrm{M}^{-1}\right\}$ asymmetric bands in the region 5000-6600 $\mathrm{cm}^{-1}$, which are composed of three underlying Gaussian components (Tables B3.18-19, Appendix B), are likely to represent spin-orbit transitions in a vibronic progression from the intense transitions in the region $2000-4000 \mathrm{~cm}^{-1}$. The nature of the mid-IR and NIR spectral features for the complexes are strikingly similar to the osmium analogue of the Creutz-Taube ion $\left[\left\{\mathrm{Os}\left(\mathrm{NH}_{3}\right)_{5}\right\}_{2}(\mu-\mathrm{pyz})\right]^{n+}(n=5,6)$, which has been interpreted on the basis of complete electronic delocalisation. ${ }^{187-190}$ In the present case, the new bands in the NIR region 6600$10000 \mathrm{~cm}^{-1}$ which have been identified as IVCT transitions appear at higher energies compared with the IVCT bands in their Ru analogues (Table 3.5).

\section{(ii) A Localised Model}

As discussed in §1.5.3.5, the appearance of diagnostic $\mathrm{d} \pi \rightarrow \mathrm{d} \pi$ IC transitions are indicative of localisation. ${ }^{187-190}$ In the fully-oxidised forms of meso- and $r a c-\left[\left\{\mathrm{O}(\mathrm{bpy})_{2}\right\}_{2}(\mu-\mathrm{BL})\right]^{6+}\{\mathrm{BL}=\mathrm{dpb}$ ', dpq', ppz, dpb, 2,3-dpp\}, IC transitions are evident in the region 4000-5500 $\mathrm{cm}^{-1}$ (Table 3.15, Figures 3.16 and B3.4, Appendix B). These transitions occur at approximately the same energies but exhibit higher intensities compared with the IC transitions at $4580 \mathrm{~cm}^{-1}\left(\varepsilon_{\max }=450 \mathrm{M}^{-1} \mathrm{~cm}^{-1}\right)$ and $5090 \mathrm{~cm}^{-1}\left(\varepsilon_{\max }=360\right.$ $\left.\mathrm{M}^{-1} \mathrm{~cm}^{-1}\right)$ in $\left[\mathrm{Os}(\mathrm{bpy})_{3}\right]^{3+}{ }^{144}$ The absence of the two IC transitions in the NIR region for $\left[\left\{\mathrm{Os}(\mathrm{bpy})_{2}\right\}_{2}(\mu-\right.$ $\mathrm{BL})]^{5+}$ is indicative of the high degree of electronic delocalisation in these species.

While the characteristics of the NIR mixed-valence bands support a localised-to-delocalised or delocalised classification for the species, the former assignment is strictly valid only when there is clear evidence of localisation on the IR time-scale $\left(\leq 10^{-11} \mathrm{~s}\right) .{ }^{14}$ Figure 3.17 shows the differential mid-IR spectra for meso- $\left[\left\{\mathrm{OS}(\mathrm{bpy})_{2}\right\}_{2}(\mu-\mathrm{ppz})\right]^{n+}(n=5,6)$. By comparison with the mid-IR studies on the +5 and +6 forms of related Class II-III pyz-bridged polypyridyl complexes of osmium such as cis,cis$\left[\left\{\mathrm{Os}(\mathrm{bpy})_{2}(\mathrm{Cl})\right\}_{2}(\mu-\mathrm{pyz})\right]^{3+165,166}$ (discussed in §1.5.2), the new vibration at $1625 \mathrm{~cm}^{-1}$ in meso$\left[\left\{\mathrm{Os}(\mathrm{bpy})_{2}\right\}_{2}(\mu-\mathrm{ppz})\right]^{5+}$ coincides with the expected position of a $v_{8 a}(\mathrm{pyz})$ stretching vibration. ${ }^{14}$ The appearance of this band provides evidence for electronic localisation due to a small residual barrier to electron transfer, and is consistent with a localised-to-delocalised (Class II-III) classification for the mixed-valence species.

\footnotetext{
* The behaviour of the $v$ (bpy) vibrations for the $n=4,5,6$ systems in the region $1400-1600 \mathrm{~cm}^{-1}$ would provide an additional measure of the degree of delocalisation. ${ }^{14}$ Complete mid-IR analyses for the full series of dinuclear osmium complexes in each oxidation state were beyond the scope of the present work, and a more extensive discussion of the vibrational bands in the midIR region for meso- $\left[\left\{\mathrm{Ru}(\mathrm{bpy})_{2}\right\}_{2}(\mu-\mathrm{ppz})\right]^{n+}(n=5,6)$ is not warranted at the present time.
} 
Table 3.15 Summary of the energies of the IC transitions obtained by Gaussian deconvolution for $\left[\left\{\mathrm{Os}(\mathrm{bpy})_{2}\right\}_{2}(\mu-\mathrm{BL})\right]^{6+}$ complexes. $^{\mathrm{a}, \mathrm{b}}$

\begin{tabular}{|c|c|c|c|c|c|c|}
\hline BL & Diastereoisomer & $\begin{array}{l}v_{\max } \\
\pm 10 \\
/ \mathrm{cm}^{-1} \\
\end{array}$ & $\begin{array}{c}(\varepsilon / v)_{\max } \\
\pm 0.0001 \\
/ \mathrm{M}^{-1}\end{array}$ & $\begin{array}{l}\Delta v_{1 / 2} \\
\pm 10 \\
/ \mathrm{cm}^{-1}\end{array}$ & $\begin{array}{l}\Delta v_{\text {IC }}{ }^{\mathrm{c}} \\
/ \mathrm{cm}^{-1}\end{array}$ & $\begin{array}{c}M_{0} \\
/ \mathrm{M}^{-1}\end{array}$ \\
\hline \multirow[t]{2}{*}{$\mathrm{dpb}$} & meso & $\begin{array}{l}4550 \\
5160\end{array}$ & $\begin{array}{l}0.2410 \\
0.2786\end{array}$ & $\begin{array}{l}428 \\
512\end{array}$ & 610 & $\begin{array}{l}106 \\
150\end{array}$ \\
\hline & rac & $\begin{array}{l}4550 \\
5145\end{array}$ & $\begin{array}{l}0.2482 \\
0.2601\end{array}$ & $\begin{array}{l}502 \\
477\end{array}$ & 595 & $\begin{array}{l}132 \\
132\end{array}$ \\
\hline \multirow[t]{2}{*}{ dpb' } & meso & $\begin{array}{l}4530 \\
5080\end{array}$ & $\begin{array}{l}0.2226 \\
0.1872\end{array}$ & $\begin{array}{l}426 \\
547\end{array}$ & 550 & $\begin{array}{l}100 \\
110\end{array}$ \\
\hline & rac & $\begin{array}{l}4470 \\
5045\end{array}$ & $\begin{array}{l}0.2256 \\
0.1906\end{array}$ & $\begin{array}{l}495 \\
625\end{array}$ & 575 & $\begin{array}{l}118 \\
127\end{array}$ \\
\hline \multirow[t]{2}{*}{ dpq' } & meso & $\begin{array}{l}4520 \\
5170\end{array}$ & $\begin{array}{l}0.2245 \\
0.2376\end{array}$ & $\begin{array}{l}505 \\
573\end{array}$ & 650 & $\begin{array}{l}120 \\
145\end{array}$ \\
\hline & rac & $\begin{array}{l}4505 \\
5175 \\
\end{array}$ & $\begin{array}{l}0.2019 \\
0.2133 \\
\end{array}$ & $\begin{array}{l}510 \\
575 \\
\end{array}$ & 670 & $\begin{array}{l}110 \\
130 \\
\end{array}$ \\
\hline \multirow[t]{2}{*}{ ppz } & meso & $\begin{array}{l}4395 \\
5095\end{array}$ & $\begin{array}{l}0.1312 \\
0.1549\end{array}$ & $\begin{array}{l}723 \\
725\end{array}$ & 700 & $\begin{array}{c}98.9 \\
120\end{array}$ \\
\hline & rac & $\begin{array}{l}4395 \\
5095\end{array}$ & $\begin{array}{l}0.1310 \\
0.1535\end{array}$ & $\begin{array}{l}716 \\
719\end{array}$ & 700 & $\begin{array}{l}100 \\
120\end{array}$ \\
\hline \multirow[t]{2}{*}{ 2,3-dpp } & meso & $\begin{array}{l}3320 \\
5100 \\
\end{array}$ & $\begin{array}{l}0.2273 \\
0.2449\end{array}$ & $\begin{array}{l}460 \\
615 \\
\end{array}$ & 780 & $\begin{array}{l}110 \\
160 \\
\end{array}$ \\
\hline & rac & $\begin{array}{l}4270 \\
5065\end{array}$ & $\begin{array}{l}0.2581 \\
0.2397\end{array}$ & $\begin{array}{l}433 \\
601\end{array}$ & 795 & $\begin{array}{l}117 \\
153\end{array}$ \\
\hline
\end{tabular}

${ }^{\mathrm{a}}$ The errors in the observed parameters are $\pm 10 \mathrm{~cm}^{-1}$ for $v_{\max }$ and $\pm 0.0001 \mathrm{M}^{-1}$ for $(\varepsilon / v)_{\max }$.

${ }^{\mathrm{b}}$ Full details of the bands obtained from Gaussian deconvolution are provided in Tables B3.18-3.19 (Appendix B).

${ }^{\mathrm{c}}$ Difference between the energies of the IC bands.

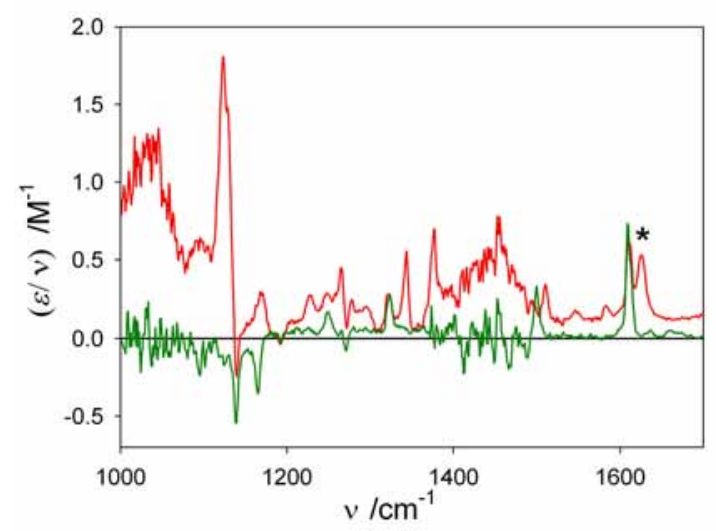

Figure 3.17 Differential mid-IR spectra for meso- $\left[\left\{\mathrm{Os}(\mathrm{bpy})_{2}\right\}_{2}(\mu-\mathrm{ppz})\right]^{n+}\{n=5(-), 6(-)\}$. The reference species is the +4 state $(-)$. The asterisk denotes the new band at $1625 \mathrm{~cm}^{-1}$.

On the basis of a localised-to-delocalised classification for the Os complexes, the spectroscopic properties of the mixed-valence states can be interpreted by assuming a small degree of residual localisation. The bands in the mid-IR region are assigned as IVCT(1), and the components under the manifold originate from multiple interactions across the bridging ligand between $\operatorname{Os}(\mathrm{d} \pi)$ orbitals that are highly mixed with $\pi, \pi^{*}(\mathrm{BL})$. While three IVCT and two IC transitions ${ }^{14}$ are predicted from a two-site model which includes spin-orbit coupling (§1.5.3.5), the present results suggest that the excited states 
exhibit multiplicities that are higher than single order. This demonstrates that at least a three-site description is necessary to describe the systems. A vibronic progression associated with IVCT(1) could also explain the multiple underlying components. Since the characteristics of IVCT(1) determine the nature of the ground state, the significant intensity of this band is consistent with the high degree of electronic delocalisation. The relatively weak bands in the region $5000-6600 \mathrm{~cm}^{-1}$ are assigned as $\mathrm{d} \pi \rightarrow$ $\mathrm{d} \pi$ IC transitions, and occur in the same range in the +6 state.

The new bands in the NIR region $6600-10000 \mathrm{~cm}^{-1}$ for the mixed-valence species are assigned as IVCT bands. On the basis of the semi-quantitative relationships in equation $1.33,{ }^{14}$ if the transition at $\sim 8300 \mathrm{~cm}^{-1}$ in $\left[\left\{\mathrm{OS}(\mathrm{bpy})_{2}\right\}_{2}(\mu-\mathrm{BL})\right]^{5+}\{\mathrm{BL}=\mathrm{ppz}, 2,3-\mathrm{dpp}\}$ is assigned as IVCT(2), then IVCT(1) should occur at IVCT(2) - $\Delta \mathrm{E}_{\mathrm{so}(1)} \approx 4000 \mathrm{~cm}^{-1}\left(\Delta \mathrm{E}_{\mathrm{so}(1)}\right.$ is approximated as the energy of the first IC band in the +6 state from Table 3.15). However, this predicted position does not correlate with the observed energies of the mid-IR or NIR bands. According to equation 1.33, the separation between IVCT(2) and (3) is equated with the separation of the IC states, $\Delta v_{\mathrm{IC}}$, and IVCT(3) would be assigned as the transition which overlaps IVCT(2) on the higher energy side.

Clearly, a localised argument is insufficient to rationalise the properties of the dinuclear Os systems. While the mixed-valence species $\left[\left\{\mathrm{O}(\mathrm{bpy})_{2}\right\}_{2}(\mu-\mathrm{BL})\right]^{5+}\{\mathrm{BL}=\mathrm{dpb}, 2,3-\mathrm{dpp}\}$ have previously been assigned as localised systems by Brewer and coworkers, ${ }^{139}$ it is evident that the full series of complexes in the present study lie at the borderline between the localised-to-delocalised and delocalised regimes.

\section{Stereochemical Effects on the IC Transitions in $\left[\left\{\mathrm{OS}(\mathrm{bpy})_{2}\right\}_{2}(\mu-\mathrm{BL})\right]^{6+}$}

In the +6 state, the trends in the IC bands also provide evidence for stereochemical and structural influences on the $\mathrm{d} \pi(\mathrm{Os})$ energy levels. Figure 3.18 provides a schematic illustration of the energies of the IC states. $\Delta v_{\mathrm{IC}}$ increases as BL is varied in the order $\mathrm{dpb}^{\prime}<\mathrm{dpb}<\mathrm{dpq}<\mathrm{ppz}<2,3-\mathrm{dpp}$. This splitting is greater for the diastereoisomers incorporating the unfused bridging ligands dpb and 2,3-dpp, compared with their fused analogues dpb' and ppz, due to the influence of the structural distortion in the former cases. The $\Delta v_{1 / 2}$ values for the IC transitions also differ most significantly between the diastereoisomeric forms of the dpb- and 2,3-dpp-bridged complexes. The IC bands occur at higher energies in the meso forms for the complexes incorporating the unfused ligands due to the larger magnitude of the stereochemically-induced redox asymmetry, $\Delta \mathrm{E}_{\text {struct }}$, relative to the rac forms. The IC energies are comparable for both diastereoisomers in the complexes incorporating the fused bridging ligands. These observations are illustrated by the overlays of the IC bands for $\left[\left\{\mathrm{Os}(\mathrm{bpy})_{2}\right\}_{2}(\mu-\mathrm{BL})\right]^{6+}\{\mathrm{BL}=\mathrm{dpb}$ ', dpb $\}$ in Figure 3.19 and for the full series of complexes in Figure B3.4 (Appendix B). 


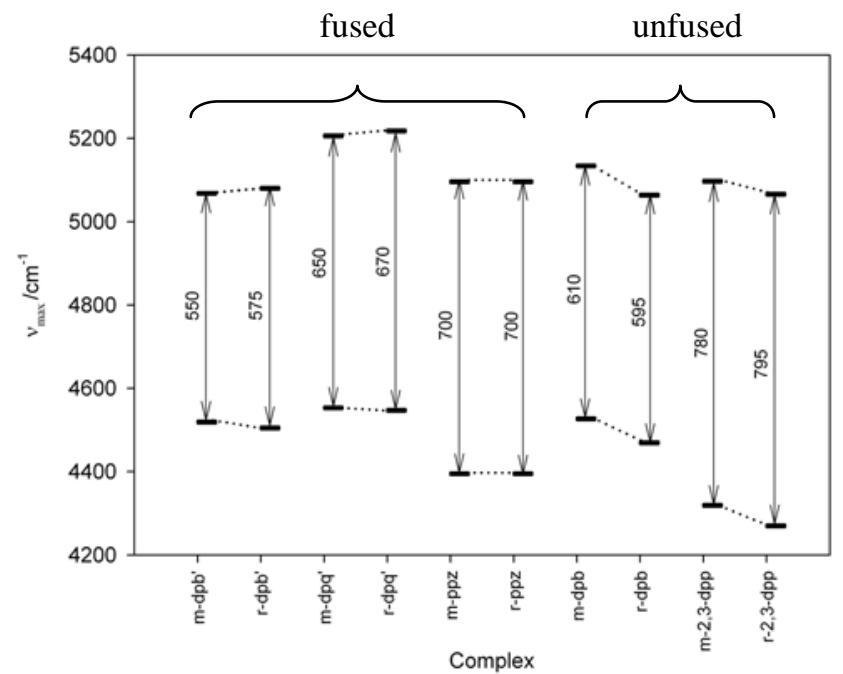

Figure 3.18 Schematic diagram of the energies of the IC bands (obtained by spectral deconvolution) for the meso and rac diastereoisomers of $\left[\left\{\mathrm{O}(\mathrm{bpy})_{2}\right\}_{2}(\mu-\mathrm{BL})\right]^{6+}\{\mathrm{BL}=\mathrm{dpb}$ ', dpq', ppz, dpb, 2,3-dpp $\}$, abbreviated m-BL or r-BL, respectively.

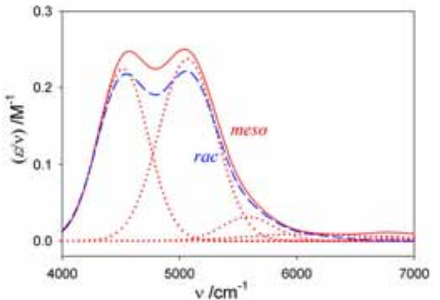

(a)

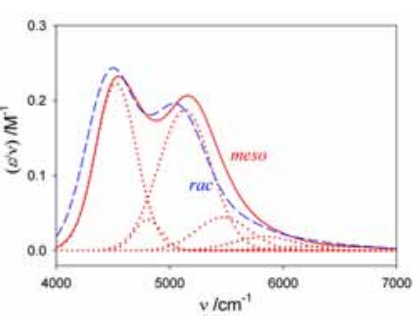

(b)

Figure 3.19 Overlay of the NIR spectra for meso- and rac- $\left[\left\{\mathrm{OS}(\mathrm{bpy})_{2}\right\}_{2}(\mu-\mathrm{BL})\right]^{6+}\{\mathrm{BL}=\mathrm{dpb}$ (a), dpb (b) $\}$ at $-35^{\circ} \mathrm{C}$ with the bands obtained by Gaussian deconvolution ( $\cdots$ of of the meso diastereoisomers.

From the trends in the characteristics of the IC bands in the +6 states, three major conclusions may be drawn regarding the contribution of $\Delta \mathrm{E}^{\prime}$ to the activation barrier for electron transfer in equation 3.1. Firstly, since the local site symmetry at each Os centre is similar over the series of complexes incorporating the fused bridging ligands, the energies and splittings of the IC bands are dependent on the degree of delocalisation. Secondly, $\Delta v_{\mathrm{IC}}$ increases in the presence of structural distortions, and the absolute energies of the IC transitions are influenced by stereochemically-induced structural distortions. Thirdly, the relative magnitudes of $\Delta v_{\mathrm{IC}}$ in the +6 states of the Os complexes are reflected by the splittings between the components of the IVCT bands in their Ru analogues (§3.3.2.4). The broadening of the IVCT bands for the latter as BL is varied in the order dpb', dpq' and ppz (Table 3.5), arises from the corresponding increase in $\Delta v_{\text {IC }}$ through the series. Since both diastereoisomers of a given Os complex exhibit similar $\Delta v_{\text {IC }}$ values, the separation of the components in the Ru systems should also be similar for both diastereoisomers of a given complex. Additional contributions to the differential IVCT bandwidths for the diastereoisomeric forms of each complex arise from solvent coupling, ion-pairing and thermochromic effects. These issues are the subject of §3.3.7 and §3.3.8. 


\subsubsection{The Effective Electron Transfer Distance: Stark Absorption Spectroscopy}

Stark absorption spectra were obtained for the dinuclear complexes meso-[\{Ru(bpy $\left.\left.)_{2}\right\}_{2}(\mu-B L)\right]^{5+}$ $\{\mathrm{BL}=\mathrm{dpb}, \mathrm{dpq}\}$ and meso- $\left[\left\{\mathrm{Os}(\mathrm{bpy})_{2}\right\}_{2}(\mu-\mathrm{BL})\right]^{5+}\{\mathrm{BL}=\mathrm{dpb}, \mathrm{dpb}, \mathrm{dpq}, \mathrm{ppz}\}$ in $n$-butyronitrile glass at $77 \mathrm{~K}$ and a field strength $\mathrm{F}_{\text {ext }}$ of $4.0 \mathrm{MVcm}^{-1}$. The quantitative Liptay analysis ${ }^{118}$ of the Stark response $^{114,116,119}$ yielded the dipole moment change $(|\Delta \mu|)$, the angle between the transition moment and the dipole moment change $(\xi)$, the difference polarisability $(\Delta \alpha)$, and magnitude of the trace of the difference polarisability $\{\operatorname{Tr}(\Delta \alpha)\}$. The results for the absorption $\{\mathrm{A}(v)$ vs. $v\}$ and Stark absorption $\{\Delta \mathrm{A}(v)$ vs. $v\}$ spectra for the dinuclear ruthenium and osmium complexes are presented in Tables 3.16 and 3.17, respectively. The spectra for meso- $\left[\left\{\mathrm{Ru}(\mathrm{bpy})_{2}\right\}_{2}(\mu-\mathrm{dpb})\right]^{5+}$ and meso- $\left[\left\{\mathrm{O}(\mathrm{bpy})_{2}\right\}_{2}(\mu-\mathrm{ppz})\right]^{5+}$ are shown in Figures 3.20 and 3.21, respectively, where panel (a) shows the unperturbed absorption spectrum, and panels (b) and (c) show the first- and second-order derivatives of the absorption spectrum, respectively. The Stark spectrum and the best fit to the spectrum are shown in panel (d). The corresponding spectra for meso-[\{Os(bpy $\left.\left.)_{2}\right\}_{2}(\mu-\mathrm{BL})\right]^{5+}\{\mathrm{BL}=\mathrm{dpq}$ ', dpb' $\}$ are shown in Figures B3.5 and B3.6 (Appendix B).

The most notable feature of the Stark signals for the IVCT bands in meso-[ $\left.\left\{\mathrm{Ru}(\mathrm{bpy})_{2}\right\}_{2}(\mu-\mathrm{dpb})\right]^{5+}$ (Figure 3.20) and meso- $\left[\left\{\mathrm{Ru}(\mathrm{bpy})_{2}\right\}_{2}(\mu-\mathrm{dpq})\right]^{5+}$ in the range $3050-12500 \mathrm{~cm}^{-1}$ is the absence of a strong second-derivative component. This is indicative of a minimal dipole moment change, $\left|\Delta \mu_{12}\right|$, accompanying the IVCT excitation, and a negligible adiabatic charge transfer distance, $r_{12}$ (equation 1.26). As shown in Figure 3.20, the Stark spectrum for meso-[\{Ru(bpy) $\left.\}_{2}(\mu-d p b)\right]^{5+}$ strongly resembles the negative of the unperturbed (zeroth-derivative) absorption spectrum at energies below the maximum of the overall IVCT manifold, and the low-energy side of the Stark signal was modelled well by a negative zeroth-derivative. At energies above the absorption maximum, the Stark spectrum exhibits a slightly positive $\Delta \mathrm{A}(\mathrm{v})$ component which is indicative of a contribution from the first-order derivative.

Table 3.16 Absorption and electroabsorption properties of the IVCT bands for meso- $\left[\left\{\mathrm{Ru}(\mathrm{bpy})_{2}\right\}_{2}(\mu-\mathrm{BL})\right]^{5+}\{\mathrm{BL}=$ dpb, dpq'\} in $n$-butyronitrile glass at 77K and a field strength of $4.0 \mathrm{MVcm}^{-1}$.

\begin{tabular}{|c|c|c|c|c|c|c|c|}
\hline $\mathrm{BL}$ & $\begin{array}{c}v_{\max } \\
/ \mathrm{cm}^{-1}\end{array}$ & $\begin{array}{l}\Delta v_{1 / 2} \\
/ \mathrm{cm}^{-1}\end{array}$ & $\begin{array}{c}\left|\Delta \mu_{12}\right| \\
/ \mathrm{e} \AA\end{array}$ & $\begin{array}{c}\left|\Delta \mu_{12}\right| \\
/ \mathrm{D}\end{array}$ & $\begin{array}{c}\xi \\
\rho^{\circ}\end{array}$ & $\begin{array}{c}\operatorname{Tr}(\Delta \alpha) \\
/ \AA^{3}\end{array}$ & $\begin{array}{c}\Delta \alpha \\
/ \AA^{3}\end{array}$ \\
\hline $\mathrm{dpb}$ & 4810 & 1030 & $0.386(2)$ & $1.84(2)$ & $-22^{\mathrm{b}}$ & -200 & -466 \\
& 5515 & 1330 & $2.2(5) \times 10^{-4}$ & $8.1(1) \times 10^{-4}$ & 0 & 657 & 1300 \\
& 6165 & 2250 & $4.2(2) \times 10^{-4}$ & $2.0(2) \times 10^{-3}$ & 0 & -1080 & -577 \\
\hline $\mathrm{dpq}$ & 5195 & 1110 & $0.324(3)$ & $1.54(2)$ & 0 & -796 & -1193 \\
& 6050 & 1130 & $4.2(5) \times 10^{-4}$ & $2.0(2) \times 10^{-3}$ & $17^{\mathrm{b}}$ & -8095 & -10530 \\
& 6830 & 1500 & $7.1(4) \times 10^{-5}$ & $3.38(2) \times 10^{-3}$ & 0 & -21195 & -34510 \\
\hline
\end{tabular}

${ }^{a}$ The errors in the observed and calculated parameters (not tabulated) are $\pm 10 \mathrm{~cm}^{-1}$ for $v_{\max }$ and $\Delta v_{1 / 2}, \pm 2^{\circ}$ for $\xi$, and $\pm 10 \AA^{3}$ for $\operatorname{Tr}(\Delta \alpha)$ and $\Delta \alpha$.

${ }^{\mathrm{b}}$ The non-zero values for $\xi$ are unusual and indicate that the direction of charge displacement in the excited state is not aligned with the transition moment. 
(a)

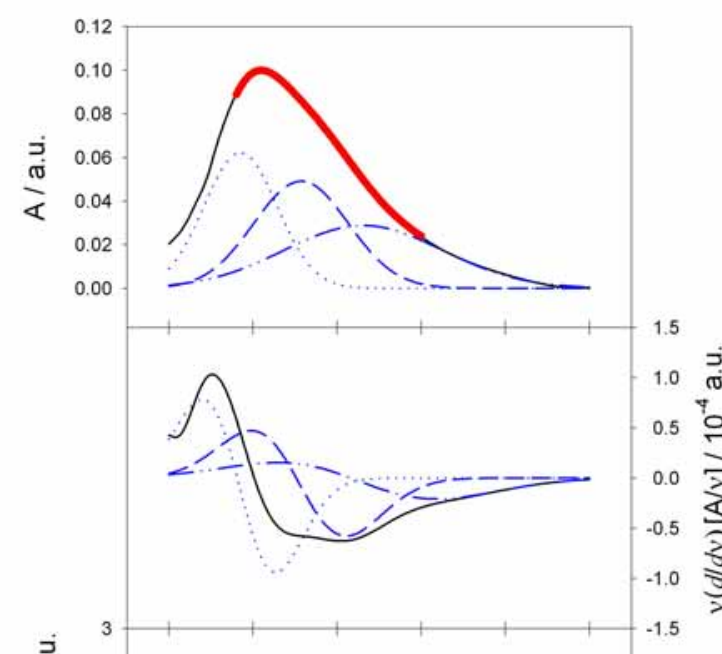

(c)

(b)

(d)

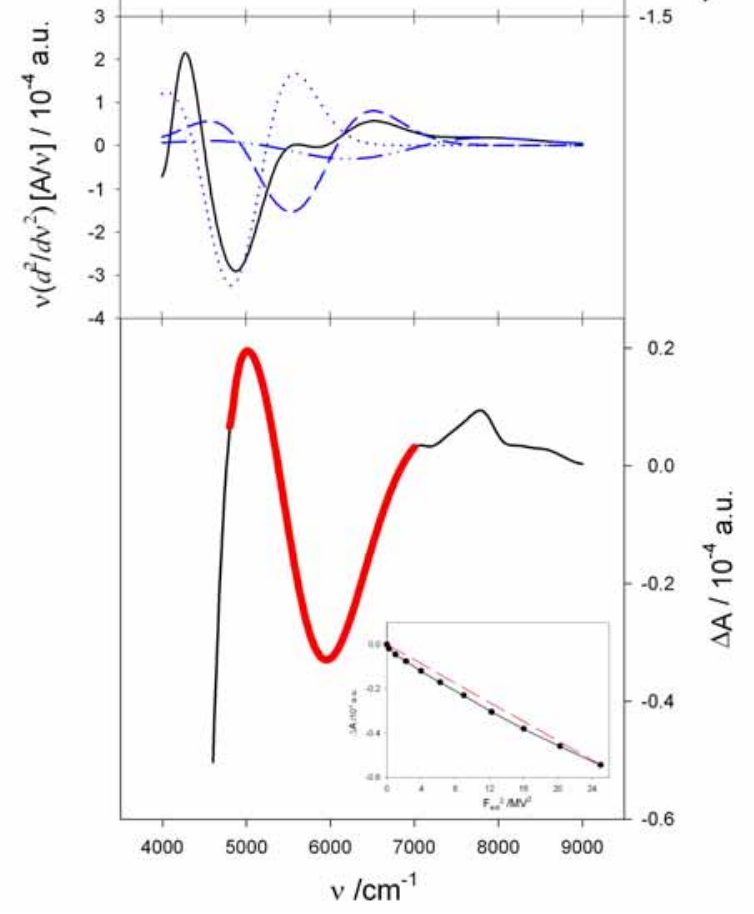

Figure 3.20 Liptay analysis of the Stark absorption spectrum (A vs. v) for meso-[ $\left.[\text { Ru(bpy) }\}_{2}\right\}_{2}(\mu \text {-dpb) }]^{5+}$ in $n$-butyronitrile glass at $77 \mathrm{~K}$, with a field strength of $4.0 \mathrm{MVcm}^{-1}$. (a) Unperturbed absorption spectrum with the bands obtained by Gaussian deconvolution and energy weighted first (b) and second (c) derivatives of the absorption spectrum. (d) Measured (-) and fitted (-) Stark signals ( $\triangle \mathrm{A} v$ vs. $v$ ) at $45^{\circ}$. The inset shows the Stark response at $6500 \mathrm{~cm}^{-1}$ as a function of $\mathrm{F}_{\mathrm{ext}}{ }^{2}$ (the predicted $\Delta \mathrm{A} v \mathrm{vs} . \mathrm{F}_{\mathrm{ext}}{ }^{2}$ dependence is ---).

Poor fits were obtained from attempts to fit the Stark signal over the entire IVCT manifold as the high energy side of the signal behaved in a complicated manner, suggestive of multiple underlying bands with different field dependencies. The inset in Figure 3.20(d) shows the dependence of $\Delta \mathrm{A}(\mathrm{v})$ on $\mathrm{F}_{\mathrm{ext}}{ }^{2}$ at $6500 \mathrm{~cm}^{-1}$. While equation 1.11 assumes a linear variation between these parameters, the deviation from linearity supports the presence of overlapping components. The best fits were obtained using three underlying components (previously assigned as spin-orbit components in §3.3.2.4). The results for the parameters obtained from the Liptay analysis of each component are presented in Table 3.16. The lowest energy component at $4810 \mathrm{~cm}^{-1}$ exhibits a dipole moment change of $0.386 \pm 0.002 \mathrm{e} \AA$, compared with negligible dipole moment changes for the higher-energy components. These compare with the value of 
$6.870 \pm 0.003$ e $\AA$ expected for unit electron transfer across the geometric metal-metal distance (i.e. average of the crystallographic $\mathrm{Ru} \cdots \mathrm{Ru}$ distances for the $\mathrm{PF}_{6}{ }^{-}$and $\left[\mathrm{ZnCl}_{4}\right]^{2-}$ structures in Table 3.2).

The IVCT Stark signal for meso-[ $\left\{\mathrm{Ru}(\mathrm{bpy})_{2}\right\}_{2}(\mu \text {-dpq') }]^{5+}$ was similar to that observed for meso$\left[\left\{\mathrm{Ru}(\mathrm{bpy})_{2}\right\}_{2}(\mu-\mathrm{dpb})\right]^{5+}$ in Figure 3.20(d), and was also modelled in terms of three Gaussian-shaped components. The lowest energy component at $5195 \mathrm{~cm}^{-1}$ exhibits a dipole moment change of $0.324 \pm$ $0.003 \mathrm{e} \AA$, while negligible changes were obtained for the higher-energy components. These values compare with the expected value of $6.818 \pm 0.001 \mathrm{e} \AA$ for unit electron transfer across the geometric metal-metal distance (Table 3.2).

IVCT(1) characterises the ground state of the mixed-valence species, and the relative magnitudes of the dipole moment changes for the three IVCT transitions in meso- $\left[\left\{\mathrm{Ru}(\mathrm{bpy})_{2}\right\}_{2}(\mu-\mathrm{BL})\right]^{5+}\{\mathrm{BL}=\mathrm{dpb}$, $\mathrm{dpq}^{\prime}$ \} suggest that the ground states exhibit a small degree of residual localisation, while the excited states are relatively more delocalised. The dpq'-bridged system possesses a smaller dipole moment change for IVCT(1) relative to the dpb-bridged complex, due to the shorter $\mathrm{Ru} \cdots \mathrm{Ru}$ distance, and the greater degree of electronic coupling in the former. For both complexes, the results demonstrate that the "actual" $\mathrm{H}_{\mathrm{ab}}$ values for the systems are at least a factor of three larger than those implied by calculations based solely on the geometrical distances (Table 3.5), and are closer to the values obtained from equation 1.11 in the delocalised limit.

The negative $\Delta \alpha$ and $\operatorname{Tr}(\Delta \alpha)$ values for the components (Table 3.16) are unusual, although not unprecedented, ${ }^{114,119,191}$ and also signal substantial electronic coupling in the meso- $\left[\{\operatorname{Ru}(\mathrm{bpy})\}_{2}(\mu-\mathrm{BL})\right]^{5+}$ systems. In the two-state limit (i.e. a ground state and single excited state) with superexchange coupling, significant upper-excited state configuration interactions exist which should result in greater polarisability in this state, and large positive $\Delta \alpha$ and $\operatorname{Tr}(\Delta \alpha)$ values. ${ }^{191}$ The negative polarisability changes suggest that a three-site delocalisation mechanism is operative, as proposed earlier in §3.2.2.4. This situation is illustrated schematically in Figure 1.4, where the doubly-occupied bonding orbital distributes charge simultaneously across both of the metals and the bridging ligand in the ground state. In the excited state, charge redistribution occurs from the bridging ligand to both metal centres, thus diminishing the molecular polarisability and leading to negative $\Delta \alpha$ and $\operatorname{Tr}(\Delta \alpha)$ values.

The Stark spectra for meso-[\{Os(bpy) $\left.\}_{2}(\mu-\mathrm{BL})\right]^{5+}\{\mathrm{BL}=\mathrm{dpb}, \mathrm{dpb}$ ', dpq', ppz $\}$ were obtained over the range $3850-20000 \mathrm{~cm}^{-1}$, and were fitted to a combination of multiple Gaussian components. In each case, the relative energies, intensities and bandwidths for the underlying transitions in the spectra were consistent with those reported in §3.3.5.3. Due to the errors involved in fitting the Stark spectra for the weak transitions in the NIR region $3850-6000 \mathrm{~cm}^{-1}$, and the significantly convoluted nature of the spectra, Table 3.17 presents the results for the major absorption bands only.

Figure 3.21(a) shows the IVCT band and spectral components in the region $6000-10000 \mathrm{~cm}^{-1}$ for meso-[\{Os(bpy) $\left.\}_{2}(\mu-\mathrm{ppz})\right]^{5+}$, and Figures 3.21(b) and (c) show the first and second-order derivatives of the IVCT band. The IVCT Stark signal \{Figure 3.21(d)\} was modelled in terms of a negative zerothderivative on the low energy side, and a positive $\Delta \mathrm{A}(v)$ contribution on the higher energy side. The 
negligible dipole moment changes reported in Table 3.17 are consistent with the previous classification of the system (§3.3.5.3) at the borderline between the localised-to-delocalised and delocalised regimes. The negative polarisability changes also serve to reinforce the contention that the two-site model is inapplicable for the description of the system. Interestingly, the absorption and Stark absorption lineshapes are reminiscent of those observed for the IVCT band at $6410 \mathrm{~cm}^{-1}\left(\right.$ in $\mathrm{D}_{2} \mathrm{O}$ ) in the Creutz-Taube ion, ${ }^{192}$ where the change in dipole moment of $0.7 \pm 0.1 \mathrm{D}$ was significantly smaller than the calculated value of $32.7 \mathrm{D}$ for unit electron transfer across the $6.9 \AA$ pyz bridge (1:1 glycerol:water at $77 \mathrm{~K} ; 1$ $\mathrm{MVcm}^{-1}$ field strength).

(a)

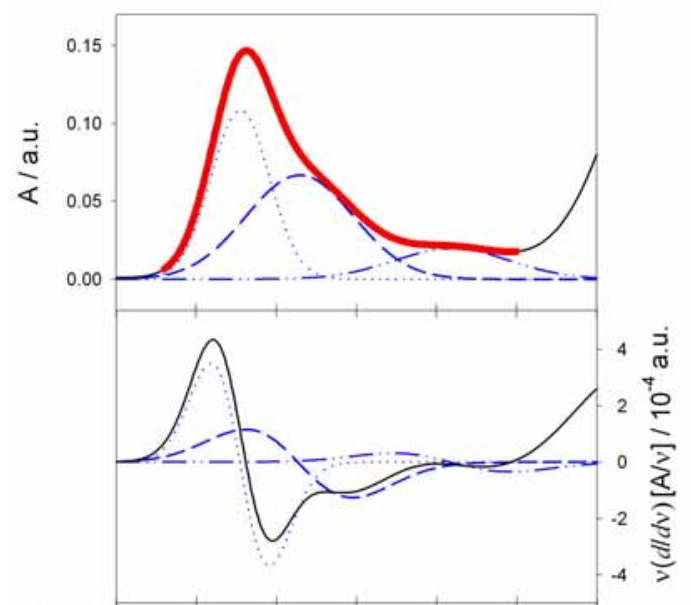

(c)

(b)

(d)

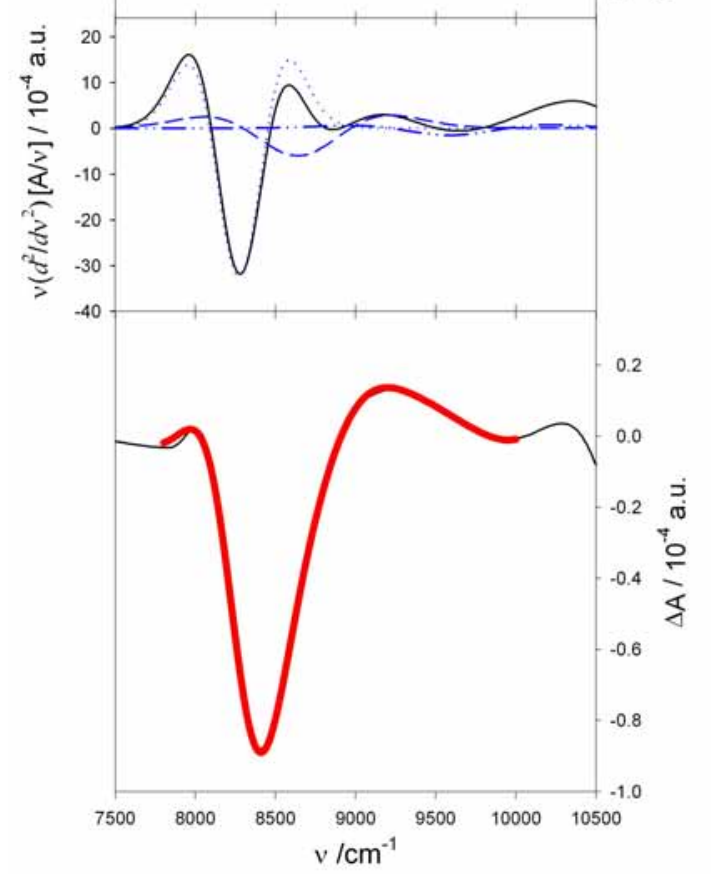

Figure 3.21 Liptay analysis of the Stark absorption spectrum (A vs. v) for meso-[(Os(bpy $\left.\left.)_{2}\right)_{2}(\mu-p p z)\right]^{5+}$ in $n$-butyronitrile glass at $77 \mathrm{~K}$, with a field strength of $4.0 \mathrm{MVcm}^{-1}$. (a) Unperturbed absorption spectrum with the bands obtained by Gaussian deconvolution and energy weighted first (b) and second (c) derivatives of the absorption spectrum. (d) Measured (一) and fitted (一) Stark signals ( $\Delta$ A vs. v) at $45^{\circ}$. 
Table 3.17 Absorption and electroabsorption properties of the NIR bands for meso-[ $\left.\left[\mathrm{OS}(\mathrm{bpy})_{2}\right\}_{2}(\mu-\mathrm{BL})\right]^{5+}\{\mathrm{BL}=$ dpb, dpq', dpb', ppz $\}$ in $n$-butyronitrile glass at $77 \mathrm{~K}$ and a field strength of $4.0 \mathrm{MVcm}^{-1}$.

\begin{tabular}{|c|c|c|c|c|c|c|}
\hline $\mathrm{BL}$ & $\begin{array}{c}v_{\max } \\
/ \mathrm{cm}^{-1}\end{array}$ & $\begin{array}{c}\Delta v_{1 / 2} \\
/ \mathrm{cm}^{-1}\end{array}$ & $\begin{array}{c}\left|\Delta \mu_{12}\right| \\
/ \mathrm{e} \AA\end{array}$ & $\begin{array}{c}\left|\Delta \mu_{12}\right| \\
/ \mathrm{D}\end{array}$ & $\begin{array}{c}\operatorname{Tr}(\Delta \alpha) \\
/ \AA^{3}\end{array}$ & $\begin{array}{c}\Delta \alpha \\
/ \AA^{3}\end{array}$ \\
\hline $\mathrm{dpb}$ & $7485^{\mathrm{b}}$ & 833 & $1.3(1) \times 10^{-4}$ & $6.5(1) \times 10^{-4}$ & 957 & 831 \\
& 8930 & 1358 & $7.46(2) \times 10^{-2}$ & $0.35(5)$ & 572 & -221 \\
& 10030 & 1698 & $2.7(1) \times 10^{-4}$ & $1.28(1) \times 10^{-3}$ & 336 & 130 \\
& 12290 & 1152 & $0.106(3)$ & $0.50(5)$ & -84.8 & -214 \\
\hline $\mathrm{dpb}^{\prime}$ & $6820^{\mathrm{b}}$ & 659 & $0.23(4)$ & $1.12(2)$ & -380 & -408 \\
& 8470 & 656 & $0.54(1)$ & $2.57(3)$ & -305 & -343 \\
& 11590 & 980 & $4.7(1) \times 10^{-4}$ & $2.24(1) \times 10^{-4}$ & -215 & 770 \\
& 12615 & 816 & $0.63(3)$ & $3.01(5)$ & -325 & -770 \\
\hline $\mathrm{dpq}$ & $7650^{\mathrm{b}}$ & 814 & $0.51(5)$ & $2.45(7)$ & 256 & -876 \\
& 9105 & 1187 & $1.3(4)$ & $6.39(3)$ & -1860 & 360 \\
& 9860 & 882 & $0.38(4)$ & $1.83(4)$ & -370 & 385 \\
& 10550 & 905 & $3.1(1) \times 10^{-4}$ & $1.48(3) \times 10^{-3}$ & -264 & -50.7 \\
\hline $\mathrm{ppz}$ & $8280^{\mathrm{b}}$ & 439 & $9(5) \times 10^{-6}$ & $4.4(1) \times 10^{-4}$ & -26.3 & -76.0 \\
& $8620^{\mathrm{b}}$ & 338 & $4.9(2) \times 10^{-2}$ & $0.23(7)$ & 102 & 256 \\
& $8900^{\mathrm{b}}$ & 576 & $1.3(1) \times 10^{-4}$ & $6.3(5) \times 10^{-4}$ & -32.9 & -5.49 \\
\hline
\end{tabular}

${ }^{a}$ The errors in the observed and calculated parameters (not tabulated) are $\pm 10 \mathrm{~cm}^{-1}$ for $v_{\max }$ and $\Delta v_{1 / 2}$ and $\pm 10 \AA^{3}$ for $\operatorname{Tr}(\Delta \alpha)$ and $\Delta \alpha$. $\xi$ was $0 \pm 2^{\circ}$ in all cases.

${ }^{\mathrm{b}}$ Absorption bands definitively assigned as IVCT transitions.

As shown in Table 3.17, the small magnitudes of the dipole moment changes are a characteristic feature of the full series of meso- $\left[\{\mathrm{Os}(\mathrm{bpy})\}_{2}(\mu-\mathrm{BL})\right]^{5+}$ complexes. Since the definitive assignment of the IVCT bands in these systems is not possible at the present time, a detailed discussion of the relative magnitudes of the molecular parameters obtained from the Liptay analysis is inappropriate. However, the results support significant delocalisation in all cases.

In summary, Stark effect measurements on the meso diastereoisomers of $\left[\left\{\mathrm{Ru}(\mathrm{bpy})_{2}\right\}_{2}(\mu-\mathrm{BL})\right]^{5+}$ $\{\mathrm{BL}=\mathrm{dpq}$ ', dpb $\}$ and $\left[\left\{\mathrm{OS}(\mathrm{bpy})_{2}\right\}_{2}(\mu-\mathrm{BL})\right]^{5+}\{\mathrm{BL}=\mathrm{dpb}$ ', dpq', ppz, dpb $\}$ reveal that the effective electron transfer distances in the mixed-valence forms of the complexes are a small fraction only of their geometrical metal-metal distances. This contradicts the previous Class II assignment for the complexes $\left[\left\{\mathrm{Os}(\mathrm{bpy})_{2}\right\}_{2}(\mu-\mathrm{BL})\right]^{5+}\{\mathrm{BL}=\mathrm{dpb}, 2,3-\mathrm{dpp}\}$ by Brewer and coworkers ${ }^{139,140}$ which was based solely on a classical two-state analysis of the NIR spectra.

\section{Shortcomings of the Experimental and Theoretical Analysis}

Boxer and coworkers ${ }^{120}$ have detailed the shortcomings of the experimental, analytical and theoretical analysis of Stark spectra, and the major issues which are relevant to the present analysis only are discussed here. Firstly, the analysis assumes that the local field correction factor ${ }^{193-196} f$ is 1.3 , however the absolute magnitude is uncertain as this depends on the solvent, chromophore and counter-ion concentrations which are known to differ between the different complexes and between the diastereoisomeric forms of the same complex. Secondly, the physical significance of the molecular parameters extracted for the multiple overlapping bands is questionable as small variations in the 
characteristics of the bands can lead to dramatic variations in the parameters derived from the Stark fitting procedure. In the present case, the deconvolutions were consistent with those reported in §3.3.2.4 and §3.3.5.3. Since one band usually dominates each manifold, a single set of Liptay parameters were initially used to extract the parameters that predominantly reflected the major component. Additional data sets were subsequently included with fixed parameters for the dominant transition to discern reliable trends in the deduced parameters. ${ }^{120,197}$ Thirdly, the formalism which underlies the Liptay analysis ${ }^{118}$ is invalid for the treatment of complexes in the localised-to-delocalised region as the band-shapes are fielddependent. The latter was exemplified in Figure 3.20(d) for meso- $\left[\left\{\operatorname{Ru}(\mathrm{bpy})_{2}\right\}_{2}(\mu-\mathrm{dpb})\right]^{5+}$.

The development of theoretical methods for the analysis of Stark effects in the localised-todelocalised and delocalised regimes represents an important thrust of current research in the area. Treynor and Boxer, ${ }^{198}$ and Reimers and Hush. ${ }^{48,199}$ have recently emphasised the importance of the contribution of the symmetric vibration mode in modelling the Stark absorption spectra of such systems. The three-state (one- and two-mode) and four-state classical models of Brunschwig, Creutz and Sutin ${ }^{38}$ have also been extended to the treatment of Stark absorption spectra in this regime. ${ }^{38,200}$ While the detailed testing of such theories is beyond the scope of the present study, the data provide a basis for assessing the validity of these methods.

\subsubsection{Diastereoisomers as Probes for Redox Asymmetry Contributions: Ion-Pairing and IVCT Thermochromism}

\subsubsection{Differential Ion-Pairing in the Diastereoisomers of $\left[\left\{\mathrm{Ru}(\mathrm{bpy})_{2}\right\}_{2}(\mu-\mathrm{BL})\right]^{5+}$}

\section{$\left\{B L=d p b, d p b^{\prime}\right.$ and $\left.d p q '\right\}$}

A comparison of the IVCT bands for the diastereoisomers of $\left[\left\{\mathrm{Ru}(\mathrm{bpy})_{2}\right\}_{2}(\mu-\mathrm{BL})\right]^{5+}\{\mathrm{BL}=\mathrm{dpb}$, dpb', dpq'\} in acetonitrile solution containing $0.1 \mathrm{M}\left[\left(n-\mathrm{C}_{4} \mathrm{H}_{9}\right)_{4} \mathrm{~N}\right] \mathrm{PF}_{6}$ and $0.02 \mathrm{M}\left[\left(n-\mathrm{C}_{4} \mathrm{H}_{9}\right)_{4} \mathrm{~N}\right]\left\{\mathrm{B}\left(\mathrm{C}_{6} \mathrm{~F}_{5}\right)_{4}\right\}$ electrolytes is provided in Figure 3.22. An overlay of the IVCT bands for the series of complexes in the latter medium is shown in Figure 3.23 and the corresponding band parameters are summarised in Table 3.18 .

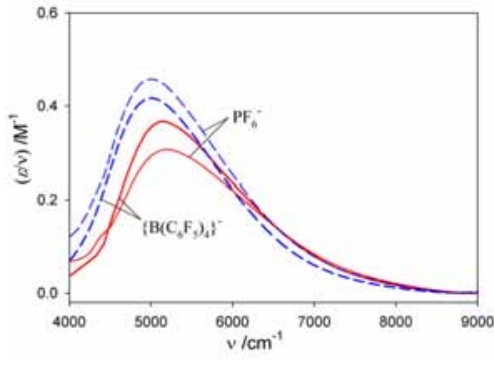

(a)

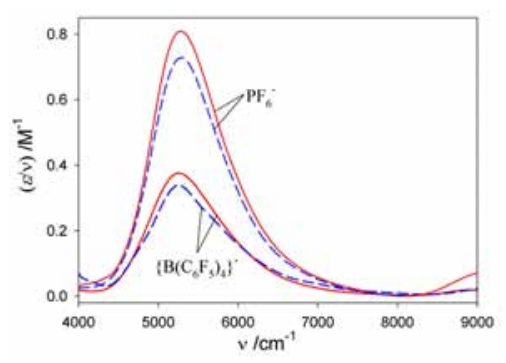

(b)

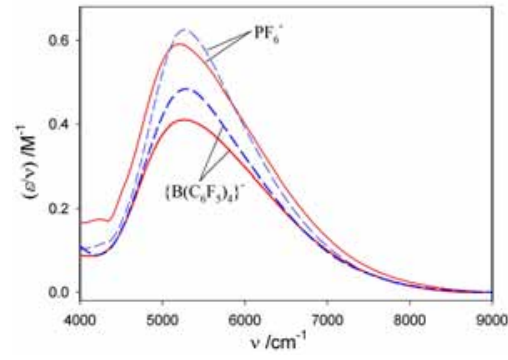

(c)

Figure 3.22 Overlay of IVCT bands in $0.1 \mathrm{M}\left[\left(n-\mathrm{C}_{4} \mathrm{H}_{9}\right)_{4} \mathrm{~N}\right] \mathrm{PF}_{6}$ and $0.02 \mathrm{M}\left[\left(n-\mathrm{C}_{4} \mathrm{H}_{9}\right)_{4} \mathrm{~N}\right]\left\{\mathrm{B}\left(\mathrm{C}_{6} \mathrm{~F}_{5}\right)_{4}\right\} / \mathrm{CH}_{3} \mathrm{CN}$ electrolytes for the diastereoisomeric forms of $\left[\left\{\mathrm{Ru}(\mathrm{bpy})_{2}\right\}_{2}(\mu-\mathrm{BL})\right]^{5+}\{\mathrm{BL}=\mathrm{dpb}(\mathrm{a}), \mathrm{dpb}(\mathrm{b})$ and dpq' (c) $\}$ at $-35^{\circ} \mathrm{C}$. 


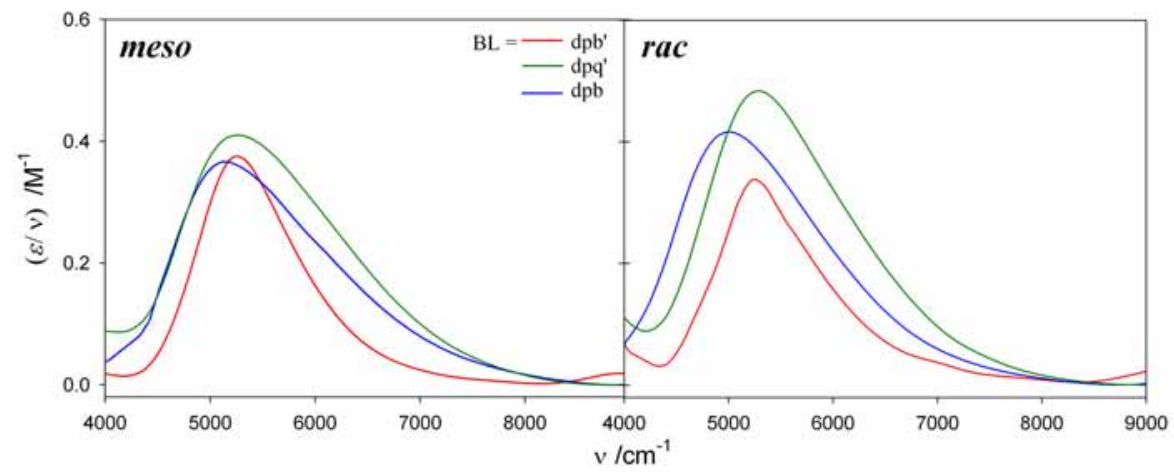

Figure 3.23 Overlay of the IVCT bands for meso- and rac- $\left[\left\{\mathrm{Ru}(\mathrm{bpy})_{2}\right\}_{2}(\mu-\mathrm{BL})\right]^{5+}\{\mathrm{BL}=\mathrm{dpb}$, dpb' and dpq' $\}$ in $0.02 \mathrm{M}\left[\left(n-\mathrm{C}_{4} \mathrm{H}_{9}\right)_{4} \mathrm{~N}\right]\left\{\mathrm{B}\left(\mathrm{C}_{6} \mathrm{~F}_{5}\right)_{4}\right\} / \mathrm{CH}_{3} \mathrm{CN}$ at $-35^{\circ} \mathrm{C}$.

Table 3.18 IVCT spectral data of the reduced absorption spectra ( $\varepsilon / v v s . v)$ for the diastereoisomeric forms of $\left[\left\{\mathrm{Ru}(\mathrm{bpy})_{2}\right\}_{2}(\mu-\mathrm{BL})\right]^{5+}\{\mathrm{BL}=\mathrm{dpb}, \mathrm{dpb}$ and dpq' $\}$ in $0.02 \mathrm{M}\left[\left(n-\mathrm{C}_{4} \mathrm{H}_{9}\right)_{4} \mathrm{~N}\right]\left\{\mathrm{B}\left(\mathrm{C}_{6} \mathrm{~F}_{5}\right)_{4}\right\} / \mathrm{CH}_{3} \mathrm{CN}$ at $-35^{\circ} \mathrm{C}$. ${ }^{\mathrm{a}}$

\begin{tabular}{|c|c|c|c|c|c|c|c|c|c|c|}
\hline BL & Diastereoisomer & $\begin{array}{c}v_{\max } \\
/ \mathrm{cm}^{-1}\end{array}$ & $\begin{array}{c}(\varepsilon / v)_{\max } \\
/ \mathrm{M}^{-1}\end{array}$ & $\begin{array}{c}\Delta v_{1 / 2} \\
/ \mathrm{cm}^{-1}\end{array}$ & $\begin{array}{c}M_{0} \\
/ \mathrm{M}^{-1}\end{array}$ & $\begin{array}{c}M_{1} \\
/ \mathrm{cm}^{-1}\end{array}$ & $\begin{array}{c}\left|\mu_{12}\right| \\
/ \mathrm{e} \AA\end{array}$ & $\begin{array}{c}\Delta v_{1 / 2}{ }^{\mathrm{o}} \\
/ \mathrm{cm}^{-1}\end{array}$ & $\begin{array}{c}\mathrm{H}_{\mathrm{ab}}^{\mathrm{b}} \\
/ \mathrm{cm}^{-1}\end{array}$ & $\Gamma$ \\
\hline $\mathrm{dpb}$ & meso & 5125 & 0.3668 & 1720 & 688 & 5615 & 0.5403 & 3070 & 403 & 0.440 \\
& rac & 4990 & 0.4206 & 1635 & 753 & 5130 & 0.5653 & 3026 & 410 & 0.460 \\
\hline $\mathrm{dpb}^{\prime}$ & meso & 5250 & 0.3928 & 1050 & 470 & 5495 & 0.4466 & 3105 & 340 & 0.662 \\
& rac & 5295 & 0.3000 & 1250 & 460 & 5540 & 0.4418 & 3118 & 340 & 0.599 \\
\hline dpq' & meso & 5230 & 0.4182 & 1755 & 802 & 5645 & 0.5834 & 3100 & 447 & 0.434 \\
& rac & 5265 & 0.4979 & 1565 & 871 & 5630 & 0.6080 & 3110 & 470 & 0.496 \\
\hline
\end{tabular}

${ }^{a}$ The errors in the observed parameters are $\pm 10 \mathrm{~cm}^{-1}$ for $v_{\max }, M_{1}$ and $\Delta v_{1 / 2}, \pm 0.0001 \mathrm{M}^{-1}$ for $(\varepsilon / v)_{\max }, \pm 5 \mathrm{M}^{-1}$ for $M_{0}$ and \pm 0.0001 eÅ for $\left|\mu_{12}\right|$.

${ }^{\mathrm{b}} \mathrm{H}_{\mathrm{ab}}$ determined from equation 3.2, where $r_{a b}$ is equated with the $\mathrm{Ru} \cdots \mathrm{Ru}$ distances from Table 3.2. For $\mathrm{BL}=\mathrm{dpb}, r_{a b}$ is taken as the average $\mathrm{Ru} \cdots \mathrm{Ru}$ distance of $6.870(3) \AA$ from the structures involving $\mathrm{PF}_{6}{ }^{-}$and $\left[\mathrm{ZnCl}_{4}\right]^{2-}$ counter-ions.

The trends in the IVCT band parameters for the series of complexes in the presence of $\left\{\mathrm{B}\left(\mathrm{C}_{6} \mathrm{~F}_{5}\right)_{4}\right\}^{-}$differ from those reported previously in $\mathrm{PF}_{6}{ }^{-}$medium (§3.3.2.4). In general, the IVCT bands are narrower for the full series of complexes in the $\left\{\mathrm{B}\left(\mathrm{C}_{6} \mathrm{~F}_{5}\right)_{4}\right\}^{-}$electrolyte, and the parameters $(\varepsilon / v)_{\max }$, $\left|\mu_{12}\right|, M_{0}$ and $\mathrm{H}_{\mathrm{ab}}$ are decreased for all species except meso-[\{Ru(bpy $\left.\left.)_{2}\right\}_{2}(\mu-\mathrm{dpb})\right]^{5+}$. The bands are redshifted for the dpb- and dpb'-bridged diastereoisomers, and blue-shifted for the dpq'-bridged diastereoisomers relative to their positions in $\mathrm{PF}_{6}{ }^{-}$electrolyte. For $\left[\left\{\mathrm{Ru}(\mathrm{bpy})_{2}\right\}_{2}(\mu-\mathrm{BL})\right]^{5+}\{\mathrm{BL}=\mathrm{dpq}$, $d p b '\}$, small differences are observed in the IVCT energies between the diastereoisomers of the same complex. The difference of $205 \mathrm{~cm}^{-1}$ between the IVCT energies for the diastereoisomers of $\left[\left\{\mathrm{Ru}(\mathrm{bpy})_{2}\right\}_{2}(\mu-\mathrm{dpb})\right]^{5+}$ in $\mathrm{PF}_{6}{ }^{-}$electrolyte (Table 3.5) is maintained in $\left\{\mathrm{B}\left(\mathrm{C}_{6} \mathrm{~F}_{5}\right)_{4}\right\}^{-}$medium, where $v_{\max }$ and $M_{1}$ differ by 135 and $487 \mathrm{~cm}^{-1}$, respectively. The $\Gamma$ parameter exhibits the same trend in both electrolytes: for the meso diastereoisomers, $\Gamma$ increases as BL is varied from dpq' to dpb to dpb', while $\Gamma$ increases as BL is varied from dpb to dpq' to dpb' for the rac forms. The results indicate that the systems lie close to the localised-to-delocalised transition.

The significant difference in the IVCT energies between the diastereoisomers of $\left[\left\{\mathrm{Ru}(\mathrm{bpy})_{2}\right\}_{2}(\mu-\right.$ $\mathrm{dpb})]^{5+}$ is consistent with the presence of a differential redox asymmetry contribution, $\Delta \mathrm{E}_{\text {struct. }}$ The magnitude of $\Delta \mathrm{E}_{\text {struct }}$ is also dependent on the identity of the counter-ion, which influences the degree of 
structural distortion. Indeed, the presence of ion-pairing induced structural distortions between the diastereoisomeric forms was observed in the different solid-state structures of the meso diastereoisomer with $\mathrm{PF}_{6}{ }^{-}$and $\left[\mathrm{ZnCl}_{4}\right]^{2-}$ counter-ions (§3.3.2.1). The dependence of the IVCT characteristics on the identity of the electrolyte anion for the diastereoisomers of the dpq'- and dpb'-bridged complexes, which exhibit relatively less structural distortion, suggests that ion-pairing has additional microscopic consequences on the factors which contribute to the electron transfer barrier (equation 3.1).

While the dependence of the IVCT parameters on the identity of the electrolyte anion is unexpected on the basis of equation 3.1, ion-pairing effects have been shown to influence IVCT energies and bandwidths for a number of mixed-valence systems. ${ }^{34,36,37,95,102,112}$ Qualitatively, the observations in the present study are similar to previous literature reports: the addition of the relatively strongly ionpairing electrolyte $\mathrm{PF}_{6}{ }^{-}$is accompanied by blue-shifts in the IVCT bands. The formation of the ion-pair may be regarded as a redox asymmetry contribution, or as a component of the reorganisational energy due to translation of the counter-ion between the donor and acceptor. ${ }^{17}$

IVCT thermochromism measurements for the diastereoisomeric forms of the series of complexes $\left[\left\{\mathrm{Ru}(\mathrm{bpy})_{2}\right\}_{2}(\mu-\mathrm{BL})\right]^{5+}\{\mathrm{BL}=\mathrm{dpb}, \mathrm{dpb}$ ', dpq' and 2,3-dpp $\}$ offer additional insights into ion-pairing and temperature-dependent effects on the IVCT transitions.

\subsubsection{IVCT Thermochromism in the Diastereoisomers of $\left[\left\{\operatorname{Ru}(\mathbf{b p y})_{2}\right\}_{2}(\mu-d p b)\right]^{5+}$}

A comparative study of IVCT thermochromism in the diastereoisomers of $\left[\left\{\mathrm{Ru}(\mathrm{bpy})_{2}\right\}_{2}(\mu-\mathrm{dpb})\right]^{5+}$ was performed in $n$-butyronitrile solution containing $0.1 \mathrm{M}\left[\left(n-\mathrm{C}_{4} \mathrm{H}_{9}\right)_{4} \mathrm{~N}\right] \mathrm{PF}_{6}$ or $0.02 \mathrm{M}$ $\left[\left(n-\mathrm{C}_{4} \mathrm{H}_{9}\right)_{4} \mathrm{~N}\right]\left\{\mathrm{B}\left(\mathrm{C}_{6} \mathrm{~F}_{5}\right)_{4}\right\}$ over the temperature range $-45^{\circ} \mathrm{C}(228 \mathrm{~K})$ to $-15^{\circ} \mathrm{C}(258 \mathrm{~K})$. The results for the temperature dependence of $v_{\max }, M_{1}$ and $\Delta v_{1 / 2}$ are presented in Figures 3.24, Figure B3.7 and Tables B3.20-21 (Appendix B).

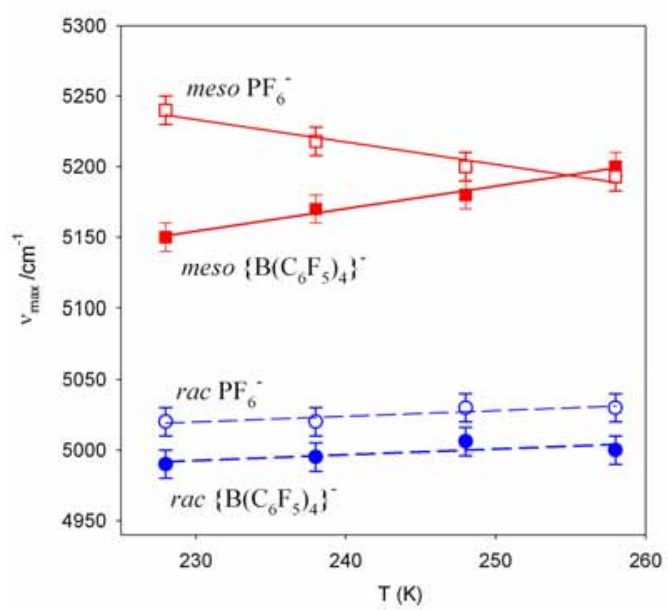

Figure 3.24 Temperature dependence of $v_{\max }$ for the meso (-) and rac (---) diastereoisomers of $\left[\left\{\mathrm{Ru}(\mathrm{bpy})_{2}\right\}_{2}(\mu-\right.$ $\mathrm{dpb})]^{5+}$ in $0.1 \mathrm{M}\left[\left(n-\mathrm{C}_{4} \mathrm{H}_{9}\right)_{4} \mathrm{~N}\right] \mathrm{PF}_{6}$ or $0.02 \mathrm{M}\left[\left(n-\mathrm{C}_{4} \mathrm{H}_{9}\right)_{4} \mathrm{~N}\right]\left\{\mathrm{B}\left(\mathrm{C}_{6} \mathrm{~F}_{5}\right)_{4}\right\} / n$-butyronitrile.

As shown in Figure 3.24, the energies of the IVCT bands for the diastereoisomeric forms of $\left[\left\{\mathrm{Ru}(\mathrm{bpy})_{2}\right\}_{2}(\mu-\mathrm{dpb})\right]^{5+}$ exhibit different temperature dependencies in the presence of $\mathrm{PF}_{6}{ }^{-}$and 
$\left\{\mathrm{B}\left(\mathrm{C}_{6} \mathrm{~F}_{5}\right)_{4}\right\}^{-}$, which also differ between the diastereoisomeric forms of the complex. The energies of the IVCT maxima for the rac diastereoisomers are relatively insensitive to temperature $\left(\mathrm{d} v_{\max } / \mathrm{dT}=0.41 \pm\right.$ $0.24 \mathrm{~cm}^{-1} \mathrm{~K}^{-1}$ in $\mathrm{PF}_{6}{ }^{-}$and $0.40 \pm 0.14 \mathrm{~cm}^{-1} \mathrm{~K}^{-1}$ in $\left.\left\{\mathrm{B}\left(\mathrm{C}_{6} \mathrm{~F}_{5}\right)_{4}\right\}^{-}\right)$, and the band is blue-shifted by $30 \mathrm{~cm}^{-1}$ in the presence of $\mathrm{PF}_{6}{ }^{-}$. For the meso diastereoisomers, $v_{\max }$ exhibits a red-shift with increasing temperature in $\mathrm{PF}_{6}{ }^{-}$electrolyte, compared with a blue-shift in $\left\{\mathrm{B}\left(\mathrm{C}_{6} \mathrm{~F}_{5}\right)_{4}\right\}^{-}$electrolyte $\left(\mathrm{d} v_{\max } / \mathrm{dT}=-1.59 \pm 0.24 \mathrm{~cm}^{-1} \mathrm{~K}^{-1}\right.$ in $\mathrm{PF}_{6}{ }^{-}$and $1.60 \pm 0.14 \mathrm{~cm}^{-1} \mathrm{~K}^{-1}$ in $\left.\left\{\mathrm{B}\left(\mathrm{C}_{6} \mathrm{~F}_{5}\right)_{4}\right\}^{-}\right)$.

IVCT thermochromism measurements have been used to assess the degree of delocalisation in mixed-valence systems. ${ }^{9}$ According to the semi-classical adiabatic two-state treatment ${ }^{40}$ in equation 3.8 , the temperature dependence of $v_{\max }$ is given by $\mathrm{d}\left(\Delta \mathrm{E}_{0}\right) / \mathrm{dT}$ in the localised limit (i.e. $\lambda_{i}+\lambda_{0}+\Delta \mathrm{E}+\Delta \mathrm{E}^{\prime}$ » $2 \mathrm{H}_{\mathrm{ab}}$ ), but should approach zero in the delocalised limit (i.e. $\lambda_{i}+\lambda_{o}+\Delta \mathrm{E}+\Delta \mathrm{E}^{\prime}$ « $2 \mathrm{H}_{\mathrm{ab}}$ ), regardless of the magnitude of $\mathrm{d}\left(\Delta \mathrm{E}_{0}\right) / \mathrm{dT}$ (assuming that $\lambda_{i}, \lambda_{0}$ and $\mathrm{H}_{\mathrm{ab}}$ are temperature independent quantities) ${ }^{98}$

$$
v_{\max }=\left[\left(\lambda_{i}+\lambda_{0}+\Delta \mathrm{E}_{0}+\Delta \mathrm{E}^{\prime}\right)^{2}+4 \mathrm{H}_{\mathrm{ab}}^{2}\right]^{1 / 2}
$$

The negligible temperature dependence of $v_{\max }$ for $\left.r a c-\left\{\mathrm{Ru}(\mathrm{bpy})_{2}\right\}_{2}(\mu-\mathrm{dpb})\right]^{5+}$ in both electrolytes is suggestive of a near-delocalised classification. By comparison, $v_{\max }$ exhibits a greater temperature dependence for the meso diastereoisomer in both electrolytes, which is indicative of localisation, and a temperature dependence of the redox asymmetry.

Two sources of redox asymmetry have been identified as the inherent structural asymmetry, $\Delta \mathrm{E}_{\text {struct, }}$, and ion-pairing asymmetry. ${ }^{36,37,201,202}$ The latter is more pronounced at lower temperatures, and is likely to occur preferentially for the meso compared with the rac diastereoisomeric form. Indeed, the stronger interaction of the meso form with tosylate anions accounts for the increased rate of elution of this diastereoisomer in the cation-exchange separation process. ${ }^{65,66,129}$ At $\mathrm{T}=0 \mathrm{~K}$, the differences between

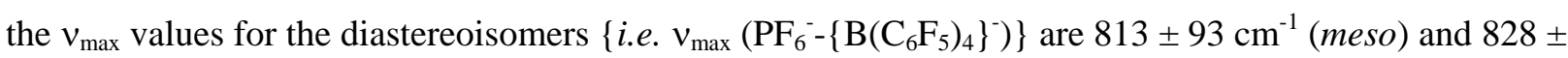
$92 \mathrm{~cm}^{-1}$ (rac), which indicates that ion-pairing induces an equivalent ground-state redox asymmetry contribution $\left(\Delta \mathrm{E}_{0}\right)$ for both diastereoisomers. The ion-pairing contribution may alternatively be treated as a reorganisational energy rather than a source of redox asymmetry. For the meso diastereoisomer a decrease in ion-pairing and a red-shift in $v_{\max }$ occur with increasing temperature as the associated ion is driven away from the complex. However, a temperature dependence of opposite sign is also evident for this diastereoisomer in the lower ion-pairing electrolyte $\left\{\mathrm{B}\left(\mathrm{C}_{6} \mathrm{~F}_{5}\right)_{4}\right\}^{-}$, which demonstrates that a temperature-dependent ion-pairing contribution only partly accounts for the trends observed.

The residual temperature dependence of $v_{\max }$ for the meso diastereoisomer in $\left\{\mathrm{B}\left(\mathrm{C}_{6} \mathrm{~F}_{5}\right)_{4}\right\}^{-}$medium suggests that the temperature variation leads to structural changes. The inherent stereochemicallyinduced redox asymmetry difference between the diastereoisomers $\left\{\right.$ i.e. $v_{\max }$ (meso-rac) $\}$ is evaluated from Figure 3.24 at $\mathrm{T}=0 \mathrm{~K}$ as $671 \pm 93 \mathrm{~cm}^{-1}$ and $686 \pm 93 \mathrm{~cm}^{-1}$ in $\mathrm{PF}_{6}{ }^{-}$and $\left\{\mathrm{B}\left(\mathrm{C}_{6} \mathrm{~F}_{5}\right)_{4}\right\}^{-}$electrolytes, respectively. While the temperature dependence may arise from a $\mathrm{d}\left(\Delta \mathrm{E}_{\text {struct }}\right) / \mathrm{dT}$ component, a more likely explanation is the presence of conformational isomers, the proportions of which differ with temperature. 
If the structures of the conformers differ sufficiently, their IVCT bands will overlap. According to equation $1.8,{ }^{1,2} \Delta v_{1 / 2}$ should vary linearly with $\mathrm{T}^{1 / 2}$, and the bands should narrow with decreasing temperature. The absence of this correlation (as shown in Figure B3.7(a), Appendix B), and the greater deviation from linearity for the meso diastereoisomer, supports an explanation based on the contribution of conformational isomers.

An examination of the temperature variation of $M_{1}{ }^{17}$ reveals weak dependencies for both diastereoisomers, as shown in Figure B3.7(b) \{Appendix B\}. This dependence arises due to changes in the frequencies and quantum spacings for the coupled vibrations and solvent modes which introduce an entropic change, $\Delta S^{\circ}$, due to the dependence on the free energy, ${ }^{17,95} \Delta \mathrm{G}^{\circ}$, according to equation 3.9:

$$
M_{1}=\sum_{j} \lambda_{j}^{\prime}+\Delta \mathrm{G}^{\circ}+\Delta \mathrm{E}^{\prime}=\lambda+\Delta \mathrm{H}^{\circ}-\mathrm{T} \Delta \mathrm{S}^{\circ}+\Delta \mathrm{E}^{\prime}
$$

Here, $\Delta \mathrm{H}^{\circ}$ and $\Delta \mathrm{S}^{\circ}$ are the enthalpy and entropy, respectively, and $\lambda_{j}^{\prime}$ is the reorganisational energy for mode $j$ along the excited-state energy surface. Accordingly, the temperature dependence of $M_{1}$ is given by $\mathrm{d} M_{1} / \mathrm{dT}=-\Delta \mathrm{S}^{\circ}$. As shown in Figure A3.7, $M_{1}$ exhibits a non-linear dependence on $\mathrm{T}$, which is opposite in sign for the diastereoisomers in both $\mathrm{PF}_{6}{ }_{6}$ and $\left\{\mathrm{B}\left(\mathrm{C}_{6} \mathrm{~F}_{5}\right)_{4}\right\}^{-}$electrolytes. This suggests that the enhanced temperature dependent ion-pairing contributions for the meso relative to the rac form are manifested primarily in the temperature dependence of the redox-asymmetry component of $v_{\max }$, rather than in the $\Delta \mathrm{S}^{\circ}$ component of $M_{1}$.

In summary, the present results serve to reinforce the findings of previous studies which have demonstrated the influence of ion-pairing on the energies of the IVCT bands, ${ }^{17,36,37,95,201,202}$ and reveal the subtle influence of stereochemically-directed anion effects on the redox asymmetry contribution in equation 3.1. In addition to the differential ion-pairing interactions, the inherent stereochemicallyinduced redox asymmetry difference between the diastereoisomers $\left(\Delta \mathrm{E}_{\text {struct }}\right)$ has been evaluated from the temperature dependence of $v_{\max }$. The origin of the remaining temperature-dependent component of $v_{\max }$ for the meso diastereoisomer in $\left\{\mathrm{B}\left(\mathrm{C}_{6} \mathrm{~F}_{5}\right)_{4}\right\}^{-}$medium may arise from the greater influence of conformational isomerism relative to the rac form, or from a temperature dependence of the solvent reorganisational energy. ${ }^{203}$

\subsubsection{IVCT Thermochromism in the Diastereoisomers of $\left[\left\{\operatorname{Ru}(\mathrm{bpy})_{2}\right\}_{2}(\mu-\mathrm{BL})\right]^{5+}$ $\{B L=d p b, d p b ', d p q ', 2,3-d p p\}$}

The IVCT thermochromism properties of meso- and rac-[\{Ru(bpy $\left.\left.)_{2}\right\}_{2}(\mu-\mathrm{BL})\right]^{5+}\{\mathrm{BL}=\mathrm{dpb}, \mathrm{dpb}$, dpq', 2,3-dpp \} were investigated to further elucidate the origins of the temperature-dependent contributions to the IVCT parameters. Figures 3.25(a) and (b) show the temperature variation of the IVCT bands for meso- and rac-[\{Ru(bpy $\left.\left.)_{2}\right\}_{2}(\mu-\mathrm{dpq})\right]^{5+}$, and a summary of the temperature dependence of $v_{\max }$ for the full series of complexes in $0.02 \mathrm{M}\left[\left(n-\mathrm{C}_{4} \mathrm{H}_{9}\right) \mathrm{N}\right]\left\{\mathrm{B}\left(\mathrm{C}_{6} \mathrm{~F}_{5}\right)_{4}\right\} / n$-butyronitrile over the temperature range $-45^{\circ} \mathrm{C}(228 \mathrm{~K})$ to $-15^{\circ} \mathrm{C}(258 \mathrm{~K})$. The temperature dependence of $\Delta v_{1 / 2}$ and $M_{1}$ are 
shown in Figure B3.8 (Appendix B), and the IVCT parameters for the series of complexes are reported in Tables B3.22-24 (Appendix B). The comparison of the IVCT thermochromism results for the dpbbridged diastereoisomers with those incorporating the relatively rigid ligands dpb' and dpq' permits an assessment of the thermochromic effect on the degree of delocalisation for the systems that lie in the localised-to-delocalised regime.

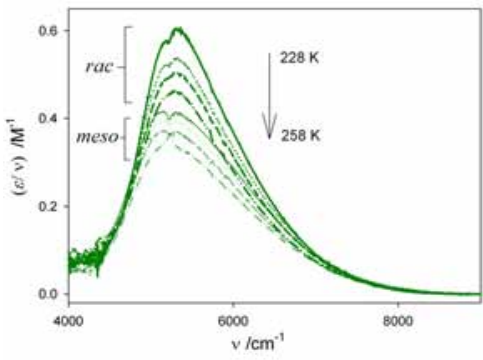

(a)

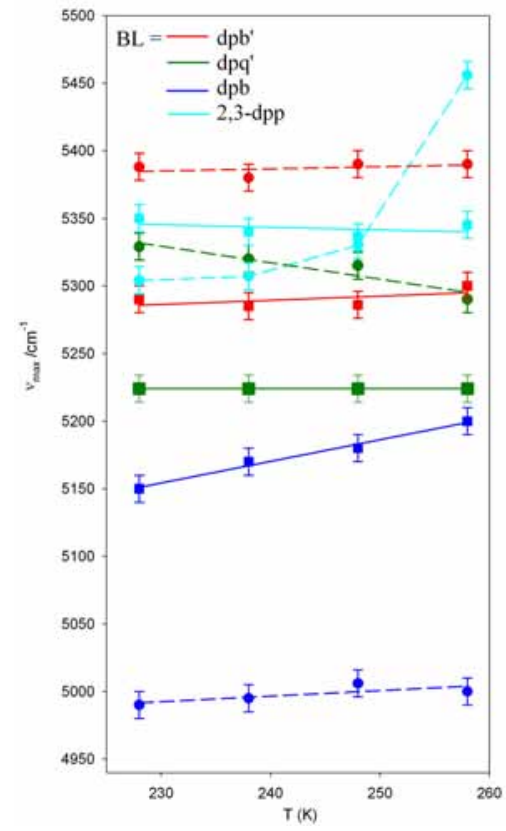

(b)

Figure 3.25 (a) Temperature variation of the IVCT band for the diastereoisomers of $\left[\left\{\mathrm{Ru}(\mathrm{bpy})_{2}\right\}_{2}(\mu \text {-dpq') }]^{5+}\right.$ in $0.02 \mathrm{M}\left[\left(n-\mathrm{C}_{4} \mathrm{H}_{9}\right)_{4} \mathrm{~N}\right]\left\{\mathrm{B}\left(\mathrm{C}_{6} \mathrm{~F}_{5}\right)_{4}\right\} / n$-butyronitrile. The arrow indicates the direction of the band shift with increasing temperature (228 K to $258 \mathrm{~K})$. (b) Temperature dependence of $v_{\max }$ for the meso (solid line) and rac (dashed line) diastereoisomers of $\left[\left\{\mathrm{Ru}(\mathrm{bpy})_{2}\right\}_{2}(\mu-\mathrm{BL})\right]^{5+}\{\mathrm{BL}=\mathrm{dpb}$, dpb', dpq', 2,3-dpp $\}$ in $0.02 \mathrm{M}\left[\left(n-\mathrm{C}_{4} \mathrm{H}_{9}\right)_{4} \mathrm{~N}\right]\left\{\mathrm{B}\left(\mathrm{C}_{6} \mathrm{~F}_{5}\right)_{4}\right\} / n-$ butyronitrile.

As shown in Figure 3.25(b), the temperature dependencies of the IVCT parameters differ between the series of complexes, and between the diastereoisomeric forms of the same complex. For both diastereoisomers of $\left[\left\{\mathrm{Ru}(\mathrm{bpy})_{2}\right\}_{2}\left(\mu-\mathrm{dpb}^{\prime}\right)\right]^{5+}, v_{\max }$ and $\Delta v_{1 / 2}$ exhibit a negligible temperature dependence, which is consistent with a delocalised classification. A $100 \mathrm{~cm}^{-1}$ difference between the $v_{\max }$ values for the diastereoisomers is maintained over the temperature range examined. For meso- $\left[\left\{\mathrm{Ru}(\mathrm{bpy})_{2}\right\}_{2}(\mu-\right.$ $\left.\left.\mathrm{dpq}^{\prime}\right)\right]^{5+}, v_{\max }$ is invariant with temperature, while $v_{\max }$ decreases with increasing temperature for the rac form $\left(\mathrm{d} v_{\max } / \mathrm{dT}=-1.22 \pm 0.31 \mathrm{~cm}^{-1} \mathrm{~K}^{-1}\right) . \Delta \mathrm{E}_{0}$ is zero for the complexes incorporating the fused dpq' and dpb' bridging ligands, and ion-pairing influences are minimised by the use of $\left[\left(n-\mathrm{C}_{4} \mathrm{H}_{9}\right)_{4} \mathrm{~N}\right]\left\{\mathrm{B}\left(\mathrm{C}_{6} \mathrm{~F}_{5}\right)_{4}\right\}$ electrolyte, so that the weak temperature dependence of meso- $\left[\left\{\mathrm{Ru}(\mathrm{bpy})_{2}\right\}_{2}(\mu-\mathrm{dpq})\right]^{5+}$ may arise from a temperature dependence of the solvent reorganisational energy. ${ }^{203}$ Temperature-dependent measurements in a range of solvents would serve to clarify this issue, but were beyond the scope of the present study.

A comparison between the IVCT thermochromism properties of the complexes incorporating the $\mathrm{dpb}$ and 2,3-dpp bridging ligands addresses the contribution of conformational isomerism discussed in §3.3.7.2. An overlay of the IVCT bands for the latter is provided in Figure B3.9 (Appendix B). Variable 
temperature ${ }^{1} \mathrm{H}$ NMR measurements ${ }^{65}$ on the diastereoisomers of $\left[\left\{\mathrm{Ru}(\mathrm{bpy})_{2}\right\}_{2}(\mu \text {-2,3-dpp) }]^{4+}\right.$ revealed the presence of two conformers in approximately equal proportions for the meso form at $-50^{\circ} \mathrm{C}$, compared with a ca. 4:1 ratio for the rac form. The interconversion between the conformers increased as the temperature was raised. In the present case, the IVCT thermochromism measurements reveal that $v_{\max }$ and $\Delta v_{1 / 2}$ are relatively invariant with temperature for the meso diastereoisomer, while the rac form exhibits a blue-shift in $v_{\max }$ and a narrowing of the bandwidth with increasing temperature. The latter may reflect the transition towards a single averaged conformation with increasing temperature. The blueshift in $v_{\max }$ and the greater temperature dependence of the IVCT parameters for the rac diastereoisomer would reflect the greater internal reorganisational energy attending electron transfer due to the conformational fluctuation in the bridging ligand in this form. Similar variations in the IVCT parameters are also evident for the dpb-bridged diastereoisomers, although in this case the meso form exhibits a more pronounced temperature dependence compared with the rac form. Temperature-dependent NMR measurements would serve to clarify the contribution of conformational isomers for the dpb-bridged case.

For complexes in the localised-to-delocalised regime, the classical two-state model attributes the asymmetry of the IVCT bands to the "band cut-off effect" on the low energy side due to thermal population at the top of the electron transfer barrier. ${ }^{14}$ According to this model, the IVCT band should attain a more symmetrical appearance with decreasing temperature. In the present case, the band asymmetry for all complexes is maintained over the temperature range examined and an explanation for the band asymmetry based on the "cut-off effect" does not appear to be valid.

\subsubsection{Diastereoisomers as Probes for Solvent Reorganisational Contributions}

\subsubsection{IVCT Solvatochromism in a Series of Angular Complexes}

IVCT solvatochromism measurements on the diastereoisomers of $\left[\left\{\mathrm{Ru}(\mathrm{bpy})_{2}\right\}_{2}(\mu-\mathrm{BL})\right]^{5+}$ $\{\mathrm{BL}=\mathrm{dpb}, \mathrm{dpb}$ ' and dpq' $\}$ were performed in a series of solvents including acetonitrile $\left\{\mathrm{CH}_{3} \mathrm{CN}, \mathrm{AN}\right\}$, propionitrile $\left\{\mathrm{CH}_{3} \mathrm{CH}_{2} \mathrm{CN}, \mathrm{PN}\right\}, n$-butyronitrile $\left\{\mathrm{CH}_{3}\left(\mathrm{CH}_{2}\right)_{2} \mathrm{CN}, \mathrm{BN}\right\}$, iso-butyronitrile $\left\{\left(\mathrm{CH}_{3}\right)_{2} \mathrm{HCCN}\right.$, $\left.{ }^{\mathrm{i}} \mathrm{BN}\right\}$, acetone $\left\{\left(\mathrm{CH}_{3}\right)_{2} \mathrm{CO}, \mathrm{AO}\right\}$ and dichloromethane $\left\{\mathrm{CH}_{2} \mathrm{Cl}_{2}, \mathrm{DCM}\right\}$ at $-35^{\circ} \mathrm{C}$. Experiments were performed in $0.02 \mathrm{M}\left[\left(n-\mathrm{C}_{4} \mathrm{H}_{9}\right)_{4} \mathrm{~N}\right]\left\{\mathrm{B}\left(\mathrm{C}_{6} \mathrm{~F}_{5}\right)_{4}\right\}$ electrolyte ${ }^{100}$ with the solution containing a uniform low concentration of the given diastereoisomer $\left(0.40 \times 10^{-3} \mathrm{M}\right)$ to eliminate ion-pairing artefacts which are known to influence the IVCT characteristics (§3.3.7). The results for the IVCT characteristics as a function of $1 / \mathrm{D}_{\mathrm{op}}-1 / \mathrm{D}_{\mathrm{s}}$ are reported in Tables B3.25-27 (Appendix B). Panel (A) in Figure 3.26 displays an overlay of the solvent dependence of $v_{\max }, \Delta v_{1 / 2}$ and $M_{1}$, and panel (B) displays the difference between the IVCT parameters for diastereoisomeric forms of each complex. 
(A)

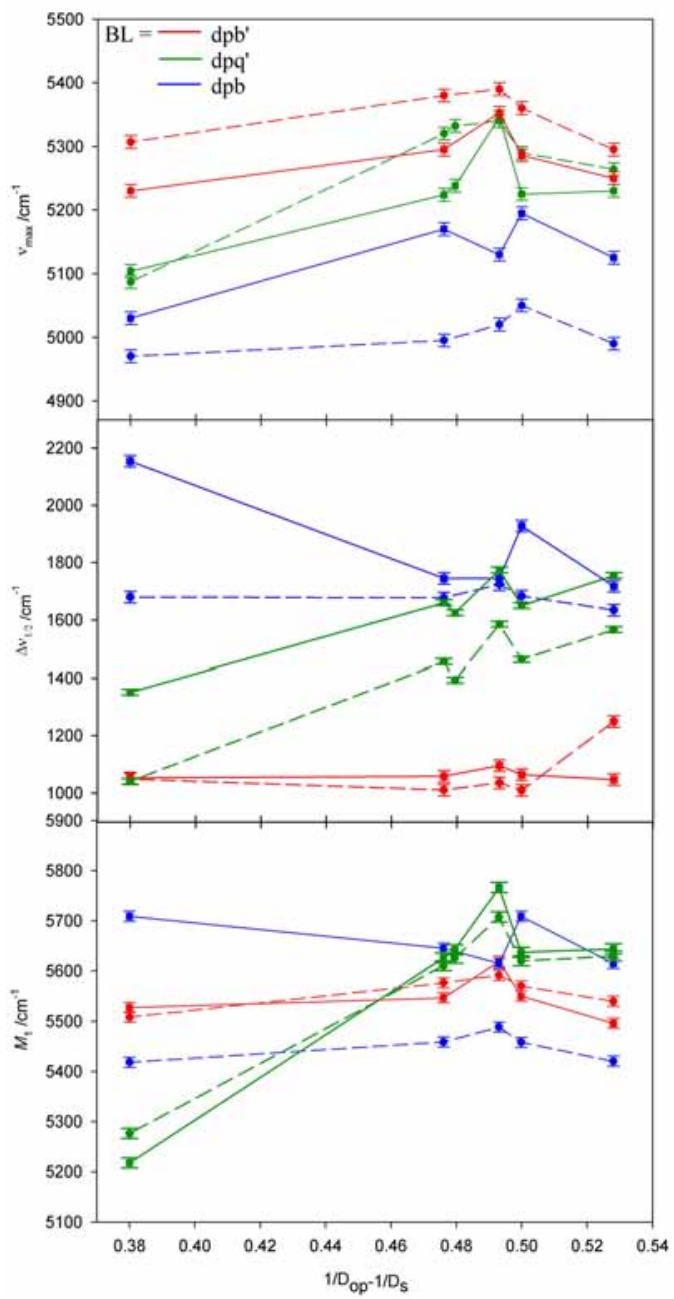

(B)

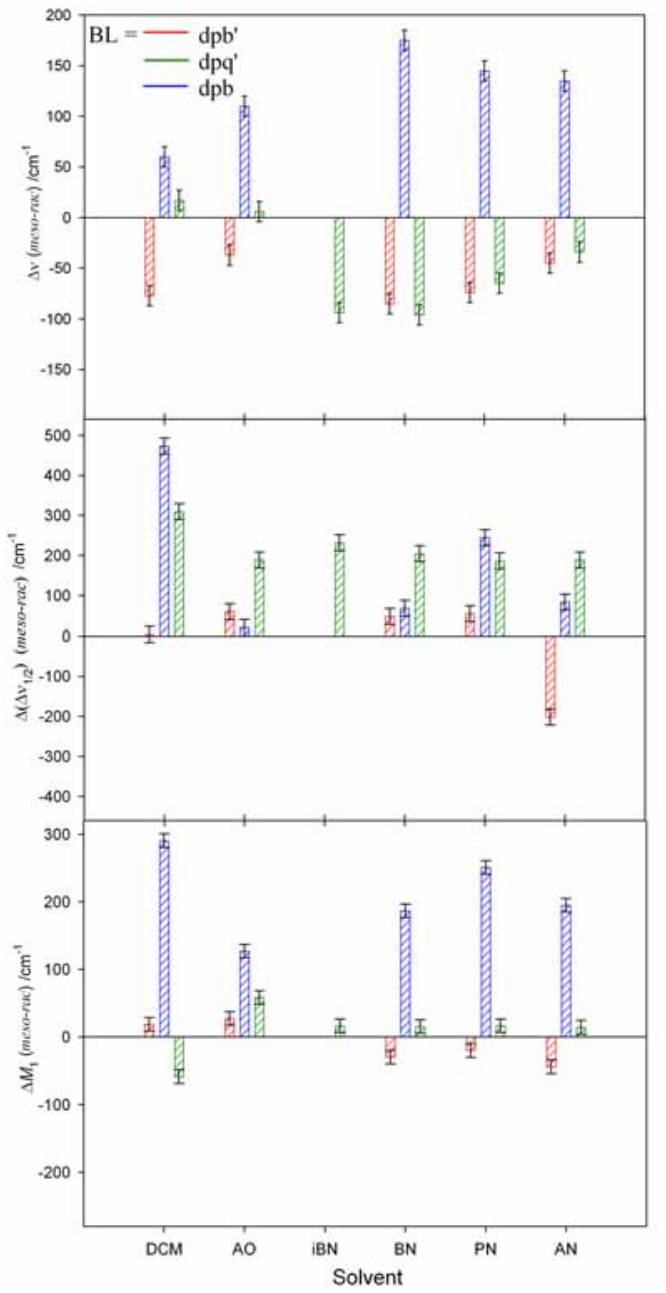

Figure 3.26 (A) Solvent dependence of $v_{\max }, \Delta v_{1 / 2}$ and $M_{1}$ for the meso (solid line) and rac (dashed line) diastereoisomers of $\left[\left\{\mathrm{Ru}(\mathrm{bpy})_{2}\right\}_{2}(\mu-\mathrm{BL})\right]^{5+}\{\mathrm{BL}=\mathrm{dpb}, \mathrm{dpb}$ ', dpq' $\}$ in $0.02 \mathrm{M}\left[\left(n-\mathrm{C}_{4} \mathrm{H}_{9}\right) \mathrm{N}\right]\left\{\mathrm{B}\left(\mathrm{C}_{6} \mathrm{~F}_{5}\right)_{4}\right\} /$ solvent at $35^{\circ} \mathrm{C}$. (B) Differential energies, bandwidths and first moments of the IVCT transitions for the diastereoisomers. Note that only the dpq'-bridged complex was measured in ${ }^{\mathrm{i}} \mathrm{BN}$.

In accordance with the dielectric continuum model (equation 1.15), $v_{\max }$ should exhibit a linear dependence on $1 / \mathrm{D}_{\mathrm{op}}-1 / \mathrm{D}_{\mathrm{s}}$, with slope $e^{2}\left(1 / \mathrm{D}_{\mathrm{op}}-1 / \mathrm{D}_{\mathrm{s}}\right)$ and intercept $\lambda_{i}+\Delta \mathrm{E}_{0}+\Delta \mathrm{E}^{\prime}{ }^{1,2}$ In general, the IVCT parameters for the series of complexes exhibit a weak dependence on $1 / \mathrm{D}_{\mathrm{op}}-1 / \mathrm{D}_{\mathrm{s}}$, as shown in panel (A) of Figure 3.26, which is consistent with charge transfer transitions involving a minimal dipole moment change, a small residual barrier to intramolecular electron transfer, and the classification of the complexes as borderline localised-to-delocalised systems. ${ }^{14}$ Table B3.28 (Appendix B) summarises the results for the slopes and intercepts of the solvatochromism plots of $v_{\max }$ as a function of $1 / \mathrm{D}_{\mathrm{op}}-1 / \mathrm{D}_{\mathrm{s}}$.

Despite the weak solvent dependence of $v_{\max }$, differences are evident in the solvatochromism plots of $M_{1}$ as a function of $1 / \mathrm{D}_{\mathrm{op}}-1 / \mathrm{D}_{\mathrm{s}}$ between the complexes, and between the diastereoisomeric forms of the same complex. The $M_{1}$ values are relatively invariant to solvent for the dpb'- and dpb-bridged complexes, while the dpq'-bridged diastereoisomers exhibit a greater solvent dependence, as given by the slope $1093 \pm 526 \mathrm{~cm}^{-1} \AA^{-1}$ (for both diastereoisomers). The approximately linear trends in $M_{1}$ as a function of $1 / \mathrm{D}_{\mathrm{op}}-1 / \mathrm{D}_{\mathrm{s}}$ for the latter are striking, as the validity of the dielectric continuum model ${ }^{1,2}$ for 
the present analysis is questionable on the following bases: (i) the two-state model is inappropriate for complexes exhibiting moderately strong electronic coupling, and (ii) the Ru(bpy) ${ }_{2}$ coordination spheres are likely to interpenetrate, and invalidate the assumption $d » 2 a^{*}$.

The different IVCT solvatochromism properties arise from the variation in the electronic and structural characteristics of the bridging ligands, since the coordination geometries of the constituent metal centres are identical for a given diastereoisomer across the series of complexes. The negligible slopes of the $v_{\max }$ and $M_{1}$ solvatochromism plots for the dpb- and dpb'-bridged diastereoisomers demonstrate that both complexes are "solvent delocalised", while the weak solvent dependence for the dpq'-bridged diastereoisomers suggests that the systems exhibit a small degree of residual localisation. The trends in $M_{1}$ are also mirrored by the solvent dependencies of $\Delta v_{1 / 2}$ shown in panel (A) of Figure 3.26. The dpb'-bridged diastereoisomers exhibit the narrowest (solvent invariant) bands, while $\Delta v_{1 / 2}$ decreases with $1 / \mathrm{D}_{\mathrm{op}}-1 / \mathrm{D}_{\mathrm{s}}$ for the dpq'-bridged forms. The $\Delta v_{1 / 2}$ values for $\mathrm{rac}-\left[\left\{\mathrm{Ru}(\mathrm{bpy})_{2}\right\}_{2}(\mu-\mathrm{dpb})\right]^{5+}$ are also solvent invariant, while a more scattered variation in $\Delta v_{1 / 2}$ is evident for the meso form.

From a classical approach, ${ }^{1,2}$ the influence of the electronic coupling on the IVCT solvatochromism may be considered from two perspectives. Firstly, an increase in the electronic coupling decreases the effective charge transfer distance relative to the geometrical distance. The results of the Stark effect measurements for meso-[ $\left.\left.[\text { Ru(bpy })_{2}\right\}_{2}(\mu-\mathrm{BL})\right]^{5+}\{\mathrm{BL}=\mathrm{dpb}$ and dpq' $\}$ in §3.3.6 revealed effective charge transfer distances that were appreciably smaller than the geometrical metal-metal distances. This effect should be manifested by a decrease in the slope of the $v_{\max }$ and $M_{1}$ solvatochromism plots due to the inverse dependence of $\lambda_{0}$ and $\mathrm{H}_{\mathrm{ab}}$ on $d$, at fixed $d$ and $a$ (equation 1.15). A similar explanation was proposed for the lower than expected solvent dependence of $\left[\left\{\mathrm{Ru}\left(\mathrm{NH}_{3}\right)_{5}\right\}_{2}(\mu-\right.$ $4,4^{\prime}$-bpy) $]^{5+}{ }^{204}$ Secondly, the influence of appreciable electronic delocalisation may be considered through the introduction of "effective amount of charge transfer", $\left(1-2 b^{2}\right) e$, rather than $e$. The parameter $b^{2}$ has been defined previously in $\$ 3.3 .4 .2$ as the mixing coefficient. ${ }^{40}$

While electronic coupling effects provide a qualitative rationale for the weak solvent dependence of $v_{\max }$ across the series of complexes, the trends in $M_{1}$ appear to signal a transition between the localised (Class II) and localised-to-delocalised (Class II-III) regimes. ${ }^{14}$ The slight solvent dependence for the dpq'-bridged diastereoisomers indicates that the systems lie in the Class II region, approaching the Class II-III borderline, while the dpb- and dpb'-bridged diastereoisomers lie in the Class II-III regime. For the latter, electron transfer occurs faster than solvent reorganisation, and the solvent motions are uncoupled dynamically from the electron transfer. For the dpq'-bridged forms, the intramolecular electron transfer time-scale is slightly lower than the solvent relaxation time-scale and the solvent motions are weakly-coupled. The larger $\Delta v_{1 / 2}$ values for the dpq'-bridged diastereoisomers relative to the dpb'-bridged forms support this rationale. The broader bandwidths for the dpb-bridged diastereoisomers relative to the dpb'- and dpq'-bridged forms can be rationalised primarily on the basis of

\footnotetext{
${ }^{*} a$ is approximated as $5.43 \AA$ from $§ 2.3 .4 .1$. According to the geometric Ru $\cdots$ Ru distances in Table 3.2, $d<2 a$.
} 
the greater splitting between the three underlying components of the IVCT band due to the inherent structural distortion - as established from the analysis of the dinuclear osmium systems in §3.3.5.3 rather than solvent coupling. The assignment of the dpb'- and dpb-bridged systems as more delocalised than their dpq'-bridged counterparts, appears to contradict the trends in the $\mathrm{H}_{\mathrm{ab}}$ values (Tables 3.5 and 3.18) which suggest that the former are less strongly coupled. A possible rationalisation for this anomaly is that solvent coupling with the symmetric mode, rather than the anti-symmetric modes dominates for the dpq'-bridged diastereoisomers.

Specific solvation effects are reflected in the plots of the difference between the IVCT parameters for the diastereoisomers $\left\{\Delta v_{\max }\right.$ (meso-rac), $\Delta v_{1 / 2}$ (meso-rac) and $\Delta M_{1}$ (meso-rac) $\}$ in panel (B), Figure 3.26. Such effects arise due to solvent penetration within the clefts between the terminal bpy rings, as discussed previously for the complexes incorporating linear bridging ligands in Chapter 2 . The plots of $\Delta v_{\max }$ and $\Delta M_{1}$ for the dpb'- and dpq'-bridged complexes exhibit similar trends across the series of solvents. The magnitudes of $\Delta v_{\max }$ and $\Delta M_{1}$ are larger for the dpb-bridged complex due to the inherent stereochemically-induced redox asymmetry. Stereochemically-dependent specific solvation effects are present between the diastereoisomers of each complex, and act primarily to vary $\Delta v_{\max }$ rather than $\Delta M_{1}$. These observations demonstrate that the specific interactions are likely to couple the solvent and electronic coordinates and invalidate the Franck-Condon approximation for this series of systems. ${ }^{17}$

\section{Predictions for the Inner-Sphere Reorganisational Barrier}

According to the classical model, ${ }^{1,2}$ the intercepts of the IVCT solvatochromism plots of $v_{\max }$ as a function of $1 / \mathrm{D}_{\mathrm{op}}-1 / \mathrm{D}_{\mathrm{s}}$ given in Table B3.28 (Appendix B) provide estimates of the $\lambda_{i}$ contributions in equation 3.1. For the dpb'- and dpq'-bridged systems, the intercept is given by $\lambda_{i}+\Delta \mathrm{E}^{\prime}$, and for the dpbbridged system, by $\lambda_{i}+\Delta \mathrm{E}^{\prime}+\Delta \mathrm{E}_{\text {struct }}$ (assuming that $\Delta \mathrm{E}^{\prime}$ and $\Delta \mathrm{E}_{\text {struct }}$ are solvent invariant). While the $\lambda_{i}$ values for the three IVCT components may be extracted from Gaussian deconvolution of the IVCT manifolds, the errors in obtaining unique fits to the IVCT manifolds precluded such analyses. $\lambda_{i}$ was therefore obtained from equation $2.5^{205}$ (§2.3.4.1), where $\Delta$ represents the energy difference between $v_{\max }$ and IVCT(1). $\Delta$ estimates in the range $1590-1620 \mathrm{~cm}^{-1}$ were obtained from the energies of the IC bands in the dinuclear osmium complexes (§3.3.5.3), as reported in Table B3.29 (Appendix B). $\lambda_{i}$ was subsequently determined as $c a$. $3000 \mathrm{~cm}^{-1}$ for the series of complexes.

The significant magnitudes of $\lambda_{i}$ are surprising in view of the small bond distance changes that occur with oxidation. ${ }^{9,206}$ Estimates based on Ru-N bond distance changes between $\left[\mathrm{Ru}(\mathrm{bpy})_{3}\right]^{2+}$ and $\left[\mathrm{Ru}(\mathrm{bpy})_{3}\right]^{3+}$ lead to a prediction of less than $400 \mathrm{~cm}^{-1}$ for $\lambda_{i \cdot}{ }^{207}$ As discussed in §2.3.4.1, such anomalies may be attributed to appreciable intra-metal electronic delocalisation which invalidates the weak-coupling assumption. 


\subsubsection{IVCT Solvatochromism in a Series of Stepped-Parallel Complexes}

IVCT solvatochromism measurements for the diastereoisomeric forms of $\left[\left\{\mathrm{Ru}(\mathrm{bpy})_{2}\right\}_{2}(\mu-\mathrm{BL})\right]^{5+}$ $\{\mathrm{BL}=2,5$-dpp, dpop and apy $\}$ were performed in the homologous series of nitrile solvents AN, PN, BN, ${ }^{\mathrm{i}} \mathrm{BN}$ and BzN \{benzonitrile, $\left(\mathrm{C}_{6} \mathrm{H}_{5}\right) \mathrm{CN}$ \} containing $0.02 \mathrm{M}\left[\left(n-\mathrm{C}_{4} \mathrm{H}_{9}\right)_{4} \mathrm{~N}\right]\left\{\mathrm{B}\left(\mathrm{C}_{6} \mathrm{~F}_{5}\right)_{4}\right\}^{100}$ electrolyte. ${ }^{*}$ The complete electrochemical and UV/Vis/NIR spectral characteristics of the complexes are reported in Tables B3.30 and B3.31, respectively, and the spectra in $n$-butyronitrile solution are shown in Figure B3.10 (Appendix B). The electrochemical and UV/Vis characteristics of $\left[\left\{\mathrm{Ru}(\mathrm{bpy})_{2}\right\}_{2}(\mu-\mathrm{BL})\right]^{4+}(\mathrm{BL}=$ 2,5-dpp ${ }^{72,83-85}$ and dpop $\left.{ }^{77,86,208}\right)$ as diastereoisomeric mixtures, and meso- and rac-[\{Ru(bpy $\left.\left.)_{2}\right\}_{2}(\mu-\mathrm{apy})\right]^{n+}$ $(n=4,5,6)^{70}$ have been reported previously.

The lowest energy absorption bands for the series of complexes are assigned as $d \pi(\mathrm{Ru}) \rightarrow$ $\pi^{*}(\mathrm{BL})$ MLCT transitions, and exhibit similar trends upon oxidation to those reported for the systems incorporating angular bridging ligands in §3.3.2.3. The mixed-valence species exhibit asymmetricallyshaped IVCT bands in the region 4000-9000 $\mathrm{cm}^{-1}$ for $\left[\left\{\mathrm{Ru}(\mathrm{bpy})_{2}\right\}_{2}(\mu-\mathrm{BL})\right]^{5+}(\mathrm{BL}=2,5-\mathrm{dpp}$, dpop) and $6000-9000 \mathrm{~cm}^{-1}$ for $\left[\left\{\mathrm{Ru}(\mathrm{bpy})_{2}\right\}_{2}(\mu \text {-apy })\right]^{5+}$, as shown in Figure 3.27. On the basis of the magnitudes of the IVCT parameters reported in Tables B3.32-34 (Appendix B) and the parameters $\mathrm{H}_{\mathrm{ab}}{ }^{\dagger}$ and $\Gamma$ (equation $1.21),{ }^{38}$ the complexes lie in the localised-to-delocalised regime. Due to the shorter inter-metal separation in the apy-bridged systems (ca. $4.9 \AA$ ) the IVCT process is facilitated by direct overlap of the $\mathrm{Ru}(\mathrm{d} \pi)$ orbitals, ${ }^{83-85}$ in addition to superexchange interactions via the $\pi$-system of the bridging ligand. ${ }^{72,77,81,86}$ These factors may explain the higher energy of the IVCT bands for the apy-bridged systems relative to the 2,5-dpp- and dpop-bridged complexes (in which the inter-metal separation is ca. $6.9 \AA$ ). Since the IVCT bands are overlapped on the high energy side by the lowest energy MLCT transition for the apybridged diastereoisomers, accurate determinations of $M_{1}$ were not possible, and the $\Delta v_{1 / 2}$ values were obtained by fitting the low energy side of the IVCT manifold with a single Gaussian-shaped band.

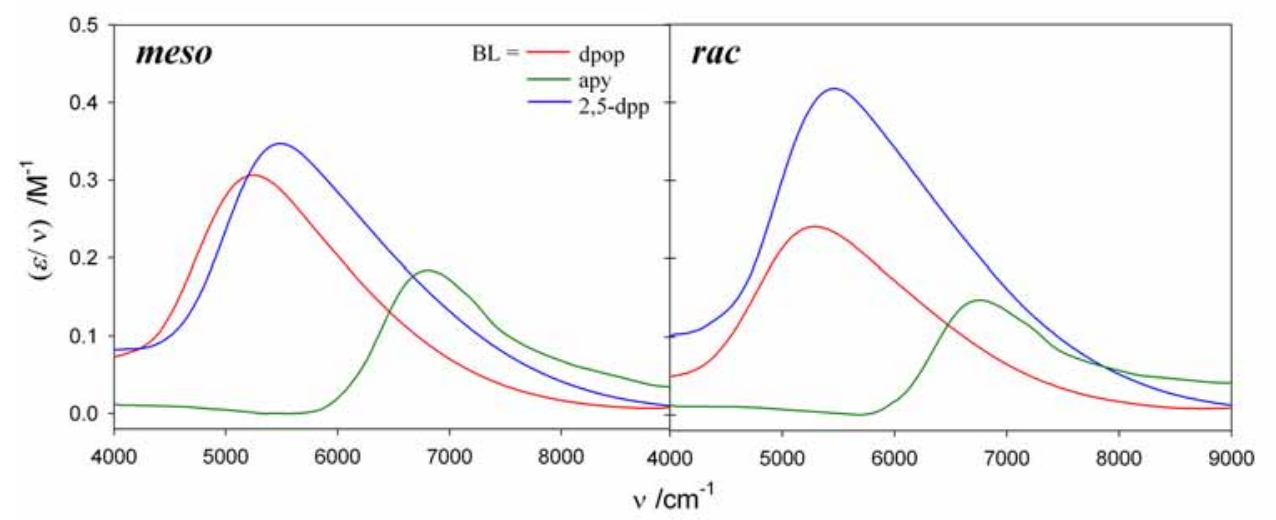

Figure 3.27 Overlay of the IVCT bands for the stepped-parallel bridging ligands for meso (a) and rac (b) $\left[\left\{\mathrm{Ru}(\mathrm{bpy})_{2}\right\}_{2}(\mu-\mathrm{BL})\right]^{5+}$ in $0.02 \mathrm{M}\left[\left(n-\mathrm{C}_{4} \mathrm{H}_{9}\right) \mathrm{N}\right]\left\{\mathrm{B}\left(\mathrm{C}_{6} \mathrm{~F}_{5}\right)_{4}\right\} / \mathrm{CH}_{3} \mathrm{CN}$ at $+25^{\circ} \mathrm{C}$ for $\mathrm{BL}=2,5$-dpp, dpop and $-35^{\circ} \mathrm{C}$ for $\mathrm{BL}=$ apy.

\footnotetext{
* (a) The spectroelectrochemical oxidation of the 2,5-dpp- and dpop-bridged systems was performed at $+25^{\circ} \mathrm{C}$. The apy-bridged diastereoisomers were unstable at room temperature and required low temperature spectroelectrochemical generation at $-35^{\circ} \mathrm{C}$ (i.e. below the freezing point of $\mathrm{BzN}$ ). (b) Solvatochromism measurements in AO and DCM were not performed.

${ }^{\dagger} \mathrm{H}_{\mathrm{ab}}$ was determined from equation 3.2 with $r_{\mathrm{ab}}$ estimated as the geometric Ru$\cdots \mathrm{Ru}$ distance of $6.9 \AA$ for $\left[\left\{\mathrm{Ru}(\mathrm{bpy})_{2}\right\}_{2}(\mu-\mathrm{BL})\right]^{5+}$ $\{\mathrm{BL}=2,5$-dpp, dpop $\}$ and $4.9 \AA$ for $\left[\left\{\mathrm{Ru}(\mathrm{bpy})_{2}\right\}_{2}(\mu \text {-apy) }]^{5+}\right.$ from molecular models.
} 
Panel (A) in Figure 3.28 displays an overlay of the solvent dependence of the IVCT band parameters $v_{\max }, \Delta v_{1 / 2}$ and $M_{1}$, and Table B3.35 (Appendix B) summarises the results for the slopes and intercepts of the solvatochromism plots. The data reveal differences in the solvent dependencies of the IVCT parameters between the different complexes, and between the diastereoisomers of the same complex. The weaker solvent dependence of $M_{1}$ and the narrower bandwidths for the dpop-bridged diastereoisomers relative to the 2,5-dpp-bridged forms are consistent with a "solvent delocalised" (Class II-III) classification in the former case. By comparison, the slight solvent dependence of $M_{1}$ for the latter indicates that the systems lie at the borderline between the solvent localised (Class II) and solvent delocalised (Class II-III) regimes.

(A)

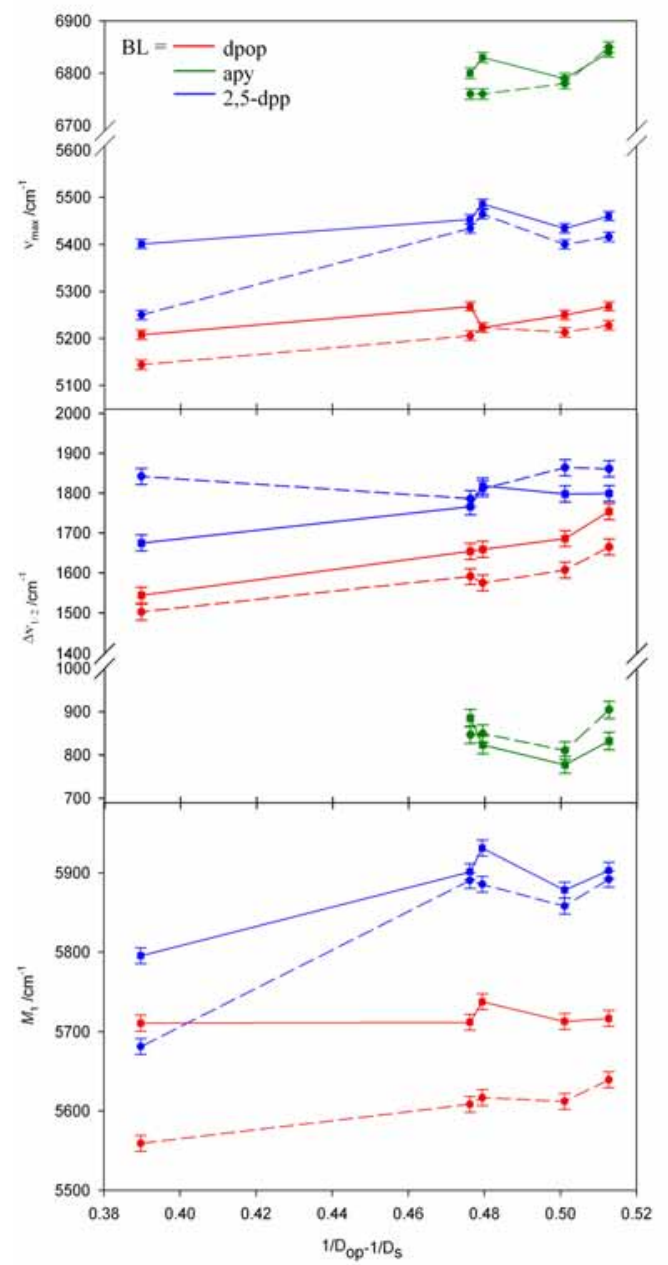

(B)

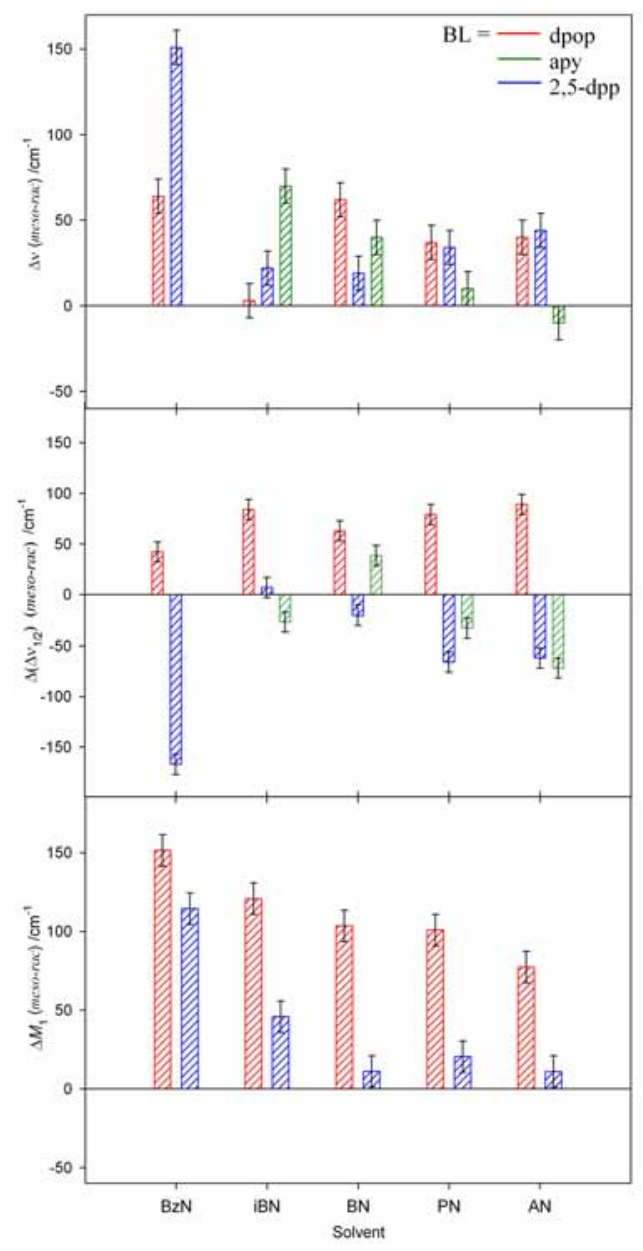

Figure 3.28 (A) Solvent dependence of $v_{\max }, \Delta v_{1 / 2}$ and $M_{1}$ for the meso (solid line) and rac (dashed line) diastereoisomers of $\left[\left\{\mathrm{Ru}(\mathrm{bpy})_{2}\right\}_{2}(\mu-\mathrm{BL})\right]^{5+}$ in $0.02 \mathrm{M}\left[\left(n-\mathrm{C}_{4} \mathrm{H}_{9}\right) \mathrm{N}\right]\left\{\mathrm{B}\left(\mathrm{C}_{6} \mathrm{~F}_{5}\right)_{4}\right\} /$ solvent at $+25^{\circ} \mathrm{C}$ for $\mathrm{BL}=2,5$-dpp, dpop and $-35^{\circ} \mathrm{C}$ for $\mathrm{BL}=$ apy. (B) Differential energies, bandwidths and first moments of the IVCT transitions for the diastereoisomers.

The influence of specific solvation effects are shown in panel (B) of Figure 3.28, as the difference in the IVCT parameters between the diastereoisomeric forms of the same complex. The $M_{1}$ values for meso- $\left[\left\{\mathrm{Ru}(\mathrm{bpy})_{2}\right\}_{2}(\mu-\mathrm{BL})\right]^{5+}(\mathrm{BL}=2,5-\mathrm{dpp}$, dpop) are consistently greater than those for the corresponding rac forms across the series of solvents. In addition, the magnitude of $\Delta M_{1}$ varies to a 
lesser extent for the dpop- relative to the 2,5-dpp-bridged complex, consistent with the greater degree of "solvent delocalisation" in the former case.

For the 2,5-dpp- and dpop-bridged complexes, the $\Delta v_{\max }$ and $\Delta M_{1}$ values increase as the solvent is varied through the series AN, PN, BN, ${ }^{\mathrm{i}} \mathrm{BN}$ and BzN for each complex. The magnitudes of these parameters reflect differential solvent interactions with the interior clefts of the diastereoisomeric forms. The largest specific interaction occurs in BzN, as the discrete solvent molecules may be accommodated preferentially within the parallel-shaped interior cleft of the rac diastereoisomers of the complexes i.e. "below" the plane of the bridging ligand, as shown in Figures 3.2(a) for the 2,5-dpp-bridged forms.

The dimensions of the interior clefts are reduced for the apy-bridged diastereoisomers (shown in Figure 3.29) relative to the 2,5-dpp- and dpop-bridged forms due to the closer proximity of the metal centres. The more open interior clefts in the meso form are more ideally disposed to accommodate aliphatic solvent molecules compared with the rac form. This may account for the increase in $\Delta v_{\max }$ as the size of the aliphatic nitrile solvent is increased through the series $\mathrm{AN}, \mathrm{PN}, \mathrm{BN}$ and ${ }^{\mathrm{i}} \mathrm{BN}$.

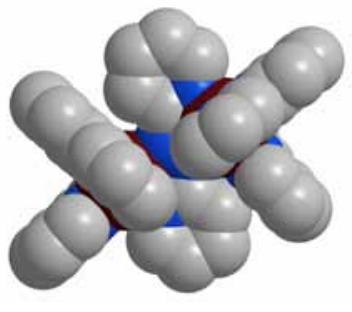

meso $(\Lambda \Delta)$

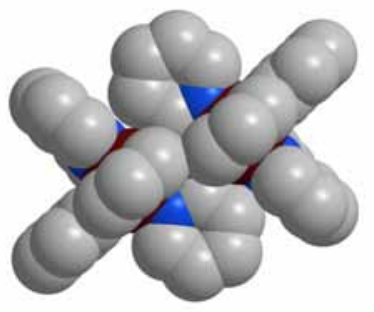

$\operatorname{rac}(\Delta \Delta)$

Figure 3.29 Chem3D representations (view from above the plane of the bridging ligand) of the meso $(\Lambda \Delta)$ and rac $(\Delta \Delta)$ diastereoisomers of $\left[\left\{\mathrm{Ru}(\mathrm{bpy})_{2}\right\}_{2}(\mu \text {-apy })\right]^{4+}$ showing the different dimensions of the clefts "above" and "below" the plane of the bridging ligand.

\subsubsection{Consolidating the IVCT Solvatochromism Properties of the Series of Angular and Stepped-Parallel Complexes}

The weak solvent dependencies of the IVCT parameters for the diastereoisomers of $\left[\left\{\mathrm{Ru}(\mathrm{bpy})_{2}\right\}_{2}(\mu-\mathrm{BL})\right]^{5+}(\mathrm{BL}=\mathrm{dpb}, \mathrm{dpb}$ ', dpq' and 2,5-dpp, dpop, apy) are consistent with significant electronic delocalisation, and the classification of the systems at the borderline of the localised (Class II) and localised-to-delocalised (Class II-III) regimes. The consideration of the trends in the first moments of the IVCT bands according to the dielectric continuum model provides a qualitative basis for explaining the relative degree of coupling, with the allowance for stereochemically-induced redox asymmetry and specific solvation effects. The specific solvent effect in acetonitrile described in Chapter 2 for $\left[\left\{\mathrm{Ru}(\mathrm{bpy})_{2}\right\}_{2}(\mu \text {-bpm) }]^{5+}\right.$ (§2.3.4.1) is absent for the series of angular and stepped-parallel complexes. This demonstrates that the interior clefts in the linear complex accommodate the small acetonitrile molecules more readily. 


\subsection{Conclusions and Future Prospects}

The present study has detailed the first observation of stereochemical effects on the IVCT transitions in a series of dinuclear polypyridyl complexes of ruthenium and osmium. The systematic variation in the electronic properties of the bridging ligands in the series of complexes $\left[\left\{\mathrm{M}(\mathrm{bpy})_{2}\right\}_{2}(\mu-\right.$ $\mathrm{BL})]^{5+}(\mathrm{M}=\mathrm{Ru}, \mathrm{Os})$ incorporating the angular and stepped-parallel bridging ligands shown in Figure 3.1 has demonstrated the subtle influence of stereochemical and structural factors on the reorganisational energies $\left(\lambda_{0}\right.$ and $\left.\lambda_{i}\right)$, the redox asymmetry $\left(\Delta \mathrm{E}_{0}\right)$ and the spin-orbit coupling ( $\left.\Delta \mathrm{E}^{\prime}\right)$ contributions to the electron transfer barrier, as expressed in equation 3.1.

\section{(1) Electronic and Structural Consequences of Bridging Ligand Modification}

(i) Electronic Aspects. The destabilisation in the energy of the LUMO of the bridging ligands through the series $\left[\left\{\mathrm{Ru}(\mathrm{bpy})_{2}\right\}_{2}(\mu-\mathrm{BL})\right]^{5+}$ (where $\mathrm{BL}=\mathrm{dpb}$ ', dpq', ppz) increases the degree of electronic coupling in these borderline localised/delocalised systems. This trend is surprising and reflects the localisation of the LUMO away from the nitrogen atoms coordinated to the metal centres as BL is varied through the series ppz, dpq' and dpb'. Minor differences are evident between the diastereoisomeric forms of each complex due to differential ion-pairing and specific solvent effects.

The IVCT characteristics of meso- and rac- $\left[\left\{\mathrm{Ru}(\mathrm{bpy})_{2}\right\}_{2}(\mu-\mathrm{BL})\right]^{5+}\{\mathrm{BL}=\mathrm{dpo}, \mathrm{dpt}\}$ are consistent with a delocalised classification and reveal the influence of the symmetric vibration mode. Accordingly, a three-state delocalisation mechanism is appropriate, in which the bridging ligand is explicitly included as a third electronic state. Stronger ion-pairing interactions with the meso diastereoisomer of each complex are proposed to explain the higher intensity IVCT bands relative to the corresponding rac forms.

(ii) Structural Aspects. The X-ray crystal structures of the dinuclear cations in meso$\left[\left\{\mathrm{Ru}(\mathrm{bpy})_{2}\right\}_{2}(\mu-\mathrm{dpb})\right]\left(\mathrm{PF}_{6}\right)_{4} .5 \mathrm{H}_{2} \mathrm{O}$ and meso- $\left[\left\{\mathrm{Ru}(\mathrm{bpy})_{2}\right\}_{2}(\mu-\mathrm{dpb})\right]\left(\mathrm{ZnCl}_{4}\right)_{2} .5 \mathrm{H}_{2} \mathrm{O}$ exhibit significant distortions in the conformations of the bridging ligands, in comparison with the cations in meso$\left[\left\{\mathrm{Ru}(\mathrm{bpy})_{2}\right\}_{2}\left(\mu-\mathrm{dpb}^{\prime}\right)\right]\left(\mathrm{PF}_{6}\right)_{4} \cdot 2 \mathrm{H}_{2} \mathrm{O} .2\left\{\left(\mathrm{CH}_{3}\right)_{2} \mathrm{CO}\right\}$ and meso-[\{Ru(bpy $\left.\left.)_{2}\right\}_{2}\left(\mu-\mathrm{dpq} \mathrm{q}^{\prime}\right)\right]\left(\mathrm{ZnCl}_{4}\right)_{2} \cdot 3 \mathrm{H}_{2} \mathrm{O}$. The distortions in the former case are dependent on the identity of the counter-ion, and are manifested by differences in the IVCT characteristics between the diastereoisomeric forms of the same complex. The stereochemically-induced structural distortion contributes an effective redox asymmetry contribution, $\Delta \mathrm{E}_{\text {struct }}$ to the electron transfer barrier. The degree of electronic coupling is reduced for both diastereoisomers of the dpb-bridged complex relative to their dpb'-bridged analogues.

\section{(2) Redox Asymmetry $\left(\Delta E_{0}\right)$ Contributions}

The degree of delocalisation in the symmetrical complexes $\left[\left\{\mathrm{Ru}(\mathrm{bpy})_{2}\right\}_{2}(\mu-\mathrm{dpb})\right]^{5+}$ and $\left[\left\{\mathrm{Ru}(\mathrm{bpy})_{2}\right\}_{2}(\mu-\mathrm{dpb})\right]^{5+}$ is diminished by the substitution of the terminal bpy ligands at one end of the complex. The results of a classical analysis for the series of complexes $\left[\left\{\operatorname{Ru}(b p y)_{2}\right\}(\mu-B L)\left\{R u(p p)_{2}\right\}\right]^{5+}$ ( $\mathrm{BL}=\mathrm{dpb}$ ', dpb; pp = bpy, $\mathrm{Me}_{2} \mathrm{bpy}, \mathrm{Me}_{4} \mathrm{bpy}$ ) according to the electrochemical method proposed by Curtis 
and coworkers ${ }^{88-90,178}$ indicate that a greater degree of ground state delocalisation exists in the complexes incorporating the fused bridging ligand dpb' compared with the unfused dpb analogue. A two-state analysis in which $\Delta \mathrm{E}_{0}$ is varied at constant $\mathrm{H}_{\mathrm{ab}}$ and $\lambda$ does not adequately describe the properties of the systems due to the importance of a third electronic state corresponding to the bridging ligand. The findings reinforce the contention by Reimers and $\mathrm{Hush}^{48}$ that the accurate treatment of mixed-valence systems in the localised-to-delocalised regime requires a sophisticated model incorporating both symmetric and anti-symmetric modes in which $\lambda$ and $\mathrm{H}_{\mathrm{ab}}$ are permitted to vary with $\Delta \mathrm{E}_{0}$.

\section{(3) IVCT Solvatochromism, Thermochromism and Ion-Pairing Contributions}

(i) Ion-pairing Studies. The IVCT properties of the diastereoisomers of $\left[\left\{\mathrm{Ru}(\mathrm{bpy})_{2}\right\}_{2}(\mu-\mathrm{BL})\right]^{5+}$ $\left\{\mathrm{BL}=\mathrm{dpb} \mathrm{d}^{\prime}, \mathrm{dpq}\right.$ and $\left.\mathrm{dpb}\right\}$ in the presence of relatively strong, $\mathrm{PF}_{6}^{-}$, and weak, $\left\{\mathrm{B}\left(\mathrm{C}_{6} \mathrm{~F}_{5}\right)_{4}\right\}^{-}$, ion-pairing electrolytes reveals that the trends in the relative magnitudes of the IVCT parameters are comparable in both electrolyte media. However, the IVCT bands are narrower for the full series of complexes in $\left\{\mathrm{B}\left(\mathrm{C}_{6} \mathrm{~F}_{5}\right)_{4}\right\}^{-}$electrolyte. A significant difference between the IVCT energies for the diastereoisomers of $\left[\left\{\mathrm{Ru}(\mathrm{bpy})_{2}\right\}_{2}(\mu-\mathrm{dpb})\right]^{5+}$ is evident in both media due to the inherent stereochemically-induced redox asymmetry.

Electrochemical studies of the diastereoisomeric forms of $\left[\left\{\mathrm{Ru}(\mathrm{bpy})_{2}\right\}_{2}(\mu-\mathrm{BL})\right]^{5+}\{\mathrm{BL}=\mathrm{bpm}$, dbneil, dpb, dpo, bpt $\left.{ }^{-}\right\}$in the two electrolytes reveal the significant and sometimes unpredictable dependence of the redox potentials for the metal-based oxidation processes on the identity of the electrolyte anion. This emphasises the need for standard conditions for the comparison of electrochemical data, and the danger of over-interpretation of the comproportionation constants derived from such data.

(ii) IVCT thermochromism studies on the diastereoisomers of $\left[\left\{\mathrm{Ru}(\mathrm{bpy})_{2}\right\}_{2}(\mu-\mathrm{BL})\right]^{5+}$ $\{\mathrm{BL}=\mathrm{dpb}$, dpq', dpb and 2,3-dpp $\}$ in $\left\{\mathrm{B}\left(\mathrm{C}_{6} \mathrm{~F}_{5}\right)_{4}\right\}^{-}$medium reveal that both diastereoisomers of the dpb'bridged complex are delocalised, while the meso diastereoisomer of the dpq'-bridged complex is more delocalised than its rac analogue. For the dpb-bridged diastereoisomers, the rac form is delocalised while the more localised meso form exhibits a slight temperature dependence due to stronger ion-pairing interactions with $\mathrm{PF}_{6}^{-}$versus $\left\{\mathrm{B}\left(\mathrm{C}_{6} \mathrm{~F}_{5}\right)_{4}\right\}^{-}$.

(iii) IVCT solvatochromism studies on the diastereoisomers of $\left[\left\{\mathrm{Ru}(\mathrm{bpy})_{2}\right\}_{2}(\mu-\mathrm{BL})\right]^{5+}$ $\{B L=\mathrm{dpb}, \mathrm{dpb}$ ', dpq' (angular) and 2,5-dpp, dpop and apy (stepped-parallel) $\}$ reveal that the systems bridged by dpb, dpb', 2,5-dpp, dpop and apy are solvent delocalised, while a small residual barrier to solvent exists in the diastereoisomers of the dpq'-bridged complex. This is consistent with a Class II-III classification for the former systems, while the weak solvent dependence of the latter supports a borderline Class II to Class II-III classification. The differential stereochemically-induced redox asymmetry between the diastereoisomers of $\left[\left\{\mathrm{Ru}(\mathrm{bpy})_{2}\right\}_{2}(\mu-\mathrm{dpb})\right]^{5+}$ is maintained over the full series of solvents. 


\section{(4) Spin-Orbit Coupling ( $\left.\triangle E^{\prime}\right)$ Contributions}

The mixed-valence systems $\left[\left\{\mathrm{O}(\mathrm{bpy})_{2}\right\}_{2}(\mu-\mathrm{BL})\right]^{5+}\{\mathrm{BL}=\mathrm{dpb}$ ', dpq', ppz, dpb, 2,3-dpp $\}$ are characterised by multiple IVCT and IC bands in the mid-IR and NIR regions, and the lowest energy IVCT band occurs in the mid-IR region 2000-4000 $\mathrm{cm}^{-1}$. Differences in the relative energies of the IC transitions in the NIR region for the fully-oxidised (+6) states, demonstrate that stereochemical effects lead to fundamental changes in the energy levels of the metal-based $d \pi$ orbitals which are split by spinorbit coupling and ligand field asymmetry. While the separation between the IC bands is relatively invariant for the diastereoisomeric forms of each species, their absolute energies are lower for the rac relative to the meso forms of the complexes incorporating the unfused bridging ligands dpb and 2,3-dpp. The results have important implications for the analysis of the underlying spin-orbit coupling transitions in the analogous dinuclear ruthenium systems:

(i) Meso- $\left[\left\{\mathrm{Ru}(\mathrm{bpy})_{2}\right\}_{2}(\mu \text {-dpb) }]^{n+}\right.$ exhibits a slightly higher degree of localisation than the rac form due to the larger magnitude of the inherent stereochemically-induced redox asymmetry, $\Delta \mathrm{E}_{\text {struct }}$. The separation of the IC bands is comparable for both diastereoisomers, however the $\Delta \mathrm{E}_{\text {struct }}$ contribution increases the orbital energies of the IC states in the former case. The larger bandwidth for the meso diastereoisomer relative to the rac form arises from preferential ion-pairing and solvent coupling (due to specific rather than continuum solvation effects).

(ii) For the series of complexes incorporating the fused bridging ligands, the increase in the separation between the IC bands as BL is varied through the series dpb', dpq' and ppz reflects the increase in the degree of electronic coupling through the series of complexes. The increase in the IVCT bandwidths through the series is attributed to the increase in the separation of the three underlying components of the bands. In addition, the IVCT bands are broader for the dpq'- relative to the dpb'bridged complexes (Tables 3.5 and 3.19) due to solvent coupling in the former case.

IVCT solvatochromism and thermochromism studies on the diastereoisomers of the ppz-bridged complex are required to determine the generality of this trend. This is the subject of current research efforts in our laboratory which aim to provide a coherent picture of the IVCT properties of the full series of complexes incorporating the fused bridging ligands dpb', dpq' and ppz, and their unfused dpb, dpq \{2,3-bis(2-pyridyl)quinoxaline $\}$ and 2,3-dpp analogues.

\section{(5) The Effective Electron Transfer Distance}

Stark effect measurements on the meso diastereoisomers of $\left[\left\{\mathrm{Ru}(\mathrm{bpy})_{2}\right\}_{2}(\mu-\mathrm{BL})\right]^{5+}\{\mathrm{BL}=\mathrm{dpq}$, $\mathrm{dpb}\}$ and $\left[\left\{\mathrm{Os}(\mathrm{bpy})_{2}\right\}_{2}(\mu-\mathrm{BL})\right]^{5+}\{\mathrm{BL}=\mathrm{dpb}$ ', dpq', ppz, dpb $\}$ reveal that the effective electron transfer distances are a small fraction only of the geometrical metal-metal distances. This provides independent support for the classification of the dinuclear ruthenium and osmium complexes as borderline localisedto-delocalised or delocalised systems and contradicts the previous localised assignment for

$\left[\left\{\mathrm{Os}(\mathrm{bpy})_{2}\right\}_{2}(\mu-\mathrm{BL})\right]^{5+}\{\mathrm{BL}=\mathrm{dpb}, 2,3-\mathrm{dpp}\}{ }^{139,140}$ 


\section{The Analysis IVCT Transitions in Mixed-Valence Complexes}

While the majority of IVCT studies to date have focused on the comparison of the "raw" parameters of the IVCT bands (i.e. $v_{\max }, \varepsilon_{\max }$ and $\Delta v_{1 / 2}$ ), the present analysis emphasises the importance of the following factors in obtaining meaningful comparisons of the IVCT properties for mixed-valence complexes: (i) consideration of the reduced absorption spectrum (scaled as $\int_{\varepsilon(v) / v} \mathrm{~d} v^{1,164}$ ); (ii) the definition of an accurate baseline for the IVCT band; (iii) consideration of the multiple IVCT and IC transitions which exist due to spin-orbit coupling; ${ }^{14}$ and (iv) calculation of the moments of the IVCT bands. The importance of the latter has been highlighted by Hush and Reimers, ${ }^{1,164}$ although this aspect appears not to have been generally appreciated by experimentalists. ${ }^{17}$ Such analyses are particularly crucial for the elucidation of trends in the electronic coupling, solvatochromism and thermochromism properties of mixed-valence systems between the fully localised and delocalised regimes, where the asymmetric shapes of the bands frequently preclude the accurate determination of the component spinorbit transitions.

Meyer and coworkers ${ }^{14}$ have described the gradation in behaviour between the limiting cases of the localised (Class II) and delocalised (Class III) regimes by invoking the Class II-III classification for complexes within the transition region. The present results serve to reinforce the contention ${ }^{14}$ that a gradual variation in the IVCT properties accompanies the transition between the regimes. While classical band-shape analyses are not strictly valid for systems in the intermediate region, the IVCT bands for the dinuclear ruthenium systems in the present study were reasonably deconvoluted into three Gaussianshaped components (although the fits were often not unique). Reliable conclusions regarding the trends in the parameters extracted from the IVCT analyses are permitted when the widths of the individual vibronic components of the bands are larger than their vibrational spacing.

\section{Limitations of the Present Study and Future Prospects}

A major shortcoming of the present analysis is the absence of detailed IR data which would provide more definitive evidence for the extent of localisation (or delocalisation) from the behaviour of marker bands in the IR. These include the observation of symmetrical bridging ligand vibrations and the extent of averaging of the terminal ligand vibrations. ${ }^{14}$ While preliminary mid-IR studies on selected systems have been reported herein, an important thrust of future work involves the extension of the midIR and NIR IVCT studies (including solvatochromism and thermochromism) to the full series of dinuclear ruthenium and osmium complexes incorporating the fused ligands dpb', dpq' and ppz, and the analogous unfused ligands dpb, dpq and 2,3-dpp. The comparison of the IR properties for the complexes with the existing IVCT data will provide a coherent picture of the factors governing the transitions between the localised (Class II), localised-to-delocalised (Class II-III) and delocalised (Class III) regimes. In addition, the experimental data will provide an extensive platform for testing existing vibronic coupling theories for IVCT (such as the PKS model ${ }^{47}$ and the vibronic coupling model of Reimers and 
$\mathrm{Hush}^{48}$ ) and guiding developing theories which explicitly include symmetric vibration modes. ${ }^{48}$ Resonance Raman spectroscopy would permit the analysis of the modes which are coupled to IVCT. ${ }^{176}$

The series of complexes $\left[\left\{\mathrm{Ru}(\mathrm{bpy})_{2}\right\}_{2}(\mu-\mathrm{BL})\right]^{4+}$ based on the bridging ligands shown in Figure 3.1 have been investigated extensively over the past two decades as the basis of novel molecular materials capable of performing useful light- and/or redox-induced functions. ${ }^{60-64}$ While these studies were performed without regard for the inherent stereochemistry of the systems, the present work demonstrates that stereochemical influences provide a significant contribution to the barrier to intramolecular electron transfer, and are indeed manifested in the electrochemical, spectral and IVCT properties of the systems. The realisation that metal-metal interactions in dinuclear polypyridyl complexes can be modified by the variation of their stereochemical properties has significant consequences for controlling such interactions in higher nuclearity polymetallic assemblies. 


\subsection{References}

1. Hush, N. S. Prog. Inorg. Chem. 1967, 8, 391-444.

2. Hush, N. S. Electrochim. Acta 1968, 13, 1005-1023.

3. $\quad$ Marcus, R. A. J. Chem. Phys. 1957, 26, 867-871.

4. $\quad$ Marcus, R. A. J. Chem. Phys. 1956, 24, 966-978.

5. Robin, M. B.; Day, P. Adv. Inorg. Chem. Radiochem. 1967, 10, 247-403.

6. $\quad$ Creutz, C.; Newton, M. D.; Sutin, N. J. Photochem. 1994, 82, 47-59.

7. $\quad$ Cave, R. J.; Newton, M. D. Chem. Phys. Lett. 1996, 249, 15-19.

8. Hush, N. S. Coord. Chem. Rev. 1985, 64, 135-157.

9. $\quad$ Creutz, C. Prog. Inorg. Chem. 1983, 30, 1-73.

10. Crutchley, R. J. Adv. Inorg. Chem. 1994, 41, 273-325.

11. Kalyanasundaram, K.; Nazeeruddin, M. K. Inorg. Chim. Acta 1994, 226, 213-230.

12. Ward, M. D. Chem. Soc. Rev. 1995, 24, 121-134.

13. Barbara, P. F.; Meyer, T. J.; Ratner, M. A. J. Phys. Chem. 1996, 100, 13148-13168.

14. Demadis, K. D.; Hartshorn, C. M.; Meyer, T. J. Chem. Rev. 2001, 101, 2655-2685.

15. Launay, J.-P. Chem. Soc. Rev. 2001, 30, 386-397.

16. Kaim, W.; Klein, A.; Glöckle, M. Acc. Chem. Res. 2000, 33, 755-763.

17. Chen, P.; Meyer, T. J. Chem. Rev. 1998, 98, 1439-1477.

18. Nelsen, S. F. Chem. Eur. J. 2000, 6, 581-588.

19. Nelsen, S. F.; Trieber, D. A., II; Ismagilov, R. F.; Teki, Y. J. Am. Chem. Soc. 2001, 123, 5684-5694.

20. Hupp, J. T.; Weaver, M. J. Inorg. Chem. 1984, 23, 3639-3644.

21. Katriel, J.; Ratner, M. A. J. Phys. Chem. 1989, 93, 5065-5070.

22. $\quad$ Drago, R. S.; Richardson, D. E.; George, J. E. Inorg. Chem. 1997, 36, 25-32.

23. Barthel, E. R.; Martini, I. B.; Schwartz, B. J. J. Phys. Chem. B 2001, 2001, 12230-12241.

24. Matyushov, D. V.; Schmid, R. J. Phys. Chem. 1994, 98, 5152-5159.

25. Sullivan, B. P.; Curtis, J. C.; Kober, E. M.; Meyer, T. J. Nouv. J. Chim. 1980, 4, 643-650.

26. Roberts, J. A.; Hupp, J. T. Inorg. Chem. 1992, 31, 157-160.

27. $\quad$ Blackbourn, R. L.; Hupp, J. T. J. Phys. Chem. 1988, 92, 2817-2820.

28. Blackbourn, R. L.; Hupp, J. T. Inorg. Chem. 1989, 28, 3786-3790.

29. Hupp, J. T.; Weydert, J. Inorg. Chem. 1987, 26, 2657-2660.

30. $\quad$ Ennix, K. S.; McMahon, P. T.; de la Rosa, R.; Curtis, J. C. Inorg. Chem. 1987, 26, 2660-2666.

31. $\quad$ Brunschwig, B. S.; Ehrenson, S.; Sutin, N. J. Phys. Chem. 1986, 90, 3657-3668.

32. Nelsen, S. F.; Ismagilov, R. F. J. Phys. Chem. A 1999, 103, 5373-5378.

33. $\quad$ Marcus, R. A. J. Phys. Chem. B 1998, 102, 10071-10077.

34. Pereztejeda, P.; Neto-Ponce, P.; Sánchez, F. J. Chem. Soc., Dalton Trans. 2001, 1686-1691.

35. Lau, K. W.; Hu, A. M. H.; Yen, M. H. J.; Fung, E. Y.; Grzybicki, S.; Matamoros, R.; Curtis, J. C. Inorg. Chim. Acta 1994, 226, 137-143.

36. $\quad$ Lewis, N. A.; Obeng, Y. S.; Purcell, W. L. Inorg. Chem. 1989, 28, 3796-3799.

37. Lewis, N. A.; Obeng, Y. S. J. Am. Chem. Soc. 1988, 110, 2306-2307.

38. $\quad$ Brunschwig, B. S.; Creutz, C.; Sutin, N. Chem. Soc. Rev. 2002, 31, 168-184.

39. Brunschwig, B. S.; Sutin, N. Electron Transfer in Chemistry; Wiley-VCH Verlag GmbH, Germany: 2001; Vol. 2, pp 583-617.

40. Brunschwig, B. S.; Sutin, N. Coord. Chem Rev. 1999, 187, 233-254.

41. Sutin, N. Inorg. Chem. 1988, 30, 441-498.

42. Sutin, N.; Brunschwig, B. S. ACS Symp. Ser. 1982, 198, 105-135.

43. Brunschwig, B. S.; Logan, J.; Newton, M. D.; Sutin, N. J. Am. Chem. Soc. 1980, 102, 5798-5809.

44. Piepho, S. B.; Krausz, E. R.; Schatz, P. N. J. Am. Chem. Soc. 1978, 100, 2996-3005.

45. Wong, K. Y.; Schatz, P. N. Prog. Inorg. Chem. 1981, 28, 369-449.

46. Piepho, S. B. J. Am. Chem. Soc. 1988, 110, 6319-6326.

47. Piepho, S. B. J. Am. Chem. Soc. 1990, 112, 4197-4206.

48. Reimers, J. R.; Hush, N. S. Chem. Phys. 1996, 208, 177-193.

49. $\quad$ Root, L. J.; Ondrechen, M. J. Chem. Phys. Lett. 1982, 93, 421-424.

50. $\quad$ Ko, J.; Ondrechen, M. J. Chem. Phys. Lett. 1984, 112, 507-512.

51. Ondrechen, M. J.; Ko, J.; Root, L. J. J. Phys. Chem. 1984, 88, 5919-5923.

52. Ko, J.; Ondrechen, M. J. J. Am. Chem. Soc. 1985, 107, 6161-6167.

53. Ondrechen, M. J.; Ko, J.; Zhang, L.-T. J. Am. Chem. Soc. 1987, 109, 1672-1676.

54. Kober, E. M.; Goldsby, K. A.; Narayana, D. N. S.; Meyer, T. J. J. Am. Chem. Soc. 1983, 105, $4303-4309$.

55. Powers, M. J. J. Am. Chem. Soc. 1980, 102, 1289-1297.

56. Meyer, T. J. Acc. Chem. Res. 1978, 11, 94-100.

57. Lambert, C.; Nöll, G. J. Am. Chem. Soc. 1999, 121, 8434-8442. 
58. Creutz, C.; Taube, H. J. Am. Chem. Soc. 1969, 91, 3988-3989.

59. $\quad$ Creutz, C.; Taube, H. J. Am. Chem. Soc. 1973, 95, 1086-1094.

60. Garcia, C. G.; de Lima, J. F.; Iha, N. Y. M. Coord. Chem. Rev. 2000, 196, 219-247.

61. Belser, P.; Bernhard, S.; Blum, C.; Beyeler, A.; De Cola, L.; Balzani, V. Coord. Chem. Rev 1999, 190-192, 155-169.

62. Balzani, V.; Juris, A.; Venturi, M.; Campagna, S.; Serroni, S. Chem. Rev. 1996, 96, 759-833.

63. Belser, P.; Dux, R.; Baak, M.; De Cola, L.; Balzani, V. Angew. Chem., Int. Ed. Engl. 1995, 34, 595-598.

64. Balzani, V.; Scandola, F., Supramolecular Photochemistry; Ellis Horwood: Chichester, 1991.

65. Keene, F. R. Chem. Soc. Rev. 1998, 27, 185-193.

66. Keene, F. R. Coord. Chem. Rev. 1997, 166, 122-159.

67. Yeomans, B. D.; Kelso, L. S.; Tregloan, P. A.; Keene, F. R. Eur. J. Inorg. Chem. 2001, 239-246.

68. Kelso, L. S. Ph.D. Thesis, James Cook University (Townsville, Australia), 2000.

69. D'Alessandro, D. M.; Kelso, L. S.; Keene, F. R. Inorg. Chem. 2001, 40, 6841-6844.

70. Kelso, L. S.; Reitsma, D. A.; Keene, F. R. Inorg. Chem. 1996, 35, 5144-5153.

71. Richardson, C.; Steel, P. J.; D'Alessandro, D. M.; Junk, P. C.; Keene, F. R. J. Chem. Soc., Dalton Trans. 2002, 2775-2785.

72. Denti, G.; Campagna, S.; Sabatino, L.; Serroni, S.; Ciano, M.; Balzani, V. Inorg. Chem. 1990, 29, 47504758.

73. Seneviratne, D. S.; Uddin, M. J.; Swayambunathan, V.; Schlegel, H. B.; Endicott, J. F. Inorg. Chem. 2002, 41, 1502-1517.

74.

Molnar, S. M.; Neville, K. R.; Jensen, G. E.; Brewer, K. J. Inorg. Chim. Acta 1993, 206, 69-76.

Wallace, A. W.; Murphy, W. R.; Petersen, J. D. Inorg. Chim. Acta 1989, 166, 47-54.

Scott, S. M.; Gordon, K. C. Inorg. Chim. Acta 1997, 254, 267-272.

Johnson, J. E. B.; Ruminski, R. R. Inorg. Chim. Acta 1993, 208, 231-237.

Braunstein, C. H.; Baker, A. D.; Strekas, T. C.; Gafney, H. D. Inorg. Chem. 1984, 23, 857-864.

Rillema, P.; Mack, K. B. Inorg. Chem. 1982, 21, 3849-3854.

Berger, R. M. Inorg. Chem. 1990, 29, 1920-1924.

Giuffrida, G.; Campagna, S. Coord. Chem. Rev. 1994, 135, 517-531.

Juris, A.; Barigelletti, S.; Campagna, S.; Balzani, V.; Belser, P.; von Zelewsky, A. Coord. Chem. Rev.

1988, 84, 85-277.

Ernst, S.; Kasack, V.; Kaim, W. Inorg. Chem. 1988, 27, 1146-1148.

Ernst, S. D.; Kaim, W. Inorg. Chem. 1989, 28, 1520-1528.

Kaim, W.; Kohlmann, S. Inorg. Chem. 1987, 26, 68-77.

Treadway, J. A.; Strouse, G. F.; Ruminski, R. R.; Meyer, T. J. Inorg. Chem. 2001, 40, 4508-4509.

Curtis, J. C.; Blackbourn, R. L.; Ennix, K. S.; Hu, S.; Roberts, J. A.; Hupp, J. T. Inorg. Chem. 1989, 28, 3791-3795.

88. $\quad$ Salaymeh, F.; Berhane, S.; Yusof, R.; Delarosa, R.; Fung, E. Y.; Matamoros, R.; Lau, K. W.; Zheng, Q.; Kober, E. M.; Curtis, J. C. Inorg. Chem. 1993, 32, 3895-3908.

89. $\quad$ Dong, Y.; Hupp, J. T. Inorg. Chem. 1992, 31, 3170-3172.

90. Mines, G. A.; Roberts, J. A.; Hupp, J. T. Inorg. Chem. 1992, 31, 125-128.

91. Chatterjee, D.; Bajaj, H. C.; Das, A. Inorg. Chim. Acta 1994, 224, 189-192.

92. Zhao, X.; Burt, J. A.; Knorr, F. J.; McHale, J. L. J. Phys. Chem. A 2001, 105, 11110-11117.

93. Dong, Y.; Hupp, J. T. Inorg. Chem. 1992, 31, 3322-3324.

94. Prassides, K.; Day, P. J. Chem. Soc., Faraday Trans. 2 1984, 80, 85-95.

95. Hupp, J. T.; Neyhart, G. A.; Meyer, T. J.; Kober, E. M. J. Phys. Chem. 1992, 96, 10820-10830.

96. Chatterjee, D.; Bajaj, H. C.; Das, A. Inorg. Chem. 1993, 32, 4049-4052.

97. Catterjee, D.; Bajaj, H. C.; Das, A. Inorg. Chim. Acta 1994, 224, 189-192.

98. Hupp, J. T.; Dong, Y. H. J. Am. Chem. Soc. 1993, 115, 6428-6429.

99. $\quad$ Coropceanu, V.; Lambert, C.; Nöll, G.; Brédas, J. L. Chem. Phys. Lett. 2003, 373, 153-160.

100. LeSuer, R.; Geiger, W. E. Angew. Chem., Int. Ed. Engl. 2000, 39, 248-250.

101. Khoshtariya, D. E.; Bajaj, H. C.; Tregloan, P. A.; van Eldik, R. J. Phys. Chem. A 2000, 104, 5535-5544.

102. Blackbourn, R. L.; Dong, Y.; Lyon, L. A.; Hupp, J. T. Inorg. Chem. 1994, 33, 4446-4452.

103. Blackbourn, R. L.; Hupp, J. T. J. Phys. Chem. 1990, 94, 1788-1793.

104. Coll, J. C.; Bowden, B. F. J. Nat. Prod. 1986, 49, 934-936.

105. Armarego, W. L.; Perrin, D. D. Purification of Laboratory Chemicals. 4th ed.; Butterworth-Heinemann: Oxford, 1996.

106. Vogel, A. I. A Text-Book of Practical Organic Chemistry. 3rd ed.; Longmans, Green and Co. Ltd.: London, 1962; p 189.

107. Patterson, B. T.; Keene, F. R. Inorg. Chem. 1998, 37, 645-650.

108. Borg, S. J.; Best, S. P. J. Electroanal. Chem. 2002, 535, 57-64.

109. Best, S. P.; Clark, R. J. H.; McQueen, R. C. S. Rev. Sci. Instrum. 1987, 58, 2071-2074.

110. Ohrenberg, C.; Geiger, W. E. Inorg. Chem. 2000, 39, 2948-2950. 
111. Hill, M. G.; Lamanna, W. M.; Mann, K. R. Inorg. Chem. 1991, 30, 4687-4690.

112. Lowery, M. D.; Hammack, W. S.; Drickamer, H. G.; Hendrickson, D. N. J. Am. Chem. Soc. 1987, 109, 8019-8024.

113. Andrews, S. S.; Boxer, S. G. Rev. Sci. Instrum. 2000, 71, 3567-3569.

114. Dinolfo, P. H.; Hupp, J. T. J. Am. Chem. Soc. 2004, 126, 16814-16819.

115. Abboud, J.-L. M.; Notario, R. Pure Appl. Chem. 1999, 71, 645-718.

116. Karki, L.; Hupp, J. T. Inorg. Chem. 1997, 36, 3318-3321.

117. Walters, K. A. In Comprehensive Coordination Chemistry II; Meyer, T. J., McCleverty, J. A. Eds.; Elsevier: Oxford, 2004; Vol.2, pp 303-313.

118. Liptay, W. Angew. Chem., Int. Ed. Engl. 1969, 8, 177-188.

119. Shin, Y.-g. K.; Brunschwig, B. S.; Creutz, C.; Sutin, N. J. Phys. Chem. 1996, 100, 8157-8169.

120. Bublitz, G. U.; Boxer, S. G. Annu. Rev. Phys. Chem. 1997, 48, 213-242.

121. Imor, S.; Morgan, R. J.; Wang, S. H.; Morgan, O.; Baker, A. D. Syn. Commun. 1996, 26, 2197-2203.

122. Bonhôte, P.; Wrighton, M. S. Synlett 1997, 897-898.

123. Evans, I. P.; Spencer, A.; Wilkinson, G. J. Chem. Soc., Dalton Trans. 1973, 204-209.

124. Togano, T.; Nagao, N.; Tsuchida, M.; Kumakura, H.; Hisamatsu, K.; Howell, F. S.; Mukaida, M. Inorg. Chim. Acta 1992, 195, 221-225.

125. Anderson, P. A.; Anderson, R. F.; Furue, M.; Junk, P. C.; Keene, F. R.; Patterson, B. T.; Yeomans, B. D. Inorg. Chem. 2000, 39, 2721-2728.

126. Buckingham, D. A.; Dwyer, F. P.; Goodwin, H. A.; Sargeson, A. M. Aust. J. Chem. 1964, 17, 325-336.

127. Jandrasics, E. Z.; Keene, F. R. J. Chem. Soc., Dalton Trans. 1997, 153-159.

128. Fletcher, N. C.; Junk, P. C.; Reitsma, D. A.; Keene, F. R. J. Chem. Soc., Dalton Trans. 1998, 133-138.

129. Fletcher, N. C.; Keene, F. R. J. Chem. Soc., Dalton Trans. 1999, 683-689.

130. Rutherford, T. J.; Van Gijte, O.; Kirsch - De Mesmaeker, A.; Keene, F. R. Inorg. Chem. 1997, 36, 44654474.

131. D'Alessandro, D. M.; Keene, F. R. Dalton Trans. 2004, 3950-3954.

132. Hua, X. Ph.D. Thesis, University of Fribourg (Fribourg, Switzerland), 1993.

133. Blessing, R. H. Acta. Cryst. 1995, A51, 33-38.

134. Sheldrick, G. M. SHELXL-97, Program for Crystal Structure Solution; University of Göttingen: Germany, 1997; Sheldrick, G. M. SHELXS-97, Program for Crystal Structure Refinement; University of Göttingen: Germany, 1997.

135. Barbour, L. J. X-SEED Crystallographic Interface; University of Missouri, USA, 1999.

136. Rutherford, T. J.; Keene, F. R. J. Chem. Soc., Dalton Trans. 1998, 1155-1162.

137. Fuchs, Y.; Lofters, S.; Dieter, T.; Shi, W.; Morgan, T. C.; Strekas, T. C.; Gafney, H. D.; Baker, A. D. J. Am. Chem. Soc. 1987, 109, 2691-2697.

138. Morgan, O.; Wang, S.; Bae, S.-A.; Morgan, R. J.; Baker, A. D.; Strekas, T. C.; Engel, R. J. Chem. Soc., Dalton Trans. 1997, 3773-3776.

139. Richter, M. M.; Brewer, K. J. Inorg. Chem. 1993, 32, 2827-2834.

140. Richter, M. M.; Brewer, K. J. Inorg. Chim. Acta 1991, 180, 125-131.

141. Richter, M. M.; Brewer, K. J. Inorg. Chem. 1992, 31, 1594-1598.

142. Rutherford, T. J.; Keene, F. R. Inorg. Chem. 1997, 36, 3580-3581.

143. Constable, E. C.; Lewis, J. Inorg. Chim. Acta 1983, 70, 251-253.

144. Kober, E. M.; Meyer, T. J. Inorg. Chem. 1983, 22, 1614-1616.

145. Bardwell, D.; Jeffery, J. C.; Joulie, L.; Ward, M. D. J. Chem. Soc., Dalton Trans. 1993, 2255-2256.

146. Bardwell, D. A.; Horsburgh, L.; Jeffery, J. C.; Joulié, L. F.; Ward, M. D.; Webster, I.; Yellowlees, L. J. J. Chem. Soc., Dalton Trans. 1996, 2527-2531.

147. Hage, R.; Haasnoot, J. G.; Nieuwenhuis, H. A.; Reedijk, J.; De Ridder, D. J. A.; Vos, J. G. J. Am. Chem. Soc. 1990, 112, 9245-9251.

148. Balzani, V.; Bardwell, D. A.; Barigelletti, F.; Cleary, F. L.; Guardigli, M.; Jeffery, J. C.; Sovrani, T.; Ward, M. D. J. Chem. Soc., Dalton Trans. 1995, 3601-3608.

149. Baitalik, S.; Flörke, U.; Nag, K. J. Chem. Soc., Dalton Trans. 1999, 719-727.

150. Masui, H.; Freda, A. L.; Zerner, M. C.; Lever, A. B. P. Inorg. Chem. 2000, 39, 141-152.

151. Dejehet, F.; Debuyst, R.; Declercq, J. P. J. Chim. Phys., Phys-Chim. Biol. 1986, 83, 85-90.

152. Goldsby, K. A.; Meyer, T. J. Inorg. Chem. 1984, 23, 3002-3010.

153. Richardson, D. E.; Taube, H. J. Am. Chem. Soc. 1983, 105, 40-51.

154. Haga, M.-A.; Ali, M. M.; Koseki, S.; Yoshimura, A.; Nozaki, K.; Ohno, T. Inorg. Chim. Acta 1994, 226, 17-24.

155. Bean, G. P. J. Org. Chem. 1998, 63, 2497-2506.

156. Barriere, F.; Camire, N.; Geiger, W. E.; Mueller-Westerhoff, U. T.; Sanders, R. J. Am. Chem. Soc. 2002, 124, 7262-7263.

157. Noviandri, I.; Brown, K. N.; Fleming, D. S.; Gulyas, P. T.; Lay, P. A.; Masters, A. F.; Phillips, L. J. Phys. Chem. B 1999, 103, 6713-6722. 
158. Hage, R.; Dijkhuis, A. H. J.; Haasnoot, J. G.; Prins, R.; Reedijk, J.; Buchanan, B. E.; Vos, J. G. Inorg. Chem. 1988, 27, 2185-2189.

159. Barigelletti, F.; De Cola, L.; Balzani, V.; Hage, R.; Haasnoot, J. G.; Reedijk, J.; Vos, J. G. Inorg. Chem. 1989, 28, 4344-4350.

160. Su, H.; Kincaid, J. R. J. Raman Spect. 2003, 34, 907-916.

161. Sherborne, J.; Scott, S. M.; Gordon, K. C. Inorg. Chim. Acta. 1997, 260, 199-205.

162. Cooper, J. B.; MacQueen, D. B.; Petersen, J. D.; Wertz, D. W. Inorg. Chem. 1990, 29, 3701-3705.

163. Pourtois, G.; Beljonne, D.; Moucheron, C.; Schumm, S.; Mesmaeker, A. K.-D.; Lazzaroni, R.; Brédas, J.-L. J. Am. Chem. Soc. 2004, 126, 683-692.

164. Reimers, J. R.; Hush, N. S. Inorg. Chem. 1990, 29, 3686-3697.

165. Demadis, K. D.; El-Samanody, E.-S.; Coia, G. M.; Meyer, T. J. J. Am. Chem. Soc. 1999, 121, $535-544$.

166. Demadis, K. D.; Neyhart, G. A.; Kober, E. M.; White, P. S.; Meyer, T. J. Inorg. Chem. 1999, 38, 59485959.

167. Neyhart, G. A.; Timpson, C. J.; Bates, W. D.; Meyer, T. J. J. Am. Chem. Soc. 1996, 118, 3730-3737.

168. Best, S. P.; Clark, R. J. H.; McQueen, R. C. S.; Joss, S. J. Am. Chem. Soc. 1989, 111, 548-550.

169. Rocha, R. C.; Shreve, A. P. Inorg. Chem. 2004, 43, 2231-2233.

170. Hornung, F. M.; Baumann, F.; Kaim, W.; Olabe, J. A.; Slep, L. D.; Fiedler, J. Inorg. Chem. 1998, 37, 311316.

171. Demadis, K. D.; Neyhart, G. A.; Kober, E. M.; Meyer, T. J. J. Am. Chem. Soc. 1998, 120, 7121-7122.

172. Talaga, D. S.; Zink, J. I. J. Phys. Chem. A 2001, 105, 10511-10519.

173. Zhang, L.-T.; Ko, J.; Ondrechen, M. J. J. Phys. Chem. 1989, 93, 3030-3034.

174. Zhang, L.-T.; Ko, J.; Ondrechen, M. J. J. Am. Chem. Soc. 1987, 109, 1666-1671.

175. Petrov, V.; Hupp, J. T.; Mottley, C.; Mann, L. C. J. Am. Chem. Soc. 1994, 116, 2171-2172.

176. Hupp, J. T.; Williams, R. D. Acc. Chem. Res. 2001, 34, 808-817.

177. Kalyanasundaram, K.; Zakeeruddin, S. M.; Nazeeruddin, M. K. Coord. Chem. Rev. 1994, 132, $259-264$.

178. Curtis, J. C.; Bernstein, J. S.; Schmehl, R. H.; Meyer, T. J. Chem. Phys. Lett. 1981, 81, 48-52.

179. de la Rossa, R.; Chang, P. J.; Salaymeh, F.; Curtis, J. C. Inorg. Chem. 1985, 24, 4229-4231.

180. Bino, A.; Lay, P. A.; Taube, H.; Wishart, J. F. Inorg. Chem. 1985, 24, 3969-3971.

181. Richardson, D. E.; Taube, H. Coord. Chem. Rev. 1984, 60, 107-129.

182. Kober, E. M.; Sullivan, B. P.; Dressick, W. J.; Caspar, J. V.; Meyer, T. J. J. Am. Chem. Soc. 1980, 102, 7383-7385.

183. Caspar, J. V.; Kober, E. M.; Sullivan, B. P.; Meyer, T. J. J. Am. Chem. Soc. 1982, 104, 630-632.

184. Dodsworth, E. S.; Lever, A. B. P. Chem. Phys. Lett. 1985, 119, 61-66.

185. Saji, T.; Aoyagui, S. J. Electroanal. Chem. 1975, 60, 1-10.

186. Richardson, D. E.; Sen, J. P.; Buhr, J. D.; Taube, H. Inorg. Chem. 1982, 21, 3136-3140.

187. Magnuson, R. H.; Lay, P. A.; Taube, H. J. Am. Chem. Soc. 1982, 105, 2507-2509.

188. Magnuson, R. H.; Lay, P. A.; Taube, H. J. Am. Chem. Soc. 1983, 105, 2507-2509.

189. Lay, P. A.; Magnuson, R. H.; Taube, H. Inorg. Chem. 1988, 27, 2364-2371.

190. Dubicki, L.; Ferguson, J.; Krausz, E. R.; Lay, P. A.; Maeder, M.; Taube, H. J. Phys. Chem. 1984, 88, 39403941.

191. Karki, L.; Williams, R. D.; Hupp, J. T. Inorg. Chem. 1998, 37, 2837-2840.

192. Oh, D. H.; Boxer, S. G. J. Am. Chem. Soc. 1990, 112, 8161-8162.

193. Oh, D. H.; Sano, M.; Boxer, S. G. J. Am. Chem. Soc. 1991, 113, 6880-6890.

194. Bublitz, G. U.; Boxer, S. G. J. Am. Chem. Soc. 1998, 120, 3988-3992.

195. Shin, Y.-G. K.; Brunschwig, B. S.; Creutz, C.; Sutin, N. J. Phys. Chem. 1996, 100, 8157-8169.

196. Shin, Y. G. K.; Brunschwig, B. S.; Creutz, C.; Sutin, N. J. Am. Chem. Soc. 1995, 117, 8668-8669.

197. Bublitz, G. U.; Laidlaw, W. M.; Denning, R. G.; Boxer, S. G. J. Am. Chem. Soc. 1998, 120, 6068-6075.

198. Treynor, T. P.; Boxer, S. G. J. Phys. Chem. B 2004, 108, 13513-13522.

199. $\quad$ Reimers, J. R.; Hush, N. S. J. Phys. Chem. 1991, 95, 9773-9781.

200. Sando, G. M. 2003.

201. Blackbourn, R. L.; Hupp, J. T. Chem. Phys. Lett. 1988, 150, 399-405.

202. Johnson, R. C.; Hupp, J. T. J. Am. Chem. Soc. 2001, 123, 2053-2057.

203. Vath, P.; Zimmt, M. B.; Matyushov, D. V.; Voth, G. A. J. Phys. Chem. B 1999, 103, 9130-9140.

204. Hupp, J. T.; Dong, Y. H.; Blackbourn, R. L.; Lu, H. J. Phys. Chem. 1993, 97, 3278-3282.

205. Hupp, J. T.; Meyer, T. J. Inorg. Chem. 1987, 26, 2332-2334.

206. Creutz, C. Inorg. Chem. 1978, 17, 3723-3725.

207. Brunschwig, B. S.; Creutz, C.; Macartney, D. H.; Sham, T.-K.; Sutin, N. Faraday Discuss. Chem. Soc. 1982, 74, 113-127.

208. Marcaccio, M.; Paolucci, F.; Paradisi, C.; Roffia, S.; Fontanesi, C.; Yellowlees, L. J.; Serroni, S.; Campagna, S.; Denti, G.; Balzani, V. J. Am. Chem. Soc 1999, 121, 10081-10091. 
ChAPTER 4 
Chapter 4

\section{EXTENDING THE IVCT PROBE TO Trinuclear Polymetallic Assemblies}

\subsection{Introduction}

The elucidation of fundamental electron transfer phenomena in higher nuclearity tri- and tetranuclear complexes provides the link between the understanding of such processes in dinuclear species, and in extended arrays and metallosupramolecular systems. IVCT studies provide a particularly valuable insight into intramolecular electron transfer processes; however, studies of this type are relatively scarce, and the degree to which such processes are influenced by the nuclearity, oxidation state and overall geometry are not clear. The extension of the IVCT probe to stereochemically-unambiguous trinuclear assemblies represents a key step in the rational design of higher nuclearity systems in which the electron transfer processes may be controlled and ultimately exploited.

\subsubsection{IVCT in Trinuclear Complexes}

The limited number of IVCT studies on higher nuclearity polymetallic assemblies is due in part to the presence of multiple, electronically-coupled metal centres which often complicate theoretical analyses. To date, these studies have focused predominantly on complexes incorporating polypyridyl iron, ruthenium and osmium components, linked by cyanide, pyrazine ${ }^{1,2}$ and aromatic N-heterocyclic bridging ligands such as 2,3-dpp \{2,3-bis(2-pyridyl)pyrazine.$^{3}$ A few reports of IVCT in higher nuclearity systems including cyclic and star-burst cyano-bridged molecular squares and clusters, ${ }^{4,5}$ and polyferrocenes $^{6-9}$ have also appeared.

In principle, the partially-oxidised mixed-valence forms of trinuclear complexes can exhibit multi-site interactions, which may be manifested by multiple IVCT transitions in the mid-IR or NIR spectral regions. As an illustrative example, Bignozzi and coworkers ${ }^{10-18}$ have investigated the IVCT properties of a series of cyano-bridged systems such as $\left[\left(\mathrm{NH}_{3}\right)_{5} \mathrm{Ru}_{\text {term }}-\mathrm{NC}-\mathrm{M}_{\text {cent }}(\mathrm{bpy})_{2}-\mathrm{CN}-\right.$ $\left.\mathrm{Ru}_{\text {term }}\left(\mathrm{NH}_{3}\right)_{5}\right]^{4+}\{\mathrm{M}=\mathrm{Ru}$, Os; “cent” and “term” denote the central and terminal metals, respectively $\}^{10-12,15}$ and their analogues $\left[(\mathrm{py})\left(\mathrm{NH}_{3}\right)_{4} \mathrm{Ru}_{\text {term }}-\mathrm{NC}-\mathrm{Ru}_{\text {cent }}(\text { bpy })_{2}-\mathrm{CN}-\mathrm{Ru}_{\text {term }}\left(\mathrm{NH}_{3}\right)_{4}(\mathrm{X})\right]^{4+12,15}$ $\left\{\mathrm{X}=\mathrm{NH}_{3}\right.$ or py (pyridine) $\}$. The metal-based oxidation processes for $\left[\left(\mathrm{NH}_{3}\right)_{5} \mathrm{Ru}_{\text {term }}-\mathrm{NC}-\mathrm{Ru}_{\text {cent }}(\mathrm{bpy})_{2}-\right.$ $\left.\mathrm{CN}-\mathrm{Ru}_{\text {term }}\left(\mathrm{NH}_{3}\right)_{5}\right]^{4+}$ (represented by [2,2,2] to denote the valencies of the metal centres) were assigned to two sequential one-electron processes corresponding to oxidation of the terminal $\mathrm{Ru}(\mathrm{NH})_{3}$ moieties, followed by one-electron oxidation of the central Ru(bpy $)_{2}$ unit. 
(a)

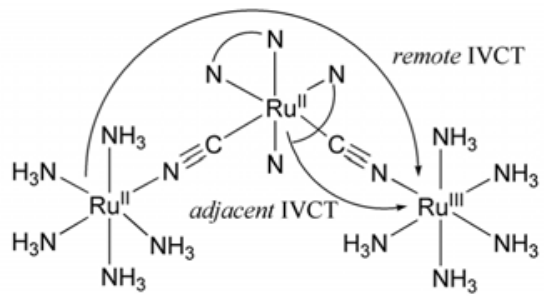

(b)

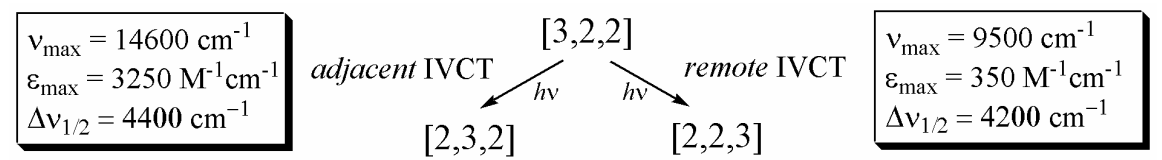

Figure 4.1 (a) Structure of $\left[\left(\mathrm{NH}_{3}\right)_{5} \mathrm{Ru}-\mathrm{NC}-\mathrm{Ru}(\mathrm{bpy})_{2}-\mathrm{CN}-\mathrm{Ru}\left(\mathrm{NH}_{3}\right)_{5}\right]^{5+}$ illustrating the adjacent and remote IVCT transitions. (b) IVCT band parameters for the IVCT transitions. ${ }^{10-12,15}$

The partially-oxidised forms of the trinuclear complexes [3,2,2] and [3,2,3] are mixed-valence species, and exhibited a rich combination of electronic transitions in their UV/Vis and NIR absorption spectra, which were ascribed to transitions of both MLCT and IVCT origin. In a cyano-bridged trinuclear system comprising the $\mathrm{M}(\mu-\mathrm{CN}) \mathrm{M}^{\prime}(\mu-\mathrm{CN}) \mathrm{M}^{\prime}$ core, two forms of IVCT may be envisaged: one that

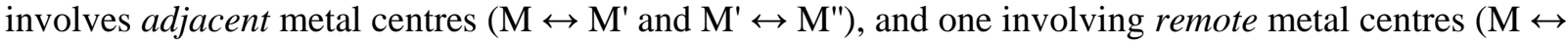
$\left.\mathrm{M}^{\prime \prime}\right)$. In the [3,2,2] system, the transitions were assigned as (i) $\mathrm{Ru}_{\text {cent }}{ }^{\mathrm{II}} \rightarrow$ bpy and (ii) $\mathrm{Ru}_{\text {cent }} \stackrel{\mathrm{II}}{\rightarrow} \mathrm{Ru}_{\text {term }}{ }^{\text {III }}$ IVCT between adjacent cyano-bridged units, in addition to the intriguing observation of two types of remote transitions, assigned as (iii) $\mathrm{Ru}_{\text {term }}{ }^{\text {II }} \rightarrow$ bpy MLCT, and (iv) $\mathrm{Ru}_{\text {term }}{ }^{\text {II }} \rightarrow \mathrm{Ru}_{\text {term }}{ }^{\text {III IVCT transitions }}$ between the remote $\mathrm{Ru}\left(\mathrm{NH}_{3}\right)_{5}$ units. The origins of the IVCT transitions are depicted in Figure 4.1(a), and a comparison of their parameters is shown in Figure 4.1(b). The remote transitions engage in an "intensity stealing” mechanism from the relatively higher intensity adjacent transitions. ${ }^{15}$ The doublyoxidised $[3,2,3]$ mixed-valence species, $\left[\left(\mathrm{NH}_{3}\right)_{5} \mathrm{Ru}{ }^{\mathrm{II}}-\mathrm{NC}-\mathrm{Ru}^{\mathrm{III}}(\mathrm{bpy})_{2}-\mathrm{CN}-\mathrm{Ru}^{\mathrm{II}}\left(\mathrm{NH}_{3}\right)_{5}\right]^{6+}$, exhibited a single $\mathrm{Ru}_{\text {cent }}{ }^{\text {II }} \rightarrow \mathrm{Ru}_{\text {term }}{ }^{\text {III }}$ IVCT band. ${ }^{10-12,15}$

The overall geometries of these "chain-like” assemblies based on the central Ru(bpy $)_{2}$ moiety are limited by the invariably cis-configured central metal. The influence of the cis or trans configuration of trinuclear assemblies was elucidated in IVCT studies of the complexes $\left[\left\{\mathrm{Fe}^{\mathrm{III}}(\mathrm{dppe}) \mathrm{Cp}\right\}-\mathrm{NC}-\left\{\mathrm{Pt}^{\mathrm{II}}(\mathrm{L})_{2}\right\}-\mathrm{CN}-\left\{\mathrm{Ru}^{\mathrm{II}}\left(\mathrm{PPh}_{3}\right)_{2} \mathrm{Cp}\right\}\right]^{n+}\{\mathrm{Cp}=$ cyclopentadienyl anion; dppe = 1,2bis(diphenylphosphine)ethane; $\mathrm{L}=$ py, $\left.\mathrm{CN}^{-}\right\} .{ }^{19}$ Remote IVCT transitions $\left(\mathrm{Ru}^{\mathrm{II}} \rightarrow \mathrm{Fe}^{\mathrm{III}}\right)$ were observed for the trans-configured species only, such that the metal-metal interactions were facilitated by a polynuclear backbone in which the metals and intervening bridging ligands provided a linear (rather than angular) conduit for electron transfer.

While a number of important aspects relating to the influence of the nuclearity and overall oxidation-state of the assembly on the IVCT properties have been investigated, the majority of studies on the physical characteristics of polymetallic assemblies have been conducted without regard for the inherent stereochemical complexities in such systems. ${ }^{20,21}$ Three major factors are crucial to extend the IVCT probe to higher nuclearity systems: firstly, the effect of increasing the number of electronicallycoupled metal centres on the IVCT transitions (specifically, their energy, intensity and bandwidth); 
secondly, the effect of the relative distances and orientations of the centres; and thirdly, their stereochemical relationship. ${ }^{20,21}$ Given the diverse range of metallosupramolecular architectures, these factors underlie the need for a systematic understanding of how the nuclearity and geometry of these systems influences their electron transfer properties. Knowledge of the electron-transfer properties of the components, the bridging ligands - and their interdependence as a result of the molecular architecture of the assembly - gives rise to the potential for control of useful processes involving multiple-electron transfer phenomena. These aspects are addressed in a review article titled "IVCT in Tri- and Tetranuclear Complexes of Iron, Ruthenium and Osmium” submitted for publication by D. M. D’Alessandro and F. R. Keene, which is included on the CD accompaniment to this thesis.

\subsubsection{Trinuclear Polymetallic Assemblies: Molecular Architectures and Applications}

A number of comprehensive reviews over the last decade have addressed the design and synthetic routes to tri-, tetra- and higher nuclearity complexes possessing a range of architectural motifs. ${ }^{22-29}$ Due to their multicomponent nature, these structures have considerable design potential which exploits the cooperation between the metals and/or other redox-active centres. Novel photochemical molecular devices (PMDs) have been constructed which are capable of performing useful light- and redox-induced functions including artificial photosynthesis and photoinduced energy and electron transfer processes in light-harvesting "antenna" systems. ${ }^{30-32}$ Metallosupramolecular assemblies have also been designed to mimic the photoinduced charge separation function in photosynthetic organisms, in an attempt to elucidate the complex electron and energy transfer mechanisms which occur in natural systems. ${ }^{30,33-37}$ The possibility of multiple electron transfer - arising from absorption of several photons by linked chromophores or the design of systems which generate more than one electron upon absorption of one photon - has significant implications in catalytic schemes, for understanding long-range electron transfer in biological systems, and the conductivity of "molecular wires". In molecules involving delocalised unpaired electrons, polarisability may be present so that the species exhibit interesting nonlinear optical or magnetic properties. Clearly, the realisation of such diverse practical applications of polymetallic assemblies is critically dependent on the understanding of the intramolecular electron transfer processes that occur between the constituent metal-based chromophores.

The polypyridyl bridging ligands 2,2'-bipyrimidine (bpm), 2,3-bis(2-pyridyl)pyrazine (2,3-dpp), 2,3-bis(2-pyridyl)quinoxaline (dpq) and 2,3-bis(2-pyridyl)-1,4-benzoquinoxaline (dpb) have been utilised extensively as the basis of homo- and hetero-trinuclear complexes of the form $\left[\left\{\mathrm{M}(\mathrm{bpy})_{2}\right\}_{2}\left\{\mathrm{M}^{\prime}(\mathrm{Cl})_{2}(\mu-\right.\right.$ $\left.\left.\mathrm{BL})_{2}\right\}\right]^{4+}\left\{\mathrm{M}, \mathrm{M}^{\prime}=\mathrm{Ru}^{\mathrm{II}}, \mathrm{Os}^{\mathrm{II}}, \mathrm{Ir}^{\mathrm{III}}, \mathrm{Rh}^{\mathrm{IIII}}\right.$; $\mathrm{BL}=$ bpm, 2,3-dpp, dpq, dpb; bpy = 2,2'-bipyridine , since the variation of the bridging ligands and metal centres permits extensive tuning of the ground and excitedstate properties. ${ }^{38-52}$ The trinuclear complexes $\left[\left\{\operatorname{Ru}(\mathrm{bpy})_{2}\right\}_{2}\left\{\operatorname{Ir}(\mathrm{Cl})_{2}(\mu-\mathrm{BL})\right\}\right]^{5+}\{\mathrm{BL}=\mathrm{dpq}$ and dpb $\}$ have been implicated in novel schemes for the reduction of carbon dioxide, ${ }^{53}$ where the bridging ligands serve as multiple electron reservoirs for the central catalytically-active $\operatorname{Ir}^{\mathrm{III}}(\mathrm{Cl})_{2}(\mathrm{BL})_{2}$ core. The related species $\left[\left\{\mathrm{Ru}(\mathrm{bpy})_{2}\right\}_{2}\left\{\mathrm{Rh}(\mathrm{Cl})_{2}(\mu-2,3-\mathrm{dpp})\right\}\right]^{5+},\left[\left\{\mathrm{Os}(\mathrm{bpy})_{2}\right\}_{2}\left\{\mathrm{Rh}(\mathrm{Cl})_{2}(\mu-2,3-\mathrm{dpp})\right]^{5+}\right.$ and 
$\left[\{\mathrm{Ru}(\mathrm{tpy}) \mathrm{Cl}\}_{2}\left\{\mathrm{Rh}(\mathrm{Cl})_{2}(\mu-2,3-\mathrm{dpp})\right]^{3+}\left\{\text { tpy }=2,2^{\prime}: 6^{\prime}, 2^{\prime \prime} \text {-terpyridine }\right\}^{54}\right.$ have been investigated for the visible light-induced photocleavage of DNA. By taking advantage of the lability of the chloride ligands at the central metal, these "active trinuclear dendrons" have provided the basis of higher-nuclearity dendritic species containing up to twenty-two metal sub-units., ${ }^{3,55-74}$ The polymetallic systems described here exhibit additional complexities due to the potential chirality ( $\Delta$ or $\Lambda$ ) of the constituent metal centres, and the unsymmetrical nature or lack of structural rigidity in the bridging ligands. While "flexible" bridging ligands based on the $\alpha, \alpha^{\prime}$-diimine moiety have been utilised extensively as the basis of polymetallic assemblies, the possibility of bond rotation in the phenyl or alkynyl linkage between the ligating groups leads to structurally ambiguous assemblies due to variations in the inter-component distances and orientations. ${ }^{1,75-83}$

\subsubsection{Stereochemical Ambiguities in Higher Nuclearity Polymetallic Assemblies}

As discussed in §1.6, the majority of studies concerning the physical characteristics of polymetallic assemblies have been conducted on systems which may comprise many stereoisomeric forms. ${ }^{20,21}$ The stereoisomeric complexity increases exponentially with the number of metal centres, and with the introduction of unsymmetrical terminal and/or bridging ligands. While all the possible stereoisomers may not be represented in a synthesised mixture, the presence of as few as two diastereoisomers introduces considerable complexity into the structural characterisation process by conventional NMR, IR and UV-Vis techniques. For higher nuclearity complexes, electrospray ${ }^{51,84-86}$ and fast-atom bombardment ${ }^{65,87-89}$ mass spectrometry have been utilised as the predominant means of characterisation of nuclearity, however the stereochemical composition remains ambiguous. While X-ray crystallography has permitted the unambiguous stereochemical identification of a number of dinuclear complexes, ${ }^{88,90-97}$ few solid state structures of higher nuclearity polypyridyl complexes exist due to difficulties in obtaining crystalline materials suitable for X-ray analysis. ${ }^{98}$

Significant differences in the overall geometry of the assembly may arise depending on the relative stereochemistries of the component metal-based chromophores. In the trinuclear complexes, $\left[\left\{\mathrm{Ru}(\mathrm{bpy})_{2}\right\}_{2}\left\{\mathrm{Os}(\mathrm{Cl})_{2}(\mu-\mathrm{BL})\right\}\right]^{4+}\{\mathrm{BL}=2,3-\mathrm{dpp}, \mathrm{dpq}, \mathrm{dpb}\}^{43}$ the bridging Os centre may possess either cis $(\mathrm{Cl})$ or trans $(\mathrm{Cl})$ geometry, and since the 2,3-dpp ligand is unsymmetrical, the trans and cis forms exhibit two and three different geometric isomers, respectively. The cis isomers each possess an enantiomeric form, as do both ruthenium centres. As a result, the complex may exist in sixteen different diastereoisomeric forms, of which only two possess enantiomers. ${ }^{20}$ The $\Delta(\mathrm{Ru}) \Delta(\mathrm{Os}) \Delta(\mathrm{Ru})$ forms, for example, differ only in that the geometric arrangement about the Os centre is cis(Cl)cis(pyz) and cis(Cl)trans(pyz) \{pyz = pyrazine $\}$, giving rise to an "angular" or "linear" disposition of the two terminal metal centres respectively, as shown in Figure 4.2. 


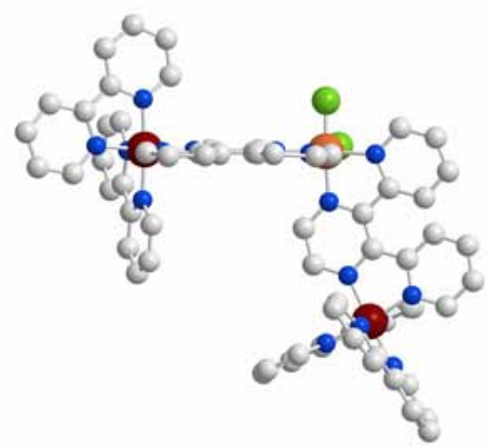

(a)

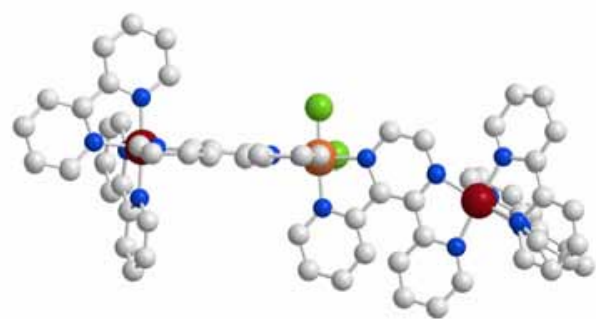

(b)

Figure 4.2 The $\Delta(\mathrm{Ru}) \Delta(\mathrm{Os}) \Delta(\mathrm{Ru})$ forms of $\left[\left\{\mathrm{Ru}(\mathrm{bpy})_{2}\right\}_{2}\left\{\mathrm{Os}(\mathrm{Cl})_{2}(\mu-2,3-\mathrm{dpp})\right\}\right]^{4+}$ illustrating the consequences of the (a) $\operatorname{cis}(\mathrm{Cl}) \operatorname{cis}(\mu-2,3-\mathrm{dpp})$ and (b) $\operatorname{cis}(\mathrm{Cl}) \operatorname{trans}(\mu-2,3-\mathrm{dpp})$ arrangement about the Os centres.

Recently, the development of synthetic and chromatographic methodologies ${ }^{20,21}$ for the isolation of the individual stereoisomers of polymetallic assemblies has permitted the investigation of stereochemical influences on their intramolecular electron transfer characteristics. This has provided the first examples of differences in the physical properties of the stereoisomeric forms of mono-, di- and trinuclear complexes, as discussed in §1.6.3. ${ }^{99-102}$

\subsubsection{Scope and Objectives of the Present Study}

The present study represents the first systematic investigation of IVCT in di- and trinuclear polypyridyl complexes of ruthenium(II) and osmium(II) based on the bridging ligands HAT (1,4,5,8,9,12hexaazatriphenylene) and ppz (4,7-phenanthrolino-5,6:5',6'-pyrazine). The study of rigid, stereochemically-unambiguous complexes addresses the often-neglected stereochemical complexities inherent in such systems, and their influence on the electrochemical, spectral and IVCT properties. Specifically, the study addresses the following influences on IVCT:

(i) increasing nuclearity of the assembly (from dinuclear to trinuclear);

(ii) the relative distances and orientations of the electronically-coupled metal centres;

(iii) the oxidation state of the assembly;

(iv) the global geometry of the assembly; and

(v) the stereochemical relationship of the component metal centres.

The implications of the results for the theoretical analysis of IVCT in trinuclear complexes are also discussed.

\section{(1) Mono-, Di- and Trinuclear Assemblies Based on the Bridging Ligand HAT}

The bridging ligand HAT (Figure 4.3) represents a particularly attractive basis for polymetallic assemblies as the planar delocalised $\pi$-electron system facilitates a strong electronic interaction between the metal centres which are coordinated at the three potential bidentate sites. ${ }^{20,21,101}$ Mono-, di- and trinuclear complexes of HAT have been synthesised, ${ }^{101,103-114}$ as well as elaborate three-dimensional 
assemblies such as molecular squares ${ }^{115}$ and microporous networks. ${ }^{105,116,117}$ These have been recognised for their potential applications as biological probes ${ }^{106,112,113}$ and as components in novel molecular materials. ${ }^{118}$

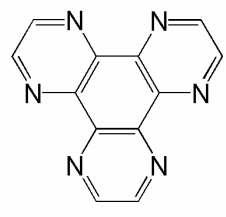

HAT

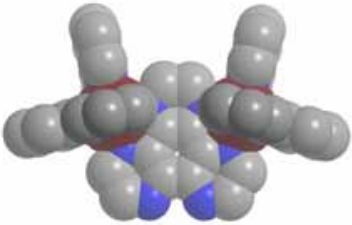

(a) $\Delta \Lambda$

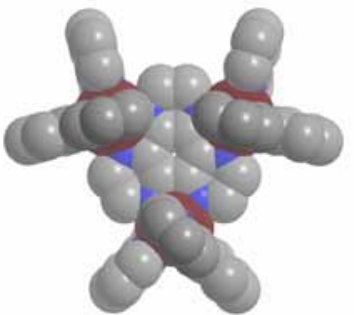

(c) $\Delta_{2} \Lambda$

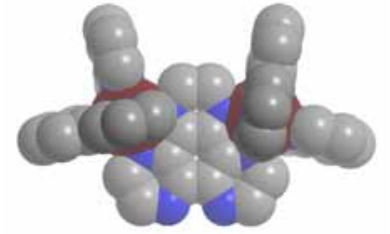

(b) $\Delta \Delta$

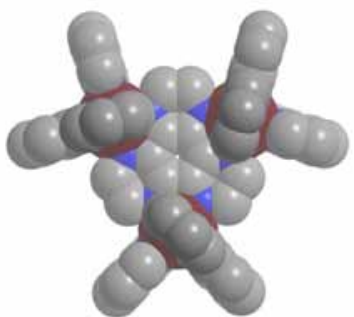

(d) $\Delta_{3}$

Figure 4.3 The bridging ligand HAT, and Chem 3D representations of (a) the meso $(\Delta \Lambda)$ and (b) rac $(\Delta \Delta)$ diastereoisomers of $\left[\left\{\mathrm{Ru}(\mathrm{bpy})_{2}\right\}_{2}(\mu \text {-HAT) }]^{4+}\right.$, and (c) the heterochiral $\left(\Delta_{2} \Lambda\right)$ and (d) homochiral $\left(\Delta_{3}\right)$ diastereoisomers of $\left[\left\{\mathrm{Ru}(\mathrm{bpy})_{2}\right\}_{3}(\mu-\mathrm{HAT})\right]^{6+}$. Hydrogen atoms are omitted for clarity.

The present work reports the first study on the IVCT properties of the homo-dinuclear and homotrinuclear ruthenium complexes meso- $(\Delta \Lambda)$ - and $\operatorname{rac}-(\Delta \Delta / \Lambda \Lambda)-\left[\left\{\mathrm{Ru}(\mathrm{bpy})_{2}\right\}_{2}(\mu-\mathrm{HAT})\right]^{4+}$, and homochiral$\left(\Delta_{3} / \Lambda_{3}\right)$ - and heterochiral- $\left(\Delta_{2} \Lambda / \Lambda_{2} \Delta\right)-\left[\left\{\mathrm{Ru}(\mathrm{bpy})_{2}\right\}_{3}(\mu \text {-HAT })\right]^{6+}$, shown in Figure 4.3. The analysis is subsequently broadened to the diastereoisomers of the homo- and hetero-dinuclear osmium systems $\left[\left\{\mathrm{Os}(\mathrm{bpy})_{2}\right\}_{2}(\mu \text {-HAT })\right]^{4+}$ and $\left[\left\{\mathrm{Ru}(\mathrm{bpy})_{2}\right\}(\mu \text {-HAT })\left\{\mathrm{Os}(\mathrm{bpy})_{2}\right\}\right]^{4+}$, respectively, and to the heterotrinuclear complex heterochiral- $\left(\Delta_{2} \Lambda^{\prime} / \Lambda_{2} \Delta^{\prime}\right)-\left[\left\{\mathrm{Ru}(\mathrm{bpy})_{2}\right\}_{2}\left\{\mathrm{Os}(\mathrm{bpy})_{2}\right\}(\mu \text {-HAT) }]^{6+}\right.$ (where the prime denotes the stereochemistry of the Os centre).

\section{(2) Mono-, Di- and Trinuclear Assemblies Based on the Bridging Ligand ppz}

The bridging ligand ppz (Figure 4.4) is closely related electronically and structurally to HAT and the bridging ligand 2,3-dpp which have been utilised extensively in the construction of tri-, tetra- and higher nuclearity assemblies. ${ }^{30,55,59}$ The planarity of ppz overcomes the problem of conformational lability in complexes incorporating 2,3-dpp, ${ }^{21}$ which has impeded attempts to obtain stereochemicallypure higher nuclearity complexes based on the latter bridging ligands. ${ }^{119}$ In the present investigation, the nature of the IVCT characteristics in the "chain-like" trinuclear complex $\Delta \Delta^{t} \Delta-\left[\left\{\operatorname{Ru}(\mathrm{bpy})_{2}\right\}_{2}\left\{\mathrm{Ru}(\mathrm{bpy})_{2}(\mu-\right.\right.$ ppz) $\}]^{6+}$ are compared with the "cluster-like" systems based on HAT.

Due to the unsymmetrical nature of the ppz bridging ligand, the mononuclear "core" $\left[\mathrm{Ru}(\mathrm{bpy})(\mathrm{ppz})_{2}\right]^{2+}$ possesses two geometric isomers, trans and cis, shown in Figure 4.4, in addition to the $\Lambda$ and $\Delta$ enantiomeric forms of each. While symmetrical bridges such as bpm provide the basis for structurally-rigid assemblies such as $\left[\left\{\mathrm{Ru}(\mathrm{bpy})_{2}\right\}_{2}\left\{\mathrm{Ru}(\mathrm{Cl})_{2}(\mathrm{bpm})_{2}\right\}\right]^{4+}, 38$ these systems are restricted to an overall "angular" geometry due to the cis configuration at the central metal. However, by the 
incorporation of the cis and trans geometric isomers of $\left[\mathrm{Ru}(\mathrm{bpy})(\mathrm{ppz})_{2}\right]^{2+}$ as the central unit, the construction of assemblies with overall "angular" or "linear" geometries is permitted. The use of unsymmetrical bridging ligands such as ppz thus provides for subtle variations in the overall geometry of assemblies, beyond that which may be achieved using linear bridging ligands such as bpm.

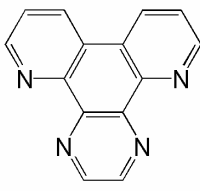

$\mathrm{ppz}$

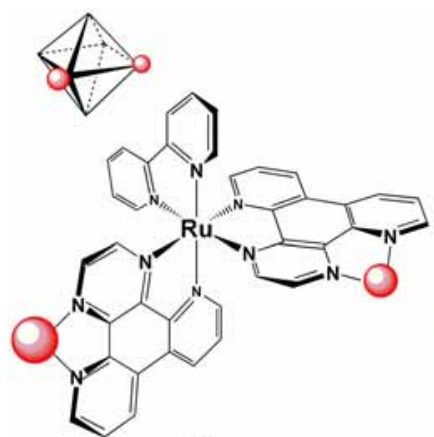

cis

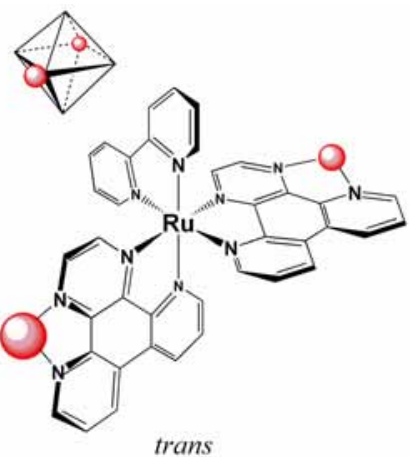

$\Lambda^{\mathrm{t}}-\left[\mathrm{Ru}(\mathrm{bpy})(\mathrm{ppz})_{2}\right]^{2+}$

Figure 4.4 The bridging ligand ppz, and schematic representations of the geometric isomers of $\Lambda$ $\left[\mathrm{Ru}(\mathrm{bpy})(\mathrm{ppz})_{2}\right]^{2+}$ illustrating the relative cis or trans disposition of the bidentate sites available for the potential coordination of metal centres, $\bigcirc$ (e.g. $\Lambda$ - or $\Delta$-[Ru(bpy) $\left.\left.)_{2}\right]^{2+}\right)$.

A combined stereoretentive synthetic and chromatographic strategy is employed for the construction of the trinuclear complex $\Delta \Delta^{t} \Delta-\left[\left\{\operatorname{Ru}(\text { bpy })_{2}\right\}_{2}\left\{\operatorname{Ru}(\text { bpy })(\mu-\mathrm{ppz})_{2}\right\}\right]^{6+}$, shown in Figure 4.5. The IVCT characteristics of the novel "chain-like" trinuclear assembly are compared with the analogous dinuclear systems meso- $(\Delta \Lambda)$ - and $\operatorname{rac}-(\Delta \Delta / \Lambda \Lambda)-\left[\left\{\operatorname{Ru}(\mathrm{bpy})_{2}\right\}_{2}(\mu-\mathrm{ppz})\right]^{4+}$, and with the "cluster-like" assemblies based on HAT.

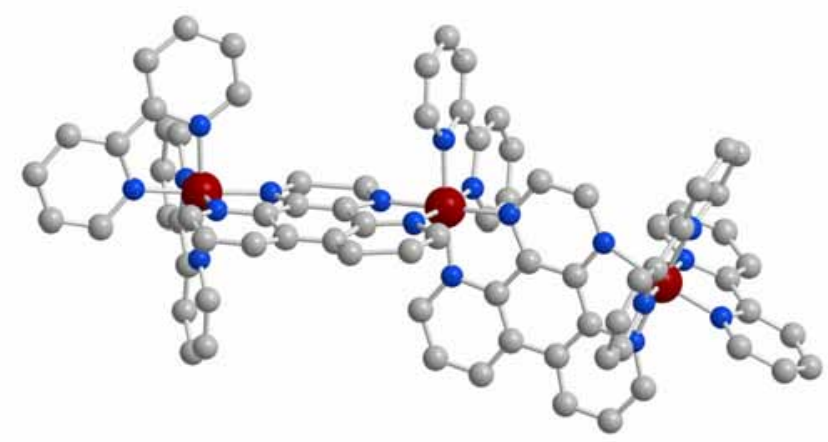

Figure 4.5 Chem 3D representation of $\Delta \Delta^{\mathrm{t}} \Delta-\left[\left\{\mathrm{Ru}(\mathrm{bpy})_{2}\right\}_{2}\left\{\mathrm{Ru}(\mathrm{bpy})(\mu-\mathrm{ppz})_{2}\right\}\right]^{6+}$ investigated in the present study. Hydrogen atoms are omitted for clarity. 


\subsection{Experimental}

\subsubsection{Materials}

The materials used in this chapter were identical to those reported earlier in §3.2.1, with the following additions: pyridine (py; Aldrich, 98\%), 1,2-dichlorobenzene (Aldrich, 99\%) and 2-methoxyethanol (Fluka, 99.5\%) were distilled prior to use. Trifluoromethanesulfonic acid (3 M) was distilled in an all-glass apparatus under vacuum prior to use. All other solvents were of laboratory grade unless otherwise specified. Trimethylamine $N$-oxide dihydrate (TMNO; Fluka) was purified by sublimation at $120^{\circ} \mathrm{C}$ under vacuum and stored under argon. Aqueous solutions of the sodium salts of (-)-O,O'-dibenzoyl-L-tartrate and (+)-di-O,O'-4-toluoyl-D-tartrate were prepared by neutralisation of the corresponding acids (Fluka, 98+\%) using sodium hydroxide $(\mathrm{NaOH})$.

The bridging ligand HAT (1,4,5,8,9,12-hexxazatriphenylene) was supplied by Dr Nicholas Fletcher, ${ }^{109}$ and the synthesis of ppz (4,7-phenanthrolino-5,6:5',6'-pyrazine) was described previously in §3.2.3.1.

\subsubsection{Instrumentation and Physical Methods}

The chromatographic procedures, structural characterisation methods (elemental microanalyses, circular dichroism) and instrumentation and physical measurements (electronic spectroscopy, electrochemistry, UV/Vis/NIR spectroelectrochemistry) were identical to those detailed in §2.2.2 and $\S 3.2 .2$.

\section{UV/Vis/NIR Spectroelectrochemistry}

Solutions for the spectroelectrochemical experiments contained $0.1 \mathrm{M}\left[\left(n-\mathrm{C}_{4} \mathrm{H}_{9}\right)_{4} \mathrm{~N}\right] \mathrm{PF}_{6}$ supporting electrolyte in $\mathrm{CH}_{3} \mathrm{CN}$ and the complex $\left(\mathrm{ca} .1 \times 10^{-3} \mathrm{M}\right)$. The low temperature facility enabled in situ spectroelectrochemical characterisation of the electrogenerated species which were otherwise unstable at room temperature. The dinuclear systems required approximately $6 \mathrm{~h}$ for data collection at $-35^{\circ} \mathrm{C}$, while the trinuclear systems required approximately $10 \mathrm{~h}$ and were conducted at $-15^{\circ} \mathrm{C}$ due to limitations in the availability of a continuous supply of coolant gas.

The analysis and Gaussian deconvolution of the spectral data were performed according to the methods described previously in §2.2.2 and §3.2.2.

\subsubsection{Synthesis of Mono-, Di- and Trinuclear Complexes}

cis-[Ru(bpy) $\left.{ }_{2} \mathrm{Cl}_{2}\right] \cdot 2 \mathrm{H}_{2} \mathrm{O}^{120}$ was prepared according to the literature procedure and cis[Os(bpy $\left.)_{2} \mathrm{Cl}_{2}\right] .2 \mathrm{H}_{2} \mathrm{O}$ was prepared as described previously (§3.2.3.2). $\left[\left\{\mathrm{Ru}(\mathrm{bpy})_{2}\right\}_{2}\left\{\mathrm{Os}(\mathrm{bpy})_{2}\right\}(\mu-\right.$ HAT $)]\left(\mathrm{PF}_{6}\right)_{6}$ was supplied by Dr Todd Rutherford, ${ }^{48,103}\left(\mathrm{NH}_{4}\right)_{2}\left[\mathrm{Os}^{\mathrm{IV}} \mathrm{Cl}_{6}\right]$ was supplied by Dr Eric Jandrasics and $\left[\mathrm{Ru}(\mathrm{bpy}) \mathrm{Cl}_{4}\right]^{121}$ was supplied by Dr Bradley Patterson. 


\subsubsection{Mono-, Di- and Trinuclear Assemblies Based on HAT}

The synthesis and characterisation of $\left[\left\{\mathrm{Ru}(\mathrm{bpy})_{2}\right\}_{2}(\mu-\mathrm{HAT})\right]\left(\mathrm{PF}_{6}\right)_{4},\left[\left\{\mathrm{Ru}(\mathrm{bpy})_{2}\right\}_{3}(\mu-\mathrm{HAT})\right]\left(\mathrm{PF}_{6}\right)_{6}$, and $\left[\left\{\mathrm{Ru}(\mathrm{bpy})_{2}\right\}_{2}\left\{\mathrm{Os}(\mathrm{bpy})_{2}\right\}(\mu-\mathrm{HAT})\right]\left(\mathrm{PF}_{6}\right)_{6}$, as well as the separation and purification of the respective diastereoisomers, were performed using the techniques reported previously. ${ }^{48,101,103}$

[Ru(bpy)(HAT) $\left.)_{2}\right]\left(\mathbf{P F}_{6}\right)_{2}$ was synthesised via a procedure analogous to that reported previously for [Ru(bpy)(2,3-dpp) $\left.)_{2}\right]\left(\mathrm{PF}_{6}\right)_{2}{ }^{122}$ A suspension of HAT (200 mg, $0.854 \mathrm{mmol}$ ) was heated in ethylene glycol $\left(10 \mathrm{~cm}^{3}\right)$ for $5 \mathrm{~min}$ via microwave heating to complete dissolution. [Ru(bpy)Cl ${ }_{4}$ ( $45 \mathrm{mg}, 0.113 \mathrm{mmol}$ ) was added in four portions over $10 \mathrm{~min}$, during which time the solution attained an orange colouration. After further heating (high power) for $5 \mathrm{~min}$, the mixture was quenched with distilled water $\left(\mathrm{ca} .50 \mathrm{~cm}^{3}\right)$ and filtered to recover the excess HAT ligand. One major band was observed in the gradient elution procedure on SP Sephadex C-25 cation exchanger $(20 \times 2.5 \mathrm{~cm})$, and was eluted with $0.15 \mathrm{M} \mathrm{NaCl}$. Following the addition of a saturated solution of aqueous $\mathrm{KPF}_{6}$, the mixture was chilled by refrigeration overnight. The orange precipitate was isolated by filtration, washed with chilled water $\left(3 \mathrm{~cm}^{3}\right)$, diethyl ether $\left(3 \times 10 \mathrm{~cm}^{3}\right)$ and dried in vacuo. Yield: $70.2 \mathrm{mg}(61 \%)$. Anal. Calcd for $\mathrm{C}_{34} \mathrm{H}_{20} \mathrm{~F}_{12} \mathrm{~N}_{14} \mathrm{P}_{2} \mathrm{Ru}$ : C, 40.2; H, 1.98; N, 19.3\%. Found: C, 40.0; H, 2.05; N, 19.2\%. Further structural characterisation was performed following separation and chiral resolution of the geometrical isomers and their enantiomeric forms.

Resolution of $\left[\mathbf{R u}(\mathbf{b p y})(\mathbf{H A T})_{2}\right]\left(\mathbf{P F}_{6}\right)_{2}$. The mononuclear complex was resolved via an analogous procedure to that described previously for $\left[\mathrm{Ru}(\mathrm{bpy})_{2}(\mathrm{HAT})\right]^{2+}{ }^{101}$ The $\mathrm{PF}_{6}{ }^{-}$salt was converted to the corresponding aqueous $\mathrm{Cl}^{-}$form by stirring with $\operatorname{DOWEX}^{\circledR}\left(1 \times 8,50-100\right.$ mesh, $\mathrm{Cl}^{-}$form $)$anion exchange resin. The aqueous solution was sorbed onto SP Sephadex C-25 cation exchanger $(96 \times 2 \mathrm{~cm})$ and chromatographed using $0.10 \mathrm{M}(+)$-di-O,O'-4-toluoyl-D-tartrate solution. Resolution of the enantiomeric forms was achieved with $\sim 50 \mathrm{~cm}$ of travel down the column. The products from the two bands were precipitated on addition of aqueous $\mathrm{KPF}_{6}$. The solutions were refrigerated overnight and the solids were isolated by filtration, washed with chilled water $\left(3 \mathrm{~cm}^{3}\right)$, diethyl ether $\left(3 \times 10 \mathrm{~cm}^{3}\right)$ and dried in vacuo. By comparison with similar complexes of known configuration, ${ }^{101}$ Band 1 was assigned as $\Delta-(-)-\left[\mathrm{Ru}(\mathrm{bpy})(\mathrm{HAT})_{2}\right]^{2+}$ and Band 2 as $\Lambda$-(+)-[Ru(bpy)(HAT) $\left.)_{2}\right]^{2+}$. UV/Vis in $\mathrm{CH}_{3} \mathrm{CN}, \lambda / \mathrm{nm}$ $\left\{\varepsilon_{\max } / \mathrm{M}^{-1} \mathrm{~cm}^{-1}\right.$ (Band $1=$ Band 2) \}: 210 (52089), 275 (54956), 408 (11326), 466 (11089). CD spectra in $\mathrm{CH}_{3} \mathrm{CN}, \lambda / \mathrm{nm}\left\{\Delta \varepsilon / \mathrm{M}^{-1} \mathrm{~cm}^{-1}\right.$ (Band 1, Band 2)\}: 206 (-18.0, +19.6), 224 (+8.85, -8.51), 259 (+7.58, -8.02), $297(-49.4,+47.2), 318(-53.0,+52.0), 392(-15.8,+15.6), 482(-9.73,+10.1)$. The ${ }^{1} \mathrm{H}$ NMR spectra are reported in Table 4.8, §4.3.3.1. 
rac- $\left[\mathbf{O s}(\mathbf{b p y})_{2}(\mathrm{HAT})\right]\left(\mathbf{P F}_{6}\right)_{2}$ and $\left[\left\{\mathbf{O s}(\mathbf{b p y})_{2}\right\}_{2}(\mu-\mathrm{HAT})\right]\left(\mathbf{P F}_{6}\right)_{4}$. The synthesis and purification were performed via an analogous procedure to that reported previously for $\left[\left\{\mathrm{Ru}(\mathrm{bpy})_{2}\right\}_{2}(\mu-\mathrm{HAT})\right]\left(\mathrm{PF}_{6}\right)_{4}{ }^{101}$ using cis-[Os(bpy) $\left.{ }_{2} \mathrm{Cl}_{2}\right] \cdot 2 \mathrm{H}_{2} \mathrm{O}$ as the precursor instead of cis-[Ru(bpy $\left.)_{2} \mathrm{Cl}_{2}\right] \cdot 2 \mathrm{H}_{2} \mathrm{O}$. A suspension of HAT (14.6 mg, $0.0623 \mathrm{mmol})$ in ethylene glycol $\left(3 \mathrm{~cm}^{3}\right)$ was heated in a modified microwave oven on medium high power for $3 \mathrm{~min}$ to complete dissolution. cis-[Os(bpy $\left.)_{2} \mathrm{Cl}_{2}\right] \cdot 2 \mathrm{H}_{2} \mathrm{O}(71.5 \mathrm{mg}, 0.117 \mathrm{mmol})$ was added in four portions over 10 min during which time the solution attained a purple colouration. The crude mixture was diluted with distilled water $\left(50 \mathrm{~cm}^{3}\right)$ and loaded onto a column of SP Sephadex C-25 (dimensions $3 \times 25 \mathrm{~cm}$ ). Separation of the desired dinuclear product from the mixture was achieved via the gradient elution procedure described by Masschelein et al. ${ }^{50}$ A dark orange band of the mononuclear complex eluted first $(0.20 \mathrm{M} \mathrm{NaCl})$ followed by a dark purple band of the dinuclear complex $(0.40 \mathrm{M}$ $\mathrm{NaCl}$ ). The complexes were precipitated as their $\mathrm{PF}_{6}{ }^{-}$salts by addition of a saturated solution of $\mathrm{KPF}_{6}$ and the solids were isolated by vacuum filtration and washed with diethyl ether $\left(3 \times 10 \mathrm{~cm}^{3}\right)$. Yields: mononuclear complex: $27.0 \mathrm{mg}$; dinuclear complex: $67.7 \mathrm{mg}(60 \%) . \mathbf{R a c}-\left[\mathbf{O s}(\mathbf{b p y})_{\mathbf{2}}(\mathbf{H A T}) \mathbf{( P F}_{\mathbf{6}} \mathbf{2}_{\mathbf{2}}\right.$. ${ }^{1} \mathrm{H}$ NMR ( $\delta$ ppm; $\mathrm{CD}_{3} \mathrm{CN}$ ): 7.18 (H5', 2H, $J=8,5 \mathrm{~Hz}$, dd), 7.48 (H5, 2H, $J=8,5 \mathrm{~Hz}$, dd), 7.50 (H6', 2H, $J=$ 5, $1.5 \mathrm{~Hz}, \mathrm{dd}), 7.67$ (H6, 2H, $J=5,1.5 \mathrm{~Hz}, \mathrm{dd}), 7.93$ (H4', 2H, $J=8,8 \mathrm{~Hz}, \mathrm{dd}$ ), 8.00 (H4, 2H, $J=8,8 \mathrm{~Hz}$, dd), 8.34 (H2/H11 HAT, 2H, $J=3$ Hz, d), 8.50 (H3', 2H, $J=8,1.5 \mathrm{~Hz}, \mathrm{dd}), 8.57$ (H3, 2H, $J=8,1.5 \mathrm{~Hz}$,

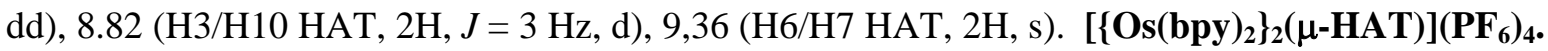
Anal. Calcd for $\mathrm{C}_{52} \mathrm{H}_{38} \mathrm{~F}_{24} \mathrm{~N}_{14} \mathrm{P}_{4} \mathrm{Os}_{2}$ : C, 34.3; H, 2.11; N, 10.8\%. Found: C, 34.4; H, 2.50; N, 10.8\%.

Separation of the diastereoisomers of $\left[\left\{\mathrm{Os}(\mathrm{bpy})_{2}\right\}_{2}(\mu-\mathrm{HAT})\right]\left(\mathrm{PF}_{6}\right)_{4}$ was achieved as detailed previously for the related complexes $\left[\left\{\mathrm{Ru}(\mathrm{bpy})_{2}\right\}_{2}(\mu-\mathrm{BL})\right]^{4+}\{$ e.g. BL $=\mathrm{dpb}, \mathrm{ppz}\}$ in §3.2.3.3 using SP Sephadex C-25 support with 0.25 M sodium tosylate solution as the eluent. Bands 1 and 2 were determined to be the meso and rac diastereoisomers, respectively, as established by ${ }^{1} \mathrm{H}$ and COSY NMR characterisation and by comparison with their ruthenium analogues. ${ }^{1} \mathrm{H}$ NMR ( $\left.\delta \mathrm{ppm} ; \mathrm{CD}_{3} \mathrm{CN}\right)$ : (Band 1; meso) 7.20 (H5d, 2H, $J$ = 8, 5 Hz, dd), 7.36 (H5b, 2H, $J=8,5$ Hz, dd), 7.44 (H5c, 2H, $J=8,5 \mathrm{~Hz}$, dd), 7.50 (H5a, 2H, $J=8,5 \mathrm{~Hz}$, dd), 7.54 (H6d, 2H, $J=5,1.5 \mathrm{~Hz}, \mathrm{dd}$ ), 7.62 (H6a, 2H, $J=5,1.5 \mathrm{~Hz}, \mathrm{dd}$ ), 7.65 (H6c, 2H, $J=5,1.5 \mathrm{~Hz}, \mathrm{dd}$ ), 7.76 (H6b, 2H, $J=5,1.5 \mathrm{~Hz}, \mathrm{dd}$ ), 7.82 (H10/H11 HAT, 2H, s), 7.89 (H4d, $2 \mathrm{H}, J=8,8 \mathrm{~Hz}, \mathrm{dd}), 7.93(\mathrm{H} 4 \mathrm{c}, 2 \mathrm{H}, J=8,8 \mathrm{~Hz}, \mathrm{dd}), 8.00$ (H4b, 2H, $J=8,8 \mathrm{~Hz}, \mathrm{dd}), 8.02$ (H4a, 2H, $J=$ 8, $8 \mathrm{~Hz}, \mathrm{dd}$ ), 8.40 (H3d, 2H, $J=8,1.5 \mathrm{~Hz}, \mathrm{dd}$ ), 8.44 (H2/H7 HAT, 2H, $J=3 \mathrm{~Hz}, \mathrm{~d}), 8.54$ (H3c, 2H, $J=8$, $1.5 \mathrm{~Hz}, \mathrm{dd}$ ), 8.52 (H3b, 2H, $J=8,1.5 \mathrm{~Hz}, \mathrm{dd}$ ), 8.42 (H3a, 2H, $J=8,1.5 \mathrm{~Hz}, \mathrm{dd}$ ), 8.87 (H3/H6 HAT, 2H, $J=3 \mathrm{~Hz}, \mathrm{~d}$ ); (Band 2; rac) 7.08 (H5b, 2H, $J=8,5 \mathrm{~Hz}, \mathrm{dd}$ ), 7.32 (H5d, 2H, $J=8,5 \mathrm{~Hz}, \mathrm{dd}$ ), 7.36 (H6b, 2H, $J=5,1.5 \mathrm{~Hz}, \mathrm{dd}), 7.41$ (H5c, 2H, $J=8,5 \mathrm{~Hz}, \mathrm{dd}), 7.52$ (H5a, 2H, $J=8,5 \mathrm{~Hz}, \mathrm{dd}), 7.52$ (H6c, 2H, $J$ = 5, $1.5 \mathrm{~Hz}, \mathrm{dd}), 7.62$ (H6d, 2H, $J=$ 5, $1.5 \mathrm{~Hz}, \mathrm{dd}), 7.65$ (H6a, 2H, $J=5,1.5 \mathrm{~Hz}$, dd), 7.88 (H10/H11 HAT, 2H, s), 7.94 (H4b, 2H, $J=8,8$ Hz, dd), 7.95 (H4c, 2H, $J=8,8$ Hz, dd), 8.03 (H4b, 2H, $J=8,8$ Hz, dd), 8.06 (H4a, 2H, $J=8,8$ Hz, dd), 8.46 (H2/H7 HAT, 2H, $J=3$ Hz, d), 8.51 (H3b, 2H, $J=8,1.5$ Hz, dd), 8.55 (H3c, 2H, $J=8,1.5$ Hz, dd), 8.55 (H3d, 2H, $J=8,1.5$ Hz, dd), 8.60 (H3a, 2H, $J=8,1.5 \mathrm{~Hz}$, dd), 8.89 (H6/H7 HAT, 2H, $J=3$ Hz, d). 
$\left[\left\{\mathbf{R u}(\mathbf{b p y})_{2}\right\}(\mu-\mathbf{H A T})\left\{\mathbf{O s}(\mathbf{b p y})_{2}\right\}\right]\left(\mathbf{P F}_{6}\right)_{4}$ was synthesised in a similar manner to that described previously for the unsymmetrical compound $\left[\left\{\mathrm{Ru}(\mathrm{bpy})_{2}\right\}(\mu-\mathrm{HAT})\left\{\mathrm{Ru}(\mathrm{phen})_{2}\right\}\right]\left(\mathrm{PF}_{6}\right)_{4}{ }^{48,103}\left[\mathrm{Os}(\mathrm{bpy})_{2}(\mathrm{HAT})\right]\left(\mathrm{PF}_{6}\right)_{2}$ (25.0 mg, $0.023 \mathrm{mmol})$ was added to a nitrogen-purged solution of methanol/water $\left(1: 1,60 \mathrm{~cm}^{3}\right)$ and brought to reflux. [Ru(bpy $\left.)_{2} \mathrm{Cl}_{2}\right] \cdot 2 \mathrm{H}_{2} \mathrm{O}(11.2 \mathrm{mg}, 0.023 \mathrm{mmol})$ was dissolved in methanol/water and added dropwise over several hours. The reaction mixture refluxed for a further $12 \mathrm{~h}$. The methanol was removed in vacuo and the crude product purified on SP Sephadex C-25 via the gradient elution procedure described above. A dark purple band of the dinuclear complex eluted with $0.40 \mathrm{M} \mathrm{NaCl}$ and was precipitated as the $\mathrm{PF}_{6}{ }^{-}$salt by addition of a saturated solution of $\mathrm{KPF}_{6}$. The solid was isolated by vacuum filtration and washed with diethyl ether $\left(3 \times 10 \mathrm{~cm}^{3}\right)$. Yield: $35.0 \mathrm{mg}(88 \%)$. Anal. Calcd for $\mathrm{C}_{52} \mathrm{H}_{38} \mathrm{~F}_{24} \mathrm{~N}_{14} \mathrm{P}_{4} \mathrm{OsRu}$ C, 36.1; H, 2.21; N, 11.3\%. Found: C, 36.2; H, 2.53; N, 11.1\%.

Separation of the diastereoisomers was achieved as detailed previously for the related complexes $\left[\left\{\mathrm{Ru}(\mathrm{bpy})_{2}\right\}_{2}(\mu-\mathrm{BL})\right]^{4+}\{$ e.g. $\mathrm{BL}=\mathrm{dpb}, \mathrm{ppz}\}$ in §3.2.3.3. Bands 1 and 2 were determined to be the $\Delta \Lambda / \Lambda \Delta$ and $\Delta \Delta / \Lambda \Lambda$ diastereoisomers, respectively, as established by ${ }^{1} \mathrm{H}$ and COSY NMR characterisation and by comparison with its homo-nuclear ruthenium and osmium analogues. ${ }^{1} \mathrm{H} \mathrm{NMR}\left(\delta \mathrm{ppm} ; \mathrm{CD}_{3} \mathrm{CN}\right)$ : (Band 1; $\Delta \Lambda / \Lambda \Delta) 7.21(1 \mathrm{H}, J=8,5 \mathrm{~Hz}, \mathrm{dd}), 7.28(1 \mathrm{H}, J=8,5 \mathrm{~Hz}, \mathrm{dd}), 7.36$ (1H, $J=8,5 \mathrm{~Hz}, \mathrm{dd}), 7.45$ (1H, $J=8,5 \mathrm{~Hz}, \mathrm{dd}), 7.45-7.63(10 \mathrm{H}, \mathrm{m}), 7.66(1 \mathrm{H}, J=5,1.5 \mathrm{~Hz}, \mathrm{dd}), 7.72(2 \mathrm{H}, J=5,1.5 \mathrm{~Hz}, \mathrm{dd}), 7.75$ $(1 \mathrm{H}, J=5,1.5 \mathrm{~Hz}, \mathrm{dd}), 7.90(1 \mathrm{H}, \mathrm{s}), 7.95(1 \mathrm{H}, J=8,8 \mathrm{~Hz}, \mathrm{dd}), 8.01(1 \mathrm{H}, J=8,8 \mathrm{~Hz}, \mathrm{dd}), 8.04(2 \mathrm{H}, J=$ 8, $8 \mathrm{~Hz}, \mathrm{dd}), 8.10(1 \mathrm{H}, \mathrm{s}), 8.13(1 \mathrm{H}, J=8,8 \mathrm{~Hz}, \mathrm{dd}), 8.18(1 \mathrm{H}, J=8,8 \mathrm{~Hz}, \mathrm{dd}), 8.23(1 \mathrm{H}, J=3 \mathrm{~Hz}, \mathrm{~d})$, $8.43(1 \mathrm{H}, J=3 \mathrm{~Hz}, \mathrm{~d}), 8.45$ (2H, $J=8,1.5 \mathrm{~Hz}, \mathrm{dd}), 8.47$ (2H, $J=8,1.5 \mathrm{~Hz}, \mathrm{dd}), 8.54(1 \mathrm{H}, J=8,1.5 \mathrm{~Hz}$, dd), 8.55 (1H, $J=8,1.5 \mathrm{~Hz}, \mathrm{dd}), 8.59$ (1H, $J=8,1.5 \mathrm{~Hz}, \mathrm{dd}), 8.62$ (1H, $J=8,1.5 \mathrm{~Hz}, \mathrm{dd}), 8.91(1 \mathrm{H}, J=$ $3 \mathrm{~Hz}, \mathrm{~d}), 9.22$ (1H, $J=3 \mathrm{~Hz}, \mathrm{~d}$ ); (Band 2; $\Delta \Delta / \Lambda \Lambda) 7.07$ (1H, $J=8,5 \mathrm{~Hz}, \mathrm{dd}), 7.14$ (1H, $J=8,5 \mathrm{~Hz}, \mathrm{dd}$ ), $7.31(1 \mathrm{H}, J=8,5 \mathrm{~Hz}, \mathrm{dd}), 7.31(1 \mathrm{H}, J=5,1.5 \mathrm{~Hz}, \mathrm{dd}), 7.39$ (1H, $J=8,5 \mathrm{~Hz}, \mathrm{dd}), 7.41(2 \mathrm{H}, J=5,1.5$ $\mathrm{Hz}, \mathrm{dd}), 7.63-7.47$ (6H, m), 7.64 (2H, $J=5,1.5 \mathrm{~Hz}, \mathrm{dd}), 7.75$ (2H, $J=5,1.5 \mathrm{~Hz}, \mathrm{dd}), 7.94$ (1H, $J=8,8$ Hz, dd), 7.96 (1H, $J=8,8 \mathrm{~Hz}, \mathrm{dd}), 8.05(1 \mathrm{H}, J=8,8 \mathrm{~Hz}, \mathrm{dd}), 8.06$ (1H, $J=8,8 \mathrm{~Hz}, \mathrm{dd}), 8.05(1 \mathrm{H}, J=8$, $8 \mathrm{~Hz}, \mathrm{dd}), 8.10(1 \mathrm{H}, \mathrm{s}), 8.12$ (1H, $J=8,8 \mathrm{~Hz}, \mathrm{dd}), 8.17$ (2H, $J=8,8 \mathrm{~Hz}, \mathrm{dd}), 8.43$ (1H, $J=3 \mathrm{~Hz}, \mathrm{~d}), 8.47$ (1H, $J=3 \mathrm{~Hz}, \mathrm{~d}), 8.48-8.50$ (3H, m), 8.51 (2H, $J=8,1.5 \mathrm{~Hz}, \mathrm{dd}), 8.57$ (2H, $J=8,1.5 \mathrm{~Hz}, \mathrm{dd}), 8.61(1 \mathrm{H}$, $J=8,1.5 \mathrm{~Hz}, \mathrm{dd}), 8.90$ (1H, $J=3 \mathrm{~Hz}, \mathrm{~d}), 9.21(1 \mathrm{H}, J=3 \mathrm{~Hz}, \mathrm{~d})$.

\section{$X$-ray Crystallography}

Single crystals of $\Lambda \Delta / \Delta \Lambda$-[\{Ru(bpy $\left.)_{2}\right\}$ ( $\mu$-HAT) $\left.\left\{\mathrm{Os}(\text { bpy })_{2}\right\}\right]\left(\mathrm{PF}_{6}\right)_{3}$. Cl were grown by slow evaporation of a 70:30 2,2,2-trifluoroethanol/ $\mathrm{H}_{2} \mathrm{O}$ solution of the complex under ambient conditions in the absence of light. A green/purple dichroic crystal of dimensions $0.5 \times 0.1 \times 0.1 \mathrm{~mm}$ was coated with paratone-N and mounted onto a glass fibre.

The collection and refinement of X-ray data were performed by Dr Murray Davies (Department of Chemistry, James Cook University). Data was collected using a Bruker SMART CCD diffractometer, and the data sets were corrected for absorption using the program SADABS. ${ }^{123}$ The structure was solved using direct methods and refined on $F^{2}$ using SHELXL-97 $7^{124}$ within the X-SEED ${ }^{125}$ interface. All non- 
hydrogen atoms were located and were refined with anisotropic thermal parameters. Hydrogen atoms were placed in calculated positions (riding model) and were not refined. A summary of data collection and refinement details is provided in Table C4.2 (Appendix C) and the full details are included on the CD accompaniment to this thesis.

\subsubsection{Mono-, Di- and Trinuclear Assemblies Based on ppz}

The synthesis of $\left[\left\{\mathrm{Ru}(\mathrm{bpy})_{2}\right\}_{2}(\mu-\mathrm{ppz})\right]\left(\mathrm{PF}_{6}\right)_{4}$ was reported in §3.2.3.3.

$\left[\mathbf{R u}(\mathbf{b p y})_{2}(\mathbf{p y})_{2}\right]\left(\mathbf{P F}_{\mathbf{6}}\right)_{2}$ was prepared according to a minor variation on the literature procedure. ${ }^{126}$ $\left[\mathrm{Ru}(\mathrm{bpy}){ }_{2} \mathrm{Cl}_{2}\right] .2 \mathrm{H}_{2} \mathrm{O}(0.164 \mathrm{~g}, 0.315 \mathrm{mmol})$ and pyridine $\left(0.655 \mathrm{~cm}^{3}, 8.10 \mathrm{mmol}\right)$ were combined in aqueous methanol $\left(13 \mathrm{~cm}^{3}, 1: 1 \mathrm{v} / \mathrm{v}\right)$ and the mixture heated at reflux for $4 \mathrm{~h}$ during which time the solution attained an orange-red colouration. The mixture was cooled to room temperature and the solvent removed by rotary evaporation. The resultant red residue was dissolved in a minimum volume of methanol $\left(\mathrm{ca} .1 \mathrm{~cm}^{3}\right)$ and the complex precipitated by the addition of diethyl ether. The precipitate was collected by vacuum filtration and washed with diethyl ether $\left(3 \times 10 \mathrm{~cm}^{3}\right)$.

The complex was obtained following purification by cation-exchange chromatography (SP Sephadex C-25). The solid was converted to the chloride salt by stirring an aqueous suspension with DOWEX ${ }^{\circledR}$ anion exchange resin $\left(1 \times 8,50-100\right.$ mesh, $\mathrm{Cl}^{-}$form), and the solution was loaded onto the column $(20 \times 2 \mathrm{~cm})$ and eluted with $0.2 \mathrm{M} \mathrm{NaCl}$. The complex from the orange band was precipitated as the $\mathrm{PF}_{6}{ }^{-}$salt by the addition of aqueous $\mathrm{KPF}_{6}$. The solid was isolated by filtration, washed with copious amounts of diethyl ether and dried in vacuo. Yield: $0.290 \mathrm{~g}(80 \%)$. The ${ }^{1} \mathrm{H}$ NMR was identical to that reported previously. ${ }^{126}$

Resolution of $\left[\mathbf{R u}(\mathbf{b p y})_{2}(\mathbf{p y})_{2}\right]\left(\mathbf{P F}_{\mathbf{6}}\right)_{2}$. $\quad\left[\mathrm{Ru}(\mathrm{bpy})_{2}(\mathrm{py})_{2}\right]\left(\mathrm{PF}_{6}\right)_{2}$ was converted to the $\mathrm{Cl}^{-}$salt by stirring the complex with DOWEX ${ }^{\circledR}$ anion exchange resin $\left(1 \times 8,50-100\right.$ mesh, $\mathrm{Cl}^{-}$form), and the solution was applied to a cation-exchange column (SP Sephadex C-25; $96 \times 1.6 \mathrm{~cm}$ ). The two enantiomers were resolved on elution with an aqueous solution of sodium (-)-O,O'-dibenzoyl-L-tartrate (0.10 M). The complex in each band was precipitated using a saturated solution of $\mathrm{KPF}_{6}$ and collected by filtration.

Purification was achieved on silica gel as described previously. The complex was washed with water $\left(3 \times 5 \mathrm{~cm}^{3}\right)$ then copious amounts of diethyl ether and dried in vacuo. Bands 1 and 2 were assigned as the $\Delta-(-)$ and $\Lambda-(+)$ enantiomers, respectively, based on exciton analysis of their CD spectra ${ }^{127,128}$ and by comparison with related compounds with known absolute configurations. ${ }^{129,} 130$

\section{Synthesis of Dicarbonyl Ruthenium(II) Complexes}

$\left[\mathrm{Ru}(\mathrm{CO})_{2} \mathrm{Cl}_{2}\right]_{\mathrm{n}}$, $\left[\mathrm{Ru}(\mathrm{bpy})(\mathrm{CO})_{2} \mathrm{Cl}_{2}\right]$ and $\left[\mathrm{Ru}(\mathrm{bpy})(\mathrm{CO})_{2}\left(\mathrm{CF}_{3} \mathrm{SO}_{3}\right)_{2}\right]$ were prepared according to the literature procedures. ${ }^{131}$ 
[Ru(bpy)(ppz)(CO) $\left.)_{2}\right]\left(\mathbf{P F}_{\mathbf{6}}\right)_{2}$ was prepared according to an adaptation of the literature method. ${ }^{131}$ [Ru(bpy) $\left.(\mathrm{CO})_{2}\left(\mathrm{CF}_{3} \mathrm{SO}_{3}\right)_{2}\right](0.180 \mathrm{~g}, 0.294 \mathrm{mmol})$ and ppz (136.7 mg, $\left.0.589 \mathrm{mmol}\right)$ were dissolved in 95\% ethanol $\left(30 \mathrm{~cm}^{3}\right)$ under $\mathrm{N}_{2}$. The solution was refluxed for 30 min during which time the mixture attained a yellow colouration. The mixture was refluxed for a further $60 \mathrm{~min}$ and evaporated to dryness by rotary evaporation. The beige residue was dissolved in boiling water and filtered to remove unreacted ligand (ppz). A saturated aqueous solution of $\mathrm{NH}_{4} \mathrm{PF}_{6}\left(5 \mathrm{~cm}^{3}\right)$ was added to yield a white precipitate which was isolated by filtration and washed with copious amounts of chilled water followed by diethyl ether. Yield: 240 mg (98\%) Anal. Calcd for $\mathrm{C}_{40} \mathrm{H}_{24} \mathrm{~F}_{12} \mathrm{~N}_{10} \mathrm{O}_{4} \mathrm{P}_{2} \mathrm{Ru}$ : C, 55.4; H, 2.77; N, 16.2\%. Found: C, 55.2; H, 2.83; N, 16.3\%.

[Ru(bpy)(ppz) $\mathbf{~}_{2}\left(\mathbf{P F}_{\mathbf{6}}\right)_{2}$. The bridging ligand ppz (225 mg, $0.970 \mathrm{mmol}$ ) was dissolved in 2methoxyethanol $\left(40 \mathrm{~cm}^{3}\right)$ and the solution purged with $\mathrm{N}_{2}$ for 20 mins. [Ru(bpy)(ppz)(CO) $\left.)_{2}\right]\left(\mathrm{PF}_{6}\right)_{2}(270$ $\mathrm{mg}, 0.323 \mathrm{mmol}$ ) and a three-fold excess of TMNO (31 $\mathrm{mg}, 0.413 \mathrm{mmol}$ ) were added and the mixture heated to reflux for $3 \mathrm{~h}$ in subdued light. During this time the solution attained a deep red colouration with the concurrent production of trimethylamine which was trapped using a bubbler containing $\mathrm{HCl}$ solution. The crude reaction mixture was dissolved in water and purified via a gradient elution procedure on SP Sephadex C-25 cation exchanger with aqueous $\mathrm{NaCl}$ (0.1-0.15 M) eluent. Precipitation of the desired product from the major orange band was achieved by addition of a saturated aqueous solution of $\mathrm{NH}_{4} \mathrm{PF}_{6}$. The orange solid was isolated by vacuum filtration, washed with chilled water $\left(2 \times 5 \mathrm{~cm}^{3}\right)$ and copious diethyl ether and dried in vacuo. Yield: $140 \mathrm{mg}$ (46\%). Anal. Calcd for $\mathrm{C}_{38} \mathrm{H}_{24} \mathrm{~F}_{12} \mathrm{~N}_{10} \mathrm{P}_{2} \mathrm{Ru}$ : C, 45.1; H, 2.39; N, 13.8\%. Found: C, 45.0; H, 2.20; N, 13.5\%.

Separation and resolution of trans-[Ru(bpy)(ppz $\left.)_{2}\right]\left(\mathbf{P F}_{6}\right)_{2}$. The separation of the geometric isomers and chiral resolution of their enantiomeric forms was achieved during a single column chromatographic procedure. The complex (ca. $90 \mathrm{mg}$ ) was absorbed onto a column of SP Sephadex C-25 cation exchanger \{as the $\mathrm{Cl}^{-}$form, obtained by stirring an aqueous suspension with DOWEX ${ }^{\circledR}$ anion exchange resin $(1 \times 8$, 50-100 mesh, $\mathrm{Cl}^{-}$form) $\}$and eluted using aqueous $0.10 \mathrm{M}$ sodium tosylate solution. The column was sealed at the top and bottom to permit recycling of the broadening band and small portions of the front and back of the band were collected on each pass down the column. The complex was extracted from each fraction using dichloromethane following the addition of a saturated solution of aqueous $\mathrm{KPF}_{6}$. The organic layers were dried over $\mathrm{Na}_{2} \mathrm{SO}_{4}$, filtered, and the solvent removed by rotary evaporation. Purification of the solids was achieved on a short column of silica gel $(3 \times 2 \mathrm{~cm})$. A solution of the complex in acetone was loaded onto the column and washed alternately with acetone, water and acetone and eluted with acetone containing $5 \% \mathrm{NH}_{4} \mathrm{PF}_{6}$. Addition of water and removal of the acetone under reduced pressure afforded an orange solid which was collected by filtration through Celite and washed with diethyl ether $\left(3 \times 5 \mathrm{~cm}^{3}\right)$. The precipitate was washed off the Celite into a test tube using acetone (ca. $2 \mathrm{~cm}^{3}$ ), evaporated under a stream of dry nitrogen and dried in vacuo for $3 \mathrm{~h}$ at $50^{\circ} \mathrm{C}$. By using ${ }^{1} \mathrm{H}$ NMR and CD techniques, as well as by comparison with similar complexes of known configuration, ${ }^{101,104}$ 
the identities of the front and back fractions of the eluting band (Bands 1 and 4, respectively) were established as the $\Delta^{\mathrm{t}}-\left[\mathrm{Ru}(\mathrm{bpy})(\mathrm{ppz})_{2}\right]^{2+}$ and $\Lambda^{\mathrm{t}}-\left[\mathrm{Ru}(\mathrm{bpy})(\mathrm{ppz})_{2}\right]^{2+}$ isomers, respectively $(\mathrm{t}=$ trans $)$. The other fractions contained various admixtures of the $\Delta^{\mathrm{c}}-\left[\mathrm{Ru}(\mathrm{bpy})(\mathrm{ppz})_{2}\right]^{2+}$ and $\Lambda^{\mathrm{c}}-\left[\mathrm{Ru}(\mathrm{bpy})(\mathrm{ppz})_{2}\right]^{2+}$ isomers $(\mathrm{c}=\mathrm{cis})$.

By comparison with similar complexes of known configuration, ${ }^{101}$ Band 1 was assigned as $\Delta^{\mathrm{t}}$ $\left[\mathrm{Ru}(\mathrm{bpy})(\mathrm{ppz})_{2}\right]^{2+}$ and Band 4 as $\Lambda^{\mathrm{t}}-\left[\mathrm{Ru}(\mathrm{bpy})(\mathrm{ppz})_{2}\right]^{2+}$. UV/Vis in $\mathrm{CH}_{3} \mathrm{CN}, v / \mathrm{cm}^{-1}\left\{\varepsilon_{\max } / \mathrm{M}^{-1} \mathrm{~cm}^{-1}\right.$ (Band 1 = Band 4)\}: 464 (12850), 426 (13110), 279 (53333), 264 (60520), 257 (60580), 243 (54710). CD spectra in $\mathrm{CH}_{3} \mathrm{CN}, \lambda / \mathrm{nm}\left\{\Delta \varepsilon / \mathrm{M}^{-1} \mathrm{~cm}^{-1}\right.$ (Band 1, Band 4)\}: 250 (10.0, -8.00), 270 (29.4, -26.8), 294 (-81.5, 80.5), 380 (11.8, -10.20), 420 (17.7, -16.6), $470(-13.9,14.5)$. The ${ }^{1} \mathrm{H}$ NMR spectra are reported in Table 4.8, §4.3.3.1.

Stereoselective synthesis of $\left[\left\{\Delta-\mathbf{R u}(\mathbf{b p y})_{2}\right\}_{2}\left\{\Delta^{\mathrm{t}}-\mathbf{R u}(\mathbf{b p y})(\mu-\mathbf{p p z})_{2}\right\}\right]\left(\mathbf{P F}_{6}\right)_{6} \cdot \Delta^{\mathrm{t}}-\left[\mathrm{Ru}(\mathrm{bpy})(\mathrm{ppz})_{2}\right]\left(\mathrm{PF}_{6}\right)_{2}(7.0$ mg, $6.92 \mu \mathrm{mol})$ and $\Delta$-[Ru(bpy $\left.)_{2}(\mathrm{py})_{2}\right]\left(\mathrm{PF}_{6}\right)_{2}(17.4 \mathrm{mg}, 15.2 \mu \mathrm{mol})$ were combined in ethylene glycol (1 $\mathrm{cm}^{3}$ containing $10 \%$ water) and the mixture heated at $120^{\circ} \mathrm{C}$ for $5 \mathrm{~h}$ in the absence of light. During this time the initially orange solution attained a deep purple colouration. The reaction mixture was cooled to room temperature, and water $\left(\mathrm{ca} .5 \mathrm{~cm}^{3}\right)$ was added. The isolation of the desired trinuclear product from the crude mixture was achieved via a gradient elution procedure with SP Sephadex C-25 cation exchanger $(20 \times 2 \mathrm{~cm})$ using aqueous $\mathrm{NaCl}$ solution $(0.2-0.8 \mathrm{M})$. Two bands were observed: a faint orange band (presumably containing unreacted excess $\left[\mathrm{Ru}(\mathrm{bpy})_{2}(\mathrm{py})_{2}\right]^{2+}$ ) which eluted with $0.2 \mathrm{M} \mathrm{NaCl}$, followed by a major purple band which eluted with $0.5 \mathrm{M} \mathrm{NaCl}$. The product from the latter was precipitated by addition of saturated aqueous $\mathrm{KPF}_{6}$, and chilled by refrigeration overnight. The dark purple solid was isolated by filtration and washed with copious diethyl ether. Yield: $12.4 \mathrm{mg}$ (74\%) Anal. Calcd for $\mathrm{C}_{78} \mathrm{H}_{56} \mathrm{~F}_{36} \mathrm{~N}_{18} \mathrm{P}_{4} \mathrm{Ru}_{3}$ : C, 38.7; H, 2.33; N, 10.4\%. Found: C, 38.6; H, 2.37; N, 10.2\%. The ${ }^{1} \mathrm{H}$ NMR spectra are provided in Table 4.9, §4.3.3.1. 


\subsection{Results and Discussion}

\subsubsection{Homo-nuclear Ruthenium Complexes based on the Bridging Ligand HAT}

\subsubsection{Synthesis and Stereoisomer Separation}

The synthetic strategies for the stereoisomerically pure dinuclear fmeso $(\Delta \Lambda)$ and $\operatorname{rac}(\Delta \Delta$ and $\Lambda \Lambda)$ \} and trinuclear \{homochiral ( $\Delta_{3}$ and $\Lambda_{3}$ ) and heterochiral $\left(\Delta_{2} \Lambda\right.$ and $\left.\Lambda_{2} \Delta\right)$ \} systems have been reported previously, ${ }^{48,101,103}$ and were based upon a combination of stereoretentive and chromatographic methodologies. ${ }^{20,21}$

\subsubsection{Electrochemistry and Electronic Spectroscopy}

\section{Electrochemistry}

The electrochemical properties of the stereoisomeric forms ${ }^{103}$ of $\left[\left\{\mathrm{Ru}(\mathrm{bpy})_{2}\right\}_{2}(\mu-\mathrm{HAT})\right]^{4+}$ and $\left[\left\{\mathrm{Ru}(\mathrm{bpy})_{2}\right\}_{3}(\mu \text {-HAT })\right]^{6+}$ as well as the stereoisomeric mixtures ${ }^{50,110}$ have been reported previously. Electrochemical data obtained by differential pulse voltammetry (in addition to cyclic voltammetry used in the earlier measurements) for the metal-based oxidation processes are provided in Tables 4.1 and 4.2.

The di- and trinuclear systems are characterised by two and three reversible one-electron redox processes, respectively, corresponding to successive oxidation of the metal centres. The first two ruthenium-based oxidation processes in $\Delta_{3} / \Lambda_{3}$ and $\Delta_{2} \Lambda / \Lambda_{2} \Delta$-[\{Ru(bpy $\left.\left.)_{2}\right\}_{3}(\mu-\mathrm{HAT})\right]^{6+}$ occur at similar potentials to those for their dinuclear analogues. This has been rationalised on the basis that the highest occupied molecular $\mathrm{Ru}(\mathrm{d} \pi)$ orbital is relatively unaffected by the additional $\mathrm{Ru}(\mathrm{bpy})_{2}{ }^{2+}$ moiety in the trinuclear systems. ${ }^{50,52}$

All complexes exhibited multiple reversible ligand-based reductions in the cathodic region (Table C4.1, Appendix C). Previous electrochemical studies ${ }^{50}$ have established that the first reduction process is localised on the HAT ligand due to the lower $\pi^{*}$ level relative to the peripheral bpy ligands. This was confirmed by the anodic shift of the first reduction process with an increase in the number of coordinated ruthenium centres, since the increase in nuclearity of the assembly is expected to influence the HAT reduction relatively more than the bpy reductions. On this basis, the second reduction was assigned to the HAT ligand in the di- and trinuclear complexes; the third reduction is also localised on HAT in the trinuclear systems. ${ }^{52}$

As discussed in §1.3.1.2, the potential differences between the successive metal-based oxidation processes $\left(\Delta \mathrm{E}_{\mathrm{ox}}\right)$ and comproportionation constants $\left(\mathrm{K}_{\mathrm{c}}\right)$ have been used previously as semi-quantitative criteria to assess the extent of electronic delocalisation and stability of the dinuclear mixed-valence systems. ${ }^{132,134,135}$ A summary of $\Delta \mathrm{E}_{\text {ox }}$ and the resultant $\mathrm{K}_{\mathrm{c}}$ values for the di- and trinuclear complexes is also provided in Tables 4.1 and 4.2. The separation between the oxidation processes allowed the electrochemical generation of the singly-oxidised $(+5)$ mixed-valence forms of the dinuclear complexes, and the singly- $(+7)$ and doubly-oxidised (+8) mixed-valence forms of the trinuclear complexes. 
Table 4.1 UV/Vis/NIR spectral ${ }^{\mathrm{a}}$ data of the reduced absorption spectra $(\varepsilon / v v s . v)$ for the dinuclear complexes at $-35^{\circ} \mathrm{C}$, and electrochemical ${ }^{\mathrm{b}}$ data (in $\mathrm{mV}$ ), and comproportionation constants $\left(\mathrm{K}_{\mathrm{c}}\right)^{\mathrm{c}}$ for the complexes. The NIR spectral data are indicated in bold type. ${ }^{\mathrm{d}}$

\begin{tabular}{|c|c|c|c|c|c|}
\hline Complex & $n$ & $\begin{array}{c}v_{\max } \pm 10 / \mathrm{cm}^{-1} \\
\left\{(\varepsilon / v)_{\max } \pm 0.0001 / \mathrm{M}^{-1}\right\}\end{array}$ & $E_{\text {ox1 }}$ & $E_{\text {ox2 }}$ & $\begin{array}{l}\Delta \mathrm{E}_{\mathrm{ox}(2-1)} \\
\left(\mathrm{K}_{\mathrm{c}}\right)^{\mathrm{C}}\end{array}$ \\
\hline \multirow[t]{3}{*}{ meso- $\left[\left\{\mathrm{Ru}(\mathrm{bpy})_{2}\right\}_{2}(\mu-\mathrm{HAT})\right]^{n+}$} & 4 & $\begin{array}{l}17820(0.8505) \\
20750(0.6301) \\
24160(0.6696)\end{array}$ & \multirow[t]{3}{*}{1168} & \multirow[t]{3}{*}{1392} & \multirow[t]{3}{*}{$\begin{array}{c}224 \\
\left(6.26 \times 10^{3}\right)\end{array}$} \\
\hline & 5 & $\begin{array}{l}\mathbf{5 2 5 0}(\mathbf{0 . 8 2 4 0 )} \\
11780(0.0816) \\
17170(0.5540) \\
25670(0.2760) \\
\end{array}$ & & & \\
\hline & 6 & $\begin{array}{l}11640(1.321) \\
\text { sh } 12975(0.6289) \\
16110(0.1311) \\
24380(0.2601) \\
\end{array}$ & & & \\
\hline \multirow[t]{3}{*}{$\operatorname{rac}-\left[\left\{\mathrm{Ru}(\mathrm{bpy})_{2}\right\}_{2}(\mu-\mathrm{HAT})\right]^{n+}$} & 4 & $\begin{array}{l}17850(0.9944) \\
20630(0.7478) \\
24150(0.7807) \\
\end{array}$ & \multirow[t]{3}{*}{1156} & \multirow[t]{3}{*}{1380} & \multirow[t]{3}{*}{$\begin{array}{c}224 \\
\left(6.26 \times 10^{3}\right)\end{array}$} \\
\hline & 5 & $\begin{array}{l}\mathbf{5 2 4 0}(\mathbf{0 . 6 8 6 2}) \\
11780(0.04510) \\
13100(0.1116) \\
17250(0.5310) \\
\end{array}$ & & & \\
\hline & 6 & $\begin{array}{l}11500(1.304) \\
\text { sh } 12980(0.5918)\end{array}$ & & & \\
\hline
\end{tabular}

\section{Electronic Spectroscopy}

The complete UV/Vis/NIR spectral data for the un-oxidised, partially-oxidised and fully-oxidised forms of the di- and trinuclear systems (for the range 3050-30000 $\mathrm{cm}^{-1}$ ) are provided in Tables 4.1 and 4.2. The UV/Vis spectra for the diastereoisomeric mixtures of the di- and trinuclear complexes $\left[\left\{\mathrm{Ru}(\mathrm{bpy})_{2}\right\}_{2}(\mu-\mathrm{HAT})\right]^{4+}$ and $\left[\left\{\mathrm{Ru}(\mathrm{bpy})_{2}\right\}_{3}(\mu-\mathrm{HAT})\right]^{6+}$ have been described previously, ${ }^{50,52}$ and in previous work from our laboratory ${ }^{101,103}$ no significant differences were observed in the spectral properties of the diastereoisomers of either of the un-oxidised systems.

The spectra of the un-oxidised systems in the region from $30000-50000 \mathrm{~cm}^{-1}$ are characterised by $\pi \rightarrow \pi^{*}(\mathrm{HAT})$ and $\pi \rightarrow \pi^{*}$ (bpy) transitions, where the intensities of the latter are proportional to the number of coordinated Ru(bpy $)_{2}{ }^{2+}$ moieties. The UV/Vis spectra over the region $15000-30000 \mathrm{~cm}^{-1}$ for the un-oxidised forms of the dinuclear systems are characterised by a combination of overlapping $\mathrm{d} \pi\left(\mathrm{Ru}^{\mathrm{II}}\right) \rightarrow \pi^{*}(\mathrm{HAT})$ and $\mathrm{d} \pi\left(\mathrm{Ru}^{\mathrm{II}}\right) \rightarrow \pi^{*}$ (bpy) singlet metal-to-ligand charge transfer ( $\left.{ }^{1} \mathrm{MLCT}\right)$ transitions. The spectra have been completely assigned on the basis of comparisons with the electrochemical data, ${ }^{50,52}$ and resonance Raman measurements. ${ }^{110}$ 
Table 4.2 UV/Vis/NIR spectral ${ }^{\text {a }}$ data of the reduced absorption spectra ( $\varepsilon / v$ vs. $v$ ) for the trinuclear complexes at $-15^{\circ} \mathrm{C}$ and electrochemical ${ }^{\mathrm{b}}$ data (in $\mathrm{mV}$ ), and comproportionation constants $\left(\mathrm{K}_{\mathrm{c}}\right)^{\mathrm{c}}$ for the complexes. The NIR spectral data are indicated in bold type. ${ }^{\mathrm{d}}$

\begin{tabular}{|c|c|c|c|c|c|c|c|}
\hline Complex & $n$ & $\begin{array}{c}v_{\max } \pm 10 / \mathrm{cm}^{-1} \\
\left\{(\varepsilon / \mathrm{v})_{\max } \pm 0.0001 / \mathrm{M}^{-1}\right\}\end{array}$ & $E_{o x 1}$ & $E_{\text {ox2 }}$ & $E_{\text {ox3 }}$ & $\begin{array}{l}\Delta \mathrm{E}_{\mathrm{ox}(2-1)} \\
\left(\mathrm{K}_{\mathrm{c}}\right)^{\mathrm{c}}\end{array}$ & $\begin{array}{l}\Delta \mathrm{E}_{\mathrm{ox}(3-2)} \\
\left(\mathrm{K}_{\mathrm{c}}\right)^{\mathrm{c}}\end{array}$ \\
\hline \multirow[t]{4}{*}{$\begin{array}{l}\text { heterochiral- } \\
{\left[\left\{\operatorname{Ru}(\mathrm{bpy})_{2}\right\}_{3}(\mu-\mathrm{HAT})\right]^{n+}}\end{array}$} & 6 & $\begin{array}{l}17575(1.725) \\
19410(1.357) \\
24545(0.9280) \\
\operatorname{sh} 26700(0.6680)\end{array}$ & \multirow[t]{4}{*}{1256} & \multirow[t]{4}{*}{1476} & \multirow[t]{4}{*}{1720} & \multirow[t]{4}{*}{$\begin{array}{c}220 \\
(5.36 \times \\
\left.10^{3}\right)\end{array}$} & \multirow[t]{4}{*}{$\begin{array}{c}244 \\
(13.7 \times \\
\left.10^{3}\right)\end{array}$} \\
\hline & 7 & $\begin{array}{l}\mathbf{4 6 4 0}(\mathbf{0 . 7 7 4 3 )} \\
\mathbf{5 8 2 0}(\mathbf{0 . 4 5 8 1}) \\
18325(1.380) \\
\operatorname{sh} 25735(0.6448) \\
\end{array}$ & & & & & \\
\hline & 8 & $\begin{array}{l}\mathbf{4 9 1 0}(\mathbf{0 . 4 4 9 8 )} \\
\mathbf{8 1 9 0}(\mathbf{0 . 6 1 0 4 )} \\
11575(0.6060) \\
13610(0.5780) \\
\text { sh } 17650(0.8257) \\
18745(0.8627)\end{array}$ & & & & & \\
\hline & 9 & $\begin{array}{l}13495(1.4616) \\
16025(0.7759)\end{array}$ & & & & & \\
\hline \multirow[t]{4}{*}{$\begin{array}{l}\text { homochiral- } \\
\left.\left[\{\text { Ru(bpy })_{2}\right\}_{3}(\mu-\mathrm{HAT})\right]^{n+}\end{array}$} & 6 & $\begin{array}{l}17620(1.700) \\
19450(1.350) \\
24490(0.8954) \\
\text { sh } 26830(0.6713)\end{array}$ & \multirow[t]{4}{*}{1256} & \multirow[t]{4}{*}{1486} & \multirow[t]{4}{*}{1726} & \multirow[t]{4}{*}{$\begin{array}{c}230 \\
(7.91 \times \\
\left.10^{3}\right)\end{array}$} & \multirow[t]{4}{*}{$\begin{array}{c}240 \\
(11.7 \times \\
\left.10^{3}\right)\end{array}$} \\
\hline & 7 & $\begin{array}{l}\mathbf{4 6 5 0}(\mathbf{0 . 5 6 6 1}) \\
\mathbf{6 0 2 0}(\mathbf{0 . 2 7 7 0 )} \\
11070(0.4015) \\
18080(0.8630) \\
\end{array}$ & & & & & \\
\hline & 8 & $\begin{array}{l}\mathbf{4 6 9 0}(\mathbf{0 . 4 0 9 3 )} \\
\mathbf{7 1 1 0 - 8 0 6 0 ( 0 . 4 5 1 6 )} \\
\text { sh } 11170(0.2480) \\
13560(0.4524) \\
18310(1.225)\end{array}$ & & & & & \\
\hline & 9 & $\begin{array}{l}13420(1.460) \\
17600(0.7730)\end{array}$ & & & & & \\
\hline
\end{tabular}

sh = shoulder of band.

b All potentials $( \pm 3 \mathrm{mV})$ measured in $0.1 \mathrm{M}\left[\left(n-\mathrm{C}_{4} \mathrm{H}_{9}\right)_{4} \mathrm{~N}\right] \mathrm{PF}_{6} / \mathrm{CH}_{3} \mathrm{CN}$ at $+25^{\circ} \mathrm{C} v \mathrm{~s} . \mathrm{Fc}^{+} / \mathrm{Fc}^{0}$.

${ }^{\mathrm{c}} \mathrm{K}_{\mathrm{c}}$ is given by equation $1.18(\mathrm{a}){ }^{132}$

${ }^{\mathrm{d}}$ Band parameters for the reduced absorption spectra ( $\left.\varepsilon / v v s . v\right)$, rather than parameters for the unreduced spectra ( $\varepsilon v$ s. $\left.v\right)$ which were reported previously. ${ }^{133}$

In the present report, minor differences were observed in the band energies and intensities between the diastereoisomers of the partially- and fully-oxidised di- and trinuclear systems. The IVCT spectra for meso- and rac-[\{Ru(bpy $\left.\left.)_{2}\right\}_{2}(\mu-\mathrm{HAT})\right]^{5+}$ are shown in Figures 4.6(a) and (b).

Spectroelectrochemical generation of the +5 and +6 forms of the dinuclear diastereoisomers at $-35^{\circ} \mathrm{C}$ revealed stable isosbestic points in the spectral progressions accompanying both oxidation processes. The ${ }^{1}$ MLCT absorption bands decreased in intensity following one electron-oxidation and eventually collapsed on further oxidation to the +6 species. The latter was characterised by intense $\pi(\mathrm{bpy}) \rightarrow$ 
$\mathrm{d} \pi\left(\mathrm{Ru}^{\mathrm{III}}\right)$ and $\pi(\mathrm{HAT}) \rightarrow \mathrm{d} \pi\left(\mathrm{Ru}^{\mathrm{III}}\right)$ ligand-to-metal charge transfer (LMCT) absorptions in the region $10000-16000 \mathrm{~cm}^{-1}$. The energies of these bands are consistent with the previously assigned $\pi$ (bpy) $\rightarrow$ $\mathrm{d} \pi\left(\mathrm{Ru}^{\mathrm{III}}\right)$ LMCT transitions at 14815 and $17160 \mathrm{~cm}^{-1}$ in $\left[\mathrm{Ru}^{\mathrm{III}}(\mathrm{bpy})_{3}\right]^{3+}{ }^{136}$ Spectral deconvolution was performed to reveal the behaviour of the underlying transitions responsible for the variation in the spectra with the extent of oxidation of the assemblies.

In the un-oxidised (+6) forms of the trinuclear systems, the presence of the third $\mathrm{Ru}(\mathrm{bpy})_{2}{ }^{2+}$ moiety has the effect of increasing the absorptivity, and shifting the ${ }^{1}$ MLCT transition to lower energy relative to their dinuclear congeners. Masschelein and coworkers ${ }^{50}$ have rationalised this observation on the basis of spectral-electrochemical correlations: the energy of the highest occupied $\mathrm{d} \pi$ molecular orbital is relatively invariant to the addition of an $\mathrm{Ru}(\mathrm{bpy})_{2}{ }^{2+}$ moiety, whereas at the same time the lowest unoccupied $\pi^{*}$ molecular orbital is stabilised.
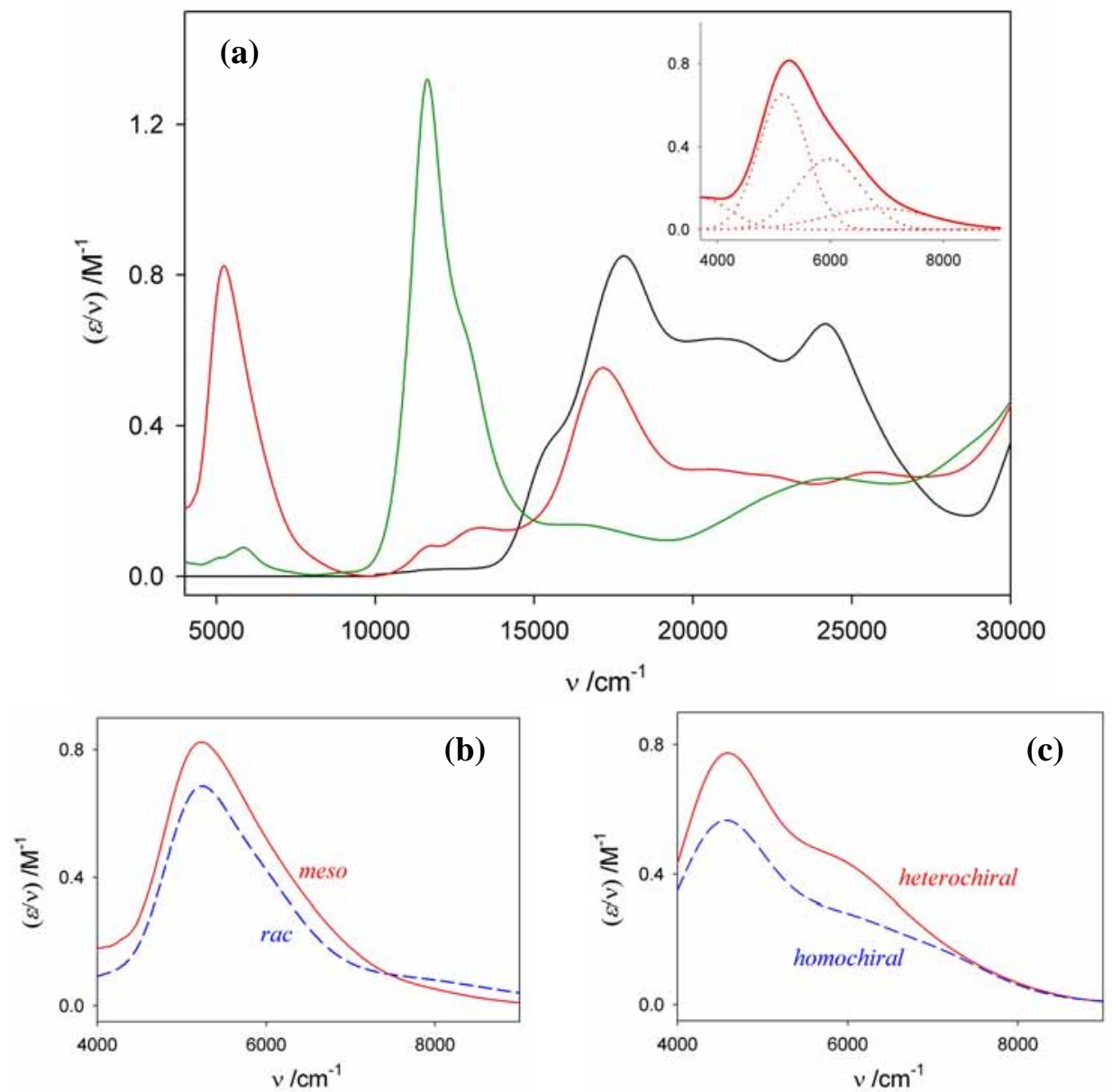

Figure 4.6 (a) UV/Vis/NIR spectra of meso- $\left[\left\{\mathrm{Ru}(\mathrm{bpy})_{2}\right\}_{2}(\mu-\mathrm{HAT})\right]^{n+}\{n=4(-), 5(-), 6(-)\}$ at $-35^{\circ} \mathrm{C}$. The inset shows the best fit of the bands obtained by Gaussian deconvolution of the IVCT band. Overlay of the IVCT bands for (b) meso- and rac- $\left[\left\{\mathrm{Ru}(\mathrm{bpy})_{2}\right\}_{2}(\mu-\mathrm{HAT})\right]^{5+}$ at $-35^{\circ} \mathrm{C}$, and (c) heterochiral- and homochiral$\left[\left\{\mathrm{Ru}(\mathrm{bpy})_{2}\right\}_{3}(\mu-\mathrm{HAT})\right]^{7+}$ at $-15^{\circ} \mathrm{C}$.

Spectroelectrochemical oxidation of the trinuclear complexes allowed the generation of the mixed-valence +7 and +8 forms, and the fully-oxidised +9 forms at $-15^{\circ} \mathrm{C}$ (Figures 4.6 (c) and 4.7). Stable isosbestic points were observed in the spectral progressions accompanying the three stages of oxidation. The ${ }^{1}$ MLCT absorption bands decreased in intensity following one- and two-electron 
oxidation, and collapsed on further oxidation to the +9 species. Oxidation of the formally $\mathrm{Ru}^{\mathrm{II}}$ to $\mathrm{Ru}^{\mathrm{III}}$ centres was accompanied by an increase in the intensity of the transitions in the region $10000-16000 \mathrm{~cm}^{-1}$. These are assigned as ligand-to-metal $\pi(\mathrm{bpy}) \rightarrow \mathrm{d} \pi\left(\mathrm{Ru}^{\mathrm{III}}\right)$ and $\pi(\mathrm{HAT}) \rightarrow \mathrm{d} \pi\left(\mathrm{Ru}^{\mathrm{III}}\right)$ LMCT transitions by comparison with the behaviour of the dinuclear analogues.

(a)

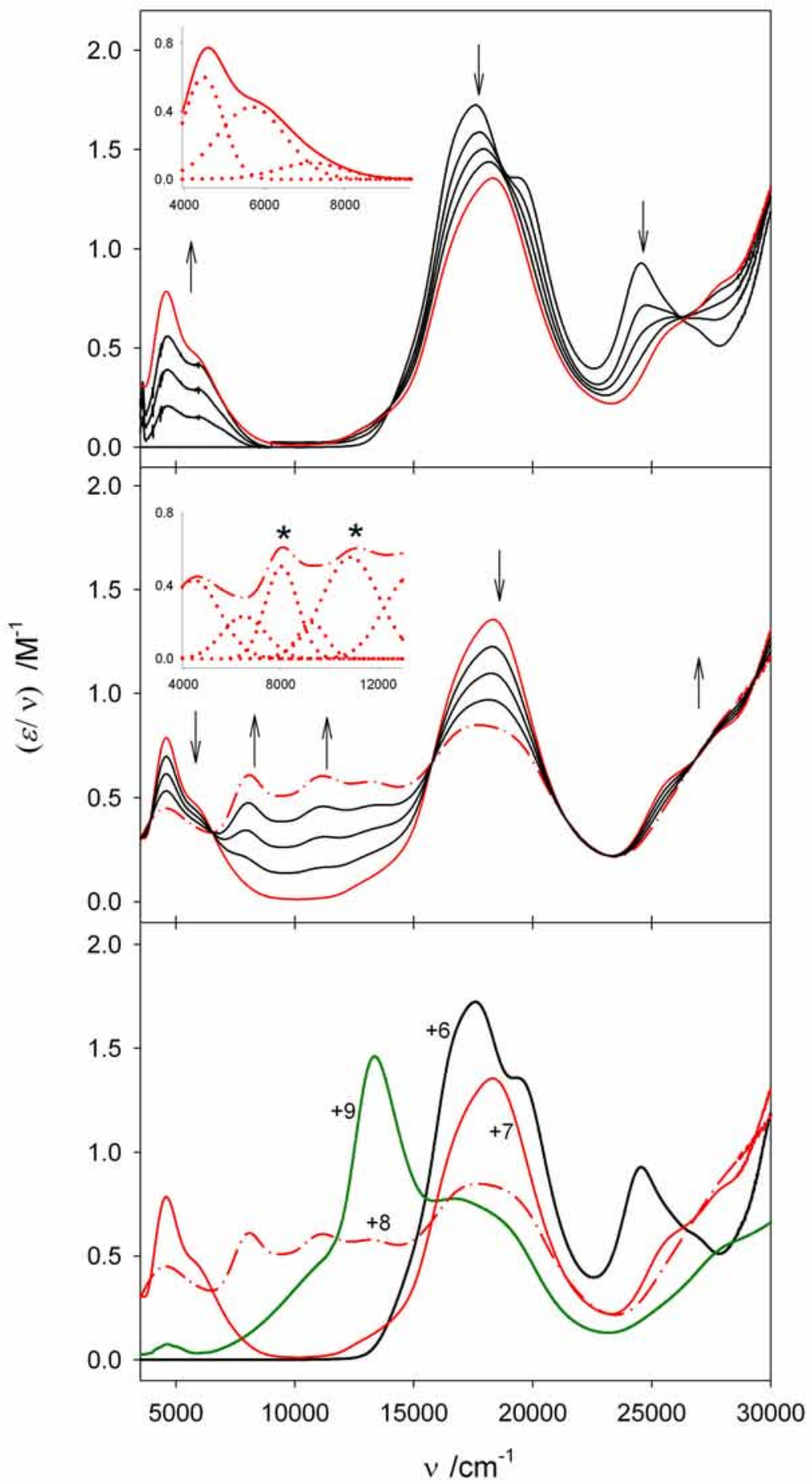

Figure 4.7 Spectroelectrochemistry for the oxidation of the trinuclear complex heterochiral- $\left[\left\{\mathrm{Ru}(\mathrm{bpy})_{2}\right\}_{3}(\mu-\right.$ HAT $)]^{n+}(n=6-9)$ at $-15^{\circ} \mathrm{C}$. (a) Spectroelectrochemical changes for the oxidation reaction heterochiral$\left[\left\{\mathrm{Ru}(\mathrm{bpy})_{2}\right\}_{3}(\mu \text {-HAT })\right]^{6+} \rightarrow$ heterochiral-[ $\left\{\mathrm{Ru}(\mathrm{bpy})_{2}\right\}_{3}(\mu$-HAT $\left.)\right]^{7+}$. The inset shows IVCT band (一) of the latter in addition to the bands obtained from Gaussian deconvolution (…). (b) Spectroelectrochemical changes for the oxidation reaction heterochiral-[\{Ru(bpy $\left.\left.)_{2}\right\}_{3}(\mu-\mathrm{HAT})\right]^{7+} \rightarrow$ heterochiral- $\left[\left\{\mathrm{Ru}(\mathrm{bpy})_{2}\right\}_{3}(\mu-\mathrm{HAT})\right]^{8+}$. The inset shows the NIR spectrum $\left(-\cdot{ }^{-}\right)$of the latter in addition to the bands obtained from Gaussian deconvolution ( $\left.\cdots \cdot\right)$. The asterisks indicate the IVCT bands. (c) Summary of the spectra of heterochiral-[\{Ru(bpy $\left.\left.)_{2}\right\}_{3}(\mu-\mathrm{HAT})\right]^{n+}$. 


\subsubsection{Intervalence Charge Transfer}

The NIR spectra of the di- and trinuclear systems spectra were scaled as $\int \varepsilon(v) / v d v^{137,138}$ and deconvoluted by use of the software package GRAMS32. The results for the IVCT parameters $\left\{v_{\max }\right.$, $(\varepsilon / v)_{\max }$ and $\left.\Delta v_{1 / 2}\right\}$ derived from the deconvolution procedure are summarised in Table 4.3.

Table 4.3 NIR spectral data of the reduced absorption spectra for the di- and trinuclear complexes at -35 and $-15^{\circ} \mathrm{C}$, respectively. For the dinuclear species, the parameters for the overall NIR band envelopes are shown in bold type, and the details of the deconvoluted bands are in normal type. ${ }^{2}$

\begin{tabular}{|c|c|c|c|c|c|}
\hline Complex & Component & $\begin{array}{l}v_{\max } \\
\pm 10 \\
/ \mathrm{cm}^{-1}\end{array}$ & $\begin{array}{c}(\varepsilon / v)_{\max } \\
\pm 0.0001 \\
/ \mathrm{M}^{-1}\end{array}$ & $\begin{array}{l}\Delta v_{1 / 2} \\
\pm 10 \\
/ \mathrm{cm}^{-1}\end{array}$ & $\begin{array}{c}\Delta v_{1 / 2}{ }^{\circ b} \\
/ \mathrm{cm}^{-1}\end{array}$ \\
\hline meso- $\left[\left\{\mathrm{Ru}(\mathrm{bpy})_{2}\right\}_{2}(\mu-\mathrm{HAT})\right]^{5+}$ & $\begin{array}{l}1 \\
2 \\
3\end{array}$ & $\begin{array}{c}\mathbf{5 2 5 0} \\
3870^{\mathrm{d}} \\
5150 \\
5950 \\
6985\end{array}$ & $\begin{array}{l}\mathbf{0 . 8 2 4 1} \\
0.1524 \\
0.6533 \\
0.3416 \\
0.1025\end{array}$ & $\begin{array}{c}\mathbf{1 6 0 0} \\
827 \\
1020 \\
1410 \\
2560\end{array}$ & $\begin{array}{l}3100 \\
3075 \\
3305 \\
3580\end{array}$ \\
\hline rac- $\left[\left\{\mathrm{Ru}(\mathrm{bpy})_{2}\right\}_{2}(\mu-\mathrm{HAT})\right]^{5+}$ & $\begin{array}{l}1 \\
2 \\
3\end{array}$ & $\begin{array}{c}5240 \\
4030^{d} \\
5110 \\
5710 \\
7615\end{array}$ & $\begin{array}{l}\mathbf{0 . 6 8 6 2} \\
0.1500 \\
0.6030 \\
0.2415 \\
0.0975\end{array}$ & \begin{tabular}{|c|}
$\mathbf{1 4 8 5}$ \\
708 \\
824 \\
1515 \\
2295
\end{tabular} & $\begin{array}{l}3100 \\
\\
3065 \\
3240 \\
3740\end{array}$ \\
\hline heterochiral-[\{Ru(bpy $\left.\left.)_{2}\right\}_{3}(\mu-\mathrm{HAT})\right]^{7+}$ & $\begin{array}{l}1 \\
2 \\
3\end{array}$ & $\begin{array}{l}4515 \\
5820 \\
7160\end{array}$ & $\begin{array}{l}0.7484 \\
0.4539 \\
0.1438\end{array}$ & $\begin{array}{l}1225 \\
1610 \\
1715\end{array}$ & \\
\hline heterochiral-[\{Ru(bpy $\left.\left.)_{2}\right\}_{3}(\mu-\mathrm{HAT})\right]^{8+\mathrm{c}}$ & & $\begin{array}{c}4715 \\
6455 \\
7980^{*} \\
9350 \\
10900^{*} \\
13145 \\
14770 \\
16790 \\
19660\end{array}$ & $\begin{array}{l}0.4419 \\
0.1305 \\
0.5654 \\
0.2179 \\
0.5464 \\
0.4542 \\
0.1058 \\
0.7367 \\
0.5914\end{array}$ & $\begin{array}{l}2735 \\
1260 \\
1740 \\
1440 \\
2515 \\
2445 \\
1725 \\
3735 \\
1655\end{array}$ & \\
\hline 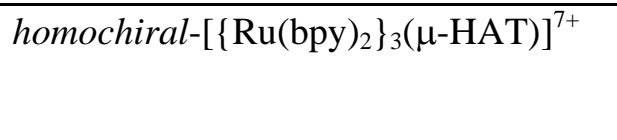 & $\begin{array}{l}1 \\
2 \\
3\end{array}$ & $\begin{array}{l}4475 \\
5750 \\
7180\end{array}$ & $\begin{array}{l}0.6273 \\
0.2993 \\
0.1186\end{array}$ & $\begin{array}{l}1255 \\
1930 \\
1680\end{array}$ & \\
\hline homochiral-[\{Ru(bpy $\left.\left.)_{2}\right\}_{3}(\mu-\mathrm{HAT})\right]^{8+\mathrm{c}}$ & & $\begin{array}{c}4460 \\
6745^{*} \\
11060^{*} \\
13270 \\
17890\end{array}$ & $\begin{array}{c}0.4093 \\
0.4516 \\
0.2485 \\
0.4524 \\
1.225\end{array}$ & $\begin{array}{l}1460 \\
5670 \\
2450 \\
2035 \\
5490\end{array}$ & \\
\hline
\end{tabular}

\footnotetext{
reported previously. ${ }^{133}$

${ }^{\mathrm{b}} \Delta \mathrm{v}_{1 / 2}{ }^{\mathrm{o}}$ is given by equation $1.8^{139}$ where $16 \mathrm{RT} \ln 2=1836 \mathrm{~cm}^{-1}$ at $238 \mathrm{~K}$.

${ }^{\mathrm{c}}$ Band characteristics for the transitions of IVCT origin are indicated by asterisks.

${ }^{\mathrm{d}}$ Artefact peak at detector limit.
}

${ }^{\mathrm{a}}$ Band parameters for the reduced absorption spectra are now reported, rather than parameters for the unreduced spectra 


\section{Dinuclear Systems}

The first oxidation process for the diastereoisomers of $\left[\left\{\mathrm{Ru}(\mathrm{bpy})_{2}\right\}_{2}(\mu-\mathrm{HAT})\right]^{4+}$ was characterised by the appearance of an intense new band in the region $3500-8000 \mathrm{~cm}^{-1}\left\{v_{\max }=5250\right.$ (meso) and $5240 \mathrm{~cm}^{-1}$ (rac) $\}$, which collapsed on removal of the second electron: on this basis, the band was assigned as an IVCT transition \{Table 4.3, Figures 4.6(a) and (b) \}. The bands are asymmetrically-shaped and narrower on the lower energy side with $\Delta v_{1 / 2}$ values of 1600 (meso) and $1485 \mathrm{~cm}^{-1}$ (rac). Compared with the theoretical bandwidths $\left(\Delta v_{1 / 2}{ }^{\circ}\right)$ of $3100 \mathrm{~cm}^{-1}$ for both diastereoisomers \{equation 1.8, where 16RTIn2 $=1836 \mathrm{~cm}^{-1}$ at $\left.238 \mathrm{~K}\right\},{ }^{139}$ the relatively narrow observed bandwidths point towards significant electronic communication between the metal centres. The $\Gamma$ values $^{140}$ (equation 1.21) of 0.484 (meso) and 0.521 (rac) suggest that the systems lie close to the localised-delocalised transition, with the rac diastereoisomer exhibiting a marginally greater degree of electronic delocalisation relative to the meso form. From equation 1.9, $\mathrm{H}_{\mathrm{ab}} \geq 584$ and $514 \mathrm{~cm}^{-1}$ for the meso and rac diastereoisomers, respectively, where $r_{\mathrm{ab}}$ is equated with the approximate geometric metal-metal separation of $6.65 \AA$.

The interpretation of the IVCT data for the dinuclear HAT-bridged systems is challenging since they appear to lie at transition between the localised and delocalised regimes, where classical models $^{137,140-142}$ break down due to the failure of the Born-Oppenheimer approximation upon which they are based. ${ }^{140,143}$ The treatment of the full vibronic coupling problem ${ }^{144-147}$ is central to the quantitative analysis of these borderline localised-to-delocalised systems; however, the application of such methods is beyond the scope of the present work. Instead, we seek to investigate the qualitative correspondence between the IVCT properties of the di- and trinuclear complexes on the basis of alternate localised and delocalised descriptions.

\section{(i) A Localised Model for IVCT}

From a localised viewpoint, the asymmetric appearance of the IVCT bands is attributed to the "band cut-off" effect which occurs at $h v=2 \mathrm{H}_{\mathrm{ab}}$, and is more pronounced for systems lying closer to the localised-to-delocalised transition. ${ }^{140,148,149}$ Alternatively, the asymmetry of the IVCT bands may be attributed to a manifold of discrete, underlying transitions which are identified as spin-orbit components. ${ }^{143,150-152}$

Spectral deconvolution of the NIR bands reveals three underlying Gaussian-shaped components (Table 4.3). The differences observed between the energies, intensities and bandwidths for the diastereoisomers lie well outside the limits of experimental error. The lowest energy components at 3870 and $4030 \mathrm{~cm}^{-1}$ in the meso and rac diastereoisomers, respectively, appear to be artefacts which arise near the limit of the NIR spectrometer at $\sim 3000 \mathrm{~cm}^{-1}$. For both diastereoisomers, IVCT(1) and (2) dominate the IVCT manifolds, although the $M_{0}$ values differ between the two forms: IVCT(1) dominates IVCT(2) for the meso form \{i.e. IVCT(1):(2) = 1.35:1\}, while the opposite is observed for the rac form \{i.e. IVCT(2): $(1)=2.25: 1\}$. For the overall IVCT manifolds, the band area (i.e. the zeroth-moment, $M_{0}$ ) for the meso diastereoisomer is greater than that for the rac form, and suggests a greater degree of metal- 
metal interaction in the former. This contradicts the previous assessment of the relative extent of metalmetal coupling on the $\Gamma$ parameter, where the coupling was greater in the rac diastereoisomer. Since $M_{0}$ is directly proportional to the extent of delocalisation in the mixed-valence ground state, ${ }^{139}$ these values are expected to provide a more accurate estimate of delocalisation rather than the $\Gamma$ parameter which is based on bandwidth measurements alone.

The intervalence process occurs through an electron transfer superexchange mechanism via the $\pi, \pi^{*}(\mathrm{HAT})$ orbitals as discussed in §3.3.2.4 for the complexes incorporating a series of angular bridging ligands. In accordance with the coordinate designation in Figure 3.11 which is also applicable to the HAT-bridged systems, the $\mathrm{d}_{x z}, \mathrm{~d}_{y z}\left(\mathrm{~d} \pi_{1}, \mathrm{~d} \pi_{2}\right)$ orbitals are stabilised to a relatively greater extent (and will thus lie lower in energy) than the $d_{x y}$ orbitals that lie orthogonal to the $\pi, \pi^{*}(\mathrm{HAT})$ system in the plane of the bridging ligand. In order of increasing energy, the three $\mathrm{d} \pi$ orbitals may be more closely represented by $d_{x z}, d_{y z}\left(d \pi_{1}, d \pi_{2}\right)$ and $d_{x y}\left(d \pi_{3}\right)$. Three IVCT transitions arise due to separate electronic excitations from any $d \pi_{n}(n=1-3)$ orbital at $\mathrm{Ru}^{\mathrm{II}}$ to the hole in the corresponding $\mathrm{d} \pi_{\mathrm{n}}$ orbital which is present in any of the three spin-orbit states at $\mathrm{Ru}^{\mathrm{III}}$ (Figure 1.9). ${ }^{143}$ These transitions are identified with the three underlying components in the IVCT band, separated by the energy differences between the ground and two excited spin-orbit states, $\Delta \mathrm{E}_{\mathrm{so}(1)}$ and $\Delta \mathrm{E}_{\mathrm{so}(2)}$, as expressed in equation 1.33. From Table $4.3, \Delta \mathrm{E}_{\mathrm{so}(1)}$ and $\Delta \mathrm{E}_{\mathrm{so}(2)}$ are 800 and $1035 \mathrm{~cm}^{-1}$ for the meso form, and 600 and $1905 \mathrm{~cm}^{-1}$ for the rac form, respectively. The energy splittings between the underlying components are within the range expected for spin-orbit coupling between $\mathrm{Ru}^{\mathrm{II}}$ and $\mathrm{Ru}^{\mathrm{III}}$ centres (i.e. $\sim 800-1250 \mathrm{~cm}^{-1}$ ) for the meso diastereoisomer, although for the rac form the splitting between IVCT(2) and IVCT(3) is significantly greater than anticipated. The differences between the diastereoisomers indicate that streochemical factors such as differential solvent and anion interactions may also influence the inter-metal electronic interaction. ${ }^{153}$ For IVCT(1), $v_{\max }$ is comparable for the two diastereoisomers (5150 and $5110 \mathrm{~cm}^{-1}$ for the meso and rac forms, respectively); however $\mathrm{H}_{\mathrm{ab}}$ for the meso form is considerably greater than that for the rac form (405 vs. $275 \mathrm{~cm}^{-1}$ ).

The intensities of the three IVCT components are proportional to the degree of orbital overlap between the $\mathrm{d} \pi$ orbitals at the formally $\mathrm{Ru}^{\mathrm{II}}$ centre and the $\mathrm{d} \pi$ orbital containing the hole at the formally $\mathrm{Ru}^{\text {III }}$ centre. The $M_{0}$ and $\mathrm{H}_{\mathrm{ab}}$ values provide an indication of inherent differences in the extent of overlap between the donor and acceptor orbitals in the diastereoisomeric forms. IVCT(3) is assigned as a $\left(\mathrm{Ru}^{\mathrm{II}}\right) \mathrm{d} \pi_{3}-\pi^{*}(\mathrm{HAT})-\left(\mathrm{Ru}^{\mathrm{III}}\right) \mathrm{d} \pi_{3}$ transition abbreviated $\mathrm{d} \pi_{3}-\pi^{*}(\mathrm{HAT})-\mathrm{d} \pi_{3}$ \} and exhibits the lowest intensity of the three IVCT components. The low absorptivity arises because electronic coupling occurs between donor and acceptor orbitals which are predominantly $\mathrm{d}_{x y}$ in character, and are oriented orthogonal to the $\pi, \pi^{*}$ (HAT) system. For the rac form, IVCT(2) dominates the IVCT manifold \{i.e. the ratio of the $M_{0}$ values is IVCT(2):(1) = 2.25:1 , while IVCT(1) dominates for the meso form \{i.e. $\operatorname{IVCT}(1):(2)=1.35: 1$ \} Based on ligand field considerations, IVCT(1) and (2) are associated with $\mathrm{d} \pi_{1}-\pi^{*}(\mathrm{HAT})-\mathrm{d} \pi_{1}$ and $\mathrm{d} \pi_{2^{-}}$ $\pi^{*}(\mathrm{HAT})-\mathrm{d} \pi_{2}$ interactions across the bridge, where $\mathrm{d} \pi_{1}$ and $\mathrm{d} \pi_{2}$ are predominantly $\mathrm{d}_{x z}$ and $\mathrm{d}_{y z}$ in character. The enhanced $\mathrm{d} \pi_{2}-\pi^{*}(\mathrm{HAT})-\mathrm{d} \pi_{2}$ interaction relative to the $\mathrm{d} \pi_{1}-\pi^{*}(\mathrm{HAT})$ - $\mathrm{d} \pi_{1}$ interaction in the rac form 
suggests that the $\mathrm{d} \pi_{2}$ orbitals of the formally $\mathrm{Ru}^{\mathrm{II}}$ and $\mathrm{Ru}{ }^{\mathrm{III}}$ centres are overlapped more effectively when the stereochemistries of the two metal centres are equivalent.

\section{(ii) A Delocalised Model for IVCT}

The bridging HAT ligand plays an integral role in mediating the intervalence electron transfer process between the metal centres via a superexchange-assisted mechanism. ${ }^{154}$ This suggests that the explicit inclusion of a third electronic state associated with the bridge may be required to rationalise the underlying electronic structure of the IVCT bands. In this case, the IVCT transition arises from electron transfer between the bonding and non-bonding molecular orbitals within the molecular orbital manifold of the system (Figure 1.4). ${ }^{155-161}$

\section{Trinuclear Systems}

The two mixed-valence states of the trinuclear diastereoisomers were generated upon one- and two-electron oxidation of the +6 species at $-15^{\circ} \mathrm{C}$. The NIR spectra of the +7 and +8 mixed-valence forms (Figure 4.7) exhibit striking differences from one another (which lie well outside the limits of experimental error), and from their dinuclear analogues.

The +7 mixed-valence species for both diastereoisomers exhibit new absorption bands in the range 3500-9000 $\mathrm{cm}^{-1}$ \{Figures 4.6(c) and 4.7, Table 4.3\}. Spectral deconvolution revealed the presence of three underlying transitions, separated by 1305 and $1340 \mathrm{~cm}^{-1}$ (heterochiral) and 1275 and $1430 \mathrm{~cm}^{-1}$ (homochiral). The $\mathrm{M}_{0}$ values of the components indicate that the transitions denoted IVCT(1) and IVCT(2) dominate the IVCT manifold \{i.e. IVCT(1) $>(2)>(3)\}$, with ratios of 3.87:2.84:1 (heterochiral) and 2.81:2.48:1 (homochiral). This relative energy ordering differs from that found for the dinuclear diastereoisomers where IVCT(2) dominated the IVCT manifold. The overall $M_{0}$ of the IVCT manifold for the heterochiral form is slightly greater than that for the homochiral form, and is approximately twice the value for the related dinuclear complexes. The difference may be rationalised on the basis of the doubly-degenerate nature of the transition; i.e. electron transfer occurs from the two formally $\mathrm{Ru}^{\mathrm{II}}$ centres to the hole at the formally $\mathrm{Ru}^{\mathrm{III}}$ centre in the trinuclear species. For both diastereoisomers, IVCT(2) and IVCT(3) exhibit comparable energies and separations relative to the same components in the dinuclear diastereoisomers, although IVCT(1) is significantly red-shifted (by $~ 600$ $\mathrm{cm}^{-1}$ ) relative to the same component in the dinuclear systems.

While vibronic coupling models may provide a more quantitative understanding of the NIR bands, the magnitude of the splitting observed between the two dominant underlying transitions in the NIR band manifold is still consistent with a localised description. On this basis, the bands are assigned as separate optically-induced transitions between energy levels within the molecular orbital manifold of the trinuclear complexes which are split predominantly by spin-orbit coupling of the metal centres. Relative to the dinuclear diastereoisomers, IVCT(1) experiences the most pronounced energy shift, while IVCT(2) and IVCT(3) appear relatively unperturbed. 
Based on the coordinate description invoked for the dinuclear diastereoisomers, the incorporation of a third $\mathrm{Ru}(\mathrm{bpy})_{2}{ }^{2+}$ moiety will perturb the $\mathrm{d} \pi$ orbitals oriented towards the additional Ru centre to the greatest extent. Since electronic delocalisation within the assembly is governed by superexchange coupling through the $\pi^{*}$ orbitals of the bridging ligand, the orbitals perturbed most significantly are predominantly $\mathrm{d}_{x z}$ and $\mathrm{d}_{y z}$ in character, and have already been assigned as the dominant Cartesian components of $\mathrm{d} \pi_{1}$ and $\mathrm{d} \pi_{2}$ for the dinuclear complexes.

The overlap of the $\mathrm{d}_{x z}, \mathrm{~d}_{y z}\left(\mathrm{~d} \pi_{1}, \mathrm{~d} \pi_{2}\right)$ orbitals of the two formally Ru${ }^{\mathrm{II}}$ centres with the hole at the formally $\mathrm{Ru}^{\mathrm{III}}$ centre should be equivalent since they are equidistant to the acceptor. This contrasts the situation in the dinuclear system, where greater overlap is expected for the orbitals directed towards the $\mathrm{Ru}^{\mathrm{II}}$ centre compared with those directed towards the vacant coordination site. Based on this localised argument, IVCT(1) in the trinuclear complex - which is the component shifted most significantly relative to the dinuclear diastereoisomers - would involve optically-induced electron transfer between molecular orbitals centred over the core of the bridging HAT ligand. Accordingly, IVCT(1), (2) and (3) represent, respectively, IVCT transitions which originate from $\mathrm{d} \pi_{1}-\pi^{*}(\mathrm{HAT})-\mathrm{d} \pi_{1}, \mathrm{~d} \pi_{2}-\pi^{*}(\mathrm{HAT})-\mathrm{d} \pi_{2}$ and $\mathrm{d} \pi_{3^{-}}$ $\pi^{*}$ (HAT)-d $\pi_{3}$ interactions across the bridge, where $\mathrm{d} \pi_{1}$ and $\mathrm{d} \pi_{2}$ are predominantly $\mathrm{d}_{x z}$ and $\mathrm{d}_{y z}$, and $\mathrm{d} \pi_{3}$ is predominantly $\mathrm{d}_{x y}$ in character. As was the situation with the dinuclear forms, the low intensity of the $\mathrm{d} \pi_{3}-\pi^{*}(\mathrm{HAT})-\mathrm{d} \pi_{3}$ interaction is consistent with the orthogonal orientation of the $\mathrm{d}_{x y}\left(\mathrm{~d} \pi_{3}\right)$ orbitals relative to the $\pi, \pi^{*}(\mathrm{HAT})$ system.

On the basis of a localised analysis for the spectral properties of the +5 forms of the dinuclear mixed-valence complexes, and +7 forms of the trinuclear systems, the presence of a third Ru(bpy $)_{2}{ }^{2+}$ chromophore in the trinuclear assembly amplifies the energy splitting between the spin-orbit coupling states, and leads to a two-fold enhancement in the electronic interaction mediated by HAT. It is interesting to consider this result in light of previous IVCT measurements on the mixed-valence forms of the trinuclear cyano-bridged chain $\left[\mathrm{NC}-\mathrm{Ru}^{\mathrm{II}}(\mathrm{bpy})_{2}-\mathrm{CN}-\mathrm{Ru}^{\mathrm{III}}(\mathrm{bpy})_{2}-\mathrm{NC}-\mathrm{Ru}^{\mathrm{II}}(\mathrm{bpy})_{2}-\mathrm{CN}\right]^{3+} \cdot{ }^{13-15}{ }^{17}$ The mixed-valence species exhibited a broad band (assigned as an IVCT transition) in the region 4500-12000 $\mathrm{cm}^{-1}$ which appeared to comprise two underlying components of approximately equal intensity. By comparison, the analogous dinuclear complex $\left[\mathrm{NC}-\mathrm{Ru}^{\mathrm{II}}(\mathrm{bpy})_{2}-\mathrm{CN}-\mathrm{Ru}^{\mathrm{III}}(\mathrm{bpy})_{2}-\mathrm{CN}\right]^{2+}$ revealed a single absorption band at $5700 \mathrm{~cm}^{-1}$. The apparent splitting of the IVCT transition into two components in the trinuclear case was attributed to non-negligible superexchange-assisted through-bond coupling between the remote metal centres, and the result was rationalised by the construction of adiabatic energy surfaces for the trinuclear system. ${ }^{13-15,17}$ For the HAT-bridged systems in the present study, the three metal centres share the same bridging ligand, and are equivalently disposed from one another, such that a remote superexchange mechanism cannot be invoked.

The intensities of the IVCT bands for the diastereoisomers of $\left[\left\{\mathrm{Ru}(\mathrm{bpy})_{2}\right\}_{3}(\mu-\mathrm{HAT})\right]^{7+}$ decreased on subsequent oxidation to the +8 mixed-valence species, with the appearance of new bands at $\sim 8000$ and $11500 \mathrm{~cm}^{-1}$ (Figure 4.7, Table 4.3). The transitions in the region at higher energies than $13000 \mathrm{~cm}^{-1}$ were assigned as ligand-to-metal (LMCT) and metal-to-ligand (MLCT) absorptions by comparison with the 
related dinuclear species, and by their behaviour on oxidation to the +9 species. The assignments of the bands in the region at energies lower than $7000 \mathrm{~cm}^{-1}$ are ambiguous due to the presence of complicating comproportionation equilibria, although these may be reasonably ascribed to the residual absorptions of the mono-oxidised +7 species. For the heterochiral diastereoisomer, the new absorption bands at $\sim 8000$ and $11500 \mathrm{~cm}^{-1}$ are assigned as IVCT transitions. These transitions are expected to occur at higher energy than those in the +7 mixed-valence form due to the decreased electronic delocalisation in the system, and hence the destabilisation of the $\mathrm{Ru}^{\mathrm{III}}$ acceptor orbitals. Accordingly, the predicted blue-shift in the energies of the absorption bands is observed, and is consistent with previous literature reports for IVCT transitions in related di- and trinuclear systems. ${ }^{162}$ The $\sim 3000 \mathrm{~cm}^{-1}$ separation between the IVCT bands is not consistent with their interpretation as spin-orbit coupling components however, and indicates that a molecular orbital approach which includes vibronic coupling may be essential.

The possibility also exists that the multiple IVCT transitions originate from optically-induced electron transfer between different “exciton” states. The doubly-oxidised (+8) species comprises two formally $\mathrm{Ru}^{\mathrm{III}}$ and one formally $\mathrm{Ru}^{\mathrm{II}}$ centre and therefore possesses two unpaired electrons. These may be aligned in a parallel or antiparallel fashion, giving rise to an overall singlet or triplet state for the trinuclear complex. The energy required for optical excitation to these two different "exciton" states will differ, in which case the two new transitions observed in the NIR region for the +8 species may be assigned as transitions to singlet and triplet “exciton" states in a valence-delocalised trinuclear mixedvalence species. A similar interpretation was proposed by Weyland et al. ${ }^{9}$ to account for the observation of multiple IVCT transitions in valence-localised trinuclear cyclopentadienyliron complexes with meta connections around a phenylene ring.

\subsubsection{Conclusions}

The IVCT properties of the mixed-valence forms of the diastereoisomers (meso and rac) of the dinuclear complex $\left[\left\{\mathrm{Ru}(\mathrm{bpy})_{2}\right\}_{2}(\mu-\mathrm{HAT})\right]^{4+}$, and of the trinuclear species $\left[\left\{\mathrm{Ru}(\mathrm{bpy})_{2}\right\}_{3}(\mu-\mathrm{HAT})\right]^{6+}$ (homochiral and heterochiral), display a marked dependence on the nuclearity and extent of oxidation of the mixed-valence assemblies, while small differences are also observed between the diastereoisomers of the same complex. The significant differences in IVCT properties between the di- and trinuclear species are attributed to the extensive electronic communication between the Ru(bpy $)_{2}{ }^{2+}$ chromophores, which gives rise to novel properties of the two mixed-valence states in the trinuclear case. The splitting of the IVCT transitions in the +7 species is attributed to the influence of spin-orbit coupling, while the two new dominant transitions in the NIR spectra of the +8 (diradical) species may arise from to transitions to separate delocalised "exciton" states of singlet and triplet character. The origins of the IVCT transitions in the mixed-valence states of $\left[\left\{\mathrm{Ru}(\mathrm{bpy})_{2}\right\}_{3}(\mu-\mathrm{HAT})\right]^{n+}(n=7,8)$ are depicted in Figure 4.8.

Alternative localised and delocalised approaches are discussed for the interpretation of the mixedvalence characteristics of the dinuclear systems; however, treatment of the full vibronic coupling problem is necessary in order to provide a quantitative analysis of these borderline localised-to-delocalised 
systems. Qualitatively, a localised description based on the geometrical properties of the $\mathrm{d} \pi\left(\mathrm{Ru}^{\mathrm{II} / I I I}\right)$ orbitals provides a reasonable rationale for the IVCT behaviour in the di- and trinuclear systems.

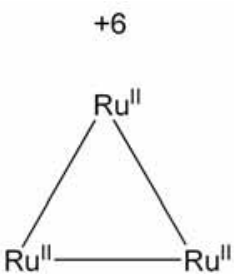

$[2,2,2]$

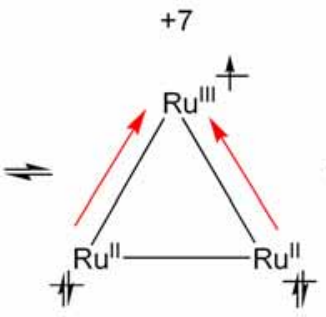

$[3,2,2]$

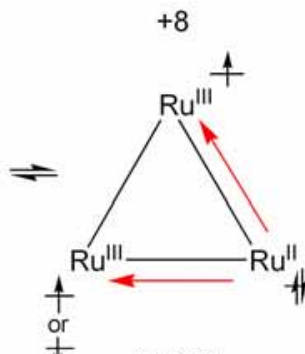

$[3,3,2]$

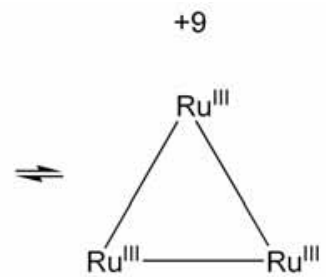

$[3,3,3]$

Figure 4.8 Schematic illustration of the proposed origins of the IVCT transitions (denoted by red arrows) in the +7 and +8 mixed-valence forms of $\left[\left\{\mathrm{Ru}(\mathrm{bpy})_{2}\right\}_{3}(\mu-\mathrm{HAT})\right]^{n+}(n=6-9)$. The formal oxidation states of the metal centres are shown in square brackets. The occupancies of the highest occupied molecular orbitals of the formally $\mathrm{Ru}^{2+}$ \{doubly-occupied, spin-paired $\}$ and $\mathrm{Ru}^{3+}$ \{singly-occupied, spin-unpaired $\}$ centres are also indicated.

\subsubsection{Homo- and Hetero-nuclear Osmium Complexes based on HAT}

\subsubsection{Synthesis, Separation and Structural Characterisation}

The syntheses of the mononuclear $\left[\mathrm{Os}(\mathrm{bpy})_{2}(\mathrm{HAT})\right]^{2+}$ and dinuclear $\left[\left\{\mathrm{Os}(\mathrm{bpy})_{2}\right\}_{2}(\mu-\mathrm{HAT})\right]^{4+}$ species were performed using the microwave methodology reported for the synthesis of the analogous ruthenium complexes. ${ }^{101}$ The hetero-dinuclear complex $\left[\left\{\mathrm{Ru}(\mathrm{bpy})_{2}\right\}(\mu \text {-HAT })\left\{\mathrm{Os}(\mathrm{bpy})_{2}\right\}\right]^{4+}$ was prepared by the thermal reaction of the precursor complexes $\left[\mathrm{Os}(\mathrm{bpy})_{2}(\mathrm{HAT})\right]^{2+}$ with $\left[\mathrm{Ru}(\mathrm{bpy})_{2} \mathrm{Cl}_{2}\right] \cdot 2 \mathrm{H}_{2} \mathrm{O}$ in methanol/water for $12 \mathrm{~h}$. The reaction was attempted using microwave conditions but with limited success.

The separation of the diastereoisomeric forms of the dinuclear complexes [ $\left\{\mathrm{Os}(\mathrm{bpy}){ }_{2}\right\}_{2}(\mu-$ HAT $)]^{4+}$ and $\left[\left\{\mathrm{Ru}(\mathrm{bpy})_{2}\right\}\left(\mu \text {-HAT) }\left\{\mathrm{Os}(\mathrm{bpy})_{2}\right\}\right]^{4+}\right.$ was achieved by cation exchange chromatography using SP Sephadex C-25 as the support with aqueous 0.25 M sodium tosylate solution as eluent. The Band 1 and 2 eluates were determined to be the meso and rac diastereoisomers, respectively, in the former case, and the $\Delta \Lambda / \Lambda \Delta$ and $\Delta \Delta / \Lambda \Lambda$ diastereoisomers in the latter case, as established by NMR characterisation (see below). A crystal structure was obtained for the hetero-dinuclear complex isolated from the Band 1 eluate which confirmed its assignment as the $\Lambda \Delta / \Lambda \Delta$ ("pseudo-meso") diastereoisomer.

The stereoisomerically-pure trinuclear species $\left.\left.\Delta_{2} \Lambda^{\prime} / \Lambda_{2} \Delta^{\prime}-\left[\{\text { Ru(bpy })_{2}\right\}_{2}\{\text { Os(bpy })_{2}\right\}(\mu-H A T)\right]^{6+}$ was prepared by a combination of stereoretentive and chromatographic methodologies as reported previously by Rutherford. ${ }^{48,103}$

\section{${ }^{1}$ H NMR Studies}

One- and two-dimensional NMR techniques permitted structural characterisation of the diastereoisomers of $\left[\left\{\mathrm{Os}(\mathrm{bpy})_{2}\right\}_{2}(\mu-\mathrm{HAT})\right]^{4+}$ and $\left[\left\{\mathrm{Ru}(\mathrm{bpy})_{2}\right\}(\mu \text {-HAT })\left\{\mathrm{Os}(\mathrm{bpy})_{2}\right\}\right]^{4+}$. The assignment of the ${ }^{1} \mathrm{H}$ NMR spectra for the meso and rac diastereoisomers of mono-, di- and trinuclear assemblies based 
on HAT is well established. ${ }^{48,103}$ Accordingly, the present discussion is restricted to the pertinent resonances which are distinctive for the two diastereoisomers. A complete listing of the ${ }^{1} \mathrm{H} N M R$ assignments was provided in §4.2.3.1.

The atom-labelling employed in the assignment of the ${ }^{1} \mathrm{H}$ NMR chemical shifts for the mononuclear complex $\left[\mathrm{Os}(\mathrm{bpy})_{2}(\mathrm{HAT})\right]^{2+}$ and dinuclear complexes $\left[\left\{\mathrm{Os}(\mathrm{bpy})_{2}\right\}_{2}(\mu-\mathrm{HAT})\right]^{4+}$ and $\left[\left\{\mathrm{Ru}(\mathrm{bpy})_{2}\right\}(\mu-\right.$ HAT $\left.\left\{\mathrm{Os}(\mathrm{bpy})_{2}\right\}\right]^{4+}$ is provided in Figure 4.9. The coordinated bpy ligands exhibit the expected coupling constant values $^{163}\left\{J_{3,4}=8 \mathrm{~Hz}, J_{3,5}=1.5 \mathrm{~Hz}, J_{4,5}=8 \mathrm{~Hz}, J_{4,6}=1.5 \mathrm{~Hz}\right.$ and $\left.J_{5,6}=5 \mathrm{~Hz}\right\}$ and coupling patterns based on the symmetry requirements of the complexes.
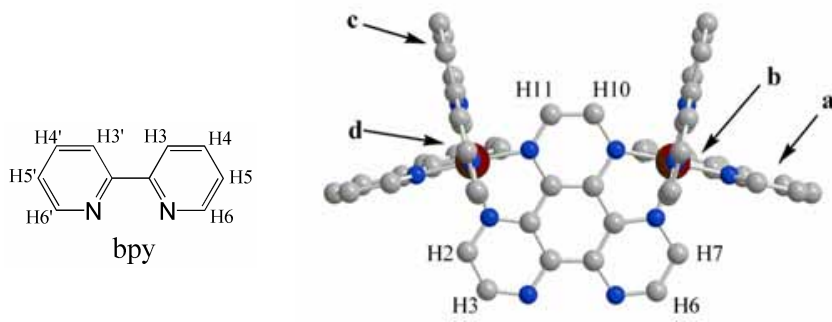

(a)

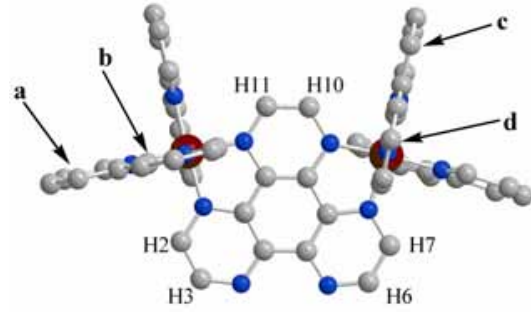

(b)

Figure 4.9 Proton numbering schemes for (a) $\Lambda \Delta$ - and (b) $\Delta \Delta-\left[\left\{\mathrm{M}(\mathrm{bpy})_{2}\right\}(\mu-\mathrm{HAT})\left\{\mathrm{M}^{\prime}(\mathrm{bpy})_{2}\right\}\right]^{4+}\left\{\mathrm{M}, \mathrm{M}^{\prime}=\mathrm{Os}, \mathrm{Os}\right.$ or $\mathrm{Ru}, \mathrm{Os}\}$ (Hydrogen atoms are omitted for clarity). Left: proton numbering scheme for bpy.

$\left[\mathrm{Os}(\text { bpy })_{2}(\mathrm{HAT})\right]^{2+}$

The $\mathbf{C}_{2}$ point group symmetry of [Os(bpy $\left.)_{2}(\mathrm{HAT})\right]^{2+}$ requires two non-equivalent pyridyl rings for each bpy ligand (which are themselves related by the two-fold axis), in addition to three proton resonances for HAT. The assignment of the chemical shifts was based on previous assignments for $\left[\mathrm{Ru}(\mathrm{bpy})_{2}(\mathrm{HAT})\right]^{2+}{ }^{103}$ The H5' and H5 bpy protons are oriented over the planes of the adjacent bpy and HAT ligands, respectively, and are thus assigned to the most upfield resonances at 7.18 and $7.48 \mathrm{ppm}$ ( $J=8,5 \mathrm{~Hz}$, dd), respectively, due to the increased anisotropic interactions. The H6/H7 HAT protons give rise to the most downfield singlet resonance at $9.36 \mathrm{ppm}$ as they are oriented away from the shielding influence from the adjacent ligands.

$\left[\left\{\mathrm{Os}(\text { bpy })_{2}\right\}_{2}(\mu-H A T)\right]^{4+}$

The meso and rac diastereoisomers possess $\mathbf{C}_{\mathrm{s}}$ and $\mathbf{C}_{2}$ point group symmetries, respectively, and may be distinguished on the basis of differential anisotropic interactions experienced by the bpy protons (particularly H5/H5' and H6/H6') depending upon the stereochemical relationship of the two metal centres. In the rac diastereoisomer, bpy ring $b$ is oriented over the plane of the HAT ligand and the bpy ligand across the bridge such that the H5' and H6' protons of this ring experience increased diamagnetic anisotropic effects. The H5' proton of ring $b$ was assigned as the most upfield resonance at $7.08 \mathrm{ppm}$ ( $J=8,5 \mathrm{~Hz}$, dd) while H6' (ring $b$ ) was assigned as the $7.36 \mathrm{ppm}(J=5,1.5 \mathrm{~Hz}$, dd) resonance. By comparison, in the meso diastereoisomer, the H5' and H6' protons of bpy ring $b$ are oriented approximately in the plane (and thus in the deshielding cone) of the equivalent bpy across the bridge. The 
H5' proton of ring $d$ which is situated over the plane of the HAT ligand and approximately parallel to the magnetically equivalent bpy across the bridge experiences the largest anisotropic effect and is assigned as the most upfield resonance at $7.20 \mathrm{ppm}\left(J=8,5 \mathrm{~Hz}\right.$, dd). ${ }^{1} \mathrm{H}$ COSY spectra permitted the assignments of the remaining resonances.

\section{$\left[\left\{R u(b p y)_{2}\right\}(\mu-H A T)\left\{O s(\text { bpy })_{2}\right\}\right]^{4+}$}

The $\Delta \Lambda / \Lambda \Delta$ and $\Delta \Delta / \Lambda \Lambda$ diastereoisomers both possess $\mathbf{C}_{\mathbf{1}}$ point group symmetry and exhibit ${ }^{1} \mathrm{H}$ NMR spectra comprising four non-equivalent bpy ligands (i.e. 40 magnetically non-equivalent proton resonances) in addition to 6 non-equivalent HAT resonances. The spectrum for each diastereoisomer of the hetero-dinuclear complex appeared as a convolution of the spectra of its homo-dinuclear Ru and Os analogues, with additional resonances due to the non-equivalence of the six HAT protons. Due to the coincidental equivalence of certain proton resonances, the assignment of the chemical shifts to specific protons was ambiguous in some cases: however, the chemical shifts of the bpy ligands in the two diastereoisomers of the hetero-dinuclear complex were approximately identical to those of the corresponding bpy ligands in the homo-dinuclear complexes with identical relative stereochemistries. In general, the proton resonances occurred slightly upfield of the analogous positions in the diastereoisomers of $\left[\left\{\mathrm{Ru}(\mathrm{bpy})_{2}\right\}_{2}(\mu-\mathrm{HAT})\right]^{4+}$, due to the increased shielding influence of the Os centre compared with Ru. ${ }^{164}$ The $\Delta \Delta / \Lambda \Lambda$ diastereoisomer exhibits the most upfield resonance at $7.07 \mathrm{ppm}(J=8,5 \mathrm{~Hz}$, dd $)$ as the H5' (ring b) proton of the bpy ligand coordinated to Os is oriented over the plane of the HAT ligand and the bpy ligand across the bridge. By comparison, in the $\Delta \Lambda / \Lambda \Delta$ diastereoisomer, the H5' (ring d) bpy proton of the Os centre is assigned to the most upfield resonance at $7.21 \mathrm{ppm}(\mathrm{J}=8,5 \mathrm{~Hz}$, dd).

\section{X-ray Crystallography}

$\Lambda \Delta / \Delta \Lambda$-[\{Ru(bpy $\left.\left.)_{2}\right\}(\mu-\mathrm{HAT})\left\{\mathrm{Os}(\mathrm{bpy})_{2}\right\}\right]\left(\mathrm{PF}_{6}\right)_{3}$.Cl crystallised in the monoclinic space group $\mathrm{P} 2_{1} / n$ with four dinuclear cations in the unit cell. A perspective view of the dinuclear cation is shown in Figure 4.10(a), and the crystal structure and refinement details are provided in Table C4.2 (Appendix C). The average M-N distances and N-M-N angles at each centre are consistent with those reported previously for polypyridyl complexes of Ru and Os. ${ }^{88,92-97}$ The unambiguous assignment of the identities of $\mathrm{M}(1)$ and $\mathrm{M}(2)$ was not possible during the structural analysis procedure, and the refinement was performed by assigning $50 \%$ occupancy of Ru and Os to both sites. The inability to assign the metal centres is probably a consequence of the racemic nature of the compound, where the two enantiomeric forms $\Lambda \Delta$ and $\Delta \Lambda$ crystallise in equal proportions. ${ }^{165}$ Assuming that the crystal is representative of the bulk solution, the first major band eluted from the column during diastereoisomer separation is confirmed as the $\Lambda \Delta / \Delta \Lambda$ ("pseudo-meso") diastereoisomer.

The bridging HAT ligand maintains planarity and each metal centre resides in a slightly distorted octahedral environment with an $\mathrm{M}(1) \cdots \mathrm{M}(2)$ separation of $6.834(10) \AA$. Two $\mathrm{PF}_{6}{ }^{-}$counter-ions were located within the clefts between the bipyridine rings at the pyrazine end of the bridging ligand 
rather than the end of the free nitrogen atoms, at approximately equivalent distances on either side of the plane of the bridging ligand, as shown in Figure 4.10(b). The anions associate in closer proximity to the pyrazine end of the bridge which is farthest from the un-coordinated nitrogen atoms of HAT due to unfavourable electron repulsions with the $\mathrm{PF}_{6}{ }^{-}$anions which would occur in the latter case. The additional $\mathrm{PF}_{6}{ }^{-}$and $\mathrm{Cl}^{-}$counter-ions reside outside the clefts and do not engage in any intermolecular interactions with the complex.

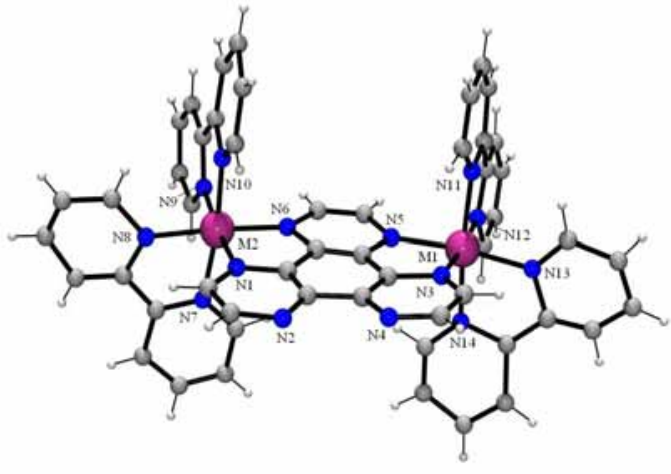

(a)

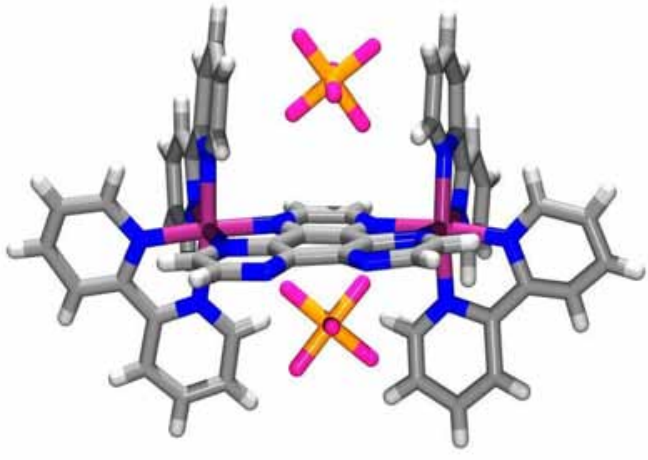

(b)

Figure 4.10 (a) X-ray crystal structure of the cation in $\Lambda \Delta-\left[\left\{\mathrm{M}(\mathrm{bpy})_{2}\right\}(\mu-\mathrm{HAT})\left\{\mathrm{M}^{\prime}(\mathrm{bpy})_{2}\right\}\right]\left(\mathrm{PF}_{6}\right)_{3} \cdot \mathrm{Cl}\left\{\mathrm{M}, \mathrm{M}^{\prime}=\mathrm{Ru}\right.$, Os $\}$ with atom labelling. (b) The $\Lambda \Delta-\left[\left\{\mathrm{M}(\mathrm{bpy})_{2}\right\}(\mu-\mathrm{HAT})\left\{\mathrm{M}^{\prime}(\mathrm{bpy})_{2}\right\}\right]^{4+}$ cation showing the association of two $\mathrm{PF}_{6}^{-}$ anions within the clefts between the bpy ligands.

\subsubsection{Electrochemistry and Electronic Spectroscopy}

\section{Electrochemistry}

The electrochemical properties of $\left[\mathrm{Os}(\mathrm{bpy})_{2}(\mathrm{HAT})\right]^{2+}$, the diastereoisomers of the dinuclear species $\left[\left\{\mathrm{Os}(\mathrm{bpy})_{2}\right\}_{2}(\mu-\mathrm{HAT})\right]^{4+}$ and $\left[\left\{\mathrm{Ru}(\mathrm{bpy})_{2}\right\}(\mu \text {-HAT })\left\{\mathrm{Os}(\mathrm{bpy})_{2}\right\}\right]^{4+}$, and the trinuclear complex $\Delta_{2} \Lambda^{\prime} / \Lambda_{2} \Delta^{\prime}-\left[\left\{\mathrm{Ru}(\mathrm{bpy})_{2}\right\}_{2}\left\{\mathrm{Os}(\mathrm{bpy})_{2}\right\}(\mu \text {-HAT })\right]^{6+}$ were investigated by cyclic and differential pulse voltammetry in acetonitrile containing $0.10 \mathrm{M}\left[\left(n-\mathrm{C}_{4} \mathrm{H}_{9}\right)_{4} \mathrm{~N} \mathrm{PF}_{6}\right.$ and are provided in Tables 4.4 and 4.5. The electrochemical properties of the diastereoisomeric forms of $\left[\left\{\mathrm{Ru}(\mathrm{bpy})_{2}\right\}_{2}\left\{\mathrm{Os}(\mathrm{bpy})_{2}\right\}(\mu-\mathrm{HAT})\right]^{6+}$ have been reported previously. ${ }^{103}$

The mono-, di- and trinuclear systems are characterised by one, two and three reversible oneelectron redox processes, respectively, corresponding to successive oxidation of the metal centres. The first oxidation in the hetero-nuclear systems is based at the osmium centre due to the raised $\mathrm{d} \pi$ level of Os versus Ru. ${ }^{164}$ The first two metal-based redox processes in $\Delta_{2} \Lambda^{\prime} / \Lambda_{2} \Delta^{\prime}-\left[\left\{\operatorname{Ru}(\mathrm{bpy})_{2}\right\}_{2}\left\{\mathrm{Os}(\mathrm{bpy})_{2}\right\}(\mu-\right.$ HAT $)]^{6+}$, occur at similar potentials to those in $\left[\left\{\mathrm{Ru}(\mathrm{bpy})_{2}\right\}(\mu \text {-HAT })\left\{\mathrm{Os}(\mathrm{bpy})_{2}\right\}\right]^{4+}$. The Ru-centred oxidation processes in $\left[\left\{\operatorname{Ru}(\text { bpy })_{2}\right\}(\mu \text {-HAT })\left\{\mathrm{Os}(\text { bpy })_{2}\right\}\right]^{4+}$, and $\Delta_{2} \Lambda^{\prime} / \Lambda_{2} \Delta^{\prime}-\left[\left\{\operatorname{Ru}(\text { bpy })_{2}\right\}_{2}\left\{\mathrm{Os}(\text { bpy })_{2}\right\}(\mu-\right.$ $\mathrm{HAT})]^{6+}$ occur at potentials slightly cathodic of the corresponding processes in $\left[\left\{\mathrm{Ru}(\mathrm{bpy})_{2}\right\}_{2}(\mu-\mathrm{HAT})\right]^{4+}$ and $\left[\left\{\mathrm{Ru}(\mathrm{bpy})_{2}\right\}_{3}(\mu-\mathrm{HAT})\right]^{6+}$, respectively. A schematic illustration of the relative potentials of the metalbased oxidation processes for the series of mono-, di- and trinuclear complexes is provided in Figure 4.11. 


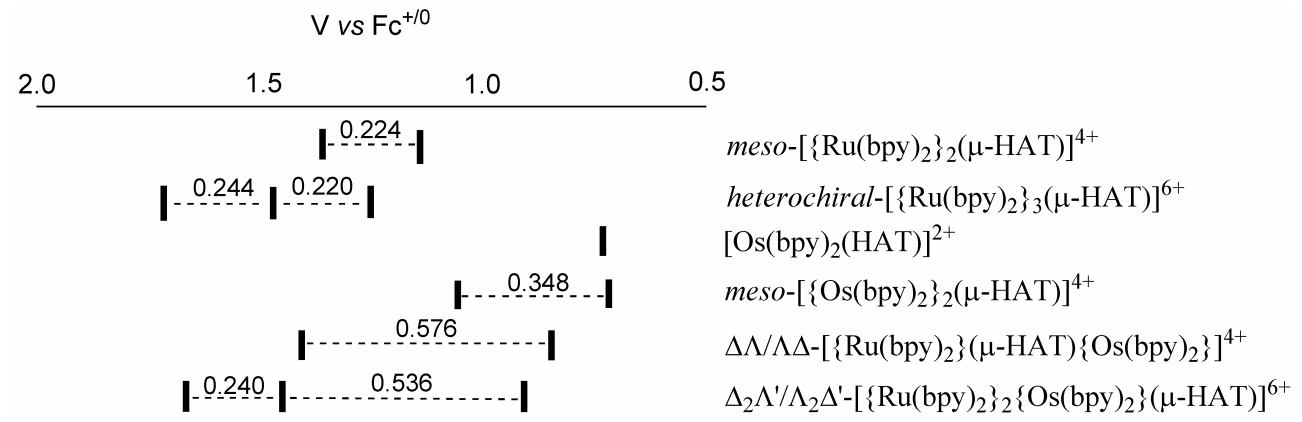

Figure 4.11 Schematic illustration of the relative potentials of the metal-based oxidation processes in selected mono-, di- and trinuclear ruthenium and osmium complexes in this study.

Table 4.4 UV/Vis/NIR spectral ${ }^{\mathrm{a}}$ data of the reduced absorption spectra for the mono- and dinuclear osmium complexes at $-35^{\circ} \mathrm{C}$, and electrochemical ${ }^{\mathrm{b}}$ data (in $\mathrm{mV}$ ), and $\mathrm{K}_{\mathrm{c}}$ values ${ }^{\mathrm{c}}$ for the complexes. The NIR spectral data are indicated in bold type.

\begin{tabular}{|c|c|c|c|c|c|}
\hline Complex & $n$ & $\begin{array}{c}v_{\max } \pm 10 / \mathrm{cm}^{-1} \\
\left\{(\varepsilon / \mathrm{v})_{\max } \pm 0.0001 / \mathrm{M}^{-1}\right\}\end{array}$ & $E_{\text {ox1 }}$ & $E_{0 \times 2}$ & $\begin{array}{l}\Delta \mathrm{E}_{\mathrm{ox}(2-1)} \\
\left(\mathrm{K}_{\mathrm{c}}\right)^{\mathrm{c}}\end{array}$ \\
\hline \multirow[t]{2}{*}[\mathrm{OS}(\mathrm{bpy})_{2}(\mu-\mathrm{HAT})]{$^{n+}$} & 2 & $\begin{array}{l}13990(0.1397) \\
\text { sh17710 (0.2896) } \\
20200(0.7398) \\
22500(0.7751)\end{array}$ & \multirow[t]{2}{*}{736} & & \\
\hline & 3 & $\begin{array}{l}\mathbf{4 4 0 0}(\mathbf{0 . 0 6 4 6 7}) \\
\mathbf{5 1 1 0}(\mathbf{0 . 0 5 5 7 9}) \\
10410(0.02731) \\
18045(0.2117) \\
20227(0.2647) \\
22210(0.2829) \\
\end{array}$ & & & \\
\hline \multirow[t]{3}{*}{ meso- $\left[\left\{\mathrm{OS}(\mathrm{bpy})_{2}\right\}_{2}(\mu-\mathrm{HAT})\right]^{n+}$} & 4 & $\begin{array}{l}12105(0.8051) \\
17615(1.1569) \\
22600(0.7927)\end{array}$ & \multirow[t]{3}{*}{712} & \multirow[t]{3}{*}{1060} & \multirow[t]{3}{*}{$\begin{array}{c}348 \\
\left(7.91 \times 10^{5}\right)\end{array}$} \\
\hline & 5 & $\begin{array}{l}4960(\mathbf{0 . 0 8 2 2 7}) \\
\mathbf{8 1 9 5 ( 0 . 2 9 8 0 )} \\
11100(0.4065) \\
\text { sh } 15670(0.3826) \\
17485(0.7274)\end{array}$ & & & \\
\hline & 6 & $\begin{array}{l}4325(\mathbf{0 . 2 7 5 7}) \\
\mathbf{5 0 8 0}(\mathbf{0 . 1 6 2 9 )} \\
6630(0.0420) \\
14185(0.3953) \\
\end{array}$ & & & \\
\hline \multirow[t]{3}{*}{ rac- $\left[\left\{\mathrm{Os}(\mathrm{bpy})_{2}\right\}_{2}(\mu-\mathrm{HAT})\right]^{n+}$} & 4 & $\begin{array}{l}12075(0.7960) \\
17585(1.1569) \\
22490(0.7780)\end{array}$ & \multirow[t]{3}{*}{720} & \multirow[t]{3}{*}{1072} & \multirow[t]{3}{*}{$\begin{array}{c}352 \\
\left(9.25 \times 10^{5}\right)\end{array}$} \\
\hline & 5 & 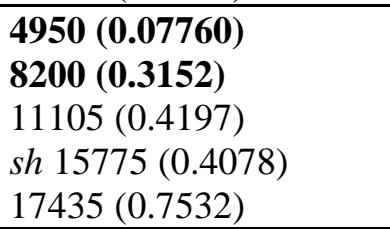 & & & \\
\hline & 6 & $\begin{array}{l}4270(0.2855) \\
5030(0.1409) \\
6790(0.02851) \\
14125(0.4474)\end{array}$ & & & \\
\hline
\end{tabular}

${ }^{\mathrm{a}}$ sh $=$ shoulder of band.

${ }^{\mathrm{b}}$ All potentials $( \pm 3 \mathrm{mV})$ in $0.1 \mathrm{M}\left[\left(n-\mathrm{C}_{4} \mathrm{H}_{9}\right)_{4} \mathrm{~N}\right] \mathrm{PF}_{6} / \mathrm{CH}_{3} \mathrm{CN}$ at $+25^{\circ} \mathrm{C} v \mathrm{~s} . \mathrm{Fc}^{+} / \mathrm{Fc}^{0}$.

${ }^{c} \mathrm{~K}_{\mathrm{c}}$ values determined from equation 1.18(a). ${ }^{132}$ 
Table 4.5 UV/Vis/NIR spectral ${ }^{\mathrm{a}}$ data of the reduced absorption spectra for the hetero-dinuclear and heterotrinuclear complexes at -35 and $-15^{\circ} \mathrm{C}$, respectively, and electrochemical ${ }^{\mathrm{b}}$ data (in $\mathrm{mV}$ ), and $\mathrm{K}_{\mathrm{c}}$ values $^{\mathrm{c}}$ for the complexes. The NIR spectral data are indicated in bold type.

\begin{tabular}{|c|c|c|c|c|c|c|c|}
\hline Complex & $n$ & $\begin{array}{c}v_{\max } \pm 10 / \mathrm{cm}^{-1} \\
\left\{(\varepsilon / v)_{\max } \pm 0.0001\right. \\
\left./ \mathrm{M}^{-1}\right\}\end{array}$ & $\mathrm{E}_{\mathrm{ox} 1}$ & $\mathrm{E}_{\mathrm{ox} 2}$ & $\mathrm{E}_{\mathrm{ox} 3}$ & $\begin{array}{l}\Delta \mathrm{E}_{\mathrm{ox}(2-1)} \\
\left(\mathrm{K}_{\mathrm{c}}\right)^{\mathrm{C}}\end{array}$ & $\begin{array}{l}\Delta \mathrm{E}_{\mathrm{ox}(3-2)} \\
\left(\mathrm{K}_{\mathrm{c}}\right)^{\mathrm{C}}\end{array}$ \\
\hline \multirow[t]{3}{*}{$\begin{aligned} & \Delta \Lambda / \Lambda \Delta- {\left[\left\{\mathrm{Ru}(\mathrm{bpy})_{2}\right\}\right.} \\
&\left.\quad(\mu-\mathrm{HAT})\left\{\mathrm{Os}(\mathrm{bpy})_{2}\right\}\right]^{n+}\end{aligned}$} & 4 & $\begin{array}{l}12730(0.3302) \\
17660(0.9994) \\
23610(0.6971) \\
\end{array}$ & \multirow[t]{3}{*}{832} & \multirow[t]{3}{*}{1408} & & \multirow[t]{3}{*}{$\begin{array}{c}576 \\
(5.79 \times \\
\left.10^{9}\right)\end{array}$} & \\
\hline & 5 & $\begin{array}{l}4090(\mathbf{0 . 4 8 3 7}) \\
4863(\mathbf{0 . 1 1 7 2}) \\
\mathbf{8 6 4 0}(\mathbf{0 . 1 7 5 7 )} \\
13990(0.1220) \\
17690(0.6203) \\
25830(0.3897)\end{array}$ & & & & & \\
\hline & 6 & $\begin{array}{l}\mathbf{4 4 3 0}(\mathbf{0 . 2 6 6 9 )} \\
\mathbf{5 1 7 0}(\mathbf{0 . 1 1 0 2}) \\
10760(0.7979) \\
13260(0.5192) \\
17810(0.1614)\end{array}$ & & & & & \\
\hline \multirow[t]{3}{*}{$\begin{aligned} & \Delta \Delta / \Lambda \Lambda- {\left[\left\{\mathrm{Ru}(\mathrm{bpy})_{2}\right\}\right.} \\
&\left.(\mu-\mathrm{HAT})\left\{\mathrm{Os}(\mathrm{bpy})_{2}\right\}\right]^{n+}\end{aligned}$} & 4 & $\begin{array}{l}12760(0.3403) \\
17650(0.9993) \\
23580(0.6765)\end{array}$ & \multirow[t]{3}{*}{834} & \multirow[t]{3}{*}{1410} & & \multirow[t]{3}{*}{$\begin{array}{c}576 \\
(5.79 \times \\
\left.10^{9}\right)\end{array}$} & \\
\hline & 5 & 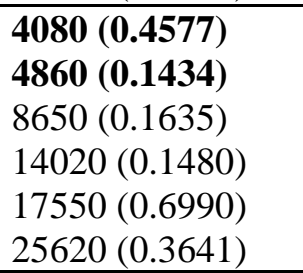 & & & & & \\
\hline & 6 & 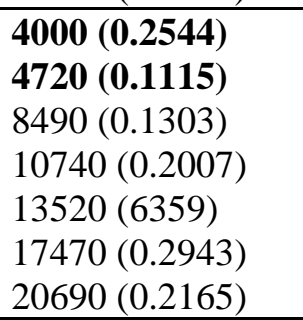 & & & & & \\
\hline \multirow[t]{4}{*}{$\begin{aligned} \Delta_{2} \Lambda^{\prime} / \Lambda_{2} \Delta^{\prime}-[ & {\left.[\text { Ru(bpy })_{2}\right\}_{2} } \\
& \left.\left\{\text { Os }(\text { bpy })_{2}\right\}(\mu-\mathrm{HAT})\right]^{n+}\end{aligned}$} & 6 & $\begin{array}{l}\text { sh12235 (0.3644) } \\
16935(1.88623) \\
\text { sh19285 (1.2245) } \\
24155(0.6670) \\
25875(0.5779)\end{array}$ & \multirow[t]{4}{*}{904} & \multirow[t]{4}{*}{1440} & \multirow[t]{4}{*}{1680} & \multirow[t]{4}{*}{$\begin{array}{c}536 \\
(1.22 \times \\
\left.10^{9}\right)\end{array}$} & \multirow[t]{4}{*}{$\begin{array}{c}240 \\
(1.17 \times \\
\left.10^{4}\right)\end{array}$} \\
\hline & 7 & $\begin{array}{l}3966 \text { (0.5169) } \\
7707(\mathbf{0 . 2 2 2 1}) \\
18055(1.3438) \\
\text { sh } 25845(0.4681)\end{array}$ & & & & & \\
\hline & 8 & $\begin{array}{l}\mathbf{4 0 7 0}(\mathbf{0 . 4 5 1 2}) \\
10585(0.5417) \\
13500(0.6279) \\
16203(12173) \\
19050(0.8669)\end{array}$ & & & & & \\
\hline & 9 & $13625(0.9870)$ & & & & & \\
\hline
\end{tabular}

\footnotetext{
sh $=$ shoulder of band

${ }^{\mathrm{b}}$ All potentials $( \pm 3 \mathrm{mV})$ in $0.1 \mathrm{M}\left[\left(n-\mathrm{C}_{4} \mathrm{H}_{9}\right)_{4} \mathrm{~N}\right] \mathrm{PF}_{6} / \mathrm{CH}_{3} \mathrm{CN}$ at $+25^{\circ} \mathrm{C} v \mathrm{~s} . \mathrm{Fc}^{+} / \mathrm{Fc}^{0}$.

${ }^{\mathrm{c}} \mathrm{K}_{\mathrm{c}}$ values determined from equation 1.18(a). ${ }^{132}$
} 
All complexes exhibited multiple reversible ligand-based reductions in the cathodic region (Table C4.3, Appendix C) which were assigned by analogy with the electrochemical properties of the complexes $\left[\left\{\mathrm{Ru}(\mathrm{bpy})_{2}\right\}_{n}(\mu-\mathrm{HAT})\right]^{2 n+}(n=1-3)$ reported in $\S 4.3 .1 .2 .^{103,166}$ In all cases, the first reduction process is localised on the HAT ligand. The second reduction was also localised on the HAT ligand in the dinuclear complexes and both the second and third reductions are HAT-based in the trinuclear complexes. ${ }^{52}$ In the diastereoisomers of $\left[\left\{\mathrm{Os}(\mathrm{bpy})_{2}\right\}_{2}(\mu-\mathrm{HAT})\right]^{4+}$ and $\left[\left\{\mathrm{Ru}(\mathrm{bpy})_{2}\right\}(\mu \text {-HAT })\left\{\mathrm{Os}(\mathrm{bpy})_{2}\right\}\right]^{4+}$, the subsequent four processes are assigned to sequential reductions of the terminal bpy ligands. The first one-electron bpy reduction occurs for a ligand coordinated to the osmium centre in $\left[\left\{\mathrm{Ru}(\mathrm{bpy})_{2}\right\}(\mu-\mathrm{HAT})\left\{\mathrm{Os}(\mathrm{bpy})_{2}\right\}\right]^{4+}$, followed by one-electron oxidation of a bpy coordinated to Ru. These assignments are supported by electrochemical studies for related di- and trinuclear osmium complexes incorporating polypyridyl bridging ligands such as 2,3-dpp. ${ }^{68,167-170}$

The $\Delta \mathrm{E}_{\text {ox }}$ and the resultant $\mathrm{K}_{\mathrm{c}}$ values for the complexes reported in Tables 4.4 and 4.5 provide a semi-quantitative assessment of the extent of electronic delocalisation and stability of the mixed-valence species. ${ }^{132,134,135}$ The $\mathrm{K}_{\mathrm{c}}$ values for $\left[\left\{\mathrm{Os}(\mathrm{bpy})_{2}\right\}_{2}(\mu \text {-HAT })\right]^{4+}$ suggest a two-orders of magnitude increase in the stability of the mixed-valence species compared with the analogous $\left[\left\{\operatorname{Ru}(\mathrm{bpy})_{2}\right\}_{2}(\mu \text {-HAT })\right]^{4+}$ diastereoisomers. ${ }^{135,170}$ Similar observations have been reported previously for related dinuclear osmium complexes incorporating polypyridyl bridging ligands such as 2,3-dpp (§3.3.5.2) ${ }^{68,167-170}$ For the heterodinuclear and hetero-trinuclear systems, $\Delta \mathrm{E}_{\mathrm{ox}}$ includes an additional energy contribution due to the redox asymmetry $\left(\Delta \mathrm{E}_{0}\right)$ of the complexes.

The separations between the oxidation processes were sufficient to permit the electrochemical generation of the mono-oxidized +5 mixed-valence forms of the dinuclear complexes, in addition to the singly- (+7) and doubly- (+8) forms of the trinuclear complexes.

\section{Electronic Spectroscopy}

The complete UV/Vis/NIR spectral data for the un-oxidised, partially-oxidised and fully-oxidised forms of the mono-, di- and trinuclear systems (for the range 3050-30000 $\mathrm{cm}^{-1}$ ) are provided in Tables 4.4 and 4.5, and the spectra of $\left[\left\{\mathrm{Ru}(\mathrm{bpy})_{2}\right\}(\mu-\mathrm{HAT})\left\{\mathrm{Os}(\mathrm{bpy})_{2}\right\}\right]^{n+}$, [\{Os(bpy $\left.\left.)_{2}\right\}_{2}(\mu-\mathrm{HAT})\right]^{n+}$ and $\Delta_{2} \Lambda^{\prime} / \Lambda_{2} \Delta^{\prime}-$ $\left[\left\{\mathrm{Ru}(\mathrm{bpy})_{2}\right\}_{2}\left\{\mathrm{Os}(\mathrm{bpy})_{2}\right\}(\mu-\mathrm{HAT})\right]^{n+}$ are shown in Figures 4.12-4.14. The spectrum of $\left[\mathrm{Os}(\mathrm{bpy})_{2}(\mathrm{HAT})\right]^{n+}$ is provided in Figure C4.1, Appendix C.

The spectra of the un-oxidised homo- and hetero-dinuclear systems exhibit similar features over the region 15000-30000 $\mathrm{cm}^{-1}$ which are assigned as overlapping $\mathrm{d} \pi\left(\mathrm{M}^{\mathrm{II}}\right) \rightarrow \pi^{*}(\mathrm{HAT})$ and $\mathrm{d} \pi\left(\mathrm{M}^{\mathrm{II}}\right) \rightarrow \pi^{*}$ (bpy) ${ }^{1}$ MLCT transitions $\{\mathrm{M}=\mathrm{Ru}$ and/or Os $\}$. The $\pi \rightarrow \pi^{*}(\mathrm{HAT})$ and $\pi \rightarrow \pi^{*}$ (bpy) transitions appear in the region 30000-50000 $\mathrm{cm}^{-1}$. For the dinuclear osmium systems, an additional band appears in the region $10000-15000 \mathrm{~cm}^{-1}$ compared with the spectra of $\left[\left\{\mathrm{Ru}(\mathrm{bpy})_{2}\right\}_{2}(\mu-\mathrm{HAT})\right]^{4+}$, which is assigned as a $\mathrm{d} \pi\left(\mathrm{Os}{ }^{\mathrm{II}}\right)$ $\rightarrow \pi^{*}(\mathrm{HAT})$ transition to the lowest triplet excited state $\left({ }^{3} \mathrm{MLCT}\right)$. Transitions of the same origin are also evident in the spectrum of $\left[\mathrm{Os}^{\mathrm{II}}(\mathrm{bpy})_{2}(\mathrm{HAT})\right]^{2+}$. The relative intensities of the ${ }^{3} \mathrm{MLCT}$ transitions are 
approximately doubled in the $\left[\left\{\mathrm{Os}(\mathrm{bpy})_{2}\right\}_{2}(\mu \text {-HAT })\right]^{4+}$ diastereoisomers compared with $\left[\left\{\mathrm{Ru}(\mathrm{bpy})_{2}\right\}(\mu-\right.$ HAT $\left.\left.\{\text { Os (bpy })_{2}\right\}\right]^{4+}$, consistent with the number of Os centres present. For the hetero-dinuclear complex, the lowest energy bands in the visible region consist of overlapping $\mathrm{d} \pi(\mathrm{Os}) \rightarrow \pi^{*}(\mathrm{HAT})$ and $\mathrm{d} \pi(\mathrm{Ru}) \rightarrow$ $\pi^{*}$ (HAT) ${ }^{3}$ MLCT and ${ }^{1}$ MLCT transitions with the Os component occurring at lower energy in both cases. The ${ }^{3}$ MLCT and ${ }^{1}$ MLCT transitions are progressively red-shifted as the nuclearity of the assembly increases through the series $\left[\mathrm{Os}(\mathrm{bpy})_{2}(\mathrm{HAT})\right]^{2+},\left[\left\{\mathrm{Ru}(\mathrm{bpy})_{2}\right\}(\mu-\mathrm{HAT})\left\{\mathrm{Os}(\mathrm{bpy})_{2}\right\}\right]^{4+}$ and $\left[\left\{\mathrm{Ru}(\mathrm{bpy})_{2}\right\}_{2}\left\{\mathrm{Os}(\mathrm{bpy})_{2}\right\}(\mu-\mathrm{HAT})\right]^{6+}$, due to the progressive stabilisation of the $\pi^{*}$ LUMO of the bridging ligand. ${ }^{68,167-170}$ Minor differences were observed in the band energies and intensities between the diastereoisomers of the partially- and fully-oxidised dinuclear systems.

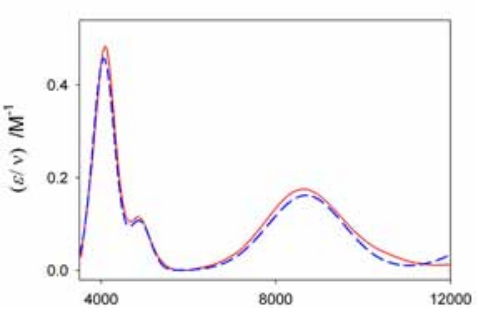

(a)

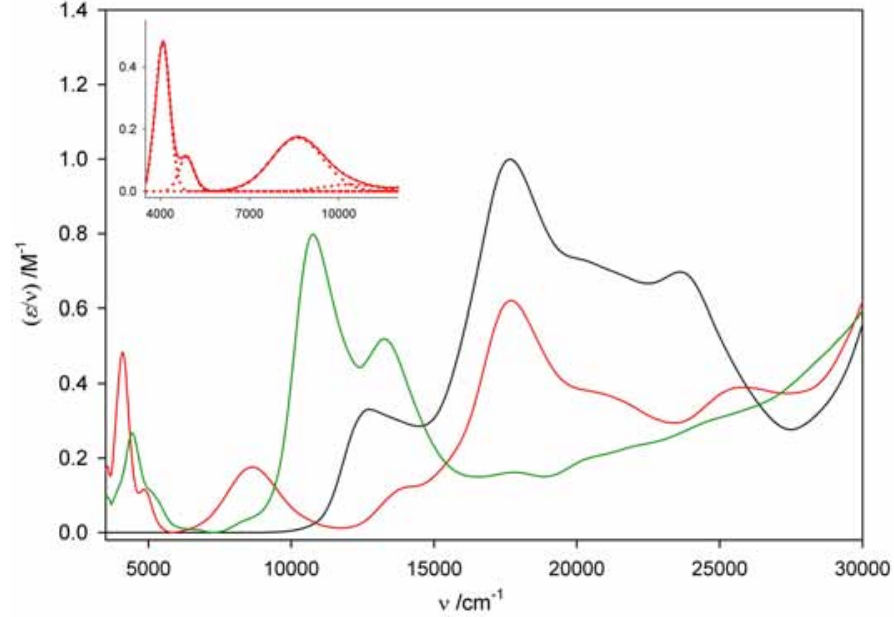

(b)

Figure 4.12 (a) Overlay of the NIR spectra of $\Delta \Lambda / \Lambda \Delta-(-)$ and $\Delta \Lambda / \Lambda \Delta-\left[\left\{\mathrm{Ru}(\mathrm{bpy})_{2}\right\}(\mu-\mathrm{HAT})\left\{\mathrm{Os}(\mathrm{bpy})_{2}\right\}\right]^{5+}(---)$ at $-35^{\circ} \mathrm{C}$, and (b) UV/Vis/NIR spectra of $\Delta \Lambda / \Lambda \Delta$-[\{Os(bpy) $\left.\left.)_{2}\right\}(\mu-\mathrm{HAT})\left\{\mathrm{Ru}(\mathrm{bpy})_{2}\right\}\right]^{n+}\{n=4(-), 5(-), 6(-)\}$ at $-35^{\circ} \mathrm{C}$. The inset shows the bands obtained by Gaussian deconvolution of the NIR spectrum for the mixed-valence species.

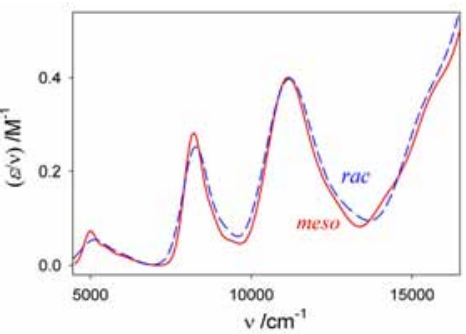

(a)

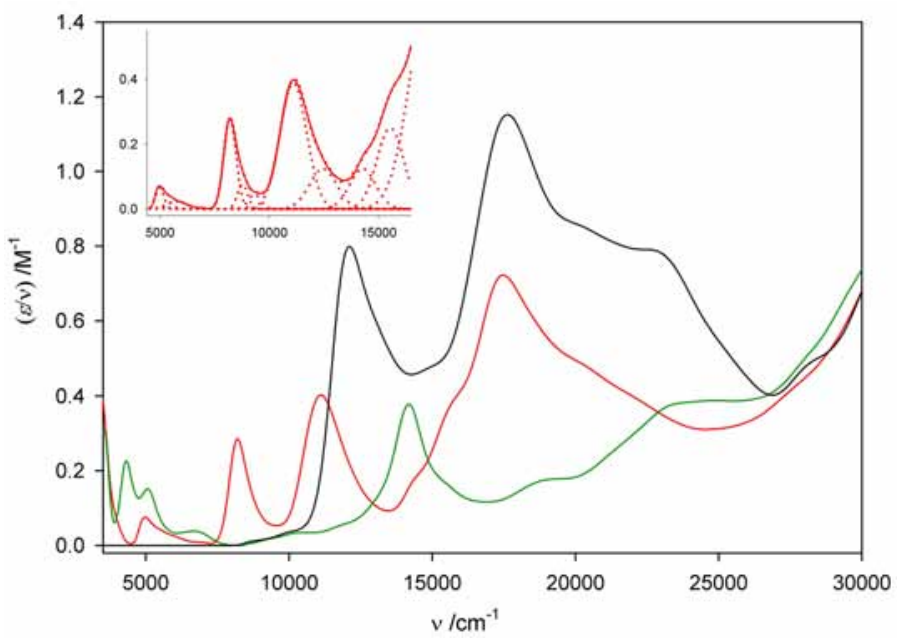

(b)

Figure 4.13 (a) Overlay of the NIR spectra of meso- (-) and rac - $\left[\left\{\mathrm{Os}(\mathrm{bpy})_{2}\right\}_{2}(\mu-\mathrm{HAT})\right]^{5+}(---)$ at $-35^{\circ} \mathrm{C}$, and (b) UV/Vis/NIR spectra of meso- $\left[\left\{\mathrm{Os}(\mathrm{bpy})_{2}\right\}_{2}(\mu-\mathrm{HAT})\right]^{n+}\{n=4(-), 5(-), 6(-)\}$ at $-35^{\circ} \mathrm{C}$. The inset shows the bands obtained by Gaussian deconvolution of the NIR spectrum for the mixed-valence species. 
(a)

(b)

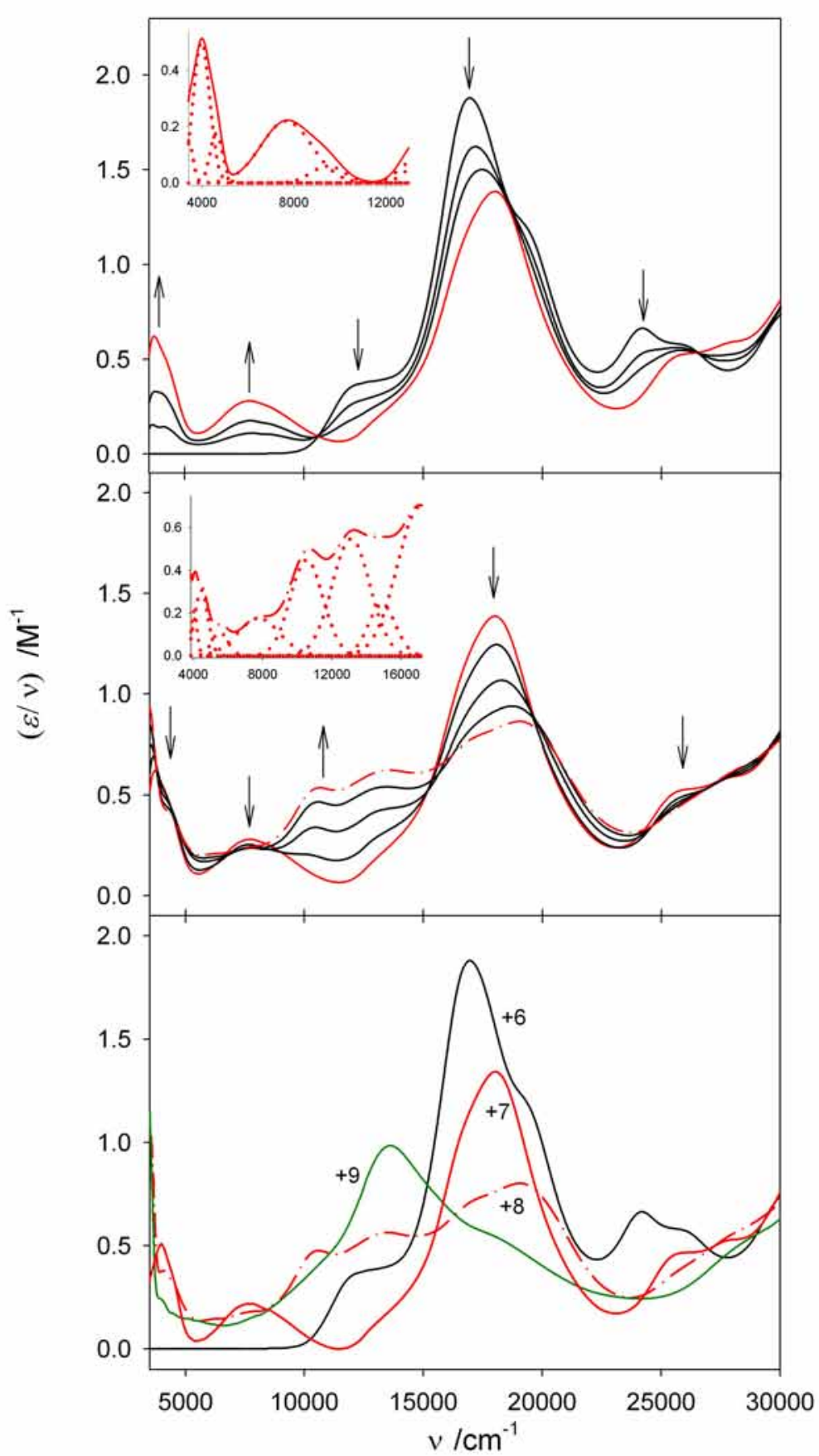

Figure 4.14 Spectroelectrochemistry for the oxidation of $\Delta_{2} \Lambda^{\prime} / \Lambda_{2} \Delta^{\prime}-\left[\left\{\mathrm{Ru}(\mathrm{bpy})_{2}\right\}_{2}\left\{\mathrm{Os}(\mathrm{bpy})_{2}\right\}(\mu-\mathrm{HAT})\right]^{n+}(n=6-9)$ at $-15^{\circ} \mathrm{C}$. (a) Spectroelectrochemical changes for the oxidation reaction $\Delta_{2} \Lambda^{\prime} / \Lambda_{2} \Delta^{\prime}-\left[\left\{\operatorname{Ru}(\text { bpy })_{2}\right\}_{2}\left\{\mathrm{Os}(\mathrm{bpy}){ }_{2}\right\}(\mu-\right.$ HAT $)]^{6+} \rightarrow \Delta_{2} \Lambda^{\prime} / \Lambda_{2} \Delta^{\prime}-\left[\left\{\mathrm{Ru}(\mathrm{bpy})_{2}\right\}_{2}\left\{\mathrm{Os}(\mathrm{bpy})_{2}\right\}(\mu-\mathrm{HAT})\right]^{7+}$. The inset shows NIR band (一) of the latter in addition to the bands obtained from Gaussian deconvolution (…). (b) Spectroelectrochemical changes for the oxidation reaction $\Delta_{2} \Lambda^{\prime} / \Lambda_{2} \Delta^{\prime}-\left[\left\{\mathrm{Ru}(\mathrm{bpy})_{2}\right\}_{2}\left\{\mathrm{Os}(\mathrm{bpy})_{2}\right\}(\mu-\mathrm{HAT})\right]^{7+} \rightarrow \Delta_{2} \Lambda^{\prime} / \Lambda_{2} \Delta^{\prime}-\left[\left\{\mathrm{Ru}(\mathrm{bpy})_{2}\right\}_{2}\left\{\mathrm{Os}(\mathrm{bpy})_{2}\right\}(\mu-\mathrm{HAT})\right]^{8+}$. The inset shows the NIR spectrum (- $\cdot-$ ) of the latter in addition to the bands obtained from Gaussian deconvolution

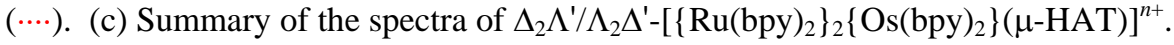

Spectroelectrochemical generation of the mixed-valence (+5), and fully oxidised (+6) forms of the dinuclear complexes revealed stable isosbestic points in the spectral progressions accompanying both oxidation processes. The ${ }^{1}$ MLCT absorption bands decreased in intensity and experienced a slight redshift following one electron-oxidation and eventually collapsed on further oxidation to the +6 species. The ${ }^{3}$ MLCT absorption bands also decreased in intensity and experienced a red-shift in the homo- 
dinuclear osmium species, and collapsed completely following two-electron oxidation. The ${ }^{3}$ MLCT absorption band collapsed completely following the one-electron oxidation in the hetero-dinuclear complexes, consistent with the presence of a single Os centre. LMCT transitions $\left\{\pi(\mathrm{bpy}) \rightarrow \mathrm{d} \pi\left(\mathrm{Os}^{\mathrm{III}}\right)\right\}$ in the +5 spectra of the dinuclear complexes appear at $\sim 14000 \mathrm{~cm}^{-1}$ as low energy shoulders on the ${ }^{1} \mathrm{MLCT}$ transitions.

The spectra of the +6 states of the dinuclear complexes are characterised by intense $\pi($ bpy $) \rightarrow$ $\mathrm{d} \pi\left(\mathrm{M}^{\mathrm{III}}\right)$ and $\pi(\mathrm{HAT}) \rightarrow \mathrm{d} \pi\left(\mathrm{M}^{\mathrm{III}}\right)$ LMCT absorptions in the region $10000-16000 \mathrm{~cm}^{-1}$. The homodinuclear diastereoisomers exhibit a single LMCT transition at $\sim 14200 \mathrm{~cm}^{-1}$, while the hetero-dinuclear diastereoisomers exhibit two LMCT transitions at $\sim 10760$ and $13260 \mathrm{~cm}^{-1}$ which involve the Ru and Os centres, respectively. The latter is consistent with the LMCT transition at $10400 \mathrm{~cm}^{-1}$ in $\left[\mathrm{Os}(\mathrm{bpy})_{2}(\mathrm{HAT})\right]^{3+}$.

Spectroelectrochemical oxidation of the trinuclear complexes allowed the generation of the mixedvalence +7 and +8 forms, and fully-oxidised +9 forms at $-15^{\circ} \mathrm{C}$ (Figure 4.14). Stable isosbestic points were observed in the spectral progressions accompanying the three stages of oxidation. The ${ }^{1}$ MLCT absorption bands decreased in intensity following one- and two-electron oxidation, and collapsed completely on further oxidation to the +9 species. The $\mathrm{d} \pi\left(\mathrm{Os}^{\mathrm{II}}\right) \rightarrow \pi^{*}(\mathrm{HAT}){ }^{3} \mathrm{MLCT}$ transition at $12235 \mathrm{~cm}^{-1}$ disappeared completely following oxidation of the Os ${ }^{\mathrm{II}}$ centre. The bands in the region $10000-16000 \mathrm{~cm}^{-1}$ which gained intensity upon oxidation were assigned as LMCT transitions by comparison with the spectra of the mono- and dinuclear analogues.

\subsubsection{NIR Spectroelectrochemistry and Intervalence Charge Transfer}

The IVCT band parameters obtained from Gaussian deconvolution of the NIR spectra for the partially-oxidised mixed-valence and the fully-oxidised species are provided in Tables C4.4 and C4.5 (Appendix C), respectively.

\section{Dinuclear Systems}

The NIR spectra of the homo- and hetero-dinuclear osmium systems $\left[\left\{\mathrm{M}(\mathrm{bpy})_{2}\right\}(\mu-\right.$ HAT $\left.\left.\{\text { Os(bpy })_{2}\right\}\right]^{n+}\{\mathrm{M}=$ Os or Ru; $n=5,6\}$ exhibit an added complexity in behaviour compared with the analogous homo-dinuclear ruthenium systems due to the larger spin-orbit coupling constant for $\mathrm{Os}^{\text {III }}$ relative to $\mathrm{Ru}^{\mathrm{III}}\left(\xi_{\mathrm{Os}} \sim 3000 \mathrm{~cm}^{-1} v s\right.$. $\xi_{\mathrm{Ru}} \sim 1000 \mathrm{~cm}^{-1}$ ), ${ }^{171}$ which is manifested by multiple IVCT and IC (interconfigurational) transitions in the NIR region of the +5 and +6 spectra (Figures 4.12 and 4.13).

The one-electron oxidation of $\left[\left\{\mathrm{Os}(\mathrm{bpy})_{2}\right\}_{2}(\mu-\mathrm{HAT})\right]^{4+}$ resulted in the appearance of two new bands in the region 3500-10000 $\mathrm{cm}^{-1}\left\{v_{\max }=4960\right.$ and $8195 \mathrm{~cm}^{-1}$ (meso), 4950 and $8200 \mathrm{~cm}^{-1}$ (rac) $\}$. The more intense, higher energy band collapsed completely on removal of the second electron and was assigned as an IVCT transition. This band appears asymmetrical and narrower on the lower energy side; Gaussian deconvolution revealed the presence of two underlying transitions at 8200 and $8780 \mathrm{~cm}^{-1}$ (meso) 
and 9260 and $9130 \mathrm{~cm}^{-1}$ (rac), with the transition moment for the first component a factor of four greater than the second.

Compared with the theoretical bandwidths $\left\{\Delta v_{1 / 2}{ }^{\circ}\right.$ in equation (1.8) $\}$ of $3880 \mathrm{~cm}^{-1}$ for the transitions at 8200 and $8260 \mathrm{~cm}^{-1}$ in meso- and rac-[\{Os(bpy) $\left.\left.\}_{2}\right\}_{2}(\mu-\mathrm{HAT})\right]^{5+}$, respectively, the relatively narrow observed bandwidths in addition to the value of $\sim 0.80$ for the $\Gamma$ parameter (equation $1.21^{140}$ ) are indicative of significant electronic communication between the metal centres. Indeed, the electrochemical and spectral features of diastereoisomers of $\left[\left\{\mathrm{Os}(\mathrm{bpy})_{2}\right\}_{2}(\mu-\mathrm{HAT})\right]^{n+}$ are strikingly similar to those described for the Os analogue of the Creutz-Taube ion, $\left[\left\{\mathrm{Os}\left(\mathrm{NH}_{3}\right)_{5}\right\}_{2}(\mu \text {-pyz) }]^{5+}\right.$, which has been analysed by assuming complete delocalisation. ${ }^{172-175}$ The greater $\mathrm{d} \pi$ orbital extension for Os enhances the inter-metal electronic coupling in $\left[\left\{\mathrm{Os}(\mathrm{bpy})_{2}\right\}_{2}(\mu-\mathrm{HAT})\right]^{5+}$ relative to its Ru analogue, and gives rise to a delocalised ground state. The electronic coupling parameter, $\mathrm{H}_{\mathrm{ab}}$, is estimated as 4100 and $4130 \mathrm{~cm}^{-1}$ for the IVCT transitions in the meso $\left(8200 \mathrm{~cm}^{-1}\right.$ band) and rac $\left(8260 \mathrm{~cm}^{-1}\right.$ band) diastereoisomers, respectively, since $H_{a b}=1 / 2 v_{\max }$ for delocalised complexes (equation 1.11). ${ }^{139}$ In this delocalised description, the explicit consideration of a third electronic state identified with the bridging ligand is essential, and the IVCT process originates from a transition between bonding and antibonding orbitals within the molecular manifold of the system, as described by Ondrechen and coworkers, and depicted in Figure 1.4. ${ }^{155-161}$

The appearance of $\mathrm{d} \pi \rightarrow \mathrm{d} \pi$ transitions between the $\mathrm{d} \pi\left(\mathrm{Os}^{\mathrm{III}}\right)$ orbitals which are split by spin-orbit coupling have been used as a diagnostic marker for localised oxidation states. ${ }^{172-175}$ The NIR spectra of $\left[\left\{\mathrm{Os}(\mathrm{bpy})_{2}\right\}_{2}(\mu \text {-HAT })\right]^{6+}$ exhibit IC transitions in the region $4000-5500 \mathrm{~cm}^{-1}$ which are slightly red-shifted and of greater intensities compared with those in the mononuclear analogue $\left[\mathrm{Os}(\mathrm{bpy})_{2}(\mathrm{HAT})\right]^{3+}$ (Figure C4.1 and Table C4.5, Appendix C). In the dinuclear complex, electronic delocalisation across the bridging ligand decreases the energy splittings between the $\mathrm{d} \pi\left(\mathrm{Os}^{\mathrm{III}}\right)$ levels and enhances the intensities of the IC transitions by mixing the charge-transfer character of the adjacent Os(II) centre into the nominally $\mathrm{d} \pi \rightarrow \mathrm{d} \pi$ transitions. ${ }^{143,150-152,164,171,176,177}$ The mixed-valence species lack the IC bands in their NIR spectra, thus supporting a delocalised classification. The relatively low intensity, asymmetric band in the region 5000-6600 $\mathrm{cm}^{-1}$ is tentatively assigned as a vibronic component associated with intense absorptions that are expected to occur outside the limit of detection in the IR region.

Due to the enhanced spin-orbit coupling in the homo-dinuclear osmium complex relative the $\left[\left\{\mathrm{Ru}(\mathrm{bpy})_{2}\right\}_{2}(\mu-\mathrm{HAT})\right]^{5+}$ systems (\$4.3.2.3), larger energy separations between the three IVCT transitions are expected. In the homo-dinuclear Ru complex, the three underlying components in the IVCT band \{IVCT(1)-(3), in order of increasing energy $\}$ were identified as spin-orbit transitions in a localised description. The separation between the components $\left\{\Delta \mathrm{E}_{\mathrm{so}(1)}\right.$ and $\left.\Delta \mathrm{E}_{\mathrm{so}(2)}\right\}$ represent the $\Delta \mathrm{E}^{\prime}$ contribution to $v_{\max }$ in equation 1.1. ${ }^{143,171}$ The IC bands for the analogous Ru complex are not observed over the spectral range investigated as the lesser magnitude of $\xi_{\mathrm{Ru}}$ has the effect of shifting the IC transitions into the infrared region and decreasing their intensities. ${ }^{143,164}$ In the localised limit, equation 1.33 expresses 
the semi-quantitative energy relationship between the IVCT and IC bands, ${ }^{143}$ where $\Delta \mathrm{E}_{\mathrm{so}(1)}$ and $\Delta \mathrm{E}_{\mathrm{so}(2)}$ are the energies of the IC bands and their separation is approximately equal to the energy difference between IVCT(2) and IVCT(3). On this basis, if the transitions at 8200 and $8780 \mathrm{~cm}^{-1}$ in meso- $\left[\left\{\mathrm{Os}(\mathrm{bpy})_{2}\right\}_{2}(\mu-\right.$ HAT) $]^{5+}$ are assigned as IVCT(2) and IVCT(3), respectively, then IVCT(1) is expected to occur at $~ 3880$ $\mathrm{cm}^{-1}\left\{\right.$ i.e. IVCT(2) $\left.-\Delta \mathrm{E}_{\mathrm{so}(1)}\right\}$. Indeed, the increased absorptivity in the range near the detector limit raises the possibility that IVCT(1) lies at this limit.

In the diastereoisomers of $\left[\left\{\mathrm{Ru}(\mathrm{bpy})_{2}\right\}(\mu-\mathrm{HAT})\left\{\mathrm{Os}(\mathrm{bpy})_{2}\right\}\right]^{4+}$, one-electron oxidation gives rise to the mixed-valence species which is characterised by a broad Gaussian-shaped IVCT band at 8610 and $8695 \mathrm{~cm}^{-1}$, in the $\Delta \Lambda / \Lambda \Delta$ and $\Delta \Delta / \Lambda \Lambda$ diastereoisomers, respectively. Two bands are also evident at 4080 and $4880 \mathrm{~cm}^{-1}(\Delta \Lambda / \Lambda \Delta)$ and 4060 and $4880 \mathrm{~cm}^{-1}(\Delta \Delta / \Lambda \Lambda)$, with the lower energy band exhibiting a factor of three intensity enhancement compared with the higher energy transition. The latter coincides with the position of the vibronic transitions in $\left[\left\{\mathrm{Os}(\mathrm{bpy})_{2}\right\}_{2}(\mu-\mathrm{HAT})\right]^{5+}$, while the former provides evidence for a localised Os ${ }^{\mathrm{III}}$ site in the hetero-dinuclear complex, and the mixed-valence formulation $\left[\left\{\mathrm{Ru}^{\mathrm{II}}(\mathrm{bpy})_{2}\right\}(\mu-\right.$ $\left.\mathrm{HAT})\left\{\mathrm{Os}^{\mathrm{III}}(\mathrm{bpy})_{2}\right\}\right]^{5+}$. The IC bands experience a slight blue-shift following two-electron oxidation due to the destabilisation, and increased splitting of the $\mathrm{d} \pi\left(\mathrm{Os}^{\mathrm{III}}\right)$ orbitals.

The IVCT band in the hetero-dinuclear complex occurs at higher energy than the analogous homo-dinuclear systems due to the additional redox asymmetry $\left(\Delta \mathrm{E}_{0}\right)$ contribution to $v_{\max }$. According to Meyer and Goldsby, ${ }^{170} \Delta \mathrm{E}_{0}$ may be estimated from the semi-quantitative relationship in equation 4.1, assuming that the additional contributions to $\Delta \mathrm{E}_{\text {ox }}$ (e.g. solvation energies) are similar for the closely structurally-related systems. ${ }^{170}$

$$
\Delta\left(\Delta \mathrm{E}_{\mathrm{ox}}\right)=\Delta \mathrm{E}_{\mathrm{ox}}\left(\mathrm{Ru}^{\mathrm{II}} / \mathrm{Os}^{\mathrm{III}}\right)-\Delta \mathrm{E}_{\mathrm{ox}}\left(\mathrm{Ru}^{\mathrm{II}} / \mathrm{Ru}^{\mathrm{III}}\right)
$$

The estimate of $2840 \mathrm{~cm}^{-1}$ (viz. $352 \mathrm{mV}$ ) for both diastereoisomers is in poor agreement with the difference of $3330 \mathrm{~cm}^{-1}$ between the maxima of the IVCT manifolds in the $\left[\left\{\mathrm{Ru}(\mathrm{bpy})_{2}\right\}_{2}(\mu-\mathrm{HAT})\right]^{5+}$ and $\left[\left\{\mathrm{Ru}(\mathrm{bpy})_{2}\right\}(\mu \text {-HAT })\left\{\mathrm{Os}(\mathrm{bpy})_{2}\right\}\right]^{5+}$ systems. The discrepancy is likely to originate from the extensive electronic coupling in the systems, which invalidates the weak coupling approximation \{implicit in equation 4.1 \}, in addition to differences in the solvation and ion-pairing energies for the two complexes. $^{135,170}$

On the basis of a classical analysis for $\left[\left\{\mathrm{Ru}(\mathrm{bpy})_{2}\right\}(\mu-\mathrm{HAT})\left\{\mathrm{Os}(\mathrm{bpy})_{2}\right\}\right]^{5+}$ the following parameters are obtained for $\Delta v_{1 / 2}{ }^{\circ}, \Gamma$ and $\mathrm{H}_{\mathrm{ab}}$, accounting for the non-zero $\Delta \mathrm{E}_{0}$ and taking $r_{\mathrm{ab}}$ as the crystallographic distance of 6.834(10) A: $(\Delta \Lambda / \Lambda \Delta) 3255 \mathrm{~cm}^{-1}, 0.370$ and $400 \mathrm{~cm}^{-1} ;(\Delta \Delta / \Lambda \Lambda) 3280 \mathrm{~cm}^{-1}$, 0.370 and $360 \mathrm{~cm}^{-1}$. The $\mathrm{H}_{\mathrm{ab}}$ values indicate that the degree of electronic delocalisation in the heteronuclear species is substantially reduced compared with the homo-dinuclear Ru and Os analogues. Electronic coupling is dominated by mixing between the Ru orbitals and the $\pi^{*}(\mathrm{HAT})$ orbitals in the hetero-dinuclear system, and the relatively lower energy of the $d \pi(\mathrm{Ru})$ orbital compared with $\mathrm{d} \pi(\mathrm{Os})$, gives rise to decreased coupling through the bridging ligand. ${ }^{168}$ 


\section{Trinuclear Systems}

The two mixed-valence states of $\left.\Delta_{2} \Lambda^{\prime} / \Lambda_{2} \Delta^{\prime}-\left[\{\text { Ru(bpy })_{2}\right\}_{2}\{\text { Os(bpy })_{2}\right\}(\mu$-HAT $\left.)\right]^{n+}(n=7,8)$ were generated upon one- and two-electron oxidation of the +6 species at $-15^{\circ} \mathrm{C}$ (Figure 4.14). The mixedvalence species $\left[\left\{\mathrm{Ru}^{\mathrm{II}}(\mathrm{bpy})_{2}\right\}_{2}\left\{\mathrm{Os}^{\mathrm{III}}(\mathrm{bpy})_{2}\right\}(\mu-\mathrm{HAT})\right]^{7+}$, is characterised by a broad Gaussian-shaped IVCT band at $7670 \mathrm{~cm}^{-1}$ that is slightly overlapping with a weak transition at $9480 \mathrm{~cm}^{-1}$. Gaussian deconvolution reveals the presence of two IC $\mathrm{d} \pi \rightarrow \mathrm{d} \pi$ bands at 3975 and $4620 \mathrm{~cm}^{-1}$ which were assigned by analogy with the spectra of the $\left[\left\{\mathrm{Ru}(\mathrm{bpy})_{2}\right\}(\mu-\mathrm{HAT})\left\{\mathrm{Os}(\mathrm{bpy})_{2}\right\}\right]^{5+}$ diastereoisomers. The appearance of these bands provides evidence for a localised Os ${ }^{\mathrm{III}}$ site.

The IVCT band in $\Delta_{2} \Lambda^{\prime} / \Lambda_{2} \Delta^{\prime}$-[\{Ru(bpy $\left.\left.)_{2}\right\}_{2}\left\{\mathrm{Os}(\mathrm{bpy}){ }_{2}\right\}(\mu-\mathrm{HAT})\right]^{7+}$ is expected to occur at higher energy than the $\left[\left\{\mathrm{Ru}(\mathrm{bpy})_{2}\right\}_{3}(\mu-\mathrm{HAT})\right]^{7+}$ systems. A semi-quantitative analysis yields an estimate of $2550 \mathrm{~cm}^{-1}$ for the $\Delta \mathrm{E}_{0}$ contribution in the hetero-trinuclear system \{viz. from equation $1.4, \Delta\left(\Delta \mathrm{E}_{\mathrm{ox}}\right)=$ $\Delta \mathrm{E}_{\mathrm{ox}(2-1)}\left(\mathrm{Ru}_{2} \mathrm{Os}\right)-\Delta \mathrm{E}_{\mathrm{ox}(3-2)}\left(\right.$ heterochiral-Ru $\left.\left.\mathrm{Ru}_{3}\right)\right\} .{ }^{170}$ On this basis, an IVCT transition in the homotrinuclear systems is predicted at $\sim 5120 \mathrm{~cm}^{-1}$, which does indeed correspond to the average energy of the two major components \{IVCT(1) and (2)\} of the IVCT manifold. Given the localised nature of the IVCT transitions in the hetero-trinuclear complex, the previously well-resolved IVCT(1) and (2) components in the diastereoisomers of $\left[\left\{\mathrm{Ru}(\mathrm{bpy})_{2}\right\}_{3}(\mu \text {-HAT })\right]^{7+}$ are overlapped to a greater extent in the former case, and are observed as a single Gaussian band. If the transition at $9480 \mathrm{~cm}^{-1}$ is assigned as an IVCT component in the hetero-trinuclear system, then a corresponding band at $6930 \mathrm{~cm}^{-1}$ is predicted in the homotrinuclear systems, which does indeed lie close to the observed energy for IVCT(3) at $~ 7170 \mathrm{~cm}^{-1}$ in the latter cases (Table 4.3). The component at $9480 \mathrm{~cm}^{-1}$ in the hetero-trinuclear system exhibits a relatively low intensity, as the origin of the transition is a $\mathrm{d} \pi_{3}-\pi^{*}(\mathrm{HAT})-\mathrm{d} \pi_{3}$ interaction between orbitals which are largely $\mathrm{d}_{x y}$ in character.

On the basis of a classical analysis, $\Delta v_{1 / 2}{ }^{\circ}$ and $H_{a b}$ for the IVCT band at $7670 \mathrm{~cm}^{-1}$ in $\left[\left\{\mathrm{Ru}(\mathrm{bpy})_{2}\right\}_{2}\left\{\mathrm{Os}(\mathrm{bpy})_{2}\right\}(\mu \text {-HAT) }]^{7+}\right.$ are determined as 3190 and $450 \mathrm{~cm}^{-1}$, respectively (where $r_{\mathrm{ab}}$ is equated with the geometrical metal-metal distance from the X-ray crystal structure of the hetero-dinuclear diastereoisomer). The results suggest that the degree of electronic delocalisation is slightly less than that for the diastereoisomers of $\left[\left\{\mathrm{Ru}(\mathrm{bpy})_{2}\right\}_{3}(\mu-\mathrm{HAT})\right]^{7+}$, and similar to that in the diastereoisomers of $\left[\left\{\mathrm{Ru}(\text { bpy })_{2}\right\}(\mu \text {-HAT })\left\{\mathrm{Os}(\text { bpy })_{2}\right\}\right]^{5+}$.

Deconvolution of the NIR spectrum of $\left[\left\{\mathrm{Ru}(\mathrm{bpy})_{2}\right\}_{2}\left\{\mathrm{Os}(\mathrm{bpy})_{2}\right\}(\mu-\mathrm{HAT})\right]^{8+}$ reveals the presence of IVCT transitions at 5530 and $7765 \mathrm{~cm}^{-1}$ between $\mathrm{Ru}^{\mathrm{II}}$, and the $\mathrm{Ru}^{\mathrm{III}}$ and $\mathrm{Os}{ }^{\mathrm{III}}$ centres, respectively (Figure 4.14, Table C4.4, Appendix C). The transition between adjacent $\mathrm{Ru}^{\mathrm{II}}$ and $\mathrm{Os}^{\mathrm{III}}$ centres is slightly blue-shifted compared with the transition of the same origin in $\left[\left\{\mathrm{Ru}(\mathrm{bpy})_{2}\right\}(\mu-\mathrm{HAT})\left\{\mathrm{Os}(\mathrm{bpy})_{2}\right\}\right]^{5+}$. The +8 mixed-valence state in the hetero-trinuclear complex is also characterised by two new bands at 10550 and $13110 \mathrm{~cm}^{-1}$. The latter is assigned as an LMCT transition, and occurs at a similar energy to the bands of the same origin in the hetero-dinuclear analogue. The band at $10550 \mathrm{~cm}^{-1}$ is tentatively assigned as an IVCT transition and decreases upon subsequent oxidation to the +9 state. As described previously 
for the homo-trinuclear analogue, the IVCT transitions may correspond to the formation of either singlet or triplet "exciton" states; however, the definitive identification of the bands is not possible in the present case due to the highly convoluted nature of the spectra. While the assignments for the NIR region in the +8 species are ambiguous due to the presence of complicating comproportionation equilibria, the bands in the energy region below $5500 \mathrm{~cm}^{-1}$ may be reasonably ascribed to IC transitions at Os ${ }^{\text {III }}$. In general, the presence of the third metal centre in the hetero-trinuclear system has the effect of decreasing the effective charge density available for the IVCT processes, resulting in IVCT and IC transitions that are less intense than those observed in the dinuclear $\left[\left\{\mathrm{Ru}(\mathrm{bpy})_{2}\right\}(\mu-\mathrm{HAT})\left\{\mathrm{Os}(\mathrm{bpy})_{2}\right\}\right]^{5+}$ analogue.

\subsubsection{Conclusions}

The mixed-valence forms of $\Delta_{2} \Lambda^{\prime} / \Lambda_{2} \Delta^{\prime}-\left[\left\{\operatorname{Ru}(\text { bpy })_{2}\right\}_{2}\left\{\mathrm{Os}(\text { bpy })_{2}\right\}(\mu-\mathrm{HAT})\right]^{n+}(n=7,8)$ exhibit IVCT properties that are intermediate between those of the diastereoisomeric forms of them localised hetero-dinuclear complex $\left[\left\{\mathrm{Ru}(\mathrm{bpy})_{2}\right\}(\mu \text {-HAT })\left\{\mathrm{Os}(\mathrm{bpy})_{2}\right\}\right]^{5+}$, and the borderline localised-to-delocalised homo-trinuclear complex $\left[\left\{\mathrm{Ru}(\mathrm{bpy})_{2}\right\}_{3}(\mu \text {-HAT })\right]^{n+}(n=7,8)$. The NIR spectrum of the +7 mixed-valence species exhibits both IVCT and IC transitions which are quantitatively similar to those in $\left[\left\{\mathrm{Ru}(\mathrm{bpy})_{2}\right\}(\mu \text {-HAT })\left\{\mathrm{Os}(\mathrm{bpy})_{2}\right\}\right]^{5+}$, and are indicative of the localised mixed-valence formulation $\left[\left\{\mathrm{Ru}^{\mathrm{II}}(\mathrm{bpy})_{2}\right\}_{2}\left\{\mathrm{Os}^{\mathrm{III}}(\mathrm{bpy})_{2}\right\}(\mu-\mathrm{HAT})\right]^{7+}$. The +8 state exhibits a new band attributable to an IVCT transition in the NIR region. The origins of the IVCT transitions in the mixed-valence states of $\left[\left\{\mathrm{Ru}(\mathrm{bpy})_{2}\right\}_{2}\left\{\mathrm{Os}(\mathrm{bpy})_{2}\right\}(\mu-\mathrm{HAT})\right]^{n+}$ are depicted in Figure 4.15. The localised nature of the IVCT transitions contrasts the situation in the diastereoisomers of $\left[\left\{\mathrm{Ru}(\mathrm{bpy})_{2}\right\}_{3}(\mu-\mathrm{HAT})\right]^{n+}$ which exhibit extensive electronic communication between the metal centres.

Qualitatively, a localised description based on the geometrical properties of the $d \pi\left(\mathrm{Os}^{\mathrm{II} / \mathrm{III}}\right)$ and $\mathrm{d} \pi\left(\mathrm{Ru}^{\mathrm{II} / I I I}\right)$ orbitals which accounts for the redox asymmetry contribution provides a reasonable rationale for the IC and IVCT behaviour in the hetero-dinuclear and hetero-trinuclear mixed-valence systems. However, the diastereoisomers of $\left[\left\{\mathrm{Os}(\mathrm{bpy})_{2}\right\}_{2}(\mu \text {-HAT })\right]^{5+}$ exhibit extensive electronic delocalisation, and the description of their mixed-valence properties requires a delocalised description which explicitly includes vibronic coupling.

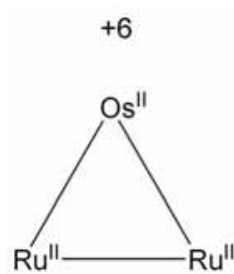

$[2,2,2]$

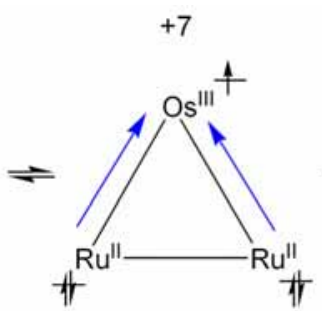

$\left[3^{\prime}, 2,2\right]$

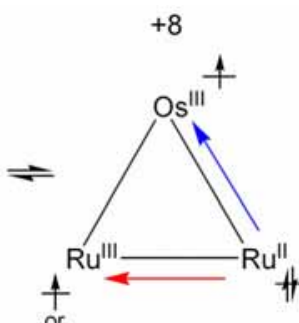

$\left[3^{\prime}, 3,2\right]$

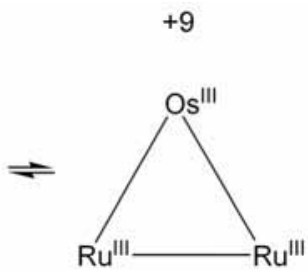

$\left[3^{\prime}, 3,3\right]$

Figure 4.15 Schematic illustration of the proposed origins of the IVCT transitions in the +7 and +8 mixed-valence forms of $\left[\left\{\mathrm{Ru}(\mathrm{bpy})_{2}\right\}_{2}\left\{\mathrm{Os}(\mathrm{bpy})_{2}\right\}(\mu-\mathrm{HAT})\right]^{n+}(n=6-9)$. The formal oxidation states of the metal centres are shown in square brackets where the primes denote the Os centre, and the blue and red arrows illustrate the Os-Ru and Ru-Ru interactions, respectively. The occupancies of the highest occupied molecular orbitals of the formally $\mathrm{Ru}^{2+}$ \{doubly-occupied, spin-paired $\}$ and $\mathrm{M}^{3+}\{\mathrm{M}=\mathrm{Ru}$ or Os, singly-occupied, spin-unpaired $\}$ centres are also indicated. 


\subsubsection{Mono-, Di- and Trinuclear Assemblies based on the Bridging Ligand ppz}

\subsubsection{Synthesis and Structural Characterisation}

Mononuclear Precursors for a Stereochemically-Pure Trinuclear Assembly. The synthesis of $\left[\mathrm{Ru}(\mathrm{bpy})(\mathrm{ppz})_{2}\right]^{2+}$ was based on the previously reported synthetic methodology for tris(heteroleptic) complexes of ruthenium(II), $\left[\mathrm{Ru}(\mathrm{pp})\left(\mathrm{pp}^{\prime}\right)\left(\mathrm{pp}^{\prime \prime}\right)\right]^{2+}$, via the dicarbonylation of $\left[\mathrm{Ru}(\mathrm{pp})(\mathrm{pp})(\mathrm{CO})_{2}\right]^{2+}$. 131, 178 The ${ }^{1} \mathrm{H}$ NMR spectrum of $\left[\mathrm{Ru}(\mathrm{bpy})(\mathrm{ppz})_{2}\right]^{2+}$ revealed a 1:1 ratio of the cis and trans geometric isomers. The isolation of the trans-[Ru(bpy)(ppz $\left.)_{2}\right]^{2+}$ geometric isomer, and chiral resolution of the corresponding $\Delta$ and $\Lambda$ enantiomeric forms, was achieved in a single step by cation-exchange chromatography (SP Sephadex C-25) using 0.10 M sodium tosylate solution as eluent. ${ }^{119,179}$ Since the anion is achiral, the concurrent resolution of the $\Delta$ and $\Lambda$ enantiomeric forms with geometric isomer separation arises from the inherent chirality of the polydextran Sephadex support.

$\left[\mathrm{Ru}(\mathrm{bpy})(\mathrm{HAT})_{2}\right]\left(\mathrm{PF}_{6}\right)_{2}$ was synthesised by the reaction of $\left[\mathrm{Ru}(\mathrm{bpy}) \mathrm{Cl}_{4}\right]$ with excess HAT under microwave conditions according to the previously reported procedure for the synthesis of [Ru(bpy)(2,3-dpp $\left.)_{2}\right]\left(\mathrm{PF}_{6}\right)_{2} .{ }^{122}$ Chiral resolution of the $\Delta$ and $\Lambda$ enantiomeric forms of $\left[\mathrm{Ru}(\mathrm{bpy})(\mathrm{HAT})_{2}\right]\left(\mathrm{PF}_{6}\right)_{2}$ was achieved by cation-exchange chromatography (SP Sephadex C-25; aqueous $0.10 \mathrm{M}(+)$-di-O, $O^{\prime}-4$-toluoyl-D-tartrate solution as eluent) in an analogous manner to that described previously for the resolution of $\left[\mathrm{Ru}(\mathrm{bpy})_{2}(\mathrm{HAT})\right]\left(\mathrm{PF}_{6}\right)_{2} \cdot{ }^{101}$

The CD spectra of $\Delta$ - and $\Lambda$-[Ru(bpy)(HAT) $\left.)_{2}\right]^{2+}$, and $\Delta^{\mathrm{t}}$ - and $\Lambda^{\mathrm{t}}-\left[\mathrm{Ru}(\mathrm{bpy})(\mathrm{ppz})_{2}\right]^{2+}$ are shown in Figure 4.16, and exhibit relatively weak circular dichroism for the $\pi \rightarrow \pi^{*}$ transitions in comparison with other polypyridylruthenium(II) complexes. ${ }^{127-129,180,181}$ A similar observation has been reported previously for $\Delta$-(-)- and $\Lambda$-(+)-[Ru(HAT) $\left.)_{3}\right]^{2+}$ and was attributed to the weak coupling of the electronic transition dipole moments localised on the ligands. ${ }^{104}$ An interpretation of the CD spectra on the basis of exciton theory ${ }^{127,128,180,181}$ was thus avoided, and the assignment of the absolute configurations for the bands obtained from resolution of $\left[\mathrm{Ru}(\mathrm{bpy})(\mathrm{BL})_{2}\right]^{2+}\{\mathrm{BL}=\mathrm{HAT}, \mathrm{ppz}\}$ was achieved by comparison with similar complexes of known absolute configuration; i.e. $\left[\mathrm{Ru}(\mathrm{bpy})_{2}(\mathrm{HAT})\right]^{2+101}$ and $\left[\mathrm{Ru}(\mathrm{HAT})_{3}\right]^{2+} .^{104}$ The Band 1 and 2 eluates obtained from the resolution of $\left[\mathrm{Ru}(\mathrm{bpy})(\mathrm{HAT})_{2}\right]^{2+}$ and the Band 1 and 4 eluates for trans-[Ru(bpy)(ppz) $)_{2}^{2+}$ were assigned as the $\Delta-(-)$ and $\Lambda-(+)$ enantiomers, respectively.
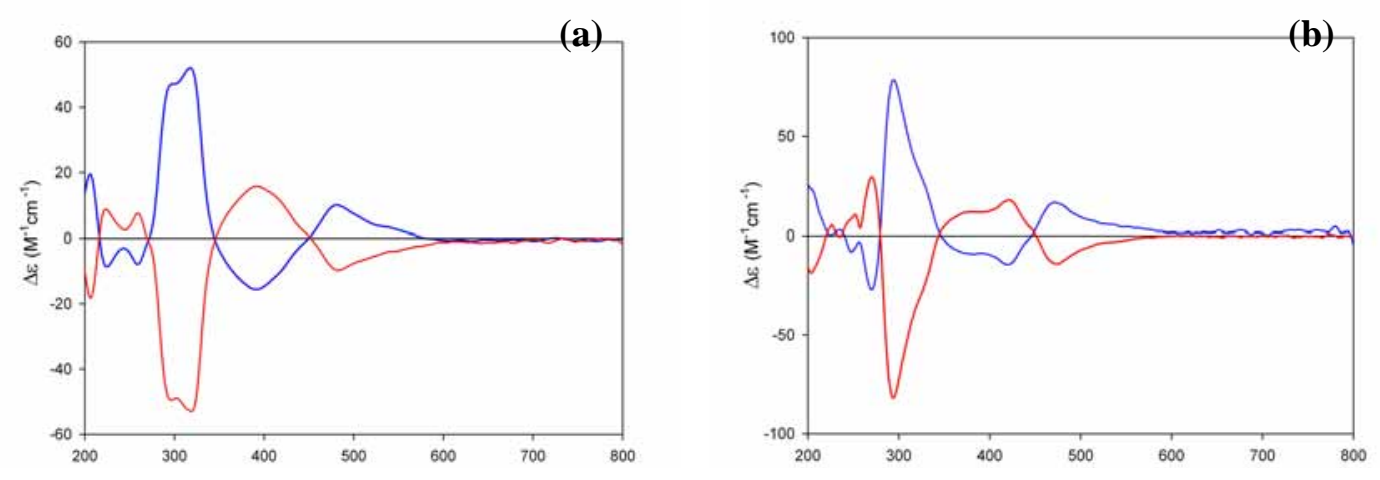

Figure 4.16 $\mathrm{CD}$ spectra $\left(\mathrm{CH}_{3} \mathrm{CN}\right)$ of (a) $\Delta$ - and $\Lambda$-[Ru(bpy)(HAT) $\left.)_{2}\right]^{2+}$, Band $1(\Delta)$ : $(-)$ and Band $2(\Lambda)$ : $(-)$; (b) $\Delta^{\mathrm{t}}$ - and $\Lambda^{\mathrm{t}}-\left[\mathrm{Ru}(\mathrm{bpy})(\mathrm{ppz})_{2}\right]^{2+}$, Band $1(\Delta)(-)$ and Band $4(\Lambda)$ : (一). 


\section{${ }^{1} \mathrm{H}$ NMR Studies}

The proton labelling schemes employed in the assignment of the ${ }^{1} \mathrm{H}$ NMR spectra of $\left[\mathrm{Ru}(\mathrm{bpy})(\mathrm{HAT})_{2}\right]^{2+}$ and trans-[Ru(bpy)(ppz $\left.)_{2}\right]^{2+}$ are shown in Figure 4.17. The coordinated bpy ligands exhibit the expected coupling constant values ${ }^{163}$ and coupling patterns based on the symmetry requirements of the complexes. The ${ }^{1} \mathrm{H}$ NMR chemical shifts of the complexes (Table 4.8) were determined by 2D COSY measurements and were assigned by comparison with the structurally-related complex $\left[\mathrm{Ru}(\mathrm{bpy})_{2}(\mathrm{HAT})\right]^{2+}$ and consideration of the relative degree of diamagnetic anisotropic interactions between the stereochemically-related ligands. ${ }^{101}$

\section{$\left[\mathrm{Ru}(\mathrm{bpy})(\mathrm{HAT})_{2}\right]^{2+}$}

As a consequence of the $\mathbf{C}_{2}$ point group symmetry of $\left[\mathrm{Ru}(\mathrm{bpy})(\mathrm{HAT})_{2}\right]^{2+}$ the proton resonances for both HAT ligands, and for each pyridyl ring of the bpy ligand are equivalent. The bpy H5 and H6 protons are oriented over the plane of the HAT ligand, and are assigned as the most upfield resonances at 7.39 and $7.83 \mathrm{ppm}$, respectively. The $\mathrm{H} 2$ proton of HAT was assigned to the resonance at $8.39 \mathrm{ppm}(\mathrm{J}=$ $3 \mathrm{~Hz}, \mathrm{~d}$ ), while the $\mathrm{H} 11$ proton of HAT was assigned to the resonance at $8.29 \mathrm{ppm}(\mathrm{J}=3 \mathrm{~Hz}, \mathrm{~d})$. The latter experiences a relatively greater shielding due to the increased anisotropic interaction with the bpy ligand compared with the HAT ligand which is relatively $\pi$-electron deficient. The most downfield resonances are attributed to the $\mathrm{H6}$ and $\mathrm{H7}$ protons of HAT, which are oriented away from the shielding influence of the adjacent bpy and HAT ligands. The remaining assignments were based on coupling constant values and comparisons with the ${ }^{1} \mathrm{H}$ NMR spectrum for $\left[\mathrm{Ru}(\mathrm{bpy})_{2}(\mathrm{HAT})\right]^{2+}{ }^{101}$

(a)
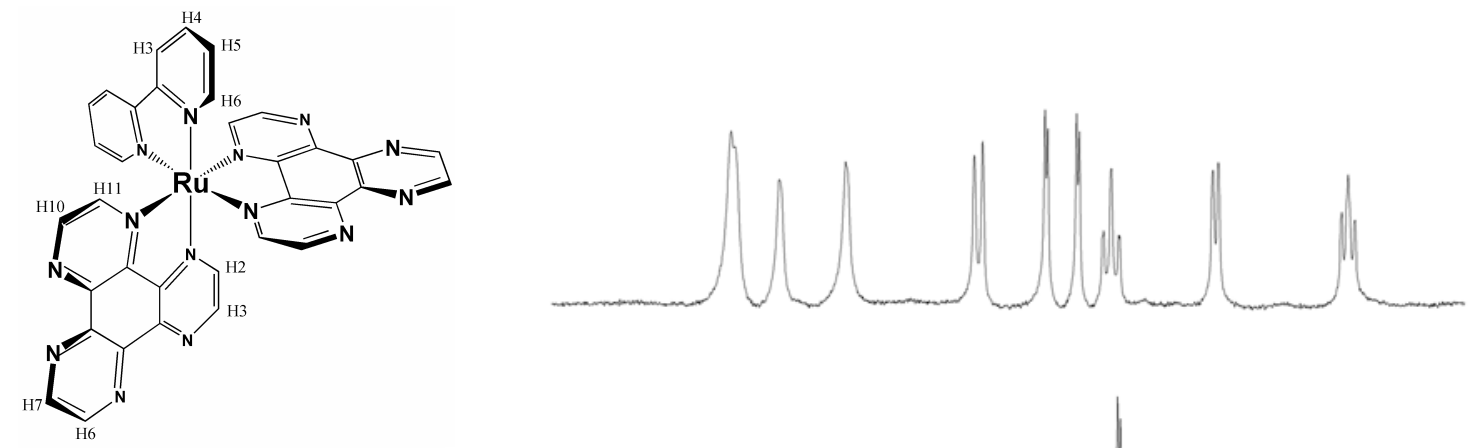

(b)
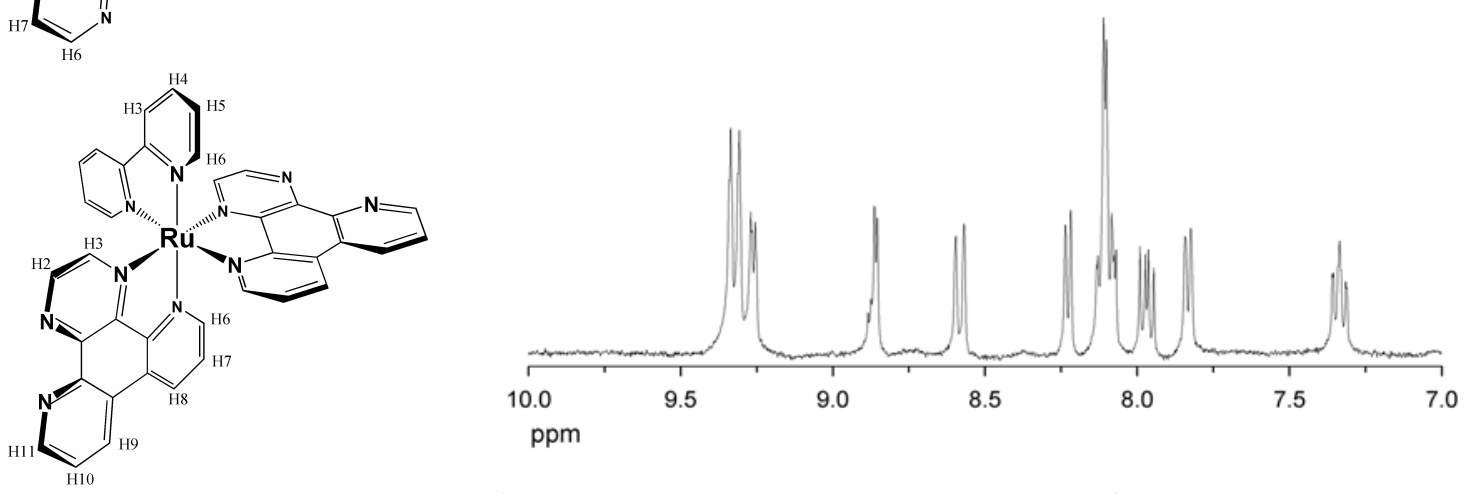

Figure 4.17 Proton numbering schemes and ${ }^{1} \mathrm{H}$ NMR spectra of (a) $\Lambda$-[Ru(bpy)(HAT) $\left.)_{2}\right]^{2+}$ and (b) $\Lambda^{\mathrm{t}-}$

$\left[\mathrm{Ru}(\mathrm{bpy})(\mathrm{ppz})_{2}\right]^{2+}\left(\mathrm{CD}_{3} \mathrm{CN}, \mathrm{PF}_{6}^{-}\right.$salts). The spectra of the $\Delta$ and $\Lambda$ enantiomers were identical in each case. 
trans- $\left[R u(b p y)(p p z)_{2}\right]^{2+}$

The $\mathbf{C}_{2}$ point group symmetry of trans-[Ru(bpy)(ppz $\left.)_{2}\right]^{2+}$ is expected to give rise to 12 nonequivalent proton resonances, while the $\mathbf{C}_{\mathbf{1}}$ point group symmetry for the corresponding cis geometric isomer would give rise to 24 non-equivalent proton resonances. As shown in Figure 4.17, the ${ }^{1} \mathrm{H}$ NMR spectrum of $\Delta^{\mathrm{t}}$-[Ru(bpy)(ppz $\left.)_{2}\right]^{2+}$ is similar to that for $\Delta-\left[\mathrm{Ru}(\mathrm{bpy})(\mathrm{HAT})_{2}\right]^{2+}$, with two additional resonances and due to the $\mathrm{H} 8$ and $\mathrm{H} 9$ protons of ppz. The chemical shifts for the bpy resonances are similar to those in $\left[\mathrm{Ru}(\mathrm{bpy})(\mathrm{HAT})_{2}\right]^{2+}$, with the $\mathrm{H} 5$ and $\mathrm{H} 4$ resonances experiencing upfield shifts of 0.05 ppm due to the increased shielding influence of ppz relative to HAT. The H3 proton of the ppz ligand is oriented over bpy ring e, and occurs slightly upfield (8.11 ppm) of the resonance at $8.22 \mathrm{ppm}$ for the H6 ppz proton which is oriented over the plane of the adjacent ppz ligand. The ppz H8 and H9 protons which are oriented away from the shielding influence of the adjacent bpy and ppz ligands, are assigned as the most downfield resonances ( $c f$. H6 and H7 HAT resonances in $\left[\mathrm{Ru}(\mathrm{bpy})(\mathrm{HAT})_{2}\right]^{2+}$ ). The remaining assignments were based on coupling constant values and comparisons with the assignments for $\left[\mathrm{Ru}(\mathrm{bpy})(\mathrm{HAT})_{2}\right]^{2+}$.

Table 4.8 ${ }^{1} \mathrm{H}$ Chemical shifts (ppm) for $\Lambda$-[Ru(bpy)(HAT) $\left.)_{2}\right]^{2+}$ and $\Lambda^{\mathrm{t}}-\left[\mathrm{Ru}(\mathrm{bpy})(\mathrm{ppz})_{2}\right]^{2+}\left(\mathrm{CD}_{3} \mathrm{CN}, \mathrm{PF}_{6}{ }^{-}\right.$salts). The ${ }^{1} \mathrm{H}$ NMR spectra of the $\Delta$ and $\Lambda$ enantiomers were identical in each case.

\begin{tabular}{|l|l|l|}
\cline { 2 - 3 } \multicolumn{1}{c|}{} & \multicolumn{1}{c|}{$\left[\mathrm{Ru}(\mathrm{bpy})(\mathrm{HAT})_{2}\right]^{2+}$} & \multicolumn{1}{|c|}{ trans-[Ru(bpy)(ppz)$\left.]_{2}\right]^{2+}$} \\
\hline bpy $^{\mathrm{a}}$ & $8.60(\mathrm{H} 3)$ & $8.58(\mathrm{H} 3)$ \\
& $8.16(\mathrm{H} 4)$ & $8.14(\mathrm{H} 4)$ \\
& $7.39(\mathrm{H} 5)$ & $7.34(\mathrm{H} 5)$ \\
& $7.83(\mathrm{H} 6)$ & $7.83(\mathrm{H} 6)$ \\
\hline HAT or ppz & $8.39(\mathrm{H} 2,2 \mathrm{H}, J=3 \mathrm{~Hz}, \mathrm{~d})$ & $8.86(\mathrm{H} 2,2 \mathrm{H}, J=3 \mathrm{~Hz}, \mathrm{~d})$ \\
& $9.24(\mathrm{H} 3,2 \mathrm{H}, \mathrm{s})$ & $8.11(\mathrm{H} 3,2 \mathrm{H}, J=3 \mathrm{~Hz}, \mathrm{~d})$ \\
& $9.39(\mathrm{H} 6,2 \mathrm{H}, \mathrm{s})$ & $8.22(\mathrm{H} 6,2 \mathrm{H}, J=5,1 \mathrm{~Hz}, \mathrm{dd})$ \\
& $9.39(\mathrm{H} 7,2 \mathrm{H}, \mathrm{s})$ & $7.97(\mathrm{H} 7,2 \mathrm{H}, J=10,8 \mathrm{~Hz}, \mathrm{dd})$ \\
& $9.02(\mathrm{H} 10,2 \mathrm{H}, \mathrm{s})$ & $9.32(\mathrm{H} 8,2 \mathrm{H}, J=8 \mathrm{~Hz}, \mathrm{~d})$ \\
& $8.29(\mathrm{H} 11,2 \mathrm{H}, J=3 \mathrm{~Hz}, \mathrm{~d})$ & $9.32(\mathrm{H} 9,2 \mathrm{H}, J=8 \mathrm{~Hz}, \mathrm{~d})$ \\
& & $8.11(\mathrm{H} 10,2 \mathrm{H}, J=10,8 \mathrm{~Hz}, \mathrm{dd})$ \\
& & $9.26(\mathrm{H} 11,2 \mathrm{H}, J=3,1.5 \mathrm{~Hz}, \mathrm{dd})$ \\
\hline
\end{tabular}
$1.5 \mathrm{~Hz}, \mathrm{dd})$.

\section{Stereoselective Synthesis of a Trinuclear Assembly}

The synthesis of $\Delta \Delta^{\mathrm{t}} \Delta-\left[\left\{\mathrm{Ru}(\mathrm{bpy})_{2}\right\}_{2}\left\{\mathrm{Ru}(\mathrm{bpy})(\mu-\mathrm{ppz})_{2}\right\}\right]^{6+}$ was performed via a modification of the stereoretentive synthetic procedure reported by Patterson ${ }^{119}$ involving the reaction of 2.2 equivalents of $\Delta-\left[\operatorname{Ru}(\mathrm{bpy})_{2}(\mathrm{py})_{2}\right]^{2+}$ with $\Delta^{\mathrm{t}}-\left[\mathrm{Ru}(\mathrm{bpy})(\mathrm{ppz})_{2}\right]^{2+}$.

\section{${ }^{1}$ H NMR Studies}

The proton labelling for the ligands employed in the assignment of the ${ }^{1} \mathrm{H}$ NMR spectrum is shown in Figure 4.18. The ${ }^{1} \mathrm{H}$ NMR chemical shifts of $\Delta \Delta^{\mathrm{t}} \Delta$-[ $\left.\left\{\mathrm{Ru}(\mathrm{bpy})_{2}\right\}_{2}\left\{\mathrm{Ru}(\mathrm{bpy})(\mu-\mathrm{ppz})_{2}\right\}\right]^{6+}$ 
\{Table 4.9, Figure 4.19(c)\} were assigned by comparisons with the structurally-related complexes $\left[\mathrm{Ru}(\mathrm{bpy})_{2}(\mathrm{ppz})\right]^{2+}$ and meso- and rac-[\{Ru(bpy)$\left.\left.\}_{2}\right\}_{2}(\mu-\mathrm{ppz})\right]^{4+}$. The ${ }^{1} \mathrm{H}$ NMR spectra of the latter are shown in Figures 4.19(a) and (b) for comparison.

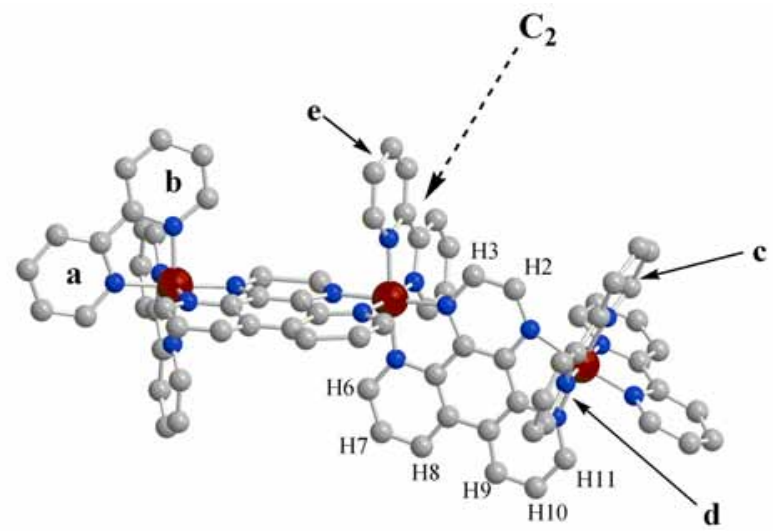

Figure 4.18 Proton numbering scheme for $\Delta \Delta^{t} \Delta-\left[\left\{\operatorname{Ru}(\mathrm{bpy})_{2}\right\}_{2}\left\{\mathrm{Ru}(\mathrm{bpy})(\mu-\mathrm{ppz})_{2}\right\}\right]^{6+}$.

(a)

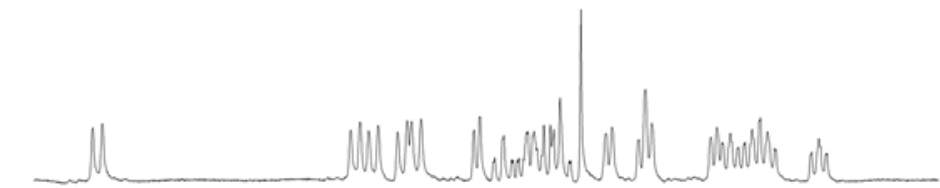

(b)

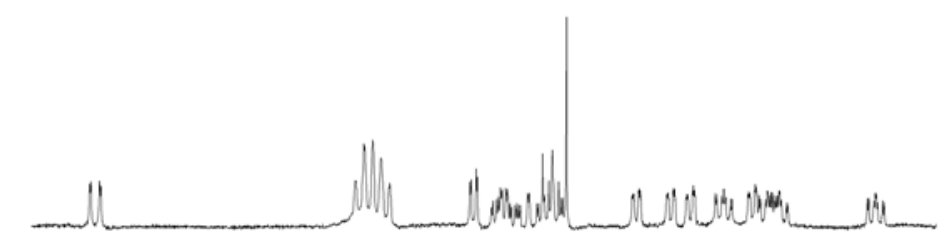

(c)

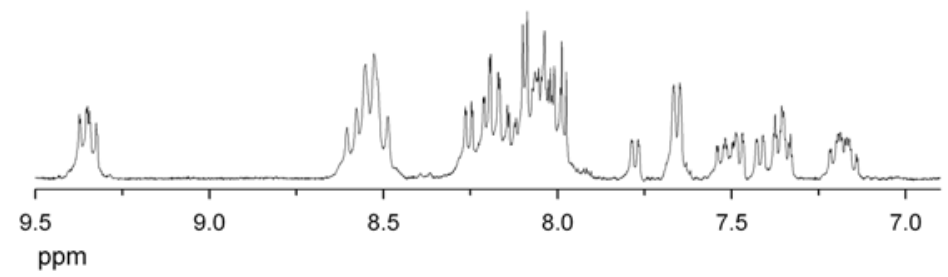

Figure 4.19 ${ }^{1} \mathrm{H}$ NMR spectra $\left(\mathrm{CH}_{3} \mathrm{CN}\right)$ for (a) meso- and (b) rac-[\{Ru(bpy) $\left.\left.\}_{2}\right\}_{2}(\mu-\mathrm{ppz})\right]^{4+}$, and (c) $\Delta \Delta^{\mathrm{t}} \Delta$ $\left[\left\{\mathrm{Ru}(\mathrm{bpy})_{2}\right\}_{2}\left\{\mathrm{Ru}(\mathrm{bpy})(\mu-\mathrm{ppz})_{2}\right\}\right]^{6+}\left(\mathrm{CD}_{3} \mathrm{CN}, \mathrm{PF}_{6}{ }^{-}\right.$salts $)$.

The $\mathbf{C}_{2}$ point group symmetry of $\Delta \Delta^{\mathrm{t}} \Delta-\left[\left\{\mathrm{Ru}(\mathrm{bpy})_{2}\right\}_{2}\left\{\mathrm{Ru}(\mathrm{bpy})(\mu-\mathrm{ppz})_{2}\right\}\right]^{6+}$ gives rise to five nonequivalent bpy pyridyl rings in addition to eight non-equivalent ppz proton resonances, resulting in a total of 36 non-equivalent proton environments. As expected, the spectrum bears a striking resemblance to that for rac- $(\Delta \Delta / \Lambda \Lambda)-\left[\left\{\mathrm{Ru}(\mathrm{bpy})_{2}\right\}_{2}(\mu-\mathrm{ppz})\right]^{4+}\{$ Figure 4.19(b) $\}$. Trans-[Ru(bpy)(ppz $\left.)_{2}\right]^{2+}$ and rac$(\Delta \Delta / \Lambda \Lambda)-\left[\left\{\operatorname{Ru}(\mathrm{bpy})_{2}\right\}_{2}(\mu-\mathrm{ppz})\right]^{4+}$ also possess $\mathbf{C}_{2}$ symmetry, and the spectrum of the trinuclear complex may be interpreted as the summation of the spectra for its mono- and dinuclear analogues. Ring $b$ is oriented over the plane of the ppz and adjacent bpy ring e \{rather than bpy (ring $d$ ) for the dinuclear case so that the $\mathrm{H} 5$ proton is assigned as the most upfield resonance at $7.17 \mathrm{ppm}$ ( $c f .7 .08 \mathrm{ppm}$ in the dinuclear complex). The ${ }^{1} \mathrm{H}$ chemical shifts for pyridyl rings $a$ and $c$ are comparable to those in the dinuclear analogues as they are oriented away from the bridging ligand, while ring $d$ exhibits slight shifts relative to the dinuclear analogue due to the influence of the ppz (rather than bpy) ligand across the bridge. The H5 
and $\mathrm{H} 6$ protons of ring $e$ are oriented over the plane of the ppz ligand in a similar environment to the corresponding protons of ring $d$. The ring $d$ and $e$ protons possess similar ${ }^{1} \mathrm{H}$ chemical shifts; however the latter experience a slightly greater shielding influence due to the adjacent bpy (rather than ppz) ligand across the bridge, and thus lie slightly upfield of the ring $d$ resonances.

The resonances for the H2, H9, H10 and H11 ppz protons experience the most pronounced shifts relative to the mononuclear analogue, as these protons are now oriented towards the terminal Ru centre, and therefore experience the shielding influence of the terminal bpy rings. For example, the H11 resonance occurs at $9.26 \mathrm{ppm}$ in the mononuclear complex compared with $8.09 \mathrm{ppm}$ in the trinuclear complex, since the proton is situated over the shielding cone of ring $a$ in the latter. In the dinuclear complex, the H2/3, H6/11, H7/10 and H8/9 protons are related by a $\mathbf{C}_{2}$ axis. In the trinuclear complex, distinct resonances are observed for each proton of the ppz ligand. For instance, the H8 and H9 protons (dd, $J=8,1.5 \mathrm{~Hz}$ ), previously observed as overlapping resonances at $9.31 \mathrm{ppm}$ in the dinuclear complex are now observed as two separate resonances at 9.33 and $9.36 \mathrm{ppm}$ with the H8 proton assigned as the most downfield resonance.

Table 4.9 ${ }^{1} \mathrm{H}$ chemical shifts (ppm) for $\Delta \Delta^{\mathrm{t}} \Delta-\left[\left\{\mathrm{Ru}(\mathrm{bpy})_{2}\right\}_{2}\left\{\mathrm{Ru}(\mathrm{bpy})(\mu-\mathrm{ppz})_{2}\right\}\right]^{6+}$ by comparison with the meso- and rac- $\left[\left\{\mathrm{Ru}(\mathrm{bpy})_{2}\right\}_{2}(\mu-\mathrm{ppz})\right]^{4+}\left(\mathrm{CD}_{3} \mathrm{CN}, \mathrm{PF}_{6}{ }^{-}\right.$salts $)$.

\begin{tabular}{|c|c|c|c|c|}
\hline & & meso $(\Delta \Lambda / \Lambda \Delta)$ & $\operatorname{rac}(\Delta \Delta / \Lambda \Lambda)$ & $\Delta \Delta^{\mathrm{t}} \Delta$ \\
\hline \multirow{4}{*}{$\begin{array}{l}\text { bpy ring } a^{\text {a }} \\
\text { (over bpy) }\end{array}$} & $\overline{\mathrm{H}} 3^{\prime}$ & 8.58 & 8.53 & 8.59 \\
\hline & H4' & 8.15 & 8.13 & 8.20 \\
\hline & H5' & 7.50 & 7.51 & 7.52 \\
\hline & H6' & 7.73 & 7.76 & 7.77 \\
\hline \multirow{4}{*}{$\begin{array}{l}\text { bpy ring } b^{\text {a }} \\
\text { (over ppz) }\end{array}$} & H3 & 8.52 & 8.51 & 8.50 \\
\hline & $\mathrm{H} 4$ & 8.09 & 8.07 & 8.10 \\
\hline & H5 & 7.39 & 7.02 & 7.17 \\
\hline & H6 & 7.85 & 7.61 & 7.42 \\
\hline \multirow{4}{*}{$\begin{array}{l}\text { bpy ring } c^{\mathrm{a}} \\
\text { (over bpy) }\end{array}$} & H3' & 8.43 & 8.55 & 8.53 \\
\hline & $\mathrm{H} 4^{\prime}$ & 8.06 & 8.15 & 8.20 \\
\hline & H5' & 7.42 & 7.38 & 7.36 \\
\hline & H6' & 7.75 & 7.66 & 7.65 \\
\hline \multirow{4}{*}{$\begin{array}{l}\text { bpy ring } d^{\mathrm{a}} \\
\text { (over ppz) }\end{array}$} & H3 & 8.39 & 8.48 & 8.53 \\
\hline & $\mathrm{H} 4$ & 7.99 & 8.00 & 8.07 \\
\hline & H5 & 7.25 & 7.36 & 7.20 \\
\hline & H6 & 7.54 & 7.42 & 7.65 \\
\hline \multirow{4}{*}{$\begin{array}{l}\text { bpy ring } e^{\mathrm{a}} \\
\text { (over ppz) }\end{array}$} & H3 & & & 8.53 \\
\hline & $\mathrm{H} 4$ & & & 8.02 \\
\hline & H5 & & & 7.36 \\
\hline & H6 & & & 7.47 \\
\hline \multirow[t]{8}{*}{$\mathrm{ppz}^{\mathrm{b}}$} & $\mathrm{H} 2$ & $7.93(\mathrm{H} 2 / 3)$ & $7.96(\mathrm{H} 2 / 3)$ & 7.98 \\
\hline & H3 & & & 8.08 \\
\hline & H6 & 8.23 (H6/11) & $8.23(\mathrm{H6} / 11)$ & 8.25 \\
\hline & $\mathrm{H} 7$ & $8.01(\mathrm{H} 7 / 10)$ & $8.01(\mathrm{H} 7 / 10)$ & 8.10 \\
\hline & H8 & $9.32(\mathrm{H} 8 / 9)$ & $9.31(\mathrm{H} / 9)$ & 9.36 \\
\hline & H9 & & & 9.33 \\
\hline & H10 & & & 8.03 \\
\hline & H11 & & & 8.09 \\
\hline
\end{tabular}

${ }^{a} \mathrm{H} 6$ (dd; $J$ = 5, $1.5 \mathrm{~Hz}$ ); H5 (dd; $J=8,5 \mathrm{~Hz}$ ); H4 (dd; $J=8,8 \mathrm{~Hz}$ ); H3 (dd; $J=8,1.5 \mathrm{~Hz}$ ).

${ }^{\mathrm{b}} \mathrm{H} 2 / 3$ (s); H 6/11 (dd, $\left.J=5,1.5 \mathrm{~Hz}\right) ; \mathrm{H} 7 / 10$ (dd, $\left.J=10,8 \mathrm{~Hz}\right)$; H8/9 (dd, $J=8,1.5 \mathrm{~Hz}$ ). 


\subsubsection{Electrochemistry and Electronic Spectroscopy}

\section{Electrochemistry}

Electrochemical data for trans-[Ru(bpy)(ppz $\left.)_{2}\right]^{2+}$, meso- and rac-[\{Ru(bpy $\left.\left.)_{2}\right\}_{2}(\mu-\mathrm{ppz})\right]^{4+}$ and $\Delta \Delta^{\mathrm{t}} \Delta-\left[\left\{\mathrm{Ru}(\mathrm{bpy})_{2}\right\}_{2}\left\{\mathrm{Ru}(\mathrm{bpy})(\mu-\mathrm{ppz})_{2}\right\}\right]^{6+}$ were obtained by cyclic and differential pulse voltammetry. The potentials of the metal-based oxidation processes and the ligand-based reduction processes are provided in Table 4.10.

The mono- and dinuclear systems exhibit one and two reversible one-electron redox processes, respectively, corresponding to successive oxidation of the metal centres. The trinuclear complex $\Delta \Delta^{t} \Delta$ $\left[\left\{\mathrm{Ru}(\mathrm{bpy})_{2}\right\}_{2}\left\{\mathrm{Ru}(\mathrm{bpy})(\mu-\mathrm{ppz})_{2}\right\}\right]^{6+}$ is characterised by two closely-spaced one-electron redox processes $\left(\mathrm{E}_{\text {ox1 }} \sim 1137 \mathrm{mV}, \Delta \mathrm{E}_{\text {ox(2-1) }}<100 \mathrm{mV}\right.$ ) corresponding to oxidation of the terminal $\mathrm{Ru}^{\text {II }}$ centres to $\mathrm{Ru}^{\mathrm{III}}$, followed by one-electron oxidation of the central $\mathrm{Ru}^{\mathrm{II}}$. The approximately simultaneous oxidation of the terminal centres contrasts to the well-separated $\left(\Delta \mathrm{E}_{\mathrm{ox}(2-1)} \approx 250 \mathrm{mV}\right)$ potentials in the trinuclear $\left[\left\{\mathrm{Ru}(\mathrm{bpy})_{2}\right\}_{3}(\mu-\mathrm{HAT})\right]^{6+}$ system (Table 4.10), and suggests that the terminal centres in the $\Delta \Delta^{\mathrm{t}} \Delta$ $\left[\left\{\mathrm{Ru}(\mathrm{bpy})_{2}\right\}_{2}\left\{\mathrm{Ru}(\mathrm{bpy})(\mu-\mathrm{ppz})_{2}\right\}\right]^{6+}$ complex are not significantly coupled electronically through the chainlike framework. The metal-based redox processes occur at more anodic potentials in the trinuclear complex versus the mononuclear analogue.

All complexes exhibited multiple reversible ligand-based reductions in the cathodic region (Table C4.6, Appendix C). In the dinuclear complexes, the first two processes are assigned to sequential oneelectron reduction of the ppz bridging ligand, due to the greater stabilisation of its $\pi^{*}$ level relative to the peripheral bpy ligands, followed by sequential one-electron reduction processes associated with the terminal bpy ligands. The first two one-electron reduction processes in $\left[\mathrm{Ru}(\mathrm{bpy})(\mathrm{ppz})_{2}\right]^{2+}$ are assigned to sequential reduction of the ppz ligands, while the third one-electron process is bpy-based (by comparison with the bpy-based reduction potentials for $\left.\left[\mathrm{Ru}(\mathrm{bpy})_{3}\right]^{2+}\right)$. The splitting of $246 \mathrm{mV}$ between the ppz reductions indicates electronic communication between the ppz ligands.

In the trinuclear complex, the first two one-electron reduction processes correspond to reduction of the two equivalent (and slightly interacting) ppz bridging ligands. The assignments for the subsequent reduction processes are ambiguous, however comparison with the related mono- and dinuclear complexes suggests that the third two-electron process consists of overlapping contributions from a second ppz reduction and a bpy-based reduction process for the bpy coordinated to the central $\left[\operatorname{Ru}(\mathrm{bpy})(\mu-\mathrm{ppz})_{2}\right]^{2+}$ chromophore. These assignments are consistent with those reported previously in extensive electrochemical studies of the related trinuclear complexes $\left[\left\{\mathrm{Ru}(\mathrm{bpy})_{2}\right\}_{2}\left\{\mathrm{Ru}(\mathrm{bpy})(\mu-\mathrm{BL})_{2}\right\}\right]^{6+}\{\mathrm{BL}=2,3-$ or 2,5-dpp (as stereoisomeric mixtures) by Denti and coworkers. ${ }^{68}$ The lesser splitting between the two ppz-based reductions (168 mV) implies decreased coupling between the ppz ligands relative to the mononuclear species as there is diminished electron density at the central Ru due to the terminal metal centres. This contrasts to the larger splittings of 592 and $596 \mathrm{mV}$ in meso and rac diastereoisomers of the dinuclear complex $\left[\left\{\mathrm{Ru}(\mathrm{bpy})_{2}\right\}_{2}(\mu-\mathrm{ppz})\right]^{n+}$ which arise from the localisation of the two electrons on the 
same ppz bridging ligand. The two ppz-based reduction processes are shifted anodically in the trinuclear complex relative to $\left[\mathrm{Ru}(\mathrm{bpy})(\mathrm{ppz})_{2}\right]^{2+}$ due to the decrease in the electron density of the ppz ligands through backbonding interactions with the terminal Ru centres.

\section{Electronic Spectroscopy}

An overlay of the spectra for the un-oxidised forms of the mono-, di- and trinuclear systems (for the range $3050-30000 \mathrm{~cm}^{-1}$ ) is provided in Figure 4.20 and the spectral characteristics for the mixedvalence states of the di- and trinuclear complexes are presented in Figures 4.21 and 4.22, respectively. The complete UV/Vis/NIR spectral data are reported in Table 4.10. The spectroelectrochemical properties of meso- and rac-[\{Ru(bpy $\left.\left.)_{2}\right\}_{2}(\mu-\mathrm{ppz})\right]^{n+}(n=4-6)$ have been detailed previously in §3.3.2.2.

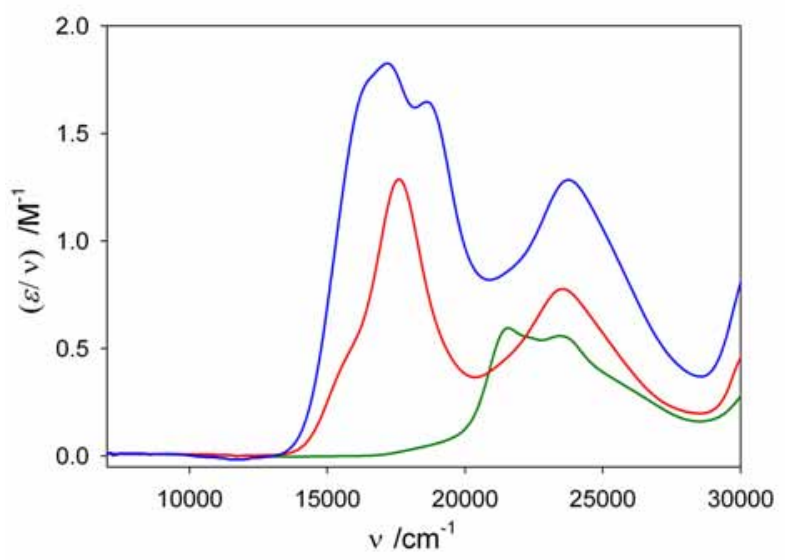

Figure 4.20 UV/Vis/NIR spectra of $\left[\mathrm{Ru}(\mathrm{bpy})(\mathrm{ppz})_{2}\right]^{2+}(-)$, meso- $\left[\left\{\mathrm{Ru}(\mathrm{bpy})_{2}\right\}_{2}(\mu-\mathrm{ppz})\right]^{4+}(-)$ and $\Delta \Delta^{\mathrm{t}} \Delta-\left[\left\{\mathrm{Ru}(\mathrm{bpy})_{2}\right\}_{2}\left\{\mathrm{Ru}(\mathrm{bpy})(\mu-\mathrm{ppz})_{2}\right\}\right]^{6+}(-)$ in $\mathrm{CH}_{3} \mathrm{CN}$ at $+25^{\circ} \mathrm{C}$.

The spectra of meso- and rac-[\{Ru(bpy $\left.\left.)_{2}\right\}_{2}(\mu-p p z)\right]^{4+}$ exhibit comparable features to the diastereoisomers of $\left[\left\{\mathrm{Ru}(\mathrm{bpy})_{2}\right\}_{2}(\mu-\mathrm{HAT})\right]^{4+}$ described previously. The spectra over the region $15000-$ $30000 \mathrm{~cm}^{-1}$ are characterised by a combination of overlapping $\mathrm{d} \pi\left(\mathrm{Ru}^{\mathrm{II}}\right) \rightarrow \pi^{*}(\mathrm{ppz})$ and $\mathrm{d} \pi\left(\mathrm{Ru}^{\mathrm{II}}\right) \rightarrow$ $\pi^{*}$ (bpy) ${ }^{1}$ MLCT transitions, with the former occurring at lower energy. The spectrum of the diastereoisomeric mixture has been completely assigned previously on the basis of resonance Raman studies. ${ }^{182}$ In the present work, minor differences were observed in the band energies and intensities between the diastereoisomeric forms. The ${ }^{1} \mathrm{MLCT}$ energies in meso- and rac- $\left[\left\{\mathrm{Ru}(\mathrm{bpy})_{2}\right\}_{2}(\mu-\mathrm{ppz})\right]^{4+}$ are red-shifted by ca. $300 \mathrm{~cm}^{-1}$ relative to the related $\left[\left\{\mathrm{Ru}(\mathrm{bpy})_{2}\right\}_{2}(\mu-\mathrm{HAT})\right]^{4+}$ species, which is consistent with the more delocalised nature of ppz (and the stabilisation of the lowest unoccupied $\pi^{*}$ molecular orbital) compared with HAT. The mixed-valence species is characterised by an IVCT band in the region $3500-5000 \mathrm{~cm}^{-1}$, as shown in Figure 4.21. The intense absorption bands in the region $10000-16000 \mathrm{~cm}^{-1}$ for the +6 species are assigned as $\pi($ bpy $) \rightarrow \mathrm{d} \pi\left(\mathrm{Ru}^{\mathrm{III}}\right)$ and $\pi(\mathrm{ppz}) \rightarrow \mathrm{d} \pi\left(\mathrm{Ru}^{\mathrm{III}}\right)$ LMCT transitions. 


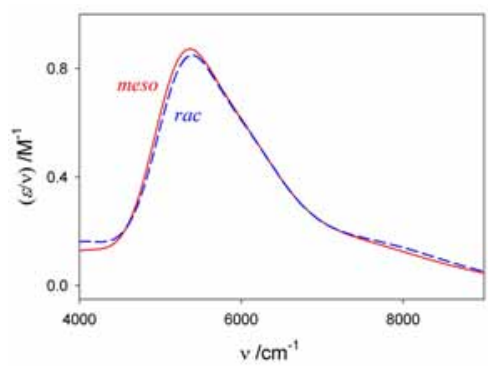

(a)

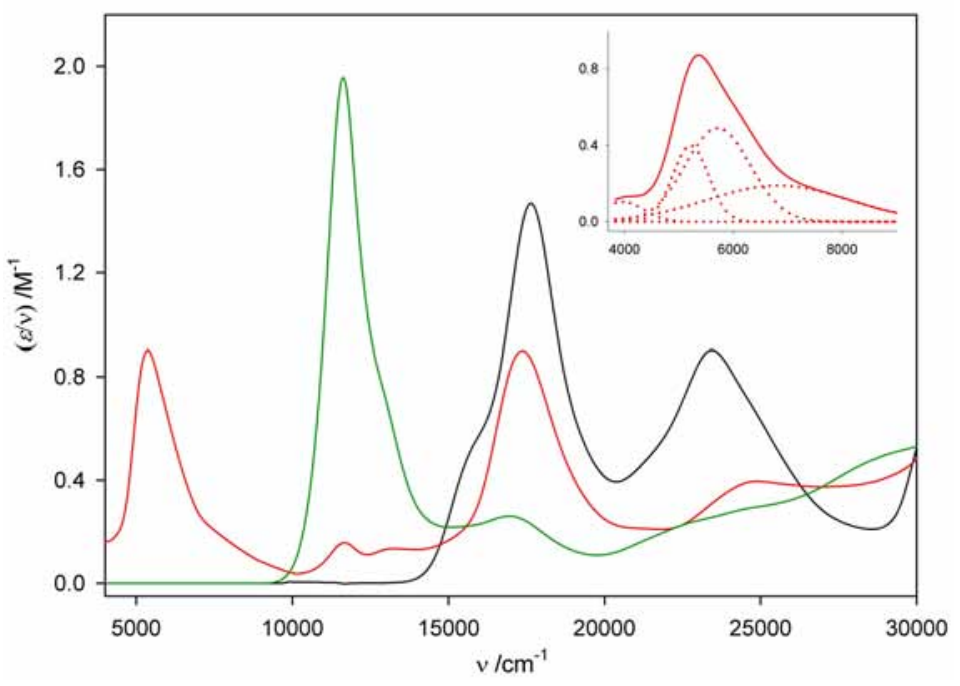

(b)

Figure 4.21 (a) Overlay of the IVCT bands for meso- and rac-[\{Ru(bpy $\left.\left.)_{2}\right\}_{2}(\mu-\mathrm{ppz})\right]^{5+}$. (b) UV/Vis/NIR spectra of meso- $\left[\left\{\mathrm{Ru}(\mathrm{bpy})_{2}\right\}_{2}(\mu-\mathrm{ppz})\right]^{n+}\{n=4(-), 5(-), 6(-)\}$ at $-35^{\circ} \mathrm{C}$. The inset shows the best fit of the bands obtained by Gaussian deconvolution of the IVCT band.

The spectrum of $\Delta \Delta^{\mathrm{t}} \Delta$-[\{Ru(bpy $\left.\left.)_{2}\right\}_{2}\left\{\mathrm{Ru}(\mathrm{bpy})(\mu-\mathrm{ppz})_{2}\right\}\right]^{6+}$ exhibits a broad absorption band between $15000-20000 \mathrm{~cm}^{-1}$, as shown in Figures 4.20 and 4.22. The band receives contributions from three underlying transitions of approximately equal intensities at 17560, 16150 and $19930 \mathrm{~cm}^{-1}$ (obtained by Gaussian deconvolution) which are assigned as $\mathrm{d} \pi\left(\mathrm{Ru}^{\mathrm{II}}\right) \rightarrow \pi^{*}(\mathrm{ppz}){ }^{1}$ MLCT transitions. The ${ }^{1}$ MLCT energy is red-shifted relative to the analogous mono- and dinuclear complexes due to the increased stabilisation of the $\pi^{*}(\mathrm{ppz})$ orbitals in the trinuclear complex. The higher energy transitions in the region 20000-30000 $\mathrm{cm}^{-1}$ involve overlapping $\mathrm{d} \pi\left(\mathrm{Ru}^{\mathrm{II}}\right) \rightarrow \pi^{*}(\mathrm{ppz})$ and $\mathrm{d} \pi\left(\mathrm{Ru}^{\mathrm{II}}\right) \rightarrow \pi^{*}(\mathrm{bpy}){ }^{1}$ MLCT transitions.

Spectroelectrochemical oxidation of the trinuclear complex allowed the generation of the mixedvalence +7 and +8 forms, and fully-oxidised +9 forms at $-15^{\circ} \mathrm{C}$ (Figure 4.22 ). Stable isosbestic points were observed in the spectral progressions accompanying each stage of oxidation. On one-electron oxidation, the lowest energy component of the ${ }^{1}$ MLCT manifold at $17560 \mathrm{~cm}^{-1}$ decreases in intensity, while the two higher energy components retain their intensities. The band at $23710 \mathrm{~cm}^{-1}$ experiences a slight blue-shift on one-electron oxidation, consistent with its $\mathrm{d} \pi\left(\mathrm{Ru}^{\mathrm{II}}\right) \rightarrow \pi^{*}(\mathrm{bpy})$ assignment. Further oxidation of the second terminal $\mathrm{Ru}^{\mathrm{II}}$ centre to $\mathrm{Ru}^{\mathrm{III}}$ generates the +8 mixed-valence state, and results in a decrease in the intensity of the lowest energy band at $17145 \mathrm{~cm}^{-1}$ in the ${ }^{1}$ MLCT manifold. A relatively narrow absorption band appears at $21860 \mathrm{~cm}^{-1}$ which is predominantly due to a $\mathrm{d} \pi\left(\mathrm{Ru}^{\mathrm{II}}\right) \rightarrow \pi^{*}(\mathrm{ppz})$ ${ }^{1}$ MLCT transition associated with the central (un-oxidised) $\mathrm{Ru}^{\mathrm{II}}$ centre. This band collapses on further oxidation to the +9 state. Oxidation of the formally $\mathrm{Ru}^{\mathrm{II}}$ to $\mathrm{Ru}^{\mathrm{III}}$ centres was accompanied by an increase in the intensity of the LMCT transitions in the region $10000-16000 \mathrm{~cm}^{-1}$. 
(a)

(b)

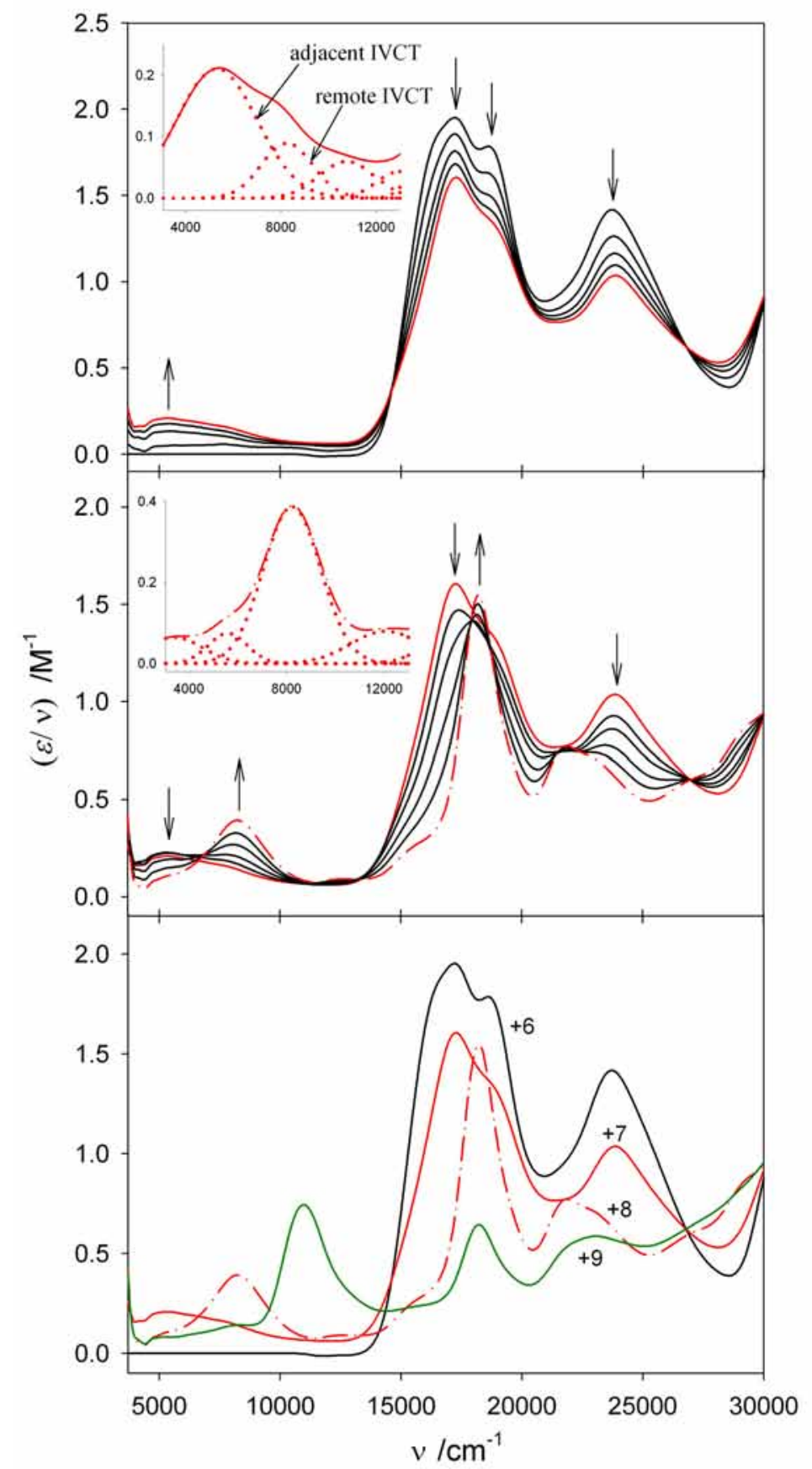

Figure 4.22 Spectroelectrochemistry for the oxidation of the trinuclear complex $\Delta \Delta^{t} \Delta-\left[\left\{\operatorname{Ru}(\mathrm{bpy})_{2}\right\}_{2}\{\mathrm{Ru}(\mathrm{bpy})(\mu-\right.$ $\left.\left.\mathrm{ppz})_{2}\right\}\right]^{n+}(n=6-9)$ at $-15^{\circ} \mathrm{C}$. (a) Spectroelectrochemical changes for the oxidation reaction $\Delta \Delta^{\mathrm{t}} \Delta$ -

$\left[\left\{\mathrm{Ru}(\mathrm{bpy})_{2}\right\}_{2}\left\{\mathrm{Ru}(\mathrm{bpy})(\mu-\mathrm{ppz})_{2}\right\}\right]^{6+} \rightarrow \Delta \Delta^{\mathrm{t}} \Delta-\left[\left\{\mathrm{Ru}(\mathrm{bpy})_{2}\right\}_{2}\left\{\mathrm{Ru}(\mathrm{bpy})(\mu-\mathrm{ppz})_{2}\right\}\right]^{7+}$. The inset shows IVCT band (一) of the latter in addition to the bands obtained from Gaussian deconvolution (‥). (b) Spectroelectrochemical changes for the oxidation reaction $\Delta \Delta^{\mathrm{t}} \Delta-\left[\left\{\mathrm{Ru}(\mathrm{bpy})_{2}\right\}_{2}\left\{\mathrm{Ru}(\mathrm{bpy})(\mu-\mathrm{ppz})_{2}\right\}\right]^{7+} \rightarrow \Delta \Delta^{\mathrm{t}} \Delta-\left[\left\{\mathrm{Ru}(\mathrm{bpy})_{2}\right\}_{2}\{\mathrm{Ru}(\mathrm{bpy})(\mu-\right.$ $\left.\left.\mathrm{ppz})_{2}\right\}\right]^{8+}$. The inset shows the NIR spectrum $\left(-^{\cdot-}\right)$ of the latter in addition to the bands obtained from Gaussian deconvolution (…). The asterisks indicate the IVCT bands. (c) Summary of the spectra of $\Delta \Delta^{\mathrm{t}} \Delta$ -

$\left[\left\{\mathrm{Ru}(\mathrm{bpy})_{2}\right\}_{2}\left\{\mathrm{Ru}(\mathrm{bpy})(\mu-\mathrm{ppz})_{2}\right\}\right]^{n+}$. 
Table 4.10 UV/Vis/NIR spectral data for the di- and trinuclear complexes at -35 and $-15^{\circ} \mathrm{C}$, respectively, and electrochemical $^{\mathrm{a}}$ data (in $\mathrm{mV}$ ), and $\mathrm{K}_{\mathrm{c}}$ values $^{\mathrm{c}}$ for the di- and trinuclear complexes. The NIR spectral data are indicated in bold type.

\begin{tabular}{|c|c|c|c|c|c|c|c|}
\hline Complex & $n$ & $\begin{array}{c}v \pm 10 / \mathrm{cm}^{-1} \\
\left\{(\varepsilon / v)_{\max } \pm 0.0001\right. \\
\left./ \mathrm{M}^{-1}\right\}\end{array}$ & $E_{0 \times 1}$ & $E_{0 \times 2}$ & $E_{0 \times 3}$ & $\begin{array}{c}\Delta \mathrm{E}_{\mathrm{ox}(2-1)} \\
\left(\mathrm{K}_{\mathrm{c}}\right)^{\mathrm{b}}\end{array}$ & $\begin{array}{l}\Delta \mathrm{E}_{\mathrm{ox}(3-2)} \\
\left(\mathrm{K}_{\mathrm{c}}\right)^{\mathrm{b}}\end{array}$ \\
\hline trans-[Ru(bpy)(ppz) $\left.)_{2}\right]^{n+}$ & 2 & $\begin{array}{l}21570(0.5958) \\
53450(0.5590)\end{array}$ & 1154 & & & & \\
\hline \multirow[t]{3}{*}{$\begin{array}{l}\text { meso- } \\
{\left[\left\{\operatorname{Ru}(\mathrm{bpy})_{2}\right\}_{2}(\mu-\mathrm{ppz})\right]^{n+c}}\end{array}$} & 4 & $\begin{array}{l}17640(1.4682) \\
23440(0.9021)\end{array}$ & \multirow[t]{3}{*}{1056} & \multirow[t]{3}{*}{1280} & & \multirow{3}{*}{$\begin{array}{c}224 \\
(6.26 \times \\
\left.10^{3}\right)\end{array}$} & \\
\hline & 5 & $\begin{array}{l}5370(\mathbf{0 . 8 7 2 1}) \\
11670(0.1566) \\
13320(0.1400) \\
17370(0.9003) \\
24900(0.3950) \\
\end{array}$ & & & & & \\
\hline & 6 & $\begin{array}{l}11630(1.9560) \\
16950(0.2601)\end{array}$ & & & & & \\
\hline \multirow{3}{*}{$\begin{array}{l}\text { rac- } \\
{\left[\left\{\mathrm{Ru}(\mathrm{bpy})_{2}\right\}_{2}(\mu-\mathrm{ppz})\right]^{n+c}}\end{array}$} & 4 & $\begin{array}{l}17630(1.4715) \\
23420(0.8987)\end{array}$ & \multirow[t]{3}{*}{1062} & \multirow[t]{3}{*}{1278} & & \multirow{3}{*}{$\begin{array}{c}216 \\
(4.58 \times \\
\left.10^{3}\right)\end{array}$} & \\
\hline & 5 & $\begin{array}{l}5390(\mathbf{0 . 8 4 9 4}) \\
11720(0.1488) \\
13090(0.2145) \\
17340(0.9043) \\
24980(0.3862) \\
\end{array}$ & & & & & \\
\hline & 6 & $\begin{array}{l}11640(1.9510) \\
16930(0.2600)\end{array}$ & & & & & \\
\hline \multirow[t]{4}{*}{$\begin{array}{l}\Delta \Delta^{\mathrm{t} \Delta-} \\
\quad\left[\left\{\mathrm{Ru}(\mathrm{bpy})_{2}\right\}_{2}\right. \\
\left.\quad\left\{\mathrm{Ru}(\mathrm{bpy})(\mu-\mathrm{ppz})_{2}\right\}\right]^{n+}\end{array}$} & 6 & $\begin{array}{l}17210(1.9525) \\
18600(1.7859) \\
23710(1.4173)\end{array}$ & \multirow[t]{4}{*}{$\begin{array}{c}\sim 1137 \\
\left(2 e^{-}\right)\end{array}$} & & \multirow[t]{4}{*}{1388} & \multirow[t]{4}{*}{$\begin{array}{l}<100 \\
(<50)\end{array}$} & \multirow[t]{4}{*}{$\begin{array}{c}250 \\
(1.73 \times \\
\left.10^{4}\right)\end{array}$} \\
\hline & 7 & $\begin{array}{l}5340(\mathbf{0 . 2 0 7 8}) \\
17270(1.6056) \\
\text { sh } 18750(1.3558) \\
23850(1.0362)\end{array}$ & & & & & \\
\hline & 8 & $\begin{array}{l}8230(\mathbf{0 . 3 9 2 0}) \\
18220(1.5478) \\
21860(0.7723) \\
\end{array}$ & & & & & \\
\hline & 9 & $\begin{array}{l}10970(0.7441) \\
18210(0.6440) \\
23050(0.5867)\end{array}$ & & & & & \\
\hline
\end{tabular}

All potentials $( \pm 3 \mathrm{mV})$ in $0.1 \mathrm{M}\left[\left(n-\mathrm{C}_{4} \mathrm{H}_{9}\right)_{4} \mathrm{~N}\right] \mathrm{PF}_{6} / \mathrm{CH}_{3} \mathrm{CN}$ at $+25^{\circ} \mathrm{C} v \mathrm{~s} . \mathrm{Fc}^{+} / \mathrm{Fc}^{0}$.

${ }^{\mathrm{b}} \mathrm{K}_{\mathrm{c}}$ values determined from equation 1.18(a). ${ }^{132}$

${ }^{c}$ Electrochemical properties reported in Table 3.3, §3.3.2.2. 


\subsubsection{Intervalence Charge Transfer}

The NIR spectra of the di- and trinuclear systems are shown in Figures 4.21 and 4.22, respectively. The results for the IVCT band parameters derived from the deconvolution procedure are summarised in Table 4.11.

Table 4.11 Spectral data for the di- and trinuclear complexes at -35 and $-15^{\circ} \mathrm{C}$, respectively. For the dinuclear species, the parameters for the overall NIR band envelopes are shown in bold type and details of the deconvoluted bands are in normal type.

\begin{tabular}{|c|c|c|c|c|c|}
\hline Complex & Component & $\begin{array}{l}v_{\max } \\
\pm 10 \\
/ \mathrm{cm}^{-1}\end{array}$ & $\begin{array}{c}(\varepsilon / \nu)_{\max } \\
\pm 0.0001 \\
/ \mathrm{M}^{-1}\end{array}$ & $\begin{array}{l}\Delta v_{1 / 2} \\
\pm 10 \\
/ \mathrm{cm}^{-1}\end{array}$ & $\begin{array}{l}\Delta v_{1 / 2}{ }^{0} \\
/ \mathrm{cm}^{-1}\end{array}$ \\
\hline meso-[\{Ru(bpy $\left.\left.)_{2}\right\}_{2}(\mu-p p z)\right]^{5+b}$ & $\begin{array}{l}1 \\
2 \\
3\end{array}$ & $\begin{array}{c}5370 \\
3960^{\text {a }} \\
5220 \\
5715 \\
6825\end{array}$ & $\begin{array}{l}\mathbf{0 . 8 7 2 1} \\
0.1037 \\
0.3981 \\
0.4893 \\
0.1885\end{array}$ & $\begin{array}{c}\mathbf{1 5 6 0} \\
920 \\
775 \\
1410 \\
3055\end{array}$ & $\begin{array}{l}3140 \\
2695 \\
3095 \\
3240 \\
3540\end{array}$ \\
\hline $\operatorname{rac}-\left[\left\{\mathrm{Ru}(\mathrm{bpy})_{2}\right\}_{2}(\mu-\mathrm{ppz})\right]^{5+\mathrm{b}}$ & $\begin{array}{l}1 \\
2 \\
3\end{array}$ & $\begin{array}{c}5390 \\
3940^{a} \\
5250 \\
5730 \\
7330\end{array}$ & $\begin{array}{l}\mathbf{0 . 8 4 9 4} \\
0.1487 \\
0.3683 \\
0.5539 \\
0.1677\end{array}$ & $\begin{array}{c}\mathbf{1 5 6 0} \\
1110 \\
960 \\
1490 \\
2560\end{array}$ & $\begin{array}{l}3150 \\
2690 \\
3100 \\
3240 \\
3670\end{array}$ \\
\hline$\Delta \Delta^{\mathrm{t}} \Delta-\left[\left\{\mathrm{Ru}(\mathrm{bpy})_{2}\right\}_{2}\left\{\mathrm{Ru}(\mathrm{bpy})(\mu-\mathrm{ppz})_{2}\right\}\right]^{+6}$ & & $\begin{array}{l}17560 \\
16150 \\
19930 \\
21230 \\
23370\end{array}$ & $\begin{array}{c}1.293 \\
1.617 \\
1.497 \\
0.5235 \\
0.6790\end{array}$ & $\begin{array}{l}1510 \\
2070 \\
1460 \\
1470 \\
2090\end{array}$ & \\
\hline$\Delta \Delta^{\mathrm{t}} \Delta-\left[\left\{\mathrm{Ru}(\mathrm{bpy})_{2}\right\}_{2}\left\{\mathrm{Ru}(\mathrm{bpy})(\mu-\mathrm{ppz})_{2}\right\}\right]^{7+\mathrm{c}}$ & & $\begin{array}{l}5310^{*} \\
8255^{*} \\
10755 \\
12970 \\
15755\end{array}$ & $\begin{array}{c}0.2099 \\
0.08924 \\
0.05986 \\
0.04347 \\
0.6550\end{array}$ & $\begin{array}{l}3980 \\
2530 \\
2730 \\
2215 \\
2415\end{array}$ & $\begin{array}{l}3250 \\
4050\end{array}$ \\
\hline$\Delta \Delta^{\mathrm{t}} \Delta-\left[\left\{\mathrm{Ru}(\mathrm{bpy})_{2}\right\}_{2}\left\{\mathrm{Ru}(\mathrm{bpy})(\mu-\mathrm{ppz})_{2}\right\}\right]^{8+\mathrm{c}}$ & & $\begin{array}{c}3410 \\
5590 \\
8230^{*} \\
12080 \\
16660\end{array}$ & $\begin{array}{c}0.06492 \\
0.07472 \\
0.3864 \\
0.07984 \\
0.3318\end{array}$ & $\begin{array}{l}2680 \\
1860 \\
2765 \\
3390 \\
3650\end{array}$ & 4050 \\
\hline$\Delta \Delta^{\mathrm{t}} \Delta-\left[\left\{\mathrm{Ru}(\mathrm{bpy})_{2}\right\}_{2}\left\{\mathrm{Ru}(\mathrm{bpy})(\mu-\mathrm{ppz})_{2}\right\}\right]^{9+}$ & & $\begin{array}{c}5090 \\
8195 \\
10940 \\
12935 \\
15690\end{array}$ & $\begin{array}{c}0.07753 \\
0.1218 \\
0.6995 \\
0.2147 \\
0.2314\end{array}$ & $\begin{array}{l}3865 \\
2710 \\
2010 \\
2410 \\
3075\end{array}$ & \\
\hline
\end{tabular}

artefact peak at detector limit.

b The parameters for the dinuclear complexes were previously reported in §3.3.2.4.

c Transitions of IVCT origin are indicated by asterisks.

\section{Dinuclear Systems}

The first oxidation process for the diastereoisomers of the dinuclear complex $\left[\left\{\mathrm{Ru}(\mathrm{bpy})_{2}\right\}_{2}(\mu-\right.$ $\mathrm{ppz})]^{4+}$ was characterised by the appearance of an intense new band in the region $3500-8000 \mathrm{~cm}^{-1}\left\{v_{\max }=\right.$ 5370 (meso) and $5390 \mathrm{~cm}^{-1}$ (rac) , which collapsed completely on removal of the second electron: on this 
basis, the band was assigned as an IVCT transition (Table 4.11, Figure 4.21). The bands appear asymmetrical and narrower on the lower energy side with bandwidths at half-height of $1560 \mathrm{~cm}^{-1}$ for both diastereoisomers. The IVCT transitions are qualitatively similar to those observed for $\left[\left\{\mathrm{Ru}(\mathrm{bpy})_{2}\right\}_{2}(\mu-\right.$ HAT) $]^{5+}$. Quantitative analysis of the bands according to the classical theory detailed in §4.3.1.3 suggests that the $\left[\left\{\mathrm{Ru}(\mathrm{bpy})_{2}\right\}_{2}(\mu-\mathrm{ppz})\right]^{5+}$ diastereoisomers may be similarly described as borderline localiseddelocalised systems on the following basis: (i) the experimental bandwidths are significantly narrower than the predicted bandwidths $\left(\Delta v_{1 / 2}{ }^{\circ}\right.$ in equation $\left.1.8^{139}\right)$ of 3140 and $3150 \mathrm{~cm}^{-1}$ for the meso and rac forms, respectively; and (ii) $\Gamma=0.503$ (meso) and 0.504 (rac). ${ }^{140}$ From equation 1.9, the lower limits for $\mathrm{H}_{\mathrm{ab}}$ are $610 \mathrm{~cm}^{-1}$ for both diastereoisomers, where $r_{\mathrm{ab}}$ is approximated as the geometrical metal-metal separation of $6.65 \AA$. Since electronic coupling decreases the effective electron transfer distance, $\mathrm{H}_{\mathrm{ab}}$ is likely to lie closer to the value in the fully-delocalised limit (i.e. $\mathrm{H}_{\mathrm{ab}}=1 / 2 v_{\max }$ from equation $1.11^{139}$ ) of 2625 and $2620 \mathrm{~cm}^{-1}$ for the meso and rac forms, respectively. On the basis of the electronic coupling values, the diastereoisomers exhibit a slightly greater degree of delocalisation compared with the corresponding diastereoisomer of $\left[\left\{\mathrm{Ru}(\mathrm{bpy})_{2}\right\}_{2}(\mu-\mathrm{HAT})\right]^{5+}$. Spectral deconvolution of the NIR bands reveals three underlying Gaussian-shaped components \{Figure 4.21(b)\} which may be assigned as the three underlying spin-orbit coupling components observed previously for the IVCT bands in $\left[\left\{\mathrm{Ru}(\mathrm{bpy})_{2}\right\}_{2}(\mu-\mathrm{HAT})\right]^{5+}$. Alternatively, according to a delocalised description in which the bridging ligand is included as a third electronic state, the IVCT transition arises from electron transfer between the bonding and non-bonding molecular orbitals within the molecular orbital manifold of the system. ${ }^{155-161}$

\section{Trinuclear System}

The two mixed-valence states of the trinuclear system were generated upon one- and two-electron oxidation of the +6 species at $-15^{\circ} \mathrm{C}$. The NIR spectra of the +7 and +8 mixed-valence forms (Figure 4.22) exhibit striking differences from their dinuclear analogues.

The generation of the +7 species was accompanied by the appearance of a broad absorption band in the range 3500-12000 $\mathrm{cm}^{-1}$ (Table 4.11, Figure 4.22). Spectral deconvolution revealed the presence of three underlying transitions at 5310, 8255 and $10755 \mathrm{~cm}^{-1}$, separated by 2945 and $2500 \mathrm{~cm}^{-1}$, respectively. The lowest energy component dominates the manifold and is assigned as an adjacent IVCT transition between the central $\mathrm{Ru}^{\mathrm{II}}$ and terminal $\mathrm{Ru}^{\mathrm{III}}$ centres. This transition occurs in the same region as the IVCT band for $\left[\left\{\mathrm{Ru}(\mathrm{bpy})_{2}\right\}(\mu-\mathrm{ppz})\right]^{5+}$. The second component is assigned as a remote IVCT transition between the two terminal Ru centres, since the $2945 \mathrm{~cm}^{-1}$ separation between IVCT(1) and (2) is inconsistent with their assignment as spin-orbit components (as was the case for $\left.\left[\left\{\mathrm{Ru}(\mathrm{bpy})_{2}\right\}_{3}(\mu-\mathrm{HAT})\right]^{7+}\right)$. The $2500 \mathrm{~cm}^{-1}$ separation between the second and third components suggests that the latter is an LMCT rather than an IVCT band; it occurs at approximately the same position as the LMCT bands at $10940 \mathrm{~cm}^{-1}$ in the +9 state, and at 11670 and $13320 \mathrm{~cm}^{-1}$ in meso-[\{Ru(bpy $\left.\left.)_{2}\right\}_{2}(\mu-\mathrm{ppz})\right]^{5+}$.

A classical analysis of the IVCT bands yields the following parameters: IVCT(1): $\Delta v_{1 / 2}{ }^{0}=3250$ $\mathrm{cm}^{-1}, \Gamma=-0.226$ and $\mathrm{H}_{\mathrm{ab}}=480 \mathrm{~cm}^{-1}$ assuming $r_{\mathrm{ab}}=6.65 \AA$; IVCT(2), $\Delta \mathrm{v}_{1 / 2}{ }^{\mathrm{o}}=4050 \mathrm{~cm}^{-1}, \Gamma=0.375$ and 
$\mathrm{H}_{\mathrm{ab}}=60 \mathrm{~cm}^{-1}$ assuming $r_{\mathrm{ab}}=13.3 \AA$ (i.e. twice the distance for the adjacent transition). The experimental bandwidths $\left(\Delta v_{1 / 2}\right)$ are comparable to those predicted from theory, and the magnitudes of $\Gamma$ and $\mathrm{H}_{\mathrm{ab}}$ indicate that both transitions are localised. For IVCT(1), $\Delta v_{1 / 2}>\Delta v_{1 / 2}{ }^{\circ}$, which suggests the presence of underlying spin-orbit components beneath the Gaussian-shaped IVCT manifold. As shown in Figure 4.23(a), the results are in marked contrast to the borderline localised-delocalised IVCT in the dinuclear analogues, and the $\left[\left\{\mathrm{Ru}(\mathrm{bpy})_{2}\right\}_{3}(\mu-\mathrm{HAT})\right]^{7+}$ mixed-valence species \{Figure 4.23(b) $\}$. From the electrochemical studies, the presence of the third Ru chromophore in the ppz-bridged trinuclear complex reduces the electron density at the central $\mathrm{Ru}$, giving rise to decreased coupling of the bridging ligands through the central metal compared with the mononuclear complex. This decreased coupling is also manifested in the IVCT properties by a significantly reduced coupling between the adjacent metal centres in the trinuclear species relative to its dinuclear analogues.

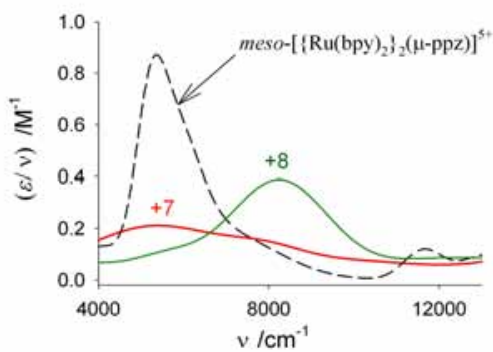

(a)

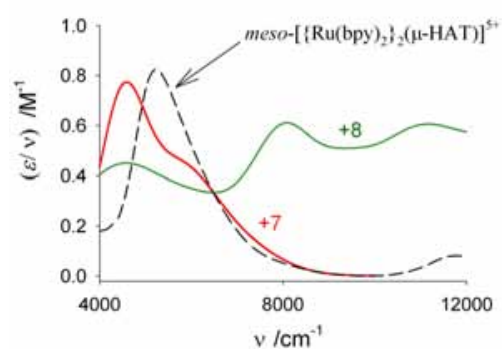

(b)

Figure 4.23 Comparison of the IVCT transitions for the di- and tri-nuclear mixed-valence systems: (a) $\Delta \Delta^{t} \Delta$ $\left[\left\{\mathrm{Ru}(\mathrm{bpy})_{2}\right\}_{2}\left\{\mathrm{Ru}(\mathrm{bpy})(\mu-\mathrm{ppz})_{2}\right\}\right]^{n+}(n=7,8)$ and meso- $\left[\left\{\mathrm{Ru}(\mathrm{bpy})_{2}\right\}_{2}(\mu-\mathrm{ppz})\right]^{5+}$; (b) heterochiral- $\left[\left\{\mathrm{Ru}(\mathrm{bpy})_{2}\right\}_{3}(\mu-\right.$ $\operatorname{HAT})]^{n+}(n=7,8)$ and meso- $\left[\left\{\mathrm{Ru}(\mathrm{bpy})_{2}\right\}_{2}(\mu-\mathrm{HAT})\right]^{5+}$.

The intensity of the IVCT bands decreased on subsequent oxidation of the second terminal $\mathrm{Ru}$ centre in the +8 mixed-valence species, with the appearance of a new band at $8230 \mathrm{~cm}^{-1}$ \{Figures 4.22 and 4.23(a), Table 4.11\} which is assigned to two degenerate adjacent IVCT transitions from the central $\mathrm{Ru}^{\mathrm{II}}$ to terminal $\mathrm{Ru}^{\mathrm{III}}$ centres. A classical analysis yields $\mathrm{H}_{\mathrm{ab}}=860 \mathrm{~cm}^{-1}$ (equation 1.9) assuming $r_{\mathrm{ab}}$ is $6.65 \AA$. The transitions are localised and the corresponding IVCT band occurs at higher energy than adjacent IVCT in the +7 mixed-valence form due to the decreased electronic delocalisation in the system, and hence the destabilisation of the acceptor $\mathrm{Ru}^{\mathrm{III}}$ orbitals. This blue-shift in the IVCT band with increasing oxidation state is consistent with previous literature reports for IVCT transitions in related di- and trinuclear systems. ${ }^{162}$ The assignment of the bands at energies lower than $7000 \mathrm{~cm}^{-1}$ is ambiguous due to the presence of comproportionation equilibria; however these may be reasonably ascribed to the residual absorptions of the mono-oxidised +7 species.

The band at $12080 \mathrm{~cm}^{-1}$ may correspond to an IVCT transition to the singlet or triplet "exciton" state, as discussed previously for $\left[\left\{\mathrm{Ru}(\mathrm{bpy})_{2}\right\}_{3}(\mu-\mathrm{HAT})\right]^{8+}$. However its assignment as an LMCT transition is more probable as LMCT bands of similar energy are present in the +9 species, and in the dinuclear analogues. Since the two holes are situated on remote localised $\mathrm{Ru}^{\mathrm{III}}$ centres, the coupling of the spins (parallel or antiparallel) should be significantly weaker given the $\sim 13.3 \AA$ separation of the 
metals, compared with the "cluster" arrangement in $\left[\left\{\mathrm{Ru}(\mathrm{bpy})_{2}\right\}_{3}(\mu-\mathrm{HAT})\right]^{8+}$. Nevertheless the possibility of an IVCT transition to the second "exciton" state cannot be discounted, and the relatively weak transition may be obscured by the low-lying LMCT and MLCT transitions.

\subsubsection{Conclusions}

The IVCT properties of the mixed-valence forms of $\Delta \Delta^{\mathrm{t}} \Delta-\left[\left\{\mathrm{Ru}(\mathrm{bpy})_{2}\right\}_{2}\left\{\mathrm{Ru}(\mathrm{bpy})(\mu-\mathrm{ppz})_{2}\right\}\right]^{n+}(n=$ $7,8)$ and the diastereoisomers of $\left[\left\{\mathrm{Ru}(\mathrm{bpy})_{2}\right\}_{2}(\mu-\mathrm{ppz})\right]^{5+}$ display a marked dependence on the nuclearity and extent of oxidation of the assemblies. The dinuclear species are classified as borderline localiseddelocalised systems while the two mixed-valence states of the trinuclear complex exhibit localised behaviour. One-electron oxidation of a terminal Ru centre in the trinuclear case gives rise to a broad, low intensity IVCT band for the +7 species which is composed of two underlying Gaussian-shaped bands. The transitions are identified as adjacent and remote IVCT transitions, with the former dominating the intensity of the IVCT manifold. The +8 species exhibits a single dominant IVCT band arising from the equivalent IVCT transitions from the central $\mathrm{Ru}^{\mathrm{II}}$ to peripheral $\mathrm{Ru}^{\mathrm{III}}$ centres. The origins of the IVCT transitions are illustrated schematically in Figure 4.24.

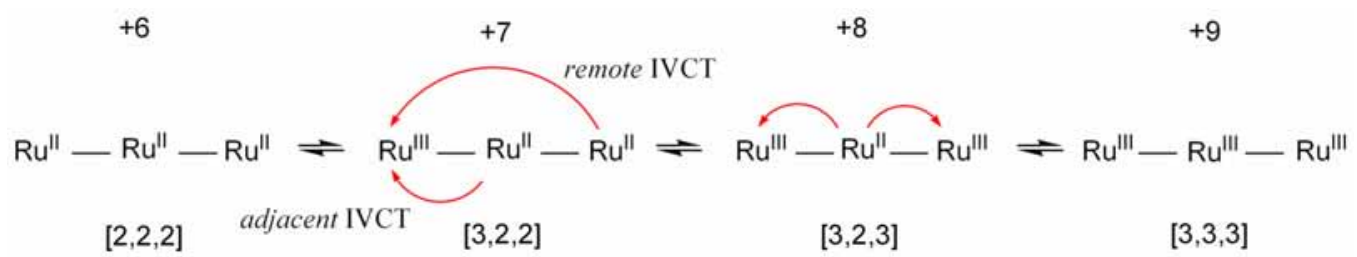

Figure 4.24 Schematic illustration of the proposed origins of the IVCT transitions (denoted by red arrows) in the +7 and +8 mixed-valence forms of $\Delta \Delta^{\mathrm{t}} \Delta-\left[\left\{\mathrm{Ru}(\mathrm{bpy})_{2}\right\}_{2}\left\{\mathrm{Ru}(\mathrm{bpy})(\mu-\mathrm{ppz})_{2}\right\}\right]^{n+}(n=6-9)$.

Despite the similarity in the IVCT properties of the dinuclear complexes $\left[\left\{\mathrm{Ru}(\mathrm{bpy})_{2}\right\}_{2}(\mu-\mathrm{ppz})\right]^{4+}$ and $\left[\left\{\mathrm{Ru}(\mathrm{bpy})_{2}\right\}_{2}(\mu-\mathrm{HAT})\right]^{5+}$, the IVCT characteristics of the mixed-valence forms of $\Delta \Delta^{\mathrm{t}} \Delta$ $\left[\left\{\operatorname{Ru}(\mathrm{bpy})_{2}\right\}_{2}\left\{\mathrm{Ru}(\mathrm{bpy})(\mu-\mathrm{ppz})_{2}\right\}\right]^{6+}$ are markedly different from those of the corresponding forms of $\left[\left\{\mathrm{Ru}(\mathrm{bpy})_{2}\right\}_{3}(\mu-\mathrm{HAT})\right]^{6+}$. As shown in Figures 4.3(c) and (d), the three Ru centres in the latter are equivalently disposed, and share in the available electron density. By comparison, a "chain-like" arrangement of the three metal centres in $\Delta \Delta^{t} \Delta-\left[\left\{\operatorname{Ru}(\mathrm{bpy})_{2}\right\}_{2}\left\{\mathrm{Ru}(\mathrm{bpy})(\mu-\mathrm{ppz})_{2}\right\}\right]^{6+}$ gives rise to a decreased coupling through the central metal. As a result, the $\left[\left\{\mathrm{Ru}(\mathrm{bpy})_{2}\right\}_{3}(\mu-\mathrm{HAT})\right]^{6+}$ complex exhibits a comparable degree of electronic coupling to its dinuclear analogue, while the degree of electronic coupling in $\Delta \Delta^{\mathrm{t}} \Delta$-[\{Ru(bpy $\left.\left.)_{2}\right\}_{2}\left\{\mathrm{Ru}(\mathrm{bpy})(\mu-\mathrm{ppz})_{2}\right\}\right]^{6+}$ is reduced relative to its dinuclear counterpart. Qualitatively, a localised description based on the geometrical properties of the $\mathrm{d} \pi\left(\mathrm{Ru}^{\mathrm{II} / I I I}\right)$ orbitals provides a reasonable rationale for the IVCT behaviour in the localised trinuclear system. 


\subsection{Conclusions and Future Prospects}

The present study has provided the first systematic investigation of IVCT in stereochemicallyunambiguous di- and trinuclear assemblies based on the HAT and ppz bridging ligands. The IVCT characteristics of the mixed-valence forms of the homo-dinuclear complexes $\left[\left\{\mathrm{Ru}(\mathrm{bpy})_{2}\right\}_{2}(\mu-\mathrm{HAT})\right]^{5+}$ and $\left[\left\{\mathrm{Ru}(\mathrm{bpy})_{2}\right\}_{2}(\mu-\mathrm{ppz})\right]^{5+}$ exhibit similar properties, which are consistent with their classification as borderline localised-delocalised mixed-valence species. The IVCT characteristics of the mixed-valence trinuclear complexes $\left[\left\{\mathrm{Ru}(\mathrm{bpy})_{2}\right\}_{3}(\mu-\mathrm{HAT})\right]^{n+}$ and $\left[\left\{\mathrm{Ru}(\mathrm{bpy})_{2}\right\}_{2}\left\{\mathrm{Ru}(\mathrm{bpy})(\mu-\mathrm{ppz})_{2}\right\}\right]^{n+}(n=7,8)$ are markedly different from those of their dinuclear analogues, and vary significantly depending on the extent of oxidation, and the overall geometry of the assembly. Small differences are also observed between the diastereoisomeric forms of the dinuclear complexes $\{$ meso $(\Delta \Lambda)$ and $\operatorname{rac}(\Delta \Delta / \Lambda \Lambda)\}$, and between the homochiral $\left(\Delta_{3} / \Lambda_{3}\right)$ and heterochiral $\left(\Delta_{2} \Lambda / \Lambda_{2} \Delta\right)$ diastereoisomers of $\left[\left\{\mathrm{Ru}(\mathrm{bpy})_{2}\right\}_{3}(\mu-\mathrm{HAT})\right]^{n+}$. The mixed-valence forms of $\Delta_{2} \Lambda^{\prime} / \Lambda_{2} \Delta^{\prime}-\left[\left\{\mathrm{Ru}(\mathrm{bpy})_{2}\right\}_{2}\left\{\mathrm{Os}(\mathrm{bpy})_{2}\right\}(\mu-\mathrm{HAT})\right]^{n+}(n=7,8)$ exhibit IVCT properties that are intermediate between those of the diastereoisomeric forms of the localised mixed-valence complex $\left[\left\{\mathrm{Ru}(\mathrm{bpy})_{2}\right\}(\mu \text {-HAT })\left\{\mathrm{Os}(\mathrm{bpy})_{2}\right\}\right]^{5+}$, and the localised-to-delocalised complex $\left[\left\{\mathrm{Ru}(\mathrm{bpy})_{2}\right\}_{3}(\mu-\right.$ $\left.\operatorname{HAT}^{n+}\right]^{n}(n=7,8)$.

The significant differences in IVCT properties between the di- and trinuclear species based on HAT are attributed to the extensive electronic communication between the Ru(bpy $)_{2}{ }^{2+}$ chromophores, which gives rise to novel properties of the two mixed-valence states in the trinuclear case. The splitting of the IVCT transitions in the +7 species is attributed to the influence of spin-orbit coupling, while the two new dominant transitions in the NIR spectra of the +8 (diradical) species may arise from transitions to separate delocalised "exciton" states of singlet and triplet character.

Despite the similarity in the IVCT properties of the dinuclear complexes $\left[\left\{\mathrm{Ru}(\mathrm{bpy})_{2}\right\}_{2}(\mu-\mathrm{ppz})\right]^{4+}$, and $\left[\left\{\mathrm{Ru}(\mathrm{bpy})_{2}\right\}_{2}(\mu-\mathrm{HAT})\right]^{5+}$, the IVCT characteristics of the mixed-valence forms of $\Delta \Delta^{\mathrm{t}} \Delta$ $\left[\left\{\mathrm{Ru}(\mathrm{bpy})_{2}\right\}_{2}\left\{\mathrm{Ru}(\mathrm{bpy})(\mu-\mathrm{ppz})_{2}\right\}\right]^{6+}$ are markedly different from those of the corresponding mixed-valence forms of $\left[\left\{\mathrm{Ru}(\mathrm{bpy})_{2}\right\}_{3}(\mu-\mathrm{HAT})\right]^{6+}$. The three Ru centres in $\left[\left\{\mathrm{Ru}(\mathrm{bpy})_{2}\right\}_{3}(\mu-\mathrm{HAT})\right]^{6+}$ are equivalently disposed, and share in the available electron density. By comparison, a "chain-like” arrangement of the three metal centres in $\Delta \Delta^{\mathrm{t}} \Delta$-[\{Ru(bpy $\left.\left.)_{2}\right\}_{2}\left\{\mathrm{Ru}(\mathrm{bpy})(\mu-\mathrm{ppz})_{2}\right\}\right]^{6+}$ gives rise to a decreased coupling through the central metal. As a result, the $\left[\left\{\mathrm{Ru}(\mathrm{bpy})_{2}\right\}_{3}(\mu-\mathrm{HAT})\right]^{6+}$ complex exhibits a comparable degree of electronic coupling to its dinuclear analogue, while the degree of electronic coupling in $\Delta \Delta^{t} \Delta$ $\left[\left\{\mathrm{Ru}(\mathrm{bpy})_{2}\right\}_{2}\left\{\mathrm{Ru}(\mathrm{bpy})(\mu-\mathrm{ppz})_{2}\right\}\right]^{6+}$ is reduced relative to its dinuclear counterpart.

Differential anion and solvent interactions which are known to influence the IVCT characteristics of closely related complexes, and the stereoisomeric forms of the same complex (as demonstrated in Chapters 2 and 3) may account in part for the differences in the IVCT characteristics for the diastereoisomeric forms of the same species. However, the major trends and conclusions from the present study are robust despite the absence of more detailed measurements of the solvent and anion dependencies of the systems. 


\section{Theoretical Implications and Considerations}

Alternative localised and delocalised approaches have been discussed for the interpretation of the mixed-valence characteristics of the dinuclear systems $\left[\left\{\mathrm{Ru}(\mathrm{bpy})_{2}\right\}_{2}(\mu-\mathrm{BL})\right]^{5+}\{\mathrm{BL}=\mathrm{HAT}$ and ppz $\}$, however treatment of the full vibronic coupling problem is necessary in order to provide a quantitative analysis of these borderline localised-to-delocalised systems. Qualitatively, a localised description based on the geometrical properties of the $\mathrm{d} \pi\left(\mathrm{Ru}^{\mathrm{II} / \mathrm{III}}\right)$ orbitals provides a reasonable rationale for the IVCT behaviour in the homo-dinuclear and homo-trinuclear ruthenium systems. A localised description based on the geometrical properties of the $\mathrm{d} \pi\left(\mathrm{Ru}^{\mathrm{II} / \mathrm{III}}\right)$ and $\mathrm{d} \pi\left(\mathrm{Os}^{\mathrm{II} / I I I}\right)$ orbitals which accounts for the redox asymmetry contribution in the hetero-dinuclear and hetero-trinuclear complexes provides a reasonable rationale for the IC and IVCT behaviour. The assumption of a two-state model and classical analysis is adequate for the IVCT transitions in the predominantly localised chain-like $\Delta \Delta^{\mathrm{t}} \Delta$ $\left[\left\{\operatorname{Ru}(\mathrm{bpy})_{2}\right\}_{2}\left\{\mathrm{Ru}(\mathrm{bpy})(\mu-\mathrm{ppz})_{2}\right\}\right]^{n+}(n=7,8)$ mixed-valence complex. For the dinuclear $\left[\left\{\mathrm{Ru}(\mathrm{bpy})_{2}\right\}_{2}(\mu-\right.$ $\mathrm{ppz})]^{5+}$ and $\left[\left\{\mathrm{M}(\mathrm{bpy})_{2}\right\}_{2}(\mu-\mathrm{HAT})\right]^{5+}(\mathrm{M}=\mathrm{Ru}, \mathrm{Os})$ complexes, as well as the trinuclear $\left[\left\{\mathrm{Ru}(\mathrm{bpy})_{2}\right\}_{3}(\mu-\right.$ HAT $)]^{n+}(n=7,8)$ complexes where delocalisation is more extensive, a molecular orbital approach which includes vibronic coupling is required. The treatment of these issues represents a challenging computational problem which is outside the scope of the present study.

Classical models ${ }^{137,141}$ are applicable for the analysis of localised trinuclear systems. Qualitatively, the IVCT bands are reminiscent of those in the analogous dinuclear mixed-valence complexes, and the trends in the IVCT characteristics are readily interpreted by considering the degeneracy of the transition and the oxidation state of the assembly. As the extent of electronic delocalisation increases, the valence-localised scenario is no longer appropriate, and the IVCT characteristics of the trinuclear complexes exhibit intriguing new properties that are distinct from their dinuclear counterparts.

Figure 4.25 provides a generalised schematic representation of the two mixed-valence states which arise following two successive one-electron oxidation processes in a chain-like trinuclear complex in which the initial site of oxidation occurs at a peripheral metal centre, and in a cluster-type trinuclear complex.

(a)

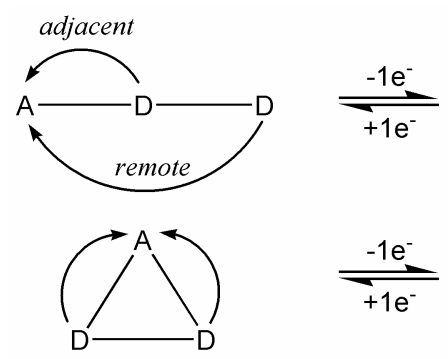

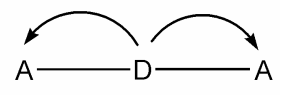

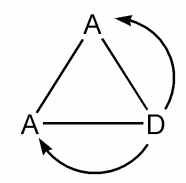

Figure 4.25 Schematic representation of the two mixed-valence states of trinuclear complexes with chain-like (a) and cluster-like (b) architectures. The arrows depict the IVCT transitions between the un-oxidised electron-donor (D) and oxidised electron-acceptor (A) centres. 
Oxidation of one of the terminal metal chromophores gives rise to separate IVCT transitions between the adjacent and remote metal centres \{Figure 4.25(a)\}. The remote IVCT transition exhibits a weak intensity due to the larger metal-metal separation between the peripheral centres. A through-bond superexchange-assisted electronic coupling mechanism may be invoked to provide a first-order semiquantitative explanation for the appearance of such remote interactions. Oxidation of the second terminal unit gives rise to equivalent IVCT transitions from the central metal to terminal metal centres.

An important difference between dinuclear and higher nuclearity systems is the possibility for additional second order spin-dependent electronic processes, due to the existence of itinerant "extra" electrons hopping over the exchange-coupled magnetic sites. For the two-electron oxidised (diradical) mixed valence system depicted in Figure 4.25(b), two IVCT transitions are anticipated due to magnetic exchange interactions between the two radical centres. The [3,3,2] species possesses two energetically distinct electronic states depending on the antiparallel or parallel alignment of the spins at the formally $\mathrm{Ru}^{\mathrm{III}}$ centres. IVCT studies alone are insufficient to determine whether the dominant coupling is ferromagnetic or antiferromagnetic. Unless the coupling is extraordinarily strong, both states will be occupied at room temperature leading to slightly different energies for the two IVCT processes. The effect will be to broaden the IVCT manifold for the $[3,3,2]$ versus the $[3,2,2]$ mixed-valence system. Unfortunately, the identification of this effect has been complicated in a number of cases due to IVCT broadening from other sources such as low symmetry, extensive orbital mixing, spin-orbit coupling of the metal $\mathrm{d} \pi$ orbitals $^{143}$ and overlap of the IVCT with low-lying LMCT and MLCT transitions. Clearly, reliable spectral deconvolution procedures are essential to discern the IVCT features of interest. Evidence for the presence of singlet and triplet IVCT transitions in mixed-valence systems has been reported for the diradical form of a localised trinuclear complex by Weyland, ${ }^{9}$ while a tentative assignment has also been made for the stereoisomers of the borderline localised-to-delocalised trinuclear complexes $\left[\left\{\mathrm{Ru}(\mathrm{bpy})_{2}\right\}_{3}(\mu-\mathrm{HAT})\right]^{8+}$ in the present study.

\section{Future Prospects}

The present study provides a platform for three important thrusts of future research:

- Synthetic strategies for the construction of stereochemically-unambiguous mono-, di- and trinuclear complexes are well established ${ }^{20,21}$ and provide for subtle and systematic stereochemical modifications in addition to extensive tuning of the electronic properties of the component metal centres as well as the bridging and terminal ligands.

- The construction of stereochemically-unambiguous trinuclear assemblies which differ only in the identity of the geometrical isomeric forms of the central metal-based chromophore such as cis- and trans-[Ru(bpy)(ppz $\left.)_{2}\right]^{2+}$ enables the construction of assemblies which exhibit an overall "angular" or "linear" disposition of the terminal metal centres. This provides a basis for the use of stereochemically-defined components to direct electron transfer in highernuclearity polymetallic assemblies. 
- The detailed analysis of IVCT in trinuclear complexes provides a foundation for the theoretical treatment and elucidation of multi-site interactions in tetra- and higher nuclearity complexes which have been proposed as the basis of molecular devices, ${ }^{30-32}$ and extended arrays and solid-state structures.

The existence of high quality experimental data that will test and guide developments in the conceptual theories for IVCT in higher nuclearity systems, and the extension of the IVCT probe to stereochemically-unambiguous trinuclear species, are extremely significant to our understanding of fundamental intramolecular electron transfer phenomena. This represents an important development in design strategies for higher nuclearity polymetallic assemblies in which the electron transfer processes may be controlled and ultimately exploited for novel practical applications. 


\subsection{References}

1. $\quad$ Powers, M. J.; Callahan, R. W.; Salmon, D. J.; Meyer, T. J. Inorg. Chem. 1976, 15, 894-900.

2. $\quad$ von Kameke, A.; Tom, G. M.; Taube, H. Inorg. Chem. 1978, 17, 1790-1796.

3. Serroni, S.; Campagna, S.; Denti, G.; Keyes, T. E.; Vos, J. G. Inorg. Chem. 1996, 35, 4513-4518.

Oshio, H.; Onodera, H.; Tamada, O.; Mizutani, H.; Hikichi, T.; Ito, T. Chem. Eur. J. 2000, 6, 2523-2530.

Oshio, H.; Onodera, H.; Ito, T. Chem. Eur. J. 2003, 9, 3946-3950.

Nishihara, H. Bull. Chem. Soc. Jpn. 2001, 74, 19-29. Chem. 1975, 14, 506-511.

8. Plenio, H.; Hermann, J.; Sehring, A. Chem. Eur. J. 2000, 6, 1820-1829.

9. Weyland, T.; Costuas, K.; Toupet, L.; Halet, J.-F.; Lapinte, C. Organometallics 2000, 19, 4228-4239.

10. Roffia, S.; Paradisi, C.; Bignozzi, C. A. J. Electroanal. Chem. 1986, 200, 105-118.

11. Bignozzi, C. A.; Roffia, S.; Scandola, F. J. Am. Chem. Soc. 1985, 107, 1644-1651.

12. Bignozzi, C. A.; Paradisi, C.; Roffia, S.; Scandola, F. Inorg. Chem. 1988, 27, 408-414.

13. Bignozzi, C. A.; Roffia, S.; Chiorboli, C.; Davila, J.; Indelli, M. T.; Scandola, F. Inorg. Chem. 1989, 28, 4350-4358.

14. Bignozzi, C. A.; Argazzi, R.; Schoonover, J. R.; Gordon, K. C.; Dyer, R. B.; Scandola, F. Inorg. Chem. 1992, 31, 5260-5267.

15. Scandola, F.; Argazzi, R.; Bignozzi, C. A.; Chiorboli, C.; Indelli, M. T.; Rampi, M. A. Coord. Chem. Rev. 1993, 125, 283-292.

16. Bignozzi, C. A.; Indelli, M. T.; Scandola, F. J. Am. Chem. Soc. 1989, 111, 5192-5198.

17. Bignozzi, C. A.; Argazzi, R.; Chiorboli, C.; Scandola, F.; Dyer, R. B.; Schoonover, J. R.; Meyer, T. J. Inorg. Chem. 1994, 33, 1652-1659.

18. Bignozzi, C. A.; Argazzi, R.; Chiorboli, C.; Roffia, S.; Scandola, F., Coord. Chem. Rev. 1991, 111, 261-266.

19. Richardson, G. N.; Brand, U.; Vahrenkamp, H. Inorg. Chem. 1999, 38, 3070-3079.

20. Keene, F. R. Coord. Chem. Rev. 1997, 166, 122-159.

21. Keene, F. R. Chem. Soc. Rev. 1998, 27, 185-193.

22. Balzani, V.; Credi, A.; Venturi, M. Chem. Eur. J. 2002, 8, 5525-5532.

23. Holliday, B. J.; Mirkin, C. A. Angew. Chem., Int. Ed. Engl. 2001, 40, 2022-2043.

24. Steed, J. W.; Atwood, J. L. Supramolecular Chemistry; Wiley: Chichester, 2000.

25. Provent, C.; Williams, A. F. In Transition Metals in Supramolecular Chemistry; Sauvage, J. P., Ed.; John Wiley and Sons Ltd.: Chichester, 1999; pp 135-191.

26. MacDonnell, F. M.; Kim, M.-J.; Bodige, S. Coord. Chem. Rev. 1999, 185-186, 535-549.

27. Zeng, F.; Zimmerman, S. C. Chem. Rev. 1997, 97, 1681-1712.

28. Constable, E. C. Chem. Commun. 1997, 1073-1080.

29. Constable, E. C. In Comprehensive Supramolecular Chemistry; Lehn, J.-M., Atwood, J. L., Davies, J. E. D., MacNicol, D. D., Vögtle, F., Sauvage, J.-P.; Hosseini, M. W., Eds.; Pergamon: Oxford, 1996; Vol. 9, pp 213-252.

30. Balzani, V.; Juris, A.; Venturi, M.; Campagna, S.; Serroni, S. Chem. Rev. 1996, 96, 759-833.

31. Belser, P.; Bernhard, S.; Blum, C.; Beyeler, A.; De Cola, L.; Balzani, V. Coord. Chem. Rev 1999, 190-192, 155-169.

32. Garcia, C. G.; de Lima, J. F.; Iha, N. Y. M. Coord. Chem. Rev. 2000, 196, 219-247.

33. Ward, M. D. Chem. Soc. Rev. 1997, 26, 365-375.

34. Barigelletti, F.; Flamigni, L. Chem. Soc. Rev. 2000, 29, 1-12.

35. $\quad$ Gust, D.; Moore, T. A.; Moore, A. L. Acc. Chem. Res. 2001, 34, 40-48.

36. Balzani, V.; Credi, A.; Raymo, F.; Stoddart, J. F. Angew. Chem., Int. Ed. Engl. 2000, 39, 3348-3391.

37. Dürr, H.; Bossmann, S. Acc. Chem. Res. 2001, 34, 905-917.

38. Hunziker, M.; Ludi, A. J. Am. Chem. Soc. 1977, 99, 7370-7371.

39. Ronco, S. E.; Thompson, D. W.; Gahan, S. L.; Petersen, J. D. Inorg. Chem. 1998, 37, 2020-2027.

40. $\quad$ Swavey, S.; Brewer, K. J. Inorg. Chem. 2002, 41, 6196-6198.

41. Campagna, S.; Serroni, S.; Puntoriero, F.; Loiseau, F.; de Cola, L.; Kleverlaan, C. J.; Becher, J.; Sørensen, A. P.; Hascoat, P.; Thorup, N. Chem. Eur. J. 2002, 8, 4461-4469.

42. Puntoriero, F.; Serroni, s.; Licciardello, A.; Venturi, M.; Juris, A.; Ricevuto, V.; Campagna, S. J. Chem. Soc., Dalton Trans. 2001, 1035-1042.

43. $\quad$ Richter, M. M.; Brewer, K. J. Inorg. Chem. 1993, 32, 5762-5768.

44. Molnar, S. M.; Nallas, G.; Bridgewater, J. S.; Brewer, K. J. J. Am. Chem. Soc. 1994, 116, 5206-5210.

45. Brauns, E.; Jones, S. W.; Clark, J. A.; Molnar, S. M.; Kawanishi, Y.; Brewer, K. J. Inorg. Chem. 1997, 36, 2861-2867.

46. Bridgewater, J. S.; Vogler, L. M.; Molnar, S. M.; Brewer, K. J. Inorg. Chim. Acta 1993, 208, 179-188. 
47. Barthram, A. M.; Cleary, R. L.; Kowallick, R.; Ward, M. D. Chem. Commun. 1998, 2695-2696.

48. $\quad$ Rutherford, T. J.; Keene, F. R. J. Chem. Soc., Dalton Trans. 1998, 1155-1162.

49. Bianchini, C.; Meli, A.; Peruzzini, M.; Vizza, F. J. Am. Chem. Soc. 1990, 112, 6726-6728.

50. Masschelein, A.; Kirsch-De Mesmaeker, A.; Verhoeven, C.; Nasielski-Hinkens, R. Inorg. Chim. Acta 1987, 129, L13-L16.

51. Warnmark, K.; Heyke, O.; Thomas, J. A.; Lehn, J. M. Chem. Commun. 1996, 2603-2604.

52. Jacquet, L.; Kirsch-De Mesmaeker, A. J. Chem. Soc., Faraday Trans. 1992, 88, 2471-2480.

53. Nallas, G. N. A.; Brewer, K. J. Inorg. Chim. Acta 1996, 253, 7-13.

54. Holder, A. A. Inorg. Chem. 2004, 43, 303-308.

55. Serroni, S.; campagna, S.; Puntoriero, F.; Di Pietro, C.; McClenaghan, N. D.; Loiseau, F. Chem. Soc. Rev. 2001, 30, 367-375.

56. Sommovigo, M.; Denti, G.; Serroni, S.; Campagna, S.; Mingazzini, C.; Mariotti, C.; Juris, A. Inorg. Chem. 2001, 40, 3318-3323.

57. Marcaccio, M.; Paolucci, F.; Paradisi, C.; Roffia, S.; Fontanesi, C.; Yellowlees, L. J.; Serroni, S.; Campagna, S.; Denti, G.; Balzani, V. J. Am. Chem. Soc. 1999, 121, 10081-10091.

58. Dixon, I. M.; Collin, J.-P.; Sauvage, J.-P.; Flamigni, L.; Encinas, S.; Barigelletti, F. Chem. Soc. Rev. 2000, 29, 385-391.

59. Balzani, V.; Juris, A. Coord. Chem. Rev. 2001, 211, 97-115.

60. Balzani, V.; Campagna, S.; Denti, G.; Juris, A.; Serroni, S.; Venturi, M. Acc. Chem. Res. 1998, $31,26-34$.

61. Serroni, S.; Juris, A.; Venturi, M.; Campagna, S.; Resino, I. R.; Denti, G.; Credi, A.; Balzani, V. J. Mater. Chem. 1997, 7, 1227-1236.

62. Juris, A.; Balzani, V.; Campagna, S.; Denti, G.; Serroni, S.; Frei, G.; Güdel, H. U. Inorg. Chem. 1994, 33, 1491-1496.

63. Juris, A.; Venturi, M.; Pontoni, L.; Resino, I. R.; Balzani, V.; Serroni, S.; Campagna, S.; Denti, G. Can. J. Chem. 1995, 73, 1875-1882.

64. Balzani, V.; Campagna, S.; Denti, G.; Juris, A.; Serroni, S.; Venturi, M. Coord. Chem. Rev. 1994, 132, $1-13$.

65. Serroni, S.; Denti, G. Inorg. Chem. 1992, 31, 4251-4255.

66. Serroni, S.; Denti, G.; Campagna, S.; Juris, A.; Ciano, M.; Balzani, V. Angew. Chem., Int. Ed. Engl. 1992, 31, 1493-1495.

67. Balzani, V. New Scientist 1994, 1951, 31-34.

68. Denti, G.; Campagna, S.; Sabatino, L.; Serroni, S.; Ciano, M.; Balzani, V. Inorg. Chem. 1990, 29, 4750-4758.

69. Serroni, S.; Juris, A.; Campagna, S.; Venturi, M.; Denti, G.; Balzani, V. J. Am. Chem. Soc. 1994, 116, 9086-9091.

70. Denti, G.; Campagna, S.; Sabatino, L.; Serroni, S.; Ciano, M.; Balzani, V. Inorg. Chim. Acta 1990, 176, 175-178.

71. Denti, G.; Campagna, S.; Serroni, S.; Ciano, M.; Balzani, V. J. Am. Chem. Soc. 1992, 114, $2944-2950$.

72. $\quad$ Campagna, S.; Denti, G.; Serroni, S.; Ciano, M.; Juris, A.; Balzani, V. Inorg. Chem. 1992, 31, $2982-2984$.

73. Denti, G.; Serroni, S.; Campagna, S.; Ricevuto, V.; Balzani, V. Coord. Chem. Rev. 1991, 111, $227-236$.

74. Campagna, S.; Denti, G.; Serroni, S.; Ciano, M. Inorg. Chem. 1991, 30, 3728-3732.

75. Grosshenny, V.; Harriman, A.; Hissler, M.; Ziessel, R. Platinum Met. Rev. 1996, 40, $26-35$.

76. $\quad$ Encinas, S.; Flamigni, L.; Barigelletti, F.; Constable, E. C.; Housecroft, C. E.; Schofield, E. R.; Figgemeier, E.; Fenske, D.; Neuburger, M.; Vos, J. G.; Zehnder, M. Chem. Eur. J. 2002, 8, 137-150.

77. Benniston, A. C.; Harriman, A.; Grosshenny, V.; Ziessel, R. New J. Chem. 1997, 21, 405-408.

78. Tzalis, D.; Tor, Y. Chem. Commun. 1996, 1043-1044.

79. $\quad$ Grosshenny, V.; Ziessel, R. J. Chem. Soc., Dalton Trans. 1993, 817-819.

80. Grosshenny, V.; Harriman, A.; Ziessel, R. Angew. Chem., Int. Ed. Engl. 1996, 34, 2705-2708.

81. Hasenknopf, B.; Hall, J.; Lehn, J. M.; Balzani, V.; Credi, A.; Campagna, S., New J. Chem. 1996, 20, 725-730.

82. Wacholtz, W. F.; Auerbach, R. A.; Schmehl, R. H. Inorg. Chem. 1987, 26, 2989-2994.

83. Schmehl, R. H.; Auerbach, R. A.; Wacholtz, W. F.; Elliott, C. M.; Freitag, R. A.; Merkert, J. W. Inorg. Chem. 1986, 25, 2440-2445.

84. Moucheron, C.; Kirsch-De Mesmaeker, A.; Dupont-Gervais, A.; Leize, E.; Van Dorsselaer, A. J. Am. Chem. Soc. 1996, 118, 12834-12835.

85. Haga, M.-A.; Ali, M. M.; Arakawa, R. Angew. Chem., Int. Ed. Engl. 1996, 35, 76-78.

86. Chao, H.; Qiu, Z.-R.; Cai, L.-R.; Zhang, H.; Li, X.-Y.; Wong, K.-S.; Ji, L.-N. Inorg. Chem. 2003, 42, 8823-8830.

87. Didier, P.; Jacquet, L.; Kirsch-De Mesmaeker, A.; Hueber, R.; van Dorsselaer, A. Inorg. Chem. 1992, 31, 4803-4809.

88. Balzani, V.; Bardwell, D. A.; Barigelletti, F.; Cleary, F. L.; Guardigli, M.; Jeffery, J. C.; Sovrani, T.; Ward, M. D. J. Chem. Soc., Dalton Trans 1995, 3601-3608. 
89. Denti, G.; Serroni, S.; Sindona, G.; Uccella, N. J. Am. Soc. Mass Spectrom. 1993, 4, 306-311.

90. Richardson, C.; Steel, P. J.; D'Alessandro, D. M.; Junk, P. C.; Keene, F. R. J. Chem. Soc., Dalton Trans. 2002, 2775-2785.

91. Z Zampese, J. A.; Keene, F. R.; Steel, P. J. Dalton Trans. 2004, 4124-4129.

92. Fletcher, N. C.; Junk, P. C.; Reitsma, D. A.; Keene, F. R. J. Chem. Soc., Dalton Trans. 1998, 133-138.

93. Hage, R.; Haasnoot, J. G.; Nieuwenhuis, H. A.; Reedijk, J.; De Ridder, D. J. A.; Vos, J. G. J. Am. Chem. Soc. 1990, 112, 9245-9251.

94. Baitalik, S.; Flörke, U.; Nag, K. J. Chem. Soc., Dalton Trans. 1999, 719-727.

95. Masui, H.; Freda, A. L.; Zerner, M. C.; Lever, A. B. P. Inorg. Chem. 2000, 39, 141-152.

96. Bardwell, D.; Jeffery, J. C.; Joulie, L.; Ward, M. D. J. Chem. Soc., Dalton Trans. 1993, 2255-2256.

97. Bardwell, D. A.; Horsburgh, L.; Jeffery, J. C.; Joulié, L. F.; Ward, M. D.; Webster, I.; Yellowlees, L. J. J. Chem. Soc., Dalton Trans. 1996, 2527-2531.

98. Polson, M. I. J.; Hanan, G. S.; Taylor, N. J.; Hasenknopf, B.; Thouvenot, R. Chem. Commun. 2004, 13141315.

99. Kelso, L. S.; Reitsma, D. A.; Keene, F. R. Inorg. Chem. 1996, 35, 5144-5153.

100. Rutherford, T. J.; Keene, F. R. Inorg. Chem. 1997, 36, 2872-2878.

101. Rutherford, T. J.; Van Gijte, O.; Kirsch-De Mesmaeker, A.; Keene, F. R. Inorg. Chem. 1997, 36, 4465-4474.

102. Treadway, J. A.; Chen, P.; Rutherford, T. J.; Keene, F. R.; Meyer, T. J. J. Phys. Chem. A 1997, 101, 6824-6826.

103. Rutherford, T. J.; Keene, F. R. Inorg. Chem. 1997, 36, 3580-3581.

104. Rutherford, T. J.; Pellegrini, P. A.; Aldrich-Wright, J.; Junk, P. C.; Keene, F. R. Eur. J. Inorg. Chem. 1998, 1677-1688.

105. Okubo, T.; Kitagawa, S.; Kondo, M.; Matsuzaka, H.; Ishii, T. Angew. Chem., Int. Ed. Engl. 1999, 38, 931-933.

106. Moucheron, C.; Kirsch-De Mesmaeker, A.; Choua, S. Inorg. Chem. 1997, 36, 584-592.

107. Ortmans, I.; Didier, P.; Kirsch-De Mesmaeker, A. Inorg. Chem. 1995, 34, 3695-3704.

108. Didier, P.; Ortmans, I.; Kirsch-De Mesmaeker, A.; Watts, R. J. Inorg. Chem. 1993, 32, 5239-5245.

109. Sahai, R.; Rillema, D. P.; Shaver, R.; Van Wellendael, S.; Jackman, D. C.; Boldaji, M. Inorg. Chem. 1989, 28, 1022-1028.

110. Kirsch-De Mesmaeker, A.; Jacquet, L.; Masschelein, A.; Vanhecke, F.; Heremans, K. Inorg. Chem. 1989, 28, 2465-2470.

111. Baxter, P. N. W.; Lehn, J.-M.; Kneisel, B. O.; Baum, G.; Fenske, D. Chem. Eur. J. 1999, 5, 113-120.

112. Lecomte, J.-P.; Mesmaeker, A. K.-D.; Feeney, M. M.; Kelly, J. M. Inorg. Chem. 1995, 34, 6481-6491.

113. Galán-Mascarós, J. R.; Dunbar, K. R. Chem. Commun. 2001, 217-218.

114. Grove, H.; Sletten, J.; Julve, M.; Lloret, F.; Cano, J. J. Chem. Soc., Dalton Trans. 2001, 259-265.

115. Marshall, S. R.; Rheingold, A. L.; Dawe, L. N.; Shum, W. W.; Kitamura, C.; Miller, J. Inorg. Chem. 2002, 41, 3599-3601.

116. Abrahams, B. F.; Jackson, P. A.; Robson, R. Angew. Chem., Int. Ed. Engl. 1998, 37, 2656-2659.

117. Masaoka, S.; Furukawa, S.; Chang, H.-C.; Mizutani, T.; Kitagawa, S. Angew. Chem., Int. Ed. Engl. 2001, 40, 3817-3819.

118. Balzani, V.; Scandola, F. Supramolecular Photochemistry; Ellis Horwood: Chichester, 1991.

119. Patterson, B. T. Ph.D. Thesis, James Cook University (Townsville, Australia), 2002.

120. Togano, T.; Nagao, N.; Tsuchida, M.; Kumakura, H.; Hisamatsu, K.; Howell, F. S.; Mukaida, M. Inorg. Chim. Acta 1992, 195, 221-225.

121. Krause, R. A. Inorg. Chim. Acta 1977, 22, 209-213.

122. Anderson, P. A.; Anderson, R. F.; Furue, M.; Junk, P. C.; Keene, F. R.; Patterson, B. T.; Yeomans, B. D. Inorg. Chem. 2000, 39, 2721-2728.

123. Blessing, R. H. Acta. Cryst. 1995, A51, 33-38.

124. Sheldrick, G. M. SHELXL-97, Program for Crystal Structure Solution; University of Göttingen: Germany, 1997; Sheldrick, G. M. SHELXS-97, Program for Crystal Structure Refinement; University of Göttingen: Germany, 1997.

125. Barbour, L. J. X-SEED Crystallographic Interface; University of Missouri, USA, 1999.

126. Hua, X. Ph.D. Thesis, University of Fribourg (Fribourg, Switzerland), 1993.

127. Bosnich, B. Inorg. Chem. 1968, 7, 2379-2386.

128. Bosnich, B. Inorg. Chem. 1968, 7, 178-180.

129. Hua, X.; von Zelewsky, A. Inorg. Chem. 1995, 34, 5791-5797.

130. Patterson, B. T.; Keene, F. R. Inorg. Chem. 1998, 37, 645-650.

131. Anderson, P. A.; Deacon, G. B.; Haarmann, K. H.; Keene, F. R.; Meyer, T. J.; Reitsma, D. A.; Skelton, B. W.; Strouse, G. F.; Thomas, N. C.; Treadway, J. A.; White, A. H. Inorg. Chem. 1995, 34, 6145-6157.

132. Creutz, C., Prog. Inorg. Chem. 1983, 30, 1-73.

133. D'Alessandro, D. M.; Keene, F. R. Chem. Eur. J. 2005, 11, 3679-3688. 
134. Crutchley, R. J. Adv. Inorg. Chem. 1994, 41, 273-325.

135. Richardson, D. E.; Taube, H. Coord. Chem. Rev. 1984, 60, 107-129.

136. Kalyanasundaram, K.; Zakeeruddin, S. M.; Nazeeruddin, M. K. Coord. Chem. Rev. 1994, 132, $259-264$.

137. Hush, N. S. Prog. Inorg. Chem. 1967, 8, 391-444.

138. Reimers, J. R.; Hush, N. S. Inorg. Chem. 1990, 29, 3686-3697.

139. Hush, N. S. Coord. Chem. Rev. 1985, 64, 135-157.

140. Brunschwig, B. S.; Creutz, C.; Sutin, N. Chem. Soc. Rev. 2002, 31, 168-184.

141. Hush, N. S. Electrochim. Acta 1968, 13, 1005-1023.

142. Brunschwig, B. S.; Sutin, N. Coord. Chem. Rev. 1999, 187, 233-254.

143. Demadis, K. D.; Hartshorn, C. M.; Meyer, T. J. Chem. Rev. 2001, 101, 2655-2685.

144. Piepho, S. B.; Krausz, E. R.; Schatz, P. N. J. Am. Chem. Soc. 1978, 100, 2996-3005.

145. Piepho, S. B. J. Am. Chem. Soc. 1988, 110, 6319-6326.

146. Wong, K. Y.; Schatz, P. N. Prog. Inorg. Chem. 1981, 28, 369-449.

147. Reimers, J. R.; Hush, N. S. Chem. Phys. 1996, 208, 177-193.

148. Nelsen, S. F. Chem. Eur. J. 2000, 6, 581-588.

149. Lambert, C.; Nöll, G. J. Am. Chem. Soc. 1999, 121, 8434-8442.

150. Demadis, K. D.; El-Samanody, E.-S.; Coia, G. M.; Meyer, T. J. J. Am. Chem. Soc. 1999, 121, 535-544.

151. Demadis, K. D.; Neyhart, G. A.; Kober, E. M.; White, P. S.; Meyer, T. J. Inorg. Chem. 1999, 38, 5948-5959.

152. Neyhart, G. A.; Timpson, C. J.; Bates, W. D.; Meyer, T. J. J. Am. Chem. Soc. 1996, 118, 3730-3737.

153. D'Alessandro, D. M.; Kelso, L. S.; Keene, F. R. Inorg. Chem. 2001, 40, 6841-6844.

154. Giuffrida, G.; Campagna, S. Coord. Chem. Rev. 1994, 135, 517-531.

155. Zhang, L.-T.; Ko, J.; Ondrechen, M. J. J. Phys. Chem. 1989, 93, 3030-3034.

156. Root, L. J.; Ondrechen, M. J. Chem. Phys. Lett. 1982, 93, 421-424.

157. Ko, J.; Ondrechen, M. J. Chem. Phys. Lett. 1984, 112, 507-512.

158. Ondrechen, M. J.; Ko, J.; Root, L. J. J. Phys. Chem. 1984, 88, 5919-5923.

159. Ko, J.; Ondrechen, M. J. J. Am. Chem. Soc. 1985, 107, 6161-6167.

160. Ondrechen, M. J.; Ko, J.; Zhang, L.-T. J. Am. Chem. Soc. 1987, 109, 1672-1676.

161. Zhang, L.-T.; Ko, J.; Ondrechen, M. J. J. Am. Chem. Soc. 1987, 109, 1666-1671.

162. Pfennig, B. W.; Cohen, J. L.; Sosnowski, I.; Novotny, N. M.; Ho, D. M. Inorg. Chem. 1999, 38, 606-612.

163. Constable, E. C.; Lewis, J. Inorg. Chim. Acta 1983, 70, 251-253.

164. Kober, E. M.; Meyer, T. J. Inorg. Chem. 1983, 22, 1614-1616.

165. von Zelewsky, A. Stereochemistry of Coordination Compounds; Wiley: Chichester, 1995.

166. Rutherford, T. J. Ph.D. Thesis, James Cook University (Townsville, Australia), 1997.

167. Richter, M. M.; Brewer, K. J. Inorg. Chem. 1992, 31, 1594-1598.

168. Richter, M. M.; Brewer, K. J. Inorg. Chem. 1993, 32, 2827-2834.

169. Richter, M. M.; Brewer, K. J. Inorg. Chim. Acta 1991, 180, 125-131.

170. Goldsby, K. A.; Meyer, T. J. Inorg. Chem. 1984, 23, 3002-3010.

171. Kober, E. M.; Goldsby, K. A.; Narayana, D. N. S.; Meyer, T. J. J. Am. Chem. Soc. 1983, 105, $4303-4309$.

172. Magnuson, R. H.; Lay, P. A.; Taube, H. J. Am. Chem. Soc. 1982, 105, 2507-2509.

173. Magnuson, R. H.; Lay, P. A.; Taube, H. J. Am. Chem. Soc. 1983, 105, 2507-2509.

174. $\quad$ Lay, P. A.; Magnuson, R. H.; Taube, H. Inorg. Chem. 1988, 27, 2364-2371.

175. Dubicki, L.; Ferguson, J.; Krausz, E. R.; Lay, P. A.; Maeder, M.; Taube, H. J. Phys. Chem. 1984, 88, 3940-3941.

176. Kober, E. M.; Meyer, T. J. Inorg. Chem. 1982, 21, 3967-3977.

177. Demadis, K. D.; Neyhart, G. A.; Kober, E. M.; Meyer, T. J. J. Am. Chem. Soc. 1998, 120, 7121-7122.

178. Strouse, G. F.; Anderson, P. A.; Schoonover, J. R.; Meyer, T. J.; Keene, F. R. Inorg. Chem. 1992, 31, 3004-3006.

179. Patterson, B. T, Honours Dissertation, James Cook University (Townsville, Australia), 1997.

180. McCaffery, A. J.; Mason, S. F. Proc. Chem. Soc. 1963, 211-212.

181. McCaffery, A. J.; Mason, S. F.; Norman, B. J. J. Chem. Soc. A 1969, 1428-1441.

182. Fuchs, Y.; Lofters, S.; Dieter, T.; Shi, W.; Morgan, T. C.; Strekas, T. C.; Gafney, H. D.; Baker, A. D. J. Am. Chem. Soc. 1987, 109, 2691-2697. 
EPILOGUE 


\section{EPILOGUE}

Experimental studies of IVCT in dinuclear mixed-valence complexes have provided crucial insights into the fundamental factors that govern electronic delocalisation and the barriers to electron transfer, through the seminal theoretical formalism pioneered by Hush. ${ }^{1,2}$ To date, these factors have been probed predominantly by the variation of "global" features of the complexes, such as the identity and coordination environments of the constituent metal centres, or through variations in the macroscopic features of the medium such as the solvent, anions and temperature. In many cases, the theoretical implications of the results have been confounded by ion-pairing and specific solvation effects, and ambiguities in the geometries of the complexes due to a lack of structural rigidity. In addition, electron transfer studies on dinuclear polypyridyl complexes of ruthenium and osmium have often neglected their inherent stereochemical identity. Keene and coworkers ${ }^{3,4}$ reported the first examples of differences in the photophysical, electrochemical and spectral properties of the stereoisomers of such systems, although the origins of the stereochemical influences were unclear.

The present study has revealed significant stereochemical effects on the IVCT characteristics of a range of di- and trinuclear mixed-valence complexes. The origins of such influences are attributed to environmental contributions such as specific solvation effects as discussed in Chapter 2, in addition to inherent structural distortions in the bridging ligands and stereochemically-directed anion and solvent interactions, as discussed in Chapter 3. Within the semi-classical formalism for IVCT, the stereochemical effects are reflected in the Franck-Condon reorganisational contributions to the electron transfer barrier, the redox asymmetry factor and the spin-orbit coupling term. The latter indicates that these influences modulate the orbital energy levels of the component metal centres themselves.

While previous literature reports have elucidated the contributions to IVCT from ion-pairing, specific solvation and spin-orbit coupling effects, as well as conformational distortions in the bridging ligands, ${ }^{5-14}$ the present observations highlight the subtle effects of such factors on the electron transfer barrier at the molecular level. In the case of diastereoisomers incorporating rigid bridging ligands, the differences between the forms can be directly related to spatially-directed anion and solvent interactions, which also depend on the temperature of the surrounding medium. Complexes incorporating non-rigid bridges pose an additional complexity, as the inherent structural distortion introduces an effective redox asymmetry contribution. For the completely delocalised mixed-valence systems incorporating the dpo \{3,4-di(2-pyridyl)-1,2,5-oxadiazole $\}$ and dpt \{3,4-di(2-pyridyl)-1,2,5-thiadiazole $\}$ bridging ligands in Chapter 3, the variations in the IVCT properties for the diastereoisomeric forms of the same complex are attributed primarily to differential anion interactions. Since the symmetric mode contributions dictate the IVCT lineshape in the delocalised limit, studies of the ionic strength and polarisability influences within this regime represent an important avenue of future research. Indeed, the systematic investigation of IVCT in delocalised mixed-valence systems has received limited attention compared with those in the 
localised regime. Clearly, meaningful comparisons between the results from IVCT studies require standard conditions of electrolyte, solvent and temperature.

While the present study is based primarily on a semi-classical two-state analysis for IVCT, a minimum of three-states is required to adequately account for the integral role of the bridging ligands in mediating the IVCT process via a superexchange mechanism. In Chapters 3 and 4, the trends in the IVCT properties are rationalised on the basis of a qualitative three-state model which includes the influence of the symmetric vibration mode. An accurate quantitative treatment of the results requires consideration of the full vibronic coupling problem, with the explicit inclusion of the symmetric and antisymmetric vibration modes. The recent success of such treatments in modelling the mixed-valence "special-pair" radical cation of the photosynthetic reaction centre is encouraging in this regard, ${ }^{15}$ and there is significant potential for the application of these methods to dinuclear mixed-valence systems. ${ }^{*}$

An important consideration for the theoretical and computational treatment of mixed-valence systems is the explicit recognition of the underlying spin-orbit components of the IVCT bands.

Identification of the first component is paramount, as this transition characterises the ground-state of the mixed-valence chromophore. ${ }^{11}$ In the present study, this issue has been considered within the context of a localised bonding scheme; however, reliable determinations of the first IVCT transition were frequently precluded by the overlapping nature of the components. A moment analysis was essential for the elucidation of trends in the IVCT data for the asymmetrically-shaped bands. While this analysis has been limited to the first moments of the IVCT bands, it is clear that the examination of higher order moments should be pursued. For example, the second-order moment would provide a measure of the standard deviation of the bands, and a more accurate insight into the trends in the line-widths. ${ }^{1,2,14}$ Such analyses may assist in clarifying the contribution of symmetric modes to IVCT broadening in the localised-todelocalised and delocalised regimes.

As noted in Chapter 3, a major shortcoming of the present work, and an aspect which deserves future attention, is the measurement of IR and resonance Raman spectra for the series of complexes. These experiments would provide a link between the trends in the NIR IVCT bands and the symmetrical bridging ligand stretches and "spectator vibrations" in the IR, as well as the mode-specific contributions to the electron transfer barrier. As detailed by Meyer and coworkers, the appearance and relative intensities of symmetrical bridging ligand stretches in the IR region provide one of the most definitive means for assessing the relative degree of electronic delocalisation, and distinguishing between the localised and delocalised regimes. ${ }^{11}$

A further important avenue of research is the consideration of more sophisticated models for the treatment of the electroabsorption (Stark effect) data presented in Chapter 3, as the classical two-state Liptay analysis ${ }^{17}$ is inappropriate for the treatment of systems in the localised-to-delocalised regime. Despite the invalidity of the quantitative analysis, the data obtained from Stark effect measurements indicate that the series of complexes exhibit extensive electronic delocalisation, which contradicts the

\footnotetext{
* Computational modelling studies of the IVCT bands in the present series of complexes have been commenced by Dr Jeffrey Reimers and Dr Zhengli Cai (University of Sydney, Australia). ${ }^{16}$
} 
previous localised assignment for systems such as $\left[\left\{\mathrm{Os}(\mathrm{bpy})_{2}\right\}_{2}(\mu-\mathrm{dpb})\right]^{5+}\{\mathrm{dpb}=2,3$-bis(2-pyridyl)-1,4benzoquinoxaline ${ }^{18,19}$ Boxer and coworkers ${ }^{20}$ have recently postulated a general theoretical formalism to treat moderately- to strongly-coupled mixed-valence systems, and the present series of complexes may provide an interesting platform to investigate the applicability of such treatments.

In addition to these fundamental insights, the extension of the IVCT probe to stereochemicallypure trinuclear assemblies in Chapter 4 has revealed the contribution of "second-order" interactions on the IVCT process due to multiple electronically-coupled metal centres. Such interactions are enhanced in the presence of strong electronic delocalisation, as exemplified by the "cluster-type" systems incorporating the tri-bidentate HAT \{1,4,5,8,9,12-hexaazatriphenylene $\}$ bridging ligand, in contrast to the "chain-like” systems incorporating the closely electronically and structurally-related bridging ligand ppz \{4,7-phenanthrolino-5,6:5',6'-pyrazine\}.

The transition between the localised and delocalised regimes in IVCT depends on a complexity of underlying factors which are governed by the relative time-scales for electron transfer, and the coupled solvent and vibrational dynamics. The elucidation of stereochemical effects on IVCT has significant implications for understanding spatial effects on electron transfer processes in nature. For example, in the photosynthetic apparatus, the "special-pair" of chlorophyll molecules that form the primary electron donor are organised into predetermined orientations within an enzyme matrix. The efficiencies of the electron migration pathways are governed by the precise structural control of the components, and the influence of environmental interactions on their electron transfer properties. The observation of stereochemically-directed solvent and anion interactions, as well as stereochemical dependencies on the redox asymmetry and spin-orbit coupling contributions, may assist in unravelling the intricate factors which govern the structure-function relationship in biological systems. At an applied level, stereochemical effects could ultimately be exploited in the rational design of higher-nuclearity assemblies which form the basis of novel molecular materials. 


\section{References}

1. Hush, N. S. Prog. Inorg. Chem. 1967, 8, 391-444.

2. Hush, N. S. Electrochim. Acta 1968, 13, 1005-1023.

3. Keene, F. R. Coord. Chem. Rev. 1997, 166, 122-159.

4. $\quad$ Keene, F. R. Chem. Soc. Rev. 1998, 27, 185-193.

5. Hush, N. S. Coord. Chem. Rev. 1985, 64, 135-157.

6. Creutz, C. Prog. Inorg. Chem. 1983, 30, 1-73.

7. Crutchley, R. J. Adv. Inorg. Chem. 1994, 41, 273-325.

8. Kalyanasundaram, K.; Nazeeruddin, M. K. Inorg. Chim. Acta 1994, 226, 213-230.

9. Ward, M. D. Chem. Soc. Rev. 1995, 24, 121-134.

10. Barbara, P. F.; Meyer, T. J.; Ratner, M. A. J. Phys. Chem. 1996, 100, 13148-13168.

11. Demadis, K. D.; Hartshorn, C. M.; Meyer, T. J. Chem. Rev. 2001, 101, 2655-2685.

12. Launay, J.-P. Chem. Soc. Rev. 2001, 30, 386-397.

13. Kaim, W.; Klein, A.; Glöckle, M. Acc. Chem. Res. 2000, 33, 755-763.

14. Chen, P.; Meyer, T. J. Chem. Rev. 1998, 98, 1439-1477.

15. Reimers, J. R.; Hush, N. S. J. Am. Chem. Soc. 2004, 126, 4123-4144.

16. Reimers, J. R.; Hush, N. S. Chem. Phys. 1996, 208, 177-193.

17. Liptay, W. Angew. Chem., Int. Ed. Engl. 1969, 8, 177-188.

18. Richter, M. M.; Brewer, K. J. Inorg. Chim. Acta 1991, 180, 125-131.

19. $\quad$ Richter, M. M.; Brewer, K. J. Inorg. Chem. 1993, 32, 2827-2834.

20. Treynor, T. P.; Boxer, S. G. J. Phys. Chem. A 2004, 108, 1764-1778. 
ApPendices 


\section{APPENDiX A}

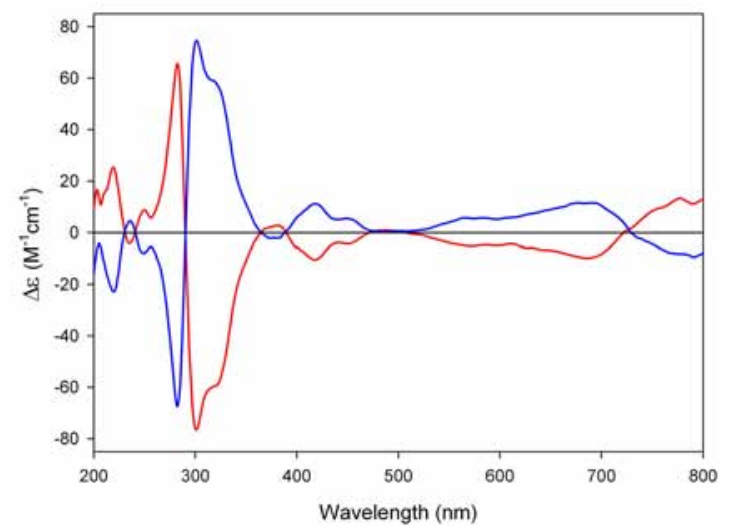

Figure A2.1 CD spectra of the chromatographically-separated Band 1, $\Delta \Delta(-)$ and Band 2, $\Lambda \Lambda(-)$ enantiomers of rac- $\left[\left\{\mathrm{Ru}(\mathrm{bpy})_{2}\right\}_{2}(\mu-\mathrm{dbneil})\right]^{4+}$ in $\mathrm{CH}_{3} \mathrm{CN}$.

Table A2.1 Crystal data and structure refinement parameters for the dinuclear complexes incorporating the bpm bridging ligand.

\begin{tabular}{|c|c|c|}
\hline Parameter & {$\left[\left\{\mathrm{Ru}\left(\mathrm{Me}_{2} \mathrm{bpy}\right)_{2}\right\}_{2}(\mu\right.$-bpm $\left.)\right](\text { tos })_{4}$} & {$\left[\left\{\mathrm{Ru}(\mathrm{bpy})_{2}\right\}_{2}(\mu\right.$-bpm $\left.)\right]\left(\mathrm{PF}_{6}\right)_{4}$} \\
\hline Formula & $\mathrm{C}_{86} \mathrm{H}_{82} \mathrm{~N}_{12} \mathrm{Ru}_{2} \mathrm{~S}_{4} \mathrm{O}_{18}$ & $\mathrm{C}_{48} \mathrm{H}_{38} \mathrm{~N}_{12} \mathrm{~F}_{24} \mathrm{P}_{4} \mathrm{Ru}_{2}$ \\
\hline Formula weight & 1902.1 & 1564.9 \\
\hline $\mathrm{T}(\mathrm{K})$ & 293 & 293 \\
\hline Crystal System & orthorhombic & monoclinic \\
\hline Space Group & $\mathrm{Pbca}$ & $\mathrm{C}_{2} / \mathrm{c}$ \\
\hline$a(\AA)$ & $19.8373(29)$ & $19.367(5)$ \\
\hline$b(\AA)$ & 13.5927(20) & 17.679(5) \\
\hline$c(\AA)$ & $32.8928(49)$ & $18.321(5)$ \\
\hline$\alpha\left(^{\circ}\right)$ & $90.0005(5)$ & $90.000(5)$ \\
\hline$\beta\left(^{\circ}\right)$ & $90.0005(5)$ & $111.228(5)$ \\
\hline$\gamma\left({ }^{\circ}\right)$ & $90.0005(5)$ & $90.000(5)$ \\
\hline$V\left(\AA^{3}\right)$ & $8869(2)$ & $5847(3)$ \\
\hline Z & 4 & 4 \\
\hline$D_{\mathrm{c}}\left(\mathrm{g} \mathrm{cm}^{-3}\right)$ & 1.424 & 1.778 \\
\hline$\mu\left(\mathrm{Mo}-\mathrm{K}_{\alpha}\right)\left(\mathrm{mm}^{-1}\right)$ & 0.51 & 0.75 \\
\hline$F(000)$ & 3911.4 & 3095.5 \\
\hline Crystal form & dark green plate & dark green/purple prism \\
\hline Crystal size $(\mathrm{mm})$ & $0.2 \times 0.2 \times 0.02$ & $0.2 \times 0.2 \times 0.15$ \\
\hline $2 \theta_{\max }\left({ }^{\circ}\right)$ & 23.4 & 26.5 \\
\hline Limiting indices & $0 \leq h \leq 21,0 \leq k \leq 15,0 \leq l \leq 36$ & $-24 \leq h \leq 22,0 \leq k \leq 22,0 \leq l \leq 22$ \\
\hline Reflections collected & $6431--5-5$ & $605 \overline{4}-5-1-5$ \\
\hline Unique reflections & 4000 & $2355\left(\mathrm{R}_{\mathrm{int}}=0.1969\right)$ \\
\hline Parameters & $573\left(\mathrm{R}_{\mathrm{int}}=0.127\right)$ & 410 \\
\hline Refinement method & Full-matrix least-squares on $F^{2}$ & Full-matrix least-squares on $F^{2}$ \\
\hline Absorption correction & none & none \\
\hline GooF & 0.964 & 0.870 \\
\hline$R[I>2 \sigma(I)]$ & 0.082 & 0.0681 \\
\hline$w R$ (all data) & 0.247 & 0.2064 \\
\hline Diffractometer & Bruker AXS CCD $(\lambda=0.71069 \AA)$ & Bruker AXS CCD $(\lambda=0.71069 \AA)$ \\
\hline
\end{tabular}




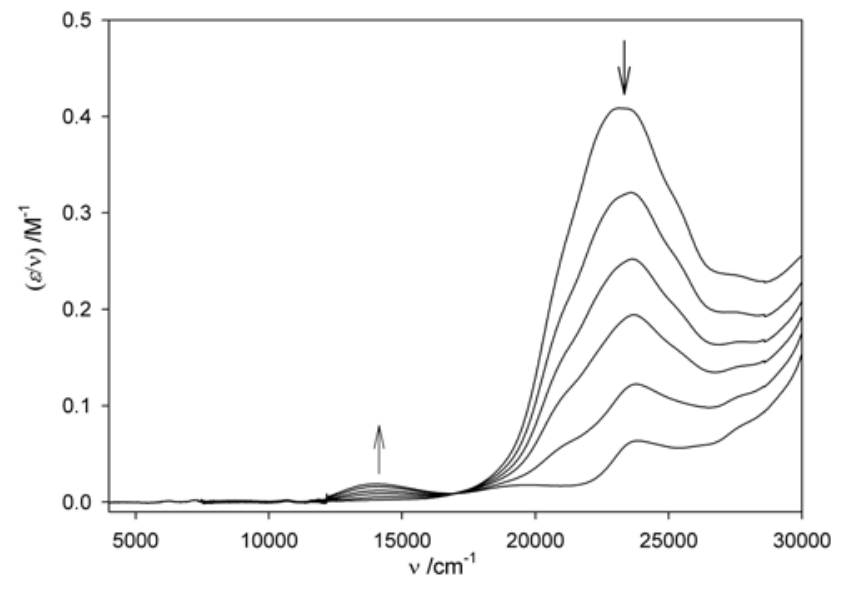

Figure A2.2 UV/Vis/NIR spectroelectrochemical progression for the oxidation reaction $\left[\mathrm{Ru}(\mathrm{bpy})_{2}(\mathrm{bpm})\right]^{2+} \rightarrow$ $\left[\mathrm{Ru}(\mathrm{bpy})_{2}(\mathrm{bpm})\right]^{3+}$ in $0.02 \mathrm{M}\left[\left(n-\mathrm{C}_{4} \mathrm{H}_{9}\right)_{4} \mathrm{~N}\right]\left\{\mathrm{B}\left(\mathrm{C}_{6} \mathrm{~F}_{5}\right)_{4}\right\} / \mathrm{CH}_{3} \mathrm{CN}$ at $+25^{\circ} \mathrm{C}$.

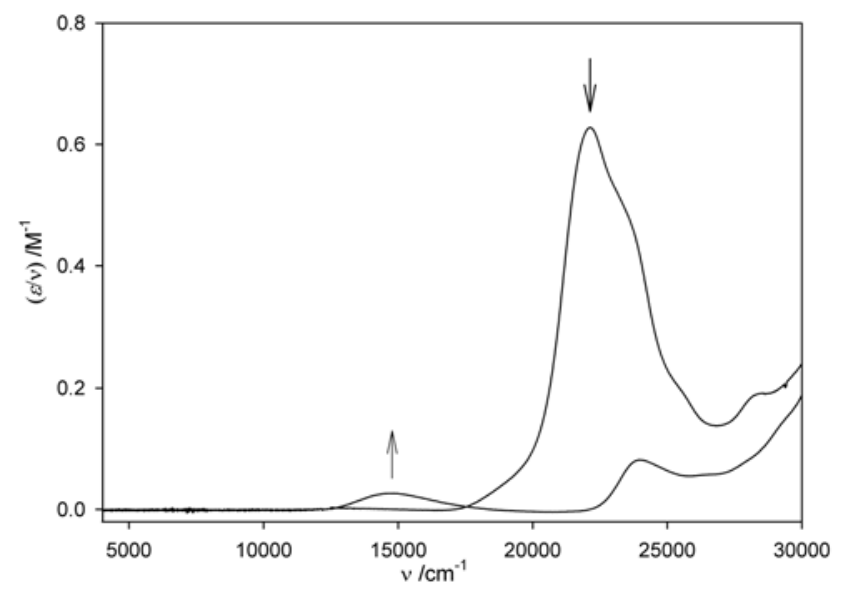

Figure A2.3 UV/Vis/NIR spectra for $\left[\mathrm{Ru}(\mathrm{bpy})_{3}\right]^{2+}$ and $\left[\mathrm{Ru}(\mathrm{bpy})_{3}\right]^{3+}$ in $0.02 \mathrm{M}\left[\left(n-\mathrm{C}_{4} \mathrm{H}_{9}\right)_{4} \mathrm{~N}\right]\left\{\mathrm{B}\left(\mathrm{C}_{6} \mathrm{~F}_{5}\right)_{4}\right\} / \mathrm{CH}_{3} \mathrm{CN}$ at $+25^{\circ} \mathrm{C}$.

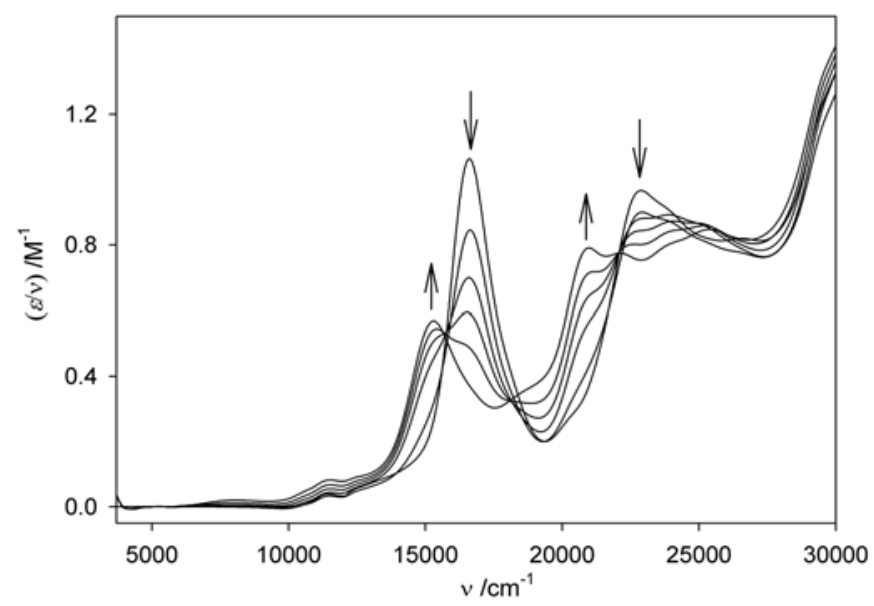

Figure A2.4 UV/Vis/NIR spectroelectrochemical progression for the oxidation reaction $\left[\mathrm{Ru}(\mathrm{bpy})_{2}(\mathrm{dbneil})\right]^{2+} \rightarrow$ $\left[\mathrm{Ru}(\text { bpy })_{2}(\mathrm{dbneil})\right]^{3+}$ in $0.02 \mathrm{M}\left[\left(n-\mathrm{C}_{4} \mathrm{H}_{9}\right)_{4} \mathrm{~N}\right]\left\{\mathrm{B}\left(\mathrm{C}_{6} \mathrm{~F}_{5}\right)_{4}\right\} / \mathrm{CH}_{3} \mathrm{CN}$ at $+25^{\circ} \mathrm{C}$. 
Table A2.2 UV/Vis/NIR spectral data of the reduced absorption spectra ( $\varepsilon / v v s . v$ ) for the diastereoisomers of $\left[\left\{\mathrm{Ru}(\text { bpy })_{2}\right\}_{2}(\mu \text {-dbneil })\right]^{n+}$ in $0.02 \mathrm{M}\left[\left(n-\mathrm{C}_{4} \mathrm{H}_{9}\right)_{4} \mathrm{~N}\right]\left\{\mathrm{B}\left(\mathrm{C}_{6} \mathrm{~F}_{5}\right)_{4}\right\} /$ solvent at $+25^{\circ} \mathrm{C}$. The NIR spectral data are indicated in bold type.

\begin{tabular}{|c|c|c|c|c|c|}
\hline \multirow[b]{2}{*}{ Solvent } & \multirow[b]{2}{*}{$n$} & \multicolumn{2}{|c|}{ meso } & \multicolumn{2}{|c|}{ rac } \\
\hline & & $\begin{array}{c}v_{\max } \\
\pm 10 / \mathrm{cm}^{-1}\end{array}$ & $\begin{aligned} & (\varepsilon / v)_{\max } \\
\pm & 0.0001 / \mathrm{M}^{-1}\end{aligned}$ & $\begin{array}{c}v_{\max } \\
\pm 10 / \mathrm{cm}^{-1}\end{array}$ & $\begin{aligned} & (\varepsilon / v)_{\max } \\
\pm & 0.0001 / \mathrm{M}^{-1}\end{aligned}$ \\
\hline \multirow[t]{6}{*}{ AN } & 4 & 14290 & 1.9748 & 14290 & 3.2190 \\
\hline & & sh 22270 & 1.1306 & sh 22195 & 1.8129 \\
\hline & & 23700 & 1.5970 & 23780 & 2.5901 \\
\hline & 5 & 4650 & 0.2112 & 4560 & 0.2064 \\
\hline & & 14045 & 1.0073 & 14070 & 1.6604 \\
\hline & & 23660 & 1.0051 & 23070 & 1.6604 \\
\hline \multirow[t]{6}{*}{ PN } & 4 & 14335 & 1.9611 & 14335 & 1.9611 \\
\hline & & sh 22299 & 1.1563 & sh 22299 & 1.1563 \\
\hline & & 23767 & 1.6107 & 23767 & 1.6107 \\
\hline & 5 & 4610 & 0.2081 & 4670 & 0.1515 \\
\hline & & 14228 & 1.0609 & 14228 & 1.0609 \\
\hline & & 23792 & 1.2583 & 23792 & 1.2583 \\
\hline \multirow[t]{6}{*}{ BN } & 4 & 14340 & 1.9642 & 14338 & 1.9642 \\
\hline & & sh 22300 & 1.1600 & sh 22300 & 1.1600 \\
\hline & & 23750 & 1.6075 & 23750 & 1.6075 \\
\hline & 5 & 4590 & 0.1890 & 4585 & 0.0855 \\
\hline & & 14307 & 1.3610 & 14307 & 1.3610 \\
\hline & & 23740 & 1.2843 & 23740 & 1.2843 \\
\hline \multirow[t]{6}{*}{${ }^{\mathrm{i}} \mathrm{BN}$} & 4 & 14350 & 1.9678 & 14353 & 1.9678 \\
\hline & & sh 22302 & 1.1500 & sh 22302 & 1.1500 \\
\hline & & 23796 & 1.6039 & 23796 & 1.6039 \\
\hline & 5 & 4610 & 0.1953 & 4600 & 0.1929 \\
\hline & & 14228 & 1.1233 & 14228 & 1.1233 \\
\hline & & 23714 & 1.1162 & 23714 & 1.1162 \\
\hline \multirow[t]{6}{*}{$\mathrm{BzN}$} & 4 & 14170 & 1.8744 & 14168 & 1.8744 \\
\hline & & sh 21994 & 1.1620 & sh 21994 & 1.1620 \\
\hline & & 23503 & 1.6973 & 23503 & 1.6973 \\
\hline & 5 & 4600 & 0.1800 & 4590 & 0.1500 \\
\hline & & 14200 & 0.9412 & 14200 & 0.9412 \\
\hline & & 23500 & 0.9111 & 23500 & 0.9111 \\
\hline
\end{tabular}




\section{APPENDIX B}
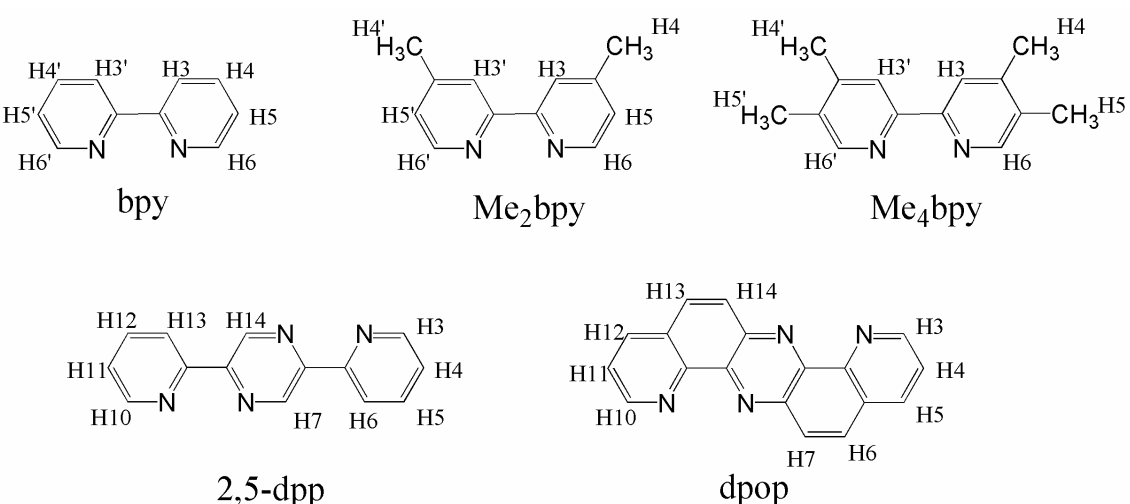

2,5-dpp dpop<smiles>C1=CNC2=c3c(ccnc3=N1)C1=C2C=CPC=N1</smiles>

ppz

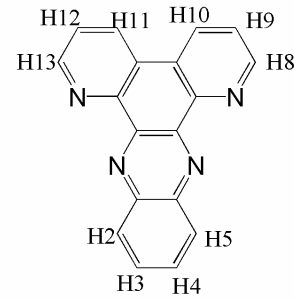

dpq'

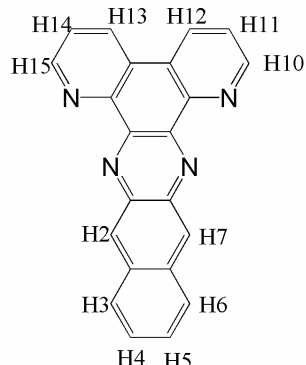

dpb'

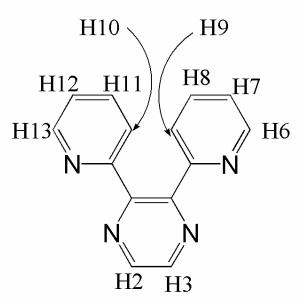

$2,3 d p p$

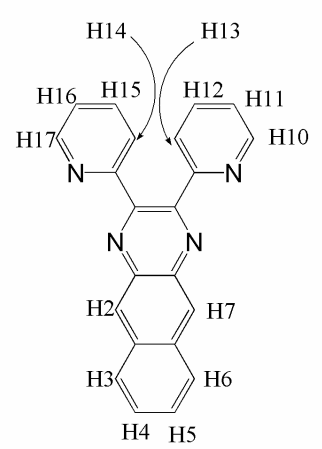

$\mathrm{dpb}$

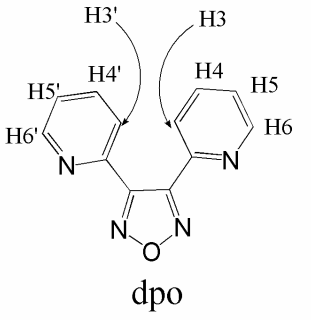

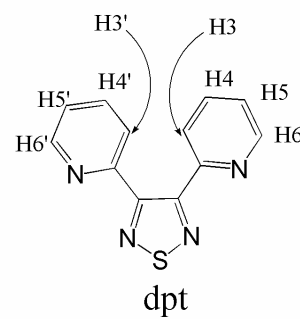

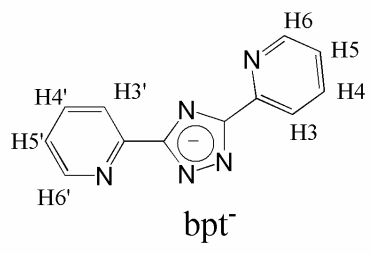

Figure B3.1 Proton numbering schemes for the terminal and bridging ligands used in the present study. 
Table B3.1 Crystal data and structure refinement parameters for the dinuclear ruthenium complexes incorporating the angular bridging ligands dpb, dpb' and dpq'.

\begin{tabular}{|c|c|c|c|c|}
\hline Parameter & $\begin{array}{c}\text { meso- }(\Lambda \Delta)- \\
{\left[\left\{\mathrm{Ru}(\mathrm{bpy})_{2}\right\}_{2}(\mu-\mathrm{dpb})\right]\left(\mathrm{PF}_{6}\right)_{4}} \\
.5 \mathrm{H}_{2} \mathrm{O}\end{array}$ & $\begin{array}{c}\text { meso- }(\Lambda \Delta)- \\
{\left[\left\{\mathrm{Ru}(\mathrm{bpy})_{2}\right\}_{2}(\mu-\mathrm{dpb})\right]\left(\mathrm{ZnCl}_{4}\right)_{2}} \\
.5 \mathrm{H}_{2} \mathrm{O}\end{array}$ & $\begin{array}{c}\text { meso- }(\Lambda \Delta)- \\
{\left[\left\{\mathrm{Ru}(\mathrm{bpy})_{2}\right\}_{2}\left(\mu-\mathrm{dpb}^{\prime}\right)\right]\left(\mathrm{PF}_{6}\right)_{4}} \\
.2 \mathrm{H}_{2} \mathrm{O} .2\left\{\left(\mathrm{CH}_{3}\right)_{2} \mathrm{CO}\right\}\end{array}$ & $\begin{array}{c}\text { meso- }(\Lambda \Delta)- \\
{\left[\left\{\mathrm{Ru}(\mathrm{bpy})_{2}\right\}_{2}(\mu-\mathrm{dpq})\right]\left(\mathrm{ZnCl}_{4}\right)_{2}} \\
.3 \mathrm{H}_{2} \mathrm{O}\end{array}$ \\
\hline Formula & $\mathrm{C}_{62} \mathrm{H}_{56} \mathrm{~F}_{24} \mathrm{~N}_{12} \mathrm{O}_{5} \mathrm{P}_{4} \mathrm{Ru}_{2}$ & $\mathrm{C}_{62} \mathrm{H}_{56} \mathrm{Cl}_{8} \mathrm{~N}_{12} \mathrm{O}_{5} \mathrm{Ru}_{2} \mathrm{Zn}_{2}$ & $\mathrm{C}_{65} \mathrm{H}_{50} \mathrm{~N}_{12} \mathrm{~F}_{24} \mathrm{O}_{5} \mathrm{P}_{4} \mathrm{Ru}_{2}$ & $\mathrm{C}_{58} \mathrm{H}_{48} \mathrm{Cl}_{8} \mathrm{~N}_{12} \mathrm{O}_{3} \mathrm{P}_{4} \mathrm{Ru}_{2} \mathrm{Zn}_{2}$ \\
\hline Formula weight & 1831.21 & 1665.67 & 1805.19 & 1577.56 \\
\hline $\mathrm{T}(\mathrm{K})$ & $296(2)$ & $296(2)$ & $123(2)$ & $296(2)$ \\
\hline Crystal System & orthorhombic & monoclinic & orthorhombic & triclinic \\
\hline Space Group & $\mathrm{F} d d 2$ & $P 2_{1} / c$ & $\mathrm{Pbca}$ & $P \overline{1}$ \\
\hline$a(\AA)$ & $39.640(2)$ & 19.608(3) & $25.283(5)$ & $10.1847(9)$ \\
\hline$b(\AA)$ & $69.321(4)$ & $16.821(2)$ & $22.151(4)$ & $16.3990(15)$ \\
\hline$c(\AA)$ & $10.8530(6)$ & 20.930(3) & $26.204(5)$ & $19.5070(17)$ \\
\hline$\alpha\left(^{\circ}\right)$ & $90.0005(5)$ & $90.0005(5)$ & $90.0005(5)$ & $96.148(2)$ \\
\hline$\beta\left({ }^{\circ}\right)$ & $90.0005(5)$ & $96.956(3)$ & $90.0005(5)$ & $104.781(2)$ \\
\hline$\gamma\left({ }^{\circ}\right)$ & $90.0005(5)$ & $90.0005(5)$ & $90.0005(5)$ & $96.216(2)$ \\
\hline$V\left(\AA^{3}\right)$ & 29823(3) & $6852.4(16)$ & $14675(5)$ & $3100.8(5)$ \\
\hline$Z$ & 16 & 4 & 8 & 2 \\
\hline$D_{\mathrm{c}}\left(\mathrm{g} \mathrm{cm}^{-3}\right)$ & 1.631 & 1.615 & 1.624 & 1.690 \\
\hline$\mu\left(\mathrm{Mo}-\mathrm{K}_{\alpha}\right)\left(\mathrm{mm}^{-1}\right)$ & 0.606 & 1.493 & 0.612 & 1.642 \\
\hline$F(000)$ & 14656 & 3344 & 7200 & 1576 \\
\hline Crystal form & dark red rod-shaped crystals & $\begin{array}{l}\text { dichroic red/green rod-shaped } \\
\text { crystals }\end{array}$ & dichroic red/green crystals & $\begin{array}{l}\text { dichroic red/green rod-shaped } \\
\text { crystals }\end{array}$ \\
\hline Crystal size (mm) & $0.4 \times 0.4 \times 0.3$ & $0.3 \times 0.15 \times 0.15$ & $0.3 \times 0.3 \times 0.2$ & $0.35 \times 0.30 \times 0.28$ \\
\hline $2 \theta_{\max }\left({ }^{\circ}\right)$ & 23.3 & 23.34 & 28.44 & 23.25 \\
\hline Limiting indices & $\begin{array}{l}-44 \leq h \leq 44,-60 \leq \mathrm{k} \leq 76 \\
-12 \leq \mathrm{l} \leq 11\end{array}$ & $\begin{array}{l}-21 \leq h \leq 21,-13 \leq \mathrm{k} \leq 18 \\
-23 \leq \mathrm{l} \leq 23\end{array}$ & $\begin{array}{l}-33 \leq h \leq 33,-29 \leq k \leq 29 \\
-34 \leq l \leq 32\end{array}$ & $\begin{array}{l}-11 \leq h \leq 11,-16 \leq \mathrm{k} \leq 18 \\
-21 \leq \mathrm{l} \leq 18\end{array}$ \\
\hline Reflections collected & 34612 & $307 \overline{84}$ & 145604 & $142 \overline{58}$ \\
\hline Unique reflections & 10479 & 9794 & 18350 & 8834 \\
\hline Parameters & $1173\left(\mathrm{R}_{\mathrm{int}}=0.1089\right)$ & $795\left(\mathrm{R}_{\mathrm{int}}=0.1882\right)$ & $977\left(\mathrm{R}_{\mathrm{int}}=0.1500\right)$ & $751\left(\mathrm{R}_{\mathrm{int}}=0.0652\right)$ \\
\hline $\begin{array}{l}\text { Refinement method } \\
\text { Absorption correction }\end{array}$ & $\begin{array}{l}\text { Full-matrix least-squares on } F^{2} \\
\text { none }\end{array}$ & $\begin{array}{l}\text { Full-matrix least-squares on } F^{2} \\
\text { none }\end{array}$ & $\begin{array}{l}\text { Full-matrix least-squares on } F^{2} \\
\text { none }\end{array}$ & $\begin{array}{l}\text { Full-matrix least-squares on } F^{2} \\
\text { none }\end{array}$ \\
\hline GooF & 0.972 & 0.826 & 0.0635 & 0.934 \\
\hline$R[I>2 \sigma(I)]$ & 0.0703 & 0.0667 & 0.063 & 0.0493 \\
\hline$w R$ (all data) & 0.2088 & 0.1707 & 0.1802 & 0.1379 \\
\hline Diffractometer & $\begin{array}{l}\text { Bruker SMART CCD } \\
(\lambda=0.71073 \AA)\end{array}$ & $\begin{array}{l}\text { Bruker SMART CCD } \\
(\lambda=0.71073 \AA)\end{array}$ & $\begin{array}{l}\text { Enraf-Nonius Kappa CCD } \\
(\lambda=0.71069 \AA)\end{array}$ & $\begin{array}{l}\text { Bruker SMART CCD } \\
(\lambda=0.71073 \AA)\end{array}$ \\
\hline
\end{tabular}


Table B3.2(a) Crystal data and structure refinement parameters for dinuclear ruthenium complexes incorporating the angular bridging ligand dpo.

\begin{tabular}{|c|c|c|}
\hline Parameter & $\begin{array}{c}\text { meso- }(\Lambda \Delta)- \\
{\left[\left\{\mathrm{Ru}(\mathrm{bpy})_{2}\right\}_{2}(\mu-\mathrm{dpo})\right]\left(\mathrm{ZnCl}_{4}\right)_{2}} \\
.6 \mathrm{H}_{2} \mathrm{O} \\
\end{array}$ & $\begin{array}{c}\operatorname{rac}-(\Delta \Delta)- \\
{\left[\left\{\mathrm{Ru}(\mathrm{bpy})_{2}\right\}_{2}(\mu-\mathrm{dpo})\right]\left(\mathrm{ZnCl}_{4}\right)_{2}} \\
.\left[\mathrm{ZnCl}_{2}\left(\mathrm{H}_{2} \mathrm{O}\right)_{2}\right]\end{array}$ \\
\hline Formula & $\mathrm{C}_{52} \mathrm{H}_{52} \mathrm{Cl}_{8} \mathrm{~N}_{12} \mathrm{O}_{7} \mathrm{Ru}_{2} \mathrm{Zn}_{2}$ & $\mathrm{C}_{52} \mathrm{H}_{44} \mathrm{Cl}_{10} \mathrm{~N}_{12} \mathrm{O}_{3} \mathrm{Ru}_{2} \mathrm{Zn}_{3}$ \\
\hline Formula weight & 1573.54 & 1637.74 \\
\hline $\mathrm{T}(\mathrm{K})$ & $296(2)$ & $296(2)$ \\
\hline Crystal System & monoclinic & triclinic \\
\hline Space Group & $P 2_{1} / n$ & $P \overline{1}$ \\
\hline$a(\AA)$ & $13.743(3)$ & $14.855(2)$ \\
\hline$b(\AA)$ & $21.685(4)$ & $16.198(2)$ \\
\hline$c(\AA)$ & 21.332(4) & $17.717(3)$ \\
\hline$\alpha\left(^{\circ}\right)$ & $90.0005(5)$ & $75.475(3)$ \\
\hline$\beta\left(^{\circ}\right)$ & $92.55(3)$ & $66.629(3)$ \\
\hline$\gamma\left({ }^{\circ}\right)$ & $90.0005(5)$ & $70.992(3)$ \\
\hline$V\left(\AA^{3}\right)$ & $6351(2) A^{\wedge} 3$ & $3663.4(9)$ \\
\hline$Z$ & 4 & 2 \\
\hline$D_{\mathrm{c}}\left(\mathrm{g} \mathrm{cm}^{-3}\right)$ & 1.646 & 1.485 \\
\hline$\mu\left(\mathrm{Mo}_{\mathrm{\alpha}} \mathrm{K}_{\alpha}\right)\left(\mathrm{mm}^{-1}\right)$ & 1.607 & 1.780 \\
\hline$F(000)$ & 3152 & 1624 \\
\hline Crystal form & deep red rod-shaped crystals & deep red rod-shaped crystals \\
\hline Crystal size (mm) & $0.2 \times 0.2 \times 0.02$ & $0.4 \times 0.3 \times 0.3$ \\
\hline $2 \theta_{\max }\left({ }^{\circ}\right)$ & 28.05 & 28.11 \\
\hline Limiting indices & $-18 \leq h \leq 17,-28 \leq k \leq 22,-28 \leq l \leq 26$ & $-19 \leq h \leq 19,-21 \leq k \leq 21,-23 \leq l \leq 21$ \\
\hline Reflections collected & 40153 & 24743 \\
\hline Unique reflections & 14867 & 16968 \\
\hline Parameters & $739\left(\mathrm{R}_{\mathrm{int}}=0.0531\right)$ & $776\left(\mathrm{R}_{\mathrm{int}}=0.0694\right)$ \\
\hline Refinement method & Full-matrix least-squares on $F^{2}$ & Full-matrix least-squares on $F^{2}$ \\
\hline Absorption correction & none & none \\
\hline GooF & 1.016 & 0.888 \\
\hline$R[I>2 \sigma(I)]$ & 0.0566 & 0.0812 \\
\hline$w R$ (all data) & 0.1840 & 0.2974 \\
\hline Diffractometer & Bruker SMART CCD $(\lambda=0.71069 \AA)$ & Bruker SMART CCD $(\lambda=0.71069 \AA)$ \\
\hline
\end{tabular}

Table B3.2(b) Selected bond lengths $(\AA)$ and angles $\left(^{\circ}\right)$ for meso- $(\Lambda \Delta)-\left[\left\{\mathrm{Ru}(\mathrm{bpy})_{2}\right\}_{2}(\mu-\mathrm{dpo})\right]\left(\mathrm{ZnCl}_{4}\right)_{2} \cdot 6 \mathrm{H}_{2} \mathrm{O}$ and rac- $(\Delta \Delta)-\left[\left\{\mathrm{Ru}(\mathrm{bpy})_{2}\right\}_{2}(\mu-\mathrm{dpo})\right]\left(\mathrm{ZnCl}_{4}\right)_{2} \cdot\left[\mathrm{ZnCl}_{2}\left(\mathrm{H}_{2} \mathrm{O}\right)_{2}\right]$.

\begin{tabular}{|c|c|c|c|c|c|c|c|}
\hline \multicolumn{4}{|c|}{ meso- $(\Lambda \Delta)$} & \multicolumn{4}{|c|}{$\operatorname{rac}-(\Delta \Delta)$} \\
\hline \multicolumn{2}{|c|}{$\begin{array}{c}\text { Bond lengths } \\
(\AA)\end{array}$} & \multicolumn{2}{|c|}{$\begin{array}{c}\text { Bond angles } \\
\left({ }^{\circ}\right)\end{array}$} & \multicolumn{2}{|c|}{$\begin{array}{c}\text { Bond lengths } \\
(\AA)\end{array}$} & \multicolumn{2}{|c|}{$\begin{array}{c}\text { Bond angles } \\
\left({ }^{\circ}\right)\end{array}$} \\
\hline Ru1-N2 & $1.979(4)$ & N2-Ru1-N6 & 172.90(17) & Ru1- N2 & 1.971(7) & N2-Ru1-N6 & $170.0(3)$ \\
\hline Ru1-N6 & $2.060(4)$ & N6-Ru1-N5 & $78.62(17)$ & Ru1-N6 & $2.046(8)$ & N6- Ru1-N5 & $78.8(3)$ \\
\hline Ru1-N5 & $2.068(4)$ & N8-Ru1-N7 & $79.06(18)$ & Ru1-N5 & $2.067(7)$ & N8-Ru1-N7 & $79.6(3)$ \\
\hline Ru1-N8 & $2.069(4)$ & N2-Ru1 N1 & $75.67(16)$ & Ru1-N8 & $2.071(7)$ & N2-Ru1-N1 & $75.3(3)$ \\
\hline Ru1-N7 & $2.073(4)$ & N3-Ru2-N10 & $173.56(17)$ & Ru1-N7 & $2.062(7)$ & N3-Ru2-N11 & 174.1(3) \\
\hline Ru1-N1 & $2.079(4)$ & N12-Ru2-N11 & $78.2(2)$ & Ru1-N1 & $2.104(7)$ & N12-Ru2-N11 & 78.8(3) \\
\hline Ru2-N3 & $1.983(4)$ & N9-Ru2-N10 & 78.52(16) & Ru2-N3 & $1.979(7)$ & N9-Ru2-N10 & $79.2(3)$ \\
\hline Ru2-N12 & 2.061(5) & N3- Ru2-N4 & 75.55(17) & Ru2-N12 & $2.046(8)$ & N3-Ru2- N4 & 75.3(3) \\
\hline Ru2- N11 & $2.062(5)$ & & & Ru2-N11 & $2.077(8)$ & & \\
\hline Ru2-N9 & $2.069(4)$ & & & Ru2-N9 & $2.091(8)$ & & \\
\hline Ru2-N10 & $2.071(4)$ & & & Ru2-N10 & $2.081(7)$ & & \\
\hline Ru2-N4 & $2.085(4)$ & & & Ru2-N4 & $2.080(7)$ & & \\
\hline
\end{tabular}


Table B3.3 Reduction potentials for $\left[\left\{\mathrm{Ru}(\mathrm{bpy})_{2}\right\}_{2}(\mu-\mathrm{BL})\right]^{4+}$ in $0.1 \mathrm{M}\left[\left(n-\mathrm{C}_{4} \mathrm{H}_{9}\right)_{4} \mathrm{~N}\right] \mathrm{PF}_{6} / \mathrm{CH}_{3} \mathrm{CN}{ }^{\mathrm{a}}$

\begin{tabular}{|c|c|c|c|c|c|c|c|}
\hline $\mathrm{BL}$ & Diastereoisomer & $\mathrm{E}_{\text {red1 }}$ & $\mathrm{E}_{\text {red2 }}$ & $\mathrm{E}_{\text {red3 }}$ & $\mathrm{E}_{\text {red4 }}$ & $\mathrm{E}_{\text {red5 }}$ & $\mathrm{E}_{\text {red6 }}$ \\
\hline $\mathrm{dpb}$ & meso & -624 & -1268 & -1888 & -2116 & -2240 & -2552 \\
& rac & -624 & -1260 & -1876 & $\mathrm{c}$ & -2252 & -2640 \\
\hline $\mathrm{dpb}^{\prime}$ & meso & -496 & -1128 & -1868 & -1981 & -2144 & -2272 \\
& rac & -464 & -1116 & -1888 & -2180 & -2716 & \\
\hline $\mathrm{dpq}$ & meso & -596 & -1332 & -1900 & -2178 & -2203 & \\
& rac & -592 & -1268 & -1956 & -2177 & -2240 & \\
\hline $\mathrm{ppz}$ & meso & -956 & -1704 & -1890 & -1956 & -2108 & -2356 \\
& rac & -944 & -1696 & -1878 & -1975 & -2116 & -2360 \\
\hline $2,3-\mathrm{dpp}$ & meso & -1044 & -1540 & $-1900^{\mathrm{b}, \mathrm{c}}$ & $-2168^{\mathrm{b}}$ & & \\
& rac & -1048 & -1560 & $-1888^{\mathrm{b}, \mathrm{c}}$ & $-2248^{\mathrm{b}}$ & & \\
\hline $\mathrm{dpo}$ & meso & $-820^{\mathrm{c}}$ & -1821 & -1880 & -2100 & & \\
& rac & $-872^{\mathrm{c}}$ & $-1892^{\mathrm{b}}$ & & -2144 & & \\
\hline $\mathrm{dpt}$ & meso & -1032 & & & & & \\
& rac & -968 & & & & & \\
\hline
\end{tabular}

Potentials are quoted $\pm 3 \mathrm{mV} .{ }^{b}$ Processes complicated by adsorption/desorption peaks. ${ }^{\mathrm{c}}$ Irreversible reduction process. ${ }^{\mathrm{d}}$ Two-electron reduction process.

Table B3.4 Electrochemical data (in $\mathrm{mV}$ relative to the $\mathrm{Fc}^{+} / \mathrm{Fc}^{0}$ couple) and comproportionation constants $\mathrm{s}^{\mathrm{a}}\left(\mathrm{K}_{\mathrm{c}}\right)$ for $\left[\left\{\mathrm{Ru}(\mathrm{bpy})_{2}\right\}_{2}(\mu-\mathrm{BL})\right]^{4+}$ in $0.1 \mathrm{M}\left[\left(n-\mathrm{C}_{4} \mathrm{H}_{9}\right)_{4} \mathrm{~N}\right] \mathrm{PF}_{6} / \mathrm{CH}_{3} \mathrm{CN}{ }^{\mathrm{b}}$

\begin{tabular}{|c|c|c|c|c|c|c|c|c|c|}
\hline $\mathrm{BL}$ & Diastereoisomer & $\mathrm{K}_{\mathrm{c}}$ & $\Delta \mathrm{E}_{\mathrm{ox}}$ & $\mathrm{E}_{\mathrm{ox} 2}$ & $\mathrm{E}_{\mathrm{ox} 1}$ & $\mathrm{E}_{\text {red1 }}$ & $\mathrm{E}_{\text {red2 }}$ & $\mathrm{E}_{\text {red3 }}$ & $\mathrm{E}_{\text {red4 }}$ \\
\hline $\mathrm{dpb}$ & meso & 2060 & 196 & 1280 & 1084 & -624 & -1268 & $-1888^{\mathrm{d}}$ & -2116 \\
& rac & 944 & 176 & 1280 & 1104 & -624 & -1260 & $-1876^{\mathrm{c}}$ & $-2252^{\mathrm{f}}$ \\
\hline $\mathrm{dpo}$ & meso & 1220000 & 360 & 1536 & 1176 & $-820^{\mathrm{e}}$ & -1821 & -1880 & -2100 \\
& rac & 478000 & 336 & 1536 & 1200 & $-872^{\mathrm{e}}$ & $-1892^{\mathrm{c}}$ & & -2144 \\
\hline $\mathrm{bpt}^{-}$ & meso & 188000 & 312 & 992 & 680 & $-1764^{\mathrm{c}}$ & -1980 & $-2034^{\mathrm{d}}$ & $-2626^{\mathrm{d}, \mathrm{e}}$ \\
& rac & 161000 & 308 & 992 & 684 & $-1776^{\mathrm{c}}$ & $-2015^{\mathrm{c}, \mathrm{e}}$ & & $-2633^{\mathrm{d}, \mathrm{e}}$ \\
\hline $\mathrm{bpm}$ & meso & 1760 & 192 & 1384 & 1192 & -794 & -1466 & $-1948^{\mathrm{c}, \mathrm{d}}$ & $-2368^{\mathrm{d}, \mathrm{e}}$ \\
& rac & 1510 & 188 & 1380 & 1192 & -792 & -1480 & $-1928^{\mathrm{c} . \mathrm{d}}$ & $-2360^{\mathrm{d}, \mathrm{e}}$ \\
\hline dbneil & meso & 1100 & 180 & 1268 & 1088 & -544 & -945 & -1676 & $-1972^{\mathrm{e}}$ \\
& rac & 1190 & 182 & 1270 & 1088 & -540 & -946 & -1668 & -1965 \\
\hline
\end{tabular}

${ }^{\mathrm{a}} \mathrm{K}_{\mathrm{c}}$ values determined from equation 1.18(a). ${ }^{\mathrm{b}} \Delta \mathrm{E}_{\mathrm{ox}}=\mathrm{E}_{\mathrm{ox} 2}-\mathrm{E}_{\mathrm{ox} 1}$. Potentials are quoted $\pm 3 \mathrm{mV}$. ${ }^{\mathrm{c}}$ Processes complicated by adsorption/desorption peaks. ${ }^{\mathrm{d}}$ Irreversible reduction process. ${ }^{\mathrm{e}}$ Two-electron reduction process.

Table B3.5 Electrochemical data (in $\mathrm{mV}$ relative to the $\mathrm{Fc}^{+} / \mathrm{Fc}^{0}$ couple) and comproportionation constants ${ }^{\mathrm{a}}\left(\mathrm{K}_{\mathrm{c}}\right)$ for $\left[\left\{\mathrm{Ru}(\mathrm{bpy})_{2}\right\}_{2}(\mu-\mathrm{BL})\right]^{4+}$ in $0.02 \mathrm{M}\left[\left(n-\mathrm{C}_{4} \mathrm{H}_{9}\right)_{4} \mathrm{~N}\right]\left\{\mathrm{B}\left(\mathrm{C}_{6} \mathrm{~F}_{5}\right)_{4}\right\} / \mathrm{CH}_{3} \mathrm{CN} .^{\mathrm{b}}$

\begin{tabular}{|c|c|c|c|c|c|c|c|c|c|}
\hline $\mathrm{BL}$ & Diastereoisomer & $\mathrm{K}_{\mathrm{c}}$ & $\Delta \mathrm{E}_{\mathrm{ox}}$ & $\mathrm{E}_{\mathrm{ox2}}$ & $\mathrm{E}_{\text {ox1 }}$ & $\mathrm{E}_{\text {red1 }}$ & $\mathrm{E}_{\text {red2 }}$ & $\mathrm{E}_{\text {red3 }}$ & $\mathrm{E}_{\text {red4 }}$ \\
\hline $\mathrm{dpb}$ & meso & 5230 & 220 & 1376 & 1156 & -601 & -1197 & -1850 & -1923 \\
& rac & 2810 & 204 & 1372 & 1168 & -603 & -1227 & $-1887^{\mathrm{d}}$ & \\
\hline $\mathrm{dpo}$ & meso & & & $\mathrm{f}$ & 1240 & $-787^{\mathrm{d}}$ & $-1844^{\mathrm{e}}$ & & $-2098^{\mathrm{e}}$ \\
& rac & & & $\mathrm{f}$ & 1288 & $-820^{\mathrm{d}}$ & $-1868^{\mathrm{e}}$ & & $-2118^{\mathrm{e}}$ \\
\hline $\mathrm{bpt}^{-}$ & meso & 409000 & 332 & 1068 & 736 & -1741 & -1800 & -2018 & -2110 \\
& rac & 857000 & 351 & 1064 & 713 & $-1769^{\mathrm{d}}$ & & -2000 & -2064 \\
\hline bpm & meso & 5230 & 220 & 1500 & 1280 & -748 & $-1396^{\mathrm{e}}$ & $-1792^{\mathrm{e}}$ & $-2066^{\mathrm{e}}$ \\
& rac & 5230 & 220 & 1520 & 1300 & -770 & $-1473^{\mathrm{e}}$ & $-1837^{\mathrm{e}}$ & $-2045^{\mathrm{e}}$ \\
\hline dbneil & meso & 733000 & 347 & 931 & 584 & -508 & -908 & -1588 & -1751 \\
& rac & 793000 & 349 & 941 & 592 & -516 & -928 & -1648 & -1828 \\
\hline
\end{tabular}

${ }^{\mathrm{a}} \mathrm{K}_{\mathrm{c}}$ values determined from equation 1.18(a). ${ }^{\mathrm{b}} \Delta \mathrm{E}_{\mathrm{ox}}=\mathrm{E}_{\mathrm{ox} 2}-\mathrm{E}_{\mathrm{ox} 1}$. Potentials are quoted $\pm 3 \mathrm{mV}$. ${ }^{\mathrm{c}}$ Processes complicated by adsorption/desorption peaks. ${ }^{\mathrm{d}}$ Irreversible reduction process. ${ }^{\mathrm{e}}$ Two-electron reduction process. ${ }^{\mathrm{f}}$ Potential more anodic than the positive potential limit of the electrolyte. 
Table B3.6 UV/Vis/NIR spectral data of the reduced absorption spectra $(\varepsilon / v$ vs. $v)$ for the diastereoisomers of $\left[\left\{\mathrm{Ru}(\mathrm{pp})_{2}\right\}_{2}(\mu-\mathrm{BL})\right]^{4+}$ in $0.1 \mathrm{M}\left[\left(n-\mathrm{C}_{4} \mathrm{H}_{9}\right)_{4} \mathrm{~N}\right] \mathrm{PF} \mathrm{F}_{6} / \mathrm{CH}_{3} \mathrm{CN}$ at $-35^{\circ} \mathrm{C}$. The parameters of the IVCT transitions are indicated in bold type. ${ }^{\text {a }}$

\begin{tabular}{|c|c|c|c|c|c|c|c|c|c|c|c|}
\hline \multicolumn{2}{|l|}{$\mathrm{BL}$} & \multicolumn{2}{|c|}{$\mathrm{dpb}$} & \multicolumn{2}{|c|}{ dpb' } & \multicolumn{2}{|c|}{ dpq' } & \multicolumn{2}{|c|}{$\mathrm{ppz}$} & \multicolumn{2}{|c|}{ 2,3-dpp } \\
\hline Diastereoisomer & $n$ & $v_{\max } / \mathrm{cm}^{-1}$ & $(\varepsilon / \nu)_{\max } / \mathrm{M}^{-1}$ & $v_{\max } / \mathrm{cm}^{-1}$ & $(\varepsilon / \nu)_{\max } / \mathrm{M}^{-1}$ & $v_{\max } / \mathrm{cm}^{-1}$ & $(\varepsilon / v)_{\max } / \mathrm{M}^{-1}$ & $v_{\max } / \mathrm{cm}^{-1}$ & $(\varepsilon / \nu)_{\max } / \mathrm{M}^{-1}$ & $v_{\max } / \mathrm{cm}^{-1}$ & $(\varepsilon / v)_{\max } / \mathrm{M}^{-1}$ \\
\hline \multirow[t]{3}{*}{ meso } & 4 & $\begin{array}{c}\text { sh } 13070 \\
15530 \\
23520 \\
24460 \\
26870 \\
29450\end{array}$ & $\begin{array}{l}0.2958 \\
1.4440 \\
1.0810 \\
0.9824 \\
1.5690 \\
1.4350\end{array}$ & $\begin{array}{c}\text { sh } 11670 \\
13880 \\
16900 \\
24780 \\
29140\end{array}$ & $\begin{array}{l}0.2646 \\
2.0415 \\
0.2133 \\
1.3524 \\
1.5091\end{array}$ & $\begin{array}{c}\text { sh } 12900 \\
15150 \\
18110 \\
23480 \\
26690\end{array}$ & $\begin{array}{l}0.2813 \\
1.9723 \\
0.1567 \\
0.8393 \\
1.0829\end{array}$ & $\begin{array}{l}17640 \\
23440\end{array}$ & $\begin{array}{l}1.4682 \\
0.9021\end{array}$ & $\begin{array}{l}19056 \\
23361\end{array}$ & $\begin{array}{l}1.3356 \\
1.0805\end{array}$ \\
\hline & 5 & $\begin{array}{c}\mathbf{5 2 0 5} \\
15120 \\
\text { sh } 17370 \\
25920\end{array}$ & $\begin{array}{l}\mathbf{0 . 3 0 6 2} \\
0.7678 \\
0.4504 \\
1.4740\end{array}$ & $\begin{array}{c}\mathbf{5 2 8 5} \\
9253 \\
11420 \\
13930 \\
21660 \\
22770 \\
24760 \\
28040\end{array}$ & $\begin{array}{l}\mathbf{0 . 8 0 8 4} \\
0.0998 \\
0.3598 \\
0.9596 \\
0.3788 \\
0.4739 \\
0.7086 \\
1.2751 \\
\end{array}$ & $\begin{array}{c}\mathbf{5 1 9 4} \\
14900 \\
25730\end{array}$ & $\begin{array}{l}\mathbf{0 . 5 9 3 4} \\
1.4369 \\
1.0688\end{array}$ & $\begin{array}{c}\mathbf{5 3 7 0} \\
11670 \\
13320 \\
17370 \\
24900\end{array}$ & $\begin{array}{l}\mathbf{0 . 8 7 2 1} \\
0.1566 \\
0.1400 \\
0.9003 \\
0.3950\end{array}$ & $\begin{array}{c}\mathbf{5 3 4 0} \\
18696 \\
28680\end{array}$ & $\begin{array}{l}\mathbf{0 . 2 8 9 0} \\
0.4676 \\
0.8088\end{array}$ \\
\hline & 6 & $\begin{array}{c}15000 \\
16870 \\
25230\end{array}$ & $\begin{array}{l}0.2253 \\
1.2709\end{array}$ & $\begin{array}{l}14210 \\
20850 \\
22140 \\
27440\end{array}$ & $\begin{array}{l}0.1497 \\
0.8348 \\
0.7751 \\
1.5330 \\
\end{array}$ & & & $\begin{array}{l}11630 \\
16950\end{array}$ & $\begin{array}{l}1.9560 \\
0.2601\end{array}$ & & \\
\hline \multirow[t]{3}{*}{ rac } & 4 & $\begin{array}{c}\text { sh } 13070 \\
15420 \\
20480 \\
23470 \\
\text { sh } 24400 \\
26820 \\
29400\end{array}$ & $\begin{array}{l}0.2269 \\
1.4152 \\
0.3499 \\
1.1100 \\
0.9878 \\
1.4918 \\
1.5252\end{array}$ & $\begin{array}{c}\text { sh } 11770 \\
13870 \\
16850 \\
24780 \\
29150\end{array}$ & $\begin{array}{l}0.2532 \\
1.8962 \\
0.1661 \\
1.2155 \\
1.3248\end{array}$ & $\begin{array}{c}\text { sh } 13090 \\
15290 \\
18280 \\
23370 \\
26720\end{array}$ & $\begin{array}{l}0.4341 \\
1.9334 \\
0.1602 \\
0.8662 \\
1.0949\end{array}$ & $\begin{array}{l}17630 \\
23420\end{array}$ & $\begin{array}{l}1.4715 \\
0.8987\end{array}$ & $\begin{array}{l}19070 \\
23286\end{array}$ & $\begin{array}{l}1.2995 \\
0.8616\end{array}$ \\
\hline & 5 & $\begin{array}{c}\mathbf{4 9 9 8} \\
14980 \\
25700\end{array}$ & $\begin{array}{l}\mathbf{0 . 4 5 7 2} \\
0.6835 \\
1.6362\end{array}$ & $\begin{array}{c}\mathbf{5 2 9 0} \\
9223 \\
11250 \\
13920 \\
21630 \\
22680 \\
24570 \\
27980 \\
\end{array}$ & $\begin{array}{l}\mathbf{0 . 7 2 2 2} \\
0.0878 \\
0.2793 \\
0.9050 \\
0.3490 \\
0.4360 \\
0.6573 \\
1.1377 \\
\end{array}$ & $\begin{array}{c}5223 \\
14980 \\
25840\end{array}$ & $\begin{array}{l}\mathbf{0 . 6 3 2 9} \\
1.2244 \\
0.8573\end{array}$ & $\begin{array}{c}\mathbf{5 3 9 0} \\
11720 \\
13090 \\
17340 \\
24980\end{array}$ & $\begin{array}{l}\mathbf{0 . 8 4 9 4} \\
0.1488 \\
0.2145 \\
0.9043 \\
0.3862\end{array}$ & $\begin{array}{c}5307 \\
18666 \\
28642\end{array}$ & $\begin{array}{l}\mathbf{0 . 5 9 8 0} \\
0.5886 \\
0.9808\end{array}$ \\
\hline & 6 & $\begin{array}{l}17760 \\
25170\end{array}$ & $\begin{array}{l}0.1270 \\
1.9485\end{array}$ & $\begin{array}{l}12730 \\
15880 \\
20850 \\
22180 \\
27430\end{array}$ & $\begin{array}{l}0.3188 \\
0.2005 \\
0.6463 \\
0.6198 \\
1.3068\end{array}$ & $\begin{array}{l}14450 \\
21330 \\
23560\end{array}$ & $\begin{array}{l}0.2372 \\
0.3455 \\
0.6253\end{array}$ & $\begin{array}{l}11640 \\
16930\end{array}$ & $\begin{array}{l}1.9510 \\
0.2600\end{array}$ & & \\
\hline
\end{tabular}

Errors in $v_{\max }$ and $(\varepsilon / v)_{\max }$ are $\pm 10 \mathrm{~cm}^{-1}$ and $\pm 0.0001 \mathrm{M}^{-1}$, respectively. ${ }^{\mathrm{b}}$ Measurements performed in $0.02 \mathrm{M}\left[\left(n-\mathrm{C}_{4} \mathrm{H}_{9}\right)_{4} \mathrm{~N}\right]\left\{\mathrm{B}\left(\mathrm{C}_{6} \mathrm{~F}_{5}\right)_{4}\right\} / \mathrm{CH}_{3} \mathrm{CN} ;+6$ state not measured. 
Table B3.7 UV/Vis/NIR spectral data for $\left[\mathrm{Ru}(\mathrm{bpy})_{2}(\mathrm{BL})\right]^{n+}(n=2,3)$ in $0.1 \mathrm{M}\left[\left(n-\mathrm{C}_{4} \mathrm{H}_{9}\right)_{4} \mathrm{~N}\right] \mathrm{PF}_{6} / \mathrm{CH}_{3} \mathrm{CN}$.

\begin{tabular}{|c|c|c|c|}
\hline BL & $n$ & $\begin{array}{c}v \\
\pm 10 / \mathrm{cm}^{-1}\end{array}$ & $\begin{array}{c}(\varepsilon / v) \\
\pm 0.0001 / \mathrm{M}^{-1}\end{array}$ \\
\hline $\mathrm{dpb}$ & 2 & $\operatorname{sh} 15750$ & 0.0873 \\
& & 18110 & 0.3459 \\
& & 24570 & 0.4069 \\
& & $s h 27560$ & 0.4952 \\
\hline & 3 & 17270 & 0.3140 \\
\hline dpb' $^{\prime}$ & 2 & 17150 & 0.5713 \\
& & 23360 & 0.5007 \\
& & 24450 & 0.4867 \\
\cline { 2 - 4 } & 3 & 16510 & 0.3500 \\
\hline
\end{tabular}

Table B3.8 NIR spectral data for meso- and rac- $\left[\left\{\mathrm{Ru}(\mathrm{bpy})_{2}\right\}_{2}(\mu-\mathrm{BL})\right]^{4+}\{\mathrm{BL}=\mathrm{dpb}$, dpb' $\}$ in $0.1 \mathrm{M}[(n-$

$\left.\left.\mathrm{C}_{4} \mathrm{H}_{9}\right)_{4} \mathrm{~N}\right] \mathrm{PF}_{6} / \mathrm{CH}_{3} \mathrm{CN}$ at $-35^{\circ} \mathrm{C}$. The parameters for the overall NIR band envelopes are shown in bold type. Details of the deconvoluted bands are in normal type.

\begin{tabular}{|c|c|c|c|c|c|c|c|c|c|}
\hline \multirow[b]{2}{*}{ Diastereoisomer } & & \multicolumn{4}{|c|}{ dpb } & \multicolumn{4}{|c|}{$\mathrm{dpb}^{\prime}$} \\
\hline & & $\begin{array}{l}v_{\max } \\
\pm 10 \\
/ \mathrm{cm}^{-1}\end{array}$ & $\begin{array}{c}(\varepsilon / v)_{\max } \\
\pm 0.0001 \\
/ \mathrm{M}^{-1}\end{array}$ & $\begin{array}{l}\Delta v_{1 / 2} \\
\pm 10 \\
/ \mathrm{cm}^{-1}\end{array}$ & $\begin{array}{c}M_{1} \\
/ \mathrm{cm}^{-1}\end{array}$ & $\begin{array}{l}v_{\max } \\
\pm 10 \\
/ \mathrm{cm}^{-1}\end{array}$ & $\begin{array}{c}(\varepsilon / v)_{\max } \\
\pm 0.0001 \\
/ \mathrm{M}^{-1}\end{array}$ & $\begin{array}{l}\Delta v_{1 / 2} \\
\pm 10 \\
/ \mathrm{cm}^{-1}\end{array}$ & $\begin{array}{c}M_{1} \\
/ \mathrm{cm}^{-1}\end{array}$ \\
\hline \multirow[t]{4}{*}{ meso } & & 5205 & 0.3062 & 1910 & 5655 & 5285 & 0.8084 & 1070 & 5510 \\
\hline & (a) & $\begin{array}{l}3773 \\
5180 \\
6196 \\
6771 \\
\end{array}$ & $\begin{array}{l}0.0472 \\
0.2943 \\
0.0760 \\
0.0712 \\
\end{array}$ & $\begin{array}{c}590 \\
1356 \\
996 \\
1796 \\
\end{array}$ & & $\begin{array}{l}3942 \\
5269 \\
5938 \\
6366 \\
\end{array}$ & $\begin{array}{l}0.0318 \\
0.7759 \\
0.1787 \\
0.1114 \\
\end{array}$ & $\begin{array}{c}1734 \\
835 \\
689 \\
1353 \\
\end{array}$ & \\
\hline & (b) & $\begin{array}{l}3552 \\
4983 \\
5490 \\
6659 \\
\end{array}$ & $\begin{array}{l}0.0506 \\
0.1076 \\
0.2170 \\
0.0650\end{array}$ & $\begin{array}{c}1291 \\
892 \\
130 \\
2017 \\
\end{array}$ & & $\begin{array}{l}3942 \\
5568 \\
5173 \\
6013 \\
\end{array}$ & $\begin{array}{l}0.0318 \\
0.3452 \\
0.4757 \\
0.1403\end{array}$ & $\begin{array}{c}1734 \\
990 \\
714 \\
1731 \\
\end{array}$ & \\
\hline & (c) & $\begin{array}{l}3991 \\
5404 \\
5023 \\
6787 \\
\end{array}$ & $\begin{array}{l}0.0165 \\
0.2471 \\
0.0700 \\
0.0547 \\
\end{array}$ & $\begin{array}{c}207 \\
1847 \\
756 \\
1941 \\
\end{array}$ & & $\begin{array}{l}3974 \\
5169 \\
5484 \\
6113 \\
\end{array}$ & $\begin{array}{l}0.0301 \\
0.3737 \\
0.4352 \\
0.1268 \\
\end{array}$ & $\begin{array}{c}2166 \\
671 \\
1053 \\
1649 \\
\end{array}$ & \\
\hline \multirow[t]{4}{*}{ rac } & & 5000 & 0.4572 & 1740 & 5450 & 5290 & 0.7222 & 1077 & 5510 \\
\hline & (d) & $\begin{array}{l}3940 \\
4979 \\
5954 \\
6573 \\
\end{array}$ & $\begin{array}{l}0.0379 \\
0.4383 \\
0.1197 \\
0.0836 \\
\end{array}$ & $\begin{array}{c}357 \\
1267 \\
1016 \\
1840 \\
\end{array}$ & & $\begin{array}{l}4160 \\
5290 \\
5997 \\
6484 \\
\end{array}$ & $\begin{array}{l}0.0222 \\
0.7169 \\
0.1436 \\
0.0885 \\
\end{array}$ & $\begin{array}{c}809 \\
883 \\
672 \\
1217 \\
\end{array}$ & \\
\hline & (e) & $\begin{array}{l}3965 \\
4792 \\
5292 \\
6145 \\
\end{array}$ & $\begin{array}{l}0.0492 \\
0.1878 \\
0.2709 \\
0.1050 \\
\end{array}$ & $\begin{array}{c}504 \\
911 \\
1525 \\
2271 \\
\end{array}$ & & $\begin{array}{l}3967 \\
5188 \\
5657 \\
5910 \\
\end{array}$ & $\begin{array}{l}0.0199 \\
0.5045 \\
0.2288 \\
0.1491 \\
\end{array}$ & $\begin{array}{c}827 \\
752 \\
883 \\
1745 \\
\end{array}$ & \\
\hline & (f) & $\begin{array}{l}4865 \\
5176 \\
6576\end{array}$ & $\begin{array}{l}0.1012 \\
0.3679 \\
0.0686\end{array}$ & $\begin{array}{c}704 \\
1762 \\
1976\end{array}$ & & $\begin{array}{l}3968 \\
5188 \\
5657 \\
5910 \\
\end{array}$ & $\begin{array}{l}0.0199 \\
0.5045 \\
0.2288 \\
0.1491 \\
\end{array}$ & $\begin{array}{c}827 \\
752 \\
883 \\
1745 \\
\end{array}$ & \\
\hline
\end{tabular}


(a)

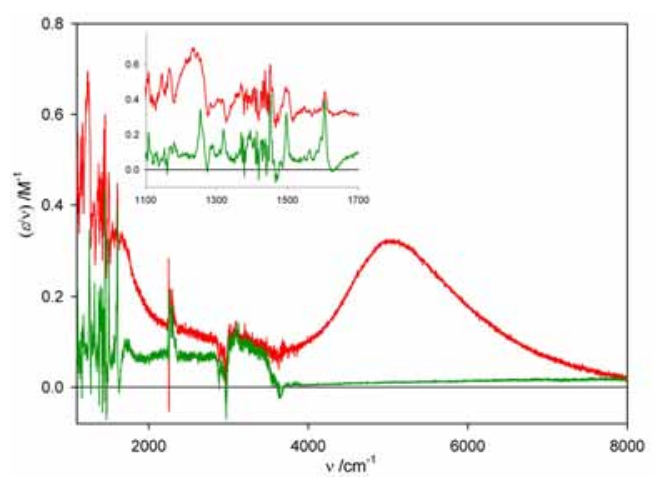

(b)

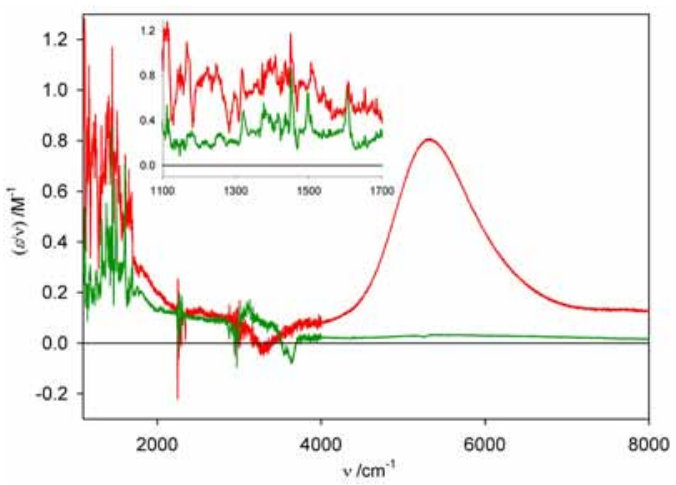

Figure B3.2 Differential mid-IR spectra of the mixed-valence $\left[\left\{\mathrm{Ru}(\mathrm{bpy})_{2}\right\}_{2}(\mu-\mathrm{BL})\right]^{5+}(-)$ and fully-oxidised $\left[\left\{\mathrm{Ru}(\mathrm{bpy})_{2}\right\}_{2}(\mu-\mathrm{BL})\right]^{6+}(-)$ forms in $0.1 \mathrm{M}\left[\left(n-\mathrm{C}_{4} \mathrm{H}_{9}\right)_{4} \mathrm{~N}\right] \mathrm{PF}_{6} / \mathrm{CH}_{3} \mathrm{CN}$ at $+25^{\circ} \mathrm{C}$, where $\mathrm{BL}=\mathrm{dpb}(\mathbf{a})$ and dpb' (b). 
Table B3.9 UV/Vis/NIR spectral data of the reduced absorption spectra $(\varepsilon / v$ vs. $v)$ for $\left[\left\{\operatorname{Ru}(b p y)_{2}\right\}_{2}(\mu-B L)\right]^{n+}$ $\{\mathrm{BL}=\mathrm{dpo}$, dpt $\}$ in $0.1 \mathrm{M}\left[\left(n-\mathrm{C}_{4} \mathrm{H}_{9}\right)_{4} \mathrm{~N}\right] \mathrm{PF}_{6} / \mathrm{CH}_{3} \mathrm{CN}$ at $-35^{\circ} \mathrm{C}$. The NIR spectral data are indicated in bold type. ${ }^{\mathrm{a}}$

\begin{tabular}{|c|c|c|c|}
\hline \multirow[b]{2}{*}{ Diastereoisomer } & \multirow[b]{2}{*}{$n$} & dpo & dpt \\
\hline & & $\begin{array}{c}v_{\max } \pm 10 / \mathrm{cm}^{-1} \\
\left\{(\varepsilon / v)_{\max } \pm 0.0001 / \mathrm{M}^{-1} \mathrm{~cm}^{-1}\right\}\end{array}$ & $\begin{array}{c}v_{\max } \pm 10 / \mathrm{cm}^{-1} \\
\left\{(\varepsilon / v)_{\max } \pm 0.0001 / \mathrm{M}^{-1} \mathrm{~cm}^{-1}\right\}\end{array}$ \\
\hline \multirow[t]{3}{*}{ meso } & 4 & $\begin{array}{c}\text { sh } 17830(0.3011) \\
20160(0.6828) \\
24590(0.8858) \\
\end{array}$ & $\begin{array}{c}\text { sh } 16702(0.3970) \\
19000(0.9673) \\
23510(0.8643) \\
\end{array}$ \\
\hline & 5 & $\begin{array}{c}\mathbf{6 5 9 0}(\mathbf{1 . 5 2 1 8}) \\
15880(0.2575) \\
18870(0.4582) \\
24920(0.4084)\end{array}$ & $\begin{array}{c}5625(\mathbf{1 . 4 9 0 8 )} \\
14380(0.1880) \\
17810(0.5303) \\
23543(0.3379)\end{array}$ \\
\hline & 6 & $\begin{array}{c}12359(0.0519) \\
18771(0.1312) \\
22671(0.2185)\end{array}$ & \\
\hline \multirow[t]{2}{*}{ rac } & 4 & $\begin{array}{c}\text { sh } 17892(0.3743) \\
20190(0.7951) \\
24620(1.0359) \\
\end{array}$ & $\begin{array}{c}\text { sh } 16690(0.3992) \\
18920(0.9629) \\
23570(0.9117)\end{array}$ \\
\hline & 5 & $\begin{array}{c}\mathbf{6 4 6 0}(\mathbf{1 . 2 7 3 7 )} \\
15895(0.1883) \\
18840(0.3703) \\
24910(0.3522)\end{array}$ & $\begin{array}{c}\mathbf{5 6 2 8}(\mathbf{0 . 8 9 9 4}) \\
14410(0.2326) \\
18370(0.5161) \\
23930(0.4854)\end{array}$ \\
\hline
\end{tabular}

${ }^{\mathrm{a}}$ meso- $\left[\left\{\mathrm{Ru}(\mathrm{bpy})_{2}\right\}_{2}(\mu-\mathrm{dpt})\right]^{6+}$ and rac-[\{Ru(bpy)$\left.\}_{2}(\mu-\mathrm{BL})\right]^{6+}\{\mathrm{BL}=\mathrm{dpo}, \mathrm{dpt}\}$ not measured.

Table B3.10 NIR spectral data of the reduced absorption spectra ( $\varepsilon / v v s . v)$ for meso- and rac- $\left[\left\{\mathrm{Ru}(\mathrm{bpy})_{2}\right\}_{2}(\mu-\mathrm{BL})\right]^{5+}\{\mathrm{BL}=\mathrm{dpo}$, dpt $\}$ in $0.1 \mathrm{M}\left[\left(n-\mathrm{C}_{4} \mathrm{H}_{9}\right)_{4} \mathrm{~N}\right] \mathrm{PF}_{6} / \mathrm{CH}_{3} \mathrm{CN}$ at $-35^{\circ} \mathrm{C}$. The parameters for the overall NIR band envelopes are shown in bold type. Details of the deconvoluted bands are in normal type.

\begin{tabular}{|c|c|c|c|c|c|c|c|}
\hline Complex & Component & $\begin{array}{l}v_{\max } \\
\pm 10 \\
/ \mathrm{cm}^{-1}\end{array}$ & $\begin{array}{c}(\varepsilon / v)_{\max } \\
\pm 0.0001 \\
/ \mathrm{M}^{-1}\end{array}$ & $\begin{array}{l}\Delta v_{1 / 2} \\
\pm 10 \\
/ \mathrm{cm}^{-1}\end{array}$ & $\begin{array}{l}\Delta v_{1 / 2}{ }^{o} \\
/ \mathrm{cm}^{-1}\end{array}$ & $\begin{array}{c}M_{0} \\
/ \mathrm{M}^{-1}\end{array}$ & $\begin{array}{c}\mathrm{H}_{\mathrm{ab}} \\
/ \mathrm{cm}^{-1}\end{array}$ \\
\hline meso- $\left[\left\{\mathrm{Ru}(\mathrm{bpy})_{2}\right\}_{2}(\mu-\mathrm{dpo})\right]^{5+}$ & $\begin{array}{l}1 \\
2 \\
3\end{array}$ & $\begin{array}{l}\mathbf{6 5 9 0} \\
5140 \\
6570 \\
7686\end{array}$ & $\begin{array}{l}\mathbf{1 . 5 2 1 8} \\
0.3339 \\
1.3959 \\
0.3742\end{array}$ & $\begin{array}{l}\mathbf{1 4 7 0} \\
1330 \\
1210 \\
1620\end{array}$ & 3480 & $\begin{array}{l}3211 \\
472.1 \\
1802 \\
645.7\end{array}$ & $\begin{array}{l}3295 \\
2570\end{array}$ \\
\hline rac- $\left[\left\{\mathrm{Ru}(\mathrm{bpy})_{2}\right\}_{2}(\mu-\mathrm{dpo})\right]^{5+}$ & $\begin{array}{l}1 \\
2 \\
3\end{array}$ & $\begin{array}{l}\mathbf{6 4 6 0} \\
3782 \\
5020 \\
6438 \\
7650\end{array}$ & $\begin{array}{l}\mathbf{1 . 2 7 3 7} \\
0.2670 \\
0.1574 \\
0.9936 \\
0.2676\end{array}$ & $\begin{array}{l}\mathbf{1 4 9 2} \\
1950 \\
1050 \\
1267 \\
1564\end{array}$ & 3440 & $\begin{array}{c}2650 \\
350.9 \\
175.9 \\
1340 \\
445.7\end{array}$ & $\begin{array}{r}3230 \\
2510\end{array}$ \\
\hline meso- $\left[\left\{\mathrm{Ru}(\mathrm{bpy})_{2}\right\}_{2}(\mu-\mathrm{dpt})\right]^{5+}$ & $\begin{array}{l}1 \\
2 \\
3\end{array}$ & $\begin{array}{l}5625 \\
4425 \\
5600 \\
6460 \\
7175\end{array}$ & $\begin{array}{l}\mathbf{1 . 4 9 0 8} \\
0.2682 \\
1.4058 \\
0.4536 \\
0.1540\end{array}$ & $\begin{array}{c}\mathbf{1 3 4 6} \\
850.4 \\
1023 \\
1016 \\
1405\end{array}$ & 3210 & $\begin{array}{c}2538 \\
241.5 \\
1530 \\
490.7 \\
230.4\end{array}$ & $\begin{array}{l}2813 \\
2800\end{array}$ \\
\hline rac $-\left[\left\{\mathrm{Ru}(\mathrm{bpy})_{2}\right\}_{2}(\mu-\mathrm{dpt})\right]^{5+}$ & $\begin{array}{l}1 \\
2 \\
3\end{array}$ & $\begin{array}{l}\mathbf{5 6 2 8} \\
4400 \\
5600 \\
6515 \\
7260\end{array}$ & $\begin{array}{l}\mathbf{0 . 8 9 9 5} \\
0.1552 \\
0.8453 \\
0.3023 \\
0.1076\end{array}$ & $\begin{array}{c}\mathbf{1 5 1 6} \\
790 \\
1093 \\
1050 \\
1543\end{array}$ & 3215 & $\begin{array}{l}\mathbf{1 6 5 6} \\
130.1 \\
984.3 \\
338.5 \\
176.8\end{array}$ & $\begin{array}{l}2814 \\
2800\end{array}$ \\
\hline
\end{tabular}


(a)

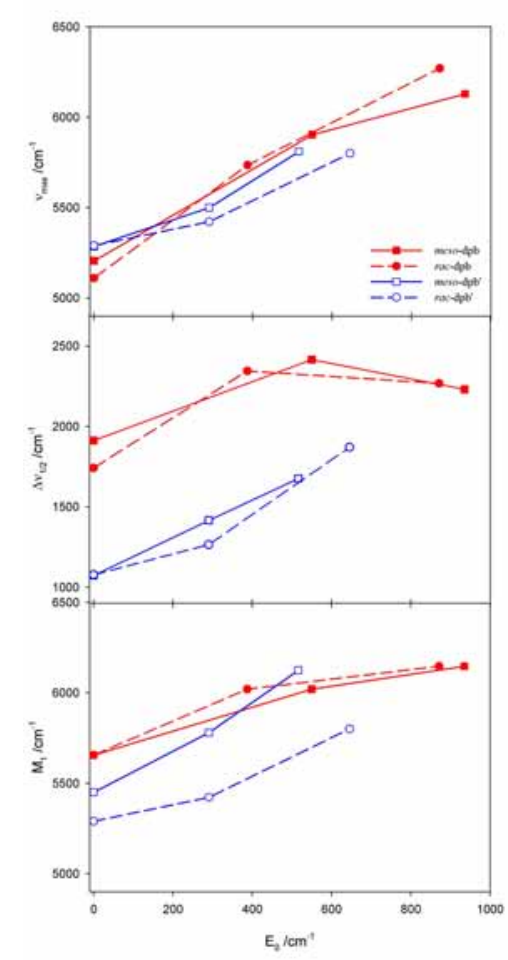

Figure B3.3 (a) $v_{\max }$ (b) $\Delta v_{1 / 2}$ and (c) $M_{1}$ as a function of $\mathrm{E}_{0}$ for the series $\left[\left\{\mathrm{Ru}(\mathrm{bpy})_{2}\right\}(\mu-\mathrm{BL})\left\{\mathrm{Ru}(\mathrm{pp})_{2}\right\}\right]^{5+}$ $\left\{\mathrm{BL}=\mathrm{dpb}, \mathrm{dpb} ; \mathrm{pp}=\mathrm{bpy}, \mathrm{Me}_{2} \mathrm{bpy}, \mathrm{Me}_{4} \mathrm{bpy}\right\}$. Error bars are omitted for clarity.

Table B3.11 Reduction potentials (in $\mathrm{mV}$ relative to the $\mathrm{Fc}^{+} / \mathrm{Fc}^{0}$ couple) for $\left[\left\{\mathrm{Ru}(\mathrm{bpy})_{2}\right\}\left(\mu \text {-dpb) }\left\{\mathrm{Ru}(\mathrm{pp})_{2}\right\}\right]^{4+}\right.$ in $0.1 \mathrm{M}\left[\left(n-\mathrm{C}_{4} \mathrm{H}_{9}\right)_{4} \mathrm{~N}\right] \mathrm{PF}_{6} / \mathrm{CH}_{3} \mathrm{CN}$.

\begin{tabular}{|c|c|c|c|c|c|c|c|}
\hline pp & Diastereoisomer & $\mathrm{E}_{\text {red1 }}$ & $\mathrm{E}_{\text {red2 }}$ & $\mathrm{E}_{\text {red3 }}$ & $\mathrm{E}_{\text {red4 }}$ & $\mathrm{E}_{\text {red5 }}$ & $\mathrm{E}_{\text {red6 }}$ \\
\hline $\mathrm{Me}_{4}$ bpy & meso & -664 & -1252 & -1604 & -2096 & -2201 & -2412 \\
& rac & -656 & -1304 & -1892 & -2125 & -2202 & -2404 \\
\hline $\mathrm{Me}_{2}$ bpy & meso & -644 & -1292 & -1880 & -1996 & -2156 & -2316 \\
& rac & -644 & -1288 & -1896 & -1996 & -2164 & -2300 \\
\hline bpy & meso & -624 & -1268 & -1888 & -2116 & -2240 & -2552 \\
& rac & -624 & -1260 & -1876 & & -2252 & -2640 \\
\hline
\end{tabular}

Table B3.12 Reduction potentials (in $\mathrm{mV}$ relative to the $\mathrm{Fc}^{+} / \mathrm{Fc}^{0}$ couple) for $\left[\left\{\mathrm{Ru}(\mathrm{bpy})_{2}\right\}\left(\mu \text {-dpb') }\left\{\mathrm{Ru}(\mathrm{pp})_{2}\right\}\right]^{4+}\right.$ in $0.1 \mathrm{M}\left[\left(n-\mathrm{C}_{4} \mathrm{H}_{9}\right)_{4} \mathrm{~N} \mathrm{PF}_{6} / \mathrm{CH}_{3} \mathrm{CN}\right.$.

\begin{tabular}{|c|c|c|c|c|c|c|c|}
\hline $\mathrm{pp}$ & Diastereoisomer & $\mathrm{E}_{\text {red1 }}$ & $\mathrm{E}_{\text {red2 }}$ & $\mathrm{E}_{\text {red3 }}$ & $\mathrm{E}_{\text {red4 }}$ & $\mathrm{E}_{\text {red5 }}$ & $\mathrm{E}_{\text {red6 }}$ \\
\hline $\mathrm{Me}_{4}$ bpy & meso & -496 & -1148 & -1880 & -2172 & -2448 & -2663 \\
& rac & -496 & -1148 & -1876 & -2160 & -2432 & -2743 \\
\hline $\mathrm{Me}_{2}$ bpy & meso & -484 & -1132 & -1883 & -1976 & -2157 & -2260 \\
& rac & -496 & -1128 & -1868 & -1981 & -2144 & -2272 \\
\hline bpy & meso & -464 & -1116 & -1888 & -2180 & -2716 & \\
& rac & -452 & -1096 & -1884 & -2192 & -2720 & \\
\hline
\end{tabular}


Table B3.13 Spectroelectrochemical data for $\left[\left\{\mathrm{Ru}(\mathrm{bpy})_{2}\right\}(\mu-\mathrm{BL})\left\{\mathrm{Ru}(\mathrm{pp})_{2}\right\}\right]^{4+}\{\mathrm{BL}=\mathrm{dpb}$ or dpb' $\}$ in $0.1 \mathrm{M}\left[\left(n-\mathrm{C}_{4} \mathrm{H}_{9}\right)_{4} \mathrm{~N}\right] \mathrm{PF}_{6} / \mathrm{CH}_{3} \mathrm{CN}$.

\begin{tabular}{|c|c|c|c|c|c|c|c|c|c|}
\hline & & \multicolumn{4}{|c|}{$\mathrm{BL}=\mathrm{dpb}$} & \multicolumn{4}{|c|}{$\mathrm{BL}=\mathrm{dpb}^{\prime}$} \\
\hline \multicolumn{2}{|l|}{ pp } & \multicolumn{2}{|c|}{$\mathrm{Me}_{4} \mathrm{bpy}$} & \multicolumn{2}{|c|}{$\mathrm{Me}_{2}$ bpy } & \multicolumn{2}{|c|}{$\mathrm{Me}_{4} \mathrm{bpy}$} & \multicolumn{2}{|c|}{$\mathrm{Me}_{2} \mathrm{bpy}$} \\
\hline Diastereoisomer & $n$ & $\begin{array}{c}v \\
\pm 10 / \mathrm{cm}^{-1}\end{array}$ & $\begin{array}{c}(\varepsilon / v) \\
\pm 0.0001 / \mathrm{M}^{-1}\end{array}$ & $\begin{array}{c}v \\
\pm 10 / \mathrm{cm}^{-1}\end{array}$ & $\begin{array}{c}(\varepsilon / v) \\
\pm 0.0001 / \mathrm{M}^{-1}\end{array}$ & $\begin{array}{c}v \\
\pm 10 / \mathrm{cm}^{-1}\end{array}$ & $\begin{array}{c}(\varepsilon / v) \\
\pm 0.0001 / \mathrm{M}^{-1}\end{array}$ & $\begin{array}{c}v \\
\pm 10 / \mathrm{cm}^{-1}\end{array}$ & $\begin{array}{c}(\varepsilon / v) \\
\pm 0.0001 / \mathrm{M}^{-1}\end{array}$ \\
\hline \multirow[t]{3}{*}{ meso } & 4 & $\begin{array}{c}14960 \\
\text { sh } 23550 \\
24300 \\
26980 \\
28240 \\
29260\end{array}$ & $\begin{array}{l}1.3255 \\
0.9462 \\
0.9870 \\
1.4240 \\
1.4327 \\
1.4428\end{array}$ & $\begin{array}{c}15310 \\
23490 \\
\text { sh } 24400 \\
26970 \\
29420\end{array}$ & $\begin{array}{l}1.1274 \\
0.9461 \\
0.8511 \\
1.3391 \\
1.3417\end{array}$ & $\begin{array}{l}13700 \\
16730 \\
24750 \\
29230\end{array}$ & $\begin{array}{l}1.8258 \\
0.2806 \\
1.2997 \\
1.4827\end{array}$ & $\begin{array}{l}13810 \\
16730 \\
24840 \\
29230\end{array}$ & $\begin{array}{l}1.9492 \\
0.2068 \\
1.2365 \\
1.4667\end{array}$ \\
\hline & 5 & $\begin{array}{c}\mathbf{6 1 2 5} \\
15100 \\
25930\end{array}$ & $\begin{array}{c}\mathbf{0 . 1 4 1 1 4} \\
0.7909 \\
1.4471\end{array}$ & $\begin{array}{c}\mathbf{5 9 0 4} \\
15130 \\
\text { sh } 25860\end{array}$ & $\begin{array}{c}\mathbf{0 . 1 7 9 1 7} \\
0.6710 \\
1.2885\end{array}$ & $\begin{array}{c}\mathbf{5 8 0 7} \\
14080 \\
\text { sh } 21820 \\
\text { sh } 22860 \\
24580 \\
28270\end{array}$ & $\begin{array}{l}\mathbf{0 . 2 2 3 8} \\
0.9164 \\
0.4592 \\
0.5765 \\
0.7882 \\
1.3037\end{array}$ & $\begin{array}{c}\mathbf{5 5 0 2} \\
14030 \\
\text { sh } 21840 \\
\text { sh } 22970 \\
24540 \\
28270\end{array}$ & $\begin{array}{l}\mathbf{0 . 5 1 8 3} \\
0.9988 \\
0.4101 \\
0.5355 \\
0.7133 \\
1.2879\end{array}$ \\
\hline & 6 & $\begin{array}{l}20400 \\
21750 \\
25270\end{array}$ & $\begin{array}{l}0.4840 \\
0.6146 \\
1.7375\end{array}$ & & & & & $\begin{array}{l}13960 \\
20870 \\
21930 \\
27390\end{array}$ & $\begin{array}{l}0.2075 \\
0.8203 \\
0.7393 \\
1.4823 \\
\end{array}$ \\
\hline \multirow[t]{3}{*}{ rac } & 4 & $\begin{array}{c}14970 \\
\text { sh } 23600 \\
24310 \\
26900 \\
28250 \\
29260\end{array}$ & $\begin{array}{l}1.2289 \\
0.8174 \\
0.8580 \\
1.2871 \\
1.2902 \\
1.2955\end{array}$ & $\begin{array}{c}15230 \\
23470 \\
\text { sh } 24380 \\
26900 \\
29190\end{array}$ & $\begin{array}{l}1.1855 \\
0.8880 \\
0.7998 \\
1.2284 \\
1.3320\end{array}$ & $\begin{array}{l}13690 \\
16660 \\
24740 \\
29280\end{array}$ & $\begin{array}{l}1.8644 \\
0.3591 \\
1.2611 \\
1.4214\end{array}$ & $\begin{array}{l}13720 \\
16690 \\
24710 \\
29130\end{array}$ & $\begin{array}{l}2.0111 \\
0.2690 \\
1.3427 \\
1.4220\end{array}$ \\
\hline & 5 & $\begin{array}{c}\mathbf{6 1 2 9} \\
15070 \\
25980\end{array}$ & $\begin{array}{c}\mathbf{0 . 1 4 4 7 8} \\
0.7315 \\
1.2724\end{array}$ & $\begin{array}{c}5543 \\
15120 \\
\text { sh } 25920\end{array}$ & $\begin{array}{l}0.2304 \\
0.6849 \\
1.3858\end{array}$ & $\begin{array}{c}\mathbf{5 7 9 9} \\
14030 \\
\text { sh } 21720 \\
22820 \\
24740 \\
28360\end{array}$ & $\begin{array}{l}\mathbf{0 . 1 6 5 0} \\
0.8983 \\
0.4869 \\
0.5891 \\
0.8230 \\
1.2046\end{array}$ & $\begin{array}{c}\mathbf{5 4 2 3} \\
13980 \\
21770 \\
\text { sh } 22850 \\
24510 \\
28140\end{array}$ & $\begin{array}{c}\mathbf{0 . 3 3 2 4 8} \\
1.1536 \\
0.4805 \\
0.5846 \\
0.8367 \\
1.2721\end{array}$ \\
\hline & 6 & $\begin{array}{l}21710 \\
20330 \\
25240\end{array}$ & $\begin{array}{l}0.4929 \\
0.3548 \\
1.5719\end{array}$ & $\begin{array}{c}20520 \\
\text { sh } 21870 \\
25270\end{array}$ & $\begin{array}{l}0.3595 \\
0.5387 \\
1.7864\end{array}$ & $\begin{array}{c}5732 \\
14130 \\
21510 \\
22700 \\
27830\end{array}$ & $\begin{array}{l}0.5359 \\
0.4708 \\
0.5030 \\
0.9834\end{array}$ & $\begin{array}{l}14050 \\
20950 \\
22020 \\
27510\end{array}$ & $\begin{array}{l}0.2186 \\
0.8933 \\
0.7639 \\
1.4088\end{array}$ \\
\hline
\end{tabular}


Table B3.14 NIR spectral data of the reduced absorption spectra $(\varepsilon / v v s$. $v)$ for $\left[\left\{\operatorname{Ru}(b p y)_{2}\right\}(\mu-B L)\left\{\operatorname{Ru}(p p)_{2}\right\}\right]^{5+}\{B L=d p b$, dpb' $\}$ at $-35^{\circ} \mathrm{C}$. The parameters for the overall NIR band envelopes are shown in bold type, and details of the deconvoluted bands are in normal type.

\begin{tabular}{|c|c|c|c|c|c|c|c|c|c|c|c|}
\hline \multirow[b]{2}{*}{ pp } & \multirow[b]{2}{*}{ Diastereoisomer } & \multicolumn{5}{|c|}{$\mathrm{BL}=\mathrm{dpb}$} & \multicolumn{5}{|c|}{$\mathrm{BL}=\mathrm{dpb}^{\prime}$} \\
\hline & & $\begin{array}{l}v_{\max } \\
\pm 10 \\
/ \mathrm{cm}^{-1}\end{array}$ & $\begin{array}{c}(\varepsilon / v)_{\max } \\
\pm 0.0001 \\
/ \mathrm{M}^{-1}\end{array}$ & $\begin{array}{l}\Delta v_{1 / 2} \\
\pm 10 \\
/ \mathrm{cm}^{-1}\end{array}$ & $\begin{array}{c}M_{0} \\
/ \mathrm{M}^{-1}\end{array}$ & $\begin{array}{c}M_{1} \\
/ \mathrm{cm}^{-1}\end{array}$ & $\begin{array}{l}v_{\max } \\
\pm 10 \\
/ \mathrm{cm}^{-1}\end{array}$ & $\begin{array}{c}(\varepsilon / v)_{\max } \\
\pm 0.0001 \\
/ \mathrm{M}^{-1}\end{array}$ & $\begin{array}{l}\Delta \mathrm{v}_{1 / 2} \\
\pm 10 \\
/ \mathrm{cm}^{-1}\end{array}$ & $\begin{array}{c}M_{0} \\
/ \mathrm{M}^{-1}\end{array}$ & $\begin{array}{c}M_{1} \\
/ \mathrm{cm}^{-1}\end{array}$ \\
\hline \multirow[t]{2}{*}{$\mathrm{Me}_{4} \mathrm{bpy}$} & meso & $\begin{array}{l}\mathbf{6 1 2 5} \\
3925 \\
4648 \\
6140 \\
7905\end{array}$ & $\begin{array}{c}\mathbf{0 . 1 4 1 1} \\
0.00564 \\
0.00678 \\
0.1376 \\
0.00410\end{array}$ & $\begin{array}{c}2230 \\
255 \\
1574 \\
2193 \\
1035\end{array}$ & $\begin{array}{l}331.7 \\
0.372 \\
9.479 \\
317.4 \\
4.495\end{array}$ & 6150 & $\begin{array}{l}5810 \\
2513 \\
5849 \\
5300 \\
7054 \\
9298 \\
\end{array}$ & $\begin{array}{l}\mathbf{0 . 2 2 3 8} \\
0.0508 \\
0.2115 \\
0.0204 \\
0.0303 \\
0.0299 \\
\end{array}$ & $\begin{array}{c}\mathbf{1 6 7 5} \\
2399 \\
1501 \\
565 \\
1429 \\
793 \\
\end{array}$ & $\begin{array}{l}\mathbf{4 8 4 . 1} \\
9.365 \\
337.5 \\
12.26 \\
46.13 \\
11.47 \\
\end{array}$ & 8640 \\
\hline & rac & $\begin{array}{l}\mathbf{6 1 3 0} \\
2398 \\
6112 \\
8061\end{array}$ & $\begin{array}{c}\mathbf{0 . 1 4 4 7 8} \\
0.0262 \\
0.1421 \\
0.00308\end{array}$ & $\begin{array}{c}2270 \\
2916 \\
2274 \\
944\end{array}$ & $\begin{array}{l}350.0 \\
7.957 \\
338.6 \\
3.297\end{array}$ & 6125 & $\begin{array}{l}\mathbf{5 8 0 0} \\
2513 \\
5849 \\
5300 \\
7054 \\
9298\end{array}$ & $\begin{array}{l}\mathbf{0 . 1 6 5 0} \\
0.0497 \\
0.2067 \\
0.0199 \\
0.0296 \\
0.0292\end{array}$ & $\begin{array}{c}\mathbf{1 8 7 0} \\
2399 \\
1501 \\
565 \\
1429 \\
793\end{array}$ & $\begin{array}{l}357.0 \\
9.153 \\
329.9 \\
11.98 \\
45.08 \\
11.21\end{array}$ & 5930 \\
\hline \multirow[t]{2}{*}{$\mathrm{Me}_{2} \mathrm{bpy}$} & meso & $\begin{array}{l}5905 \\
3897 \\
5896 \\
5022 \\
7464\end{array}$ & $\begin{array}{c}\mathbf{0 . 1 7 9 1 6 9} \\
0.0139 \\
0.1489 \\
0.0134 \\
0.0176\end{array}$ & $\begin{array}{c}2415 \\
649 \\
2182 \\
849 \\
1733\end{array}$ & $\begin{array}{l}386.2 \\
3.407 \\
338.9 \\
12.13 \\
32.22 \\
\end{array}$ & 6020 & $\begin{array}{l}5500 \\
4018 \\
5488 \\
6261 \\
6754 \\
9289 \\
\end{array}$ & $\begin{array}{l}\mathbf{0 . 5 1 8 3} \\
0.0300 \\
0.4892 \\
0.0863 \\
0.0666 \\
0.0723 \\
\end{array}$ & $\begin{array}{c}\mathbf{1 4 1 5} \\
659 \\
1099 \\
756 \\
1180 \\
976 \\
\end{array}$ & $\begin{array}{l}\mathbf{5 1 2 . 1} \\
11.05 \\
571.6 \\
69.48 \\
83.62 \\
35.42 \\
\end{array}$ & 5725 \\
\hline & rac & $\begin{array}{l}\mathbf{5 5 4 0} \\
3902 \\
4916 \\
5556 \\
7117\end{array}$ & $\begin{array}{c}\mathbf{0 . 2 3 0 3 9} \\
0.0139 \\
0.0129 \\
0.2223 \\
0.0275\end{array}$ & $\begin{array}{c}2345 \\
447 \\
629 \\
2186 \\
1944\end{array}$ & $\begin{array}{l}559.6 \\
2.384 \\
8.621 \\
494.6 \\
56.48\end{array}$ & 5780 & $\begin{array}{l}\mathbf{5 4 2 0} \\
4062 \\
5086 \\
5444 \\
6180 \\
6648 \\
9212\end{array}$ & $\begin{array}{c}\mathbf{0 . 3 3 2 5} \\
0.00605 \\
0.00810 \\
0.3141 \\
0.0830 \\
0.0587 \\
0.0340\end{array}$ & $\begin{array}{c}\mathbf{1 2 6 5} \\
330 \\
382 \\
1074 \\
795 \\
1253 \\
961\end{array}$ & $\begin{array}{l}735.8 \\
1.429 \\
3.300 \\
358.9 \\
70.29 \\
78.35 \\
19.00\end{array}$ & 7570 \\
\hline
\end{tabular}


Table B3.15 Crystal data and structure refinement parameters for dinuclear osmium complexes incorporating the dpb and dpq' bridging ligands.

\begin{tabular}{|c|c|c|}
\hline Parameter & meso- $(\Lambda \Delta)-\left[\left\{\mathrm{Os}(\mathrm{bpy})_{2}\right\}_{2}(\mu-\mathrm{dpb})\right]\left(\mathrm{ZnCl}_{4}\right)_{2} \cdot 6 \mathrm{H}_{2} \mathrm{O}$ & meso- $(\Lambda \Delta)-\left[\left\{\mathrm{Os}(\mathrm{bpy})_{2}\right\}_{2}(\mu-\mathrm{dpq})\right]\left(\mathrm{PF}_{6}\right)_{4}$ \\
\hline Formula & $\mathrm{C}_{62} \mathrm{H}_{49} \mathrm{Cl}_{8} \mathrm{~N}_{12} \mathrm{O}_{6} \mathrm{Os}_{2} \mathrm{Zn}_{2}$ & $\mathrm{C}_{58} \mathrm{H}_{42} \mathrm{~N}_{24} \mathrm{~F}_{48} \mathrm{P}_{8} \mathrm{Os}_{4}$ \\
\hline Formula weight & 1852.87 & 1867.4 \\
\hline $\mathrm{T}(\mathrm{K})$ & 293(2) K & 293 \\
\hline Crystal System & monoclinic & orthorhombic \\
\hline Space Group & $P 2_{1} / c$ & $\mathrm{Pbca}$ \\
\hline$a(\AA)$ & 19.598(5) & $26.6398(27)$ \\
\hline$b(\AA)$ & $16.755(5)$ & $25.0533(25)$ \\
\hline$c(\AA)$ & 20.799(5) & $22.0243(22)$ \\
\hline$\alpha\left(^{\circ}\right)$ & $90.000(5)$ & $90.0005(5)$ \\
\hline$\beta\left(^{\circ}\right)$ & $96.586(5)$ & $90.0005(5)$ \\
\hline$\gamma\left({ }^{\circ}\right)$ & $90.000(5)$ & $90.0005(5)$ \\
\hline$V\left(\AA^{3}\right)$ & 6785(3) & 14699(3) \\
\hline Z & 4 & 8 \\
\hline$D_{\mathrm{c}}\left(\mathrm{g} \mathrm{cm}^{-3}\right)$ & 1.814 & 1.69 \\
\hline$\mu\left(\mathrm{Mo}_{\mathrm{K}} \mathrm{K}_{\alpha}\right)\left(\mathrm{mm}^{-1}\right)$ & 4.806 & 3.65 \\
\hline$F(000)$ & 3604 & 7214.2 \\
\hline Crystal form & deep green rod-shaped crystals & deep green octahedron \\
\hline Crystal size (mm) & $0.2 \times 0.2 \times 0.15$ & $0.2 \times 0.2 \times 0.15$ \\
\hline $2 \theta_{\max }\left(^{\circ}\right)$ & 26.47 & 23.3 \\
\hline Limiting indices & $-24 \leq h \leq 24,0 \leq k \leq 20,0 \leq l \leq 26$ & $0 \leq h \leq 29,0 \leq k \leq 27,0 \leq l \leq 24$ \\
\hline Reflections collected & 14448 & 10570 \\
\hline Unique reflections & $13939\left(\mathrm{R}_{\mathrm{int}}=0.2013\right)$ & 6450 \\
\hline Parameters & 837 & $900\left(\mathrm{R}_{\mathrm{int}}=0.115\right)$ \\
\hline Refinement method & Full-matrix least-squares on $F^{2}$ & Full-matrix least-squares on $F^{2}$ \\
\hline $\begin{array}{l}\text { Absorption } \\
\text { correction }\end{array}$ & None & none \\
\hline GooF & 0.845 & 0.967 \\
\hline$R[I>2 \sigma(I)]$ & 0.0637 & 0.063 \\
\hline$w R$ (all data) & 0.1779 & 0.208 \\
\hline Diffractometer & Bruker AXS CCD $(\lambda=0.71069 \AA)$ & Bruker AXS CCD $(\lambda=0.71069 \AA)$ \\
\hline
\end{tabular}

Table B3.16 Electrochemical data (in $\mathrm{mV}$ relative to the $\mathrm{Fc}^{+} / \mathrm{Fc}^{0}$ couple) for the diastereoisomers of $\left[\left\{\mathrm{Os}(\mathrm{bpy})_{2}\right\}_{2}(\mu-\mathrm{BL})\right]^{4+}$ in $0.1 \mathrm{M}\left[\left(n-\mathrm{C}_{4} \mathrm{H}_{9}\right)_{4} \mathrm{~N}\right] \mathrm{PF}_{6} / \mathrm{CH}_{3} \mathrm{CN}$.

\begin{tabular}{|c|c|c|c|c|c|c|c|c|c|}
\hline BL & Diastereoisomer & $\begin{array}{c}\mathrm{K}_{\mathrm{c}} \\
\left(\times 10^{-3}\right)\end{array}$ & $\Delta \mathrm{E}_{\mathrm{ox}}$ & $\mathrm{E}_{\mathrm{ox} 2}$ & $\mathrm{E}_{\mathrm{ox} 1}$ & $\mathrm{E}_{\text {red1 }}$ & $\mathrm{E}_{\text {red2 }}$ & $\mathrm{E}_{\text {red3 }}$ & $\mathrm{E}_{\text {red4 }}$ \\
\hline $\mathrm{dpb}$ & meso & 256 & 320 & 956 & 636 & -664 & -1196 & -1776 & -1868 \\
& rac & 86.2 & 292 & 944 & 652 & -668 & -1200 & $-1784^{\mathrm{b}}$ & $\mathrm{b}$ \\
\hline $\mathrm{dpb}^{\prime}$ & meso & 3616 & 388 & 988 & 600 & -528 & -1064 & $-1836^{\mathrm{b}}$ & $-2180^{\mathrm{b}}$ \\
& rac & 4225 & 392 & 992 & 604 & -528 & -1056 & $-1840^{\mathrm{b}}$ & $-2188^{\mathrm{b}}$ \\
\hline $\mathrm{dpq}$ & meso & 1040 & 356 & 960 & 608 & -632 & -1240 & $-1832^{\mathrm{b}}$ & $-2208^{\mathrm{b}}$ \\
& rac & 1660 & 368 & 976 & 616 & -640 & -1232 & $-1840^{\mathrm{b}}$ & $-2184^{\mathrm{b}}$ \\
\hline $\mathrm{ppz}$ & meso & 890 & 352 & 916 & 568 & -968 & -1560 & -1794 & -1884 \\
& rac & 1040 & 356 & 924 & 568 & -960 & -1556 & -1780 & -1916 \\
\hline $2,3-\mathrm{dpp}$ & meso & 378 & 330 & 924 & 594 & -1094 & -1686 & -1800 & -1950 \\
& rac & 237 & 318 & 912 & 594 & -1097 & -1690 & -1830 & -1953 \\
\hline
\end{tabular}

\footnotetext{
${ }^{\mathrm{a}}$ Potentials are quoted $\pm 3 \mathrm{mV}$.

${ }^{\mathrm{b}}$ Processes complicated by adsorption/desorption peaks.
} 
Table B3.17(a) UV/Vis/NIR spectral data for meso- $\left[\left\{\mathrm{Os}(\mathrm{bpy})_{2}\right\}_{2}(\mu-\mathrm{BL})\right]^{n+}$ in $0.1 \mathrm{M}\left[\left(n-\mathrm{C}_{4} \mathrm{H}_{9}\right)_{4} \mathrm{~N}\right] \mathrm{PF}_{6} / \mathrm{CH}_{3} \mathrm{CN}$ at $-35^{\circ} \mathrm{C}$. The parameters of the IVCT and IC transitions in the +5 and +6 states, respectively, are indicated in bold type.

\begin{tabular}{|c|c|c|c|c|c|c|c|c|c|c|c|}
\hline \multicolumn{2}{|l|}{ BL } & \multicolumn{2}{|c|}{$\mathrm{dpb}$} & \multicolumn{2}{|c|}{ dpb' } & \multicolumn{2}{|c|}{ dpq' } & \multicolumn{2}{|c|}{ ppz } & \multicolumn{2}{|c|}{ 2,3-dpp } \\
\hline Diastereoisomer & $n$ & $\begin{array}{c}v \\
\pm 10 \\
/ \mathrm{cm}^{-1}\end{array}$ & $\begin{aligned} & (\varepsilon / v) \\
\pm & 0.0001 \\
& / \mathrm{M}^{-1}\end{aligned}$ & $\begin{array}{c}v \\
\pm 10 \\
/ \mathrm{cm}^{-1}\end{array}$ & $\begin{aligned} & (\varepsilon / v) \\
\pm & 0.0001 \\
& / \mathrm{M}^{-1}\end{aligned}$ & $\begin{array}{c}v \\
\pm 10 \\
/ \mathrm{cm}^{-1}\end{array}$ & $\begin{aligned} & (\varepsilon / v) \\
\pm & 0.0001 \\
& / \mathrm{M}^{-1}\end{aligned}$ & $\begin{array}{c}v \\
\pm 10 \\
/ \mathrm{cm}^{-1}\end{array}$ & $\begin{aligned} & (\varepsilon / v) \\
\pm & 0.0001 \\
& / \mathrm{M}^{-1}\end{aligned}$ & $\begin{array}{c}v \\
\pm 10 \\
/ \mathrm{cm}^{-1}\end{array}$ & $\begin{aligned} & (\varepsilon / v) \\
\pm & 0.0001 \\
& / \mathrm{M}^{-1}\end{aligned}$ \\
\hline meso & 4 & $\begin{array}{c}9820 \\
14880 \\
\text { sh } 16420 \\
23580 \\
27960\end{array}$ & $\begin{array}{l}0.9398 \\
1.5780 \\
0.9363 \\
0.9421 \\
1.7424\end{array}$ & $\begin{array}{c}8700 \\
13340 \\
16910 \\
22670 \\
24860 \\
29700\end{array}$ & $\begin{array}{l}1.1411 \\
2.3236 \\
0.4772 \\
0.7991 \\
1.2290 \\
1.6225\end{array}$ & $\begin{array}{c}9730 \\
14420 \\
18210 \\
22570 \\
26920\end{array}$ & $\begin{array}{l}1.4538 \\
2.4793 \\
0.6445 \\
0.9670 \\
1.2988\end{array}$ & $\begin{array}{l}12190 \\
16690 \\
22290\end{array}$ & $\begin{array}{l}1.5278 \\
1.9065 \\
1.0421\end{array}$ & $\begin{array}{r}12810 \\
13540 \\
17950 \\
23010 \\
\text { sh } 28010\end{array}$ & $\begin{array}{l}0.7952 \\
0.8155 \\
1.8422 \\
0.9795 \\
1.0916\end{array}$ \\
\hline & 5 & $\begin{array}{c}\mathbf{2 1 5 0} \\
\mathbf{4 9 2 0} \\
8930 \\
12220 \\
14700 \\
25640 \\
26970\end{array}$ & $\begin{array}{l}1.8262 \\
0.1176 \\
0.5979 \\
0.3700 \\
0.8489 \\
1.2924 \\
1.3846\end{array}$ & $\begin{array}{c}\mathbf{2 0 6 0} \\
\mathbf{4 8 7 0} \\
\mathbf{6 8 1 0} \\
8480 \\
11600 \\
13470 \\
14590 \\
17760 \\
24510 \\
28880\end{array}$ & $\begin{array}{c}3.699 \\
0.0848 \\
0.2382 \\
0.7663 \\
0.5959 \\
1.3800 \\
0.8849 \\
0.2873 \\
0.8399 \\
1.3376\end{array}$ & $\begin{array}{c}4980 \\
8840 \\
13050 \\
14880 \\
26170\end{array}$ & $\begin{array}{l}0.1399 \\
0.7750 \\
0.4783 \\
1.8796 \\
0.9090\end{array}$ & $\begin{array}{c}\mathbf{2 2 7 5} \\
\mathbf{5 0 0 0} \\
\mathbf{8 3 4 0} \\
11140 \\
\text { sh } 15800 \\
17380\end{array}$ & $\begin{array}{l}2.8906 \\
0.0930 \\
0.4142 \\
0.6074 \\
0.6082 \\
1.5311\end{array}$ & $\begin{array}{c}\mathbf{4 9 5 0} \\
\mathbf{8 3 0 0} \\
11720 \\
\text { sh } 15970 \\
18600 \\
28710\end{array}$ & $\begin{array}{l}0.1357 \\
0.5164 \\
0.4740 \\
0.3644 \\
1.2711 \\
1.1796\end{array}$ \\
\hline & 6 & $\begin{array}{c}\mathbf{4 5 5 0} \\
\mathbf{5 1 6 0} \\
21350 \\
25240\end{array}$ & $\begin{array}{l}0.2324 \\
0.2066 \\
0.5836 \\
1.6290\end{array}$ & $\begin{array}{c}\mathbf{4 5 8 0} \\
\mathbf{5 0 5 0} \\
20760 \\
27610\end{array}$ & $\begin{array}{l}0.2467 \\
0.2508 \\
0.6608 \\
1.5097\end{array}$ & $\begin{array}{c}\mathbf{4 5 6 0} \\
\mathbf{5 2 1 0} \\
19390\end{array}$ & $\begin{array}{l}0.2455 \\
0.2821 \\
0.6351\end{array}$ & $\begin{array}{c}\mathbf{4 4 9 4} \\
\mathbf{5 0 8 0} \\
14150 \\
23300\end{array}$ & $\begin{array}{l}0.1493 \\
0.1845 \\
0.2611 \\
0.6283\end{array}$ & $\begin{array}{c}\mathbf{4 3 2 0} \\
\mathbf{5 1 0 0} \\
19190 \\
23860 \\
28350\end{array}$ & $\begin{array}{l}0.2296 \\
0.2506 \\
0.2173 \\
0.5821 \\
1.3000\end{array}$ \\
\hline
\end{tabular}


Table B3.17(b) UV/Vis/NIR spectral data for $r a c-\left[\left\{\mathrm{Os}(\mathrm{bpy})_{2}\right\}_{2}(\mu-\mathrm{BL})\right]^{n+}$ in $0.1 \mathrm{M}\left[\left(n-\mathrm{C}_{4} \mathrm{H}_{9}\right)_{4} \mathrm{~N}\right] \mathrm{PF}_{6} / \mathrm{CH}_{3} \mathrm{CN}$ at $-35^{\circ} \mathrm{C}$. The parameters of the IVCT and IC transitions in the +5 and +6 states, respectively, are indicated in bold type.

\begin{tabular}{|c|c|c|c|c|c|c|c|c|c|c|c|}
\hline \multicolumn{2}{|l|}{$\mathrm{BL}$} & \multicolumn{2}{|c|}{$\mathrm{dpb}$} & \multicolumn{2}{|c|}{$\mathrm{dpb}^{\prime}$} & \multicolumn{2}{|c|}{$\mathrm{dpq}$} & \multicolumn{2}{|c|}{$\mathrm{ppz}$} & \multicolumn{2}{|c|}{ 2,3-dpp } \\
\hline Diastereoisomer & $n$ & $\begin{array}{c}v \\
\pm 10 \\
/ \mathrm{cm}^{-1} \\
\end{array}$ & $\begin{array}{l}(\varepsilon / v) \\
\pm 0.0001 \\
/ \mathrm{M}^{-1}\end{array}$ & $\begin{array}{c}v \\
\pm 10 \\
/ \mathrm{cm}^{-1} \\
\end{array}$ & $\begin{aligned} & (\varepsilon / v) \\
\pm & 0.0001 \\
& / \mathrm{M}^{-1}\end{aligned}$ & $\begin{array}{c}v \\
\pm 10 \\
/ \mathrm{cm}^{-1} \\
\end{array}$ & $\begin{aligned} &(\varepsilon / v) \\
& \pm 0.0001 \\
& / \mathrm{M}^{-1} \\
&\end{aligned}$ & $\begin{array}{c}v \\
\pm 10 \\
/ \mathrm{cm}^{-1} \\
\end{array}$ & $\begin{aligned} &(\varepsilon / v) \\
& \pm 0.0001 \\
& / \mathrm{M}^{-1} \\
&\end{aligned}$ & $\begin{array}{c}v \\
\pm 10 \\
/ \mathrm{cm}^{-1} \\
\end{array}$ & $\begin{aligned} &(\varepsilon / v) \\
& \pm 0.0001 \\
& / \mathrm{M}^{-1} \\
&\end{aligned}$ \\
\hline rac & 4 & $\begin{array}{c}9780 \\
\text { sh } 10660 \\
14840 \\
\text { sh } 16250 \\
19400 \\
23430 \\
24350 \\
27770\end{array}$ & $\begin{array}{l}0.8655 \\
0.6546 \\
1.5923 \\
1.0115 \\
0.4462 \\
0.9901 \\
0.9698 \\
1.8033\end{array}$ & $\begin{array}{c}8860 \\
13440 \\
17810 \\
22510 \\
24970 \\
29720\end{array}$ & $\begin{array}{l}1.5368 \\
2.2558 \\
0.4282 \\
0.7557 \\
1.1444 \\
1.6241\end{array}$ & $\begin{array}{c}9860 \\
14540 \\
18070 \\
22460 \\
26990\end{array}$ & $\begin{array}{l}1.8837 \\
2.8159 \\
0.5934 \\
1.0268 \\
1.3805\end{array}$ & $\begin{array}{l}12180 \\
16740 \\
22240\end{array}$ & $\begin{array}{l}1.5067 \\
1.8801 \\
1.0410\end{array}$ & $\begin{array}{c}12760 \\
13520 \\
17970 \\
23170 \\
\text { sh } 28000\end{array}$ & $\begin{array}{l}0.6986 \\
0.7022 \\
1.6977 \\
0.9091 \\
1.0471\end{array}$ \\
\hline & 5 & $\begin{array}{c}\mathbf{4 8 2 0} \\
8730 \\
12050 \\
14320 \\
25430 \\
26960\end{array}$ & $\begin{array}{l}0.0888 \\
0.5978 \\
0.3346 \\
0.7733 \\
1.2735 \\
1.2656\end{array}$ & $\begin{array}{c}\mathbf{4 8 7 0} \\
\mathbf{7 0 4 5} \\
8700 \\
11860 \\
13650 \\
14810 \\
24540 \\
29030 \\
\end{array}$ & $\begin{array}{l}0.0630 \\
0.1739 \\
0.6651 \\
0.6337 \\
1.1395 \\
0.8396 \\
0.7882 \\
1.2594 \\
\end{array}$ & $\begin{array}{c}\mathbf{4 9 8 0} \\
9190 \\
13540 \\
15100 \\
26360\end{array}$ & $\begin{array}{l}0.1079 \\
0.8203 \\
0.6290 \\
2.0797 \\
0.9148\end{array}$ & $\begin{array}{c}\mathbf{5 0 7 0} \\
\mathbf{8 4 0 0} \\
11180 \\
\text { sh } 15800 \\
17440\end{array}$ & $\begin{array}{l}0.1126 \\
0.3014 \\
0.5374 \\
0.5109 \\
1.3336\end{array}$ & $\begin{array}{c}5030 \\
8330 \\
11700 \\
\text { sh } 16070 \\
18540 \\
28940\end{array}$ & $\begin{array}{l}0.1150 \\
0.4519 \\
0.4347 \\
0.3640 \\
1.1826 \\
1.1204\end{array}$ \\
\hline & 6 & $\begin{array}{c}\mathbf{4 4 9 0} \\
\mathbf{5 0 5 0} \\
21500 \\
25320\end{array}$ & $\begin{array}{l}0.2432 \\
0.1952 \\
0.4877 \\
1.5465\end{array}$ & $\begin{array}{c}\mathbf{4 5 4 0} \\
\mathbf{5 0 6 0} \\
20830 \\
27520\end{array}$ & $\begin{array}{l}0.2176 \\
0.2206 \\
0.6172 \\
1.4275\end{array}$ & $\begin{array}{c}4540 \\
5210 \\
19550\end{array}$ & $\begin{array}{l}0.2512 \\
0.2749 \\
0.6400\end{array}$ & & & $\begin{array}{c}\mathbf{4 2 8 0} \\
\mathbf{5 0 6 0} \\
15640 \\
23540 \\
28330 \\
\end{array}$ & $\begin{array}{l}0.2647 \\
0.2441 \\
0.1377 \\
0.5290 \\
1.2642 \\
\end{array}$ \\
\hline
\end{tabular}


Table B3.18(a) Parameters obtained from Gaussian deconvolution of the UV/Vis/NIR spectra for meso- $\left[\left\{\mathrm{Os}(\mathrm{bpy})_{2}\right\}_{2}(\mu-\mathrm{BL})\right]^{n+}\left\{\mathrm{BL}=\mathrm{dpb}, \mathrm{dpb} \mathrm{b}^{\prime}\right.$ dpq' $\}$ in $0.1 \mathrm{M}\left[\left(n-\mathrm{C}_{4} \mathrm{H}_{9}\right)_{4} \mathrm{~N}\right] \mathrm{PF}_{6} / \mathrm{CH}_{3} \mathrm{CN}$ at $-35^{\circ} \mathrm{C}$.

\begin{tabular}{|c|c|c|c|c|c|c|c|c|c|c|c|c|c|}
\hline \multirow[b]{2}{*}{ Diastereoisomer } & \multirow[b]{2}{*}{$n$} & \multicolumn{4}{|c|}{$\mathrm{dpb}$} & \multicolumn{4}{|c|}{ dpb' } & \multicolumn{4}{|c|}{ dpq' } \\
\hline & & $\begin{array}{l}v_{\max } \\
\pm 10 \\
/ \mathrm{cm}^{-1}\end{array}$ & $\begin{array}{c}(\varepsilon / v) \\
\pm 0.0001 \\
/ \mathrm{M}^{-1}\end{array}$ & $\begin{array}{l}\Delta \mathrm{v}_{1 / 2} \\
\pm 10 \\
/ \mathrm{cm}^{-1}\end{array}$ & $\begin{array}{c}M_{0} \\
/ \mathrm{M}^{-1}\end{array}$ & $\begin{array}{l}v_{\max } \\
\pm 10 \\
/ \mathrm{cm}^{-1}\end{array}$ & $\begin{array}{l}(\varepsilon / v) \\
\pm 0.0001 \\
/ \mathrm{M}^{-1}\end{array}$ & $\begin{array}{l}\Delta v_{1 / 2} \\
\pm 10 \\
/ \mathrm{cm}^{-1}\end{array}$ & $\begin{array}{c}M_{0} \\
/ \mathrm{M}^{-1}\end{array}$ & $\begin{array}{l}v_{\max } \\
\pm 10 \\
/ \mathrm{cm}^{-1}\end{array}$ & $\begin{array}{c}(\varepsilon / v) \\
\pm 0.0001 \\
/ \mathrm{M}^{-1}\end{array}$ & $\begin{array}{l}\Delta v_{1 / 2} \\
\pm 10 \\
/ \mathrm{cm}^{-1}\end{array}$ & $\begin{array}{c}M_{0} \\
/ \mathrm{M}^{-1}\end{array}$ \\
\hline \multirow[t]{18}{*}{ meso } & \multirow[t]{12}{*}{5} & 4910 & 0.1128 & 533 & 63.65 & 4510 & 0.0256 & 249 & 6.772 & 4596 & 0.0307 & 274 & 8.920 \\
\hline & & 5395 & 0.0433 & 519 & 23.94 & 4757 & 0.0223 & 437 & 10.35 & 4844 & 0.0332 & 331 & 11.70 \\
\hline & & 5876 & 0.0124 & 508 & 6.714 & 5471 & 0.0355 & 811 & 30.64 & 5339 & 0.0558 & 806 & 47.85 \\
\hline & & 7385 & 0.0504 & 707 & 37.97 & 6825 & 0.2375 & 831 & 210.2 & 6070 & 0.0127 & 669 & 9.075 \\
\hline & & 8500 & 0.2611 & 969 & 405.8 & 7590 & 0.0663 & 607 & 42.91 & 7570 & 0.0638 & 1098 & 74.60 \\
\hline & & 9177 & 0.4192 & 995 & 444.0 & 8476 & 0.7359 & 791 & 620.3 & 8620 & 0.5516 & 765 & 449.4 \\
\hline & & 9986 & 0.1940 & 1085 & 224.1 & 9140 & 0.0866 & 568 & 52.39 & 9168 & 0.4908 & 842 & 440.0 \\
\hline & & 10810 & 0.0623 & 779 & 51.68 & 9786 & 0.1564 & 1647 & 274.2 & 9797 & 0.1418 & 876 & 132.3 \\
\hline & & 12220 & 0.3533 & 1289 & 484.7 & 11600 & 0.5840 & 1095 & 681.3 & 10420 & 0.0767 & 1424 & 116.2 \\
\hline & & 14130 & 0.5642 & 1643 & 983.5 & 12540 & 0.2760 & 807 & 237.1 & 13060 & 0.4800 & 1542 & 787.2 \\
\hline & & 15630 & 0.7204 & 2232 & 1123 & $\begin{array}{l}13450 \\
14600\end{array}$ & 1.3109 & $\begin{array}{c}927 \\
1148\end{array}$ & 1293 & 14900 & 1.8758 & 1130 & 1515 \\
\hline & & & & & & 14600 & 0.8666 & 1148 & 623.0 & & & & \\
\hline & \multirow[t]{6}{*}{6} & 4528 & 0.2226 & 426 & 100.2 & 4520 & 0.2245 & 505 & 120.6 & 4550 & 0.2410 & 428 & 106.2 \\
\hline & & 4810 & 0.0412 & 289 & 12.68 & 5070 & 0.2376 & 572 & 144.9 & 4845 & 0.0480 & 268 & 13.71 \\
\hline & & 5134 & 0.1872 & 547 & 110.3 & 5581 & 0.0315 & 522 & 17.51 & 5205 & 0.2786 & 512 & 151.3 \\
\hline & & 5470 & 0.0442 & 556 & 26.22 & 6096 & 0.00911 & 1215 & 11.75 & 5613 & 0.0333 & 339 & 12.03 \\
\hline & & 5840 & 0.0185 & 636 & 12.55 & 6768 & 0.00594 & 433 & 2.73 & 5860 & 0.0311 & 578 & 19.15 \\
\hline & & & & & & & & & & 6554 & 0.0164 & 1305 & 22.32 \\
\hline
\end{tabular}


Table B3.18(b) Parameters obtained from Gaussian deconvolution of the UV/Vis/NIR spectra for $r a c-\left[\left\{\mathrm{Os}(\mathrm{bpy})_{2}\right\}_{2}(\mu-\mathrm{BL})\right]^{n+}\left\{\mathrm{BL}=\mathrm{dpb}, \mathrm{dpb} \mathrm{b}^{\prime} \mathrm{dpq}\right\}$ in $0.1 \mathrm{M}\left[\left(n-\mathrm{C}_{4} \mathrm{H}_{9}\right)_{4} \mathrm{~N}\right] \mathrm{PF}_{6} / \mathrm{CH}_{3} \mathrm{CN}$ at $-35^{\circ} \mathrm{C}$.

\begin{tabular}{|c|c|c|c|c|c|c|c|c|c|c|c|c|c|}
\hline \multirow[b]{2}{*}{ Diastereoisomer } & \multirow[b]{2}{*}{$n$} & \multicolumn{4}{|c|}{$\mathrm{dpb}$} & \multicolumn{4}{|c|}{ dpb' } & \multicolumn{4}{|c|}{$\mathrm{dpq}^{\prime}$} \\
\hline & & $\begin{array}{l}v_{\max } \\
\pm 10 \\
/ \mathrm{cm}^{-1}\end{array}$ & $\begin{array}{c}(\varepsilon / v) \\
\pm 0.0001 \\
/ \mathrm{M}^{-1}\end{array}$ & $\begin{array}{l}\Delta v_{1 / 2} \\
\pm 10 \\
/ \mathrm{cm}^{-1}\end{array}$ & $\begin{array}{c}M_{0} \\
/ \mathrm{M}^{-1}\end{array}$ & $\begin{array}{l}v_{\max } \\
\pm 10 \\
/ \mathrm{cm}^{-1}\end{array}$ & $\begin{array}{c}(\varepsilon / v) \\
\pm 0.0001 \\
/ \mathrm{M}^{-1}\end{array}$ & $\begin{array}{l}\Delta v_{1 / 2} \\
\pm 10 \\
/ \mathrm{cm}^{-1}\end{array}$ & $\begin{array}{c}M_{0} \\
/ \mathrm{M}^{-1}\end{array}$ & $\begin{array}{l}v_{\max } \\
\pm 10 \\
/ \mathrm{cm}^{-1}\end{array}$ & $\begin{array}{c}(\varepsilon / v) \\
\pm 0.0001 \\
/ \mathrm{M}^{-1}\end{array}$ & $\begin{array}{l}\Delta \mathrm{v}_{1 / 2} \\
\pm 10 \\
/ \mathrm{cm}^{-1}\end{array}$ & $\begin{array}{c}M_{0} \\
/ \mathrm{M}^{-1}\end{array}$ \\
\hline \multirow[t]{18}{*}{ rac } & \multirow[t]{11}{*}{5} & 4805 & 0.0864 & 583 & 52.73 & 4622 & 0.0271 & 284 & 8.189 & 4924 & 0.0814 & 463.9 & 40.02 \\
\hline & & 5362 & 0.0347 & 577 & 52.29 & 4898 & 0.0528 & 501 & 28.03 & 5310 & 0.0444 & 721.3 & 34.06 \\
\hline & & 5858 & 0.0108 & 500 & 5.752 & 5420 & 0.0369 & 701 & 27.50 & 5865 & 0.0263 & 1171 & 32.75 \\
\hline & & 7306 & 0.0482 & 666 & 34.21 & 7011 & 0.1803 & 970 & 186.3 & 7663 & 0.0551 & 977.1 & 57.28 \\
\hline & & 8394 & 0.4495 & 885 & 423.7 & 8698 & 0.6554 & 1042 & 727.0 & 8816 & 0.5339 & 883.2 & 501.9 \\
\hline & & 9028 & 0.3984 & 863 & 366.3 & 9634 & 0.1677 & 925 & 165.2 & 9419 & 0.5366 & 915.1 & 522.6 \\
\hline & & 9710 & 0.1979 & 924 & 194.8 & 10340 & 0.0950 & 755 & 76.43 & 10110 & 0.2709 & 1117 & 322.1 \\
\hline & & 10510 & 0.0856 & 878 & 80.05 & 11850 & 0.6338 & 1440 & 971.9 & 11070 & 0.0549 & 883.3 & 51.63 \\
\hline & & 12030 & 0.3173 & 1200 & 405.2 & 12670 & 0.1360 & 668 & 98.69 & 12120 & 0.0374 & 867.9 & 34.57 \\
\hline & & 13930 & 0.5335 & 1660 & 941.6 & 13640 & 1.0955 & 1169 & 1361 & 13530 & 0.6233 & 1532 & 1013 \\
\hline & & 15480 & 0.6116 & 2364 & 1080 & 14920 & 0.8030 & 1151 & 642.4 & 15120 & 2.0561 & 1103 & 1582 \\
\hline & \multirow[t]{7}{*}{6} & 4474 & 0.2256 & 493 & 117.8 & 4510 & 0.2019 & 510 & 109.6 & 4006 & 0.0304 & 258 & 4.355 \\
\hline & & 5064 & 0.1906 & 625 & 126.8 & 5080 & 0.2133 & 575 & 130.7 & 4550 & 0.2482 & 502 & 132.3 \\
\hline & & 5660 & 0.0232 & 508 & 12.57 & 5625 & 0.0296 & 516 & 16.26 & 4897 & 0.0458 & 295 & 14.54 \\
\hline & & 6060 & 0.0149 & 744 & 11.82 & 6141 & 0.00644 & 585 & 4.009 & 5220 & 0.2601 & 477 & 132.3 \\
\hline & & 6723 & 0.00385 & 619 & 2.282 & & & & & 5586 & 0.0395 & 351 & 14.82 \\
\hline & & & & & & & & & & 5821 & 0.0318 & 590 & 19.96 \\
\hline & & & & & & & & & & 6380 & 0.0239 & 1825 & 44.36 \\
\hline
\end{tabular}


Table B3.18(c) Parameters obtained from Gaussian deconvolution of the UV/Vis/NIR spectra for meso$\left[\left\{\mathrm{Os}(\mathrm{pp})_{2}\right\}_{2}(\mu-\mathrm{BL})\right]^{n+}\{\mathrm{BL}=\mathrm{ppz}, 2,3-\mathrm{dpp}\}$ in $0.1 \mathrm{M}\left[\left(n-\mathrm{C}_{4} \mathrm{H}_{9}\right)_{4} \mathrm{~N}\right] \mathrm{PF}_{6} / \mathrm{CH}_{3} \mathrm{CN}$ at $-35^{\circ} \mathrm{C}$.

\begin{tabular}{|c|c|c|c|c|c|c|c|c|c|}
\hline \multirow[b]{2}{*}{ Diastereoisomer } & \multirow[b]{2}{*}{$n$} & \multicolumn{4}{|c|}{ ppz } & \multicolumn{4}{|c|}{ 2,3-dpp } \\
\hline & & $\begin{array}{l}v_{\max } \\
\pm 10 \\
/ \mathrm{cm}^{-1}\end{array}$ & $\begin{array}{c}(\varepsilon / v) \\
\pm 0.0001 \\
/ \mathrm{M}^{-1}\end{array}$ & $\begin{array}{l}\Delta v_{1 / 2} \\
\pm 10 \\
/ \mathrm{cm}^{-1}\end{array}$ & $\begin{array}{c}M_{0} \\
/ \mathbf{M}^{-1}\end{array}$ & $\begin{array}{l}v_{\max } \\
\pm 10 \\
/ \mathrm{cm}^{-1}\end{array}$ & $\begin{array}{c}(\varepsilon / v) \\
\pm 0.0001 \\
\\
/ \mathrm{M}^{-1}\end{array}$ & $\begin{array}{l}\Delta v_{1 / 2} \\
\pm 10 \\
/ \mathrm{cm}^{-1}\end{array}$ & $\begin{array}{c}M_{0} \\
/ \mathrm{M}^{-1}\end{array}$ \\
\hline \multirow[t]{17}{*}{ meso } & \multirow[t]{12}{*}{5} & 4980 & 0.0704 & 552 & 41.24 & 4950 & 0.1292 & 518 & 70.44 \\
\hline & & 5594 & 0.0484 & 115 & 59.10 & 5417 & 0.0380 & 436 & 17.65 \\
\hline & & 6938 & 0.0149 & 211 & 33.53 & 5798 & 0.0455 & 1018 & 49.35 \\
\hline & & 8336 & 0.4036 & 701 & 301.2 & 8295 & 0.5043 & 669 & 359.3 \\
\hline & & 8953 & 0.1229 & 601 & 78.62 & 8867 & 0.1390 & 546 & 80.91 \\
\hline & & 9521 & 0.0794 & 650 & 54.93 & 9275 & 0.1135 & 1003 & 121.2 \\
\hline & & 11140 & 0.2985 & 1562 & 995.4 & 11650 & 0.4598 & 1550 & 758.8 \\
\hline & & 12430 & 0.1565 & 1161 & 193.4 & 12740 & 0.1328 & 1186 & 167.7 \\
\hline & & 13950 & 0.1884 & 2475 & 496.3 & 13690 & 0.1542 & 1616 & 265.4 \\
\hline & & 15790 & 0.5263 & 1487 & 830.7 & 16100 & 0.3309 & 1943 & 684.1 \\
\hline & & 17370 & 1.5005 & 1380 & 1313 & 18210 & 1.0158 & 2031 & 1654 \\
\hline & & & & & & 21960 & 3.1770 & 3767 & 306.4 \\
\hline & \multirow[t]{5}{*}{6} & 4395 & 0.1312 & 723 & 98.98 & 4320 & 0.2273 & 460 & 110.3 \\
\hline & & 5095 & 0.1549 & 725 & 120.2 & 4647 & 0.0500 & 282 & 15.01 \\
\hline & & 5500 & 0.0363 & 853 & 32.92 & 5100 & 0.2449 & 615 & 160.2 \\
\hline & & 6290 & 0.0107 & 1073 & 12.20 & 5640 & 0.0331 & 496 & 17.52 \\
\hline & & & & & & 5976 & 0.0130 & 1200 & 1657 \\
\hline
\end{tabular}

Table B3.18(c) Parameters obtained from Gaussian deconvolution of the UV/Vis/NIR spectra for rac$\left[\left\{\mathrm{Os}(\mathrm{bpy})_{2}\right\}_{2}(\mu-\mathrm{BL})\right]^{n+}\{\mathrm{BL}=\mathrm{ppz}, 2,3-\mathrm{dpp}\}$ in $0.1 \mathrm{M}\left[\left(n-\mathrm{C}_{4} \mathrm{H}_{9}\right)_{4} \mathrm{~N} \mathrm{PF}_{6} / \mathrm{CH}_{3} \mathrm{CN}\right.$ at $-35^{\circ} \mathrm{C}$.

\begin{tabular}{|c|c|c|c|c|c|c|c|c|c|}
\hline \multirow[b]{2}{*}{ Diastereoisomer } & \multirow[b]{2}{*}{$n$} & \multicolumn{4}{|c|}{$\mathrm{ppz}$} & \multicolumn{4}{|c|}{ 2,3-dpp } \\
\hline & & $\begin{array}{l}v_{\max } \\
\pm 10 \\
/ \mathrm{cm}^{-1}\end{array}$ & $\begin{aligned} & (\varepsilon / v) \\
\pm & 0.0001 \\
& / \mathrm{M}^{-1}\end{aligned}$ & $\begin{array}{l}\Delta v_{1 / 2} \\
\pm 10 \\
/ \mathrm{cm}^{-1}\end{array}$ & $\begin{array}{c}M_{0} \\
/ \mathrm{M}^{-1}\end{array}$ & $\begin{array}{l}v_{\max } \\
\pm 10 \\
/ \mathrm{cm}^{-1}\end{array}$ & $\begin{aligned} & (\varepsilon / v) \\
\pm & 0.0001 \\
& / \mathrm{M}^{-1}\end{aligned}$ & $\begin{array}{l}\Delta v_{1 / 2} \\
\pm 10 \\
/ \mathrm{cm}^{-1}\end{array}$ & $\begin{array}{c}M_{0} \\
/ \mathrm{M}^{-1}\end{array}$ \\
\hline \multirow[t]{17}{*}{ rac } & \multirow[t]{12}{*}{5} & 5060 & 0.1102 & 993.5 & 114.3 & 4949 & 0.0857 & 516 & 46.54 \\
\hline & & 5970 & 0.0344 & 973.9 & 35.66 & 5342 & 0.0553 & 725 & 42.54 \\
\hline & & 8393 & 0.2986 & 910.9 & 289.5 & 5915 & 0.0355 & 1005 & 38.05 \\
\hline & & 9237 & 0.0700 & 859.6 & 64.03 & 8320 & 0.4416 & 705 & 331.7 \\
\hline & & 11190 & 0.5336 & 1686 & 957.8 & 8905 & 0.1278 & 582 & 79.25 \\
\hline & & 12490 & 0.1152 & 1207 & 148.0 & 9350 & 0.0959 & 918 & 93.72 \\
\hline & & 13630 & 0.0840 & 2056 & 183.8 & 11660 & 0.4251 & 1600 & 724.4 \\
\hline & & 15620 & 0.3752 & 1557 & 621.9 & 12780 & 0.0869 & 1150 & 106.5 \\
\hline & & 17470 & 1.3313 & 1829 & 1741 & 13630 & 0.1312 & 1830 & 266.8 \\
\hline & & & & & & 15980 & 0.3034 & 1780 & 574.5 \\
\hline & & & & & & 18110 & 0.9490 & 2090 & 1650 \\
\hline & & & & & & 20510 & 1.2492 & 2870 & 306.1 \\
\hline & \multirow[t]{5}{*}{6} & 4395 & 0.1310 & 716 & 100.3 & 4270 & 0.2581 & 433 & 117.6 \\
\hline & & 5095 & 0.1535 & 719 & 120.2 & 4626 & 0.0707 & 321 & 24.25 \\
\hline & & 5500 & 0.0365 & 856 & 34.35 & 5065 & 0.2397 & 600 & 153.4 \\
\hline & & 6290 & 0.0109 & 1080 & 12.50 & 5633 & 0.0376 & 467 & 18.73 \\
\hline & & & & & & 6038 & 0.0115 & 1385 & 16.97 \\
\hline
\end{tabular}


Table B3.19 Mid-IR spectral data of the reduced absorption spectra $(\varepsilon / v v s . v)$ for meso- $\left[\left\{\mathrm{Os}(\mathrm{bpy})_{2}\right\}_{2}(\mu-\mathrm{BL})\right]^{5+}$ $\left\{\mathrm{BL}=\mathrm{dpb}, \mathrm{dpb}^{\prime}\right.$ and $\left.\mathrm{ppz}\right\}$ at $+25^{\circ} \mathrm{C}$. The parameters for the overall NIR band envelopes are shown in bold type and details of the deconvoluted bands are in normal type.

\begin{tabular}{|c|c|c|c|c|c|c|}
\hline BL & Diastereoisomer & $\begin{array}{l}v_{\max } \\
\pm 10 \\
/ \mathrm{cm}^{-1}\end{array}$ & $\begin{array}{c}(\varepsilon / v)_{\max } \\
\pm 0.0001 \\
/ \mathrm{M}^{-1}\end{array}$ & $\begin{array}{l}\Delta v_{1 / 2} \\
\pm 10 \\
/ \mathrm{cm}^{-1}\end{array}$ & $\begin{array}{c}M_{0} \\
/ \mathrm{M}^{-1}\end{array}$ & $\begin{array}{c}M_{1} \\
/ \mathrm{cm}^{-1}\end{array}$ \\
\hline dpb & meso & $\begin{array}{l}2150 \\
2090 \\
3000 \\
4850 \\
\end{array}$ & $\begin{array}{l}\mathbf{1 . 8 2 6 2} \\
1.7085 \\
0.7485 \\
0.0693\end{array}$ & $\begin{array}{c}1240 \\
\\
963 \\
1077 \\
495\end{array}$ & 2514 & 2420 \\
\hline dpb' & meso & $\begin{array}{l}2060 \\
2015 \\
2320 \\
2936 \\
\end{array}$ & $\begin{array}{c}3.699 \\
2.7071 \\
1.5677 \\
0.5143 \\
\end{array}$ & $\begin{array}{r}630 \\
\\
426 \\
646 \\
1095 \\
\end{array}$ & 2880 & 2320 \\
\hline $\mathrm{ppz}^{\mathrm{a}}$ & $\begin{array}{l}\text { meso } \\
\text { (i) } \\
\text { (ii) }\end{array}$ & $\begin{array}{l}2275 \\
2318 \\
2798 \\
3080 \\
\\
\\
2234 \\
2535 \\
2760\end{array}$ & $\begin{array}{l}\mathbf{2 . 8 9 0 6} \\
2.8185 \\
0.6803 \\
0.4903 \\
\\
1.7624 \\
1.0830 \\
0.8324\end{array}$ & \begin{tabular}{c|}
775 \\
\\
575 \\
474 \\
921 \\
\\
460 \\
558 \\
1191
\end{tabular} & 2537 & 2529 \\
\hline
\end{tabular}

${ }^{a}$ Results for two different fits to the mid-IR band are reported. 
BL

(a)
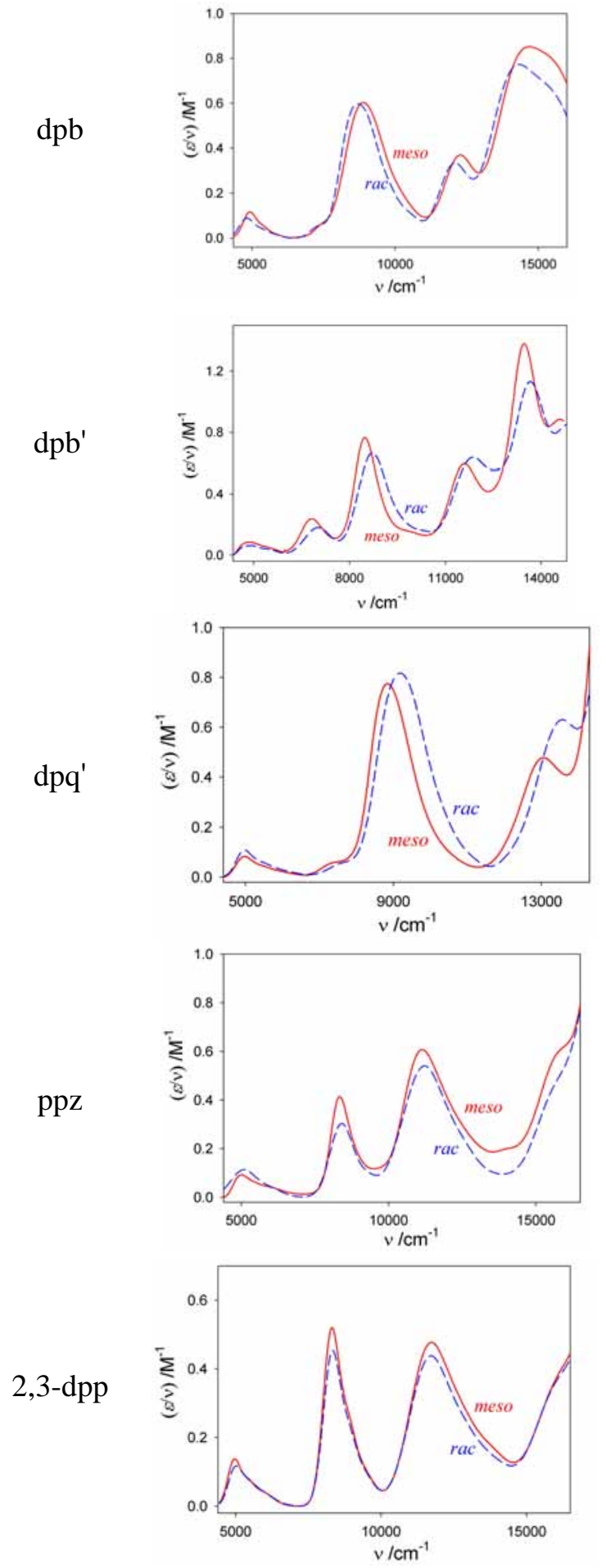

(b)
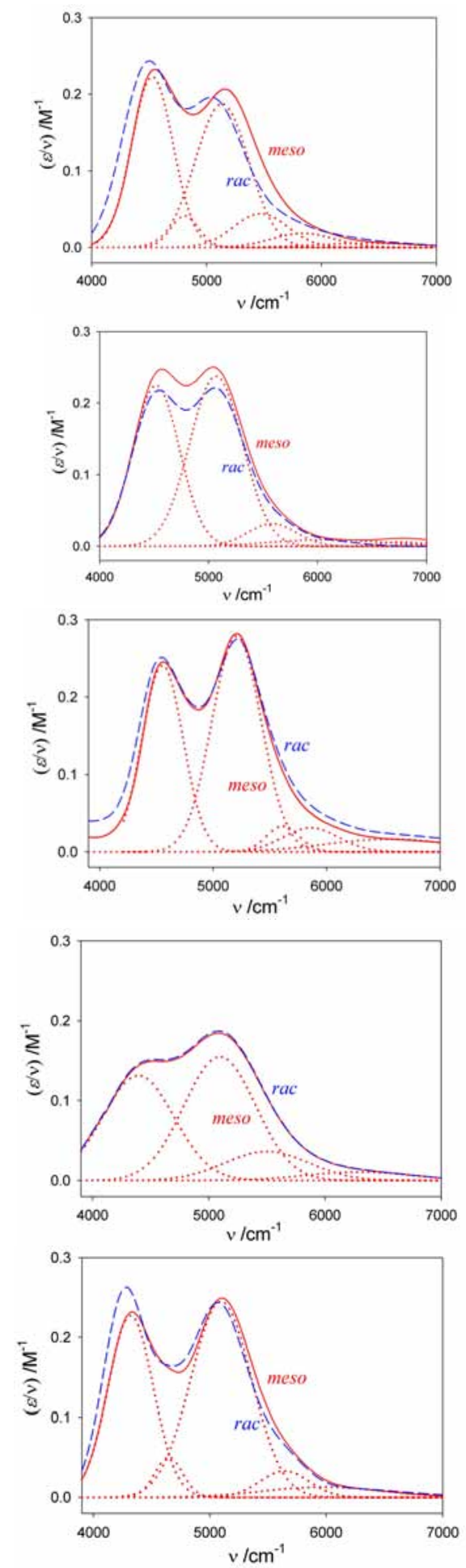

Figure B3.4 Overlay of the NIR spectra of the +5 (a) and +6 (b) forms of meso- and rac- $\left[\left\{\mathrm{Os}(\mathrm{bpy})_{2}\right\}_{2}(\mu-\mathrm{BL})\right]^{5+}$ $\{\mathrm{BL}=\mathrm{dpb}$, dpb', dpq', ppz, 2,3-dpp $\}$ in $0.1 \mathrm{M}\left[\left(n-\mathrm{C}_{4} \mathrm{H}_{9}\right)_{4} \mathrm{~N}\right] \mathrm{PF}_{6} / \mathrm{CH}_{3} \mathrm{CN}$ at $-35^{\circ} \mathrm{C}$ with the bands obtained by Gaussian deconvolution of the meso diastereoisomers of the +6 forms. 
(A)

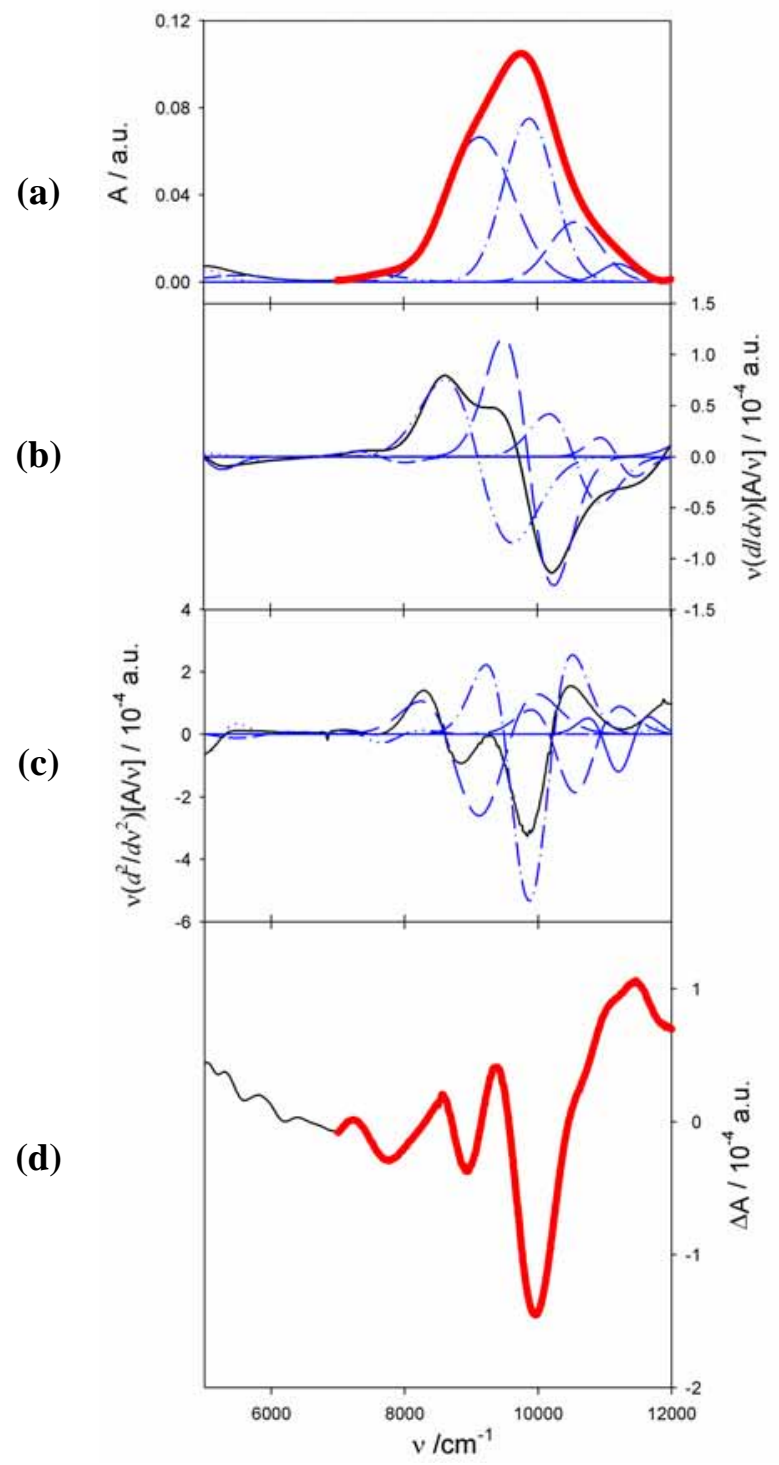

(B)

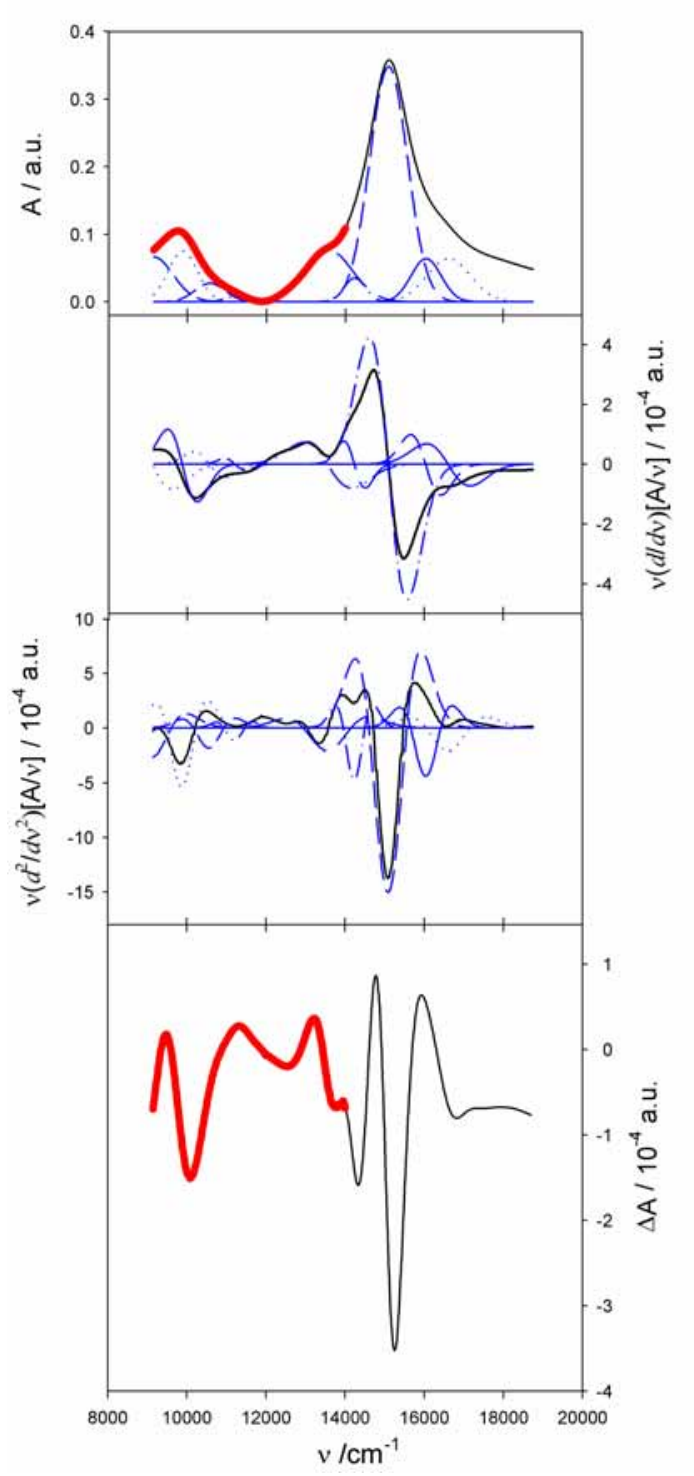

Figure B3.5 Liptay analysis of the Stark absorption spectrum (A vs. v) for meso-[(Os(bpy $\left.\left.)_{2}\right)_{2}\left(\mu-\mathrm{dpq}^{\prime}\right)\right]^{5+}$ in $n$-butyronitrile glass at $77 \mathrm{~K}$, with a field strength of $4.0 \mathrm{MVcm}^{-1}$. (a) Unperturbed absorption spectrum with the bands obtained by Gaussian deconvolution and energy weighted first (b) and second (c) derivatives of the absorption spectrum. (d) Measured (-) and fitted (-) Stark signals ( $\Delta$ A vs. v) at $45^{\circ}$. (A) and (B) show the fits for two different spectral regions. 
(A)

(a)

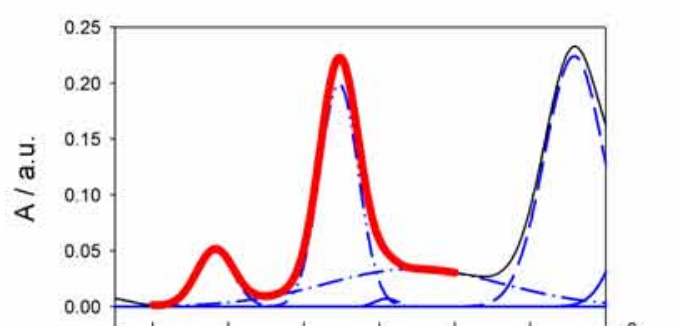

(b)

(c)

(d)

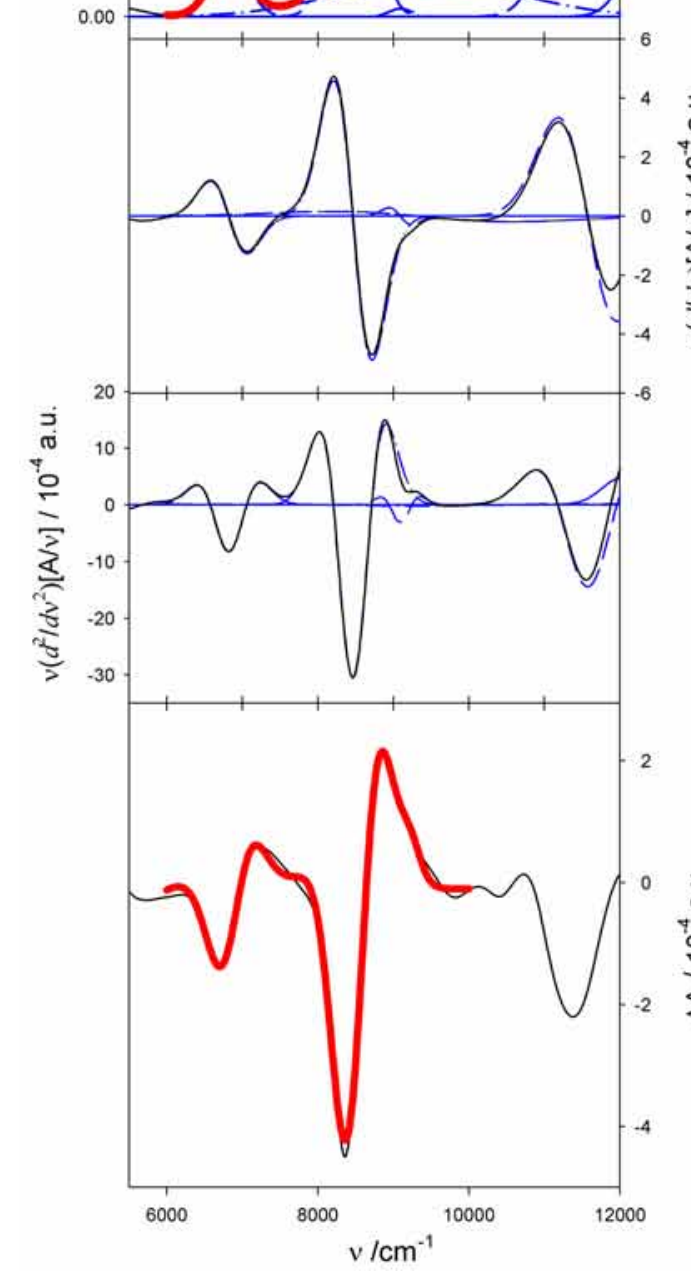

(B)

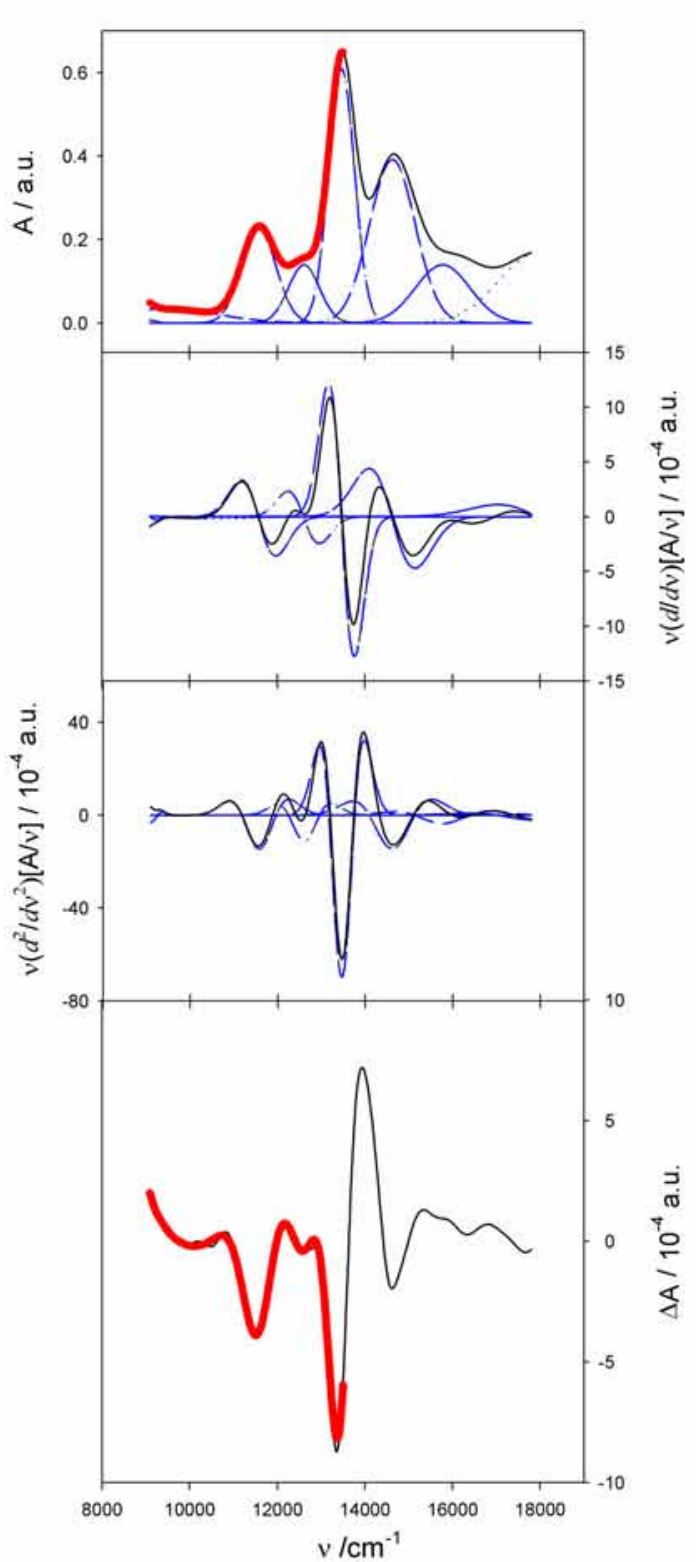

Figure B3.6 Liptay analysis of the Stark absorption spectrum (A vs. v) for meso-[(Os(bpy $\left.\left.)_{2}\right)_{2}(\mu-\mathrm{dpb} ')\right]^{5+}$ in $n$-butyronitrile glass at $77 \mathrm{~K}$, with a field strength of $4.0 \mathrm{MVcm}^{-1}$. (a) Unperturbed absorption spectrum with the bands obtained by Gaussian deconvolution and energy weighted first (b) and second (c) derivatives of the absorption spectrum. (d) Measured (-) and fitted (-) Stark signals $\left(\Delta \mathrm{A}\right.$ vs. v) at $45^{\circ}$. (A) and (B) show the fits for two different spectral regions. 


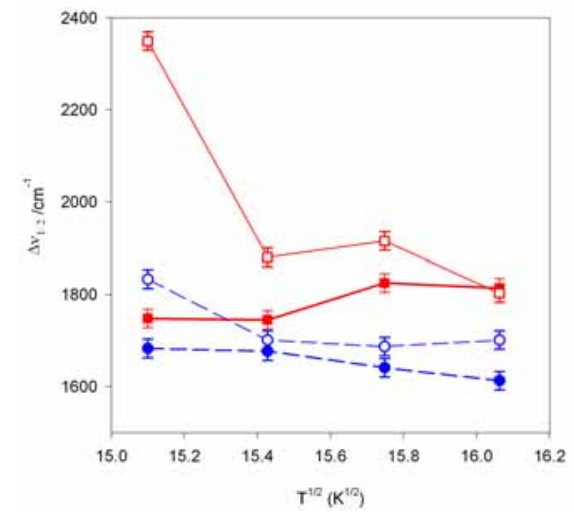

(a)

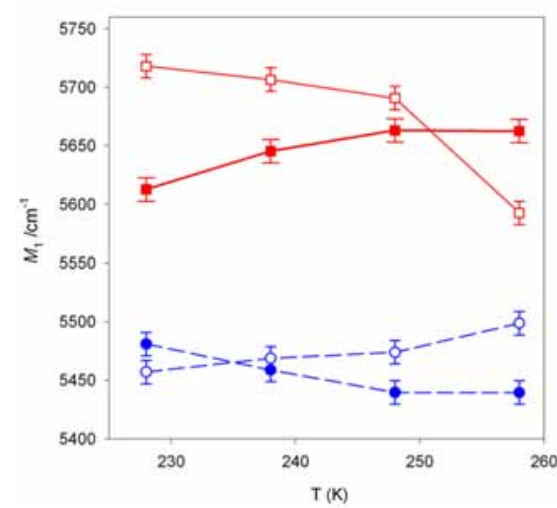

(b)

Figure B3.7 Temperature dependence of (a) $\Delta v_{1 / 2}$ and (b) $M_{1}$ for the meso (-) and rac (---) diastereoisomers of $\left[\left\{\mathrm{Ru}(\mathrm{bpy})_{2}\right\}_{2}(\mu-\mathrm{dpb})\right]^{5+}$ in $0.1 \mathrm{M}\left[\left(n-\mathrm{C}_{4} \mathrm{H}_{9}\right)_{4} \mathrm{~N}\right] \mathrm{PF}_{6}$ or $0.02 \mathrm{M}\left[\left(n-\mathrm{C}_{4} \mathrm{H}_{9}\right)_{4} \mathrm{~N}\right]\left\{\mathrm{B}\left(\mathrm{C}_{6} \mathrm{~F}_{5}\right)_{4}\right\} / n$-butyronitrile over the temperature range $-45^{\circ} \mathrm{C}(228 \mathrm{~K})$ to $-15^{\circ} \mathrm{C}(258 \mathrm{~K})$.

Table B3.20 IVCT thermochromism data of the reduced absorption spectra $(\varepsilon / v v s . v)$ for the diastereoisomers of $\left[\left\{\mathrm{Ru}(\mathrm{bpy})_{2}\right\}_{2}(\mu-\mathrm{dpb})\right]^{5+}$ in $0.1 \mathrm{M}\left[\left(n-\mathrm{C}_{4} \mathrm{H}_{9}\right)_{4} \mathrm{~N}\right]\left(\mathrm{PF}_{6}\right) / n$-butyronitrile.

\begin{tabular}{|c|c|c|c|c|c|c|c|c|c|c|}
\hline \multirow[b]{2}{*}{$\begin{array}{c}\mathrm{T} \\
/ \mathrm{K}\end{array}$} & \multicolumn{5}{|c|}{ meso } & \multicolumn{5}{|c|}{ rac } \\
\hline & $\begin{array}{l}v_{\max } \\
\pm 10 \\
/ \mathrm{cm}^{-1}\end{array}$ & $\begin{array}{c}(\varepsilon / v)_{\max } \\
\pm 0.0001 \\
/ \mathrm{M}^{-1}\end{array}$ & $\begin{array}{l}\Delta \mathrm{v}_{1 / 2} \\
\pm 10 \\
/ \mathrm{cm}^{-1}\end{array}$ & $\begin{array}{c}M_{0} \\
/ \mathrm{M}^{-1}\end{array}$ & $\begin{array}{c}M_{1} \\
/ \mathrm{cm}^{-1}\end{array}$ & $\begin{array}{l}v_{\max } \\
\pm 10 \\
/ \mathrm{cm}^{-1}\end{array}$ & $\begin{array}{c}(\varepsilon / v)_{\max } \\
\pm 0.0001 \\
/ \mathrm{M}^{-1}\end{array}$ & $\begin{array}{l}\Delta v_{1 / 2} \\
\pm 10 \\
/ \mathrm{cm}^{-1}\end{array}$ & $\begin{array}{c}M_{0} \\
/ \mathrm{M}^{-1}\end{array}$ & $\begin{array}{c}M_{1} \\
/ \mathrm{cm}^{-1}\end{array}$ \\
\hline 228 & 5240 & 0.3273 & 2349 & 798.0 & 5718 & 5020 & 0.3372 & 1833 & 664 & 5457 \\
\hline 238 & 5218 & 0.3519 & 1880 & 706.2 & 5707 & 5020 & 0.3874 & 1701 & 741 & 5469 \\
\hline 248 & 5200 & 0.2840 & 1915 & 588.0 & 5691 & 5030 & 0.3637 & 1687 & 609 & 5474 \\
\hline 258 & 5193 & 0.2744 & 1803 & 538.0 & 5593 & 5030 & 0.3607 & 1701 & 665 & 5499 \\
\hline
\end{tabular}

Table B3.21 IVCT thermochromism data of the reduced absorption spectra $(\varepsilon / v v s . v)$ for the diastereoisomers of $\left[\left\{\mathrm{Ru}(\mathrm{bpy})_{2}\right\}_{2}(\mu-\mathrm{dpb})\right]^{5+}$ in $0.02 \mathrm{M}\left[\left(n-\mathrm{C}_{4} \mathrm{H}_{9}\right)_{4} \mathrm{~N}\right]\left\{\mathrm{B}\left(\mathrm{C}_{6} \mathrm{~F}_{5}\right)_{4}\right\} / n$-butyronitrile.

\begin{tabular}{|c|c|c|c|c|c|c|c|c|c|c|}
\hline \multirow[b]{2}{*}{$\begin{array}{c}\mathrm{T} \\
/ \mathrm{K}\end{array}$} & \multicolumn{5}{|c|}{ meso } & \multicolumn{5}{|c|}{ rac } \\
\hline & $\begin{array}{l}v_{\max } \\
\pm 10 \\
/ \mathrm{cm}^{-1}\end{array}$ & $\begin{array}{c}(\varepsilon / v)_{\max } \\
\pm 0.0001 \\
/ \mathrm{M}^{-1}\end{array}$ & $\begin{array}{l}\Delta v_{1 / 2} \\
\pm 10 \\
/ \mathrm{cm}^{-1}\end{array}$ & $\begin{array}{c}M_{0} \\
/ \mathrm{M}^{-1}\end{array}$ & $\begin{array}{c}M_{1} \\
/ \mathrm{cm}^{-1}\end{array}$ & $\begin{array}{l}v_{\max } \\
\pm 10 \\
/ \mathrm{cm}^{-1}\end{array}$ & $\begin{array}{c}(\varepsilon / v)_{\max } \\
\pm 0.0001 \\
/ \mathrm{M}^{-1}\end{array}$ & $\begin{array}{l}\Delta v_{1 / 2} \\
\pm 10 \\
/ \mathrm{cm}^{-1}\end{array}$ & $\begin{array}{c}M_{0} \\
/ \mathrm{M}^{-1}\end{array}$ & $\begin{array}{c}M_{1} \\
/ \mathrm{cm}^{-1}\end{array}$ \\
\hline 228 & 5150 & 0.1620 & 1748 & 302 & 5610 & 4990 & 0.3293 & 1683 & 610 & 5480 \\
\hline 238 & 5170 & 0.2673 & 1745 & 493 & 5645 & 4995 & 0.3698 & 1677 & 680 & 5460 \\
\hline 248 & 5180 & 0.3384 & 1825 & 666 & 5665 & 5006 & 0.3712 & 1641 & 671 & 5440 \\
\hline 258 & 5200 & 0.2588 & 1814 & 507 & 5660 & 5000 & 0.2621 & 1613 & 465 & 5440 \\
\hline
\end{tabular}




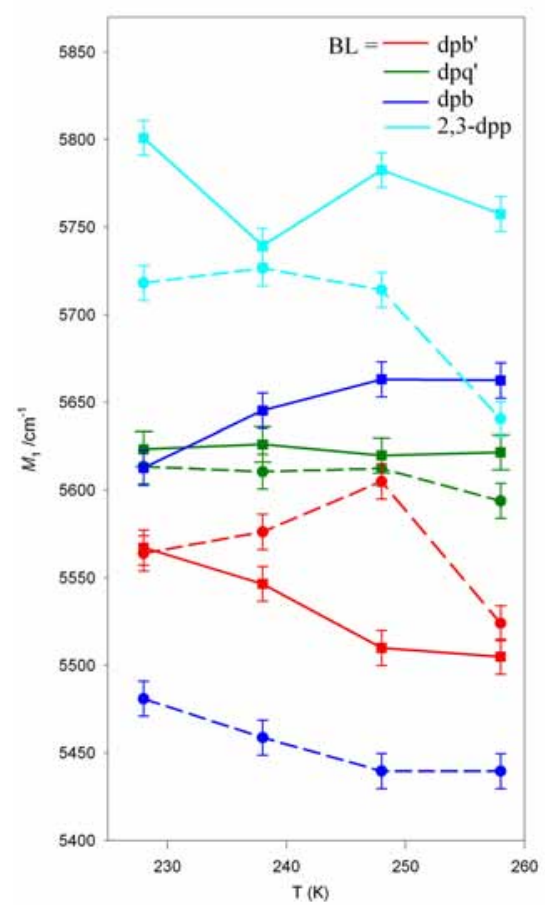

(a)

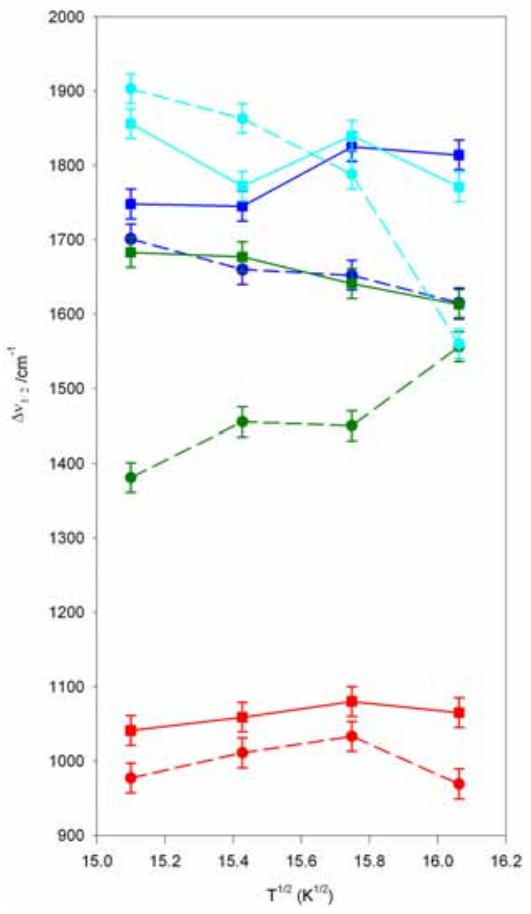

(b)

Figure B3.8 Temperature dependence of (a) and (b) $\Delta v_{1 / 2}$ for the meso (solid line) and rac (dashed line) diastereoisomers of $\left[\left\{\mathrm{Ru}(\mathrm{bpy})_{2}\right\}_{2}(\mu-\mathrm{BL})\right]^{5+}\{\mathrm{BL}=\mathrm{dpb}$, dpb', dpq', 2,3-dpp $\}$ in $0.02 \mathrm{M}\left[\left(n-\mathrm{C}_{4} \mathrm{H}_{9}\right)_{4} \mathrm{~N}\right]\left\{\mathrm{B}\left(\mathrm{C}_{6} \mathrm{~F}_{5}\right)_{4}\right\} / n-$ butyronitrile over the temperature range $-45^{\circ} \mathrm{C}(228 \mathrm{~K})$ to $-15^{\circ} \mathrm{C}(258 \mathrm{~K})$.

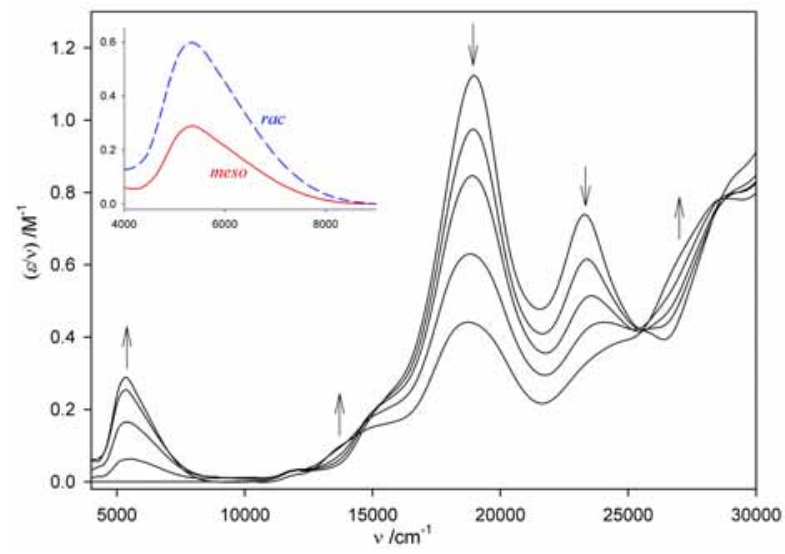

Figure B3.9 Spectral progression following the oxidation of meso- $\left[\left\{\operatorname{Ru}(\mathrm{bpy})_{2}\right\}_{2}(\mu-2,3-\mathrm{dpp})\right]^{4+} \rightarrow$ meso$\left[\left\{\mathrm{Ru}(\mathrm{bpy})_{2}\right\}_{2}(\mu-2,3-\mathrm{dpp})\right]^{5+}$ in $0.02 \mathrm{M}\left[\left(n-\mathrm{C}_{4} \mathrm{H}_{9}\right)_{4}\right]\left\{\mathrm{B}\left(\mathrm{C}_{6} \mathrm{~F}_{5}\right)_{4}\right\} / n$-butyronitrile at $-35^{\circ} \mathrm{C}$. The inset shows the overlay of the IVCT bands for the meso and rac diastereoisomers of the +5 state. 
Table B3.22 IVCT thermochromism data for the diastereoisomers of $\left.\left[\left\{\mathrm{Ru}(\mathrm{bpy})_{2}\right\}_{2}(\mu-\mathrm{dpb})^{\prime}\right)\right]^{5+}$ in $0.02 \mathrm{M}$ $\left[\left(n-\mathrm{C}_{4} \mathrm{H}_{9}\right)_{4} \mathrm{~N}\right]\left\{\mathrm{B}\left(\mathrm{C}_{6} \mathrm{~F}_{5}\right)_{4}\right\} / n$-butyronitrile.

\begin{tabular}{|c|c|c|c|c|c|c|c|c|c|c|}
\hline \multirow[b]{2}{*}{$\begin{array}{l}\mathrm{T} \\
/ \mathrm{K}\end{array}$} & \multicolumn{5}{|c|}{ meso } & \multicolumn{5}{|c|}{ rac } \\
\hline & $\begin{array}{l}v_{\max } \\
\pm 10 \\
/ \mathrm{cm}^{-1}\end{array}$ & $\begin{array}{c}(\varepsilon / v)_{\max } \\
\pm 0.0001 \\
/ \mathrm{M}^{-1}\end{array}$ & $\begin{array}{l}\Delta v_{1 / 2} \\
\pm 10 \\
/ \mathrm{cm}^{-1}\end{array}$ & $\begin{array}{c}M_{0} \\
/ \mathrm{M}^{-1}\end{array}$ & $\begin{array}{c}M_{1} \\
/ \mathrm{cm}^{-1}\end{array}$ & $\begin{array}{l}v_{\max } \\
\pm 10 \\
/ \mathrm{cm}^{-1}\end{array}$ & $\begin{array}{c}(\varepsilon / v)_{\max } \\
\pm 0.0001 \\
/ \mathrm{M}^{-1}\end{array}$ & $\begin{array}{l}\Delta v_{1 / 2} \\
\pm 10 \\
/ \mathrm{cm}^{-1}\end{array}$ & $\begin{array}{c}M_{0} \\
/ \mathrm{M}^{-1}\end{array}$ & $\begin{array}{c}M_{1} \\
/ \mathrm{cm}^{-1}\end{array}$ \\
\hline 228 & 5290 & 0.3968 & 1040 & 520 & 5570 & 5390 & 0.4210 & 980 & 550 & 5565 \\
\hline 238 & 5285 & 0.3721 & 1060 & 505 & 5550 & 5380 & 0.3405 & 1010 & 560 & 5575 \\
\hline 248 & 5285 & 0.3338 & 1080 & 450 & 5510 & 5390 & 0.2300 & 1030 & 310 & 5605 \\
\hline 258 & 5300 & 0.3160 & 1065 & 410 & 5505 & 5390 & 0.3910 & 970 & 520 & 5525 \\
\hline
\end{tabular}

Table B3.23 IVCT thermochromism data for the diastereoisomers of $\left[\left\{\mathrm{Ru}(\mathrm{bpy})_{2}\right\}_{2}\left(\mu-\mathrm{dpq} \mathrm{q}^{\prime}\right)\right]^{5+}$ in $0.02 \mathrm{M}$ $\left[\left(n-\mathrm{C}_{4} \mathrm{H}_{9}\right)_{4} \mathrm{~N}\right]\left\{\mathrm{B}\left(\mathrm{C}_{6} \mathrm{~F}_{5}\right)_{4}\right\} / n$-butyronitrile.

\begin{tabular}{|c|c|c|c|c|c|c|c|c|c|c|}
\hline \multirow[b]{2}{*}{$\begin{array}{c}\mathrm{T} \\
/ \mathrm{K}\end{array}$} & \multicolumn{5}{|c|}{ meso } & \multicolumn{5}{|c|}{ rac } \\
\hline & $\begin{array}{l}v_{\max } \\
\pm 10 \\
/ \mathrm{cm}^{-1}\end{array}$ & $\begin{array}{c}(\varepsilon / v)_{\max } \\
\pm 0.0001 \\
/ \mathrm{M}^{-1}\end{array}$ & $\begin{array}{l}\Delta v_{1 / 2} \\
\pm 10 \\
/ \mathrm{cm}^{-1}\end{array}$ & $\begin{array}{c}M_{0} \\
/ \mathrm{M}^{-1}\end{array}$ & $\begin{array}{c}M_{1} \\
/ \mathrm{cm}^{-1}\end{array}$ & $\begin{array}{l}v_{\max } \\
\pm 10 \\
/ \mathrm{cm}^{-1}\end{array}$ & $\begin{array}{c}(\varepsilon / v)_{\max } \\
\pm 0.0001 \\
/ \mathrm{M}^{-1}\end{array}$ & $\begin{array}{l}\Delta v_{1 / 2} \\
\pm 10 \\
/ \mathrm{cm}^{-1}\end{array}$ & $\begin{array}{c}M_{0} \\
/ \mathrm{M}^{-1}\end{array}$ & $\begin{array}{c}M_{1} \\
/ \mathrm{cm}^{-1}\end{array}$ \\
\hline 228 & 5224 & 0.4090 & 1701 & 768.6 & 5623 & 5329 & 0.5974 & 1381 & 941 & 5614 \\
\hline 238 & 5224 & 0.3908 & 1660 & 715.4 & 5626 & 5320 & 0.5311 & 1455 & 895 & 5611 \\
\hline 248 & 5224 & 0.3683 & 1652 & 669.6 & 5620 & 5315 & 0.4987 & 1450 & 833 & 5612 \\
\hline 258 & 5224 & 0.3476 & 1615 & 615.4 & 5622 & 5290 & 0.4574 & 1556 & 758 & 5594 \\
\hline
\end{tabular}

Table B3.24 IVCT thermochromism data for the diastereoisomers of $\left[\left\{\operatorname{Ru}(b p y)_{2}\right\}_{2}(\mu-2,3-d p p)\right]^{5+}$ in $0.02 \mathrm{M}$ $\left[\left(n-\mathrm{C}_{4} \mathrm{H}_{9}\right)_{4} \mathrm{~N}\right]\left\{\mathrm{B}\left(\mathrm{C}_{6} \mathrm{~F}_{5}\right)_{4}\right\} / n$-butyronitrile.

\begin{tabular}{|c|c|c|c|c|c|c|c|c|c|c|}
\hline \multirow{2}{*}{$\mathrm{T}$} & \multicolumn{9}{|c|}{ meso } & \multicolumn{5}{c|}{ rac } \\
\cline { 2 - 10 }$/ \mathrm{K}$ & $\begin{array}{c}v_{\max } \\
\pm 10 \\
/ \mathrm{cm}^{-1}\end{array}$ & $\begin{array}{c}(\varepsilon / v)_{\max } \\
\pm .0001 \\
/ \mathrm{M}^{-1}\end{array}$ & $\begin{array}{c}\Delta v_{1 / 2} \\
\pm 10 \\
/ \mathrm{cm}^{-1}\end{array}$ & $\begin{array}{c}M_{0} \\
/ \mathrm{M}^{-1}\end{array}$ & $\begin{array}{c}M_{1} \\
/ \mathrm{cm}^{-1}\end{array}$ & $\begin{array}{c}v_{\max } \\
\pm 10 \\
/ \mathrm{cm}^{-1}\end{array}$ & $\begin{array}{c}(\varepsilon / \mathrm{v})_{\max } \\
\pm 0.0001 \\
/ \mathrm{M}^{-1}\end{array}$ & $\begin{array}{c}\Delta v_{1 / 2} \\
\pm 10 \\
/ \mathrm{cm}^{-1}\end{array}$ & $\begin{array}{c}M_{0} \\
/ \mathrm{M}^{-1}\end{array}$ & $\begin{array}{c}M_{1} \\
/ \mathrm{cm}^{-1}\end{array}$ \\
\hline 228 & 5350 & 0.5195 & 1856 & 1050 & 5800 & 5304 & 0.5858 & 1903 & 1214 & 5718 \\
238 & 5340 & 0.2890 & 1772 & 563.1 & 5740 & 5307 & 0.5980 & 1863 & 1220 & 5727 \\
248 & 5336 & 0.6399 & 1840 & 1297 & 5782 & 5330 & 0.5282 & 1788 & 1046 & 5714 \\
258 & 5345 & 0.5649 & 1771 & 1106 & 5757 & 5456 & 0.3239 & 1560 & 585 & 5641 \\
\hline
\end{tabular}


Table B3.25 IVCT solvatochromism data for the diastereoisomers of $\left[\left\{\mathrm{Ru}(\mathrm{bpy})_{2}\right\}_{2}(\mu-\mathrm{dpb})\right]^{5+}$ in $0.02 \mathrm{M}$ $\left[\left(n-\mathrm{C}_{4} \mathrm{H}_{9}\right)_{4} \mathrm{~N}\right]\left\{\mathrm{B}\left(\mathrm{C}_{6} \mathrm{~F}_{5}\right)_{4}\right\} /$ solvent at $-35^{\circ} \mathrm{C}$.

\begin{tabular}{|c|c|c|c|c|c|c|c|c|c|c|c|}
\hline \multirow[b]{2}{*}{ Solvent } & \multirow[b]{2}{*}{$1 / \mathrm{D}_{\mathrm{op}}-1 / \mathrm{D}_{\mathrm{s}}$} & \multicolumn{5}{|c|}{ meso } & \multicolumn{5}{|c|}{ rac } \\
\hline & & $\begin{array}{l}v_{\max } \\
\pm 10 \\
/ \mathrm{cm}^{-1}\end{array}$ & $\begin{array}{c}(\varepsilon / v)_{\max } \\
\pm 0.0001 \\
/ \mathrm{M}^{-1}\end{array}$ & $\begin{array}{l}\Delta \mathrm{v}_{1 / 2} \\
\pm 10 \\
/ \mathrm{cm}^{-1}\end{array}$ & $\begin{array}{c}M_{0} \\
/ \mathrm{M}^{-1}\end{array}$ & $\begin{array}{c}M_{1} \\
/ \mathrm{cm}^{-1}\end{array}$ & $\begin{array}{l}v_{\max } \\
\pm 10 \\
/ \mathrm{cm}^{-1}\end{array}$ & $\begin{array}{c}(\varepsilon / v)_{\max } \\
\pm 0.0001 \\
/ \mathrm{M}^{-1}\end{array}$ & $\begin{array}{l}\Delta v_{1 / 2} \\
\pm 10 \\
/ \mathrm{cm}^{-1}\end{array}$ & $\begin{array}{c}M_{0} \\
/ \mathrm{M}^{-1}\end{array}$ & $\begin{array}{c}M_{1} \\
/ \mathrm{cm}^{-1}\end{array}$ \\
\hline AN & 0.5127 & 5125 & 0.3668 & 1718 & 688 & 5615 & 4990 & 0.4206 & 1634 & 753 & 5422 \\
\hline PN & 0.5011 & 5195 & 0.2862 & 1928 & 592 & 5710 & 5050 & 0.3270 & 1683 & 595 & 5460 \\
\hline $\mathrm{BN}$ & 0.4762 & 5170 & 0.2673 & 1745 & 493 & 5645 & 4995 & 0.3698 & 1677 & 680 & 5460 \\
\hline $\mathrm{AO}$ & 0.493 & 5130 & 0.3738 & 1745 & 710 & 5615 & 5020 & 0.3101 & 1724 & 580 & 5488 \\
\hline DCM & 0.380 & 5030 & 0.5082 & 2154 & 960 & 5442 & 4970 & 0.4395 & 1680 & 816 & 5418 \\
\hline
\end{tabular}

Table B3.26 IVCT solvatochromism data for the diastereoisomers of $\left[\left\{\mathrm{Ru}(\mathrm{bpy})_{2}\right\}_{2}(\mu-\mathrm{dpb})\right]^{5+}$ in $0.02 \mathrm{M}$ $\left[\left(n-\mathrm{C}_{4} \mathrm{H}_{9}\right)_{4} \mathrm{~N}\right]\left\{\mathrm{B}\left(\mathrm{C}_{6} \mathrm{~F}_{5}\right)_{4}\right\} /$ solvent at $-35^{\circ} \mathrm{C}$.

\begin{tabular}{|c|c|c|c|c|c|c|c|c|c|c|c|}
\hline & & \multicolumn{9}{|c|}{ meso } & \multicolumn{5}{c|}{ rac } \\
\cline { 3 - 11 } Solvent & & $\begin{array}{c}v_{\max } \\
\pm 10\end{array}$ & $\begin{array}{c}(\varepsilon / v)_{\max } \\
\pm 0.0001 \\
/ \mathrm{D}_{\mathrm{op}}-1 / \mathrm{D}_{\mathrm{s}}\end{array}$ & $\begin{array}{c}\Delta v_{1 / 2} \\
\pm 10\end{array}$ & $\begin{array}{c}M_{0} \\
/ \mathrm{M}^{-1}\end{array}$ & $\begin{array}{c}M_{1} \\
/ \mathrm{cm}^{-1}\end{array}$ & $\begin{array}{c}v_{\max } \\
\pm 10 \\
/ \mathrm{cm}^{-1}\end{array}$ & $\begin{array}{c}(\varepsilon / \mathrm{v})_{\max } \\
\pm 0.0001 \\
/ \mathrm{M}^{-1}\end{array}$ & $\begin{array}{c}\Delta v_{1 / 2} \\
\pm 10\end{array}$ & $\begin{array}{c}M_{0} \\
/ \mathrm{cm}^{-1}\end{array}$ & $\begin{array}{c}M_{1} \\
/ \mathrm{cm}^{-1}\end{array}$ \\
\hline $\mathrm{AN}$ & 0.5127 & 5250 & 0.3928 & 1048 & 482 & 5496 & 5295 & 0.300 & 1250 & 460 & 5608 \\
$\mathrm{PN}$ & 0.5011 & 5285 & 0.5311 & 1065 & 683 & 5636 & 5360 & 0.4000 & 1010 & 500 & 5570 \\
$\mathrm{BN}$ & 0.4762 & 5285 & 0.3721 & 1060 & 503 & 5545 & 5380 & 0.3405 & 1010 & 457 & 5575 \\
$\mathrm{AO}$ & 0.493 & 5350 & 0.3051 & 1095 & 415 & 5520 & 5390 & 0.3761 & 1035 & 520 & 5590 \\
$\mathrm{DCM}$ & 0.380 & 5230 & 0.3159 & 1050 & 437 & 5530 & 5310 & 0.1650 & 1050 & 246 & 5510 \\
\hline
\end{tabular}

Table B3.27 IVCT solvatochromism data for the diastereoisomers of $\left[\left\{\mathrm{Ru}(\mathrm{bpy})_{2}\right\}_{2}(\mu-\mathrm{dpq})\right]^{5+}$ in $0.02 \mathrm{M}$ $\left[\left(n-\mathrm{C}_{4} \mathrm{H}_{9}\right)_{4} \mathrm{~N}\right]\left\{\mathrm{B}\left(\mathrm{C}_{6} \mathrm{~F}_{5}\right)_{4}\right\} /$ solvent at $-35^{\circ} \mathrm{C}$.

\begin{tabular}{|c|c|c|c|c|c|c|c|c|c|c|c|}
\hline & & \multicolumn{9}{|c|}{ meso } & \multicolumn{5}{c|}{ rac } \\
\cline { 3 - 11 } Solvent & $1 / \mathrm{D}_{\mathrm{op}}-1 / \mathrm{D}_{\mathrm{s}}$ & $\begin{array}{c}v_{\max } \\
\pm 10 \\
/ \mathrm{cm}^{-1}\end{array}$ & $\begin{array}{c}(\varepsilon / v)_{\max } \\
\pm 0.0001 \\
/ \mathrm{M}^{-1}\end{array}$ & $\begin{array}{c}\Delta v_{1 / 2} \\
\pm 10 \\
/ \mathrm{cm}^{-1}\end{array}$ & $\begin{array}{c}M_{0} \\
/ \mathrm{M}^{-1}\end{array}$ & $\begin{array}{c}M_{1} \\
/ \mathrm{cm}^{-1}\end{array}$ & $\begin{array}{c}v_{\max } \\
\pm 10 \\
/ \mathrm{cm}^{-1}\end{array}$ & $\begin{array}{c}(\varepsilon / v)_{\max } \\
\pm 0.0001 \\
/ \mathrm{M}^{-1}\end{array}$ & $\begin{array}{c}\Delta v_{1 / 2} \\
\pm 10 \\
/ \mathrm{cm}^{-1}\end{array}$ & $\begin{array}{c}M_{0} \\
/ \mathrm{M}^{-1}\end{array}$ & $\begin{array}{c}M_{1} \\
/ \mathrm{cm}^{-1}\end{array}$ \\
\hline $\mathrm{AN}$ & 0.5127 & 5230 & 0.4182 & 1755 & 802 & 5644 & 5264 & 0.4979 & 1566 & 871 & 5630 \\
$\mathrm{PN}$ & 0.5011 & 5225 & 0.4088 & 1650 & 741 & 5637 & 5290 & 0.5062 & 1463 & 846 & 5620 \\
$\mathrm{BN}$ & 0.4762 & 5224 & 0.3908 & 1660 & 787 & 5626 & 5320 & 0.5311 & 1455 & 896 & 5611 \\
${ }^{\mathrm{i}} \mathrm{BN}$ & 0.4795 & 5238 & 0.4844 & 1625 & 864 & 5642 & 5332 & 0.5548 & 1393 & 893 & 5617 \\
$\mathrm{AO}$ & 0.493 & 5346 & 0.3674 & 1774 & 707 & 5766 & 5340 & 0.4855 & 1585 & 870 & 5708 \\
$\mathrm{DCM}$ & 0.380 & 5104 & 0.2204 & 1351 & 329 & 5218 & 5087 & 0.1562 & 1041 & 190 & 5276 \\
\hline
\end{tabular}

Table B3.28 Summary of the solvent dependence of $v_{\max }$ for $\left[\left\{\mathrm{Ru}(\mathrm{bpy})_{2}\right\}_{2}(\mu-\mathrm{BL})\right]^{5+}\{\mathrm{BL}=\mathrm{dpb}, \mathrm{dpb}$ ', dpq' $\}$ according to the dielectric continuum model.

\begin{tabular}{|l|c|c|c|}
\hline \multicolumn{1}{|c|}{ Complex } & $\begin{array}{c}\text { Slope } \\
e^{2}(1 / a-1 / d) \\
/ \mathrm{cm}^{-1}\end{array}$ & $\begin{array}{c}\text { Intercept } \\
\lambda_{i}+\Delta \mathrm{E}^{\prime}+\Delta \mathrm{E}_{0} \\
/ \mathrm{cm}^{-1}\end{array}$ & $R^{2}$ \\
\hline meso- $\left[\left\{\mathrm{Ru}(\mathrm{bpy})_{2}\right\}_{2}(\mu-\mathrm{dpb})\right]^{5+}$ & $850 \pm 415$ & $4725 \pm 200$ & 0.59 \\
rac- $\left[\left\{\mathrm{Ru}(\mathrm{bpy})_{2}\right\}_{2}(\mu-\mathrm{dpb})\right]^{5+}$ & $305 \pm 260$ & $4860 \pm 125$ & 0.31 \\
meso- $\left[\left\{\mathrm{Ru}(\mathrm{bpy})_{2}\right\}_{2}(\mu-\mathrm{dpb})\right]^{5+}$ & $363 \pm 436$ & $5110 \pm 210$ & 0.19 \\
rac- $\left[\left\{\mathrm{Ru}(\mathrm{bpy})_{2}\right\}_{2}\left(\mu-\mathrm{dpb}^{\prime}\right)\right]^{5+}$ & $170 \pm 430$ & $5265 \pm 205$ & 0.05 \\
meso- $\left[\left\{\mathrm{Ru}(\mathrm{bpy})_{2}\right\}_{2}(\mu-\mathrm{dpq})\right]^{5+}$ & $1090 \pm 525$ & $4710 \pm 250$ & 0.52 \\
rac- $\left[\left\{\mathrm{Ru}(\mathrm{bpy})_{2}\right\}_{2}(\mu-\mathrm{dpq})\right]^{5+}$ & $1495 \pm 570$ & $4560 \pm 270$ & 0.63 \\
\hline
\end{tabular}


Table B3.29 Summary of the energies of the IC transitions ( $v_{\mathrm{IC}}$ ) obtained by Gaussian deconvolution for $\left[\left\{\mathrm{Os}(\mathrm{bpy})_{2}\right\}_{2}(\mu-\mathrm{BL})\right]^{6+}$ and the predicted energies of the IC bands for $\left[\left\{\mathrm{Ru}(\mathrm{bpy})_{2}\right\}_{2}(\mu-\mathrm{BL})\right]^{5+}\left\{\mathrm{BL}=\mathrm{dpb}, \mathrm{dpb} \mathrm{d}^{\prime}, \mathrm{dpq}\right\}$.

\begin{tabular}{|c|c|c|c|c|c|}
\hline \multirow[t]{2}{*}{ Complex } & \multicolumn{2}{|c|}{$\begin{array}{c}\text { Observed } \\
\mathrm{M}=\text { Os; } n=6+\end{array}$} & \multicolumn{3}{|c|}{$\begin{array}{c}\text { Predicted } \\
\mathrm{M}=\mathrm{Ru} ; n=5+\end{array}$} \\
\hline & $\begin{array}{c}v_{\mathrm{IC}} \\
/ \mathrm{cm}^{-1}\end{array}$ & $\begin{array}{l}\Delta v_{\mathrm{IC}}^{\mathrm{a}} \\
/ \mathrm{cm}^{-1}\end{array}$ & $\begin{array}{l}v_{\mathrm{IC}}^{\mathrm{b}} \\
/ \mathrm{cm}^{-1}\end{array}$ & $\begin{array}{c}\Delta^{\mathrm{c}} \\
/ \mathrm{cm}^{-1}\end{array}$ & $\begin{array}{l}\Delta v_{\mathrm{IC}}{ }^{\mathrm{a}} \\
/ \mathrm{cm}^{-1}\end{array}$ \\
\hline meso- $\left[\left\{\mathrm{M}(\mathrm{bpy})_{2}\right\}_{2}(\mu-\mathrm{dpb})\right]^{n+}$ & $\begin{array}{l}4550 \\
5160\end{array}$ & 610 & $\begin{array}{l}1515 \\
1720 \\
\end{array}$ & 1620 & 205 \\
\hline$r a c-\left[\left\{\mathrm{M}(\mathrm{bpy})_{2}\right\}_{2}(\mu-\mathrm{dpb})\right]^{n+}$ & $\begin{array}{l}4550 \\
5145\end{array}$ & 595 & $\begin{array}{l}1515 \\
1715\end{array}$ & 1615 & 200 \\
\hline meso- $\left[\left\{\mathrm{M}(\mathrm{bpy})_{2}\right\}_{2}\left(\mu-\mathrm{dpb}^{\prime}\right)\right]^{n+}$ & $\begin{array}{l}4530 \\
5080\end{array}$ & 550 & $\begin{array}{l}1510 \\
1690\end{array}$ & 1610 & 200 \\
\hline rac- $\left[\left\{\mathrm{M}(\mathrm{bpy})_{2}\right\}_{2}\left(\mu-\mathrm{dpb}^{\prime}\right)\right]^{n+}$ & $\begin{array}{l}4470 \\
5045 \\
\end{array}$ & 575 & $\begin{array}{l}1490 \\
1680 \\
\end{array}$ & 1590 & 200 \\
\hline meso- $\left.\left[\left\{\mathrm{M}(\mathrm{bpy})_{2}\right\}_{2}(\mu-\mathrm{dpq})^{\prime}\right)\right]^{n+}$ & $\begin{array}{l}4520 \\
5170\end{array}$ & 650 & $\begin{array}{l}1505 \\
1720 \\
\end{array}$ & 1610 & 215 \\
\hline rac- $\left[\left\{\mathrm{M}(\mathrm{bpy})_{2}\right\}_{2}(\mu-\mathrm{dpq})\right]^{n+}$ & $\begin{array}{l}4505 \\
5175\end{array}$ & 670 & $\begin{array}{l}1500 \\
1725\end{array}$ & 1610 & 225 \\
\hline
\end{tabular}

${ }^{\mathrm{a}}$ Difference between the energies of the IC bands.

${ }^{\mathrm{b}}$ The predicted energies of the IC bands in the dinuclear ruthenium systems occur at $\sim 1 / 3$ the energies in the analogous dinuclear osmium systems, since $\xi_{\mathrm{Ru}}$ is $\sim 1 / 3 \xi_{\mathrm{Os}}$.

${ }^{c}$ Average energy of the IC bands and predicted energy difference between the absorption band maximum and IVCT(1).

Table B3.30 Electrochemical data (in $\mathrm{mV}$ relative to the $\mathrm{Fc}^{+} / \mathrm{Fc}^{0}$ couple) and comproportionation constants ${ }^{\mathrm{a}}\left(\mathrm{K}_{\mathrm{c}}\right)$ for $\left[\left\{\mathrm{Ru}(\mathrm{bpy})_{2}\right\}_{2}(\mu-\mathrm{BL})\right]^{4+}$ in $0.1 \mathrm{M}\left[\left(n-\mathrm{C}_{4} \mathrm{H}_{9}\right)_{4} \mathrm{~N}\right] \mathrm{PF}_{6} / \mathrm{CH}_{3} \mathrm{CN}$. $^{\mathrm{b}}$

\begin{tabular}{|c|c|c|c|c|c|c|c|c|c|}
\hline BL & Diastereoisomer & $\Delta \mathrm{E}_{\text {ox }}$ & $\mathrm{E}_{\text {ox2 }}$ & $\mathrm{E}_{\text {ox1 }}$ & $\mathrm{E}_{\text {red1 }}$ & $\mathrm{E}_{\text {red2 }}$ & $\mathrm{E}_{\text {red3 }}$ & $\mathrm{E}_{\text {red4 }}$ & $\mathrm{E}_{\text {red5 }}$ \\
\hline 2,5-dpp & meso & 215 & 1185 & 970 & -980 & -1530 & & & \\
& rac & 210 & 1186 & 976 & -978 & -1522 & & & \\
\hline dpop & meso & 207 & 1279 & 1072 & -588 & -1348 & $-1908^{\mathrm{c}}$ & -2100 & \\
& rac & 200 & 1276 & 1076 & -608 & -1332 & $-1908^{\mathrm{c}}$ & -2165 & -2218 \\
\hline
\end{tabular}

${ }^{\mathrm{a}} \mathrm{K}_{\mathrm{c}}$ values determined from equation 1.18(a).

${ }^{\mathrm{b}} \Delta \mathrm{E}_{\mathrm{ox}}=\mathrm{E}_{\mathrm{ox} 2}-\mathrm{E}_{\mathrm{ox} 1}$. Potentials are quoted $\pm 3 \mathrm{mV}$.

${ }^{\mathrm{c}}$ Two-electron reduction process.

Table B3.31 UV/Vis/NIR spectral data for the stepped-parallel dinuclear complexes $\left[\left\{\mathrm{Ru}(\mathrm{pp})_{2}\right\}_{2}(\mu-\mathrm{BL})\right]^{n+}$ in 0.02 $\mathrm{M}\left[\left(n-\mathrm{C}_{4} \mathrm{H}_{9}\right)_{4} \mathrm{~N}\right]\left\{\mathrm{B}\left(\mathrm{C}_{6} \mathrm{~F}_{5}\right)_{4}\right\} / \mathrm{CH}_{3} \mathrm{CN}$ at $+25^{\circ} \mathrm{C}$ for $\mathrm{BL}=2,5$-dpp, dpop and $-35^{\circ} \mathrm{C}$ for $\mathrm{BL}=$ apy. The parameters of the IVCT bands are indicated in bold type.

\begin{tabular}{|c|c|c|c|c|c|c|c|}
\hline \multicolumn{2}{|l|}{ BL } & \multicolumn{2}{|c|}{ 2,5-dpp } & \multicolumn{2}{|c|}{ dpop } & \multicolumn{2}{|c|}{ apy } \\
\hline Diastereoisomer & $n$ & $\begin{array}{c}v \\
\pm 10 \\
/ \mathrm{cm}^{-1}\end{array}$ & $\begin{array}{c}(\varepsilon / v) \\
\pm 0.0001 \\
\\
/ \mathrm{M}^{-1}\end{array}$ & $\begin{array}{c}v \\
\pm 10 \\
/ \mathrm{cm}^{-1}\end{array}$ & $\begin{array}{c}(\varepsilon / v) \\
\pm 0.0001 \\
\\
/ \mathrm{M}^{-1}\end{array}$ & $\begin{array}{c}v \\
\pm 10 \\
/ \mathrm{cm}^{-1}\end{array}$ & $\begin{array}{c}(\varepsilon / v) \\
\pm 0.0001 \\
\\
/ \mathrm{M}^{-1}\end{array}$ \\
\hline meso & 4 & $\begin{array}{l}17105 \\
23180 \\
28160\end{array}$ & $\begin{array}{l}0.9392 \\
0.7347 \\
1.0696\end{array}$ & $\begin{array}{l}15025 \\
24030 \\
26920\end{array}$ & $\begin{array}{l}1.8988 \\
0.7681 \\
0.9058\end{array}$ & $\begin{array}{l}12940 \\
25340\end{array}$ & $\begin{array}{l}2.083 \\
0.840\end{array}$ \\
\hline & 5 & $\begin{array}{c}\mathbf{5 4 6 0} \\
16835 \\
23700 \\
27470\end{array}$ & $\begin{array}{l}\mathbf{0 . 3 5 6 1} \\
0.6156 \\
0.3246 \\
0.9525\end{array}$ & $\begin{array}{c}\mathbf{5 2 2 8} \\
14940 \\
26000\end{array}$ & $\begin{array}{l}\mathbf{0 . 3 1 5 0} \\
1.1268 \\
0.7745\end{array}$ & $\begin{array}{c}\mathbf{6 8 4 0} \\
12800 \\
14480 \\
24540\end{array}$ & $\begin{array}{c}\mathbf{0 . 2 4 3 4} \\
0.863 \\
0.860 \\
1.055\end{array}$ \\
\hline rac & 4 & $\begin{array}{l}17100 \\
23140 \\
28220\end{array}$ & $\begin{array}{l}0.7935 \\
0.6548 \\
0.9427\end{array}$ & $\begin{array}{l}15120 \\
23890 \\
26930\end{array}$ & $\begin{array}{l}1.8033 \\
0.7360 \\
0.8898\end{array}$ & $\begin{array}{l}12865 \\
25265\end{array}$ & $\begin{array}{l}2.6607 \\
1.0525\end{array}$ \\
\hline & 5 & $\begin{array}{c}\mathbf{5 4 1 6} \\
16930 \\
23550 \\
27670\end{array}$ & $\begin{array}{l}\mathbf{0 . 4 2 7 8} \\
0.5087 \\
0.2940 \\
0.7634\end{array}$ & $\begin{array}{c}\mathbf{5 2 6 8} \\
14980 \\
26100\end{array}$ & $\begin{array}{l}\mathbf{0 . 2 4 7 2} \\
1.0880 \\
0.7242\end{array}$ & $\begin{array}{c}\mathbf{6 8 5 0} \\
12915 \\
14345 \\
24735\end{array}$ & $\begin{array}{l}\mathbf{0 . 1 4 3 2} \\
0.5919 \\
0.5050 \\
0.7377\end{array}$ \\
\hline
\end{tabular}


(a)

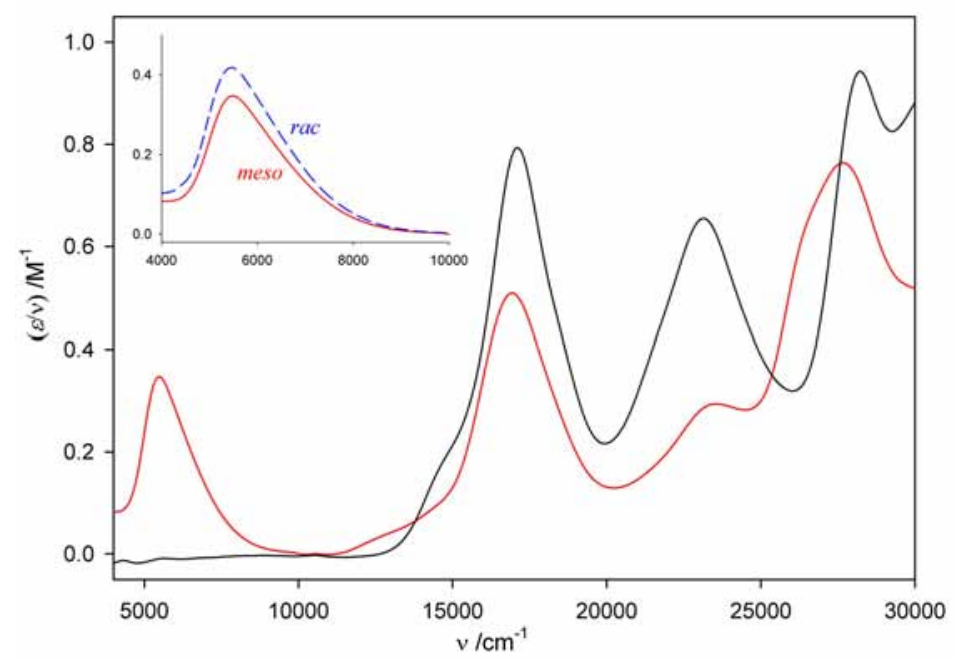

(b)

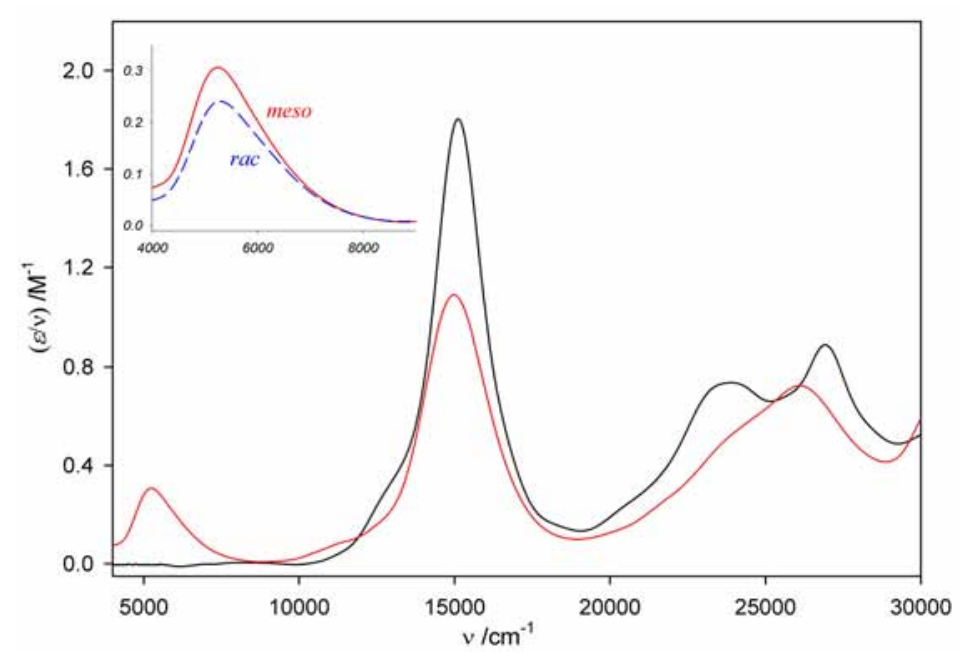

(c)

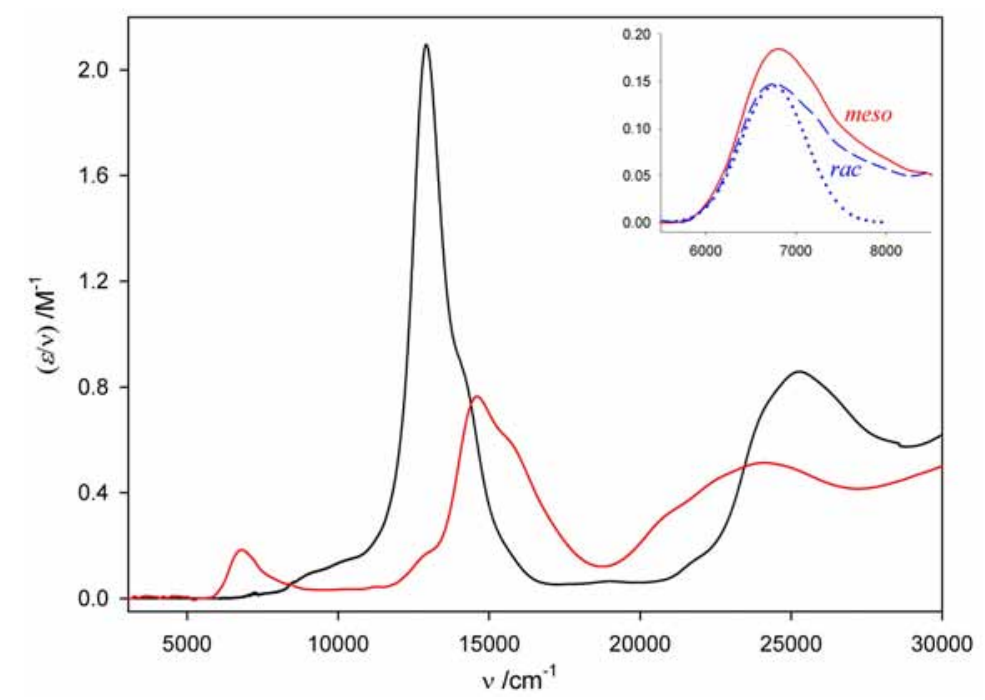

Figure B3.10 UV/Vis/NIR spectra of meso- $\left[\left\{\mathrm{Ru}(\mathrm{bpy})_{2}\right\}_{2}(\mu-\mathrm{BL})\right]^{n+}\{n=4(-), 5(-)\}$ where BL is (a) 2,5-dpp, (b) dpop or (c) apy, in $0.02 \mathrm{M}\left[\left(n-\mathrm{C}_{4} \mathrm{H}_{9}\right)_{4} \mathrm{~N}\right]\left\{\mathrm{B}\left(\mathrm{C}_{6} \mathrm{~F}_{5}\right)_{4}\right\} / n$-butyronitrile at $+25^{\circ} \mathrm{C}\{$ for $\mathrm{BL}=2,5$-dpp, dpop $\}$ and $-35^{\circ} \mathrm{C}$ $\{$ for $\mathrm{BL}=$ apy . Insets show the overlays of the IVCT bands for the meso and rac diastereoisomers of each complex. The Gaussian deconvolution of $\operatorname{rac}-\left[\left\{\mathrm{Ru}(\mathrm{bpy})_{2}\right\}_{2}(\mu \text {-apy) }]^{5+}\right.$ is also shown. 
Table B3.32 IVCT solvatochromism data for the diastereoisomers of $\left[\left\{\mathrm{Ru}(\mathrm{bpy})_{2}\right\}_{2}(\mu-2,5-\mathrm{dpp})\right]^{5+}$ in $0.02 \mathrm{M}$ $\left[\left(n-\mathrm{C}_{4} \mathrm{H}_{9}\right)_{4} \mathrm{~N}\right]\left\{\mathrm{B}\left(\mathrm{C}_{6} \mathrm{~F}_{5}\right)_{4}\right\} /$ solvent at $+25{ }^{\circ} \mathrm{C}$.

\begin{tabular}{|c|c|c|c|c|c|c|c|c|c|c|c|}
\hline & & \multicolumn{9}{|c|}{ meso } & \multicolumn{5}{c|}{ rac } \\
\cline { 3 - 12 } Solvent & $1 / \mathrm{D}_{\mathrm{op}}-1 / \mathrm{D}_{\mathrm{s}}$ & $\begin{array}{c}v_{\max } \\
\pm 10 \\
/ \mathrm{cm}^{-1}\end{array}$ & $\begin{array}{c}(\varepsilon / \mathrm{v})_{\max } \\
\pm 0.0001 \\
/ \mathrm{M}^{-1}\end{array}$ & $\begin{array}{c}\Delta v_{1 / 2} \\
\pm 10 \\
/ \mathrm{cm}^{-1}\end{array}$ & $\begin{array}{c}M_{0} \\
/ \mathrm{M}^{-1}\end{array}$ & $\begin{array}{c}M_{1} \\
/ \mathrm{cm}^{-1}\end{array}$ & $\begin{array}{c}v_{\max } \\
\pm 10 \\
/ \mathrm{cm}^{-1}\end{array}$ & $\begin{array}{c}(\varepsilon / v)_{\max } \\
\pm 0.0001 \\
/ \mathrm{M}^{-1}\end{array}$ & $\begin{array}{c}\Delta v_{1 / 2} \\
\pm 10 \\
/ \mathrm{cm}^{-1}\end{array}$ & $\begin{array}{c}M_{0} \\
/ \mathrm{M}^{-1}\end{array}$ & $\begin{array}{c}M_{1} \\
/ \mathrm{cm}^{-1}\end{array}$ \\
\hline $\mathrm{AN}$ & 0.5127 & 5460 & 0.3561 & 1799 & 829.0 & 5903 & 5416 & 0.4278 & 1861 & 918.6 & 5890 \\
$\mathrm{PN}$ & 0.5011 & 5434 & 0.2118 & 1798 & 572.2 & 5878 & 5400 & 0.2262 & 1864 & 380.2 & 5860 \\
$\mathrm{i} \mathrm{BN}$ & 0.4795 & 5486 & 0.7516 & 1818 & 792.1 & 5931 & 5464 & 0.4579 & 1811 & 835.4 & 5885 \\
$\mathrm{BN}$ & 0.4762 & 5453 & 0.5180 & 1766 & 795.6 & 5901 & 5434 & 0.2600 & 1786 & 666.4 & 5890 \\
$\mathrm{BzN}$ & 0.3897 & 5401 & 0.3406 & 1675 & 429.8 & 5795 & 5250 & 0.1655 & 1842 & 244.7 & 5680 \\
\hline
\end{tabular}

Table B3.33 IVCT solvatochromism data for the diastereoisomers of $\left[\left\{\mathrm{Ru}(\mathrm{bpy})_{2}\right\}_{2}(\mu-\mathrm{dpop})\right]^{5+}$ in $0.02 \mathrm{M}$ $\left[\left(n-\mathrm{C}_{4} \mathrm{H}_{9}\right)_{4} \mathrm{~N}\right]\left\{\mathrm{B}\left(\mathrm{C}_{6} \mathrm{~F}_{5}\right)_{4}\right\} /$ solvent at $+25{ }^{\circ} \mathrm{C}$.

\begin{tabular}{|c|c|c|c|c|c|c|c|c|c|c|c|}
\hline \multirow{2}{*}{ Solvent } & \multirow{2}{*}{} & \multicolumn{9}{|c|}{ meso } & \multicolumn{6}{c|}{ rac } \\
\cline { 3 - 11 } & $1 / \mathrm{D}_{\mathrm{op}}-1 / \mathrm{D}_{\mathrm{s}}$ & $\begin{array}{c}v_{\max } \\
\pm 10 \\
\end{array}$ & $\begin{array}{c}(\varepsilon / \mathrm{v})_{\max } \\
\pm 0.0001 \\
/ \mathrm{cm}^{-1}\end{array}$ & $\begin{array}{c}\Delta v_{1 / 2} \\
\pm 10 \\
/ \mathrm{cm}^{-1}\end{array}$ & $\begin{array}{c}M_{0} \\
/ \mathrm{M}^{-1}\end{array}$ & $\begin{array}{c}M_{1} \\
/ \mathrm{cm}^{-1}\end{array}$ & $\begin{array}{c}v_{\max } \\
\pm 10 \\
/ \mathrm{cm}^{-1}\end{array}$ & $\begin{array}{c}(\varepsilon / \mathrm{v})_{\max } \\
\pm 0.0001 \\
/ \mathrm{M}^{-1}\end{array}$ & $\begin{array}{c}\Delta v_{1 / 2} \\
\pm 10 \\
/ \mathrm{cm}^{-1}\end{array}$ & $\begin{array}{c}M_{0} \\
/ \mathrm{M}^{-1}\end{array}$ & $\begin{array}{c}M_{1} \\
/ \mathrm{cm}^{-1}\end{array}$ \\
\hline $\mathrm{AN}$ & 0.5127 & 5228 & 0.3150 & 1665 & 596 & 5639 & 5268 & 0.2472 & 1754 & 488 & 5717 \\
$\mathrm{PN}$ & 0.5011 & 5213 & 0.2861 & 1607 & 530 & 5612 & 5250 & 0.2037 & 1686 & 390 & 5713 \\
$\mathrm{i} \mathrm{BN}$ & 0.4795 & 5223 & 0.3306 & 1575 & 606 & 5617 & 5223 & 0.1183 & 1659 & 220 & 5738 \\
$\mathrm{BN}$ & 0.4762 & 5206 & 0.2131 & 1591 & 375 & 5608 & 5268 & 0.2296 & 1654 & 425 & 5712 \\
$\mathrm{BzN}$ & 0.3897 & 5144 & 0.2747 & 1502 & 472 & 5559 & 5208 & 0.1520 & 1544 & 274 & 5711 \\
\hline
\end{tabular}

Table B3.34 IVCT solvatochromism data for the diastereoisomers of $\left[\left\{\mathrm{Ru}(\mathrm{bpy})_{2}\right\}_{2}(\mu \text {-apy) }]^{5+}\right.$ in $0.02 \mathrm{M}$ $\left[\left(n-\mathrm{C}_{4} \mathrm{H}_{9}\right)_{4} \mathrm{~N}\right]\left\{\mathrm{B}\left(\mathrm{C}_{6} \mathrm{~F}_{5}\right)_{4}\right\} /$ solvent at $-35^{\circ} \mathrm{C}$.

\begin{tabular}{|c|c|c|c|c|c|c|c|}
\hline \multirow[b]{2}{*}{ Solvent } & \multirow[b]{2}{*}{$1 / \mathrm{D}_{\mathrm{op}}-1 / \mathrm{D}_{\mathrm{s}}$} & \multicolumn{3}{|c|}{ meso } & \multicolumn{3}{|c|}{ rac } \\
\hline & & $\begin{array}{l}v_{\max } \\
\pm 10 \\
/ \mathrm{cm}^{-1}\end{array}$ & $\begin{array}{c}(\varepsilon / v)_{\max } \\
\pm 0.0001 \\
/ \mathrm{M}^{-1}\end{array}$ & $\begin{array}{l}\Delta v_{1 / 2} \\
\pm 10 \\
/ \mathrm{cm}^{-1}\end{array}$ & $\begin{array}{l}v_{\max } \\
\pm 10 \\
/ \mathrm{cm}^{-1}\end{array}$ & $\begin{array}{c}(\varepsilon / v)_{\max } \\
\pm 0.0001 \\
/ \mathrm{M}^{-1}\end{array}$ & $\begin{array}{l}\Delta v_{1 / 2} \\
\pm 10 \\
/ \mathrm{cm}^{-1}\end{array}$ \\
\hline AN & 0.5127 & 6840 & 0.2434 & 832 & 6850 & 0.1432 & 904 \\
\hline PN & 0.5011 & 6790 & 0.1206 & 778 & 6780 & 0.1466 & 810 \\
\hline${ }^{\mathrm{i}} \mathrm{BN}$ & 0.4795 & 6830 & 0.1777 & 823 & 6760 & 0.1797 & 850 \\
\hline BN & 0.4762 & 6800 & 0.1834 & 885 & 6760 & 0.1473 & 847 \\
\hline
\end{tabular}

Table B3.35 Summary of the solvent dependence of $v_{\max }$ for $\left[\left\{\mathrm{Ru}(\mathrm{bpy})_{2}\right\}_{2}(\mu-\mathrm{BL})\right]^{5+}\{\mathrm{BL}=2,5-\mathrm{dpp}$, dpop, apy $\}$ according to the dielectric continuum model.

\begin{tabular}{|l|c|c|c|}
\hline \multicolumn{1}{|c|}{ Complex } & $\begin{array}{c}\text { Slope } \\
e^{2}(1 / a-1 / d) \\
/ \mathrm{cm}^{-1}\end{array}$ & $\begin{array}{c}\text { Intercept } \\
\lambda_{i}+\Delta \mathrm{E}^{\prime}+\Delta \mathrm{E}_{0} \\
/ \mathrm{cm}^{-1}\end{array}$ & $R^{2}$ \\
\hline meso- $\left[\left\{\mathrm{Ru}(\mathrm{bpy})_{2}\right\}_{2}(\mu-2,5-\mathrm{dpp})\right]^{5+}$ & $454.7 \pm 270$ & $5232 \pm 130$ & 0.482 \\
rac- $\left[\left\{\mathrm{Ru}(\mathrm{bpy})_{2}\right\}_{2}(\mu-2,5-\mathrm{dpp})\right]^{5+}$ & $1455 \pm 530$ & $4706 \pm 250$ & 0.714 \\
meso- $\left[\left\{\mathrm{Ru}(\mathrm{bpy})_{2}\right\}_{2}(\mu \text {-dpop) }]^{5+}\right.$ & $676.4 \pm 109$ & $4884 \pm 5$ & 0.927 \\
rac- $\left[\left\{\mathrm{Ru}(\mathrm{bpy})_{2}\right\}_{2}(\mu \text {-dpop) }]^{5+}\right.$ & $439.9 \pm 200$ & $5039 \pm 10$ & 0.603 \\
meso- $\left[\left\{\mathrm{Ru}(\mathrm{bpy})_{2}\right\}_{2}(\mu \text {-apy) }]^{5+}\right.$ & $368.9 \pm 925$ & $6633 \pm 455$ & 0.074 \\
rac- $\left[\left\{\mathrm{Ru}(\mathrm{bpy})_{2}\right\}_{2}(\mu \text {-apy })\right]^{5+}$ & $2185 \pm 770$ & $5712 \pm 380$ & 0.800 \\
\hline
\end{tabular}




\section{APPENDIX C}

Table C4.1 Reduction potentials based on the HAT bridging ligand for the di- and trinuclear complexes in $0.1 \mathrm{M}\left[\left(n-\mathrm{C}_{4} \mathrm{H}_{9}\right)_{4} \mathrm{~N} \mathrm{PF}_{6} / \mathrm{CH}_{3} \mathrm{CN}\right.$ at $+25^{\circ} \mathrm{C}$. ${ }^{\mathrm{a}}$

\begin{tabular}{|c|c|c|c|}
\hline Complex & $\mathrm{E}_{\text {red1 }}$ & $\mathrm{E}_{\mathrm{red} 2}$ & $E_{\text {red3 }}$ \\
\hline$\overline{\text { meso- }\left[\left\{\mathrm{Ru}(\mathrm{bpy})_{2}\right\}_{2}(\mu-\mathrm{HAT})\right]^{4+}}$ & -808 & -1304 & \\
\hline rac- $\left[\left\{\mathrm{Ru}(\mathrm{bpy})_{2}\right\}_{2}(\mu-\mathrm{HAT})\right]^{4+}$ & -798 & -1324 & \\
\hline homochiral-[\{Ru(bpy $\left.\left.)_{2}\right\}_{3}(\mu-\mathrm{HAT})\right]^{6+}$ & -584 & -930 & -1390 \\
\hline heterochiral-[\{Ru(bpy $\left.\left.)_{2}\right\}_{3}(\mu-\mathrm{HAT})\right]^{6+}$ & -600 & -940 & -1420 \\
\hline
\end{tabular}

${ }^{a}$ All potentials are quoted $\pm 3 \mathrm{mV} v$ s. $\mathrm{Fc}^{+} / \mathrm{Fc}^{0}$.

Table C4.2 Crystal data and structure refinement parameters for $\Lambda \Delta$-[\{Ru(bpy $\left.\left.)_{2}\right\}(\mathrm{HAT})\left\{\mathrm{Os}(\mathrm{bpy})_{2}\right\}\right]\left(\mathrm{PF}_{6}\right)_{3} \mathrm{Cl}$.

\begin{tabular}{|c|c|}
\hline Parameter & \\
\hline Formula & $\mathrm{C}_{52} \mathrm{H}_{38} \mathrm{~N}_{12} \mathrm{Cl}_{8} \mathrm{Zn}_{2} \mathrm{OsRu}$ \\
\hline Formula weight & 1625.8 \\
\hline $\mathrm{T}(\mathrm{K})$ & 293 \\
\hline Crystal System & monoclinic \\
\hline Space Group & $\mathrm{P} 2{ }_{1} / n$ \\
\hline$a(\AA)$ & $10.061(5)$ \\
\hline$b(\AA)$ & $17.519(5)$ \\
\hline$c(\AA)$ & $37.869(5)$ \\
\hline$\alpha\left({ }^{\circ}\right)$ & $90.0005(5)$ \\
\hline$\beta\left({ }^{\circ}\right)$ & $90.270(5)$ \\
\hline$\gamma\left({ }^{\circ}\right)$ & $90.0005(5)$ \\
\hline$V\left(\AA^{3}\right)$ & $6675(4)$ \\
\hline$Z$ & 4 \\
\hline$D_{\mathrm{c}}\left(\mathrm{g} \mathrm{cm}^{-3}\right)$ & 1.62 \\
\hline$\mu\left(\mathrm{Mo}_{-} \mathrm{K}_{\alpha}\right)\left(\mathrm{mm}^{-1}\right)$ & 4.866 \\
\hline$F(000)$ & 3127.1 \\
\hline Crystal form & green/purple dichroic crystal \\
\hline Crystal size (mm) & $0.5 \times 0.1 \times 0.1$ \\
\hline $2 \theta_{\max }\left({ }^{\circ}\right)$ & 26.5 \\
\hline Limiting indices & $-12 \leq h \leq 12,0 \leq k \leq 21,0 \leq l \leq 47$ \\
\hline Reflections collected & 13798 \\
\hline Unique reflections & 3041 \\
\hline Parameters & 812 \\
\hline Refinement method & Full-matrix least-squares on $F^{2}$ \\
\hline Absorption correction & none \\
\hline GooF & 0.886 \\
\hline$R[I>2 \sigma(I)]$ & 0.125 \\
\hline$w R$ (all data) & 0.437 \\
\hline Diffractometer & Bruker AXS CCD $(\lambda=0.71069 \AA)$ \\
\hline
\end{tabular}


Table C4.3 Reduction potentials based on the HAT bridging ligand for the di- and trinuclear complexes in $0.1 \mathrm{M}\left[\left(n-\mathrm{C}_{4} \mathrm{H}_{9}\right)_{4} \mathrm{~N}\right] \mathrm{PF}_{6} / \mathrm{CH}_{3} \mathrm{CN}$ at $+25^{\circ} \mathrm{C}$.

\begin{tabular}{|l|c|c|c|}
\hline \multicolumn{1}{|c|}{ Complex } & $E_{\text {red1 }}$ & $E_{\text {red2 }}$ & $E_{\text {red3 }}$ \\
\hline$\left[\text { Os }(\text { bpy })_{2}(\mathrm{HAT})\right]^{2+}$ & -1101 & -1276 & \\
meso- $\left[\left\{\mathrm{Os}(\mathrm{bpy})_{2}\right\}_{2}(\mu-\mathrm{HAT})\right]^{4+}$ & -844 & -1320 & \\
rac- $\left[\left\{\mathrm{Os}(\mathrm{bpy})_{2}\right\}_{2}(\mu-\mathrm{HAT})\right]^{4+}$ & -840 & -1325 & \\
$\Delta \Lambda / \Lambda \Delta-\left[\left\{\mathrm{Ru}(\mathrm{bpy})_{2}\right\}(\mu-\mathrm{HAT})\left\{\mathrm{Os}(\mathrm{bpy})_{2}\right\}\right]^{4+}$ & -804 & -1316 & \\
$\Delta \Lambda \Lambda \Lambda-\left[\left\{\mathrm{Ru}(\mathrm{bpy})_{2}\right\}(\mu-\mathrm{HAT})\left\{\mathrm{Os}(\mathrm{bpy})_{2}\right\}\right]^{4+}$ & -805 & -1320 & \\
$\Delta_{2} \Lambda^{\prime} / \Lambda_{2} \Delta^{\prime}-\left[\left\{\mathrm{Ru}(\mathrm{bpy})_{2}\right\}_{2}\left\{\mathrm{Os}(\mathrm{bpy})_{2}\right\}(\mu-\mathrm{HAT})\right]^{6+}$ & -624 & -956 & -1384 \\
\hline
\end{tabular}

All potentials are quoted $\pm 3 \mathrm{mV} v \mathrm{vs}$. $\mathrm{Fc}^{+} / \mathrm{Fc}^{0}$.

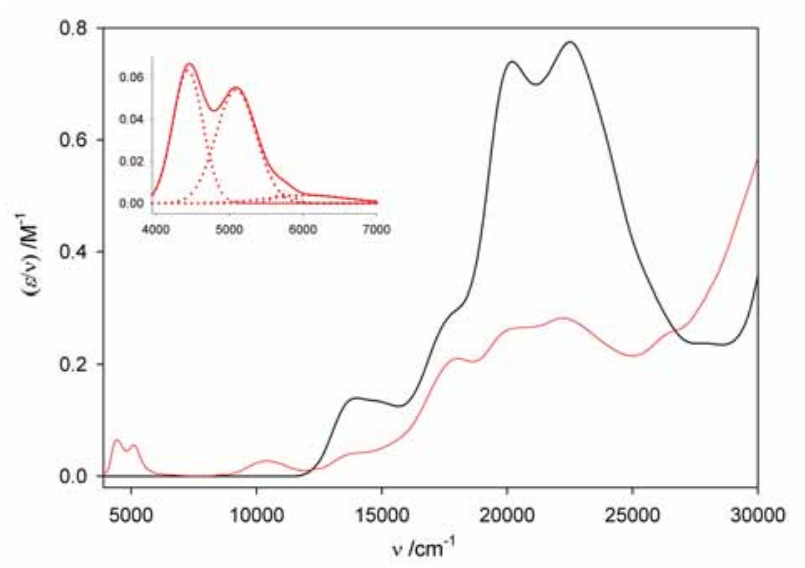

Figure C4.1 UV/Vis/NIR spectra of $\left[\mathrm{Os}(\mathrm{bpy})_{2}(\mathrm{HAT})\right]^{n+}\{n=2(-), 3(-)\}$ at $-35^{\circ} \mathrm{C}$. The inset shows the interconfigurational (IC) bands for the $n=3$ species and the components obtained from Gaussian deconvolution of the bands (…). 
Table C4.4 Deconvoluted NIR spectral data of the reduced absorption spectra ( $\varepsilon / v v s . v)$ for the di- and trinuclear complexes at -35 and $-15^{\circ} \mathrm{C}$, respectively. ${ }^{\mathrm{a}}$

\begin{tabular}{|c|c|c|c|}
\hline Complex & $\begin{array}{l}v_{\max } \\
\pm 10 \\
/ \mathrm{cm}^{-1} \\
\end{array}$ & $\begin{array}{c}(\varepsilon / v)_{\max } \\
\pm 0.0001 \\
/ \mathrm{M}^{-1}\end{array}$ & $\begin{array}{l}\Delta v_{1 / 2} \\
\pm 10 \\
/ \mathrm{cm}^{-1}\end{array}$ \\
\hline \multirow{9}{*}{ meso- $\left[\left\{\mathrm{Os}(\mathrm{bpy})_{2}\right\}_{2}(\mu-\mathrm{HAT})\right]^{5+}$} & 4980 & 0.0661 & 444 \\
\hline & 5420 & 0.0200 & 422 \\
\hline & 5770 & 0.0229 & 1150 \\
\hline & 8200 & 0.2782 & 663 \\
\hline & 8780 & 0.0774 & 540 \\
\hline & 9325 & 0.0441 & 695 \\
\hline & 11110 & 0.3878 & 1445 \\
\hline & 12470 & 0.1234 & 1482 \\
\hline & 14285 & 0.1241 & 1398 \\
\hline \multirow[t]{6}{*}{ rac- $\left[\left\{\mathrm{Os}(\mathrm{bpy})_{2}\right\}_{2}(\mu-\mathrm{HAT})\right]^{5+}$} & 5090 & 0.0575 & 932 \\
\hline & 5935 & 0.0230 & 974 \\
\hline & 8260 & 0.2726 & 920 \\
\hline & 9130 & 0.0678 & 848 \\
\hline & 11130 & 0.4123 & 1654 \\
\hline & 12715 & 0.1266 & 2030 \\
\hline \multirow[t]{5}{*}{$\Delta \Lambda / \Lambda \Delta-\left[\left\{\mathrm{Ru}(\mathrm{bpy})_{2}\right\}(\mu-\mathrm{HAT})\left\{\mathrm{Os}(\mathrm{bpy})_{2}\right\}\right]^{5+}$} & 4080 & 0.4747 & 578 \\
\hline & 4880 & 0.1107 & 596 \\
\hline & 8610 & 0.1735 & 2055 \\
\hline & 10405 & 0.0219 & 2160 \\
\hline & 13950 & 0.1145 & 1970 \\
\hline \multirow[t]{4}{*}{$\Delta \Delta / \Lambda \Lambda-\left[\left\{\mathrm{Ru}(\mathrm{bpy})_{2}\right\}(\mu-\mathrm{HAT})\left\{\mathrm{Os}(\mathrm{bpy})_{2}\right\}\right]^{5+}$} & 4060 & 0.5990 & 577 \\
\hline & 4880 & 0.1388 & 591 \\
\hline & 8695 & 0.2125 & 2060 \\
\hline & 14060 & 0.1906 & 2857 \\
\hline \multirow[t]{6}{*}{$\Delta_{2} \Lambda^{\prime} / \Lambda_{2} \Delta^{\prime}-\left[\left\{\mathrm{Ru}(\mathrm{bpy})_{2}\right\}_{2}\left\{\mathrm{Os}(\mathrm{bpy})_{2}\right\}(\mu-\mathrm{HAT})\right]^{7+}$} & 3975 & 0.5008 & 844 \\
\hline & 4620 & 0.1754 & 632.8 \\
\hline & 7670 & 0.2193 & 2597 \\
\hline & 9480 & 0.0595 & 1695 \\
\hline & 13080 & 0.0707 & 1345 \\
\hline & 14650 & 0.2518 & 2197 \\
\hline \multirow[t]{8}{*}{$\Delta_{2} \Lambda^{\prime} / \Lambda_{2} \Delta^{\prime}-\left[\left\{\mathrm{Ru}(\mathrm{bpy})_{2}\right\}_{2}\left\{\mathrm{Os}(\mathrm{bpy})_{2}\right\}(\mu-\mathrm{HAT})\right]^{8+}$} & 3530 & 1.0179 & 414 \\
\hline & 4040 & 0.2080 & 412 \\
\hline & 4470 & 0.3064 & 881 \\
\hline & 5530 & 0.1096 & 1088 \\
\hline & 7765 & 0.1750 & 2854 \\
\hline & 10550 & 0.4488 & 2235 \\
\hline & 13110 & 0.5473 & 2562 \\
\hline & 14890 & 0.2410 & 1854 \\
\hline
\end{tabular}

${ }^{a}$ Due to the convoluted nature of the NIR spectra, unambiguous determination of the band characteristics for the IC transitions in the +9 state of the trinuclear species was not possible. 
Table C4.5 Deconvoluted NIR spectral data of the fully-oxidised forms of the mono- and dinuclear complexes at $-35^{\circ} \mathrm{C}$. The parameters for the IC bands are indicated in bold type. ${ }^{a}$

\begin{tabular}{|c|c|c|c|}
\hline Complex & $\begin{array}{l}v_{\max } \\
\pm 10 \\
/ \mathrm{cm}^{-1} \\
\end{array}$ & $\begin{array}{c}(\varepsilon / v)_{\max } \\
\pm 0.0001 \\
/ \mathrm{M}^{-1}\end{array}$ & $\begin{array}{l}\Delta v_{1 / 2} \\
\pm 10 \\
/ \mathrm{cm}^{-1}\end{array}$ \\
\hline$\left[\mathrm{Os}(\mathrm{bpy})_{2}(\mathrm{HAT})\right]^{3+}$ & $\begin{array}{c}\mathbf{4 4 3 5} \\
\mathbf{5 0 9 0} \\
5735 \\
6060 \\
10460 \\
12120\end{array}$ & $\begin{array}{c}0.06315 \\
0.05405 \\
0.004250 \\
0.003798 \\
0.02746 \\
0.004165\end{array}$ & $\begin{array}{c}485 \\
645 \\
330 \\
1280 \\
1820 \\
908\end{array}$ \\
\hline meso- $\left[\left\{\mathrm{Os}(\mathrm{bpy})_{2}\right\}_{2}(\mu-\mathrm{HAT})\right]^{6+}$ & $\begin{array}{l}\mathbf{4 3 2 0} \\
\mathbf{5 0 6 0} \\
5790 \\
6645\end{array}$ & $\begin{array}{c}0.2144 \\
0.1500 \\
0.02133 \\
0.03988\end{array}$ & $\begin{array}{c}514 \\
746 \\
560 \\
1210\end{array}$ \\
\hline rac- $\left[\left\{\mathrm{Os}(\mathrm{bpy})_{2}\right\}_{2}(\mu-\mathrm{HAT})\right]^{6+}$ & $\begin{array}{l}\mathbf{4 2 8 0} \\
4600 \\
\mathbf{5 0 3 0} \\
5607 \\
6695\end{array}$ & $\begin{array}{c}0.2788 \\
0.06351 \\
0.1457 \\
0.01473 \\
0.03058\end{array}$ & $\begin{array}{l}396 \\
302 \\
600 \\
503 \\
990\end{array}$ \\
\hline$\Delta \Lambda / \Lambda \Delta-\left[\left\{\mathrm{Ru}(\mathrm{bpy})_{2}\right\}(\mu-\mathrm{HAT})\left\{\mathrm{Os}(\mathrm{bpy})_{2}\right\}\right]^{6+}$ & $\begin{array}{l}\mathbf{4 4 3 0} \\
5035 \\
5335 \\
6075 \\
9090\end{array}$ & $\begin{array}{c}0.2642 \\
0.04626 \\
0.06867 \\
0.01228 \\
0.04851\end{array}$ & $\begin{array}{c}697 \\
405 \\
600 \\
1117 \\
1736\end{array}$ \\
\hline$\Delta \Delta / \Lambda \Lambda$-[\{Ru(bpy $\left.\left.)_{2}\right\}(\mu-\mathrm{HAT})\left\{\mathrm{Os}(\mathrm{bpy})_{2}\right\}\right]^{6+}$ & $\begin{array}{c}3920 \\
4720 \\
8300 \\
10778\end{array}$ & $\begin{array}{l}0.3529 \\
0.1477 \\
0.1677 \\
0.2550\end{array}$ & $\begin{array}{c}578 \\
942 \\
2100 \\
2226\end{array}$ \\
\hline
\end{tabular}

${ }^{a}$ Due to the convoluted nature of the NIR spectra, unambiguous determination of the band characteristics for the IC transitions in the +9 state of the trinuclear species was not possible.

Table C4.6 Redox potentials for the mono-, di- and trinuclear complexes in $0.1 \mathrm{M}\left[\left(n-\mathrm{C}_{4} \mathrm{H}_{9}\right)_{4} \mathrm{~N}\right] \mathrm{PF}_{6} / \mathrm{CH}_{3} \mathrm{CN}$ at $+25^{\circ} \mathrm{C}^{\text {a }}$ The reduction potentials for $\left[\mathrm{Ru}(\mathrm{bpy})_{3}\right]^{2+}$ are included for comparison.

\begin{tabular}{|c|c|c|c|c|}
\hline Complex & $E_{\text {red1 }}$ & $\mathrm{E}_{\mathrm{red} 2}$ & $\mathrm{E}_{\mathrm{red} 3}$ & $\mathrm{E}_{\mathrm{red} 4}$ \\
\hline$\left[\mathrm{Ru}(\mathrm{bpy})_{3}\right]^{2+b}$ & -1717 & -1912 & -2160 & -2700 \\
\hline trans- $\left[\mathrm{Ru}(\mathrm{bpy})(\mathrm{ppz})_{2}\right]^{2+}$ & -1000 & -1246 & -1700 & $-1968^{c}$ \\
\hline meso- $\left[\left\{\mathrm{Ru}(\mathrm{bpy})_{2}\right\}_{2}(\mu-\mathrm{ppz})\right]^{4+}$ & -956 & -1704 & -1890 & -1956 \\
\hline$r a c-\left[\left\{\mathrm{Ru}(\mathrm{bpy})_{2}\right\}_{2}(\mu-\mathrm{ppz})\right]^{4+}$ & -944 & -1696 & -1878 & -1975 \\
\hline$\Delta \Delta^{\mathrm{t}} \Delta-\left[\left\{\mathrm{Ru}(\mathrm{bpy})_{2}\right\}_{2}\left\{\mathrm{Ru}(\mathrm{bpy})(\mu-\mathrm{ppz})_{2}\right\}\right]^{6+}$ & -868 & -1036 & $-1800^{\mathrm{C}}$ & $-2072^{\mathrm{C}}$ \\
\hline
\end{tabular}

${ }^{a}$ All potentials are quoted $\pm 3 \mathrm{mV} v \mathrm{ss} . \mathrm{Fc}^{+} / \mathrm{Fc}^{0}$.

${ }^{\mathrm{b}} \mathrm{The} \mathrm{Ru}^{\mathrm{II} / \mathrm{III}}$ oxidation occurs at $880 \mathrm{mV}$ on the same scale.

${ }^{\mathrm{c}}$ Two-electron reduction process 